Westinghouse Proprietary Class 2

NUCLEAR ENERGY RESEARCH INITIATIVE (NERI)

ANNUAL PROGRESS REPORT

\title{
Testing of Passive Safety System Performance for Higher Power Advanced Reactors
}

DE-FG0701SF22326

Project No. 01-094

Final Report

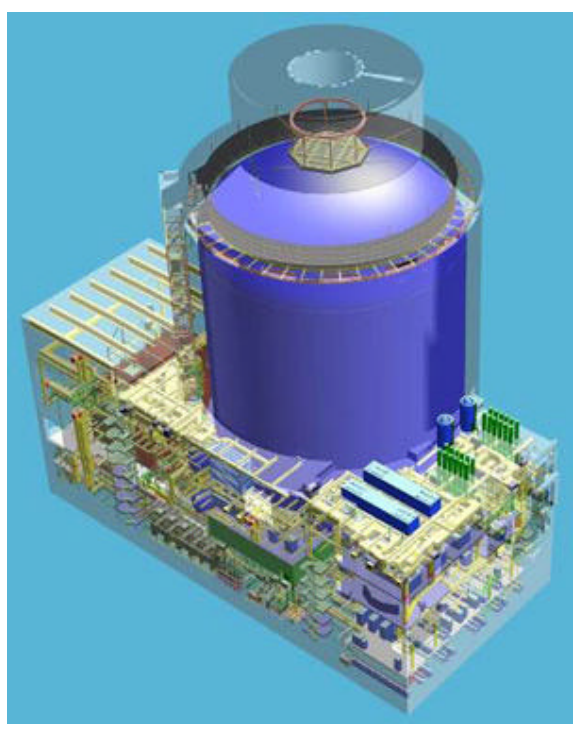

Submitted by:

Brian G. Woods, Co-Principal Investigator

Oregon State University, Department of Nuclear Engineering

Oregon State University, 128 Radiation Center

Corvallis, OR 97331-5902

(541) 737-6335

(541) 737-4678 (FAX)

woodsb@ne.orst.edu

Collaborating Organization:

Richard Wright

Westinghouse Electric, LLC, Pittsburgh, PA

Authors:

Jose Reyes, Jr., Oregon State University

Brian Woods, Oregon State University

John Groome, Oregon State University

Richard Wright, Westinghouse Electric Corporation

December 31, 2004 


\section{Table of Contents}

Executive Summary..................................................................... 1

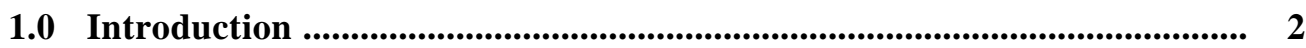

2.0 Research Objectives ….................................................................................... 5

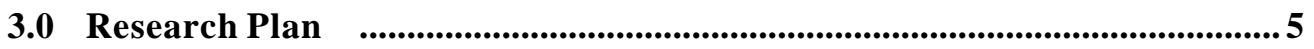

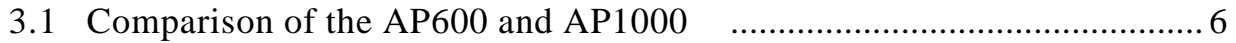

3.2 Modifications to the APEX Test Facility ……..................................... 9

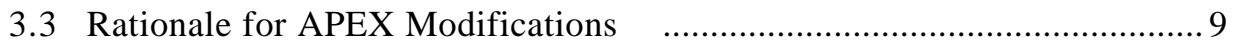

4.0 APEX Scaling Assessment Summary …...................................................11

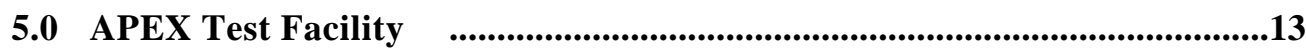

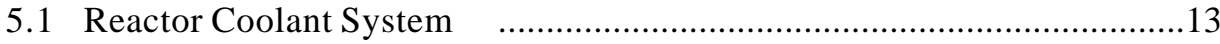

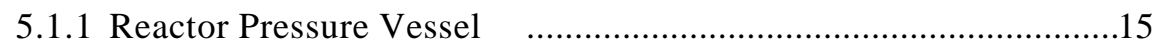

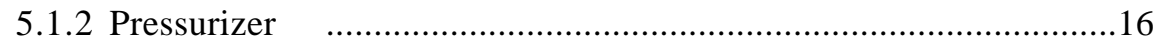

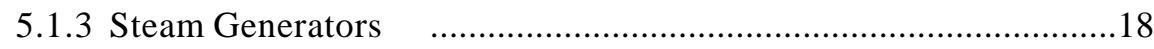

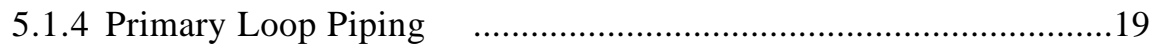

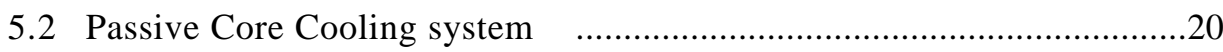

5.2.1 Automatic Depressurization System ……..............................20

5.2.2 In-containment Refueling Water Storage Tank …......................22

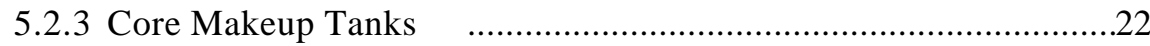

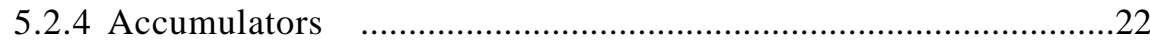

5.2.5 Passive Residual Heat Removal Heat Exchanger ......................22

5.3 Break and ADS Measurement System …..........................................23

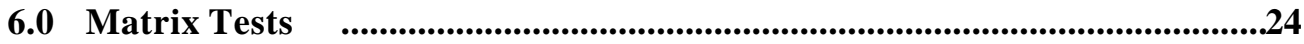

7.0 APEX 1000/600 Test Results Comparisons ................................................29

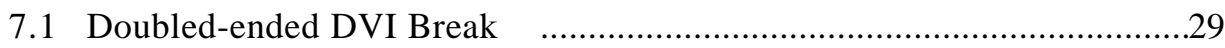

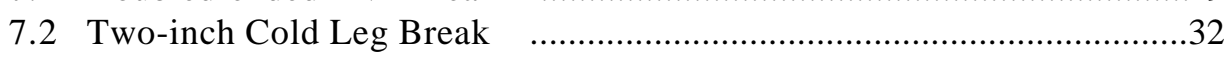

8.0 APEX1000 Test Comparison to Code Calculations …..............................34

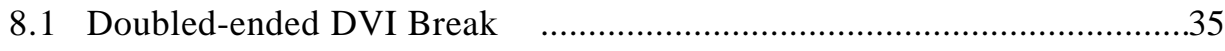

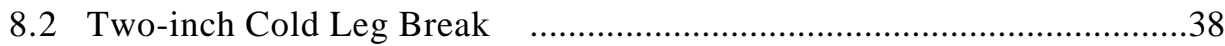




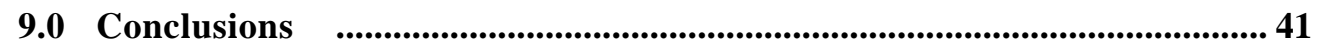

10.0 Budget and Milestones .............................................................................. 41

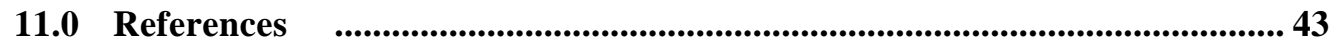

Appendix A -- OSU-AP1000-01

Appendix B -- OSU-AP1000-02

Appenidx C -- OSU-AP1000-03

Appendix D -- OSU-AP1000-04

Appendix E -- OSU-AP1000-05

Appendix F -- OSU-AP1000-06

Appendix G -- OSU-AP1000-07

Appendix H -- OSU-AP1000-08

Appendix I -- OSU-AP1000-09

Appendix J -- OSU-AP1000-10

Appendix K -- OSU-AP1000-11 


\section{List of Figures}

Section 1 Figure

FIGURE 1.1 AP1000 Passive Containment Cooling System

\section{Section 3 Figure}

FIGURE 3.1 Flow Chart for the OSU AP1000 Research Program 6

Section 5 Figures

FIGURE 5.1 APEX Test Facility 13

FIGURE 5.2 Elevation view of the Reactor Coolant System $\quad$...............................14

FIGURE 5.3 Plan view of the Reactor Coolant System …..................................15

FIGURE 5.4 Reactor Pressure Vessel Cross-sectional View …...........................16

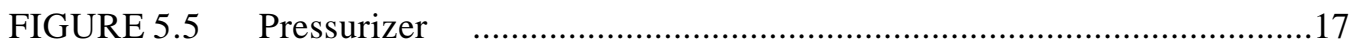

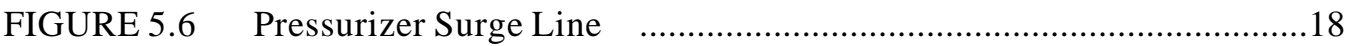

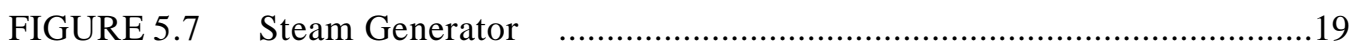

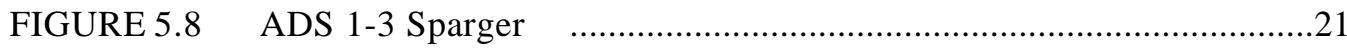

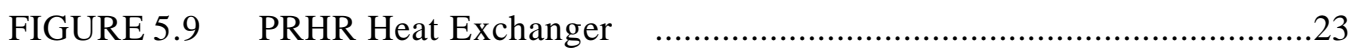

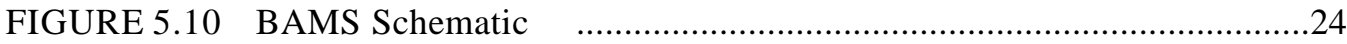

\section{Section 7 Figures}

FIGURE 7.1 APEX-1000/600 DVI Break Comparison - RCS Pressure …............30

FIGURE 7.2 APEX1000/600 DVI Break Comparison - Core Makeup Flow …...31

FIGURE 7.3 APEX1000/600 DVI Break Comparison - Core Collapsed Liquid Level

FIGURE 7.4 APEX1000/600 2-inch Cold Leg Break Comparison - RCS

Pressure

FIGURE 7.5 APEX1000/600 2-inch Cold Leg Break Comparison - Core

Makeup Flow

FIGURE 7.6 APEX1000/600 DVI Break Comparison2-inch Cold Leg Break

Comparison - Core Collapsed Liquid Level 
Page

\section{Section 8 Figures}

FIGURE 8.1 APEX1000/NOTRUMP DVI Break Comparison-RCS Pressure ...36

FIGURE 8.2 APEX1000/NOTRUMP DVI Break Comparison - Broken DVI

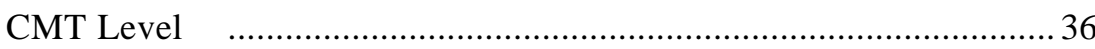

FIGURE 8.3 APEX1000/NOTRUMP DVI Break Comparison - Intact DVI

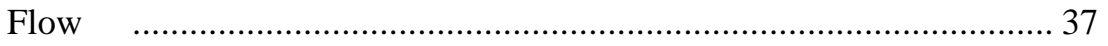

FIGURE 8.4 APEX1000/NOTRUMP DVI Break Comparison - RPV

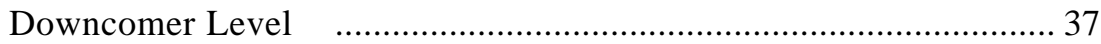

FIGURE 8.5 APEX1000/NOTRUMP 2-inch Break Comparison - RCS

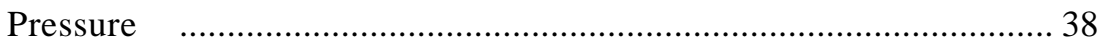

FIGURE 8.6 APEX1000/NOTRUMP 2-inch Break Comparison-CMT Level ...39

FIGURE 8.7 APEX1000/NOTRUMP 2-inch Break Comparison - RPV

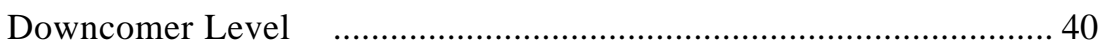

FIGURE 8.8 APEX1000/NOTRUMP 2-inch Break Comparison-CMT Level ..40

\section{Section 10 Figure}

FIGURE 10.1 Comparison of Actual Cumulative Expenses to the Cumulative

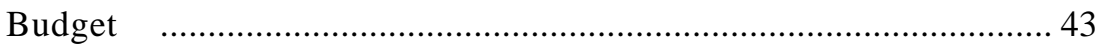




\section{List of Tables}

Page

\section{Section 3 Tables}

TABLE 3.1. Comparison of NSSS Design Parameters …......................................

TABLE 3.2. Comparison of Passive Safety System Design Features ........................ 8

TABLE 3.3. Proposed Modifications to the APEX Test Facility ……….................. 9

\section{Section 4 Table}

TABLE 4.1. Key Scaling Ratios for AP600/AP1000

\section{Section 6 Table}

TABLE 6.1. Summary of AP1000 Matrix Tests Performed

\section{Section 10 Tables}

TABLE 10.1. Student Participation in NERI Research During FY2003 …...............41

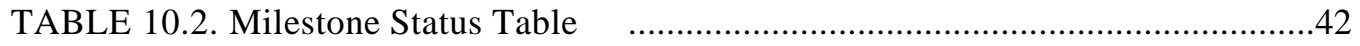

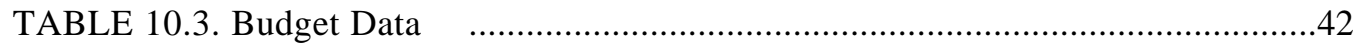




\section{Executive Summary}

This report describes the results of NERI research on the testing of advanced passive safety performance for the Westinghouse AP1000 design. The objectives of this research were: a) to assess the AP1000 passive safety system core cooling performance under high decay power conditions for a spectrum of breaks located at a variety of locations, b) to compare advanced thermal hydraulic computer code predictions to the APEX high decay power test data and c) to develop new passive safety system concepts that could be used for Generation IV higher power reactors.

During FY01, a roadmap for the AP1000 testing program was established and several meetings with Westinghouse Electric Corporation were conducted to gain insight in to the AP1000 plant design. The primary focus of FY02 was the assessment of the geometric, kinematic and dynamic similarity between the APEX test facility components and those of the AP1000 reactor design concept. The scaling analysis identified the facility modifications that were needed to adequately simulate the AP1000 passive safety systems under LOCA conditions.

The primary effort for FY03 was the design and completion of the test facility modifications in preparation of testing and the initiation of the APEX-1000 test program.

Upon completion of these facility modifications, a DOE sponsored readiness review was performed by staff from DOE, MPR Associates, Westinghouse and the NRC in the first quarter of FY03. The purpose of the readiness assessment was to verify that the test facility appropriately represents the AP1000 passive safety systems and that the testing is adequate to support DOE and NRC testing objectives for the AP1000. All action items from the readiness review report were implemented to assure testing initiation in FY03. A revised test plan was developed subsequent to the readiness review meeting. 
During FY03, 6 of the 11 planned tests were completed. Analysis of the test data began in FY03 and continued through the end of the project. During FY04, the remaining tests were completed. Selected APEX-1000 test results were compared to the APEX-600 test results to gain an understanding of the effects of high power on passive safety system performance.

This final report documents the performance of the subject research program. It includes a description of the test results, key comparisons to APEX-600 data and code comparisons to APEX-1000 data.

\subsection{Introduction}

Westinghouse Electric Corporation has designed and certified an advanced 600 MWe light water pressurized reactor plant called the AP600. The AP600 employs passive safety systems that rely on gravity, compressed gas, natural circulation, and evaporation to provide for long term cooling in the event of an accident.

A few simple valves automatically align the passive safety systems when they are needed without the use of AC power. The AP1000 is a two-loop, $1000 \mathrm{MWe}$ plant that keeps the same basic design as AP600. The core diameter, reactor vessel diameter, containment diameter and nuclear island footprint are all the same as for AP600. The main difference between the AP600 and AP1000 containment building is the building height. The AP1000 containment building was increased to add free volume and to accommodate larger components. Those component changes that impact the plant the most are the increase in size of the steam generator, from a delta-75 to a delta-125, and a larger capacity reactor coolant pump. An example of one of the added benefits of larger reactor coolant pumps is its higher inertia compared to that for AP600. This higher inertia allows increased margin for departure from nucleate boiling (DNB) during lossof-flow events by providing increased coastdown flow to the core after pump 
trip. Unique to the AP1000 is the Passive Core Cooling System (PXS) shown in Figure 1.1.

The PXS utilizes three sources of makeup water to maintain core cooling. These sources include the Core Makeup Tanks (CMT), pressurized Accumulators (ACC), and an In-containment Refueling Water Storage Tank (IRWST). Makeup water to the core is accomplish by connecting the injection nozzles directly to the Reactor Pressure Vessel (RPV). The CMTs are elevated above the core and a pressure balance line is provided to maintain the CMTs at RCS pressure. Upon receiving a safety signal, the CMT outlet valves are opened, allowing the gravity driven injection of borated makeup water to the core.

FIGURE 1.1

\section{AP1000 Passive Containment Cooling System}

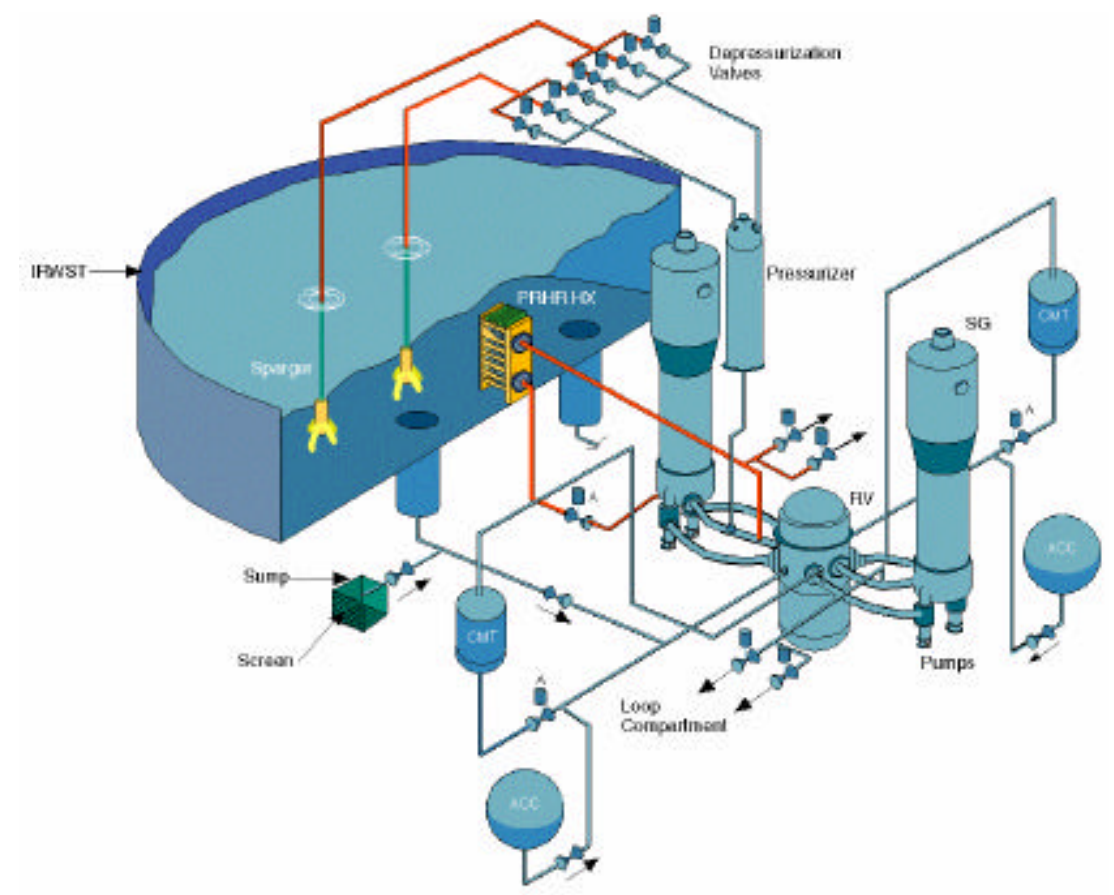

To allow the pressure/gravity injection of makeup water to the core, the AP1000 utilizes an Automatic Depressurization System (ADS) to depressurize the Reactor Coolant System (RCS). The ADS consists of a set of valves that are con- 
nected to the Pressurizer steam space and the two hot legs. The ADS valves are opened sequentially based on CMT level to provide a controlled depressurization of the primary system. The first three stages of the ADS discharge to the IRWST while the fourth stage discharges directly to the containment atmosphere.

For large Loss-of-Coolant Accidents (LOCA), accumulators are utilized to provide the higher injection flowrates necessary to prevent the core from uncovering. The gas pressure in the accumulator forces open the discharge check valves to rapid respond to a severe blowdown of the RCS.

When the RCS has depressurized to near atmospheric pressure, the head of the IRWST is sufficient to overcome the reduced RCS pressure. The IRWST provides sufficient makeup to flood the recirculation sump to provide for long term cooling.

A recirculation sump is provided to collect water discharged from the primary system and steam that condenses inside the containment. After the IRWST has injected most of its water to the RCS, and the gravity head becomes great enough, the water in the sump is allowed to recirculate to the primary system, providing long-term cooling of the core.

A Passive Residual Heat Removal heat exchanger (PRHR) is also provided to removed decay heat. The PRHR is composed of a C-shape tube bundle submerged inside the IRWST and can remove heat from the primary system at any pressure.

\subsection{Research Objectives}

The objectives of this project are:

- To assess the AP1000 passive safety system core cooling performance under high decay power conditions in the APEX test facility. 
- To compare advanced thermal hydraulic computer code predictions to the APEX high decay power test data.

The Oregon State University APEX integral test facility, simulating the AP600 design, was adapted to model the AP1000 and a series of tests were conducted to demonstrate system behavior under accident conditions. The test data was used to provide benchmarks for computer codes used in licensing of the AP1000. All test data produced meet the requirements of 10CFR50 Appendix B and NQA-2.

\subsection{Research Plan}

Figure 3.1 is a flow chart describing the research conducted. The first activity was devoted to assessing the geometric, kinematic, and dynamic similarity between the APEX test facility components and those of the AP1000 reactor design concept. The scaling analysis identified which modifications were needed to adequately simulate the AP1000 PXS under LOCA conditions. Having completed the scaling analysis, the key modifications were made to the APEX test facility and a set of "as-built" drawings were developed. The facility modification details were incorporated into the APEX RELAP5 input deck for purposes of thermal hydraulic computer code comparisons to the APEX-1000 test data. A variety of pre-test and post-test calculations was performed under separate contract to the NRC. 


\section{FIGURE 3.1}

Flow Chart for the OSU AP1000 Research Program

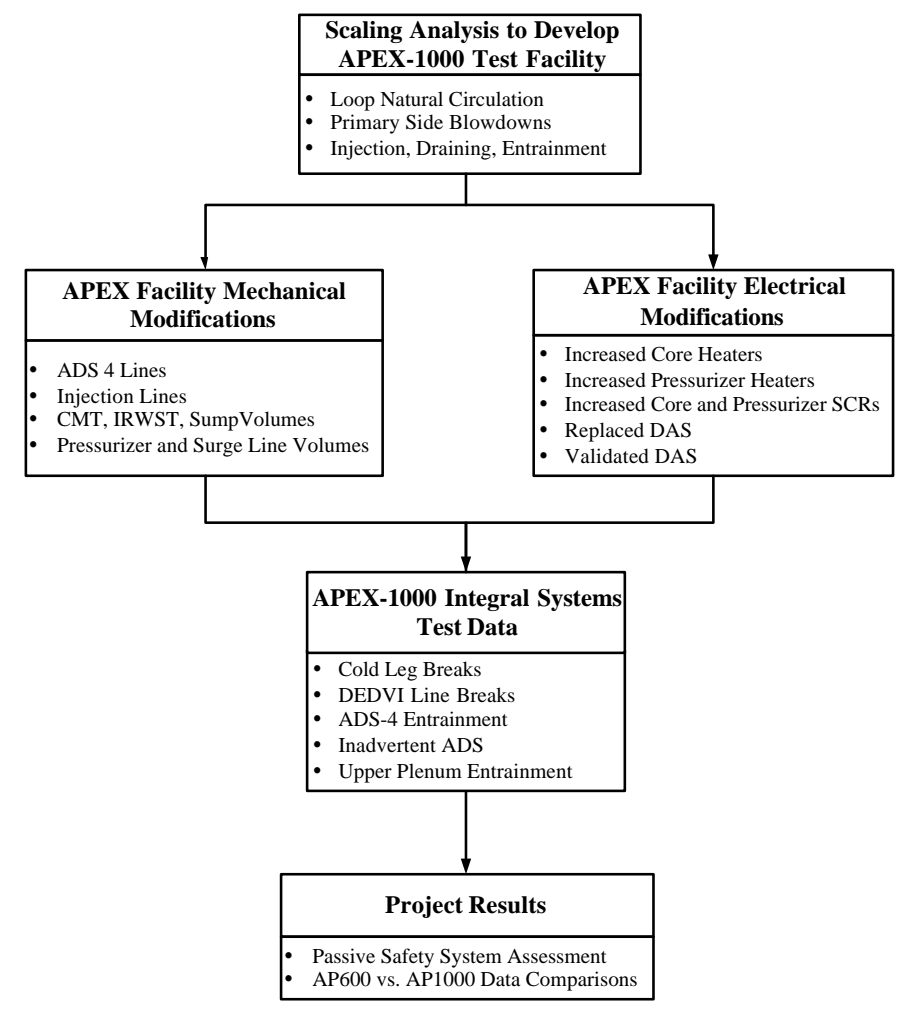

Having modified the APEX test facility, a wide range of integral systems tests were performed. The APEX-1000 test results were compared to the APEX-600 test results to gain an understanding of the effects of high power on passive safety system performance. All of the results are documented in individual test reports attached as Appendices.

\subsection{Comparison of the AP600 and AP1000}

Westinghouse has recently issued its AP1000 Plant Description and Analysis Report. ${ }^{1}$ Table 3.1 provides a summary comparison of the key design parameters of the AP1000 with those of the AP600. 
TABLE 3.1. Comparison of NSSS Design Parameters

\begin{tabular}{|c|c|c|}
\hline Parameter & AP600 & AP1000 \\
\hline Reactor Power, MWt & 1933 & 3400 \\
\hline Hot Leg Temperature, ${ }^{\circ} \mathrm{F}$ & 600 & 615 \\
\hline Number of Fuel Assemblies & 145 & 157 \\
\hline Type of Fuel Assembly & $17 \times 17$ & $17 \times 17$ \\
\hline Active Fuel Length, $\mathrm{ft}$ & 12 & 14 \\
\hline Linear Heat Length, $\mathrm{ft}$ & 4.10 & 5.707 \\
\hline Reactor Vessel Inside Diameter, inches & 157 & 157 \\
\hline Steam Generator heat Transfer Area, $\mathrm{ft}^{2}$ & 75,180 & 125,000 \\
\hline Reactor Coolant Pump Flow, gpm & 51,000 & 75,000 \\
\hline Pressurizer Volume, $\mathrm{ft}^{3}$ & 1,600 & 2,100 \\
\hline
\end{tabular}

The most significant change was the increase in core power. The reactor vessel volume remains unchanged and the steam generator tube surface area, the pressurizer volume and the reactor coolant pump flow have all increased to accommodate the increased core power. A comparison summarizing the changes to the passive safety system design is provided in Table 3.2. Several changes were made to increase the volume of the CMTs and the IRWST. The flow capacities for the IRWST, ADS-4 and the PRHR have been increased by reducing the resistance in each line. The PRHR surface area was also increased. The details of the AP1000 design are provided in the Westinghouse Plant Parameters document. $^{2}$ 
TABLE 3.2.

Comparison of Passive Safety System Design Features

\begin{tabular}{|c|c|c|c|}
\hline & AP600 & AP100 & Comment \\
\hline $\begin{array}{l}\text { Core Makeup Tanks } \\
\text { Number } \\
\text { Volume, } \mathrm{ft}^{3} \\
\text { Line Resistance, \% } \\
\text { Design Flow Rate, \% }\end{array}$ & $\begin{array}{c}2 \\
2000 \\
100 \% \\
100 \%\end{array}$ & $\begin{array}{c}2 \\
2500 \\
64 \% \\
124 \%\end{array}$ & $\begin{array}{l}\text { Core makeup tank (CMT) volume and flow rate is } \\
\text { increased to provide additional safety injection flow. } \\
\text { CMT elevations are maintained at the AP600 level. The } \\
\text { duration of CMT injection is maintained similar to } \\
\text { AP600. }\end{array}$ \\
\hline $\begin{array}{l}\text { Accumulators } \\
\text { Number } \\
\text { Volume, } \mathrm{ft}^{3} \\
\text { Pressure, psig }\end{array}$ & $\begin{array}{c}2 \\
2000 \\
700\end{array}$ & $\begin{array}{c}2 \\
2000 \\
700\end{array}$ & $\begin{array}{l}\text { The accumulators are the same as AP600. Accumulator } \\
\text { sizing is based on LBLOCA performance and is deter- } \\
\text { mined largely on reactor vessels volume. }\end{array}$ \\
\hline $\begin{array}{l}\text { IRWST } \\
\text { Volume, gallons } \\
\text { Water Level, ft } \\
\text { Line Resistance, \% } \\
\text { Design Flow Rate, \% }\end{array}$ & $\begin{array}{r}557,000 \\
130.00 " \\
100 \% \\
100 \%\end{array}$ & $\begin{array}{c}590,000 \\
131.58 " \\
32 \% \\
184 \%\end{array}$ & $\begin{array}{l}\text { The IRWST level has been increased in the AP1000 by } \\
\text { using more accurate level instruments. This permits a } \\
\text { high operating level. }\end{array}$ \\
\hline $\begin{array}{l}\text { Automatic Depressurization } \\
\text { Stages 1-3 } \\
\text { Location, } \\
\text { Configuration, } \\
\text { Vent Area, \% } \\
\text { Stage } 4 \\
\text { Location, } \\
\text { Configuration, } \\
\text { Line size, nominal } \\
\text { Vent Area, \% } \\
\text { Line Resistance } \\
\text { Capacity }\end{array}$ & $\begin{array}{c}\text { Top PZR } \\
6 \text { paths } \\
100 \% \\
\\
\text { Hot Leg } \\
4 \text { paths } \\
10 \text {-inch } \\
100 \% \\
100 \% \\
100 \%\end{array}$ & $\begin{array}{c}\text { Hot Legs } \\
4 \text { paths } \\
14 \text {-inch } \\
176 \% \\
28 \% \\
189 \%\end{array}$ & $\begin{array}{l}\text { The first three stages of ADS are the same as AP600. } \\
\text { Their sizing basis is to reduce pressure to permit ade- } \\
\text { quate injection from the accumulators and to permit tran- } \\
\text { sition to 4th stage ADS. } \\
\text { The ADS 4th stage vent area is increased more than the } \\
\text { ratio of the core power. The 4th stage ADS venting is } \\
\text { the most important design feature to allow for stable } \\
\text { IRWST/sump injection during long term core cooling. }\end{array}$ \\
\hline $\begin{array}{l}\text { Passive RHR Heat Exchanger } \\
\text { Type } \\
\text { Surface Area, \% } \\
\text { Design Flow Rate, \% } \\
\text { Design Heat Transfer, \% }\end{array}$ & $\begin{array}{l}\text { C-Tube } \\
100 \% \\
100 \% \\
100 \%\end{array}$ & $\begin{array}{l}\text { C-Tube } \\
122 \% \\
174 \% \\
172 \%\end{array}$ & $\begin{array}{l}\text { The AP1000 PRHR HX retains the AP600 configura- } \\
\text { tion. The heat transfer surface area is increased by } \\
\text { extending the length of the heat exchanger. The inlet } \\
\text { and outlet piping has been increased resulting in higher } \\
\text { flow rates. }\end{array}$ \\
\hline $\begin{array}{l}\text { Containment } \\
\text { Diameter, } \mathrm{ft} \\
\text { Overall Height, } \mathrm{ft} \\
\text { Shell Thickness, 1A } \\
\text { Design Pressure, psig } \\
\text { Net Free Volume, } \mathrm{ft}^{3}\end{array}$ & $\begin{array}{c}130 \\
189.83 \\
1.625 \\
45 \\
173 \times 10^{6}\end{array}$ & $\begin{array}{c}130 \\
215.33 \\
1.75 \\
59 \\
2.07 \times 10^{6}\end{array}$ & $\begin{array}{l}\text { The AP1000 containment volume and design pressure } \\
\text { are increased to accommodate higher mass and energy } \\
\text { releases. }\end{array}$ \\
\hline $\begin{array}{l}\text { Passive Containment Cooling } \\
\text { System Water Storage Tank Vol- } \\
\text { ume (Top of Overflow), gallons }\end{array}$ & 580,000 & 800,000 & $\begin{array}{l}\text { The PCS water storage tank was increased to accommo- } \\
\text { date higher flow rates. The PCS flow rates have been } \\
\text { increased based on the increased in core power. }\end{array}$ \\
\hline
\end{tabular}




\subsection{Modifications to the APEX Test Facility}

Based on a review of the design changes listed in Tables 3.1 and 3.2, and their detailed description in the AP1000 Plant Parameters document, the set of modifications listed in Table 3.3 were performed to APEX to best simulate AP1000 thermal hydraulic behavior.

TABLE 3.3.

Proposed Modifications to the APEX Test Facility

\begin{tabular}{|l|l|}
\hline \multicolumn{1}{|c|}{ Component } & \multicolumn{1}{c|}{ Modification to APEX } \\
\hline Reactor Power & Increase core power by 67 percent \\
\hline Pressurizer & $\begin{array}{l}\text { Increase Pressurizer volume } \\
\text { Reduce Pressurizer Surge Line Diameter }\end{array}$ \\
\hline Steam Generator Heat Transfer Area & No change required for testing \\
\hline Reactor Coolant Pump Flow & No change requried for testing \\
\hline Core Makeup Tanks & $\begin{array}{l}\text { Increase Core Makeup Tank (CMT) volumes by 25 percent } \\
\text { Reduce line resistance to 64\% of original value }\end{array}$ \\
\hline Accumulators & No changes to original design \\
\hline IRWST & Increase IRWST liquid level \\
\hline Automatic Depressurization Stages 1-3 & No changes to original design \\
\hline Automatic Depressurization Stage 4 & $\begin{array}{l}\text { Increase ADS-4 flow area by 76 percent } \\
\text { Reduce line resistance to 28 percent of original value }\end{array}$ \\
\hline Passive Residual Heat Removal (PRHR) Heat Exchanger & $\begin{array}{l}\text { Increase PRHR flow capacity by 74\% by reducing line resistance } \\
\text { No change in surface area/tube number required for testing }\end{array}$ \\
\hline Containment & $\begin{array}{l}\text { No change to original design of lower compartment heights or vol- } \\
\text { umes }\end{array}$ \\
\hline Passive Containment Cooling System & Not part of APEX Testing Program \\
\hline
\end{tabular}

\subsection{Rationale for APEX Modifications}

An increase in the reactor core power was needed to preserve the power to volume scaling ratio. This is a key feature of the original scaling analysis and is essential to simulating decay heat behavior, loop natural circulation and primary system depressurization rates. An increase in pressurizer volume was essential because it is a major source of liquid to the core during primary system blowdowns. The reduction in pressurizer surge line diameter was identified as a need 
by the NRC at the conclusion of the AP600 research program. ${ }^{3}$ This modification was needed to assure the properly scaled pressurizer draining behavior during primary system blowdowns and properly scaled surge line pressure drop behavior during ADS 1-3 operation.

Increases to the APEX steam generator tube volume and heat transfer area were not deemed necessary for several reasons. First, their tube volume is small compared to the remainder of the primary system. Secondly, they are oversized for the decay power operations involved in testing. That is, they have much more surface area than needed to remove core heat. The impact of not increasing the steam generator surface area will be an increase in the core fluid temperature rise. This is well within the design constraints of the existing facility.

An increase in reactor coolant pump flow capacity was not necessary because the pumps are tripped at the start of each transient. Their primary contribution during testing is their resistance which is preserved.

The CMT volumes were increased because they are an essential part of the PXS response and ADS actuation is dependent on the CMT liquid volume. The CMT line resistance was also reduced to simulate the scaled CMT draining rates. CMT volume and flow rate are critical to the transition from ADS 4 blowdown to IRWST injection.

The IRWST injection flow rate has a significant impact on core cooling. Therefore the IRWST lines were modified to increase their flow capacity to match the AP1000 design on a scaled basis. The onset of IRWST injection is dependent on the liquid level height in the tank. The APEX IRWST liquid level was raised to match the design change.

The fourth stage valves of the ADS assure that the primary system pressure will be reduced below the IRWST liquid level head so that injection can begin. The increased flow area is needed to properly simulate the AP1000 ADS 4 operation. In addition, ADS 4 venturis were employed to match both the choke flow area and the scaled pressure drop during non-choke flow conditions 
Increases to the PRHR heat exchanger tube volume and surface area were not deemed necessary for the experiment. As with the steam generator, the PRHR tube volume is small compared to the primary loop volume. Furthermore, it is oversized for the decay power operations involved in testing. That is, they have much more surface area than needed to remove core heat. The impact of not increasing the PRHR will be an increase in the core fluid temperature rise. This is well within the design constraints of the existing facility. Properly scaled natural circulation flow rates are assured by adjusting the PRHR loop resistance.

The ADS 1-3 valves, the containment lower compartments and the accumulators remain the same as the original AP600 design. Therefore these components will not be modified for the AP1000 testing program.

\subsection{APEX Scaling Assessment Summary}

The original scaling analysis performed in support of the design of the APEX test facility is documented in WCAP-14270 issued by Westinghouse in January 1995. ${ }^{4}$ The objective of the scaling study was to obtain the physical dimensions of a test facility that would simulate the flow and heat transfer behavior of importance to the AP600 passive safety system operation.

Westinghouse has recently issued an AP1000 Phenomena Identification and Ranking Tables (PIRT) and Scaling Assessment, which reviews the original scaling analysis effort and assesses the applicability of the original AP600 test program to the AP1000 design. ${ }^{3}$ Westinghouse concluded that the AP1000 PIRT was the same as the AP600 PIRT with the exception of the potential higher importance of hot leg entrainment during the post ADS-4 actuation phases of the small break LOCA. Therefore, scaling of post-ADS-4 actuation phases is of particular interest to the AP1000.

OSU has found that the scaling criteria presented in the original scaling analysis, can be used to design the AP1000 modifications for the APEX facility. ${ }^{5}$ The basis for this result is twofold. First, the APEX test facility will preserve geo- 
metrically similarity to the AP1000 after modification. Thus, all of the length, flow area, volume and time scale ratios remain the same. Secondly, the important thermal hydraulic phenomena, as identified in the AP1000 PIRT, are the same as assessed in the original PIRT with one exception: hot leg and upper plenum entrainment.

The following analyses have been included in the OSU APEX-1000 Scaling Report to guide the design of the APEX test facility modifications:

- Core Decay Power Scaling Analysis

- IRWST, CMT and Pressurizer Volume Scaling Analysis

- IRWST, CMT, PRHR and Sump Recirculation Line Scaling

- Presurizer Surge Line Scaling Analysis

- ADS-4 Blowdown Scaling Analysis

- Upper Plenum Entrainment Scaling Analysis

The key Scaling Ratios for the APEX-1000 Test facility are listed in Table 4.1. These values have been superceded for some components to best scale certain phenomena. For example, flow regime transitions in the hot leg and critical flow at the break use different flow area scaling than those presented in Table 4.1. Specific component scaling ratios and there basis are presented in the APEX1000 Scaling Report.

TABLE 4.1.

Key Scaling Ratios for AP600/AP1000

\begin{tabular}{|l|c|}
\hline \multicolumn{1}{|c|}{ Parameter } & Scaling Ratio \\
\hline Vertical Lengths & $1: 4$ \\
\hline Flow Areas & $1: 48$ \\
\hline Volumes & $1: 192$ \\
\hline Power & $1: 96$ \\
\hline Mass Flow Ratio & $1: 96$ \\
\hline Fluid Residence Time Ratio & $1: 2$ \\
\hline Fluid Velocity Ratio & $1: 2$ \\
\hline
\end{tabular}




\subsection{APEX Test Facility}

The APEX test facility, shown in Figure 5.1, has been specifically designed and constructed to provide high quality data for use in computer code benchmark calculations. The test facility is an one fourth height, one half time scale, reduced pressure and temperature integral systems test facility. The APEX test facility can be operated up to its design temperature and pressure limits of $450^{\circ} \mathrm{F}(505 \mathrm{~K})$ and $400 \mathrm{psig}$ (32 bar). A complete description of the APEX-1000 test facility is documented in a separate report. ${ }^{6}$

\section{FIGURE 5.1}

APEX Test Facility

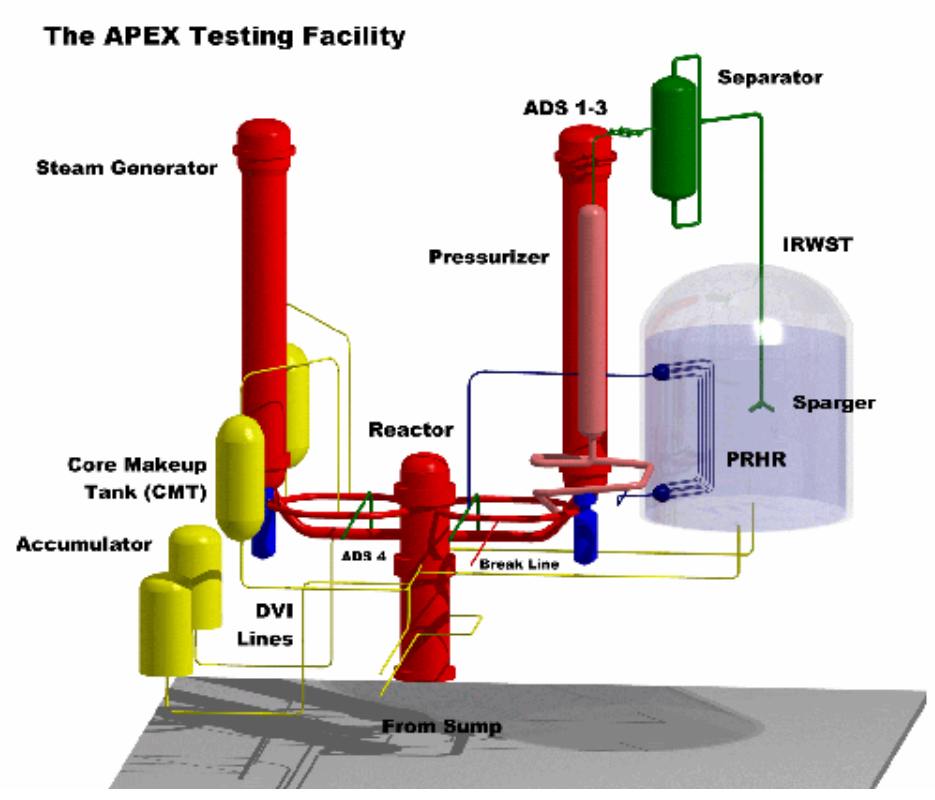

\subsection{Reactor Coolant System}

The APEX facility Reactor Coolant System (RCS) is a complete model of the AP1000 Nuclear Steam Supply System (NSSS). The RCS includes:

- a electrically heated 48-rod bundle,

- a Reactor Pressure Vessel (RPV) with upper core internals,

- a Pressurizer (pressurizer),

- two U-Tube Steam Generators (SGs), 
- four Reactor Coolant Pumps (RCPs),

- and associated primary loop piping.

See Figures 5.2 and 5.3 for elevation view and plan view of the RCS respectively.

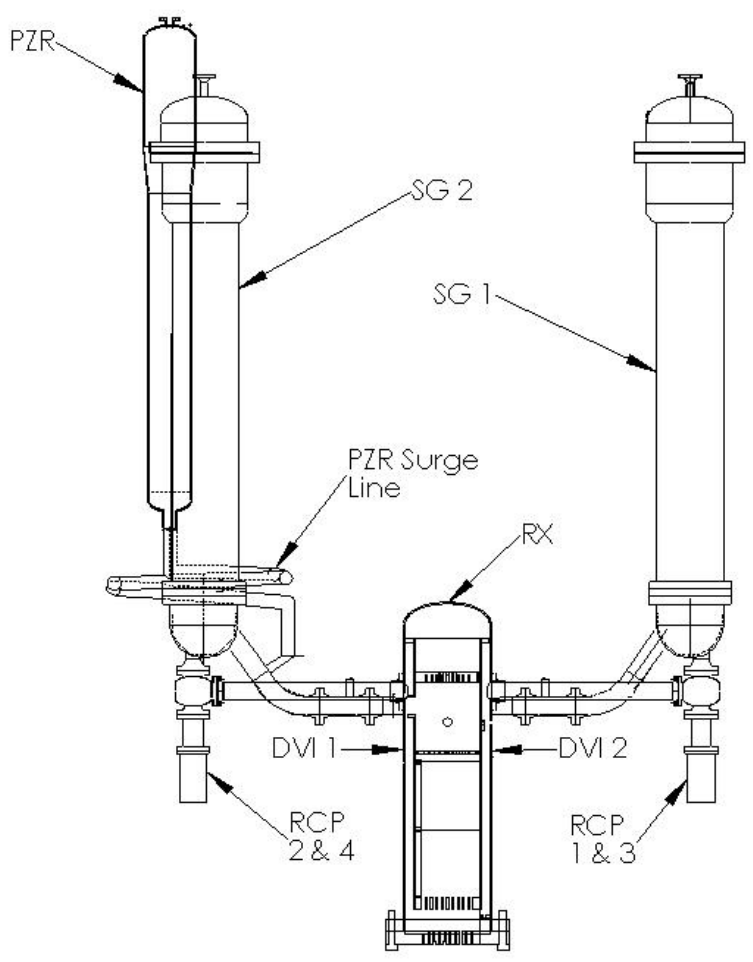




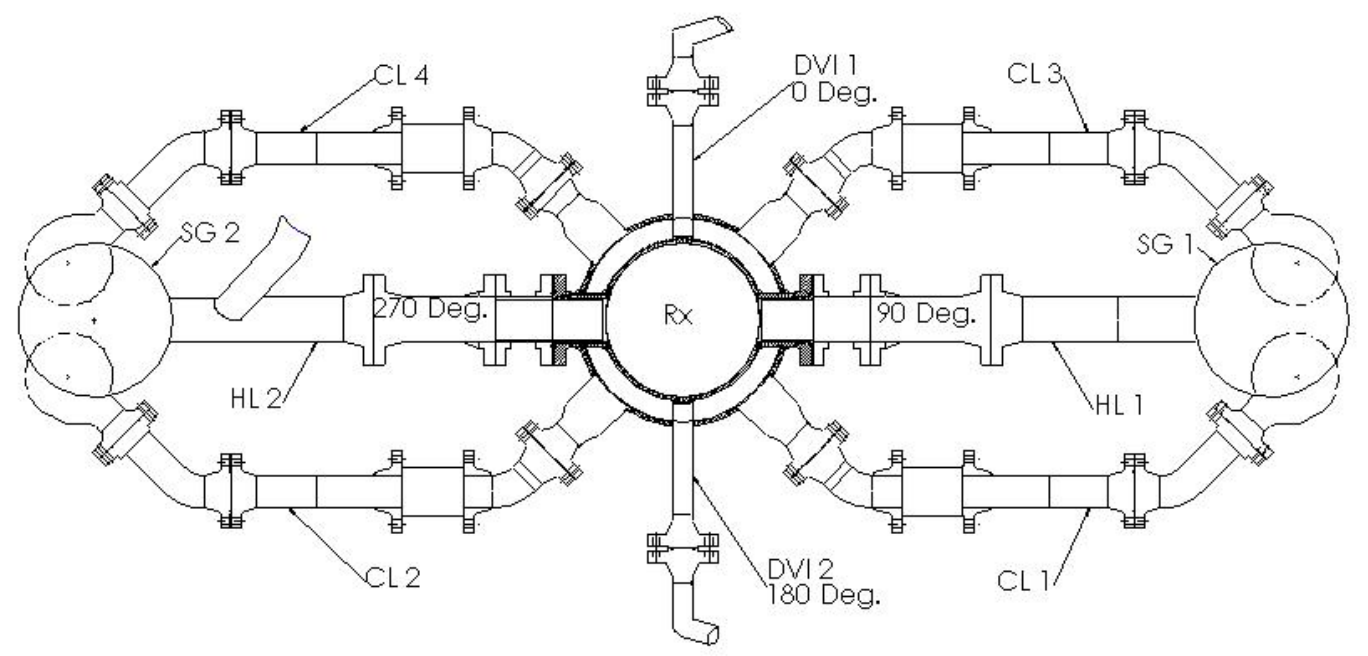

\subsubsection{Reactor Pressure Vessel}

The RPV models the upper and lower internals of the AP1000 reactor vessel, core barrel, downcomer, and core. The maximum core power is approximately $1000 \mathrm{~kW}$ and can be distributed in two radial power zones to simulate timedependent decay power. The RPV includes connections for the two Hot Legs (HLs), four Cold Legs (CLs), and two Direct Vessel Injection (DVI) lines.

During normal operation, cold water enters through four 3.5 in. $(8.89 \mathrm{~cm}) \mathrm{sch}$. 40 cold legs into an annular downcomer region that is bounded by the inside surface of the reactor vessel shell and the outside surface of the core barrel. The cold water in the downcomer flows into the lower plenum where it changes direction and travels upward through the lower core plate and into the core.

The heated zone of the core extends $36 \mathrm{in} .(91.44 \mathrm{~cm})$ from the top of the lower core plate and consists of 48 heater rods, each having a 0.875 in. $(2.22 \mathrm{~cm})$ diameter. Five fluid thermocouple $(\mathrm{T} / \mathrm{C})$ rods provide an axial and radial temperature distribution in the core region. Two spacer grids are provided for support of the heaters, one at the mid-plane and the other near the top of the heaters. 
The heater bundle is surrounded by a reflector/baffle that directs the fluid through the core, see Figure 5.4.

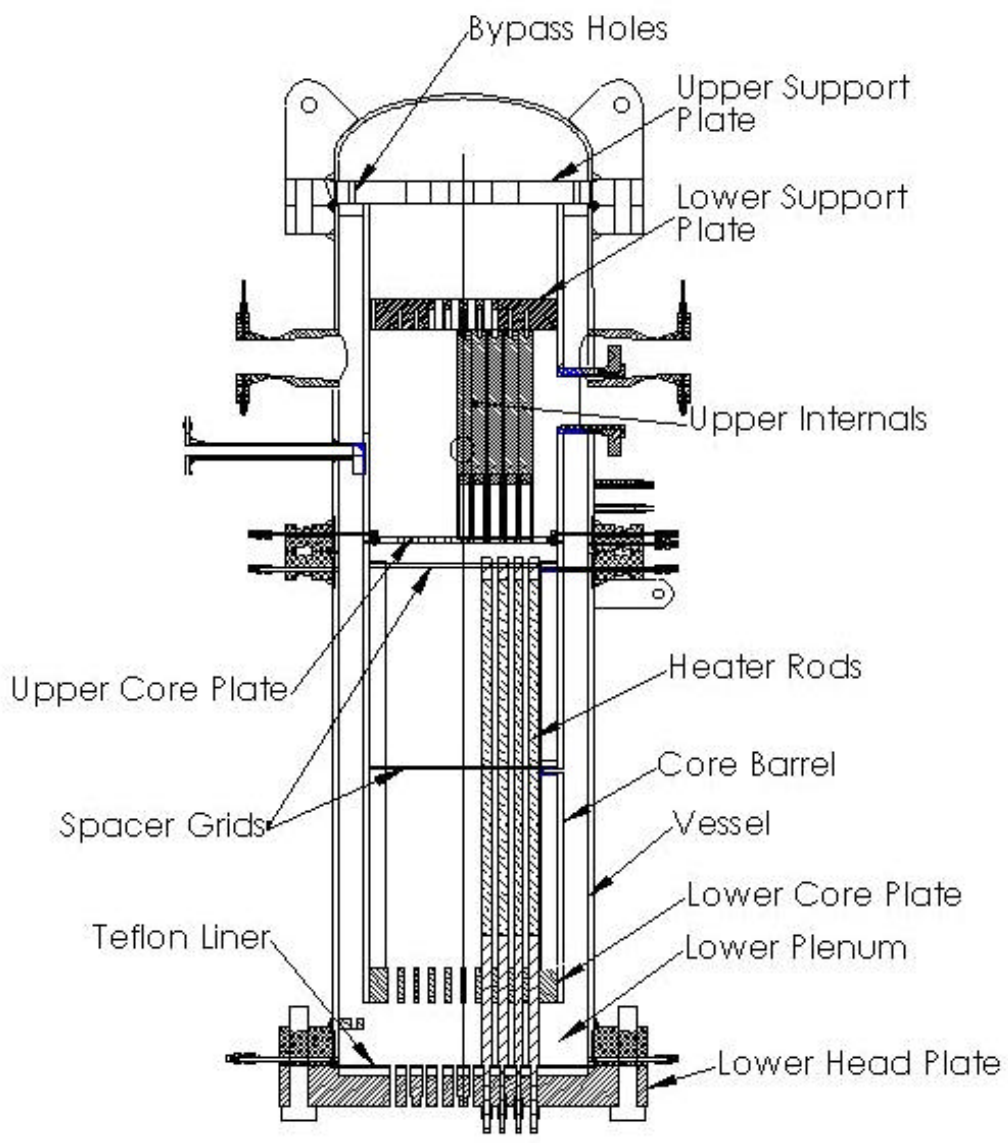

\subsubsection{Pressurizer}

A fully functional pressurizer with $20 \mathrm{~kW}$ internal heaters and a relief valve system capable of controlling the RCS pressure has been included. The pressurizer is connected to HL-2 through the pressurizer surge line. The pressurizer's diameter is not constant along its entire length, the lower cylindrical portion is constructed from 12 in. sch 40 pipe, and the upper cylindrical portion is constructed from 16 in. sch 30 pipe, see Figure 5.5. The larger diameter top on the 
pressurizer was required in the APEX facility since there was not enough vertical space in the lab to accommodate the increased height of the pressurizer if the diameter remained constant. The larger upper portion of the pressurizer is above the normal pressurizer water level, so only steam is present in the upper portion. The pressurizer in the APEX facility does not use a condensing spray for reduction of pressure; instead a vent is used to exhaust steam. A line from the first three stages of the automatic depressurization system (ADS) is connected to the top of the pressurizer for modeling the ADS system depressurization of the primary system. See Figure 5.5 for an elevation view of the pressurizer.

\section{FIGURE 5.5}

\section{Pressurizer}

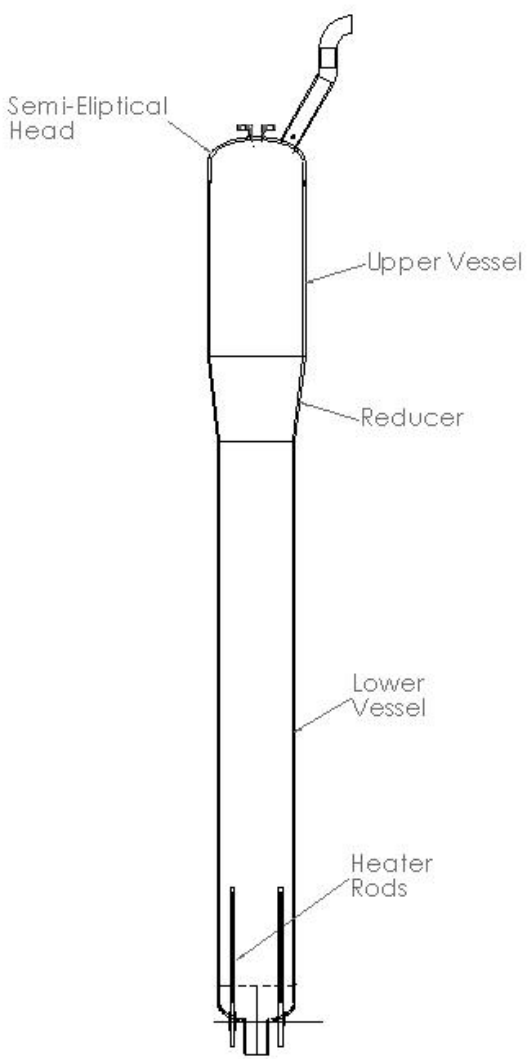


The pressurizer surge line connects the bottom of the pressurizer to the top of Hot Leg 2. The surge line enables continuous pressure adjustments between the RCS and the Pressurizer. The Pressurizer surge line geometry of the AP1000 has been preserve in APEX as show in Figure 5.6.

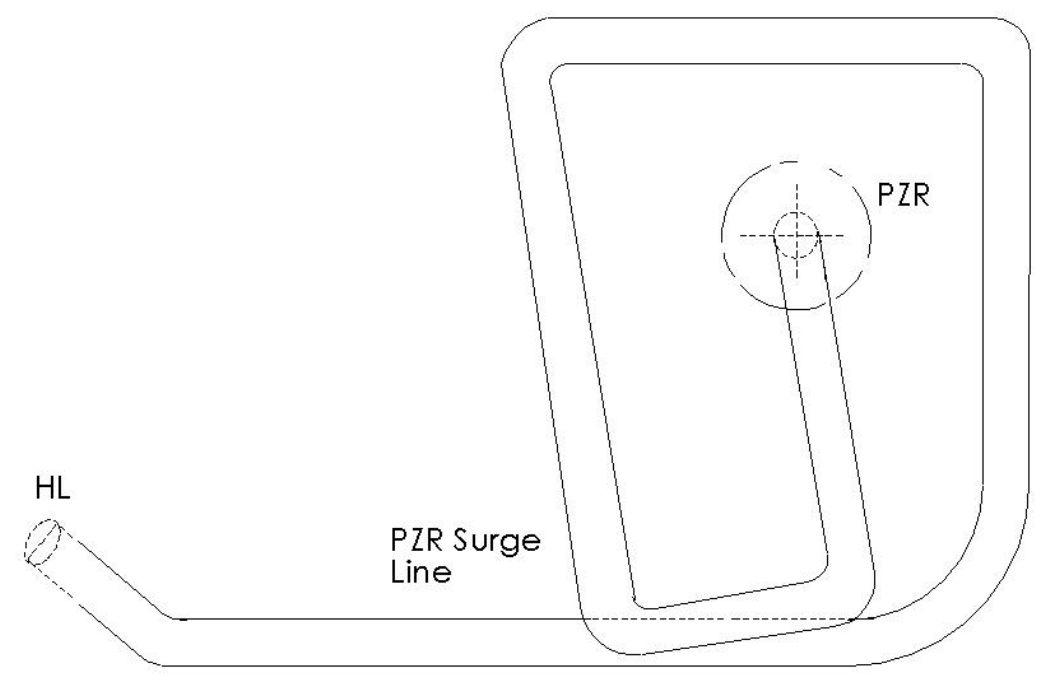

\subsubsection{Steam Generators}

Two Steam Generators (SG), one on each loop, have been included in the APEX facility. Each SG is instrumented and is a shell and tube heat exchanger that models the Westinghouse Delta-75 steam generator, see Figure 5.7. The SG lower channel head includes connections for two RCPs and a single HL. Steam Generator \#2 contains additional connections for PRHR Hx return flow and the Chemical Volume System (CVS) pump discharge. Each SG contains 133 Utubes with a 0.687 in. $(1.74 \mathrm{~cm})$ outside diameter and a single chevron type moisture separator located at the steam outlet nozzle. Moisture removed by the separator is directed to the downcomer to aid in heating the incoming feed water. Feed water is distributed inside the steam generator by the feed water nozzle which includes 8 "J" hook type nozzles to direct flow downward. 
FIGURE 5.7
Steam Generator

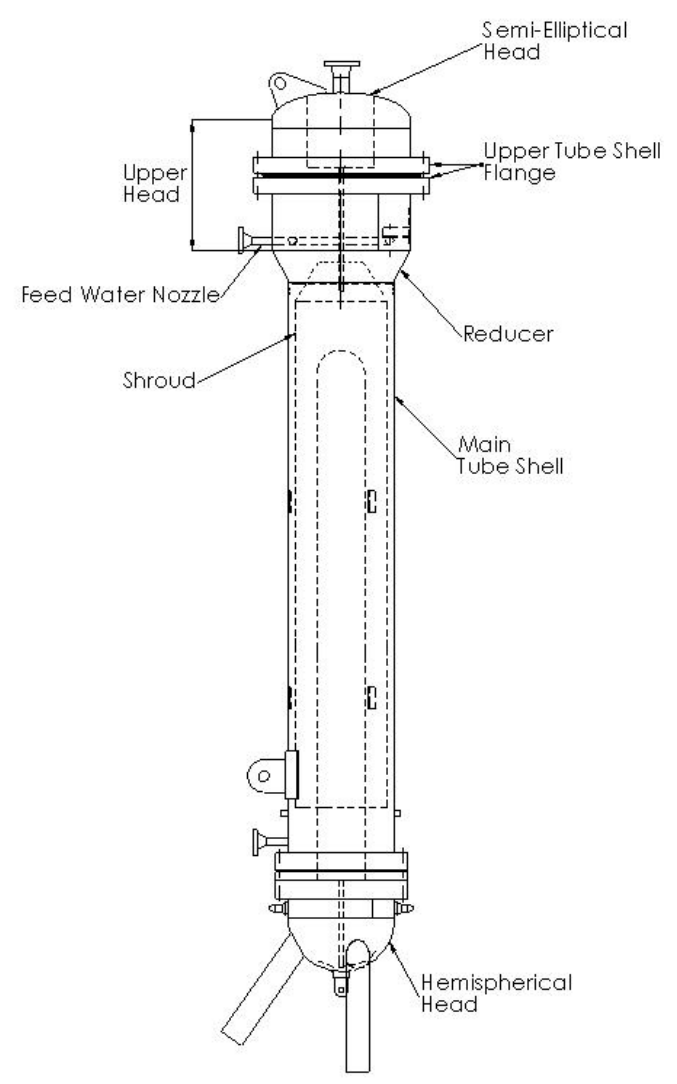

\subsubsection{Primary Loop Piping}

The primary loop piping models two primary loops, each consisting of a single hot leg and two cold legs. Break spool pieces are installed on primary loop and passive safety system piping to model various LOCA scenarios. The following LOCA breaks can be modeled in APEX:

- Top of CL \#3 break

- Bottom of CL \#3 break

- Bottom of CL \#4 break

- Bottom of HL \#2 break

- Single-ended DVI break

- Double-ended DVI break

- Single-ended CMT balance line break 
- Double-ended CMT balance line break

Each of the primary loop breaks is initiated by pneumatic operated valves connected to the associated break spool piece. A flow nozzle is used to model the size of the break. The flow from the break is discharged to the Break and ADS Measurement System (BAMS) were a separator is used to separate and measure the liquid and vapor components of the flow.

\subsection{Passive Core Cooling system}

The PXS relies on gravity and natural circulation to prevent core damage in the event of a LOCA or loss of heat sink accident. The PXS provides three sources of makeup water with Direct Vessel Injection (DVI) to the core and includes a four-stage Automatic Depressurization System (ADS), a Passive Residual Heat Removal (PRHR) heat exchanger, and a sump recirculation path for long-term cooling.

\subsubsection{Automatic Depressurization System}

The ADS provides a four stage depressurization of the RCS to allow gravity/ pressurized injection of makeup water to the core. Stages 1-3 vent from the top of the Pressurizer and discharges to the ADS 1-3 separator where the two phase flow is separated and measured. All portions of the ADS 1-3 flow are re-combined before discharging to a submerged sparger located inside the IRWST. See Figure 5.8 for details of sparger.

Each stage of ADS 1-3 in APEX models two trains in the AP1000. A flow nozzle is used to model the scaled choked flow area for each stage of ADS 1-3. To model a single valve failure, the desired flow nozzle is replaced with one having a corresponding decrease in flow area.

Two ADS 4 valves, modeling 4 valves in AP1000, are connected to the top of each Hot Leg. Both ADS 4 lines are similar with the exception that ADS 4-2 (pressurizer side) provides a connection to the PRHR Hx system. The flow from each stage of ADS 4 is discharged to a venturi. The venturi is used to model 
both the choke flow area and the scaled pressure drop. The flow is directed to a moisture separator where the steam and liquid flows are separated and measured individually. The liquid flow is then directed through a loop seal and into the primary sump, while steam flow is vented to atmosphere.

FIGURE 5.8

ADS 1-3 Sparger

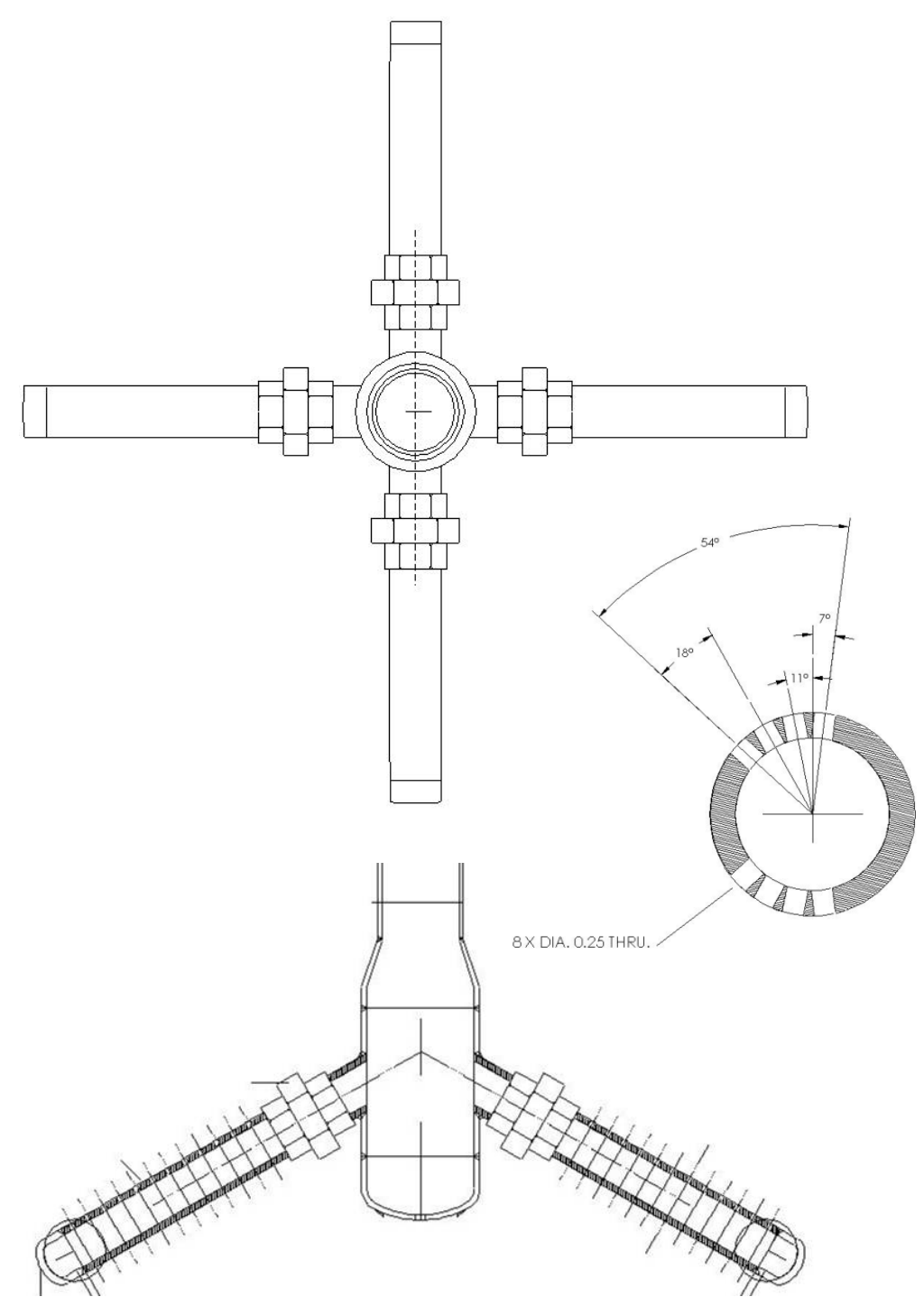




\subsubsection{In-containment Refueling Water Storage Tank}

The IRWST is modeled by a cylindrical vessel and provides connections for the ADS 1-3 sparger, two injection trains, and the PRHR Heat Exchanger. Thermocouple rakes are employed to provide a radial and axial temperature distribution of the IRWST pool. A standpipe is used to set the initial water level and a curb overflow is provided to match the scaled AP1000 curb overflow to the sump. The IRWST is capable of being pressurized to 65 psig ( 4.5 bar).

\subsubsection{Core Makeup Tanks}

Two CMTs are included in APEX. AP1000 piping geometry is preserved in APEX with each CMT connected to a cold leg (for pressure balance) and to the associated injection train. A differential pressure level detector is used to provide ADS actuation signals based on level.

\subsubsection{Accumulators}

The two accumulators are capable of being pressurized to full system pressure are included in APEX. Each accumulator employs a standpipe used to the set the water level and is pressurized with nitrogen gas. Check valves isolate the accumulators from RCS pressure and open automatically as the RCS system depressurizes during a LOCA.

\subsubsection{Passive Residual Heat Removal Heat Exchanger}

The PRHR Heat Exchanger is a C-Shape heat exchanger submerged inside the IRWST. Both the heat exchanger geometry and inlet and outlet piping geometry is preserved in APEX. The PRHR Heat Exchanger inlet is connected to ADS 42 (Hot Leg \#2), while the return is connected to the cold leg plenum of Steam Generator \#2. The PRHR Heat Exchanger constructed from of 88 "c" shaped tubes with an outside diameter of 0.375 in $(0.9525 \mathrm{~cm})$ diameter tubes, see Figure 5.9. 

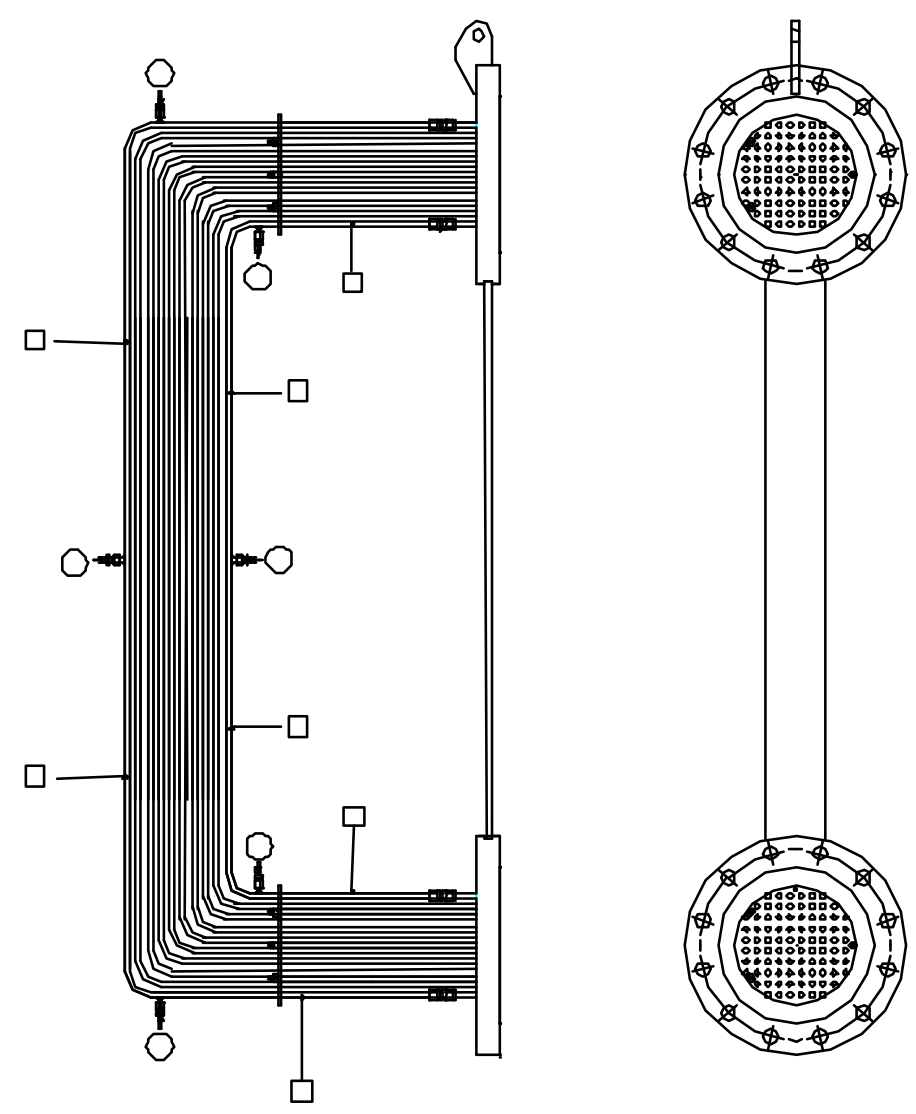

\subsection{Break and ADS Measurement System}

Break and ADS Measurement System (BAMS) is used to separate and measure two-phase volumetric flow rates from the break(s) and four stages of ADS. For the break and ADS 4 Separators, the water/steam mixture enters the separator where gravity and a cyclone separator is used to separate the two phases. The separated steam exits the top of the separator, while the liquid is collected and allowed to drain out of the bottom of the separator through a loop seal and into the primary sump. For the ADS 1-3 Separator the liquid and steam is first recombined before being discharged to the sparger located inside of the IRWST. 
The break separator and the ADS 4 separators have a maximum working pressure of $75 \mathrm{psig}(618 \mathrm{kPa})$ at $450^{\circ} \mathrm{F}(505 \mathrm{~K})$, while the ADS 1-3 separator has a maximum working pressure of $400 \mathrm{psig}(2.86 \mathrm{MPa})$ at $450^{\circ} \mathrm{F}(505 \mathrm{~K})$. The steam lines and moisture separators utilize strip heaters to minimize condensation and to maintain a constant boundary condition. See Fiqure 5.10 for a schematic of the BAMS system.

\section{FIGURE 5.10}

\section{BAMS Schematic}

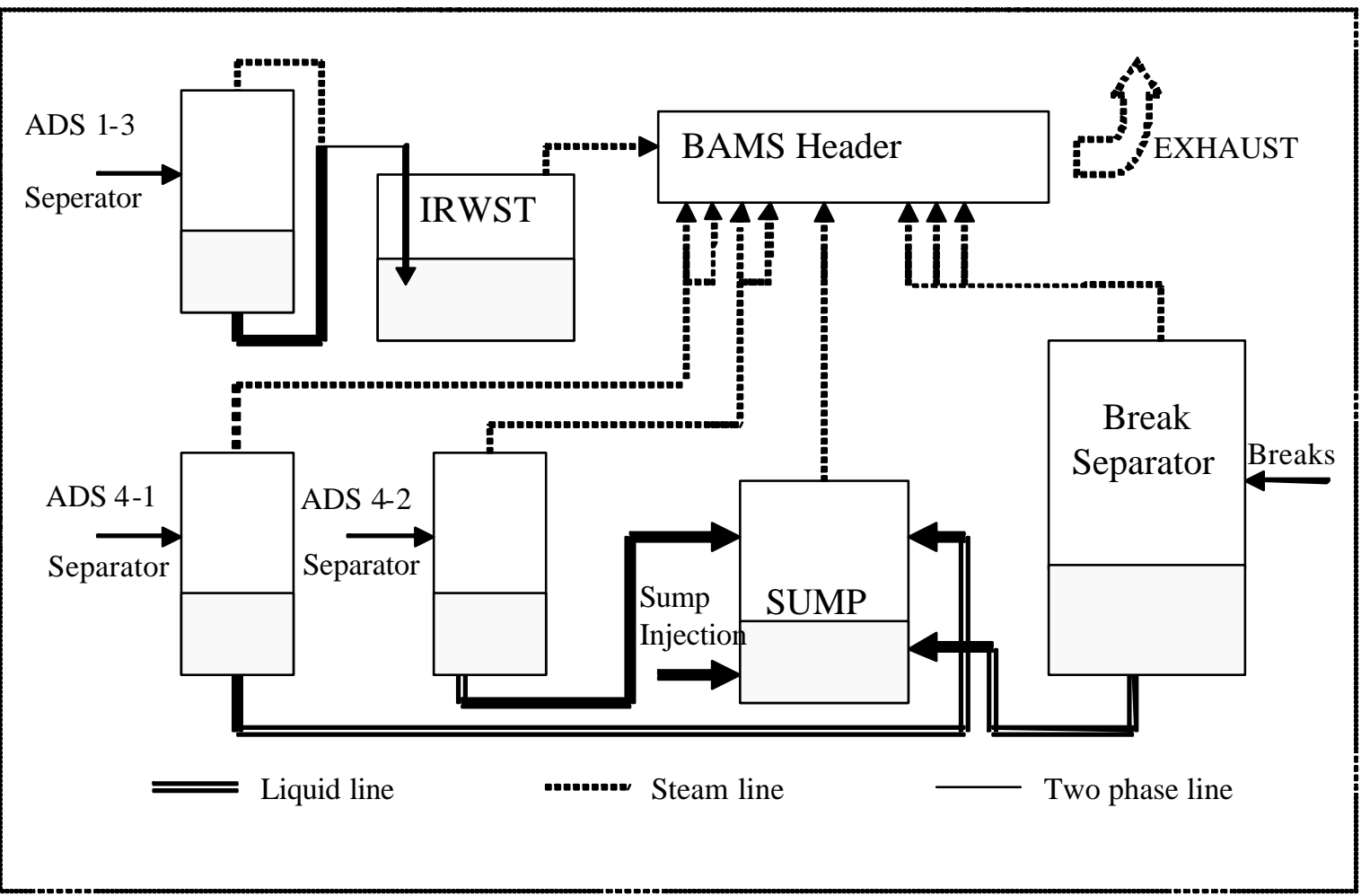

\subsection{Matrix Tests}

Eleven tests were conducted in the APEX 1000 test facility. Testing for the NRC was also performed under separate contract. The order of testing was selected to minimize plant change-outs and to expedite the test schedule. Detailed procedures for test conduct, data gathering, and storage are delineated 
in the Test Procedure for each test. The completed test procedures and data have been transmitted to Westinghouse Electric Corporation.

The general objective of the tests performed was to assess the AP1000 PXS performance for a wide range of transient conditions and system failures. A particular emphasis of the test program is low pressure ADS 4 and long-term-cooling sump recirculation operation. The data obtained from these tests was used by Westinghouse Electric Corporation to benchmark thermal hydraulic safety analysis computer codes and to aid in licensing the AP1000. All test data produced in support of the AP1000 licensing effort meet the requirements of 10 CFR 50 Appendix B, 10 CFR 21 and NQA-1.

The Double-Ended Direct Vessel Injection (DEDVI) line breaks are the largest breaks that are performed in the APEX-1000 test facility. The DEDVI break is of particular importance because it results in the loss of all injection flows from one side of the plant. That is, all flow from one CMT, one accumulator, and one IRWST injection line is directed to the break. Essentially half of the injection flows are lost with a single failure. In addition, the large break on the reactor vessel side of the DVI line break results in a rapid depressurization and a significant loss of primary liquid inventory. These are severe transients, particularly when additional safety system failures are assumed.

The "No Reserve" tests were designed to examine the limiting conditions for the transition from ADS 4 blowdown to the start of IRWST injection. The tests are initiated at full prototypic ADS 4 pressures with no liquid inventory above the bottom of the hot leg. That is, it is assumed that all the volumes above the hot leg have emptied including the CMTs. The ADS 4 flow areas, the core decay power and the initial system pressure are the test variables. The IRWST is pressurized to simulate full liquid height conditions. This test is particularly valuable because it can be performed at prototypic pressures and temperatures and at a 1:1 time scale. 
The Quasi-Steady Entrainment tests are designed to measure the pool entrainment rates during ADS 4 operation at a full range of containment backpressures. Pool entrainment conditions can potentially arise during IRWST injection or long-term sump recirculation cooling. These tests will be initiated with no liquid inventory above the hot leg elevation. A constant core power and initial DVI flow rate will be established to produce a slow boil-off. The DVI flow rate may be adjusted to assure adequate measurement times at a given upper plenum level. The tests will be conducted with a variety of ADS 4 flow areas. Entrainment rates will be measured as a function of liquid level in the upper plenum. If stable conditions can be achieved, the upper plenum draining process will be performed for a high, intermediate and low core power. An assessment of the effect of upper plenum internals on pool entrainment rate will be one of the key contributions of these tests.

Several Small Break LOCA tests, and a Mode 4 mid-loop operation test was performed to compare with the counterpart tests performed in the APEX-600 configuration. See Appendices for individual matrix test reports and Table 6.1 for a summary of tests performed. 
TABLE 6.1.

Summary of AP1000 Matrix Tests Performed

\begin{tabular}{|c|c|c|}
\hline Test ID & Description & Objective/Summary \\
\hline $\begin{array}{l}\text { OSU-AP1000-01 } \\
\text { (DBA-01) } \\
\text { 27-Feb-2003 }\end{array}$ & $\begin{array}{l}\text { AP1000 DEDVI with } 3 \text { of } \\
4 \text { ADS } 4\end{array}$ & $\begin{array}{l}\text { To obtain thermal-hydraulic data for a double-ended DVI line break } \\
\text { simulation. The top of the heater bundle was always covered during } \\
\text { this event. The test was performed for about 15,000 seconds. The } \\
\text { transient continued through Automatic Depressurization System } \\
\text { (ADS) actuation, core makeup tank (CMT), accumulator, in-contain- } \\
\text { ment refueling water storage tank (IRWST) injection, and sump } \\
\text { recirculation injection. }\end{array}$ \\
\hline $\begin{array}{l}\text { OSU-AP1000-02 } \\
\text { (TR-01-D) } \\
\text { 18-Mar-2003 }\end{array}$ & $\begin{array}{l}\text { AP1000 } 100 \text { psia Steady- } \\
\text { State Entrainment }\end{array}$ & $\begin{array}{l}\text { To obtain thermal-hydraulic data for the transition period from Auto- } \\
\text { matic Depressurization System } 4 \text { (ADS-4) actuation to stable IRWST } \\
\text { injection. For this test, the Reactor Coolant System was drained down } \\
\text { to the level of the hot legs. The RCS was heated to saturated condi- } \\
\text { tions at a pressure of } 85 \text { psig. The core power was set to a constant } \\
\text { value of } 628 \mathrm{~kW} \text {. The CMTs and ACCs were isolated for this test. } \\
\text { The ADS- } 4 \text { valves were opened to start of the test. The power was } \\
\text { held constant, and the RCS System was allowed to depressurize. } \\
\text { However, the cut-in pressure for IRWST injection was not reached } \\
\text { before the two-phase level in the upper plenum fell to the top of the } \\
\text { core. }\end{array}$ \\
\hline $\begin{array}{l}\text { OSU-AP1000-03 } \\
\text { (DBA-02) } \\
\text { 1-May-2003 }\end{array}$ & $\begin{array}{l}\text { AP1000 DEDVI with } 3 \text { of } \\
4 \text { ADS } 4\end{array}$ & $\begin{array}{l}\text { To obtain thermal-hydraulic data for a double-ended DVI line break } \\
\text { simulation. The test differs from DBA-01 (OSU-AP1000-01) in that } \\
\text { the ADS- } 4 \text { nozzles were replaced by venturis, and the ADS-4 piping } \\
\text { was modified to lower the resistance. For these tests, a single failure } \\
\text { was assumed in one of the ADS-4 valves on the hot leg opposite the } \\
\text { pressurizer. The test performed met the specified conditions. The top } \\
\text { of the heater bundle was always covered during this event. The test } \\
\text { was performed for about } 8,500 \text { seconds. The transient continued } \\
\text { through ADS actuation, CMT, ACC, IRWST injection, and sump } \\
\text { recirculation injection. }\end{array}$ \\
\hline $\begin{array}{l}\text { OSU-AP1000-04 } \\
\text { (DBA-03) } \\
\text { 15-May-2003 }\end{array}$ & $\begin{array}{l}\text { AP100 DEDVI with } 3 \text { of } 4 \\
\text { ADS } 4\end{array}$ & $\begin{array}{l}\text { To obtain thermal-hydraulic data for a double-ended DVI line break } \\
\text { simulation. The test differs from DBA-02 (OSU-AP1000-03) in that } \\
\text { for these tests, a single failure was assumed in one of the ADS- } 4 \\
\text { valves on the hot leg with the pressurizer. The test performed met the } \\
\text { specified conditions. The top of the heater bundle was always cov- } \\
\text { ered during this event. The test was performed for about } 6,600 \text { sec- } \\
\text { onds. The transient continued through ADS actuation, CMT, ACC, } \\
\text { IRWST injection, and ended just after sump recirculation injection. }\end{array}$ \\
\hline $\begin{array}{l}\text { OSU-AP1000-05 } \\
\text { (DBA-04) } \\
\text { 1-Jul-2003 }\end{array}$ & $\begin{array}{l}\text { AP1000 2" CL4 Break } \\
\text { with } 3 \text { of } 4 \text { ADS4 and } \\
\text { Degraded Containment } \\
\text { Sump }\end{array}$ & $\begin{array}{l}\text { To obtain thermal-hydraulic data for } 2 \text {-inch Cold Leg Break simula- } \\
\text { tion. The test performed met the specified conditions. The top of the } \\
\text { heater bundle was always covered during this event. The test was } \\
\text { performed for about } 17,000 \text { seconds. The transient continued through } \\
\text { ADS actuation, CMT, ACC, IRWST injection, and sump recircula- } \\
\text { tion injection. }\end{array}$ \\
\hline
\end{tabular}


TABLE 6.1.

Summary of AP1000 Matrix Tests Performed

\begin{tabular}{|c|c|c|}
\hline Test ID & Description & Objective/Summary \\
\hline $\begin{array}{l}\text { OSU-AP1000-06 } \\
\text { (TR-02) } \\
\text { 1-Jul-2003 }\end{array}$ & $\begin{array}{l}\text { AP1000 Full Pressure } \\
\text { ADS } 4 \text { Blowdown w/ } 3 \text { of } \\
4 \text { ADS4 }\end{array}$ & $\begin{array}{l}\text { To obtain thermal-hydraulic data for 2-inch Cold Leg Break simula- } \\
\text { tion with ADS- } 4 \text { actuation at plant-prototypic pressure conditions. } \\
\text { The test performed met the specified conditions. The top of the heater } \\
\text { bundle was always covered during this event. The test was performed } \\
\text { for about } 2200 \text { seconds. The transient continued through ADS, CMT, } \\
\text { ACC, and IRWST injection. }\end{array}$ \\
\hline $\begin{array}{l}\text { OSU-AP1000-07 } \\
\text { (ADS-01-D) } \\
\text { 10-Dec-2003 }\end{array}$ & $\begin{array}{l}\text { ADS } 4 \text { parametric study at } \\
\text { IRWST water level for } 18 \\
\text { inches \& } 0 \text { inches at Low } \\
\text { Decay Power }\end{array}$ & $\begin{array}{l}\text { To determine the steady-state two-phase flow and pressure drop char- } \\
\text { acteristics of the ADS- } 4 \text { lines at low-power/low-flow conditions typi- } \\
\text { cal of long-term cooling. The test was conducted with the RCS } \\
\text { drained down to the midpoint of the hot leg and the pressurizer } \\
\text { empty. Gravity injection from the IRWST was used to provide injec- } \\
\text { tion flow, and the core power was set to a constant value. The facility } \\
\text { was run in this configuration for } 15 \text { to } 30 \text { minutes as liquid and vapor } \\
\text { flows from the ADS- } 4 \text { lines were measured along with the pressure } \\
\text { drop distribution along the lines. This process was repeated by } \\
\text { changing the core power, which resulted in different void fractions in } \\
\text { the upper plenum and changed the gravity injection flow. Six power } \\
\text { levels were tested ranging from } 360 \mathrm{~kW} \text { to } 20 \mathrm{~kW} \text {. In addition, three } \\
\text { power levels ( } 20 \mathrm{~kW} \text { to } 80 \mathrm{~kW}) \text { were tested with the sump drained to } \\
\text { simulate reduced gravity flow at degraded sump conditions. }\end{array}$ \\
\hline $\begin{array}{l}\text { OSU-AP1000-08 } \\
\text { (ADS-02-D) } \\
\text { 6-Feb-2004 }\end{array}$ & ADS4 Characterization & $\begin{array}{l}\text { To determine the steady-state two-phase flow and pressure drop char- } \\
\text { acteristics of the ADS- } 4 \text { lines at high-power conditions typical of the } \\
\text { time period between ADS- } 4 \text { actuation and in-containment refueling } \\
\text { water storage tank injection. The test was conducted with the RCS } \\
\text { drained down to the midpoint of the hot leg and the pressurizer } \\
\text { empty. Core makeup flow was established at a constant rate using the } \\
\text { CVS pump with a range of } 0 \text { to } 7.5 \text { gpm. The facility was run in this } \\
\text { configuration for } 15 \text { to } 30 \text { minutes as liquid and vapor flows from the } \\
\text { ADS- } 4 \text { lines were measured along with the pressure drop distribution } \\
\text { along the lines. This process was repeated by changing the CVS flow } \\
\text { rate at a given power level. Three power levels were tested ranging } \\
\text { from } 430 \mathrm{~kW} \text { to } 60 \mathrm{~kW} \text {. In this way several injection flow/power } \\
\text { steady-state conditions were established and the data recorded. For } \\
\text { all cases, the two-phase mixture level was observed to be at or above } \\
\text { the bottom of the hot leg, and liquid entrainment was measured in the } \\
\text { ADS- } 4 \text { lines. In addition, three steady-state points were established } \\
\text { with the two-phase mixture level below the bottom of the hot leg. For } \\
\text { these cases, little or no entrainment was observed. }\end{array}$ \\
\hline $\begin{array}{l}\text { OSU-AP1000-09 } \\
\text { (DBA-03R-D) } \\
\text { 15-Apr-2004 }\end{array}$ & $\begin{array}{l}\text { AP1000 Doubled-ended } \\
\text { DVI w/ 3/4 ADS } 4\end{array}$ & $\begin{array}{l}\text { The test is a repeat of DBA-03 (OSU-AP1000-04) and was per- } \\
\text { formed to demonstrate the repeatability of the test facility. The test } \\
\text { performed met the specified conditions. The top of the heater bundle } \\
\text { was always covered during this event. The test was performed for } \\
\text { about } 2,800 \text { seconds. The transient continued through ADS actuation } \\
\text { and achieved stable IRWST injection. }\end{array}$ \\
\hline
\end{tabular}


TABLE 6.1.

Summary of AP1000 Matrix Tests Performed

\begin{tabular}{|l|l|l|}
\hline \multicolumn{1}{|c|}{ Test ID } & \multicolumn{1}{|c|}{ Description } \\
$\begin{array}{l}\text { OSU-AP1000-10 } \\
\text { (DBA-05-D) }\end{array}$ & $\begin{array}{l}\text { Inadvertent ADS Actua- } \\
\text { tion }\end{array}$ & $\begin{array}{l}\text { Objective/Summary } \\
\text { The objective of this test was to repeat a AP600 and to obtain ther- } \\
\text { fal-hydraulic data for an inadvertent ADS-1 actuation. The test per- } \\
\text { formed met the specified conditions. The top of the heater bundle } \\
\text { was always covered during this event. The test was performed for } \\
\text { about 11,000 seconds. The transient continued through ADS actua- } \\
\text { tion and achieved stable IRWST injection. }\end{array}$ \\
\hline $\begin{array}{l}\text { OSU-AP1000-11 } \\
\text { 3-Jun-2004 }\end{array}$ & $\begin{array}{l}\text { AP1000 1/2" CL4 Break } \\
\text { w/ 3 of 4 ADS4 }\end{array}$ & $\begin{array}{l}\text { The objective of this test was to obtain thermal-hydraulic data for a } \\
\text { fied conditions. The top of the heater bundle was always covered dur- } \\
\text { ing this event. The test was performed for about } 15,750 \text { seconds. The } \\
\text { transient continued through ADS actuation and achieved stable } \\
\text { IRWST injection. }\end{array}$ \\
\hline
\end{tabular}

\subsection{APEX1000/600 Test Results Comparisons}

Among the design basis events tested are the limiting small break LOCA accidents; a double-ended break of a Direct Vessel Injection (DVI) line, and a cold leg break with a 2-inch equivalent diameter. Both tests were performed assuming a single failure in the passive core cooling system. The limiting single failure for AP1000 is the loss of venting capacity from one of the four ADS Stage 4 valves. For both of these tests, the results were compared to the corresponding test from AP600. ${ }^{7}$ The comparison results are presented in non-dimensional form.

\subsection{Doubled-ended DVI Break}

For this test, the facility was operated at full power and full flow. At the start of the test, one of the two DVI lines is assumed to break. The pumps are tripped off, the steam generators are isolated and the reactor power follows a decay heat curve. In addition, plant protection logic opens the isolation valves on the CMTs and the PRHR heat exchanger. The break causes a rapid depressurization as shown in Figure 7.1. Loss of one DVI results in half of the passive injection capacity being lost. In addition, the CMT on the broken DVI line drains quickly resulting in early actuation of the ADS valves. 


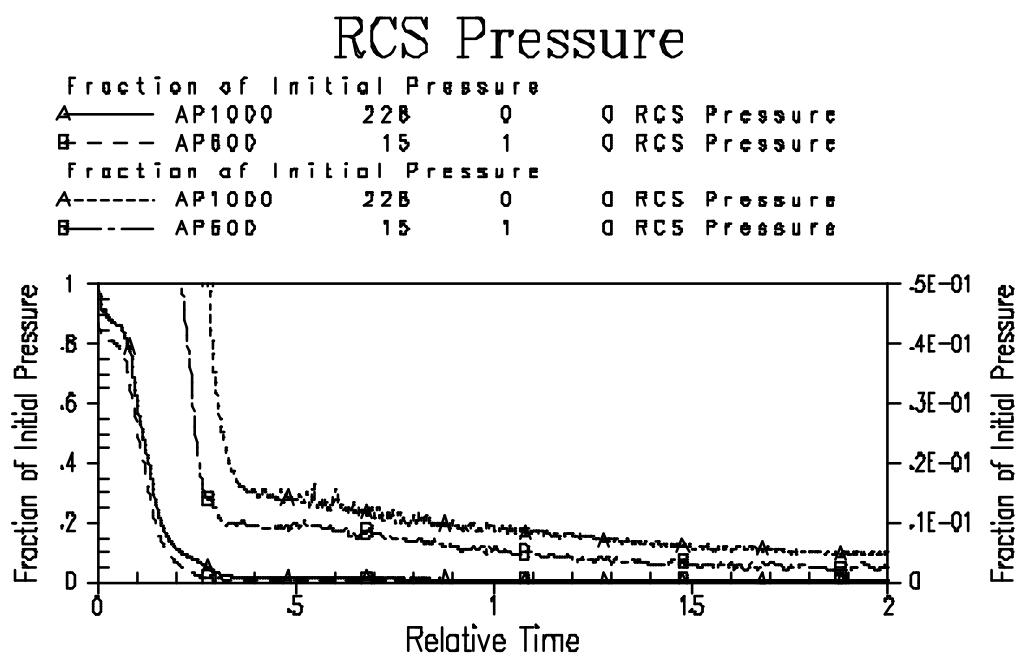

The increased venting quickly reduces the pressure in the RCS to the point where accumulator injects begins. As the CMT on the broken DVI continues to drain, the actuation setpoint is reached for the fourth stage ADS valves. This further reduces the pressure resulting in IRWST gravity injection and transition to stable long-term cooling. Figure 7.2 shows the core makeup water injection flow from the intact DVI line. There is a smooth transition from CMT flow to accumulator flow. After the accumulators are empty, CMT flow continues. The RCS pressure falls and IRWST injection is established prior to the CMT emptying. Figure 7.3 depicts the collapsed liquid level in the core for this test. The minimum core inventory occurs after blowdown and initial ADS actuation, and is lower for AP1000 due to the higher core power. Core thermocouples showed no heater rod heat up and the core remained covered for the entire test. 
FIGURE 7.2

FIGURE 7.3
APEX1000/600 DVI Break Comparison - Core Makeup Flow

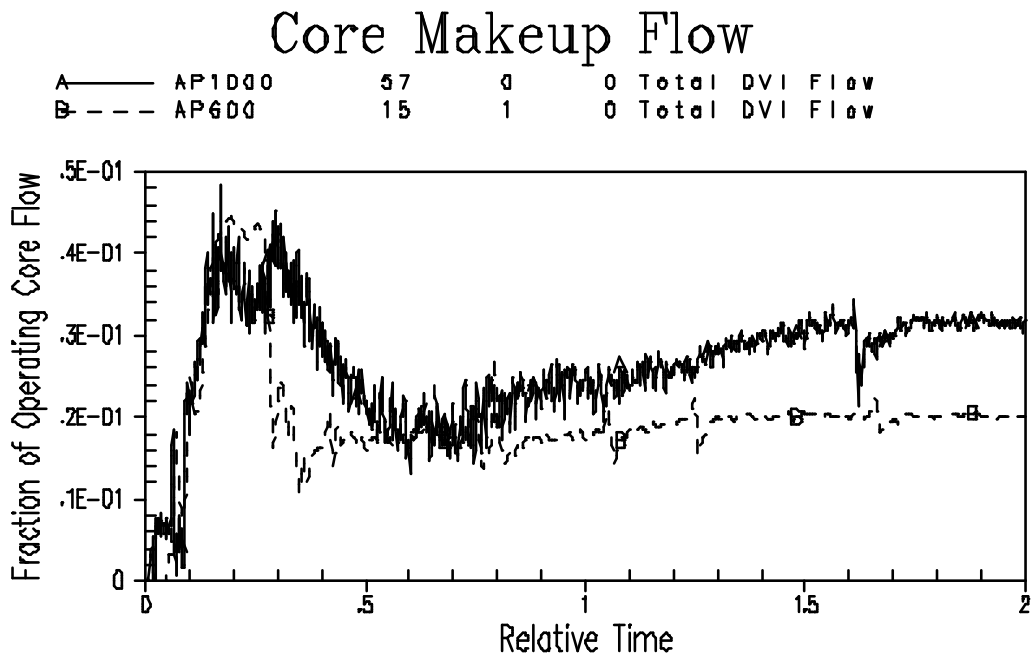

APEX1000/600 DVI Break Comparison - Core Collapsed Liquid Level

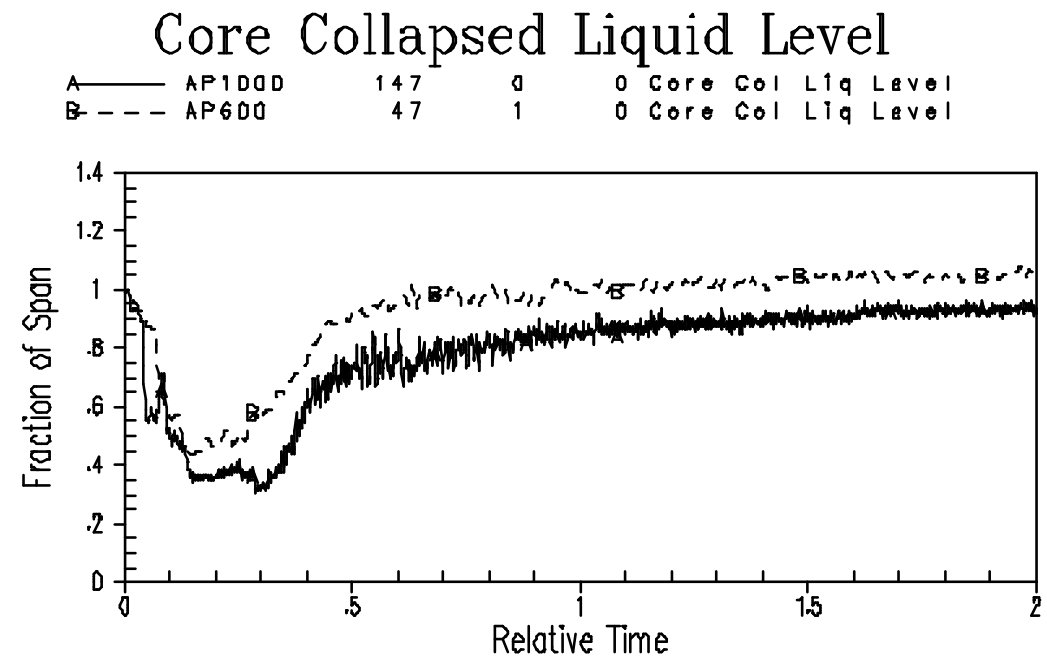




\subsection{Two-inch Cold Leg Break}

For this test, the facility was operating at full power and full flow. At the start of the test, a 2-inch break is assumed to occur in one of the four cold legs. The pumps are tripped off, the steam generators are isolated and the reactor power follows the decay heat curve. In addition, plant protection logic opens the isolation valves on the CMTs and the PRHR heat exchanger. The break causes a rapid depressurization as is shown in Figure 7.4.

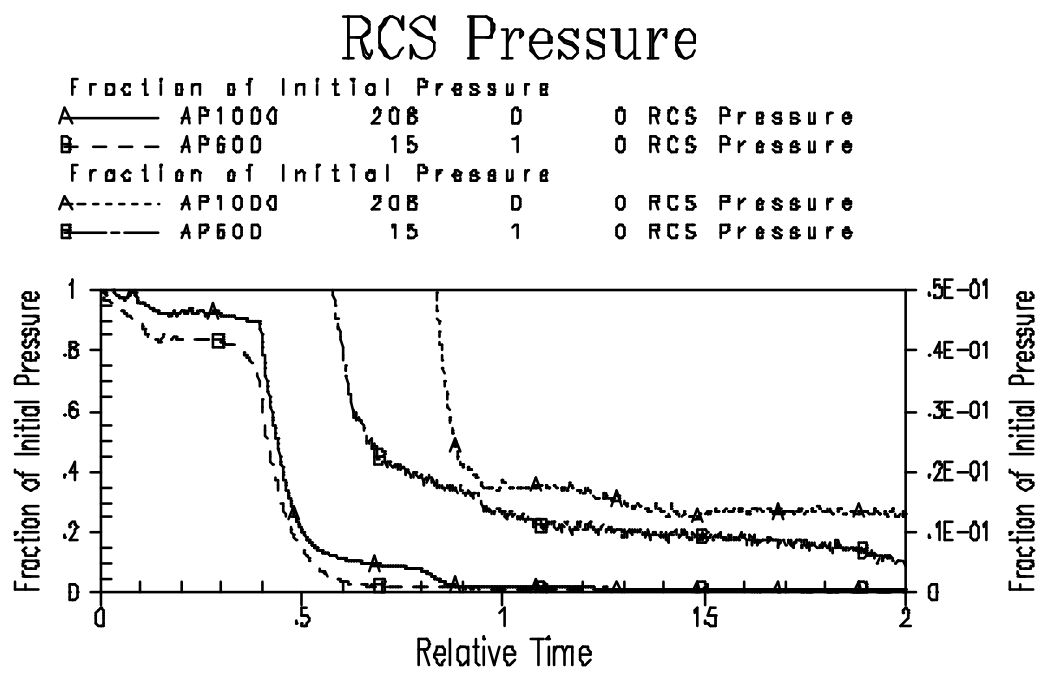

Unlike the DVI break which has early ADS actuation, the RCS loses inventory only through the break until a level is formed in the cold legs. At this time, steam enters the CMT-Cold Leg balance line and the CMTs then begin to drain. The core makeup flow through both DVI lines is shown in Figure 7.5. 


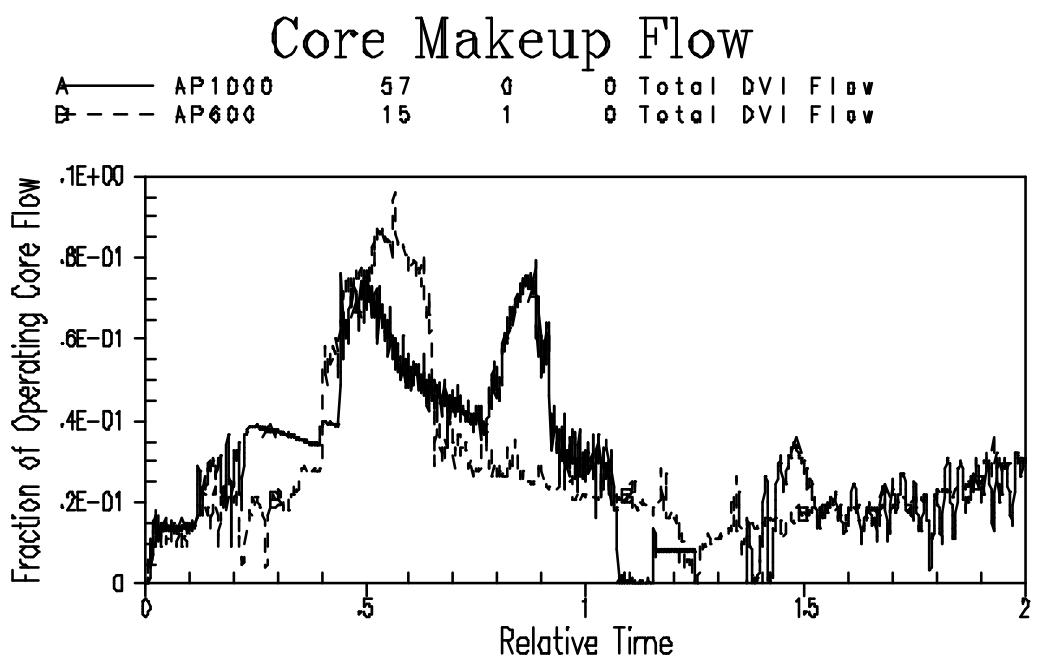

The CMT level reaches the ADS actuation point and the RCS pressure falls more rapidly causing the accumulators to inject. As the accumulators empty, the CMTs continue to inject until the ADS-4 actuation setpoint is reached. For APEX1000, the CMT flow stops before the RCS pressure reaches the cut-in point for gravity injection from the IRWST. This is different from the APEX600 test where there is a smooth transition between CMT injection and IRWST injection. Figure 7.6 shows the collapsed liquid level in the core for this test. The minimum core inventory is not as severe as for the DVI break, but is lower for AP1000. Core thermocouples showed no heater rod heat up and the core remains covered for the entire test. 
FIGURE 7.6
APEX1000/600 DVI Break Comparison2-inch Cold Leg Break Comparison - Core Collapsed Liquid Level

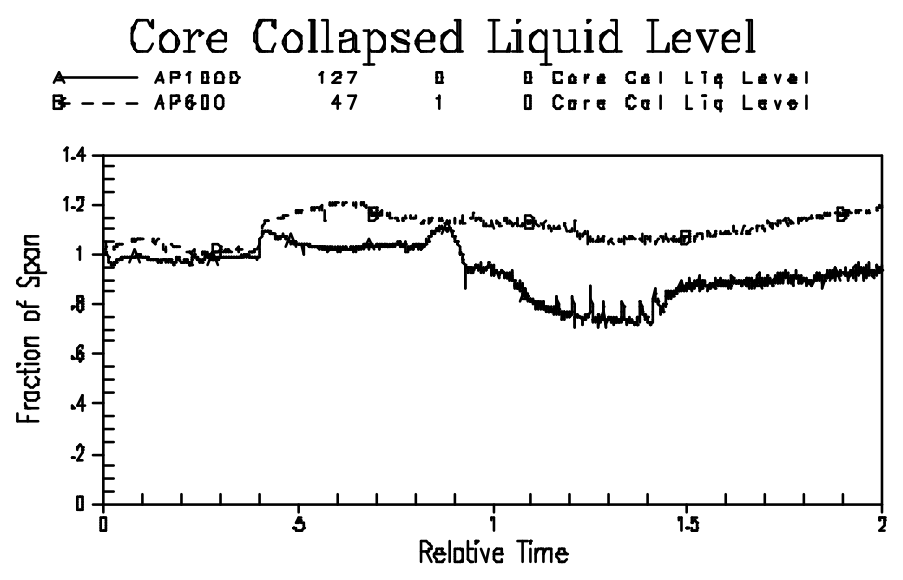

\subsection{APEX1000 Test Comparison to Code Calculations}

A 10CRF50 Appendix-K analysis of the AP1000 small-break LOCA was performed by Westinghouse Electric Corporation using the NOTRUMP computer code. NOTRUMP is a one-dimensional, two-phase thermal hydraulic analysis code that was used to support the design certification of the AP600. The vessel model consists of core nodes with a flat power profile, a multi-node downcomer, an upper plenum and an upper head. The remainder of the RCS includes the primary loop piping consisting of two hot legs and four cold legs, the surge line, pressurizer, reactor coolant pumps, and the steam generator tubes. The passive core cooling system includes two accumulators, two CMTs, the IRWST, the PRHR heat exchanger, and two DVI lines. Also modeled are the three ADS valve trains at the top of the pressurizer and the two fourth-stage ADS valves off the hot legs.

NOTRUMP was used to analyze an array of breaks including inadvertent actuation of the ADS (no break), hot leg and cold leg breaks and DVI line breaks. 
Two of the cases; the double-ended DVI and the 2-inch cold leg break were also simulated with the APEX1000 test facility. To determine the adequacy of the facility scaling and the NOTRUMP simulations, the test results were modified by the scaling ratios in Table 4-1. The scaled results for these two tests were plotted against the NOTRUMP simulations for comparison.

\subsection{Doubled-ended DVI Break}

For this test, the facility was operated at full power and full flow. At the start of the test, one of the two DVI lines is assumed to break. The pumps are tripped off, the steam generators are isolated and the reactor power follows a decay heat curve. In addition, plant protection logic opens the isolation valves on the CMTs and the PRHR heat exchanger. The break causes a rapid depressurization of the RCS. The RCS pressure for the plant and the scaled test is shown in Figure 8.1. A comparison of the plant calculation to the scaled test data shows good agreement.

Loss of one DVI results in half of the passive injection capacity being lost. In addition, the CMT on the broken DVI line quickly drains resulting in early actuation of the ADS valves at the top of the pressurizer. The increased venting quickly reduces the pressure in the RCS to the point where accumulator injection begins. As the CMT on the broken DVI continues to drain, the actuation setpoint is reached for the fourth stage ADS valves following a staged delay time. Figure 8.2 shows the CMT level on the broken DVI side. This further reduces the pressure resulting in IRWST gravity injection and transition to stable long-term cooling. Figure 8.3 shows the core makeup water injection flow from the intact DVI line for the plant and the scaled test results. The downcomer liquid level is shown in Figure 8.4. NOTRUMP overpredicts the accumulator flow, and predicts a later time for IRWST injection. Otherwise, the timing and magnitude of the injection flows is well predicted. The level in the downcomer is lower for NOTRUMP, but the timing and trends are well predicted. Core 
thermocouples showed no heater rod heat up and the core remained covered for the entire test.

FIGURE 8.1

APEX1000/NOTRUMP DVI Break Comparison - RCS Pressure
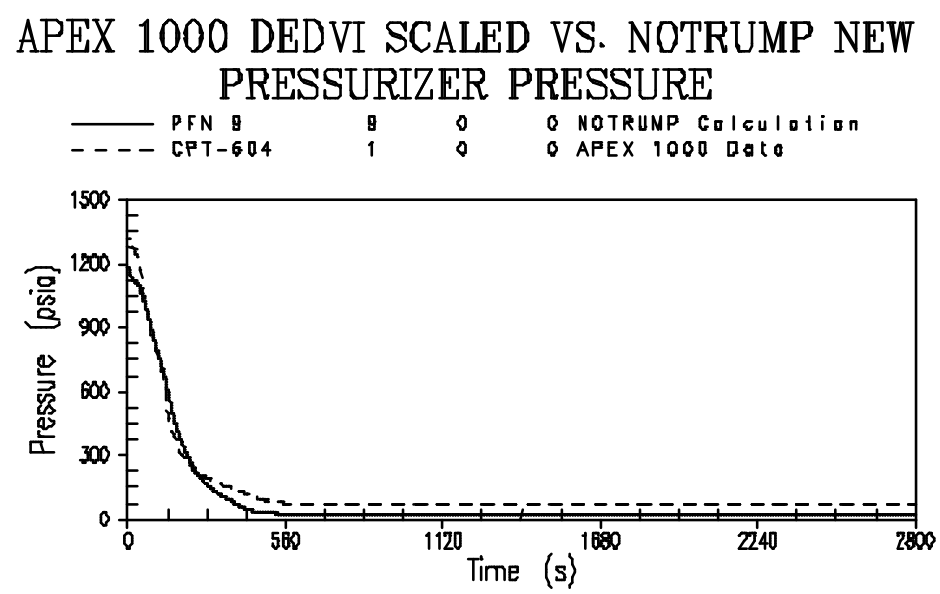

FIGURE 8.2

APEX 1000 DEDVI SCALED VS. NOTRUMP NEW CMT 1 LEVEL

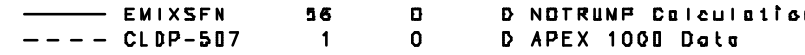

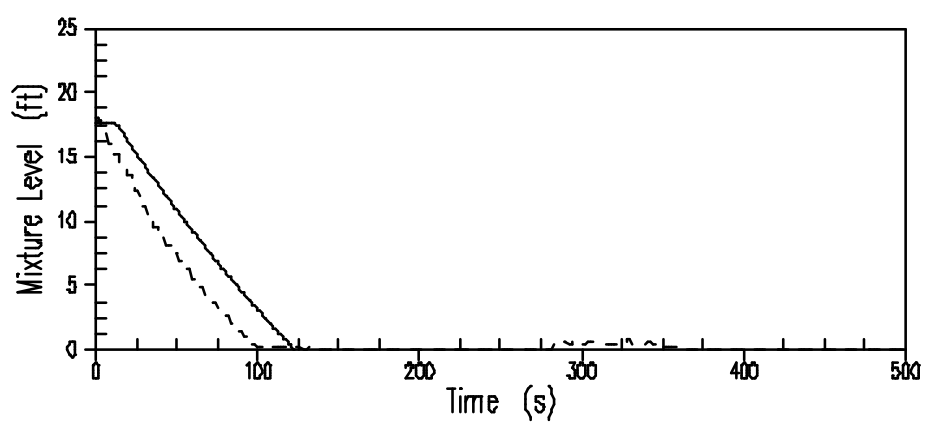


FIGURE 8.3

FIGURE 8.4
APEX1000/NOTRUMP DVI Break Comparison - Intact DVI Flow
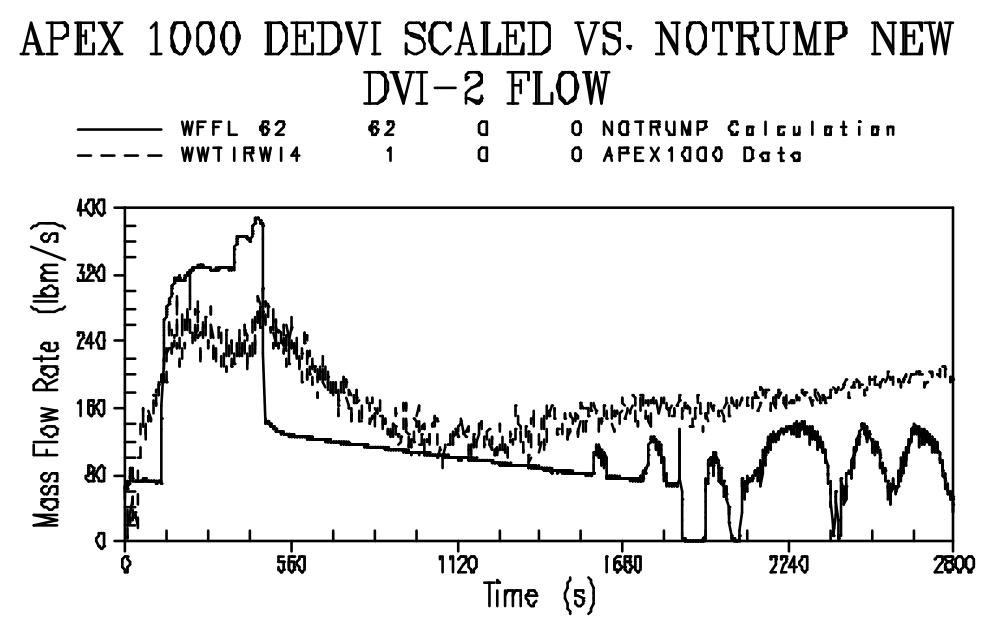

APEX1000/NOTRUMP DVI Break Comparison - RPV Downcomer Level

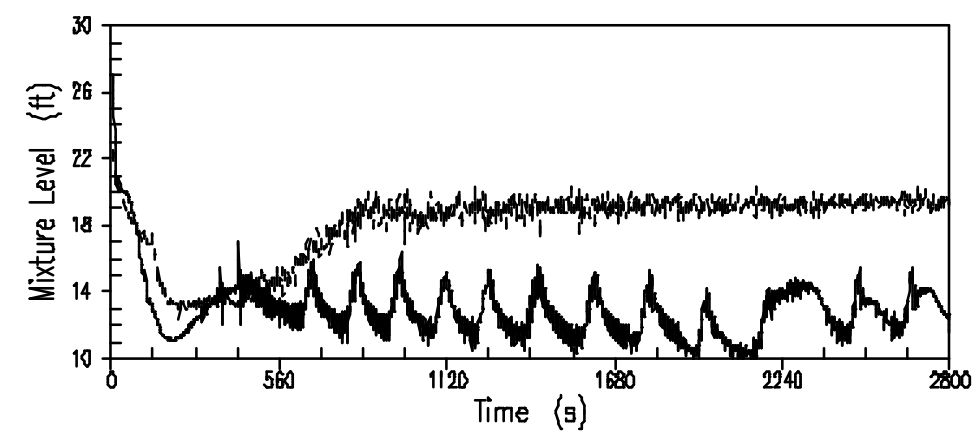




\subsection{Two-inch Cold Leg Break}

For this test, the facility was operating at full power and full flow. At the start of the test, a 2-inch equivalent diameter break is assumed in one of the four cold legs. The pumps are tripped off, the steam generators are isolated and the reactor power follows the decay heat curve. In addition, plant protection logic opens the isolation valves on the CMTs and the PRHR heat exchanger. The break causes a less rapid depressurization of the RCS compared to the larger DVI break. Figure 8.5 shows the RCS pressure for the plant and the scaled test results.
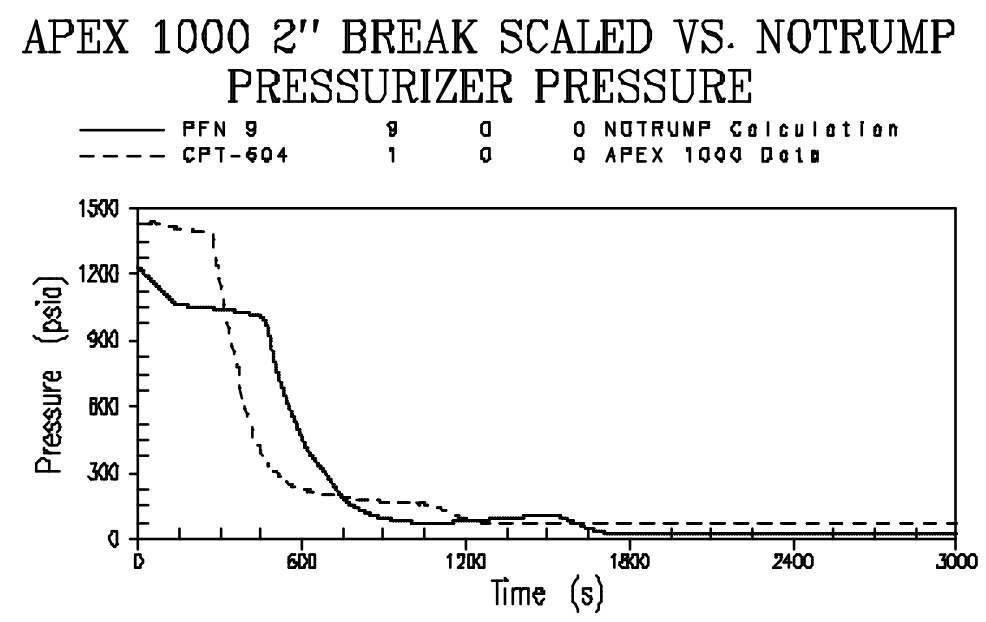

Unlike the DVI break which has early ADS actuation, the RCS loses inventory only through the break until a level is formed in the cold legs. At this time, steam enters the CMT-Cold Leg balance line and the CMTs begin to drain. The timing of the ADS depends on the rate of CMT draining. Figure 8.6 shows the CMT level. Once again there is good agreement between NOTRUMP and the scaled test results. The total core makeup flow through both DVI lines is shown 
in Figure 8.7. The CMT level reaches the ADS actuation point and the RCS pressure falls more rapidly causing the accumulators to inject. As the accumulators empty, the CMTs continue to inject until the ADS-4 actuation setpoint is reached. For both the plant simulation and the test, there is an injection gap as the CMT flow stops before the RCS pressure reaches the cut-in point for gravity injection from the IRWST. Figure 8.8 shows the downcomer level. Core thermocouples showed no heater rod heat up, thus the core remains covered for the entire test.
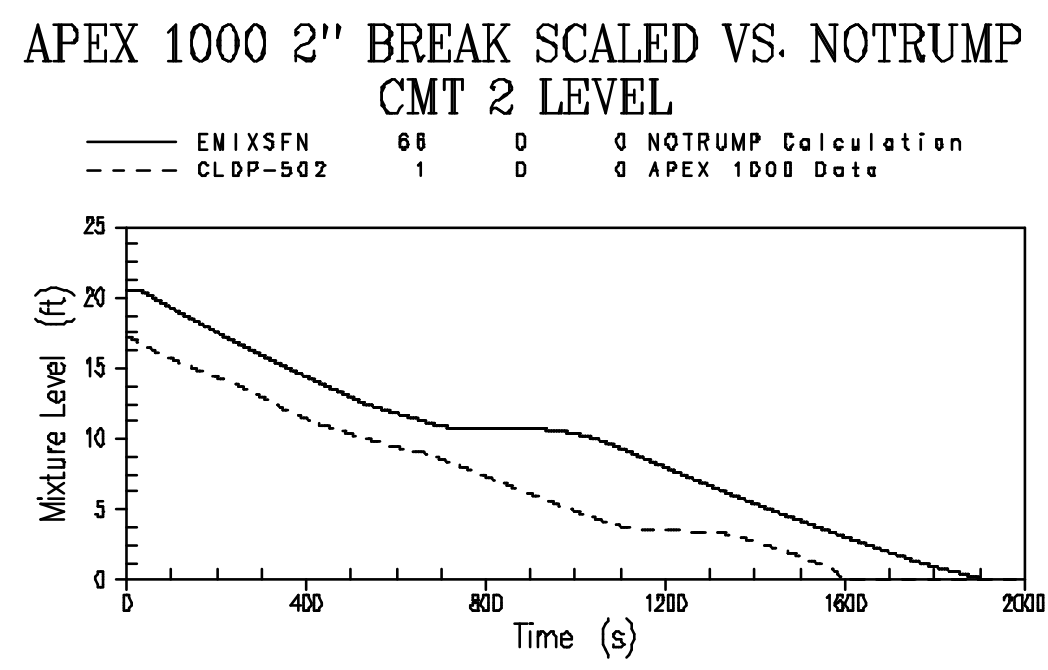
APEX $10002^{\prime \prime}$ BREAK SCALED VS. NOTRUMP TOTAL DVI FLOW
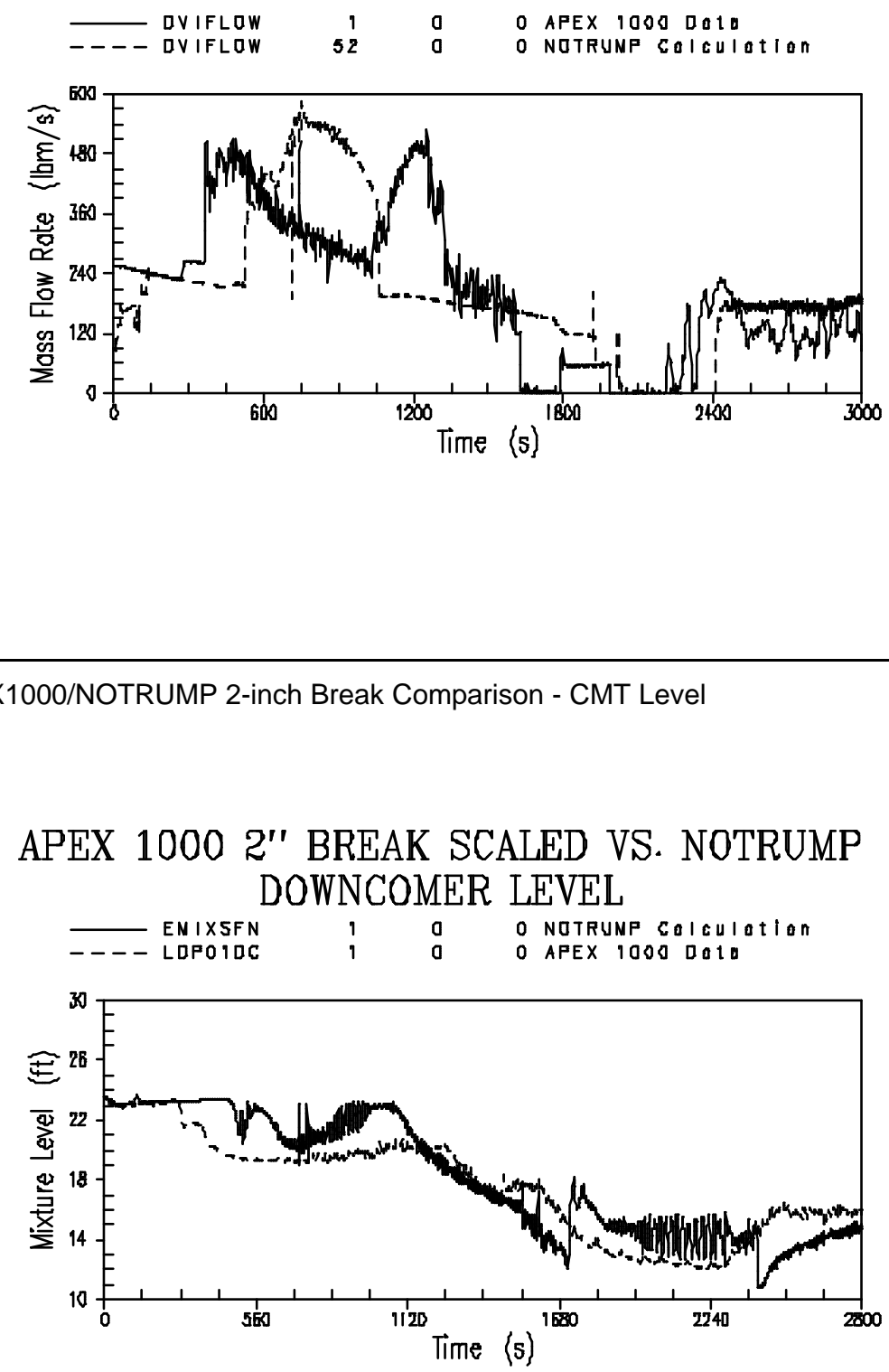


\subsection{Conclusions}

The data comparisons between AP1000 and AP600 follow roughly the same trends validating the scaling and component sizing of PXS components used in APEX 1000 despite the 67 percent increase in core power. The results of the AP1000 design basis accident tests show that even with worst-case single failures adequate core cooling was maintained throughout the duration of the test. The NOTRUMP code calculations were also in good agreement to scaled the APEX1000 test data.

\subsection{Budget and Milestones}

Table 10.1 identifies the graduate students and that participated in this research program and their tasks.

\section{TABLE 10.1.}

Student Participation in NERI Research During FY2003

\begin{tabular}{|l|l|l|}
\hline \multicolumn{1}{|c|}{ Student } & Year 03 & \multicolumn{1}{c|}{ Research Tasks } \\
\hline Kent Abel & Ph.D. & $\begin{array}{l}\text { Analysis: ADS-4 Characterization and Scaling Method- } \\
\text { ology }\end{array}$ \\
\hline Aleksey Rezvyi & Ph.D. & $\begin{array}{l}\text { Analysis: AP1000 Integral System Data Analysis and } \\
\text { Reporting }\end{array}$ \\
\hline Piyush Sabharwall & M.S. & $\begin{array}{l}\text { Analysis: AP1000 Integral System Data Analysis and } \\
\text { Reporting }\end{array}$ \\
\hline Jesse Skinner & B.S. & $\begin{array}{l}\text { Project: CFD Analysis of Pressurizer Surge Line Flood- } \\
\text { ing }\end{array}$ \\
\hline You Yao & Ph.D. & Thesis: Assessment of Upper Plenum Pool Entrainment \\
\hline Yeon-Jung Yoo & Ph.D. & $\begin{array}{l}\text { Analysis: Multi-Loop Flow Stability and AP1000 Inte- } \\
\text { gral System Data Analysis and Reporting }\end{array}$ \\
\hline Eric Young & Ph.D. & Testing: Upper Plenum Pool Entrainment Measurements \\
\hline
\end{tabular}

Table 10.2 provides the status of each major project milestone. It includes the milestone description, start date, completion date and status of completion. 


\section{TABLE 10.2.}

TABLE 10.3.

\section{Milestone Status Table}

\begin{tabular}{|c|c|c|c|}
\hline Milestone & $\begin{array}{c}\text { Start } \\
\text { Date }\end{array}$ & $\begin{array}{c}\text { Completion } \\
\text { Date }\end{array}$ & $\begin{array}{c}\text { Percent } \\
\text { Complete }\end{array}$ \\
\hline Task 1: Facility Scaling Analysis & & & \\
\hline Draft Scaling Analysis & $8 / 1 / 2001$ & $3 / 30 / 2002$ & $100 \%$ \\
\hline Pool Entrainment Scaling Analysis & $3 / 27 / 2002$ & $12 / 30 / 2002$ & $100 \%$ \\
\hline Scaling Analysis Report Issued & & $5 / 15 / 2003$ & $100 \%$ \\
\hline Task 2: APEX Facility Modification Design & & & \\
\hline Design of APEX Facility Modifications & $1 / 1 / 2002$ & $6 / 30 / 2002$ & $100 \%$ \\
\hline Design of Upper Plenum Internals & $3 / 27 / 2002$ & $12 / 30 / 2002$ & $100 \%$ \\
\hline Facility Description Report Issued & & $5 / 15 / 2003$ & $100 \%$ \\
\hline Task 3: Facility Modifications & $\mathbf{4 / 1 / 2 0 0 2}$ & $\mathbf{1 / 3 0 / 2 0 0 3}$ & $\mathbf{1 0 0 \%}$ \\
\hline Task 4: Test Plan Development & $\mathbf{3 / 3 0 / 2 0 0 2}$ & $\mathbf{1 2 / 3 0 / 2 0 0 3}$ & $\mathbf{1 0 0 \%}$ \\
\hline Task 5: Testing Program & & & \\
\hline Phase 1 & $2 / 28 / 2003$ & $9 / 30 / 2003$ & $100 \%$ \\
\hline Phase 2 & $10 / 1 / 2003$ & $7 / 30 / 2004$ & $100 \%$ \\
\hline Task 6: Test Analyses & $\mathbf{4 / 3 0 / 2 0 0 4}$ & $\mathbf{7 / 3 0 / 2 0 0 4}$ & $\mathbf{1 0 0 \%}$ \\
\hline
\end{tabular}

The actual vs. budgeted project expenses are provided in Table 10.3. As shown the project was completed on budget. The project started in the fourth quarter of FY01. The cumulative project expenses and budget through project completion are provided graphically below in Figure 10.1.

Budget Data

\begin{tabular}{|c|c|c|c|c|}
\hline \multicolumn{2}{|c|}{} & $\begin{array}{c}\text { Approved } \\
\text { Spending } \\
\text { Plan }\end{array}$ & $\begin{array}{c}\text { Actual } \\
\text { Spent } \\
\text { Date }\end{array}$ \\
\hline \multicolumn{3}{|c|}{ Phase/Budge Period } & Total & Total \\
\hline & From & To & & \\
\hline Year 1 & $8 / 1 / 2001$ & $7 / 31 / 2002$ & $\$ 865,000$ & $\$ 805,000$ \\
\hline Year 2 & $8 / 1 / 2002$ & $7 / 31 / 2003$ & $\$ 537,000$ & $\$ 595,000$ \\
\hline Year 3 & $8 / 1 / 2003$ & $7 / 31 / 2004$ & $\$ 397,000$ & $\$ 399,000$ \\
\hline \multicolumn{2}{|l|}{ Totals } & $\mathbf{\$ 1 , 7 9 9 , 0 0 0}$ & $\mathbf{\$ 1 , 7 9 9 , 0 0 0}$ \\
\hline
\end{tabular}




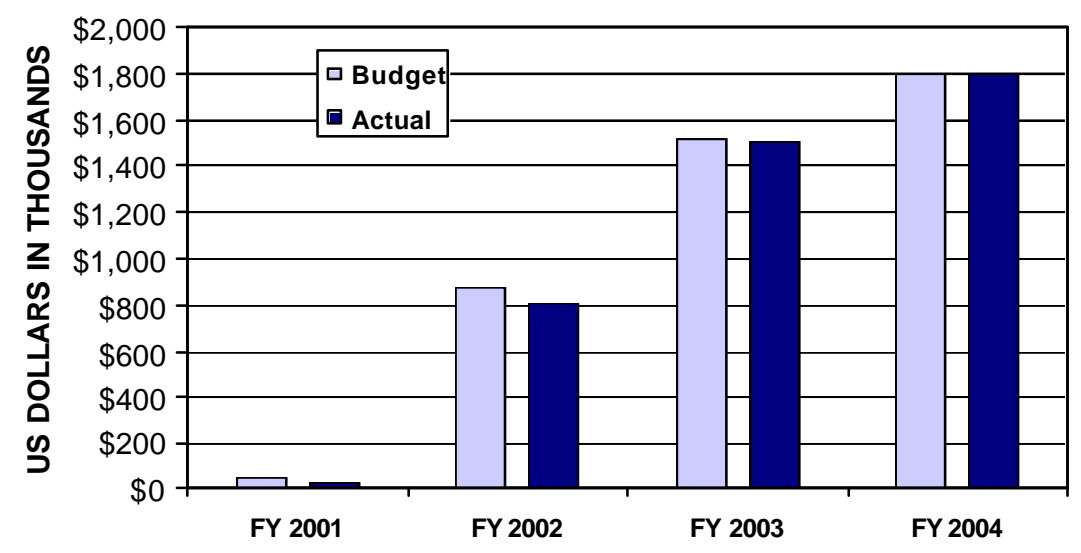

\subsection{References}

1. Westinghouse. AP1000 Plant Description and Analysis Report, WCAP-15612, December 2000.

2. Westinghouse. AP1000 Plant Parameters, APP-GW-G0-002, Revision 1, July 11, 2002

3. Westinghouse. AP1000 PIRT and Scaling Assessment, WCAP-15613, February 2001

4. Reyes, J.N. and L.E. Hochreiter, AP600 Low-Pressure Integral Systems Test at Oregon State University- Facility Scaling Report, WCAP-14270, January 1995.

5. Reyes, J.N., Scaling Assessment for the Design of the APEX-1000 Test Facility, 2002.

6. Reyes, J.N., et al, OSU APEX-1000 Test Facility Description Report, OSU-APEX03002 (Revision 0), May 2003.

7. Wright, R.F., et al, WCAP-14293, AP600 Low-Pressure Integral Systems Test at Oregon State University Test Analysis Report, July 1995. 


\section{Appendix A -- OSU-AP1000-01}




\title{
Oregon State University
}

Department of Nuclear Engineering

\author{
AdVAnCED THERMAl Hydraulic \\ RESEARCH LABORATORY
}

TEST SUMMARY REPORT

OSU-AP1000-01

AP1000 DOUbLe-Ended DVI WITH 3 OF 4 ADS 4

Revision 0 


\title{
TEST SUMMARY REPORT
}

\author{
OSU-AP1000-01
}

\section{AP1000 DOUbLE-ENDED DVI WITH 3 OF 4 ADS 4}
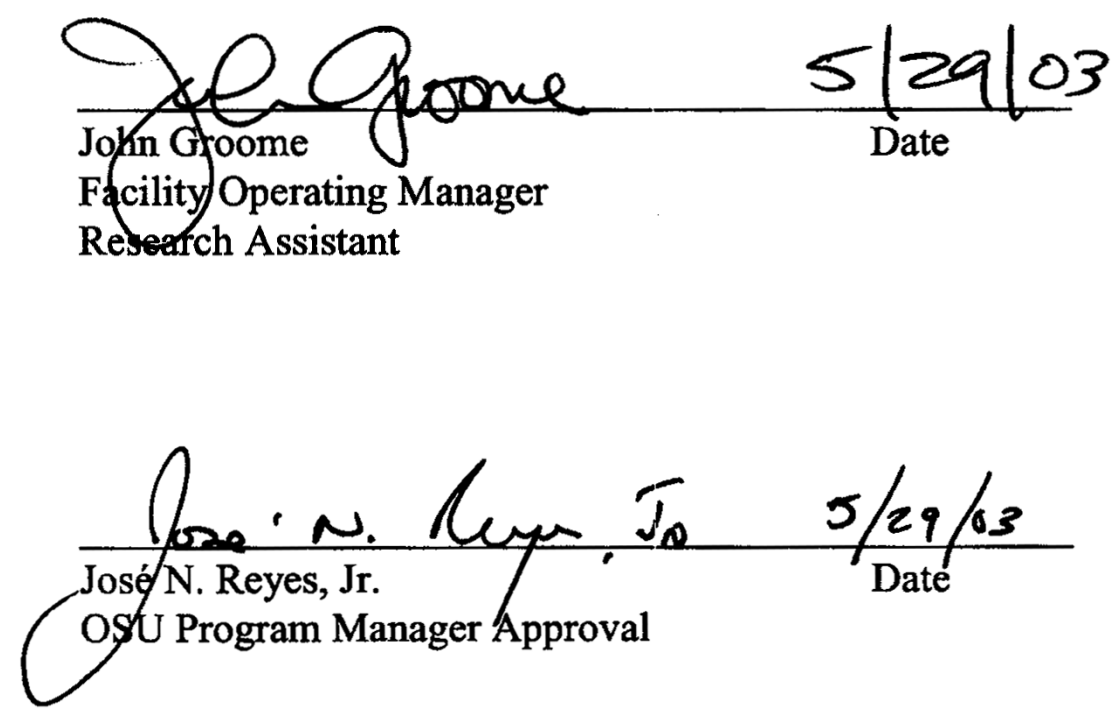

Oregon State University

116 Radiation Center

Corvallis, Oregon 97331

(C) 2003 Oregon State University

All Rights Reserved 



\section{WESTINGHOUSE PROPRIETARY INFORMATION NOTICE}

This report contains information proprietary to Westinghouse Electric Company. A non-proprietary version of this report has also been prepared for distribution to the United States Nuclear Regulatory Commission (NRC).

In order to conform to the requirements of 10 CFR 2.790 of the Commission's regulations concerning the protection of proprietary information so submitted to the $\mathrm{NRC}$, the information which is proprietary in the proprietary version of this document is contained within brackets, and where the proprietary information has been deleted in the non-proprietary versions, only the brackets remain (the information that was contained within the brackets in the proprietary versions having been deleted). The justification for claiming the information so designated as proprietary is indicated in both versions by means of lower case letters (a) through (f) located as a superscript immediately following the brackets enclosing each item of information being identified as proprietary or in the margin opposite such information. These lower case letters refer to the types of information Westinghouse customarily holds in confidence identified in Sections (4)(ii)(a) through (4)(ii)(f) of the affidavit accompanying the transmittal of these documents pursuant to 10 CFR 2.790(b)(1).

\section{COPYRIGHT NOTICE}

This document bears an Oregon State University copyright notice. The NRC is permitted to make the number of copies of the information contained in these reports which are necessary for its internal use in connection with generic and plant-specific reviews and approvals as well as the issuance, denial, amendment, transfer, renewal, modification, suspension, revocation, or violation of a license, permit, order, or regulation subject to the requirements of $10 \mathrm{CFR} 2.790$ regarding restrictions on public disclosure to the extent such information has been identified as proprietary by Westinghouse, copyright protection notwithstanding. With respect to the non-proprietary versions of these reports, the NRC is permitted to make the number of copies beyond these necessary for its internal use which are necessary in order to have one copy available for public viewing in the appropriate docket files in the public document room in Washington, DC and in local public document rooms as may be required by NRC regulations if the number of copies submitted is insufficient for this purpose. Copies made by the NRC must include the copyright notice in all instances and the proprietary notice if the original was identified as proprietary. 



\section{TABLE OF CONTENTS}

$\underline{\text { Section }}$

$\underline{\text { Title }}$

$\underline{\text { Page }}$

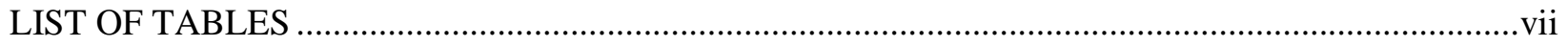

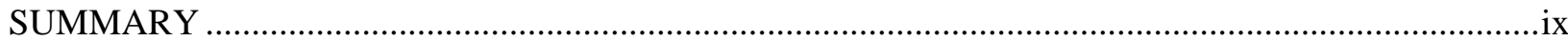

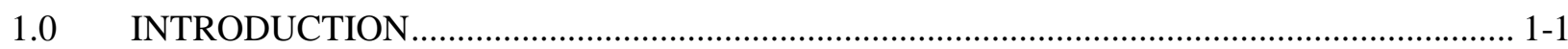

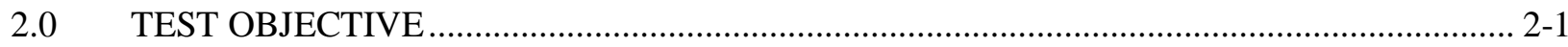

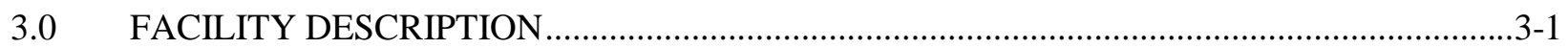

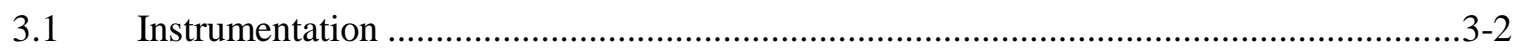

4.0 OREGON STATE UNIVERSITY TESTING PROGRAM MATRIX ................................... 4-1

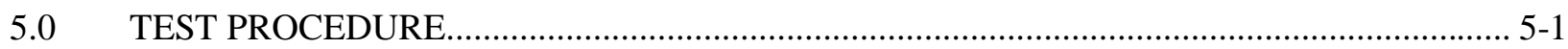

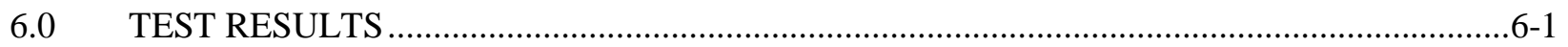

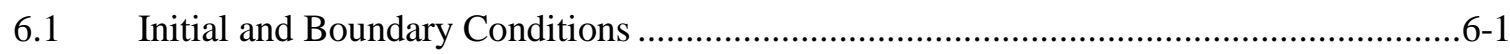

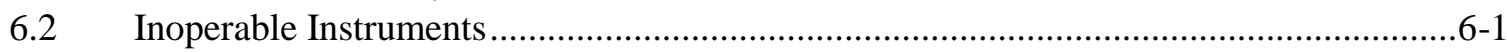

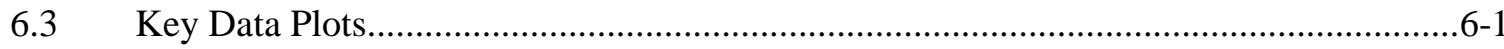

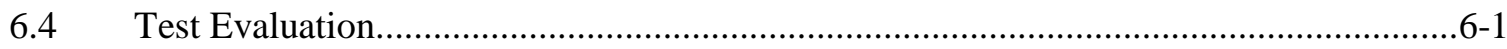

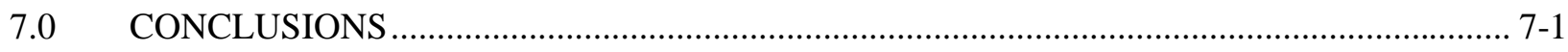

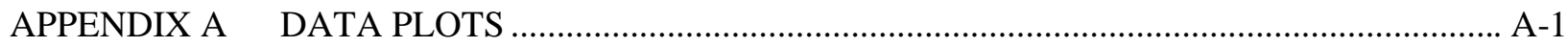

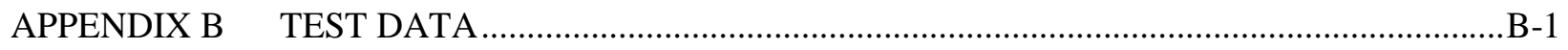





\section{LIST OF TABLES}

$\underline{\text { Table }}$

Table 4-1

Table 6-1

Table 6-2

Table 6-3

Table 6-4

\section{$\underline{\text { Title }}$}

$\underline{\text { Page }}$

OSU Test Matrix (Specified May 1, 2003)... 4-2

Actual Test Initial Conditions 6-3

Sequence of Events 6-5

Inoperable Instruments for DBA-01-D Test ........................................................ 6-7

Data Plots for Test Summary Report for DBA-01-D by Component. . $6-8$ 



\section{SUMMARY}

This report covers the test DBA-01, double-ended Direct Vessel Injection (DVI) line break simulation loss-of-coolant accident (LOCA) performed on February 27, 2003. The objective of this test was to obtain thermal-hydraulic data for a double-ended DVI line break simulation. The test performed met the specified conditions. The top of the heater bundle was always covered during this event. The test was performed for about 15,000 seconds. The transient continued through Automatic Depressurization System (ADS) actuation, core makeup tank (CMT), accumulator, in-containment refueling water storage tank (IRWST) injection, and sump recirculation injection.

This report presents the initial assessment of the test data collected. If this test is to be used by Westinghouse to support AP1000 Design Certification, additional validation of the use of this information will be documented separately. In the interim, the list of invalid data channels may change. 



\subsection{INTRODUCTION}

The Department of Nuclear Engineering at Oregon State University (OSU) is performing a series of tests for the U.S. Department of Energy (DOE). These tests are being conducted in the Advanced Plant Experiment (APEX-1000) test facility, which is a reduced pressure and height model of the two-loop Westinghouse AP1000 pressurized water reactor. The purpose of the testing is to:

- evaluate the thermal-hydraulic performance of the passive safety systems of the full-scale AP1000, and

- to assess and validate the safety analyses techniques and computer codes used in predicting the transient system behavior.

The AP1000 Long-Term Cooling Test is a 1/4 height scale, low-pressure integral systems test simulating thermal-hydraulic phenomenon for the AP1000 passive safety systems for small-break loss-of-coolant accidents (LOCAs) and long-term cooling. It accurately models the details of the AP1000 geometry, including the primary system, the passive safety systems, and a part of the non-safety grade Chemical and Volume Control System, as well as a partial non-safety grade Normal Residual Removal System. The interconnecting pipe routings are also duplicated in the model.

The overall objective of the Long-Term Cooling Test program is to obtain test data at various modes of operation. The OSU experiments will examine the passive safety system response for the small-break and large-break LOCA transition into long-term cooling. (The list of the tests to be performed is in the OSU Test Matrix provided in Section 4.0.) The facility permits a range of small-break LOCAs to be simulated at different locations on the primary system, such as the cold leg, hot leg, core makeup tank (CMT) cold leg pressure balance line, and direct vessel injection line. The break orientation (top or bottom of the cold leg) may also be studied. Selected tests continue into the long-term cooling, post-accident mode in which the passive safety injection is from the reactor sump as well as the incontainment refueling water storage tank (IRWST). A large-break, post-accident, long-term cooling situation will also be simulated. 



\subsection{TEST OBJECTIVE}

The purpose of this test is to obtain thermal-hydraulic data for a double-ended DVI line break simulation. The break is located in the DVI-1 line close to the DVI-1 nozzle as it enters the reactor vessel. The data obtained from the test will be used to verify the AP1000 thermal-hydraulic computer codes for AP1000 phenomena such as gravity injection, natural convection, and post-accident long-term core cooling behavior.

The acceptance criteria for the OSU tests are as follows:

- Test initial conditions will be achieved within a specified tolerance.

- Set points will be achieved within an acceptable tolerance band.

- All instrumentation should be operational before the test.

- Any critical instruments not operating will be identified to the test engineer before the tests. These instruments must be operational before and during the test or exceptions should be approved.

- A zero check of LDPs, DPs, and FDPs will be performed. 



\subsection{FACILITY DESCRIPTION}

A detailed facility description for the OSU test facility is documented in Reference 1. The OSU test facility has been specifically scaled, designed, and constructed to investigate the AP600 passive safety system behavior and to provide data for safety analysis computer code validation. The facility has been modified to model the AP1000.

The scaled test design accurately models the details of the AP1000 geometry, including the primary system and the pipe routings and layout for the passive safety systems. A detailed scaling report (Reference 2) was used to develop the test design modifications. The primary system consists of one hot leg and two cold legs with two active pumps and an active steam generator for each loop. Two CMTs are connected to one primary loop, and the pressurizer is connected to the other primary loop as in the AP1000 plant design. Gas-driven accumulators are connected to the DVI lines. The discharge lines from the CMT, and one-of-two IRWST and reactor sump lines are connected to each DVI line. The Automatic Depressurization System (ADS), consisting of stages 1, 2 and 3, simulates either one or two of the independent trains used in the AP1000. The two-phase flow from the ADS stages 1-to-3 is separated in a swirl-vane separator and the liquid and vapor flows are measured to obtain the total ADS flow rate. The separated flow streams are then recombined and discharged into the IRWST through a sparger, preserving the mass and energy flow into the IRWST. The injection from the reactor sump is also simulated. Note that the OSU facility models both AP1000 primary and secondary sumps. The primary sump collects the condensate return, the liquid break flow, and the liquid flow from the fourth-stage ADS; and will provide long-term injection to the reactor vessel. The secondary sump simulates the portions of containment that will remain dry during most events. This sump will collect water only when the primary sump reaches its overflow level, and provides no injection to the reactor vessel.

The time period for the experimental simulations includes not only the IRWST injection, but also the draining of the IRWST and the sump injection to simulate the long-term cooling of the AP1000. This simulation could be from several hours to a day. The time scale for the OSU test facility is one-half; that is, events occur in half the normal time. To model the long-term cooling aspects of the transient, the two-phase flow from the break is separated in a swirl-vane separator and the liquid and vapor portions of the total flow are measured. The liquid fraction of the flow is discharged to the reactor primary sump as in the AP1000 plant. The vapor is discharged to the atmosphere. The capability exists to return a portion of the equivalent liquid flow to the IRWST and primary sump to simulate the condensate return from the passive containment to the IRWST and primary sump. A similar approach is also used for the fourth-stage ADS valve on the hot leg. The two-phase flow is separated in a swirl-vane separator, the two streams are measured, and the liquid phase is discharged into the primary sump while the vapor flow is discharged to the atmosphere. Again, the capability exists to return a portion of the liquid equivalent added to the IRWST and primary sump. In addition, all other steam vents from the facility are measured (e.g., the IRWST vent), and a portion of the liquid equivalent may be added back to the facility. Note that not all of the steam discharge would be returned as liquid equivalent. A portion of the discharge would be removed to simulate the steam that is not available for recirculation because it provides containment pressurization. The IRWST and primary sump can be pressurized in the OSU facility to simulate the containment pressurization following a postulated LOCA.

A multi-tube passive residual heat removal (PRHR) heat exchanger is located in the IRWST. The heat exchanger uses the same C-tube design as the AP1000 and has two instrumented tubes to obtain wall heat 
fluxes during the tests. There are primary fluid thermocouples, wall thermocouples, and differential pressure drop measurements to determine when the heat exchanger begins to drain. The IRWST is also instrumented with strings of fluid thermocouples, to determine the degree of mixing within the tank and to assess the temperature of the coolant that is delivered to the test vessel.

The reactor vessel for the OSU tests includes a 0.914-meter (3-foot) heated core consisting of forty-eight 0.025-meter (1-inch) diameter heater rods. The heater rods have a top skewed power shape. The $1000 \mathrm{~kW}$ of electrical power available at the OSU test site will be used to simulate decay heat. Wall thermocouples are swaged inside the heater rods to measure the heater rod wall temperature.

Thermocouple rods in the heater rod bundle measure the axial coolant temperature distribution. The scaled flow volume in the core is preserved as well as the flow volume in the test vessel upper plenum. There are simulated reactor internals in the upper plenum to preserve the flow area and to correctly scale the fluid volume. The reactor vessel includes an annular downcomer into which the four cold legs and the two DVI lines are connected. The hot legs penetrate the reactor annulus and connect with the loops. The AP1000 reactor vessel neutron reflector is simulated using a ceramic liner to reduce the metal heat release to the coolant.

There were no special/unique requirements for the test other than those specified in the Initial Conditions. The specified conditions were verified on the control board prior to test implementation.

\subsection{Instrumentation}

The instrumentation has been designed to calculate a transient mass and energy balance on the test facility. All two-phase flow streams exiting the facility are separated, and each component is measured separately as a single phase flow using conventional measurement devices such as magnetic flow meters and vortex flow meters. Note that magnetic flow meters are not designed for two-phase flow and will indicate erratically. Also note, the vortex flow meters are referenced to $212^{\circ} \mathrm{F}$ and the LDPs are referenced to $60^{\circ} \mathrm{F}$. All vertical components have differential pressure cells that act as level instruments to measure the transient mass change in the component. The hot and cold leg diameters are sufficiently large in the OSU test facility so that a narrow-range differential pressure cell can be used to determine if the flow becomes stratified.

Single flow measurements are made of the CMT, accumulator, IRWST, and sump flows into the reactor vessel through the DVI lines.

Various types of instrumentation are provided in the test facility; for example, thermocouples for coolant and wall temperatures, flow meters, pressure transducers, differential pressure transducers, and weigh tanks. 


\subsection{OREGON STATE UNIVERSITY TESTING PROGRAM MATRIX}

The test matrix for the OSU test facility is shown in Table 4-1. To satisfy the test objectives, several transients will be performed to provide data on the AP1000 passive safety system response for a range of break sizes, locations, orientations, and single failure assumptions. The break size orifices are scaled based on simulating a 1-inch, 2-inch, or 4-inch pipe break.

The designation for this test is DBA-01-D, which identifies the test as a design basis double-ended DVI line break. The test matrix may be adjusted for future tests based on results and insights gained with each test. 


\begin{tabular}{|c|c|c|}
\hline \multicolumn{3}{|c|}{$\begin{array}{c}\text { TABLE 4-1 } \\
\text { OSU TEST MATRIX (SPECIFIED MAY 1, 2003) }\end{array}$} \\
\hline Test Title & Break Location and Size & Single Failure Assumed \\
\hline DBA-01-D & $\begin{array}{l}\text { Double-ended DVI Line break with } \\
\text { continuation into long-term cooling }\end{array}$ & Fail 1 of 2 lines in 1 ADS- 4 train \\
\hline DBA-02-D & $\begin{array}{l}\text { Double-ended DVI Line break with } \\
\text { continuation into long-term cooling }\end{array}$ & $\begin{array}{l}\text { Fail } 1 \text { of } 2 \text { lines in } 1 \text { ADS- } 4 \text { train } \\
\text { (Adjusted ADS-4 Resistance) }\end{array}$ \\
\hline DBA-03-D & 2-inch Cold Leg Break & Fail 1 of 2 lines in 1 ADS- 4 train \\
\hline TR-01-D & $\begin{array}{l}\text { Transition Test ADS4 opening, } 85 \text { psig initial } \\
\text { pressure and decay power } 480 \mathrm{sec}\end{array}$ & $\begin{array}{l}\text { Fail } 1 \text { of } 2 \text { lines in } 1 \text { ADS-4 train } \\
\text { (No ADS 1-3) }\end{array}$ \\
\hline TR-02-D & $\begin{array}{l}\text { Transition Test ADS4 opening, } 125 \text { psig initial } \\
\text { pressure and decay power } 480 \mathrm{sec}\end{array}$ & $\begin{array}{l}\text { Fail } 1 \text { of } 2 \text { lines in } 1 \text { ADS-4 train } \\
\text { (No ADS 1-3) }\end{array}$ \\
\hline TR-03-D & $\begin{array}{l}\text { Transition Test ADS4 opening, } 85 \text { psig initial } \\
\text { pressure and decay power } 1800 \mathrm{sec}\end{array}$ & $\begin{array}{l}\text { Fail } 1 \text { of } 2 \text { lines in } 1 \text { ADS-4 train } \\
\text { (No ADS 1-3) }\end{array}$ \\
\hline TR-04-D & $\begin{array}{l}\text { Transition Test ADS4 opening, } 85 \text { psig initial } \\
\text { pressure and decay power } 480 \mathrm{sec}\end{array}$ & $\begin{array}{l}\text { No Failure of ADS-4 lines assumed } \\
\qquad \text { (No ADS 1-3) }\end{array}$ \\
\hline EN-01-D & $\begin{array}{l}\text { Entrainment Test with Revised Upper Internals, } \\
1000 \mathrm{~kW} \text { reactor power, } 14.7 \mathrm{psi}\end{array}$ & Fail 1 of 2 lines in 1 ADS- 4 train \\
\hline EN-01-D & $\begin{array}{l}\text { Entrainment Test with Revised Upper Internals, } \\
700 \mathrm{~kW} \text { reactor power, } 14.7 \mathrm{psi}\end{array}$ & Fail 1 of 2 lines in 1 ADS- 4 train \\
\hline PRA-01-D & PRA Test - DEDVI with no accumulators & No ADS-4 Failure assumed \\
\hline PRA-02-D & $\begin{array}{l}\text { PRA Test - 3-inch Hot Leg Break with no } \\
\text { CMTs }\end{array}$ & No ADS-4 Failure assumed \\
\hline
\end{tabular}




\subsection{TEST PROCEDURE}

The test was performed as per a written procedure. There were no special/unique requirements for the test other than those specified in the initial conditions in Table 6-1. The specified conditions were checked on the control board before test implementation.

The appropriate prerequisites were completed and initial conditions were satisfied. The required break simulation piping and break instrumentation were installed per P\&ID drawing OSU 600904, Rev. 1. A break spool insert simulating the double-ended break in AP1000 was installed in the break spool in DVI horizontal line. DVI-1 nozzle flow from the reactor is directed horizontally into the break separator. The combined flow of DVI line (from CMT-1, accumulator 1, primary sump, and IRWST) is connected horizontally to the primary sump tank. The 100-percent flow nozzle was installed in the ADS 4-1 (on hot leg 1) and the 50-percent flow nozzle was installed in ADS 4-2 (on hot leg 2). Flow nozzles that simulate full flow for ADS-1, ADS-2, and ADS-3 were installed. As per the AP600 tests, ADS-3 has been scaled for full flow from all three stages, and ADS-1 and ADS-2 are closed when ADS-3 is opened.

Fill and vent was performed per APEX Operations Manual Procedure OP-B.2. Instruments were checked for required calibration.

With the break valves TS-202 and TS-203 closed, flow was used to warm up the bypass line by opening isolation valves RCS-901 and RCS-902. After the appropriate prerequisites were completed and the test facility achieved specified initial conditions, the CMT warmup bypass line isolation valves RCS-901 and RCS-902 were closed to maintain the $<80^{\circ} \mathrm{F}$ condition at the top of the CMT- 1 . With the CMT balance line valves (RCS-529 and RCS-530) placed in the open and automatic mode, both CMTs reached the reactor coolant system (RCS) pressure.

Once all other initial conditions were satisfied, a break through TS-202 and TS-203 was initiated. The transient continued through ADS actuation, and CMT, accumulator, IRWST injection, and sump injection. Per Westinghouse instructions, ADS 4-2 was actuated automatically according to the actuation logic, and ADS4-1 was actuated manually after a 30-second delay. All other actions were automatic and required no operator action. 



\subsection{TEST RESULTS}

The test results for test DBA-01-D are provided in the following subsections.

\subsection{Initial and Boundary Conditions}

Table 6-1 provides a comparison of the specified and actual conditions for test DBA-01-D. The values in this table were averaged over approximately 2 minutes preceding the test. Test initial conditions were achieved for the steam generator pressure, pressurizer pressure, pressurizer level, steam generator 01 narrow-range level, and steam generator 02 narrow-range level. Test initial conditions for the hot leg temperature were found to be acceptable, and the results will not be adversely affected.

The actual power decay curves are provided in data plots in Appendix B. The measured maximum power was $863 \mathrm{~kW}$, which was less than the facility maximum power of $1000 \mathrm{~kW}$. The programmed decay heat curve was adjusted to account for this difference, and the differences between the actual and specified power decay are considered acceptable.

PT-501 and PT-502 pressure instruments indicate the pressure changes in the CMT-1 and CMT-2. CMT-1 (PT-501) and CMT-2 (PT-502) confirm that 1 minute after the test button was pushed, both CMTs reach RCS pressure.

The sequence of events is shown in Table 6-2. This table compares the actual sequence of events with the specified timing. As can be seen in this table, all the events occurred at or very near to when the event was planned.

\subsection{Inoperable Instruments}

Table 6-3 provides a list of the instrumentation channels considered inoperable for the DBA-01-D test.

\subsection{Key Data Plots}

Table 6-4 provides a list of the instrumentation channels sorted by component, and includes instrument number, units, and Test Summary Report plot number. Appendix A contains the Test Summary Report plots for this test for selected channels. The selection of channels was based on projecting an overall picture of the test results, which would then be examined by referring to the detailed data plots or tapes. Appendix B contains a CD containing all data channels for this test.

\subsection{Test Evaluation}

The following observations were made during the test:

1. The peak power before the test was initiated was $863 \mathrm{~kW}$. The decay heat curve was adjusted for this value. 
2. The liquid flow from ADS1-3 is non-zero after ADS-4 actuation. The liquid loop seal in the ADS1-3 line may have been blown out during the initial blowdown, and the flows after ADS-4 actuation should be ignored.

3. The switch over to sump injection occurs very late relative to pre-test predictions, and the sump level at this time is significantly higher than the IRWST level. Subsequent tests should re-scale the setpoint for sump switch over according to the latest AP1000 design.

4. The ADS-4 flow is adequately scaled for choked flow, but is too restrictive for unchoked flow. Subsequent tests should use a redesigned ADS-4 nozzle that is scaled correctly for both conditions. 


\begin{tabular}{|c|c|c|c|}
\hline \multicolumn{4}{|c|}{$\begin{array}{c}\text { TABLE 6-1 } \\
\text { ACTUAL TEST INITIAL CONDITIONS }\end{array}$} \\
\hline Conditions & Instrument No. & Actual & Comment \\
\hline Pressurizer Pressure & PT-604 & 370 psig & \\
\hline $\begin{array}{l}\text { Hot Leg Temperature } \\
\quad \# 1\end{array}$ & $\begin{array}{l}\text { TF-141* } \\
\text { TF-205 } \\
\text { TF-143 }\end{array}$ & $\begin{array}{l}424^{\circ} \mathrm{F} \\
427^{\circ} \mathrm{F} \\
428^{\circ} \mathrm{F}\end{array}$ & \\
\hline $\begin{array}{l}\text { Hot Leg Temperature } \\
\quad \# 2\end{array}$ & $\begin{array}{l}\text { TF-140* } \\
\text { TF-206 } \\
\text { TF-142 }\end{array}$ & $\begin{array}{l}426^{\circ} \mathrm{F} \\
426^{\circ} \mathrm{F} \\
427^{\circ} \mathrm{F}\end{array}$ & \\
\hline $\begin{array}{l}\text { Steam Generator (SG) } \\
\text { Pressure } \\
\quad \# 1 \\
\quad \# 2 \\
\text { Header }\end{array}$ & $\begin{array}{l}\text { PT-301* } \\
\text { PT-302* } \\
\text { PT-002 }\end{array}$ & $\begin{array}{l}287 \text { psig } \\
286 \text { psig } \\
251 \text { psig }\end{array}$ & \\
\hline Pressurizer Level & $\begin{array}{l}\text { LDP-601 uncompensated } \\
\text { LDP-601 } \\
\text { Compensated by SC-608 }\end{array}$ & $\begin{array}{l}68.5 \text { inches } \\
82.3 \text { inches }\end{array}$ & $\begin{array}{l}440^{\circ} \mathrm{F} \text { used for density } \\
\text { compensation }\end{array}$ \\
\hline $\begin{array}{l}\text { SG Level } \\
\quad \# 1 \mathrm{NR}\end{array}$ & $\begin{array}{l}\text { LDP-303 uncompensated } \\
\text { LDP-303 compensated by } \\
\text { average of TF-305 and } \\
\text { TF-307 }\end{array}$ & $\begin{array}{l}20.8 \text { inches } \\
25.1 \text { inches }\end{array}$ & $\begin{array}{l}413^{\circ} \mathrm{F} \text { used for density } \\
\text { compensation }\end{array}$ \\
\hline $\begin{array}{l}\text { SG Level } \\
\text { \#2 NR }\end{array}$ & $\begin{array}{l}\text { LDP-304 uncompensated } \\
\text { LDP-304 compensated by } \\
\text { average of TF-306 and } \\
\text { TF-308 }\end{array}$ & $\begin{array}{l}21.5 \text { inches } \\
25.2 \text { inches }\end{array}$ & $\begin{array}{l}414^{\circ} \mathrm{F} \text { used for density } \\
\text { compensation }\end{array}$ \\
\hline IRWST Temperature & TF-701 & $61^{\circ} \mathrm{F}$ & Accepted $\left(<80^{\circ} \mathrm{F}\right)$ \\
\hline $\begin{array}{l}\text { CMT Temperature } \\
\# 1 \\
\# 2\end{array}$ & $\begin{array}{l}\text { TF-529 } \\
\text { TF-532 }\end{array}$ & $\begin{array}{l}71.6^{\circ} \mathrm{F} \\
72^{\circ} \mathrm{F}\end{array}$ & Accepted $\left(<80^{\circ} \mathrm{F}\right)$ \\
\hline $\begin{array}{l}\text { Accumulator } \\
\text { Temperature } \\
\# 1 \\
\# 2\end{array}$ & $\begin{array}{l}\text { TF-403 } \\
\text { TF-404 }\end{array}$ & $\begin{array}{l}70.5^{\circ} \mathrm{F} \\
71^{\circ} \mathrm{F}\end{array}$ & Accepted $\left(<80^{\circ} \mathrm{F}\right)$ \\
\hline IRWST Level & LDP-701 & 93.3 inches & \\
\hline
\end{tabular}




\begin{tabular}{||c|c|c|c||}
\hline \multicolumn{4}{|c||}{ TABLE 6-1 (Continued) } \\
\multicolumn{1}{|c||}{ ACTUAL TEST INITIAL CONDITIONS } \\
\hline \hline Conditions & Instrument No. & Actual & Comment \\
\hline Accumulator Level & & & \\
$\# 1$ & LDP-401 & 35.7 inches & \\
$\# 2$ & LDP-402 & 37.7 inches & \\
\hline Accumulator Pressure & & & \\
$\# 1$ & PT-401 & $188.9 \mathrm{psig}$ & \\
$\# 2$ & PT-402 & $189.4 \mathrm{psig}$ & \\
\hline CMT Level & & & \\
$\# 1$ & LDP-507 & $57.7 \mathrm{inches}$ & \\
$\# 2$ & LDP-502 & $57.8 \mathrm{inches}$ & \\
\hline CMT Pressure & & & \\
$\# 1$ & PT-501 & $373.5 \mathrm{psig}$ & \\
$\# 2$ & PT-502 & $374.7 \mathrm{psig}$ & \\
\hline \hline
\end{tabular}

* These instruments are used to establish initial conditions at the test site. 


\begin{tabular}{|c|c|c|}
\hline \multicolumn{3}{|c|}{$\begin{array}{c}\text { TABLE 6-2 } \\
\text { SEQUENCE OF EVENTS }\end{array}$} \\
\hline Event & Setpoint & Actual Time (sec) \\
\hline PB Depressed & N/A & -120 \\
\hline Break Valve(s) Open & 0 & 0 \\
\hline Feed Pump Trip & $3.6 \mathrm{sec}$ & 4 \\
\hline CMT01 Outlet Valve Open (RCS-535) & $6.1 \mathrm{sec}$ & 8 \\
\hline CMT02 Outlet Valve Open (RCS-536) & $6.1 \mathrm{sec}$ & 8 \\
\hline PRHR HX Outlet Valve Open (RCS-804) & $6.1 \mathrm{sec}$ & 8 \\
\hline RCP \#1 Trip & $8.6 \mathrm{sec}$ & 8 \\
\hline RCP \#2 Trip & $8.6 \mathrm{sec}$ & 8 \\
\hline RCP \#3 Trip & $8.6 \mathrm{sec}$ & 8 \\
\hline RCP \#4 Trip & $8.6 \mathrm{sec}$ & 8 \\
\hline CMT \#1 Level Low (LDP-507) & 41 inches & 72 \\
\hline CMT \#2 Level Low (LDP-502) & 41 inches & 413 \\
\hline ADS \#1 Actuation (RCS-601) & CMT Level Low $+15 \mathrm{sec}$ & 86 \\
\hline ADS \#2 Actuation (RCS-602) & CMT Level Low $+62 \mathrm{sec}$ & 132 \\
\hline ADS \#3 Actuation (RCS-603) & CMT Level Low + $122 \mathrm{sec}$ & 193 \\
\hline Low Reactor Pressure (P-107) & 40 psig & 195 \\
\hline IRWST Valve Actuation (RCS-711) & $\begin{array}{l}\text { Low Reactor Pressure } \\
\qquad(<40 \mathrm{psig})\end{array}$ & 197 \\
\hline IRWST Valve Actuation (RCS-712) & $\begin{array}{l}\text { Low Reactor Pressure } \\
\qquad(<40 \text { psig })\end{array}$ & 197 \\
\hline CMT \#1 Low Low Level (LDP-507) & 17.14 inches & 97 \\
\hline CMT \#2 Low Low Level (LDP-502) & 17.14 inches & 739 \\
\hline ADS 4-1 Actuation (RCS-615) & $\begin{array}{l}\text { CMT Low Low (17.14") and } \\
\text { CMT Low }\left(41^{\prime \prime}\right)+180 \mathrm{sec}\end{array}$ & 250 \\
\hline ADS 4-2 Actuation (RCS-616) & $\begin{array}{l}\text { CMT Low Low (17.14") and } \\
\text { CMT Low }\left(41^{\prime \prime}\right)+180 \mathrm{sec}\end{array}$ & 283 \\
\hline Sump Valve Actuation (CSS-909) & IRWST Level Low Low & 11945 \\
\hline
\end{tabular}




\begin{tabular}{|c|c|c|}
\hline \multicolumn{3}{|c|}{$\begin{array}{c}\text { TABLE 6-2 (Continued) } \\
\text { SEQUENCE OF EVENTS }\end{array}$} \\
\hline Event & Setpoint & Actual Time (sec) \\
\hline Sump Valve Actuation (CSS-910) & IRWST Level Low Low & 11945 \\
\hline $\begin{array}{l}\text { Accumulator Injection } \\
\# 1 \text { (FMM-401) }\end{array}$ & N/A & 0 \\
\hline $\begin{array}{l}\text { Accumulator Injection } \\
\# 2 \text { (FMM-402) }\end{array}$ & N/A & 107 \\
\hline $\begin{array}{l}\text { IRWST Injection } \\
\text { DVI \#1 (FMM-701) }\end{array}$ & N/A & 300 \\
\hline $\begin{array}{l}\text { IRWST Injection } \\
\text { DVI \#2 (FMM-702) }\end{array}$ & N/A & 910 \\
\hline $\begin{array}{l}\text { Accumulator Empty } \\
\text { \#1 (LDP-401) }\end{array}$ & N/A & 205 down to 1.2 inch level \\
\hline $\begin{array}{l}\text { Accumulator Empty } \\
\text { \#2 (LDP-402) }\end{array}$ & N/A & 362 down to 0.1 inch level \\
\hline $\begin{array}{l}\text { CMT Empty } \\
\quad \# 1 \text { (LDP-507) }\end{array}$ & N/A & 115 down to 0.1 inch level \\
\hline $\begin{array}{l}\text { CMT Empty } \\
\text { \#2 (LDP-502) }\end{array}$ & N/A & 920 \\
\hline Sump Injection DVI \# 1 (FMM-901) & N/A & 11945 \\
\hline Sump Injection DVI \# 2 (FMM-902) & N/A & 11945 \\
\hline
\end{tabular}

* Level indication $<2$ inches 


\begin{tabular}{|c|c|c|}
\hline \multicolumn{3}{|c|}{$\begin{array}{c}\text { TABLE 6-3 } \\
\text { INOPERABLE INSTRUMENTS FOR DBA-01-D TEST }\end{array}$} \\
\hline Instrument Number & Instrument Type & Inoperable Description \\
\hline $\begin{array}{l}\text { TW-202 } \\
\text { TW-204 } \\
\text { TW-205 } \\
\text { TW-206 } \\
\text { TW-209 } \\
\text { TW-803 } \\
\text { TW-804 }\end{array}$ & Thermocouple & Inoperative \\
\hline TH-603 & $\begin{array}{l}\text { Thermocouple measuring heater } \\
\text { temperature }\end{array}$ & Inoperative \\
\hline $\begin{array}{l}\text { FMM-202, } \\
\text { FMM-601 }\end{array}$ & Magnetic flow meter & $\begin{array}{l}\text { Inoperative } \\
\text { Measured ADS1-3 liquid flow after ADS4 } \\
\text { actuation (loop seal potentially blown) }\end{array}$ \\
\hline $\begin{array}{l}\text { TF-170 } \\
\text { TF-221 } \\
\text { TF-509 } \\
\text { TF-512 }\end{array}$ & $\begin{array}{l}\text { Thermocouple measuring fluid } \\
\text { temperature }\end{array}$ & Inoperative \\
\hline FVM-905 & Vortex flow meter & Erratic \\
\hline
\end{tabular}




\begin{tabular}{|c|c|c|c|c|}
\hline \multicolumn{5}{|c|}{$\begin{array}{c}\text { TABLE 6-4 } \\
\text { DATA PLOTS FOR TEST SUMMARY REPORT FOR DBA-01-D BY COMPONENT }\end{array}$} \\
\hline Component & Channel & Units & TSR-Plot & Comment \\
\hline $\begin{array}{l}\text { Reactor Vessel } \\
\text { Pressure }\end{array}$ & PT-107 & psig & 1 & \\
\hline Reactor Vessel Level & LDP-127 & inch of $\mathrm{H}_{2} \mathrm{O}$ & 2 & \\
\hline $\begin{array}{l}\text { Reactor Vessel } \\
\text { Downcomer Level }\end{array}$ & LDP-140 & inch of $\mathrm{H}_{2} \mathrm{O}$ & 3 & \\
\hline $\begin{array}{l}\text { Cold Leg \#1 Fluid } \\
\text { Temperature }\end{array}$ & TF-107 & ${ }^{\circ} \mathrm{F}$ & 4 & \\
\hline $\begin{array}{l}\text { Cold Leg \#2 Fluid } \\
\text { Temperature }\end{array}$ & TF-108 & ${ }^{\circ} \mathrm{F}$ & 5 & \\
\hline $\begin{array}{l}\text { Cold Leg \#3 Fluid } \\
\text { Temperature }\end{array}$ & TF-103 & ${ }^{\circ} \mathrm{F}$ & 6 & \\
\hline $\begin{array}{l}\text { Cold Leg \#4 Fluid } \\
\text { Temperature }\end{array}$ & TF-104 & ${ }^{\circ} \mathrm{F}$ & 7 & \\
\hline $\begin{array}{l}\text { Reactor Vessel Fluid } \\
\text { Temp Upper Head }\end{array}$ & TF-120 & ${ }^{\circ} \mathrm{F}$ & 8 & \\
\hline $\begin{array}{l}\text { RCS Hot Leg \#1 } \\
\text { Temperature }\end{array}$ & TF-143 & ${ }^{\circ} \mathrm{F}$ & 9 & \\
\hline $\begin{array}{l}\text { RCS Hot Leg \#2 } \\
\text { Temperature }\end{array}$ & TF-142 & ${ }^{\circ} \mathrm{F}$ & 10 & \\
\hline Pressurizer Pressure & PT-604 (WR) & psig & 11 & \\
\hline Pressurizer Pressure & PT-603 (LP Indication) & psig & 12 & \\
\hline $\begin{array}{l}\text { Pressurizer Liquid } \\
\text { Level }\end{array}$ & LDP-601 & inch of $\mathrm{H}_{2} \mathrm{O}$ & 13 & $\begin{array}{l}\text { Sharp decrease } \\
\text { followed by rapid refill }\end{array}$ \\
\hline SG \#1 Tube Level & LDP-215 & inch of $\mathrm{H}_{2} \mathrm{O}$ & 14 & \\
\hline $\begin{array}{l}\text { SG \#1 Secondary } \\
\text { Pressure }\end{array}$ & PT-301 & psig & 15 & \\
\hline SG \#1 Feed Flow Rate & FMM-001 & gpm & 16 & \\
\hline SG \#2 Tube Level & LDP-218 & inch of $\mathrm{H}_{2} \mathrm{O}$ & 17 & \\
\hline $\begin{array}{l}\text { SG \#2 Secondary } \\
\text { Pressure }\end{array}$ & PT-302 & psig & 18 & \\
\hline
\end{tabular}




\begin{tabular}{|c|c|c|c|c|}
\hline \multicolumn{5}{|c|}{$\begin{array}{l}\text { TABLE 6-4 (Continued) } \\
\text { DATA PLOTS FOR TEST SUMMARY REPORT FOR DBA-01-D BY COMPONENT }\end{array}$} \\
\hline Component & Channel & Units & TSR-Plot & Comment \\
\hline $\begin{array}{l}\text { Accumulator \#2 } \\
\text { Pressure }\end{array}$ & PT-401 & psig & 19 & \\
\hline $\begin{array}{l}\text { Accumulator \#1 } \\
\text { Pressure }\end{array}$ & PT-402 & psig & 20 & \\
\hline $\begin{array}{l}\text { Accumulator \#1 Liquid } \\
\text { Level }\end{array}$ & LDP-401 & inch of $\mathrm{H}_{2} \mathrm{O}$ & 21 & \\
\hline $\begin{array}{l}\text { Accumulator \#2 Liquid } \\
\text { Level }\end{array}$ & LDP-402 & inch of $\mathrm{H}_{2} \mathrm{O}$ & 22 & \\
\hline $\begin{array}{l}\text { Accumulator \#1 Flow } \\
\text { Rate }\end{array}$ & FMM-401 & gpm & 23 & \\
\hline $\begin{array}{l}\text { Accumulator \#2 Flow } \\
\text { Rate }\end{array}$ & FMM-402 & gpm & 24 & \\
\hline $\begin{array}{l}\text { Accumulator \#1 Liquid } \\
\text { Discharge Temperature }\end{array}$ & TF-401 & ${ }^{\circ} \mathrm{F}$ & 25 & \\
\hline $\begin{array}{l}\text { Accumulator \#2 Liquid } \\
\text { Discharge Temperature }\end{array}$ & TF-402 & ${ }^{\circ} \mathrm{F}$ & 26 & \\
\hline CMT \#1 Liquid Level & LDP-507 & inch of $\mathrm{H}_{2} \mathrm{O}$ & 27 & \\
\hline CMT \#2 Liquid Level & LDP-502 & inch of $\mathrm{H}_{2} \mathrm{O}$ & 28 & \\
\hline CMT \#1 Flow Rate & FMM-501 & gpm & 29 & \\
\hline CMT \#2 Flow Rate & FMM-504 & gpm & 30 & \\
\hline $\begin{array}{l}\text { CMT \#1 Liquid } \\
\text { Temperature }\end{array}$ & TF-501 & ${ }^{\circ} \mathrm{F}$ & 31 & \\
\hline $\begin{array}{l}\text { CMT \#1 Liquid } \\
\text { Temperature }\end{array}$ & TF-529 & ${ }^{\circ} \mathrm{F}$ & 32 & \\
\hline $\begin{array}{l}\text { CMT \#2 Liquid } \\
\text { Temperature }\end{array}$ & TF-504 & ${ }^{\circ} \mathrm{F}$ & 33 & \\
\hline $\begin{array}{l}\text { CMT \#2 Liquid } \\
\text { Temperature }\end{array}$ & TF-532 & ${ }^{\circ} \mathrm{F}$ & 34 & \\
\hline PRHR Inlet Flow Rate & FMM-802 & gpm & 35 & \\
\hline PRHR Liquid Level & LDP-802 & inch of $\mathrm{H}_{2} \mathrm{O}$ & 36 & \\
\hline
\end{tabular}




\begin{tabular}{|c|c|c|c|c|}
\hline \multicolumn{5}{|c|}{$\begin{array}{l}\text { TABLE 6-4 (Continued) } \\
\text { DATA PLOTS FOR TEST SUMMARY REPORT FOR DBA-01-D BY COMPONENT }\end{array}$} \\
\hline Component & Channel & Units & TSR-Plot & Comment \\
\hline $\begin{array}{l}\text { PRHR Outlet Flow } \\
\text { Rate }\end{array}$ & FMM-804 & gpm & 37 & \\
\hline IRWST Liquid Level & LDP-701 & inch of $\mathrm{H}_{2} \mathrm{O}$ & 38 & \\
\hline $\begin{array}{l}\text { IRWST Discharge Line } \\
\text { \#1 Flow Rate }\end{array}$ & FMM-701 & gpm & 39 & \\
\hline $\begin{array}{l}\text { IRWST Discharge Line } \\
\text { \#2 Flow Rate }\end{array}$ & FMM-702 & gpm & 40 & \\
\hline $\begin{array}{l}\text { IRWST Fluid } \\
\text { Temperature }\end{array}$ & TF-701 & ${ }^{\circ} \mathrm{F}$ & 41 & \\
\hline $\begin{array}{l}\text { IRWST Fluid } \\
\text { Temperature }\end{array}$ & TF-709 & ${ }^{\circ} \mathrm{F}$ & 42 & \\
\hline $\begin{array}{l}\text { ADS 1-3 Separator } \\
\text { Pressure }\end{array}$ & PT-605 & psig & 43 & \\
\hline $\begin{array}{l}\text { ADS 1-3 Separator } \\
\text { Steam Flow Rate }\end{array}$ & FVM-601 & $\mathrm{scfm}$ & 44 & \\
\hline $\begin{array}{l}\text { ADS 1-3 Separator } \\
\text { Liquid Flow Rate }\end{array}$ & FMM-601 & gpm & 45 & \\
\hline $\begin{array}{l}\text { ADS 4-1 Separator } \\
\text { Pressure }\end{array}$ & PT-611 & psig & 46 & \\
\hline $\begin{array}{l}\text { ADS 4-2 Separator } \\
\text { Pressure }\end{array}$ & PT-610 & psig & 47 & \\
\hline $\begin{array}{l}\text { ADS 4-1 Separator } \\
\text { Steam Flow Rate }\end{array}$ & FVM-603 & $\mathrm{scfm}$ & 48 & \\
\hline $\begin{array}{l}\text { ADS 4-2 Separator } \\
\text { Steam Flow Rate }\end{array}$ & FVM-602 & $\mathrm{scfm}$ & 49 & \\
\hline $\begin{array}{l}\text { ADS 4-1 Separator } \\
\text { Liquid Flow Rate }\end{array}$ & FMM-603 & gpm & 50 & \\
\hline $\begin{array}{l}\text { ADS 4-2 Separator } \\
\text { Liquid Flow Rate }\end{array}$ & FMM-602 & gpm & 51 & \\
\hline Primary Sump Pressure & PT-901 & psig & 52 & \\
\hline $\begin{array}{l}\text { Primary Sump Liquid } \\
\text { Level }\end{array}$ & LDP-901 & inch of $\mathrm{H}_{2} \mathrm{O}$ & 53 & \\
\hline
\end{tabular}




\begin{tabular}{|c|c|c|c|c|}
\hline \multicolumn{5}{|c|}{$\begin{array}{l}\text { TABLE 6-4 (Continued) } \\
\text { DATA PLOTS FOR TEST SUMMARY REPORT FOR DBA-01-D BY COMPONENT }\end{array}$} \\
\hline Component & Channel & Units & TSR-Plot & Comment \\
\hline $\begin{array}{l}\text { Primary Sump } \\
\text { Injection Flow Rate }\end{array}$ & FMM-901 & gpm & 54 & \\
\hline $\begin{array}{l}\text { Primary Sump } \\
\text { Injection Flow Rate }\end{array}$ & FMM-902 & gpm & 55 & \\
\hline $\begin{array}{l}\text { Secondary Sump } \\
\text { Liquid Level }\end{array}$ & LDP-902 & inch of $\mathrm{H}_{2} \mathrm{O}$ & 56 & \\
\hline $\begin{array}{l}\text { Break Separator } \\
\text { Pressure }\end{array}$ & PT-905 & psig & 57 & \\
\hline $\begin{array}{l}\text { Break Separator Liquid } \\
\text { Level }\end{array}$ & LDP-905 & inch of $\mathrm{H}_{2} \mathrm{O}$ & 58 & \\
\hline $\begin{array}{l}\text { Break Separator Flow } \\
\text { to Primary Sump }\end{array}$ & FMM-905 & gpm & 59 & \\
\hline $\begin{array}{l}\text { BAMS Steam Flow } \\
\text { Rate }\end{array}$ & FVM-901 & $\mathrm{scfm}$ & 60 & \\
\hline $\begin{array}{l}\text { BAMS Steam Flow } \\
\text { Rate }\end{array}$ & FVM-902 & $\mathrm{scfm}$ & 61 & \\
\hline $\begin{array}{l}\text { BAMS/Primary Sump } \\
\text { Steam Flow Rate }\end{array}$ & FVM-903 & $\mathrm{scfm}$ & 62 & \\
\hline $\begin{array}{l}\text { BAMS/Separator } \\
\text { Steam Flow Rate } \\
-6 \text { inch Pipe }\end{array}$ & FVM-905 & $\mathrm{scfm}$ & 63 & \\
\hline $\begin{array}{l}\text { BAMS/Exhaust Line } \\
\text { Temp. }\end{array}$ & TF-916 & ${ }^{\circ} \mathrm{F}$ & 64 & \\
\hline $\begin{array}{l}\text { BAMS/Exhaust Line } \\
\text { Temp. }\end{array}$ & SC-917 & ${ }^{\circ} \mathrm{F}$ & 65 & \\
\hline $\begin{array}{l}\text { PZR Heater Input } \\
\text { Power }\end{array}$ & KW-601 & $\mathrm{kW}$ & 66 & \\
\hline $\begin{array}{l}\text { Core Power Input } \\
\text { Power }\end{array}$ & KW-101 & $\mathrm{kW}$ & 67 & \\
\hline $\begin{array}{l}\text { Core Power Input } \\
\text { Power }\end{array}$ & KW-102 & $\mathrm{kW}$ & 68 & \\
\hline $\begin{array}{l}\text { Core Power Input } \\
\text { Power }\end{array}$ & KW-103 & $\mathrm{kW}$ & 69 & \\
\hline
\end{tabular}




\begin{tabular}{||l|c|c|c|c||}
\hline \multicolumn{5}{|c||}{ TABLE 6-4 (Continued) } \\
\multicolumn{1}{|c|}{ DATA PLOTS FOR TEST SUMMARY REPORT FOR DBA-01-D BY COMPONENT } \\
\hline \hline $\begin{array}{l}\text { Component } \\
\text { Core Power Input } \\
\text { Power }\end{array}$ & Channel & Units & TSR-Plot & Comment \\
\hline $\begin{array}{l}\text { Reactor Vessel Liquid } \\
\text { Level Between Top of } \\
\text { Vessel - Upper Support } \\
\text { Plate }\end{array}$ & LDP-115 & kW & 70 & \\
\hline $\begin{array}{l}\text { Reactor Vessel Liquid } \\
\text { Level Between bottom } \\
\text { of Upper Support Plate } \\
- \text { Upper Core Spacer } \\
\text { Grid }\end{array}$ & LDP-139 & water & 71 & \\
\hline $\begin{array}{l}\text { Inner Core } \\
\text { Thermocouple } \\
\text { Measuring Heater } \\
\text { Temperature }\end{array}$ & inches of & 72 & \\
\hline $\begin{array}{l}\text { Outer Core } \\
\text { Thermocouple } \\
\begin{array}{l}\text { Measuring Heater } \\
\text { Temperature }\end{array}\end{array}$ & TH-103-4 & ${ }^{\circ} \mathrm{F}$ & 73 & \\
\hline \hline
\end{tabular}

Note:

Data plot numbers 86 through 100 not used. 


\subsection{CONCLUSIONS}

The DBA-01-D tests were successfully completed, and the data were logged in the DAS. All critical instruments were found to operate properly with the exception of those noted in Section 6.4. The test was acceptable. 

APPENDIX A

DATA PLOTS 



\section{AP1000 Test Data Oregon State University Test DBA-01 Feb 27, 2003
$-P T-107$
231
0
o RX Up Head Press.

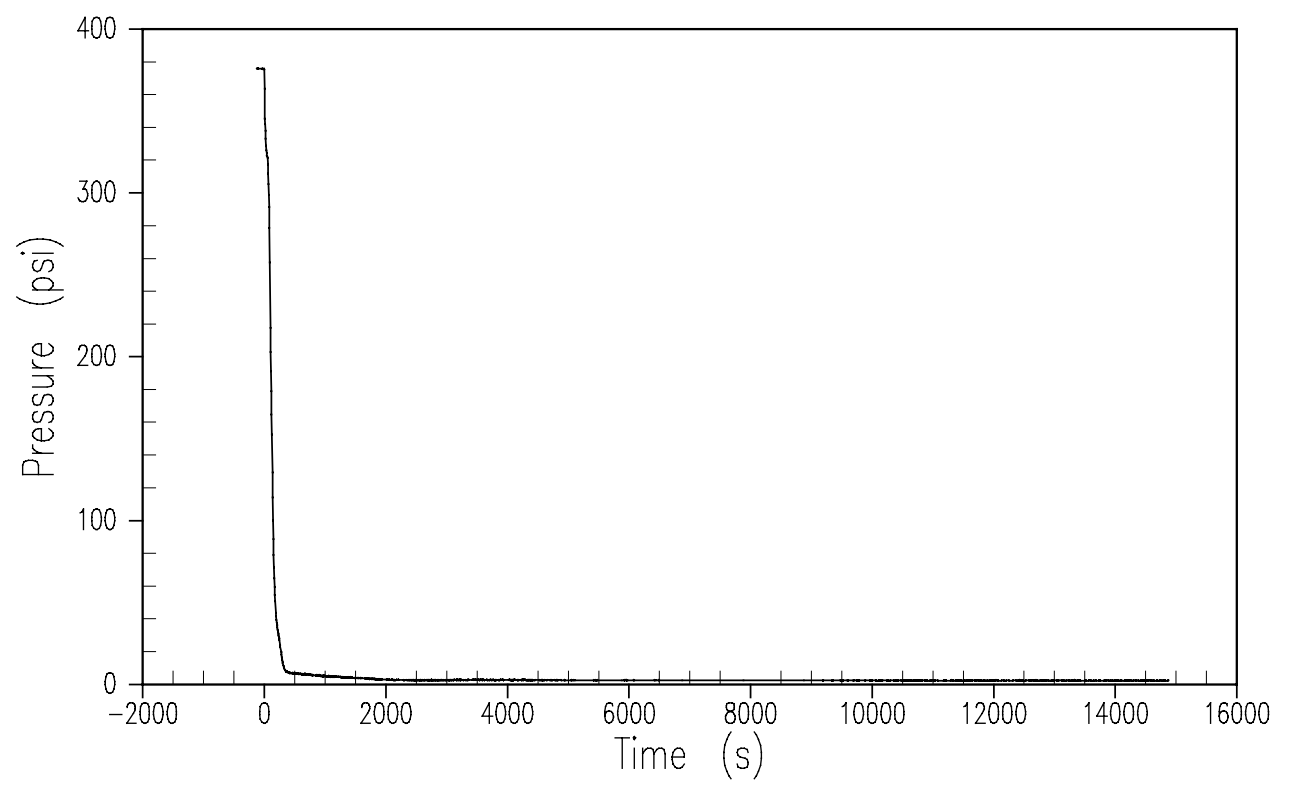

$\mathrm{a}, \mathrm{b}, \mathrm{c}$ 

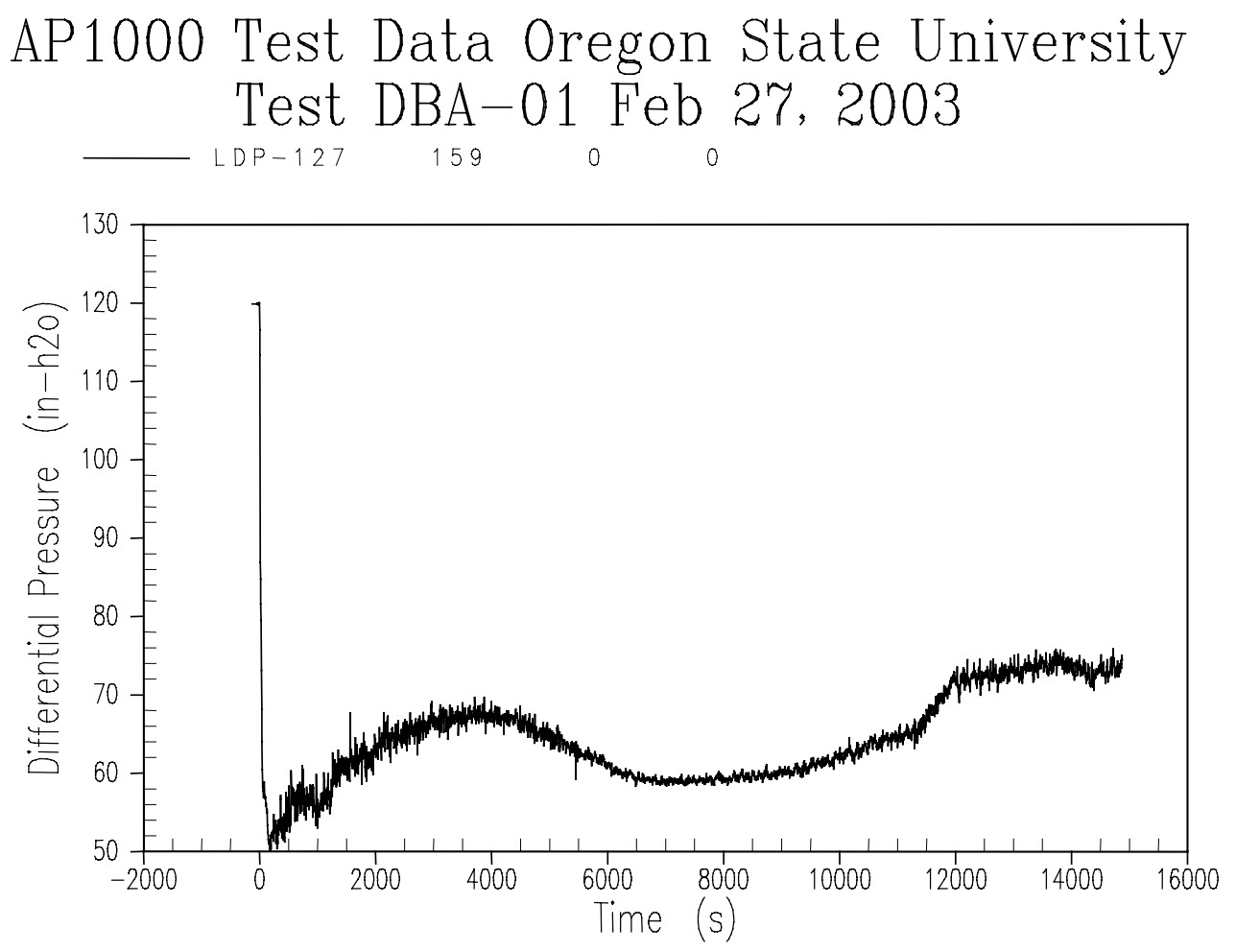

Figure A-2 Reactor Vessel Level 

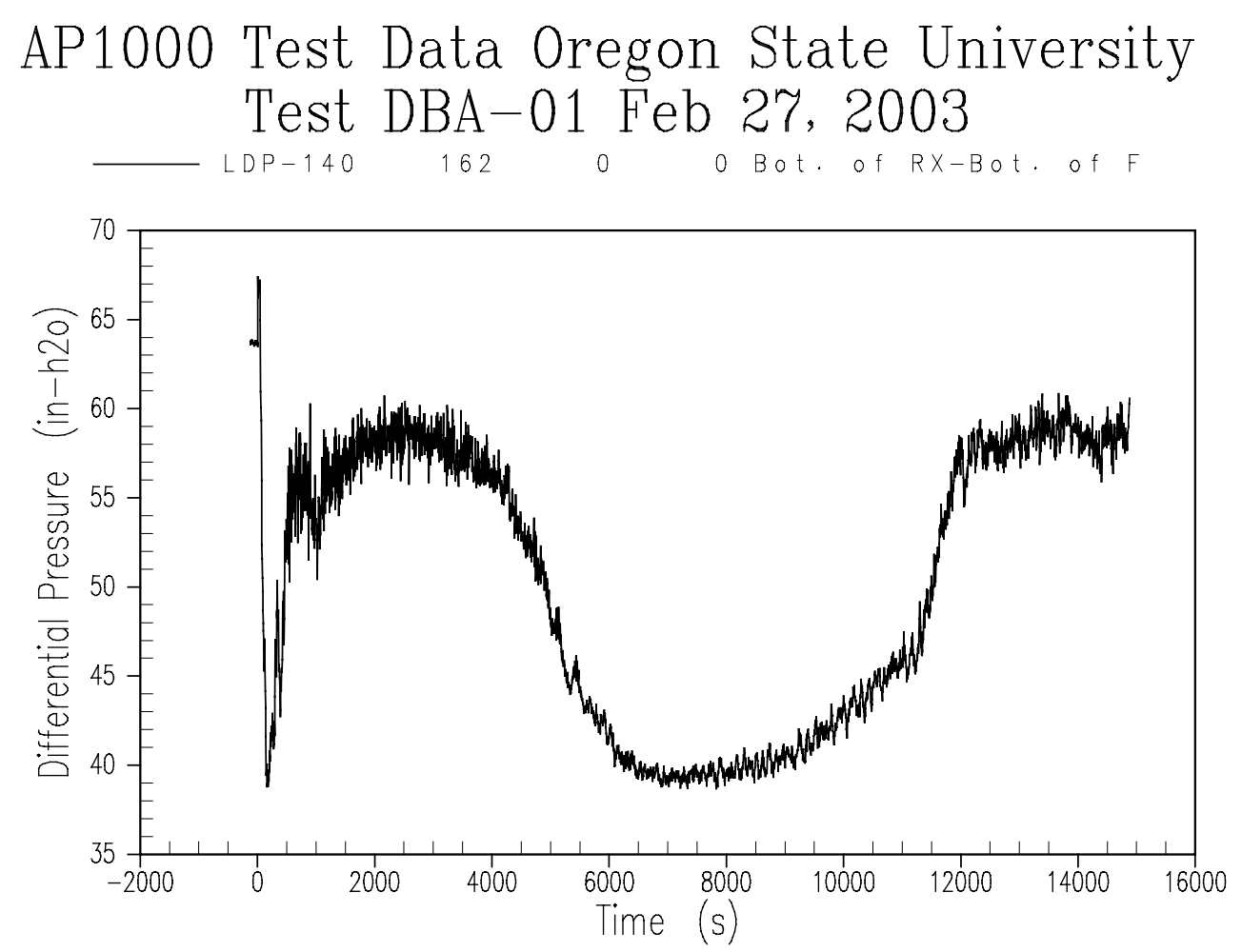

Figure A-3 Reactor Vessel Downcomer Level 

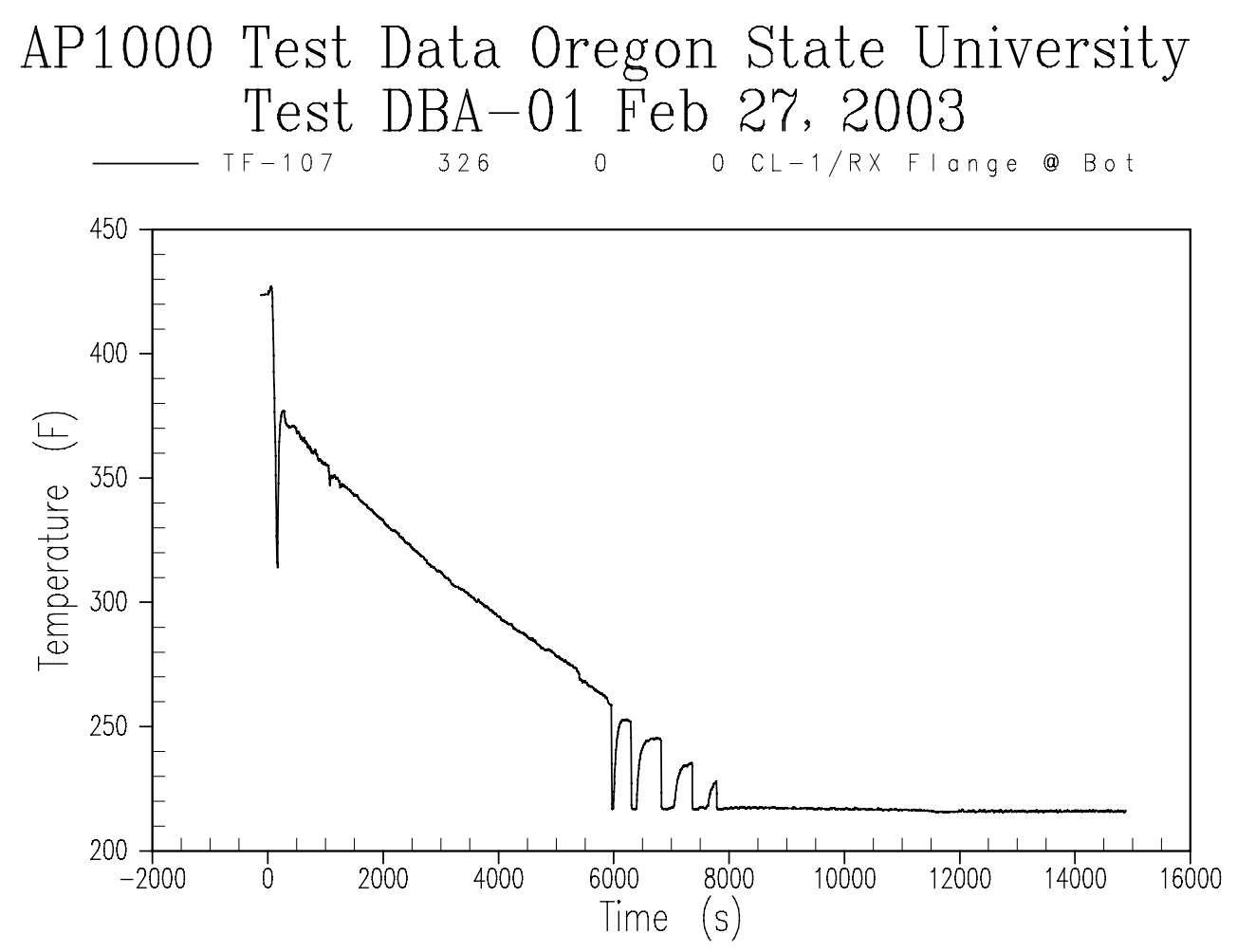

Figure A-4 Cold Leg 1 Fluid Temperature 

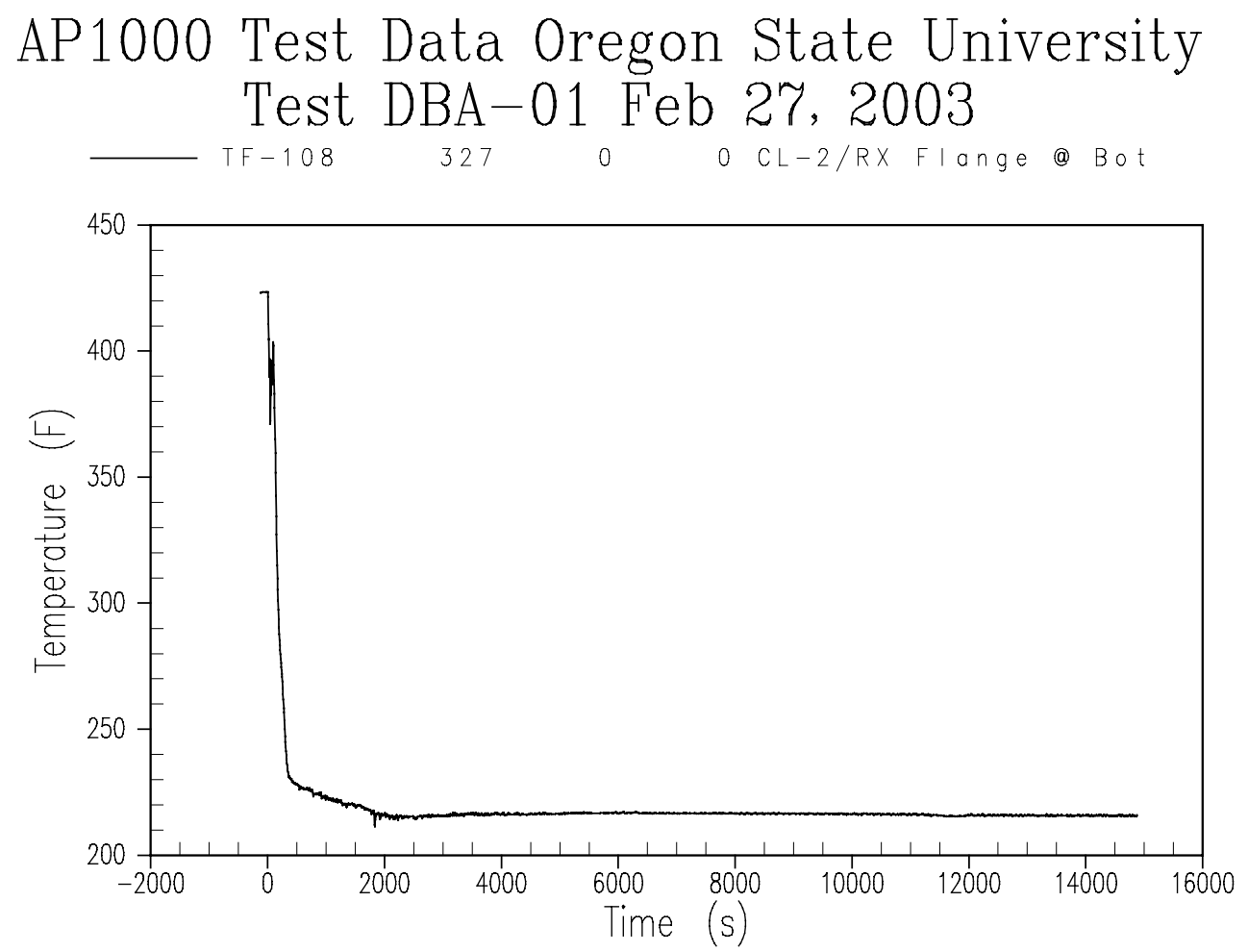

Figure A-5 Cold Leg 2 Fluid Temperature 

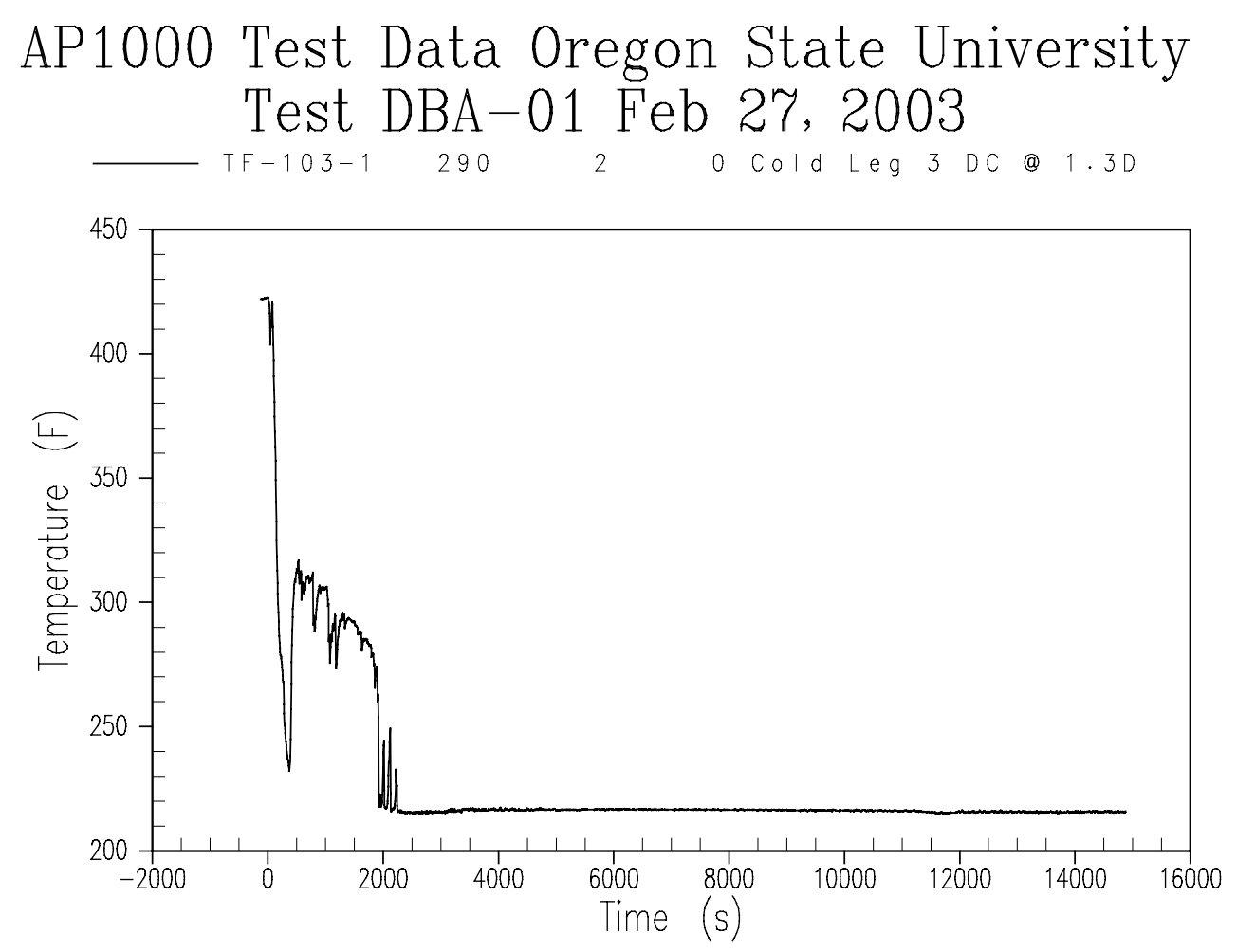

Figure A-6 Cold Leg 3 Fluid Temperature 

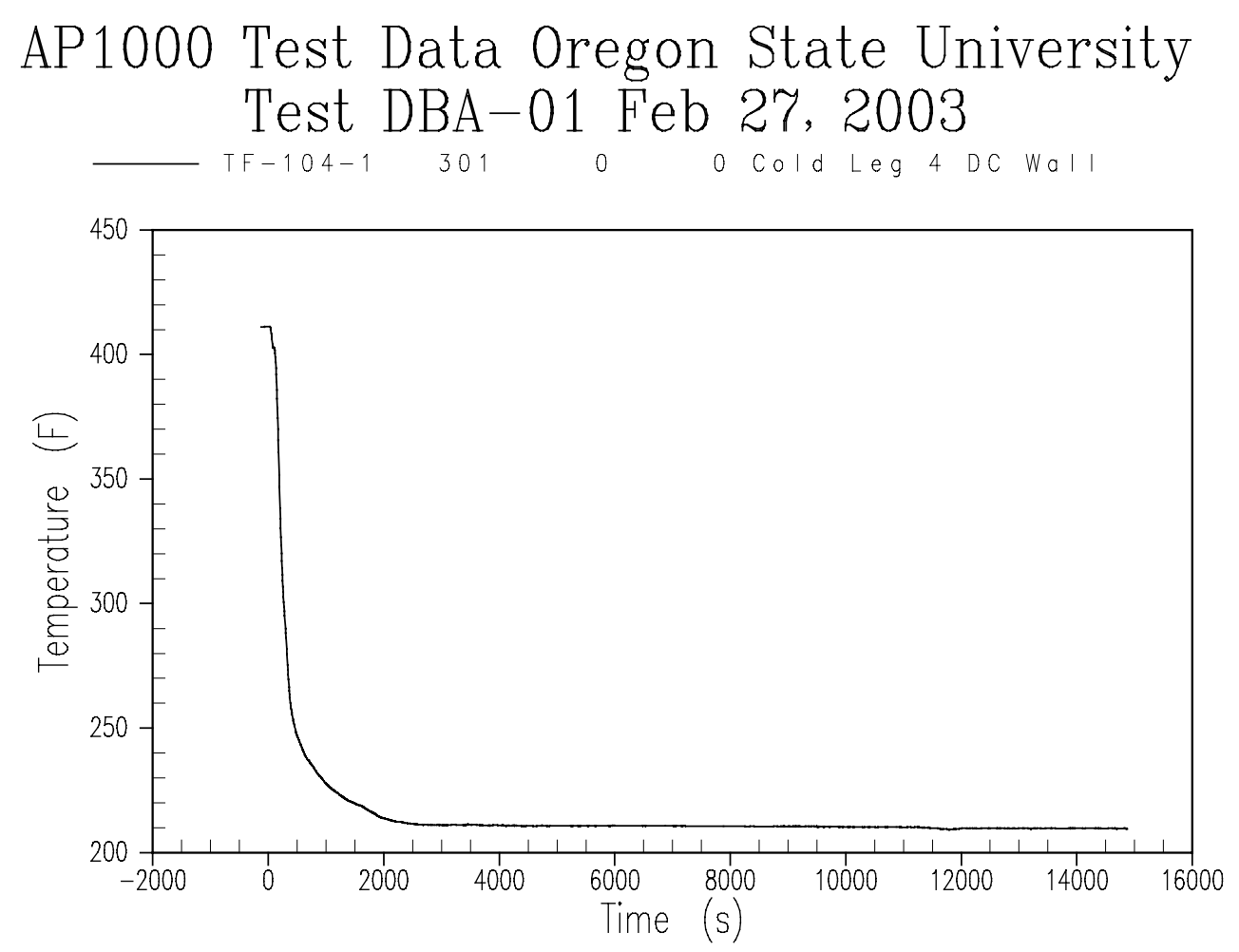

Figure A-7 Cold Leg 4 Fluid Temperature 

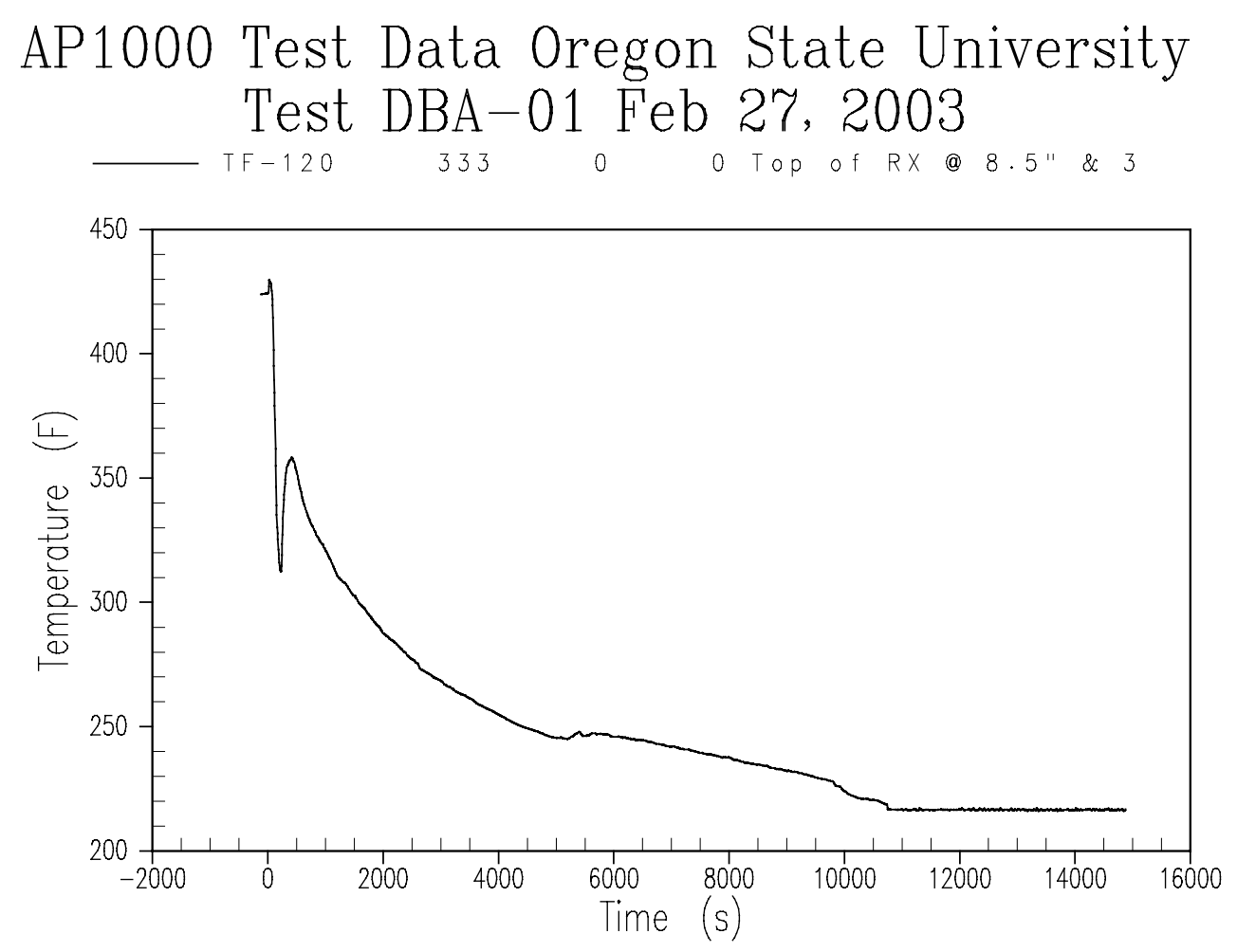

Figure A-8 Reactor Vessel Fluid Temperature Upper Head 

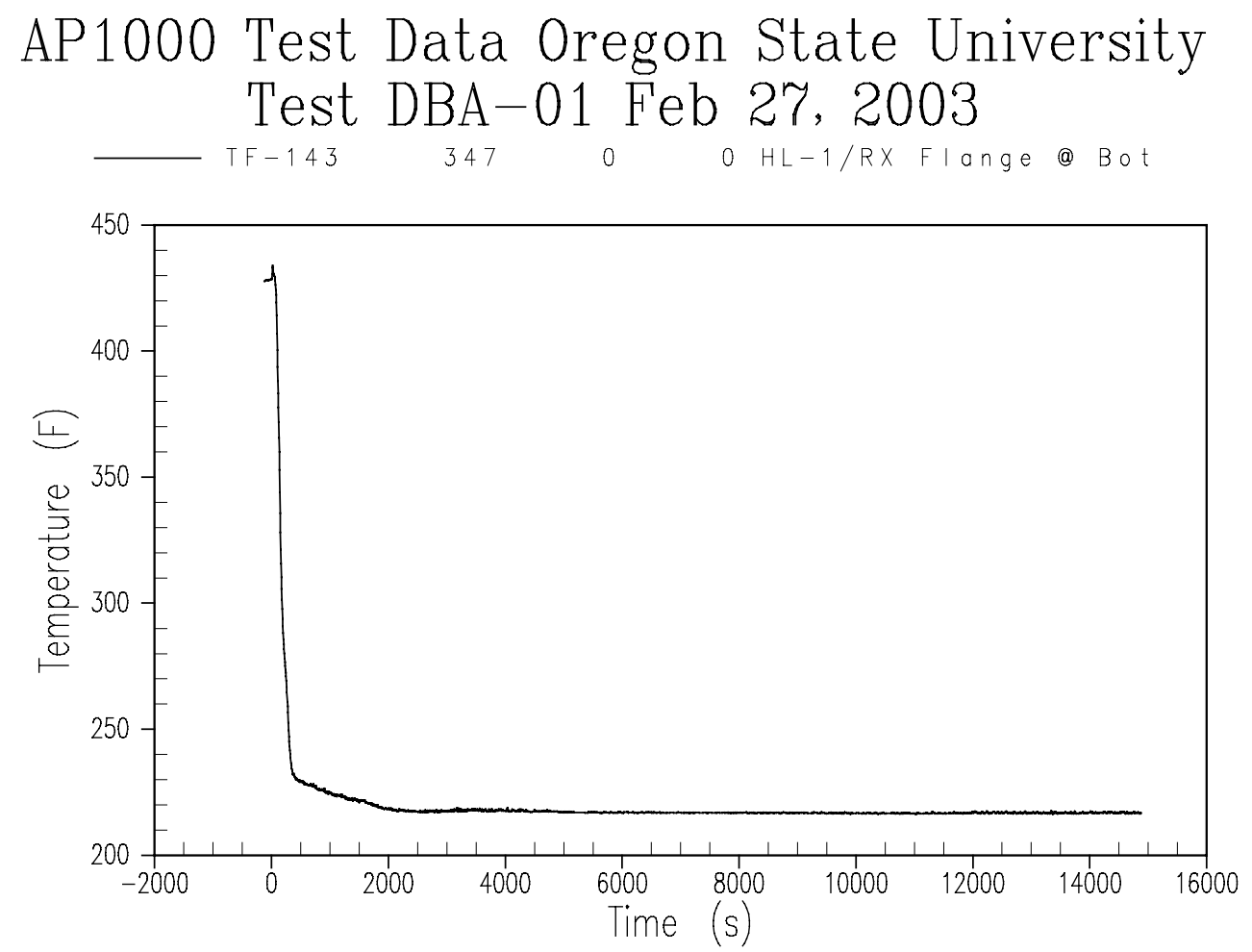

Figure A-9 Reactor Coolant System Hot Leg 1 Temperature 

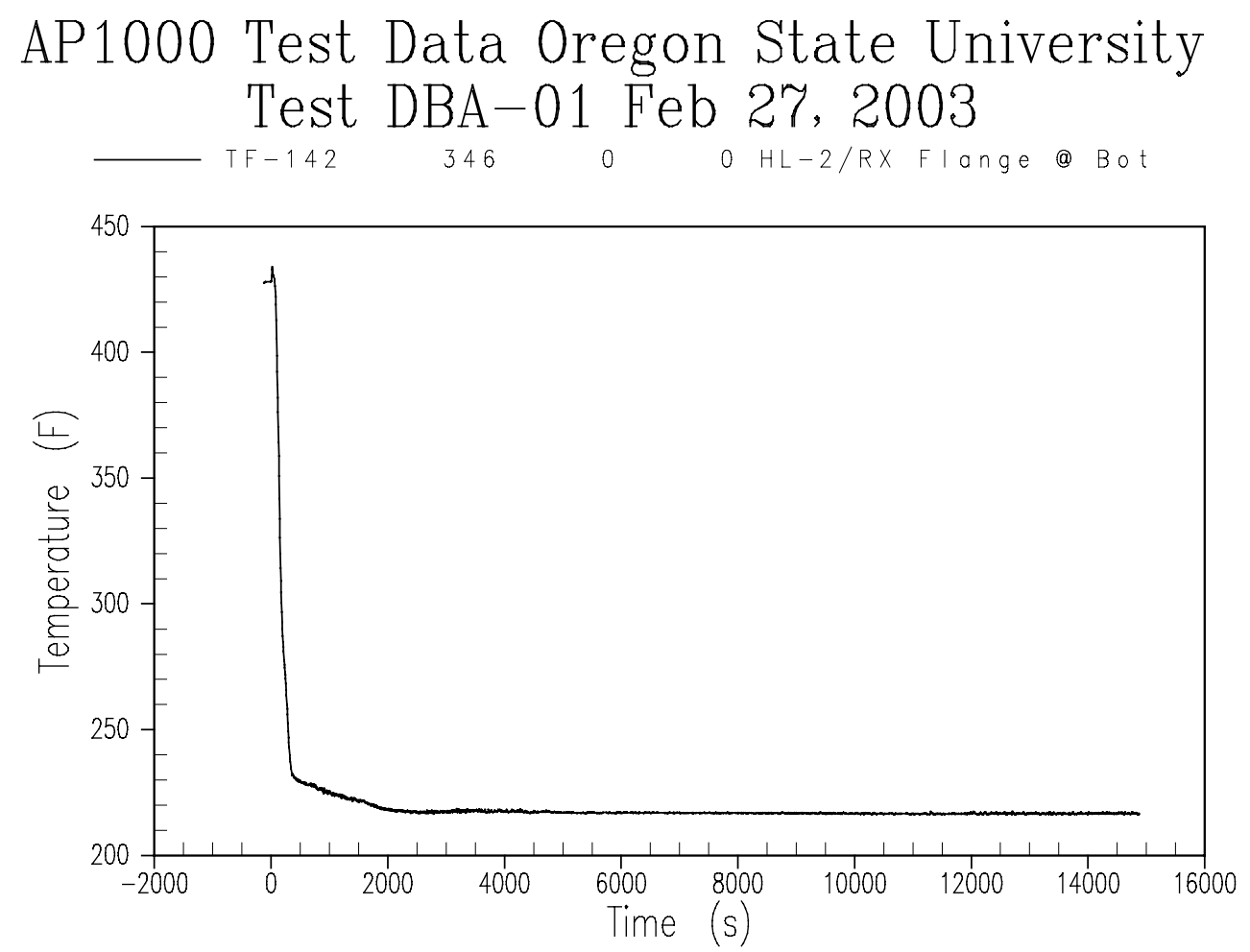

Figure A-10 Reactor Coolant System Hot Leg 2 Temperature 


\section{AP1000 Test Data Oregon State University Test DBA-01 Feb 27, 2003
$-P T-604$
252
0
o Pressurizer Press.

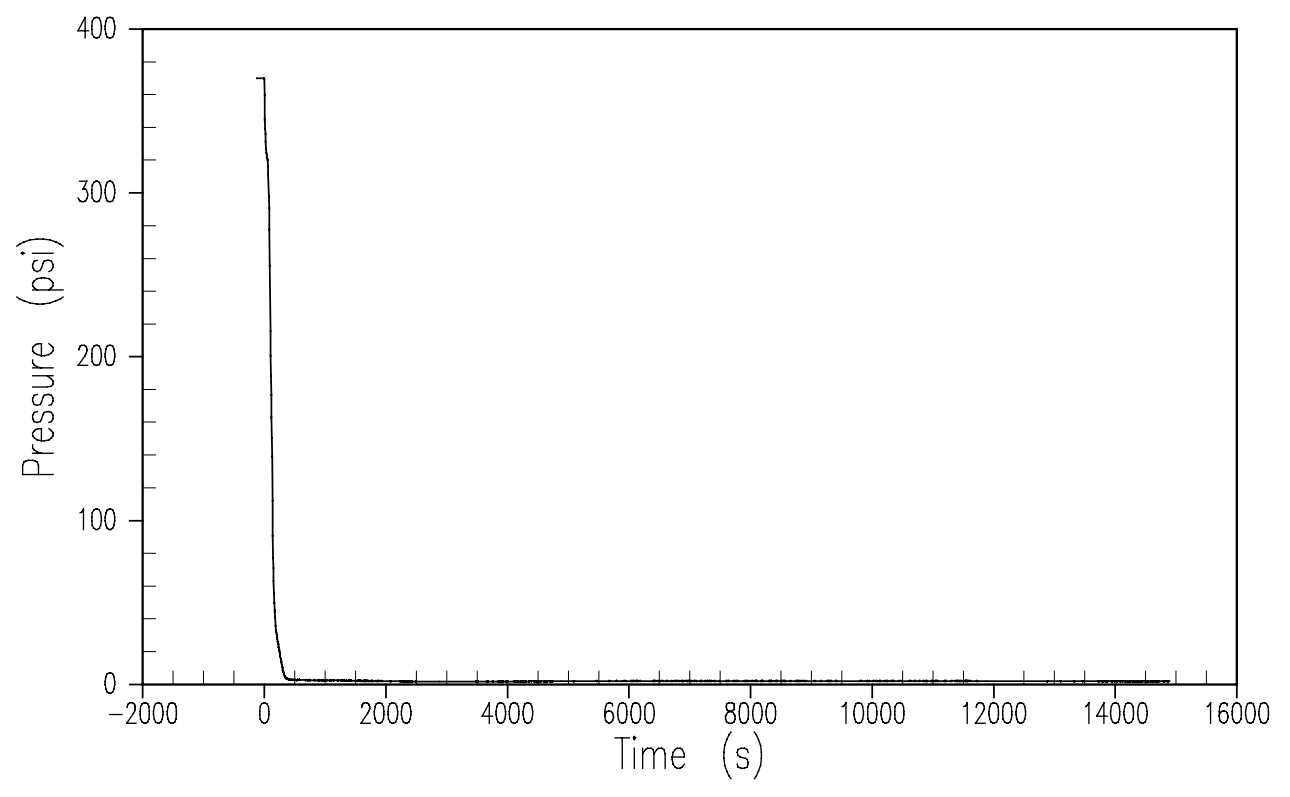

Figure A-11 Pressurizer Pressure - Wide Range 

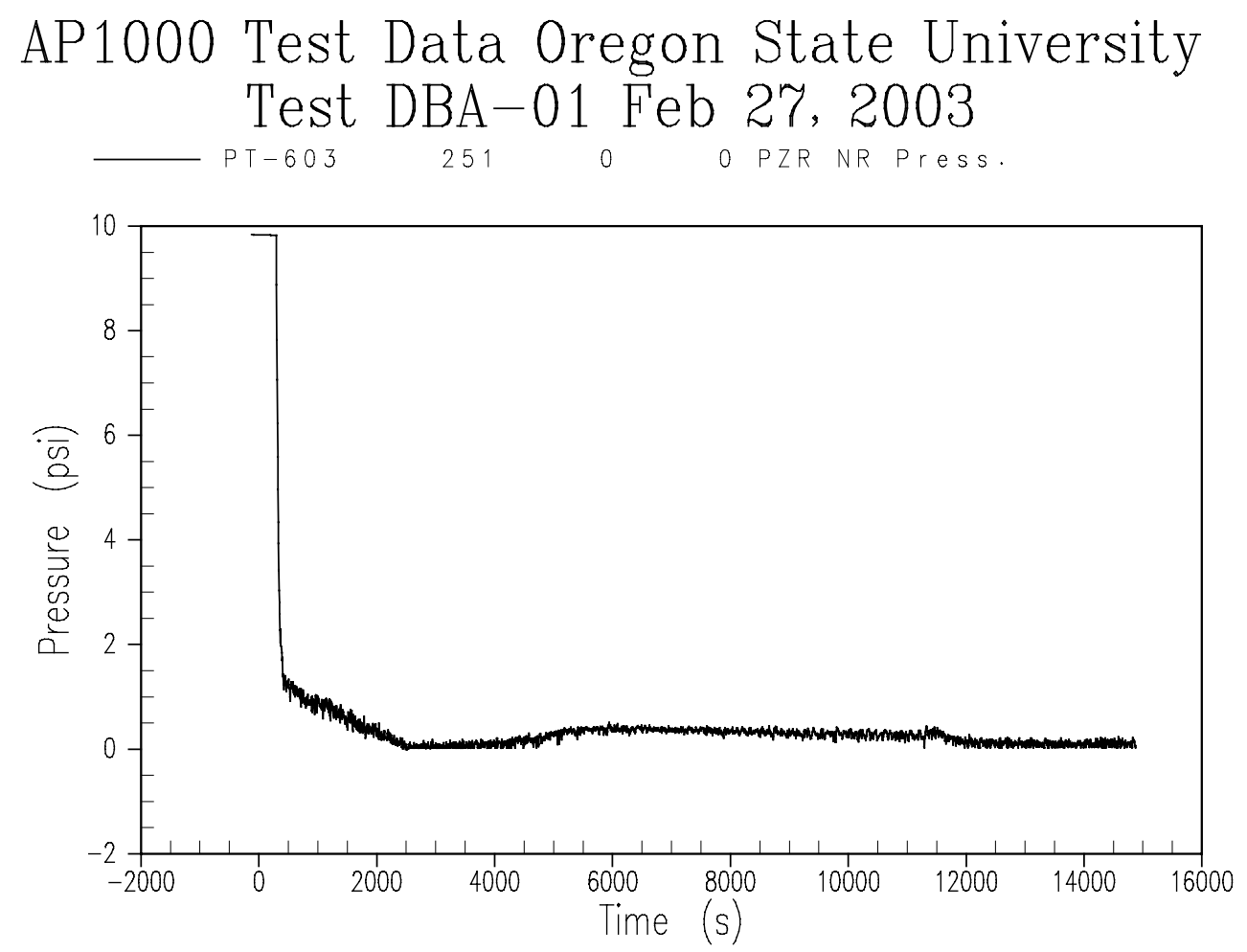

Figure A-12 Pressurizer Pressure - Narrow Range 

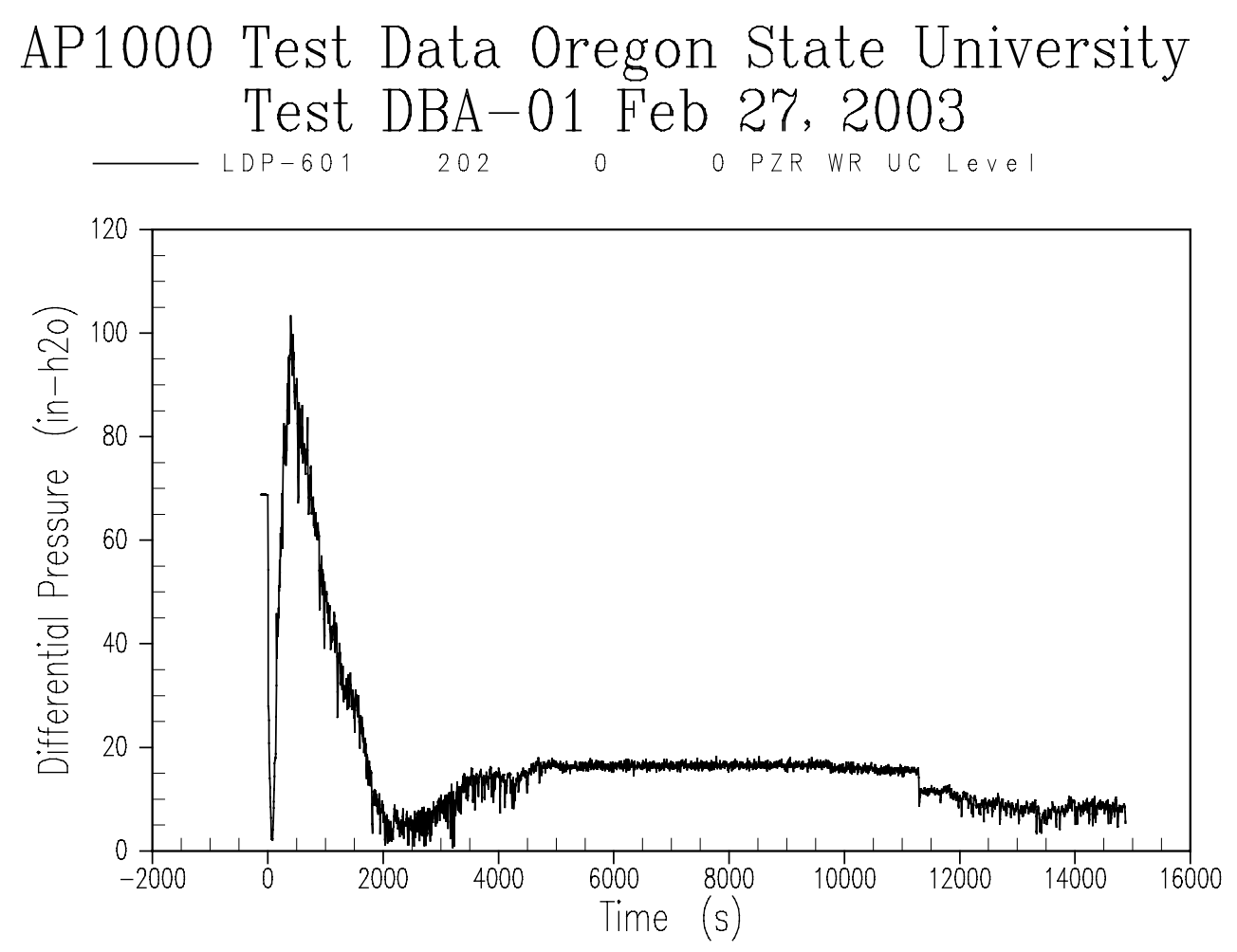

Figure A-13 Pressurizer Liquid Level 

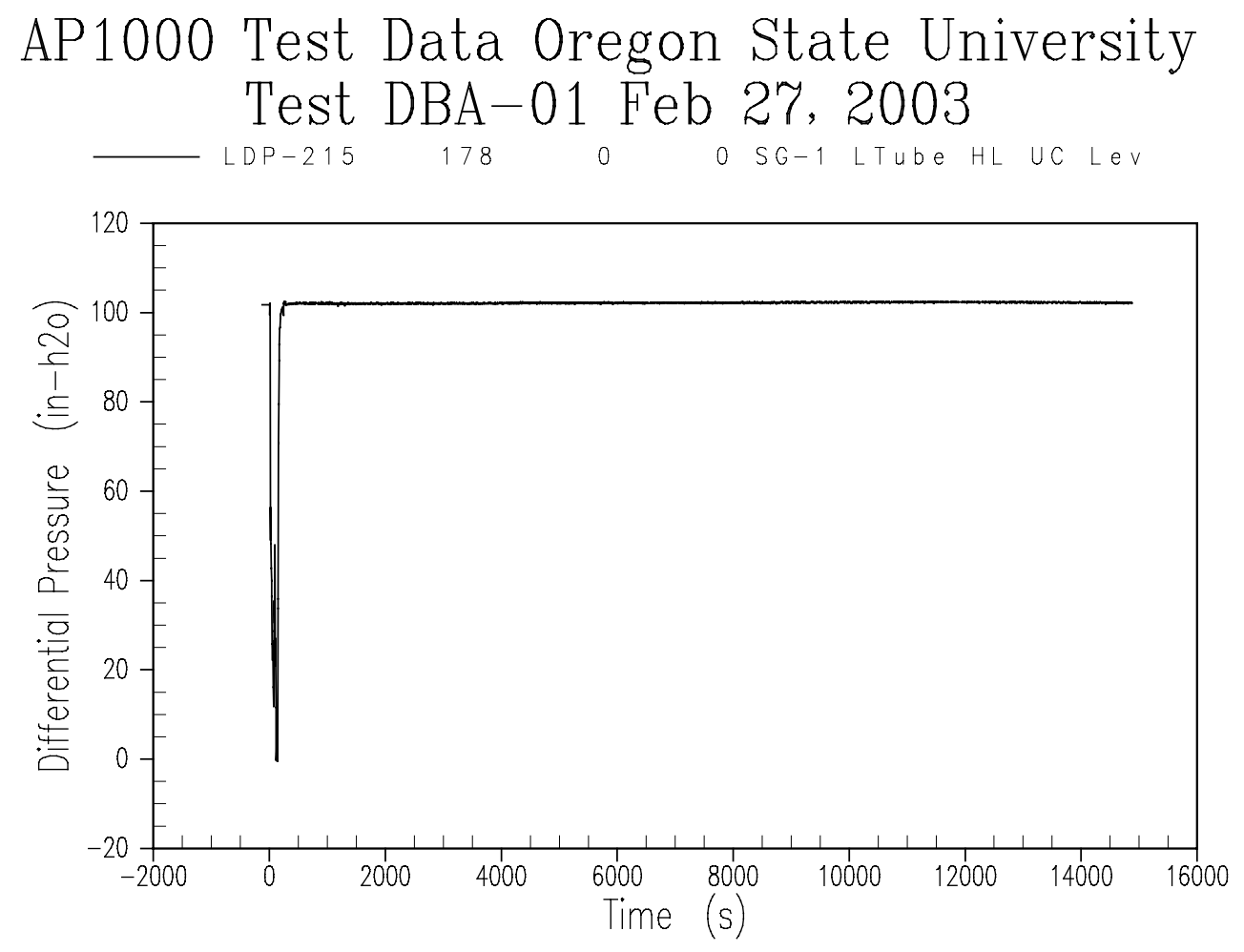

Figure A-14 Steam Generator 1 Tube Level 


\section{AP1000 Test Data Oregon State University Test DBA-01 Feb 27, 2003
$\longrightarrow$ P T -301
244
o SG-1 Press.

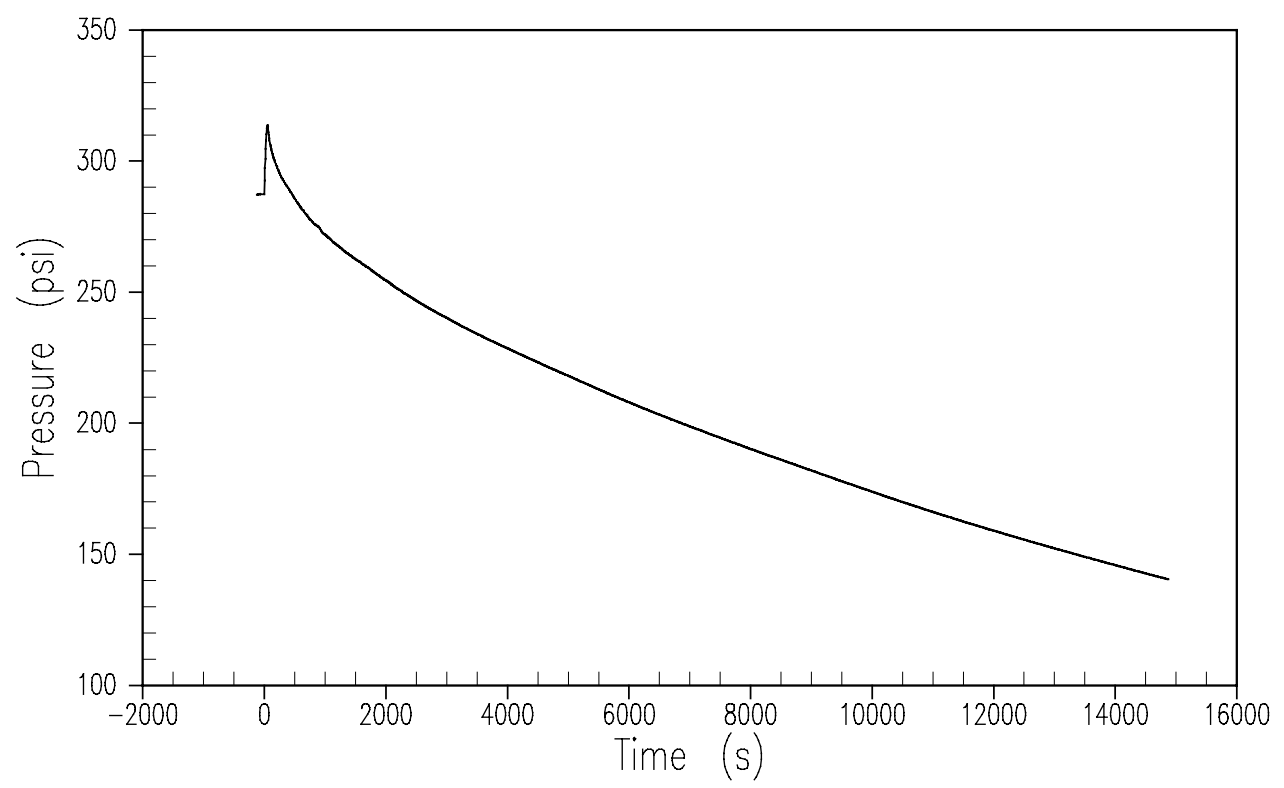

Figure A-15 Steam Generator 1 Secondary Pressure 

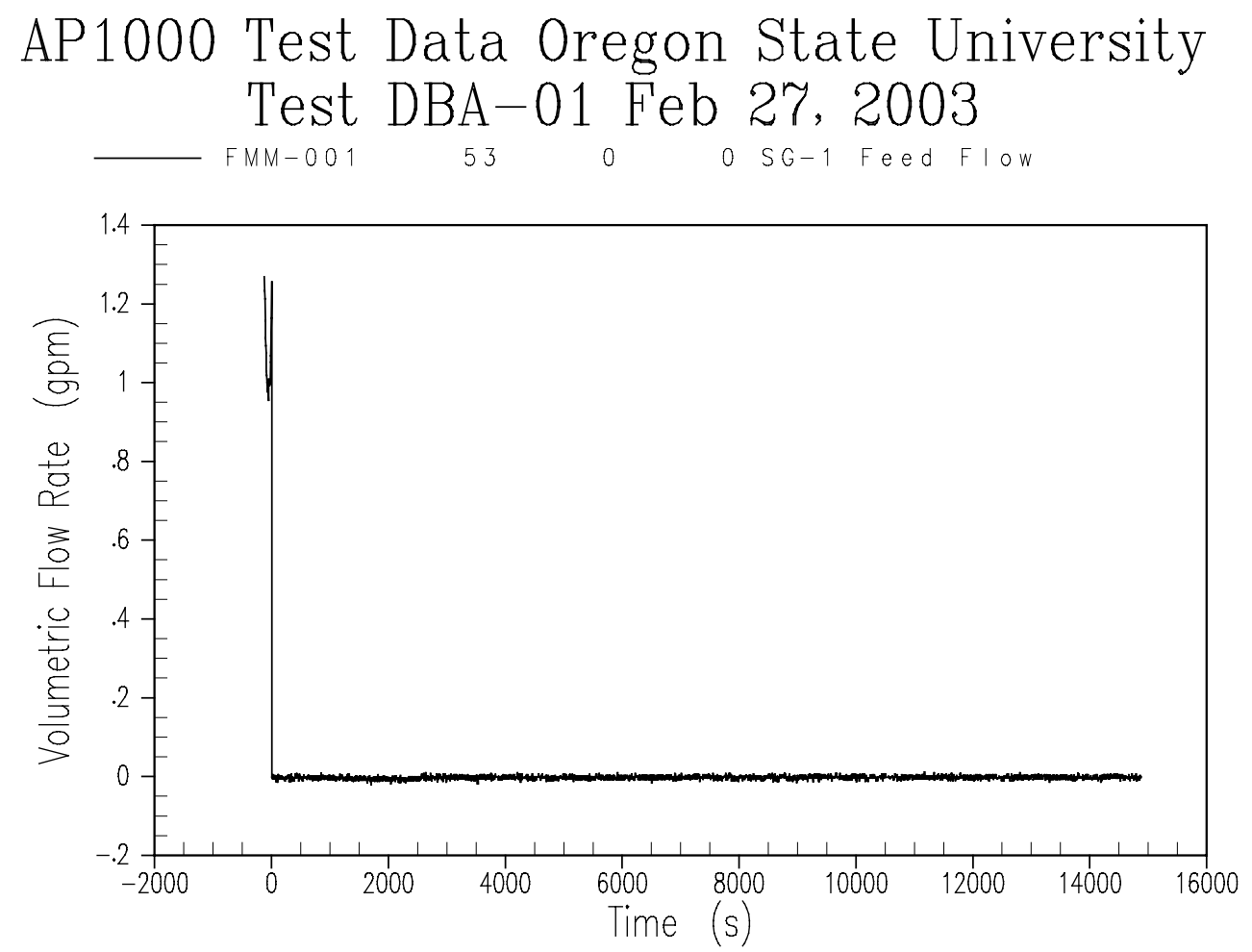

Figure A-16 Steam Generator 1 Feed Flow Rate 

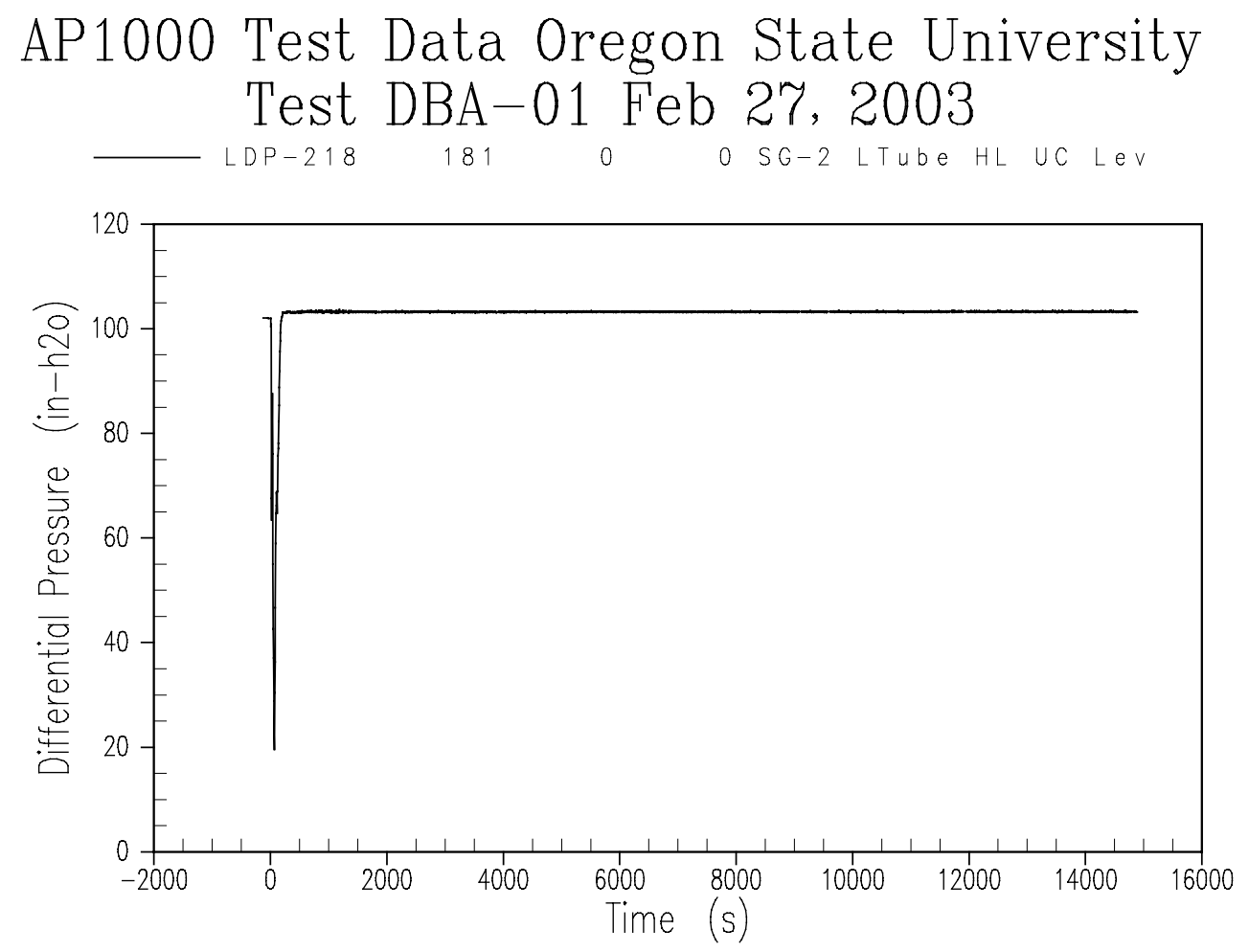

Figure A-17 Steam Generator 2 Tube Level 

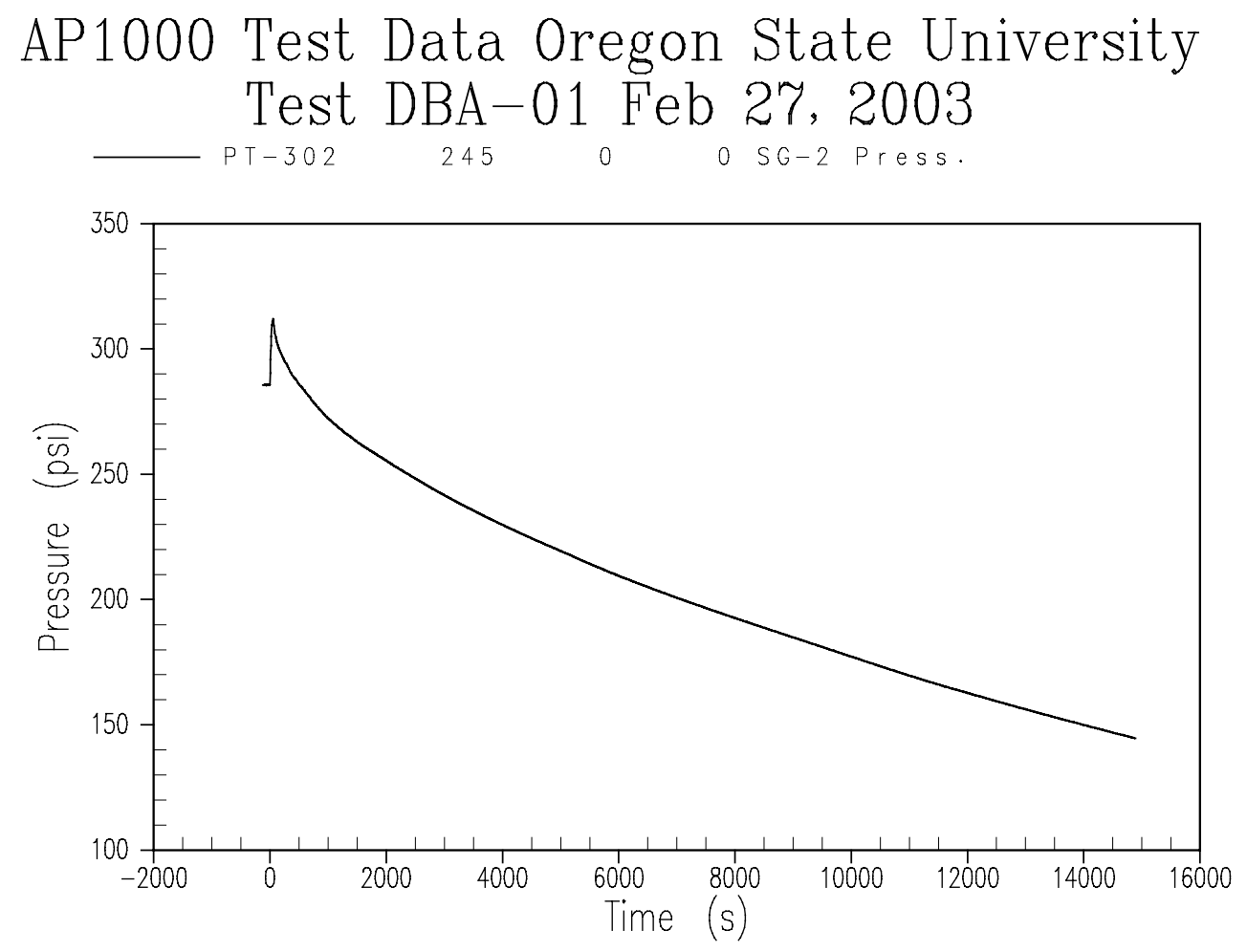

Figure A-18 Steam Generator 2 Secondary Pressure 


\section{AP1000 Test Data Oregon State University Test DBA-01 Feb 27, 2003
$-P T-401$
246
0
0 ACC-1 Press.

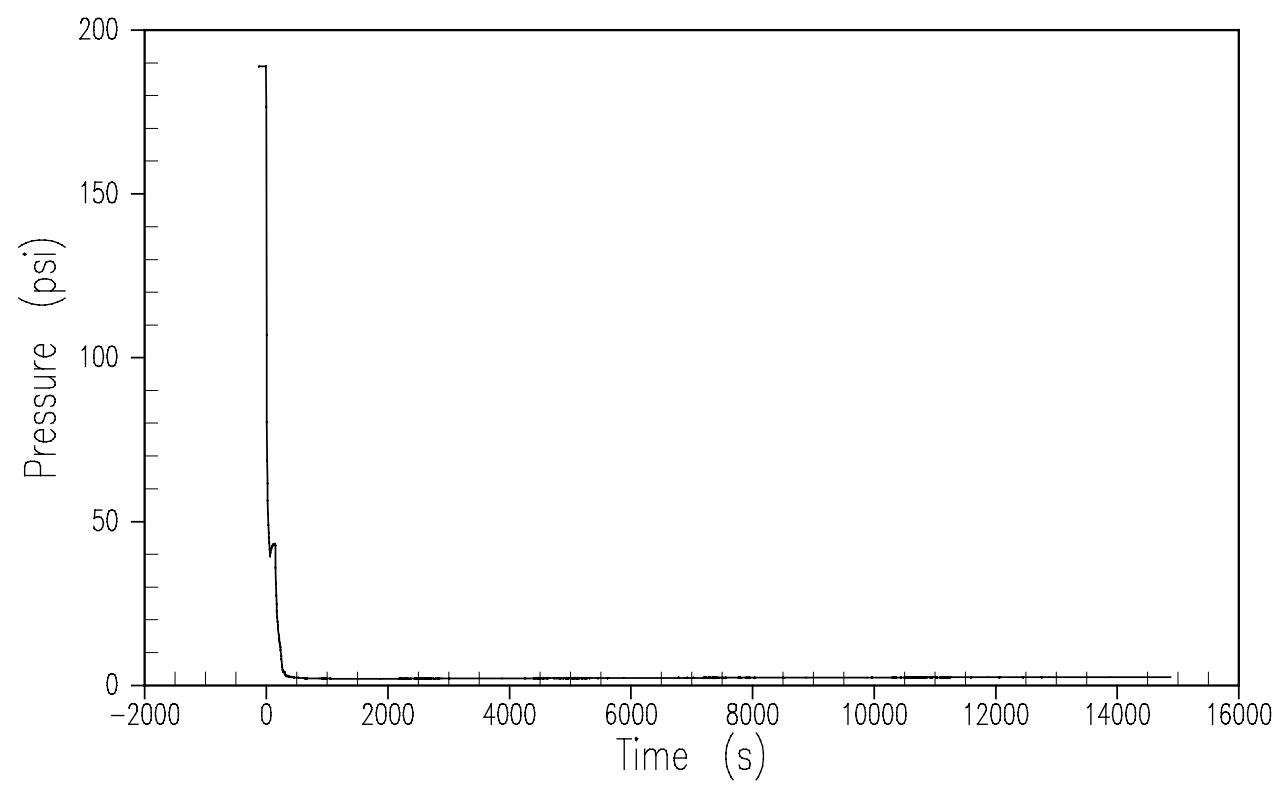

Figure A-19 Accumulator 1 Pressure 


\section{AP1000 Test Data Oregon State University Test DBA-01 Feb 27, 2003
$-P T-402$
247
0 ACC-2 Press.

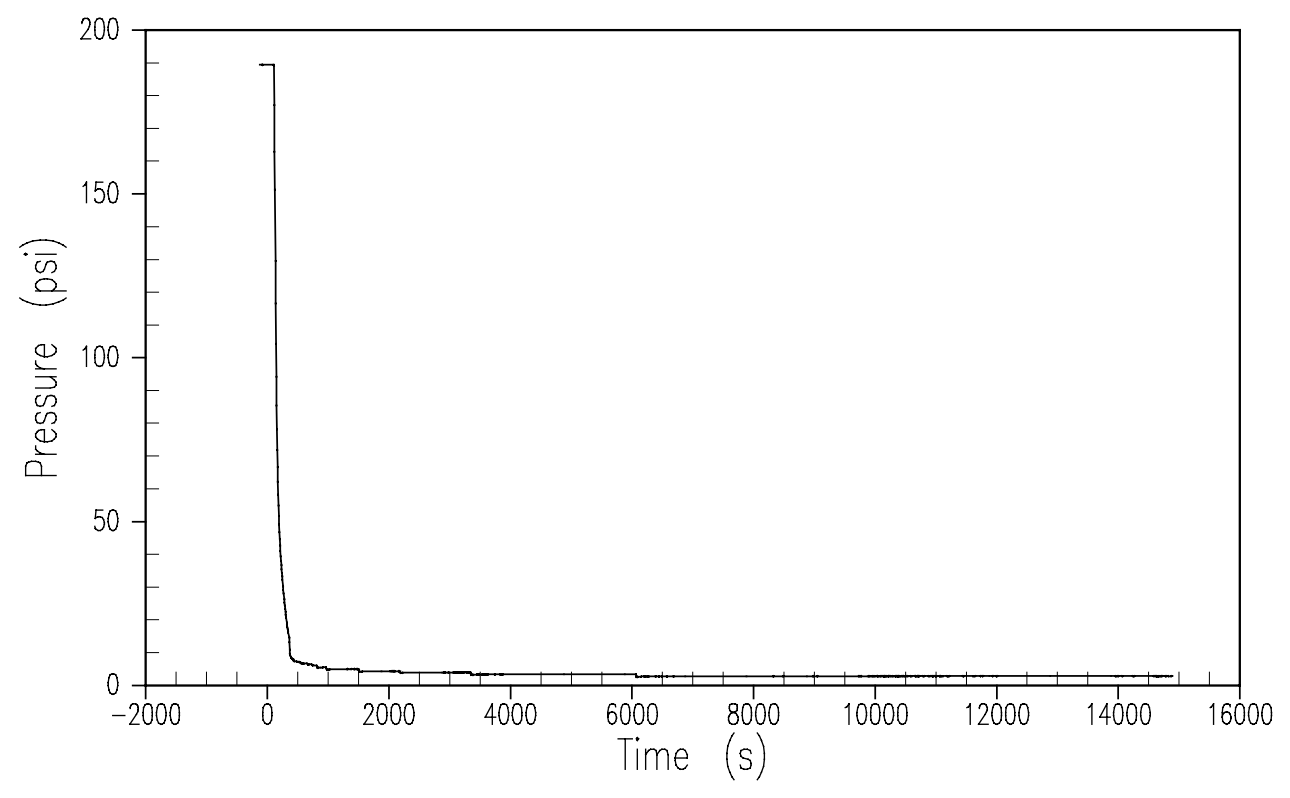

Figure A-20 Accumulator 2 Pressure 

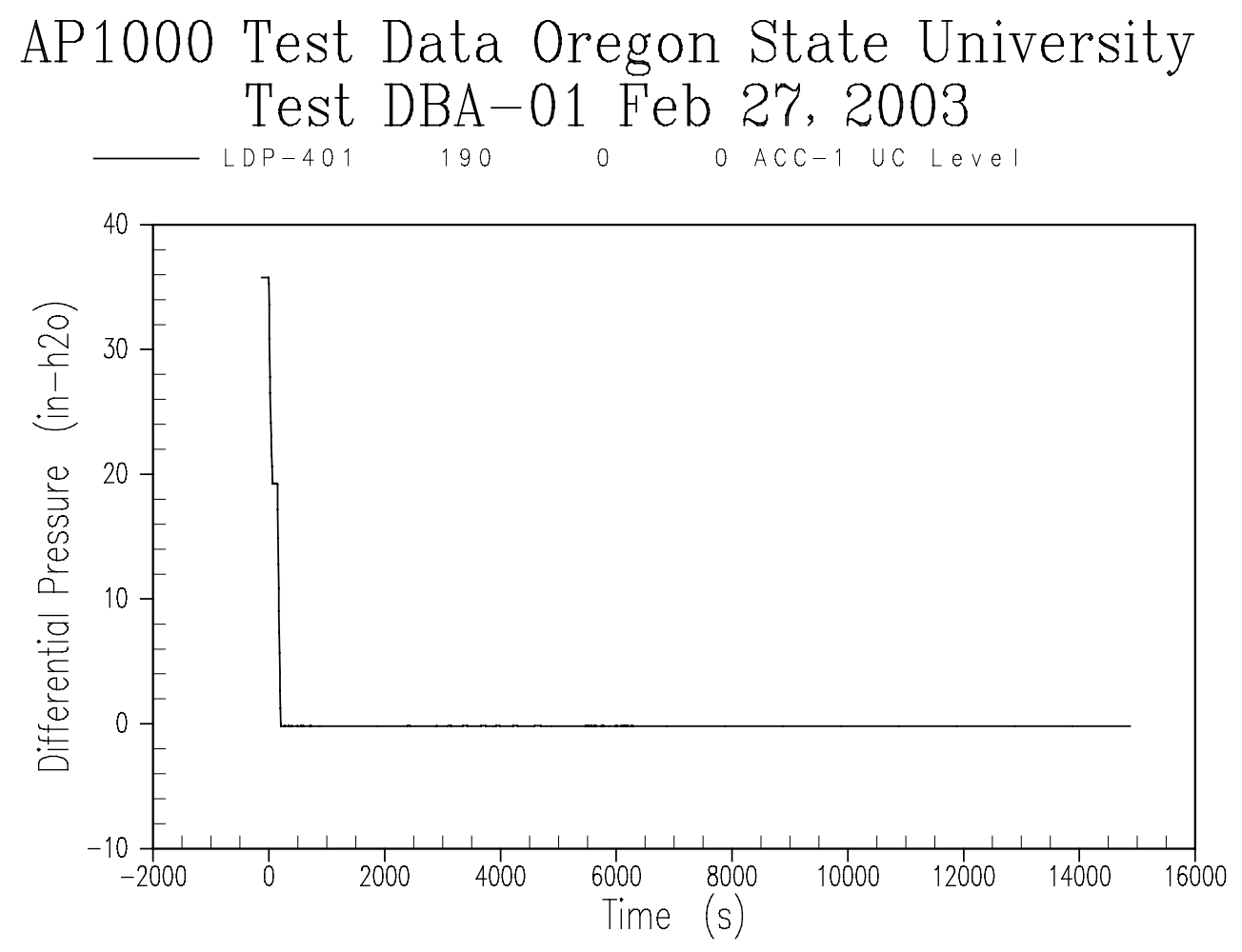

Figure A-21 Accumulator 1 Liquid Level 

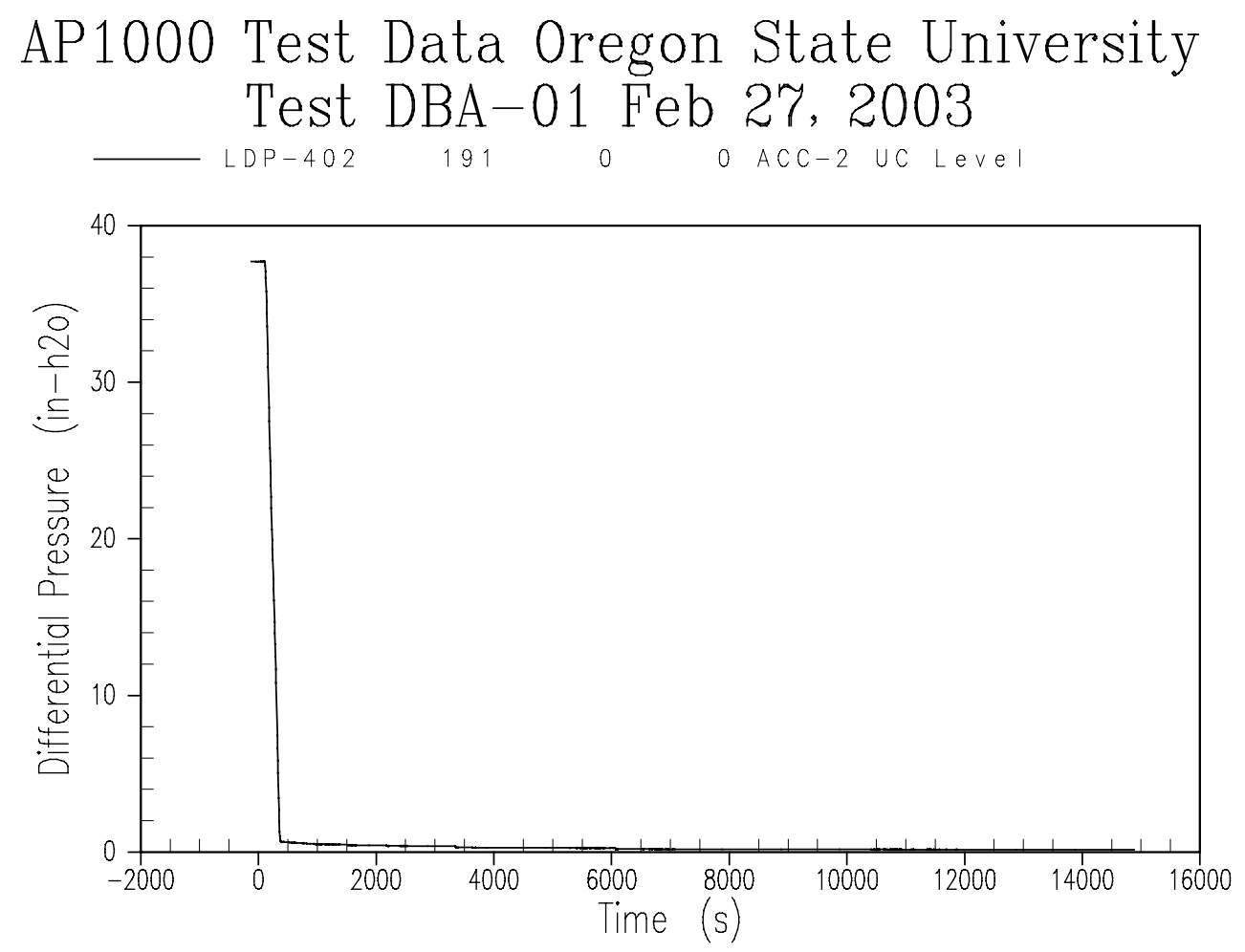

Figure A-22 Accumulator 2 Liquid Level 

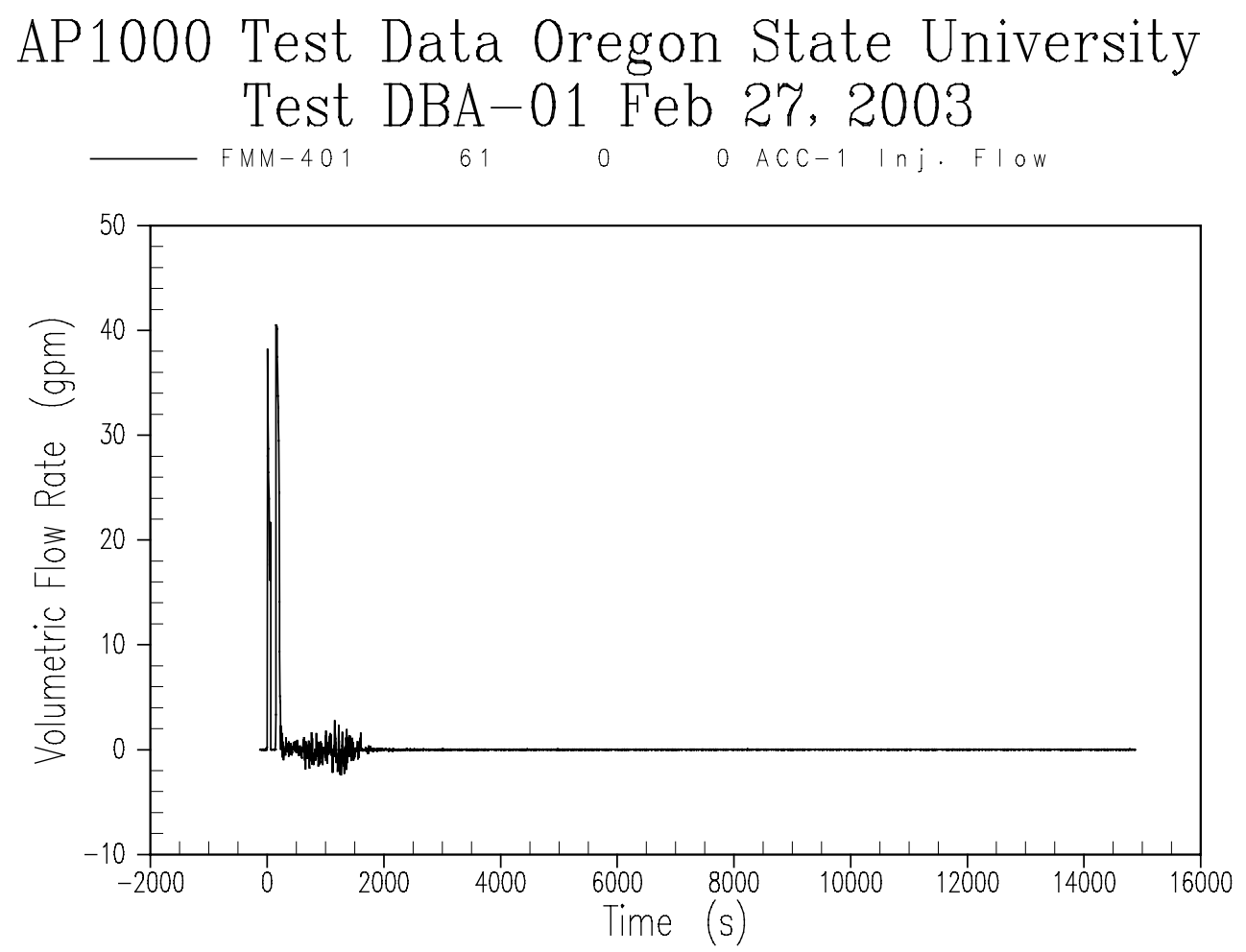

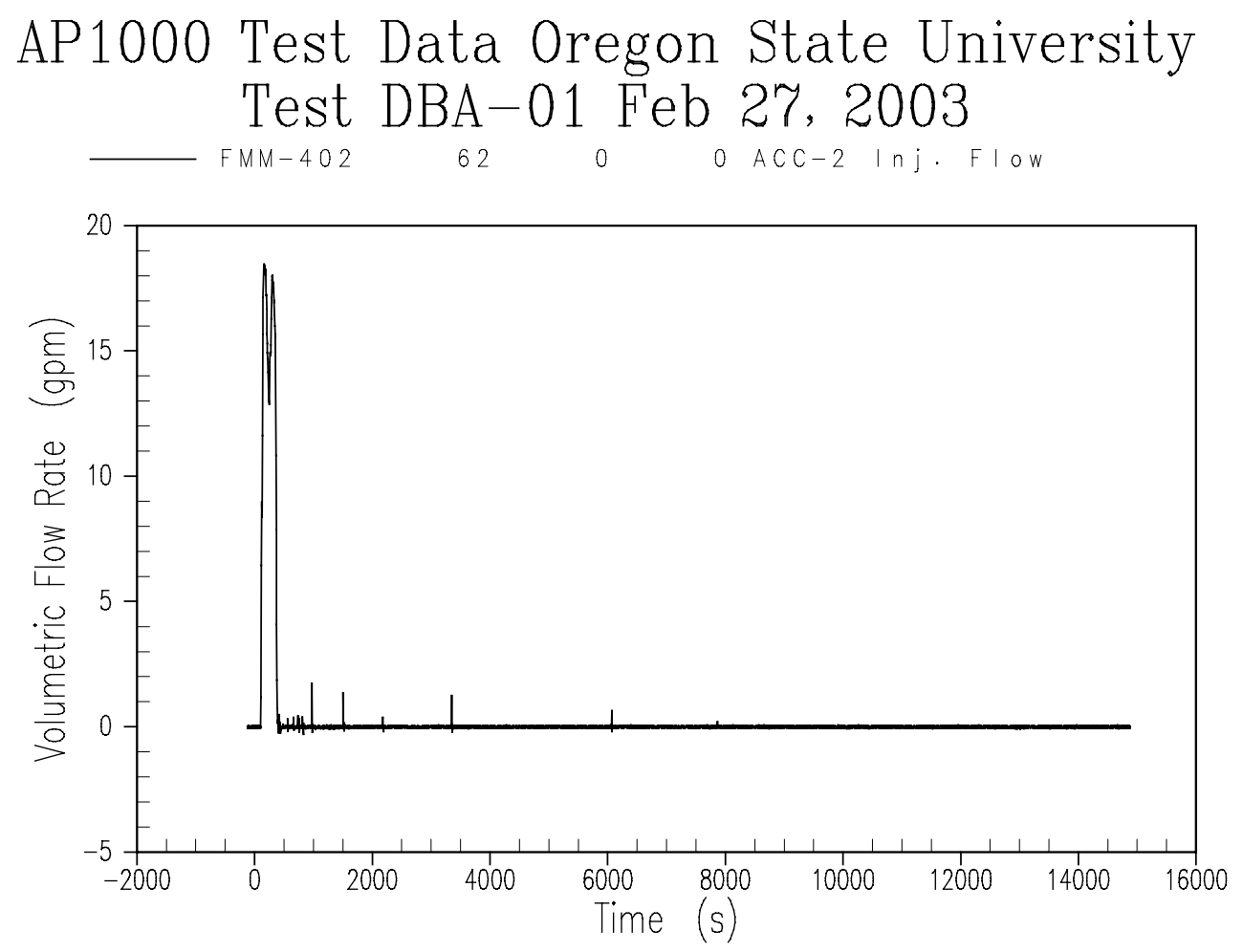

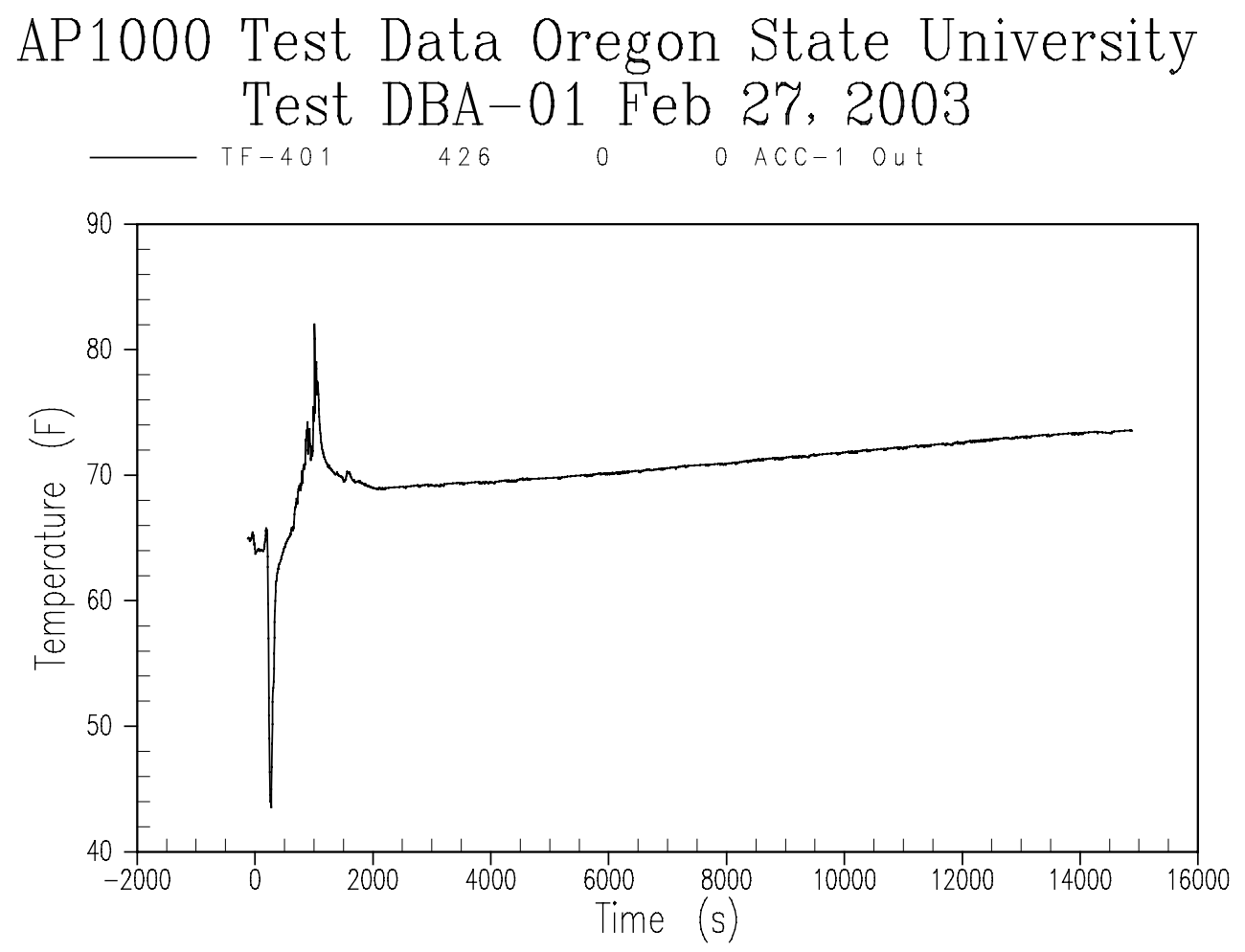

Figure A-25 Accumulator 1 Liquid Discharge Temperature 


\section{AP1000 Test Data Oregon State University Test DBA-01 Feb 27, 2003
$-T F-402$
427
0
o ACC-2 Out

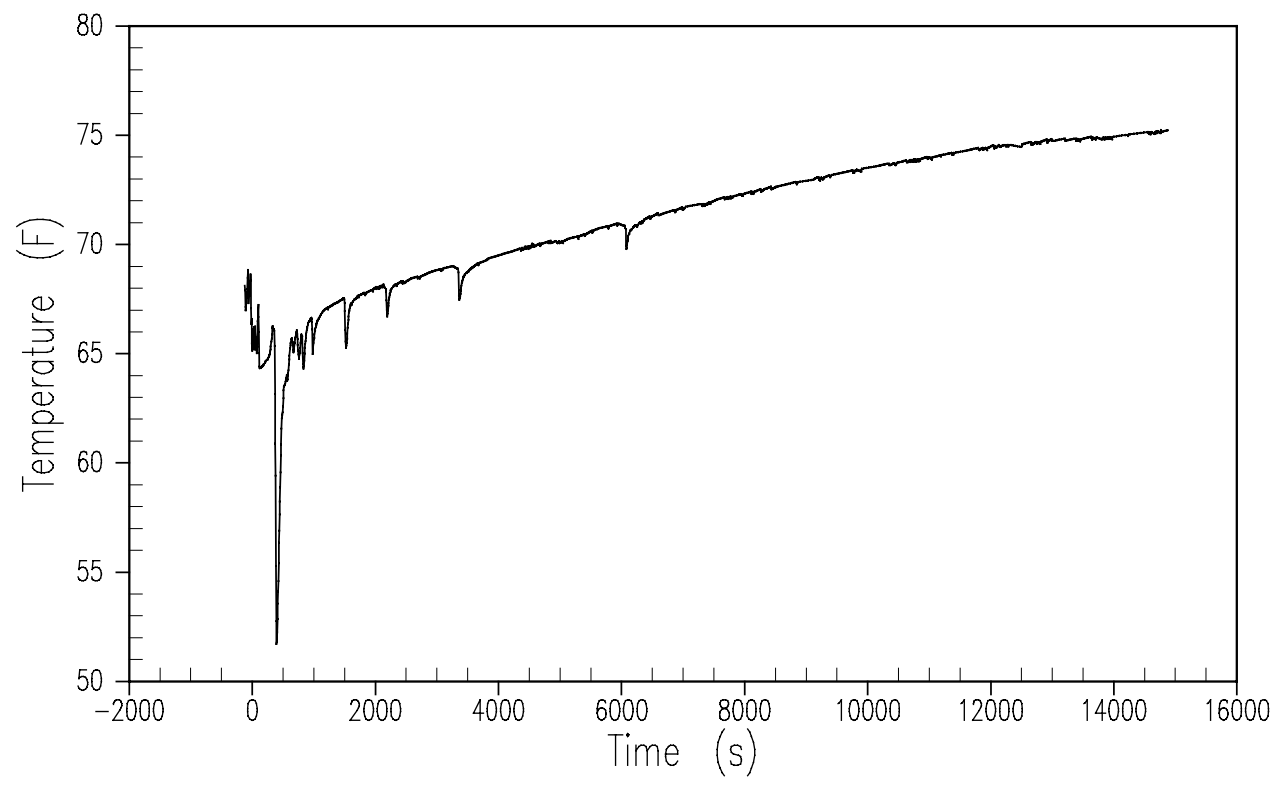

Figure A-26 Accumulator 2 Liquid Discharge Temperature 

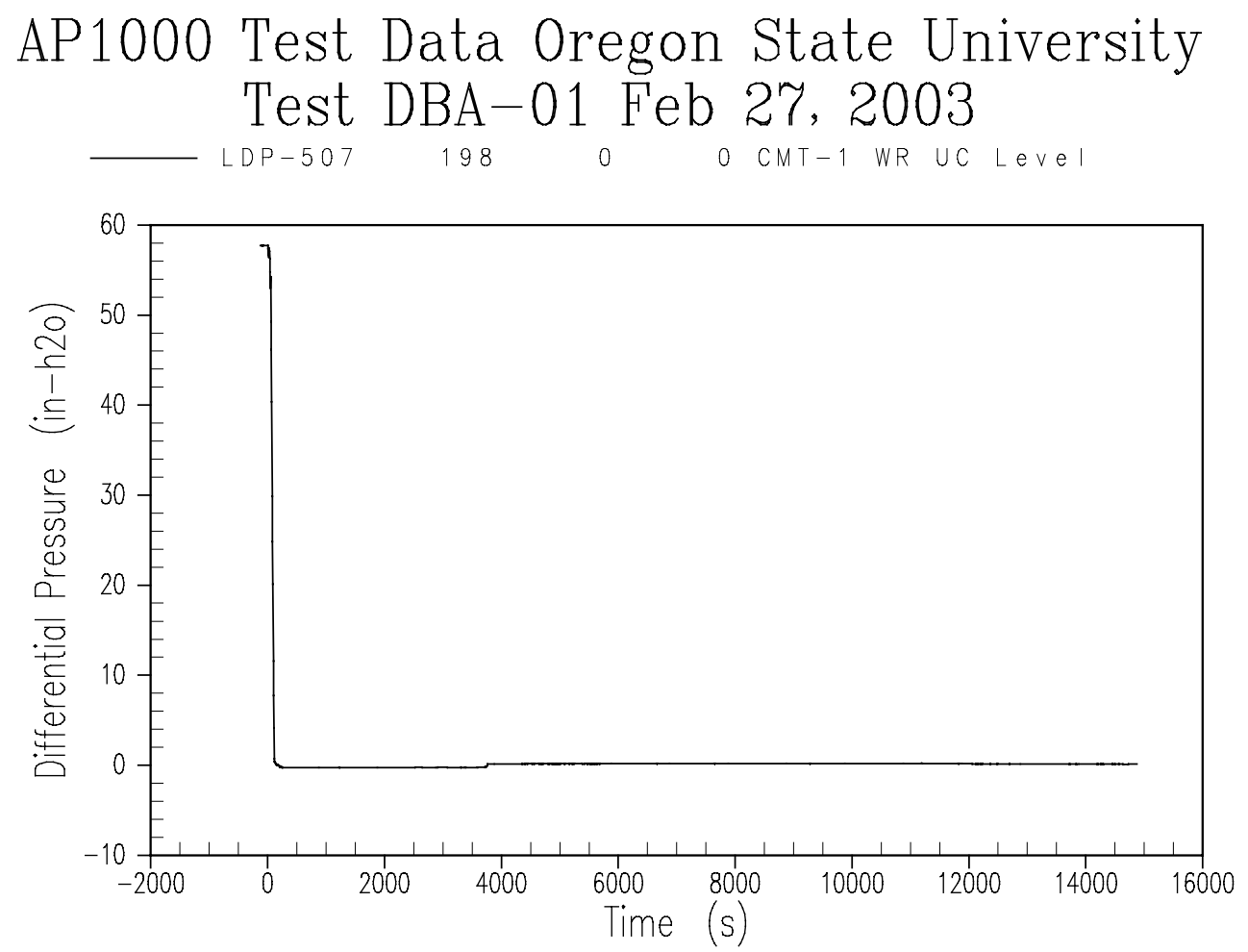

Figure A-27 Core Makeup Tank 1 Liquid Level 

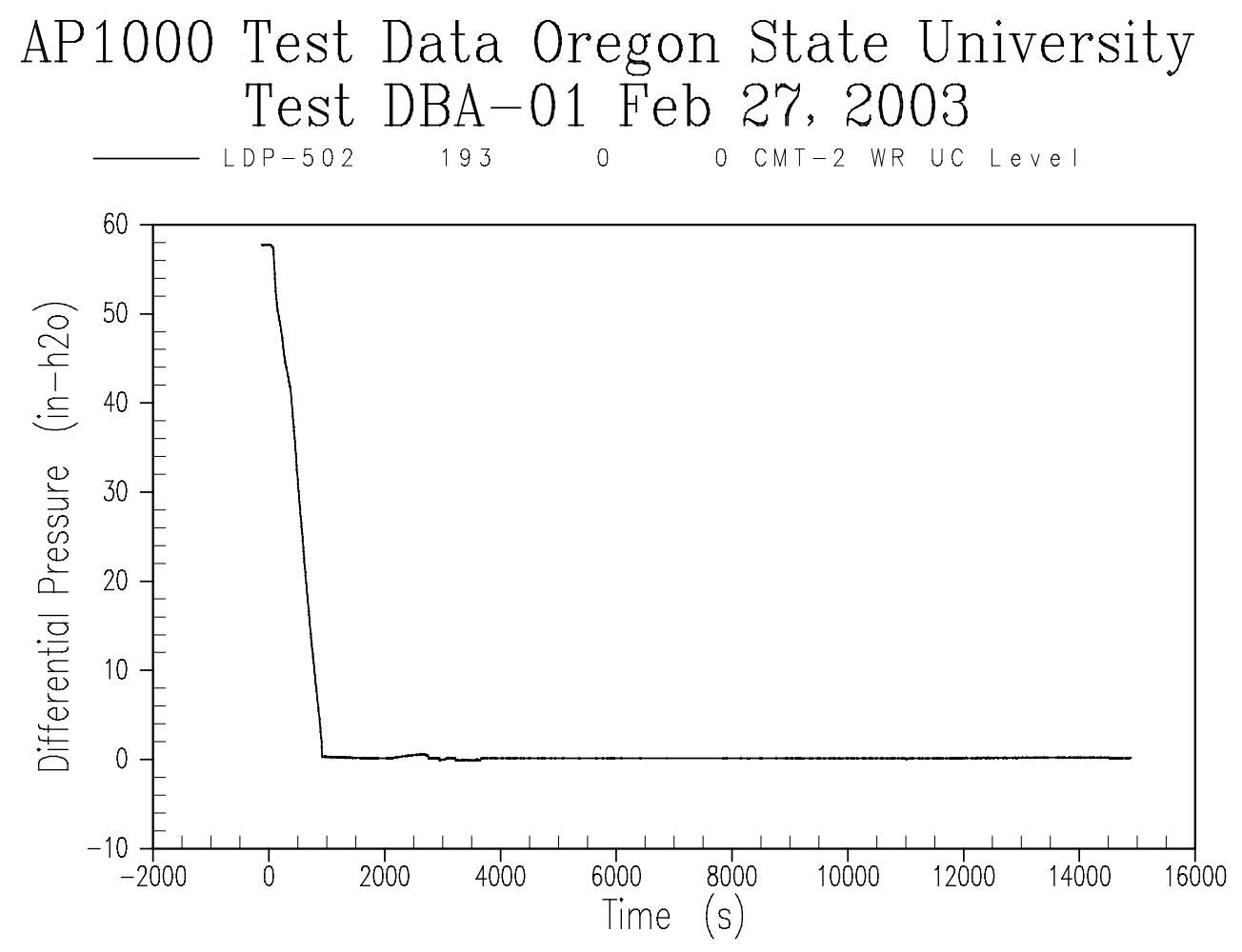

Figure A-28 Core Makeup Tank 2 Liquid Level 

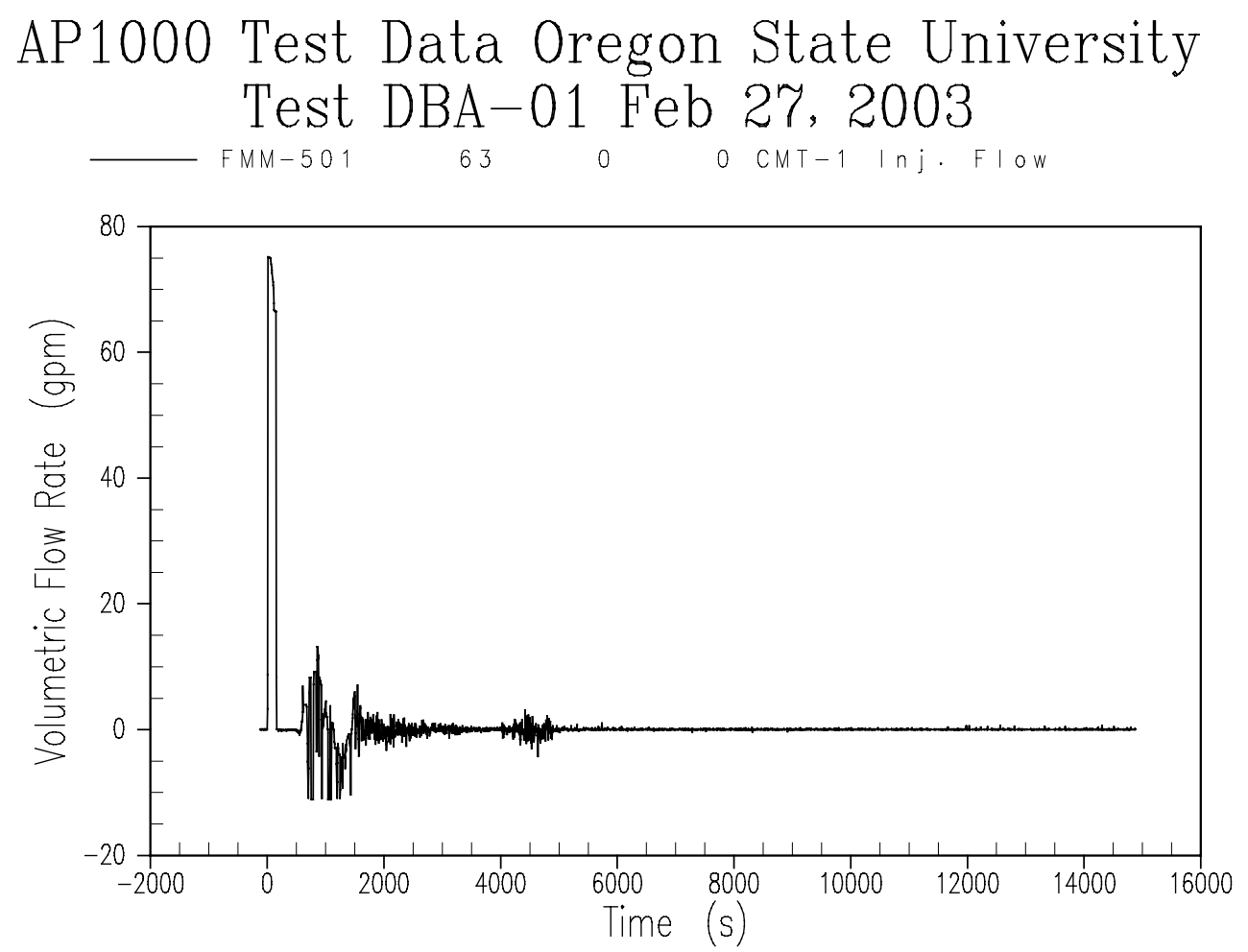

Figure A-29 Core Makeup Tank 1 Flow Rate 

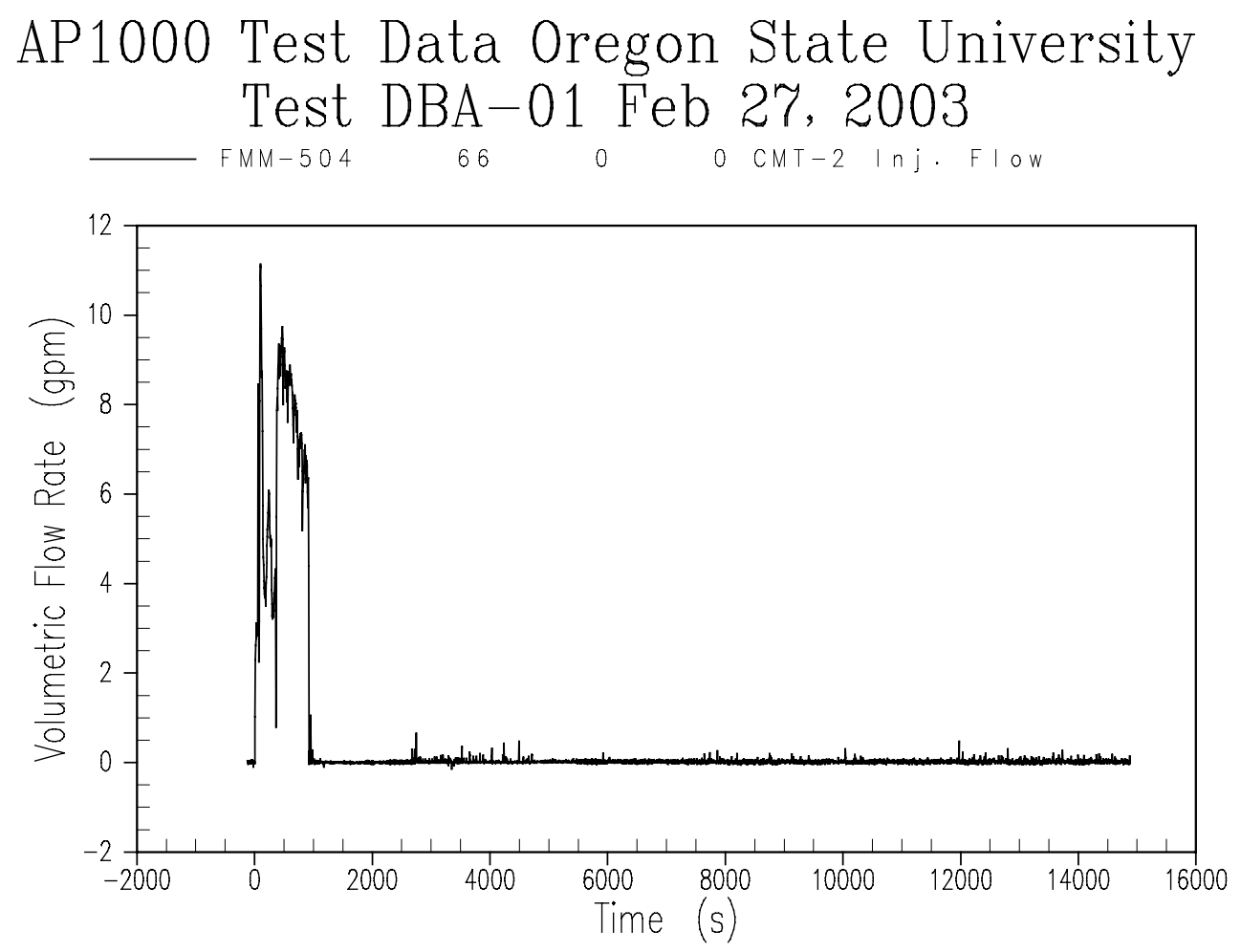

Figure A-30 Core Makeup Tank 2 Flow Rate 

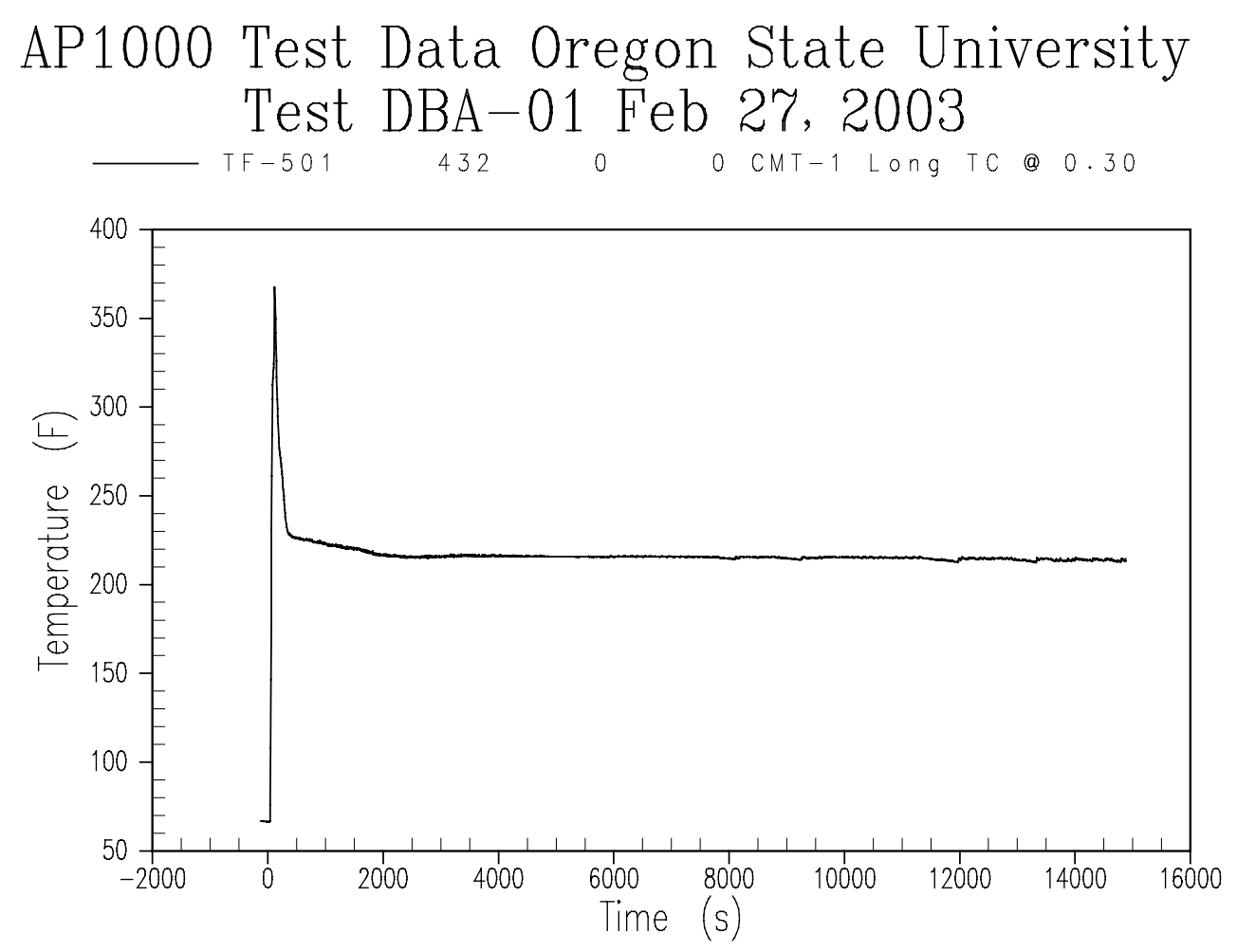

Figure A-31 Core Makeup Tank 1 Liquid Temperature - Bottom 

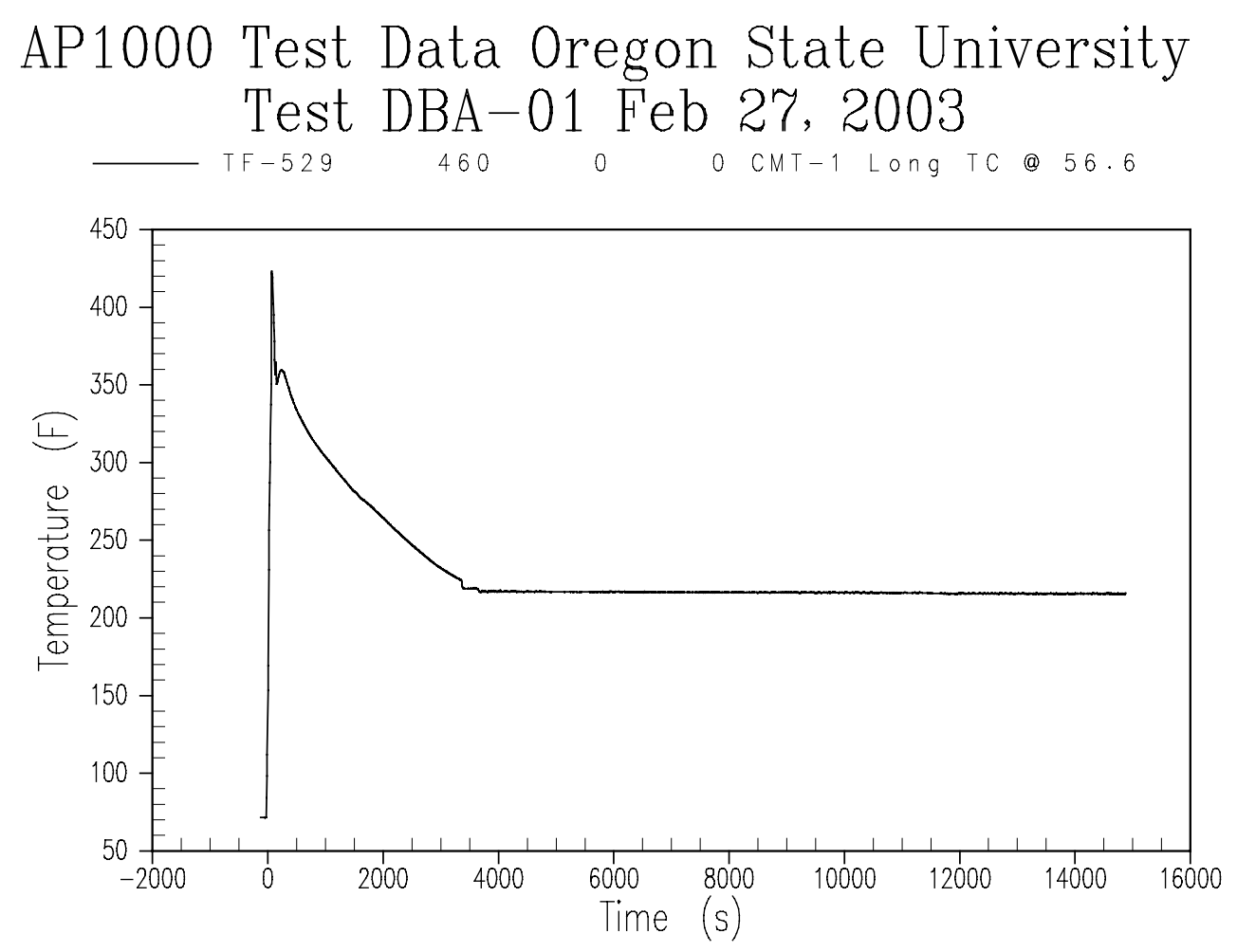

Figure A-32 Core Makeup Tank 1 Liquid Temperature - Top 

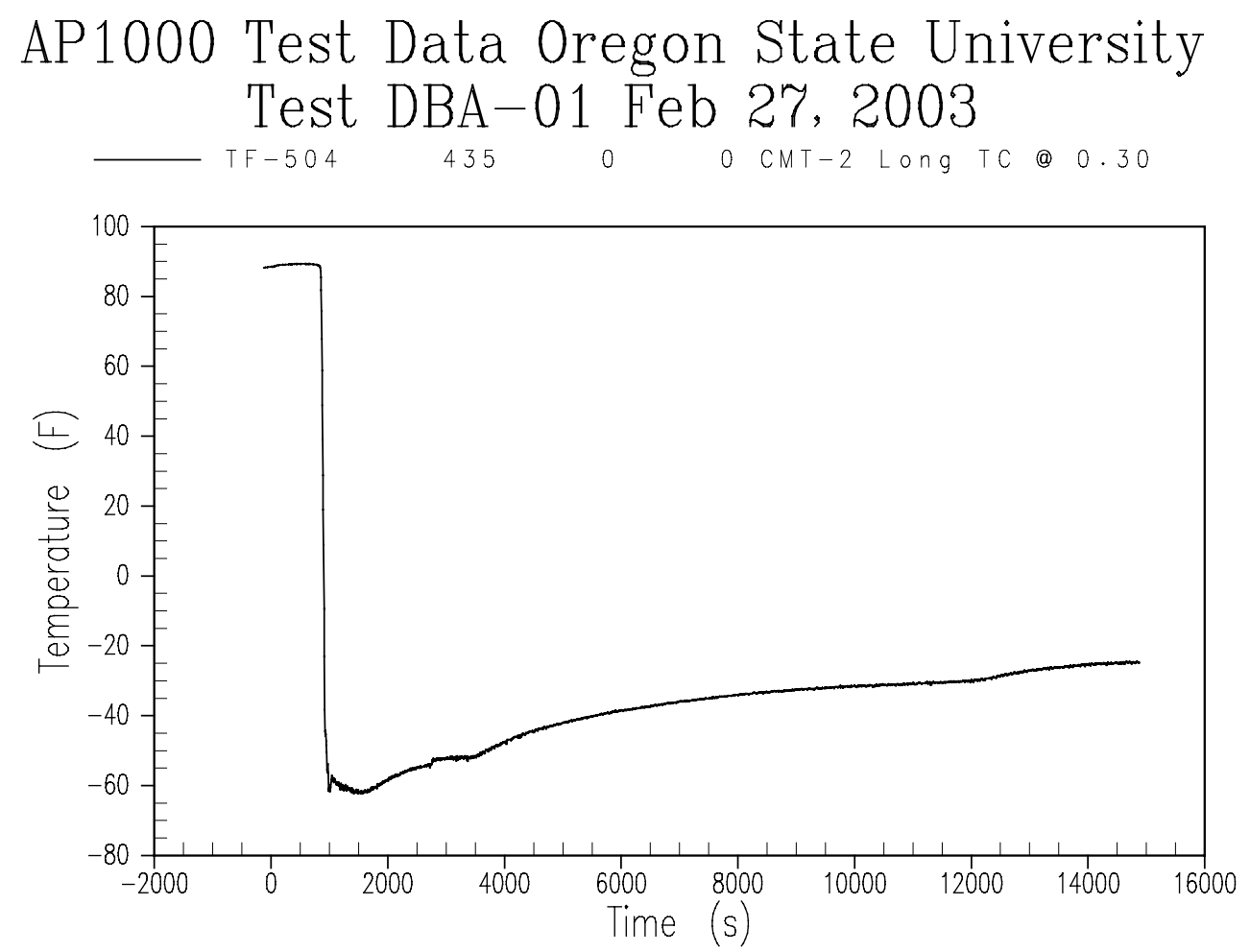

Figure A-33 Core Makeup Tank 2 Liquid Temperature - Bottom 

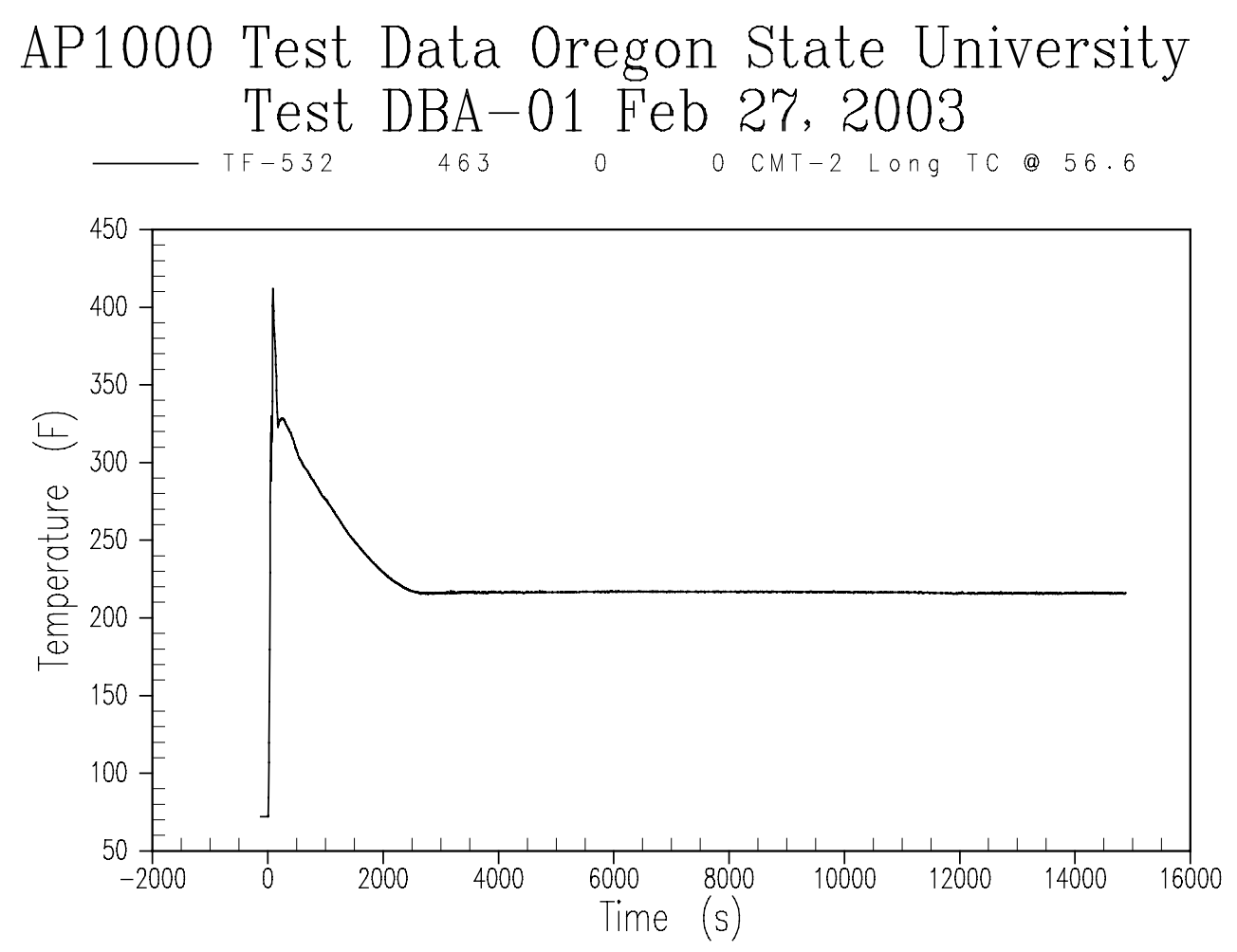

Figure A-34 Core Makeup Tank 2 Liquid Temperature - Top 

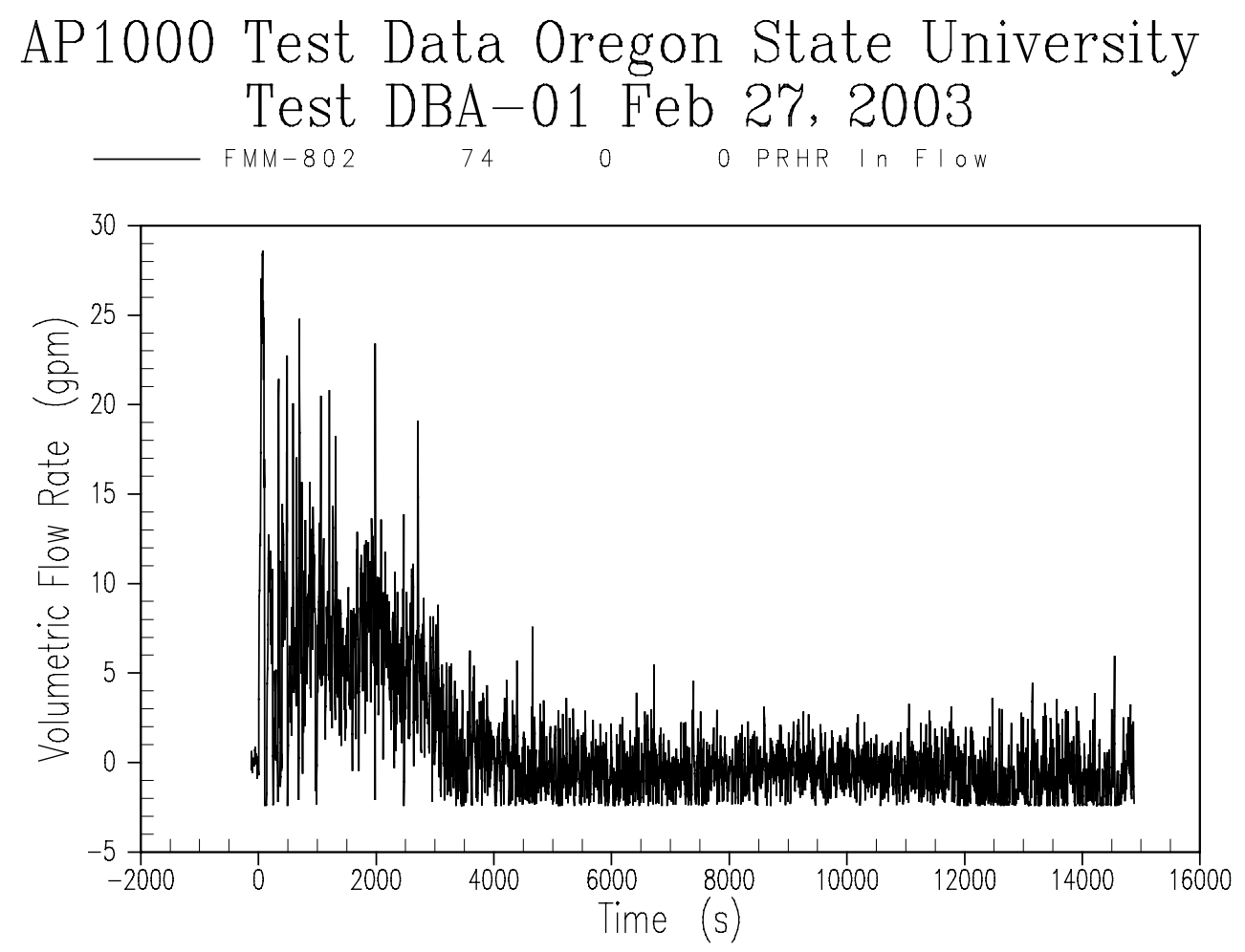

Figure A-35 Passive Residual Heat Removal Inlet Flow Rate 

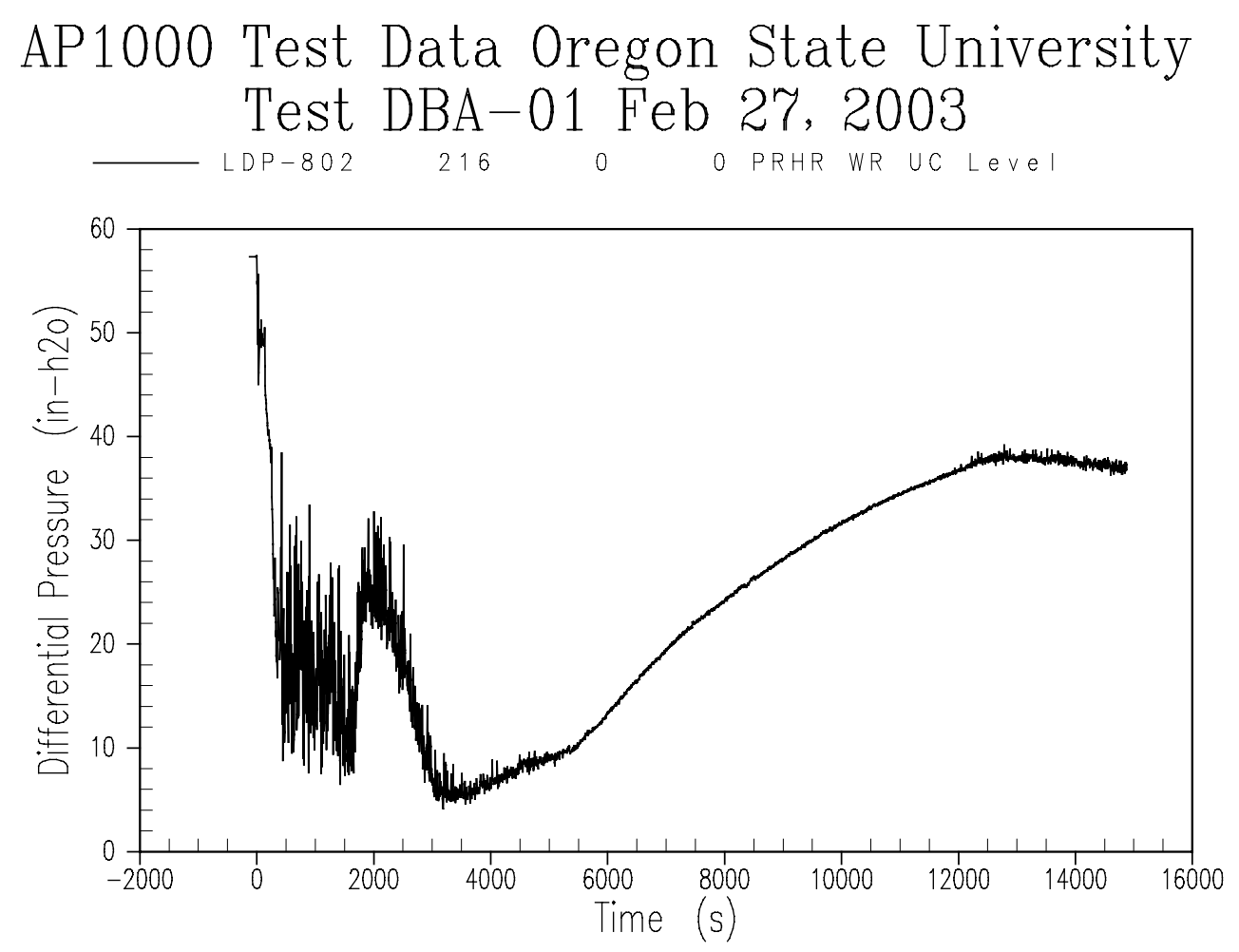

Figure A-36 Passive Residual Heat Removal Liquid Level 

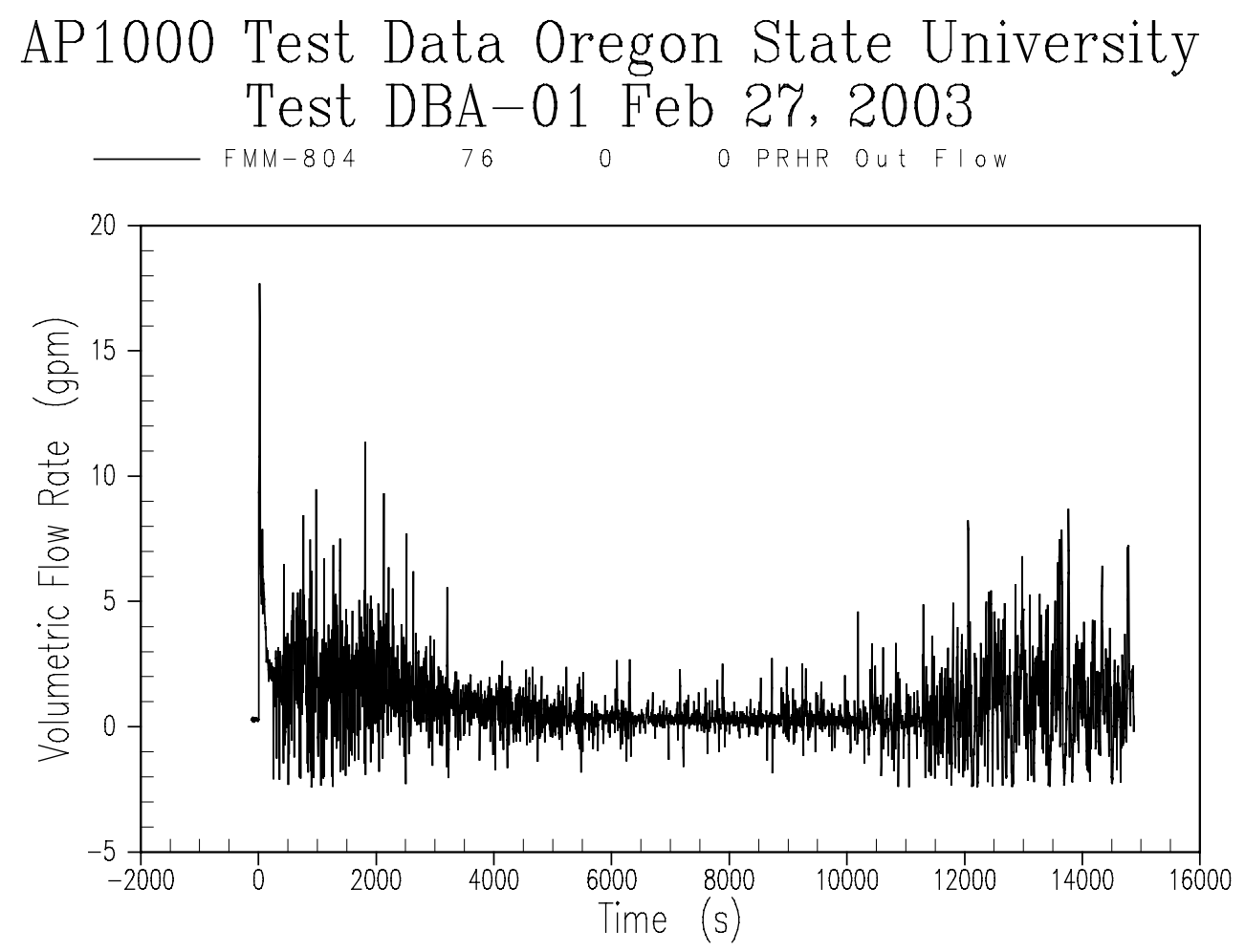

Figure A-37 Passive Residual Heat Removal Outlet Flow Rate 

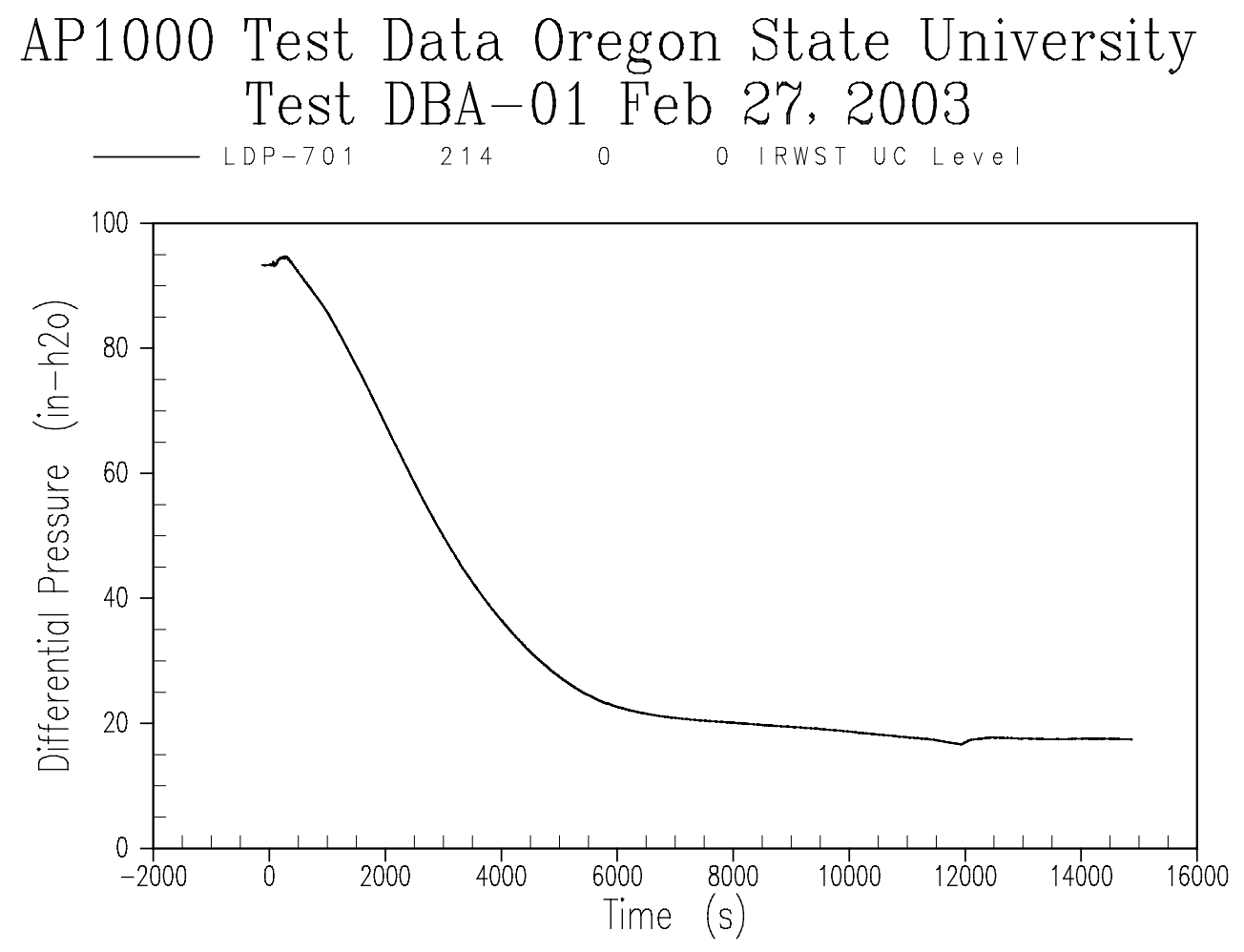

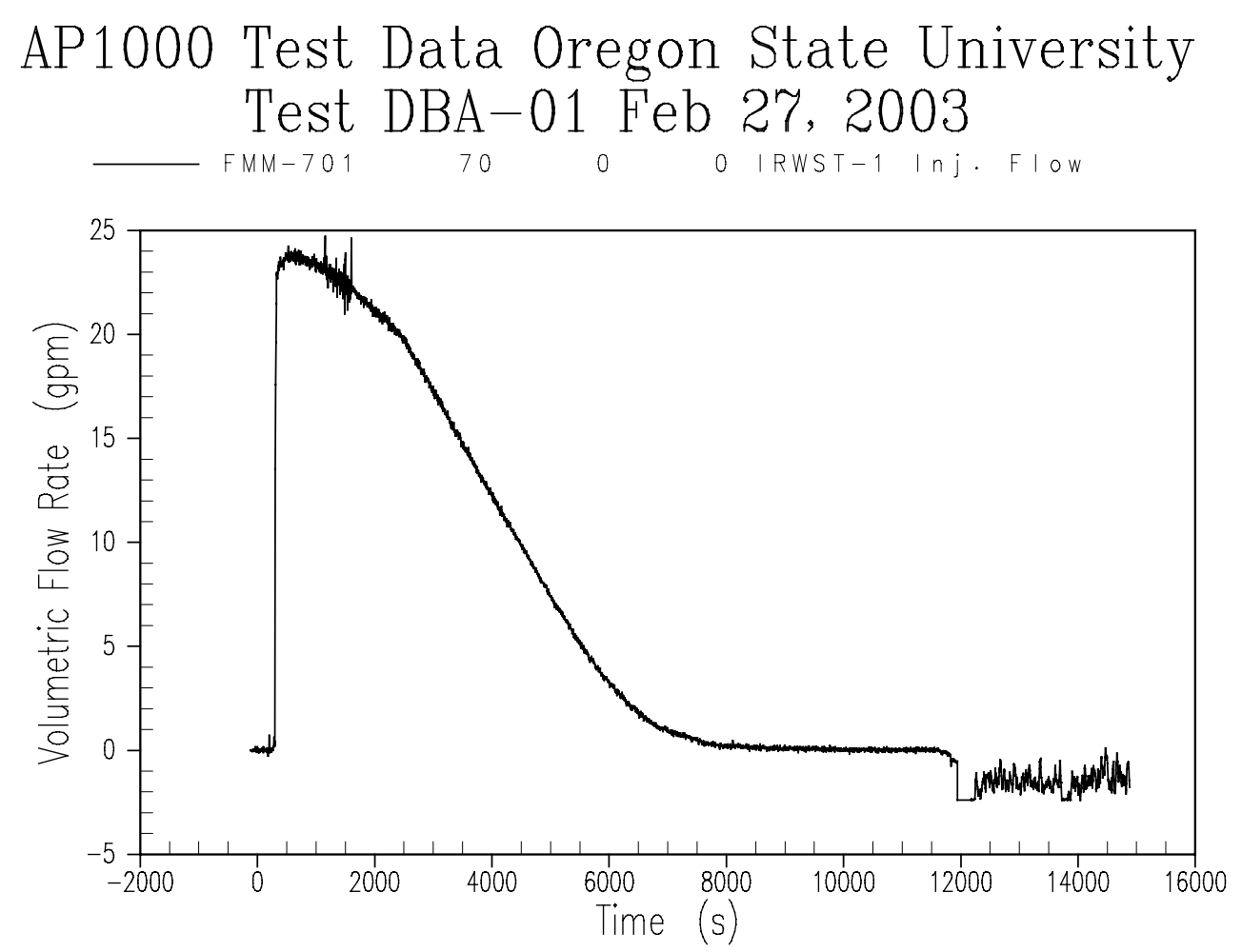

Figure A-39 IRWST Discharge Line 1 Flow Rate 

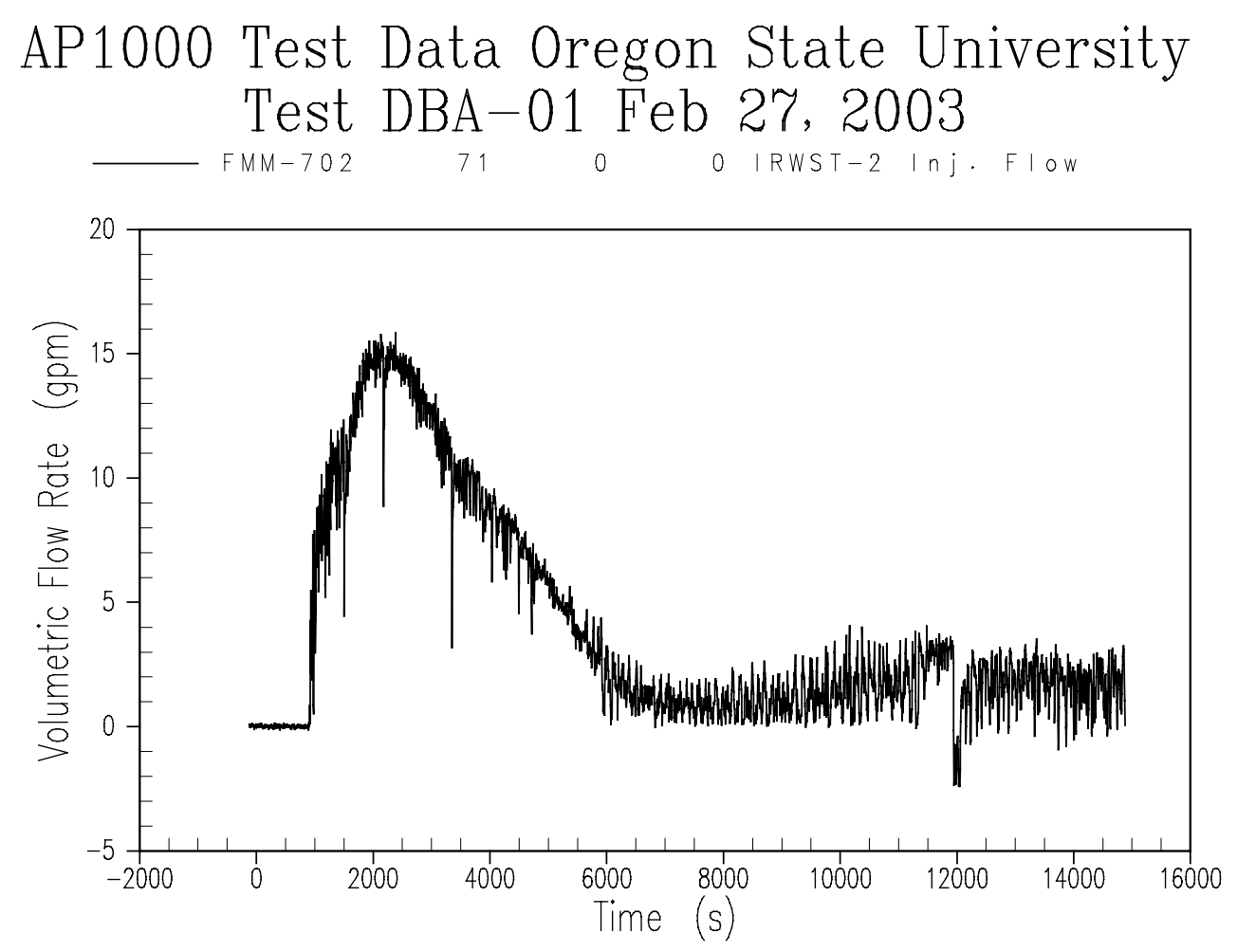

Figure A-40 IRWST Discharge Line 2 Flow Rate 

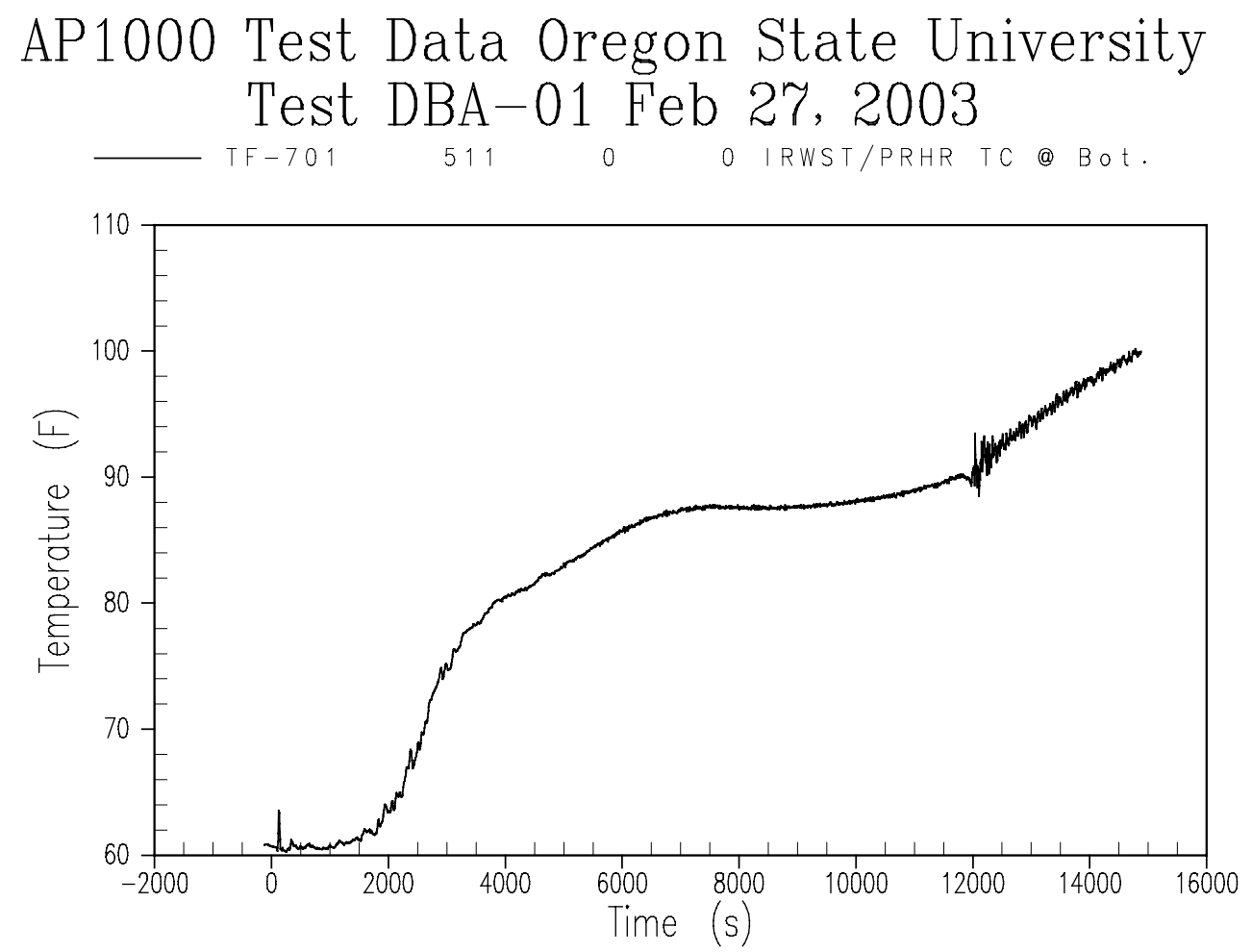

Figure A-41 IRWST Fluid Temperature 

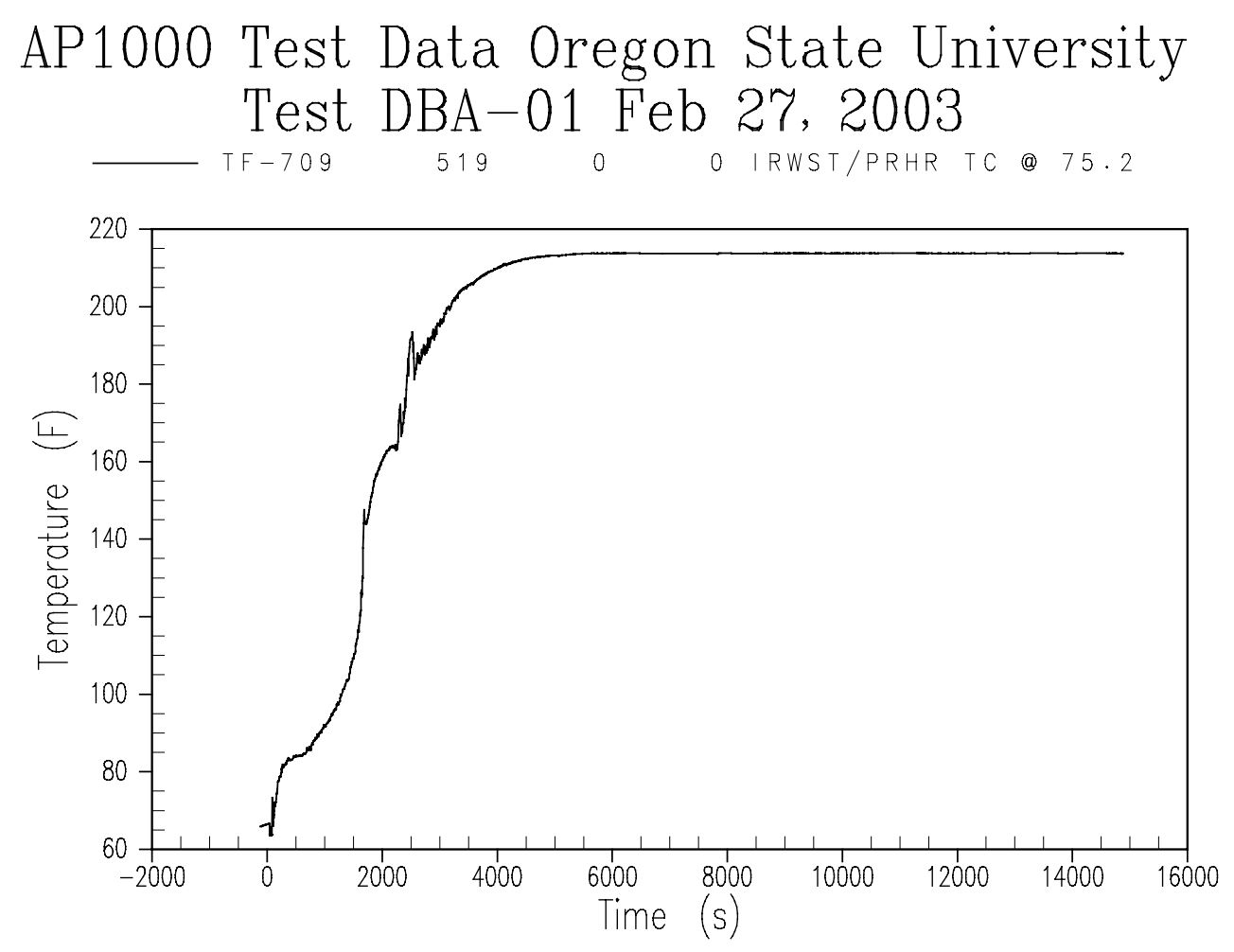

Figure A-42 IRWST Fluid Temperature 


\section{AP1000 Test Data Oregon State University Test DBA-01 Feb 27, 2003 \\ $-P T-605$ \\ 253 \\ 0 \\ o ADS1-3 sep. Press.}

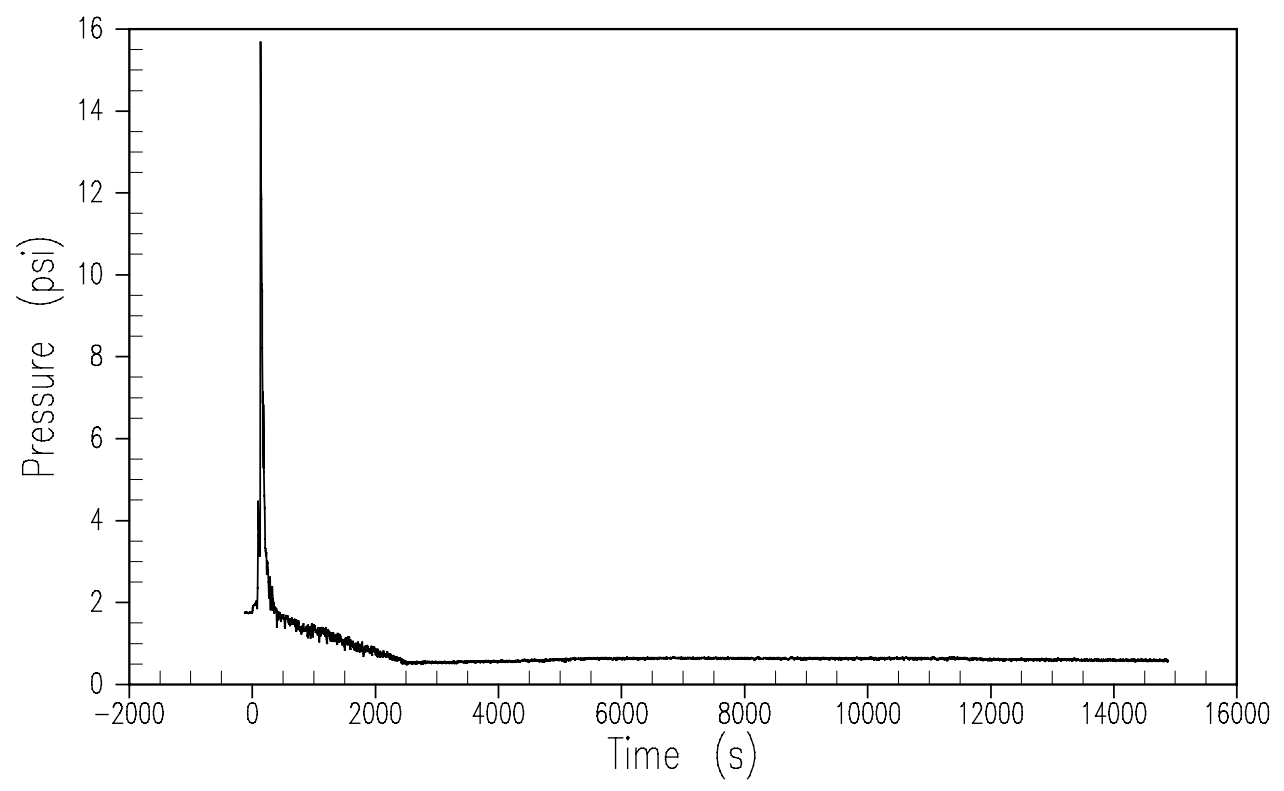

$a, b, c$

Time (s)

Figure A-43 ADS 1-3 Separator Pressure 

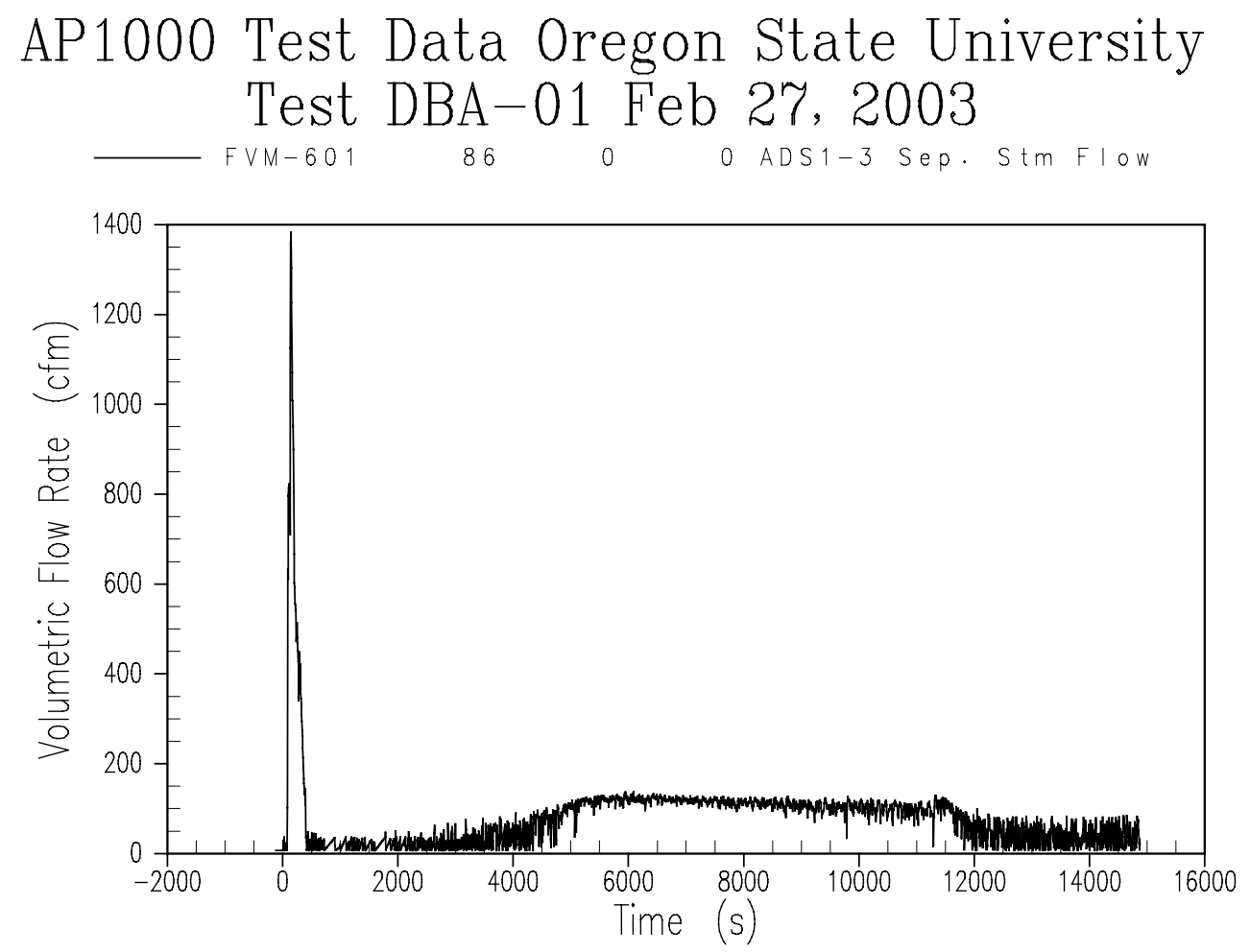

Figure A-44 ADS 1-3 Separator Steam Flow Rate 

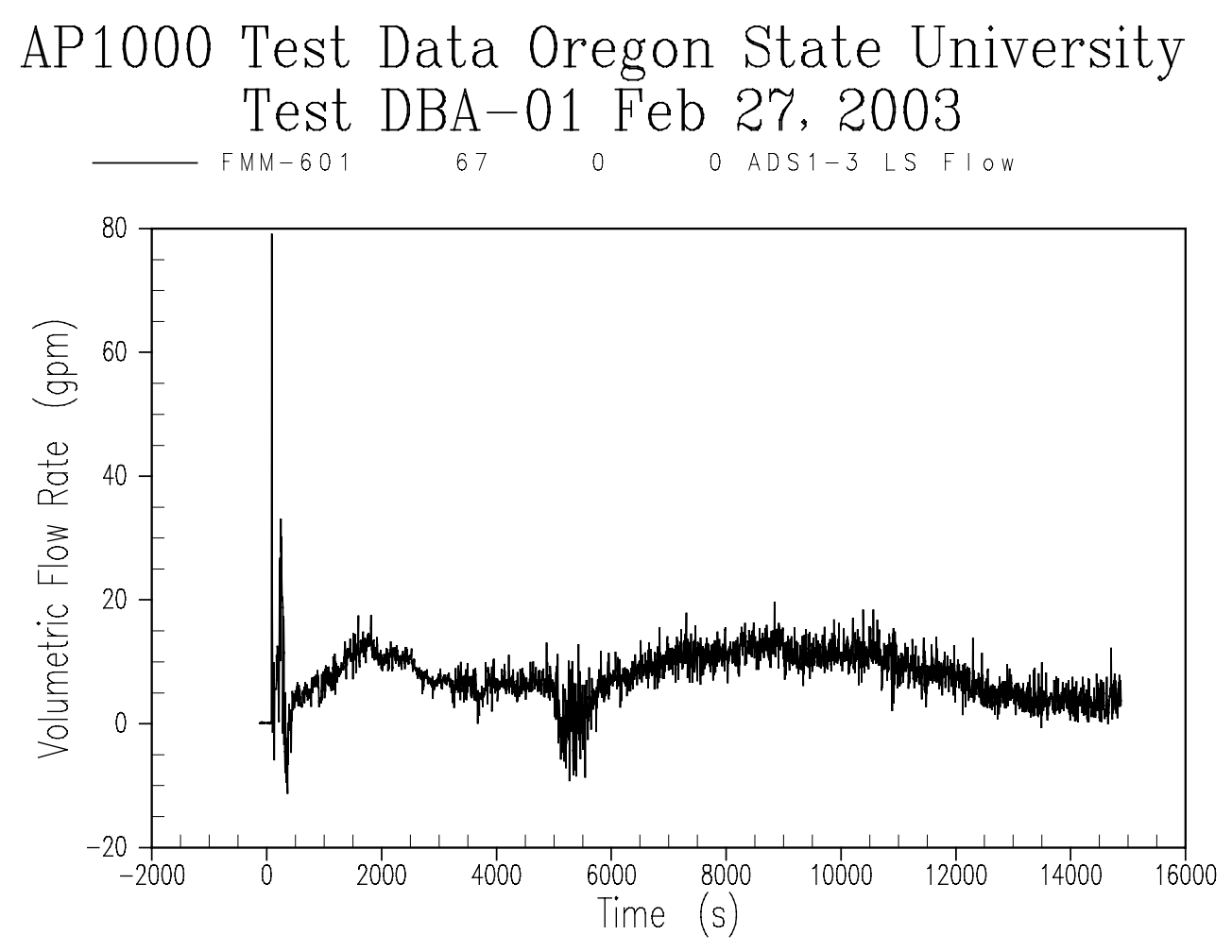

Figure A-45 ADS 1-3 Separator Liquid Flow Rate 

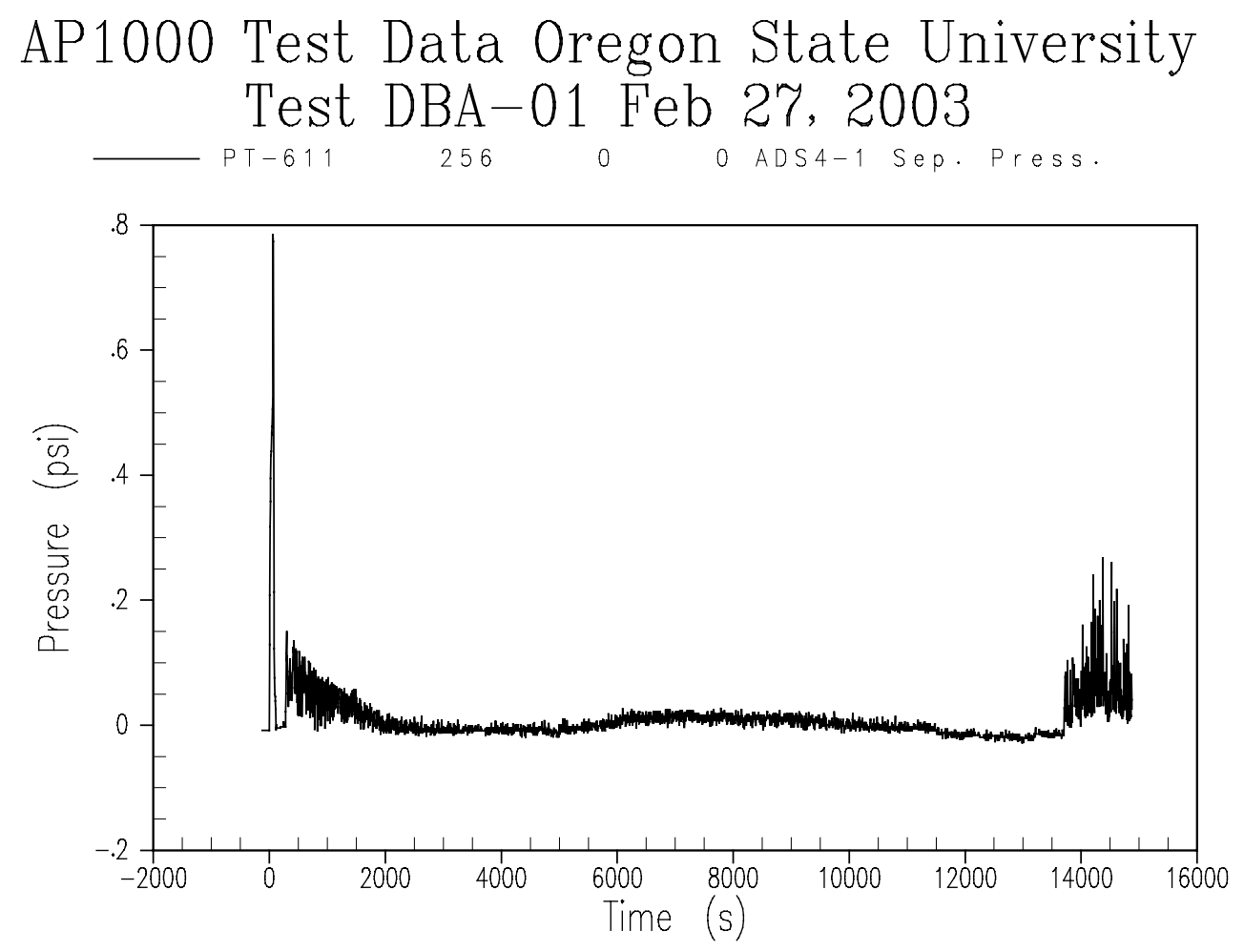

Figure A-46 ADS 4-1 Separator Pressure 

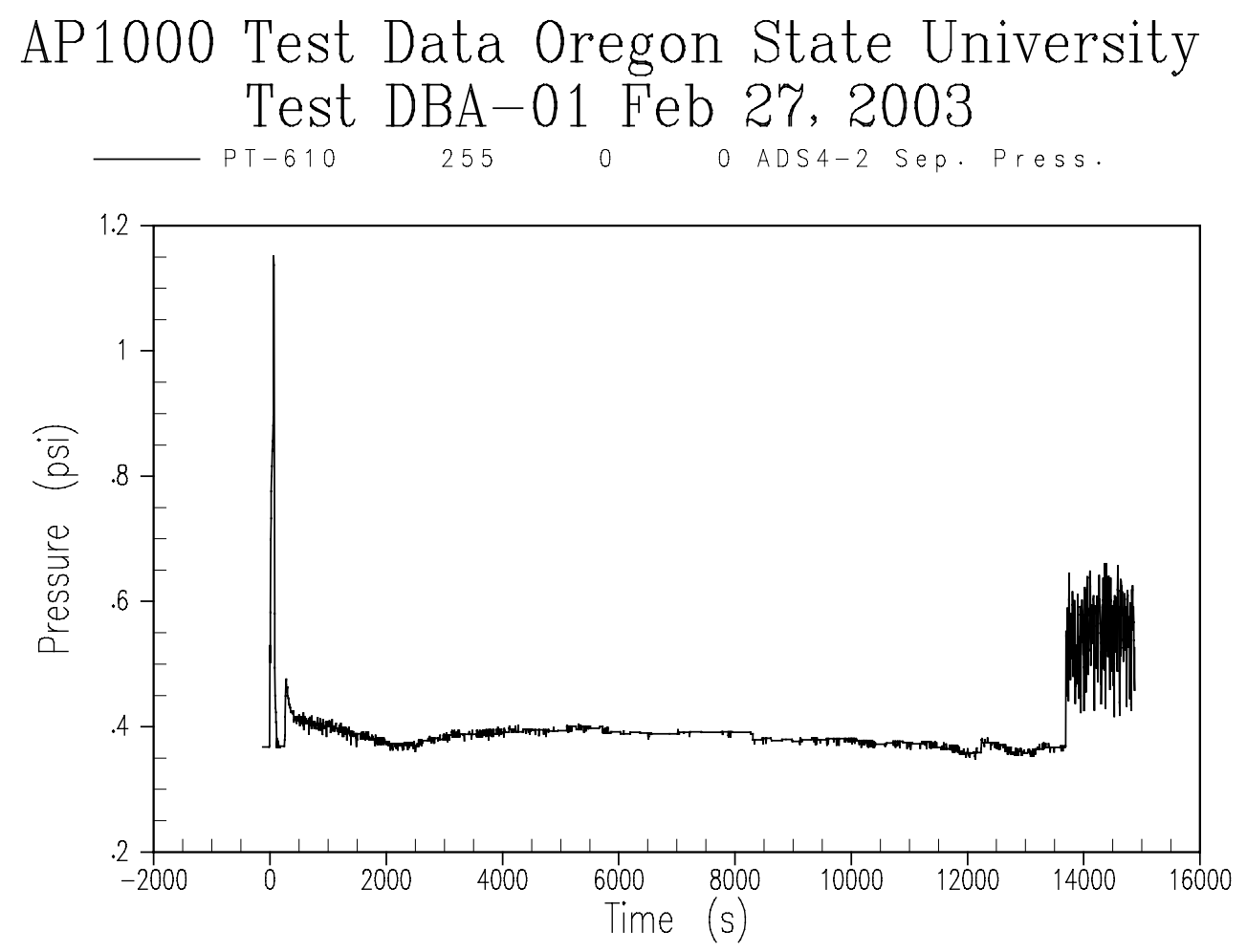

Figure A-47 ADS 4-2 Separator Pressure 

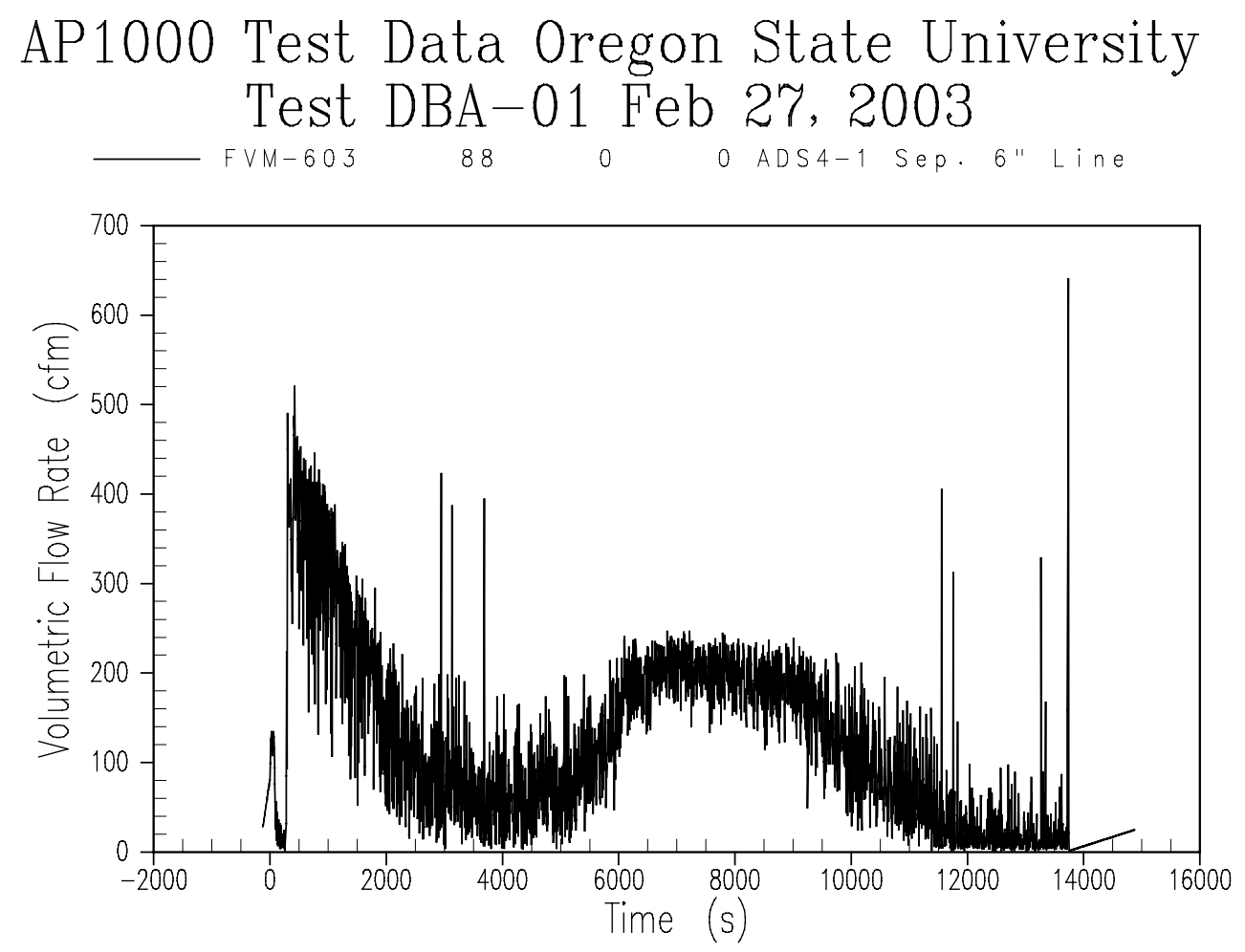

Figure A-48 ADS 4-1 Separator Steam Flow Rate 

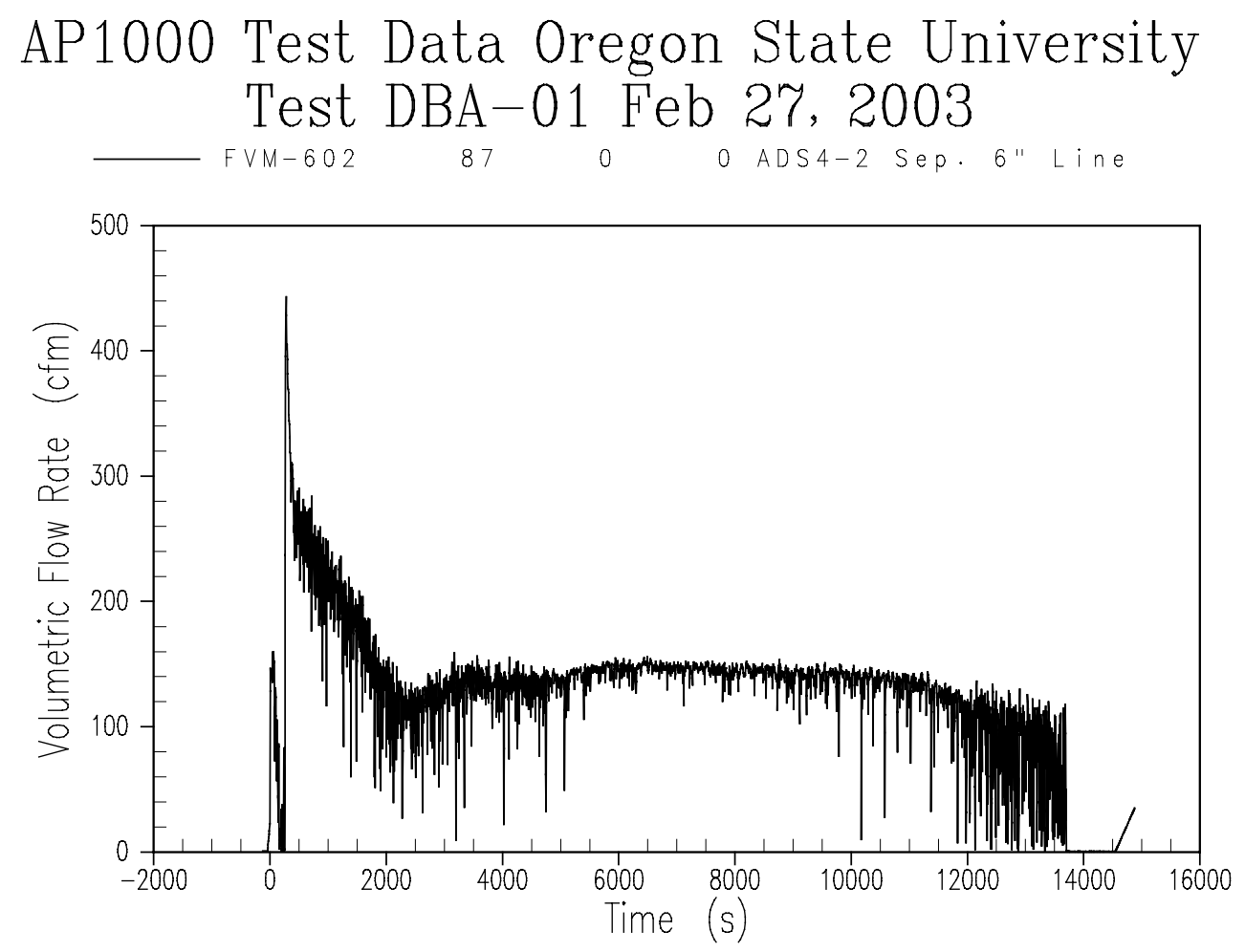

Figure A-49 ADS 4-2 Separator Steam Flow Rate 

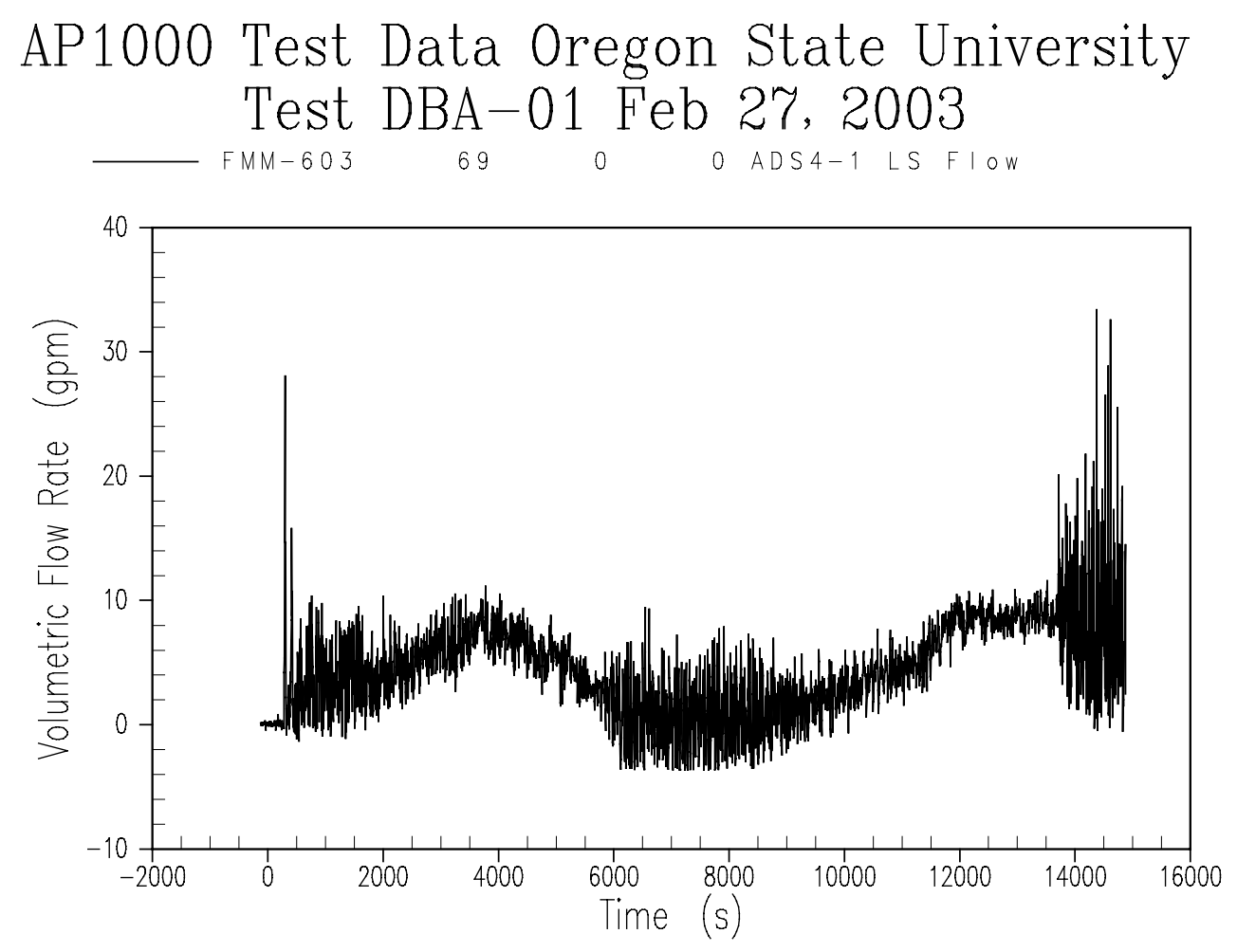

Figure A-50 ADS 4-1 Separator Liquid Flow Rate 

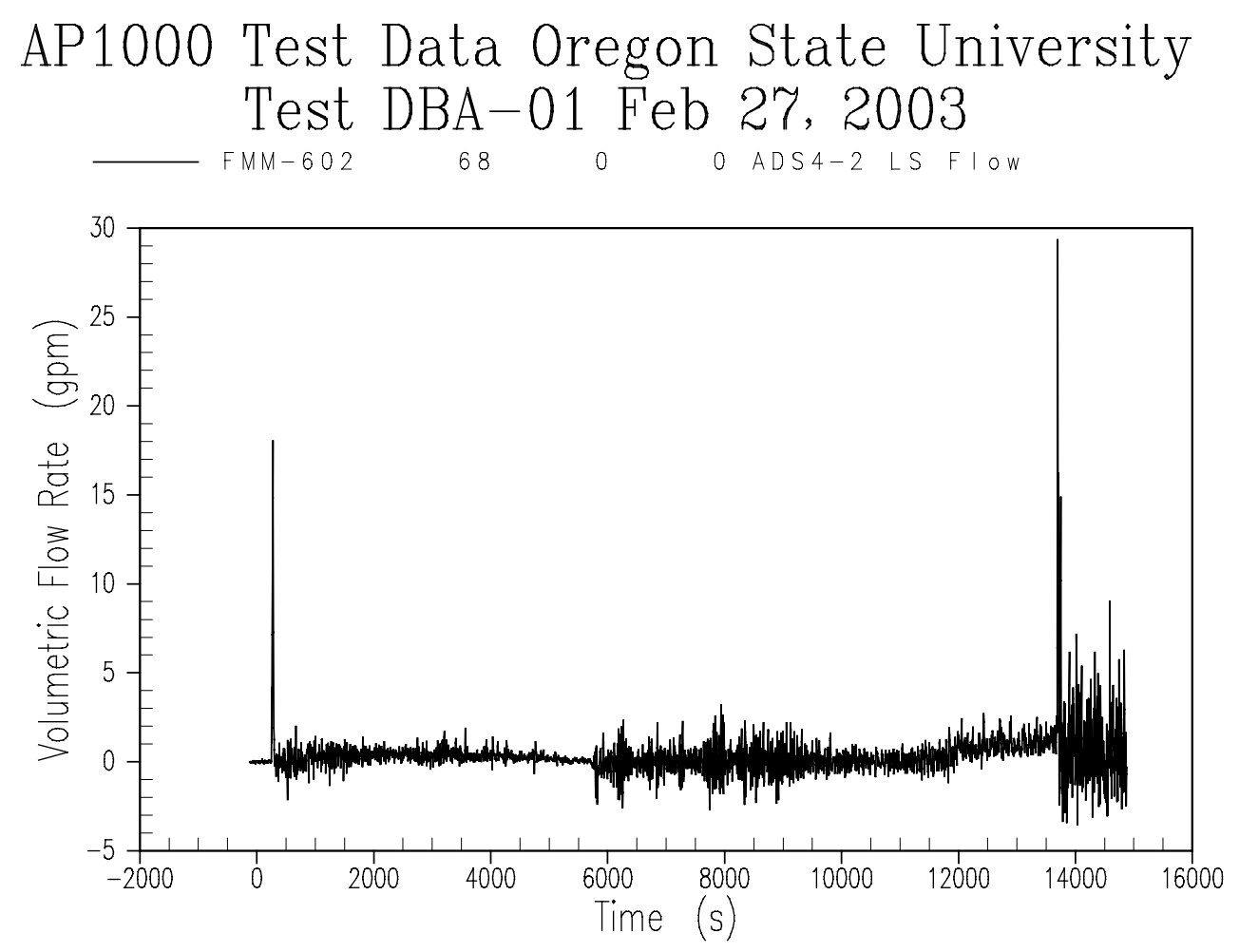

Figure A-51 ADS 4-2 Separator Liquid Flow Rate 

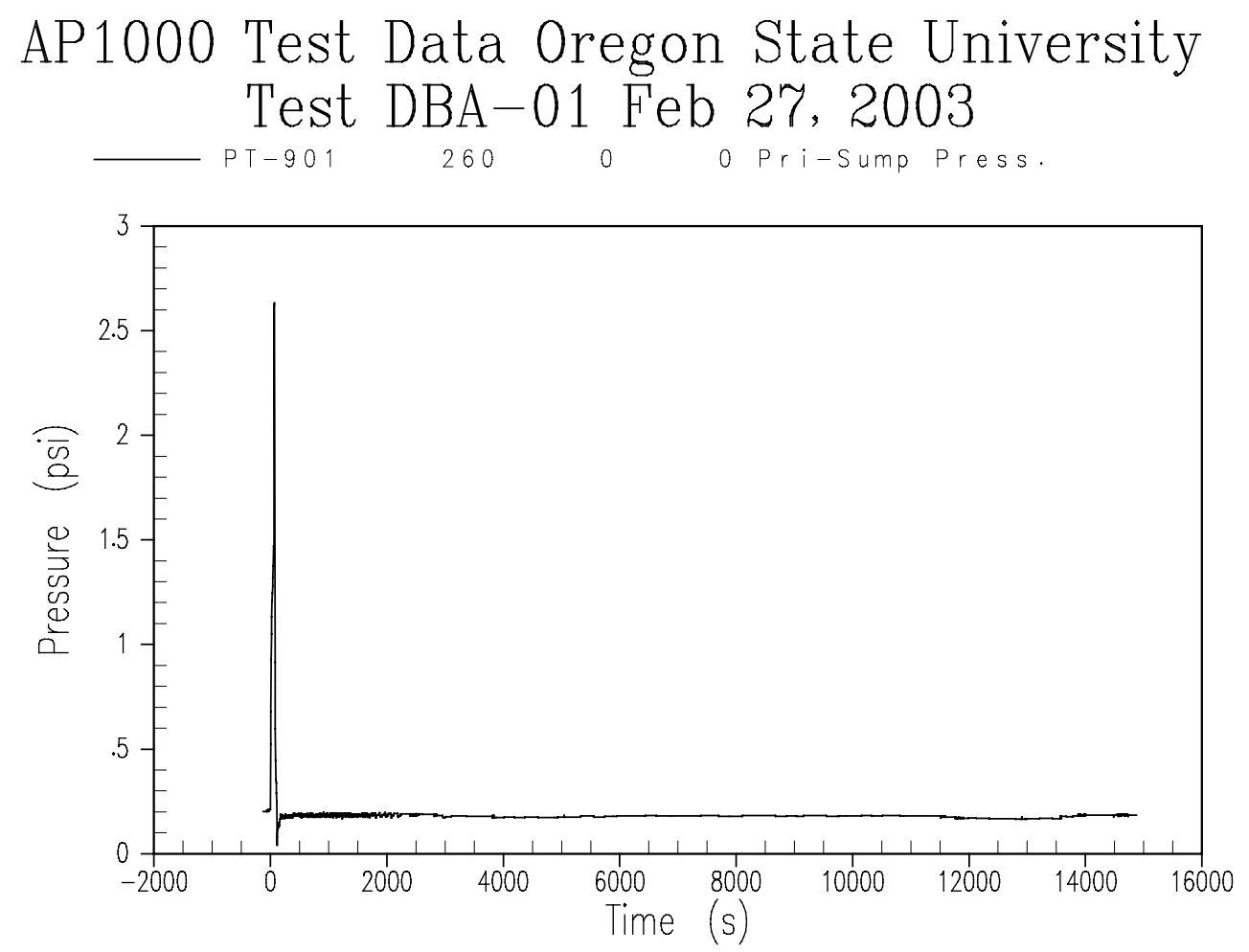

Figure A-52 Primary Sump Pressure 

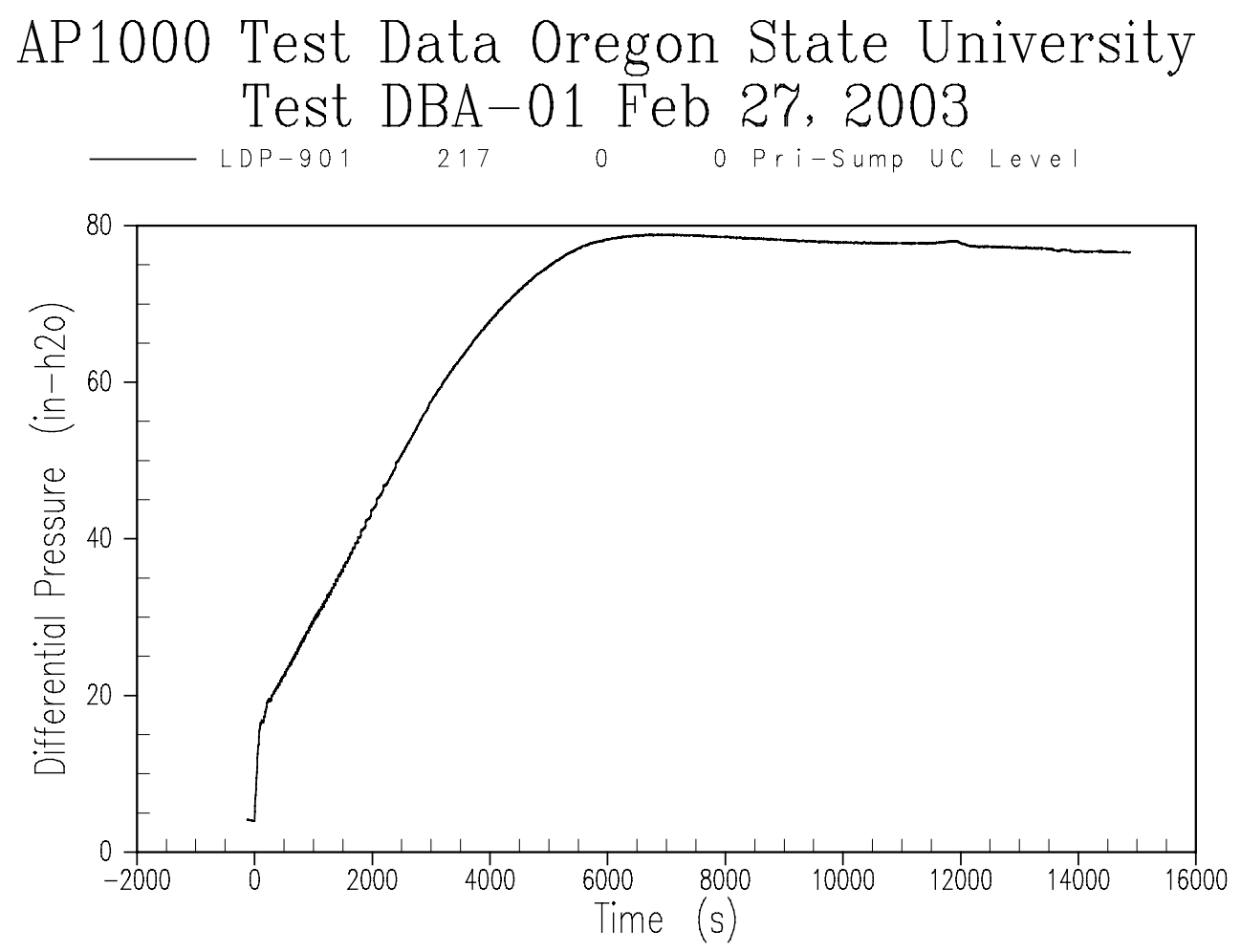

Figure A-53 Primary Sump Liquid Level 

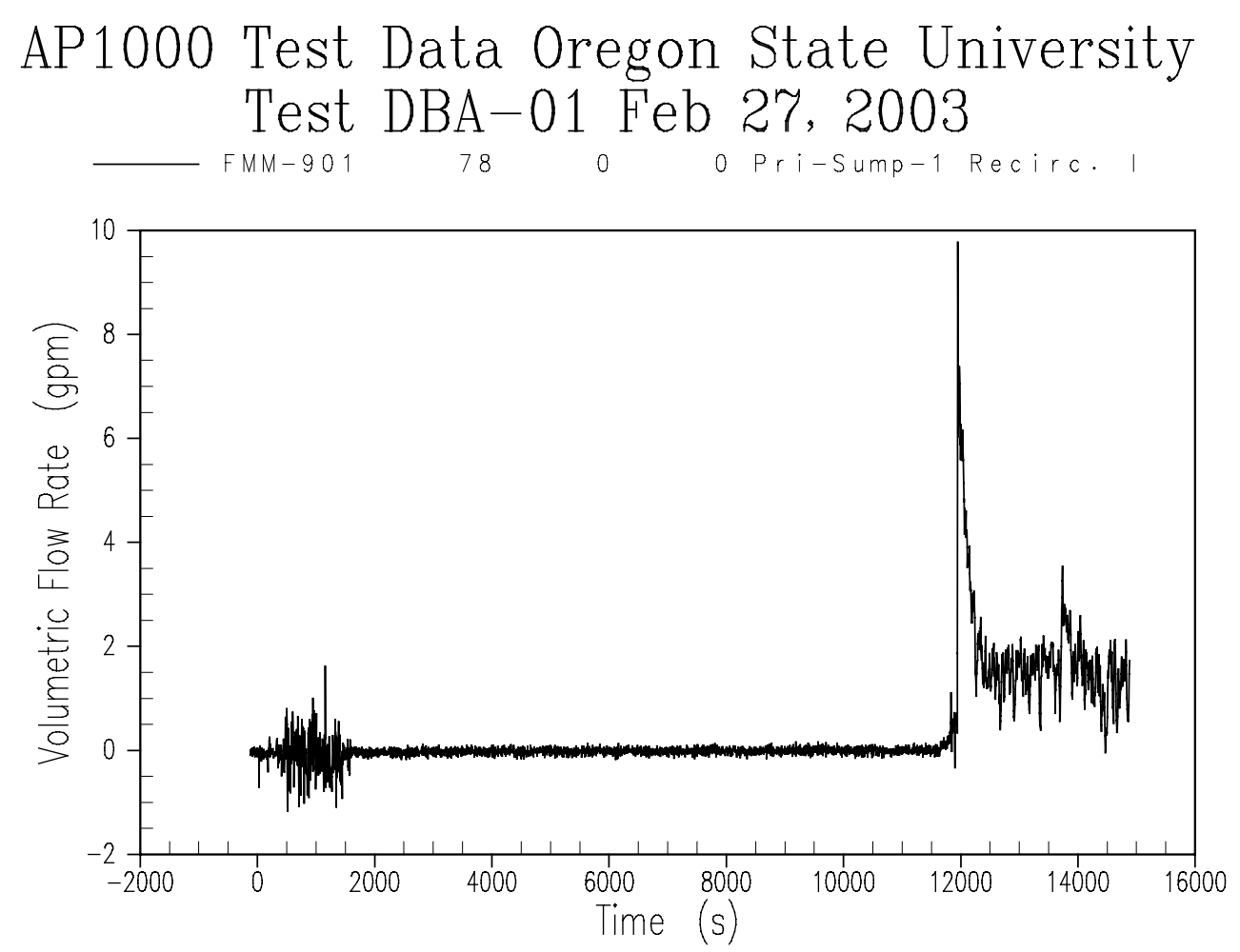

Figure A-54 Primary Sump Injection Flow Rate 

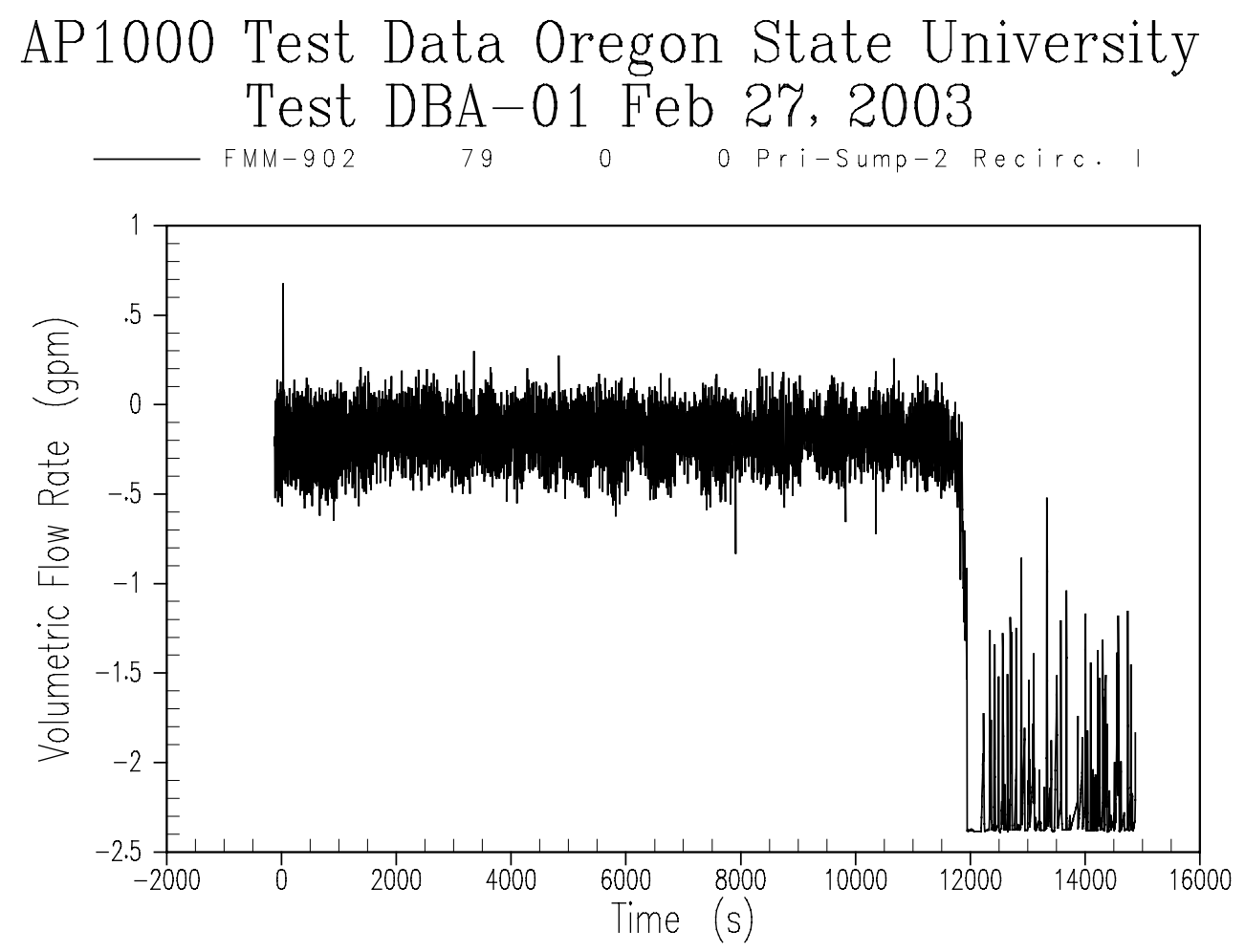

Figure A-55 Primary Sump Injection Flow Rate 

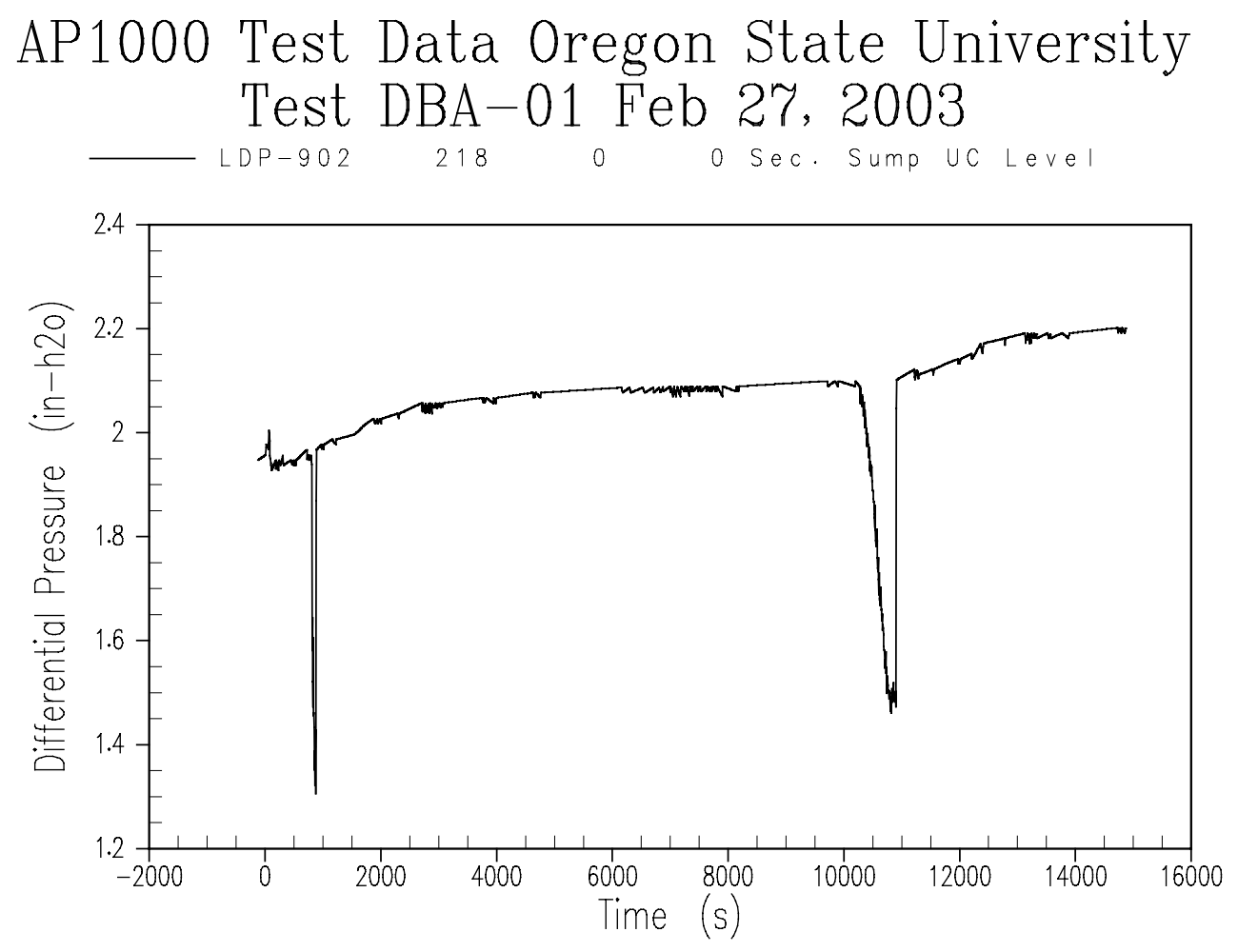

Figure A-56 Secondary Sump Liquid Level 

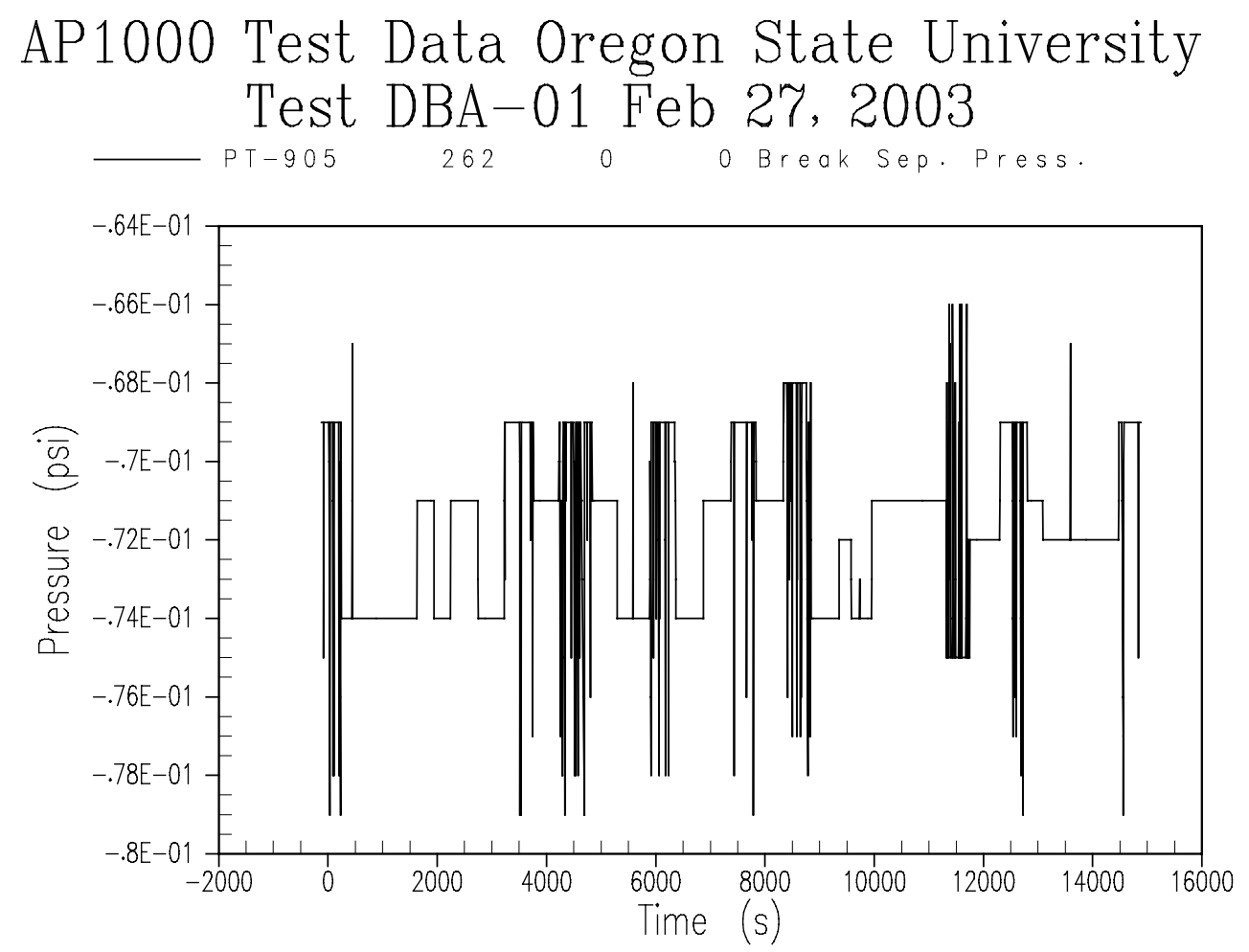

Figure A-57 Break Separator Pressure 

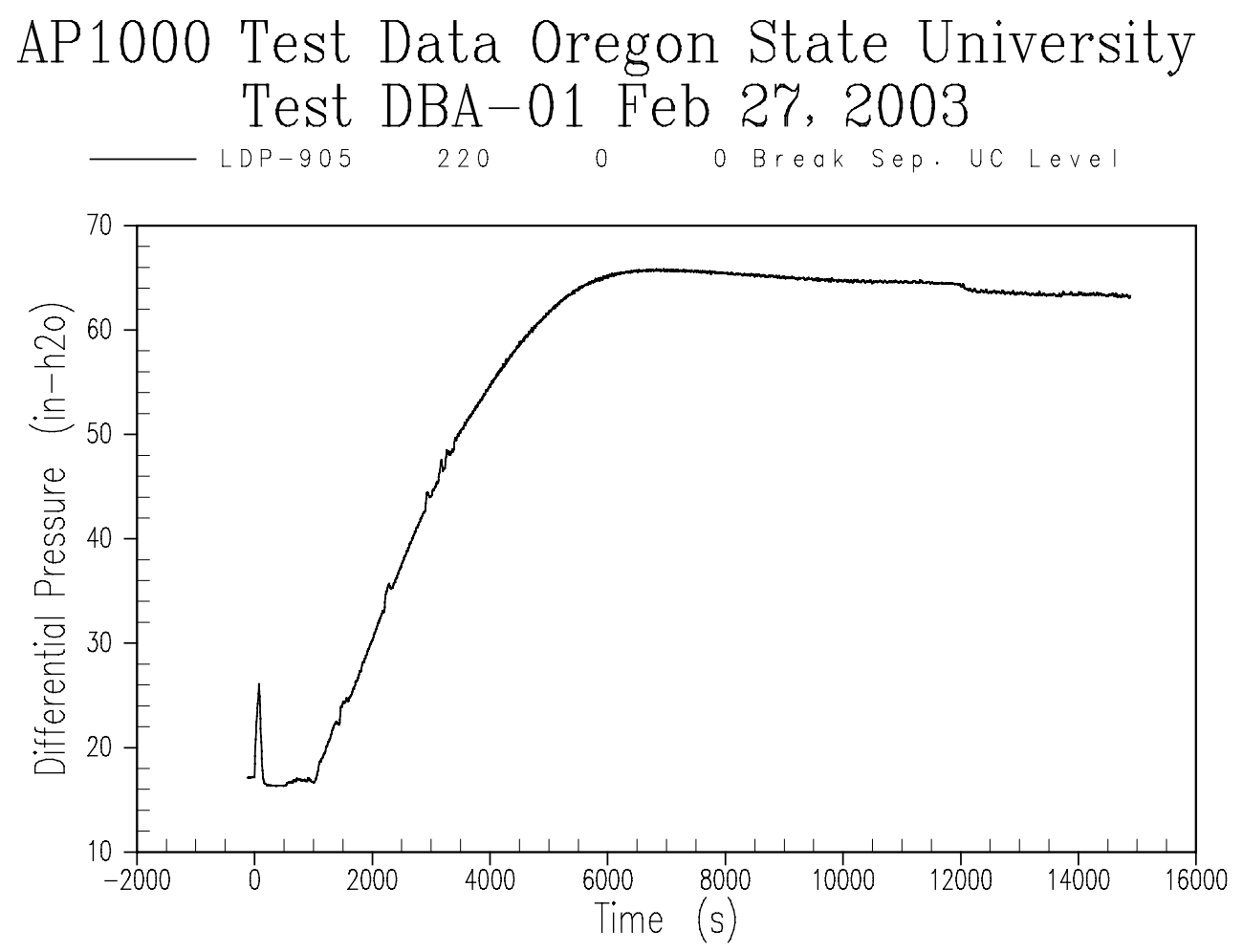

Figure A-58 Break Separator Liquid Level 

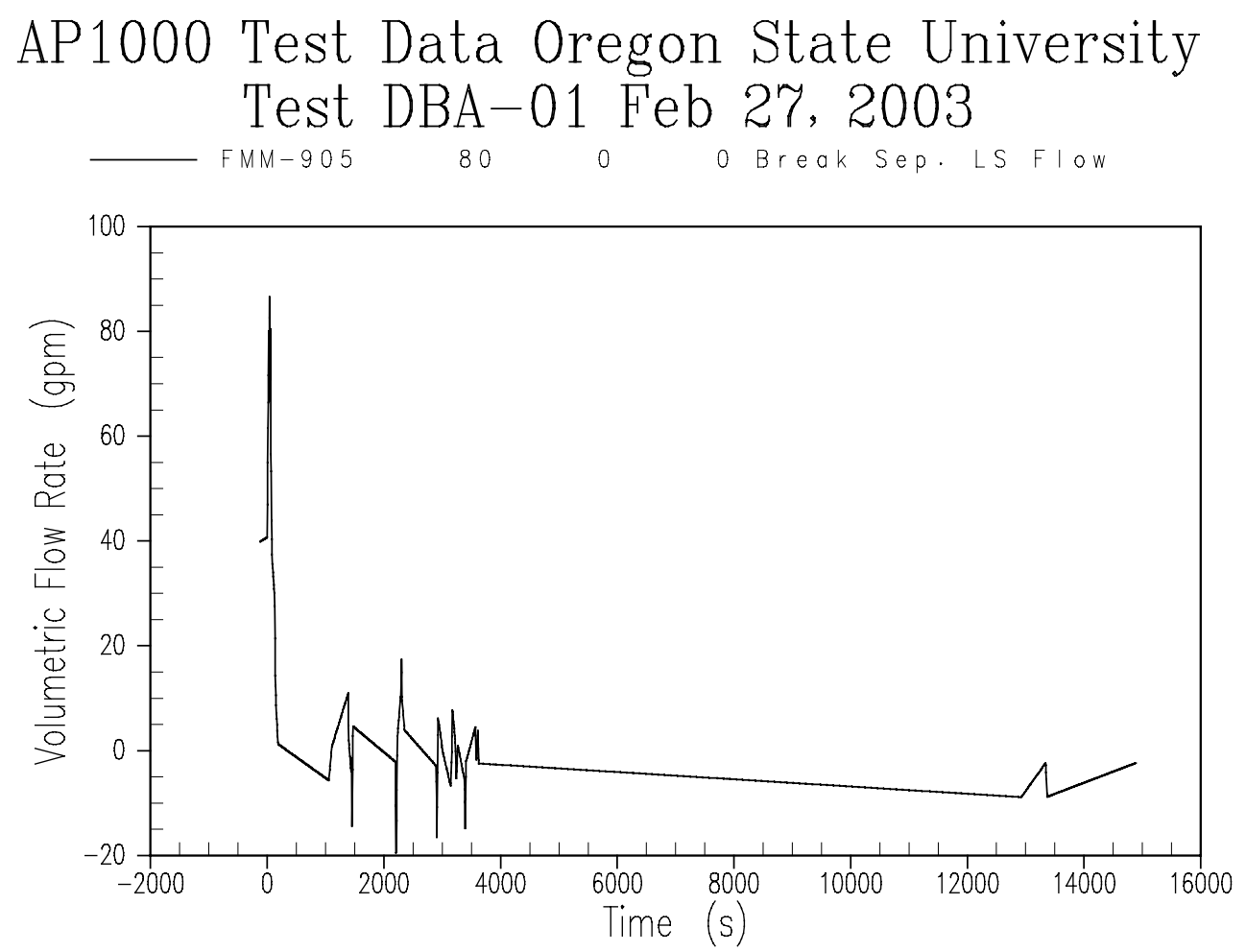

Figure A-59 Break Separator Flow to Primary Sump 

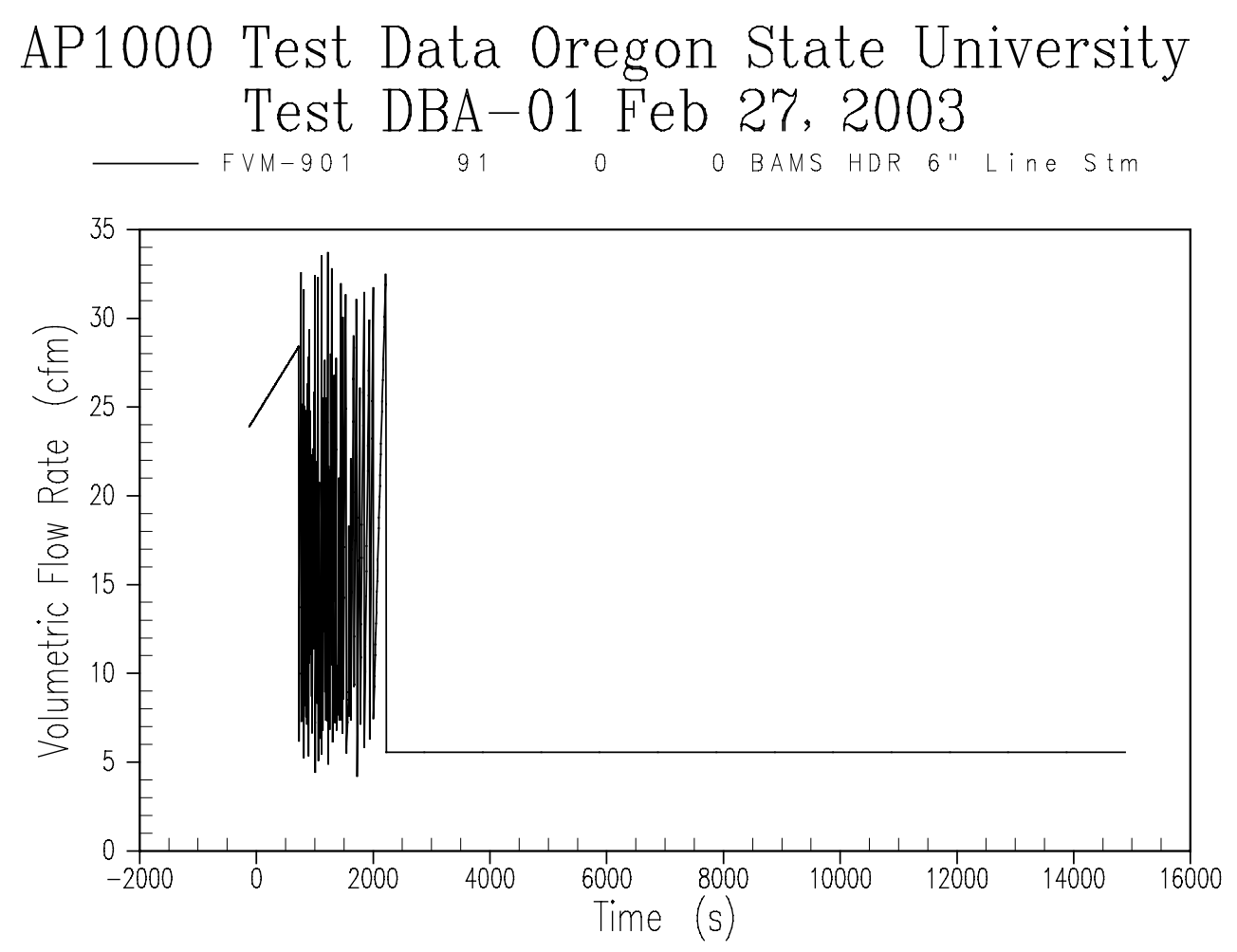

Figure A-60 BAMS Steam Flow Rate 

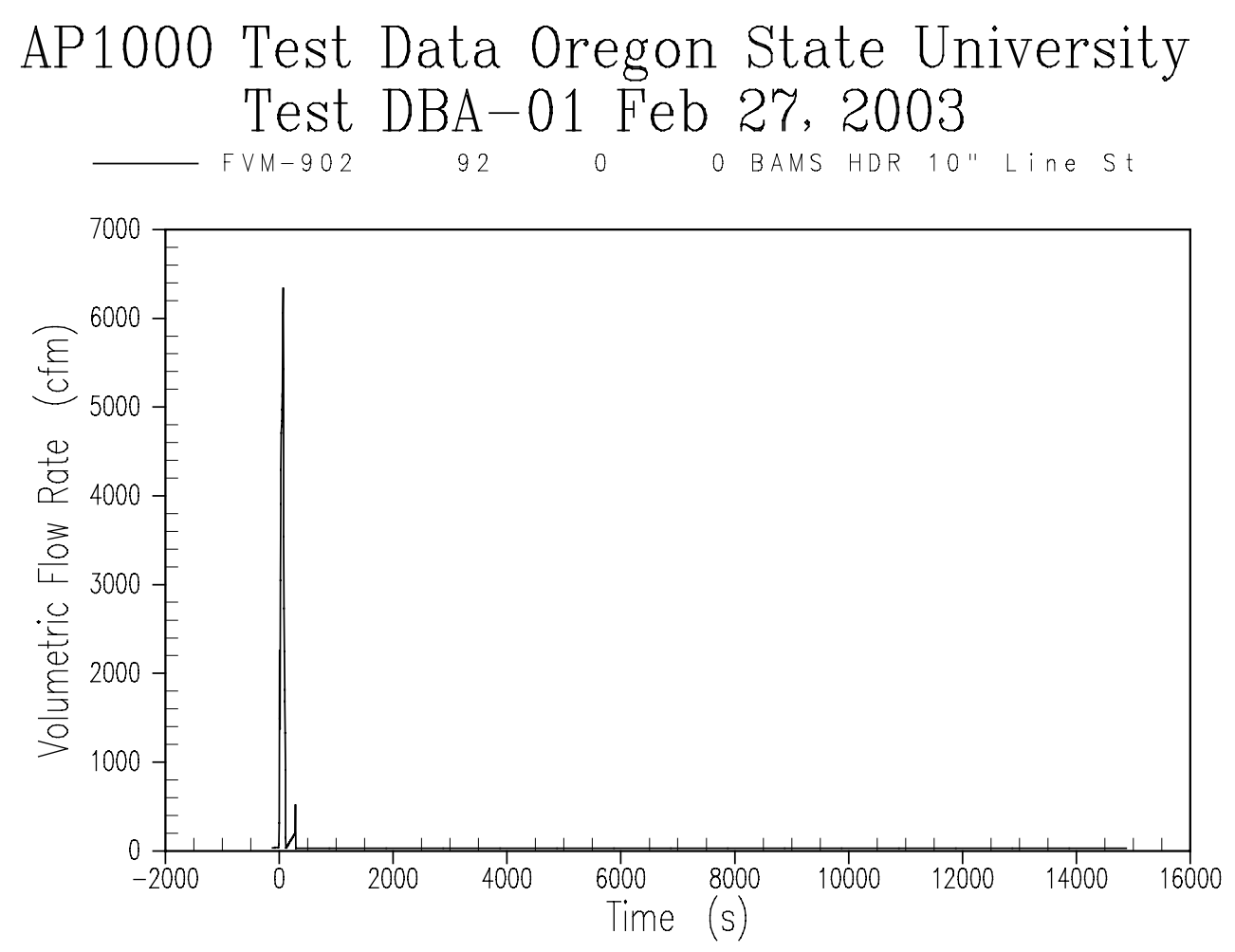

Figure A-61 BAMS Steam Flow Rate 

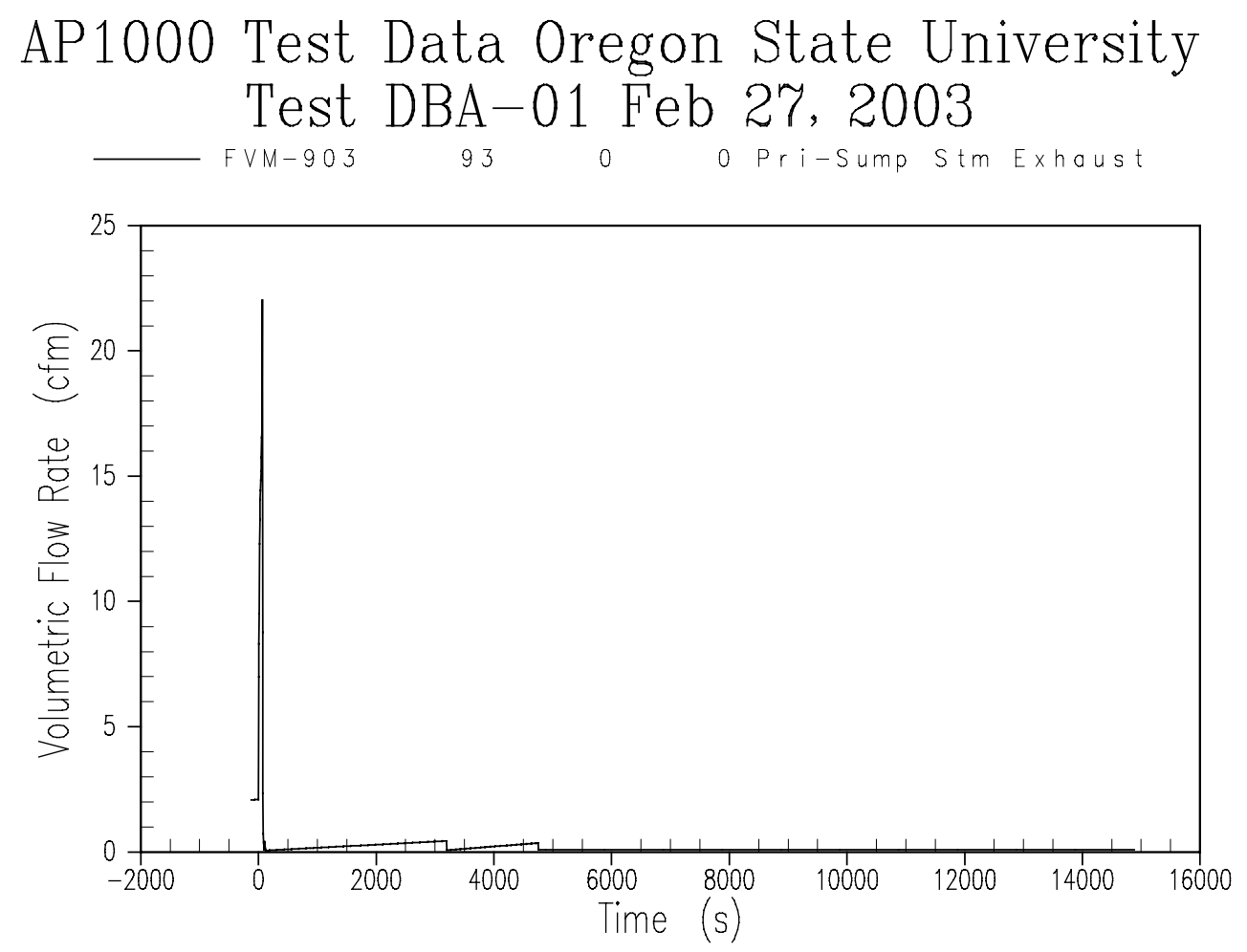

Figure A-62 BAMS/Primary Sump Steam Flow Rate 

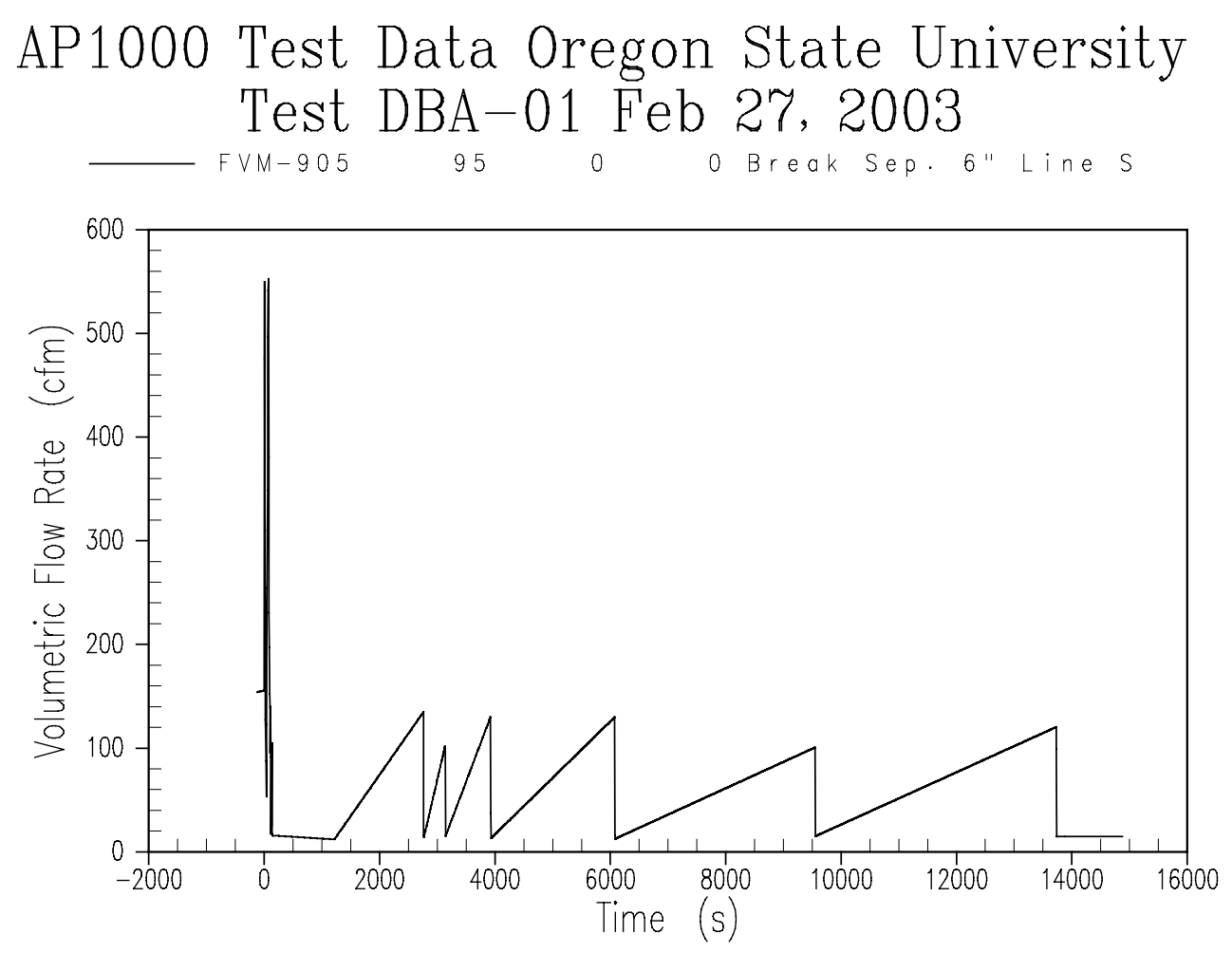

Figure A-63 BAMS/Separator Steam Flow Rate - 6-inch Pipe 

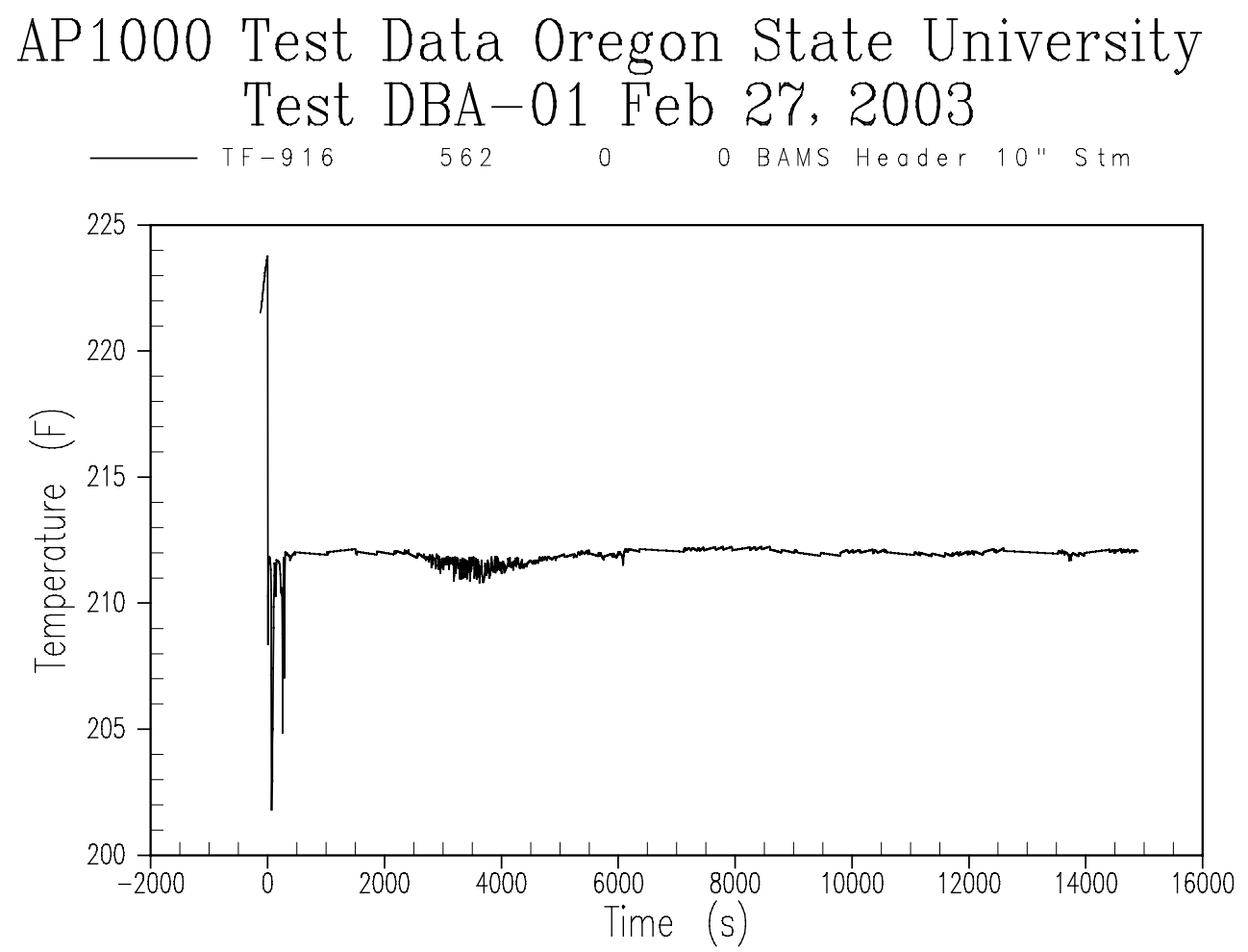

Figure A-64 BAMS/Exhaust Line Temperature 

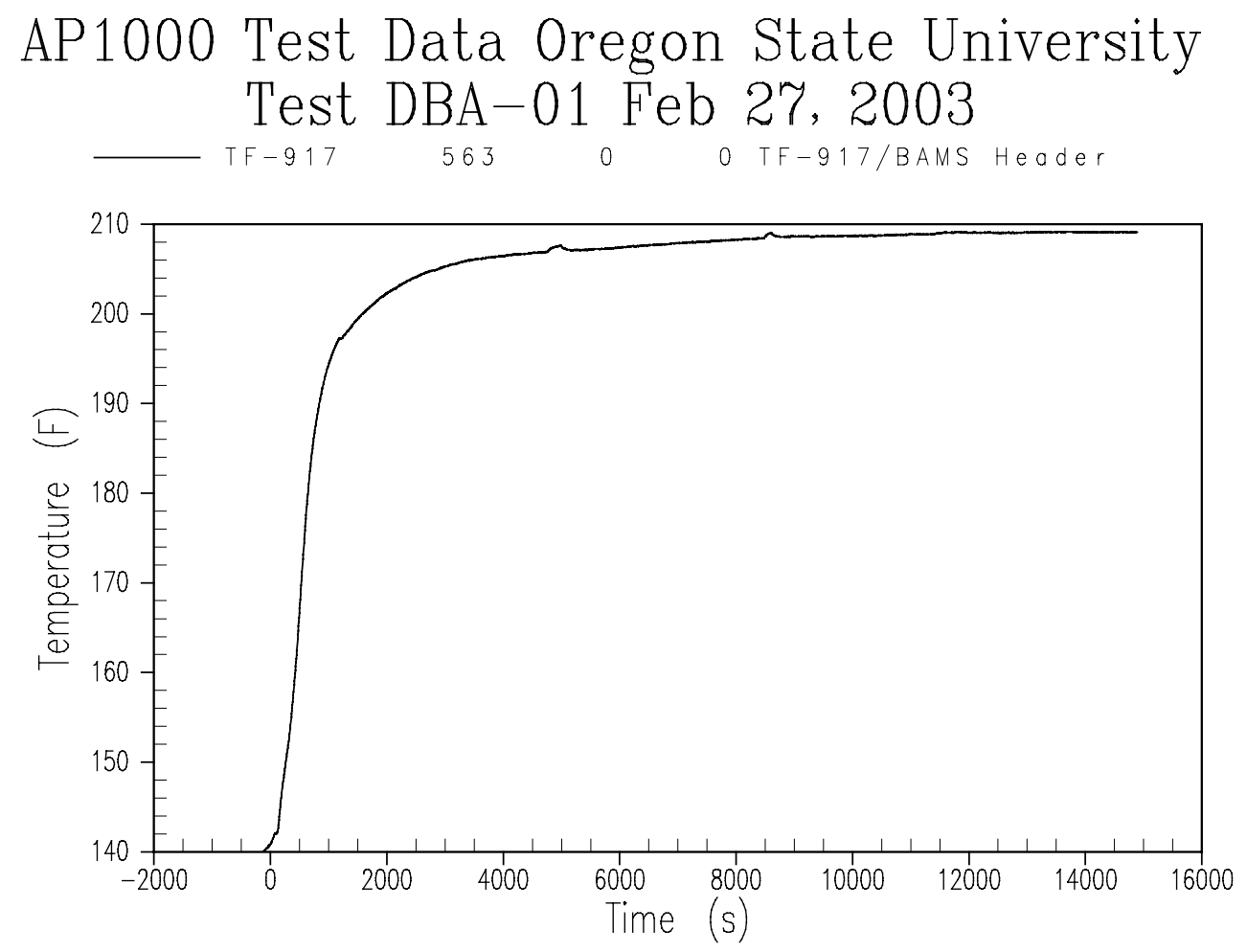

Figure A-65 BAMS/Exhaust Line Temperature 

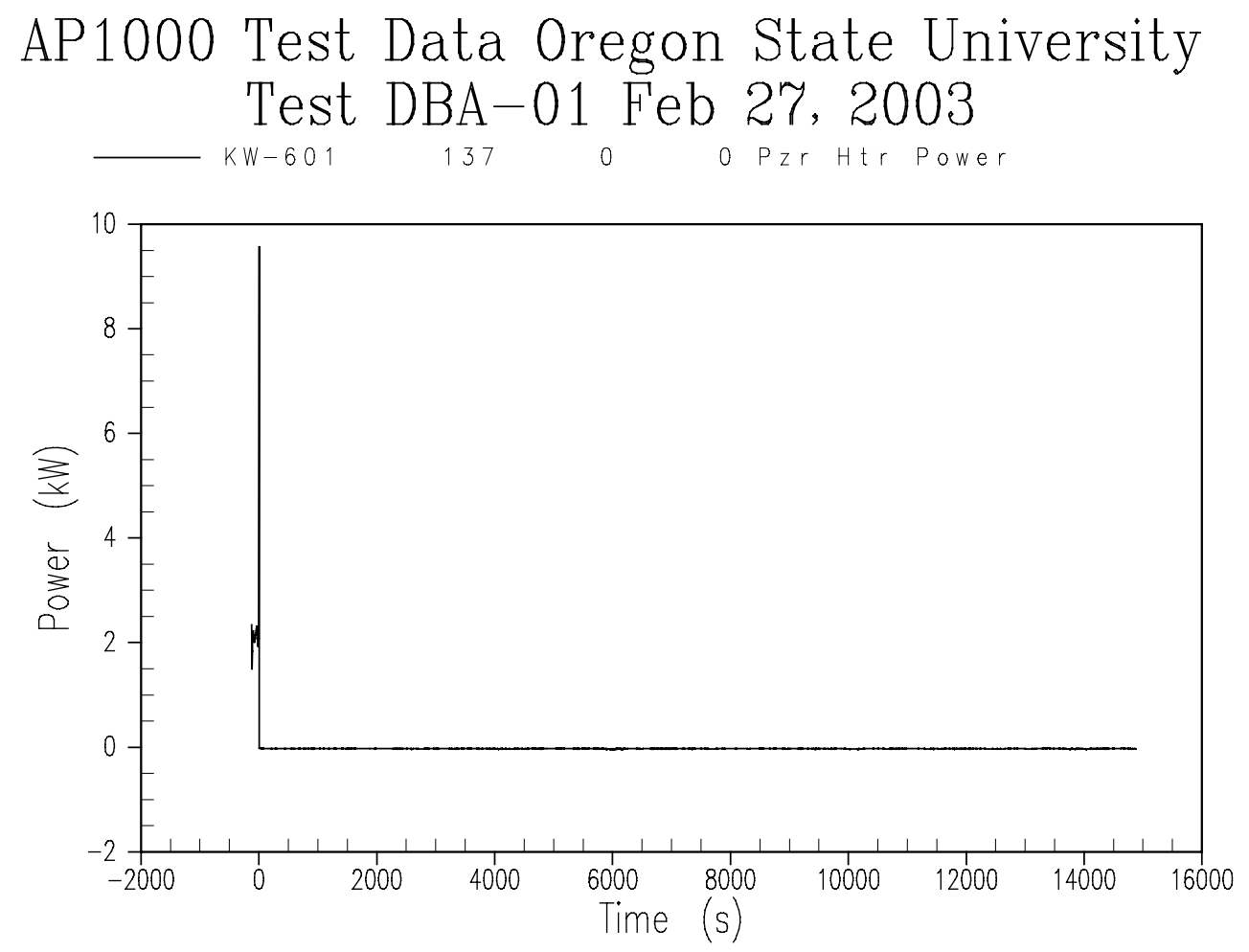

Figure A-66 Pressurizer Heater Input Power 

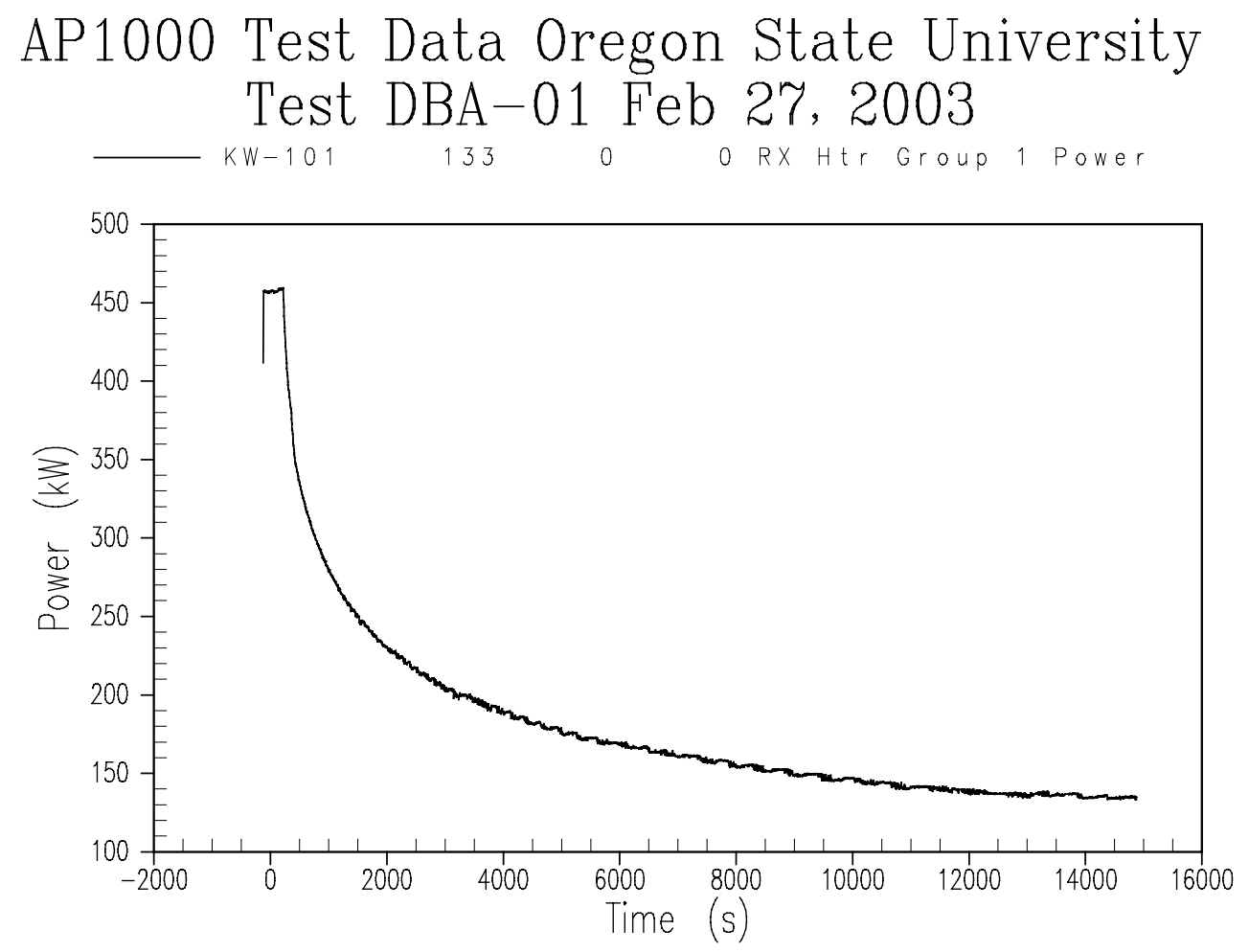

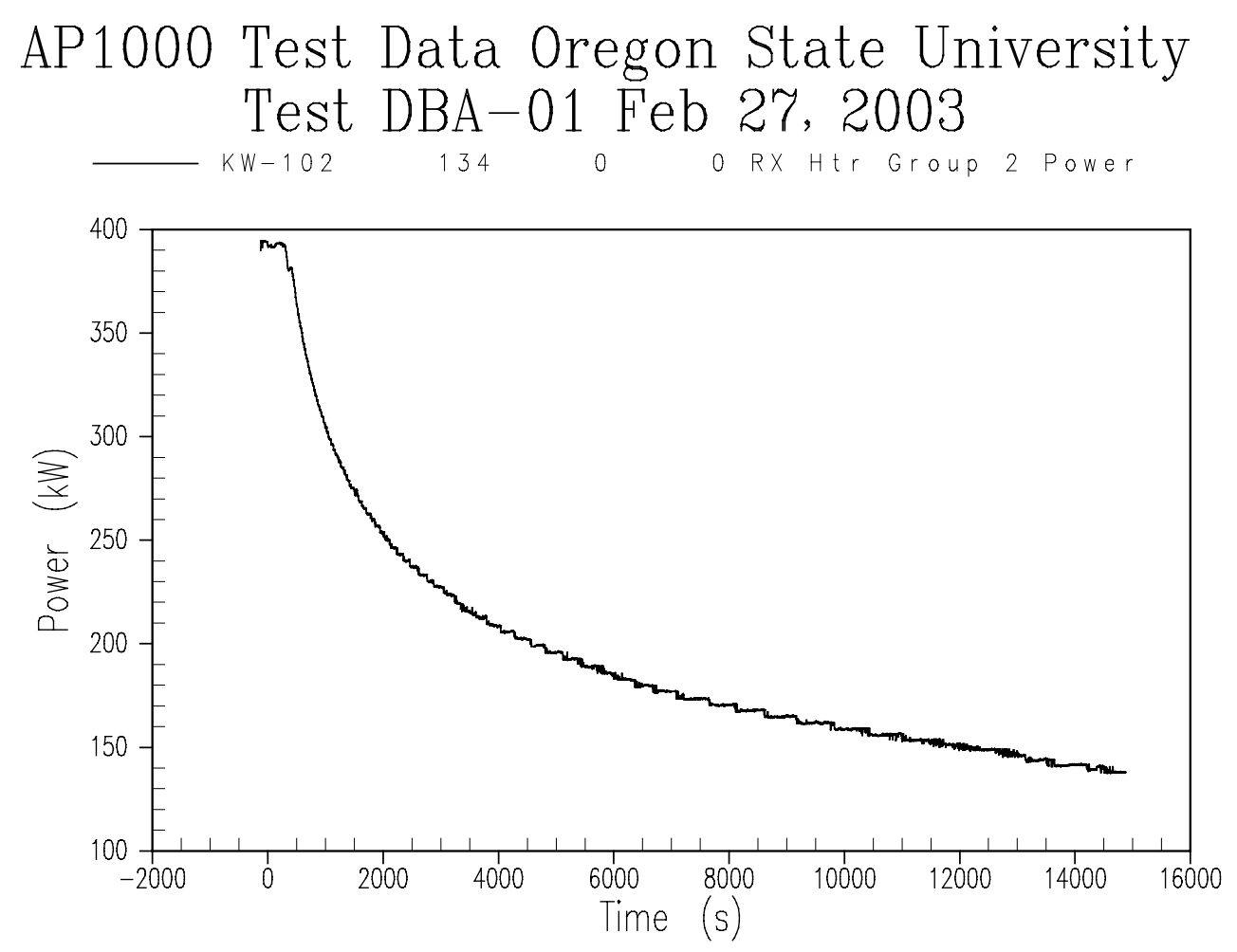

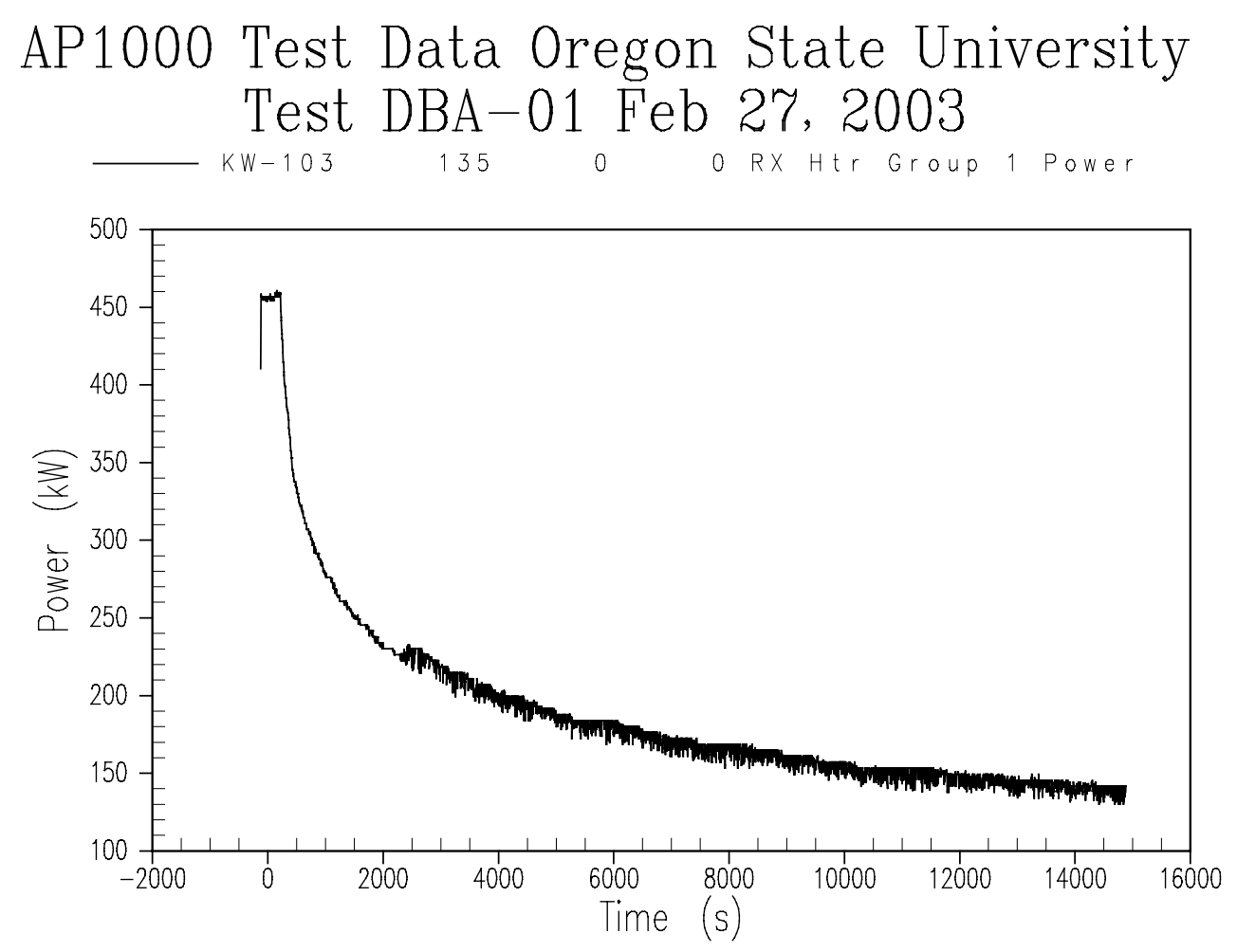

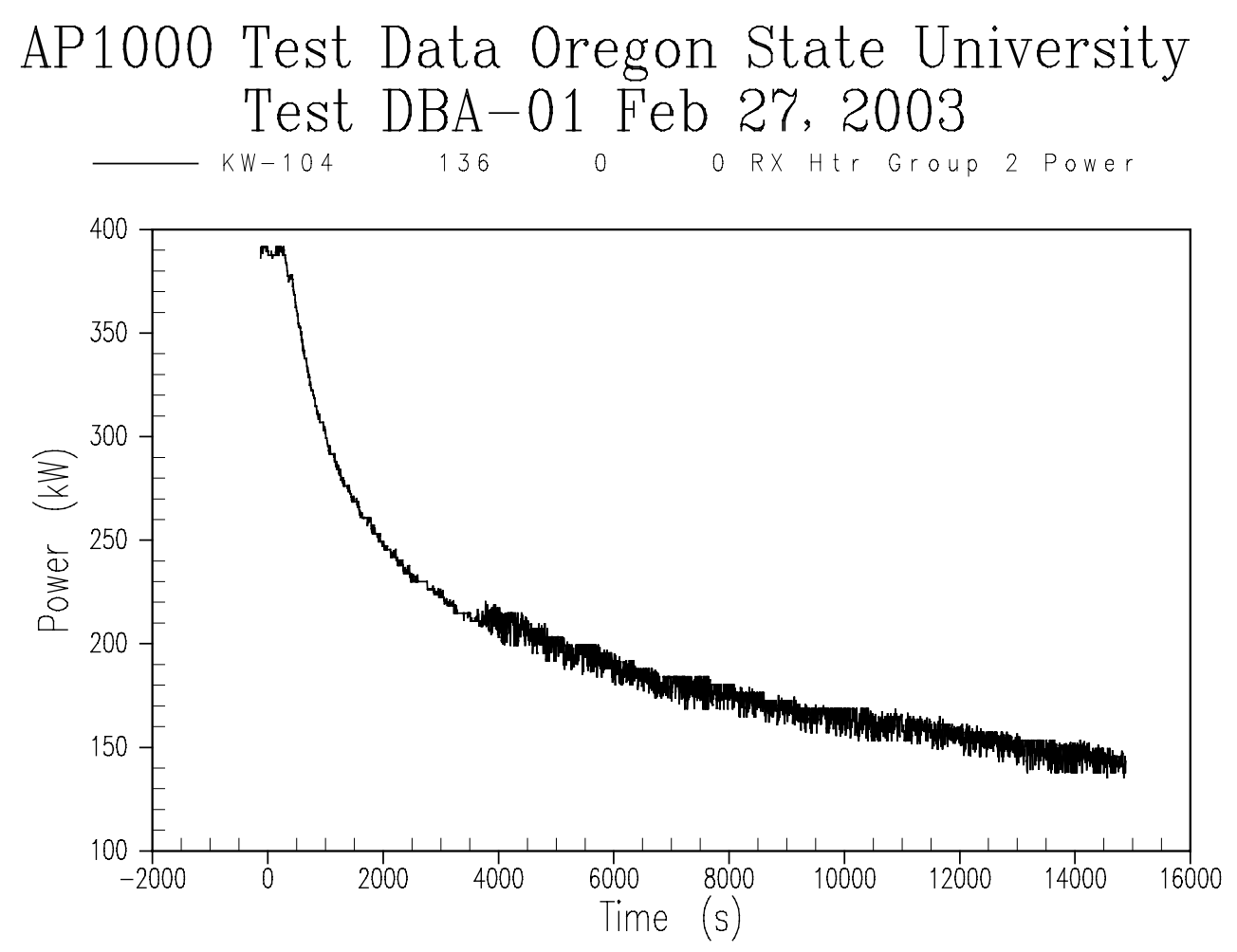

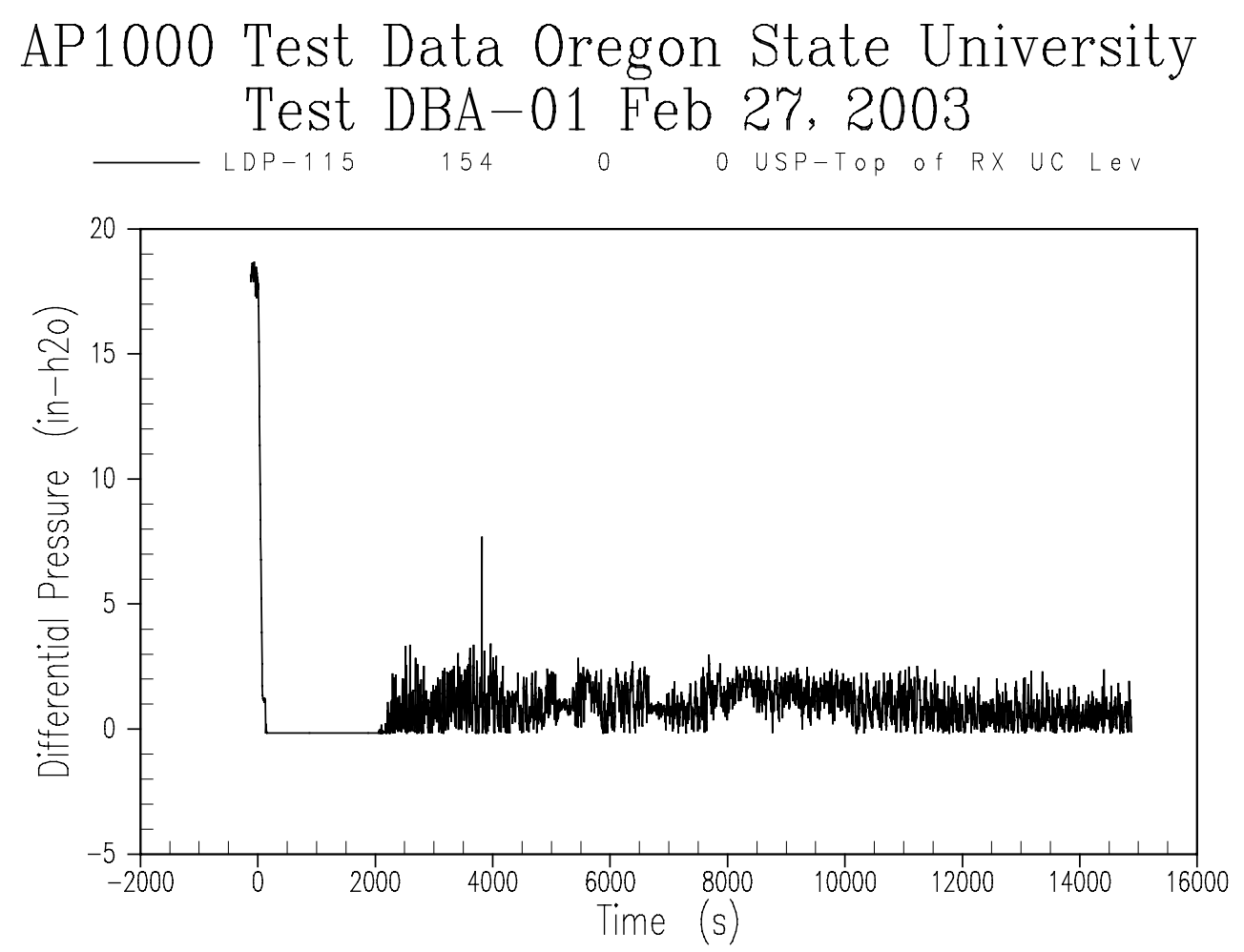

Figure A-71 Reactor Vessel Liquid Level Between Top of Vessel - Upper Support Plate 

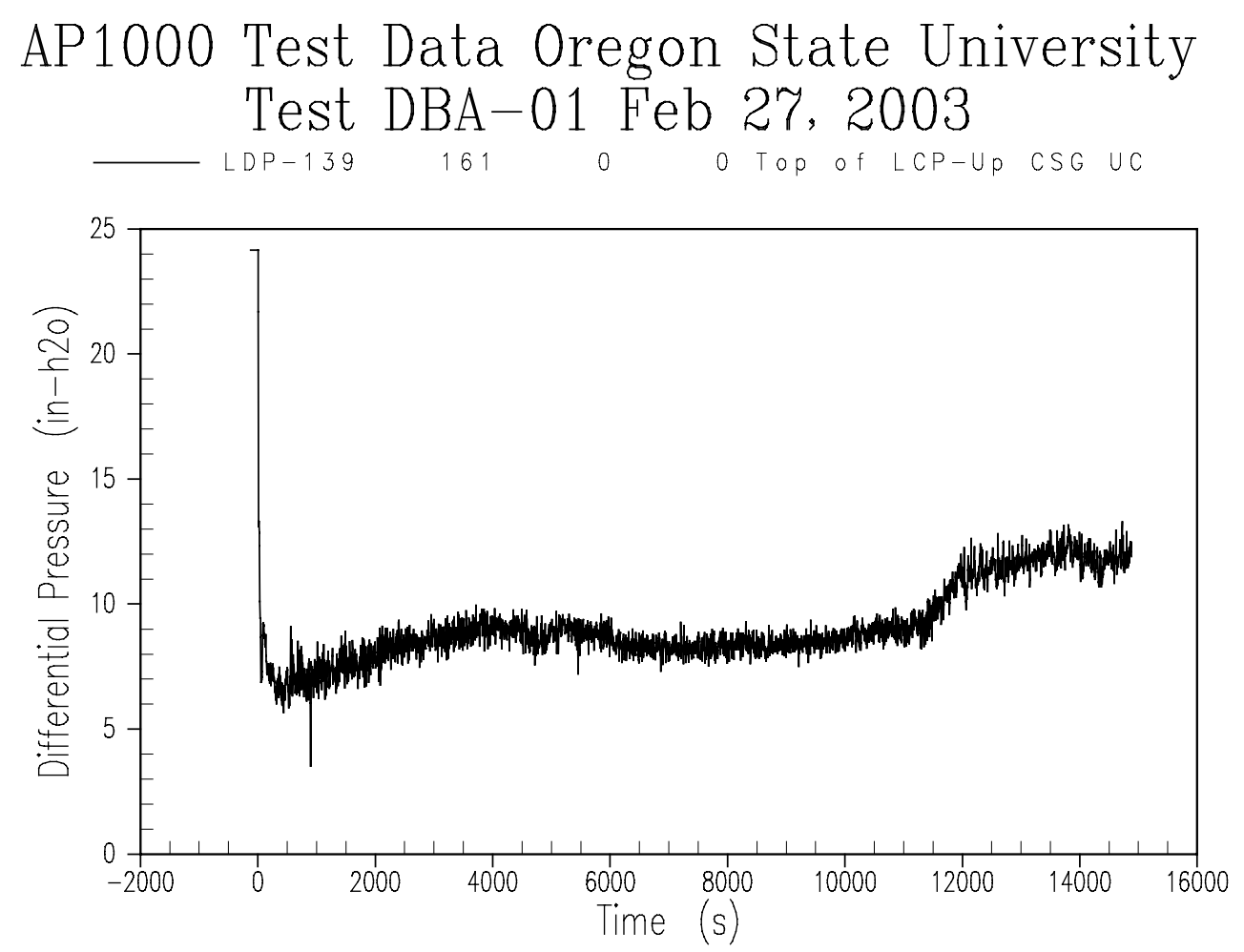

Figure A-72 Reactor Vessel Liquid Level Between Bottom of Upper Support Plate Upper Core Spacer Grid 

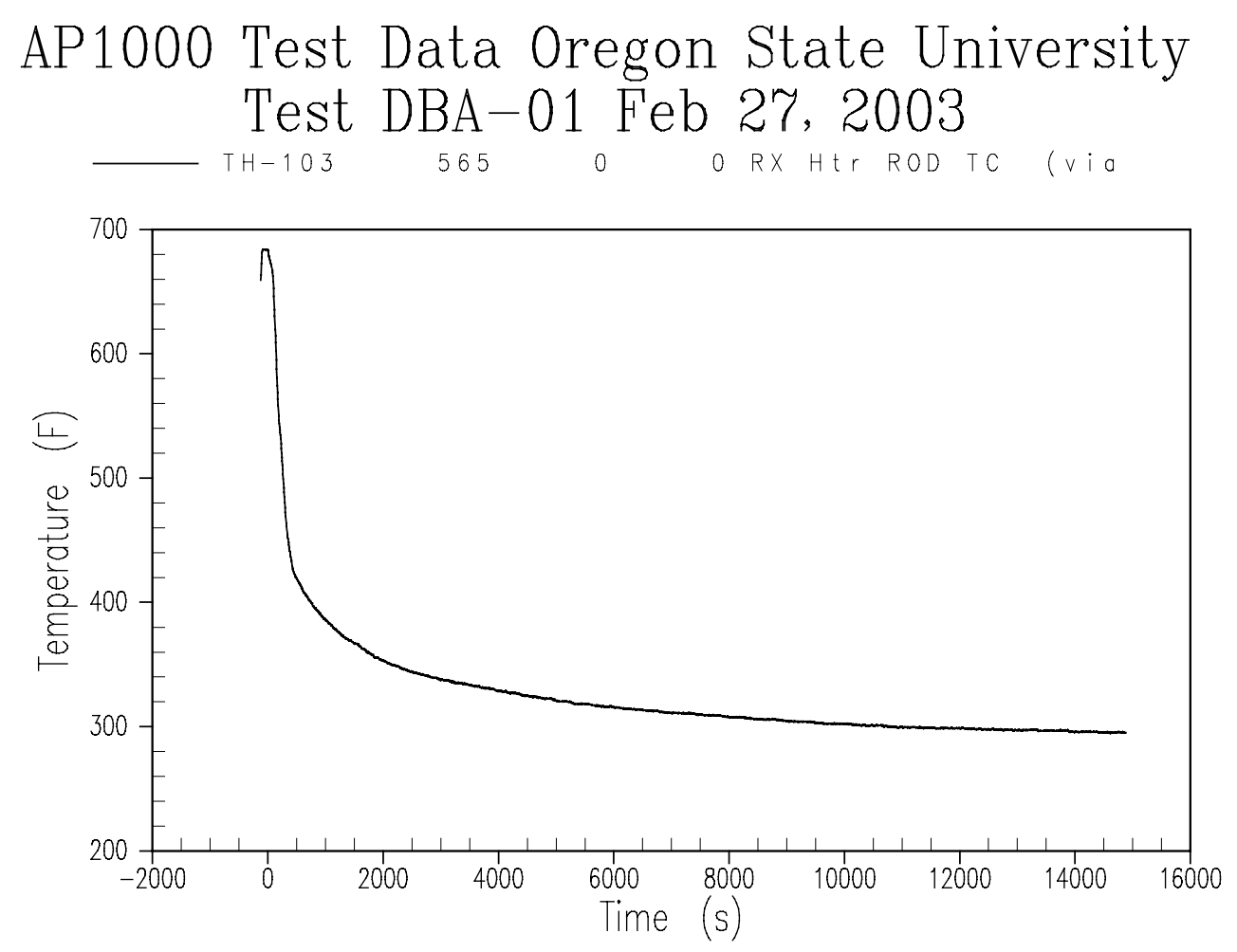

Figure A-73 Inner Core Thermocouple Measuring Heater Temperature 

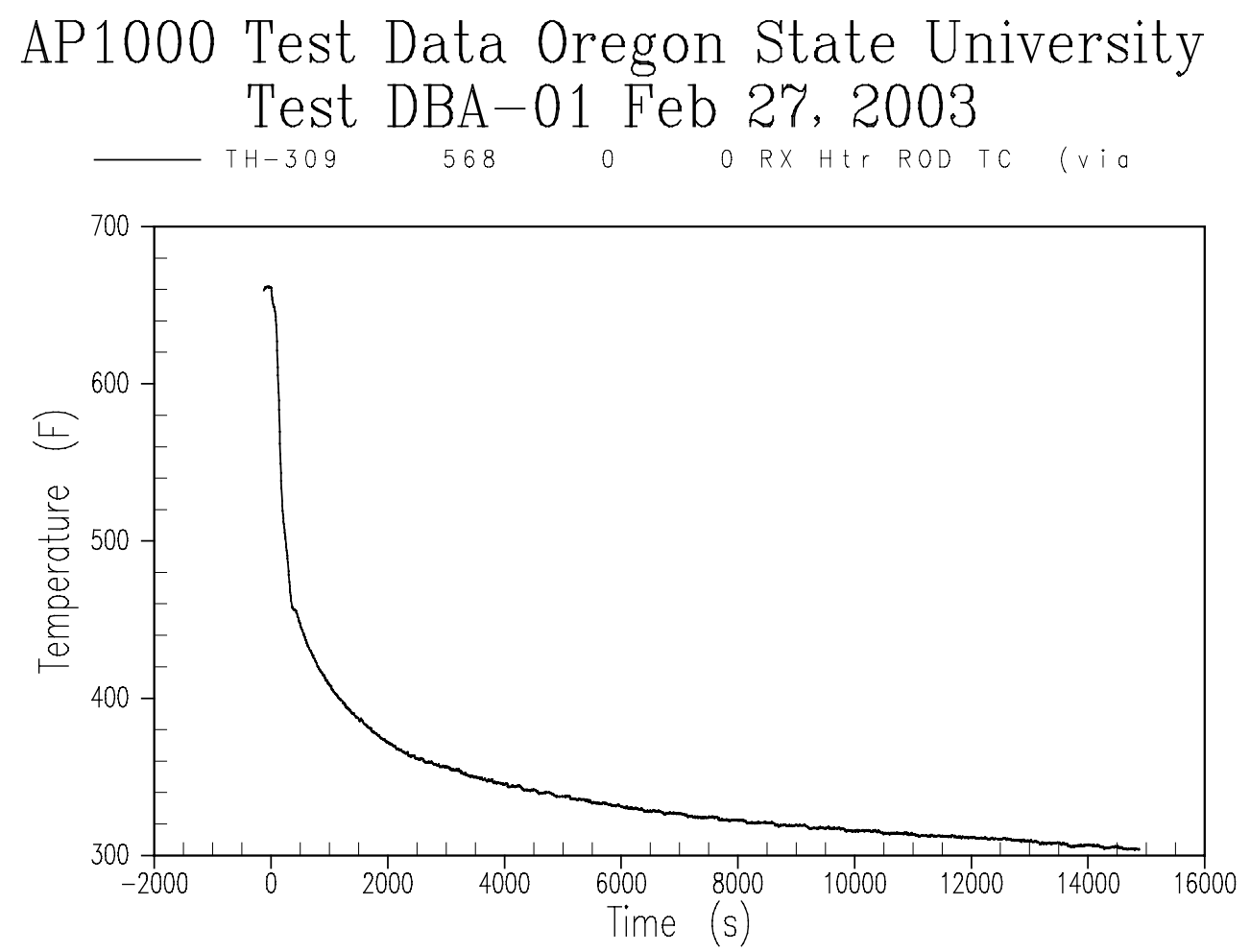

Figure A-74 Outer Core Thermocouple Measuring Heater Temperature 


\section{APPENDIX B}

TEST DATA 


\title{
Oregon State University
}

Department of Nuclear Engineering

\author{
Advanced Thermal Hydraulic \\ RESEARCH LABORATORY
}

\section{TEST SUMMARY REPORT}

OSU-AP1000-02

TR-01-D

AP1000 ADS-4 Transition TeST

Revision 0 


\title{
TEST SUMMARY REPORT
}

\author{
OSU-AP1000-02
}

TR-01-D

\section{AP1000 ADS-4 TRANSITION TEST}
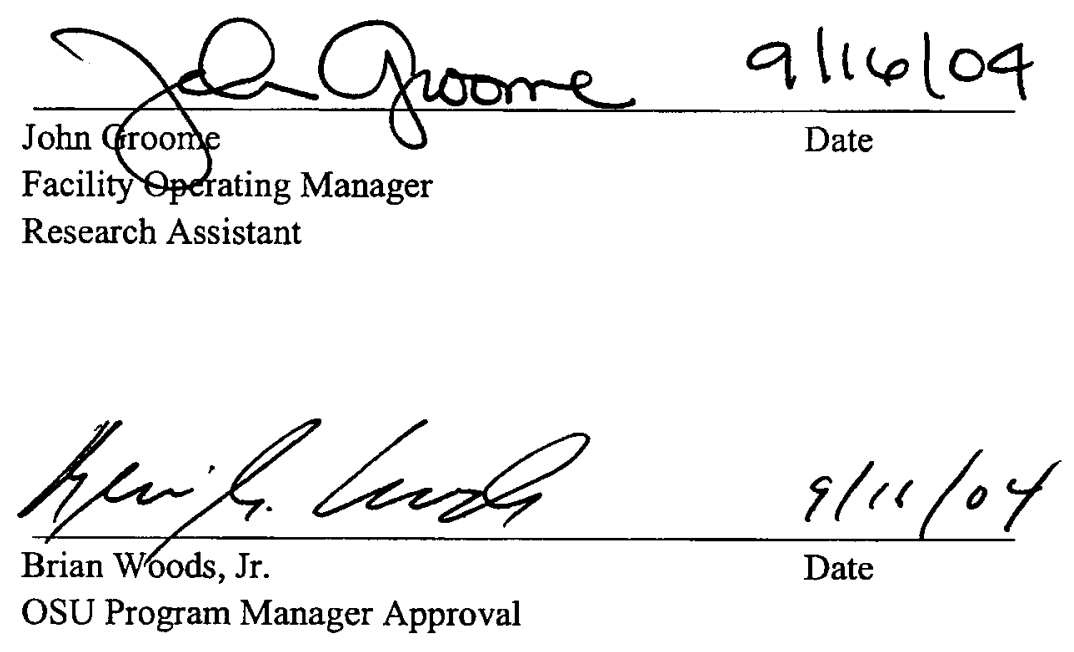

Oregon State University 116 Radiation Center

Corvallis, Oregon 97331

(C) 2004 Oregon State University

All Rights Reserved 


\section{WESTINGHOUSE PROPRIETARY INFORMATION NOTICE}

This report contains information proprietary to Westinghouse Electric Company. A non-proprietary version of this report has also been prepared for distribution to the United States Nuclear Regulatory Commission (NRC).

In order to conform to the requirements of 10 CFR 2.790 of the Commission's regulations concerning the protection of proprietary information so submitted to the NRC, the information which is proprietary in the proprietary version of this document is contained within brackets, and where the proprietary information has been deleted in the non-proprietary versions, only the brackets remain (the information that was contained within the brackets in the proprietary versions having been deleted). The justification for claiming the information so designated as proprietary is indicated in both versions by means of lower case letters (a) through (f) located as a superscript immediately following the brackets enclosing each item of information being identified as proprietary or in the margin opposite such information. These lower case letters refer to the types of information Westinghouse customarily holds in confidence identified in Sections (4)(ii)(a) through (4)(ii)(f) of the affidavit accompanying the transmittal of these documents pursuant to 10 CFR $2.790(b)(1)$.

\section{COPYRIGHT NOTICE}

This document bears an Oregon State University copyright notice. The NRC is permitted to make the number of copies of the information contained in these reports which are necessary for its internal use in connection with generic and plant-specific reviews and approvals as well as the issuance, denial, amendment, transfer, renewal, modification, suspension, revocation, or violation of a license, permit, order, or regulation subject to the requirements of 10 CFR 2.790 regarding restrictions on public disclosure to the extent such information has been identified as proprietary by Westinghouse, copyright protection notwithstanding. With respect to the non-proprietary versions of these reports, the NRC is permitted to make the number of copies beyond these necessary for its internal use which are necessary in order to have one copy available for public viewing in the appropriate docket files in the public document room in Washington, DC and in local public document rooms as may be required by NRC regulations if the number of copies submitted is insufficient for this purpose. Copies made by the NRC must include the copyright notice in all instances and the proprietary notice if the original was identified as proprietary. 
TABLE OF CONTENTS

$\underline{\text { Section }}$

Title

Page

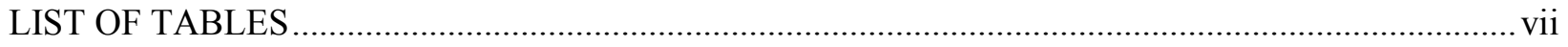

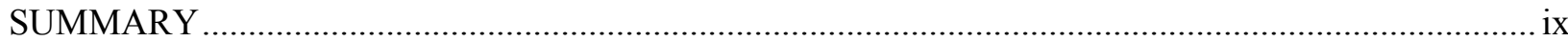

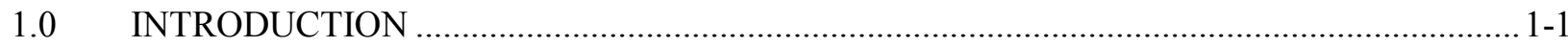

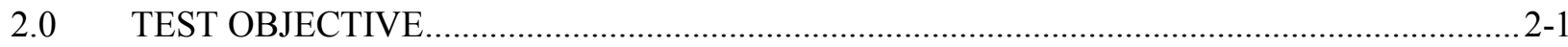

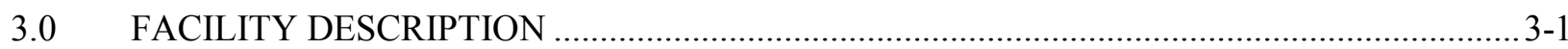

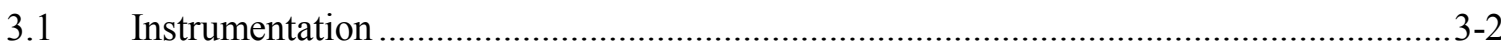

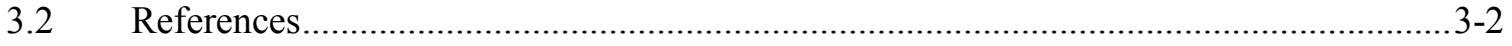

4.0 OREGON STATE UNIVERSITY TESTING PROGRAM MATRIX .......................................4-1

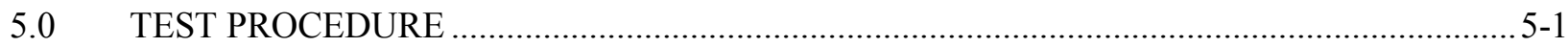

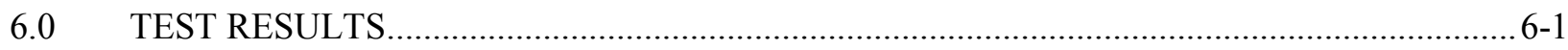

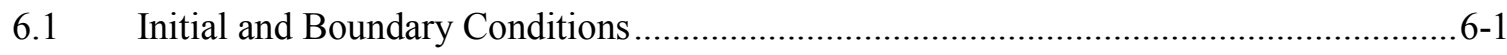

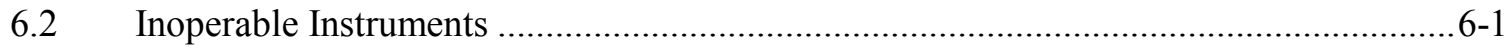

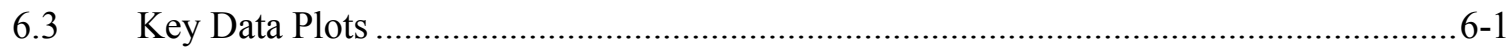

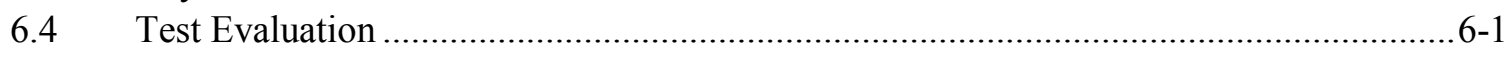

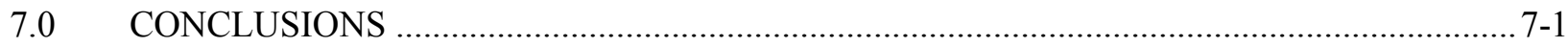

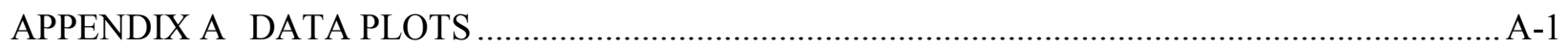

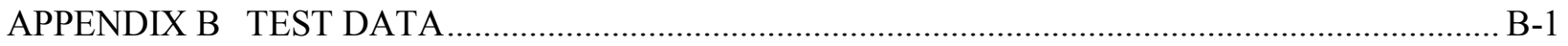




\section{LIST OF TABLES}

$\underline{\text { Table }}$

Table 4-1

Table 6-1

Table 6-2

Table 6-3

Table 6-4
Title

Page

OSU Test Matrix (as specified March 18, 2003) $4-2$

Actual Test Initial Conditions. $6-2$

Sequence of Events..... 6-4

Inoperable Instruments for TR-01-D Test ….................................................................6-5

Data Plots for QL Reports for TR-01-D by Component... 6-6 


\section{SUMMARY}

This report covers the test TR-01-D, transition from Automatic Depressurization System 4 (ADS-4) actuation to in-containment refueling water storage tank (IRWST) injection at 85 psig reactor pressure, performed on March 18, 2003. This test has been designated OSU-AP1000-02 by the Advanced Plant Experiment (APEX). The objective of this test was to demonstrate the AP1000 transition IRWST injection following actuation of the ADS-4. For this test, the Reactor Coolant System was drained down to the level of the hot legs. The Reactor Coolant System was heated to saturated conditions at a pressure of 85 psig. The core power was set to a constant value of $628 \mathrm{~kW}$. The core makeup tanks and accumulators were isolated for this test. The ADS-4 valves were opened at the start of the test. The power was held constant, and the Reactor Coolant System was depressurized. However, the cut-in pressure for IRWST injection was not reached before the two-phase level in the upper plenum fell to the top of the core. Once the thermocouples at the top of the heater rods showed a dramatic temperature increase, the test was terminated at 168 seconds. It was determined after the test that the power level was too high to allow the Reactor Coolant System to depressurize and IRWST injection to commence before the two-phase level in the upper plenum reached the top of the heater rods.

This report presents the initial assessment of the test data collected. If this test is to be used by Westinghouse to support AP1000 Design Certification, additional validation of the use of this information will be documented separately. In the interim, the list of invalid data channels may change. 


\subsection{INTRODUCTION}

The Department of Nuclear Engineering at Oregon State University (OSU) is performing a series of tests for the U.S. Department of Energy. These tests are being conducted in the Advanced Plant Experiment (APEX-1000) test facility, which is a reduced pressure and height model of the two-loop Westinghouse AP1000 pressurized water reactor. The purpose of the testing is to:

- evaluate the thermal-hydraulic performance of the passive safety systems of the full-scale AP1000, and

- assess and validate the safety analyses techniques and computer codes used in predicting the transient system behavior.

The AP1000 Long-Term Cooling Test is a 1/4 height scale, low-pressure integral systems test simulating thermal-hydraulic phenomenon for the AP1000 passive safety systems for small-break loss-of-coolant accidents (LOCAs) and long-term cooling. It accurately models the details of the AP1000 geometry, including the primary system, the passive safety systems, and a part of the non-safety grade Chemical and Volume Control System, as well as a partial non-safety grade Normal Residual Heat Removal System. The interconnecting pipe routings are also duplicated in the model.

The overall objective of the Long-Term Cooling Test program is to obtain test data at various modes of operation. The OSU experiments will examine the passive safety system response for the small-break and large-break LOCA transition into long-term cooling. (The list of the tests to be performed is in the OSU Test Matrix provided in Section 4.0.) The facility permits a range of small-break LOCAs to be simulated at different locations on the primary system, such as the cold leg, hot leg, core makeup tank (CMT) cold leg pressure balance line, and direct vessel injection (DVI) line. The break orientation (top or bottom of the cold leg) may also be studied. Selected tests continue into the long-term cooling, post-accident mode in which the passive safety injection is from the reactor sump as well as the in-containment refueling water storage tank (IRWST). 


\subsection{TEST OBJECTIVE}

The purpose of this test is to repeat the previous test TR-01-D, which was to obtain thermal-hydraulic data for the transition period from Automatic Depressurization System 4 (ADS-4) actuation to stable IRWST injection. The other sources of core makeup water, such as the CMTs and accumulators, were isolated. In addition, the pressurizer was drained and the ADS 1-3 were closed for the duration of the test. There is no break for this test. The assumed single failure is one of four ADS-4 valves and is on the non-pressurizer hot leg. The data obtained from the test will be used to verify the AP1000 thermal-hydraulic computer codes for AP1000 phenomena, such as gravity injection, natural convection, and post-accident long-term core cooling behavior.

The acceptance criteria for the OSU tests are as follows:

- Test initial conditions will be achieved within a specified tolerance.

- Setpoints will be achieved within an acceptable tolerance band.

- All instrumentation should be operational before the test.

- Any critical instruments not operating will be identified to the test engineer before the tests. These instruments must be operational before and during the test or exceptions should be approved.

- A zero check of LDPs, DPs, and FDPs will be performed. 


\subsection{FACILITY DESCRIPTION}

A detailed facility description for the OSU test facility is documented in Reference 3-1. The OSU test facility has been specifically scaled, designed, and constructed to investigate the AP600 passive safety system behavior and to provide data for safety analysis computer code validation. The facility has been modified to model the AP1000.

The scaled test design accurately models the details of the AP1000 geometry, including the primary system and the pipe routings and layout for the passive safety systems. A detailed scaling report (Reference 3-2) was used to develop the test design modifications. The primary system consists of one hot leg and two cold legs with two active pumps and an active steam generator for each loop. Two CMTs are connected to one primary loop, and the pressurizer is connected to the other primary loop as in the AP1000 plant design. Gas-driven accumulators are connected to the DVI lines. The discharge lines from the CMT, and one-of-two IRWST and reactor sump lines are connected to each DVI line. The ADS consisting of stages 1,2 , and 3 - simulates either one or two of the independent trains used in the AP1000. The two-phase flow from the ADS stages 1-to-3 is separated in a swirl-vane separator, and the liquid and vapor flows are measured to obtain the total ADS flow rate. The separated flow streams are then recombined and discharged into the IRWST through a sparger, preserving the mass and energy flow into the IRWST. The injection from the reactor sump is also simulated. Note that the OSU facility models both AP1000 primary and secondary sumps. The primary sump collects the condensate return, the liquid break flow, and the liquid flow from the fourth-stage ADS; and will provide long-term injection to the reactor vessel. The secondary sump simulates the portions of containment that will remain dry during most events. This sump collects water only when the primary sump reaches its overflow level, and provides no injection to the reactor vessel.

The time period for the experimental simulations includes not only the IRWST injection, but also the draining of the IRWST and the sump injection to simulate the long-term cooling of the AP1000. This simulation could be from several hours to a day. The time scale for the OSU test facility is one-half; that is, events occur in half the normal time. To model the long-term cooling aspects of the transient, the two-phase flow from the break is separated in a swirl-vane separator and the liquid and vapor portions of the total flow are measured. The liquid fraction of the flow is discharged to the reactor primary sump as in the AP1000 plant. The vapor is discharged to the atmosphere. The capability exists to return a portion of the equivalent liquid flow to the IRWST and primary sump to simulate the condensate return from the passive containment to the IRWST and primary sump. A similar approach is also used for the fourth-stage ADS valve on the hot leg. The two-phase flow is separated in a swirl-vane separator, the two streams are measured, and the liquid phase is discharged into the primary sump while the vapor flow is discharged to the atmosphere. Again, the capability exists to return a portion of the liquid equivalent added to the IRWST and primary sump. In addition, all other steam vents from the facility are measured (for example, the IRWST vent), and a portion of the liquid equivalent may be added back to the facility. Note that not all of the steam discharge would be returned as liquid equivalent. A portion of the discharge would be removed to simulate the steam that is not available for recirculation because it provides containment pressurization. The IRWST and primary sump can be pressurized in the OSU facility to simulate the containment pressurization following a postulated LOCA.

A multi-tube passive residual heat removal (PRHR) heat exchanger is located in the IRWST. The heat exchanger uses the same C-tube design as the AP1000 and has two instrumented tubes to obtain wall heat 
fluxes during the tests. There are primary fluid thermocouples, wall thermocouples, and differential pressure drop measurements to determine when the heat exchanger begins to drain. The IRWST is also instrumented with strings of fluid thermocouples to determine the degree of mixing within the tank and to assess the temperature of the coolant that is delivered to the test vessel.

The reactor vessel for the OSU tests includes a 0.914-meter (3-foot) heated core consisting of forty-eight 0.025 -meter (1-inch) diameter heater rods. The heater rods have a top skewed power shape. The $1000 \mathrm{~kW}$ of electrical power available at the OSU test site will be used to simulate decay heat. Wall thermocouples are swaged inside the heater rods to measure the heater rod wall temperature.

Thermocouple rods in the heater rod bundle measure the axial coolant temperature distribution. The scaled flow volume in the core is preserved as well as the flow volume in the test vessel upper plenum. There are simulated reactor internals in the upper plenum to preserve the flow area and to correctly scale the fluid volume. The reactor vessel includes an annular downcomer into which the four cold legs and the two DVI lines are connected. The hot legs penetrate the reactor annulus and connect with the loops. The AP1000 reactor vessel neutron reflector is simulated using a ceramic liner to reduce the metal heat release to the coolant.

There were no special/unique requirements for the test other than those specified in the Initial Conditions. The specified conditions were verified on the control board prior to test implementation.

\subsection{Instrumentation}

The instrumentation has been designed to calculate a transient mass and energy balance on the test facility. All two-phase flow streams exiting the facility are separated, and each component is measured separately as a single-phase flow using conventional measurement devices, such as magnetic flow meters and vortex flow meters. Note that magnetic flow meters are not designed for two-phase flow and will indicate erratically. Also, the vortex flow meters are referenced to $212^{\circ} \mathrm{F}$ and the LDPs are referenced to $60^{\circ} \mathrm{F}$. All vertical components have differential pressure cells that act as level instruments to measure the transient mass change in the component. The hot and cold leg diameters are sufficiently large in the OSU test facility so that a narrow-range differential pressure cell can be used to determine if the flow becomes stratified.

Single flow measurements are made of the CMT, accumulator, IRWST, and sump flows into the reactor vessel through the DVI lines.

Various types of instrumentation are provided in the test facility; for example, thermocouples for coolant and wall temperatures, flow meters, pressure transducers, differential pressure transducers, and weigh tanks.

\subsection{References}

3-1 APP-LTCT-T2R-005, OSU APEX-1000 Test Facility Description Report

3-2 APP-LTCT-T2R-004, Scaling Assessment for the Design of the OSU APEX-1000 Test Facility 


\subsection{OREGON STATE UNIVERSITY TESTING PROGRAM MATRIX}

The test matrix for the OSU test facility is shown in Table 4-1. To satisfy the test objectives, several transients will be performed to provide data on the AP1000 passive safety system response for a range of break sizes, locations, orientations, and single failure assumptions. The break size orifices are scaled based on simulating a 0.5 -inch, 1-inch, 2 -inch, or larger pipe break.

The designation for this test is TR-01-D, which identifies the test as an ADS-4 transition to IRWST injection test. The test matrix may be adjusted for future tests based on results and insights gained with each test. 


\begin{tabular}{|c|c|c|}
\hline \multicolumn{3}{|c|}{$\begin{array}{c}\text { TABLE 4-1 } \\
\text { OSU TEST MATRIX (AS SPECIFIED MARCH 18, 2003) }\end{array}$} \\
\hline Test Title & Break Location and Size & Single Failure Assumed \\
\hline DBA-01-D & $\begin{array}{l}\text { Double-ended DVI Line break with } \\
\text { continuation into long-term cooling }\end{array}$ & Fail 1 of 2 lines in one ADS- 4 train \\
\hline DBA-02-D & $\begin{array}{l}\text { Double-ended DVI Line break with } \\
\text { continuation into long-term cooling }\end{array}$ & $\begin{array}{l}\text { Fail } 1 \text { of } 2 \text { lines in one ADS- } 4 \text { train } \\
\text { (Adjusted ADS-4 Resistance) }\end{array}$ \\
\hline DBA-03-D & $\begin{array}{l}\text { Double-ended DVI Line break with } \\
\text { continuation into long-term cooling }\end{array}$ & $\begin{array}{l}\text { Fail } 1 \text { of } 2 \text { lines in one ADS- } 4 \text { train } \\
\text { (Failure location sensitivity) }\end{array}$ \\
\hline TR-01-D & $\begin{array}{l}\text { Transition Test ADS-4 opening, } 85 \text { psig initial } \\
\text { pressure and decay power } 480 \mathrm{sec}\end{array}$ & $\begin{array}{l}\text { Fail } 1 \text { of } 2 \text { lines in one ADS-4 train } \\
\text { (No ADS 1-3) }\end{array}$ \\
\hline TR-02-D & $\begin{array}{l}\text { Transition Test ADS-4 opening, } 100 \text { psig initial } \\
\text { pressure with prototypic plant conditions }\end{array}$ & $\begin{array}{l}\text { Fail } 1 \text { of } 2 \text { lines in one ADS-4 train } \\
\qquad \text { (No ADS 1-3) }\end{array}$ \\
\hline DBA-04-D & 2-inch Cold Leg Break & $\begin{array}{l}\text { Fail } 1 \text { of } 2 \text { lines in one ADS- } 4 \text { train } \\
\text { (Failure on non-pressurizer hot leg) }\end{array}$ \\
\hline ADS-01-D & $\begin{array}{l}\text { ADS-4 Two-Phase Characterization Test at } \\
\text { low-power conditions (steady-state points) }\end{array}$ & $\begin{array}{l}\text { Various ADS-4 line configurations } \\
\qquad \text { (No ADS 1-3) }\end{array}$ \\
\hline ADS-02-D & $\begin{array}{l}\text { ADS-4 Two-Phase Characterization Test at } \\
\text { high-power conditions (steady-state points) }\end{array}$ & $\begin{array}{l}\text { Various ADS-4 line configurations } \\
\qquad \text { (No ADS 1-3) }\end{array}$ \\
\hline DBA-03R-D & Repeat of Test DBA-03-D & $\begin{array}{l}\text { Fail } 1 \text { of } 2 \text { lines in one ADS- } 4 \text { train } \\
\text { (Failure on pressurizer hot leg) }\end{array}$ \\
\hline DBA-05-D & Inadvertent ADS-1 Actuation & $\begin{array}{l}\text { Fail } 1 \text { of } 2 \text { lines in one ADS- } 4 \text { train } \\
\text { (Failure on non-pressurizer hot leg) }\end{array}$ \\
\hline DBA-06-D & 0.5-inch Cold Leg Break & $\begin{array}{l}\text { Fail } 1 \text { of } 2 \text { lines in one ADS- } 4 \text { train } \\
\text { (Failure on non-pressurizer hot leg) }\end{array}$ \\
\hline
\end{tabular}




\subsection{TEST PROCEDURE}

The test was performed per a written procedure. There were no special/unique requirements for the test other than those specified in the initial conditions in Table 6-1. The specified conditions were checked on the control board before test implementation.

The appropriate prerequisites were completed and initial conditions were satisfied. The required break simulation piping and break instrumentation were installed per P\&ID drawing OSU 600904, Rev. 1. The 100-percent flow nozzle was installed in ADS 4-1 (on hot leg 1), and the 50-percent flow nozzle was installed in ADS 4-2 (on hot leg 2). Flow nozzles that simulate full flow for ADS-1, ADS-2, and ADS-3 were installed. As per the AP600 tests, ADS-3 has been scaled for full flow from all three stages, and ADS-1 and ADS-2 are closed when ADS-3 is opened. For this test, ADS 1-3 is closed for the duration of the test.

Fill and vent was performed per APEX Operations Manual Procedure OP-B.2. Instruments were checked for required calibration.

With the break valves TS-202 and TS-203 closed, flow was used to warm up the bypass line by opening isolation valves RCS-901 and RCS-902. After the appropriate prerequisites were completed and the test facility achieved specified initial conditions, the CMT warmup bypass line isolation valves RCS-901 and RCS-902 were closed to maintain the $<80^{\circ} \mathrm{F}$ condition at the top of the CMT-1. With the CMT balance line valves (RCS-529 and RCS-530) placed in the open and automatic mode, both CMTs reached the Reactor Coolant System pressure.

Once all other initial conditions were satisfied, ADS-4 was actuated. The test was terminated when the top of the core uncovered before IRWST injection was established. 


\subsection{TEST RESULTS}

The test results for test TR-01-D are provided in the following subsections.

\subsection{Initial and Boundary Conditions}

Table 6-1 provides a comparison of the specified and actual conditions for test TR-01-D. The values in this table were averaged over approximately 2 minutes preceding the test. Test initial conditions were achieved for the steam generator pressure, pressurizer pressure, pressurizer level, steam generator 01 narrow-range level, and steam generator 02 narrow-range level. Test initial conditions for the hot leg temperature were found to be acceptable, and the results will not be adversely affected.

The actual power curves for the heater rods are provided in data plots in Appendix B. The measured power for the test was $628 \mathrm{~kW}$, which was held constant for the duration of the test. The programmed decay heat curve was not used for this test.

The sequence of events is shown in Table 6-2. This table compares the actual sequence of events with the specified timing. As can be seen in this table, all the events occurred at or very near to when the event was planned.

\subsection{Inoperable Instruments}

Table 6-3 lists the instrumentation channels considered inoperable for the TR-01-D test.

\subsection{Key Data Plots}

Table 6-4 lists the instrumentation channels sorted by component and includes the instrument number, units, and quick look (QL) plot number. The selection of channels was based on projecting an overall picture of the test results, which would then be examined by referring to the detailed data plots or tapes.

\subsection{Test Evaluation}

The following observations were made during the test:

1. The heater rod power was held constant at $628 \mathrm{~kW}$ for the duration of the test.

2. The Reactor Coolant System pressure decreased rapidly once the ADS-4 valves were opened, but did not reach the cut-in pressure for IRWST injection before the test was terminated.

3. With no source of makeup water, the two-phase level in the upper plenum fell, reaching the top of the heater rods at approximately 494 seconds. At this time, the thermocouples at the top of the rods indicated that the rods were uncovered and the test was terminated. 


\begin{tabular}{|c|c|c|c|}
\hline \multicolumn{4}{|c|}{$\begin{array}{c}\text { TABLE 6-1 } \\
\text { ACTUAL TEST INITIAL CONDITIONS }\end{array}$} \\
\hline Conditions & Instrument No. & Actual & Comment \\
\hline Pressurizer Pressure & PT-604 & 85 psig & \\
\hline Hot Leg Temperature \#1 & $\begin{array}{l}\text { TF-141* } \\
\text { TF-205 } \\
\text { TF-143 }\end{array}$ & $\begin{array}{l}349^{\circ} \mathrm{F} \\
330^{\circ} \mathrm{F} \\
335^{\circ} \mathrm{F}\end{array}$ & \\
\hline Hot Leg Temperature $\# 2$ & $\begin{array}{l}\text { TF-140* } \\
\text { TF-206 } \\
\text { TF-142 }\end{array}$ & $\begin{array}{l}346^{\circ} \mathrm{F} \\
326^{\circ} \mathrm{F} \\
330^{\circ} \mathrm{F}\end{array}$ & \\
\hline $\begin{array}{l}\text { Steam Generator Pressure } \\
\quad \# 1 \\
\# 2 \\
\text { Header }\end{array}$ & $\begin{array}{l}\text { PT-301* } \\
\text { PT-302* } \\
\text { PT-002 }\end{array}$ & $\begin{array}{l}86 \text { psig } \\
82 \text { psig } \\
3.2 \text { psig }\end{array}$ & \\
\hline Pressurizer Level & $\begin{array}{l}\text { LDP-601 uncompensated } \\
\text { LDP-601 } \\
\text { Compensated by SC-608 }\end{array}$ & $\begin{array}{c}\text { Empty } \\
-\end{array}$ & \\
\hline $\begin{array}{l}\text { Steam Generator Level } \\
\# 1 \mathrm{NR}\end{array}$ & $\begin{array}{l}\text { LDP-303 uncompensated } \\
\text { LDP-303 compensated by } \\
\text { average of TF-305 and } \\
\text { TF-307 }\end{array}$ & $\begin{array}{l}22.5 \text { inches } \\
27.2 \text { inches }\end{array}$ & $\begin{array}{l}413^{\circ} \mathrm{F} \text { used for density } \\
\text { compensation }\end{array}$ \\
\hline $\begin{array}{l}\text { Steam Generator Level } \\
\# 2 \mathrm{NR}\end{array}$ & $\begin{array}{l}\text { LDP-304 uncompensated } \\
\text { LDP-304 compensated by } \\
\text { average of TF-306 and } \\
\text { TF-308 }\end{array}$ & $\begin{array}{l}22.2 \text { inches } \\
26 \text { inches }\end{array}$ & $\begin{array}{l}414^{\circ} \mathrm{F} \text { used for density } \\
\text { compensation }\end{array}$ \\
\hline IRWST Temperature & TF-701 & $60^{\circ} \mathrm{F}$ & Accepted $\left(<80^{\circ} \mathrm{F}\right)$ \\
\hline $\begin{array}{l}\text { CMT Temperature } \\
\text { \#1 } \\
\# 2\end{array}$ & $\begin{array}{l}\text { TF-513 } \\
\text { TF-516 }\end{array}$ & $\begin{array}{l}61.7^{\circ} \mathrm{F} \\
62.1^{\circ} \mathrm{F}\end{array}$ & Accepted $\left(<80^{\circ} \mathrm{F}\right)$ \\
\hline $\begin{array}{l}\text { Accumulator } \\
\text { Temperature } \\
\# 1 \\
\# 2\end{array}$ & $\begin{array}{l}\mathrm{TF}-403 \\
\mathrm{TF}-404\end{array}$ & $\begin{array}{l}75.8^{\circ} \mathrm{F} \\
76.5^{\circ} \mathrm{F}\end{array}$ & Accepted $\left(<80^{\circ} \mathrm{F}\right)$ \\
\hline IRWST Level & LDP-701 & 92.4 inches & \\
\hline
\end{tabular}




\begin{tabular}{|c|c|c|c|}
\hline \multicolumn{4}{|c|}{$\begin{array}{l}\text { TABLE 6-1 (Continued) } \\
\text { ACTUAL TEST INITIAL CONDITIONS }\end{array}$} \\
\hline Conditions & Instrument No. & Actual & Comment \\
\hline $\begin{array}{l}\text { Accumulator Level } \\
\text { \#1 } \\
\# 2\end{array}$ & $\begin{array}{l}\text { LDP-401 } \\
\text { LDP-402 }\end{array}$ & $\begin{array}{l}0 \text { inches } \\
0 \text { inches }\end{array}$ & \\
\hline $\begin{array}{l}\text { Accumulator Pressure } \\
\# 1 \\
\# 2\end{array}$ & $\begin{array}{l}\text { PT-401 } \\
\text { PT-402 }\end{array}$ & $\begin{array}{l}0 \text { psig } \\
0 \text { psig }\end{array}$ & \\
\hline $\begin{array}{l}\text { CMT Level } \\
\text { \#1 } \\
\# 2\end{array}$ & $\begin{array}{l}\text { LDP-507 } \\
\text { LDP-502 }\end{array}$ & $\begin{array}{l}56.9 \text { inches } \\
57.3 \text { inches }\end{array}$ & Isolated \\
\hline $\begin{array}{l}\text { CMT Pressure } \\
\text { \#1 } \\
\# 2\end{array}$ & $\begin{array}{l}\text { PT-501 } \\
\text { PT-502 }\end{array}$ & $\begin{array}{c}0.63 \mathrm{psig} \\
2.3 \mathrm{psig}\end{array}$ & \\
\hline
\end{tabular}

* These instruments are used to establish initial conditions at the test site. 


\begin{tabular}{|c|c|c|}
\hline \multicolumn{3}{|c|}{$\begin{array}{c}\text { TABLE 6-2 } \\
\text { SEQUENCE OF EVENTS }\end{array}$} \\
\hline Event & Setpoint & Actual Time (sec) \\
\hline PB Depressed & $\mathrm{N} / \mathrm{A}$ & -120 \\
\hline Break Valve(s) Open & No Break Modeled & - \\
\hline ADS 4-1 Actuation (RCS-615) & Manual & 15 \\
\hline ADS 4-2 Actuation (RCS-616) & $\begin{array}{l}\text { ADS 4-1 Actuation + } \\
30 \text { seconds }\end{array}$ & 45 \\
\hline $\begin{array}{l}\text { IRWST Injection } \\
\text { DVI \#1 (FMM-701) }\end{array}$ & N/A & Failed to reach injection \\
\hline $\begin{array}{l}\text { IRWST Injection } \\
\text { DVI \#2 (FMM-702) }\end{array}$ & N/A & Failed to reach injection \\
\hline Core Power Shutoff & $\begin{array}{c}\text { Heater Rod Temperature } \\
\text { Excursion }\end{array}$ & 168 \\
\hline
\end{tabular}




\begin{tabular}{|c|c|c|}
\hline \multicolumn{3}{|c|}{$\begin{array}{c}\text { TABLE 6-3 } \\
\text { INOPERABLE INSTRUMENTS FOR TR-01-D TEST }\end{array}$} \\
\hline Instrument Number & Instrument Type & Inoperable Description \\
\hline $\begin{array}{l}\text { TW-202 } \\
\text { TW-204 } \\
\text { TW-205 } \\
\text { TW-206 } \\
\text { TW-209 } \\
\text { TW-803 } \\
\text { TW-804 }\end{array}$ & Thermocouple & Inoperative \\
\hline TH-603 & $\begin{array}{l}\text { Thermocouple measuring heater } \\
\text { temperature }\end{array}$ & Inoperative \\
\hline FMM-202 & Magnetic flow meter & Inoperative \\
\hline $\begin{array}{l}\text { TF-170 } \\
\text { TF-221 } \\
\text { TF-509 } \\
\text { TF-512 }\end{array}$ & $\begin{array}{l}\text { Thermocouple measuring fluid } \\
\text { temperature }\end{array}$ & Inoperative \\
\hline FVM-905 & Vortex flow meter & Erratic \\
\hline
\end{tabular}




\begin{tabular}{|c|c|c|c|c|}
\hline \multicolumn{5}{|c|}{$\begin{array}{c}\text { TABLE 6-4 } \\
\text { DATA PLOTS FOR QL REPORTS FOR TR-01-D BY COMPONENT }\end{array}$} \\
\hline Component & Channel & Units & QL-Plot & Comment \\
\hline $\begin{array}{l}\text { Reactor Vessel } \\
\text { Pressure }\end{array}$ & PT-107 & psig & 1 & \\
\hline Reactor Vessel Level & LDP-127 & inch of $\mathrm{H}_{2} \mathrm{O}$ & 2 & \\
\hline $\begin{array}{l}\text { Reactor Vessel } \\
\text { Downcomer Level }\end{array}$ & LDP-140 & inch of $\mathrm{H}_{2} \mathrm{O}$ & 3 & \\
\hline $\begin{array}{l}\text { Cold Leg \#1 Fluid } \\
\text { Temperature }\end{array}$ & TF-107 & ${ }^{\circ} \mathrm{F}$ & 4 & \\
\hline $\begin{array}{l}\text { Cold Leg \#2 Fluid } \\
\text { Temperature }\end{array}$ & TF-108 & ${ }^{\circ} \mathrm{F}$ & 5 & \\
\hline $\begin{array}{l}\text { Cold Leg \#3 Fluid } \\
\text { Temperature }\end{array}$ & TF-103 & ${ }^{\circ} \mathrm{F}$ & 6 & \\
\hline $\begin{array}{l}\text { Cold Leg \#4 Fluid } \\
\text { Temperature }\end{array}$ & TF-104 & ${ }^{\circ} \mathrm{F}$ & 7 & \\
\hline $\begin{array}{l}\text { Reactor Vessel Fluid } \\
\text { Temperature Upper } \\
\text { Head }\end{array}$ & TF-120 & ${ }^{\circ} \mathrm{F}$ & 8 & \\
\hline $\begin{array}{l}\text { Reactor Coolant } \\
\text { System Hot Leg \#1 } \\
\text { Temperature }\end{array}$ & TF-143 & ${ }^{\circ} \mathrm{F}$ & 9 & \\
\hline $\begin{array}{l}\text { Reactor Coolant } \\
\text { System Hot Leg \#2 } \\
\text { Temperature }\end{array}$ & TF-142 & ${ }^{\circ} \mathrm{F}$ & 10 & \\
\hline Pressurizer Pressure & $\begin{array}{l}\text { PT-604 (WR) and PT-603 } \\
\text { (LP Indication) }\end{array}$ & psig & 11,12 & \\
\hline $\begin{array}{l}\text { Pressurizer Liquid } \\
\text { Level }\end{array}$ & LDP-601 & inch of $\mathrm{H}_{2} \mathrm{O}$ & 13 & $\begin{array}{l}\text { Sharp decrease followed } \\
\text { by rapid refill }\end{array}$ \\
\hline $\begin{array}{l}\text { Steam Generator \#1 } \\
\text { Tube Level }\end{array}$ & LDP-215 & inch of $\mathrm{H}_{2} \mathrm{O}$ & 14 & \\
\hline $\begin{array}{l}\text { Steam Generator \#1 } \\
\text { Secondary Pressure }\end{array}$ & PT-301 & psig & 15 & \\
\hline
\end{tabular}




\begin{tabular}{|c|c|c|c|c|}
\hline \multicolumn{5}{|c|}{$\begin{array}{c}\text { TABLE 6-4 (Continued) } \\
\text { DATA PLOTS FOR QL REPORTS FOR TR-01-D BY COMPONENT }\end{array}$} \\
\hline Component & Channel & Units & QL-Plot & Comment \\
\hline $\begin{array}{l}\text { Steam Generator \#1 } \\
\text { Feed Flow Rate }\end{array}$ & FMM-001 & gpm & 16 & \\
\hline $\begin{array}{l}\text { Steam Generator \#2 } \\
\text { Tube Level }\end{array}$ & LDP-218 & inch of $\mathrm{H}_{2} \mathrm{O}$ & 17 & \\
\hline $\begin{array}{l}\text { Steam Generator \#2 } \\
\text { Secondary Pressure }\end{array}$ & PT-302 & psig & 18 & \\
\hline $\begin{array}{l}\text { Accumulators \#1 and } \\
\text { \#2 Pressure }\end{array}$ & PT-401 and PT-402 & psig & 19,20 & \\
\hline $\begin{array}{l}\text { Accumulators \#1 and } \\
\text { \#2 Liquid Level }\end{array}$ & LDP-401 and LDP-402 & inch of $\mathrm{H}_{2} \mathrm{O}$ & 21,22 & \\
\hline $\begin{array}{l}\text { Accumulators \#1 and } \\
\text { \#2 Flow Rate }\end{array}$ & FMM-401 and FMM-402 & gpm & 23,24 & \\
\hline $\begin{array}{l}\text { Accumulators \#1 and } \\
\text { \#2 Liquid Discharge } \\
\text { Temperature }\end{array}$ & TF-401 and TF-402 & ${ }^{\circ} \mathrm{F}$ & 25,26 & \\
\hline $\begin{array}{l}\text { CMT \#1 and \#2 Liquid } \\
\text { Level }\end{array}$ & LDP-507 and LDP-502 & inch of $\mathrm{H}_{2} \mathrm{O}$ & 27,28 & \\
\hline $\begin{array}{l}\text { CMT \#1 and \#2 Flow } \\
\text { Rate }\end{array}$ & FMM-501 and FMM-504 & gpm & 29,30 & \\
\hline $\begin{array}{l}\text { CMT \#1 and \#2 Liquid } \\
\text { Temperature }\end{array}$ & $\begin{array}{l}\text { TF-501 and TF-529; and } \\
\text { TF-504 and TF-532 }\end{array}$ & ${ }^{\circ} \mathrm{F}$ & $31-34$ & \\
\hline PRHR Inlet Flow Rate & FMM-802 & gpm & 35 & \\
\hline PRHR Liquid Level & LDP-802 & inch of $\mathrm{H}_{2} \mathrm{O}$ & 36 & \\
\hline $\begin{array}{l}\text { PRHR Outlet Flow } \\
\text { Rate }\end{array}$ & FMM-804 & gpm & 37 & \\
\hline IRWST Liquid Level & LDP-701 & inch of $\mathrm{H}_{2} \mathrm{O}$ & 38 & \\
\hline $\begin{array}{l}\text { IRWST Discharge Line } \\
\# 1 \text { and \#2 Flow Rate }\end{array}$ & FMM-701 and FMM-702 & gpm & 39,40 & \\
\hline $\begin{array}{l}\text { IRWST Fluid } \\
\text { Temperature }\end{array}$ & TF-701 and TF-709 & ${ }^{\circ} \mathrm{F}$ & 41,42 & \\
\hline $\begin{array}{l}\text { ADS 1-3 Separator } \\
\text { Pressure }\end{array}$ & PT-605 & psig & 43 & \\
\hline
\end{tabular}




\begin{tabular}{|c|c|c|c|c|}
\hline \multicolumn{5}{|c|}{$\begin{array}{l}\text { TABLE 6-4 (Continued) } \\
\text { DATA PLOTS FOR QL REPORTS FOR TR-01-D BY COMPONENT }\end{array}$} \\
\hline Component & Channel & Units & QL-Plot & Comment \\
\hline $\begin{array}{l}\text { ADS 1-3 Separator } \\
\text { Steam Flow Rate }\end{array}$ & FVM-601 & $\operatorname{scfm}$ & 44 & \\
\hline $\begin{array}{l}\text { ADS 1-3 Separator } \\
\text { Liquid Flow Rate }\end{array}$ & FMM-601 & gpm & 45 & \\
\hline $\begin{array}{l}\text { ADS-4 Separator \#1 } \\
\text { and \#2 Pressure }\end{array}$ & PT-611 and PT-610 & psig & 46,47 & \\
\hline $\begin{array}{l}\text { ADS 4-1 Separator } \\
\text { Steam Flow Rate }\end{array}$ & FVM-603 & $\mathrm{scfm}$ & 48 & \\
\hline $\begin{array}{l}\text { ADS 4-2 Separator } \\
\text { Steam Flow Rate }\end{array}$ & FVM-602 & $\mathrm{scfm}$ & 49 & \\
\hline $\begin{array}{l}\text { ADS 4-1 Separator } \\
\text { Liquid Flow Rate }\end{array}$ & FMM-603 & gpm & 50 & \\
\hline $\begin{array}{l}\text { ADS 4-2 Separator } \\
\text { Liquid Flow Rate }\end{array}$ & FMM-602 & gpm & 51 & \\
\hline Primary Sump Pressure & PT-901 & psig & 52 & \\
\hline $\begin{array}{l}\text { Primary Sump Liquid } \\
\text { Level }\end{array}$ & LDP-901 & inch of $\mathrm{H}_{2} \mathrm{O}$ & 53 & \\
\hline $\begin{array}{l}\text { Primary Sump } \\
\text { Injection Flow Rate }\end{array}$ & FMM-901 and FMM-902 & gpm & 54,55 & \\
\hline $\begin{array}{l}\text { Secondary Sump } \\
\text { Liquid Level }\end{array}$ & LDP-902 & inch of $\mathrm{H}_{2} \mathrm{O}$ & 56 & \\
\hline $\begin{array}{l}\text { Break Separator } \\
\text { Pressure }\end{array}$ & PT-905 & psig & 57 & \\
\hline $\begin{array}{l}\text { Break Separator Liquid } \\
\text { Level }\end{array}$ & LDP-905 & inch of $\mathrm{H}_{2} \mathrm{O}$ & 58 & \\
\hline $\begin{array}{l}\text { Break Separator Flow } \\
\text { to Primary Sump }\end{array}$ & FMM-905 & gpm & 59 & \\
\hline $\begin{array}{l}\text { BAMS Steam Flow } \\
\text { Rate }\end{array}$ & FVM-901 & $\mathrm{scfm}$ & 60 & \\
\hline $\begin{array}{l}\text { BAMS Steam Flow } \\
\text { Rate }\end{array}$ & FVM-902 & $\mathrm{scfm}$ & 61 & \\
\hline
\end{tabular}




\begin{tabular}{|c|c|c|c|c|}
\hline \multicolumn{5}{|c|}{$\begin{array}{c}\text { TABLE 6-4 (Continued) } \\
\text { DATA PLOTS FOR QL REPORTS FOR TR-01-D BY COMPONENT }\end{array}$} \\
\hline Component & Channel & Units & QL-Plot & Comment \\
\hline $\begin{array}{l}\text { BAMS/Primary Sump } \\
\text { Steam Flow Rate }\end{array}$ & FVM-903 & $\mathrm{scfm}$ & 62 & \\
\hline $\begin{array}{l}\text { BAMS/Separator } \\
\text { Steam Flow Rate - } \\
\text { 6-inch Pipe }\end{array}$ & FVM-905 & $\mathrm{scfm}$ & 63 & \\
\hline $\begin{array}{l}\text { BAMS/Exhaust Line } \\
\text { Temperature }\end{array}$ & TF-916 and SC-917 & ${ }^{\circ} \mathrm{F}$ & 64,65 & \\
\hline $\begin{array}{l}\text { Pressurizer Heater } \\
\text { Input Power }\end{array}$ & KW-601 & $\mathrm{kW}$ & 66 & \\
\hline $\begin{array}{l}\text { Core Power Input } \\
\text { Power }\end{array}$ & $\begin{array}{l}\text { KW-101, KW-102, } \\
\text { KW-103, and KW-104 }\end{array}$ & $\mathrm{kW}$ & $67-70$ & \\
\hline $\begin{array}{l}\text { Reactor Vessel Liquid } \\
\text { Level Between Top of } \\
\text { Vessel - Upper Support } \\
\text { Plate }\end{array}$ & LDP-115 & inch of $\mathrm{H}_{2} \mathrm{O}$ & 71 & \\
\hline $\begin{array}{l}\text { Reactor Vessel Liquid } \\
\text { Level Between Bottom } \\
\text { of Upper Support } \\
\text { Plate - Upper Core } \\
\text { Spacer Grid }\end{array}$ & LDP-139 & inch of $\mathrm{H}_{2} \mathrm{O}$ & 72 & \\
\hline $\begin{array}{l}\text { Inner Core } \\
\text { Thermocouple } \\
\text { Measuring Heater } \\
\text { Temperature }\end{array}$ & TH-103-4 & ${ }^{\circ} \mathrm{F}$ & 73 & \\
\hline $\begin{array}{l}\text { Outer Core } \\
\text { Thermocouple } \\
\text { Measuring Heater } \\
\text { Temperature }\end{array}$ & TH-309-4 & ${ }^{\circ} \mathrm{F}$ & 74 & \\
\hline
\end{tabular}




\subsection{CONCLUSIONS}

The TR-01-D test was successfully completed, and the data was logged in the DAS. All critical instruments were found to operate properly with the exception of those noted in Section 6.4. The test was acceptable. 
APPENDIX A

DATA PLOTS 

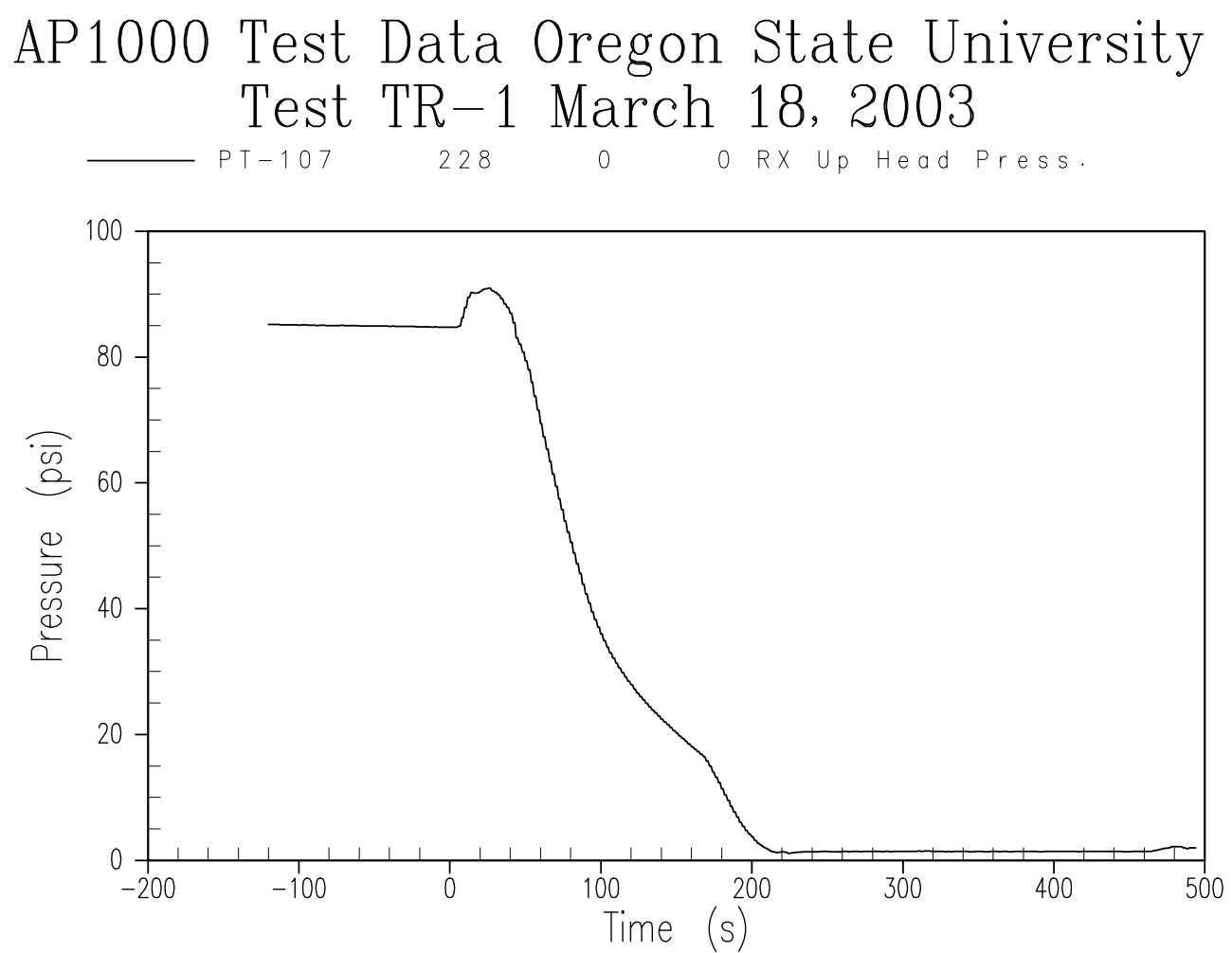

Figure A-1 Reactor Vessel Pressure 

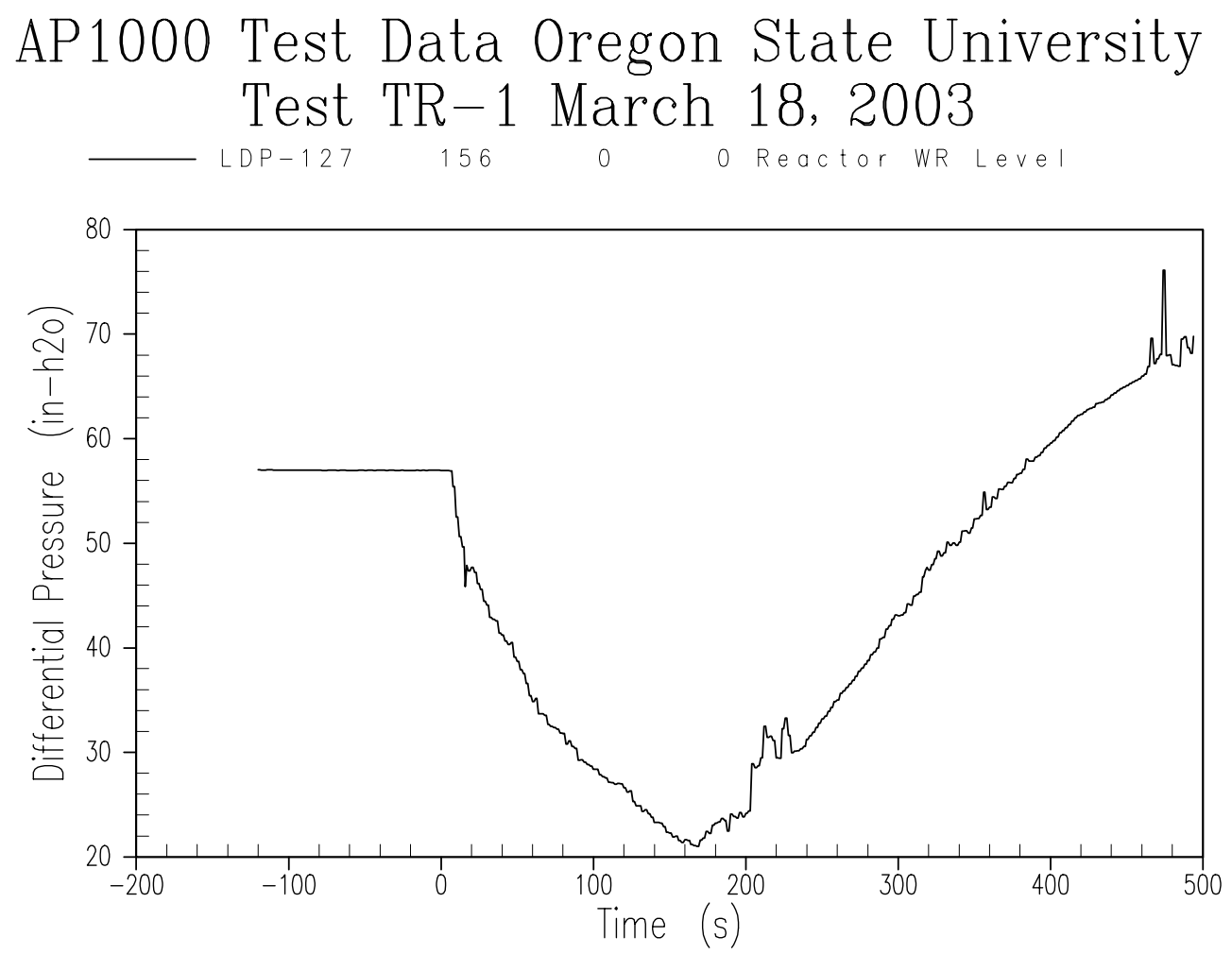

Figure A-2 Reactor Vessel Level 

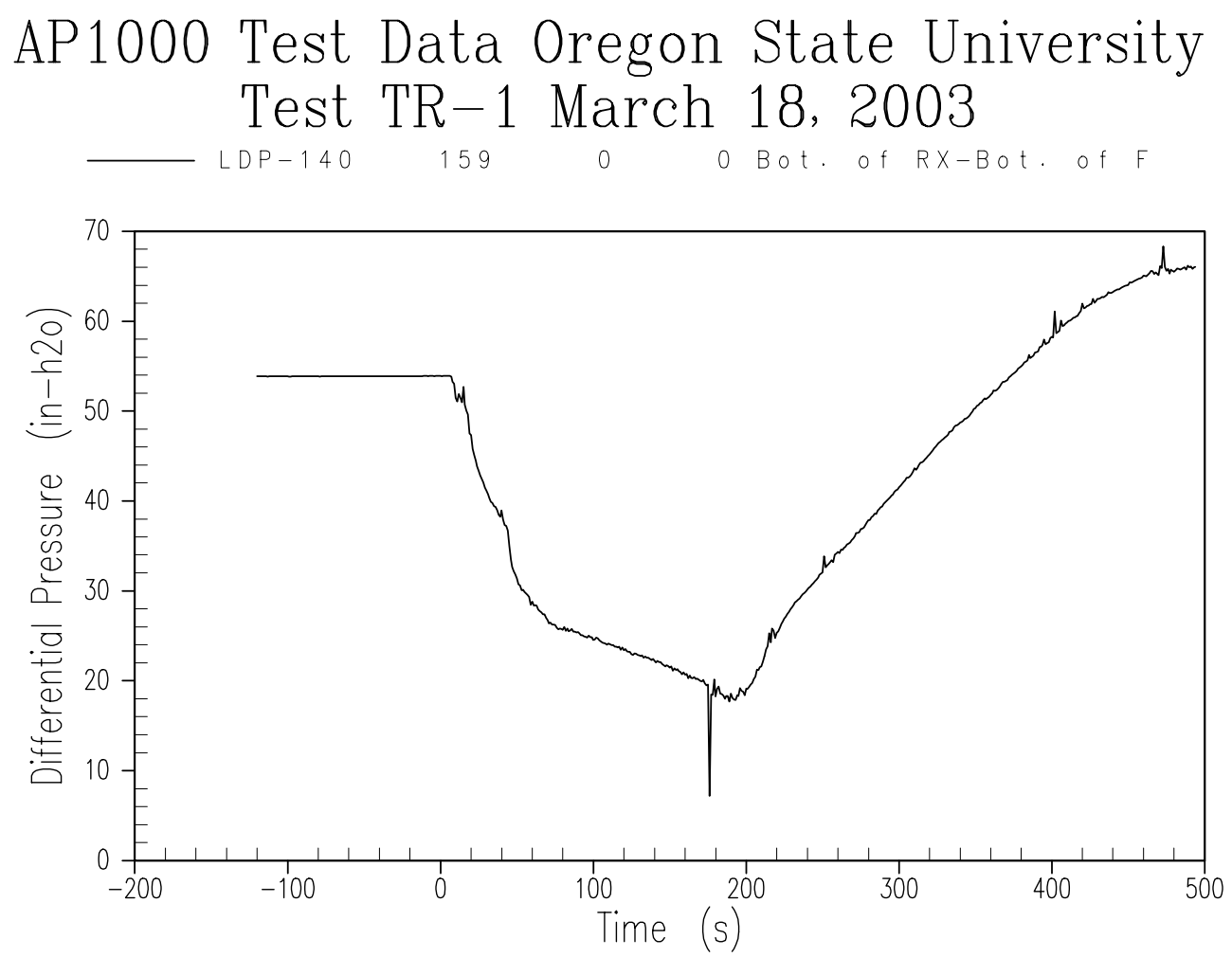

Figure A-3 Reactor Vessel Downcomer Level 
AP1000 Test Data Oregon State University Test TR-1 March 18, 2003

$\longrightarrow$ TF -107

323

0

o $C L-1 / R X F I$ ange @ Bot

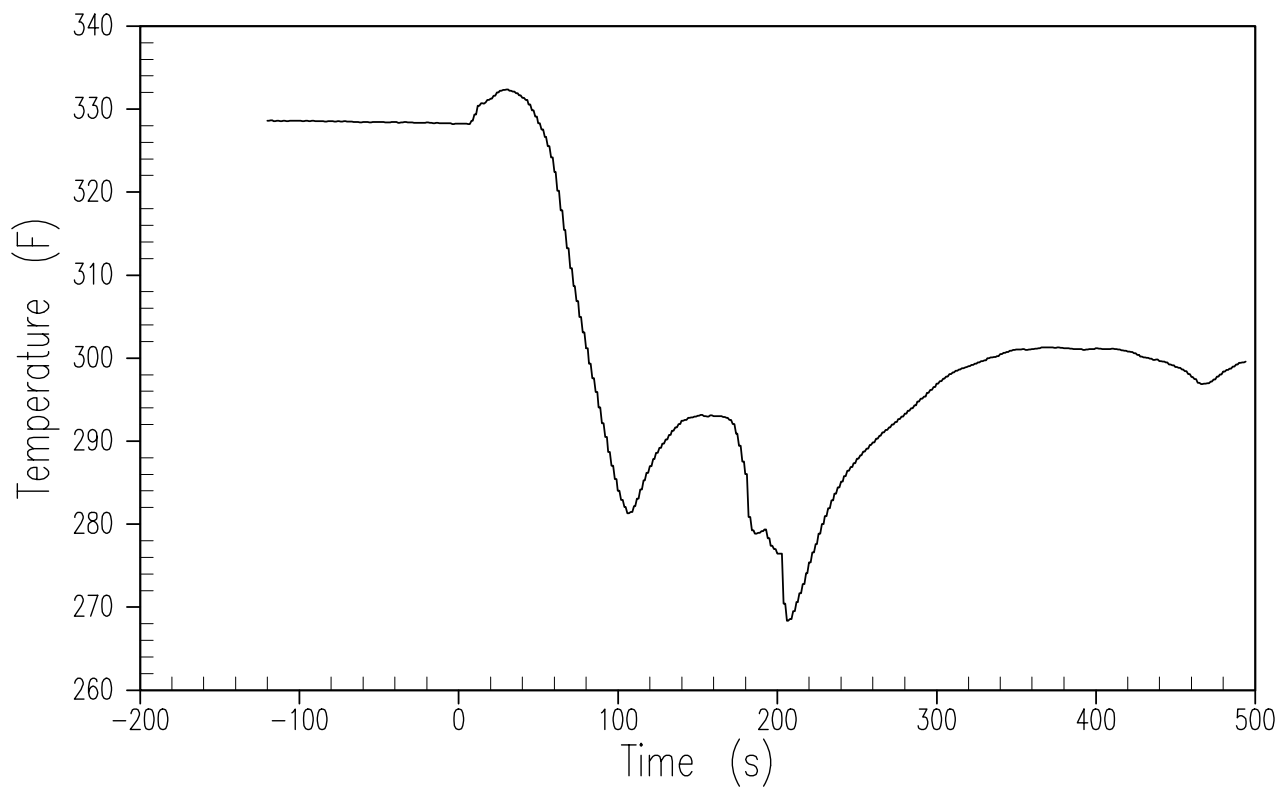

Figure A-4 Cold Leg 1 Fluid Temperature 
AP1000 Test Data Oregon State University Test TR-1 March 18, 2003

$\longrightarrow T F-108$

324

0

o $C L-2 / R X F I$ ange @ Bot

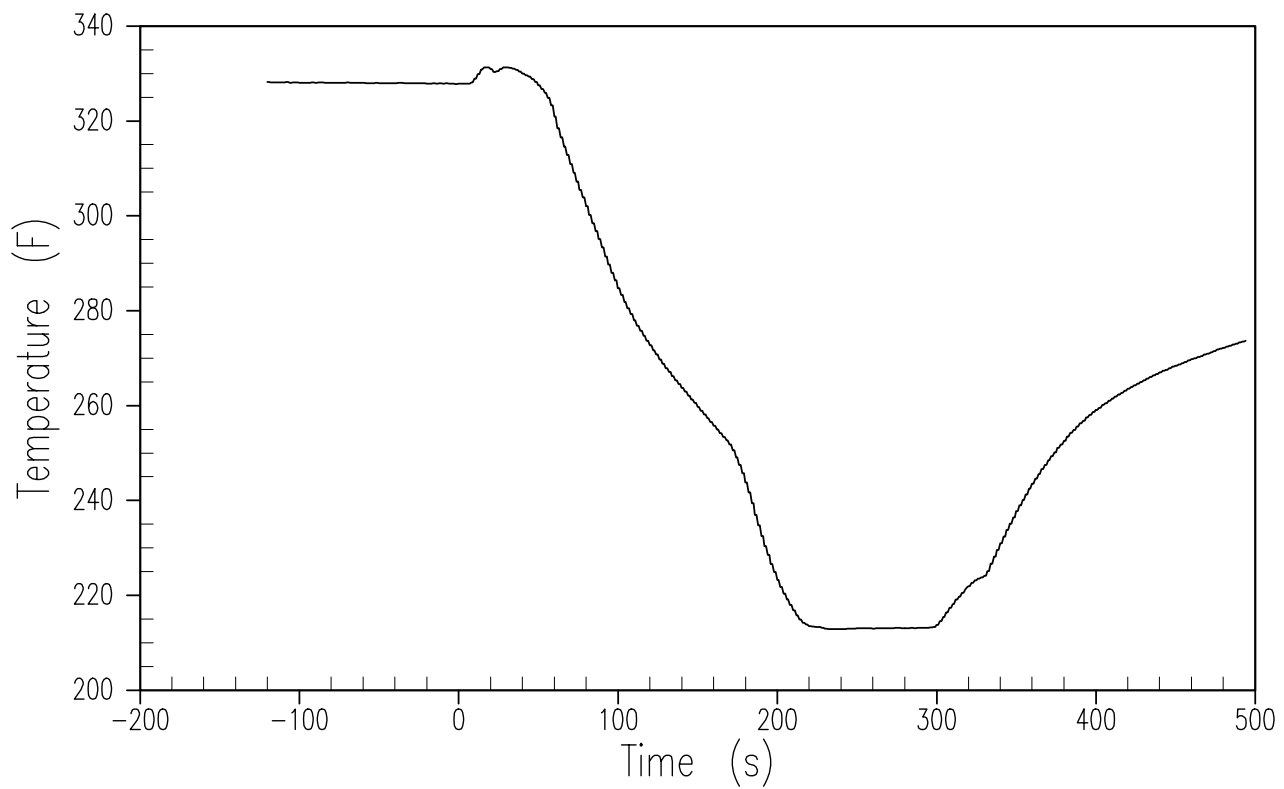

Figure A-5 Cold Leg 2 Fluid Temperature 

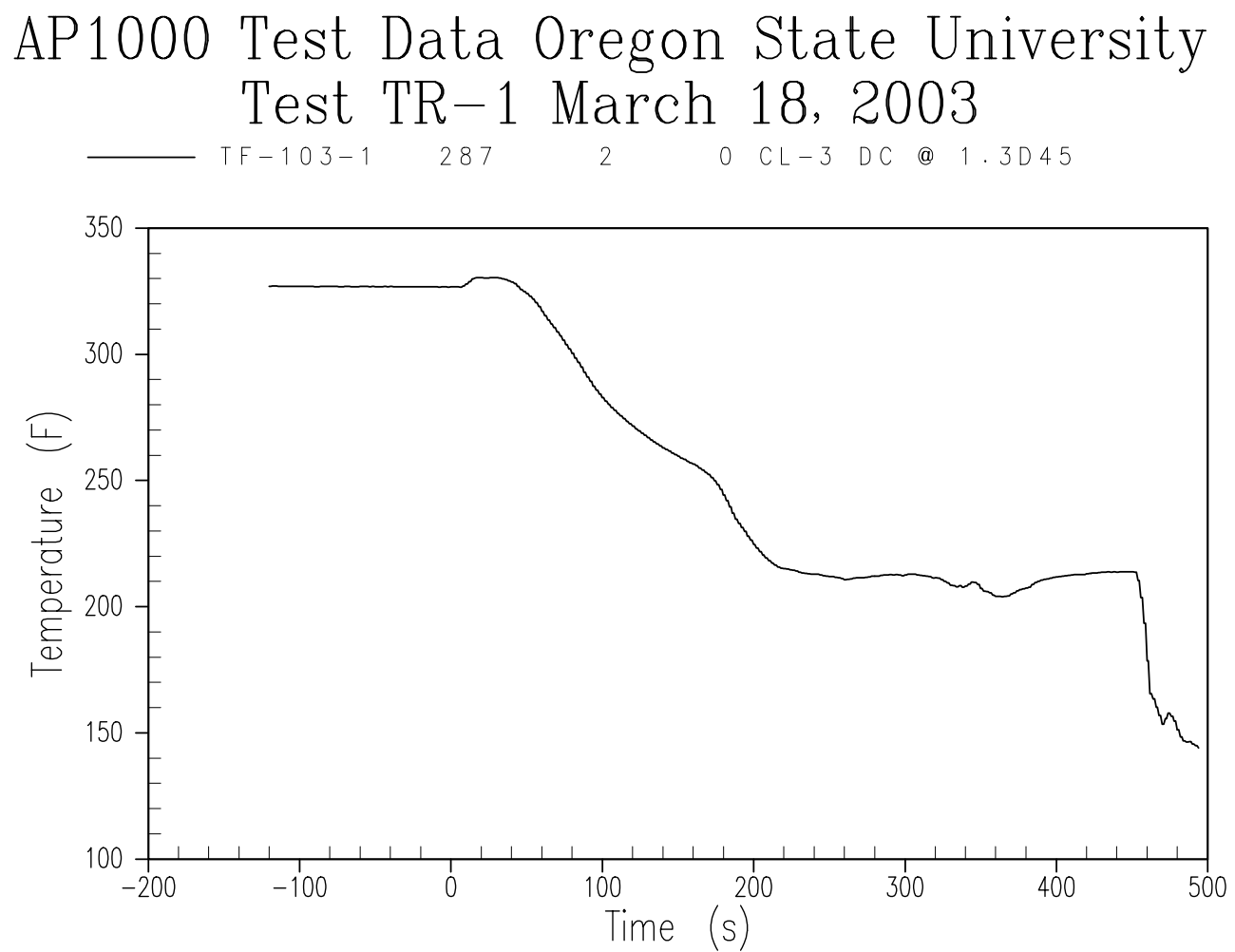

Figure A-6 Cold Leg 3 Fluid Temperature 
AP1000 Test Data Oregon State University Test TR-1 March 18, 2003
TF $-104-1$
298
0
O CL-4 DC Wa|| @ $1.5 \mathrm{D}$

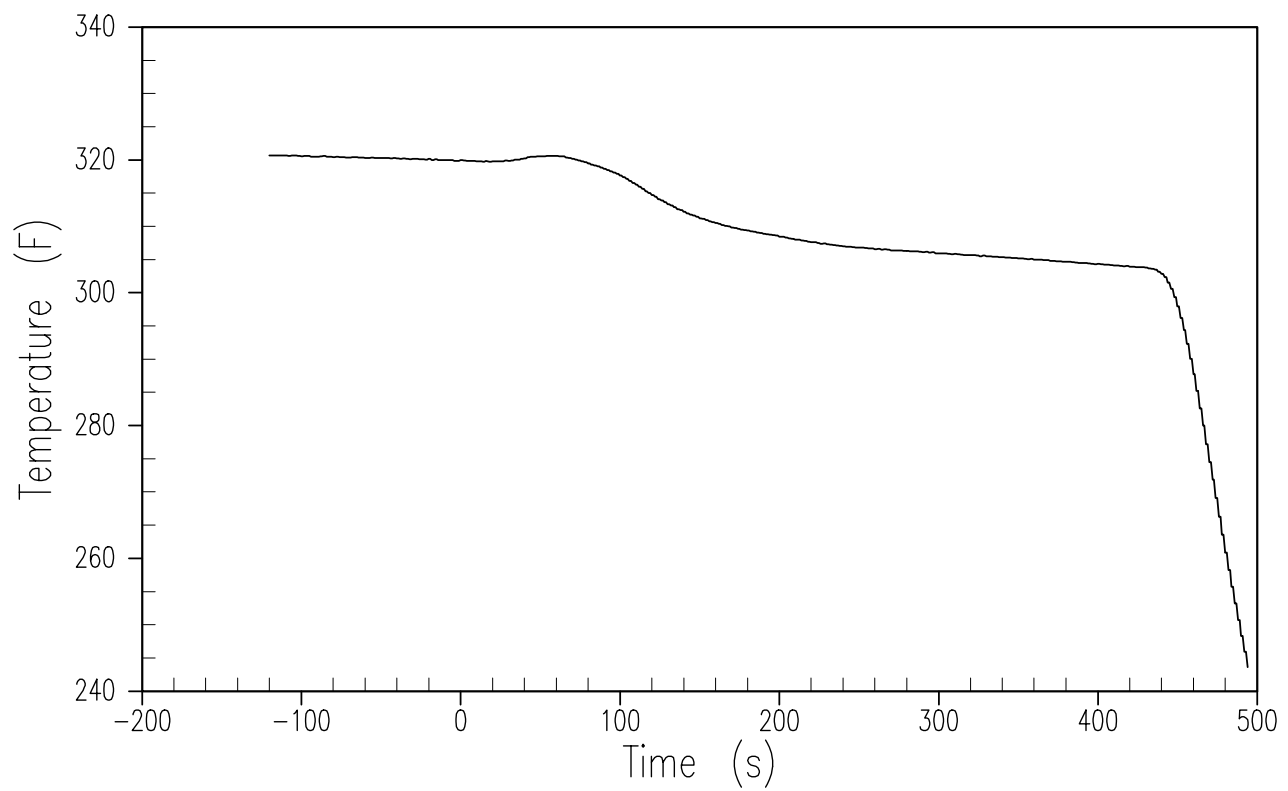

Figure A-7 Cold Leg 4 Fluid Temperature 
AP1000 Test Data Oregon State University Test TR-1 March 18, 2003

$\longrightarrow$

330

0

o Top of RX @ 8.5" \& 3

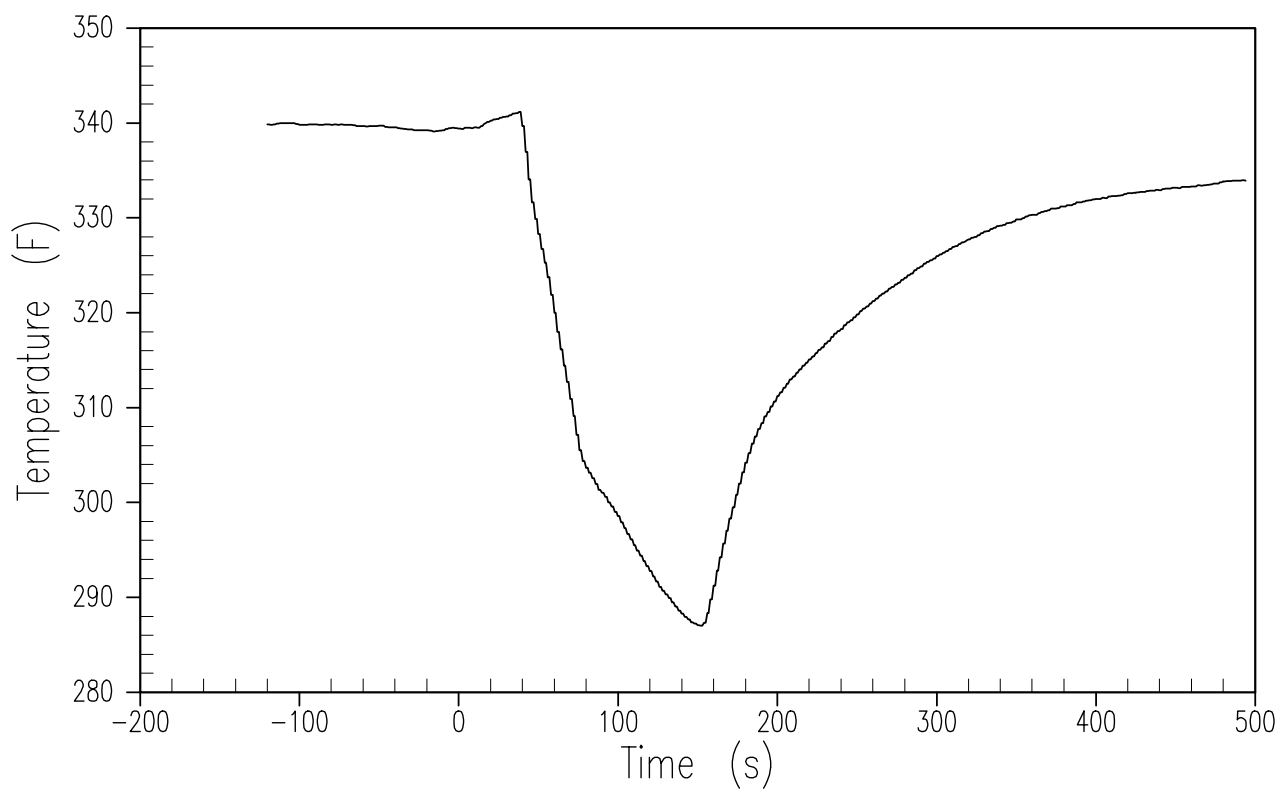

Figure A-8 Reactor Vessel Fluid Temperature Upper Head 
AP1000 Test Data Oregon State University Test TR-1 March 18, 2003

$\longrightarrow$ TF -143

344

0

o $H L-1 / R X F I$ ange @ Bot

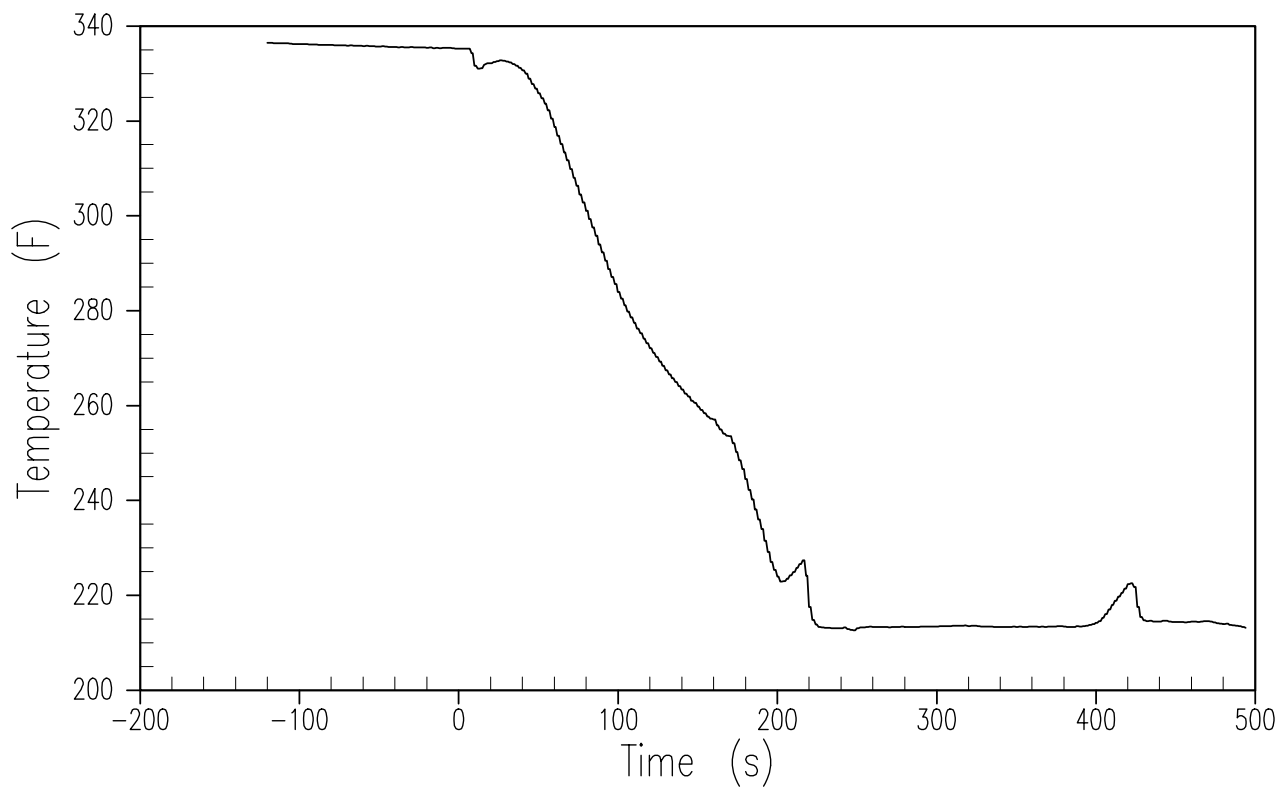

Figure A-9 Reactor Coolant System Hot Leg 1 Temperature 
AP1000 Test Data Oregon State University Test TR-1 March 18, 2003

$-T F-142$

343

0

o $H L-2 / R X F I$ ange @ Bot

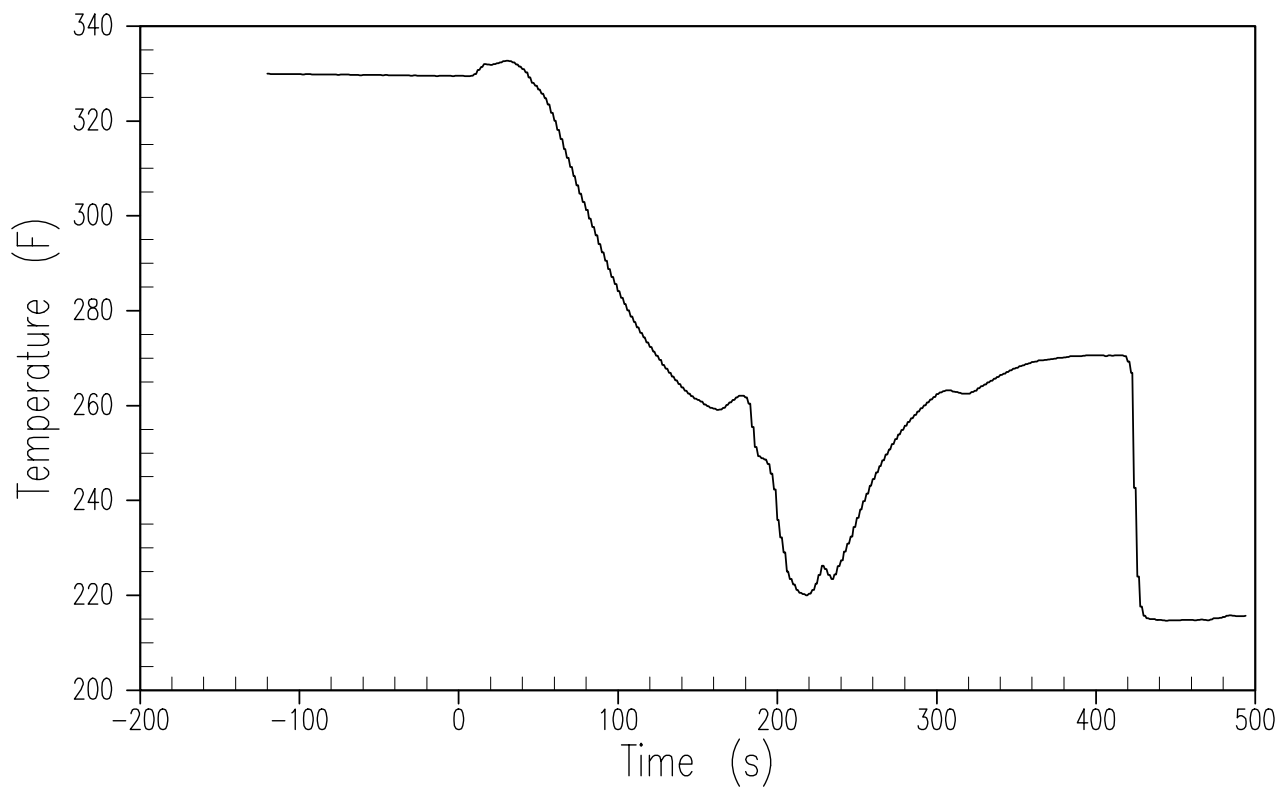

Figure A-10 Reactor Coolant System Hot Leg 2 Temperature 

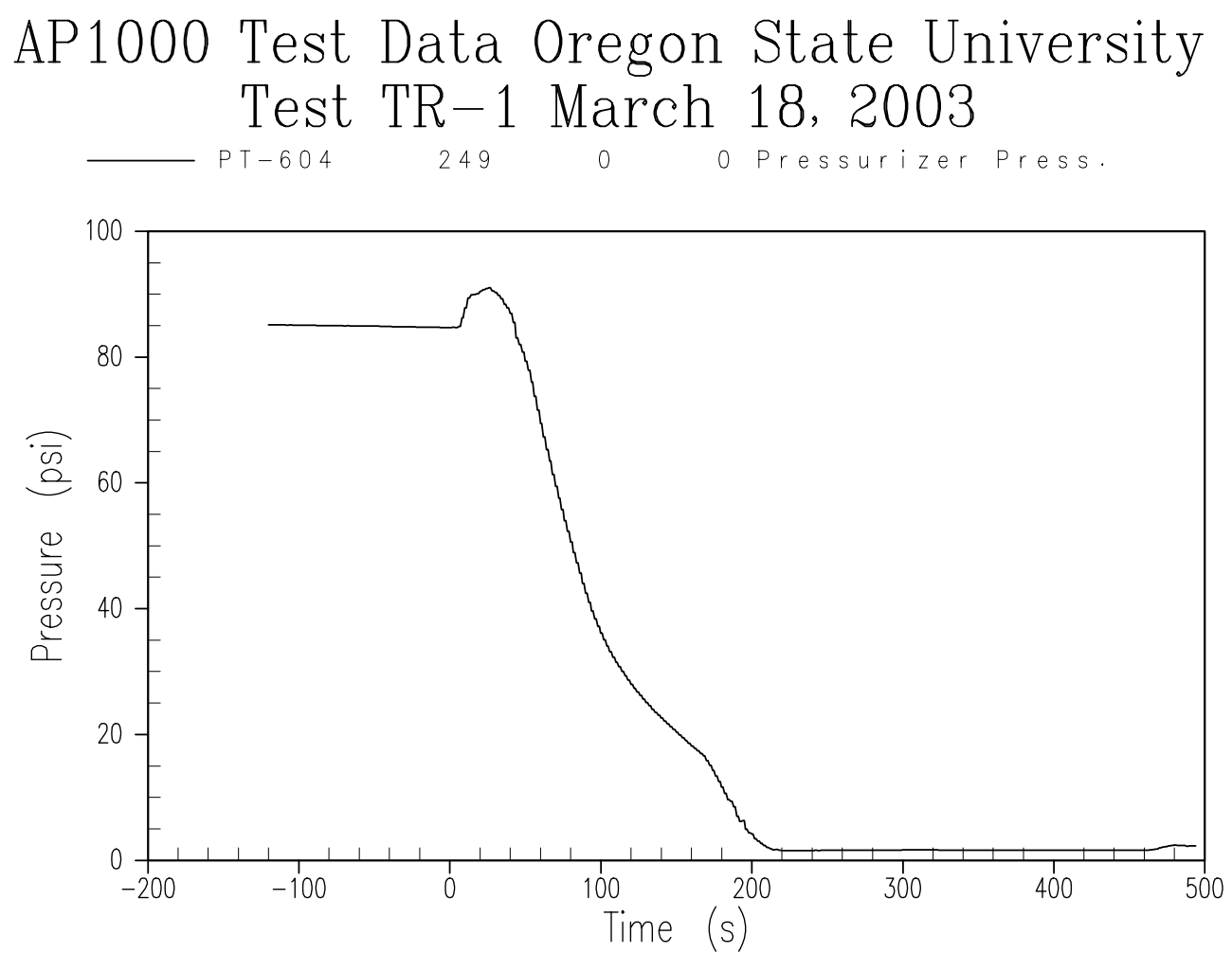

Figure A-11 Pressurizer Pressure - Wide Range 

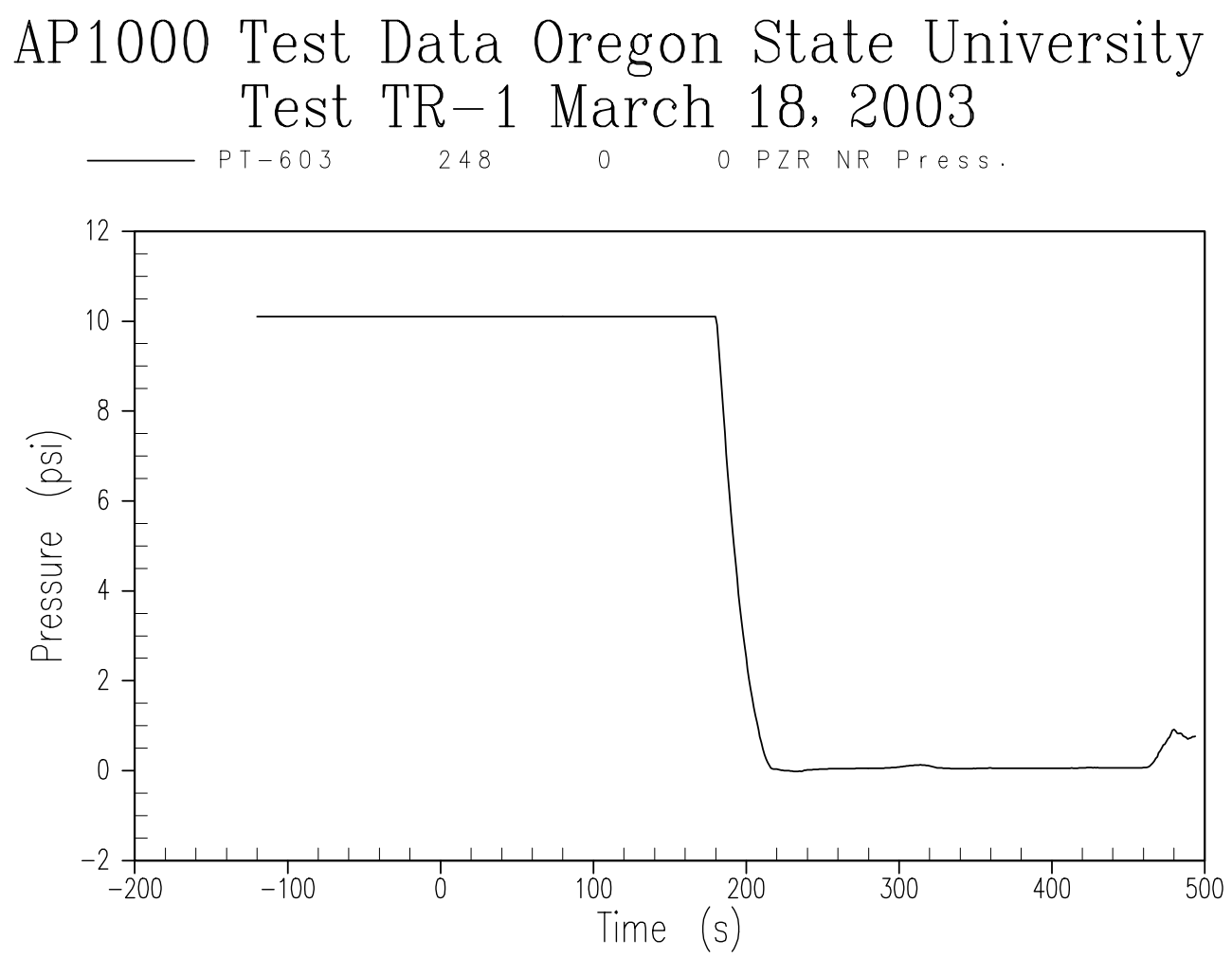

Figure A-12 Pressurizer Pressure - Narrow Range 

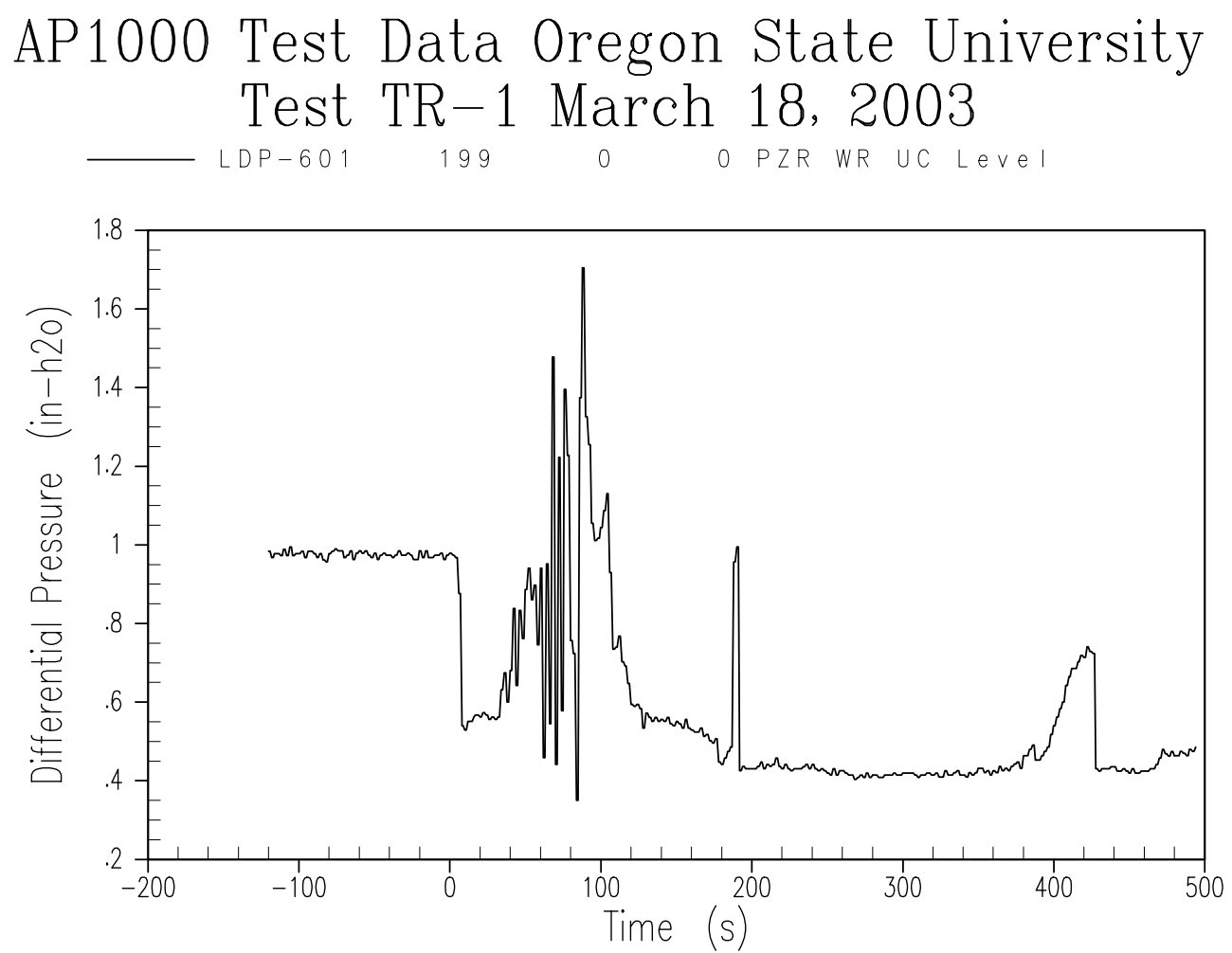

Figure A-13 Pressurizer Liquid Level 

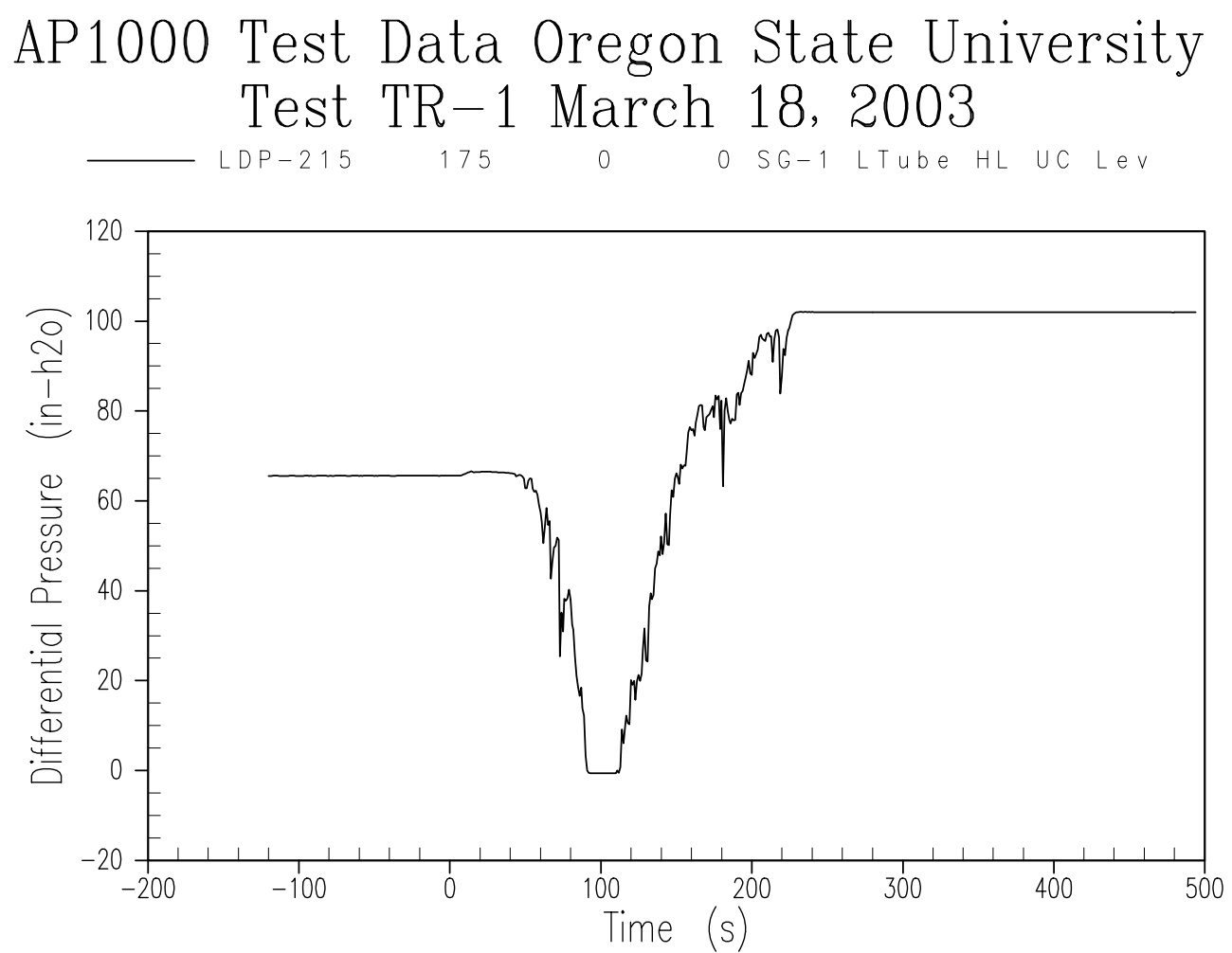

Figure A-14 Steam Generator 1 Tube Level 

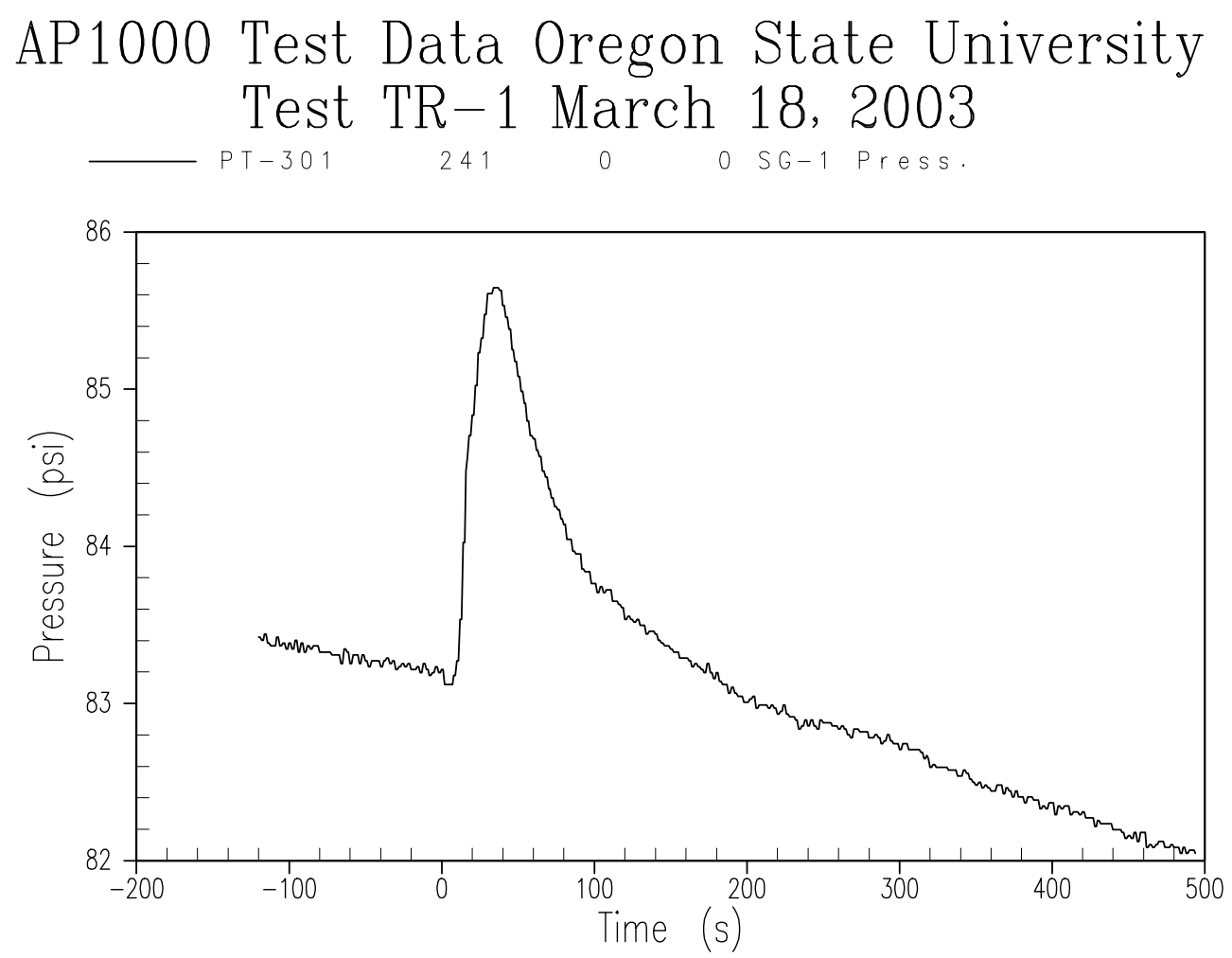

Figure A-15 Steam Generator 1 Secondary Pressure 

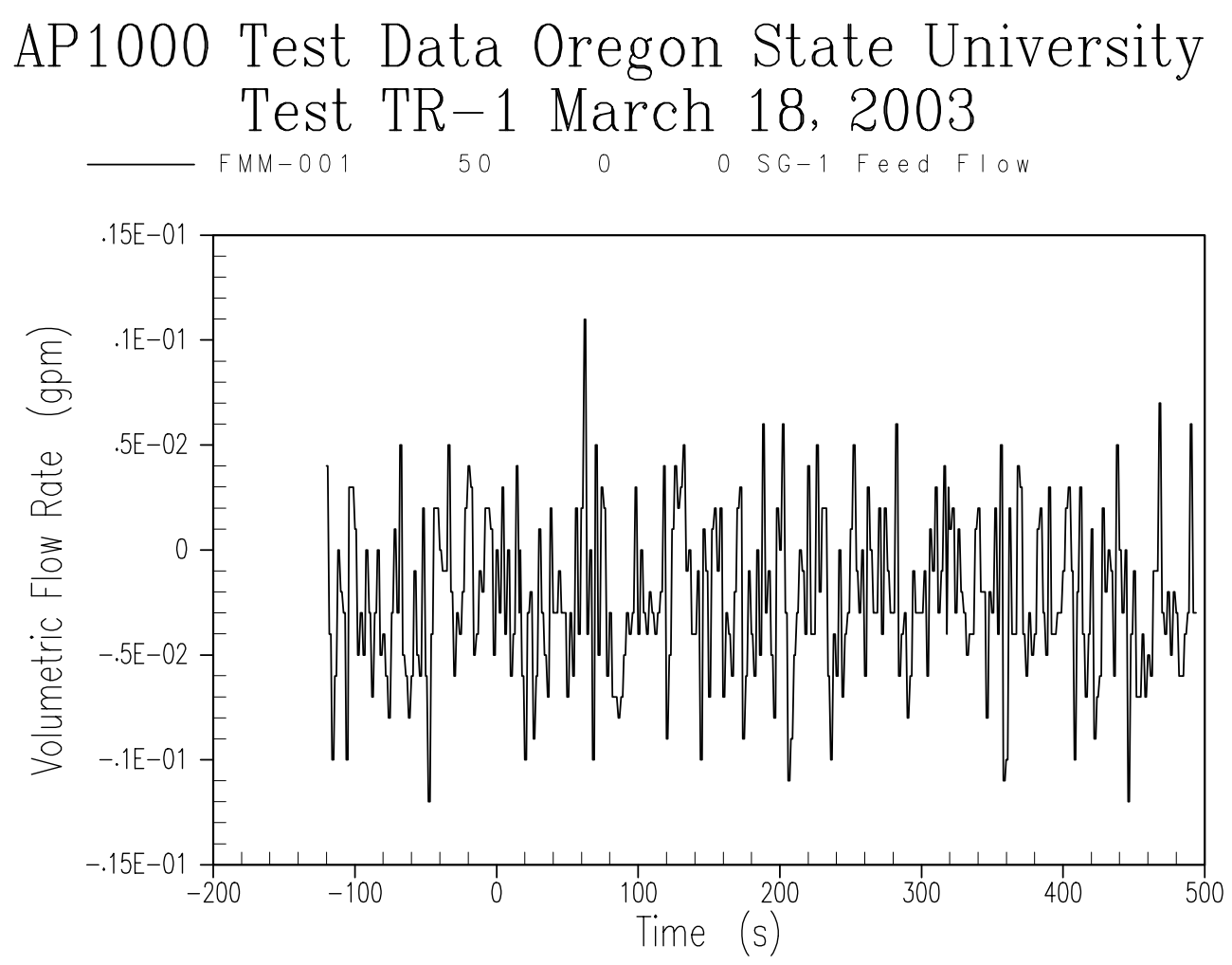

Figure A-16 Steam Generator 1 Feed Flow Rate 

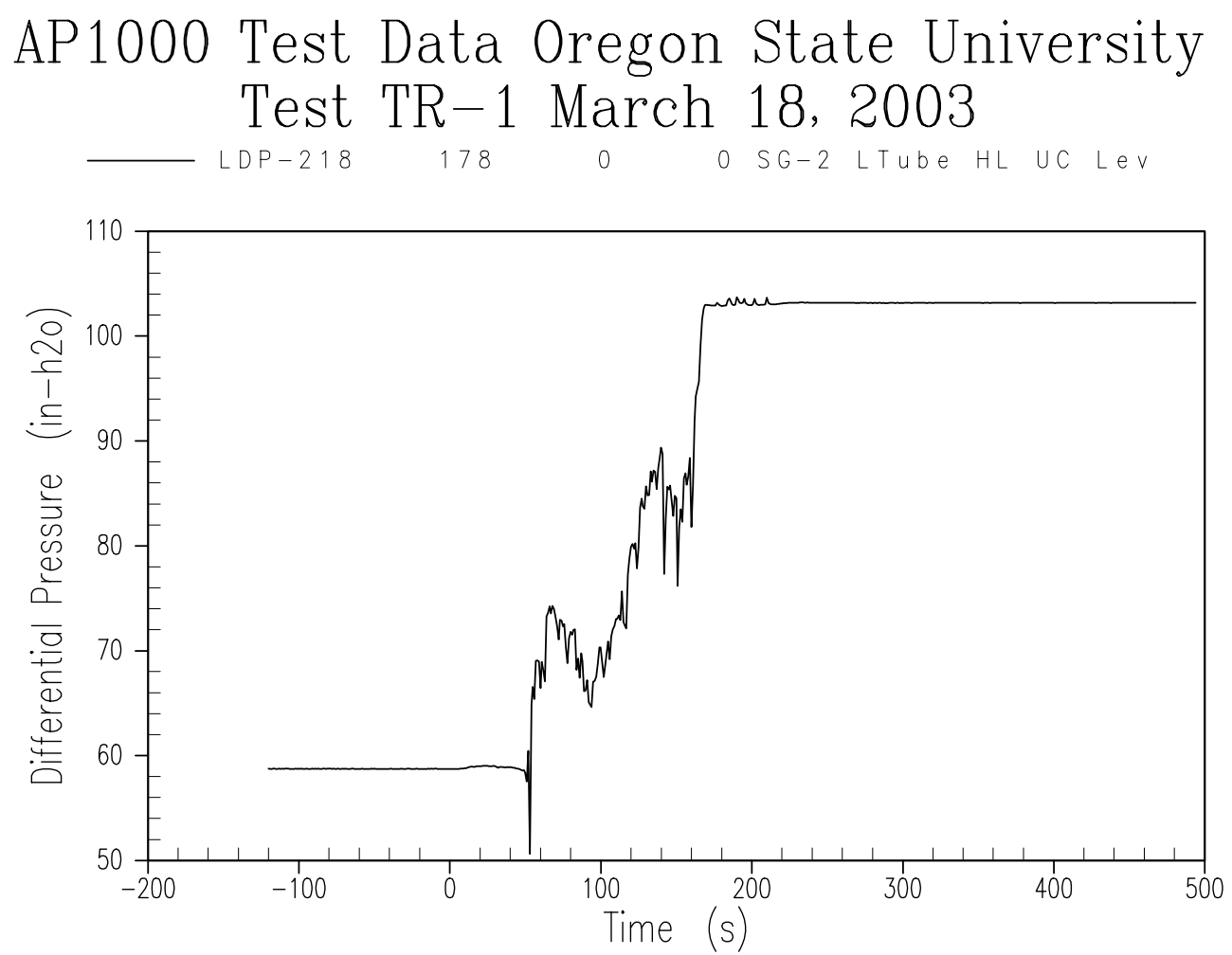

Figure A-17 Steam Generator 2 Tube Level 

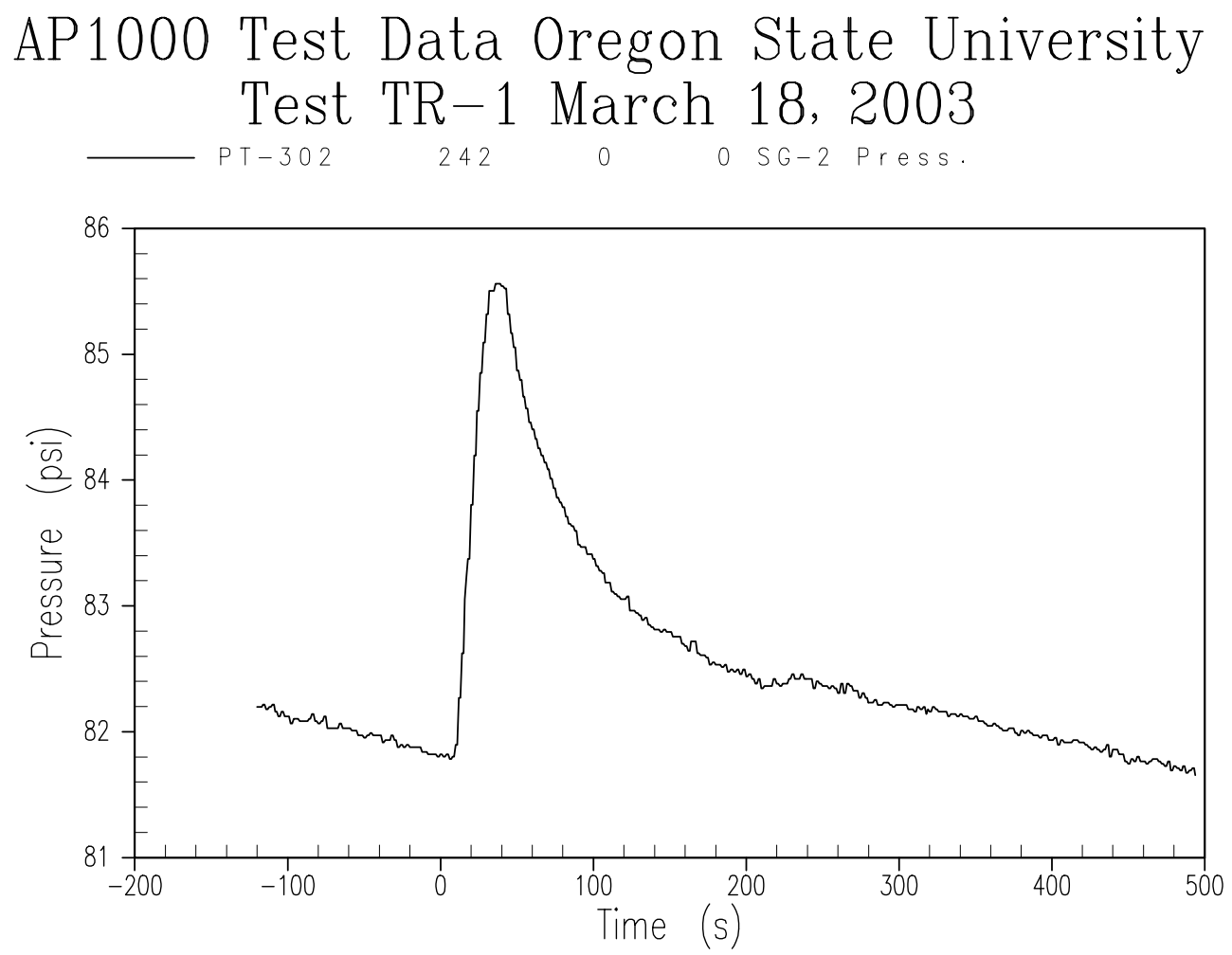

Figure A-18 Steam Generator 2 Secondary Pressure 


\section{AP1000 Test Data Oregon State University Test TR-1 March 18, 2003}

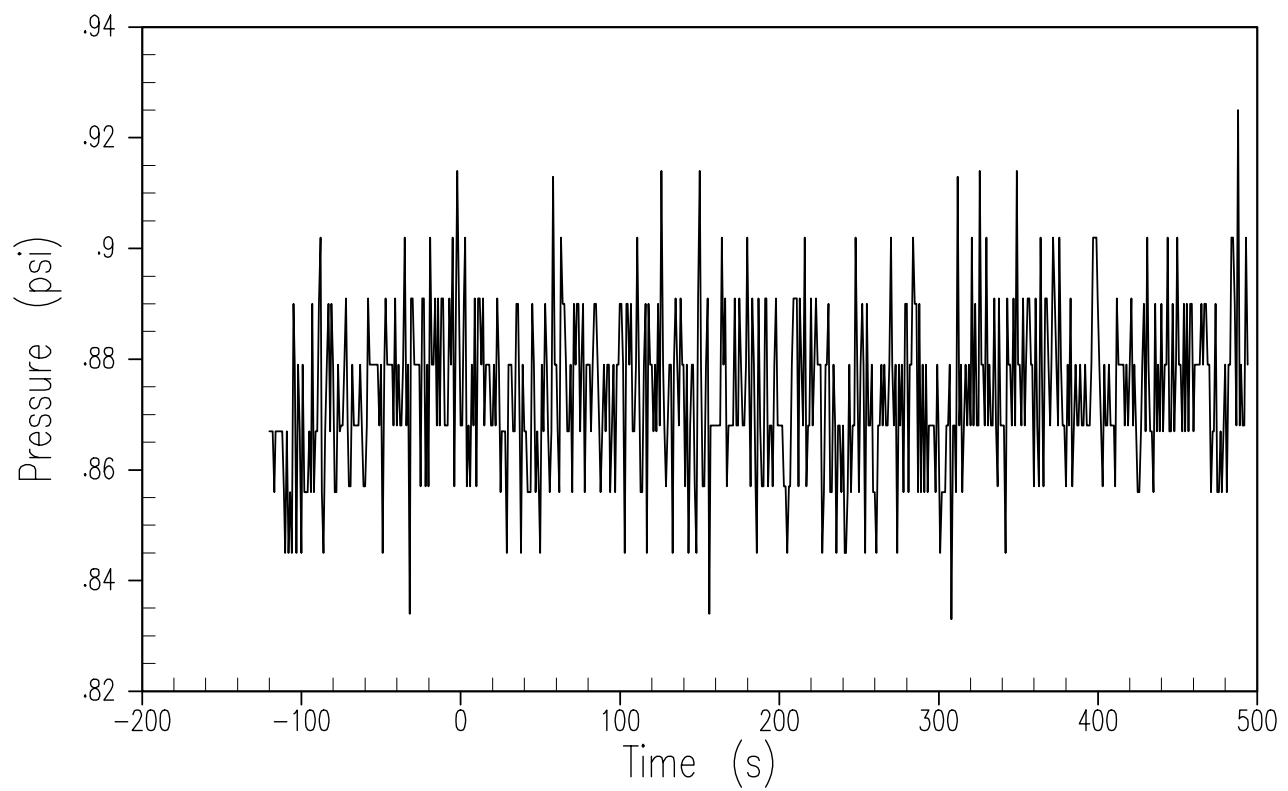

Figure A-19 Accumulator 1 Pressure 


\section{AP1000 Test Data Oregon State University Test TR-1 March 18, 2003}

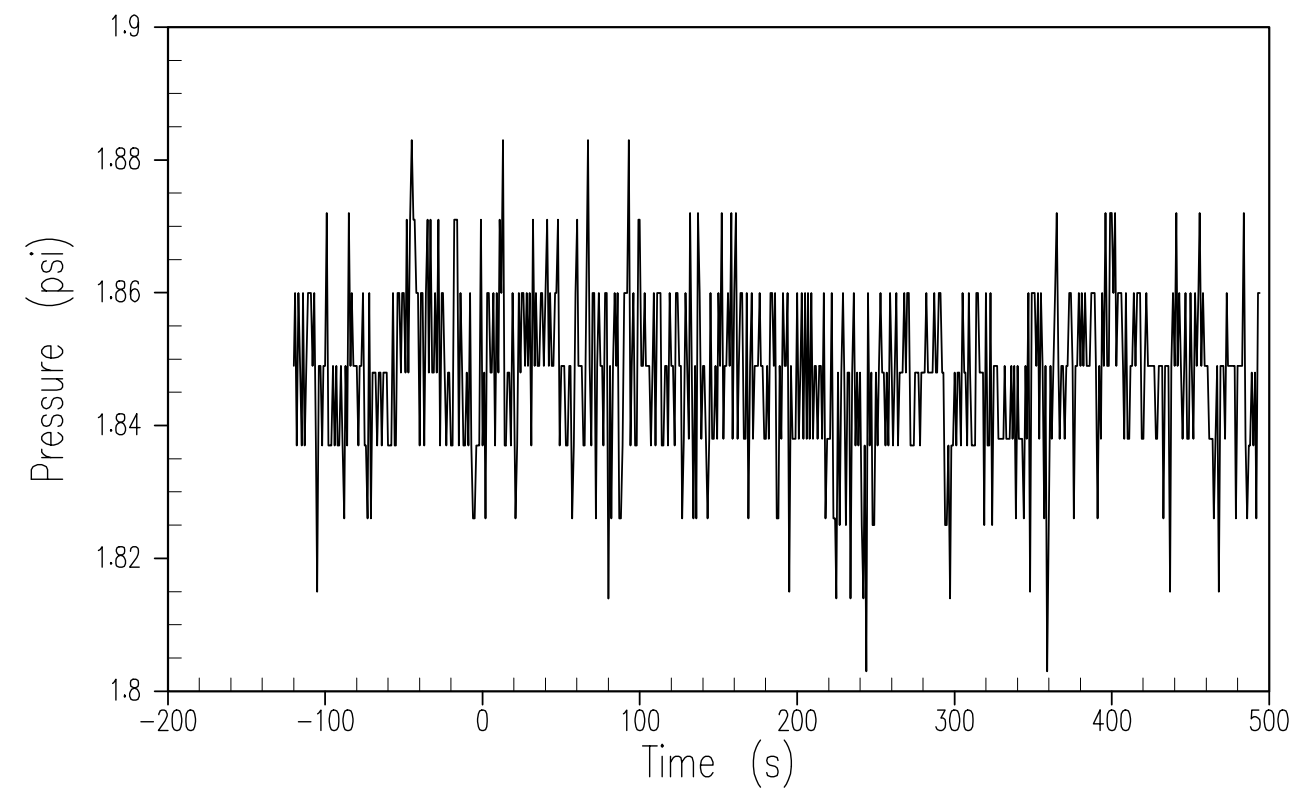

Figure A-20 Accumulator 2 Pressure 


\section{AP1000 Test Data Oregon State University Test TR-1 March 18, 2003}

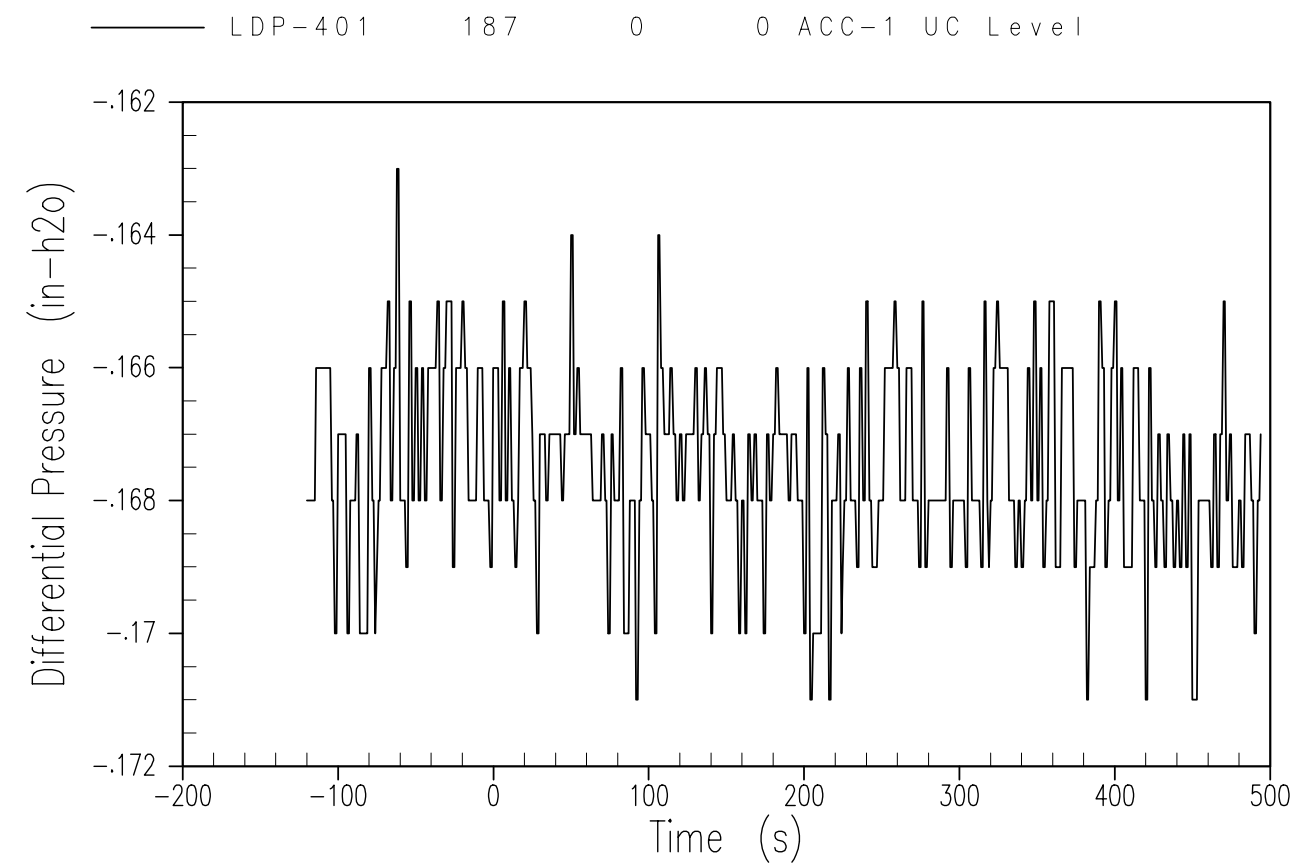

Figure A-21 Accumulator 1 Liquid Level 


\section{AP1000 Test Data Oregon State University Test TR-1 March 18, 2003 \\ $\longrightarrow$ LDP-402 $188 \quad 0 \quad 0 \quad A C C-2$ UC Leve}

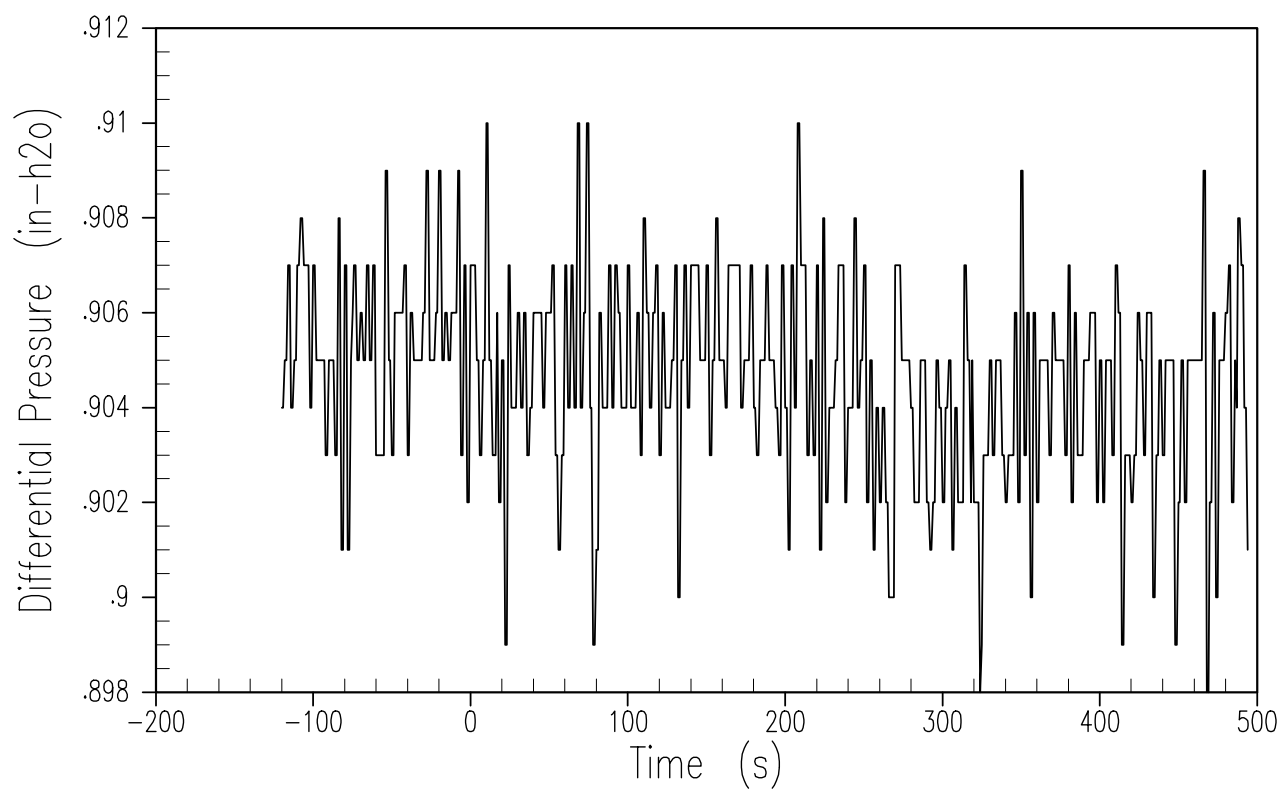

Figure A-22 Accumulator 2 Liquid Level 

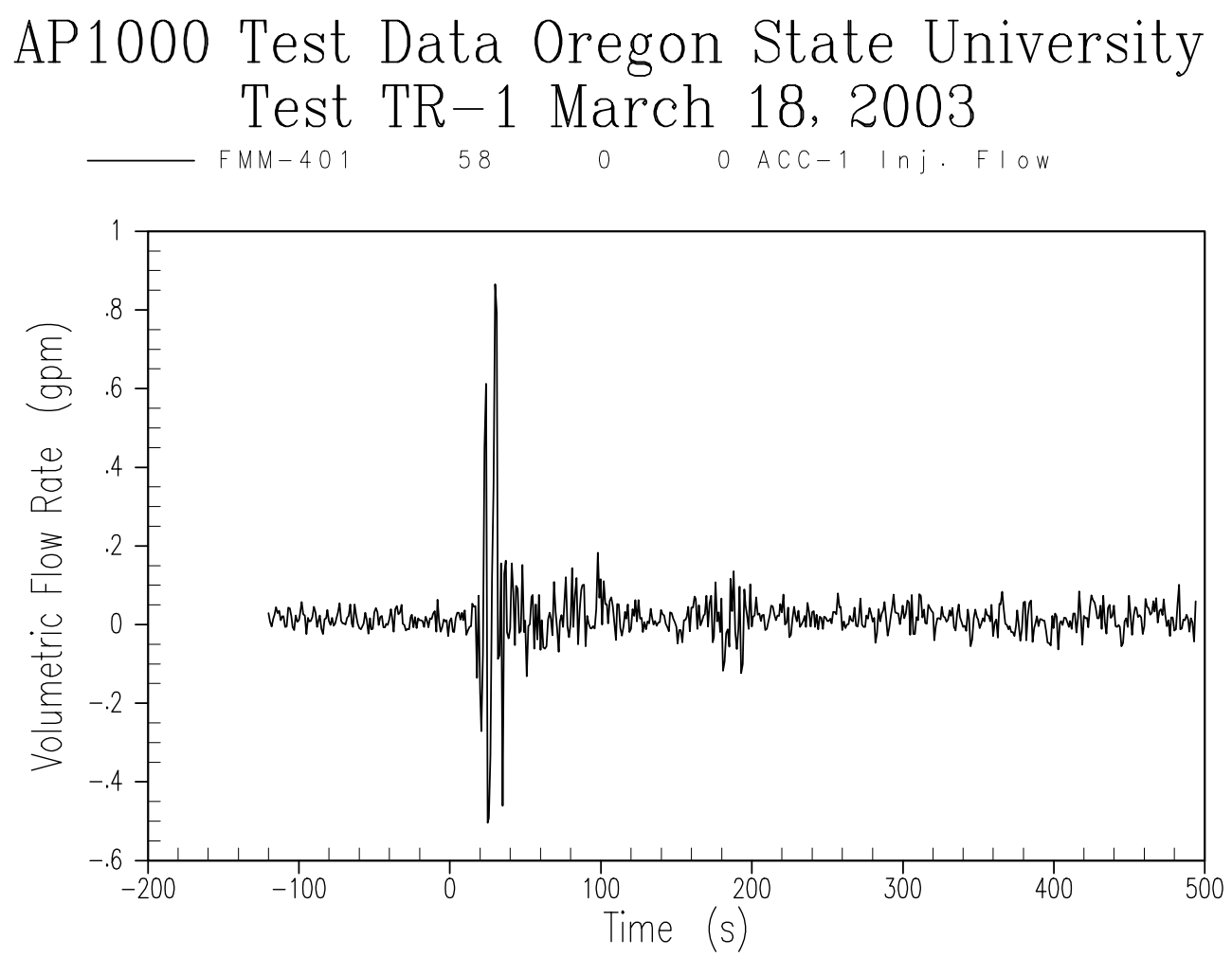

Figure A-23 Accumulator 1 Flow Rate 


\section{AP1000 Test Data Oregon State University Test TR-1 March 18, 2003 FMM-402 $59 \quad 0 \quad 0 \quad A C C-2$ Inj. F I OW}

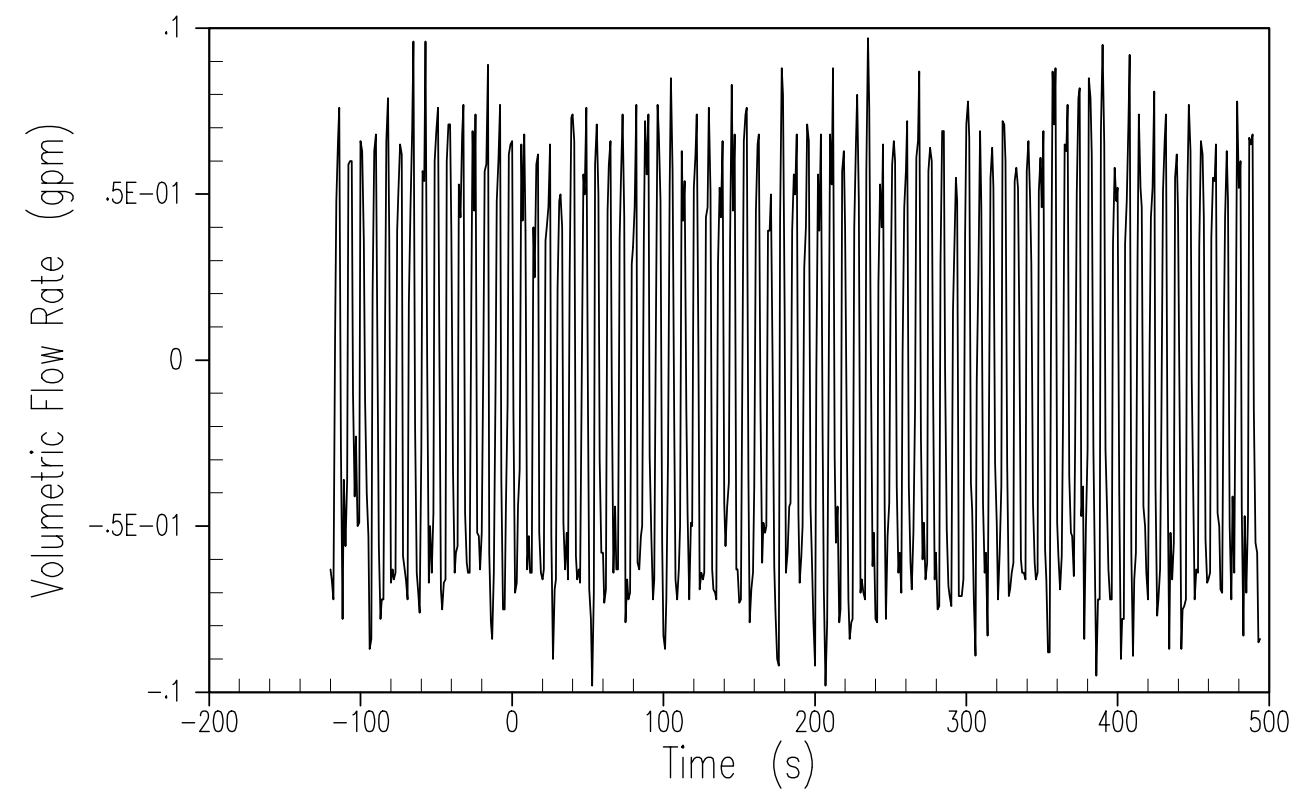

Figure A-24 Accumulator 2 Flow Rate 


\section{AP1000 Test Data Oregon State University Test TR-1 March 18, 2003}

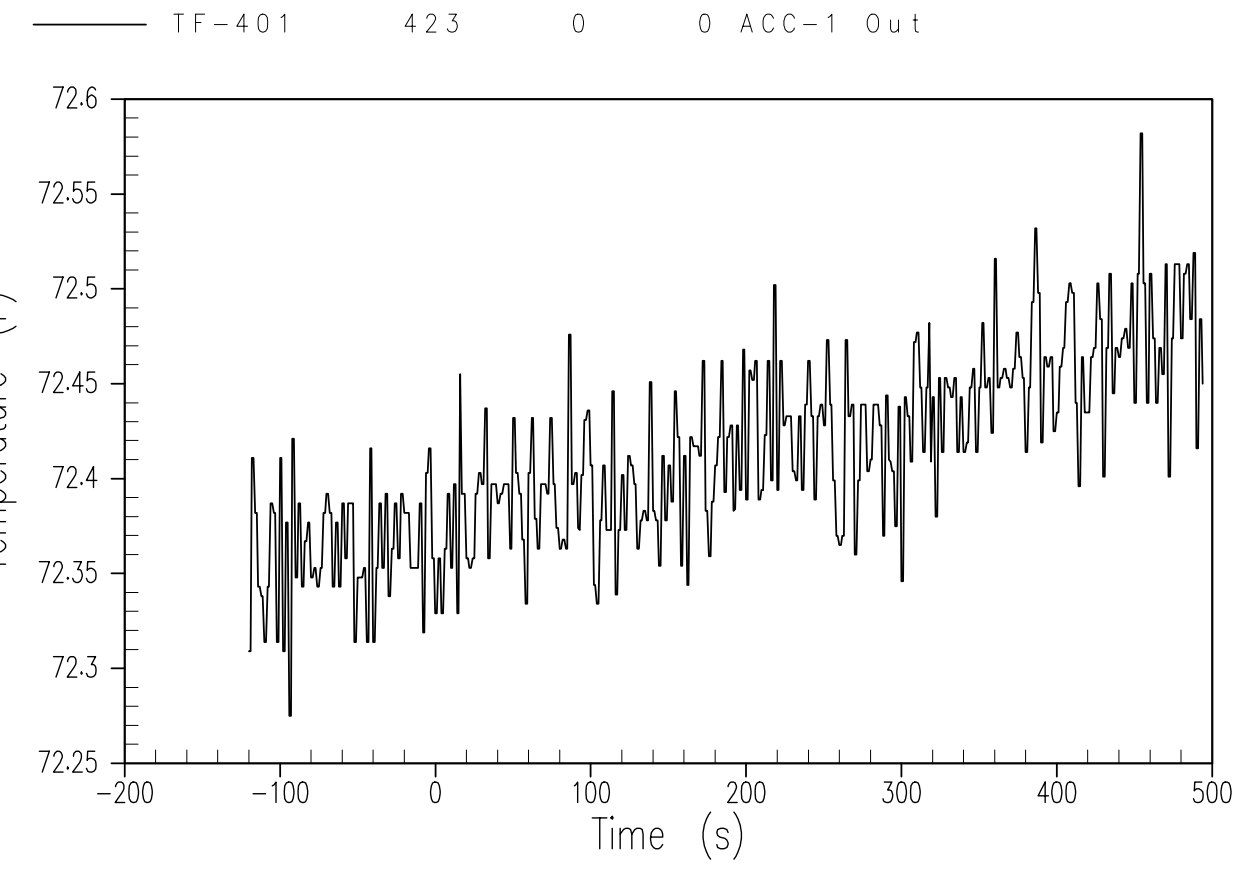

Figure A-25 Accumulator 1 Liquid Discharge Temperature 
AP1000 Test Data Oregon State University Test TR-1 March 18, 2003

TF-402

424

0

$0 \mathrm{ACC}-2 \quad \mathrm{Out}$

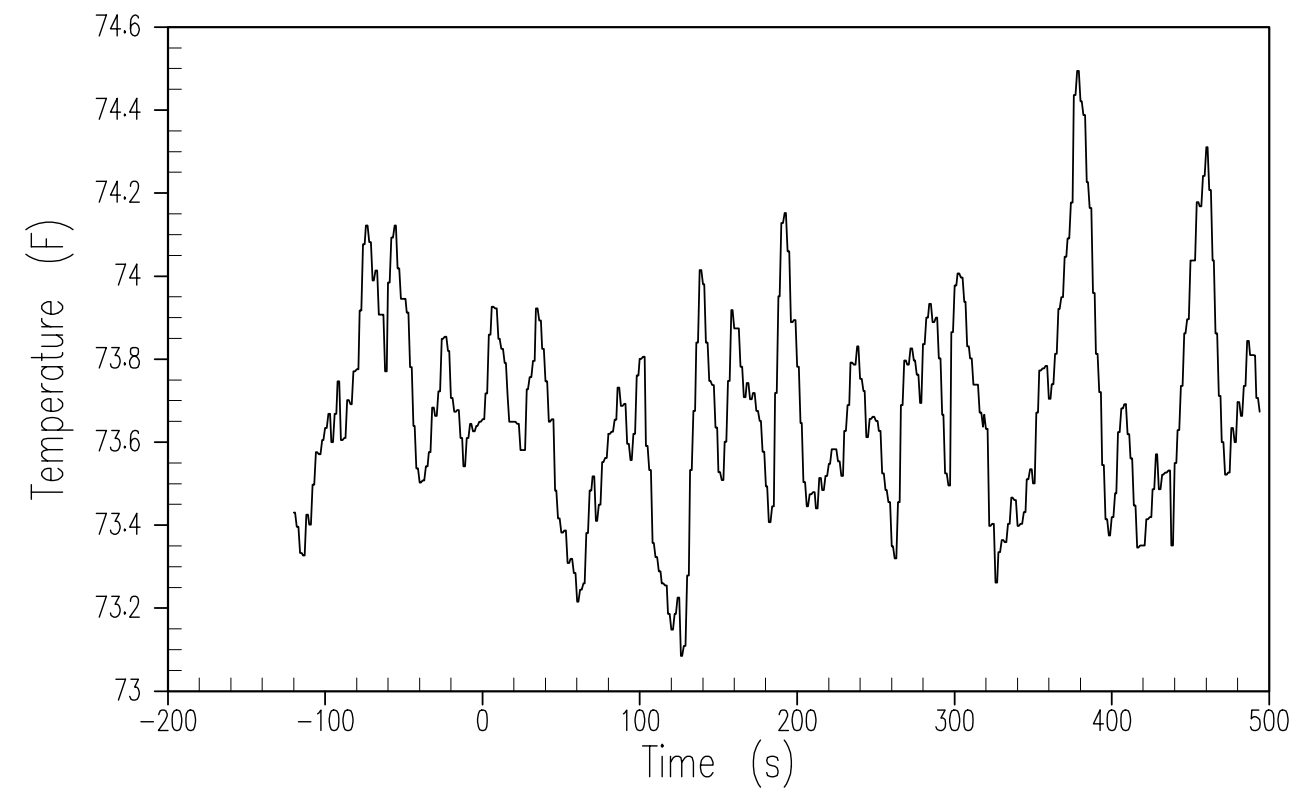

Figure A-26 Accumulator 2 Liquid Discharge Temperature 

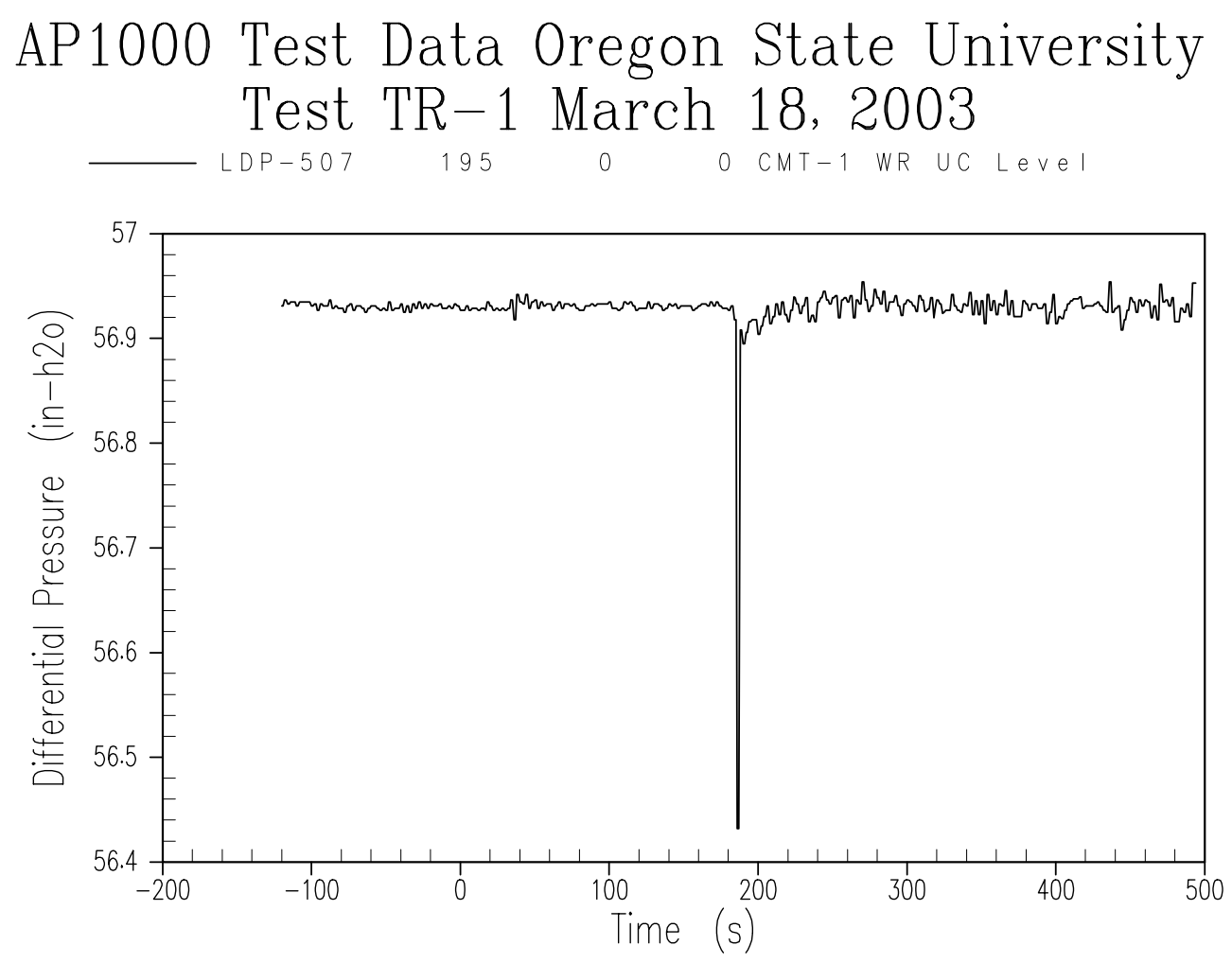

Figure A-27 Core Makeup Tank 1 Liquid Level 

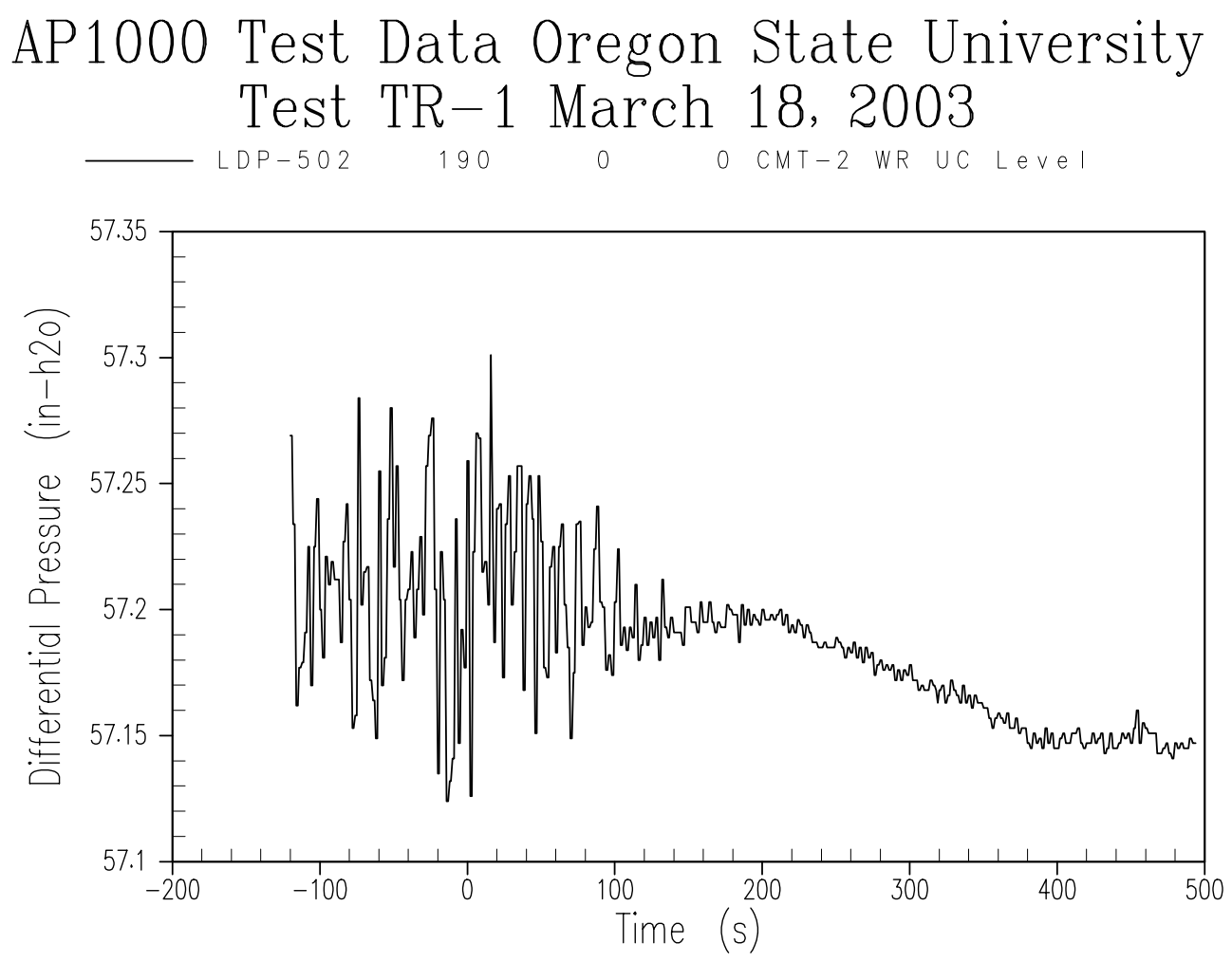

Figure A-28 Core Makeup Tank 2 Liquid Level 


\section{AP1000 Test Data Oregon State University Test TR-1 March 18, 2003}

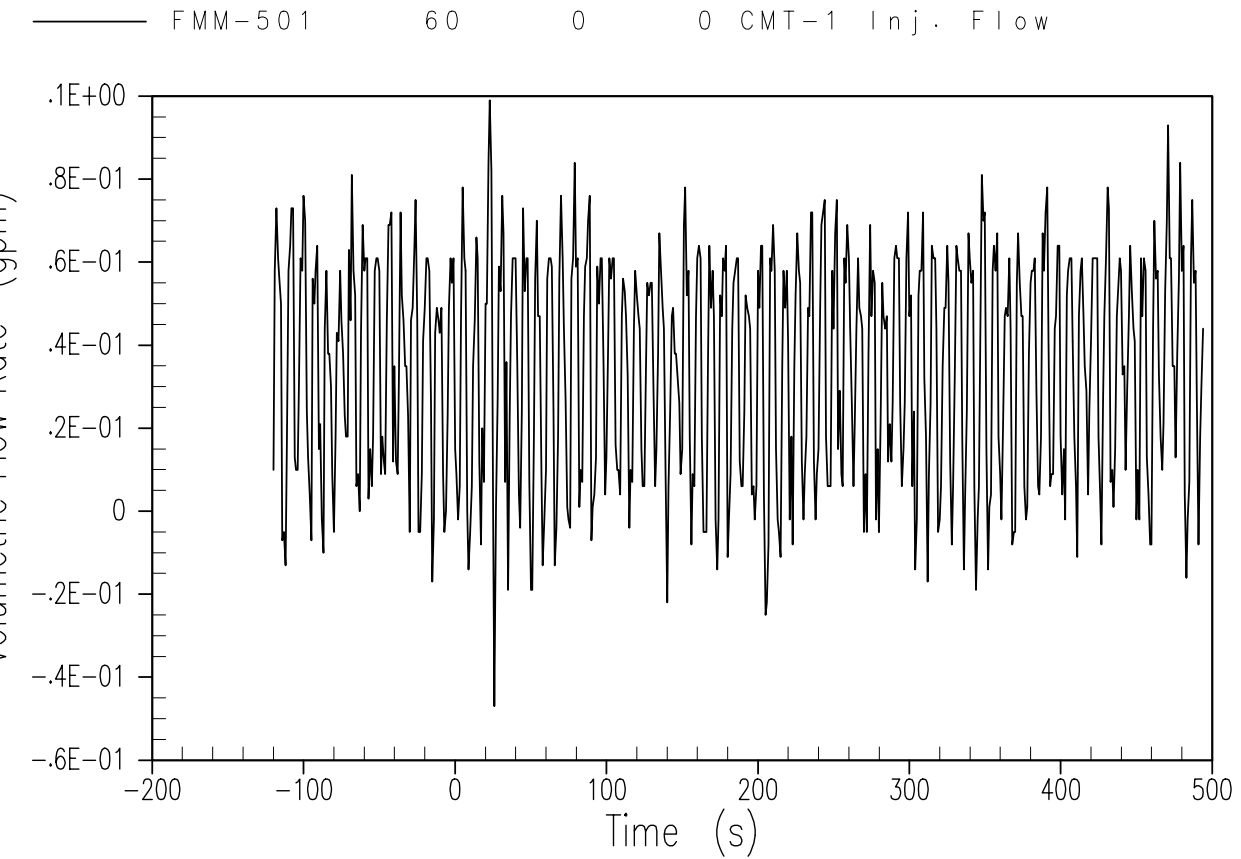

Figure A-29 Core Makeup Tank 1 Flow Rate 


\section{AP1000 Test Data Oregon State University Test TR-1 March 18, 2003 \\ FMM-504 $63 \quad 0 \quad 0$ CMT-2 Inj. FIow}

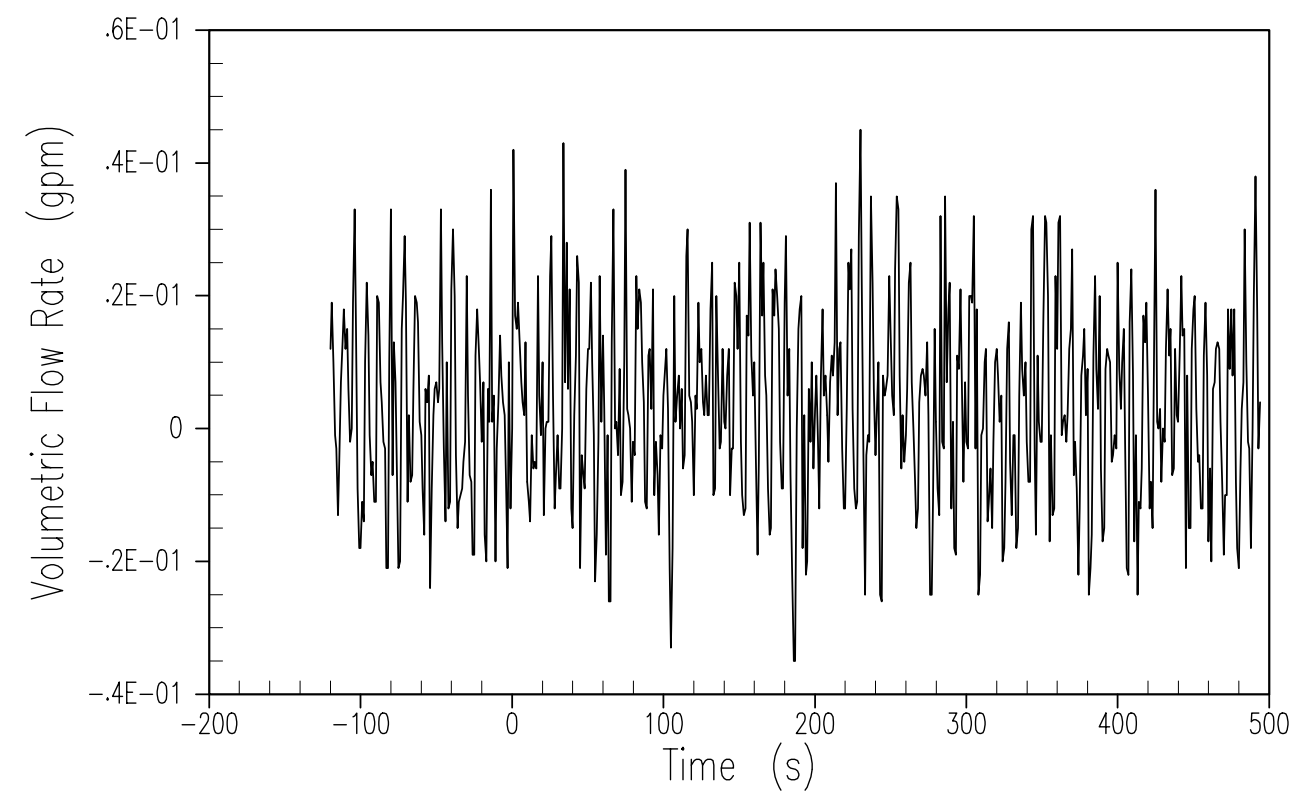

Figure A-30 Core Makeup Tank 2 Flow Rate 


\section{AP1000 Test Data Oregon State University Test TR-1 March 18, 2003

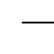
$T F-501$
429
0
o CMT-1 Long TC @ 0.30

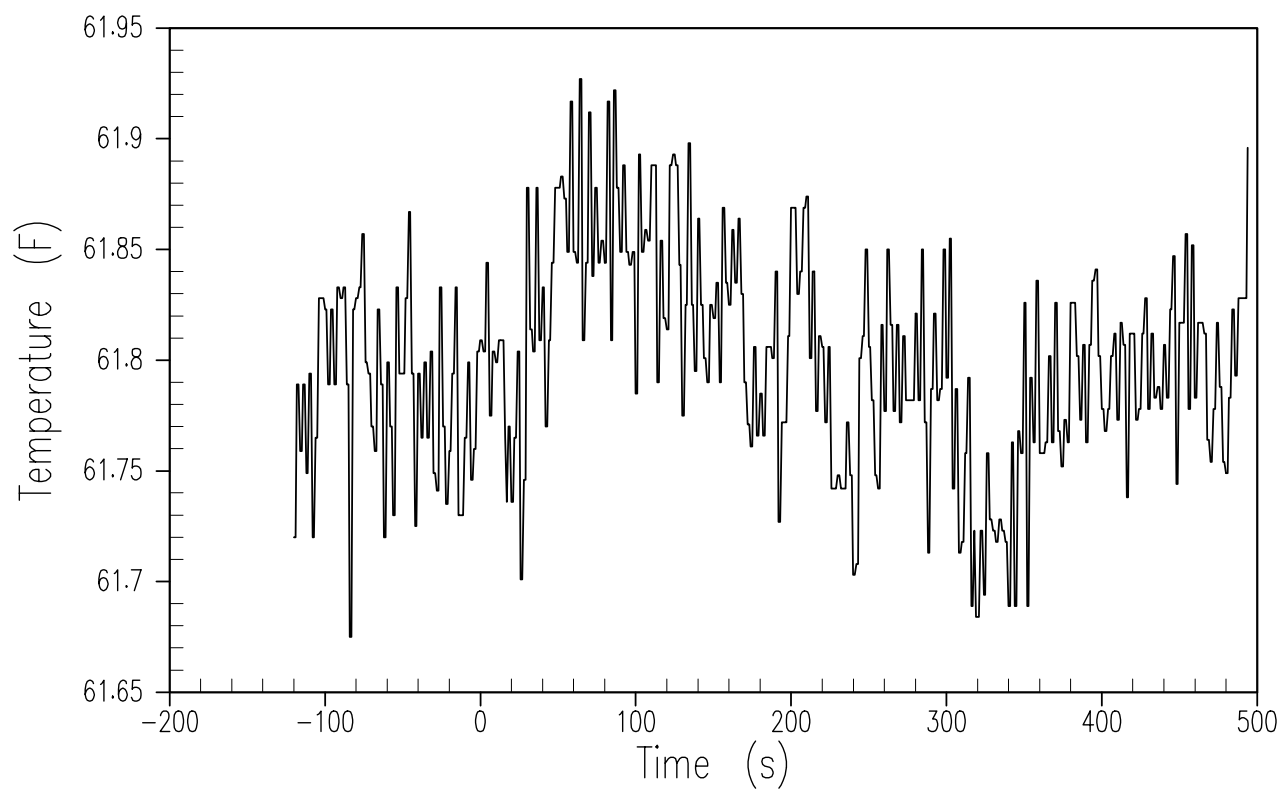

Figure A-31 Core Makeup Tank 1 Liquid Temperature - Bottom 

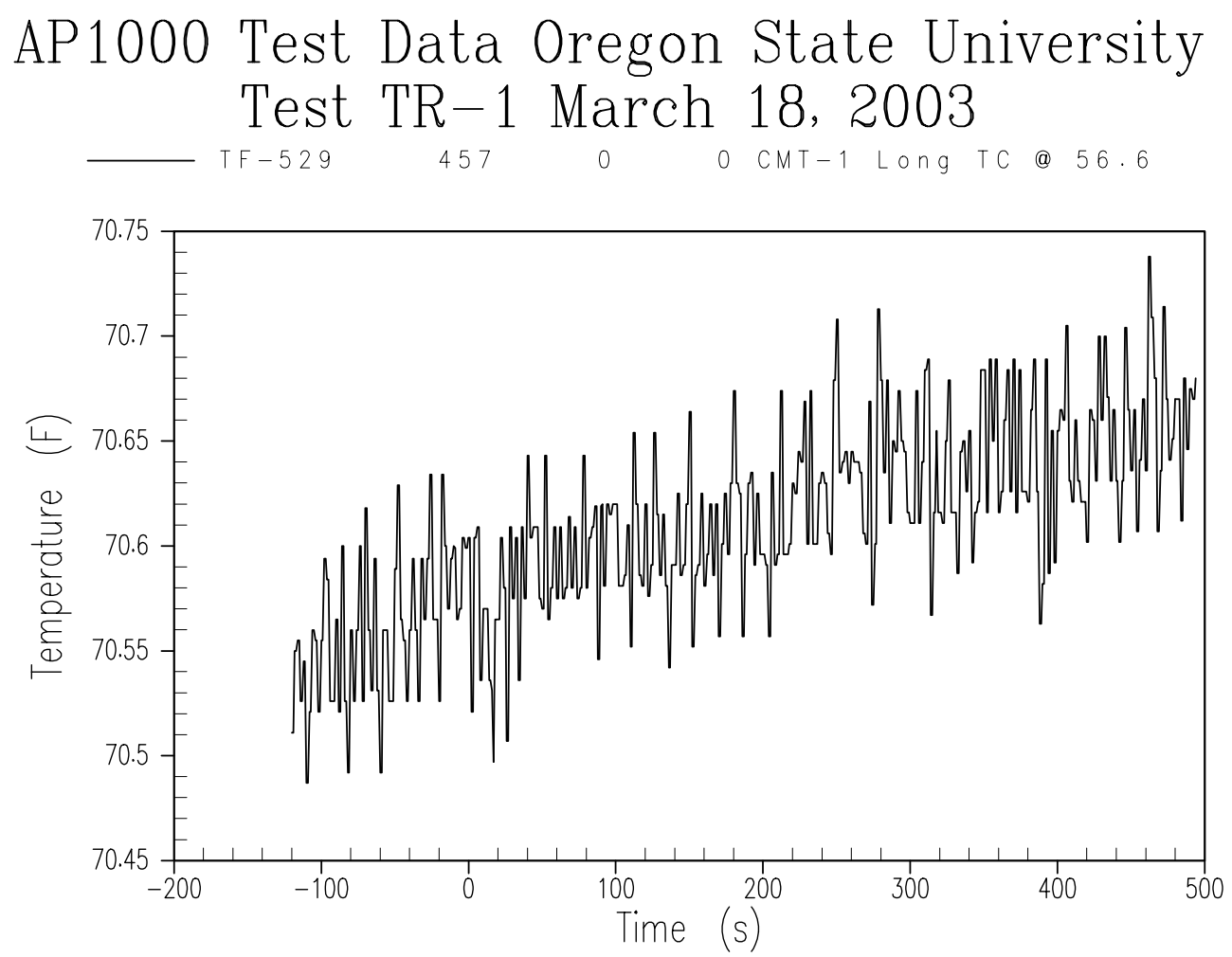

Figure A-32 Core Makeup Tank 1 Liquid Temperature - Top 
AP1000 Test Data Oregon State University Test TR-1 March 18, 2003

$-T F-$

432

0

o CMT-2 Long TC @ 0.30

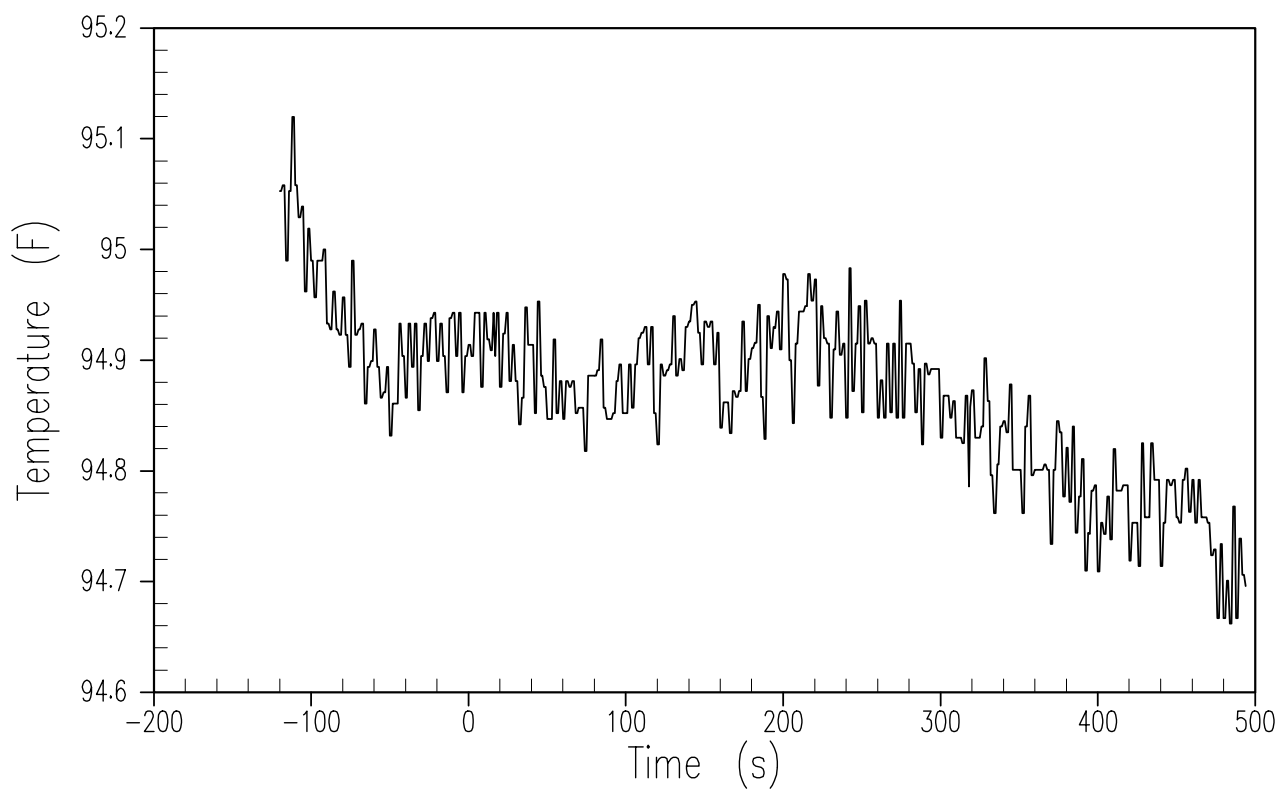

Figure A-33 Core Makeup Tank 2 Liquid Temperature - Bottom 
AP1000 Test Data Oregon State University Test TR-1 March 18, 2003

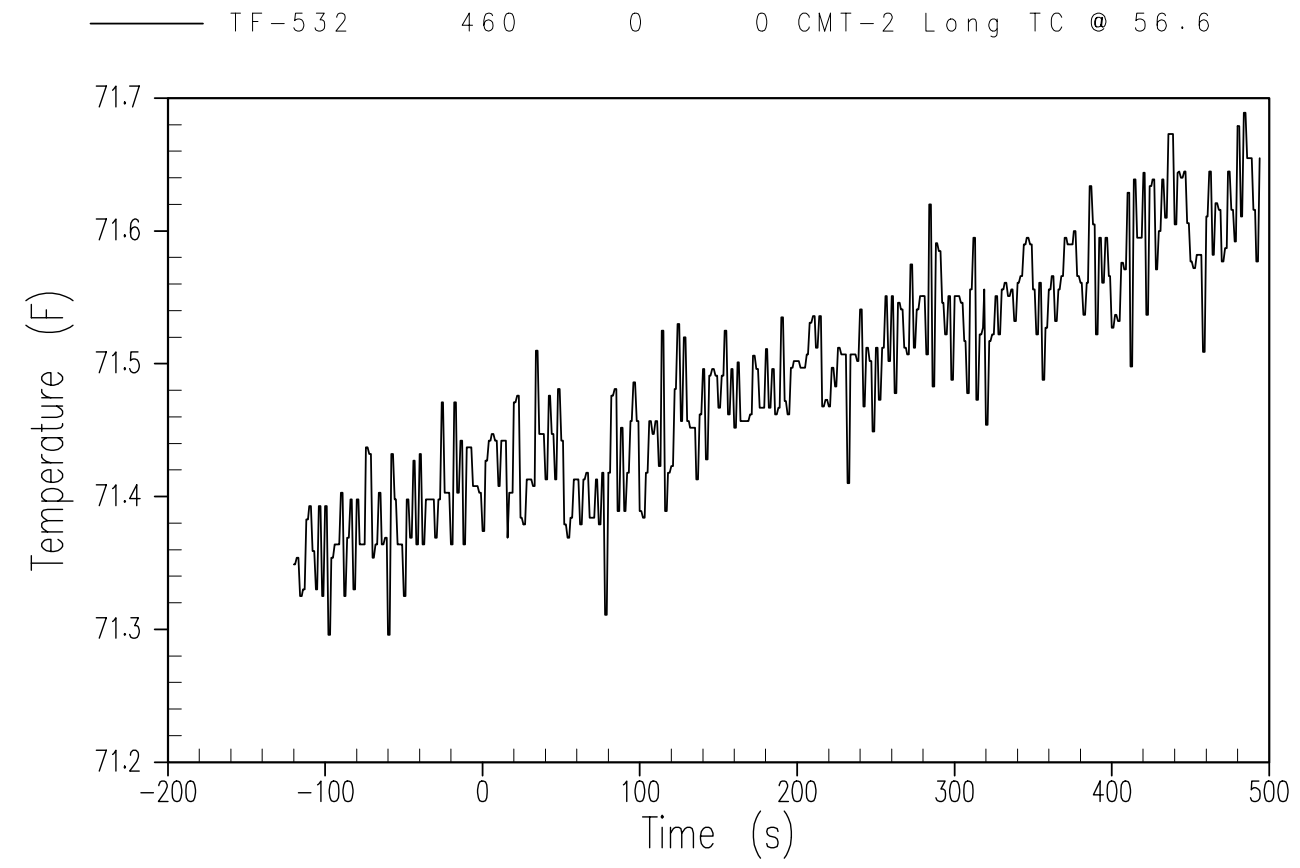

Figure A-34 Core Makeup Tank 2 Liquid Temperature - Top 

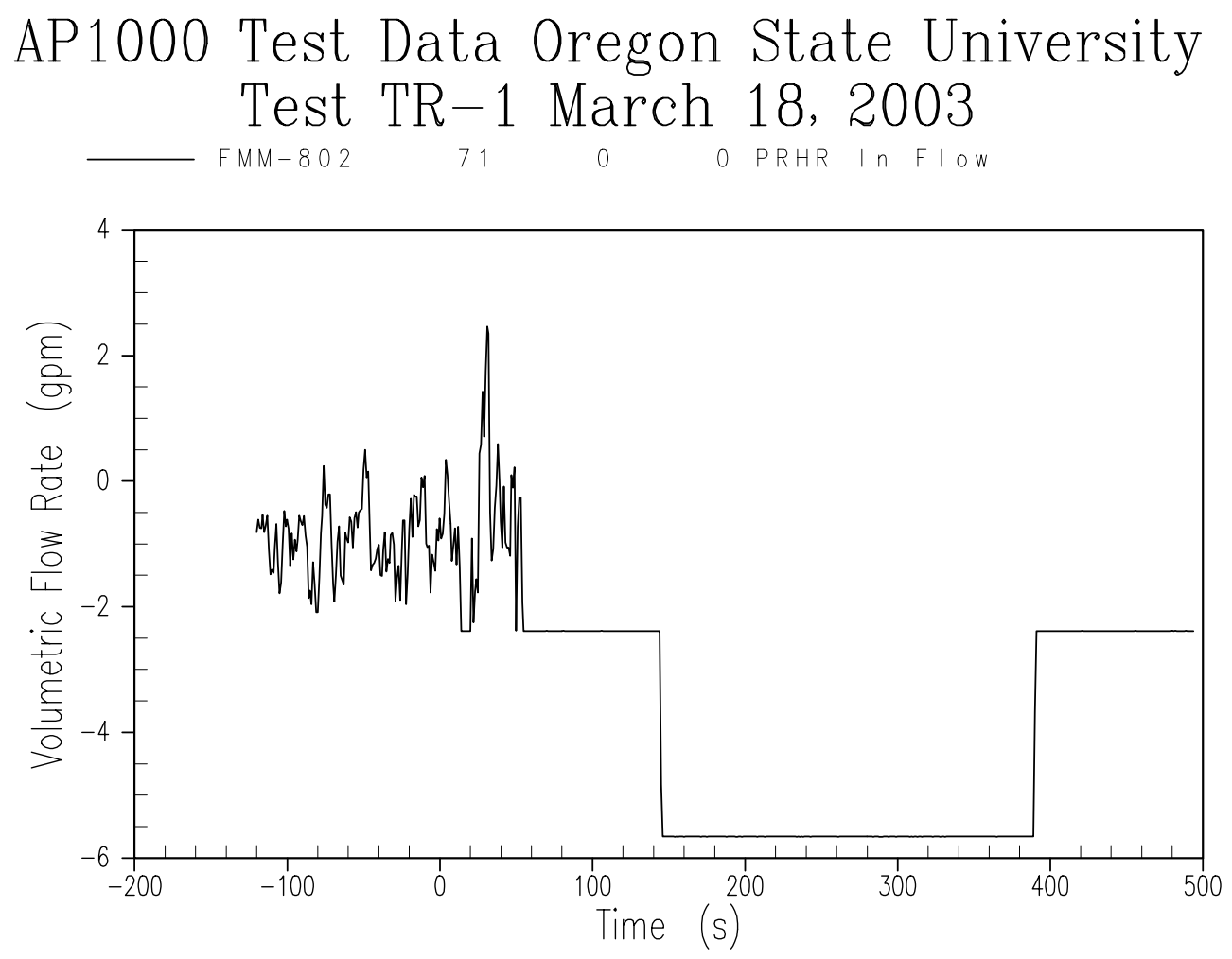

Figure A-35 Passive Residual Heat Removal Inlet Flow Rate 

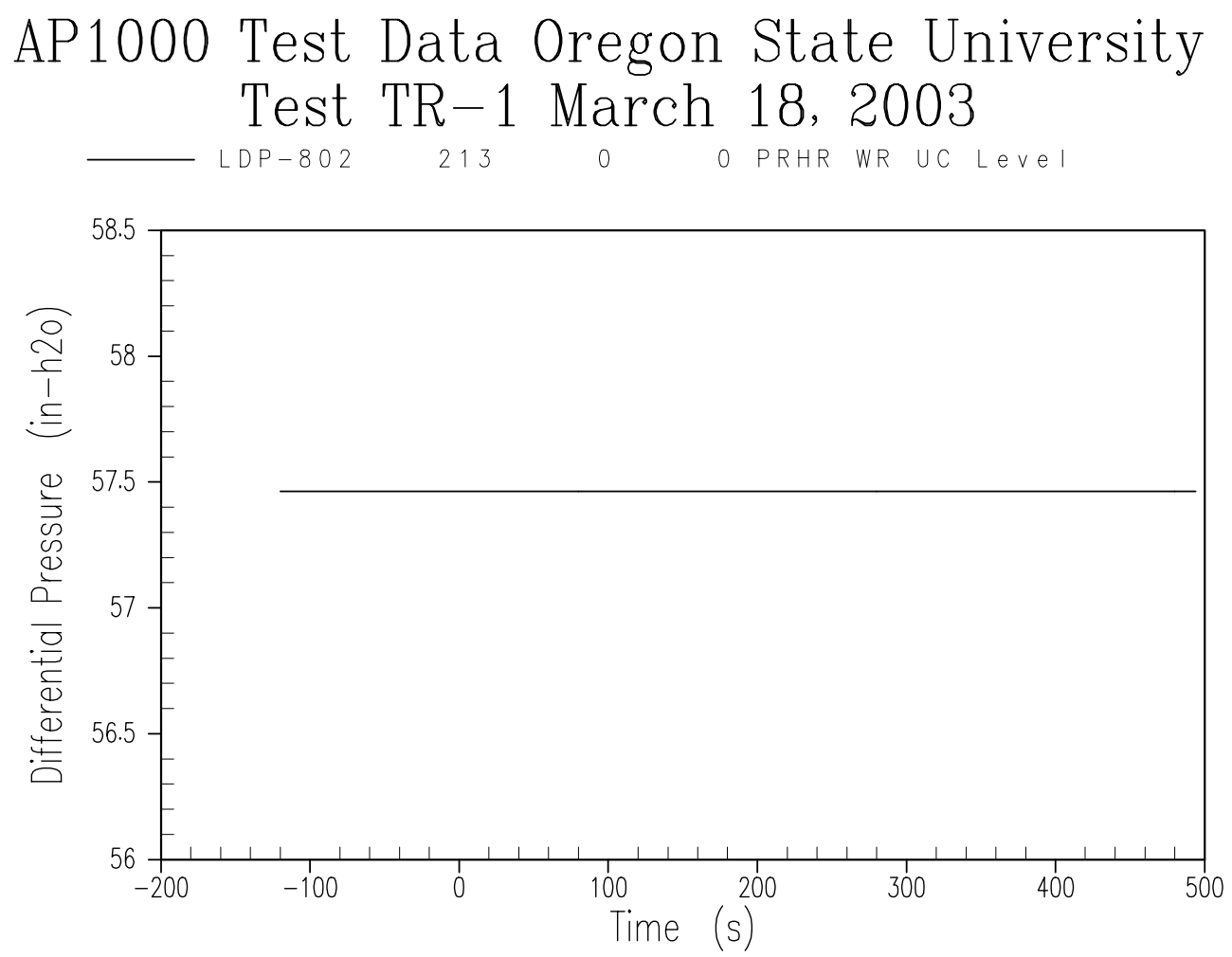

Figure A-36 Passive Residual Heat Removal Liquid Level 

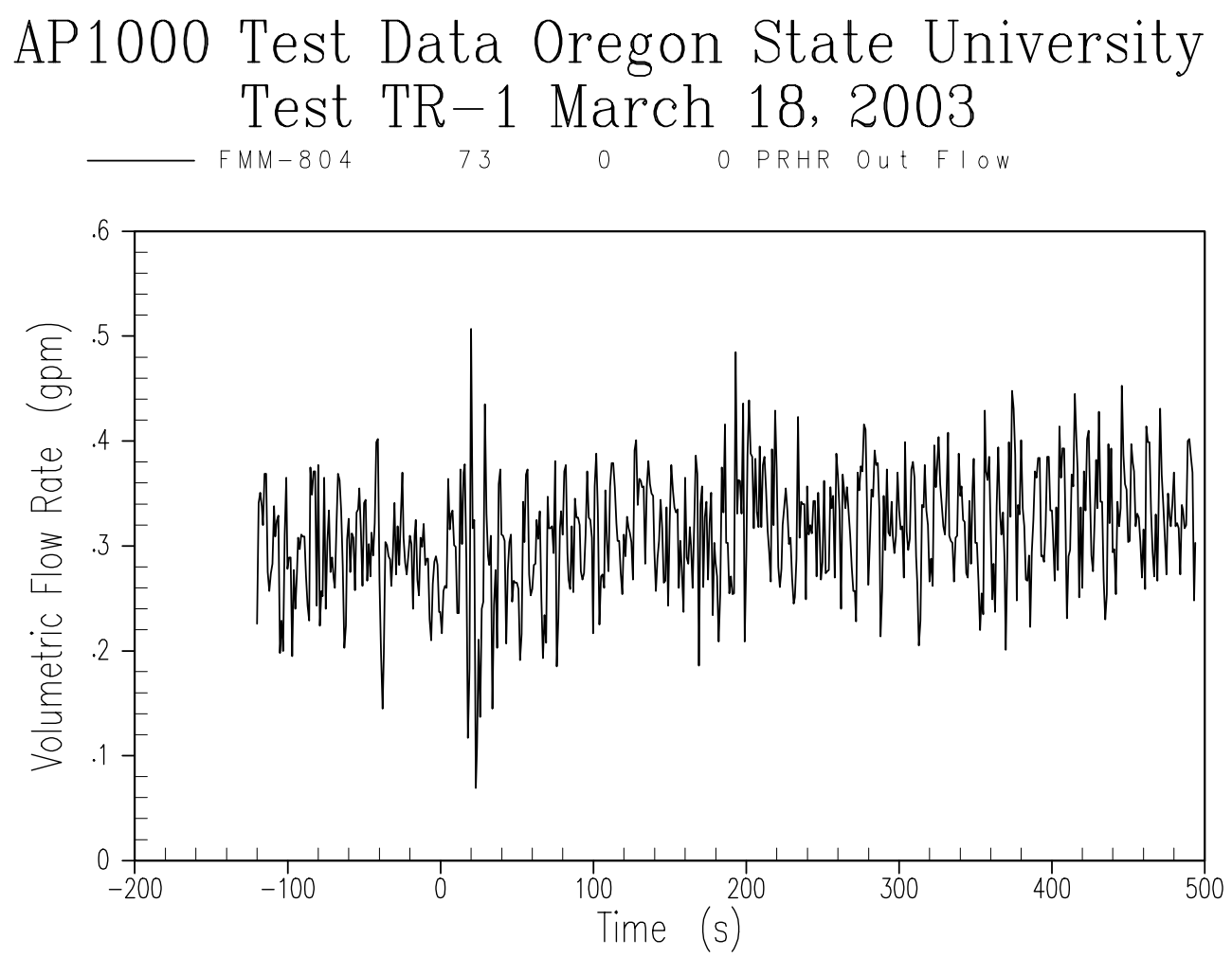

Figure A-37 Passive Residual Heat Removal Outlet Flow Rate 

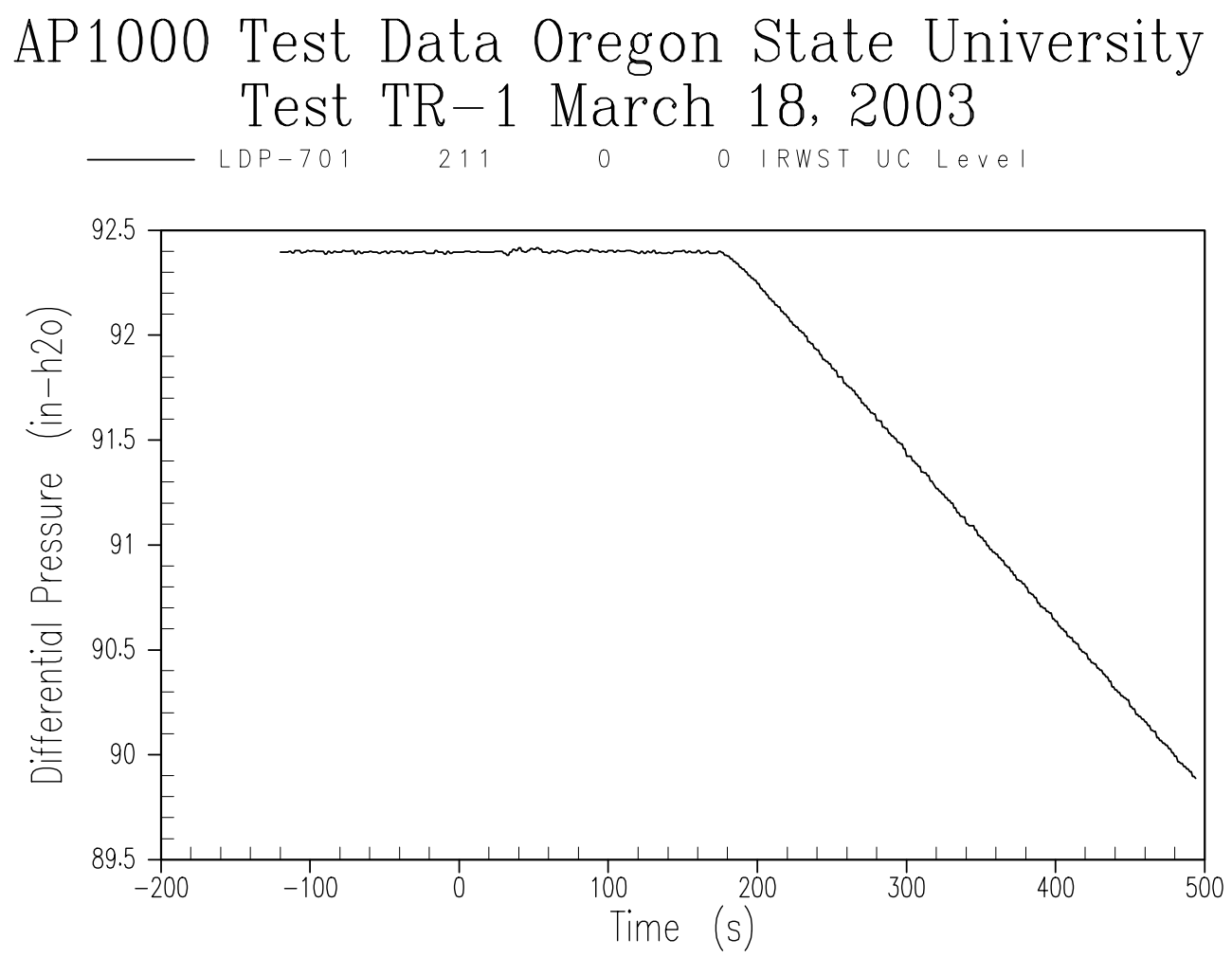

Figure A-38 IRWST Liquid Level 

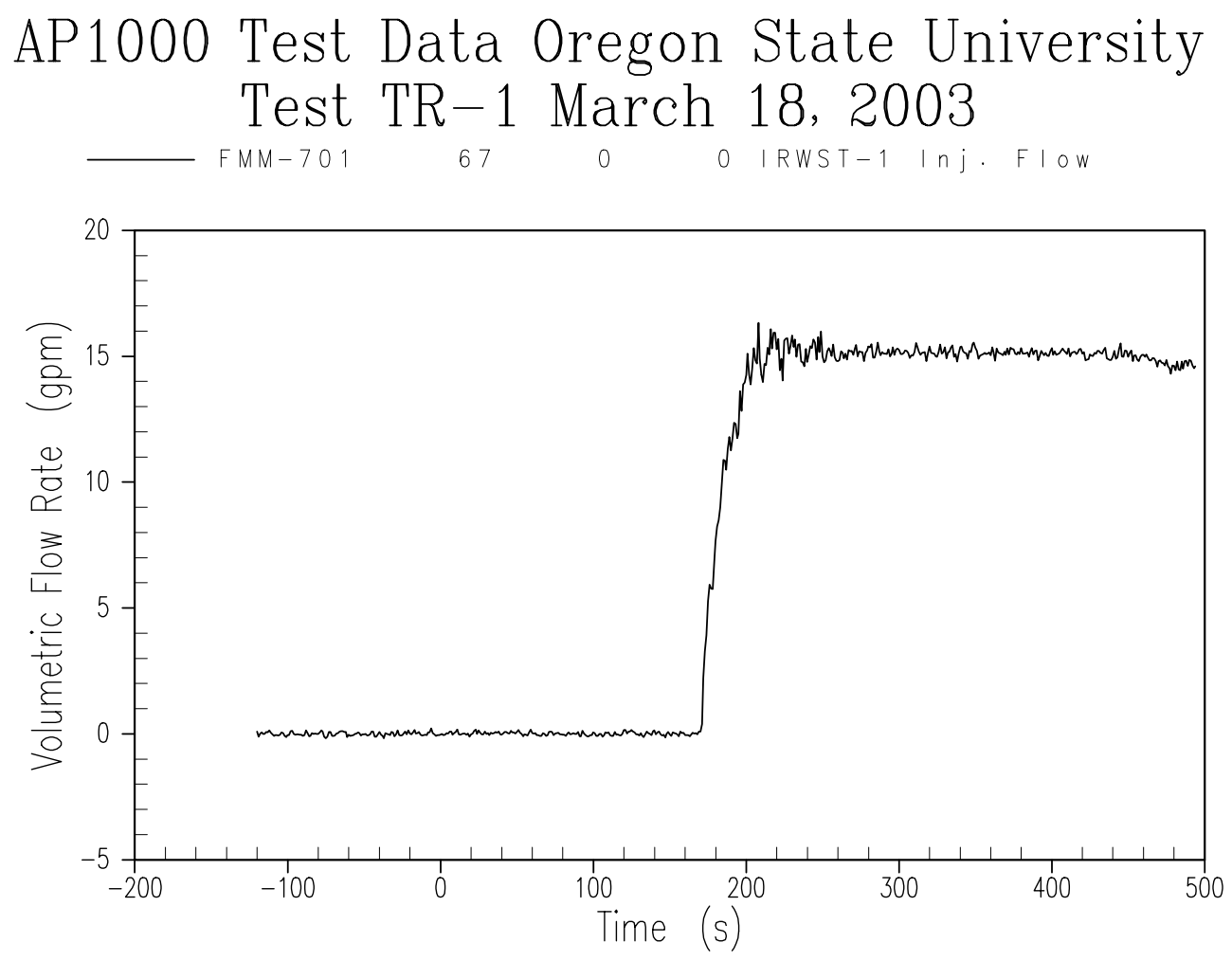

Figure A-39 IRWST Discharge Line 1 Flow Rate 

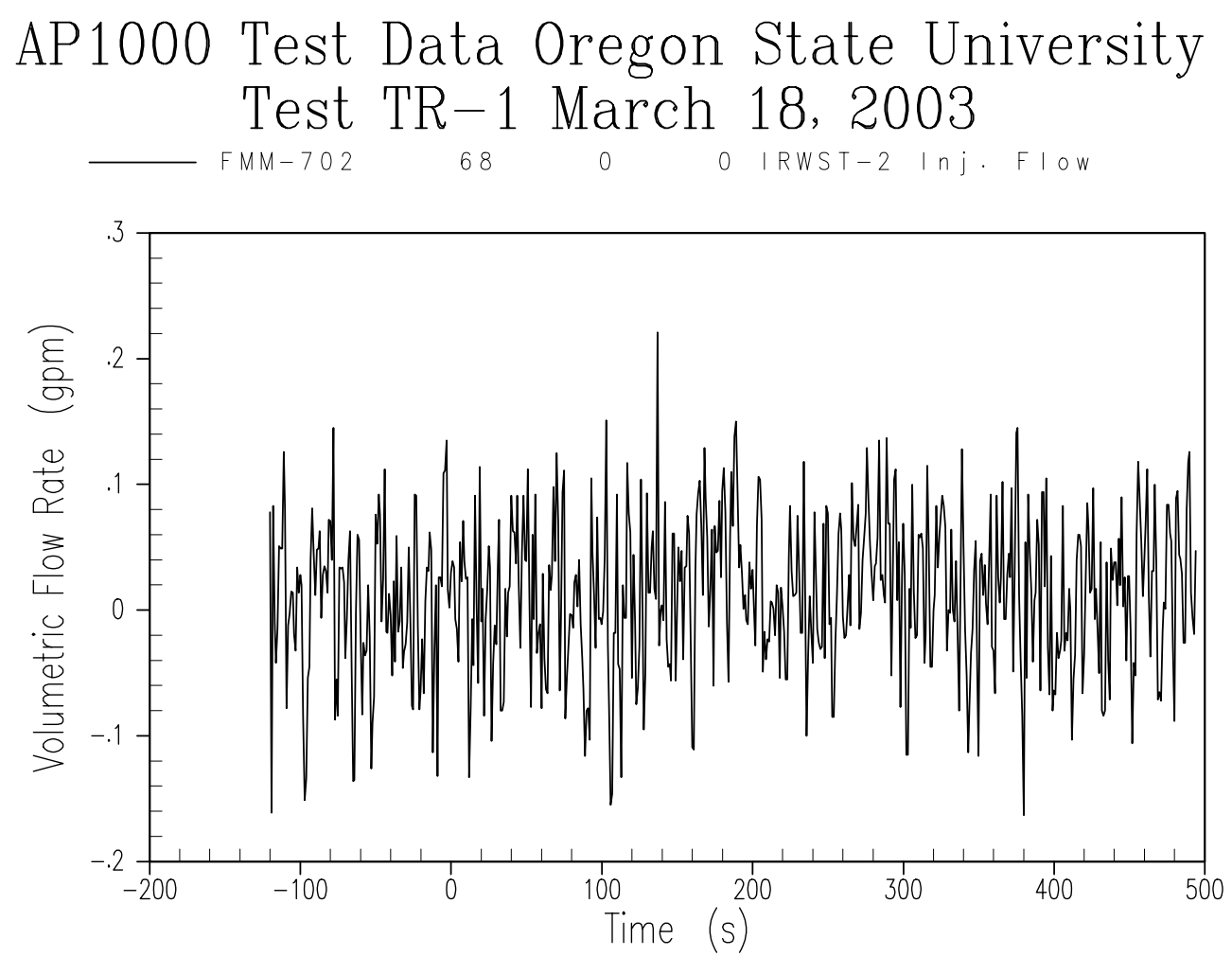

Figure A-40 IRWST Discharge Line 2 Flow Rate 


\section{AP1000 Test Data Oregon State University Test TR-1 March 18, 2003 \\ 508 \\ o IRWST/PRHR TC @ Bot.}

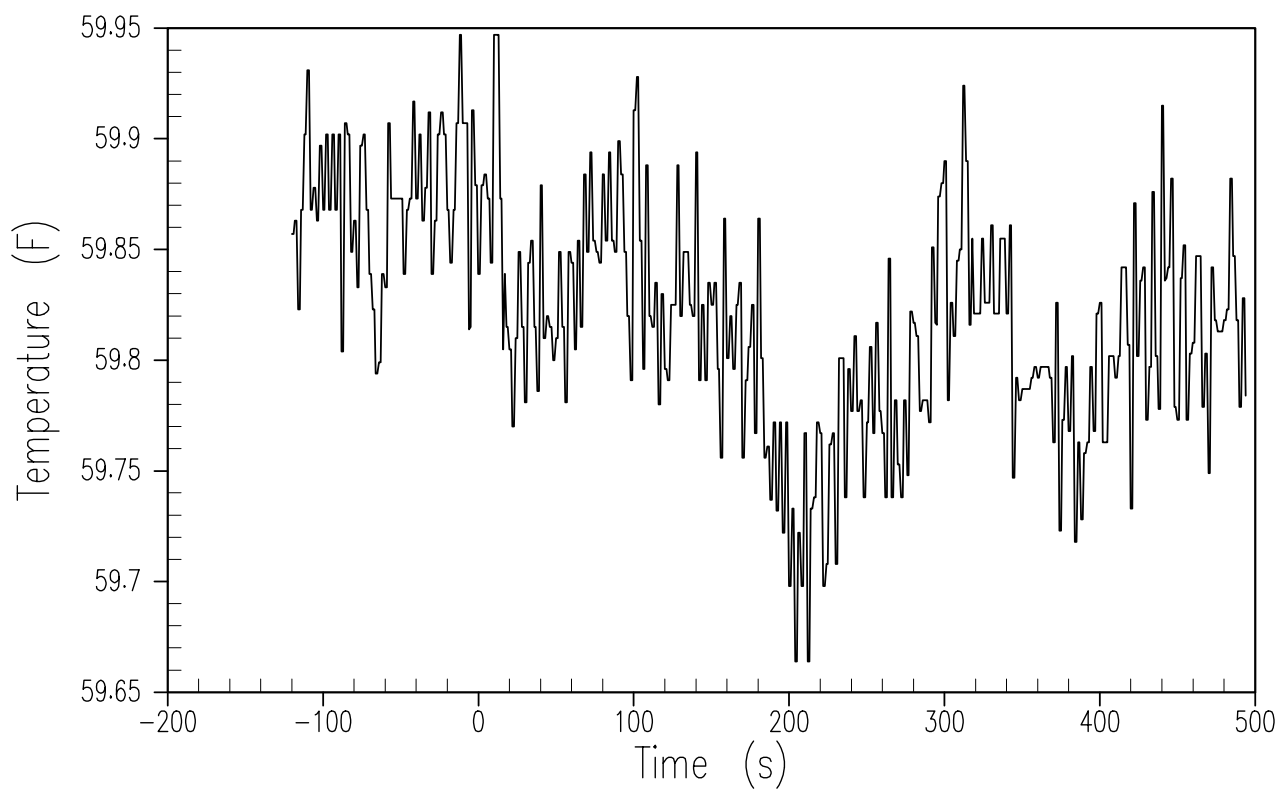

Figure A-41 IRWST Fluid Temperature - Bottom 


\section{AP1000 Test Data Oregon State University Test TR-1 March 18, 2003}

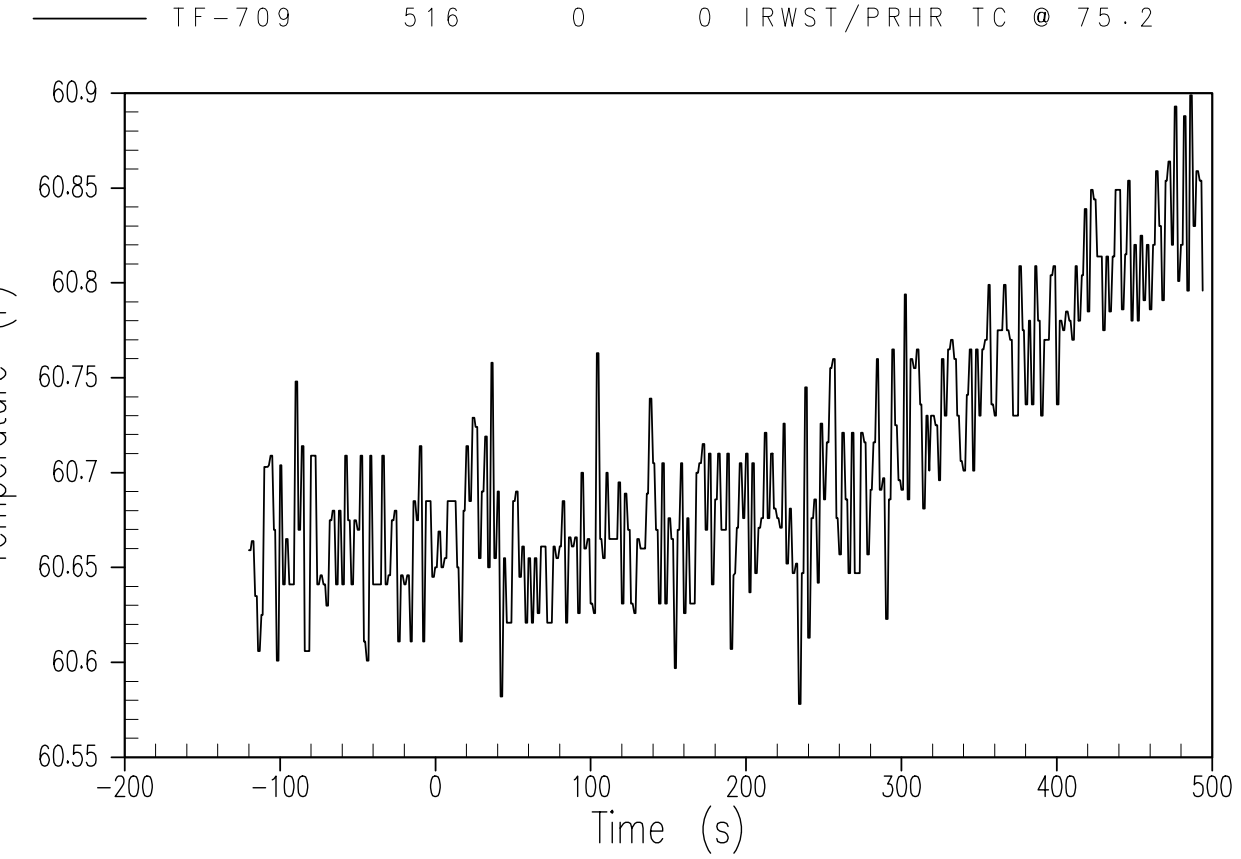

Figure A-42 IRWST Fluid Temperature - Top 

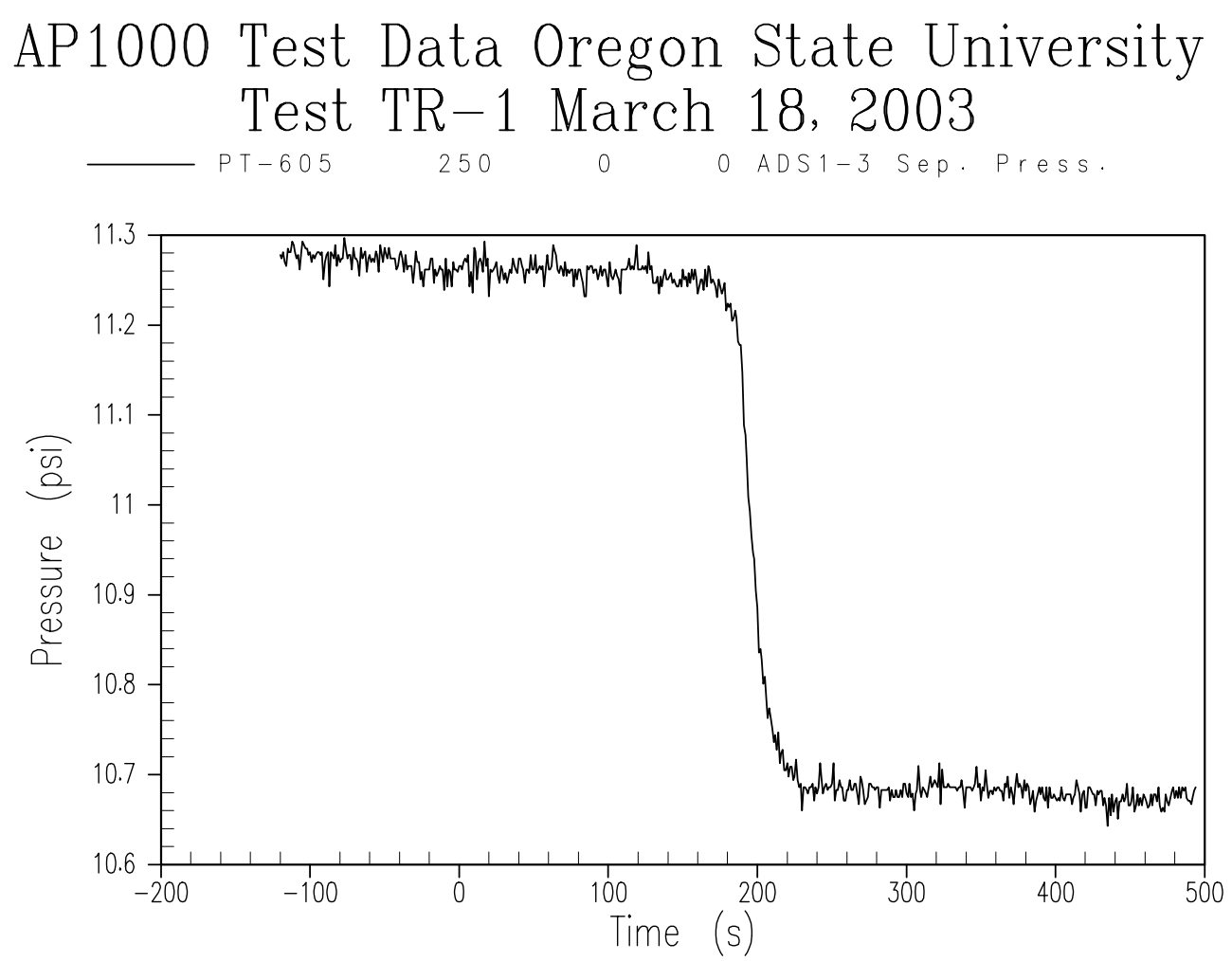

Figure A-43 ADS 1-3 Separator Pressure 

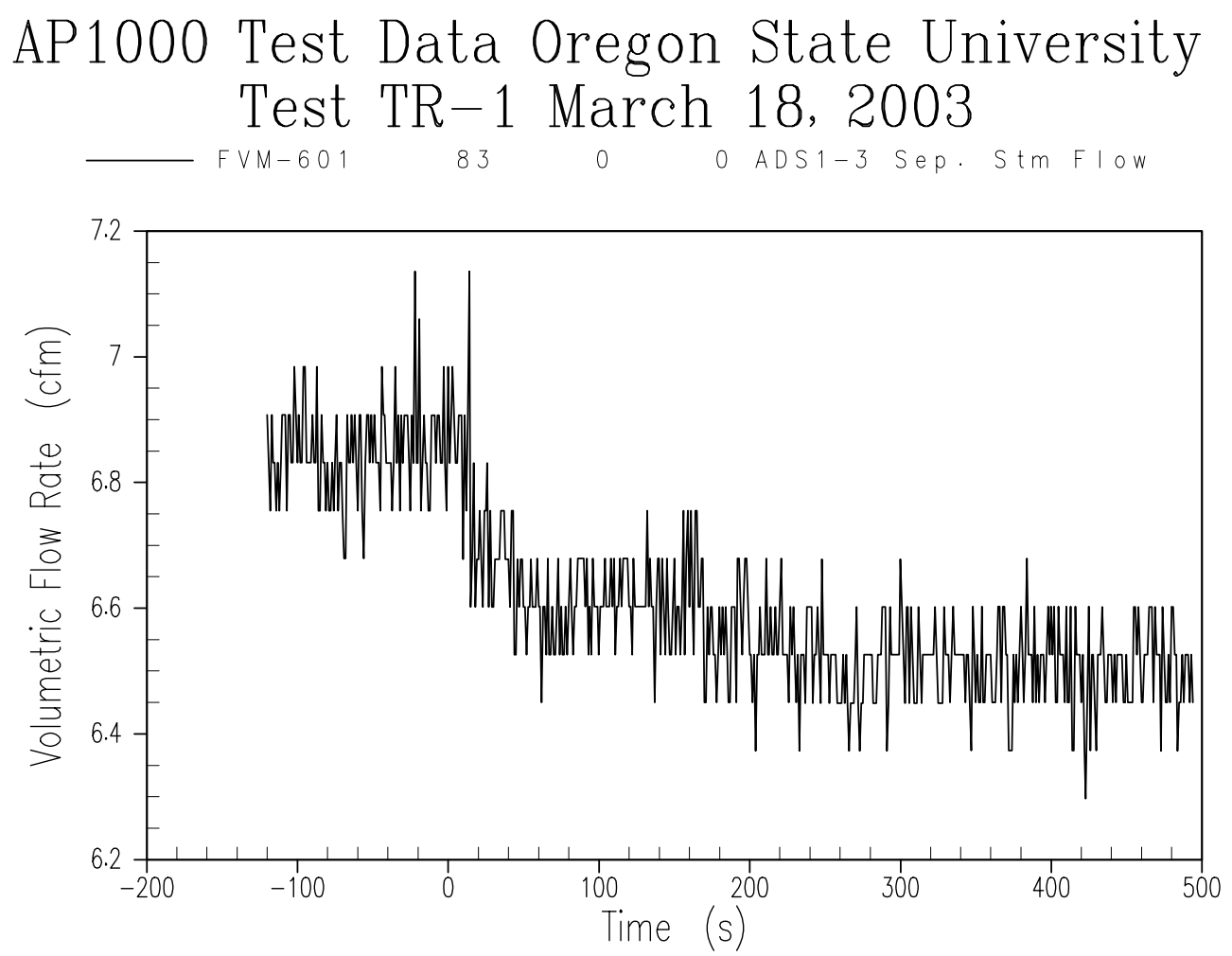

Figure A-44 ADS 1-3 Separator Steam Flow Rate 


\section{AP1000 Test Data Oregon State University Test TR-1 March 18, 2003}

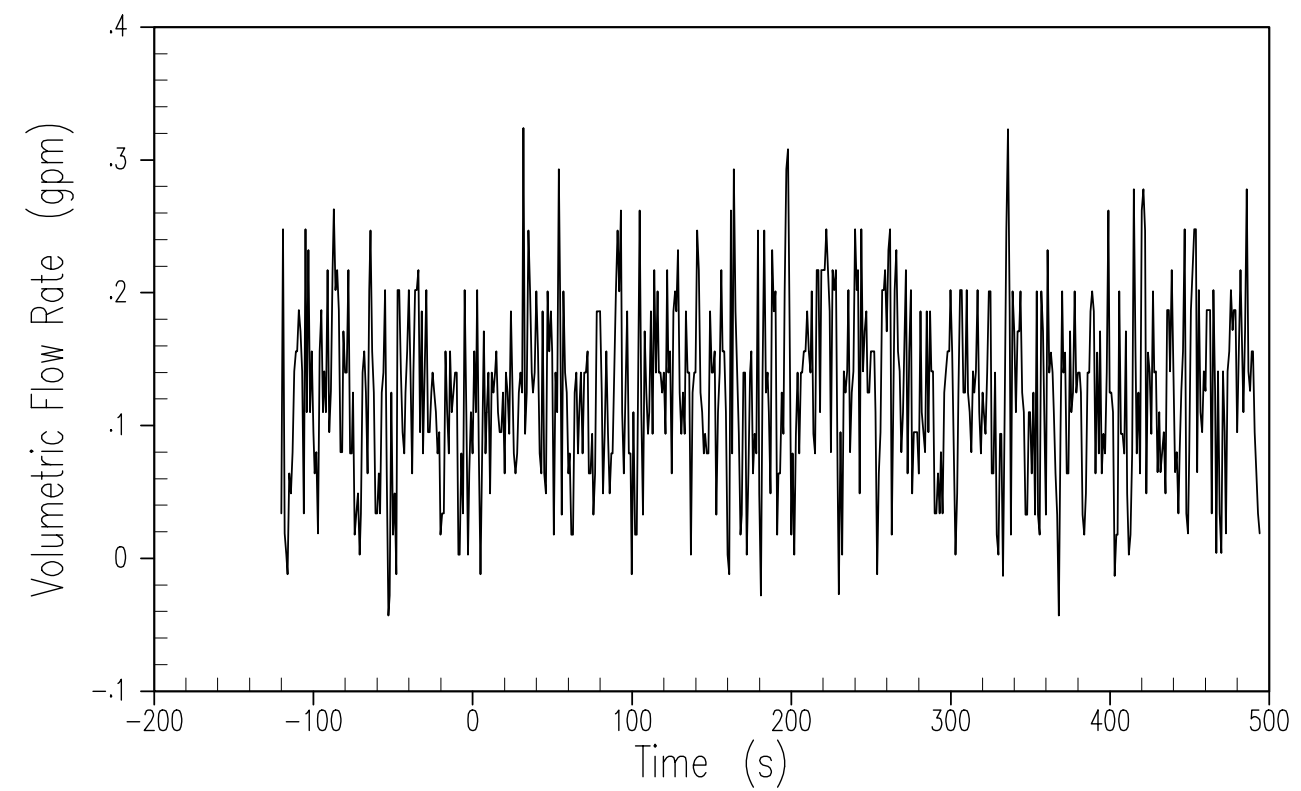

Figure A-45 ADS 1-3 Separator Liquid Flow Rate 

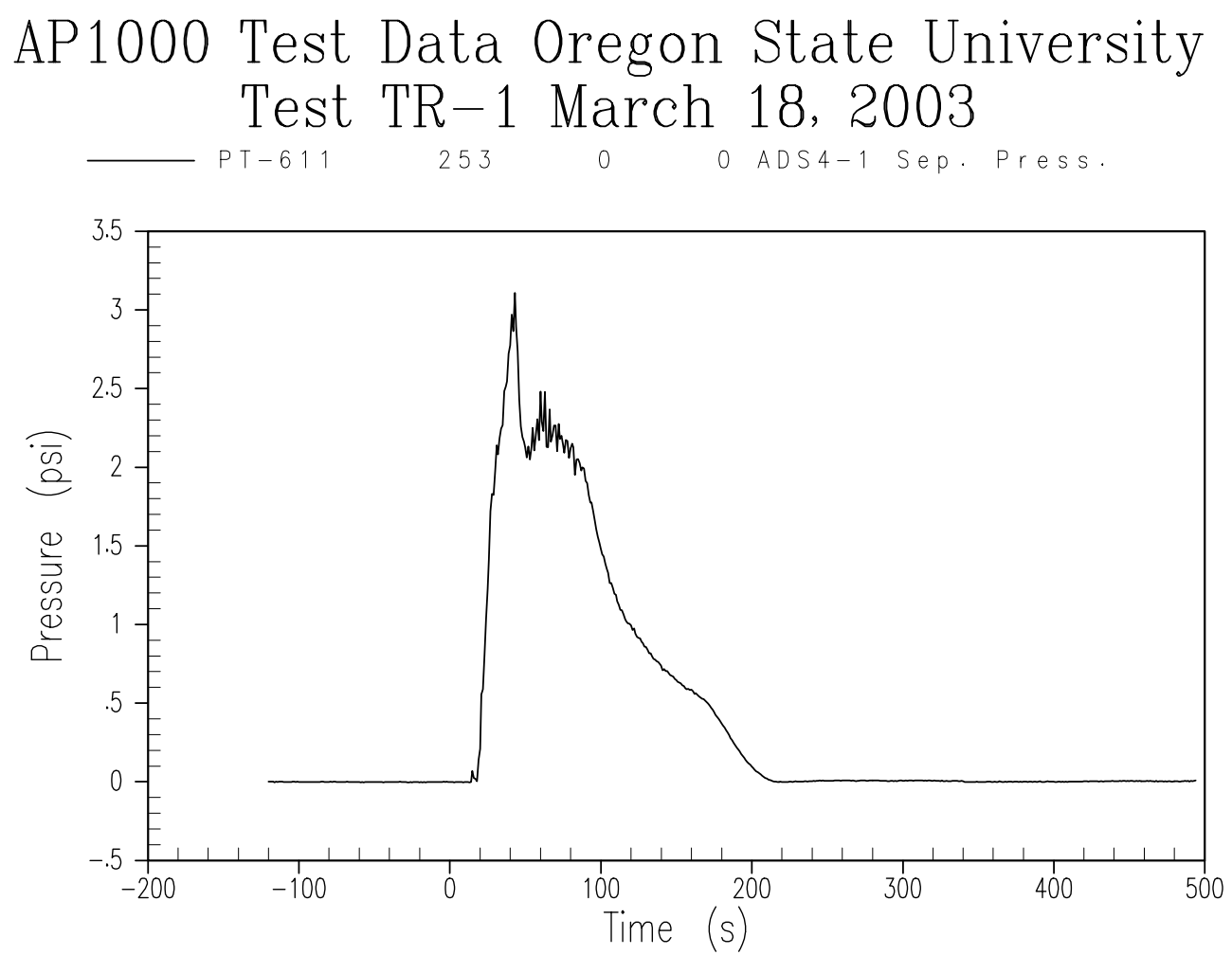

Figure A-46 ADS 4-1 Separator Pressure 

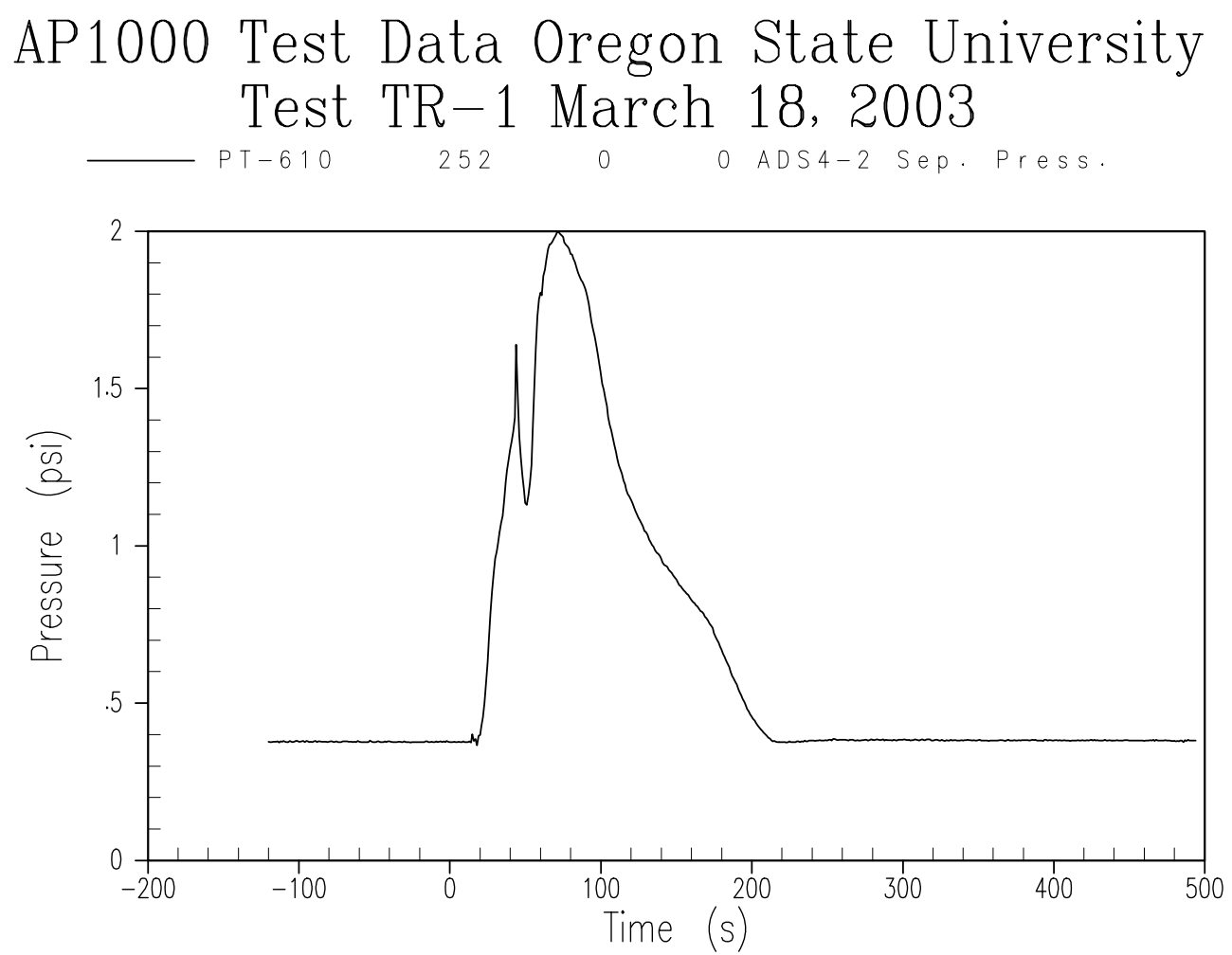

Figure A-47 ADS 4-2 Separator Pressure 

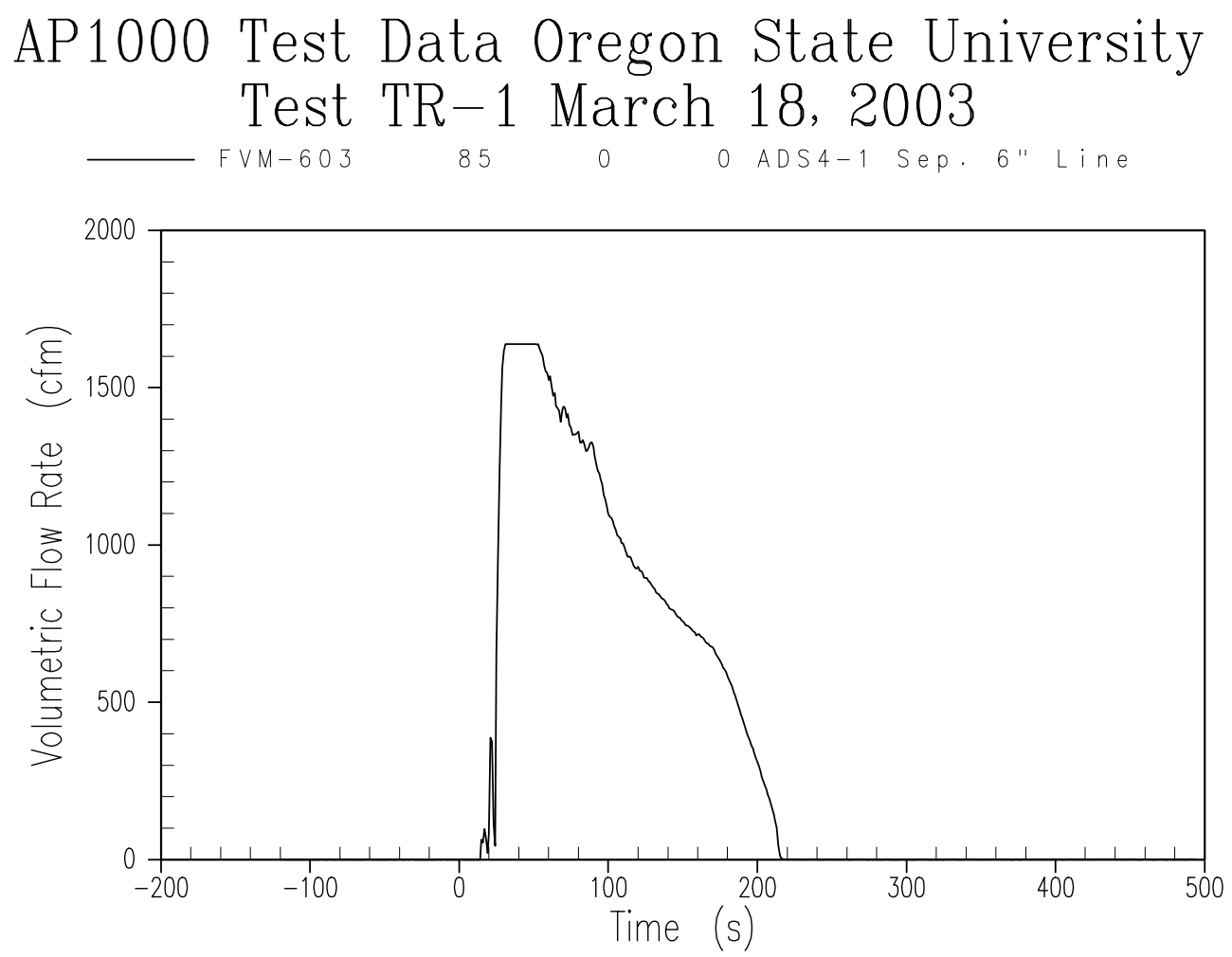

Figure A-48 ADS 4-1 Separator Steam Flow Rate 

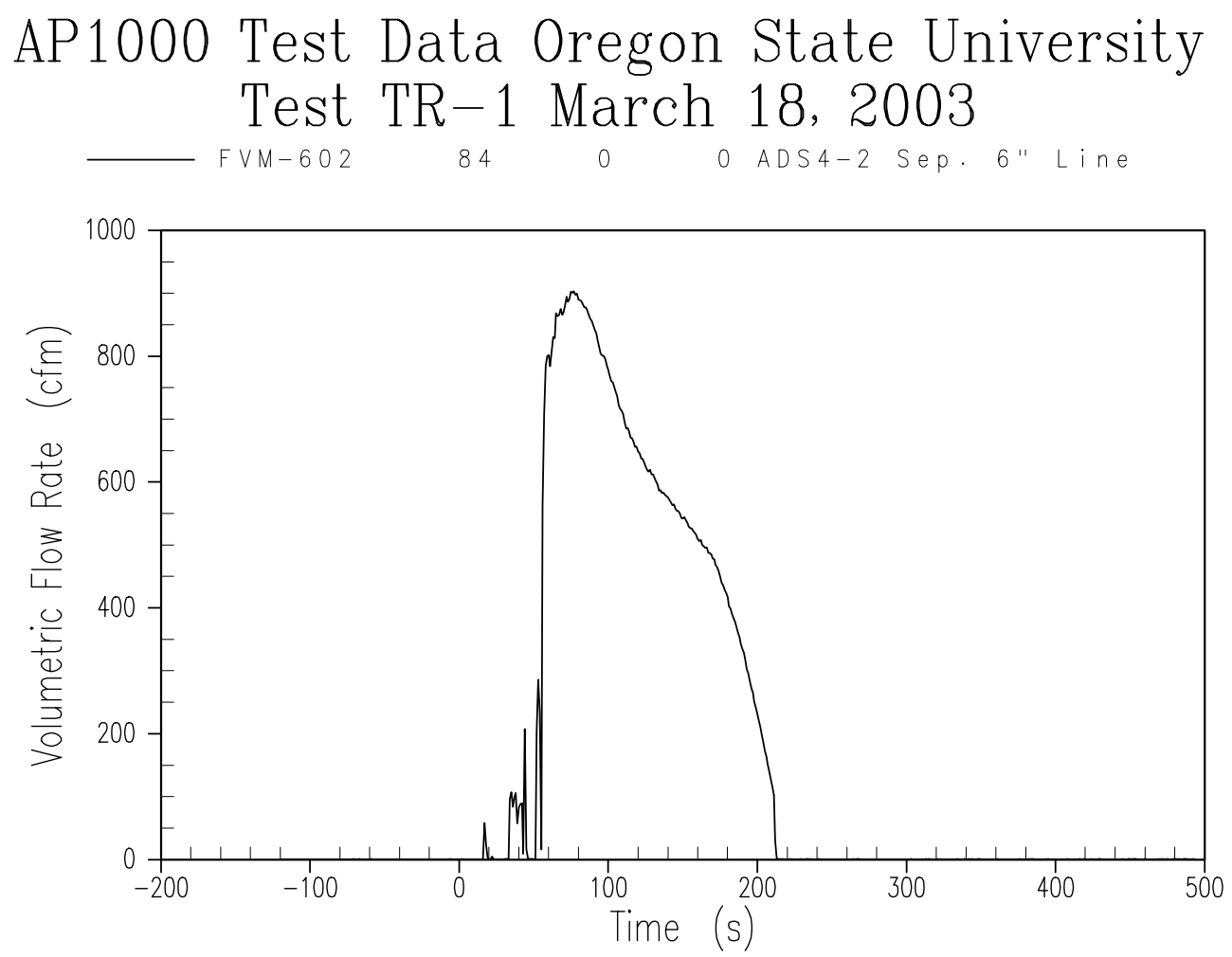

Figure A-49 ADS 4-2 Separator Steam Flow Rate 

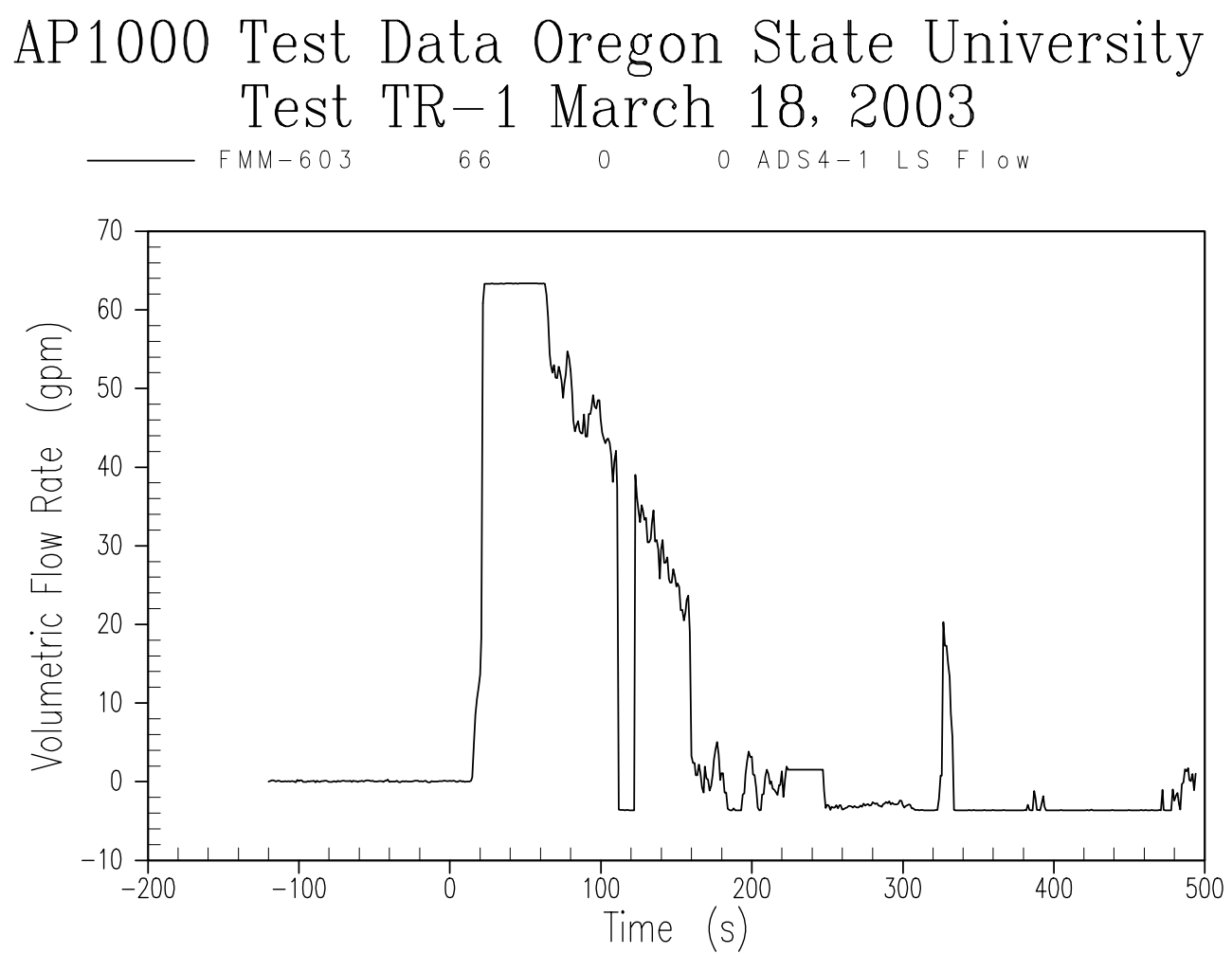

Figure A-50 ADS 4-1 Separator Liquid Flow Rate 

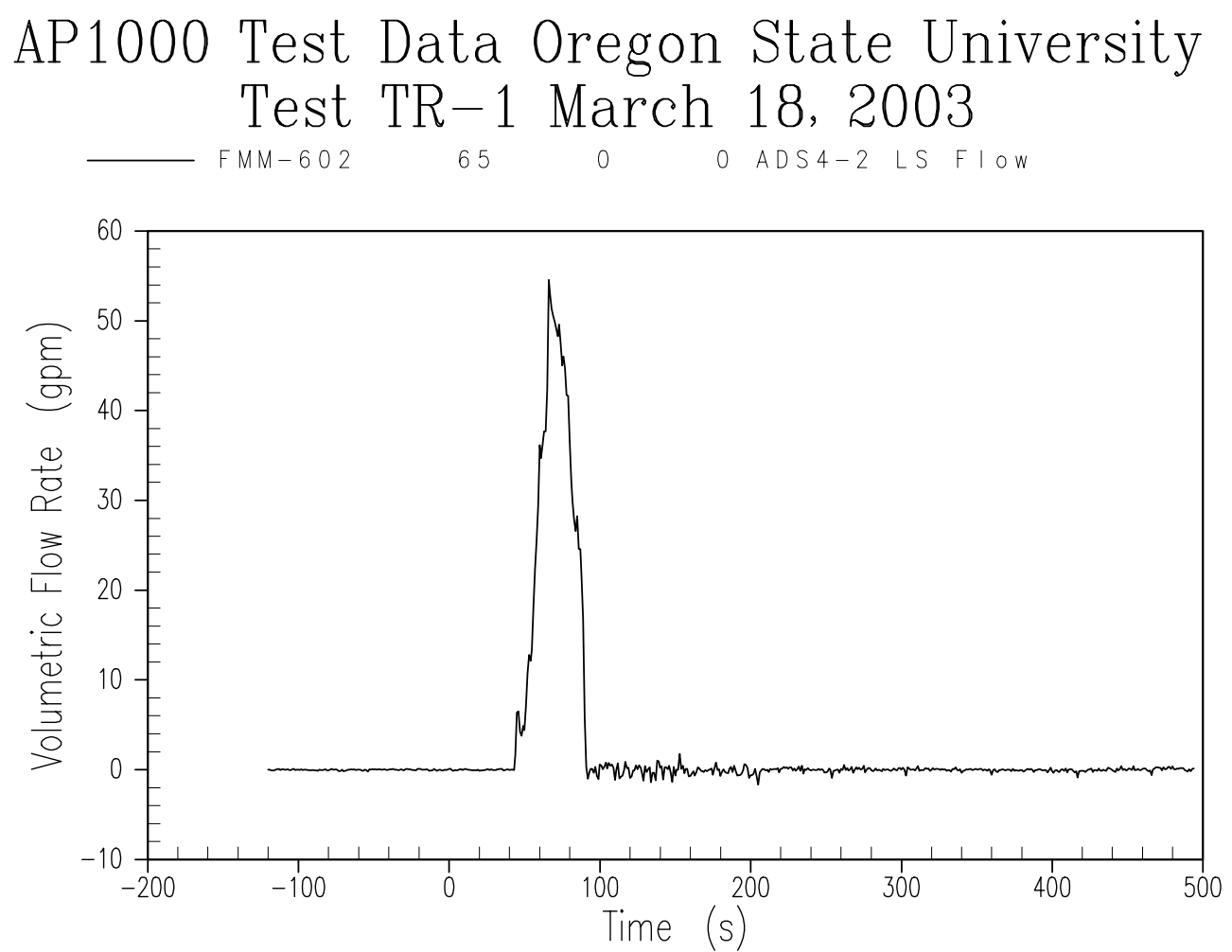

Figure A-51 ADS 4-2 Separator Liquid Flow Rate 

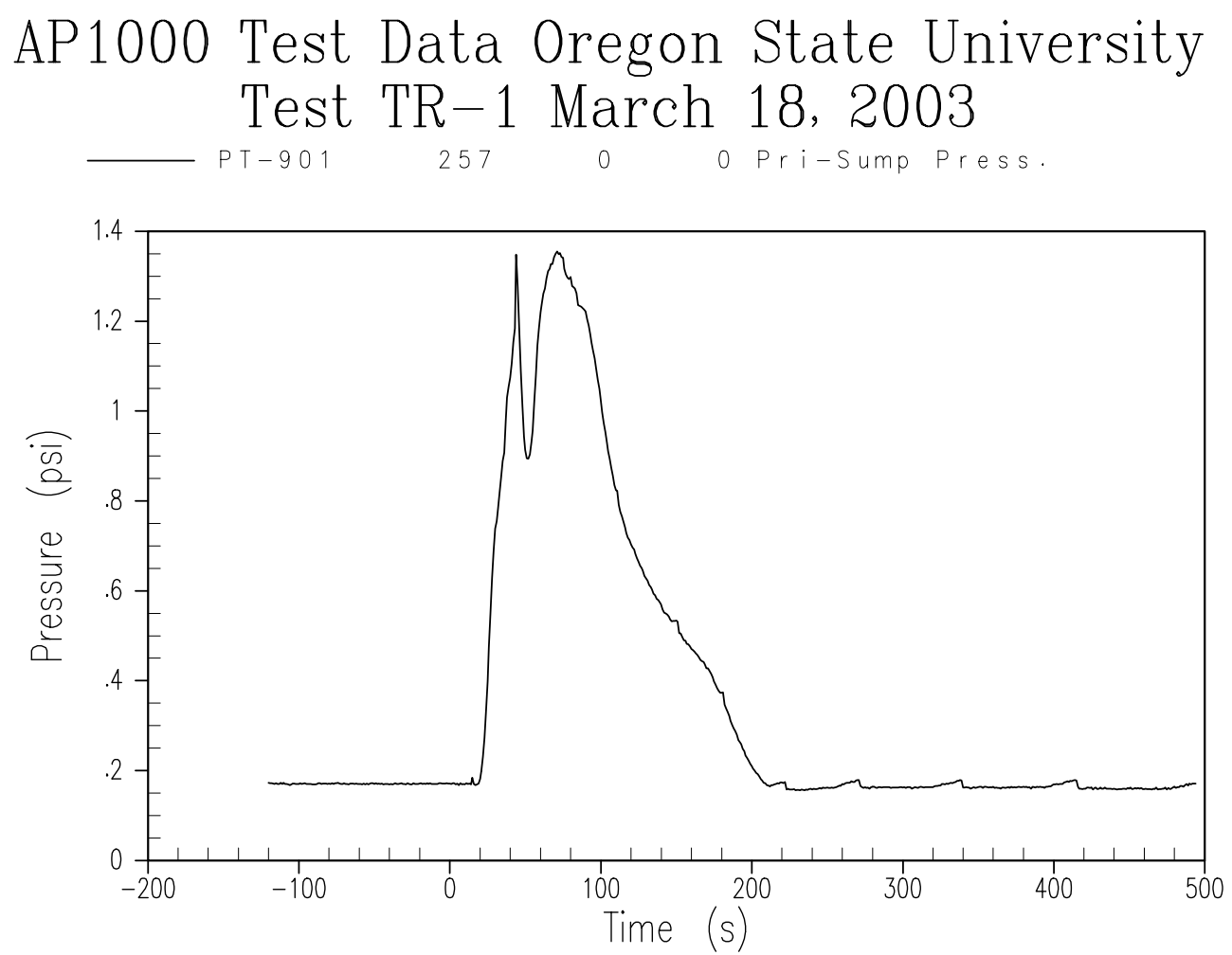

Figure A-52 Primary Sump Pressure 

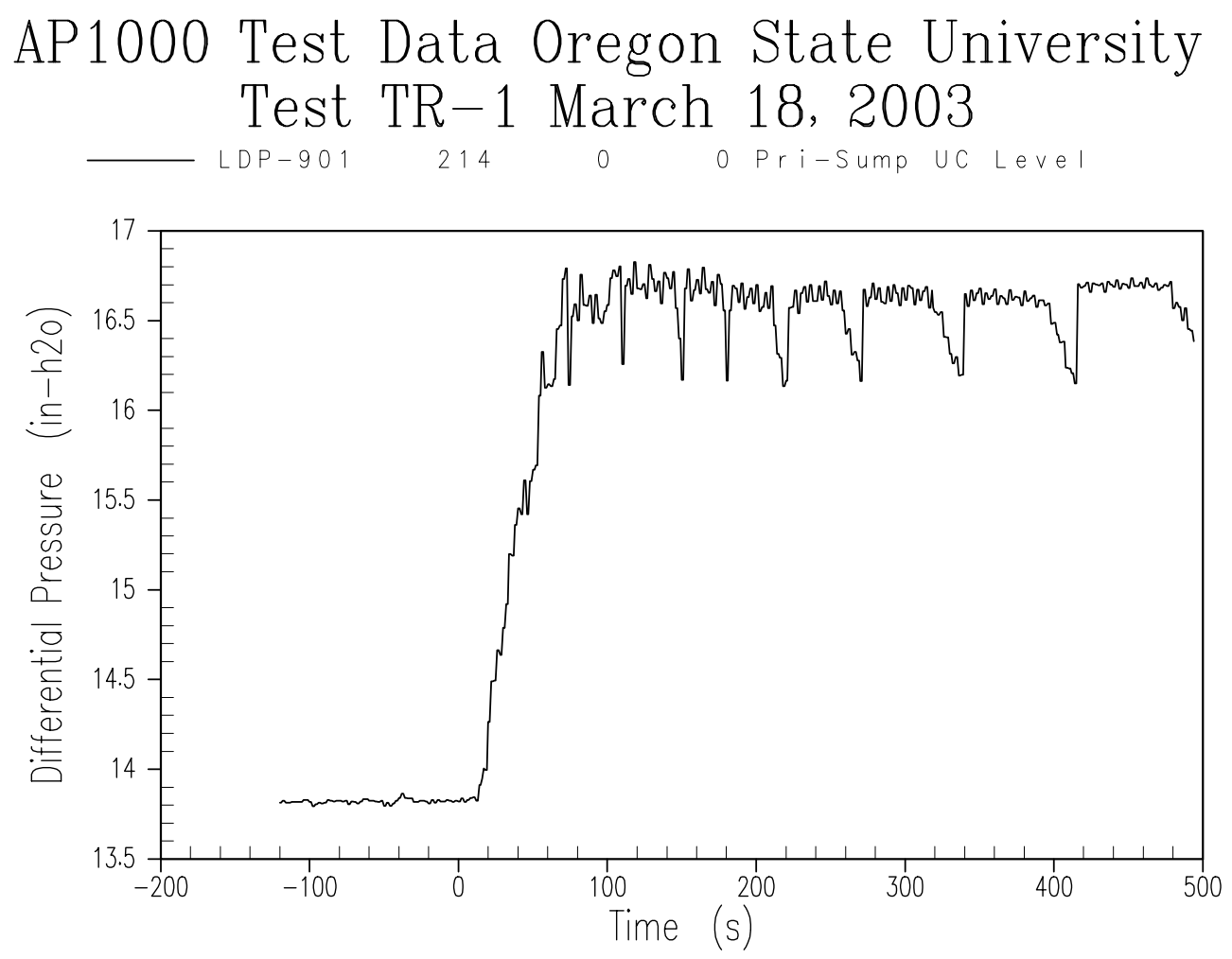

Figure A-53 Primary Sump Liquid Level 


\section{AP1000 Test Data Oregon State University Test TR-1 March 18, 2003}

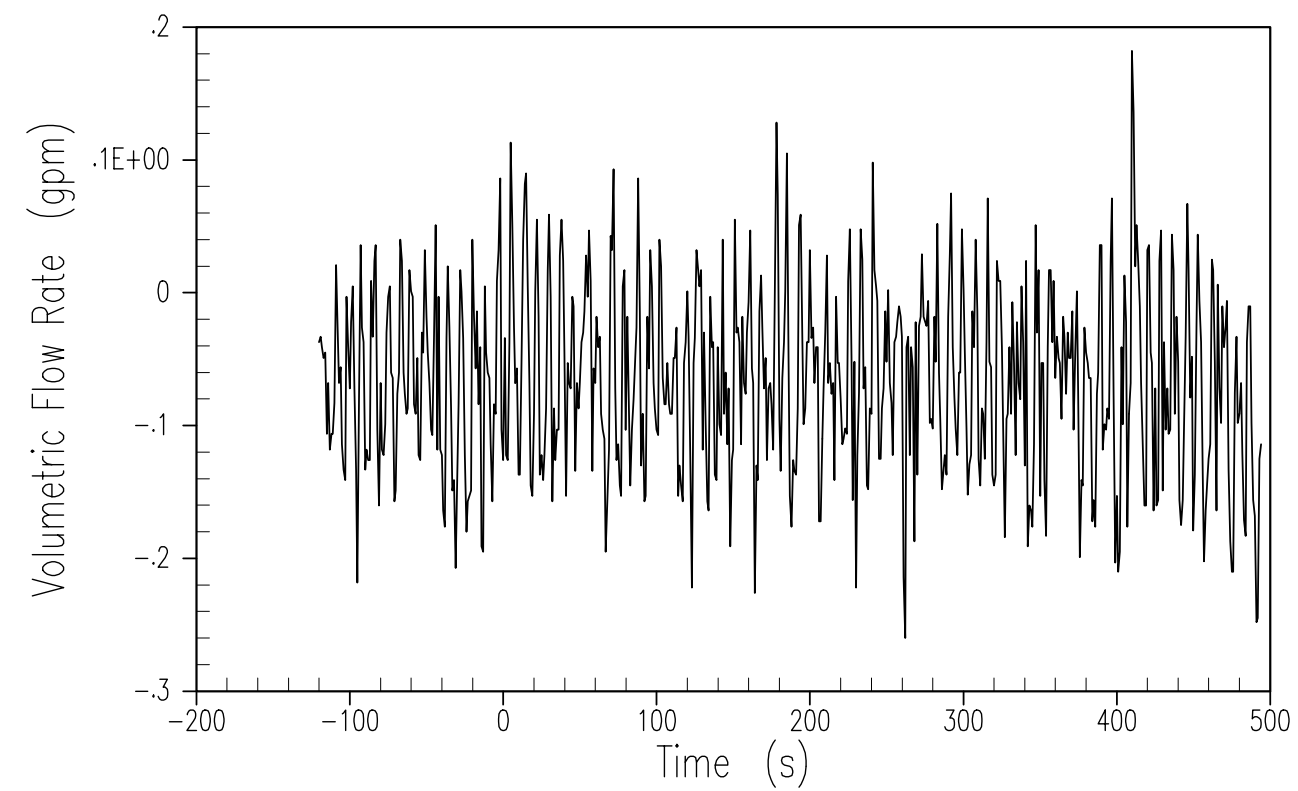

Figure A-54 Primary Sump 1 Injection Flow Rate 


\section{AP1000 Test Data Oregon State University Test TR-1 March 18, 2003}

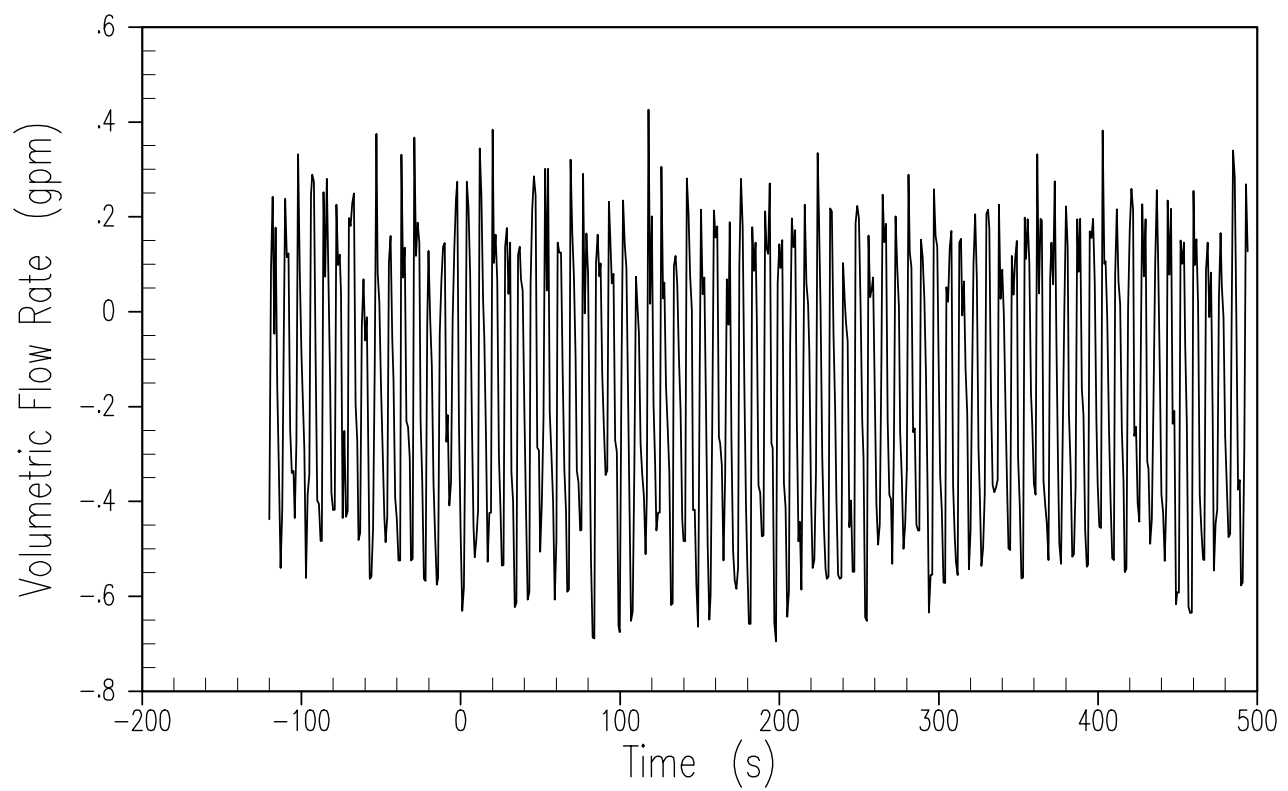

Figure A-55 Primary Sump 2 Injection Flow Rate 


\section{AP1000 Test Data Oregon State University Test TR-1 March 18, 2003}

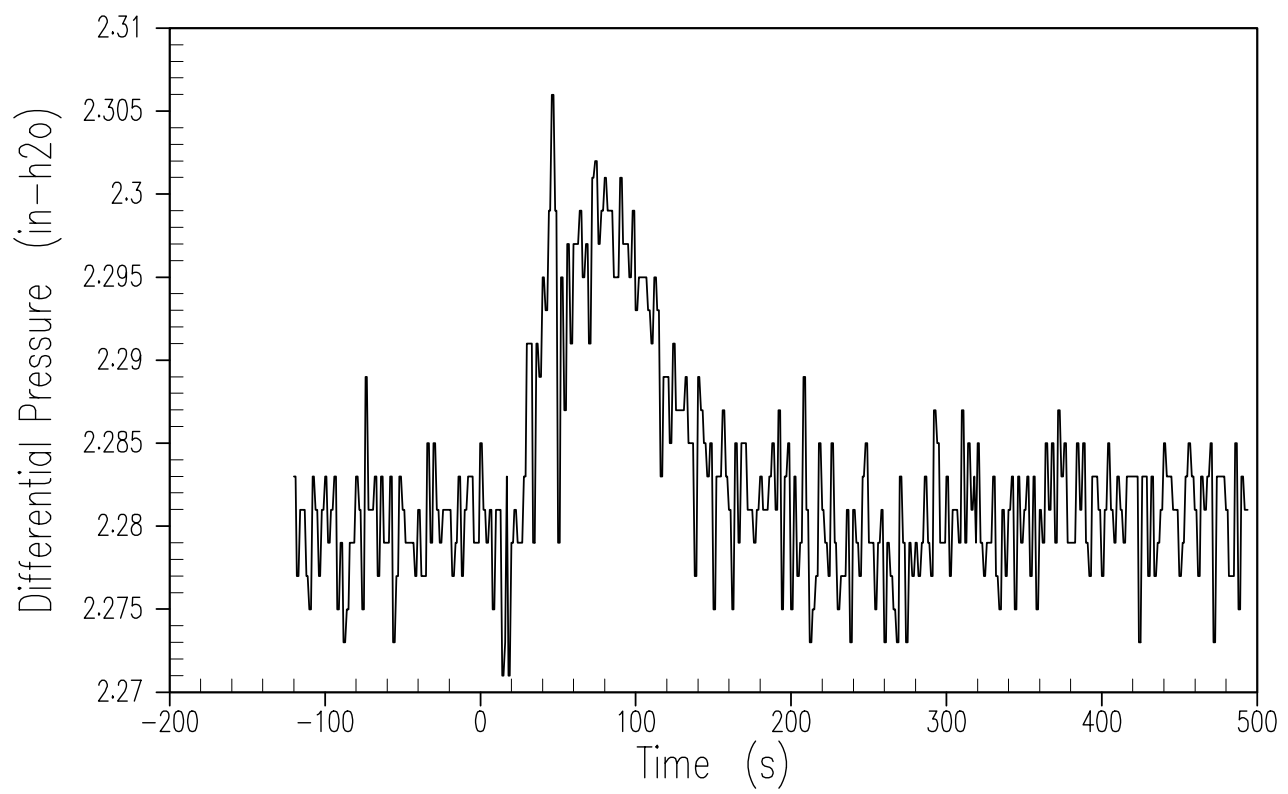

Figure A-56 Secondary Sump Liquid Level 


\section{AP1000 Test Data Oregon State University Test TR-1 March 18, 2003}

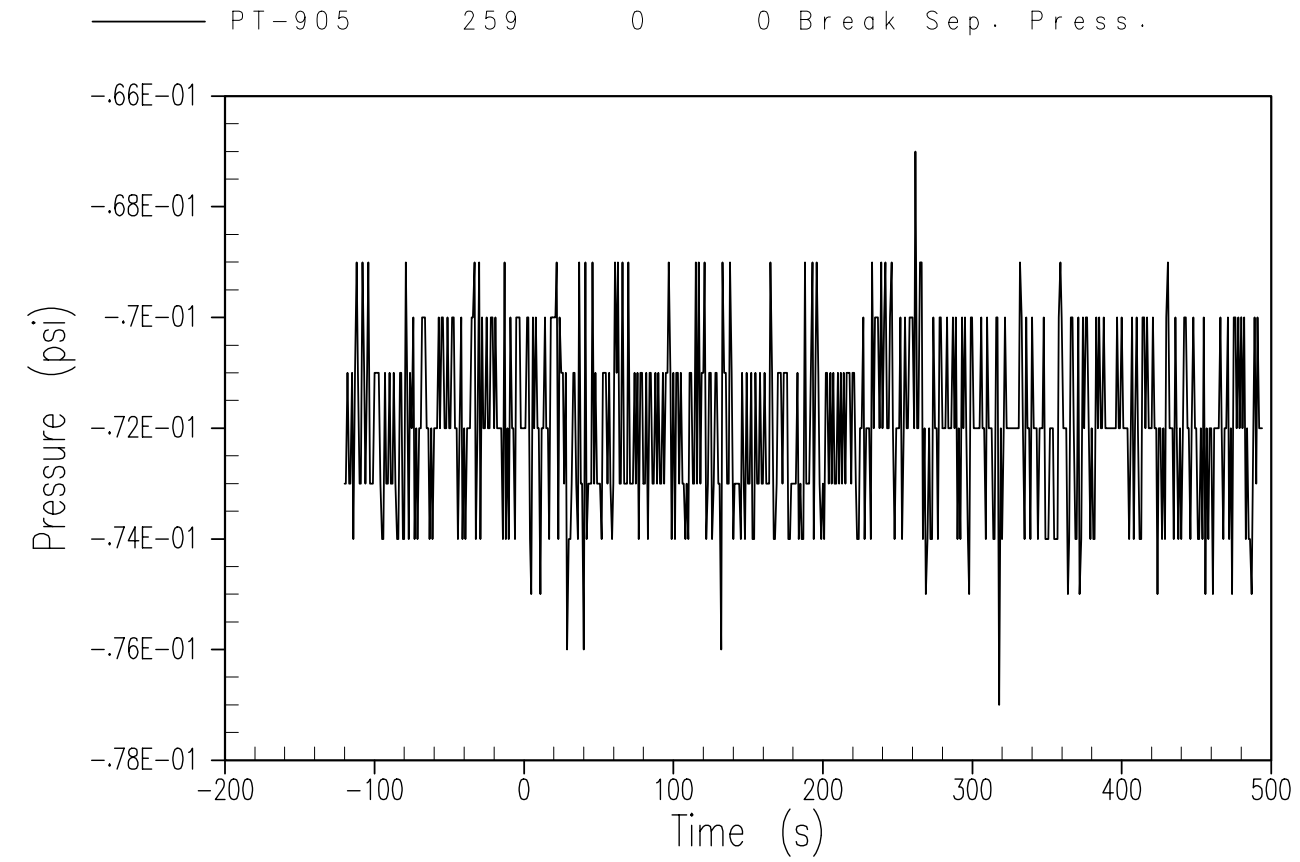

Figure A-57 Break Separator Pressure 

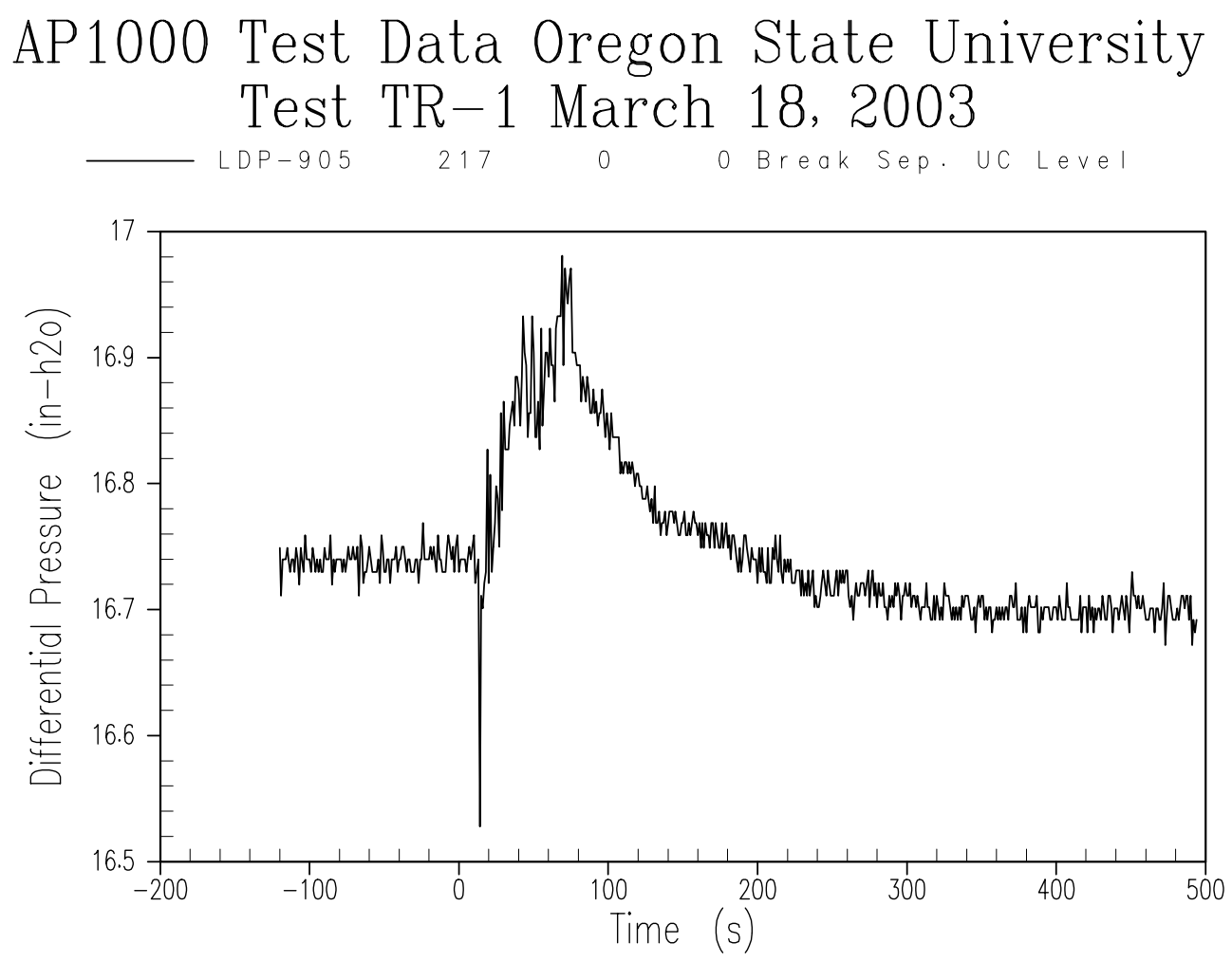

Figure A-58 Break Separator Liquid Level 

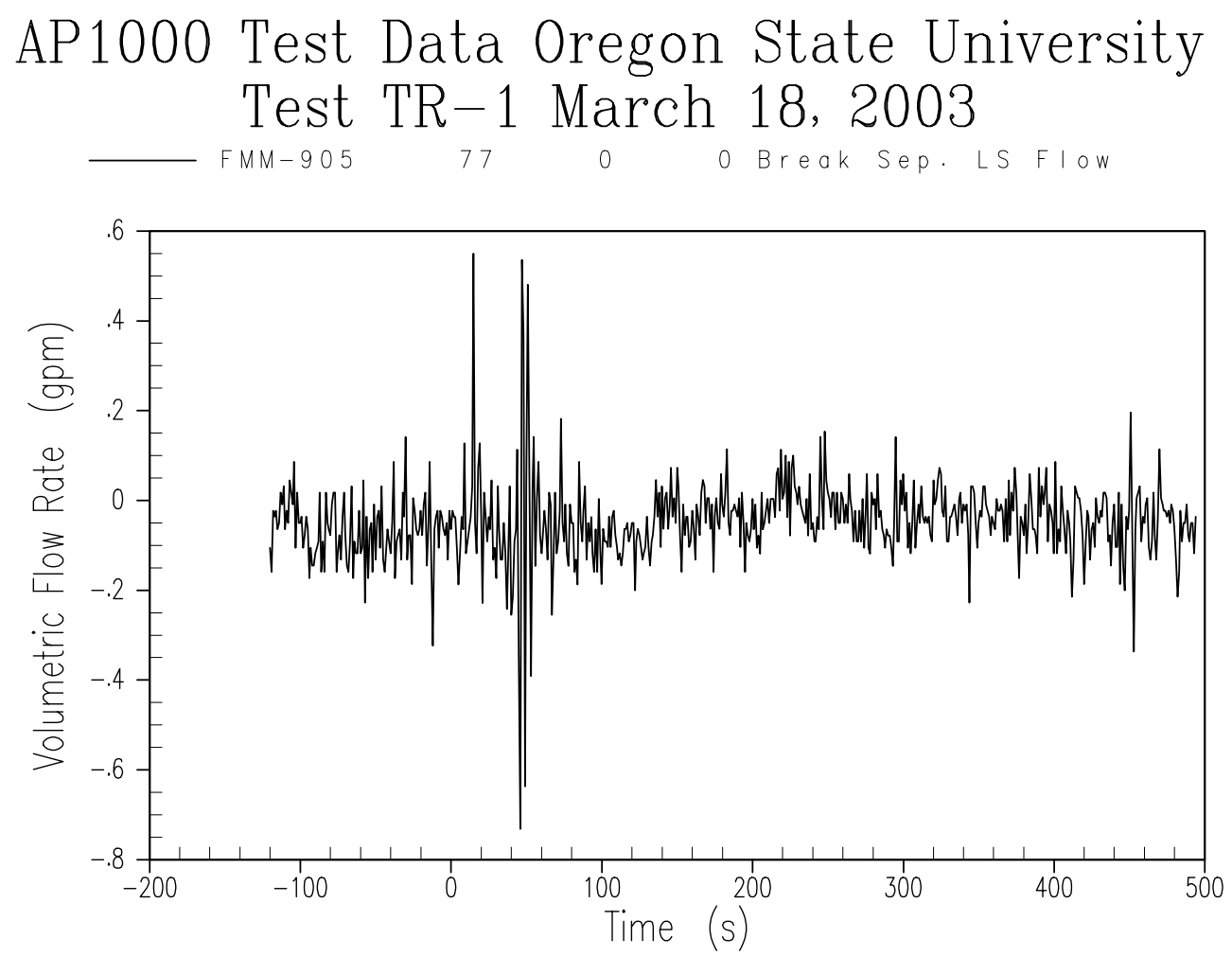

Figure A-59 Break Separator Flow to Primary Sump 

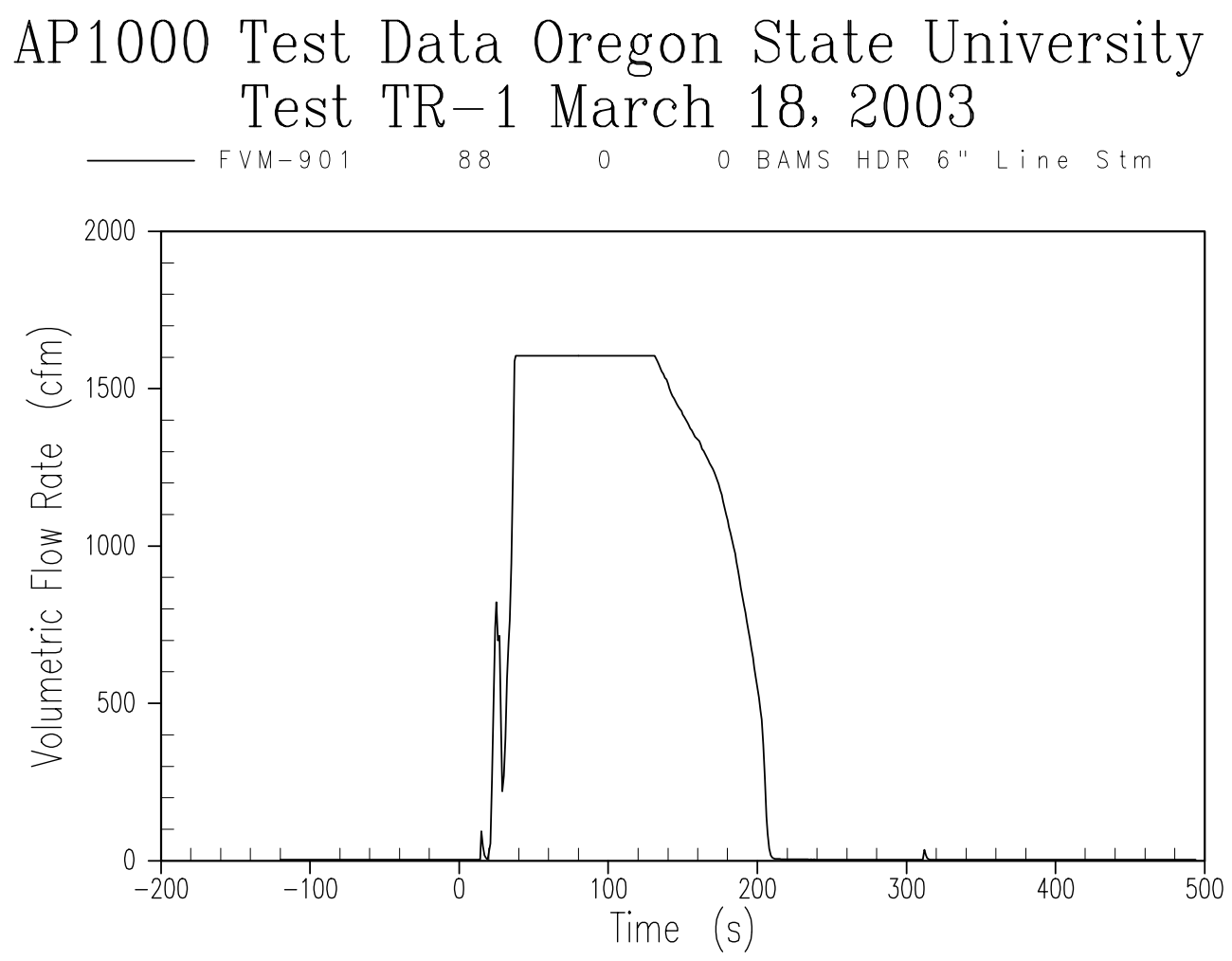

Figure A-60 BAMS Steam Flow Rate - 6-inch Line 

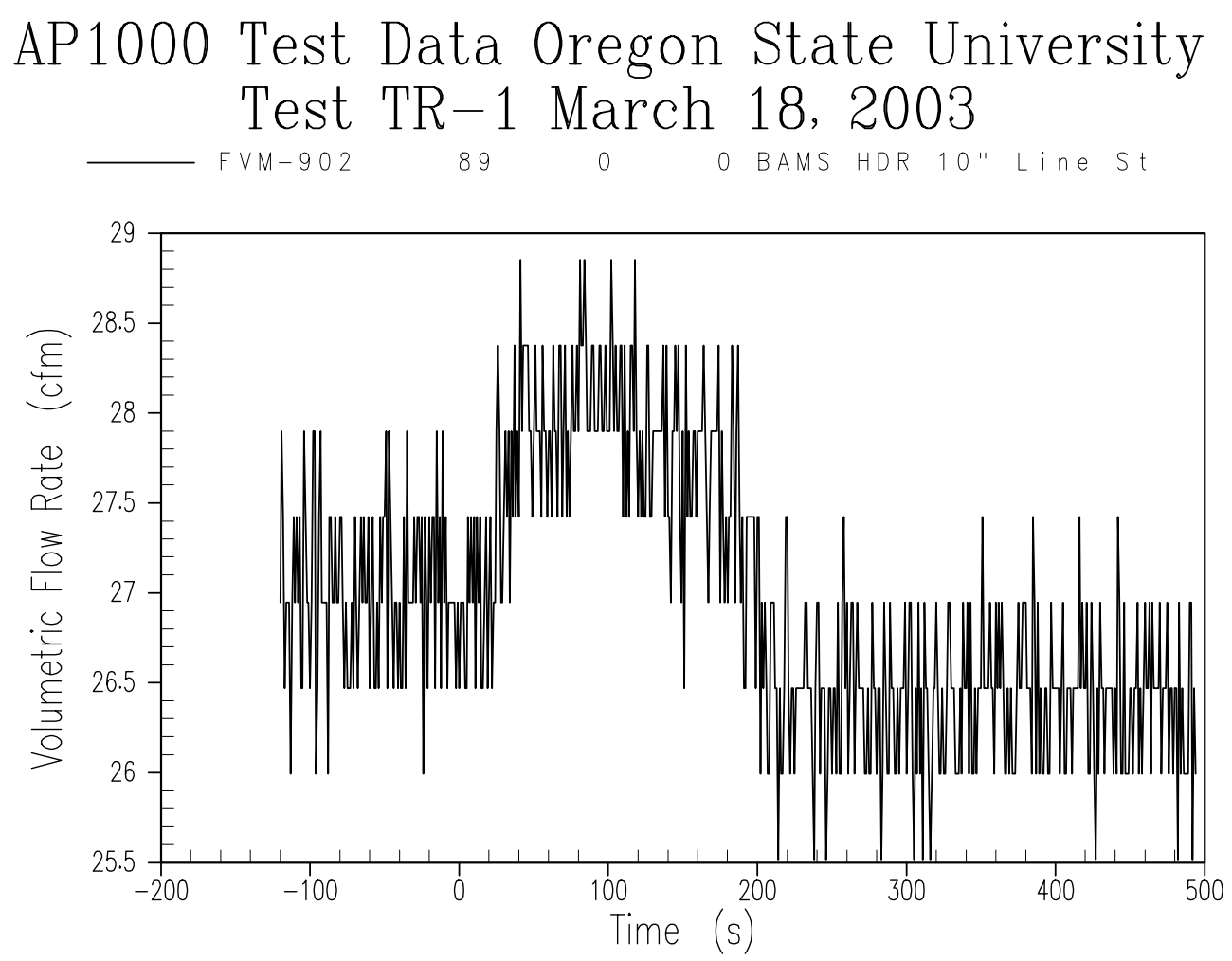

Figure A-61 BAMS Steam Flow Rate - 10-inch Line 

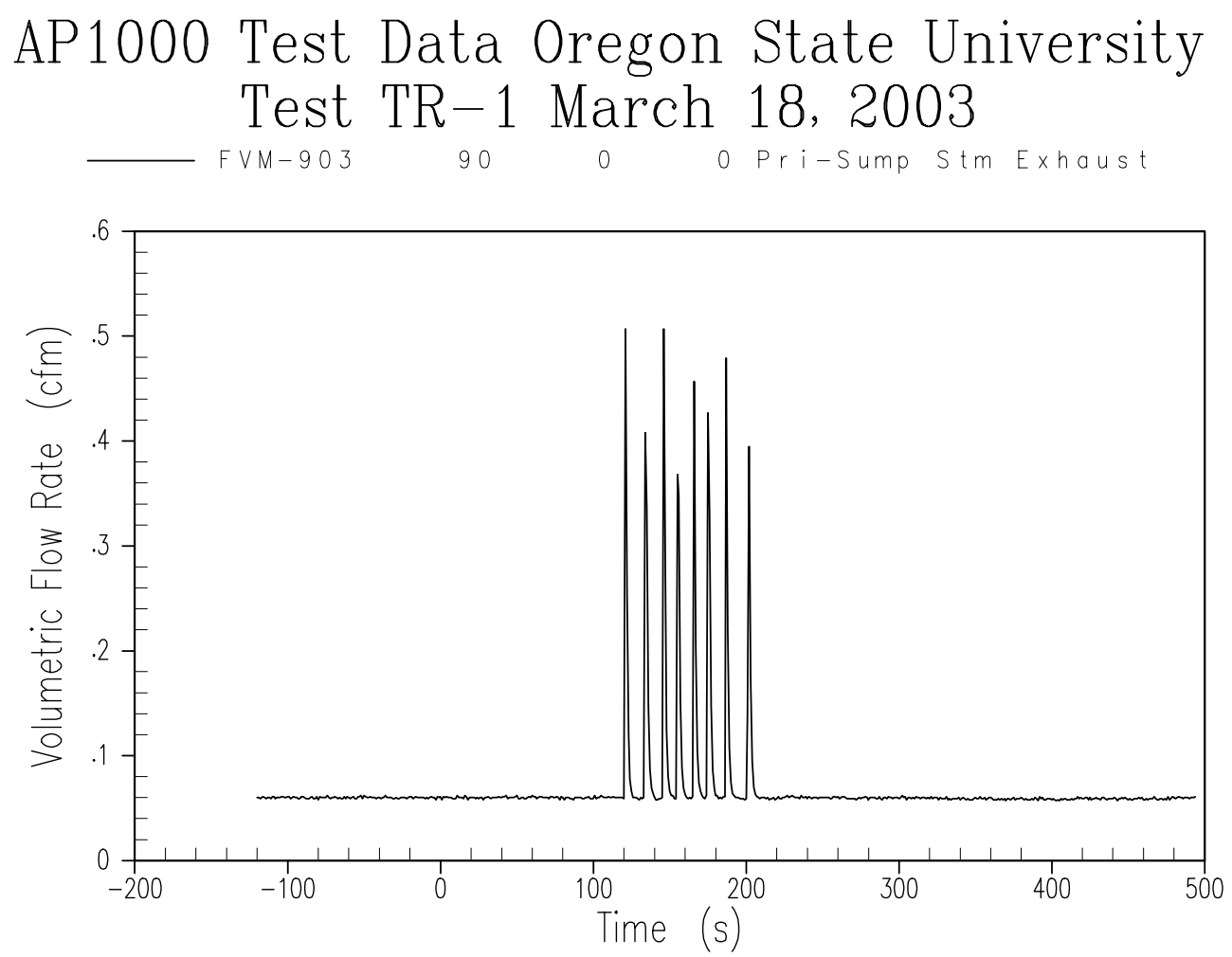

Figure A-62 BAMS/Primary Sump Steam Flow Rate 

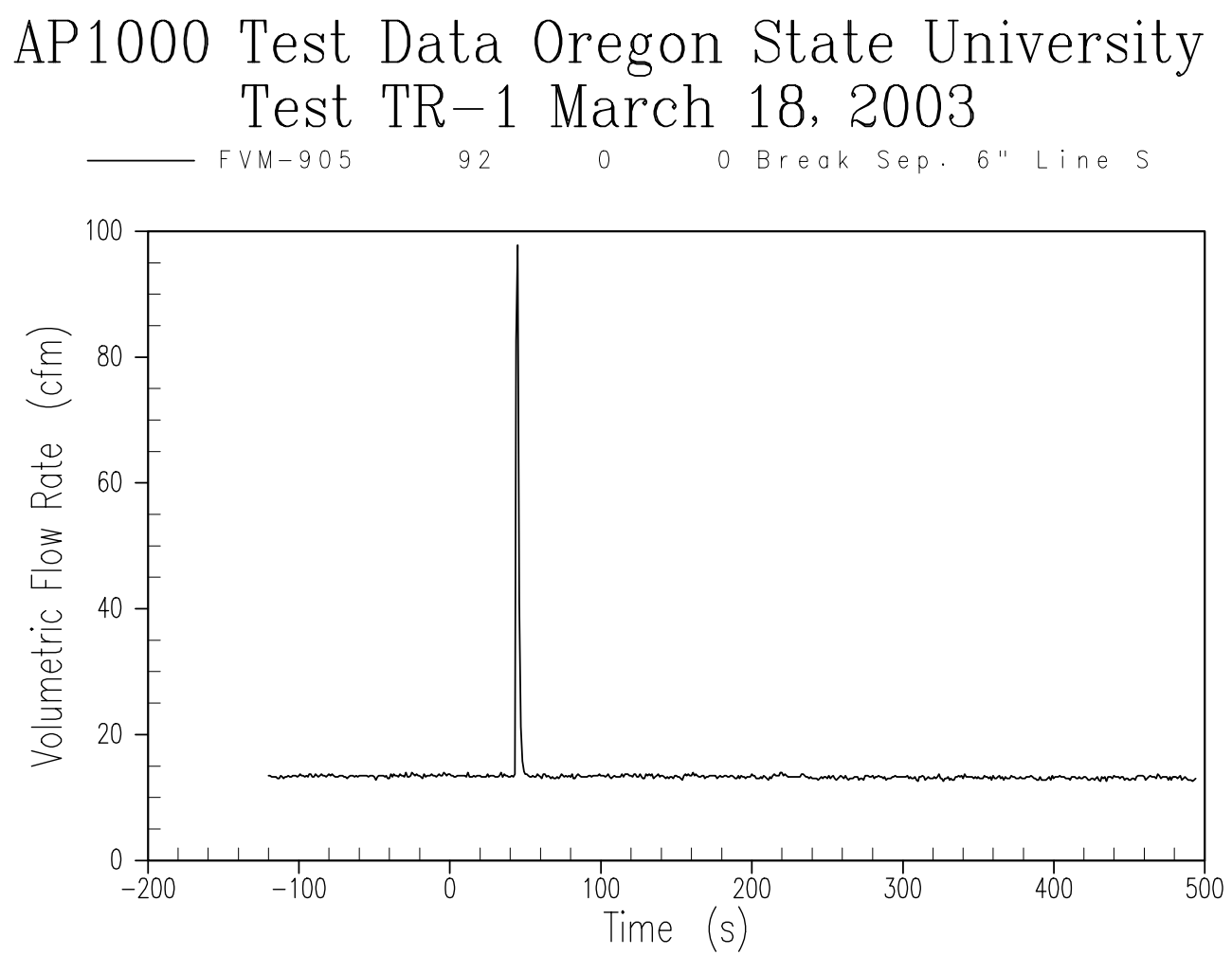

Figure A-63 BAMS/Separator Steam Flow Rate - 6-inch Pipe 

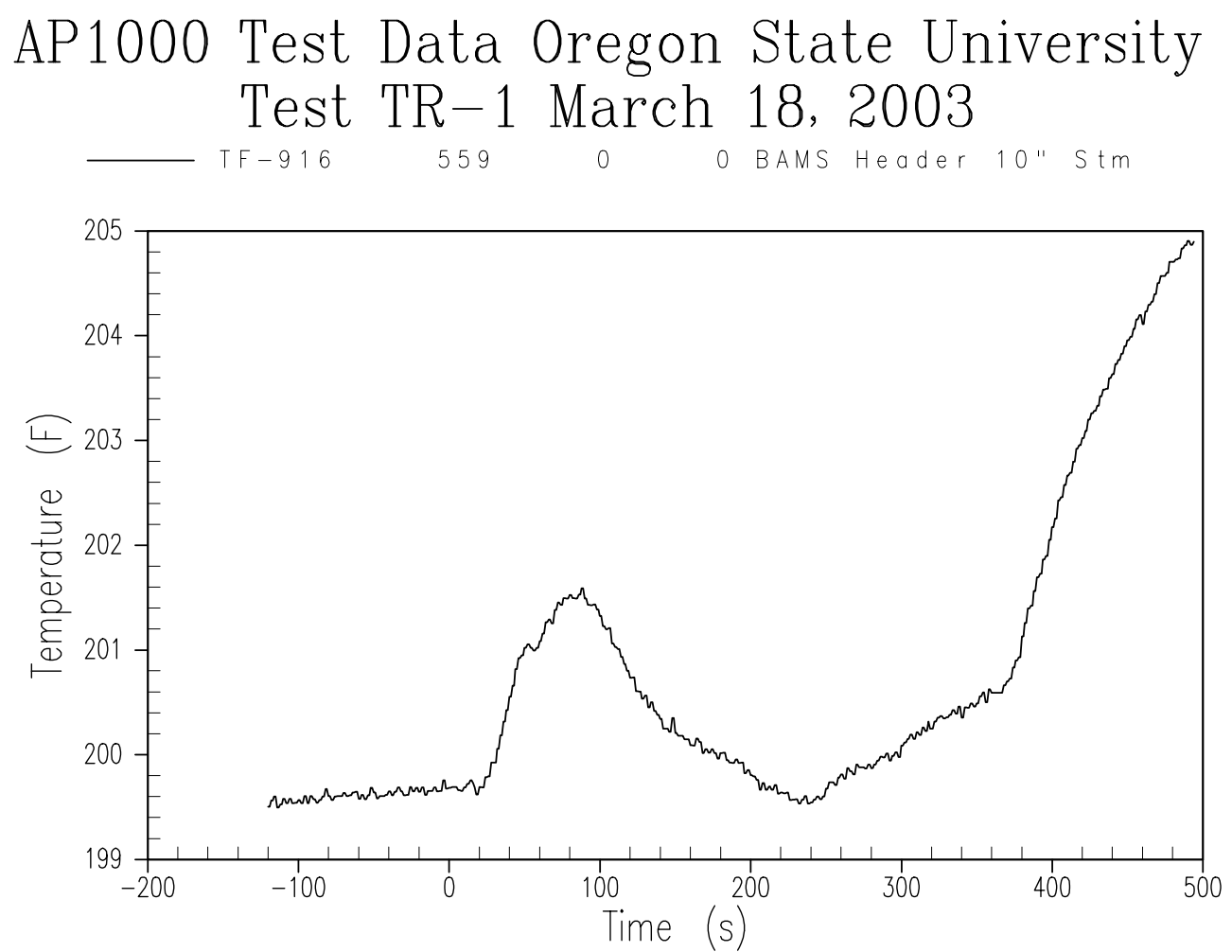

Figure A-64 BAMS/Exhaust Line Temperature - 10-inch Line 

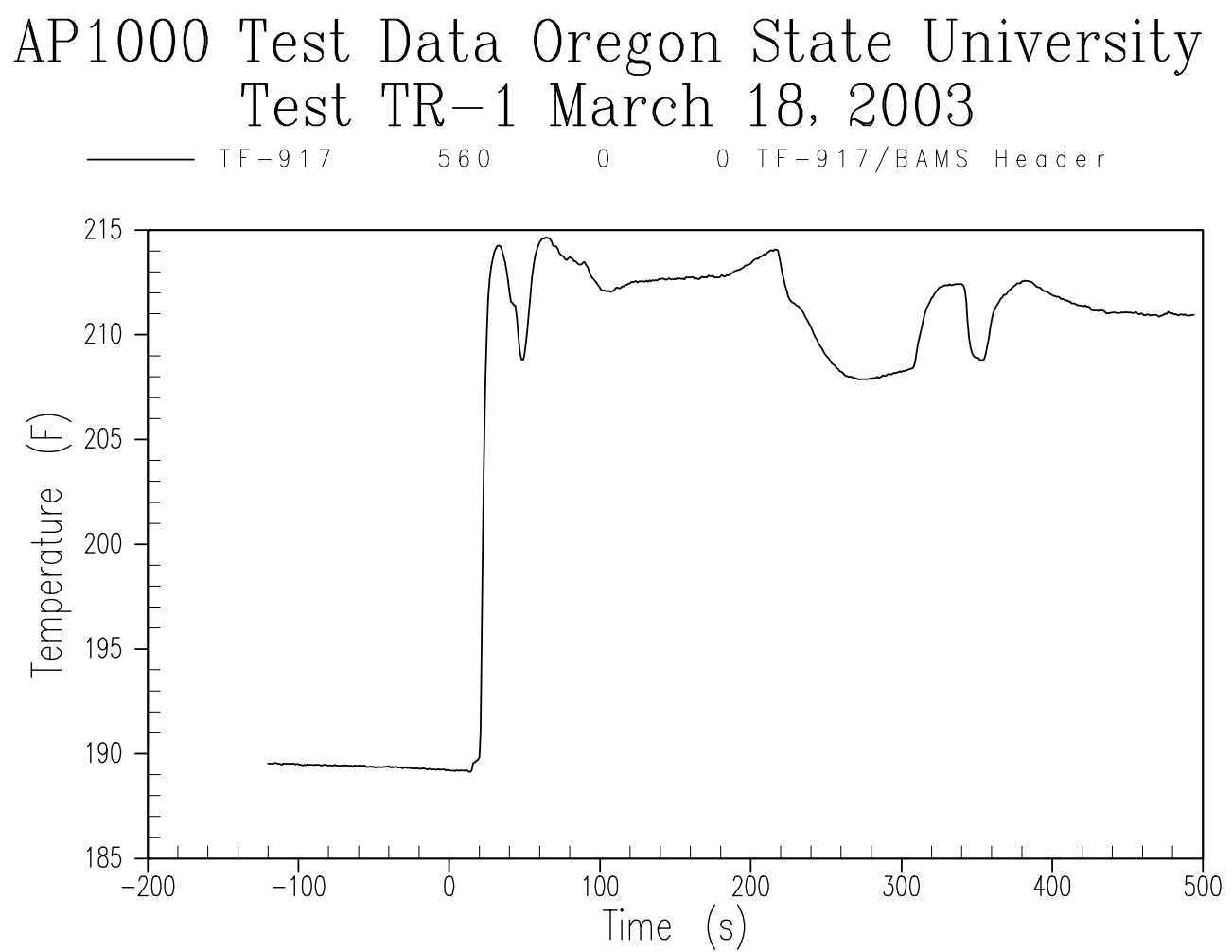

Figure A-65 BAMS/Exhaust Line Temperature - Header 


\section{AP1000 Test Data Oregon State University Test TR-1 March 18, 2003}

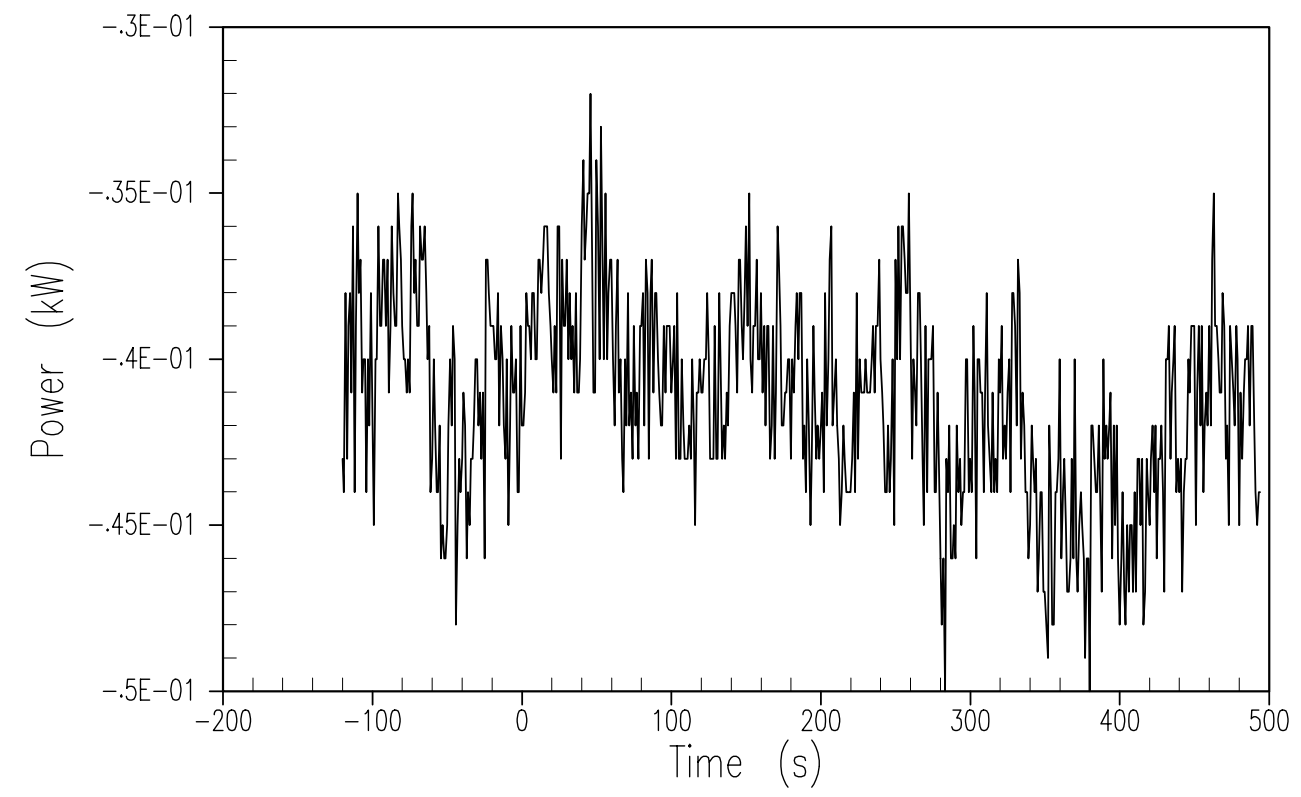

Figure A-66 Pressurizer Heater Input Power 
AP1000 Test Data Oregon State University Test TR-1 March 18, 2003

$-K W-101$

130

0

o RX Htr Group 1 Power

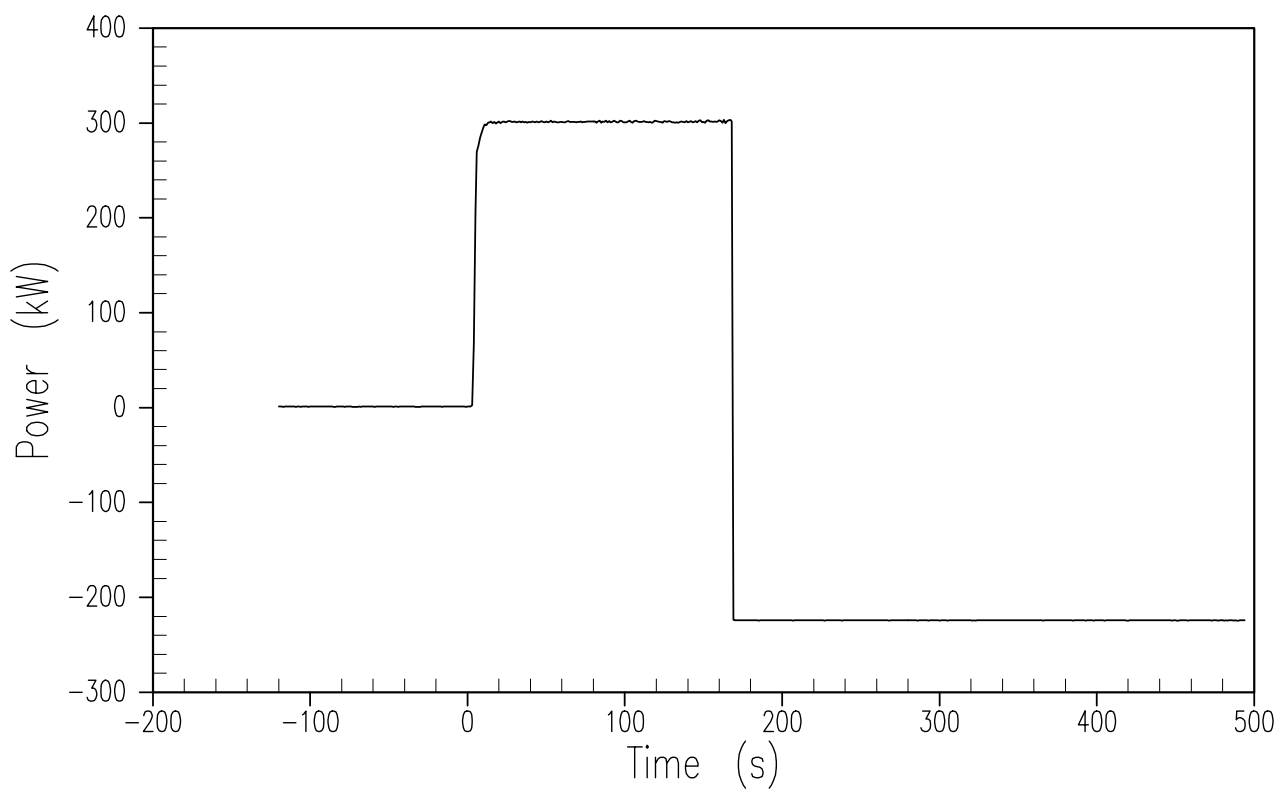

Figure A-67 Core Power Group 1 Input Power 
AP1000 Test Data Oregon State University Test TR-1 March 18, 2003

$-K W-102$

131

0

o RX Htr Group 2 Power

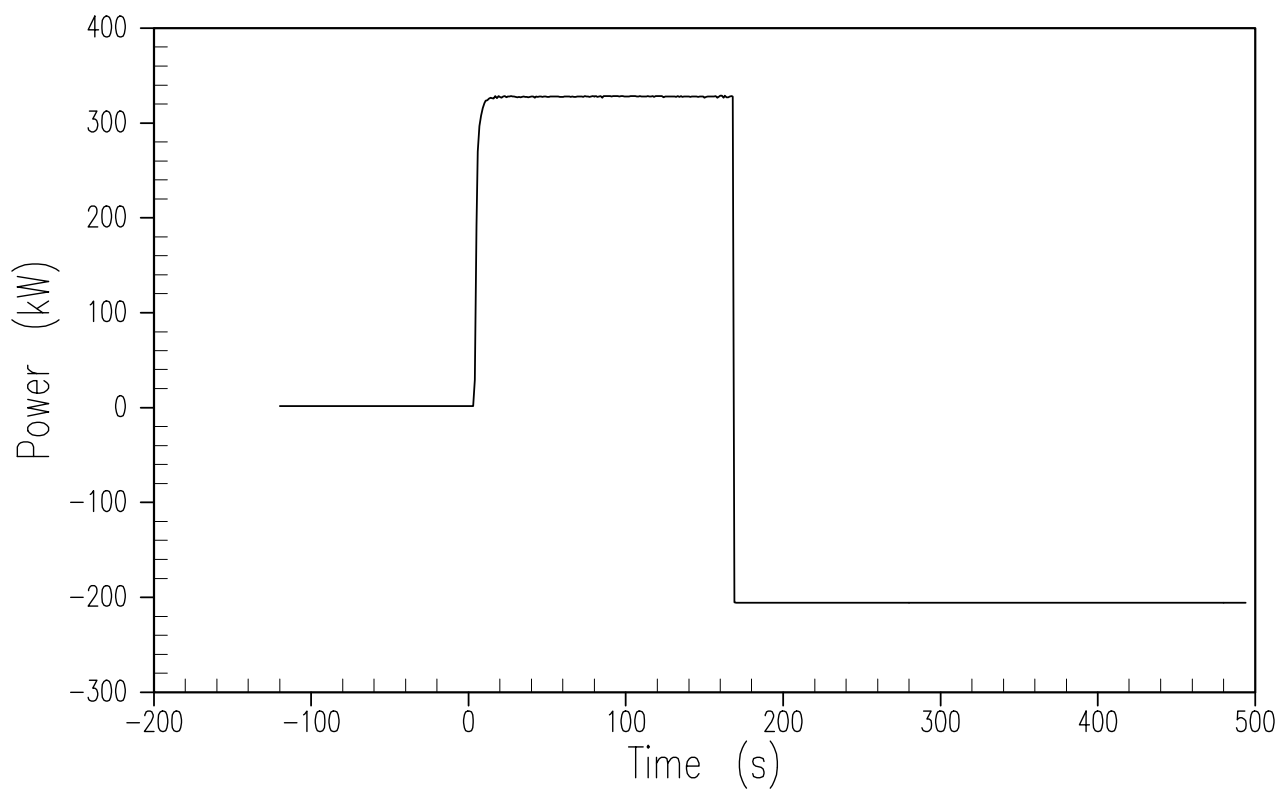

Figure A-68 Core Power Group 2 Input Power 

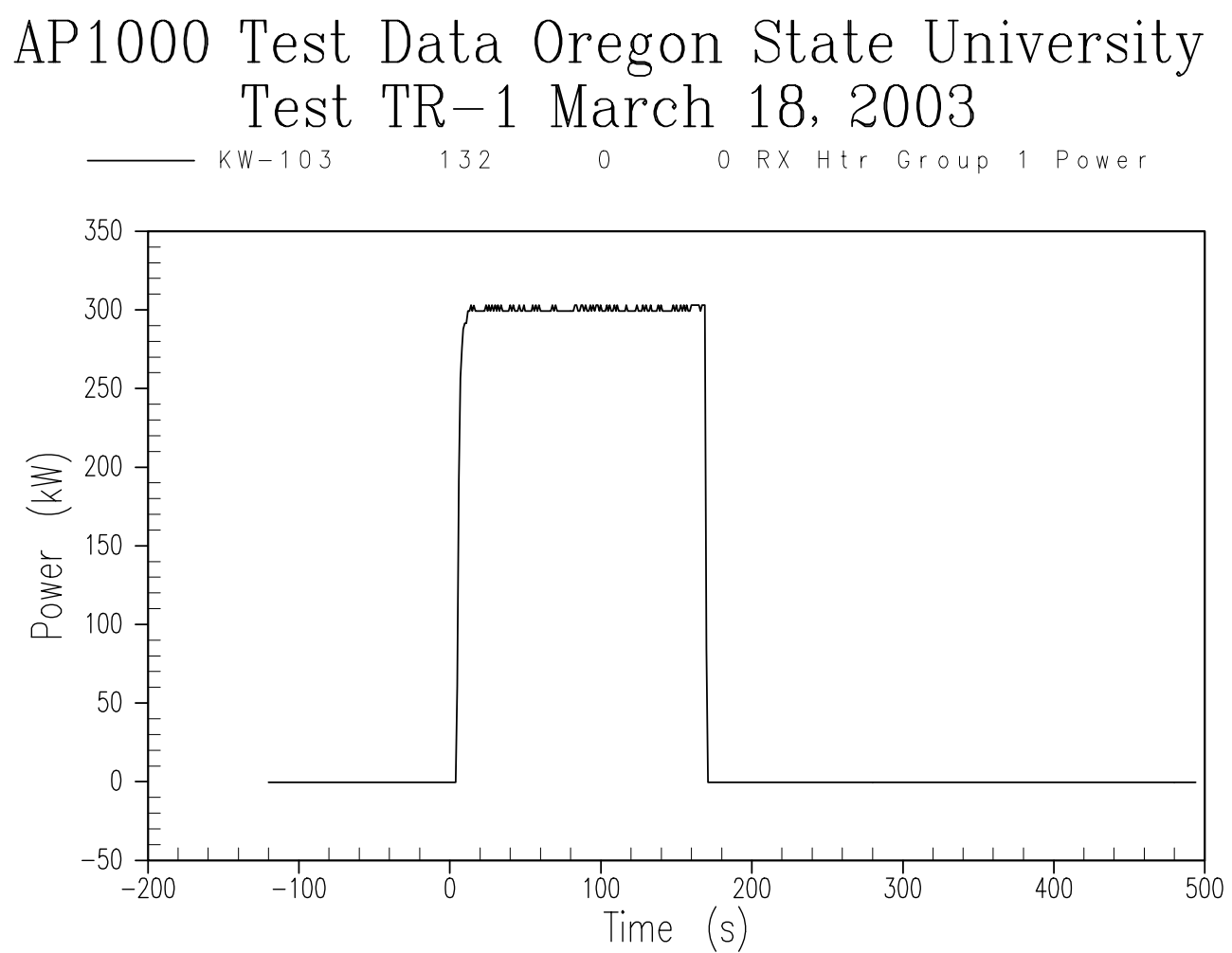

Figure A-69 Core Power Group 1 Alternate Input Power 

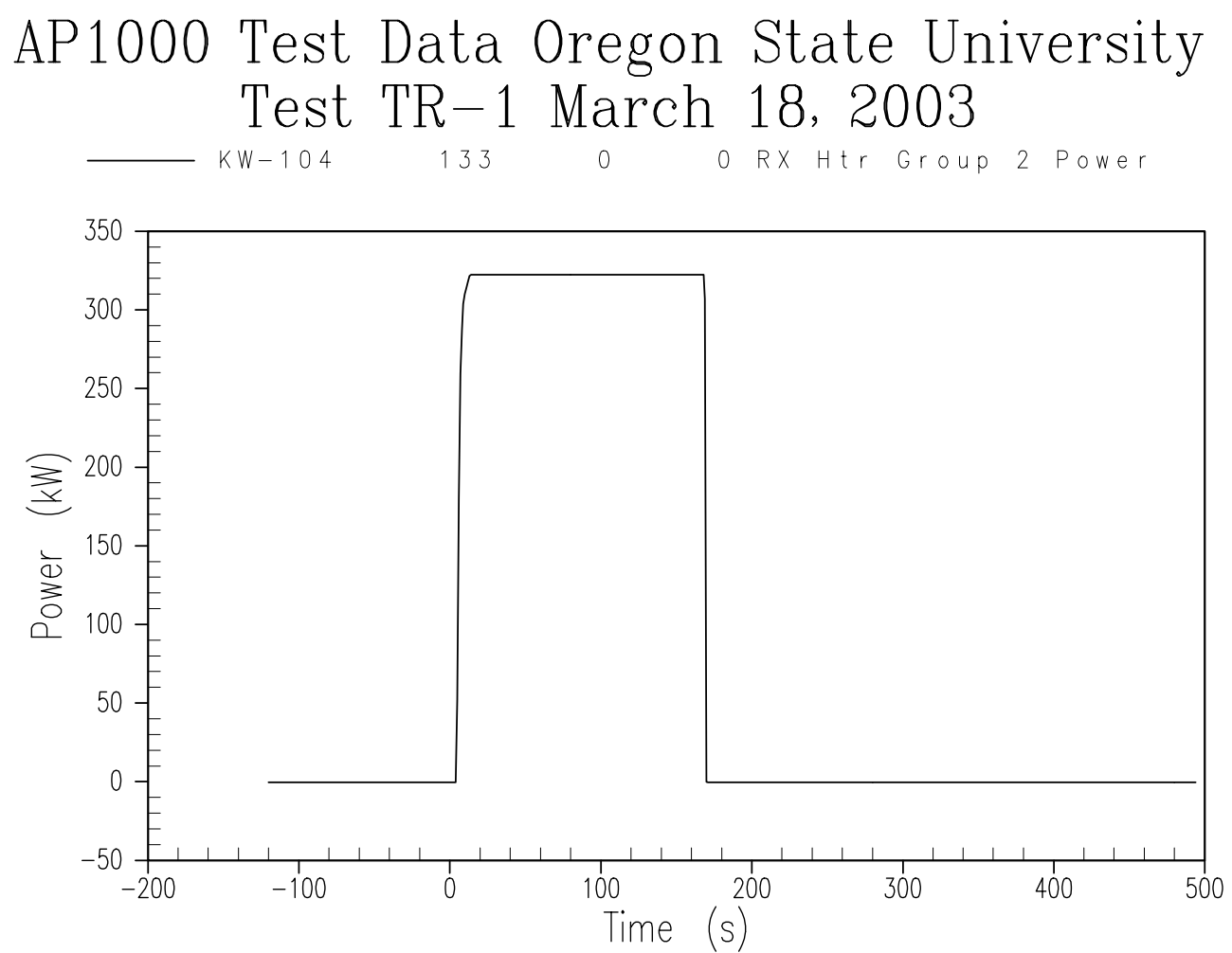

Figure A-70 Core Power Group 2 Alternate Input Power 

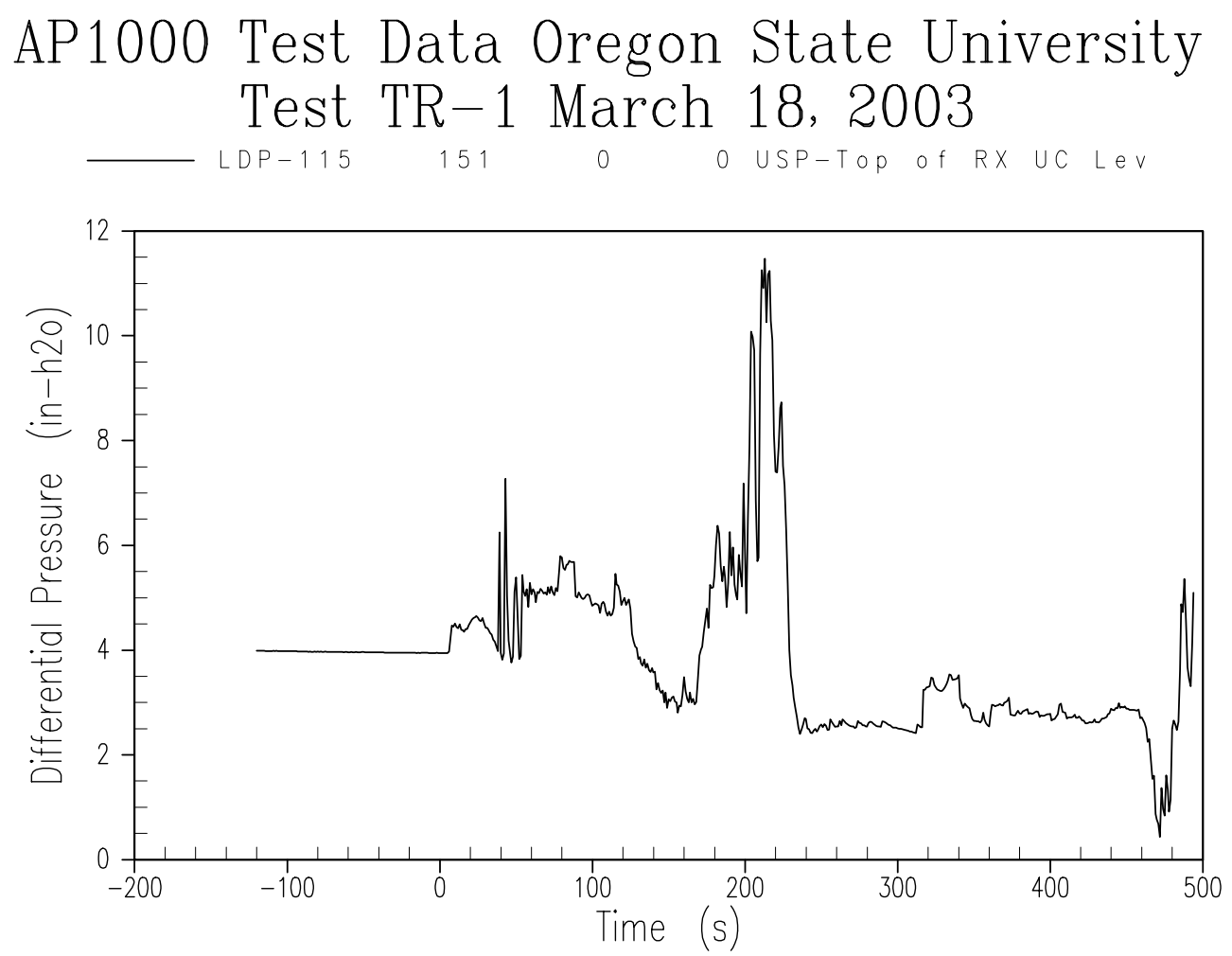

Figure A-71 Reactor Vessel Liquid Level Between Top of Vessel - Upper Support Plate 

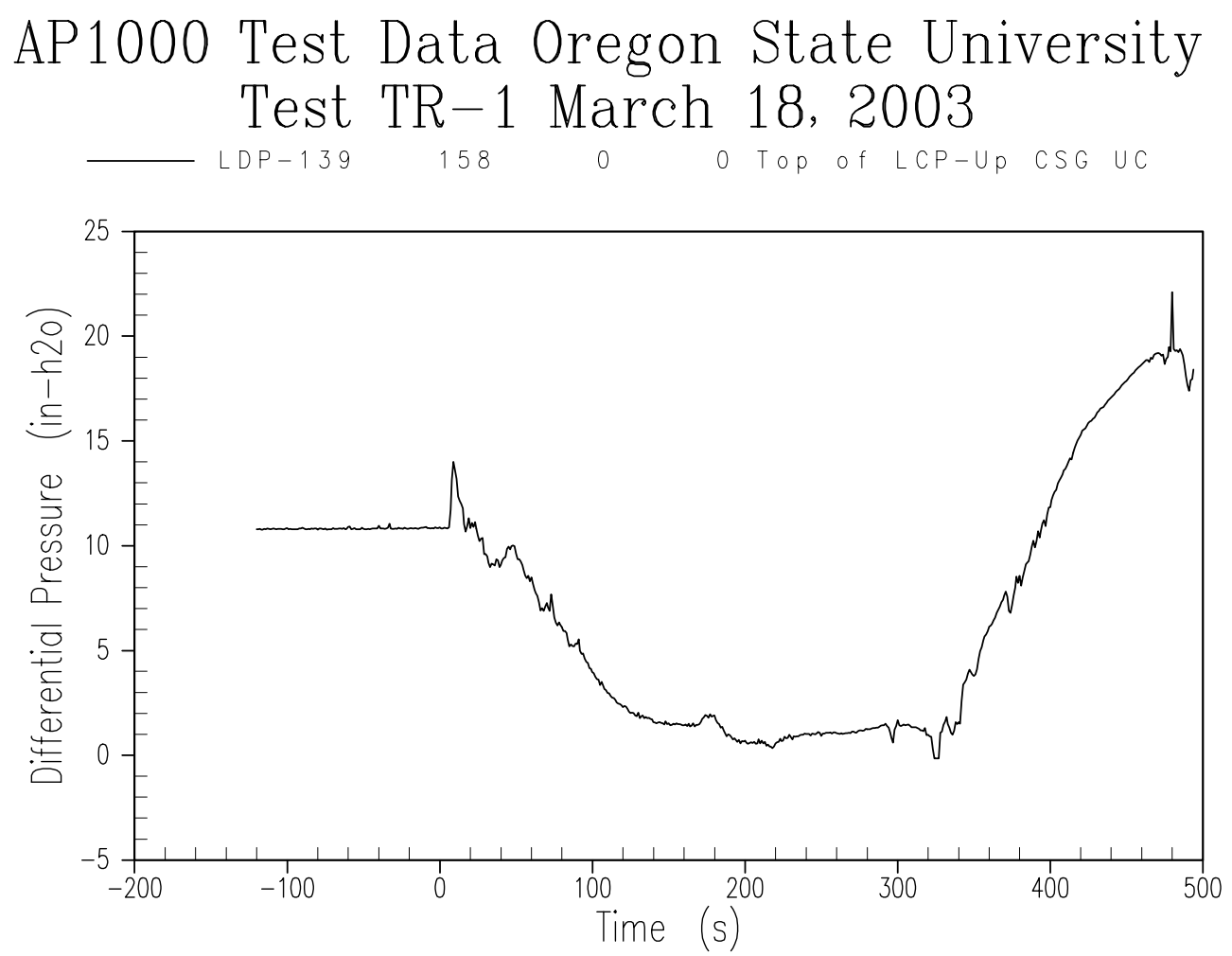

Figure A-72 Reactor Vessel Liquid Level Between Bottom of Upper Support Plate Upper Core Spacer Grid 

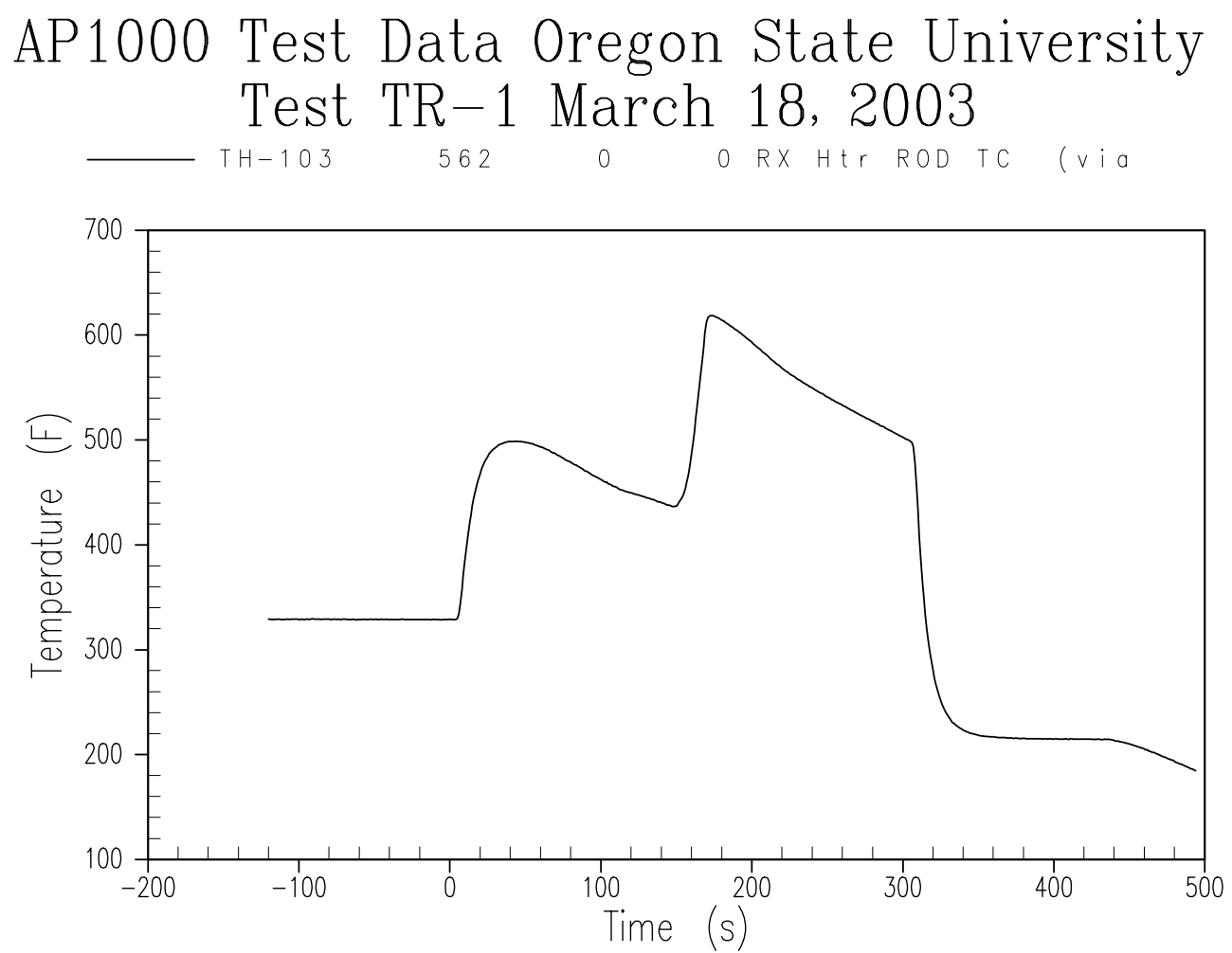

Figure A-73 Inner Core Thermocouple Measuring Heater Temperature 

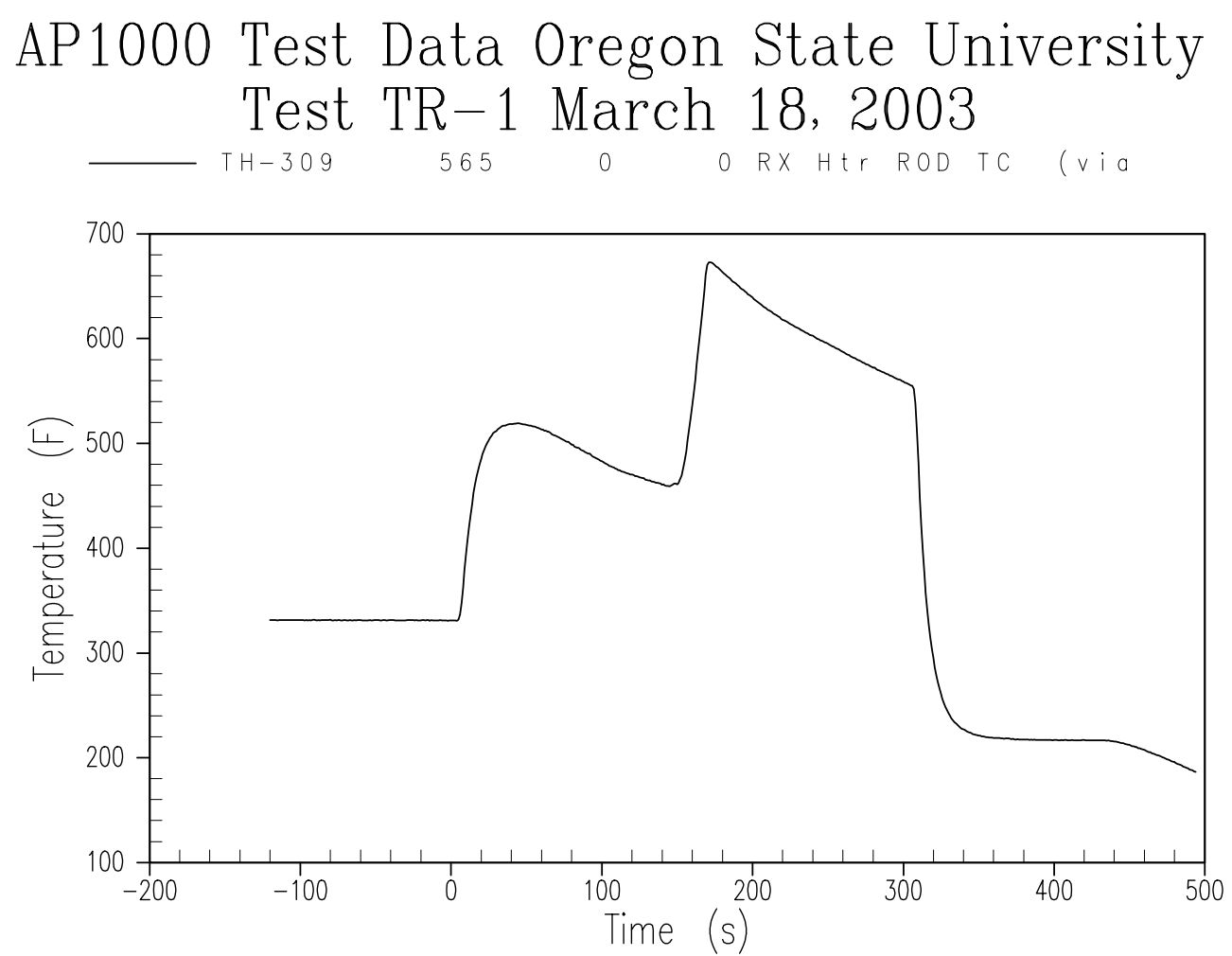

Figure A-74 Outer Core Thermocouple Measuring Heater Temperature 


\section{APPENDIX B}

TEST DATA 


\section{Appendix C -- OSU-AP1000-03}




\title{
Oregon State University
}

Department of Nuclear Engineering

\author{
Advanced Thermal Hydraulic \\ RESEARCH LABORATORY
}

\section{TEST SUMMARY REPORT}

\author{
OSU-AP1000-03
}

\section{AP1000 DOUBLE-ENDED DVI WITH 3 OF 4 ADS 4 REVISED ADS 4 PIPING AND VALVES}

Revision 0 


\title{
TEST SUMMARY REPORT
}

\author{
OSU-AP1000-03
}

\section{AP1000 DOUBLE-ENDED DVI WITH 3 OF 4 ADS 4 REVISED ADS 4 PIPING AND VAlVES}
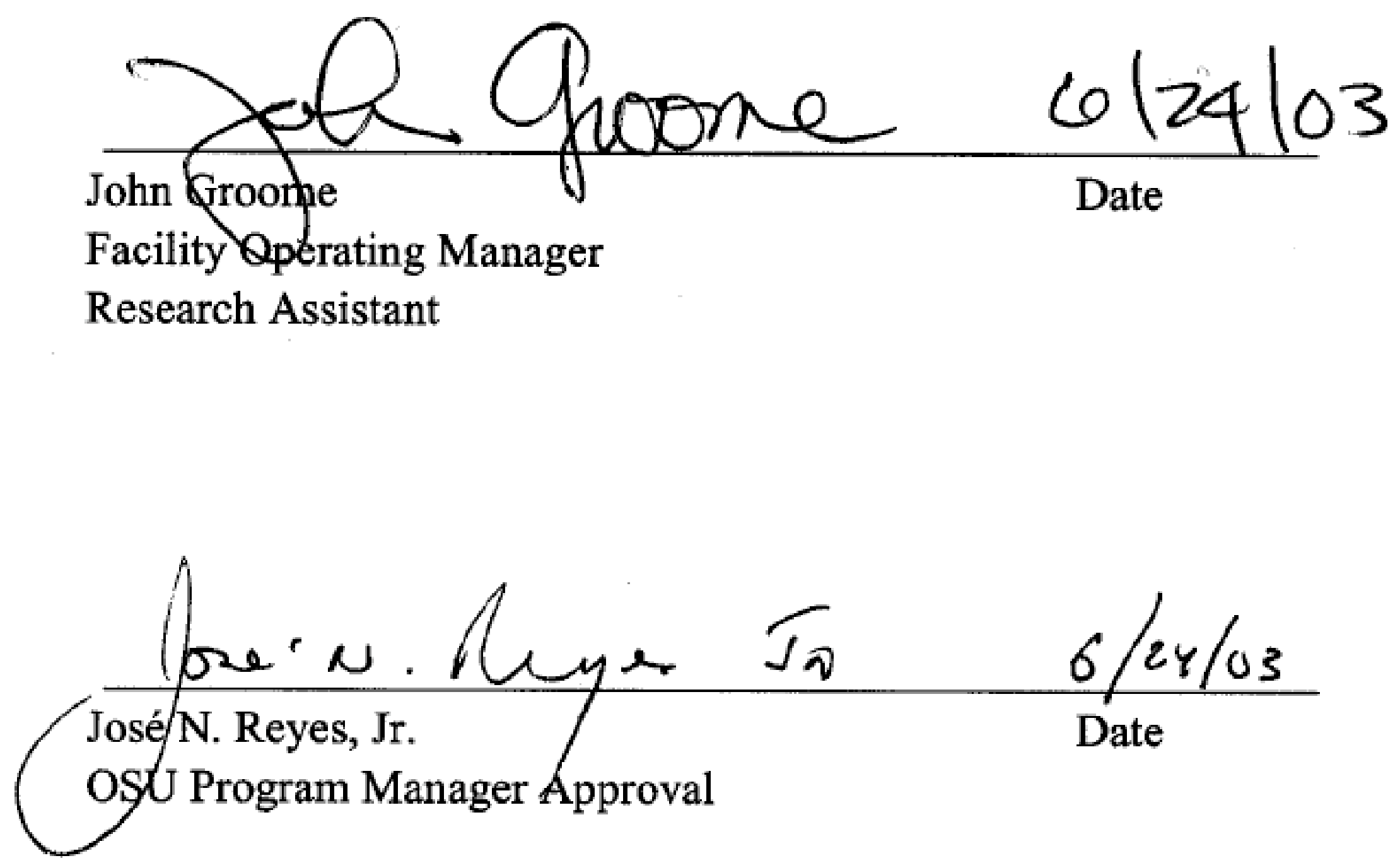

Oregon State University

116 Radiation Center

Corvallis, Oregon 97331

(C) 2003 Oregon State University

All Rights Reserved 


\section{WESTINGHOUSE PROPRIETARY INFORMATION NOTICE}

This report contains information proprietary to Westinghouse Electric Company. A non-proprietary version of this report has also been prepared for distribution to the United States Nuclear Regulatory Commission (NRC).

In order to conform to the requirements of 10 CFR 2.790 of the Commission's regulations concerning the protection of proprietary information so submitted to the NRC, the information which is proprietary in the proprietary version of this document is contained within brackets, and where the proprietary information has been deleted in the non-proprietary versions, only the brackets remain (the information that was contained within the brackets in the proprietary versions having been deleted). The justification for claiming the information so designated as proprietary is indicated in both versions by means of lower case letters (a) through (f) located as a superscript immediately following the brackets enclosing each item of information being identified as proprietary or in the margin opposite such information. These lower case letters refer to the types of information Westinghouse customarily holds in confidence identified in Sections (4)(ii)(a) through (4)(ii)(f) of the affidavit accompanying the transmittal of these documents pursuant to 10 CFR $2.790(b)(1)$.

\section{COPYRIGHT NOTICE}

This document bears an Oregon State University copyright notice. The NRC is permitted to make the number of copies of the information contained in these reports which are necessary for its internal use in connection with generic and plant-specific reviews and approvals as well as the issuance, denial, amendment, transfer, renewal, modification, suspension, revocation, or violation of a license, permit, order, or regulation subject to the requirements of 10 CFR 2.790 regarding restrictions on public disclosure to the extent such information has been identified as proprietary by Westinghouse, copyright protection notwithstanding. With respect to the non-proprietary versions of these reports, the NRC is permitted to make the number of copies beyond these necessary for its internal use which are necessary in order to have one copy available for public viewing in the appropriate docket files in the public document room in Washington, DC and in local public document rooms as may be required by NRC regulations if the number of copies submitted is insufficient for this purpose. Copies made by the NRC must include the copyright notice in all instances and the proprietary notice if the original was identified as proprietary. 
TABLE OF CONTENTS

$\underline{\text { Section }}$

Title

Page

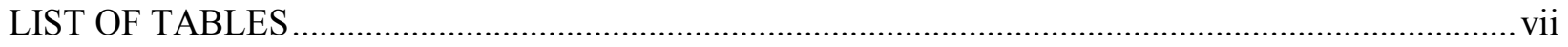

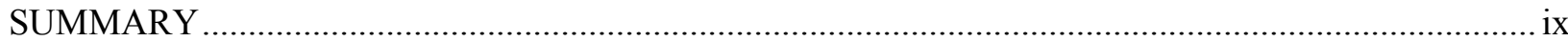

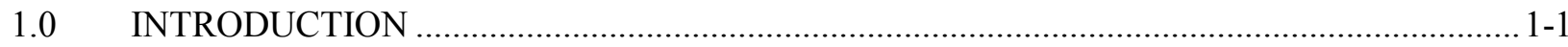

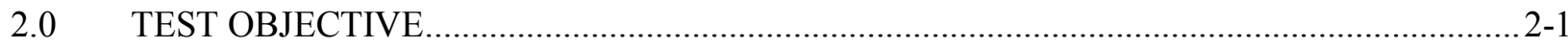

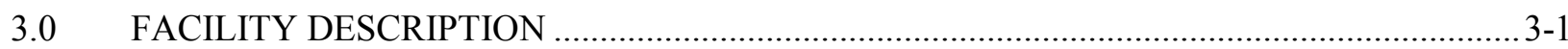

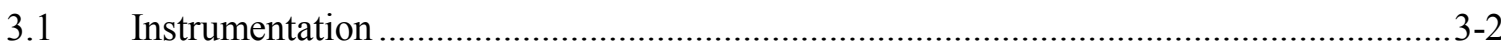

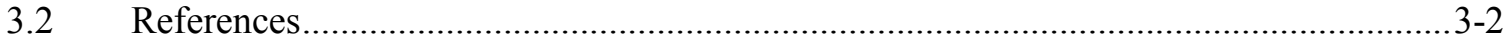

4.0 OREGON STATE UNIVERSITY TESTING PROGRAM MATRIX .......................................4-1

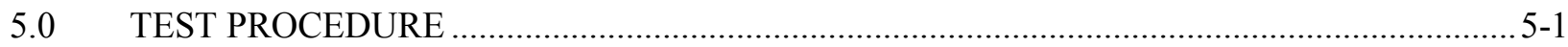

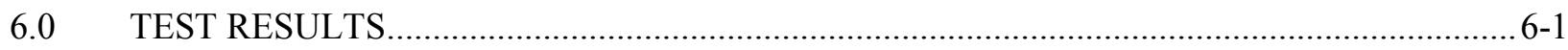

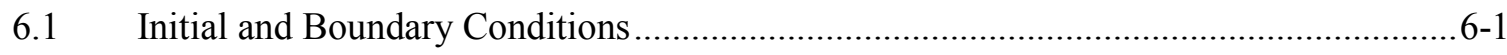

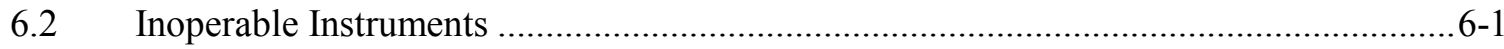

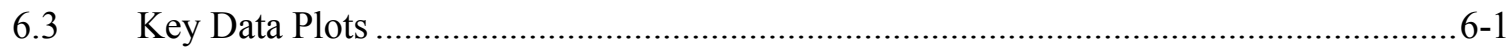

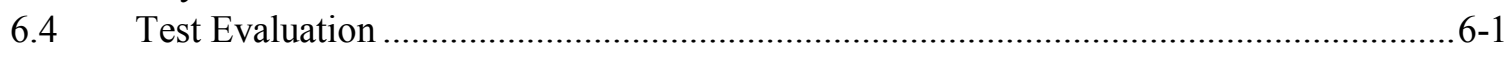

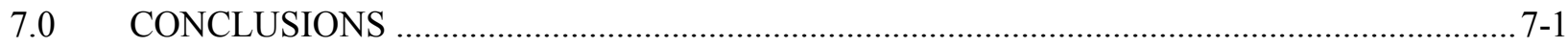

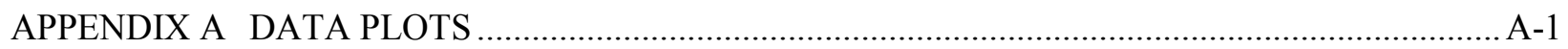

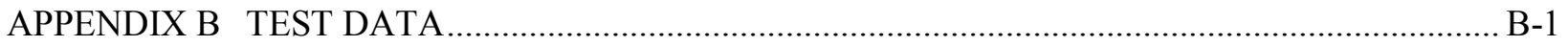




\section{LIST OF TABLES}

$\underline{\text { Table }}$

Table 4-1

Table 6-1

Table 6-2

Table 6-3

Table 6-4
Title

Page

OSU Test Matrix (as specified March 18, 2003) $4-2$

Actual Test Initial Conditions. $6-2$

Sequence of Events..... 6-4

Inoperable Instruments for TR-01-D Test ….................................................................6-5

Data Plots for QL Reports for TR-01-D by Component... 6-6 


\section{SUMMARY}

This report covers the test TR-01-D, transition from Automatic Depressurization System 4 (ADS-4) actuation to in-containment refueling water storage tank (IRWST) injection at 85 psig reactor pressure, performed on March 18, 2003. This test has been designated OSU-AP1000-02 by the Advanced Plant Experiment (APEX). The objective of this test was to demonstrate the AP1000 transition IRWST injection following actuation of the ADS-4. For this test, the Reactor Coolant System was drained down to the level of the hot legs. The Reactor Coolant System was heated to saturated conditions at a pressure of 85 psig. The core power was set to a constant value of $628 \mathrm{~kW}$. The core makeup tanks and accumulators were isolated for this test. The ADS-4 valves were opened at the start of the test. The power was held constant, and the Reactor Coolant System was depressurized. However, the cut-in pressure for IRWST injection was not reached before the two-phase level in the upper plenum fell to the top of the core. Once the thermocouples at the top of the heater rods showed a dramatic temperature increase, the test was terminated at 168 seconds. It was determined after the test that the power level was too high to allow the Reactor Coolant System to depressurize and IRWST injection to commence before the two-phase level in the upper plenum reached the top of the heater rods.

This report presents the initial assessment of the test data collected. If this test is to be used by Westinghouse to support AP1000 Design Certification, additional validation of the use of this information will be documented separately. In the interim, the list of invalid data channels may change. 


\subsection{INTRODUCTION}

The Department of Nuclear Engineering at Oregon State University (OSU) is performing a series of tests for the U.S. Department of Energy. These tests are being conducted in the Advanced Plant Experiment (APEX-1000) test facility, which is a reduced pressure and height model of the two-loop Westinghouse AP1000 pressurized water reactor. The purpose of the testing is to:

- evaluate the thermal-hydraulic performance of the passive safety systems of the full-scale AP1000, and

- assess and validate the safety analyses techniques and computer codes used in predicting the transient system behavior.

The AP1000 Long-Term Cooling Test is a 1/4 height scale, low-pressure integral systems test simulating thermal-hydraulic phenomenon for the AP1000 passive safety systems for small-break loss-of-coolant accidents (LOCAs) and long-term cooling. It accurately models the details of the AP1000 geometry, including the primary system, the passive safety systems, and a part of the non-safety grade Chemical and Volume Control System, as well as a partial non-safety grade Normal Residual Heat Removal System. The interconnecting pipe routings are also duplicated in the model.

The overall objective of the Long-Term Cooling Test program is to obtain test data at various modes of operation. The OSU experiments will examine the passive safety system response for the small-break and large-break LOCA transition into long-term cooling. (The list of the tests to be performed is in the OSU Test Matrix provided in Section 4.0.) The facility permits a range of small-break LOCAs to be simulated at different locations on the primary system, such as the cold leg, hot leg, core makeup tank (CMT) cold leg pressure balance line, and direct vessel injection (DVI) line. The break orientation (top or bottom of the cold leg) may also be studied. Selected tests continue into the long-term cooling, post-accident mode in which the passive safety injection is from the reactor sump as well as the in-containment refueling water storage tank (IRWST). 


\subsection{TEST OBJECTIVE}

The purpose of this test is to repeat the previous test TR-01-D, which was to obtain thermal-hydraulic data for the transition period from Automatic Depressurization System 4 (ADS-4) actuation to stable IRWST injection. The other sources of core makeup water, such as the CMTs and accumulators, were isolated. In addition, the pressurizer was drained and the ADS 1-3 were closed for the duration of the test. There is no break for this test. The assumed single failure is one of four ADS-4 valves and is on the non-pressurizer hot leg. The data obtained from the test will be used to verify the AP1000 thermal-hydraulic computer codes for AP1000 phenomena, such as gravity injection, natural convection, and post-accident long-term core cooling behavior.

The acceptance criteria for the OSU tests are as follows:

- Test initial conditions will be achieved within a specified tolerance.

- Setpoints will be achieved within an acceptable tolerance band.

- All instrumentation should be operational before the test.

- Any critical instruments not operating will be identified to the test engineer before the tests. These instruments must be operational before and during the test or exceptions should be approved.

- A zero check of LDPs, DPs, and FDPs will be performed. 


\subsection{FACILITY DESCRIPTION}

A detailed facility description for the OSU test facility is documented in Reference 3-1. The OSU test facility has been specifically scaled, designed, and constructed to investigate the AP600 passive safety system behavior and to provide data for safety analysis computer code validation. The facility has been modified to model the AP1000.

The scaled test design accurately models the details of the AP1000 geometry, including the primary system and the pipe routings and layout for the passive safety systems. A detailed scaling report (Reference 3-2) was used to develop the test design modifications. The primary system consists of one hot leg and two cold legs with two active pumps and an active steam generator for each loop. Two CMTs are connected to one primary loop, and the pressurizer is connected to the other primary loop as in the AP1000 plant design. Gas-driven accumulators are connected to the DVI lines. The discharge lines from the CMT, and one-of-two IRWST and reactor sump lines are connected to each DVI line. The ADS consisting of stages 1,2 , and 3 - simulates either one or two of the independent trains used in the AP1000. The two-phase flow from the ADS stages 1-to-3 is separated in a swirl-vane separator, and the liquid and vapor flows are measured to obtain the total ADS flow rate. The separated flow streams are then recombined and discharged into the IRWST through a sparger, preserving the mass and energy flow into the IRWST. The injection from the reactor sump is also simulated. Note that the OSU facility models both AP1000 primary and secondary sumps. The primary sump collects the condensate return, the liquid break flow, and the liquid flow from the fourth-stage ADS; and will provide long-term injection to the reactor vessel. The secondary sump simulates the portions of containment that will remain dry during most events. This sump collects water only when the primary sump reaches its overflow level, and provides no injection to the reactor vessel.

The time period for the experimental simulations includes not only the IRWST injection, but also the draining of the IRWST and the sump injection to simulate the long-term cooling of the AP1000. This simulation could be from several hours to a day. The time scale for the OSU test facility is one-half; that is, events occur in half the normal time. To model the long-term cooling aspects of the transient, the two-phase flow from the break is separated in a swirl-vane separator and the liquid and vapor portions of the total flow are measured. The liquid fraction of the flow is discharged to the reactor primary sump as in the AP1000 plant. The vapor is discharged to the atmosphere. The capability exists to return a portion of the equivalent liquid flow to the IRWST and primary sump to simulate the condensate return from the passive containment to the IRWST and primary sump. A similar approach is also used for the fourth-stage ADS valve on the hot leg. The two-phase flow is separated in a swirl-vane separator, the two streams are measured, and the liquid phase is discharged into the primary sump while the vapor flow is discharged to the atmosphere. Again, the capability exists to return a portion of the liquid equivalent added to the IRWST and primary sump. In addition, all other steam vents from the facility are measured (for example, the IRWST vent), and a portion of the liquid equivalent may be added back to the facility. Note that not all of the steam discharge would be returned as liquid equivalent. A portion of the discharge would be removed to simulate the steam that is not available for recirculation because it provides containment pressurization. The IRWST and primary sump can be pressurized in the OSU facility to simulate the containment pressurization following a postulated LOCA.

A multi-tube passive residual heat removal (PRHR) heat exchanger is located in the IRWST. The heat exchanger uses the same C-tube design as the AP1000 and has two instrumented tubes to obtain wall heat 
fluxes during the tests. There are primary fluid thermocouples, wall thermocouples, and differential pressure drop measurements to determine when the heat exchanger begins to drain. The IRWST is also instrumented with strings of fluid thermocouples to determine the degree of mixing within the tank and to assess the temperature of the coolant that is delivered to the test vessel.

The reactor vessel for the OSU tests includes a 0.914-meter (3-foot) heated core consisting of forty-eight 0.025 -meter (1-inch) diameter heater rods. The heater rods have a top skewed power shape. The $1000 \mathrm{~kW}$ of electrical power available at the OSU test site will be used to simulate decay heat. Wall thermocouples are swaged inside the heater rods to measure the heater rod wall temperature.

Thermocouple rods in the heater rod bundle measure the axial coolant temperature distribution. The scaled flow volume in the core is preserved as well as the flow volume in the test vessel upper plenum. There are simulated reactor internals in the upper plenum to preserve the flow area and to correctly scale the fluid volume. The reactor vessel includes an annular downcomer into which the four cold legs and the two DVI lines are connected. The hot legs penetrate the reactor annulus and connect with the loops. The AP1000 reactor vessel neutron reflector is simulated using a ceramic liner to reduce the metal heat release to the coolant.

There were no special/unique requirements for the test other than those specified in the Initial Conditions. The specified conditions were verified on the control board prior to test implementation.

\subsection{Instrumentation}

The instrumentation has been designed to calculate a transient mass and energy balance on the test facility. All two-phase flow streams exiting the facility are separated, and each component is measured separately as a single-phase flow using conventional measurement devices, such as magnetic flow meters and vortex flow meters. Note that magnetic flow meters are not designed for two-phase flow and will indicate erratically. Also, the vortex flow meters are referenced to $212^{\circ} \mathrm{F}$ and the LDPs are referenced to $60^{\circ} \mathrm{F}$. All vertical components have differential pressure cells that act as level instruments to measure the transient mass change in the component. The hot and cold leg diameters are sufficiently large in the OSU test facility so that a narrow-range differential pressure cell can be used to determine if the flow becomes stratified.

Single flow measurements are made of the CMT, accumulator, IRWST, and sump flows into the reactor vessel through the DVI lines.

Various types of instrumentation are provided in the test facility; for example, thermocouples for coolant and wall temperatures, flow meters, pressure transducers, differential pressure transducers, and weigh tanks.

\subsection{References}

3-1 APP-LTCT-T2R-005, OSU APEX-1000 Test Facility Description Report

3-2 APP-LTCT-T2R-004, Scaling Assessment for the Design of the OSU APEX-1000 Test Facility 


\subsection{OREGON STATE UNIVERSITY TESTING PROGRAM MATRIX}

The test matrix for the OSU test facility is shown in Table 4-1. To satisfy the test objectives, several transients will be performed to provide data on the AP1000 passive safety system response for a range of break sizes, locations, orientations, and single failure assumptions. The break size orifices are scaled based on simulating a 0.5 -inch, 1-inch, 2 -inch, or larger pipe break.

The designation for this test is TR-01-D, which identifies the test as an ADS-4 transition to IRWST injection test. The test matrix may be adjusted for future tests based on results and insights gained with each test. 


\begin{tabular}{|c|c|c|}
\hline \multicolumn{3}{|c|}{$\begin{array}{c}\text { TABLE 4-1 } \\
\text { OSU TEST MATRIX (AS SPECIFIED MARCH 18, 2003) }\end{array}$} \\
\hline Test Title & Break Location and Size & Single Failure Assumed \\
\hline DBA-01-D & $\begin{array}{l}\text { Double-ended DVI Line break with } \\
\text { continuation into long-term cooling }\end{array}$ & Fail 1 of 2 lines in one ADS- 4 train \\
\hline DBA-02-D & $\begin{array}{l}\text { Double-ended DVI Line break with } \\
\text { continuation into long-term cooling }\end{array}$ & $\begin{array}{l}\text { Fail } 1 \text { of } 2 \text { lines in one ADS- } 4 \text { train } \\
\text { (Adjusted ADS-4 Resistance) }\end{array}$ \\
\hline DBA-03-D & $\begin{array}{l}\text { Double-ended DVI Line break with } \\
\text { continuation into long-term cooling }\end{array}$ & $\begin{array}{l}\text { Fail } 1 \text { of } 2 \text { lines in one ADS- } 4 \text { train } \\
\text { (Failure location sensitivity) }\end{array}$ \\
\hline TR-01-D & $\begin{array}{l}\text { Transition Test ADS-4 opening, } 85 \text { psig initial } \\
\text { pressure and decay power } 480 \mathrm{sec}\end{array}$ & $\begin{array}{l}\text { Fail } 1 \text { of } 2 \text { lines in one ADS-4 train } \\
\text { (No ADS 1-3) }\end{array}$ \\
\hline TR-02-D & $\begin{array}{l}\text { Transition Test ADS-4 opening, } 100 \text { psig initial } \\
\text { pressure with prototypic plant conditions }\end{array}$ & $\begin{array}{l}\text { Fail } 1 \text { of } 2 \text { lines in one ADS-4 train } \\
\qquad \text { (No ADS 1-3) }\end{array}$ \\
\hline DBA-04-D & 2-inch Cold Leg Break & $\begin{array}{l}\text { Fail } 1 \text { of } 2 \text { lines in one ADS- } 4 \text { train } \\
\text { (Failure on non-pressurizer hot leg) }\end{array}$ \\
\hline ADS-01-D & $\begin{array}{l}\text { ADS-4 Two-Phase Characterization Test at } \\
\text { low-power conditions (steady-state points) }\end{array}$ & $\begin{array}{l}\text { Various ADS-4 line configurations } \\
\qquad \text { (No ADS 1-3) }\end{array}$ \\
\hline ADS-02-D & $\begin{array}{l}\text { ADS-4 Two-Phase Characterization Test at } \\
\text { high-power conditions (steady-state points) }\end{array}$ & $\begin{array}{l}\text { Various ADS-4 line configurations } \\
\qquad \text { (No ADS 1-3) }\end{array}$ \\
\hline DBA-03R-D & Repeat of Test DBA-03-D & $\begin{array}{l}\text { Fail } 1 \text { of } 2 \text { lines in one ADS- } 4 \text { train } \\
\text { (Failure on pressurizer hot leg) }\end{array}$ \\
\hline DBA-05-D & Inadvertent ADS-1 Actuation & $\begin{array}{l}\text { Fail } 1 \text { of } 2 \text { lines in one ADS- } 4 \text { train } \\
\text { (Failure on non-pressurizer hot leg) }\end{array}$ \\
\hline DBA-06-D & 0.5-inch Cold Leg Break & $\begin{array}{l}\text { Fail } 1 \text { of } 2 \text { lines in one ADS- } 4 \text { train } \\
\text { (Failure on non-pressurizer hot leg) }\end{array}$ \\
\hline
\end{tabular}




\subsection{TEST PROCEDURE}

The test was performed per a written procedure. There were no special/unique requirements for the test other than those specified in the initial conditions in Table 6-1. The specified conditions were checked on the control board before test implementation.

The appropriate prerequisites were completed and initial conditions were satisfied. The required break simulation piping and break instrumentation were installed per P\&ID drawing OSU 600904, Rev. 1. The 100-percent flow nozzle was installed in ADS 4-1 (on hot leg 1), and the 50-percent flow nozzle was installed in ADS 4-2 (on hot leg 2). Flow nozzles that simulate full flow for ADS-1, ADS-2, and ADS-3 were installed. As per the AP600 tests, ADS-3 has been scaled for full flow from all three stages, and ADS-1 and ADS-2 are closed when ADS-3 is opened. For this test, ADS 1-3 is closed for the duration of the test.

Fill and vent was performed per APEX Operations Manual Procedure OP-B.2. Instruments were checked for required calibration.

With the break valves TS-202 and TS-203 closed, flow was used to warm up the bypass line by opening isolation valves RCS-901 and RCS-902. After the appropriate prerequisites were completed and the test facility achieved specified initial conditions, the CMT warmup bypass line isolation valves RCS-901 and RCS-902 were closed to maintain the $<80^{\circ} \mathrm{F}$ condition at the top of the CMT-1. With the CMT balance line valves (RCS-529 and RCS-530) placed in the open and automatic mode, both CMTs reached the Reactor Coolant System pressure.

Once all other initial conditions were satisfied, ADS-4 was actuated. The test was terminated when the top of the core uncovered before IRWST injection was established. 


\subsection{TEST RESULTS}

The test results for test TR-01-D are provided in the following subsections.

\subsection{Initial and Boundary Conditions}

Table 6-1 provides a comparison of the specified and actual conditions for test TR-01-D. The values in this table were averaged over approximately 2 minutes preceding the test. Test initial conditions were achieved for the steam generator pressure, pressurizer pressure, pressurizer level, steam generator 01 narrow-range level, and steam generator 02 narrow-range level. Test initial conditions for the hot leg temperature were found to be acceptable, and the results will not be adversely affected.

The actual power curves for the heater rods are provided in data plots in Appendix B. The measured power for the test was $628 \mathrm{~kW}$, which was held constant for the duration of the test. The programmed decay heat curve was not used for this test.

The sequence of events is shown in Table 6-2. This table compares the actual sequence of events with the specified timing. As can be seen in this table, all the events occurred at or very near to when the event was planned.

\subsection{Inoperable Instruments}

Table 6-3 lists the instrumentation channels considered inoperable for the TR-01-D test.

\subsection{Key Data Plots}

Table 6-4 lists the instrumentation channels sorted by component and includes the instrument number, units, and quick look (QL) plot number. The selection of channels was based on projecting an overall picture of the test results, which would then be examined by referring to the detailed data plots or tapes.

\subsection{Test Evaluation}

The following observations were made during the test:

1. The heater rod power was held constant at $628 \mathrm{~kW}$ for the duration of the test.

2. The Reactor Coolant System pressure decreased rapidly once the ADS-4 valves were opened, but did not reach the cut-in pressure for IRWST injection before the test was terminated.

3. With no source of makeup water, the two-phase level in the upper plenum fell, reaching the top of the heater rods at approximately 494 seconds. At this time, the thermocouples at the top of the rods indicated that the rods were uncovered and the test was terminated. 


\begin{tabular}{|c|c|c|c|}
\hline \multicolumn{4}{|c|}{$\begin{array}{c}\text { TABLE 6-1 } \\
\text { ACTUAL TEST INITIAL CONDITIONS }\end{array}$} \\
\hline Conditions & Instrument No. & Actual & Comment \\
\hline Pressurizer Pressure & PT-604 & 85 psig & \\
\hline Hot Leg Temperature \#1 & $\begin{array}{l}\text { TF-141* } \\
\text { TF-205 } \\
\text { TF-143 }\end{array}$ & $\begin{array}{l}349^{\circ} \mathrm{F} \\
330^{\circ} \mathrm{F} \\
335^{\circ} \mathrm{F}\end{array}$ & \\
\hline Hot Leg Temperature $\# 2$ & $\begin{array}{l}\text { TF-140* } \\
\text { TF-206 } \\
\text { TF-142 }\end{array}$ & $\begin{array}{l}346^{\circ} \mathrm{F} \\
326^{\circ} \mathrm{F} \\
330^{\circ} \mathrm{F}\end{array}$ & \\
\hline $\begin{array}{l}\text { Steam Generator Pressure } \\
\quad \# 1 \\
\# 2 \\
\text { Header }\end{array}$ & $\begin{array}{l}\text { PT-301* } \\
\text { PT-302* } \\
\text { PT-002 }\end{array}$ & $\begin{array}{l}86 \text { psig } \\
82 \text { psig } \\
3.2 \text { psig }\end{array}$ & \\
\hline Pressurizer Level & $\begin{array}{l}\text { LDP-601 uncompensated } \\
\text { LDP-601 } \\
\text { Compensated by SC-608 }\end{array}$ & $\begin{array}{c}\text { Empty } \\
-\end{array}$ & \\
\hline $\begin{array}{l}\text { Steam Generator Level } \\
\# 1 \mathrm{NR}\end{array}$ & $\begin{array}{l}\text { LDP-303 uncompensated } \\
\text { LDP-303 compensated by } \\
\text { average of TF-305 and } \\
\text { TF-307 }\end{array}$ & $\begin{array}{l}22.5 \text { inches } \\
27.2 \text { inches }\end{array}$ & $\begin{array}{l}413^{\circ} \mathrm{F} \text { used for density } \\
\text { compensation }\end{array}$ \\
\hline $\begin{array}{l}\text { Steam Generator Level } \\
\# 2 \mathrm{NR}\end{array}$ & $\begin{array}{l}\text { LDP-304 uncompensated } \\
\text { LDP-304 compensated by } \\
\text { average of TF-306 and } \\
\text { TF-308 }\end{array}$ & $\begin{array}{l}22.2 \text { inches } \\
26 \text { inches }\end{array}$ & $\begin{array}{l}414^{\circ} \mathrm{F} \text { used for density } \\
\text { compensation }\end{array}$ \\
\hline IRWST Temperature & TF-701 & $60^{\circ} \mathrm{F}$ & Accepted $\left(<80^{\circ} \mathrm{F}\right)$ \\
\hline $\begin{array}{l}\text { CMT Temperature } \\
\text { \#1 } \\
\# 2\end{array}$ & $\begin{array}{l}\text { TF-513 } \\
\text { TF-516 }\end{array}$ & $\begin{array}{l}61.7^{\circ} \mathrm{F} \\
62.1^{\circ} \mathrm{F}\end{array}$ & Accepted $\left(<80^{\circ} \mathrm{F}\right)$ \\
\hline $\begin{array}{l}\text { Accumulator } \\
\text { Temperature } \\
\# 1 \\
\# 2\end{array}$ & $\begin{array}{l}\mathrm{TF}-403 \\
\mathrm{TF}-404\end{array}$ & $\begin{array}{l}75.8^{\circ} \mathrm{F} \\
76.5^{\circ} \mathrm{F}\end{array}$ & Accepted $\left(<80^{\circ} \mathrm{F}\right)$ \\
\hline IRWST Level & LDP-701 & 92.4 inches & \\
\hline
\end{tabular}




\begin{tabular}{|c|c|c|c|}
\hline \multicolumn{4}{|c|}{$\begin{array}{l}\text { TABLE 6-1 (Continued) } \\
\text { ACTUAL TEST INITIAL CONDITIONS }\end{array}$} \\
\hline Conditions & Instrument No. & Actual & Comment \\
\hline $\begin{array}{l}\text { Accumulator Level } \\
\text { \#1 } \\
\# 2\end{array}$ & $\begin{array}{l}\text { LDP-401 } \\
\text { LDP-402 }\end{array}$ & $\begin{array}{l}0 \text { inches } \\
0 \text { inches }\end{array}$ & \\
\hline $\begin{array}{l}\text { Accumulator Pressure } \\
\# 1 \\
\# 2\end{array}$ & $\begin{array}{l}\text { PT-401 } \\
\text { PT-402 }\end{array}$ & $\begin{array}{l}0 \text { psig } \\
0 \text { psig }\end{array}$ & \\
\hline $\begin{array}{l}\text { CMT Level } \\
\text { \#1 } \\
\# 2\end{array}$ & $\begin{array}{l}\text { LDP-507 } \\
\text { LDP-502 }\end{array}$ & $\begin{array}{l}56.9 \text { inches } \\
57.3 \text { inches }\end{array}$ & Isolated \\
\hline $\begin{array}{l}\text { CMT Pressure } \\
\text { \#1 } \\
\# 2\end{array}$ & $\begin{array}{l}\text { PT-501 } \\
\text { PT-502 }\end{array}$ & $\begin{array}{c}0.63 \mathrm{psig} \\
2.3 \mathrm{psig}\end{array}$ & \\
\hline
\end{tabular}

* These instruments are used to establish initial conditions at the test site. 


\begin{tabular}{|c|c|c|}
\hline \multicolumn{3}{|c|}{$\begin{array}{c}\text { TABLE 6-2 } \\
\text { SEQUENCE OF EVENTS }\end{array}$} \\
\hline Event & Setpoint & Actual Time (sec) \\
\hline PB Depressed & $\mathrm{N} / \mathrm{A}$ & -120 \\
\hline Break Valve(s) Open & No Break Modeled & - \\
\hline ADS 4-1 Actuation (RCS-615) & Manual & 15 \\
\hline ADS 4-2 Actuation (RCS-616) & $\begin{array}{l}\text { ADS 4-1 Actuation + } \\
30 \text { seconds }\end{array}$ & 45 \\
\hline $\begin{array}{l}\text { IRWST Injection } \\
\text { DVI \#1 (FMM-701) }\end{array}$ & N/A & Failed to reach injection \\
\hline $\begin{array}{l}\text { IRWST Injection } \\
\text { DVI \#2 (FMM-702) }\end{array}$ & N/A & Failed to reach injection \\
\hline Core Power Shutoff & $\begin{array}{c}\text { Heater Rod Temperature } \\
\text { Excursion }\end{array}$ & 168 \\
\hline
\end{tabular}




\begin{tabular}{|c|c|c|}
\hline \multicolumn{3}{|c|}{$\begin{array}{c}\text { TABLE 6-3 } \\
\text { INOPERABLE INSTRUMENTS FOR TR-01-D TEST }\end{array}$} \\
\hline Instrument Number & Instrument Type & Inoperable Description \\
\hline $\begin{array}{l}\text { TW-202 } \\
\text { TW-204 } \\
\text { TW-205 } \\
\text { TW-206 } \\
\text { TW-209 } \\
\text { TW-803 } \\
\text { TW-804 }\end{array}$ & Thermocouple & Inoperative \\
\hline TH-603 & $\begin{array}{l}\text { Thermocouple measuring heater } \\
\text { temperature }\end{array}$ & Inoperative \\
\hline FMM-202 & Magnetic flow meter & Inoperative \\
\hline $\begin{array}{l}\text { TF-170 } \\
\text { TF-221 } \\
\text { TF-509 } \\
\text { TF-512 }\end{array}$ & $\begin{array}{l}\text { Thermocouple measuring fluid } \\
\text { temperature }\end{array}$ & Inoperative \\
\hline FVM-905 & Vortex flow meter & Erratic \\
\hline
\end{tabular}




\begin{tabular}{|c|c|c|c|c|}
\hline \multicolumn{5}{|c|}{$\begin{array}{c}\text { TABLE 6-4 } \\
\text { DATA PLOTS FOR QL REPORTS FOR TR-01-D BY COMPONENT }\end{array}$} \\
\hline Component & Channel & Units & QL-Plot & Comment \\
\hline $\begin{array}{l}\text { Reactor Vessel } \\
\text { Pressure }\end{array}$ & PT-107 & psig & 1 & \\
\hline Reactor Vessel Level & LDP-127 & inch of $\mathrm{H}_{2} \mathrm{O}$ & 2 & \\
\hline $\begin{array}{l}\text { Reactor Vessel } \\
\text { Downcomer Level }\end{array}$ & LDP-140 & inch of $\mathrm{H}_{2} \mathrm{O}$ & 3 & \\
\hline $\begin{array}{l}\text { Cold Leg \#1 Fluid } \\
\text { Temperature }\end{array}$ & TF-107 & ${ }^{\circ} \mathrm{F}$ & 4 & \\
\hline $\begin{array}{l}\text { Cold Leg \#2 Fluid } \\
\text { Temperature }\end{array}$ & TF-108 & ${ }^{\circ} \mathrm{F}$ & 5 & \\
\hline $\begin{array}{l}\text { Cold Leg \#3 Fluid } \\
\text { Temperature }\end{array}$ & TF-103 & ${ }^{\circ} \mathrm{F}$ & 6 & \\
\hline $\begin{array}{l}\text { Cold Leg \#4 Fluid } \\
\text { Temperature }\end{array}$ & TF-104 & ${ }^{\circ} \mathrm{F}$ & 7 & \\
\hline $\begin{array}{l}\text { Reactor Vessel Fluid } \\
\text { Temperature Upper } \\
\text { Head }\end{array}$ & TF-120 & ${ }^{\circ} \mathrm{F}$ & 8 & \\
\hline $\begin{array}{l}\text { Reactor Coolant } \\
\text { System Hot Leg \#1 } \\
\text { Temperature }\end{array}$ & TF-143 & ${ }^{\circ} \mathrm{F}$ & 9 & \\
\hline $\begin{array}{l}\text { Reactor Coolant } \\
\text { System Hot Leg \#2 } \\
\text { Temperature }\end{array}$ & TF-142 & ${ }^{\circ} \mathrm{F}$ & 10 & \\
\hline Pressurizer Pressure & $\begin{array}{l}\text { PT-604 (WR) and PT-603 } \\
\text { (LP Indication) }\end{array}$ & psig & 11,12 & \\
\hline $\begin{array}{l}\text { Pressurizer Liquid } \\
\text { Level }\end{array}$ & LDP-601 & inch of $\mathrm{H}_{2} \mathrm{O}$ & 13 & $\begin{array}{l}\text { Sharp decrease followed } \\
\text { by rapid refill }\end{array}$ \\
\hline $\begin{array}{l}\text { Steam Generator \#1 } \\
\text { Tube Level }\end{array}$ & LDP-215 & inch of $\mathrm{H}_{2} \mathrm{O}$ & 14 & \\
\hline $\begin{array}{l}\text { Steam Generator \#1 } \\
\text { Secondary Pressure }\end{array}$ & PT-301 & psig & 15 & \\
\hline
\end{tabular}




\begin{tabular}{|c|c|c|c|c|}
\hline \multicolumn{5}{|c|}{$\begin{array}{c}\text { TABLE 6-4 (Continued) } \\
\text { DATA PLOTS FOR QL REPORTS FOR TR-01-D BY COMPONENT }\end{array}$} \\
\hline Component & Channel & Units & QL-Plot & Comment \\
\hline $\begin{array}{l}\text { Steam Generator \#1 } \\
\text { Feed Flow Rate }\end{array}$ & FMM-001 & gpm & 16 & \\
\hline $\begin{array}{l}\text { Steam Generator \#2 } \\
\text { Tube Level }\end{array}$ & LDP-218 & inch of $\mathrm{H}_{2} \mathrm{O}$ & 17 & \\
\hline $\begin{array}{l}\text { Steam Generator \#2 } \\
\text { Secondary Pressure }\end{array}$ & PT-302 & psig & 18 & \\
\hline $\begin{array}{l}\text { Accumulators \#1 and } \\
\text { \#2 Pressure }\end{array}$ & PT-401 and PT-402 & psig & 19,20 & \\
\hline $\begin{array}{l}\text { Accumulators \#1 and } \\
\text { \#2 Liquid Level }\end{array}$ & LDP-401 and LDP-402 & inch of $\mathrm{H}_{2} \mathrm{O}$ & 21,22 & \\
\hline $\begin{array}{l}\text { Accumulators \#1 and } \\
\text { \#2 Flow Rate }\end{array}$ & FMM-401 and FMM-402 & gpm & 23,24 & \\
\hline $\begin{array}{l}\text { Accumulators \#1 and } \\
\text { \#2 Liquid Discharge } \\
\text { Temperature }\end{array}$ & TF-401 and TF-402 & ${ }^{\circ} \mathrm{F}$ & 25,26 & \\
\hline $\begin{array}{l}\text { CMT \#1 and \#2 Liquid } \\
\text { Level }\end{array}$ & LDP-507 and LDP-502 & inch of $\mathrm{H}_{2} \mathrm{O}$ & 27,28 & \\
\hline $\begin{array}{l}\text { CMT \#1 and \#2 Flow } \\
\text { Rate }\end{array}$ & FMM-501 and FMM-504 & gpm & 29,30 & \\
\hline $\begin{array}{l}\text { CMT \#1 and \#2 Liquid } \\
\text { Temperature }\end{array}$ & $\begin{array}{l}\text { TF-501 and TF-529; and } \\
\text { TF-504 and TF-532 }\end{array}$ & ${ }^{\circ} \mathrm{F}$ & $31-34$ & \\
\hline PRHR Inlet Flow Rate & FMM-802 & gpm & 35 & \\
\hline PRHR Liquid Level & LDP-802 & inch of $\mathrm{H}_{2} \mathrm{O}$ & 36 & \\
\hline $\begin{array}{l}\text { PRHR Outlet Flow } \\
\text { Rate }\end{array}$ & FMM-804 & gpm & 37 & \\
\hline IRWST Liquid Level & LDP-701 & inch of $\mathrm{H}_{2} \mathrm{O}$ & 38 & \\
\hline $\begin{array}{l}\text { IRWST Discharge Line } \\
\# 1 \text { and \#2 Flow Rate }\end{array}$ & FMM-701 and FMM-702 & gpm & 39,40 & \\
\hline $\begin{array}{l}\text { IRWST Fluid } \\
\text { Temperature }\end{array}$ & TF-701 and TF-709 & ${ }^{\circ} \mathrm{F}$ & 41,42 & \\
\hline $\begin{array}{l}\text { ADS 1-3 Separator } \\
\text { Pressure }\end{array}$ & PT-605 & psig & 43 & \\
\hline
\end{tabular}




\begin{tabular}{|c|c|c|c|c|}
\hline \multicolumn{5}{|c|}{$\begin{array}{l}\text { TABLE 6-4 (Continued) } \\
\text { DATA PLOTS FOR QL REPORTS FOR TR-01-D BY COMPONENT }\end{array}$} \\
\hline Component & Channel & Units & QL-Plot & Comment \\
\hline $\begin{array}{l}\text { ADS 1-3 Separator } \\
\text { Steam Flow Rate }\end{array}$ & FVM-601 & $\operatorname{scfm}$ & 44 & \\
\hline $\begin{array}{l}\text { ADS 1-3 Separator } \\
\text { Liquid Flow Rate }\end{array}$ & FMM-601 & gpm & 45 & \\
\hline $\begin{array}{l}\text { ADS-4 Separator \#1 } \\
\text { and \#2 Pressure }\end{array}$ & PT-611 and PT-610 & psig & 46,47 & \\
\hline $\begin{array}{l}\text { ADS 4-1 Separator } \\
\text { Steam Flow Rate }\end{array}$ & FVM-603 & $\mathrm{scfm}$ & 48 & \\
\hline $\begin{array}{l}\text { ADS 4-2 Separator } \\
\text { Steam Flow Rate }\end{array}$ & FVM-602 & $\mathrm{scfm}$ & 49 & \\
\hline $\begin{array}{l}\text { ADS 4-1 Separator } \\
\text { Liquid Flow Rate }\end{array}$ & FMM-603 & gpm & 50 & \\
\hline $\begin{array}{l}\text { ADS 4-2 Separator } \\
\text { Liquid Flow Rate }\end{array}$ & FMM-602 & gpm & 51 & \\
\hline Primary Sump Pressure & PT-901 & psig & 52 & \\
\hline $\begin{array}{l}\text { Primary Sump Liquid } \\
\text { Level }\end{array}$ & LDP-901 & inch of $\mathrm{H}_{2} \mathrm{O}$ & 53 & \\
\hline $\begin{array}{l}\text { Primary Sump } \\
\text { Injection Flow Rate }\end{array}$ & FMM-901 and FMM-902 & gpm & 54,55 & \\
\hline $\begin{array}{l}\text { Secondary Sump } \\
\text { Liquid Level }\end{array}$ & LDP-902 & inch of $\mathrm{H}_{2} \mathrm{O}$ & 56 & \\
\hline $\begin{array}{l}\text { Break Separator } \\
\text { Pressure }\end{array}$ & PT-905 & psig & 57 & \\
\hline $\begin{array}{l}\text { Break Separator Liquid } \\
\text { Level }\end{array}$ & LDP-905 & inch of $\mathrm{H}_{2} \mathrm{O}$ & 58 & \\
\hline $\begin{array}{l}\text { Break Separator Flow } \\
\text { to Primary Sump }\end{array}$ & FMM-905 & gpm & 59 & \\
\hline $\begin{array}{l}\text { BAMS Steam Flow } \\
\text { Rate }\end{array}$ & FVM-901 & $\mathrm{scfm}$ & 60 & \\
\hline $\begin{array}{l}\text { BAMS Steam Flow } \\
\text { Rate }\end{array}$ & FVM-902 & $\mathrm{scfm}$ & 61 & \\
\hline
\end{tabular}




\begin{tabular}{|c|c|c|c|c|}
\hline \multicolumn{5}{|c|}{$\begin{array}{c}\text { TABLE 6-4 (Continued) } \\
\text { DATA PLOTS FOR QL REPORTS FOR TR-01-D BY COMPONENT }\end{array}$} \\
\hline Component & Channel & Units & QL-Plot & Comment \\
\hline $\begin{array}{l}\text { BAMS/Primary Sump } \\
\text { Steam Flow Rate }\end{array}$ & FVM-903 & $\mathrm{scfm}$ & 62 & \\
\hline $\begin{array}{l}\text { BAMS/Separator } \\
\text { Steam Flow Rate - } \\
\text { 6-inch Pipe }\end{array}$ & FVM-905 & $\mathrm{scfm}$ & 63 & \\
\hline $\begin{array}{l}\text { BAMS/Exhaust Line } \\
\text { Temperature }\end{array}$ & TF-916 and SC-917 & ${ }^{\circ} \mathrm{F}$ & 64,65 & \\
\hline $\begin{array}{l}\text { Pressurizer Heater } \\
\text { Input Power }\end{array}$ & KW-601 & $\mathrm{kW}$ & 66 & \\
\hline $\begin{array}{l}\text { Core Power Input } \\
\text { Power }\end{array}$ & $\begin{array}{l}\text { KW-101, KW-102, } \\
\text { KW-103, and KW-104 }\end{array}$ & $\mathrm{kW}$ & $67-70$ & \\
\hline $\begin{array}{l}\text { Reactor Vessel Liquid } \\
\text { Level Between Top of } \\
\text { Vessel - Upper Support } \\
\text { Plate }\end{array}$ & LDP-115 & inch of $\mathrm{H}_{2} \mathrm{O}$ & 71 & \\
\hline $\begin{array}{l}\text { Reactor Vessel Liquid } \\
\text { Level Between Bottom } \\
\text { of Upper Support } \\
\text { Plate - Upper Core } \\
\text { Spacer Grid }\end{array}$ & LDP-139 & inch of $\mathrm{H}_{2} \mathrm{O}$ & 72 & \\
\hline $\begin{array}{l}\text { Inner Core } \\
\text { Thermocouple } \\
\text { Measuring Heater } \\
\text { Temperature }\end{array}$ & TH-103-4 & ${ }^{\circ} \mathrm{F}$ & 73 & \\
\hline $\begin{array}{l}\text { Outer Core } \\
\text { Thermocouple } \\
\text { Measuring Heater } \\
\text { Temperature }\end{array}$ & TH-309-4 & ${ }^{\circ} \mathrm{F}$ & 74 & \\
\hline
\end{tabular}




\subsection{CONCLUSIONS}

The TR-01-D test was successfully completed, and the data was logged in the DAS. All critical instruments were found to operate properly with the exception of those noted in Section 6.4. The test was acceptable. 
APPENDIX A

DATA PLOTS 

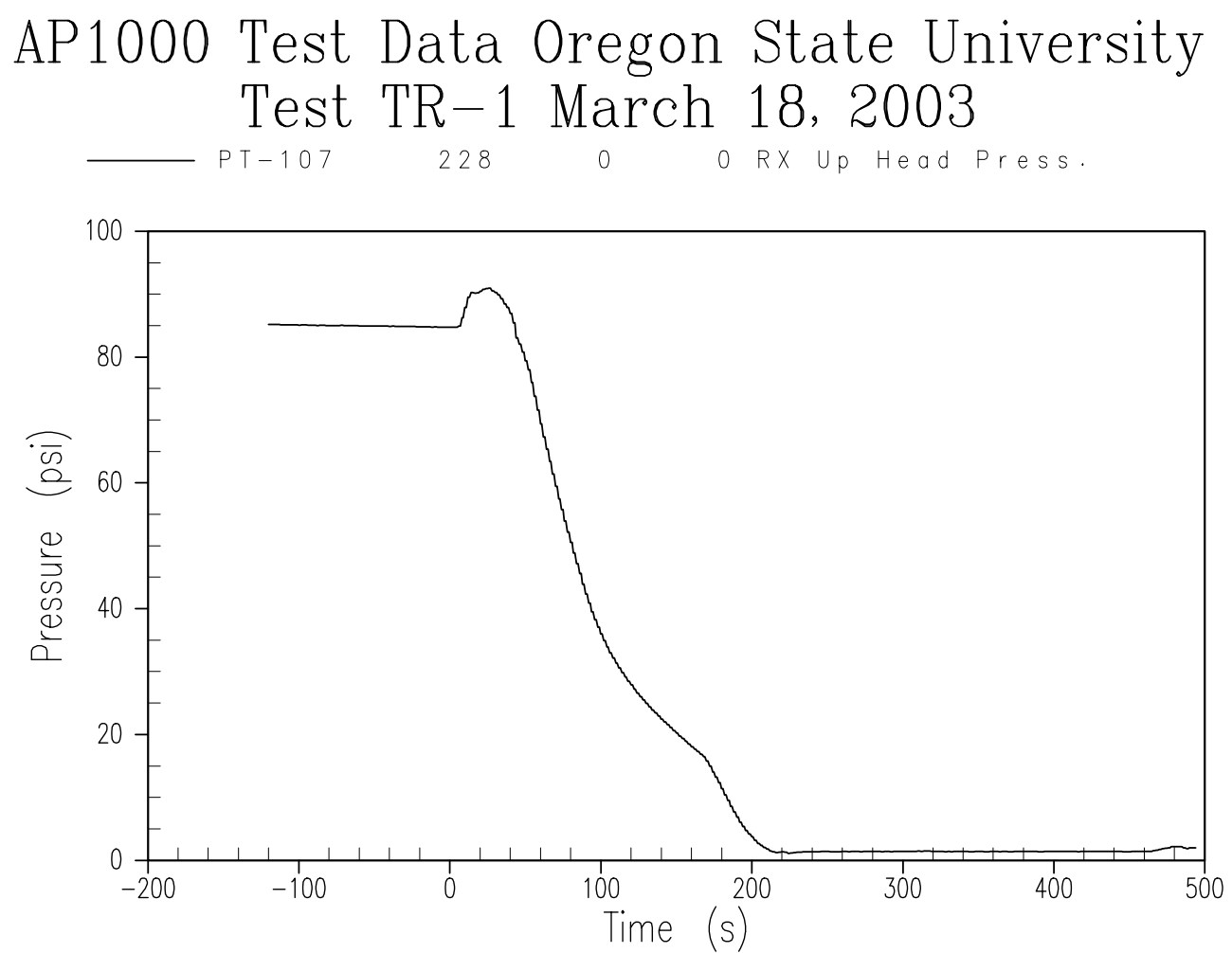

Figure A-1 Reactor Vessel Pressure 

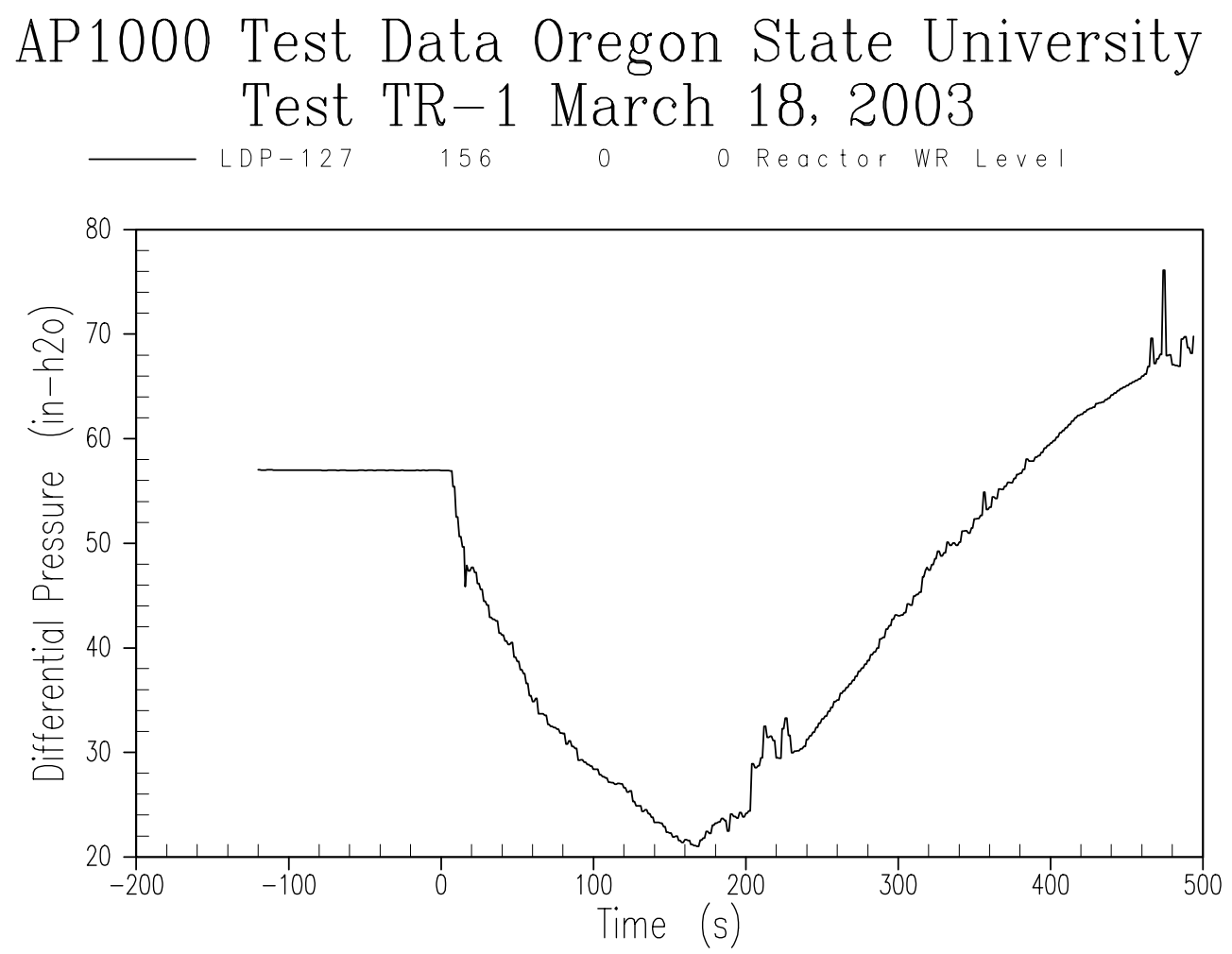

Figure A-2 Reactor Vessel Level 

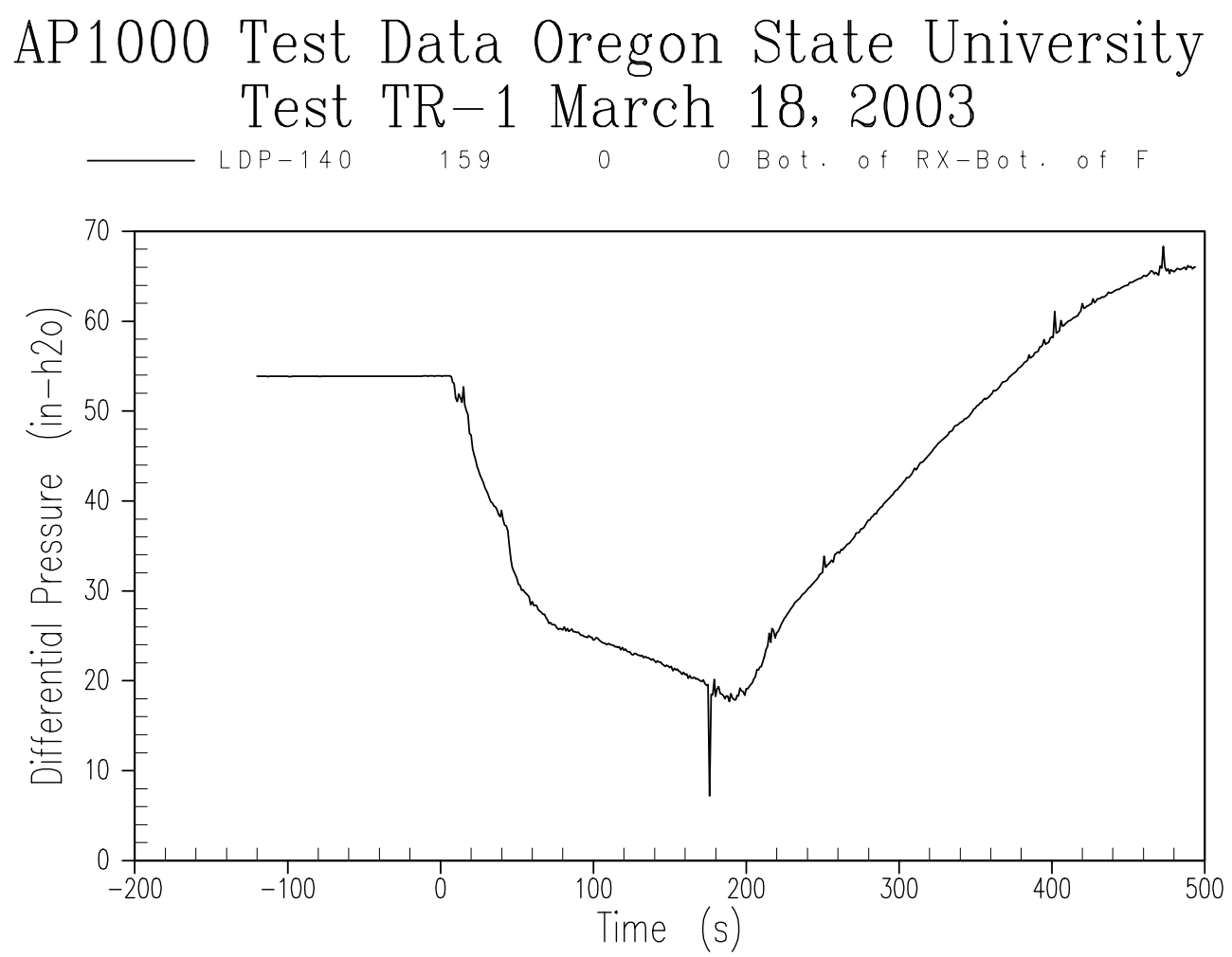

Figure A-3 Reactor Vessel Downcomer Level 
AP1000 Test Data Oregon State University Test TR-1 March 18, 2003

$\longrightarrow$ TF -107

323

0

o $C L-1 / R X F I$ ange @ Bot

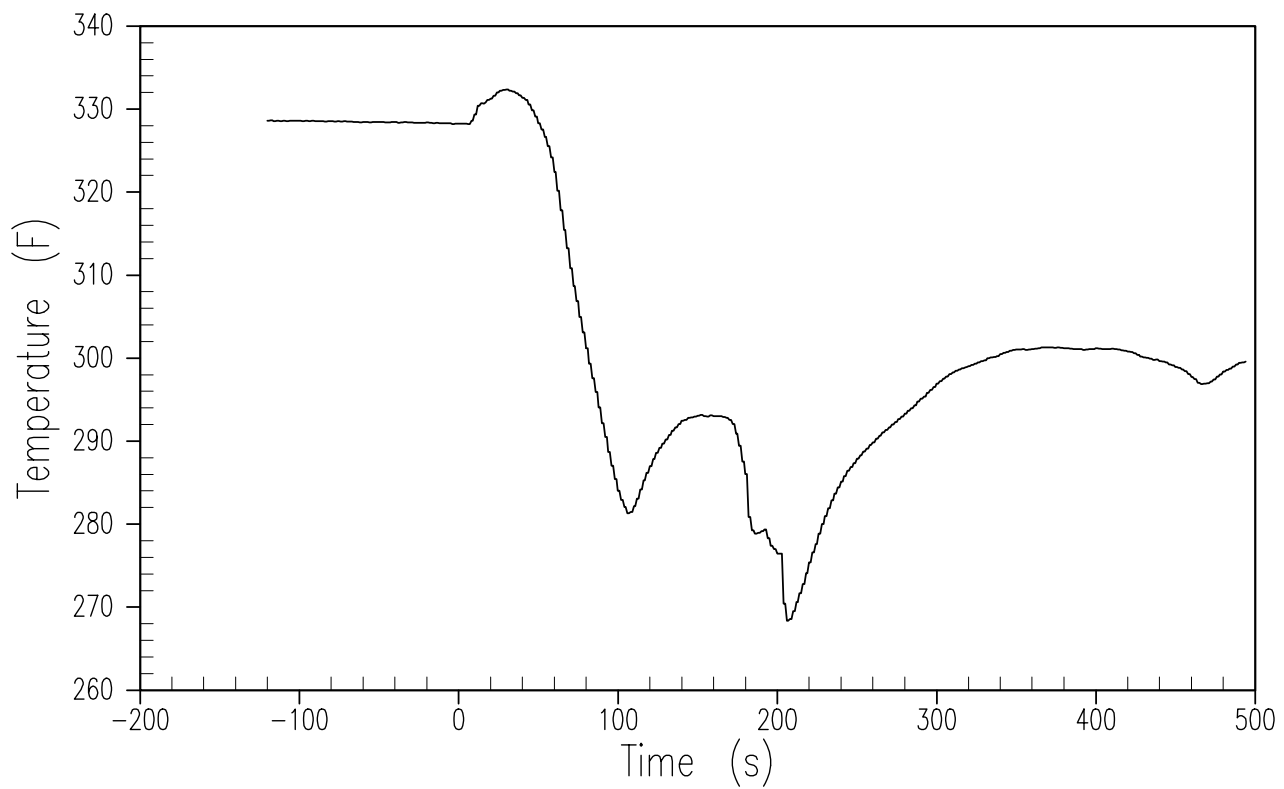

Figure A-4 Cold Leg 1 Fluid Temperature 
AP1000 Test Data Oregon State University Test TR-1 March 18, 2003

$\longrightarrow T F-108$

324

0

o $C L-2 / R X F I$ ange @ Bot

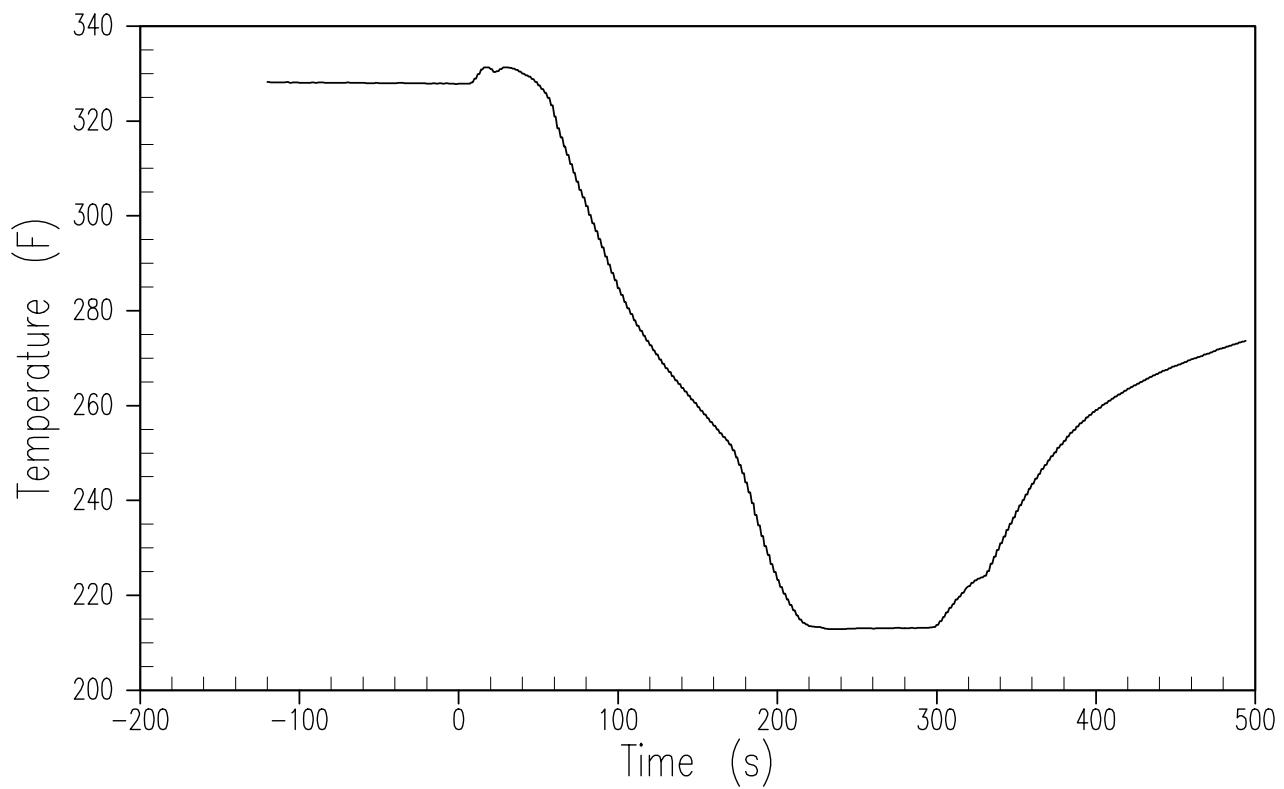

Figure A-5 Cold Leg 2 Fluid Temperature 

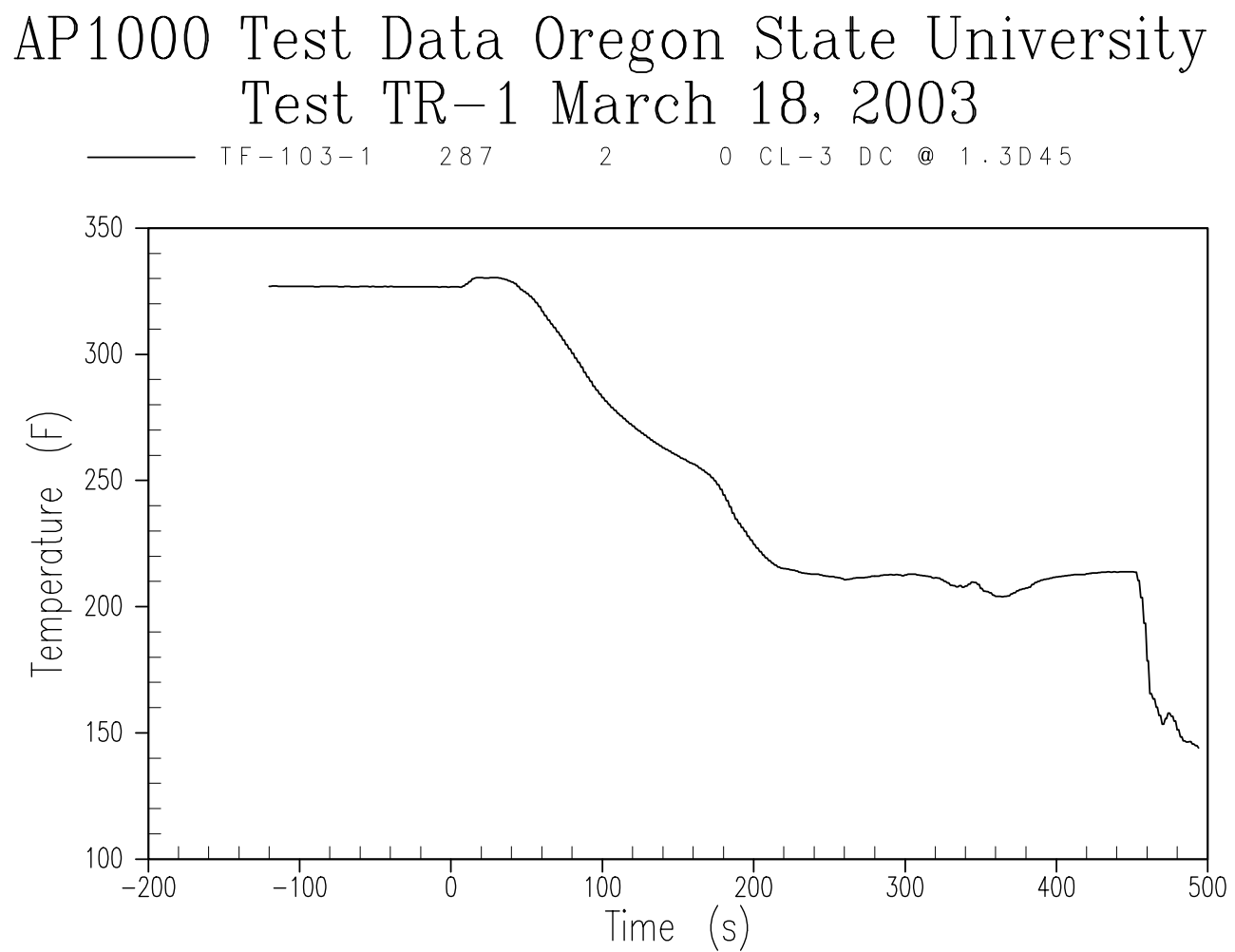

Figure A-6 Cold Leg 3 Fluid Temperature 
AP1000 Test Data Oregon State University Test TR-1 March 18, 2003
TF $-104-1$
298
0
O CL-4 DC Wa|| @ $1.5 \mathrm{D}$

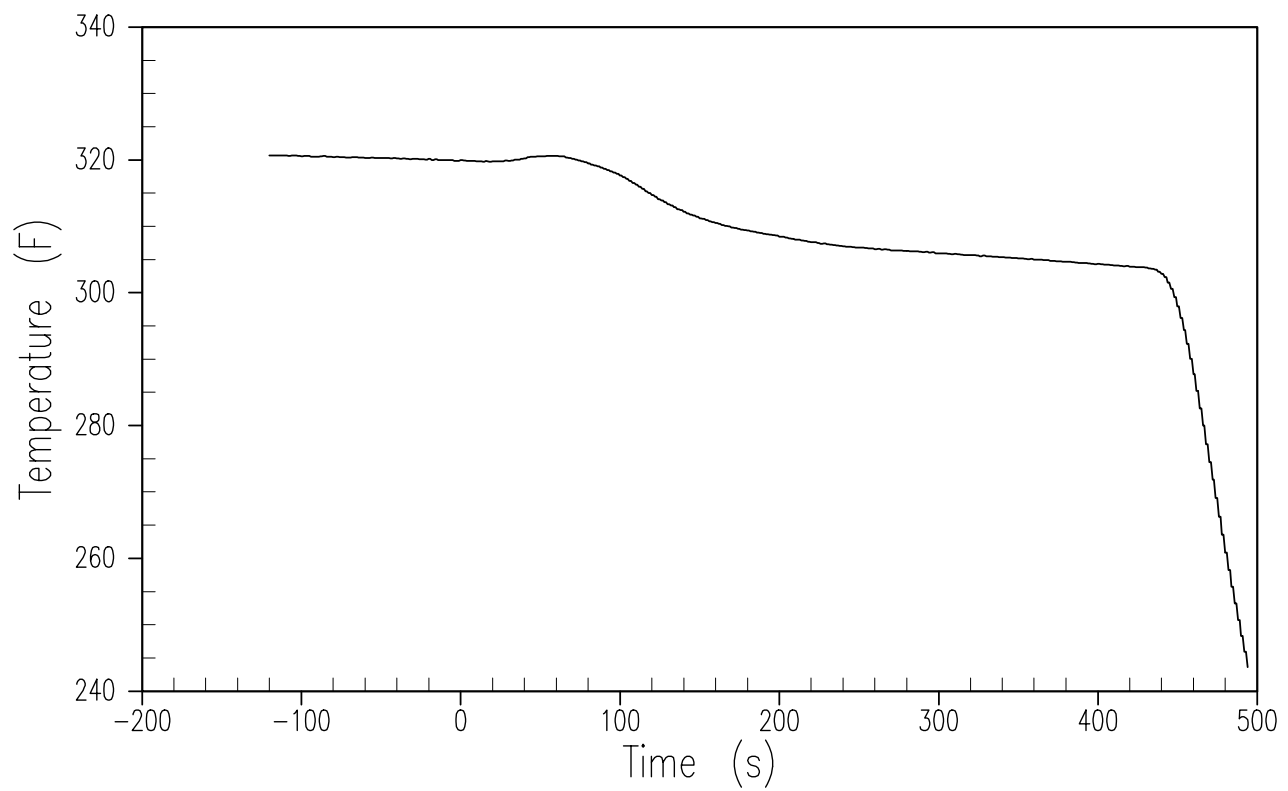

Figure A-7 Cold Leg 4 Fluid Temperature 
AP1000 Test Data Oregon State University Test TR-1 March 18, 2003

$\longrightarrow$

330

0

o Top of RX @ 8.5" \& 3

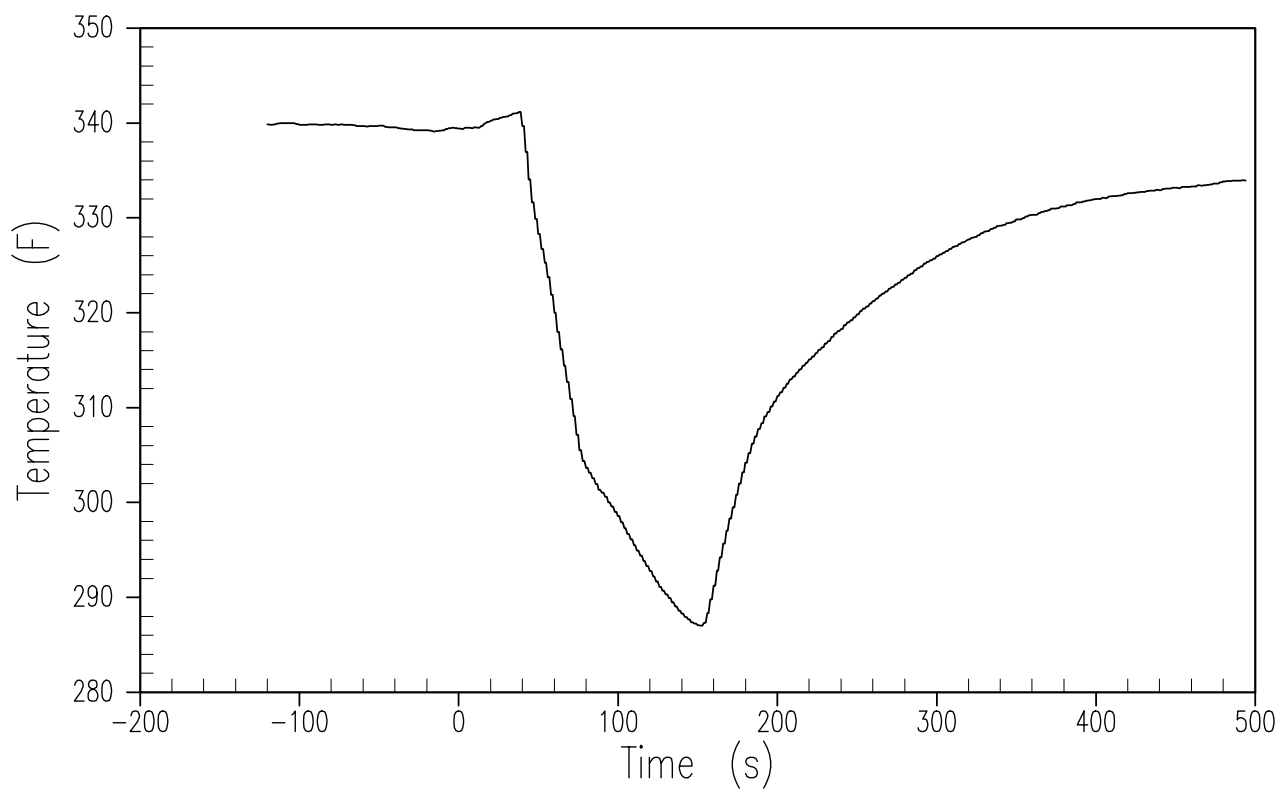

Figure A-8 Reactor Vessel Fluid Temperature Upper Head 
AP1000 Test Data Oregon State University Test TR-1 March 18, 2003

$\longrightarrow$ TF -143

344

0

o $H L-1 / R X F I$ ange @ Bot

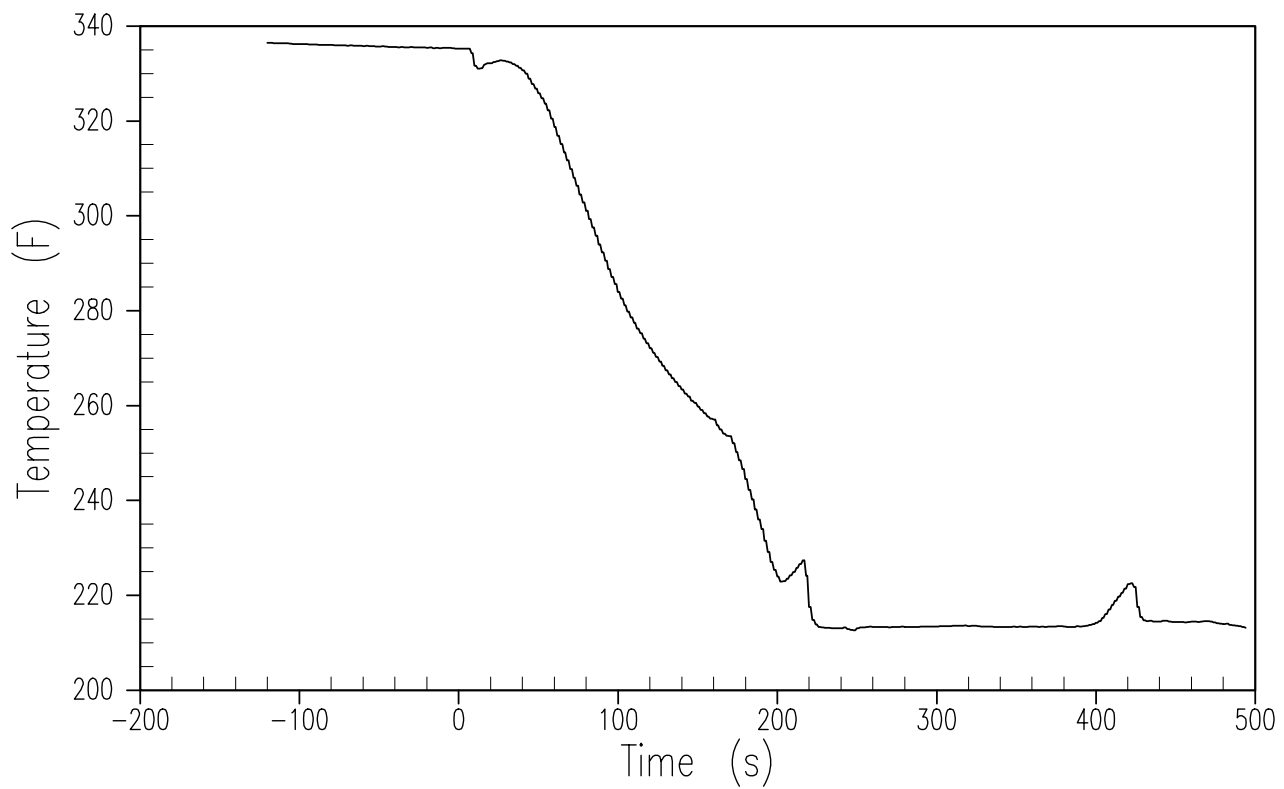

Figure A-9 Reactor Coolant System Hot Leg 1 Temperature 
AP1000 Test Data Oregon State University Test TR-1 March 18, 2003

$-T F-142$

343

0

o $H L-2 / R X F I$ ange @ Bot

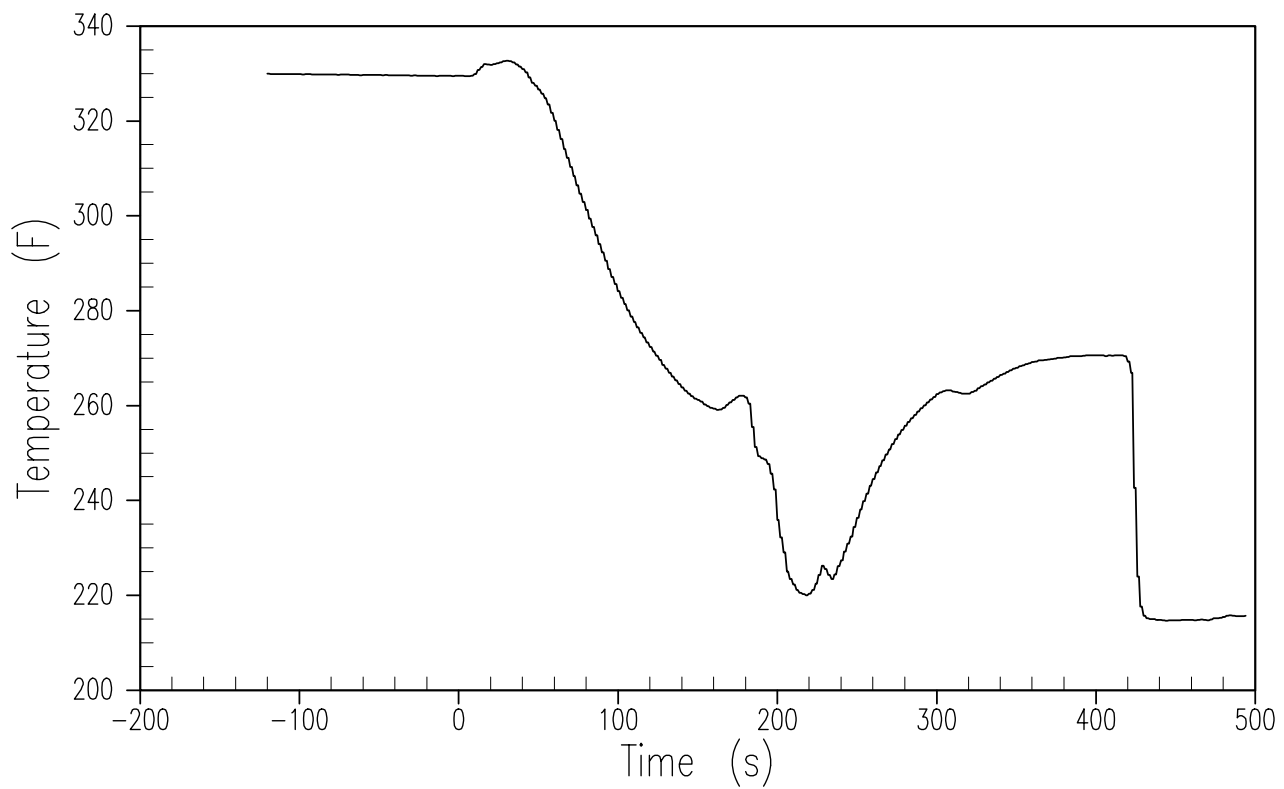

Figure A-10 Reactor Coolant System Hot Leg 2 Temperature 

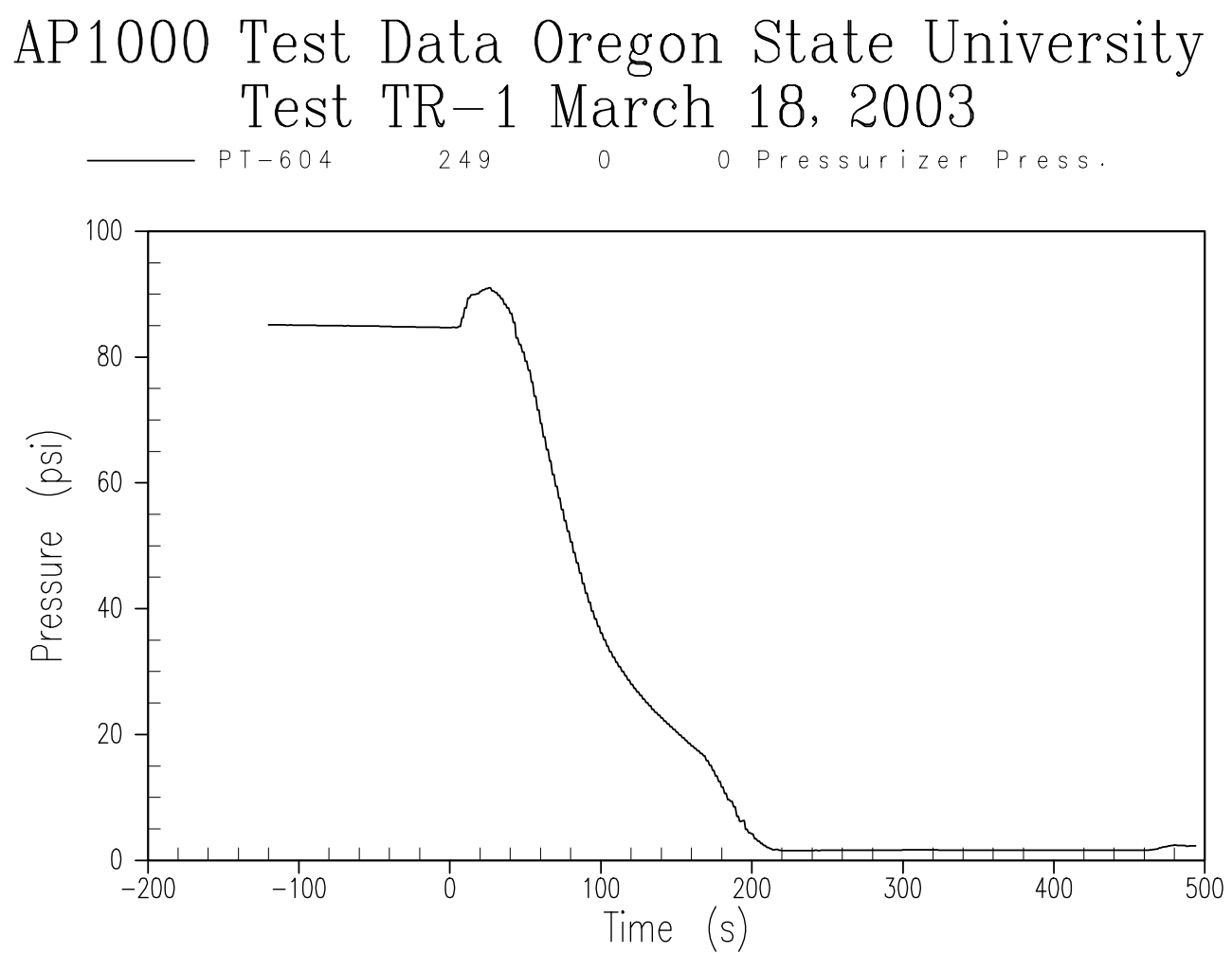

Figure A-11 Pressurizer Pressure - Wide Range 

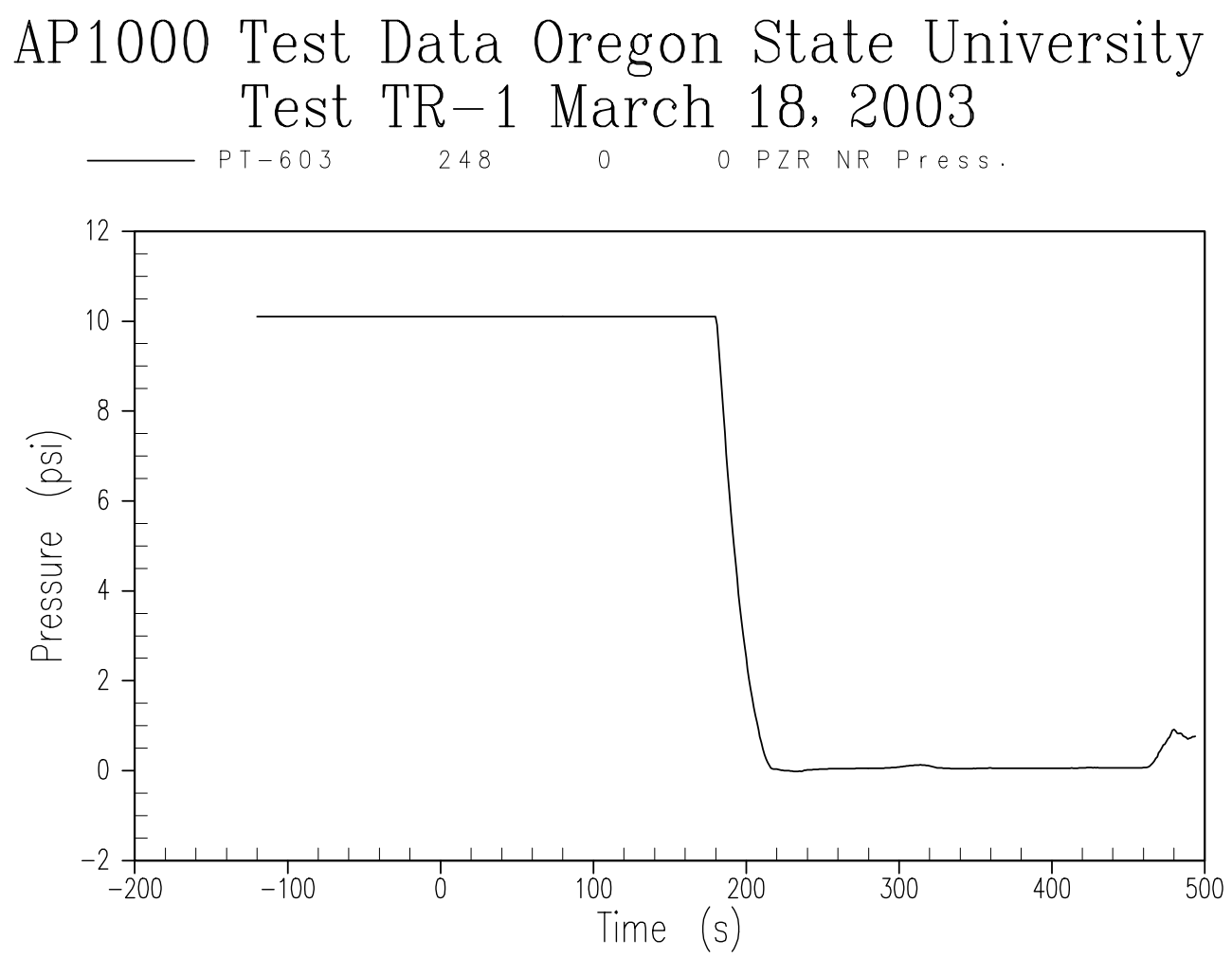

Figure A-12 Pressurizer Pressure - Narrow Range 

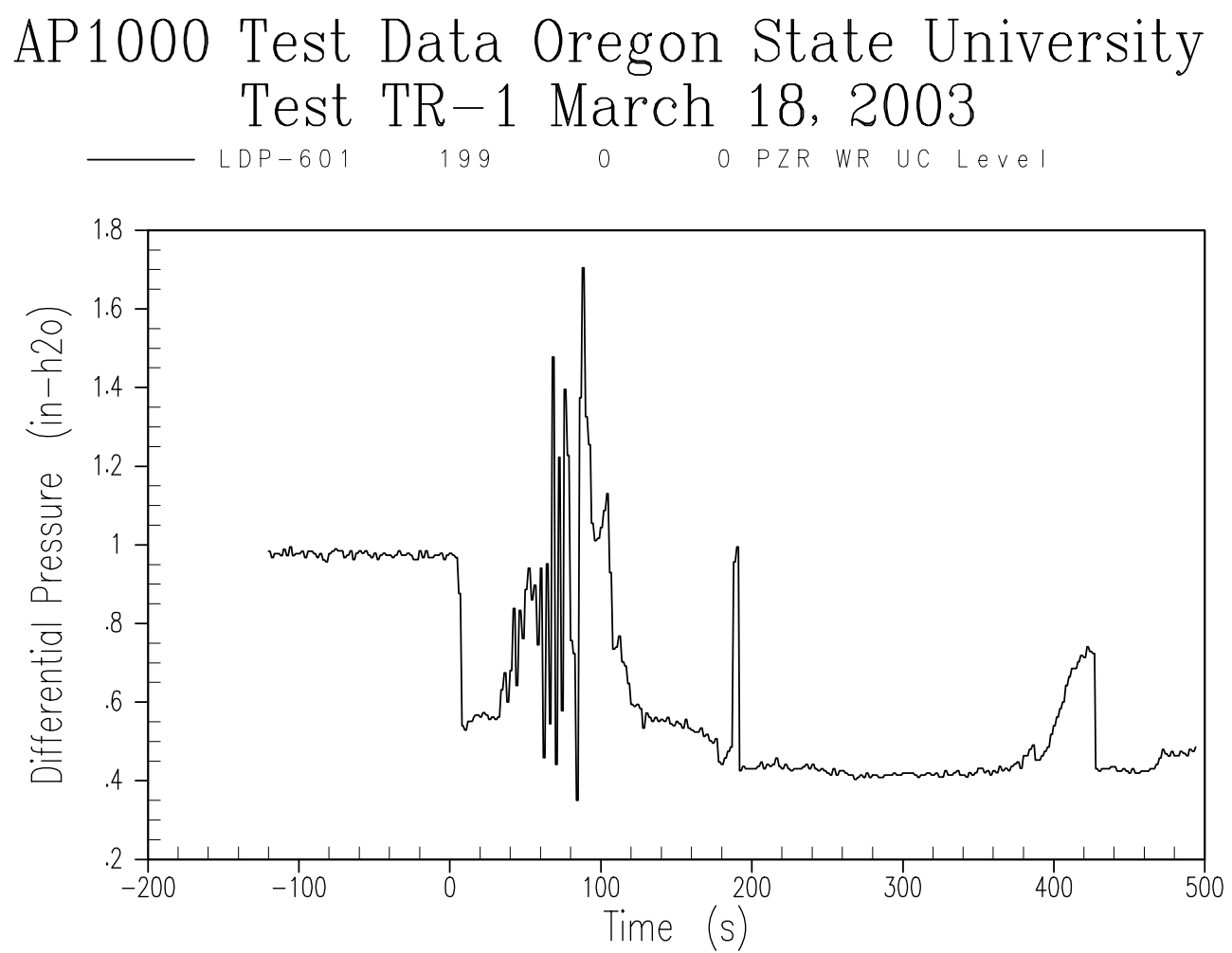

Figure A-13 Pressurizer Liquid Level 

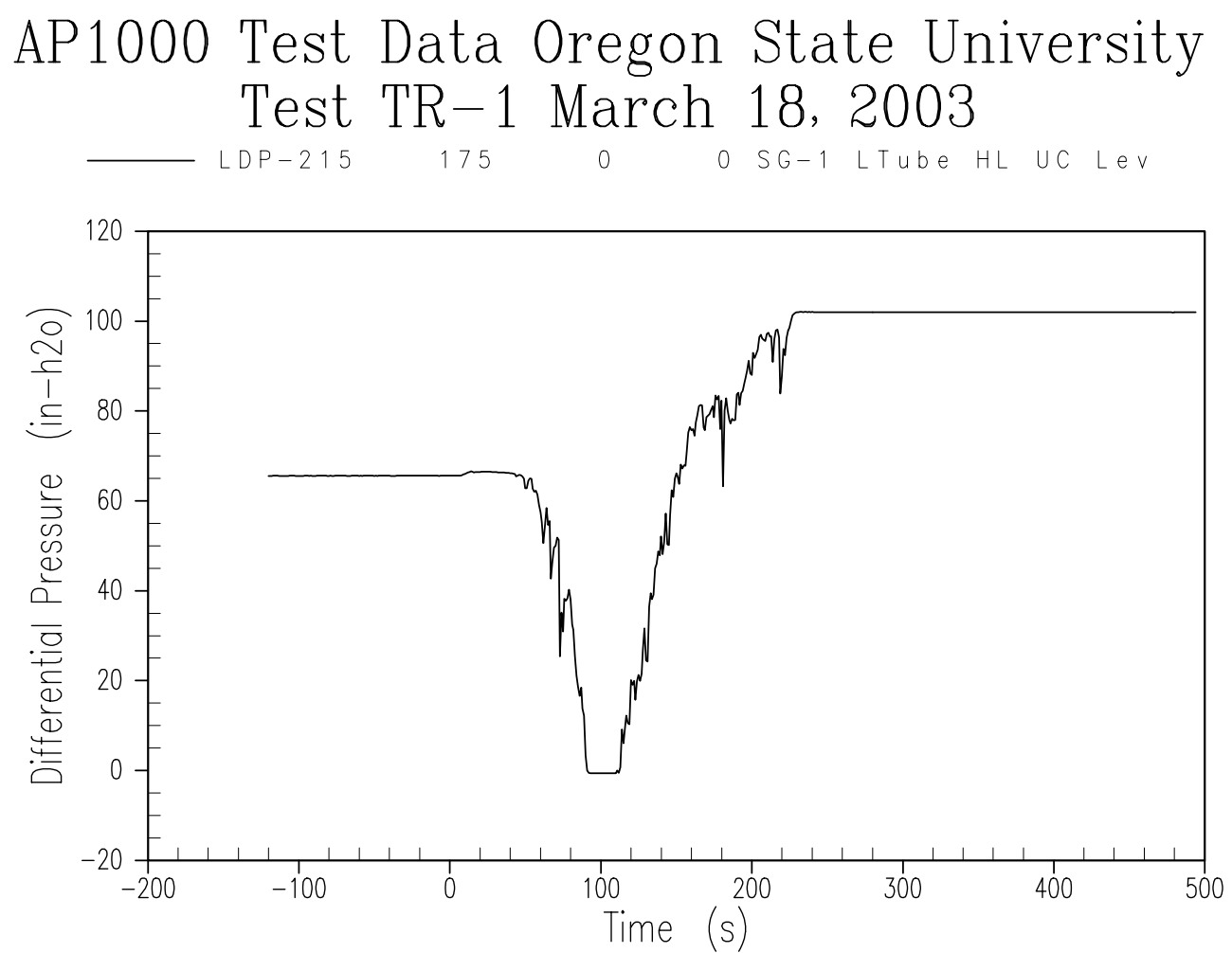

Figure A-14 Steam Generator 1 Tube Level 

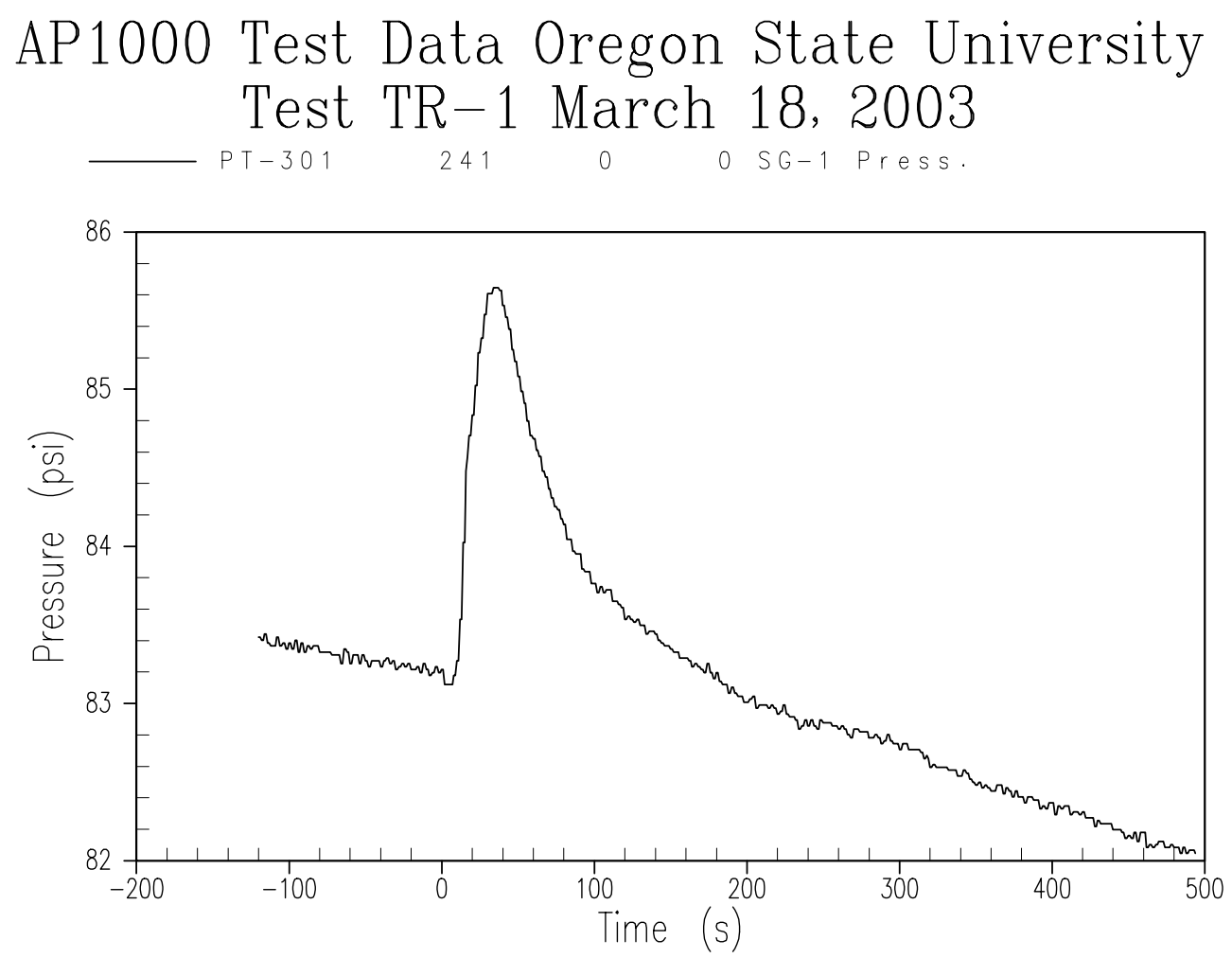

Figure A-15 Steam Generator 1 Secondary Pressure 

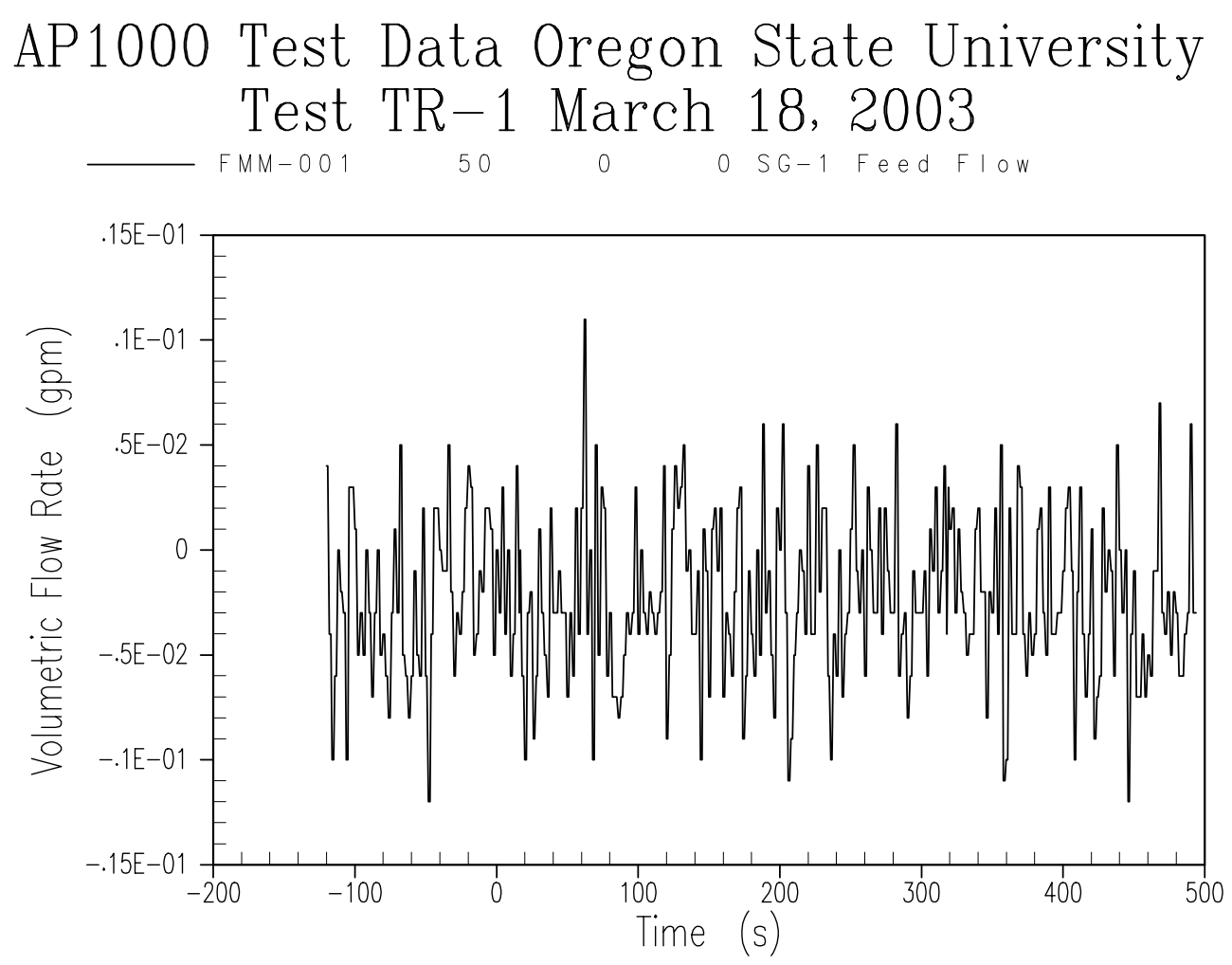

Figure A-16 Steam Generator 1 Feed Flow Rate 

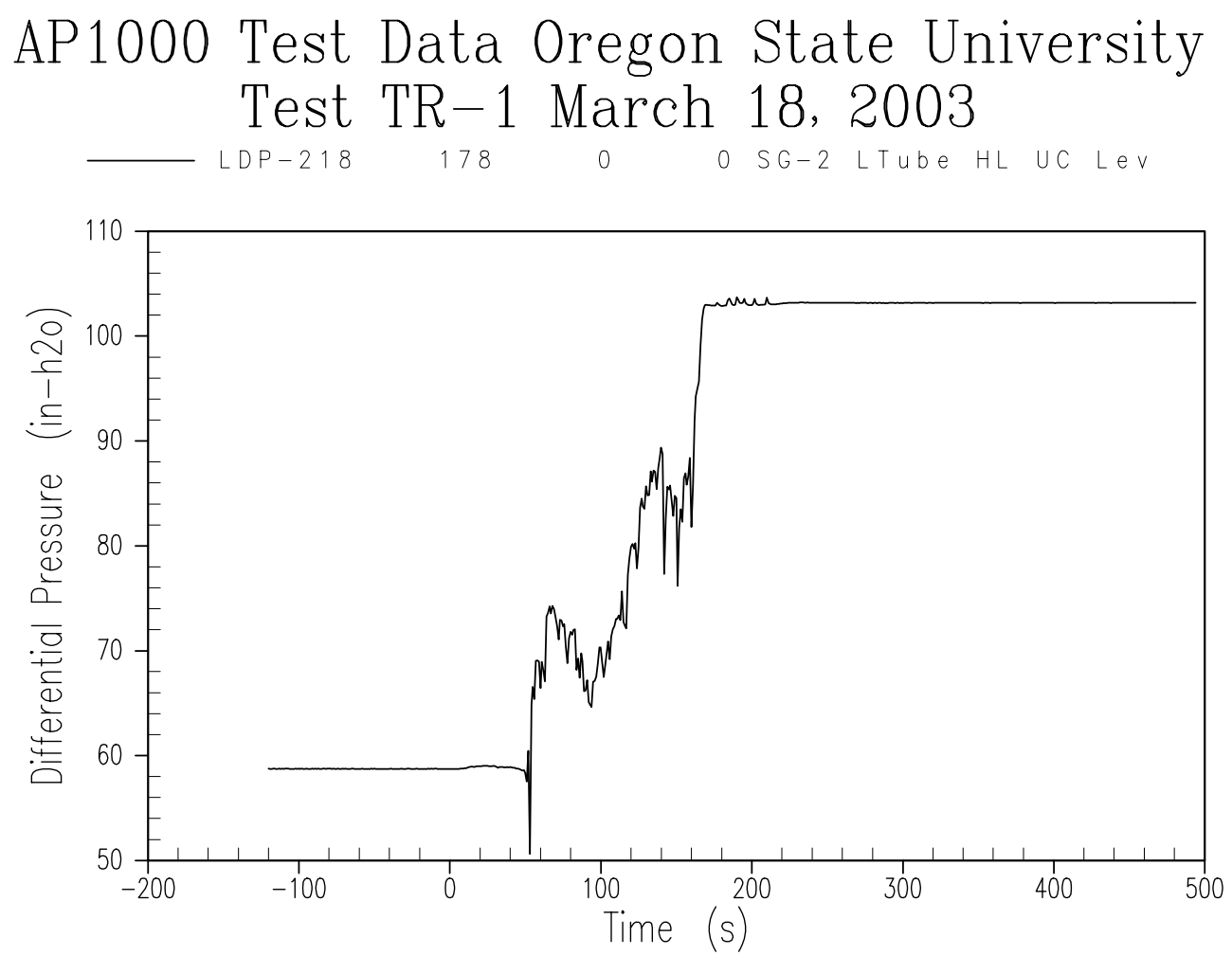

Figure A-17 Steam Generator 2 Tube Level 

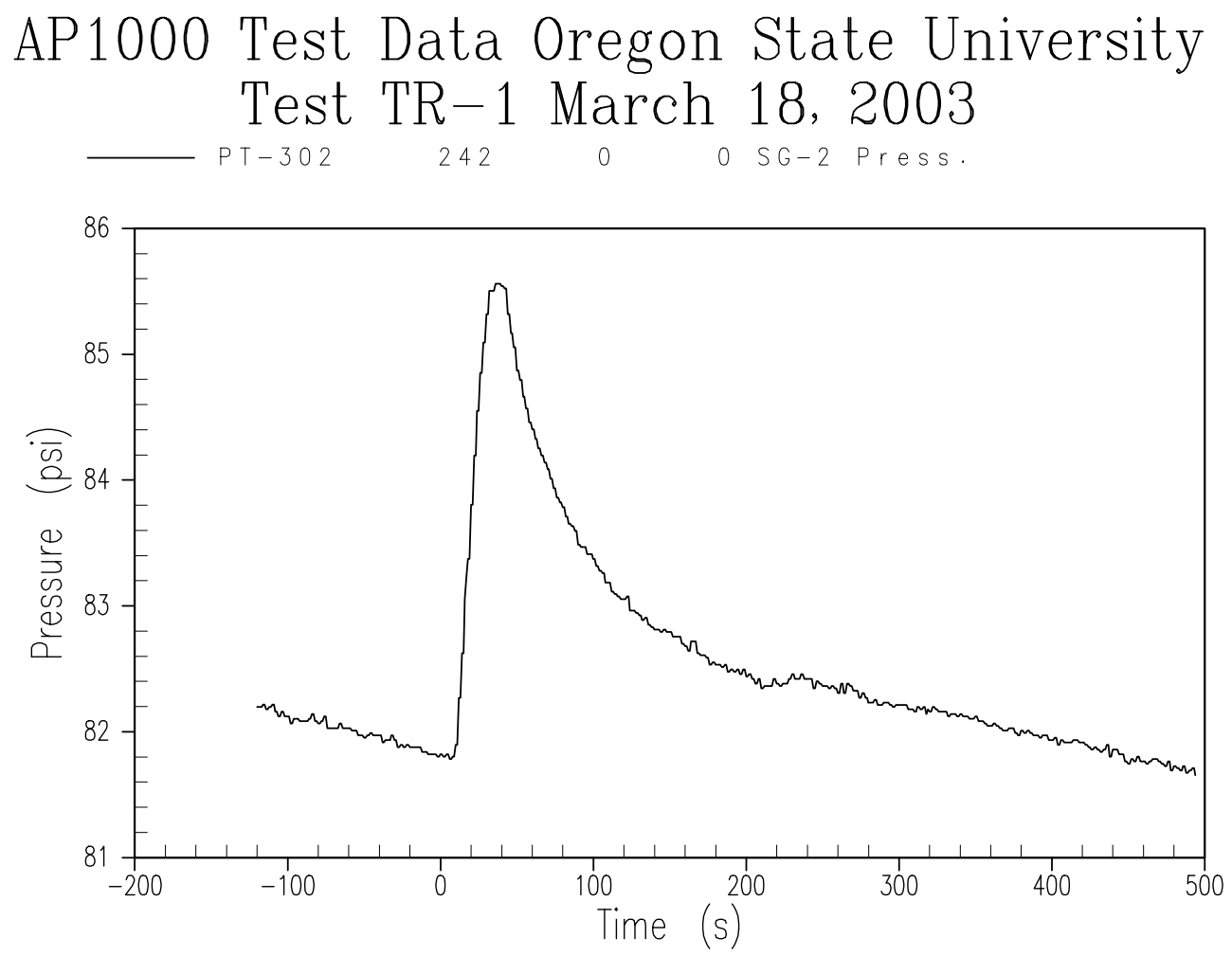

Figure A-18 Steam Generator 2 Secondary Pressure 


\section{AP1000 Test Data Oregon State University Test TR-1 March 18, 2003}

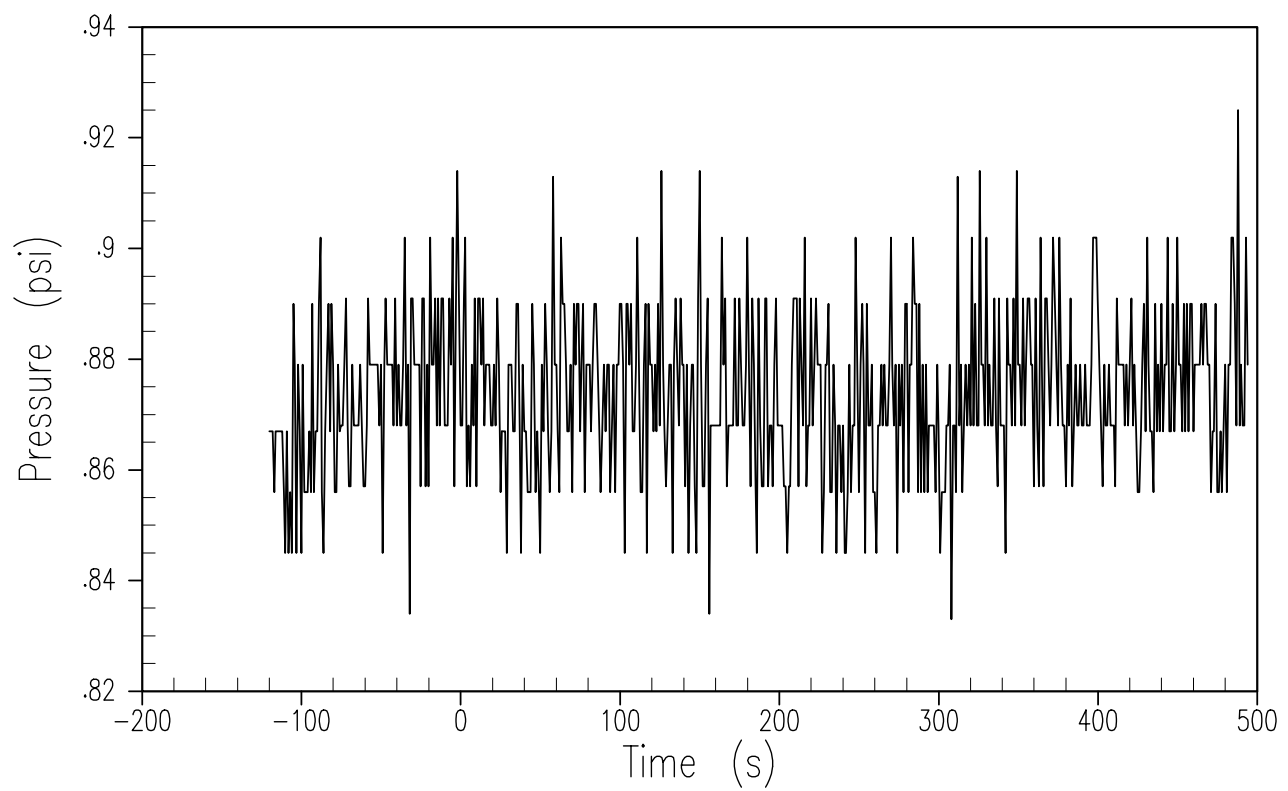

Figure A-19 Accumulator 1 Pressure 


\section{AP1000 Test Data Oregon State University Test TR-1 March 18, 2003}

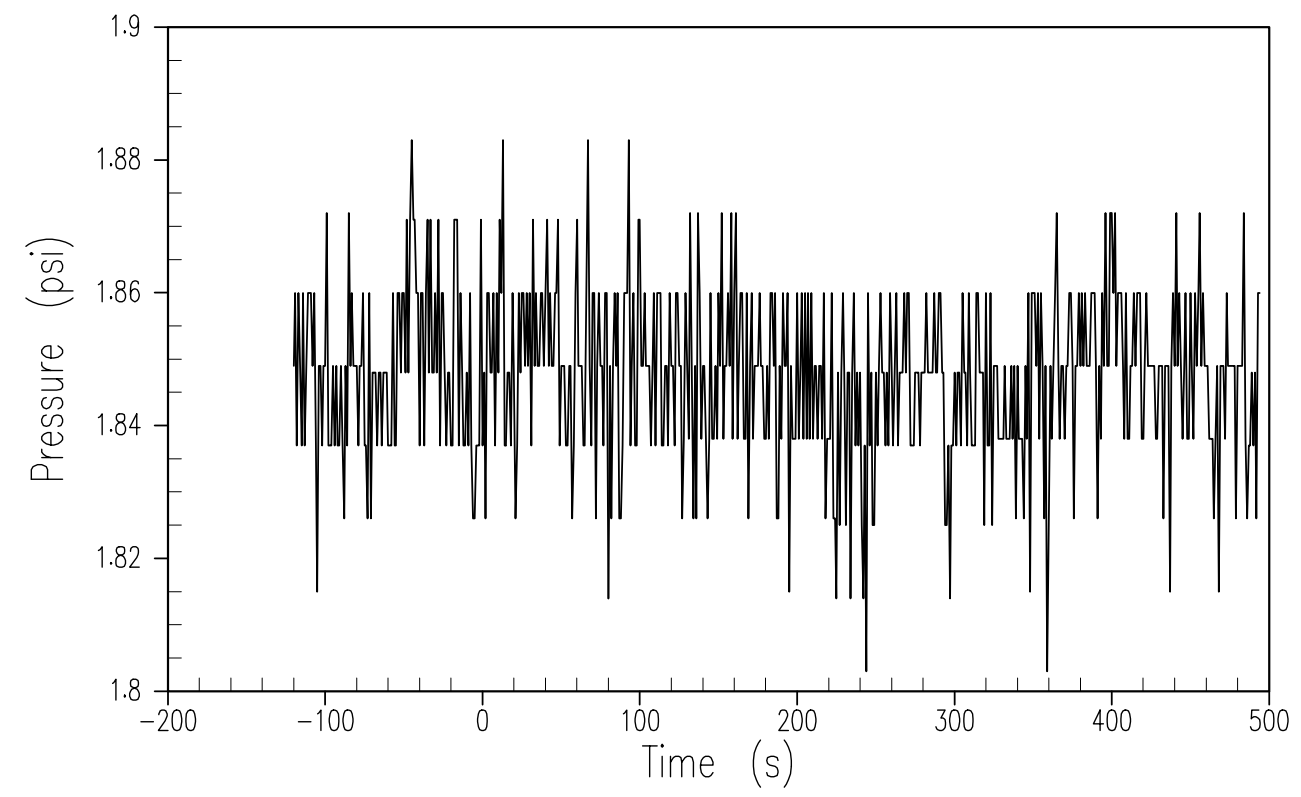

Figure A-20 Accumulator 2 Pressure 


\section{AP1000 Test Data Oregon State University Test TR-1 March 18, 2003}

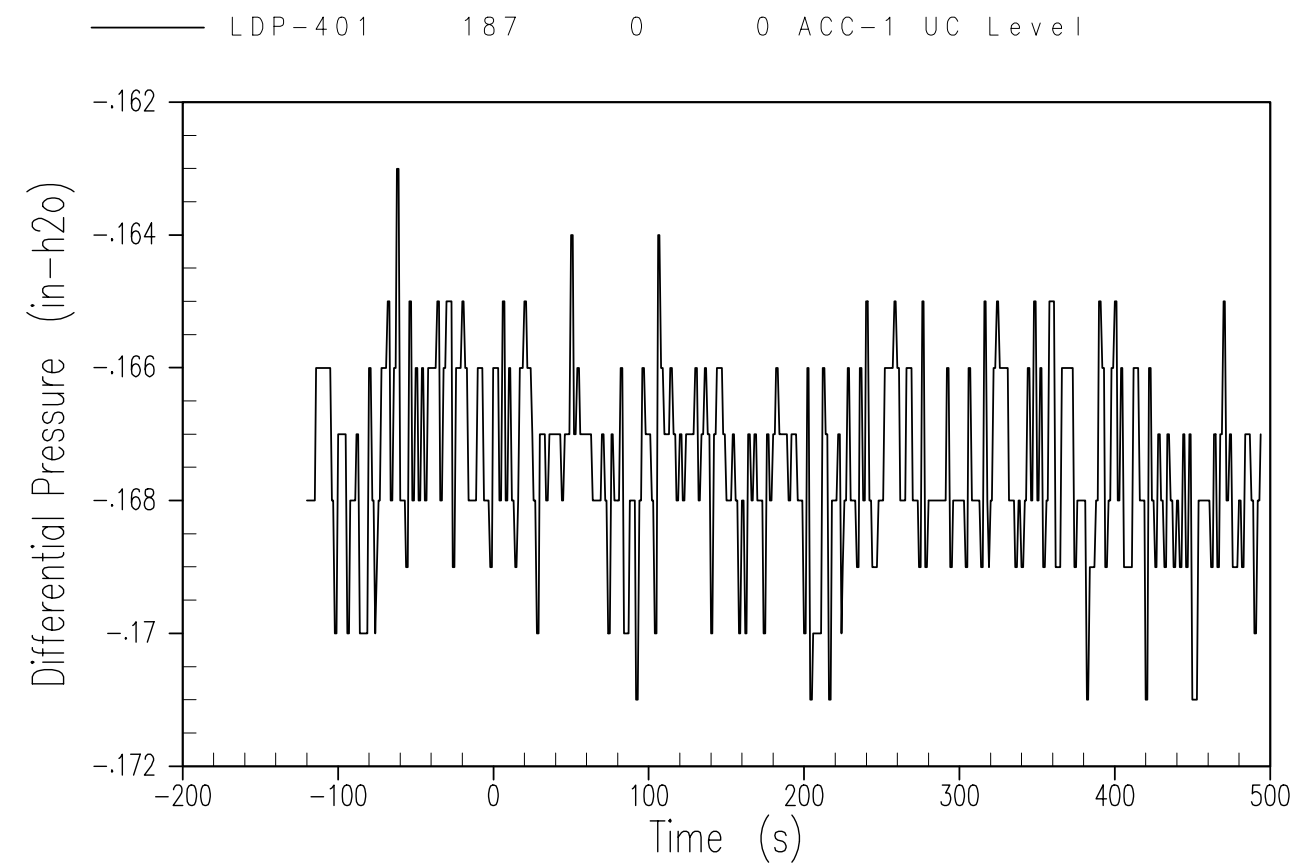

Figure A-21 Accumulator 1 Liquid Level 


\section{AP1000 Test Data Oregon State University Test TR-1 March 18, 2003 \\ $\longrightarrow$ LDP-402 $188 \quad 0 \quad 0 \quad A C C-2$ UC Leve}

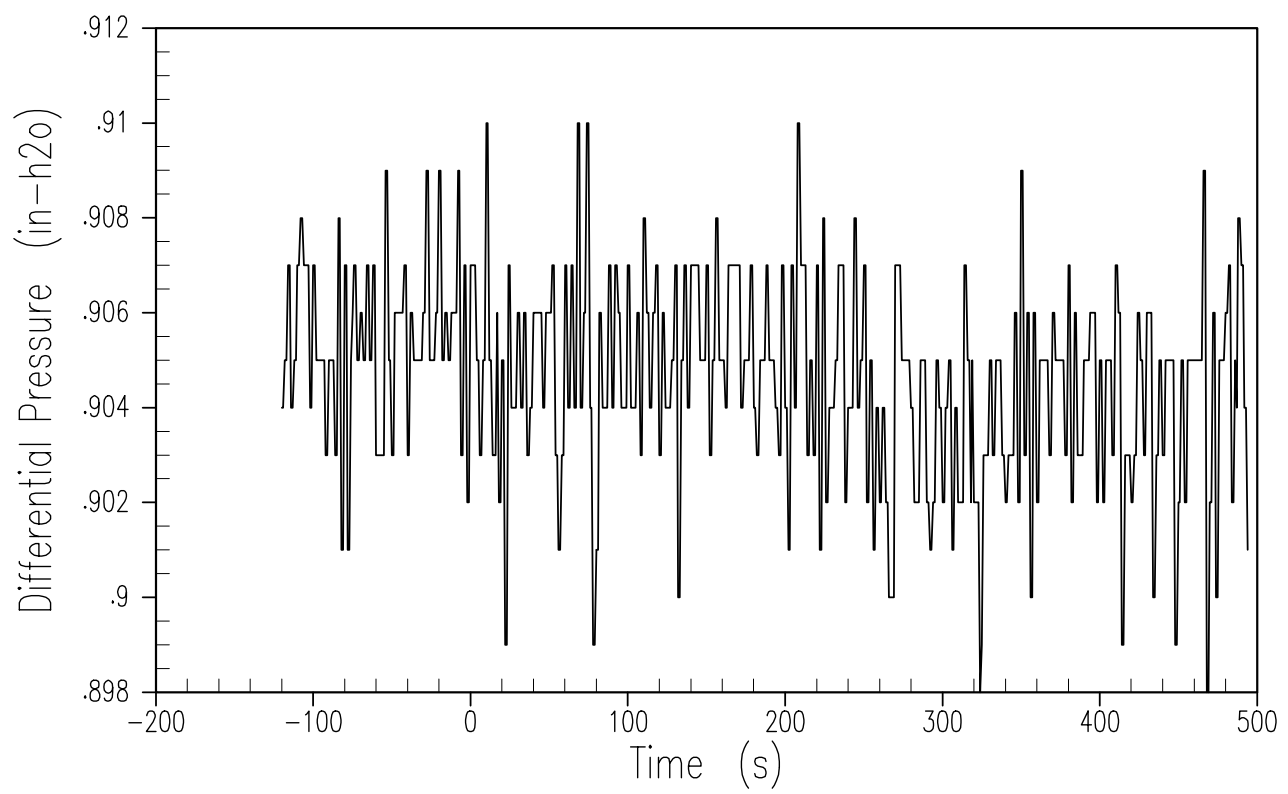

Figure A-22 Accumulator 2 Liquid Level 

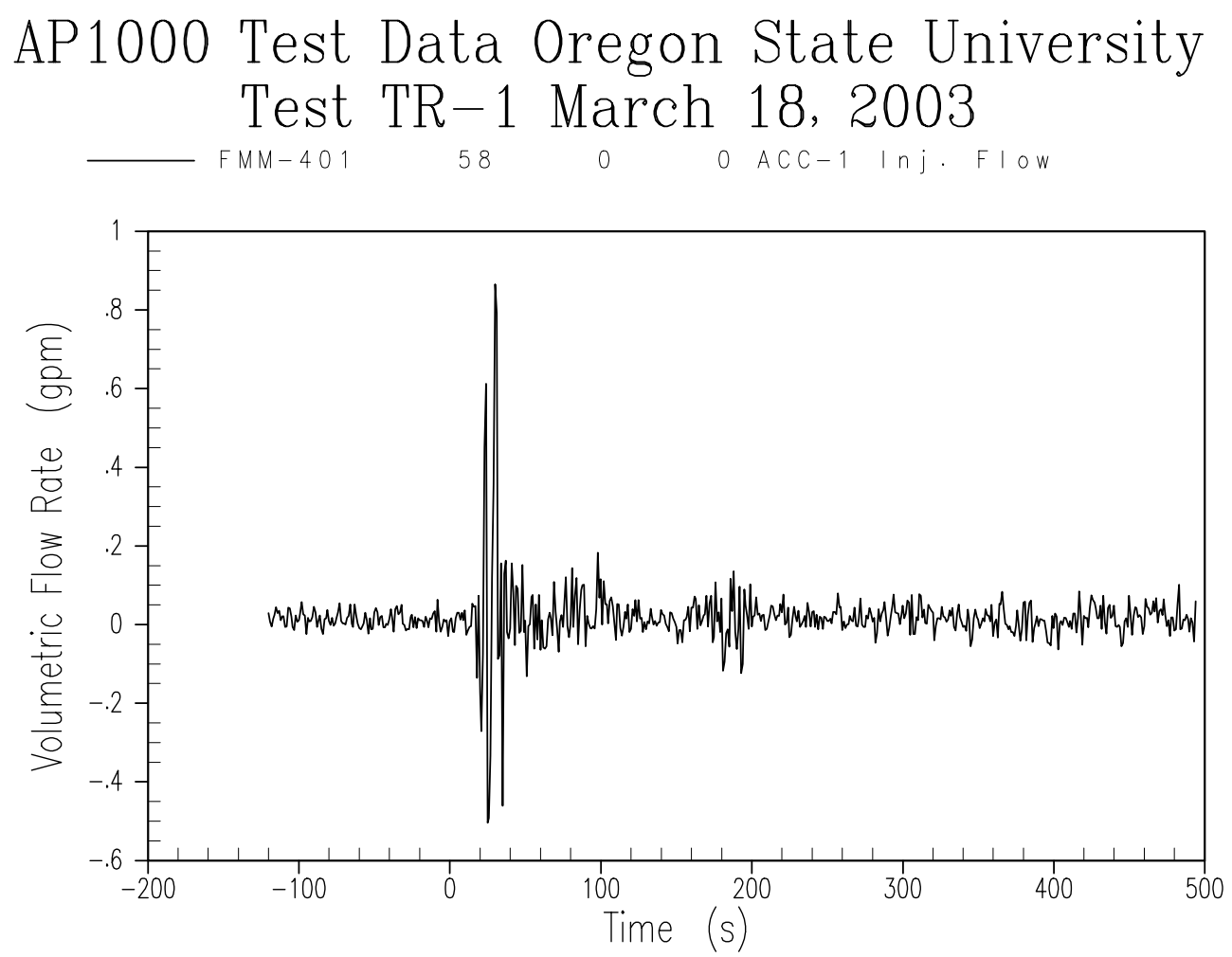

Figure A-23 Accumulator 1 Flow Rate 


\section{AP1000 Test Data Oregon State University Test TR-1 March 18, 2003 FMM-402 $59 \quad 0 \quad 0 \quad A C C-2$ Inj. F I OW}

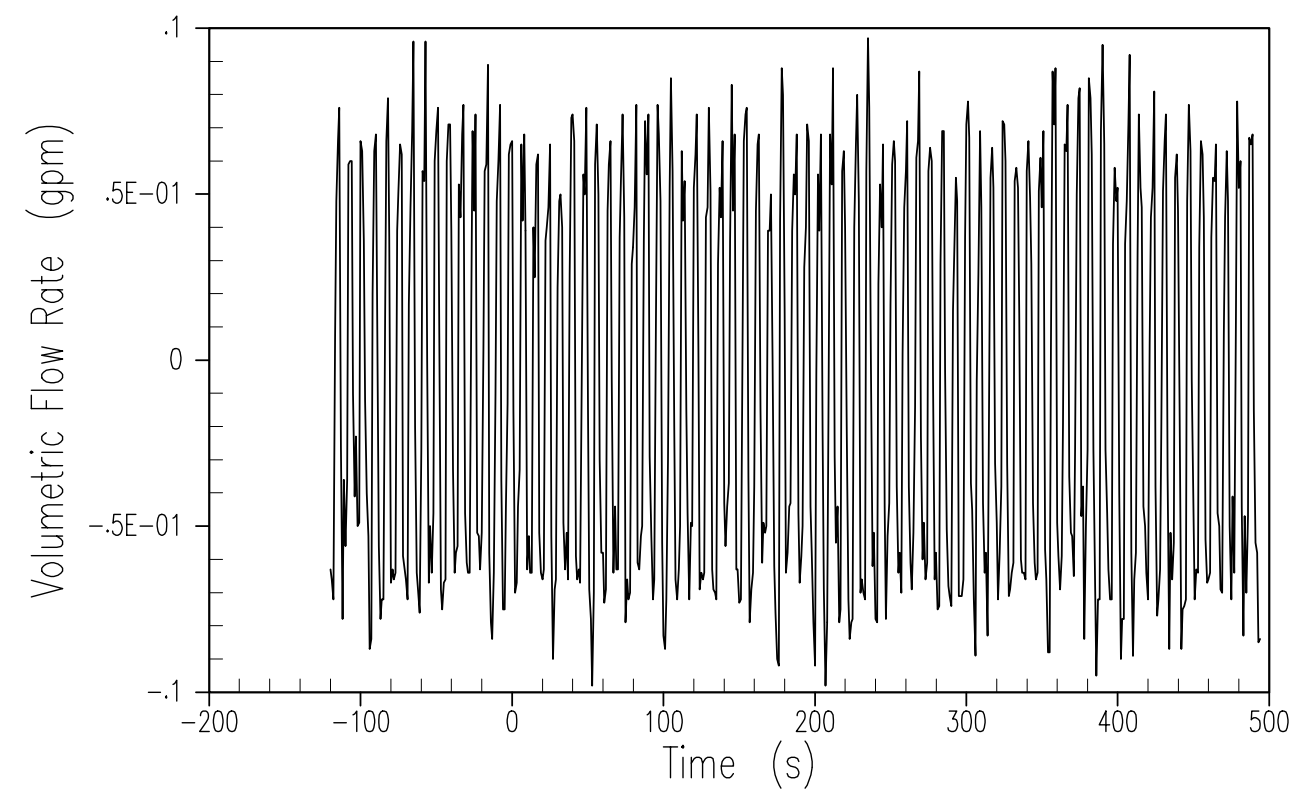

Figure A-24 Accumulator 2 Flow Rate 


\section{AP1000 Test Data Oregon State University Test TR-1 March 18, 2003}

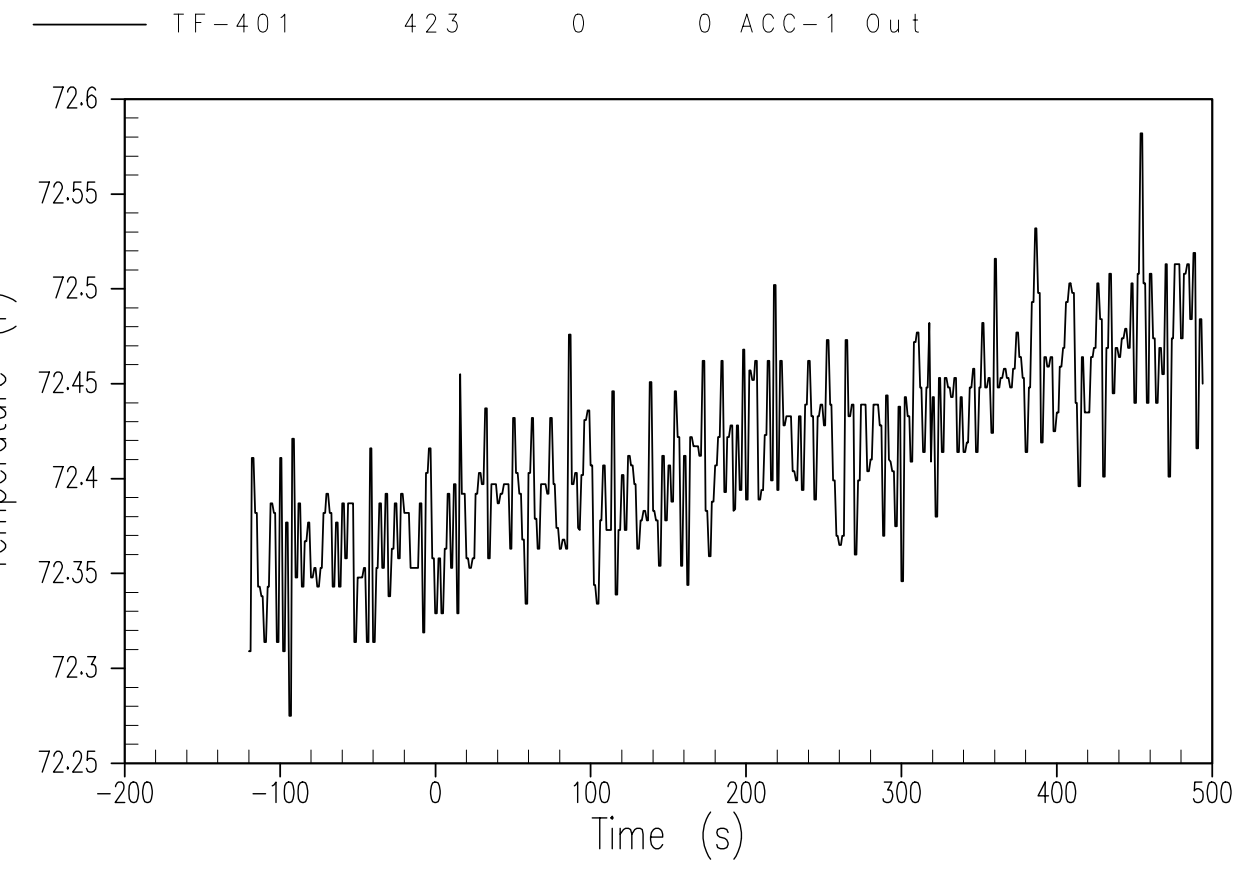

Figure A-25 Accumulator 1 Liquid Discharge Temperature 
AP1000 Test Data Oregon State University Test TR-1 March 18, 2003

TF-402

424

0

$0 \mathrm{ACC}-2 \quad \mathrm{Out}$

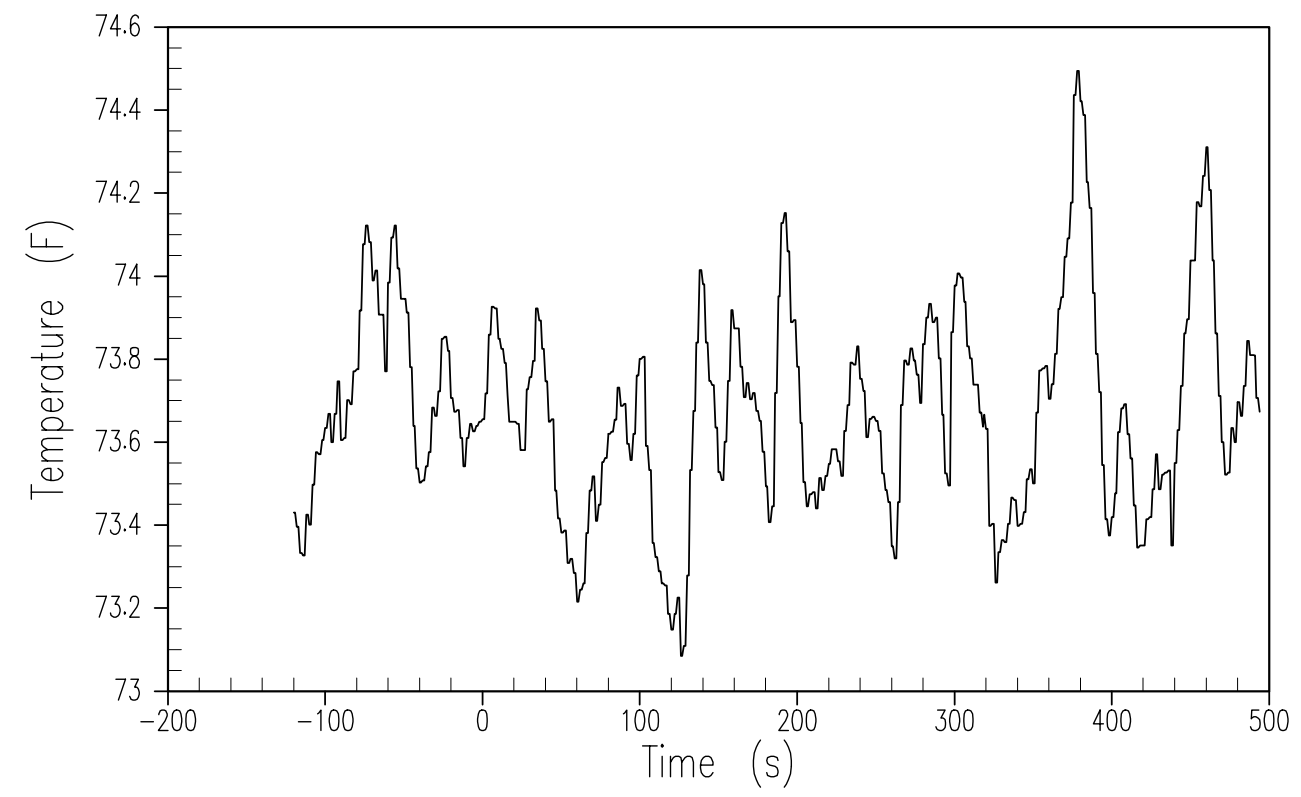

Figure A-26 Accumulator 2 Liquid Discharge Temperature 

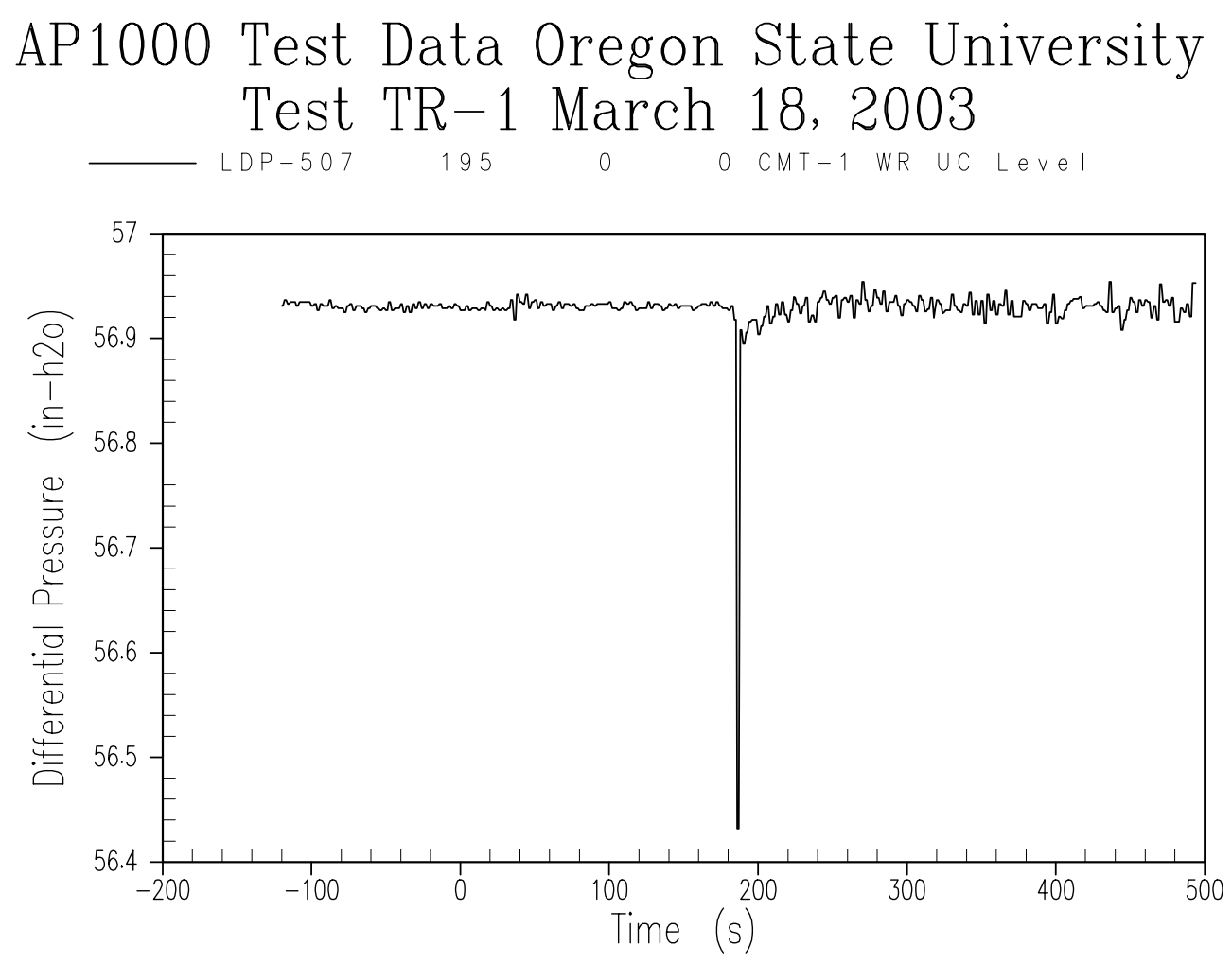

Figure A-27 Core Makeup Tank 1 Liquid Level 

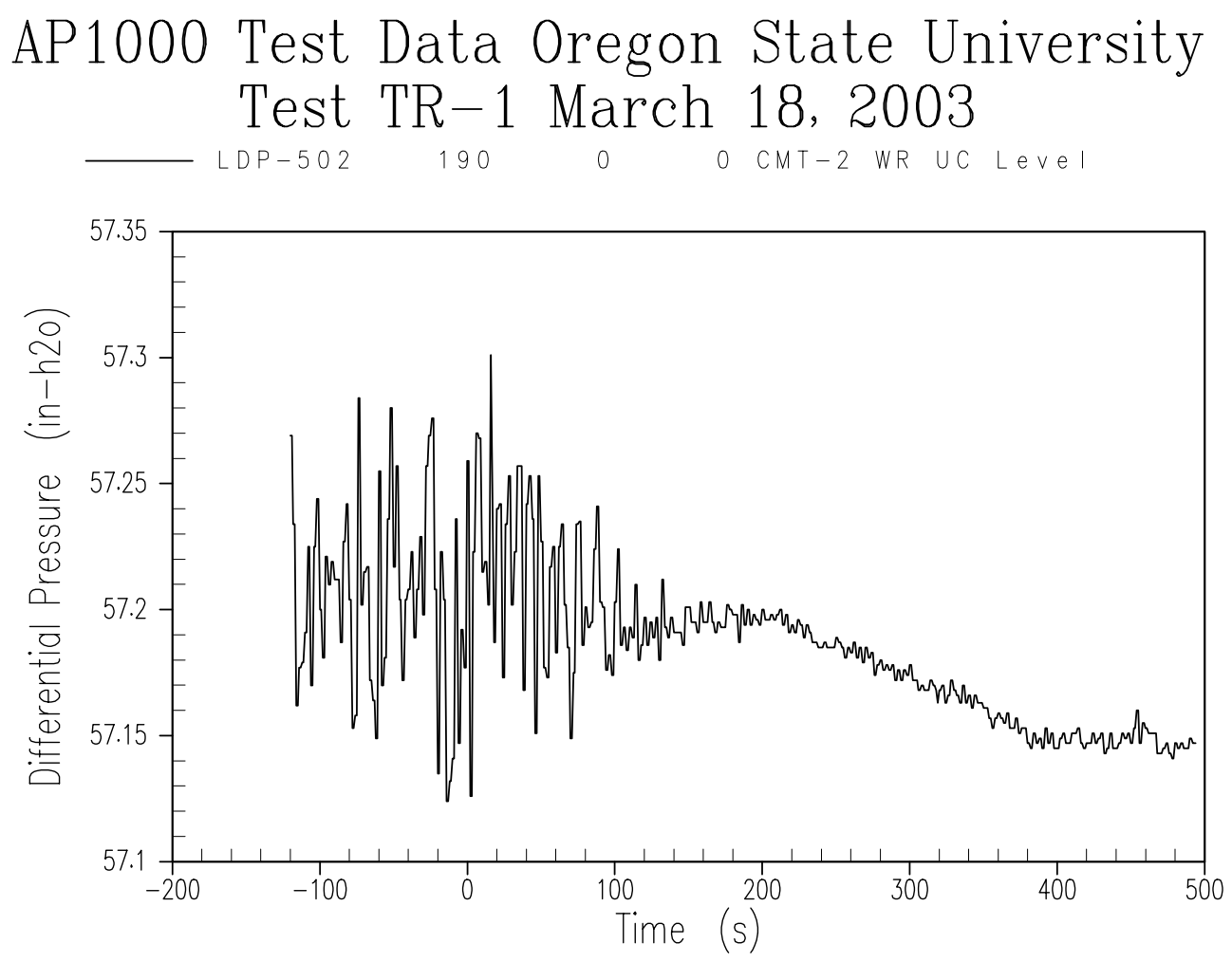

Figure A-28 Core Makeup Tank 2 Liquid Level 


\section{AP1000 Test Data Oregon State University Test TR-1 March 18, 2003}

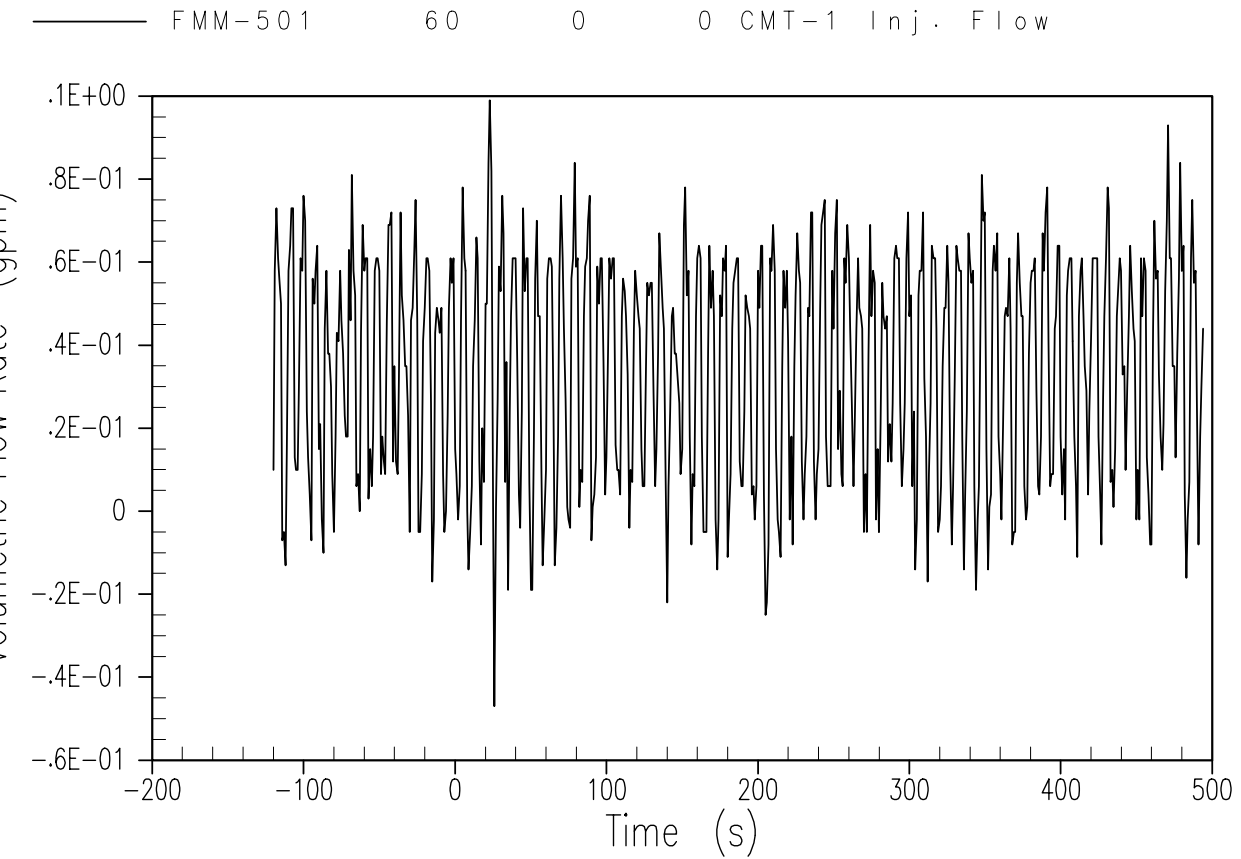

Figure A-29 Core Makeup Tank 1 Flow Rate 


\section{AP1000 Test Data Oregon State University Test TR-1 March 18, 2003 \\ FMM-504 $63 \quad 0 \quad 0$ CMT-2 Inj. FIow}

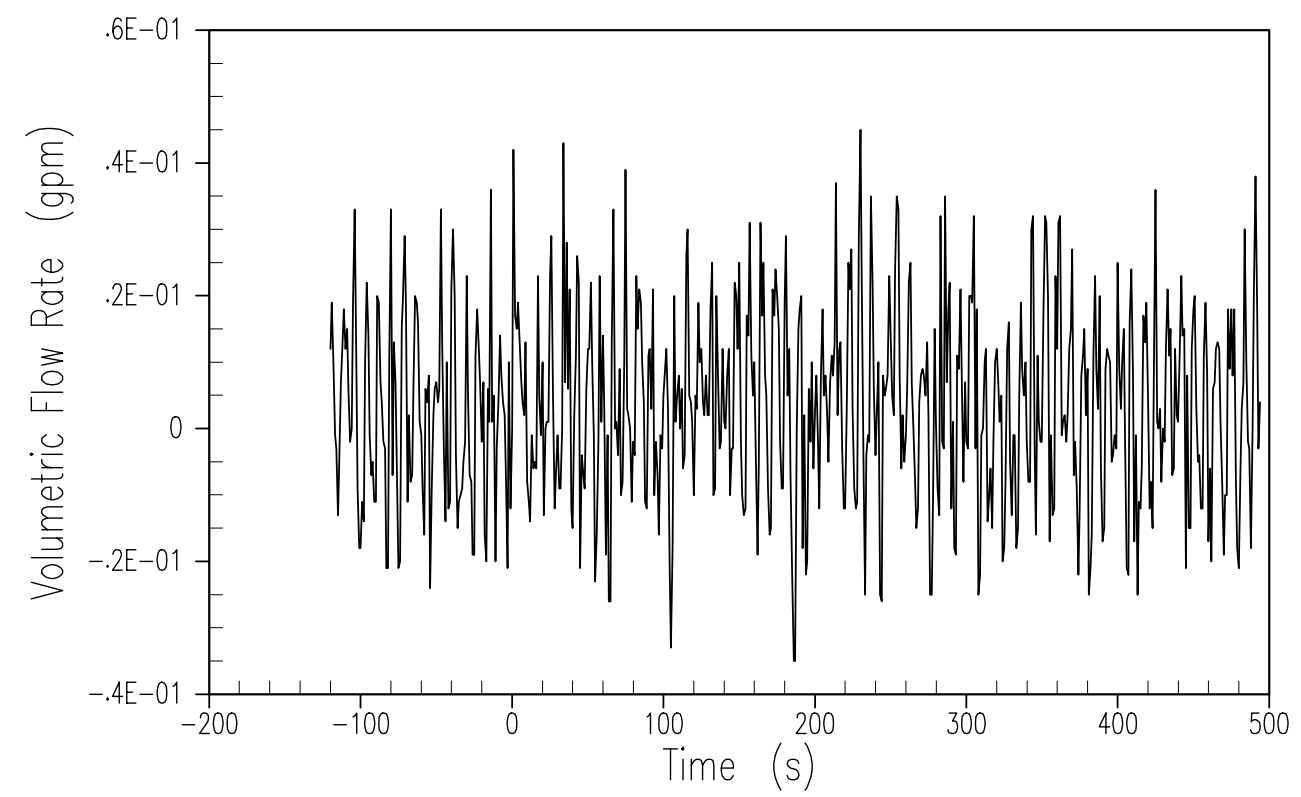

Figure A-30 Core Makeup Tank 2 Flow Rate 


\section{AP1000 Test Data Oregon State University Test TR-1 March 18, 2003

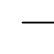
$T F-501$
429
0
o CMT-1 Long TC @ 0.30

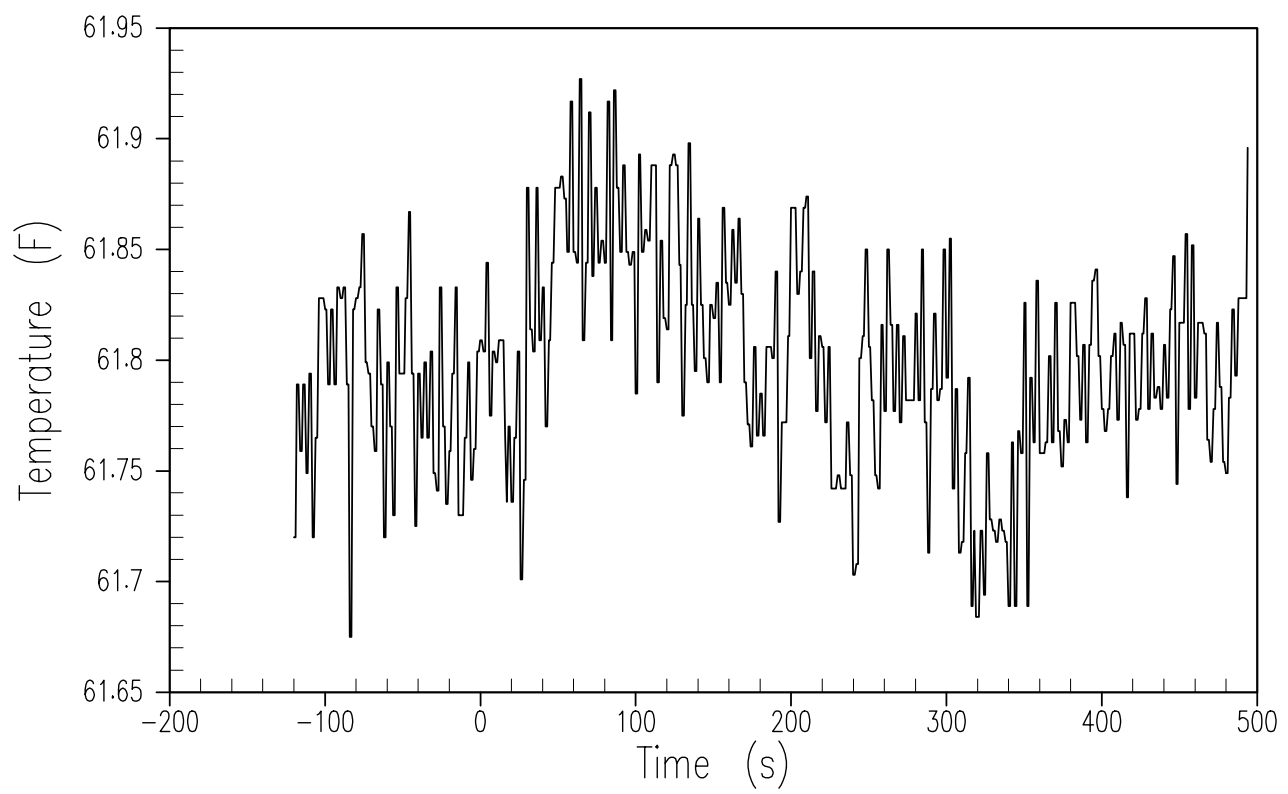

Figure A-31 Core Makeup Tank 1 Liquid Temperature - Bottom 

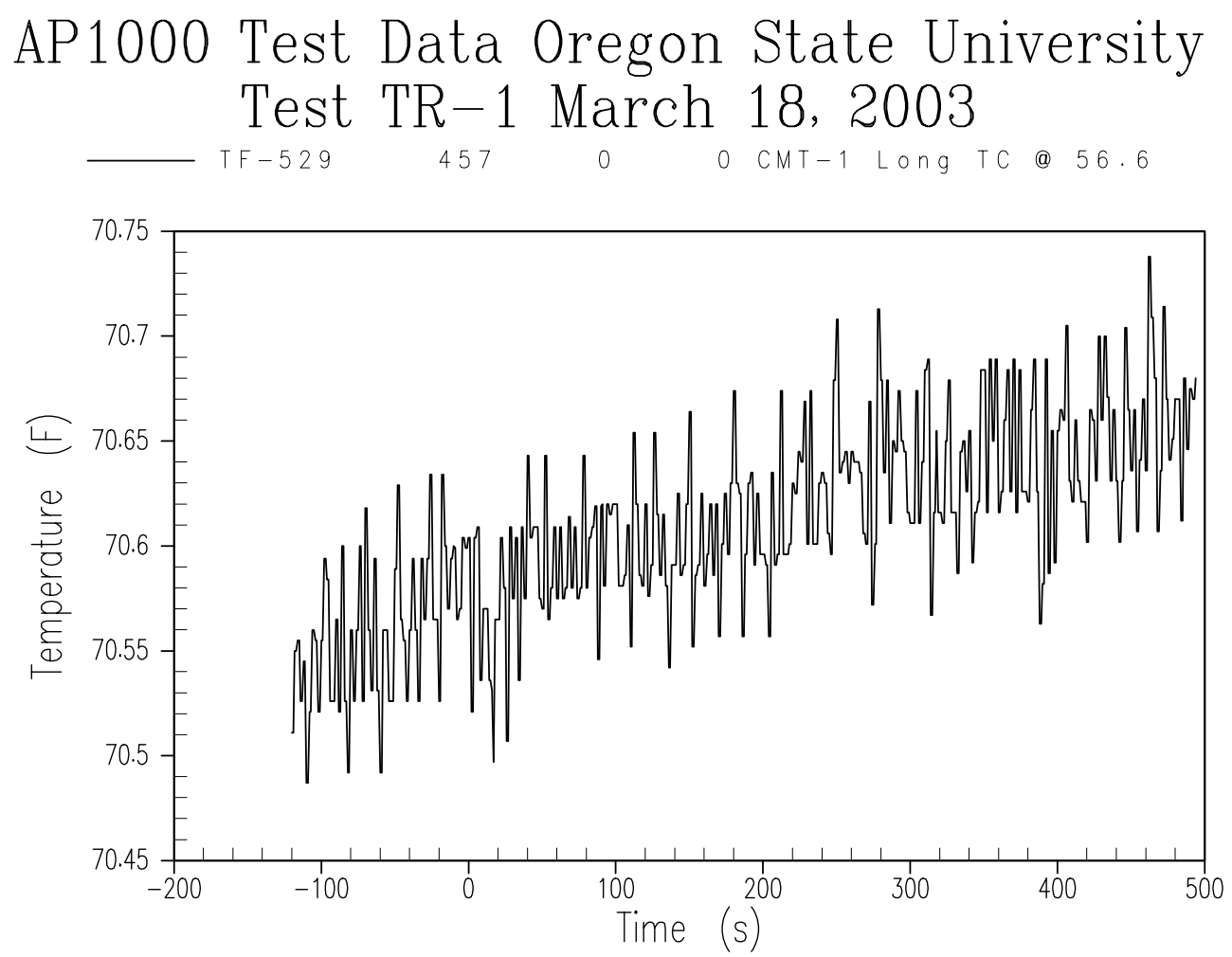

Figure A-32 Core Makeup Tank 1 Liquid Temperature - Top 
AP1000 Test Data Oregon State University Test TR-1 March 18, 2003

$-T F-$

432

0

o CMT-2 Long TC @ 0.30

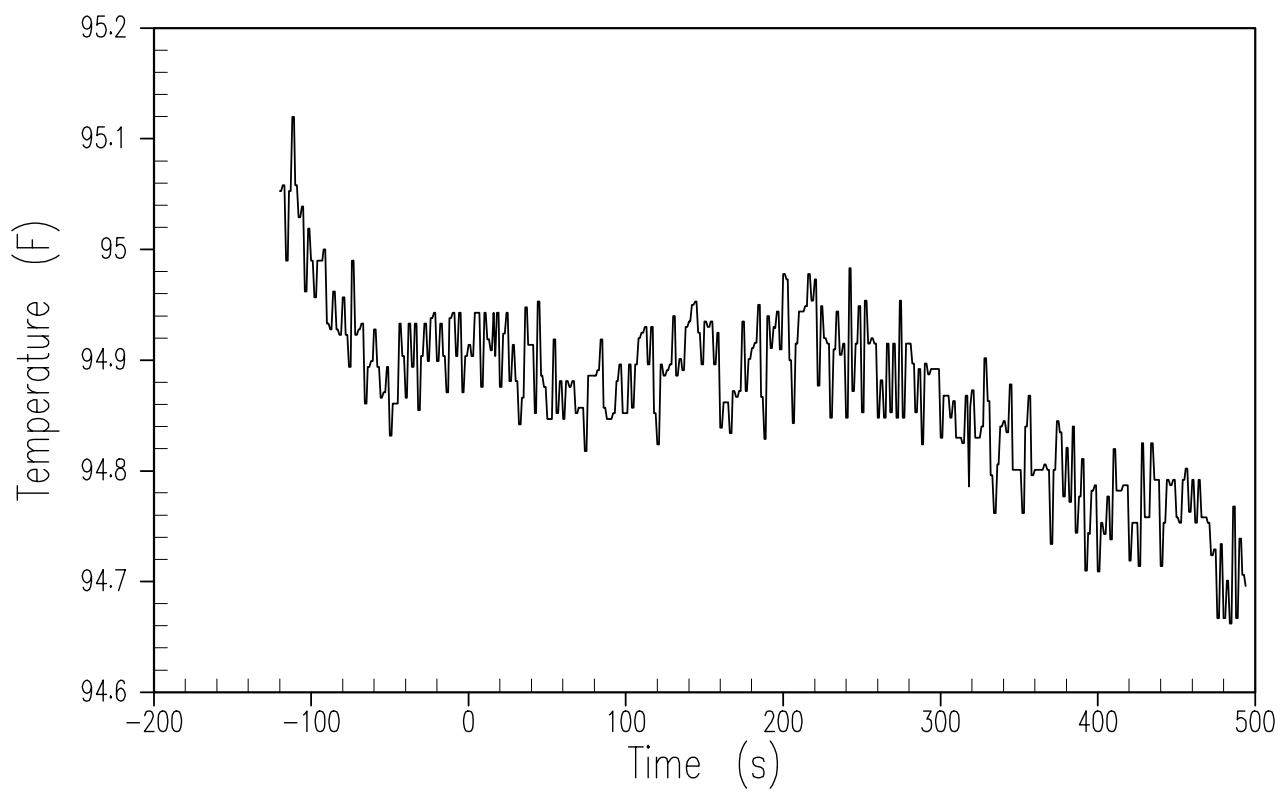

Figure A-33 Core Makeup Tank 2 Liquid Temperature - Bottom 
AP1000 Test Data Oregon State University Test TR-1 March 18, 2003

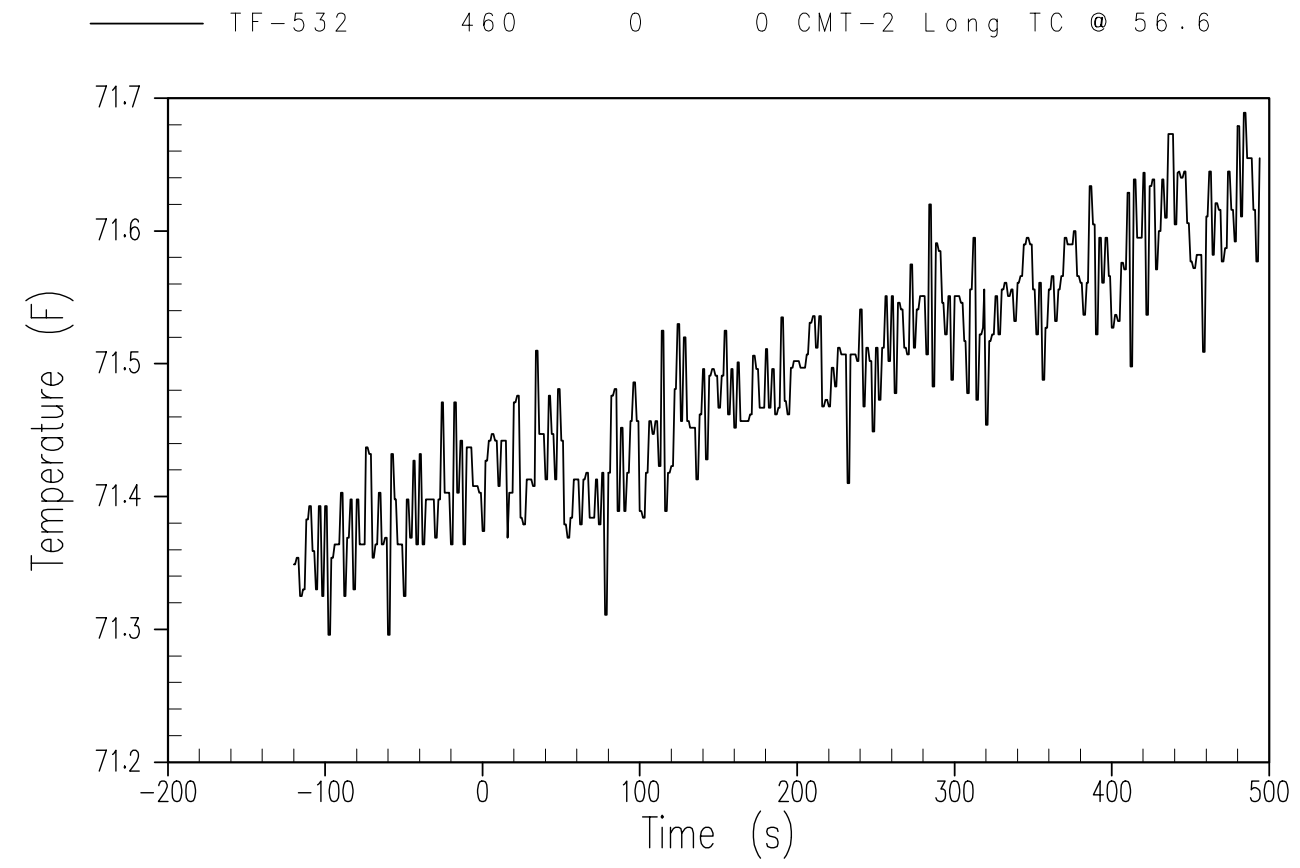

Figure A-34 Core Makeup Tank 2 Liquid Temperature - Top 

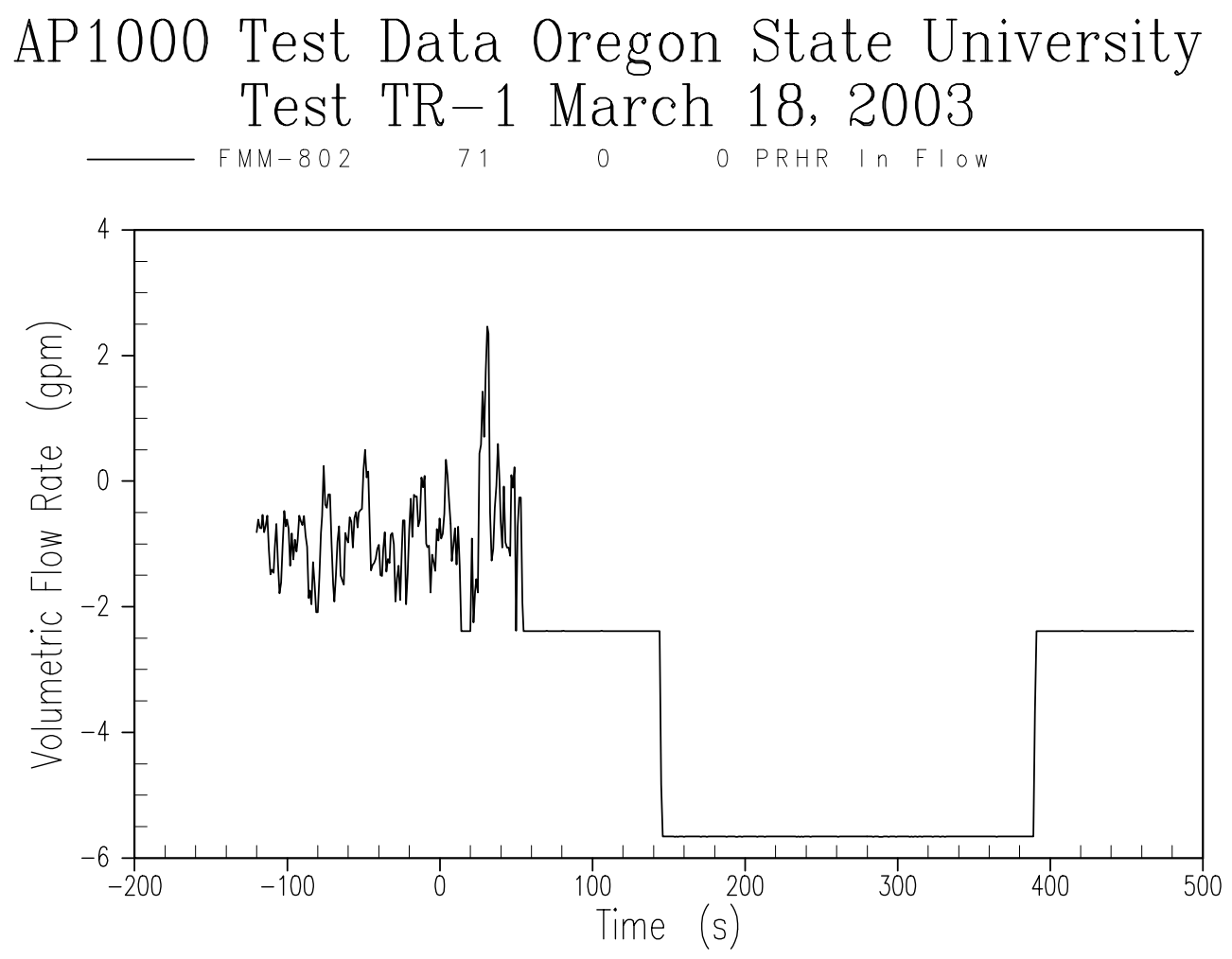

Figure A-35 Passive Residual Heat Removal Inlet Flow Rate 

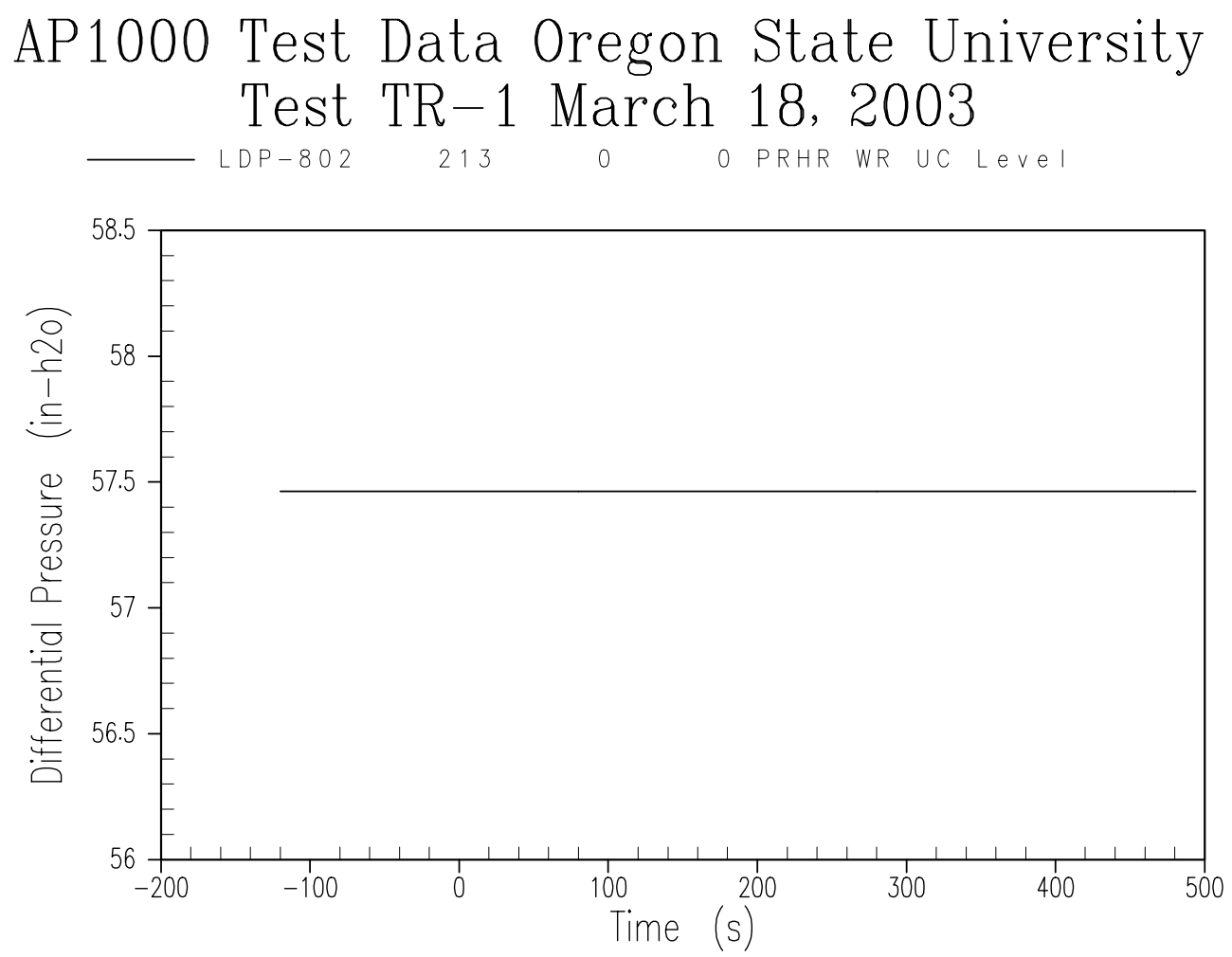

Figure A-36 Passive Residual Heat Removal Liquid Level 

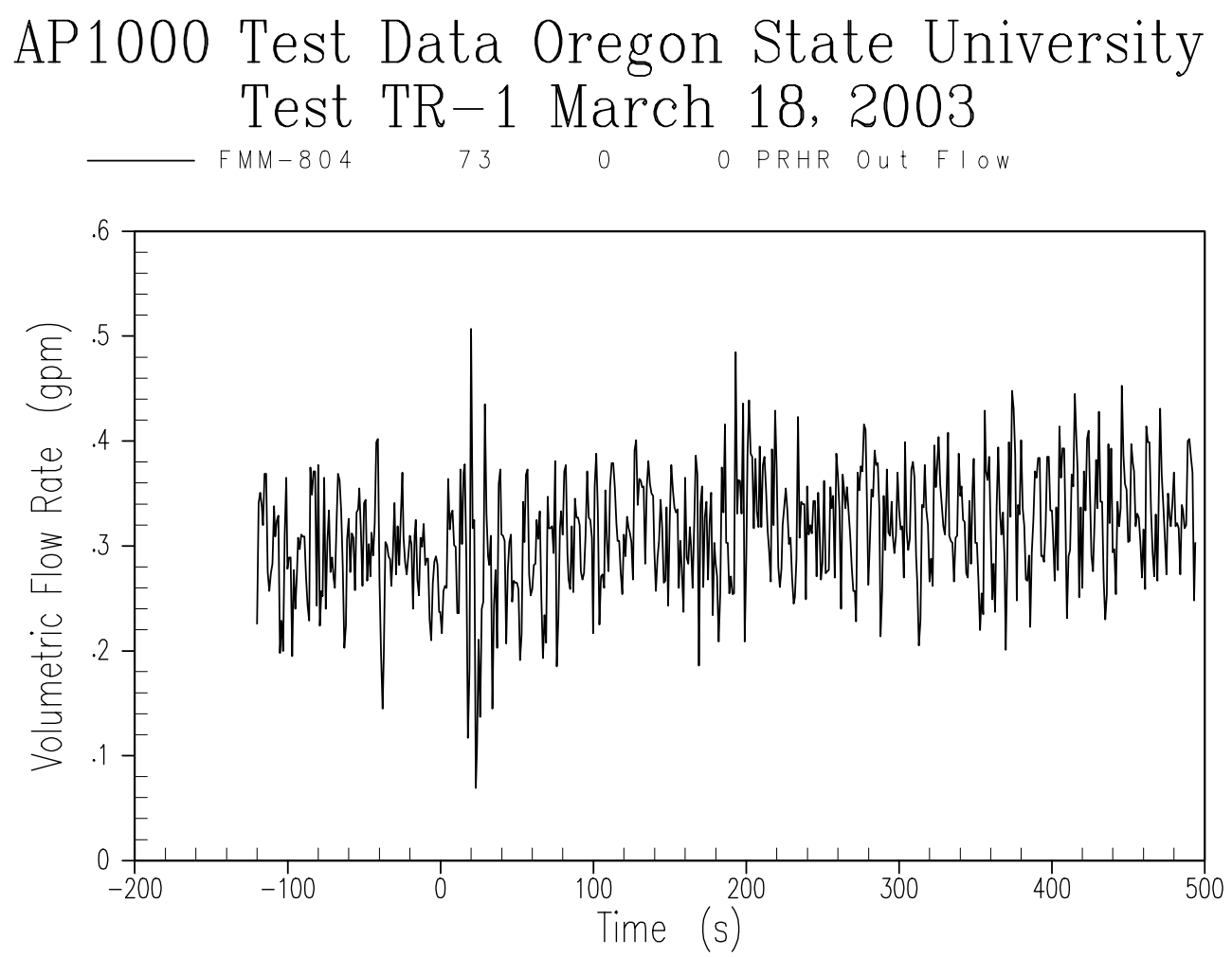

Figure A-37 Passive Residual Heat Removal Outlet Flow Rate 

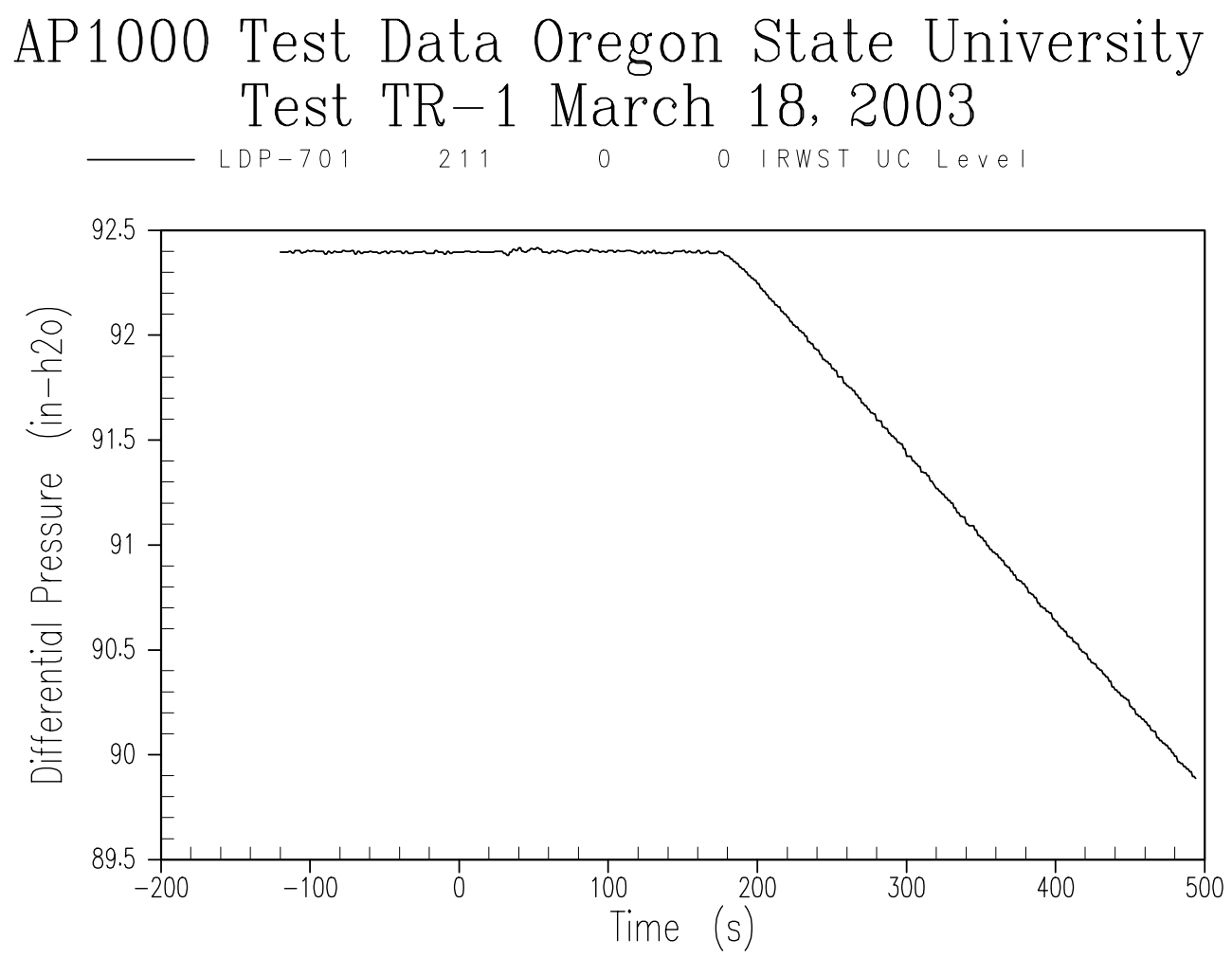

Figure A-38 IRWST Liquid Level 

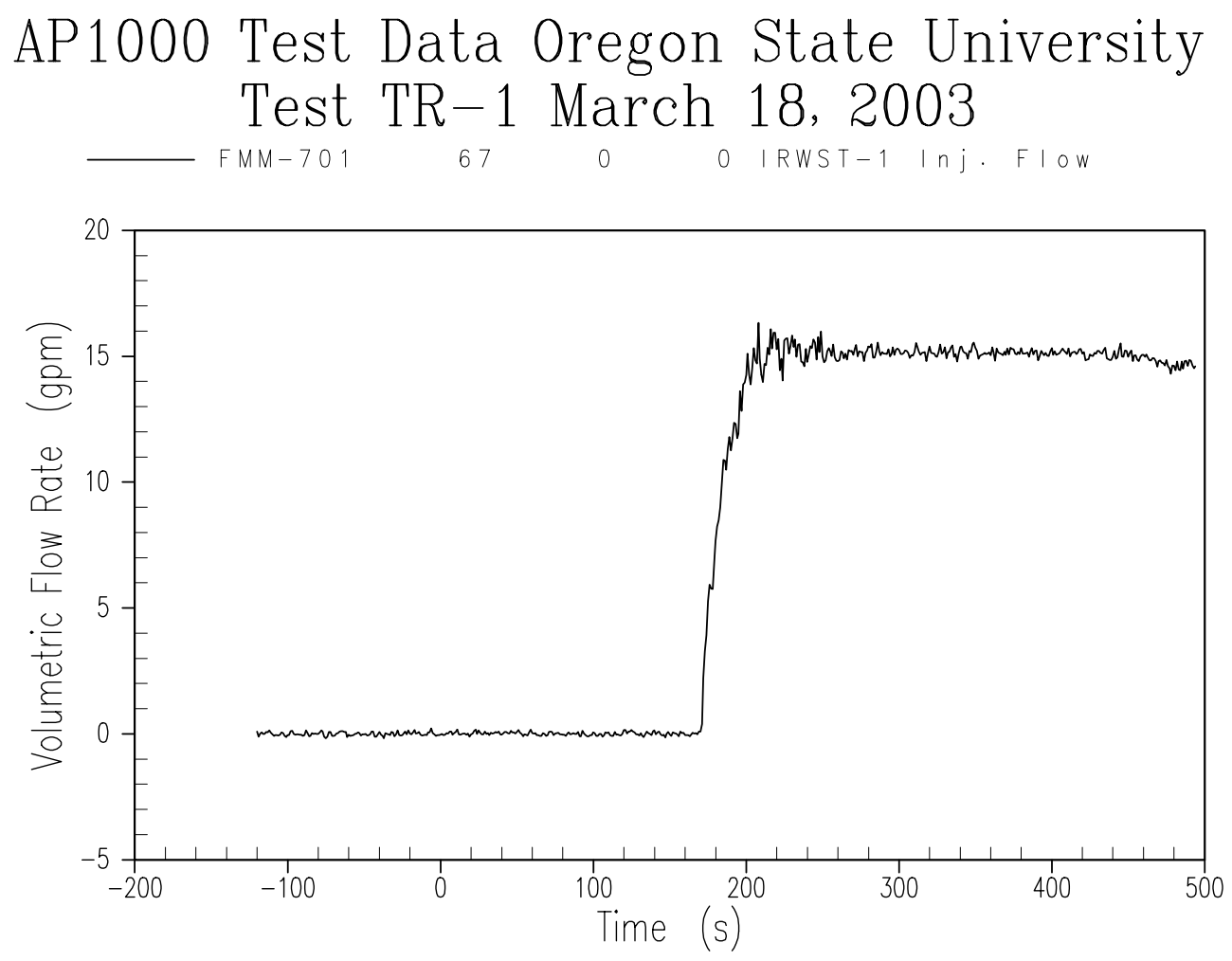

Figure A-39 IRWST Discharge Line 1 Flow Rate 

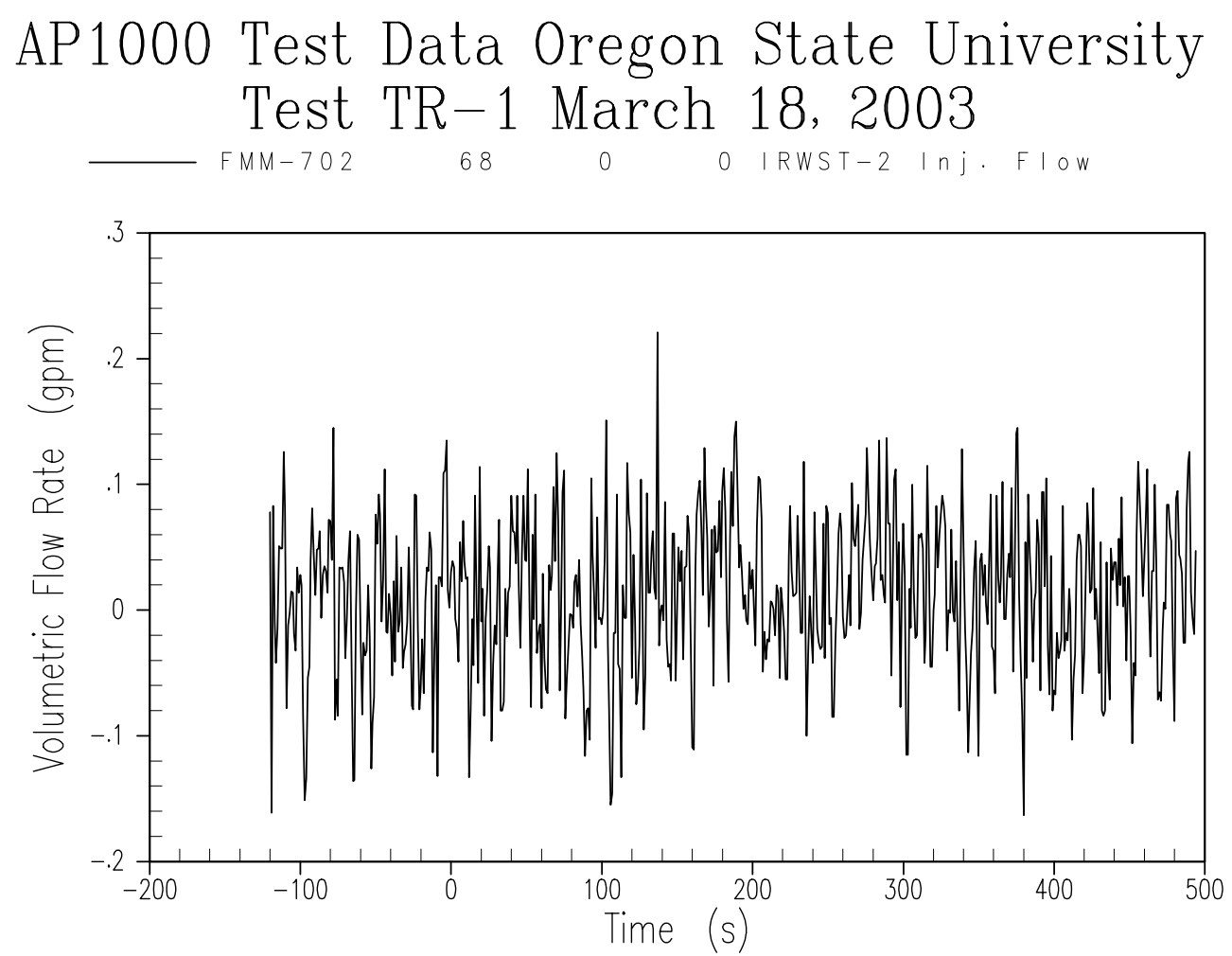

Figure A-40 IRWST Discharge Line 2 Flow Rate 


\section{AP1000 Test Data Oregon State University Test TR-1 March 18, 2003 \\ 508 \\ o IRWST/PRHR TC @ Bot.}

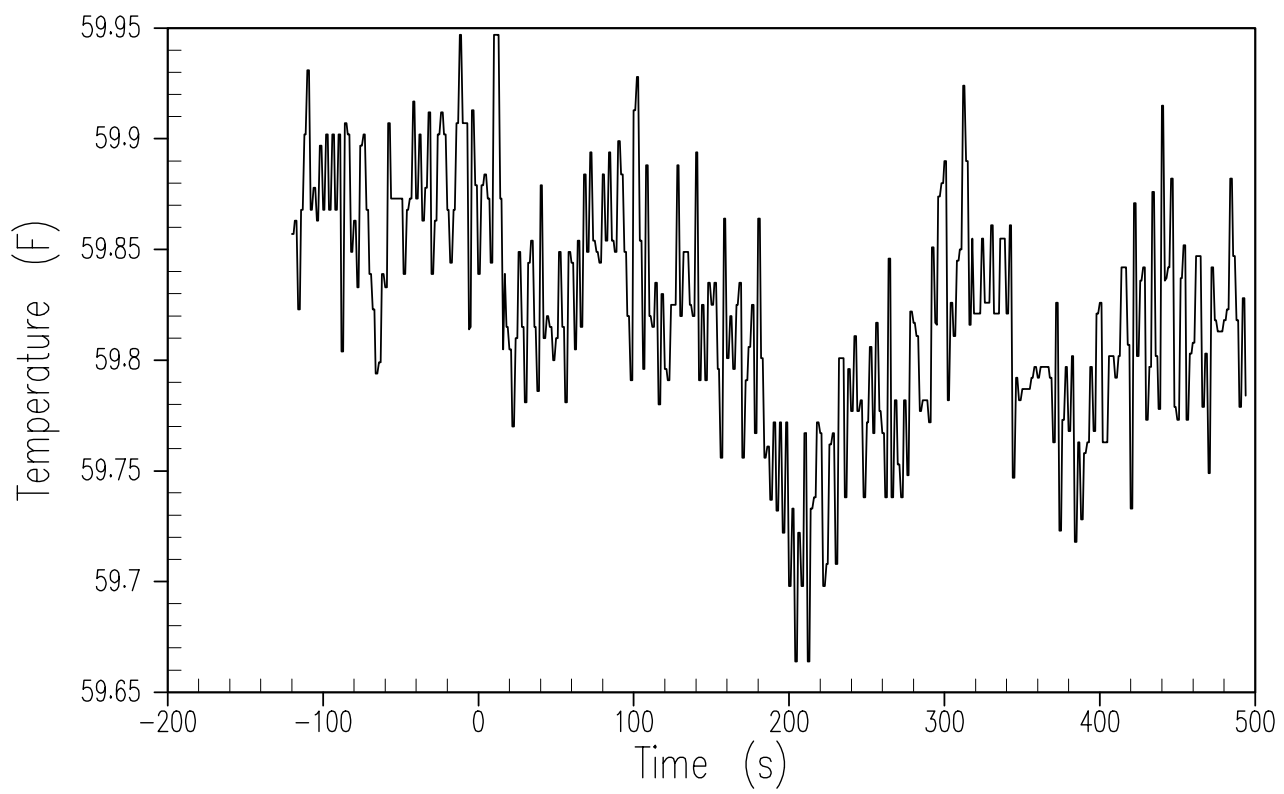

Figure A-41 IRWST Fluid Temperature - Bottom 


\section{AP1000 Test Data Oregon State University Test TR-1 March 18, 2003}

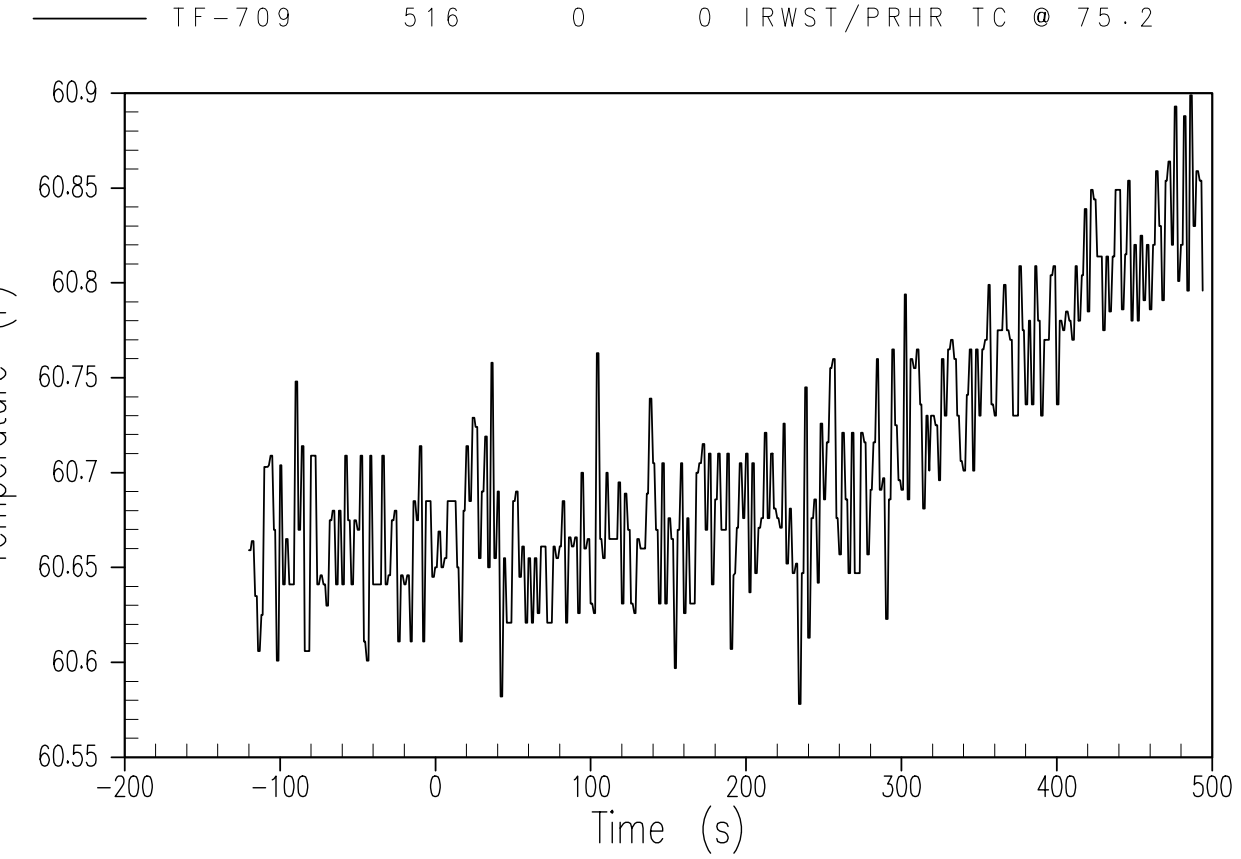

Figure A-42 IRWST Fluid Temperature - Top 

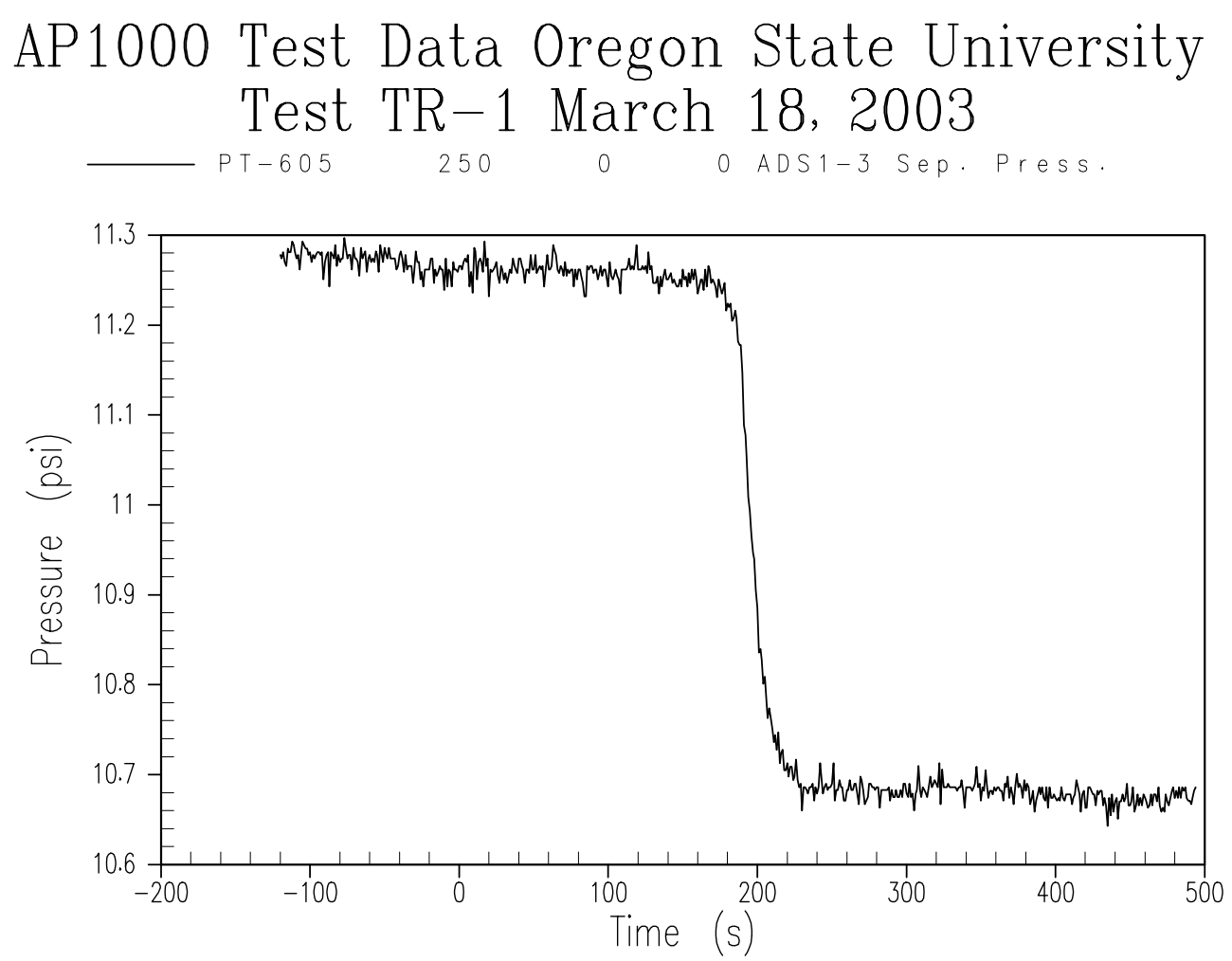

Figure A-43 ADS 1-3 Separator Pressure 

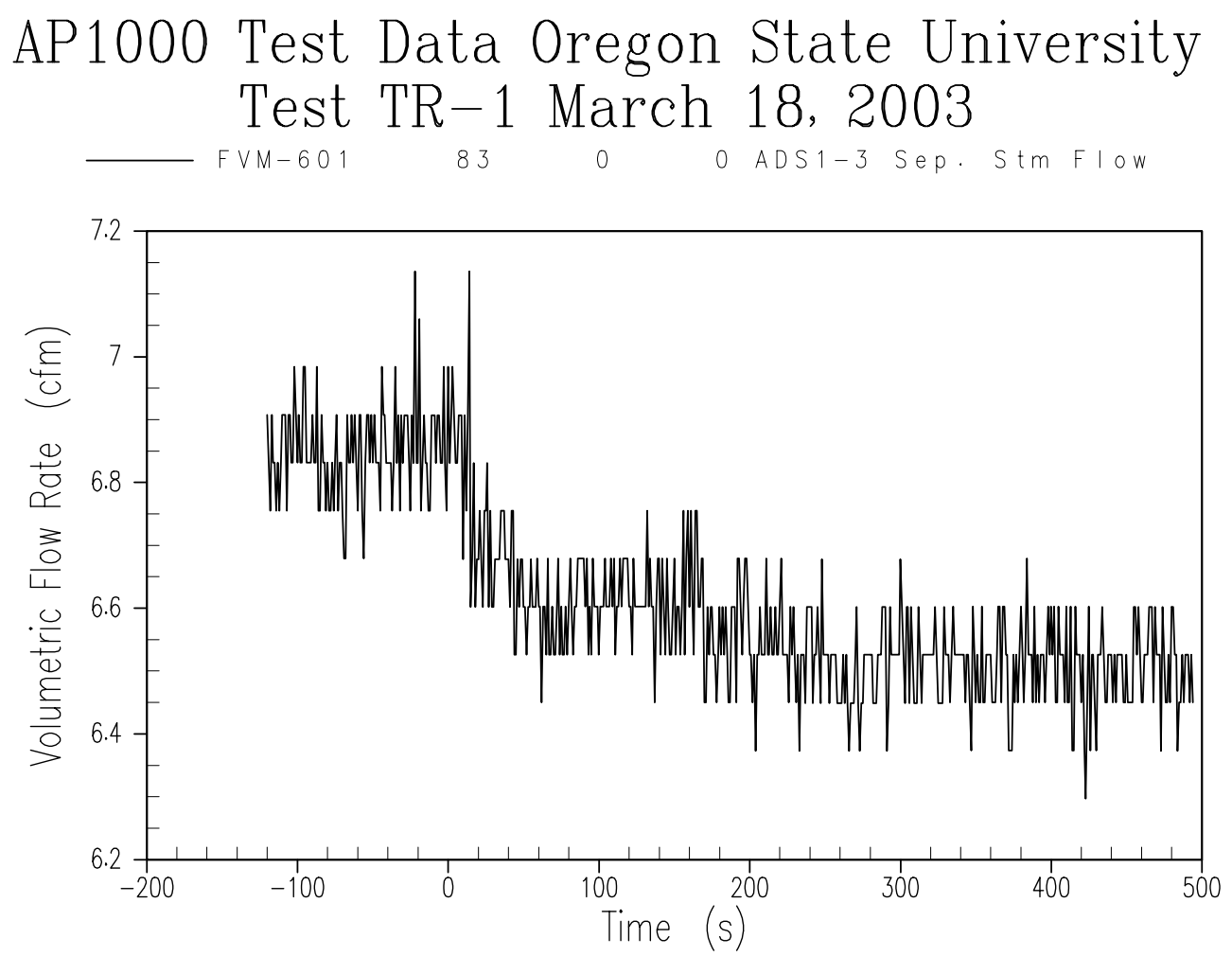

Figure A-44 ADS 1-3 Separator Steam Flow Rate 


\section{AP1000 Test Data Oregon State University Test TR-1 March 18, 2003}

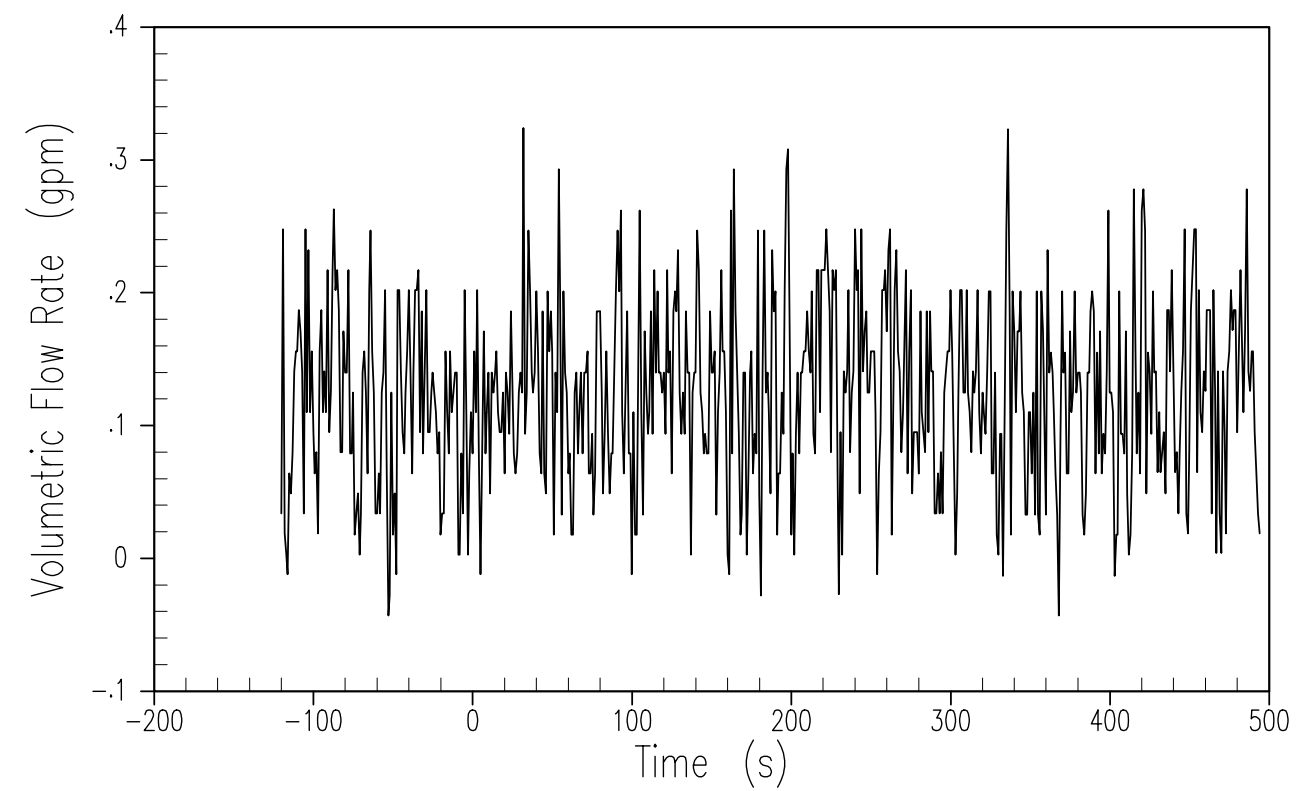

Figure A-45 ADS 1-3 Separator Liquid Flow Rate 

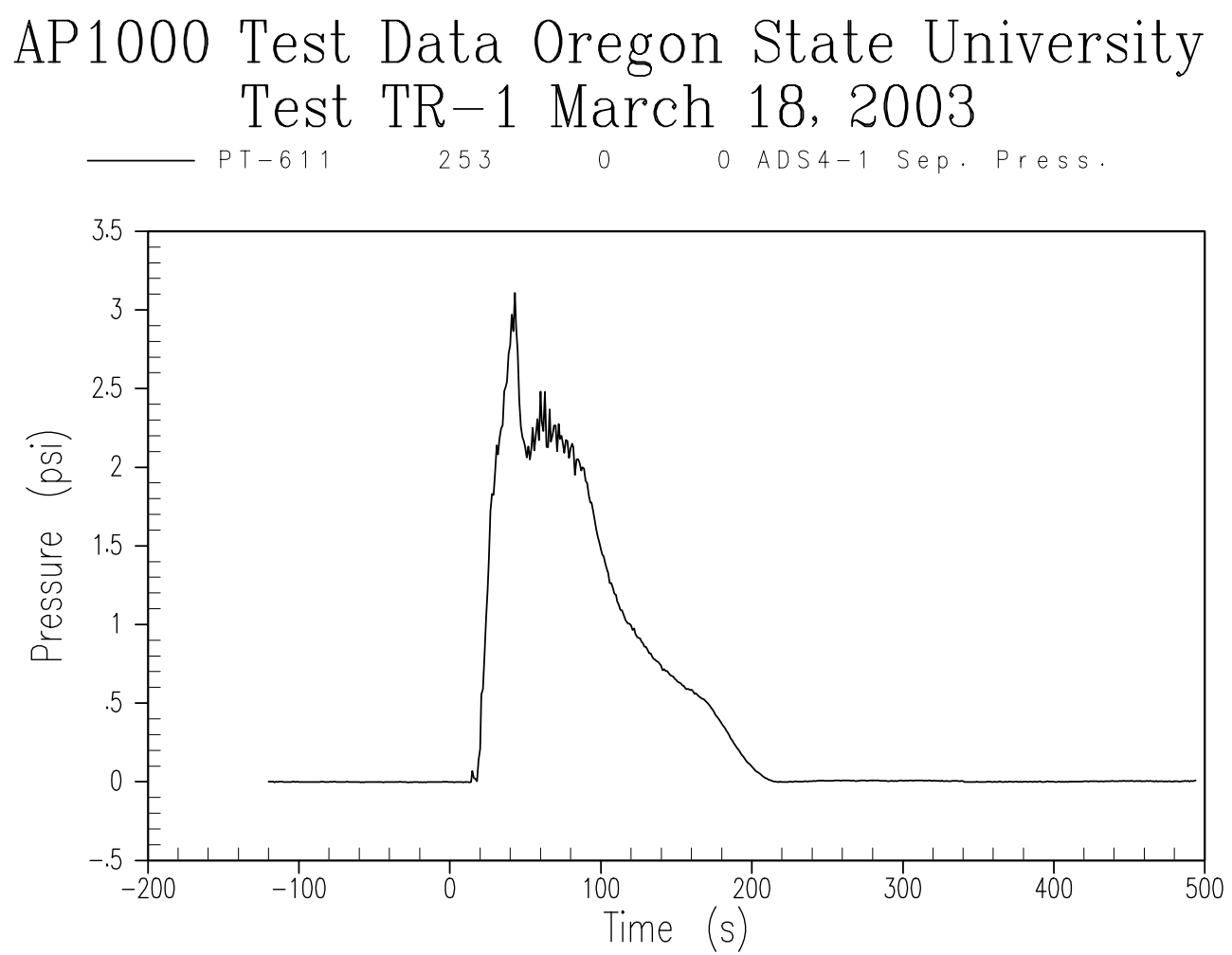

Figure A-46 ADS 4-1 Separator Pressure 

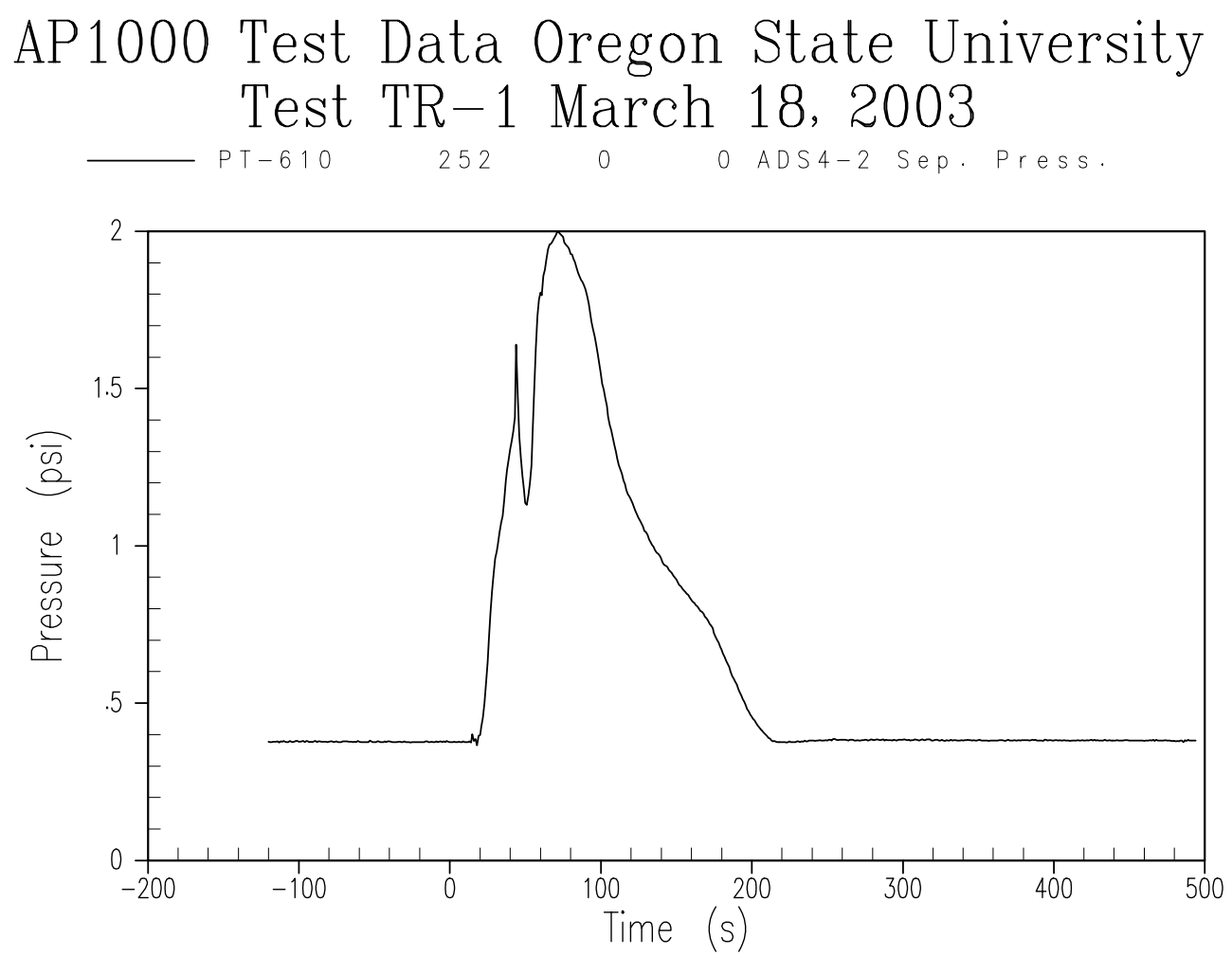

Figure A-47 ADS 4-2 Separator Pressure 

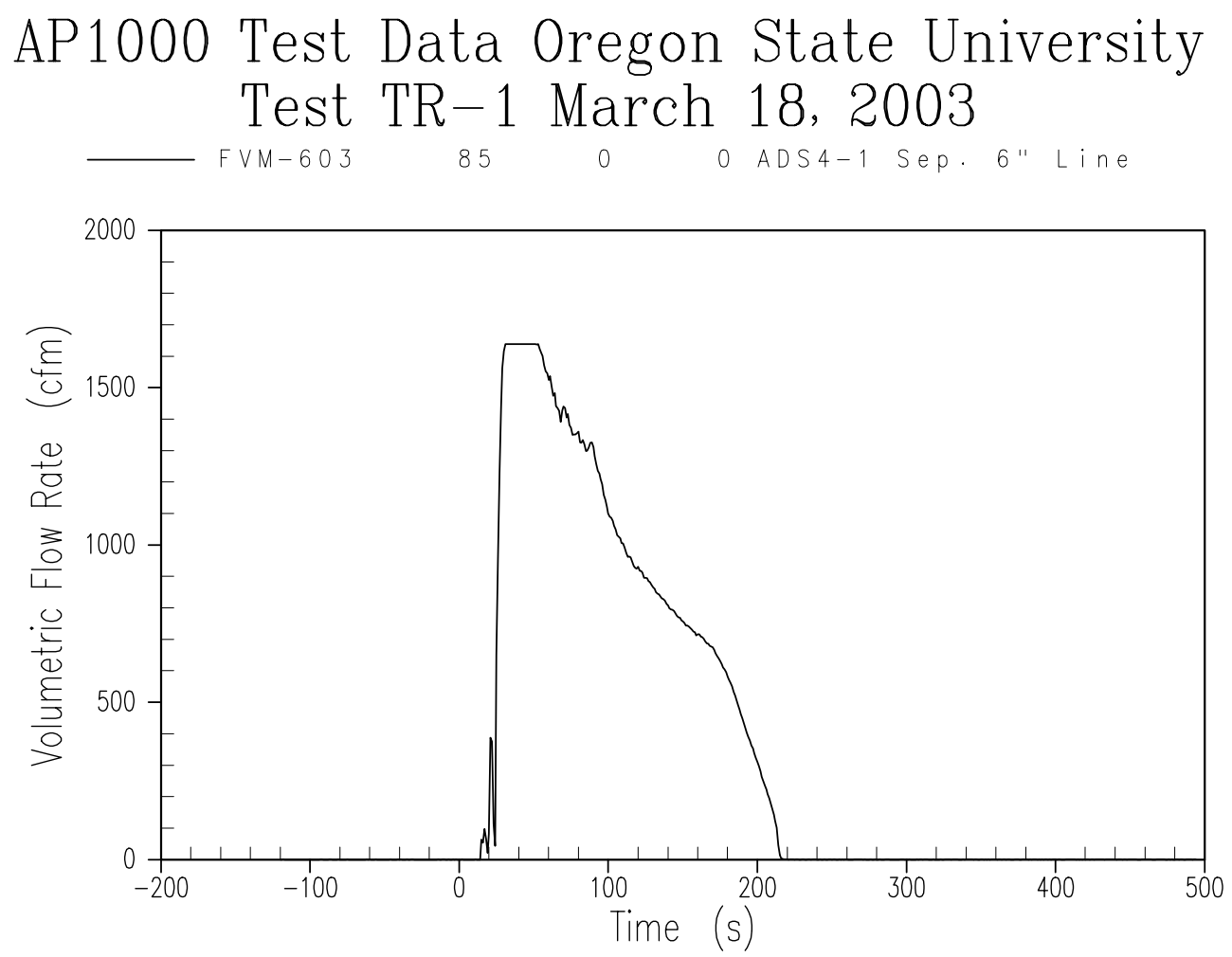

Figure A-48 ADS 4-1 Separator Steam Flow Rate 

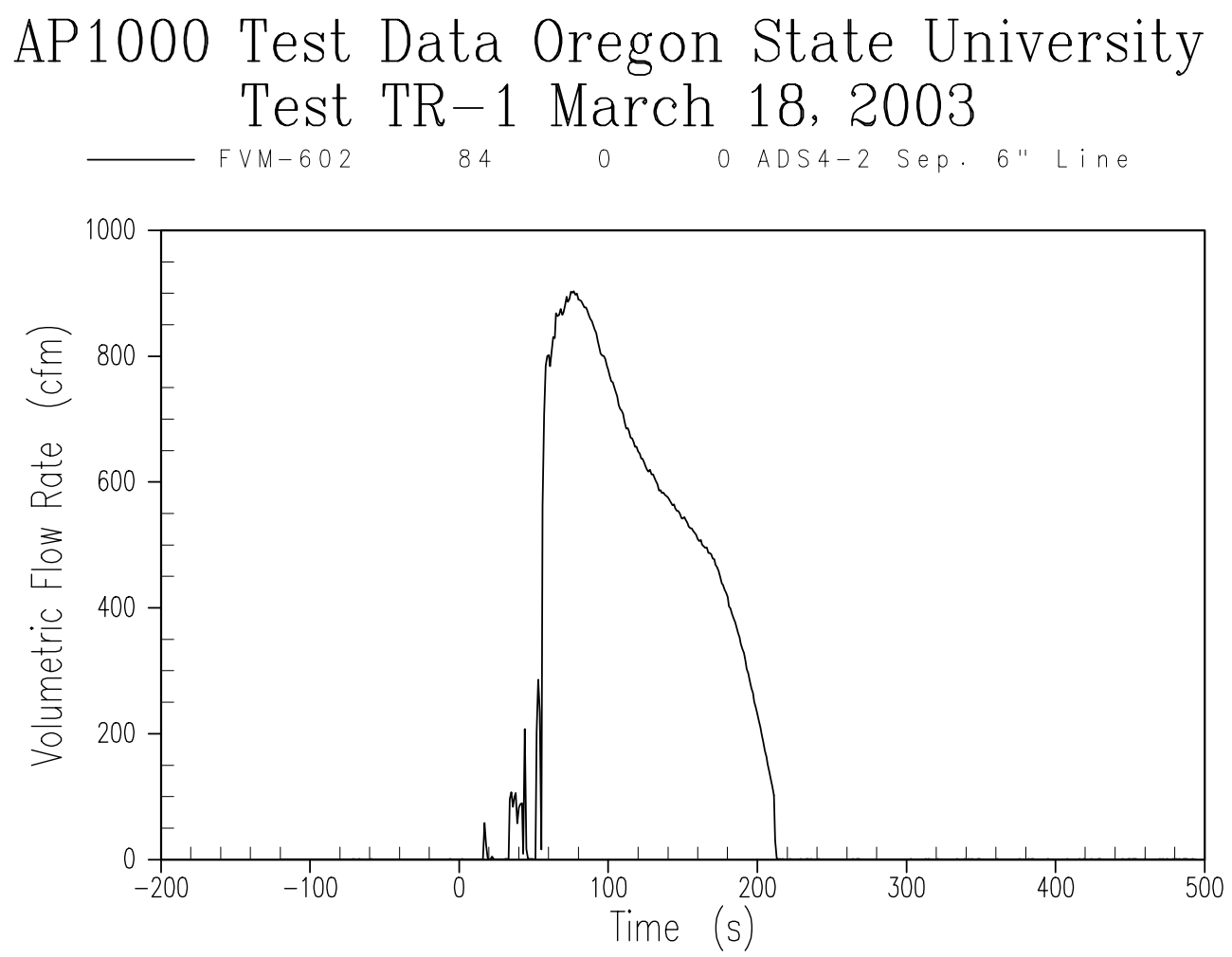

Figure A-49 ADS 4-2 Separator Steam Flow Rate 

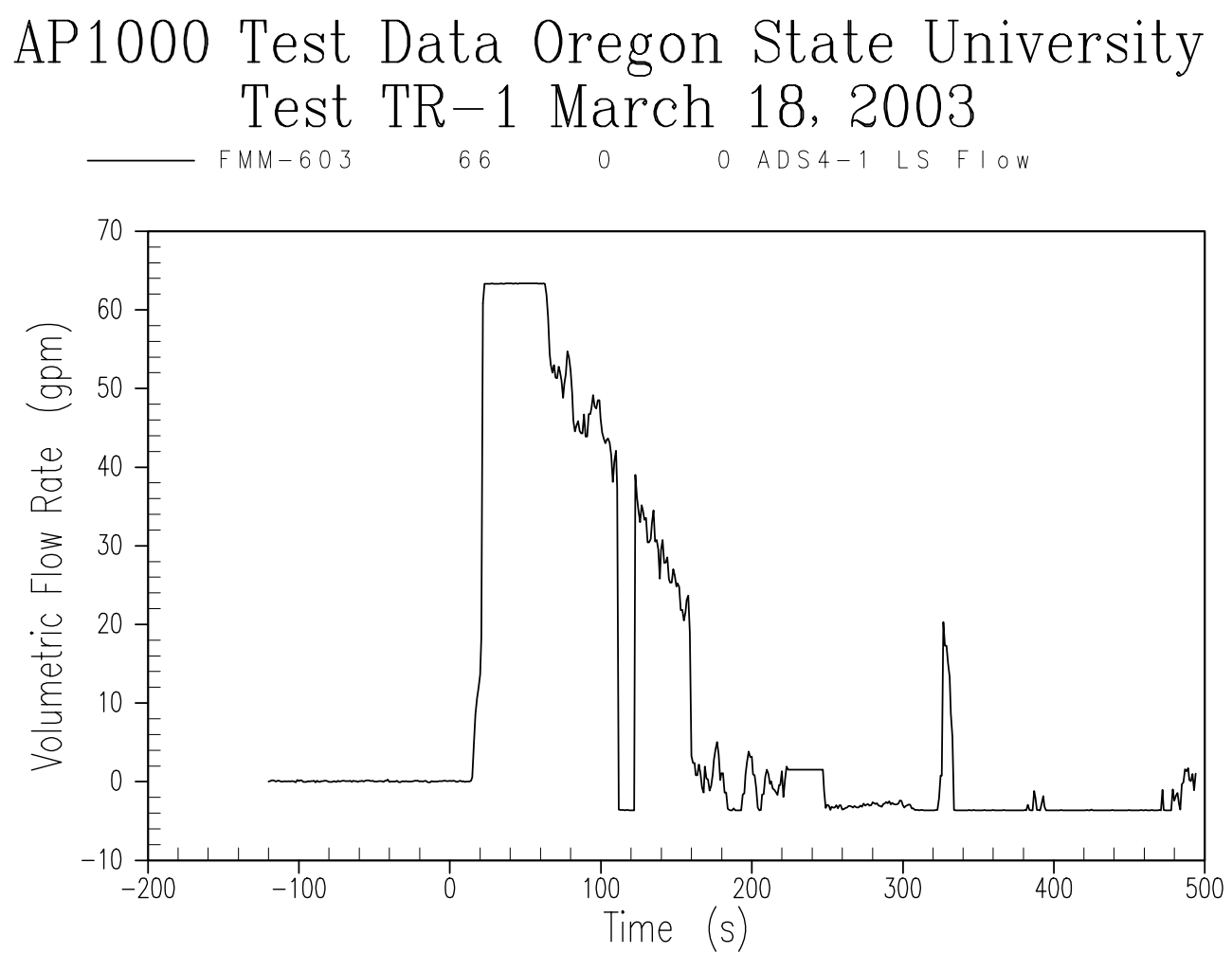

Figure A-50 ADS 4-1 Separator Liquid Flow Rate 

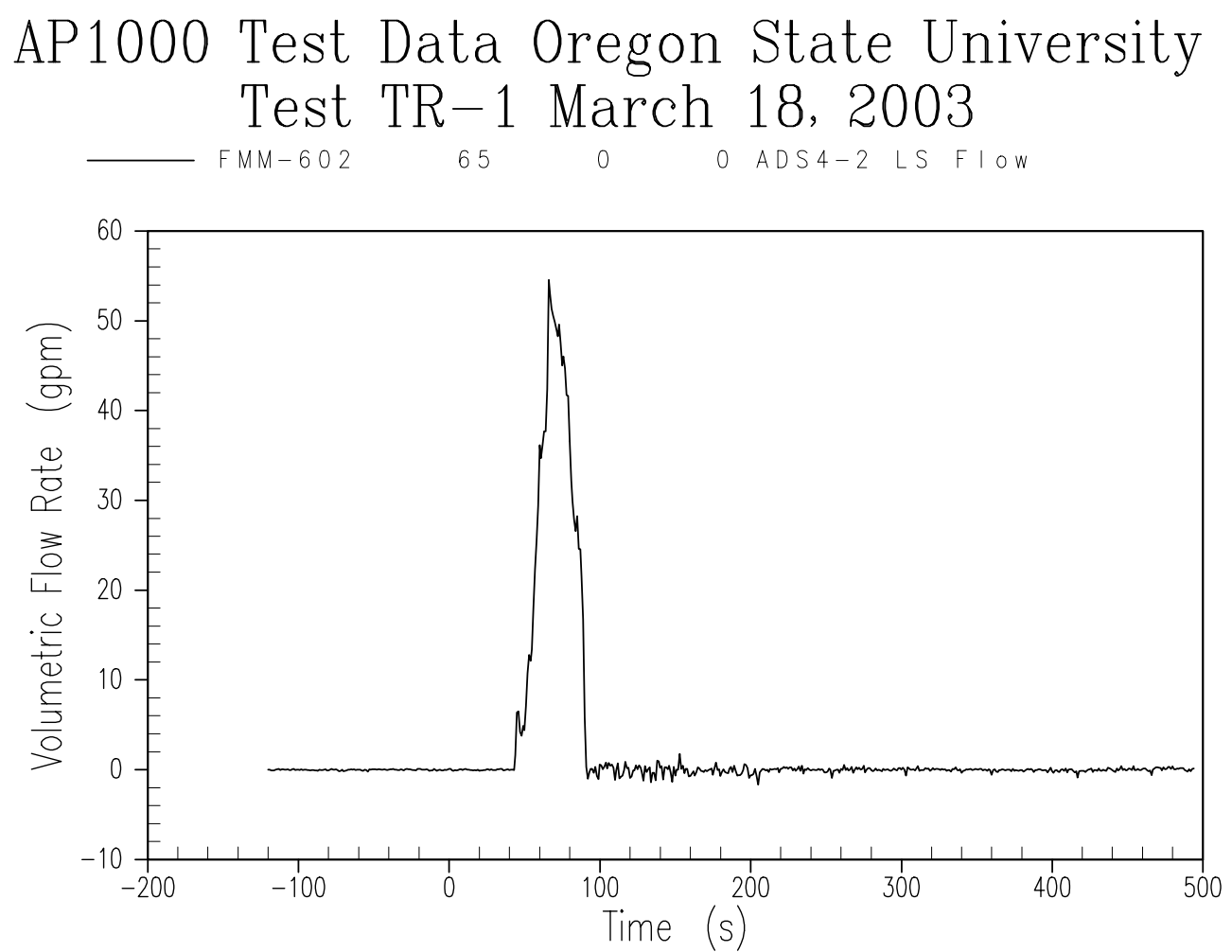

Figure A-51 ADS 4-2 Separator Liquid Flow Rate 

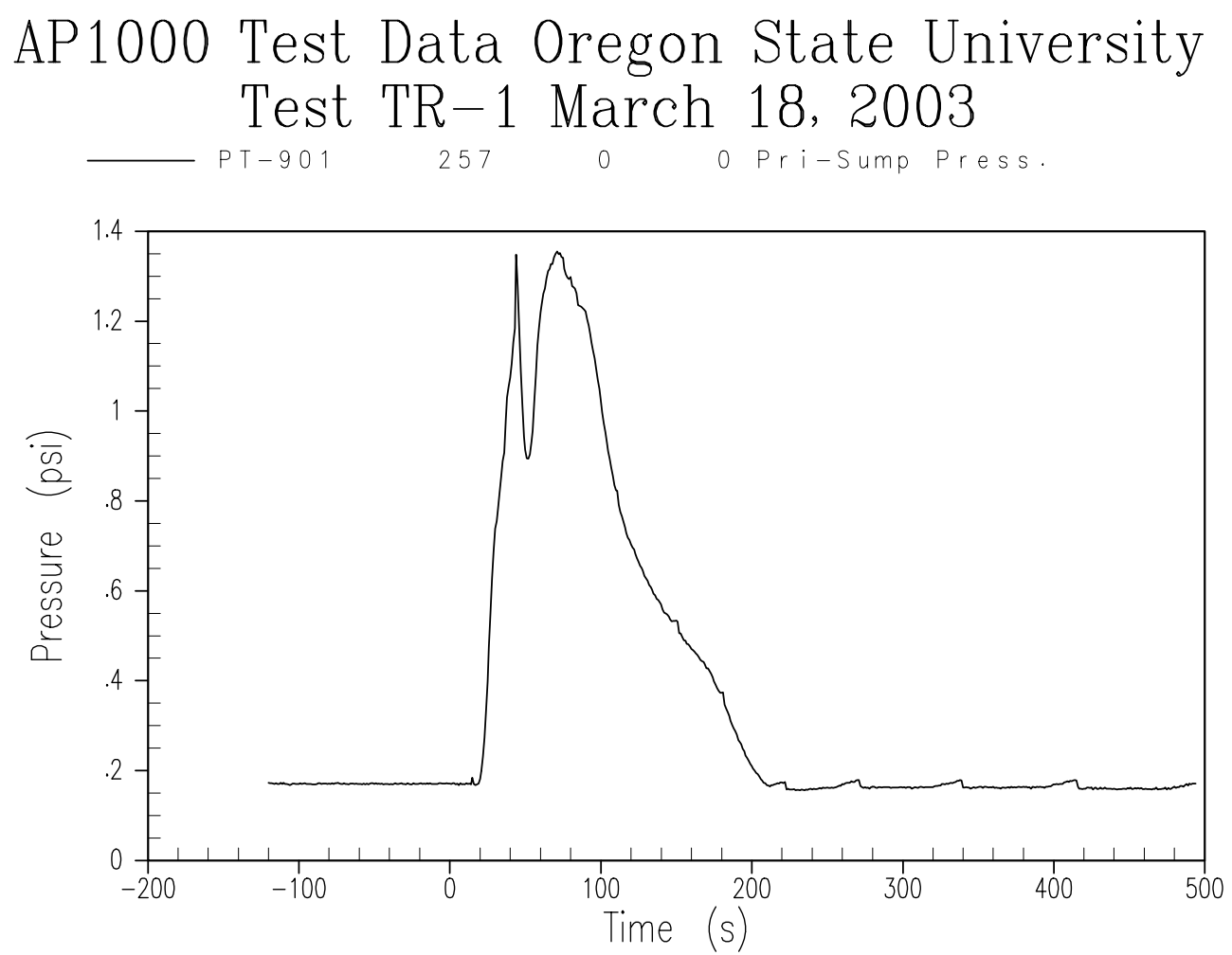

Figure A-52 Primary Sump Pressure 

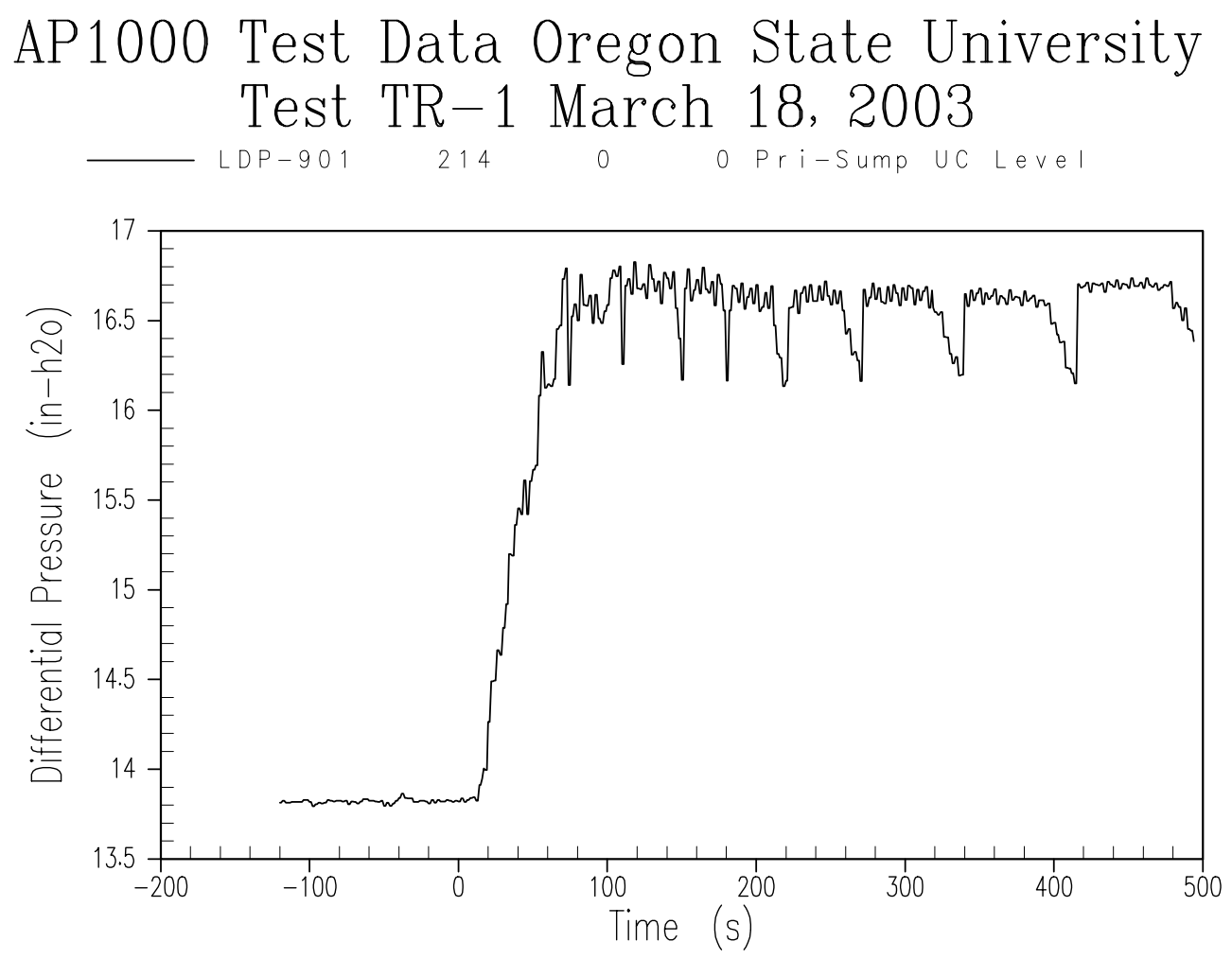

Figure A-53 Primary Sump Liquid Level 


\section{AP1000 Test Data Oregon State University Test TR-1 March 18, 2003}

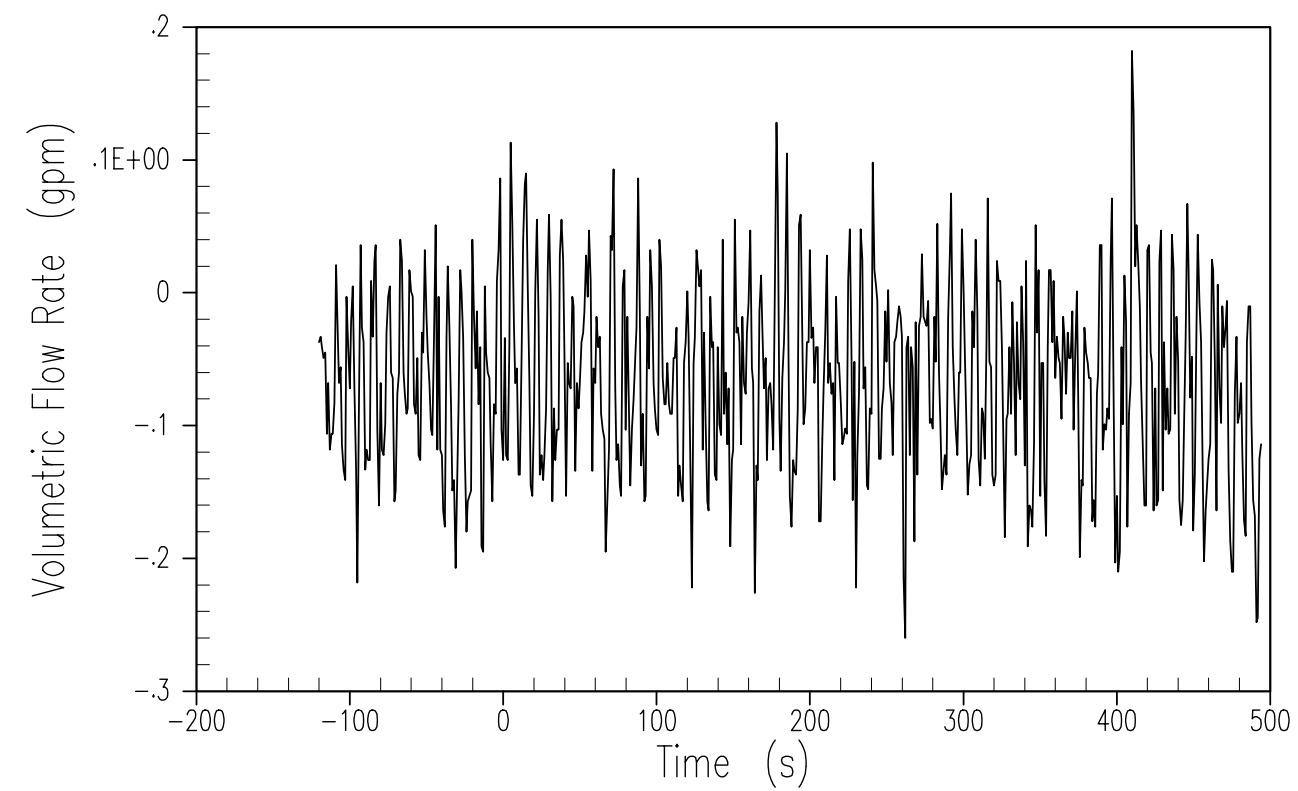

Figure A-54 Primary Sump 1 Injection Flow Rate 


\section{AP1000 Test Data Oregon State University Test TR-1 March 18, 2003}

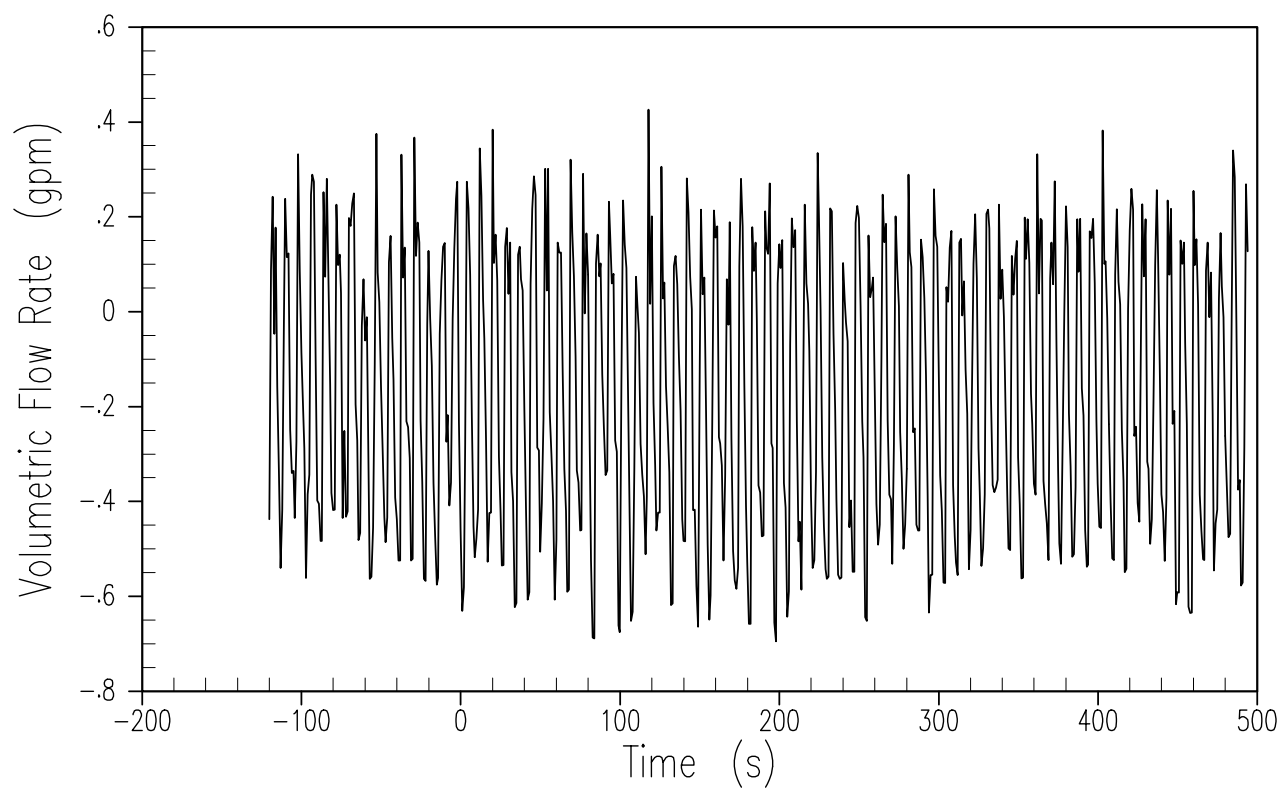

Figure A-55 Primary Sump 2 Injection Flow Rate 


\section{AP1000 Test Data Oregon State University Test TR-1 March 18, 2003}

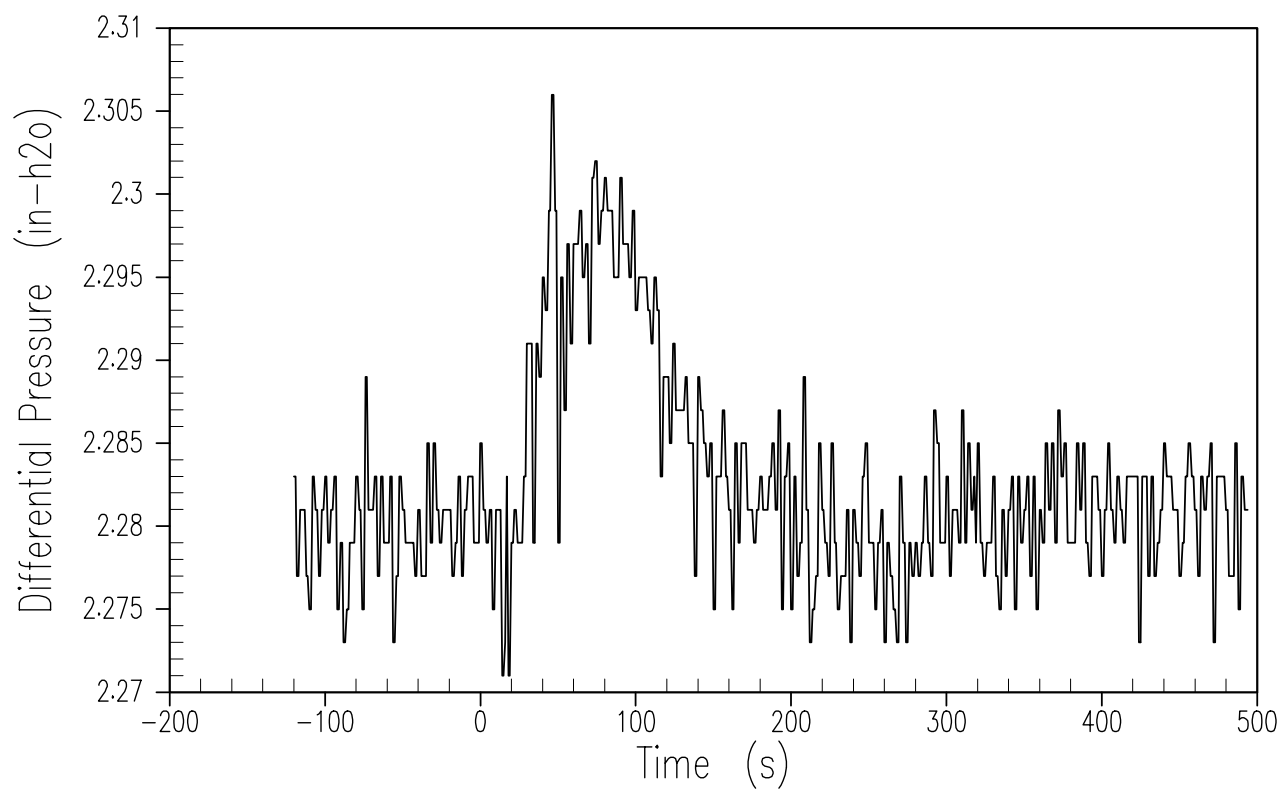

Figure A-56 Secondary Sump Liquid Level 


\section{AP1000 Test Data Oregon State University Test TR-1 March 18, 2003}

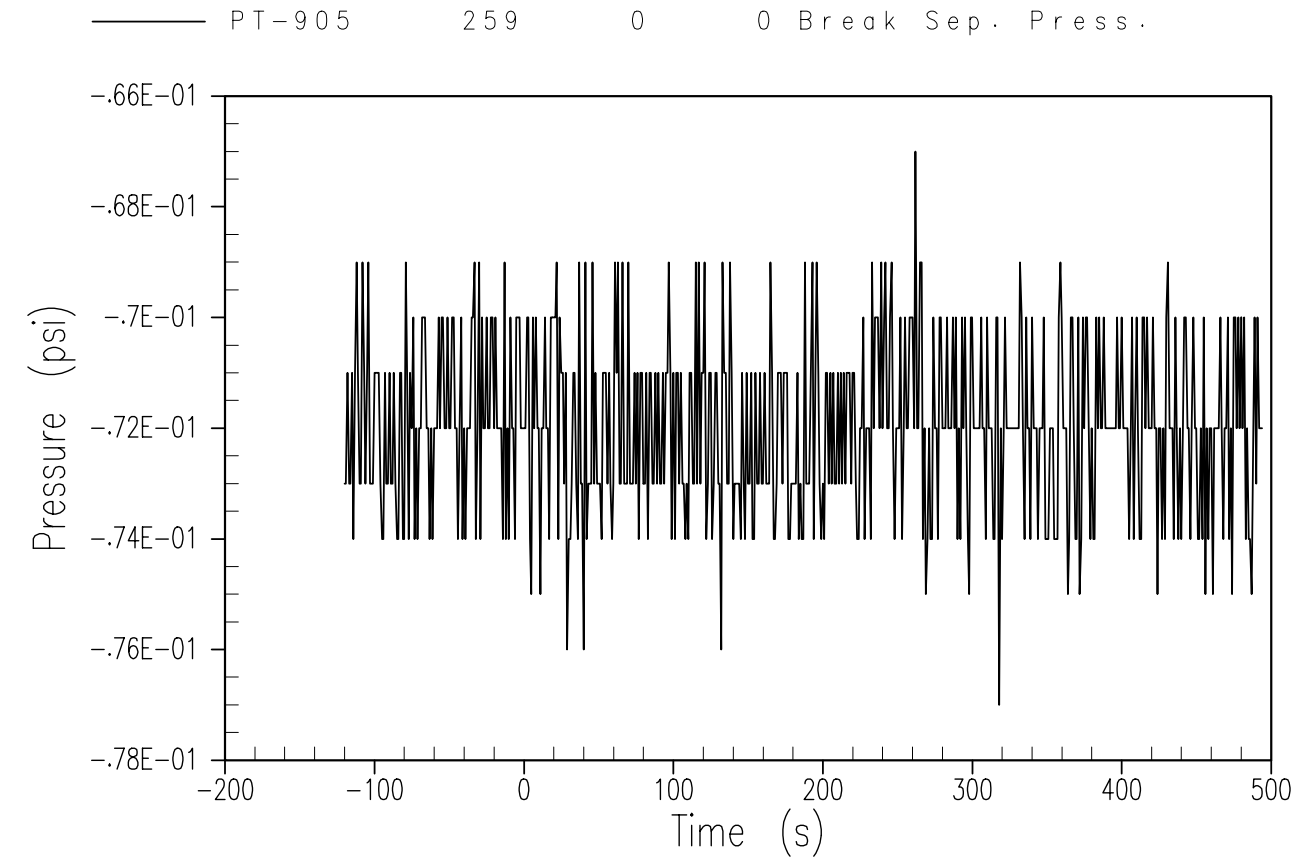

Figure A-57 Break Separator Pressure 

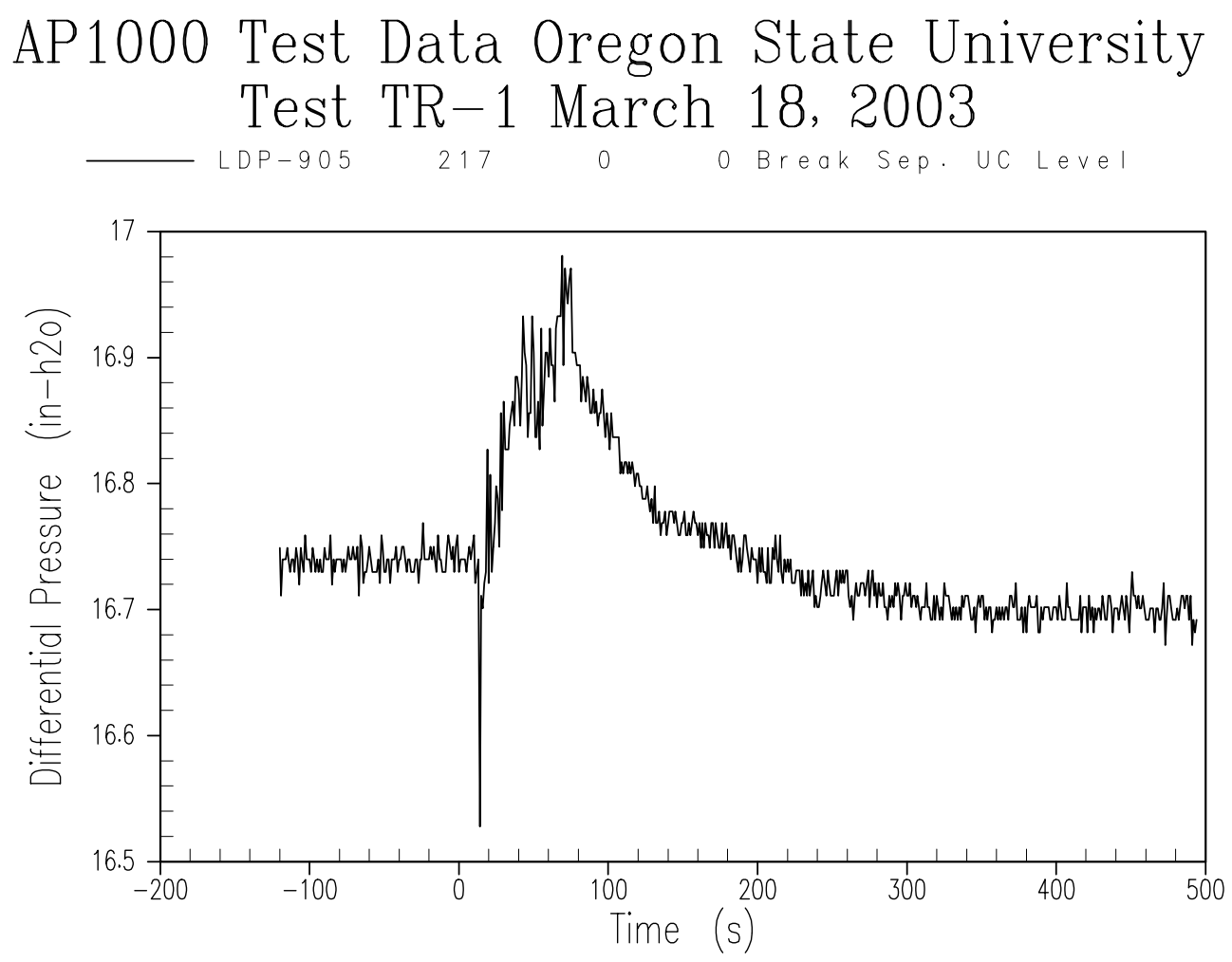

Figure A-58 Break Separator Liquid Level 

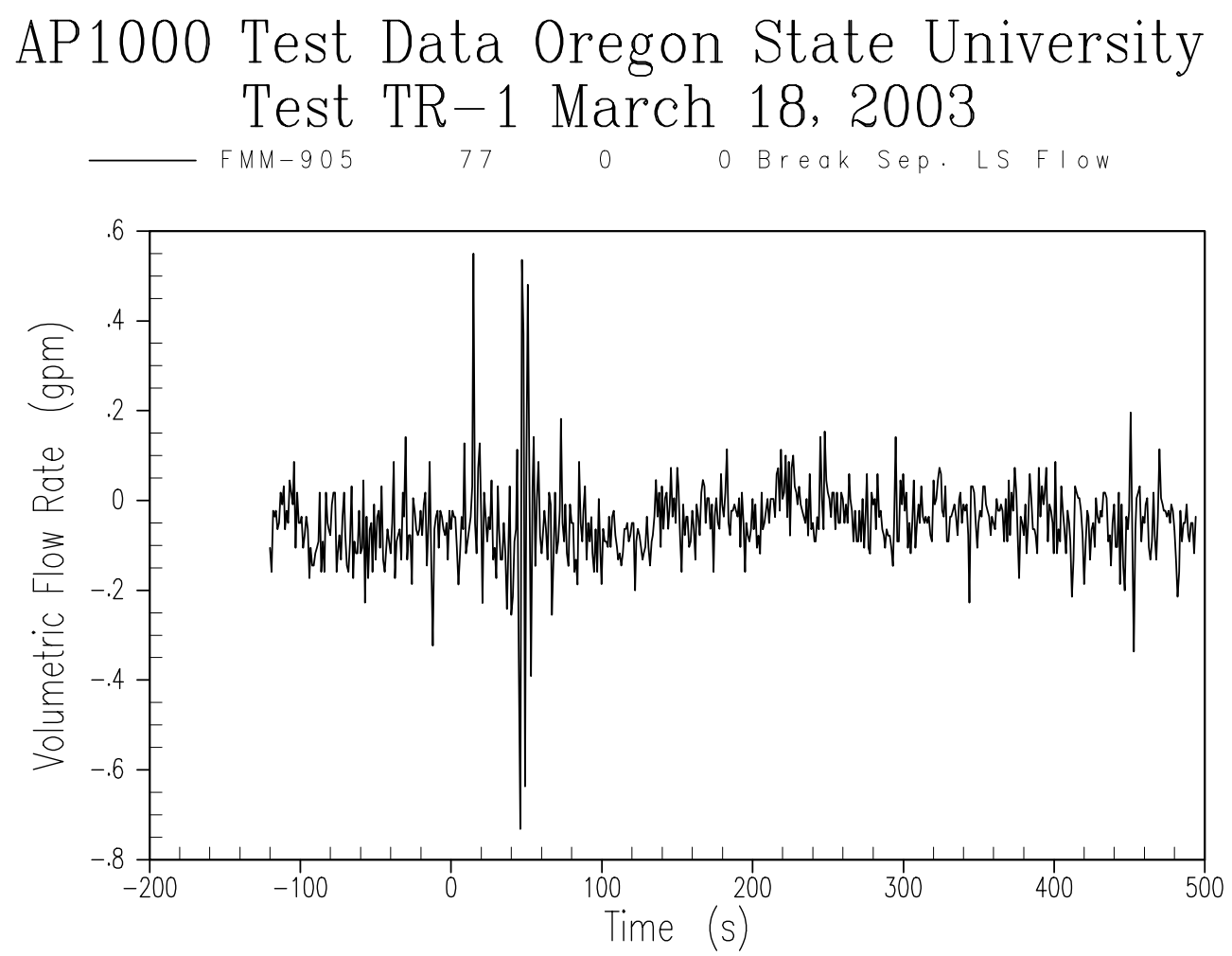

Figure A-59 Break Separator Flow to Primary Sump 

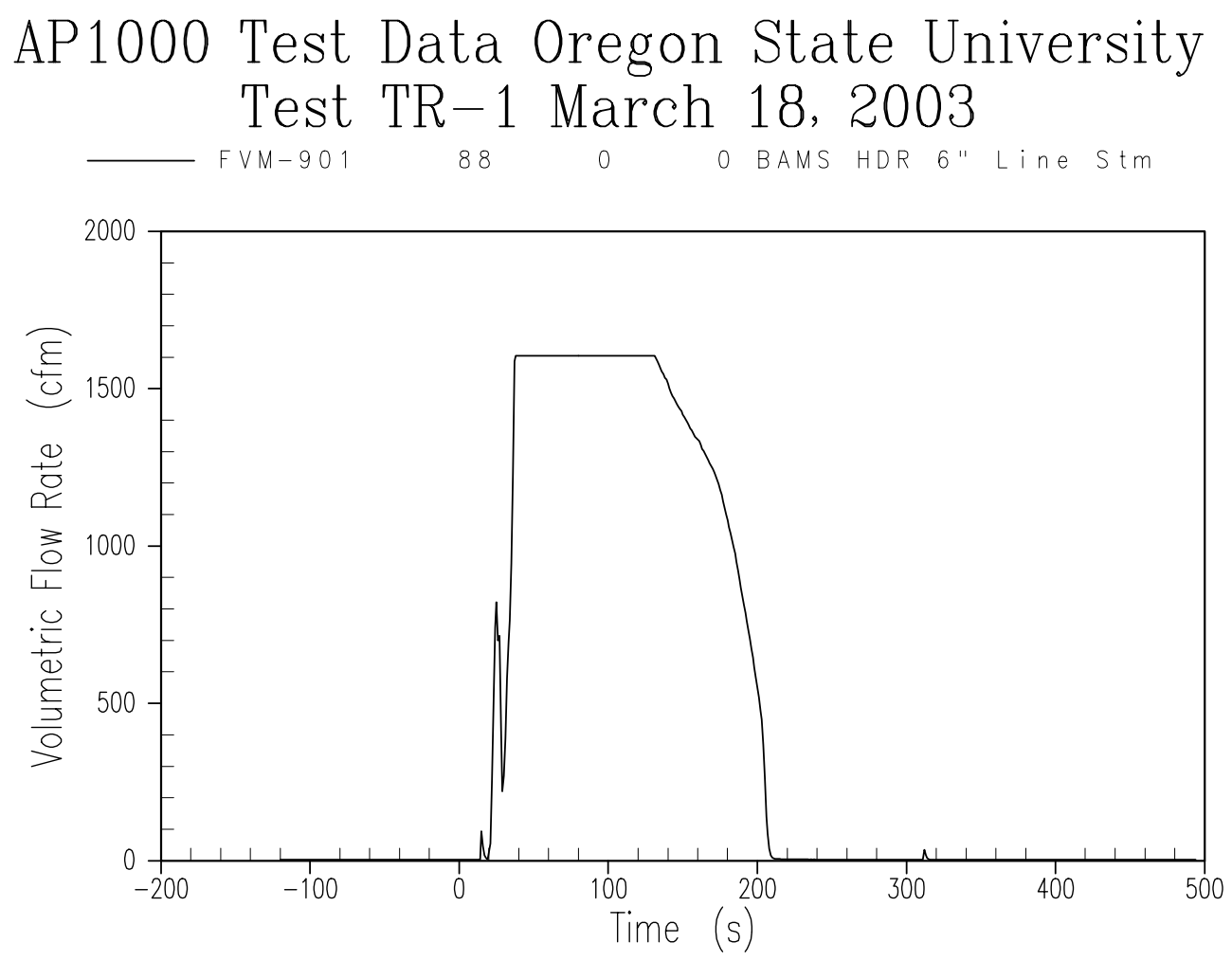

Figure A-60 BAMS Steam Flow Rate - 6-inch Line 

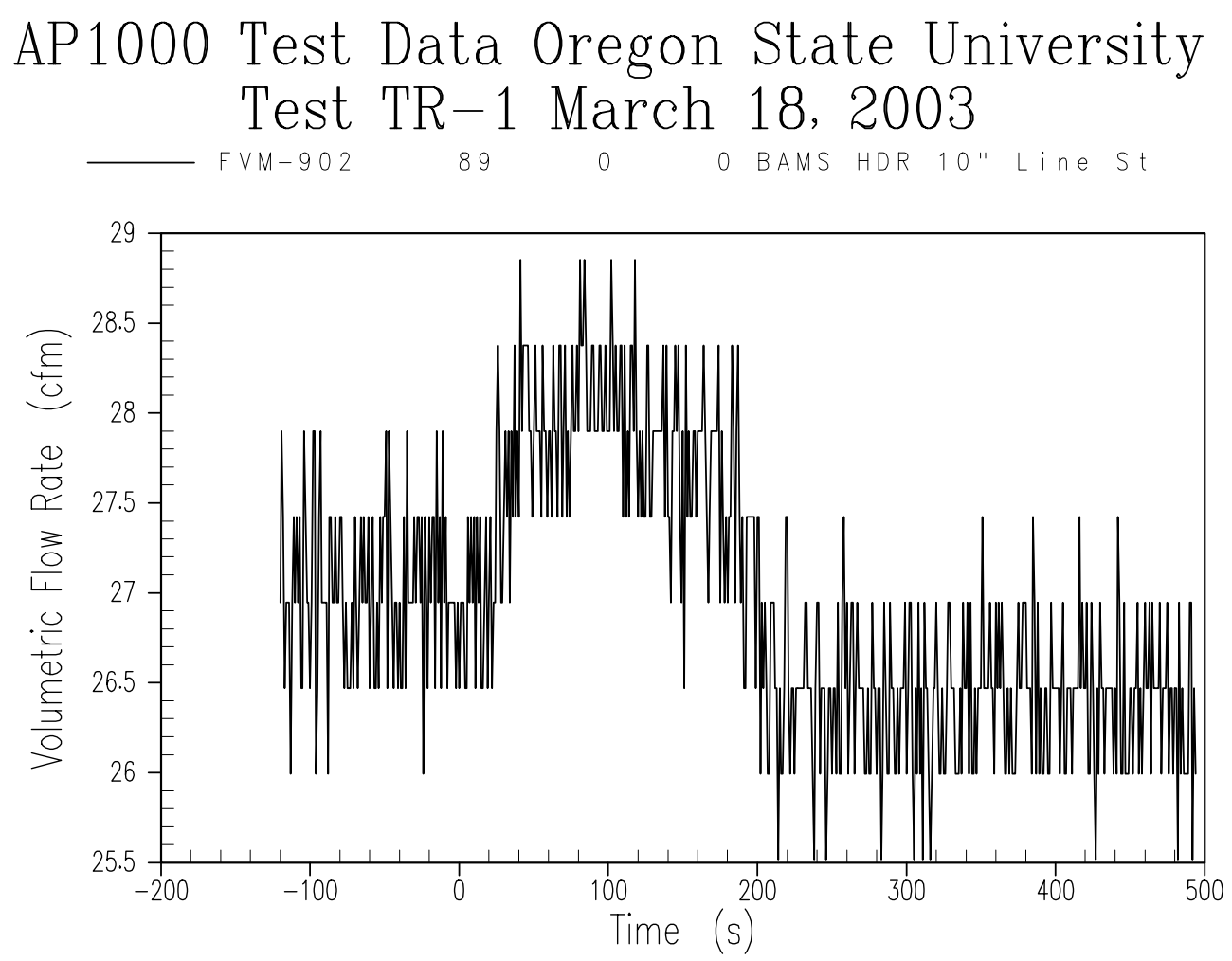

Figure A-61 BAMS Steam Flow Rate - 10-inch Line 

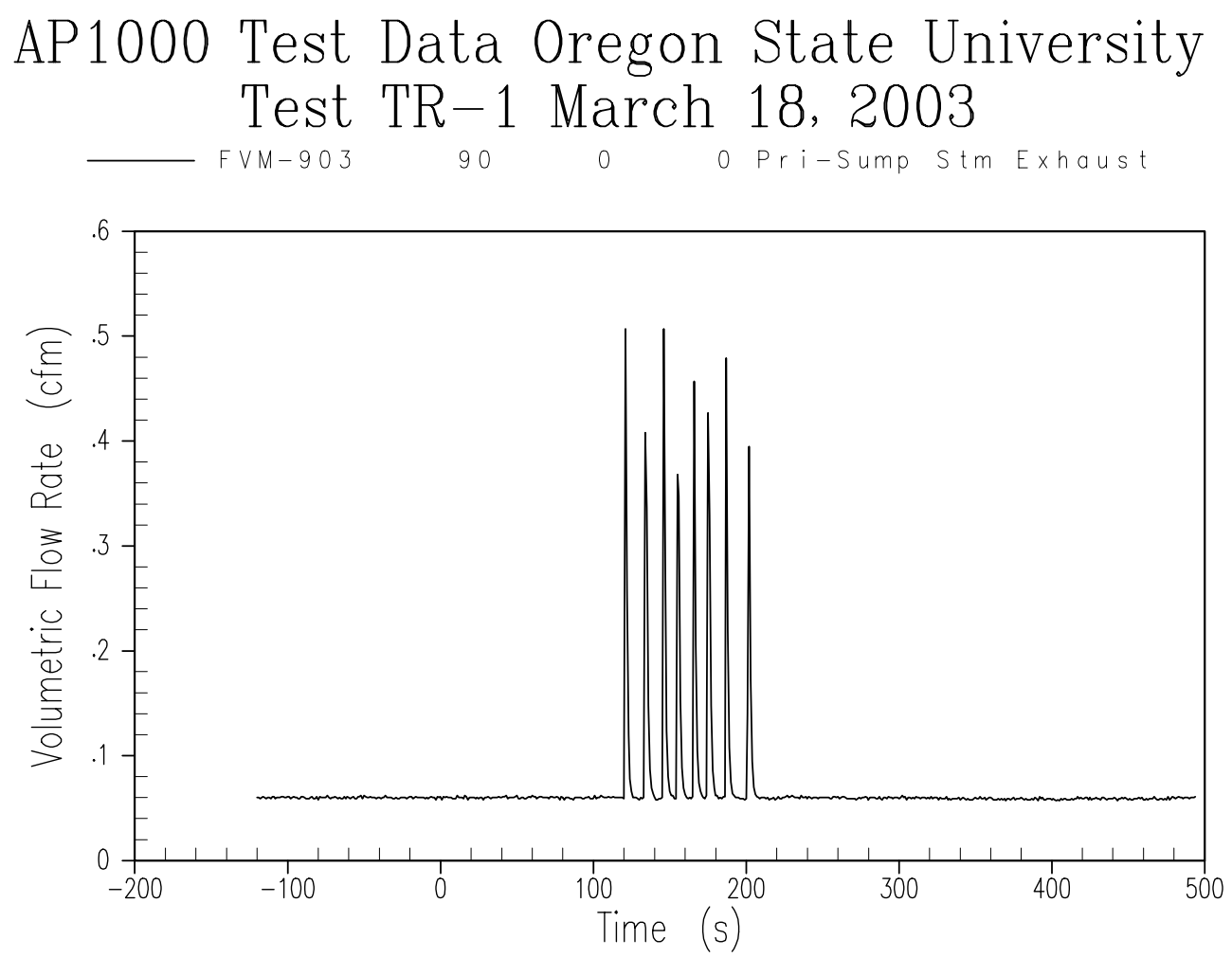

Figure A-62 BAMS/Primary Sump Steam Flow Rate 

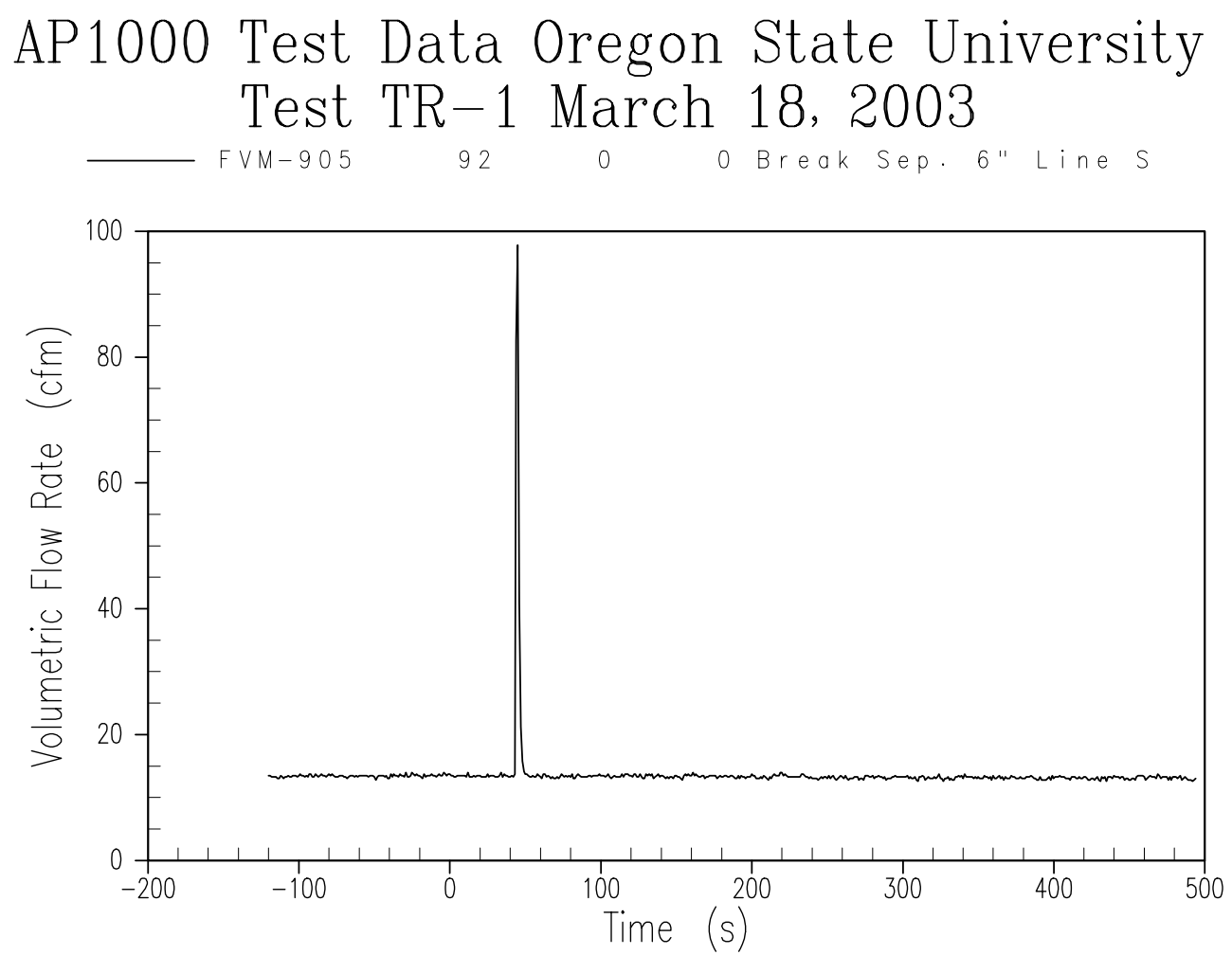

Figure A-63 BAMS/Separator Steam Flow Rate - 6-inch Pipe 

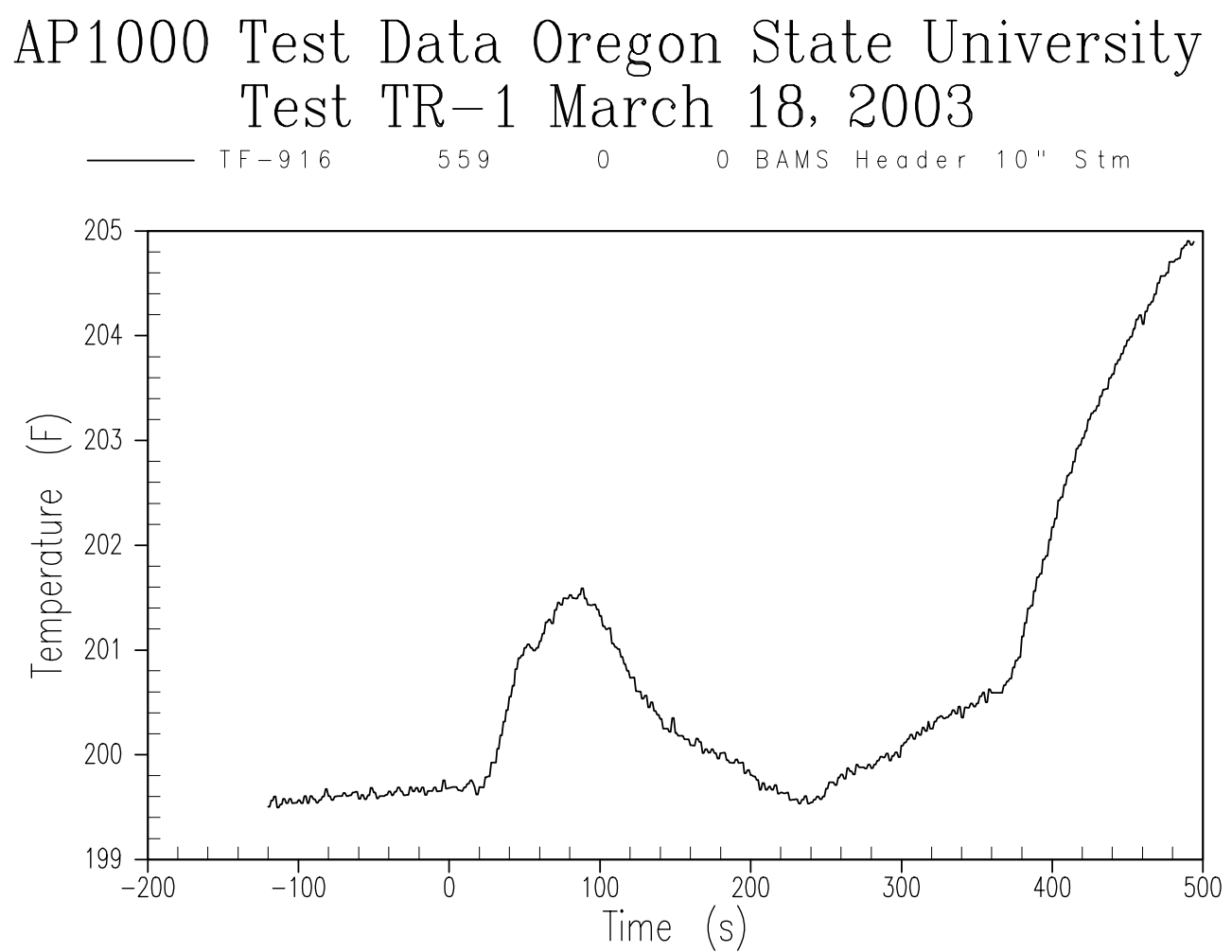

Figure A-64 BAMS/Exhaust Line Temperature - 10-inch Line 

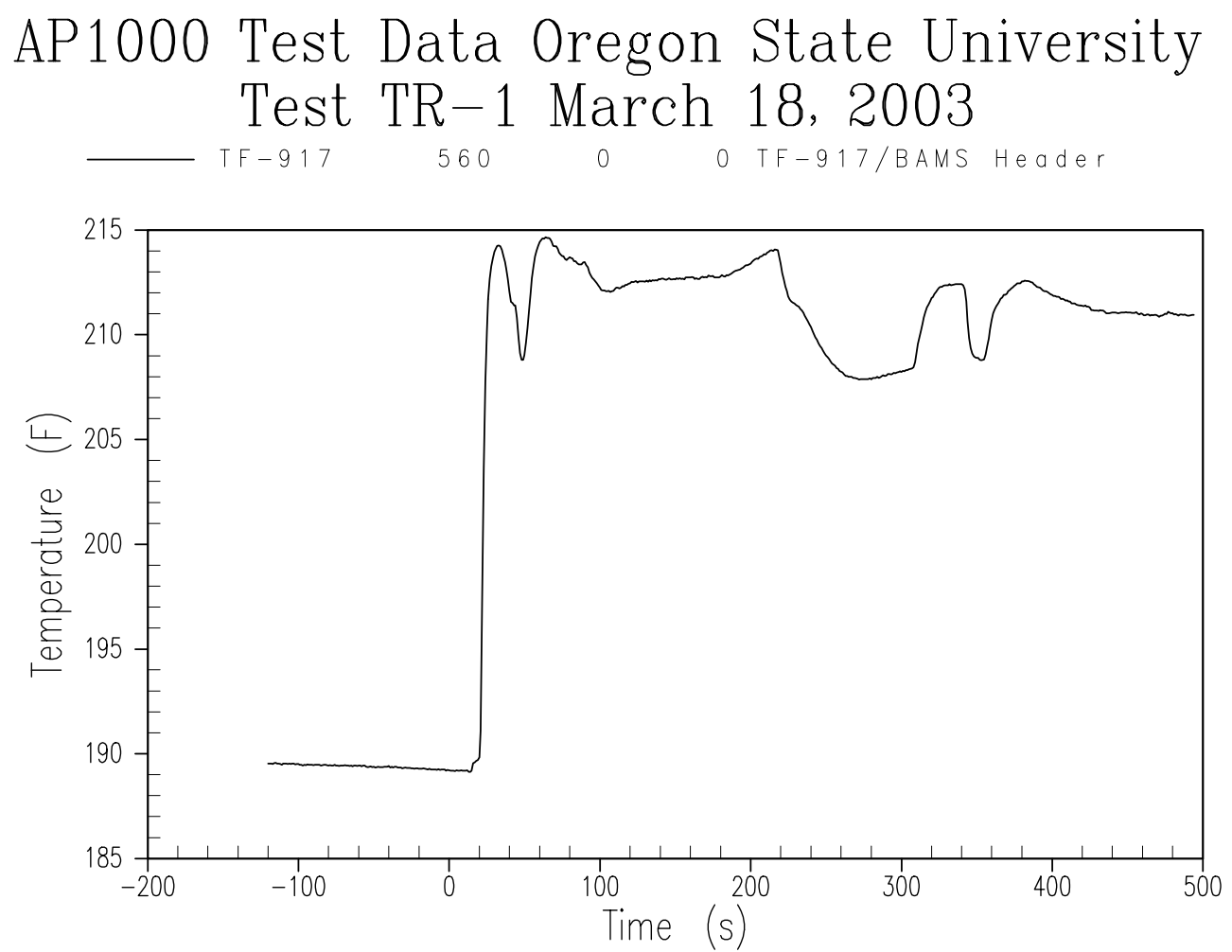

Figure A-65 BAMS/Exhaust Line Temperature - Header 


\section{AP1000 Test Data Oregon State University Test TR-1 March 18, 2003}

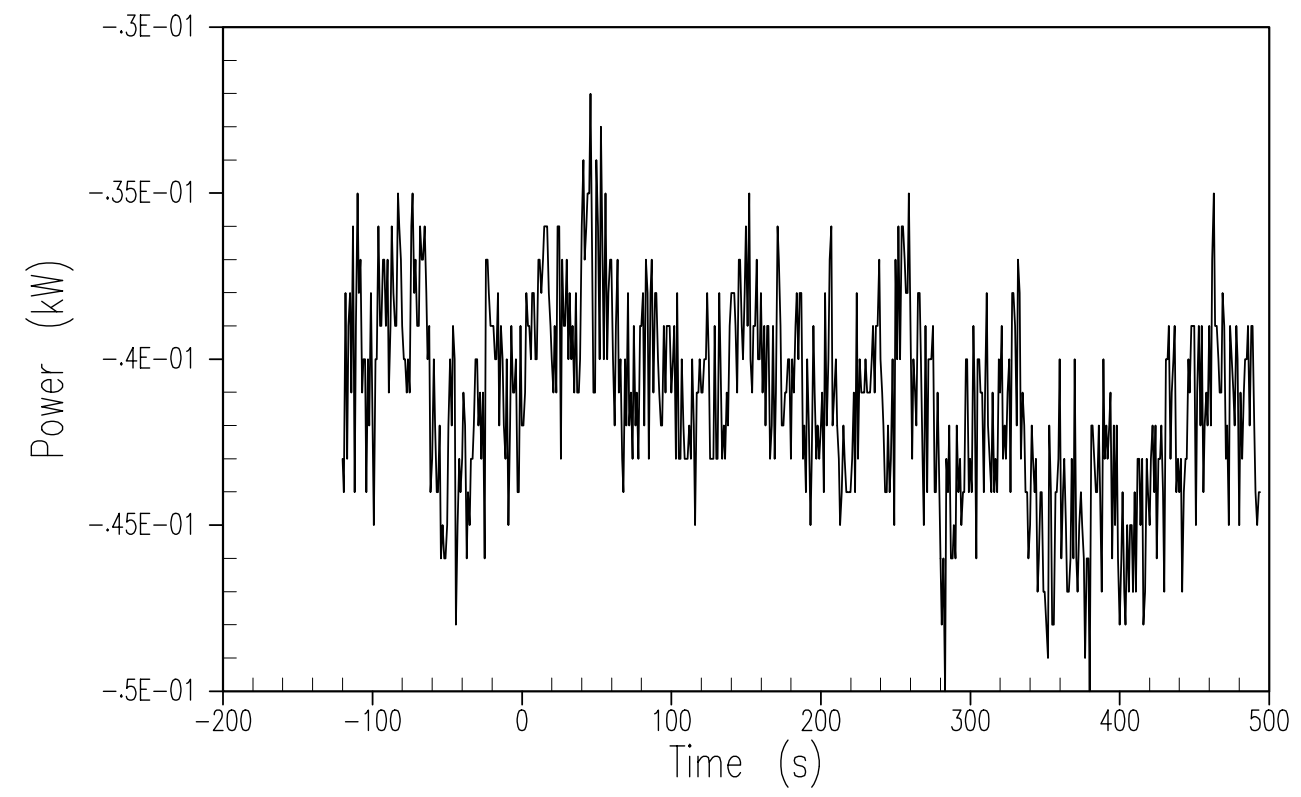

Figure A-66 Pressurizer Heater Input Power 
AP1000 Test Data Oregon State University Test TR-1 March 18, 2003

$-K W-101$

130

0

o RX Htr Group 1 Power

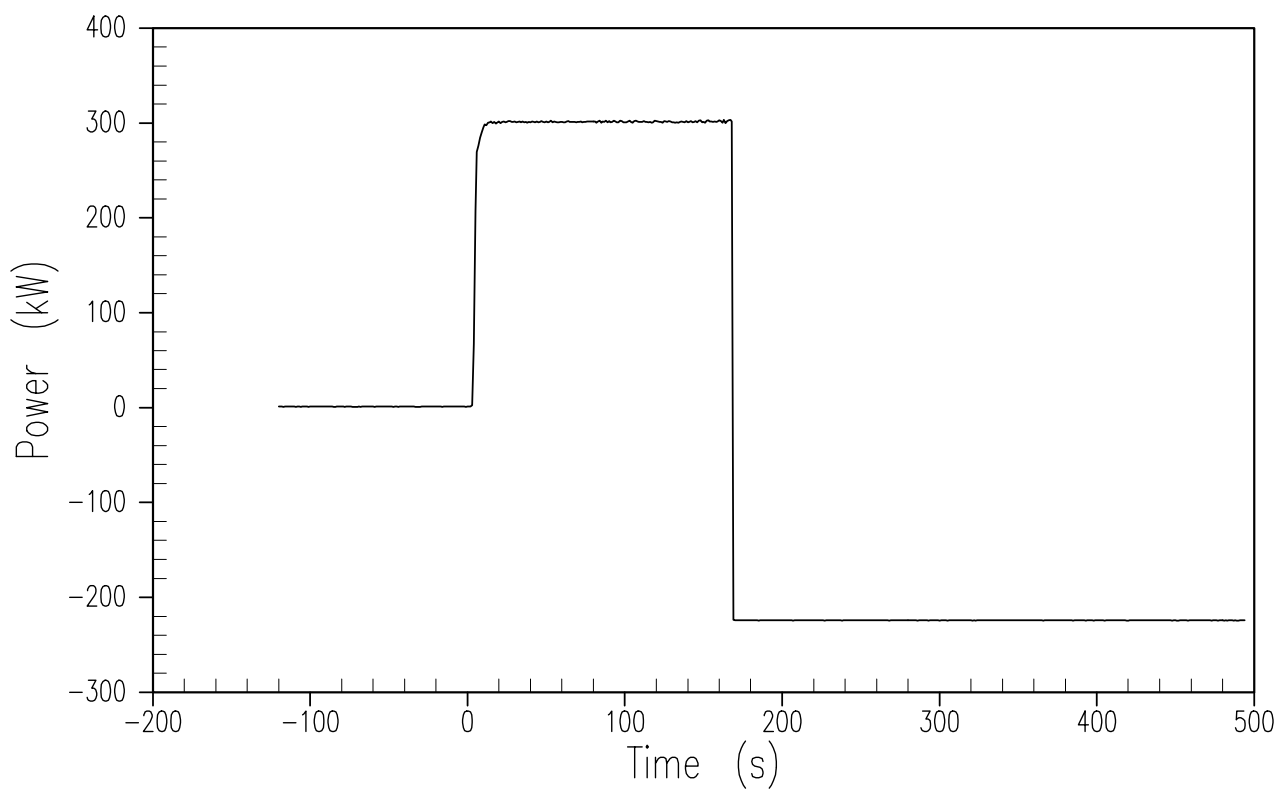

Figure A-67 Core Power Group 1 Input Power 
AP1000 Test Data Oregon State University Test TR-1 March 18, 2003

$-K W-102$

131

0

o RX Htr Group 2 Power

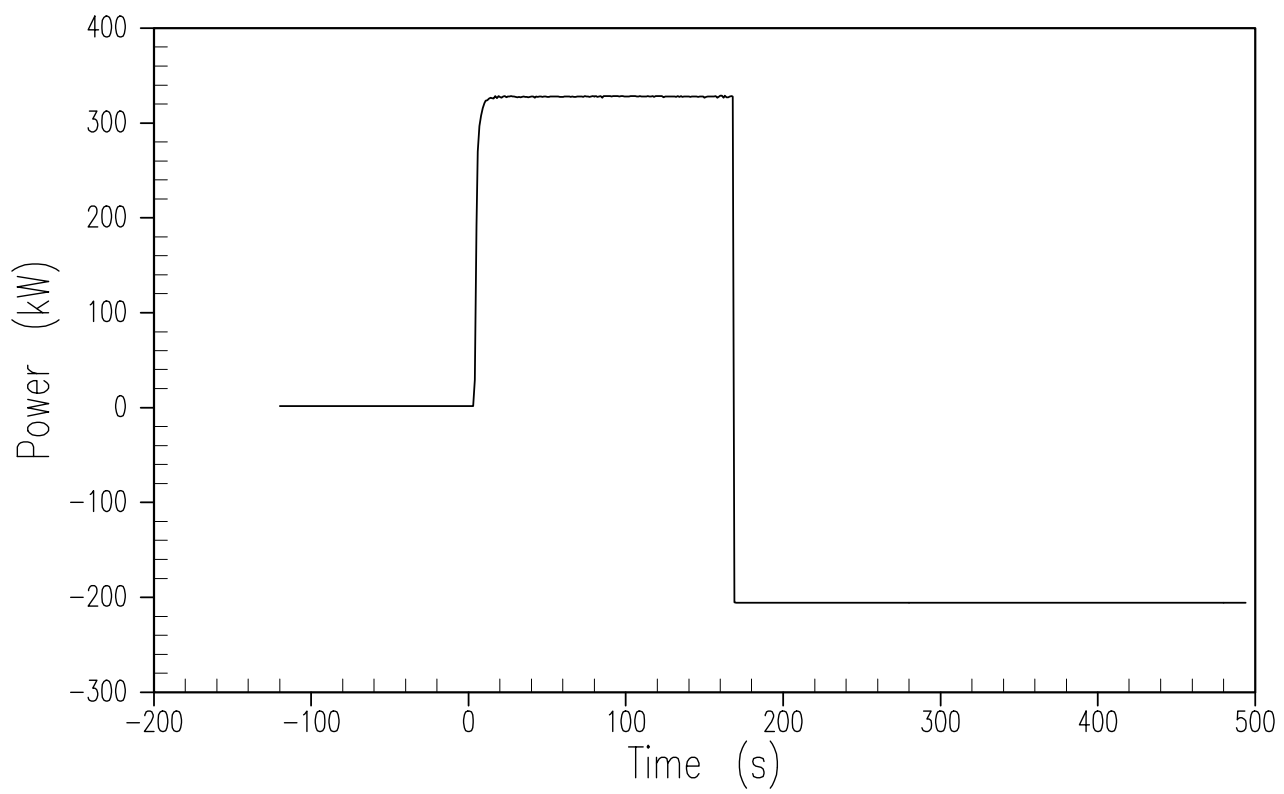

Figure A-68 Core Power Group 2 Input Power 

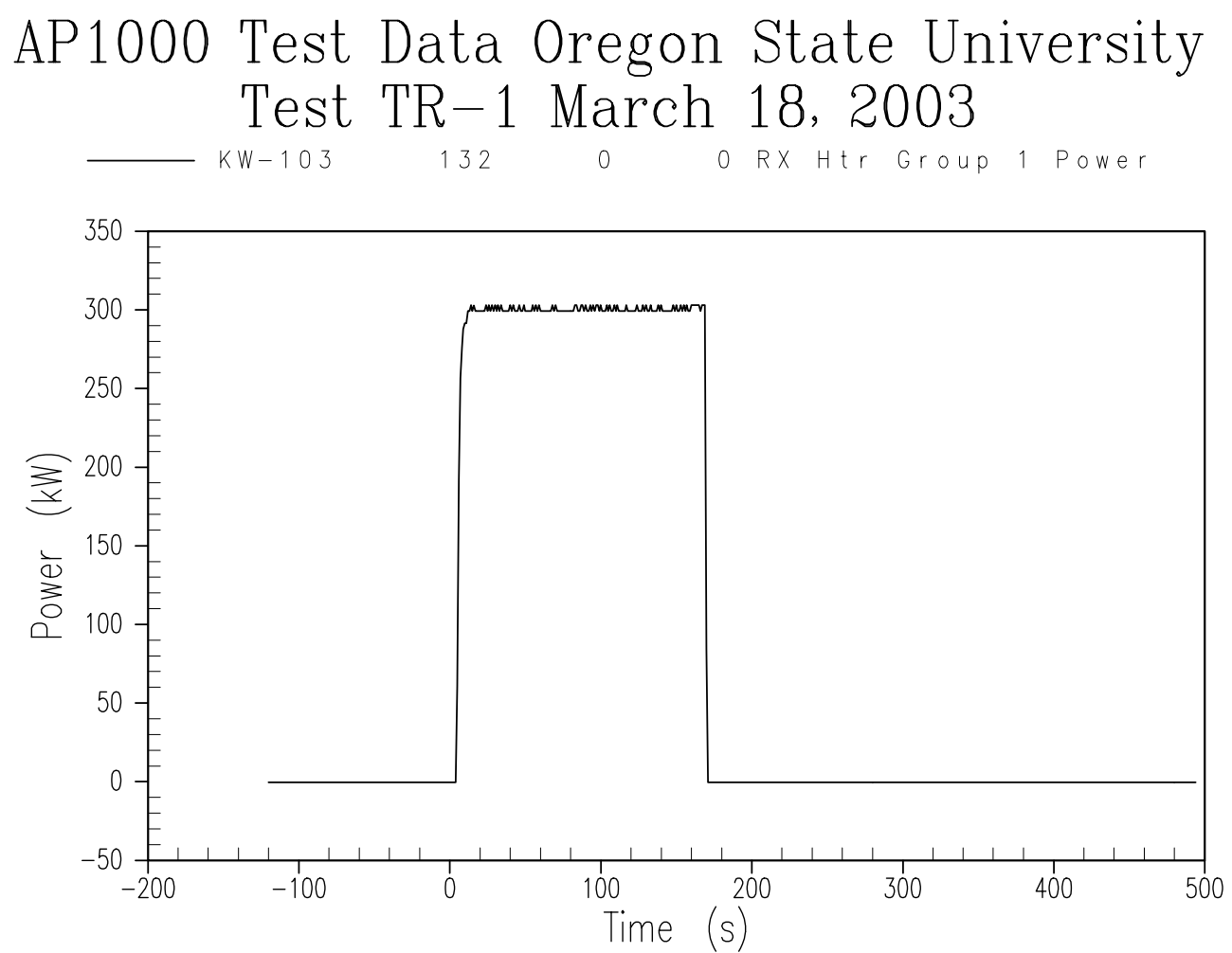

Figure A-69 Core Power Group 1 Alternate Input Power 

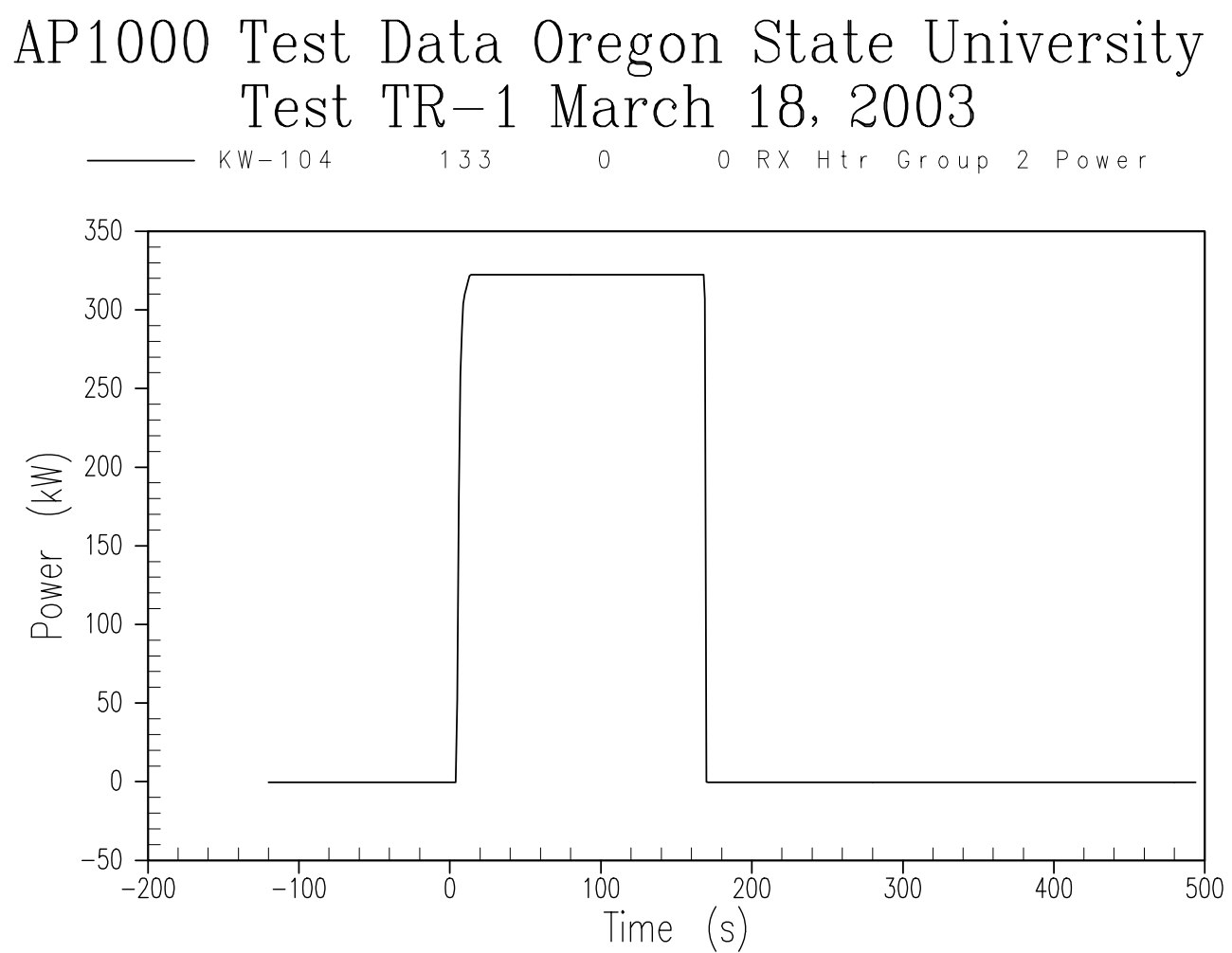

Figure A-70 Core Power Group 2 Alternate Input Power 

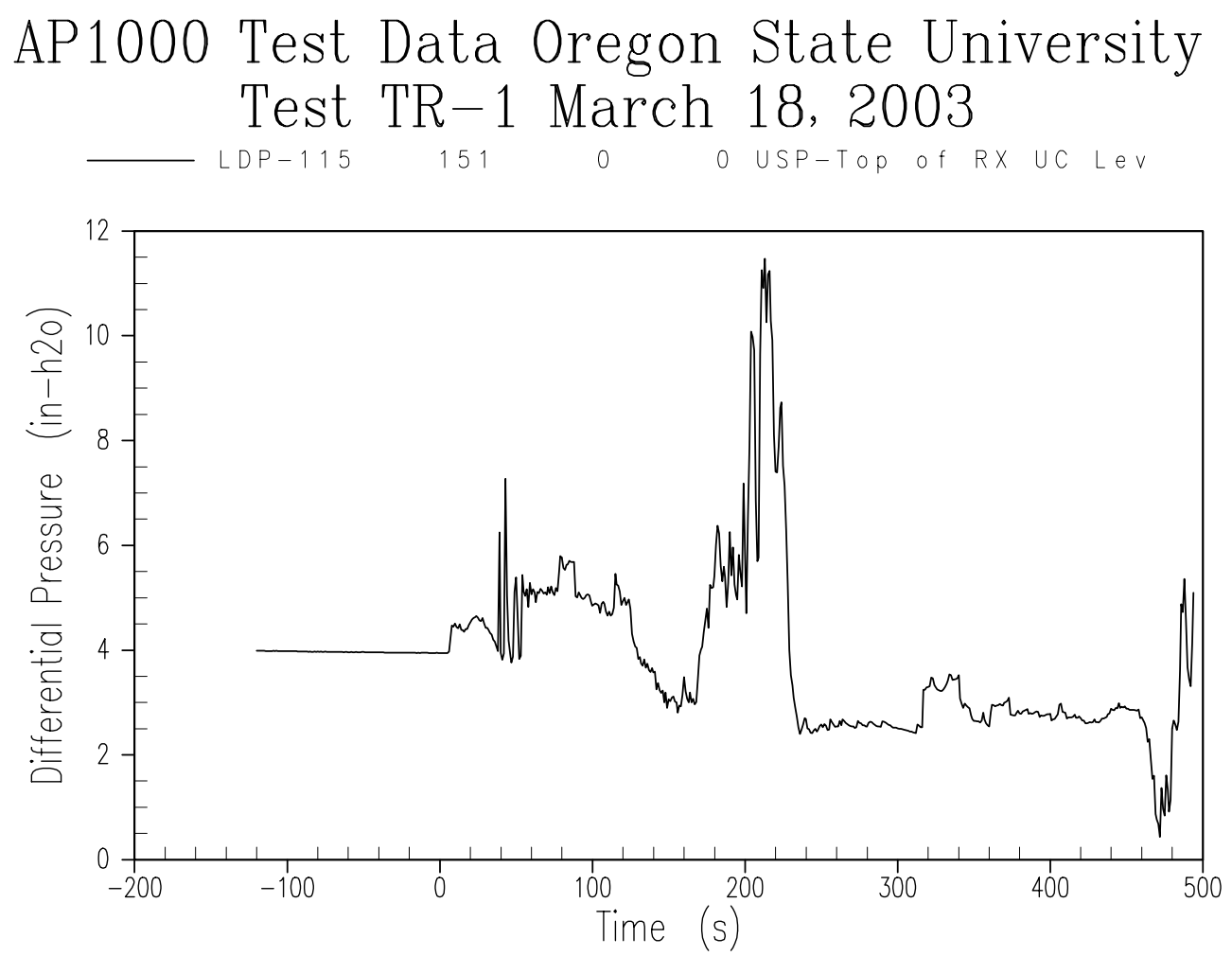

Figure A-71 Reactor Vessel Liquid Level Between Top of Vessel - Upper Support Plate 

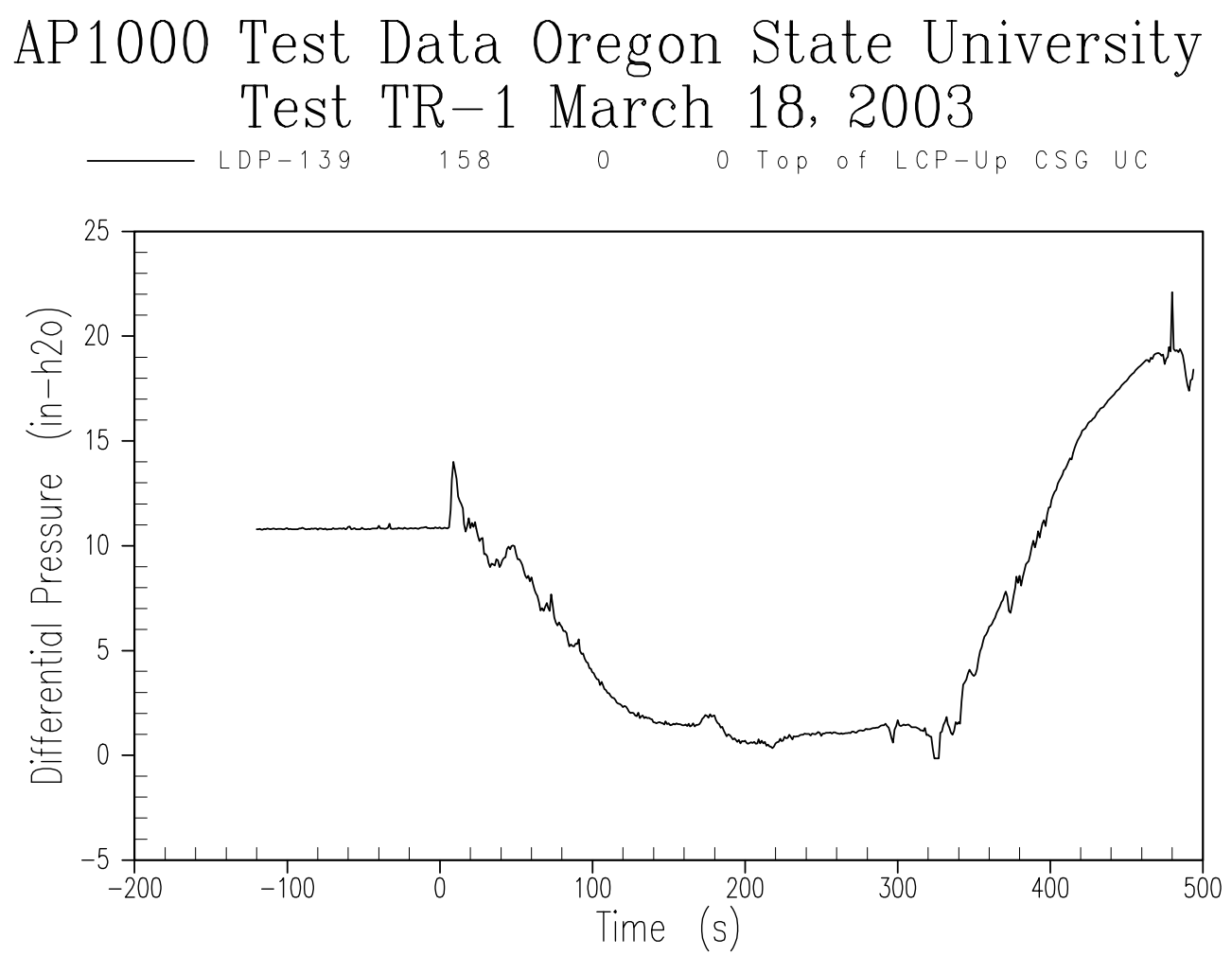

Figure A-72 Reactor Vessel Liquid Level Between Bottom of Upper Support Plate Upper Core Spacer Grid 

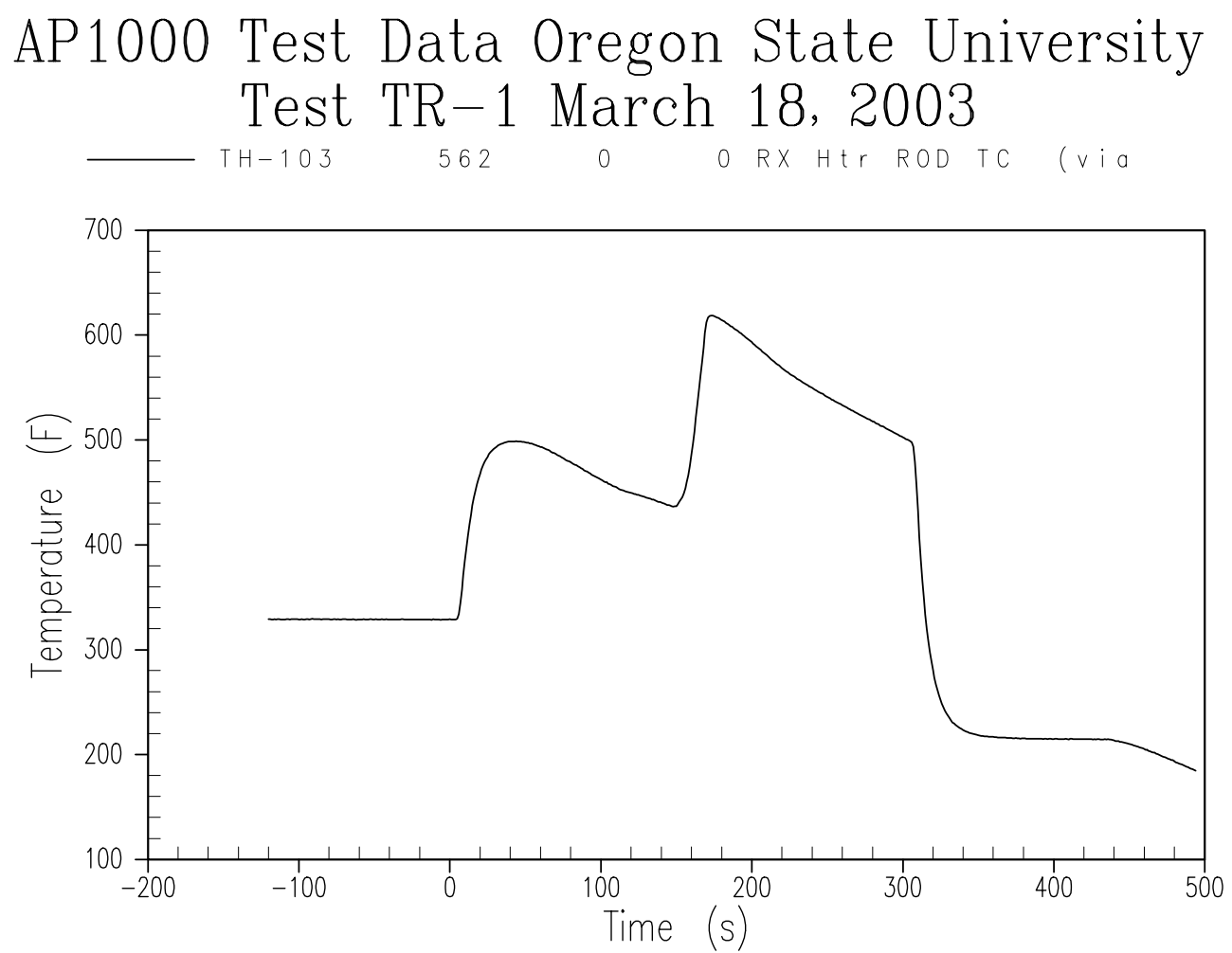

Figure A-73 Inner Core Thermocouple Measuring Heater Temperature 

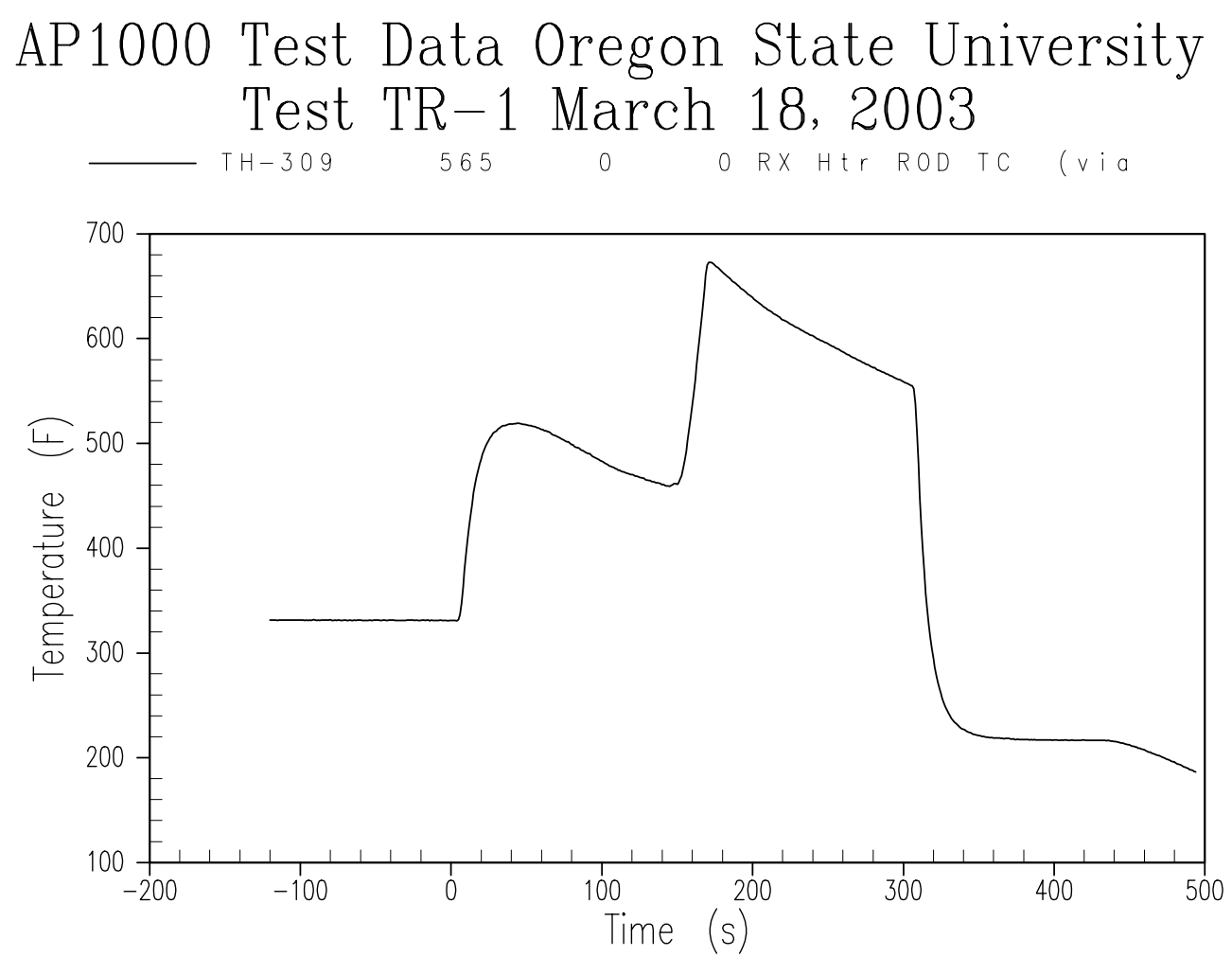

Figure A-74 Outer Core Thermocouple Measuring Heater Temperature 


\section{APPENDIX B}

TEST DATA 


\section{Appendix D -- OSU-AP1000-04}




\title{
Oregon State University
}

Department of Nuclear Engineering

\author{
AdvanCEd Thermal Hydraulic \\ RESEARCH LABORATORY
}

\section{TEST SUMMARY REPORT}

\author{
OSU-AP1000-04
}

\section{AP1000 DOUBLE-ENDED DVI WITH 3 OF 4 ADS 4 REVISED ADS 4 PIPING AND VALVES}

Revision 0 


\title{
TEST SUMMARY REPORT
}

\author{
OSU-AP1000-04
}

\section{AP1000 DOUBLE-ENDED DVI WITH 3 OF 4 ADS 4 REVISED ADS 4 PIPING AND VALVES}

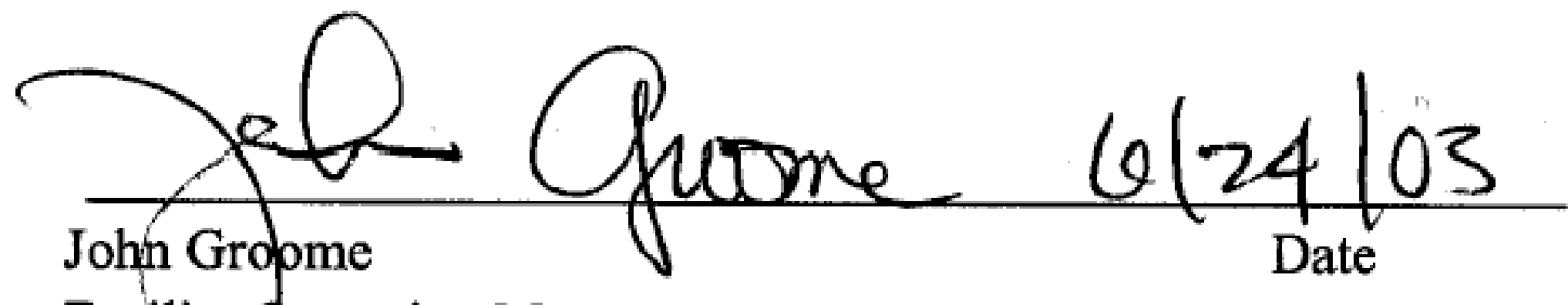

Facility $\varnothing$ perating Manager

Research Assistant

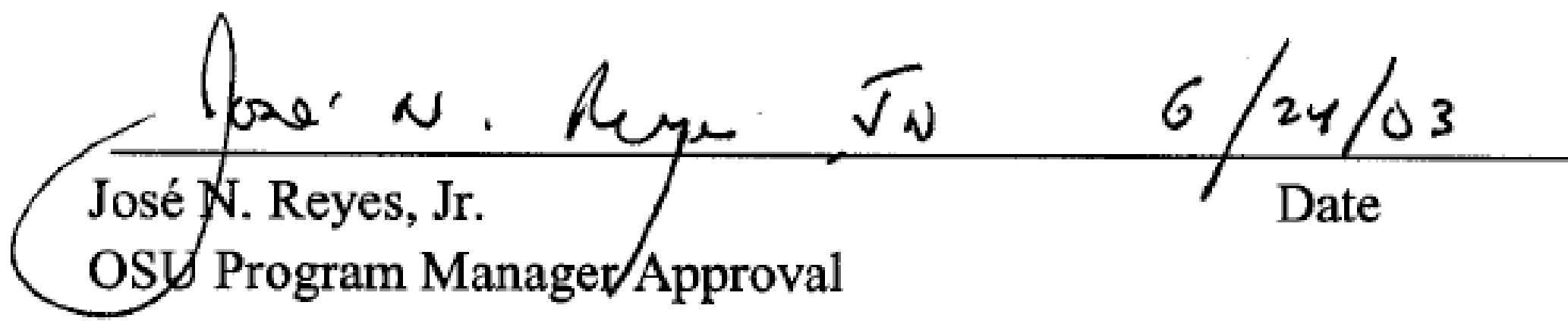

\footnotetext{
Oregon State University

116 Radiation Center

Corvallis, Oregon 97331

(C) 2003 Oregon State University

All Rights Reserved
} 


\section{WESTINGHOUSE PROPRIETARY INFORMATION NOTICE}

This report contains information proprietary to Westinghouse Electric Company. A non-proprietary version of this report has also been prepared for distribution to the United States Nuclear Regulatory Commission (NRC).

In order to conform to the requirements of 10 CFR 2.790 of the Commission's regulations concerning the protection of proprietary information so submitted to the $\mathrm{NRC}$, the information which is proprietary in the proprietary version of this document is contained within brackets, and where the proprietary information has been deleted in the non-proprietary versions, only the brackets remain (the information that was contained within the brackets in the proprietary versions having been deleted). The justification for claiming the information so designated as proprietary is indicated in both versions by means of lower case letters (a) through (f) located as a superscript immediately following the brackets enclosing each item of information being identified as proprietary or in the margin opposite such information. These lower case letters refer to the types of information Westinghouse customarily holds in confidence identified in Sections (4)(ii)(a) through (4)(ii)(f) of the affidavit accompanying the transmittal of these documents pursuant to 10 CFR 2.790(b)(1).

\section{COPYRIGHT NOTICE}

This document bears an Oregon State University copyright notice. The NRC is permitted to make the number of copies of the information contained in these reports which are necessary for its internal use in connection with generic and plant-specific reviews and approvals as well as the issuance, denial, amendment, transfer, renewal, modification, suspension, revocation, or violation of a license, permit, order, or regulation subject to the requirements of $10 \mathrm{CFR} 2.790$ regarding restrictions on public disclosure to the extent such information has been identified as proprietary by Westinghouse, copyright protection notwithstanding. With respect to the non-proprietary versions of these reports, the NRC is permitted to make the number of copies beyond these necessary for its internal use which are necessary in order to have one copy available for public viewing in the appropriate docket files in the public document room in Washington, DC and in local public document rooms as may be required by NRC regulations if the number of copies submitted is insufficient for this purpose. Copies made by the NRC must include the copyright notice in all instances and the proprietary notice if the original was identified as proprietary. 
TABLE OF CONTENTS

$\underline{\text { Section }}$

$\underline{\text { Title }}$

$\underline{\text { Page }}$

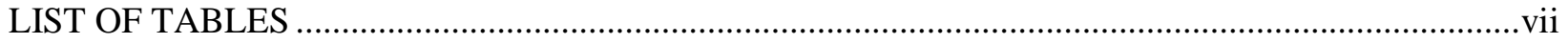

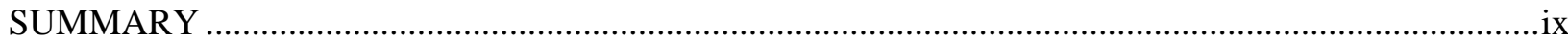

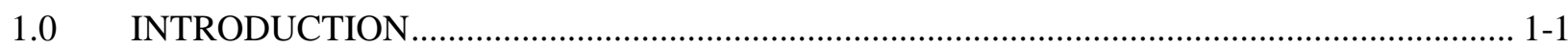

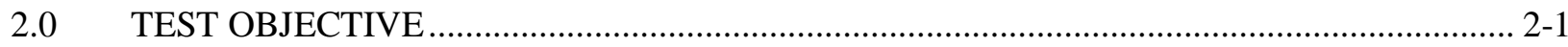

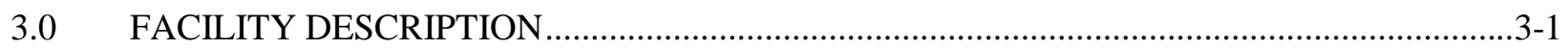

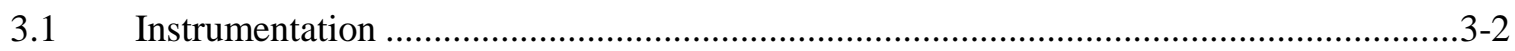

4.0 OREGON STATE UNIVERSITY TESTING PROGRAM MATRIX ................................... 4-1

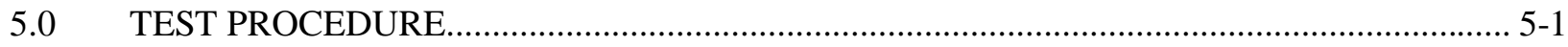

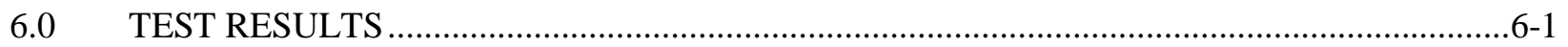

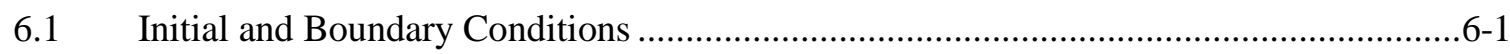

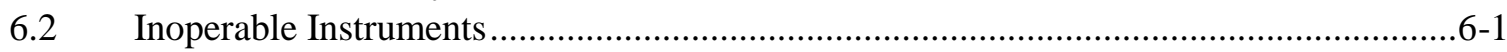

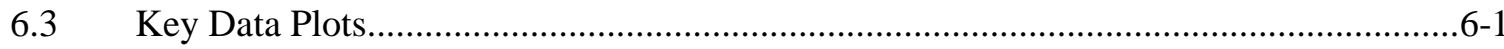

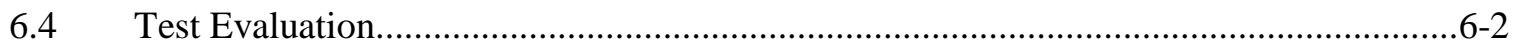

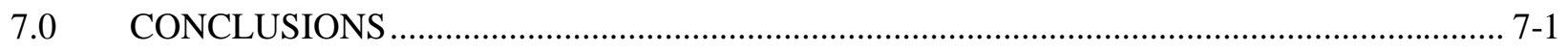

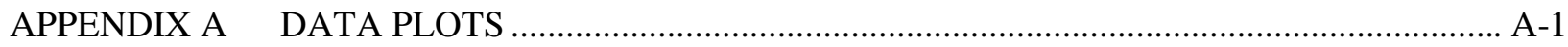

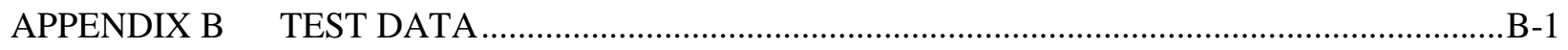




\section{LIST OF TABLES}

$\underline{\text { Table }}$

Table 4-1

Table 6-1

Table 6-2

Table 6-3

Table 6-4 $\underline{\text { Title }}$

$\underline{\text { Page }}$

$$
\text { OSU Test Matrix (Specified May 15, 2003)..... }
$$

Actual Test Initial Conditions 6-3

Sequence of Events 6-5

Inoperable Instruments for DBA-03-D Test ....................................................... 6-7

Data Plots for Test Summary Report for DBA-03-D by Component .... . $6-8$ 


\section{SUMMARY}

This report covers the test DBA-03, double-ended direct vessel injection (DVI) line break simulation loss-of-coolant accident (LOCA) performed on May 15, 2003. The objective of this test was to obtain thermal-hydraulic data for a double-ended DVI line break simulation. The test differs from DBA-02 in that for these tests, a single failure was assumed in one of the ADS-4 valves on the hot leg with the pressurizer. The test performed met the specified conditions. The top of the heater bundle was always covered during this event. The test was performed for about 6,600 seconds. The transient continued through Automatic Depressurization System (ADS) actuation, core makeup tank (CMT), accumulator, in-containment refueling water storage tank (IRWST) injection, and ended just after sump recirculation injection.

This report presents the initial assessment of the test data collected. If this test is to be used by Westinghouse to support AP1000 Design Certification, additional validation of the use of this information will be documented separately. In the interim, the list of invalid data channels may change. 


\subsection{INTRODUCTION}

The Department of Nuclear Engineering at Oregon State University (OSU) is performing a series of tests for the U.S. Department of Energy (DOE). These tests are being conducted in the Advanced Plant Experiment (APEX-1000) test facility, which is a reduced pressure and height model of the two-loop Westinghouse AP1000 pressurized water reactor. The purpose of the testing is to:

- $\quad$ evaluate the thermal-hydraulic performance of the passive safety systems of the full-scale AP1000, and

- $\quad$ to assess and validate the safety analyses techniques and computer codes used in predicting the transient system behavior.

The AP1000 Long-Term Cooling Test is a 1/4 height scale, low-pressure integral systems test simulating thermal-hydraulic phenomenon for the AP1000 passive safety systems for small-break loss-of-coolant accidents (LOCAs) and long-term cooling. It accurately models the details of the AP1000 geometry, including the primary system, the passive safety systems, and a part of the non-safety grade Chemical and Volume Control System, as well as a partial non-safety grade Normal Residual Removal System. The interconnecting pipe routings are also duplicated in the model.

The overall objective of the Long-Term Cooling Test program is to obtain test data at various modes of operation. The OSU experiments will examine the passive safety system response for the small-break and large-break LOCA transition into long-term cooling. (The list of the tests to be performed is in the OSU Test Matrix provided in Section 4.0.) The facility permits a range of small-break LOCAs to be simulated at different locations on the primary system, such as the cold leg, hot leg, core makeup tank (CMT) cold leg pressure balance line, and direct vessel injection line. The break orientation (top or bottom of the cold leg) may also be studied. Selected tests continue into the long-term cooling, post-accident mode in which the passive safety injection is from the reactor sump as well as the incontainment refueling water storage tank (IRWST). A large-break, post-accident, long-term cooling situation will also be simulated. 


\subsection{TEST OBJECTIVE}

The purpose of this test is to obtain thermal-hydraulic data for a double-ended DVI line break simulation. The break is located in the DVI-1 line close to the DVI-1 nozzle as it enters the reactor vessel. The data obtained from the test will be used to verify the AP1000 thermal-hydraulic computer codes for AP1000 phenomena such as gravity injection, natural convection, and post-accident long-term core cooling behavior.

The acceptance criteria for the OSU tests are as follows:

- $\quad$ Test initial conditions will be achieved within a specified tolerance.

- $\quad$ Set points will be achieved within an acceptable tolerance band.

- $\quad$ All instrumentation should be operational before the test.

- Any critical instruments not operating will be identified to the test engineer before the tests. These instruments must be operational before and during the test or exceptions should be approved.

- $\quad$ A zero check of LDPs, DPs, and FDPs will be performed. 


\subsection{FACILITY DESCRIPTION}

A detailed facility description for the OSU test facility is documented in Reference 1. The OSU test facility has been specifically scaled, designed, and constructed to investigate the AP600 passive safety system behavior and to provide data for safety analysis computer code validation. The facility has been modified to model the AP1000.

The scaled test design accurately models the details of the AP1000 geometry, including the primary system and the pipe routings and layout for the passive safety systems. A detailed scaling report (Reference 2) was used to develop the test design modifications. The primary system consists of one hot leg and two cold legs with two active pumps and an active steam generator for each loop. Two CMTs are connected to one primary loop, and the pressurizer is connected to the other primary loop as in the AP1000 plant design. Gas-driven accumulators are connected to the DVI lines. The discharge lines from the CMT, and one-of-two IRWST and reactor sump lines are connected to each DVI line. The Automatic Depressurization System (ADS), consisting of stages 1, 2 and 3, simulates either one or two of the independent trains used in the AP1000. The two-phase flow from the ADS stages 1-to-3 is separated in a swirl-vane separator and the liquid and vapor flows are measured to obtain the total ADS flow rate. The separated flow streams are then recombined and discharged into the IRWST through a sparger, preserving the mass and energy flow into the IRWST. The injection from the reactor sump is also simulated. Note that the OSU facility models both AP1000 primary and secondary sumps. The primary sump collects the condensate return, the liquid break flow, and the liquid flow from the fourth-stage ADS; and will provide long-term injection to the reactor vessel. The secondary sump simulates the portions of containment that will remain dry during most events. This sump will collect water only when the primary sump reaches its overflow level, and provides no injection to the reactor vessel.

The time period for the experimental simulations includes not only the IRWST injection, but also the draining of the IRWST and the sump injection to simulate the long-term cooling of the AP1000. This simulation could be from several hours to a day. The time scale for the OSU test facility is one-half; that is, events occur in half the normal time. To model the long-term cooling aspects of the transient, the two-phase flow from the break is separated in a swirl-vane separator and the liquid and vapor portions of the total flow are measured. The liquid fraction of the flow is discharged to the reactor primary sump as in the AP1000 plant. The vapor is discharged to the atmosphere. The capability exists to return a portion of the equivalent liquid flow to the IRWST and primary sump to simulate the condensate return from the passive containment to the IRWST and primary sump. A similar approach is also used for the fourth-stage ADS valve on the hot leg. The two-phase flow is separated in a swirl-vane separator, the two streams are measured, and the liquid phase is discharged into the primary sump while the vapor flow is discharged to the atmosphere. Again, the capability exists to return a portion of the liquid equivalent added to the IRWST and primary sump. In addition, all other steam vents from the facility are measured (e.g., the IRWST vent), and a portion of the liquid equivalent may be added back to the facility. Note that not all of the steam discharge would be returned as liquid equivalent. A portion of the discharge would be removed to simulate the steam that is not available for recirculation because it provides containment pressurization. The IRWST and primary sump can be pressurized in the OSU facility to simulate the containment pressurization following a postulated LOCA.

A multi-tube passive residual heat removal (PRHR) heat exchanger is located in the IRWST. The heat exchanger uses the same C-tube design as the AP1000 and has two instrumented tubes to obtain wall heat 
fluxes during the tests. There are primary fluid thermocouples, wall thermocouples, and differential pressure drop measurements to determine when the heat exchanger begins to drain. The IRWST is also instrumented with strings of fluid thermocouples, to determine the degree of mixing within the tank and to assess the temperature of the coolant that is delivered to the test vessel.

The reactor vessel for the OSU tests includes a 0.914-meter (3-foot) heated core consisting of forty-eight 0.025 -meter (1-inch) diameter heater rods. The heater rods have a top skewed power shape. The $1000 \mathrm{~kW}$ of electrical power available at the OSU test site will be used to simulate decay heat. Wall thermocouples are swaged inside the heater rods to measure the heater rod wall temperature.

Thermocouple rods in the heater rod bundle measure the axial coolant temperature distribution. The scaled flow volume in the core is preserved as well as the flow volume in the test vessel upper plenum. There are simulated reactor internals in the upper plenum to preserve the flow area and to correctly scale the fluid volume. The reactor vessel includes an annular downcomer into which the four cold legs and the two DVI lines are connected. The hot legs penetrate the reactor annulus and connect with the loops. The AP1000 reactor vessel neutron reflector is simulated using a ceramic liner to reduce the metal heat release to the coolant.

There were no special/unique requirements for the test other than those specified in the Initial Conditions. The specified conditions were verified on the control board prior to test implementation.

\subsection{Instrumentation}

The instrumentation has been designed to calculate a transient mass and energy balance on the test facility. All two-phase flow streams exiting the facility are separated, and each component is measured separately as a single phase flow using conventional measurement devices such as magnetic flow meters and vortex flow meters. Note that magnetic flow meters are not designed for two-phase flow and will indicate erratically. Also note, the vortex flow meters are referenced to $212^{\circ} \mathrm{F}$ and the LDPs are referenced to $60^{\circ} \mathrm{F}$. All vertical components have differential pressure cells that act as level instruments to measure the transient mass change in the component. The hot and cold leg diameters are sufficiently large in the OSU test facility so that a narrow-range differential pressure cell can be used to determine if the flow becomes stratified.

Single flow measurements are made of the CMT, accumulator, IRWST, and sump flows into the reactor vessel through the DVI lines.

Various types of instrumentation are provided in the test facility; for example, thermocouples for coolant and wall temperatures, flow meters, pressure transducers, differential pressure transducers, and weigh tanks. 


\subsection{OREGON STATE UNIVERSITY TESTING PROGRAM MATRIX}

The test matrix for the OSU test facility is shown in Table 4-1. To satisfy the test objectives, several transients will be performed to provide data on the AP1000 passive safety system response for a range of break sizes, locations, orientations, and single failure assumptions. The break size orifices are scaled based on simulating a 1-inch, 2-inch, or 4-inch pipe break.

The designation for this test is DBA-03-D, which identifies the test as a design basis double-ended DVI line break. The test matrix may be adjusted for future tests based on results and insights gained with each test. 


\begin{tabular}{|c|c|c|}
\hline \multicolumn{3}{|c|}{$\begin{array}{c}\text { TABLE 4-1 } \\
\text { OSU TEST MATRIX (SPECIFIED MAY 15, 2003) }\end{array}$} \\
\hline Test Title & Break Location and Size & Single Failure Assumed \\
\hline DBA-01-D & $\begin{array}{l}\text { Double-ended DVI Line break with } \\
\text { continuation into long-term cooling }\end{array}$ & Fail 1 of 2 lines in 1 ADS- 4 train \\
\hline DBA-03-D & $\begin{array}{l}\text { Double-ended DVI Line break with } \\
\text { continuation into long-term cooling }\end{array}$ & $\begin{array}{l}\text { Fail } 1 \text { of } 2 \text { lines in } 1 \text { ADS- } 4 \text { train } \\
\text { Single failure ADS- } 4 \text { opposite pressurizer } \\
\text { (Adjusted ADS- } 4 \text { Resistance) }\end{array}$ \\
\hline DBA-03-D & $\begin{array}{l}\text { Double-ended DVI Line break with } \\
\text { continuation into long-term cooling }\end{array}$ & $\begin{array}{l}\text { Fail } 1 \text { of } 2 \text { lines in } 1 \text { ADS-4 train } \\
\text { Single failure ADS-4 pressurizer side } \\
\text { (Adjusted ADS-4 Resistance) }\end{array}$ \\
\hline DBA-04-D & 2-inch Cold Leg Break & Fail 1 of 2 lines in 1 ADS- 4 train \\
\hline TR-01-D & $\begin{array}{l}\text { Transition Test ADS4 opening, } 85 \text { psig initial } \\
\text { pressure and decay power } 480 \mathrm{sec}\end{array}$ & $\begin{array}{l}\text { Fail } 1 \text { of } 2 \text { lines in } 1 \text { ADS-4 train } \\
\text { (No ADS 1-3) }\end{array}$ \\
\hline TR-02-D & $\begin{array}{l}\text { Transition Test ADS4 opening, } 125 \text { psig initial } \\
\text { pressure and decay power } 480 \mathrm{sec}\end{array}$ & $\begin{array}{l}\text { Fail } 1 \text { of } 2 \text { lines in } 1 \text { ADS-4 train } \\
\text { (No ADS 1-3) }\end{array}$ \\
\hline TR-03-D & $\begin{array}{l}\text { Transition Test ADS4 opening, } 85 \text { psig initial } \\
\text { pressure and decay power } 1800 \mathrm{sec}\end{array}$ & $\begin{array}{l}\text { Fail } 1 \text { of } 2 \text { lines in } 1 \text { ADS-4 train } \\
\text { (No ADS 1-3) }\end{array}$ \\
\hline TR-04-D & $\begin{array}{l}\text { Transition Test ADS4 opening, } 85 \text { psig initial } \\
\text { pressure and decay power } 480 \mathrm{sec}\end{array}$ & $\begin{array}{l}\text { No Failure of ADS-4 lines assumed } \\
\text { (No ADS 1-3) }\end{array}$ \\
\hline EN-01-D & $\begin{array}{l}\text { Entrainment Test with Revised Upper Internals, } \\
1000 \mathrm{~kW} \text { reactor power, } 14.7 \mathrm{psi}\end{array}$ & Fail 1 of 2 lines in 1 ADS- 4 train \\
\hline EN-01-D & $\begin{array}{l}\text { Entrainment Test with Revised Upper Internals, } \\
700 \mathrm{~kW} \text { reactor power, } 14.7 \mathrm{psi}\end{array}$ & Fail 1 of 2 lines in 1 ADS- 4 train \\
\hline PRA-02-D & $\begin{array}{l}\text { PRA Test - 3-inch Hot Leg Break with no } \\
\text { CMTs }\end{array}$ & No ADS-4 Failure assumed \\
\hline
\end{tabular}




\subsection{TEST PROCEDURE}

The test was performed as per a written procedure. There were no special/unique requirements for the test other than those specified in the initial conditions in Table 6-1. The specified conditions were checked on the control board before test implementation.

The appropriate prerequisites were completed and initial conditions were satisfied. The required break simulation piping and break instrumentation were installed per P\&ID drawing OSU 600904, Rev. 1. A break spool insert simulating the double-ended break in AP1000 was installed in the break spool in DVI horizontal line. DVI-1 nozzle flow from the reactor is directed horizontally into the break separator. The combined flow of DVI line (from CMT-1, accumulator 1, primary sump, and IRWST) is connected horizontally to the primary sump tank. The 100-percent flow nozzle was installed in the ADS 4-1 (on hot leg 1) and the 50-percent flow nozzle was installed in ADS 4-2 (on hot leg 2). Flow nozzles that simulate full flow for ADS-1, ADS-2, and ADS-3 were installed. As per the AP600 tests, ADS-3 has been scaled for full flow from all three stages, and ADS-1 and ADS-2 are closed when ADS-3 is opened.

Fill and vent was performed per APEX Operations Manual Procedure OP-B.2. Instruments were checked for required calibration.

With the break valves TS-202 and TS-203 closed, flow was used to warm up the bypass line by opening isolation valves RCS-901 and RCS-902. After the appropriate prerequisites were completed and the test facility achieved specified initial conditions, the CMT warmup bypass line isolation valves RCS-901 and RCS- 902 were closed to maintain the $<80^{\circ} \mathrm{F}$ condition at the top of the CMT-1. With the CMT balance line valves (RCS-529 and RCS-530) placed in the open and automatic mode, both CMTs reached the reactor coolant system (RCS) pressure.

Once all other initial conditions were satisfied, a break through TS-202 and TS-203 was initiated. The transient continued through ADS actuation, and CMT, accumulator, IRWST injection, and sump injection. Per Westinghouse instructions, ADS 4-2 was actuated automatically according to the actuation logic, and ADS4-1 was actuated manually after a 30-second delay. All other actions were automatic and required no operator action. 


\subsection{TEST RESULTS}

The test results for test DBA-03-D are provided in the following subsections.

\subsection{Initial and Boundary Conditions}

Table 6-1 provides a comparison of the specified and actual conditions for test DBA-03-D. The values in this table were averaged over approximately 2 minutes preceding the test. Test initial conditions were achieved for the steam generator pressure, pressurizer pressure, pressurizer level, steam generator 01 narrow-range level, and steam generator 02 narrow-range level. Test initial conditions for the hot leg temperature were found to be acceptable, and the results will not be adversely affected.

The actual power decay curves are provided in data plots in Appendix B. The measured maximum power was $920 \mathrm{~kW}$, which was less than the facility maximum power of $1000 \mathrm{~kW}$. The programmed decay heat curve was adjusted to account for this difference, and the differences between the actual and specified power decay are considered acceptable.

PT-501 and PT-502 pressure instruments indicate the pressure changes in the CMT-1 and CMT-2. CMT-1 (PT-501) and CMT-2 (PT-502) confirm that 1 minute after the test button was pushed, both CMTs reach RCS pressure.

The initial temperature in the CMTs is to be limited to $\angle 80^{\circ} \mathrm{F}$. However, the CMT isolation valves were left open during the heatup of the test facility, and were at RCS temperature $\left(\sim 420^{\circ} \mathrm{F}\right)$. Subsequent draining and refilling cycles were used to reduce the temperature, but the heat capacity of the thick tank walls caused the initial temperatures to be above the $80^{\circ} \mathrm{F}$ limit. The test results were deemed not to be sensitive to this initial condition, and the test was accepted.

The sequence of events is shown in Table 6-2. This table compares the actual sequence of events with the specified timing. As can be seen in this table, all the events occurred at or very near to when the event was planned.

\subsection{Inoperable Instruments}

Table 6-3 provides a list of the instrumentation channels considered inoperable for the DBA-03-D test.

\subsection{Key Data Plots}

Table 6-4 provides a list of the instrumentation channels sorted by component, and includes instrument number, units, and Test Summary Report plot number. Appendix A contains the Test Summary Report plots for this test for selected channels. The selection of channels was based on projecting an overall picture of the test results, which would then be examined by referring to the detailed data plots or tapes. Appendix B contains a CD containing all data channels for this test. 


\subsection{Test Evaluation}

The following observations were made during the test:

1. The peak power before the test was initiated was $920 \mathrm{~kW}$. The decay heat curve was adjusted for this value.

2. Modifications to the ADS-4 piping and flow nozzles result in more prototypic behavior for both choked and unchoked flow conditions. For DBA-02, the location of the single failure on the ADS-4 valves in the hot leg opposite the pressurizer resulted in a delay in the IRWST injection time. This was attributed to system effects from the pressurizer draining. For DBA-03, locating the single failure on the ADS-4 valves in the hot leg on the same side as the pressurizer resulted less interaction between the pressurizer draining and the venting of steam through the ADS-4. This resulted in more effective depressurization and IRWST injection sooner than in DBA-02. 


\begin{tabular}{|c|c|c|c|}
\hline \multicolumn{4}{|c|}{$\begin{array}{c}\text { TABLE 6-1 } \\
\text { ACTUAL TEST INITIAL CONDITIONS }\end{array}$} \\
\hline Conditions & Instrument No. & Actual & Comment \\
\hline Pressurizer Pressure & PT-604 & 370 psig & \\
\hline $\begin{array}{l}\text { Hot Leg Temperature } \\
\quad \# 1\end{array}$ & $\begin{array}{l}\text { TF-141* } \\
\text { TF-205 } \\
\text { TF-143 }\end{array}$ & $\begin{array}{l}426^{\circ} \mathrm{F} \\
428^{\circ} \mathrm{F} \\
429^{\circ} \mathrm{F}\end{array}$ & \\
\hline $\begin{array}{l}\text { Hot Leg Temperature } \\
\quad \# 2\end{array}$ & $\begin{array}{l}\text { TF-140* } \\
\text { TF-206 } \\
\text { TF-142 }\end{array}$ & $\begin{array}{c}427.5^{\circ} \mathrm{F} \\
426.5^{\circ} \mathrm{F} \\
429^{\circ} \mathrm{F}\end{array}$ & \\
\hline $\begin{array}{l}\text { Steam Generator (SG) } \\
\text { Pressure } \\
\quad \# 1 \\
\quad \# 2 \\
\text { Header }\end{array}$ & $\begin{array}{l}\text { PT-301* } \\
\text { PT-302* } \\
\text { PT-002 }\end{array}$ & $\begin{array}{l}285.5 \mathrm{psig} \\
285.5 \mathrm{psig} \\
246 \mathrm{psig}\end{array}$ & \\
\hline Pressurizer Level & $\begin{array}{l}\text { LDP-601 uncompensated } \\
\text { LDP-601 } \\
\text { Compensated by SC-608 }\end{array}$ & $\begin{array}{l}66 \text { inches } \\
79.3 \text { inches }\end{array}$ & $\begin{array}{l}440^{\circ} \mathrm{F} \text { used for density } \\
\text { compensation }\end{array}$ \\
\hline $\begin{array}{l}\text { SG Level } \\
\quad \# 1 \mathrm{NR}\end{array}$ & $\begin{array}{l}\text { LDP-303 uncompensated } \\
\text { LDP-303 compensated by } \\
\text { average of TF-305 and } \\
\text { TF-307 }\end{array}$ & $\begin{array}{l}21.2 \text { inches } \\
25.6 \text { inches }\end{array}$ & $\begin{array}{l}413^{\circ} \mathrm{F} \text { used for density } \\
\text { compensation }\end{array}$ \\
\hline $\begin{array}{l}\text { SG Level } \\
\text { \#2 NR }\end{array}$ & $\begin{array}{l}\text { LDP-304 uncompensated } \\
\text { LDP-304 compensated by } \\
\text { average of TF-306 and } \\
\text { TF-308 }\end{array}$ & $\begin{array}{l}21.6 \text { inches } \\
26.0 \text { inches }\end{array}$ & $\begin{array}{l}414^{\circ} \mathrm{F} \text { used for density } \\
\text { compensation }\end{array}$ \\
\hline IRWST Temperature & TF-701 & $65^{\circ} \mathrm{F}$ & Accepted $\left(<80^{\circ} \mathrm{F}\right)$ \\
\hline $\begin{array}{l}\text { CMT Temperature } \\
\# 1 \\
\# 2\end{array}$ & $\begin{array}{l}\text { TF-529 } \\
\text { TF-532 }\end{array}$ & $\begin{array}{l}67.7^{\circ} \mathrm{F} \\
65.7^{\circ} \mathrm{F}\end{array}$ & Accepted (qualified) \\
\hline $\begin{array}{l}\text { Accumulator } \\
\text { Temperature } \\
\# 1 \\
\# 2\end{array}$ & $\begin{array}{l}\text { TF-403 } \\
\text { TF-404 }\end{array}$ & $\begin{array}{c}69.2^{\circ} \mathrm{F} \\
67^{\circ} \mathrm{F}\end{array}$ & Accepted $\left(<80^{\circ} \mathrm{F}\right)$ \\
\hline IRWST Level & LDP-701 & 95.9 inches & \\
\hline
\end{tabular}




\begin{tabular}{|c|c|c|c|}
\hline \multicolumn{4}{|c|}{$\begin{array}{c}\text { TABLE 6-1 (Continued) } \\
\text { ACTUAL TEST INITIAL CONDITIONS }\end{array}$} \\
\hline Conditions & Instrument No. & Actual & Comment \\
\hline $\begin{array}{l}\text { Accumulator Level } \\
\text { \#1 } \\
\# 2\end{array}$ & $\begin{array}{l}\text { LDP-401 } \\
\text { LDP-402 }\end{array}$ & $\begin{array}{l}35.6 \text { inches } \\
38.4 \text { inches }\end{array}$ & \\
\hline $\begin{array}{l}\text { Accumulator Pressure } \\
\# 1 \\
\# 2\end{array}$ & $\begin{array}{l}\text { PT-401 } \\
\text { PT-402 }\end{array}$ & $\begin{array}{l}189.5 \text { psig } \\
194.7 \text { psig }\end{array}$ & \\
\hline $\begin{array}{l}\text { CMT Level } \\
\# 1 \\
\# 2\end{array}$ & $\begin{array}{l}\text { LDP-507 } \\
\text { LDP-502 }\end{array}$ & $\begin{array}{l}57.6 \text { inches } \\
57.6 \text { inches }\end{array}$ & \\
\hline $\begin{array}{l}\text { CMT Pressure } \\
\text { \#1 } \\
\# 2\end{array}$ & $\begin{array}{l}\text { PT-501 } \\
\text { PT-502 }\end{array}$ & $\begin{array}{c}373.3 \text { psig } \\
374 \text { psig }\end{array}$ & \\
\hline
\end{tabular}

* These instruments are used to establish initial conditions at the test site. 


\begin{tabular}{|c|c|c|}
\hline \multicolumn{3}{|c|}{$\begin{array}{c}\text { TABLE 6-2 } \\
\text { SEQUENCE OF EVENTS }\end{array}$} \\
\hline Event & Setpoint & Actual Time (sec) \\
\hline PB Depressed & N/A & -120 \\
\hline Break Valve(s) Open & 0 & 0 \\
\hline Feed Pump Trip & $3.6 \mathrm{sec}$ & 4 \\
\hline PRHR HX Outlet Valve Open (RCS-804) & $6.1 \mathrm{sec}$ & 5 \\
\hline CMT01 Outlet Valve Open (RCS-535) & $6.1 \mathrm{sec}$ & 6 \\
\hline CMT02 Outlet Valve Open (RCS-536) & $6.1 \mathrm{sec}$ & 6 \\
\hline RCP \#1 Trip & $8.6 \mathrm{sec}$ & 8 \\
\hline RCP \#2 Trip & $8.6 \mathrm{sec}$ & 8 \\
\hline RCP \#3 Trip & $8.6 \mathrm{sec}$ & 8 \\
\hline RCP \#4 Trip & $8.6 \mathrm{sec}$ & 8 \\
\hline CMT \#1 Level Low (LDP-507) & 41 inches & 69 \\
\hline CMT \#2 Level Low (LDP-502) & 41 inches & 350 \\
\hline ADS \#1 Actuation (RCS-601) & CMT Level Low $+15 \mathrm{sec}$ & 83 \\
\hline ADS \#2 Actuation (RCS-602) & CMT Level Low $+62 \mathrm{sec}$ & 130 \\
\hline ADS \#3 Actuation (RCS-603) & CMT Level Low + $122 \mathrm{sec}$ & 191 \\
\hline Low Reactor Pressure (P-107) & 40 psig & 199 \\
\hline IRWST Valve Actuation (RCS-711) & $\begin{array}{l}\text { Low Reactor Pressure } \\
\qquad(<40 \mathrm{psig})\end{array}$ & 201 \\
\hline IRWST Valve Actuation (RCS-712) & $\begin{array}{l}\text { Low Reactor Pressure } \\
\qquad(<40 \text { psig })\end{array}$ & 201 \\
\hline CMT \#1 Low Low Level (LDP-507) & 17.14 inches & 94 \\
\hline CMT \#2 Low Low Level (LDP-502) & 17.14 inches & 706 \\
\hline ADS 4-2 Actuation (RCS-616) & $\begin{array}{l}\text { CMT Low Low (17.14") and } \\
\text { CMT Low }\left(41^{\prime \prime}\right)+180 \mathrm{sec}\end{array}$ & 251 \\
\hline ADS 4-1 Actuation (RCS-615) & $\begin{array}{l}\text { CMT Low Low (17.14") and } \\
\text { CMT Low }\left(41^{\prime \prime}\right)+180 \mathrm{sec}\end{array}$ & 281 \\
\hline Sump Valve Actuation (CSS-909) & IRWST Level Low Low & 6889 \\
\hline
\end{tabular}




\begin{tabular}{|c|c|c|}
\hline \multicolumn{3}{|c|}{$\begin{array}{c}\text { TABLE 6-2 (Continued) } \\
\text { SEQUENCE OF EVENTS }\end{array}$} \\
\hline Event & Setpoint & Actual Time (sec) \\
\hline Sump Valve Actuation (CSS-910) & IRWST Level Low Low & 6892 \\
\hline $\begin{array}{l}\text { Accumulator Injection } \\
\# 1 \text { (FMM-401) }\end{array}$ & N/A & 0 \\
\hline $\begin{array}{l}\text { Accumulator Injection } \\
\# 2 \text { (FMM-402) }\end{array}$ & N/A & 110 \\
\hline $\begin{array}{l}\text { IRWST Injection } \\
\text { DVI \#1 (FMM-701) }\end{array}$ & N/A & 305 \\
\hline $\begin{array}{l}\text { IRWST Injection } \\
\text { DVI \#2 (FMM-702) }\end{array}$ & N/A & 700 \\
\hline $\begin{array}{l}\text { Accumulator Empty } \\
\text { \#1 (LDP-401) }\end{array}$ & N/A & 209 down to 1.2 inch level \\
\hline $\begin{array}{l}\text { Accumulator Empty } \\
\text { \#2 (LDP-402) }\end{array}$ & N/A & 800 down to 0.1 inch level \\
\hline $\begin{array}{l}\text { CMT Empty } \\
\quad \# 1 \text { (LDP-507) }\end{array}$ & N/A & 115 down to 0.1 inch level \\
\hline $\begin{array}{l}\text { CMT Empty } \\
\text { \#2 (LDP-502) }\end{array}$ & N/A & 1020 \\
\hline Sump Injection DVI \#1 (FMM-901) & N/A & 6420 \\
\hline Sump Injection DVI \#2 (FMM-902) & N/A & 6420 \\
\hline
\end{tabular}

* Level indication $<2$ inches 


\begin{tabular}{|c|c|c|}
\hline \multicolumn{3}{|c|}{$\begin{array}{c}\text { TABLE 6-3 } \\
\text { INOPERABLE INSTRUMENTS FOR DBA-03-D TEST }\end{array}$} \\
\hline Instrument Number & Instrument Type & Inoperable Description \\
\hline $\begin{array}{l}\text { TW-202 } \\
\text { TW-204 } \\
\text { TW-205 } \\
\text { TW-206 } \\
\text { TW-209 } \\
\text { TW-803 } \\
\text { TW-804 }\end{array}$ & Thermocouple & Inoperative \\
\hline TH-603 & $\begin{array}{l}\text { Thermocouple measuring heater } \\
\text { temperature }\end{array}$ & Inoperative \\
\hline $\begin{array}{l}\text { FMM-202, } \\
\text { FMM-601 }\end{array}$ & Magnetic flow meter & $\begin{array}{l}\text { Inoperative } \\
\text { Measured ADS1-3 liquid flow after ADS4 } \\
\text { actuation (loop seal potentially blown) }\end{array}$ \\
\hline $\begin{array}{l}\text { TF-170 } \\
\text { TF-221 } \\
\text { TF-509 } \\
\text { TF-512 }\end{array}$ & $\begin{array}{l}\text { Thermocouple measuring fluid } \\
\text { temperature }\end{array}$ & Inoperative \\
\hline FVM-905 & Vortex flow meter & Erratic \\
\hline
\end{tabular}




\begin{tabular}{|c|c|c|c|c|}
\hline \multicolumn{5}{|c|}{$\begin{array}{c}\text { TABLE 6-4 } \\
\text { DATA PLOTS FOR TEST SUMMARY REPORT FOR DBA-03-D BY COMPONENT }\end{array}$} \\
\hline Component & Channel & Units & TSR-Plot & Comment \\
\hline $\begin{array}{l}\text { Reactor Vessel } \\
\text { Pressure }\end{array}$ & PT-107 & psig & 1 & \\
\hline Reactor Vessel Level & LDP-127 & inch of $\mathrm{H}_{2} \mathrm{O}$ & 2 & \\
\hline $\begin{array}{l}\text { Reactor Vessel } \\
\text { Downcomer Level }\end{array}$ & LDP-140 & inch of $\mathrm{H}_{2} \mathrm{O}$ & 3 & \\
\hline $\begin{array}{l}\text { Cold Leg \#1 Fluid } \\
\text { Temperature }\end{array}$ & TF-107 & ${ }^{\circ} \mathrm{F}$ & 4 & \\
\hline $\begin{array}{l}\text { Cold Leg \#2 Fluid } \\
\text { Temperature }\end{array}$ & TF-108 & ${ }^{\circ} \mathrm{F}$ & 5 & \\
\hline $\begin{array}{l}\text { Cold Leg \#3 Fluid } \\
\text { Temperature }\end{array}$ & TF-103 & ${ }^{\circ} \mathrm{F}$ & 6 & \\
\hline $\begin{array}{l}\text { Cold Leg \#4 Fluid } \\
\text { Temperature }\end{array}$ & TF-104 & ${ }^{\circ} \mathrm{F}$ & 7 & \\
\hline $\begin{array}{l}\text { Reactor Vessel Fluid } \\
\text { Temp Upper Head }\end{array}$ & TF-120 & ${ }^{\circ} \mathrm{F}$ & 8 & \\
\hline $\begin{array}{l}\text { RCS Hot Leg \#1 } \\
\text { Temperature }\end{array}$ & TF-143 & ${ }^{\circ} \mathrm{F}$ & 9 & \\
\hline $\begin{array}{l}\text { RCS Hot Leg \#2 } \\
\text { Temperature }\end{array}$ & TF-142 & ${ }^{\circ} \mathrm{F}$ & 10 & \\
\hline Pressurizer Pressure & PT-604 (WR) & psig & 11 & \\
\hline Pressurizer Pressure & PT-603 (LP Indication) & psig & 12 & \\
\hline $\begin{array}{l}\text { Pressurizer Liquid } \\
\text { Level }\end{array}$ & LDP-601 & inch of $\mathrm{H}_{2} \mathrm{O}$ & 13 & $\begin{array}{l}\text { Sharp decrease } \\
\text { followed by rapid refill }\end{array}$ \\
\hline SG \#1 Tube Level & LDP-215 & inch of $\mathrm{H}_{2} \mathrm{O}$ & 14 & \\
\hline $\begin{array}{l}\text { SG \#1 Secondary } \\
\text { Pressure }\end{array}$ & PT-301 & psig & 15 & \\
\hline SG \#1 Feed Flow Rate & FMM-001 & gpm & 16 & \\
\hline SG \#2 Tube Level & LDP-218 & inch of $\mathrm{H}_{2} \mathrm{O}$ & 17 & \\
\hline $\begin{array}{l}\text { SG \#2 Secondary } \\
\text { Pressure }\end{array}$ & PT-302 & psig & 18 & \\
\hline
\end{tabular}




\begin{tabular}{|c|c|c|c|c|}
\hline \multicolumn{5}{|c|}{$\begin{array}{l}\text { TABLE 6-4 (Continued) } \\
\text { DATA PLOTS FOR TEST SUMMARY REPORT FOR DBA-03-D BY COMPONENT }\end{array}$} \\
\hline Component & Channel & Units & TSR-Plot & Comment \\
\hline $\begin{array}{l}\text { Accumulator \#2 } \\
\text { Pressure }\end{array}$ & PT-401 & psig & 19 & \\
\hline $\begin{array}{l}\text { Accumulator \#1 } \\
\text { Pressure }\end{array}$ & PT-402 & psig & 20 & \\
\hline $\begin{array}{l}\text { Accumulator \#1 Liquid } \\
\text { Level }\end{array}$ & LDP-401 & inch of $\mathrm{H}_{2} \mathrm{O}$ & 21 & \\
\hline $\begin{array}{l}\text { Accumulator \#2 Liquid } \\
\text { Level }\end{array}$ & LDP-402 & inch of $\mathrm{H}_{2} \mathrm{O}$ & 22 & \\
\hline $\begin{array}{l}\text { Accumulator \#1 Flow } \\
\text { Rate }\end{array}$ & FMM-401 & gpm & 23 & \\
\hline $\begin{array}{l}\text { Accumulator \#2 Flow } \\
\text { Rate }\end{array}$ & FMM-402 & gpm & 24 & \\
\hline $\begin{array}{l}\text { Accumulator \#1 Liquid } \\
\text { Discharge Temperature }\end{array}$ & TF-401 & ${ }^{\circ} \mathrm{F}$ & 25 & \\
\hline $\begin{array}{l}\text { Accumulator \#2 Liquid } \\
\text { Discharge Temperature }\end{array}$ & TF-402 & ${ }^{\circ} \mathrm{F}$ & 26 & \\
\hline CMT \#1 Liquid Level & LDP-507 & inch of $\mathrm{H}_{2} \mathrm{O}$ & 27 & \\
\hline CMT \#2 Liquid Level & LDP-502 & inch of $\mathrm{H}_{2} \mathrm{O}$ & 28 & \\
\hline CMT \#1 Flow Rate & FMM-501 & gpm & 29 & \\
\hline CMT \#2 Flow Rate & FMM-504 & gpm & 30 & \\
\hline $\begin{array}{l}\text { CMT \#1 Liquid } \\
\text { Temperature }\end{array}$ & TF-501 & ${ }^{\circ} \mathrm{F}$ & 31 & \\
\hline $\begin{array}{l}\text { CMT \#1 Liquid } \\
\text { Temperature }\end{array}$ & TF-529 & ${ }^{\circ} \mathrm{F}$ & 32 & \\
\hline $\begin{array}{l}\text { CMT \#2 Liquid } \\
\text { Temperature }\end{array}$ & TF-504 & ${ }^{\circ} \mathrm{F}$ & 33 & \\
\hline $\begin{array}{l}\text { CMT \#2 Liquid } \\
\text { Temperature }\end{array}$ & TF-532 & ${ }^{\circ} \mathrm{F}$ & 34 & \\
\hline PRHR Inlet Flow Rate & FMM-802 & gpm & 35 & \\
\hline PRHR Liquid Level & LDP-802 & inch of $\mathrm{H}_{2} \mathrm{O}$ & 36 & \\
\hline
\end{tabular}




\begin{tabular}{|c|c|c|c|c|}
\hline \multicolumn{5}{|c|}{$\begin{array}{l}\text { TABLE 6-4 (Continued) } \\
\text { DATA PLOTS FOR TEST SUMMARY REPORT FOR DBA-03-D BY COMPONENT }\end{array}$} \\
\hline Component & Channel & Units & TSR-Plot & Comment \\
\hline $\begin{array}{l}\text { PRHR Outlet Flow } \\
\text { Rate }\end{array}$ & FMM-804 & gpm & 37 & \\
\hline IRWST Liquid Level & LDP-701 & inch of $\mathrm{H}_{2} \mathrm{O}$ & 38 & \\
\hline $\begin{array}{l}\text { IRWST Discharge Line } \\
\text { \#1 Flow Rate }\end{array}$ & FMM-701 & gpm & 39 & \\
\hline $\begin{array}{l}\text { IRWST Discharge Line } \\
\text { \#2 Flow Rate }\end{array}$ & FMM-702 & gpm & 40 & \\
\hline $\begin{array}{l}\text { IRWST Fluid } \\
\text { Temperature }\end{array}$ & TF-701 & ${ }^{\circ} \mathrm{F}$ & 41 & \\
\hline $\begin{array}{l}\text { IRWST Fluid } \\
\text { Temperature }\end{array}$ & TF-709 & ${ }^{\circ} \mathrm{F}$ & 42 & \\
\hline $\begin{array}{l}\text { ADS 1-3 Separator } \\
\text { Pressure }\end{array}$ & PT-605 & psig & 43 & \\
\hline $\begin{array}{l}\text { ADS 1-3 Separator } \\
\text { Steam Flow Rate }\end{array}$ & FVM-601 & $\mathrm{scfm}$ & 44 & \\
\hline $\begin{array}{l}\text { ADS 1-3 Separator } \\
\text { Liquid Flow Rate }\end{array}$ & FMM-601 & gpm & 45 & \\
\hline $\begin{array}{l}\text { ADS 4-1 Separator } \\
\text { Pressure }\end{array}$ & PT-611 & psig & 46 & \\
\hline $\begin{array}{l}\text { ADS 4-2 Separator } \\
\text { Pressure }\end{array}$ & PT-610 & psig & 47 & \\
\hline $\begin{array}{l}\text { ADS 4-1 Separator } \\
\text { Steam Flow Rate }\end{array}$ & FVM-603 & $\mathrm{scfm}$ & 48 & \\
\hline $\begin{array}{l}\text { ADS 4-2 Separator } \\
\text { Steam Flow Rate }\end{array}$ & FVM-602 & $\mathrm{scfm}$ & 49 & \\
\hline $\begin{array}{l}\text { ADS 4-1 Separator } \\
\text { Liquid Flow Rate }\end{array}$ & FMM-603 & gpm & 50 & \\
\hline $\begin{array}{l}\text { ADS 4-2 Separator } \\
\text { Liquid Flow Rate }\end{array}$ & FMM-602 & gpm & 51 & \\
\hline Primary Sump Pressure & PT-901 & psig & 52 & \\
\hline $\begin{array}{l}\text { Primary Sump Liquid } \\
\text { Level }\end{array}$ & LDP-901 & inch of $\mathrm{H}_{2} \mathrm{O}$ & 53 & \\
\hline
\end{tabular}




\begin{tabular}{|c|c|c|c|c|}
\hline \multicolumn{5}{|c|}{$\begin{array}{l}\text { TABLE 6-4 (Continued) } \\
\text { DATA PLOTS FOR TEST SUMMARY REPORT FOR DBA-03-D BY COMPONENT }\end{array}$} \\
\hline Component & Channel & Units & TSR-Plot & Comment \\
\hline $\begin{array}{l}\text { Primary Sump } \\
\text { Injection Flow Rate }\end{array}$ & FMM-901 & gpm & 54 & \\
\hline $\begin{array}{l}\text { Primary Sump } \\
\text { Injection Flow Rate }\end{array}$ & FMM-902 & gpm & 55 & \\
\hline $\begin{array}{l}\text { Secondary Sump } \\
\text { Liquid Level }\end{array}$ & LDP-902 & inch of $\mathrm{H}_{2} \mathrm{O}$ & 56 & \\
\hline $\begin{array}{l}\text { Break Separator } \\
\text { Pressure }\end{array}$ & PT-905 & psig & 57 & \\
\hline $\begin{array}{l}\text { Break Separator Liquid } \\
\text { Level }\end{array}$ & LDP-905 & inch of $\mathrm{H}_{2} \mathrm{O}$ & 58 & \\
\hline $\begin{array}{l}\text { Break Separator Flow } \\
\text { to Primary Sump }\end{array}$ & FMM-905 & gpm & 59 & \\
\hline $\begin{array}{l}\text { BAMS Steam Flow } \\
\text { Rate }\end{array}$ & FVM-901 & $\mathrm{scfm}$ & 60 & \\
\hline $\begin{array}{l}\text { BAMS Steam Flow } \\
\text { Rate }\end{array}$ & FVM-902 & $\mathrm{scfm}$ & 61 & \\
\hline $\begin{array}{l}\text { BAMS/Primary Sump } \\
\text { Steam Flow Rate }\end{array}$ & FVM-903 & $\mathrm{scfm}$ & 62 & \\
\hline $\begin{array}{l}\text { BAMS/Separator } \\
\text { Steam Flow Rate } \\
-6 \text { inch Pipe }\end{array}$ & FVM-905 & $\mathrm{scfm}$ & 63 & \\
\hline $\begin{array}{l}\text { BAMS/Exhaust Line } \\
\text { Temp. }\end{array}$ & TF-916 & ${ }^{\circ} \mathrm{F}$ & 64 & \\
\hline $\begin{array}{l}\text { BAMS/Exhaust Line } \\
\text { Temp. }\end{array}$ & SC-917 & ${ }^{\circ} \mathrm{F}$ & 65 & \\
\hline $\begin{array}{l}\text { PZR Heater Input } \\
\text { Power }\end{array}$ & KW-601 & $\mathrm{kW}$ & 66 & \\
\hline $\begin{array}{l}\text { Core Power Input } \\
\text { Power }\end{array}$ & KW-101 & $\mathrm{kW}$ & 67 & \\
\hline $\begin{array}{l}\text { Core Power Input } \\
\text { Power }\end{array}$ & KW-102 & $\mathrm{kW}$ & 68 & \\
\hline $\begin{array}{l}\text { Core Power Input } \\
\text { Power }\end{array}$ & KW-103 & $\mathrm{kW}$ & 69 & \\
\hline
\end{tabular}




\begin{tabular}{|c|c|c|c|c|}
\hline \multicolumn{5}{|c|}{$\begin{array}{l}\text { TABLE 6-4 (Continued) } \\
\text { DATA PLOTS FOR TEST SUMMARY REPORT FOR DBA-03-D BY COMPONENT }\end{array}$} \\
\hline Component & Channel & Units & TSR-Plot & Comment \\
\hline $\begin{array}{l}\text { Core Power Input } \\
\text { Power }\end{array}$ & KW-104 & $\mathrm{kW}$ & 70 & \\
\hline $\begin{array}{l}\text { Reactor Vessel Liquid } \\
\text { Level Between Top of } \\
\text { Vessel - Upper Support } \\
\text { Plate }\end{array}$ & LDP-115 & $\begin{array}{c}\text { inches of } \\
\text { water }\end{array}$ & 71 & \\
\hline $\begin{array}{l}\text { Reactor Vessel Liquid } \\
\text { Level Between Bottom } \\
\text { of Upper Support Plate } \\
\text { - Upper Core Spacer } \\
\text { Grid }\end{array}$ & LDP-139 & $\begin{array}{c}\text { inches of } \\
\text { water }\end{array}$ & 72 & \\
\hline $\begin{array}{l}\text { Inner Core } \\
\text { Thermocouple } \\
\text { Measuring Heater } \\
\text { Temperature }\end{array}$ & TH-103-4 & ${ }^{\circ} \mathrm{F}$ & 73 & \\
\hline $\begin{array}{l}\text { Outer Core } \\
\text { Thermocouple } \\
\text { Measuring Heater } \\
\text { Temperature }\end{array}$ & TH-309-4 & ${ }^{\circ} \mathrm{F}$ & 74 & \\
\hline
\end{tabular}




\subsection{CONCLUSIONS}

The DBA-03-D tests were successfully completed, and the data were logged in the DAS. All critical instruments were found to operate properly with the exception of those noted in Section 6.4. The test was acceptable. 
APPENDIX A

DATA PLOTS 

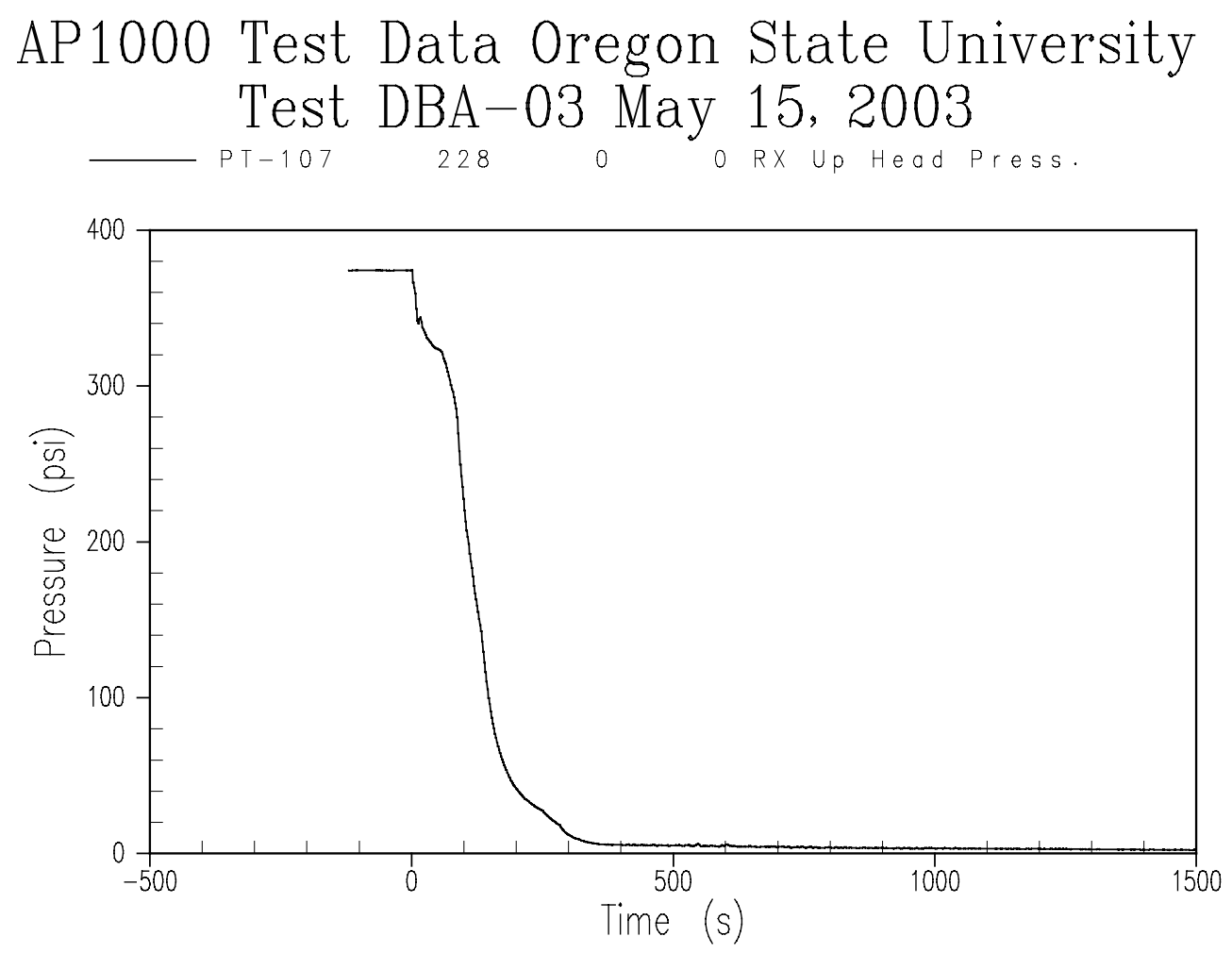

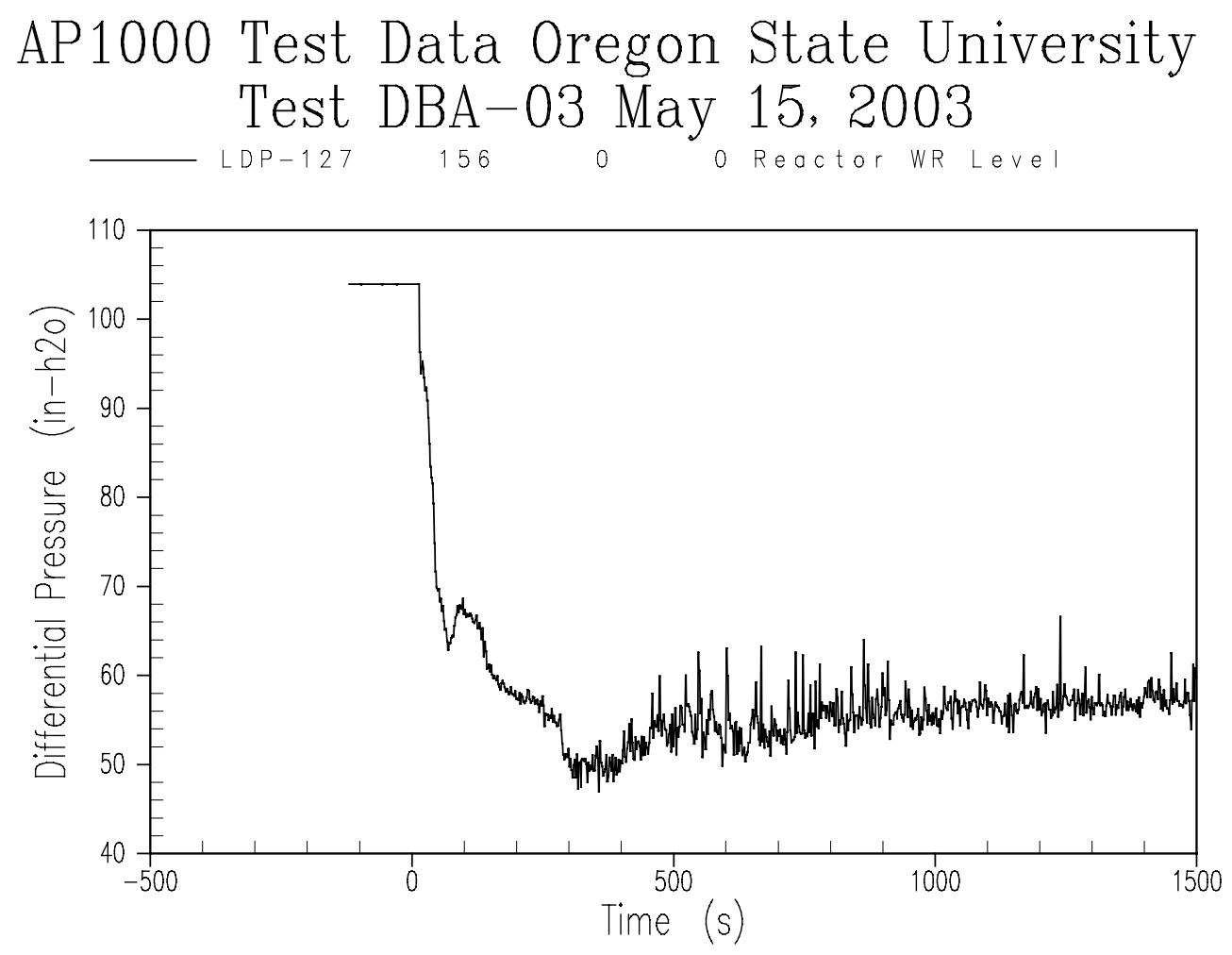

Figure A-2 Reactor Vessel Level 

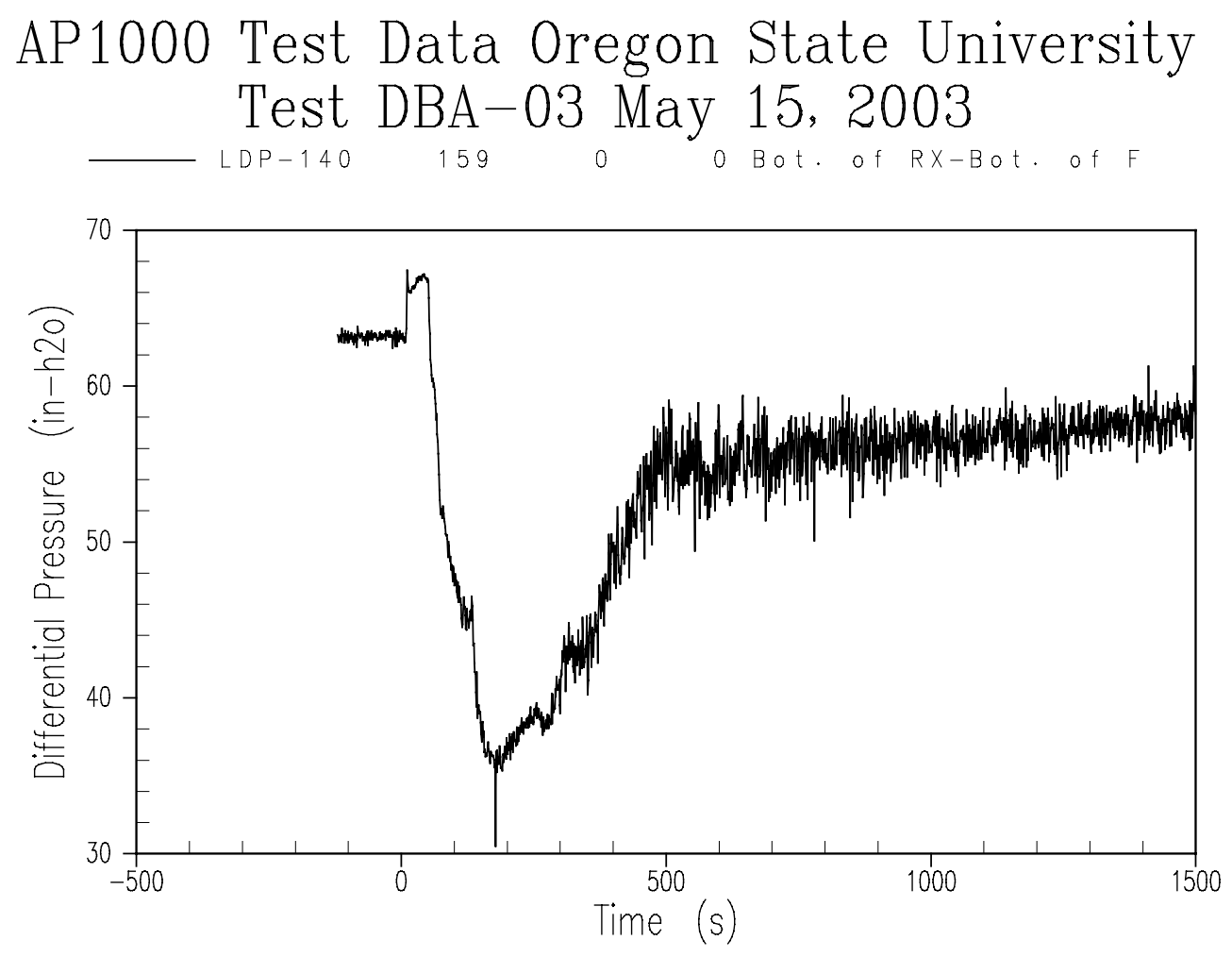

Figure A-3 Reactor Vessel Downcomer Level 

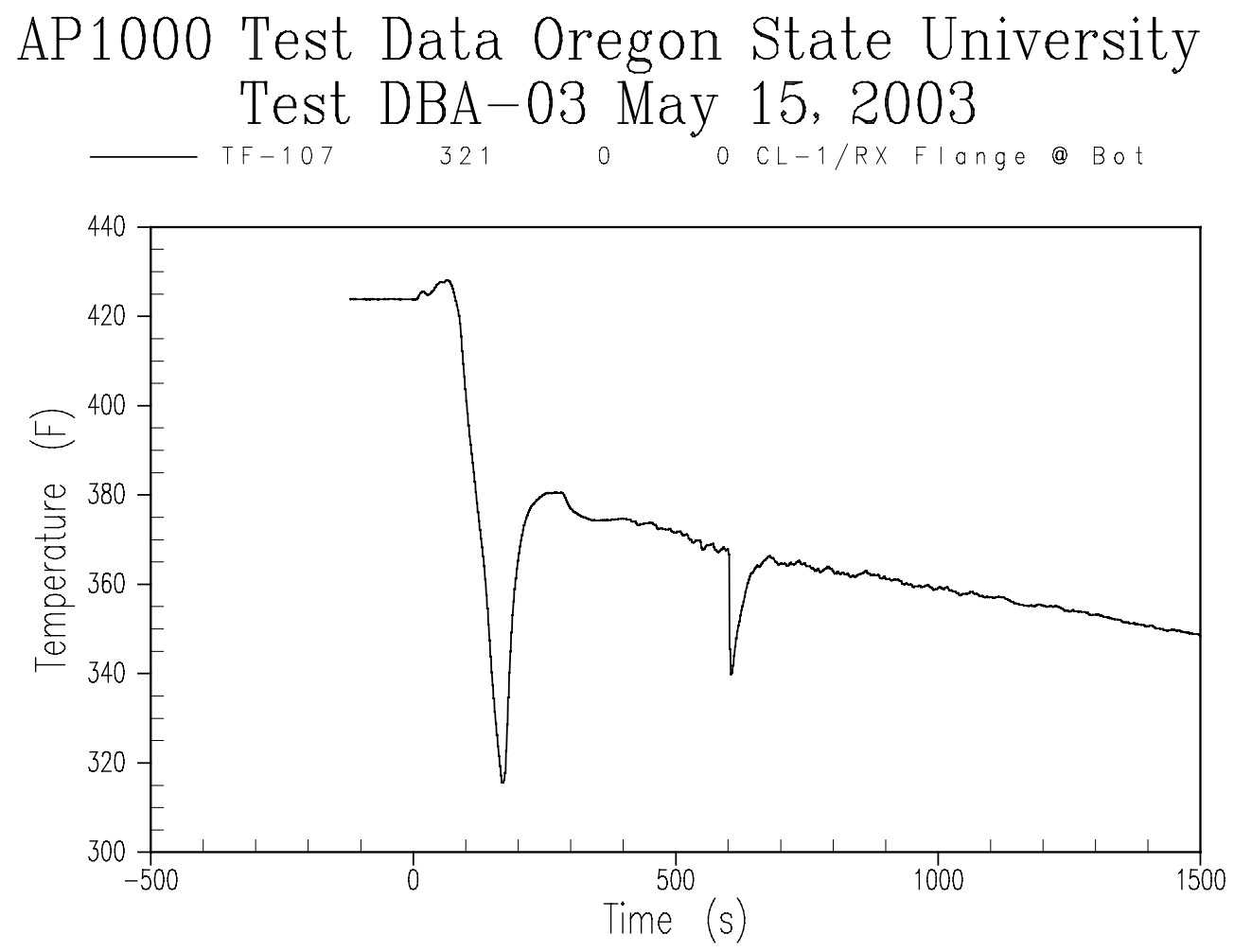

Figure A-4 Cold Leg 1 Fluid Temperature 

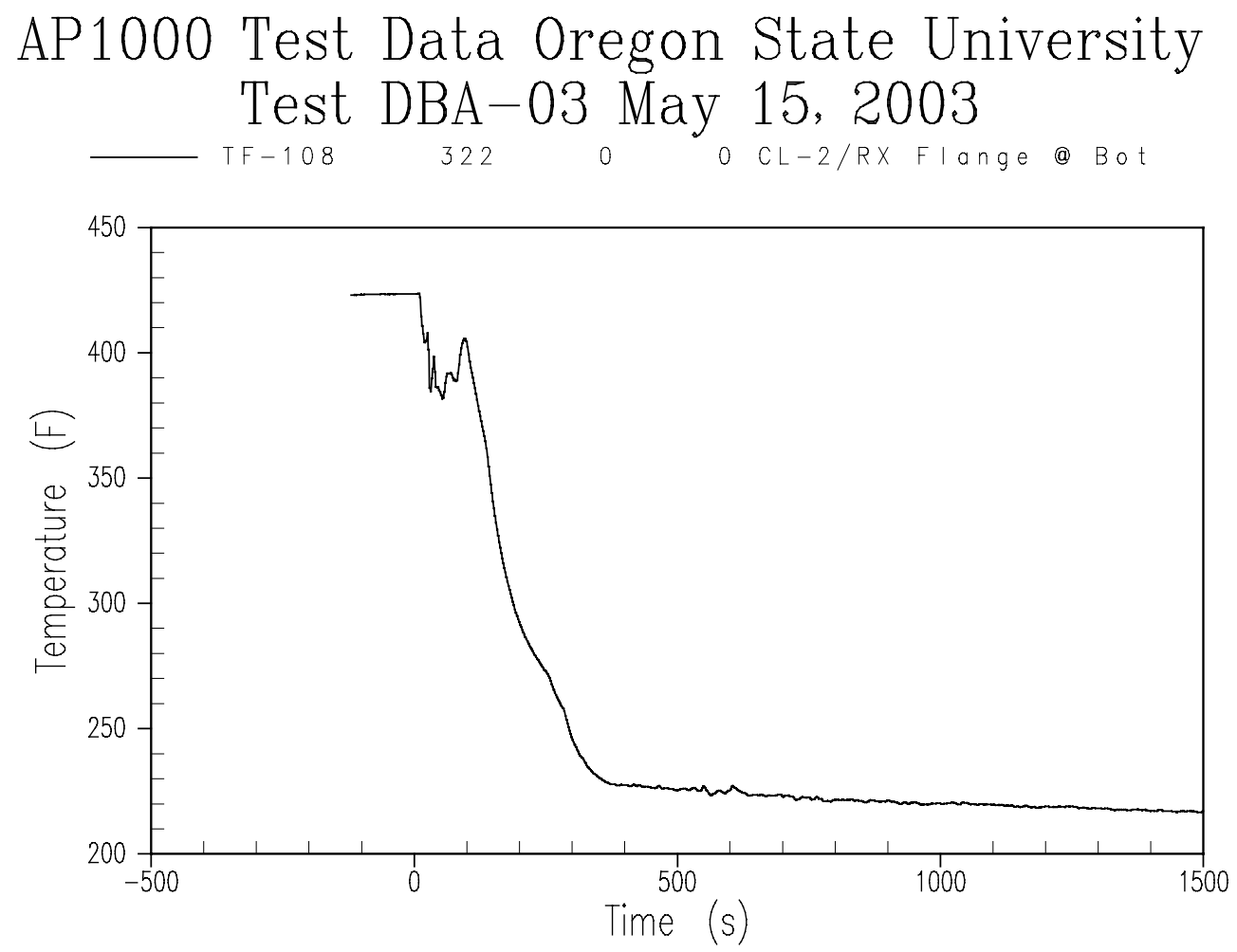

Figure A-5 Cold Leg 2 Fluid Temperature 

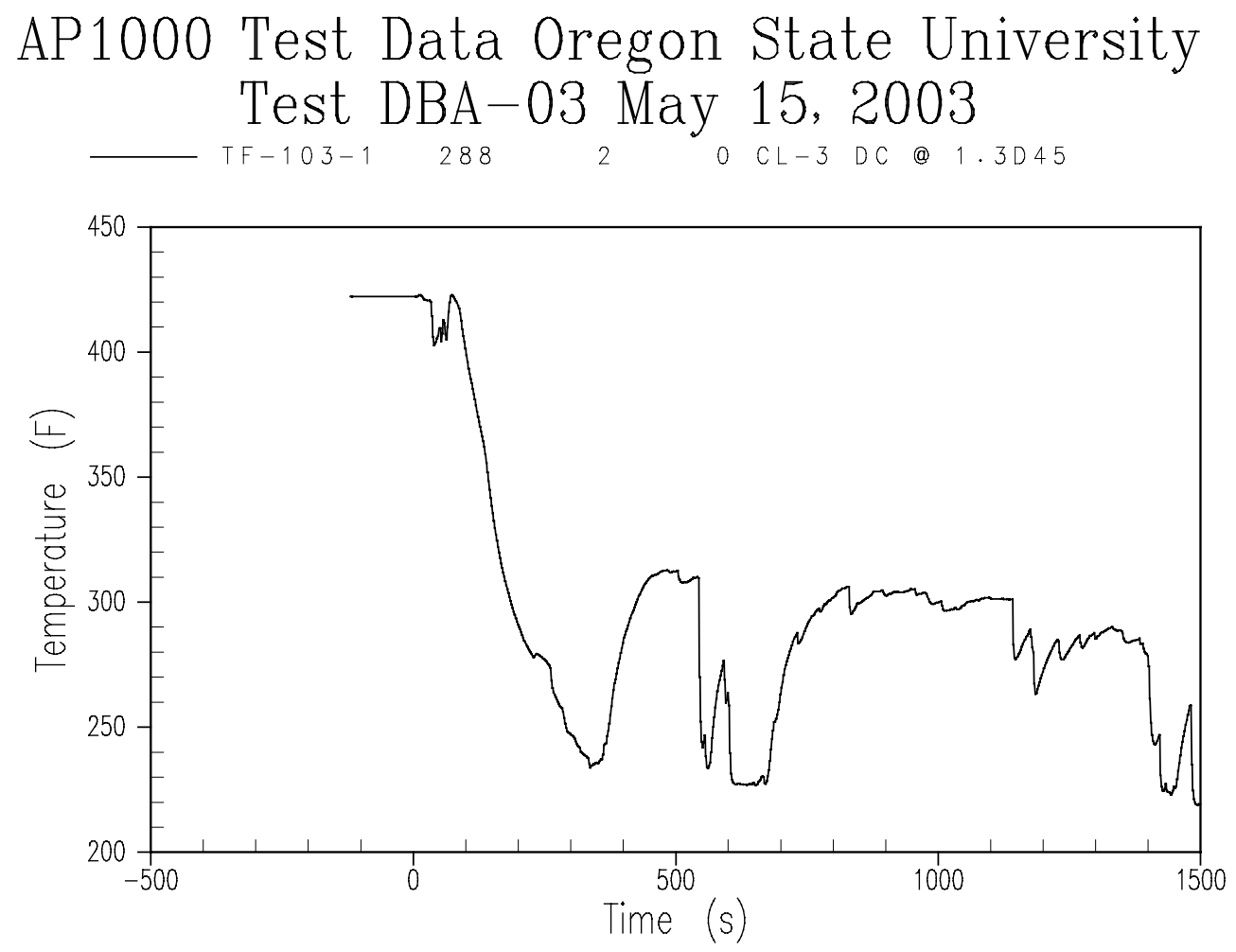

Figure A-6 Cold Leg 3 Fluid Temperature 

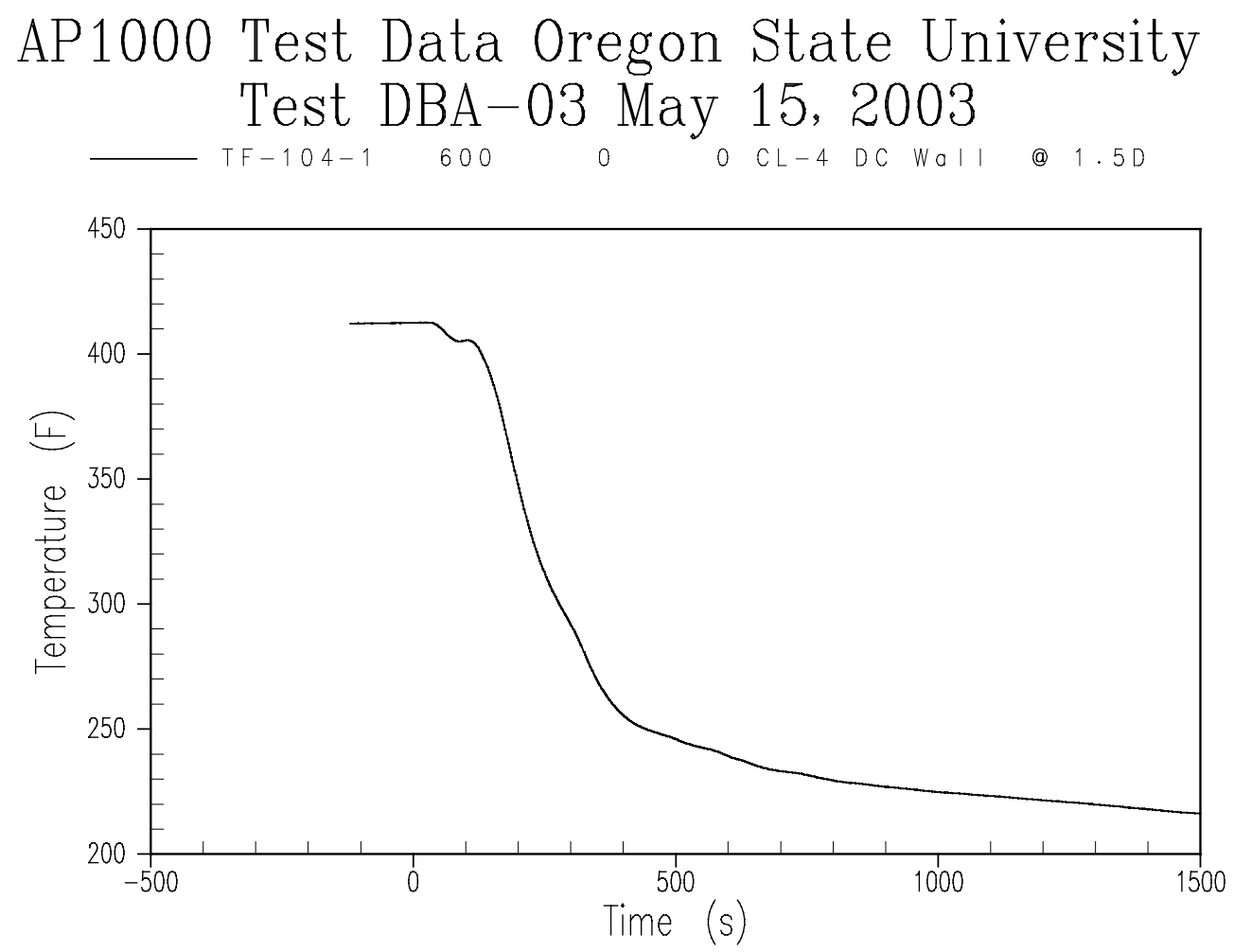

Figure A-7 Cold Leg 4 Fluid Temperature 

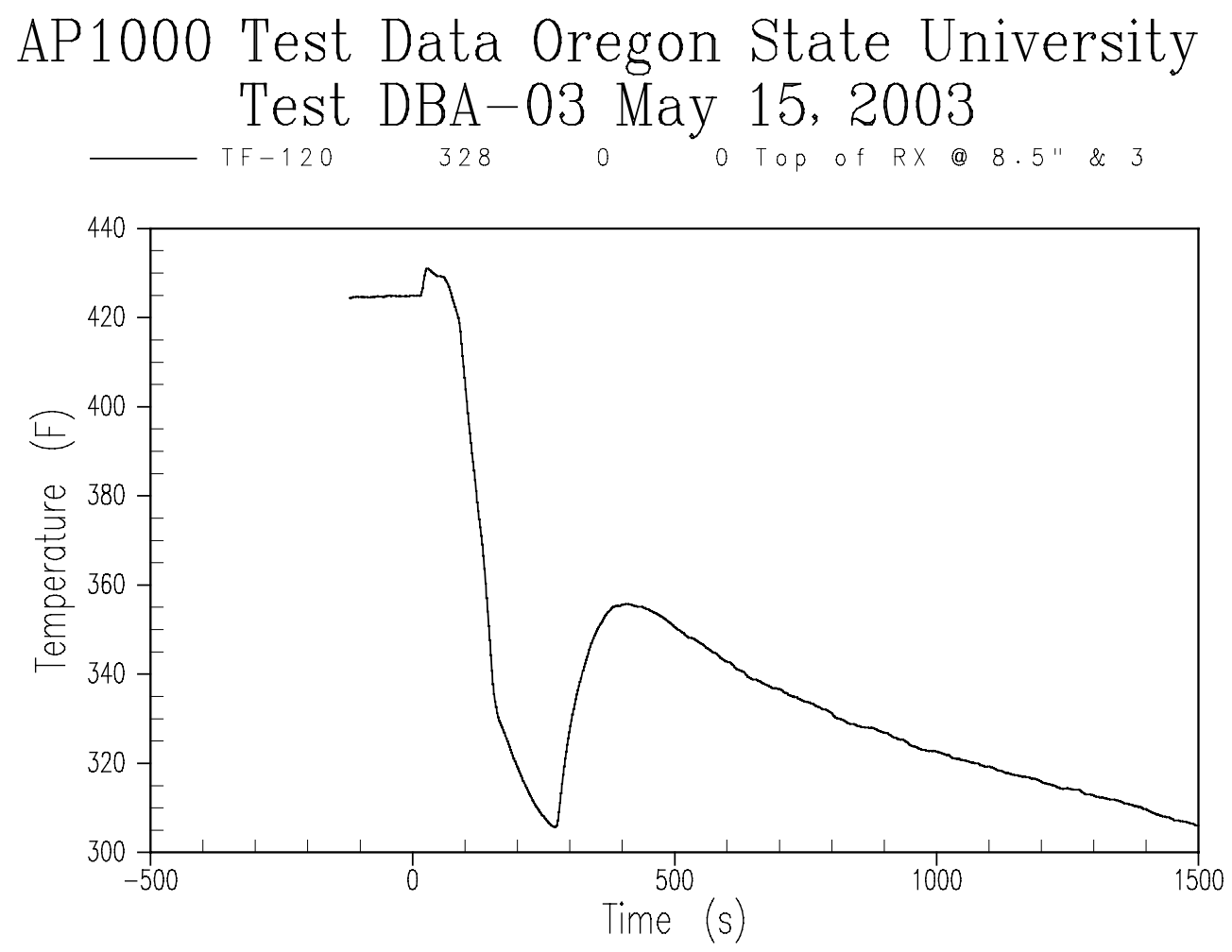

Figure A-8 Reactor Vessel Fluid Temperature Upper Head 

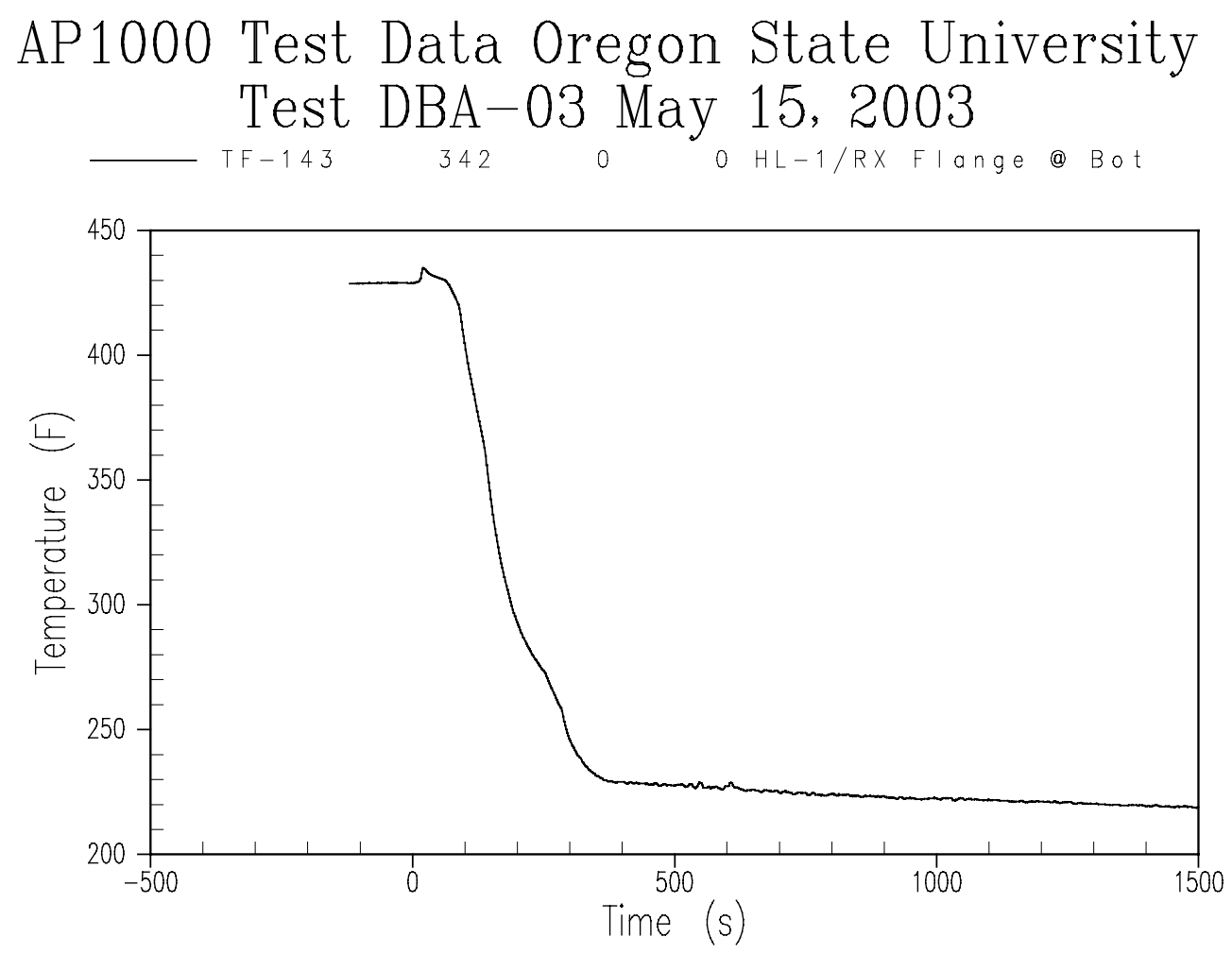

Figure A-9 Reactor Coolant System Hot Leg 1 Temperature 

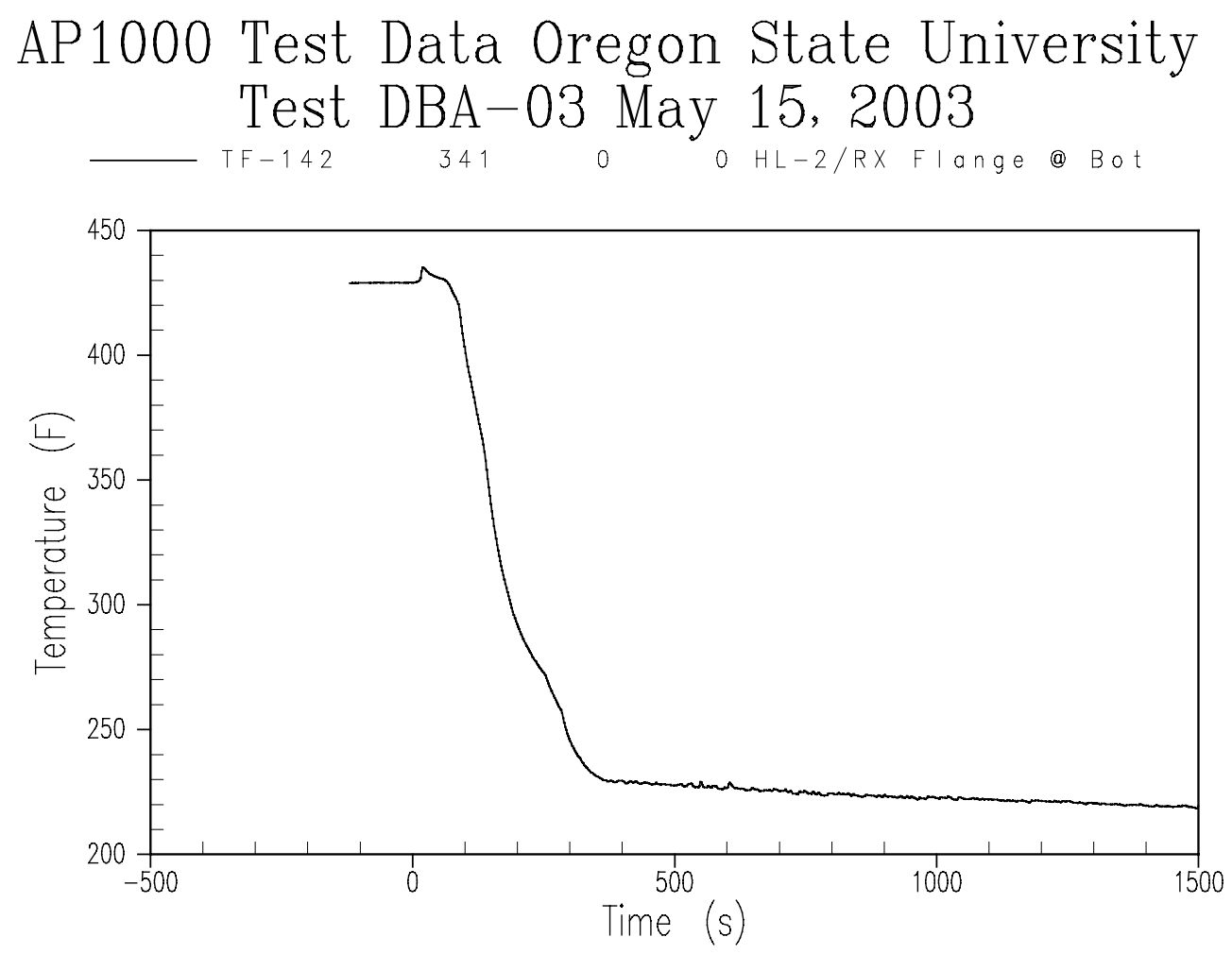

Figure A-10 Reactor Coolant System Hot Leg 2 Temperature 

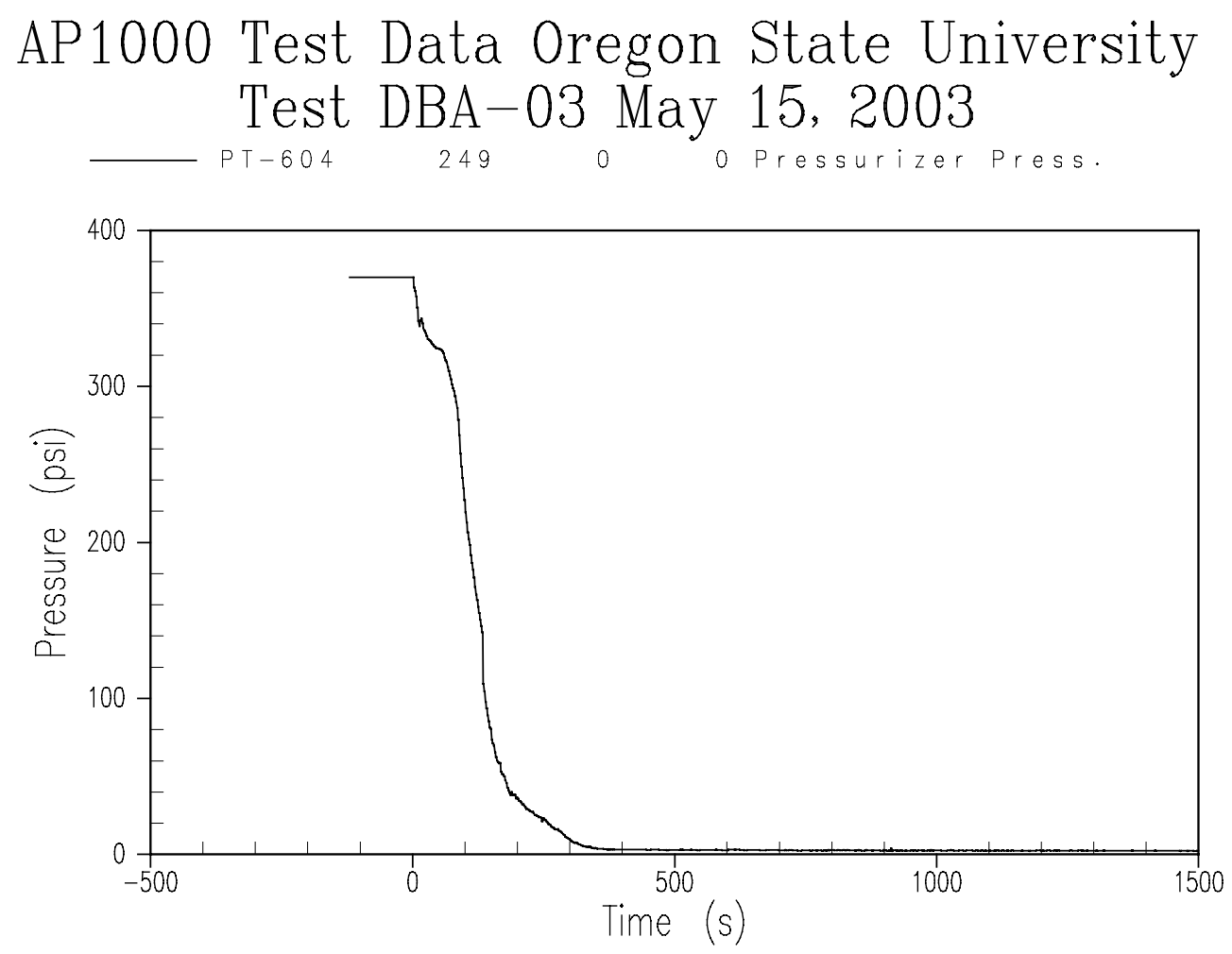

Figure A-11 Pressurizer Pressure - Wide Range 

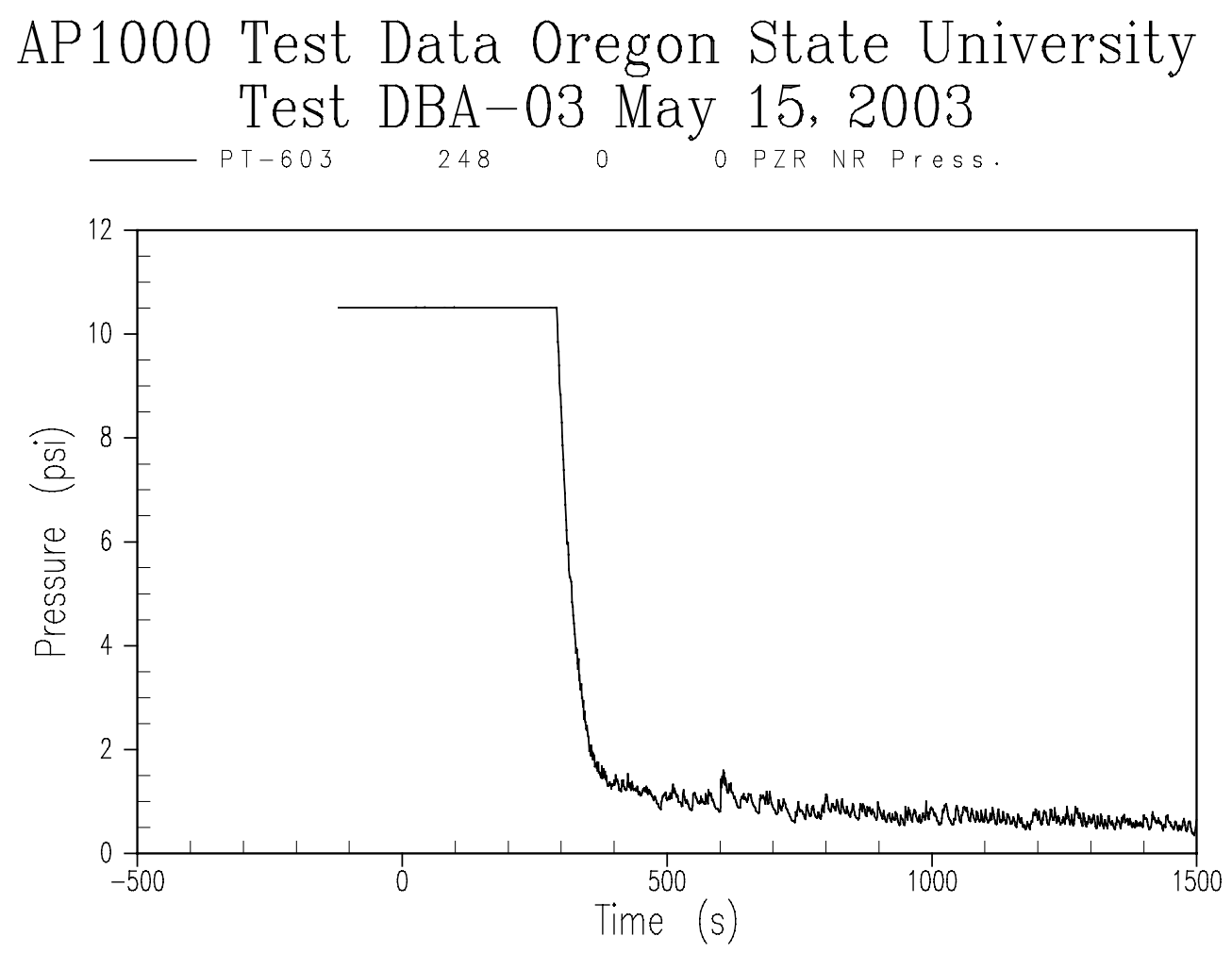

Figure A-12 Pressurizer Pressure - Narrow Range 

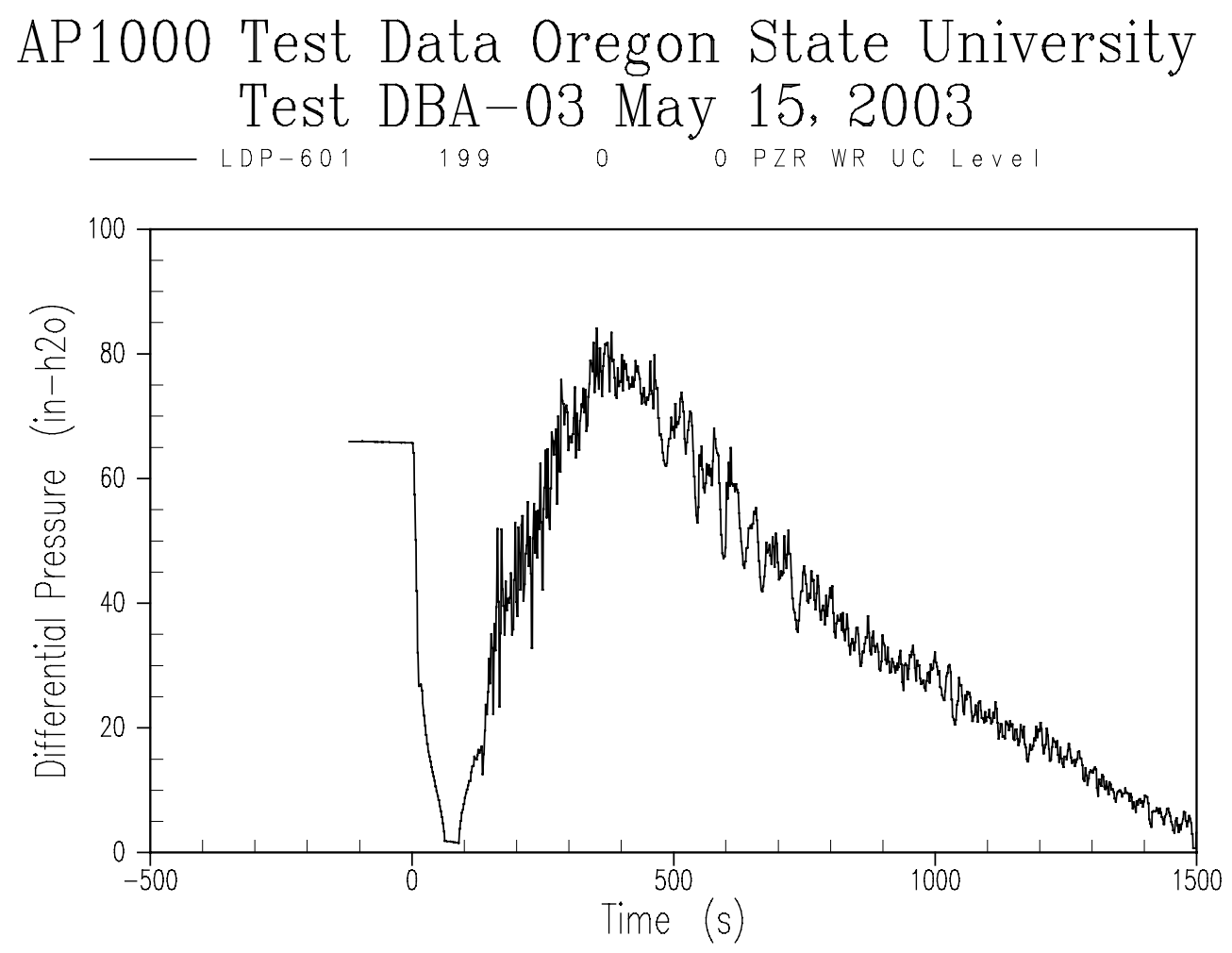

Figure A-13 Pressurizer Liquid Level 

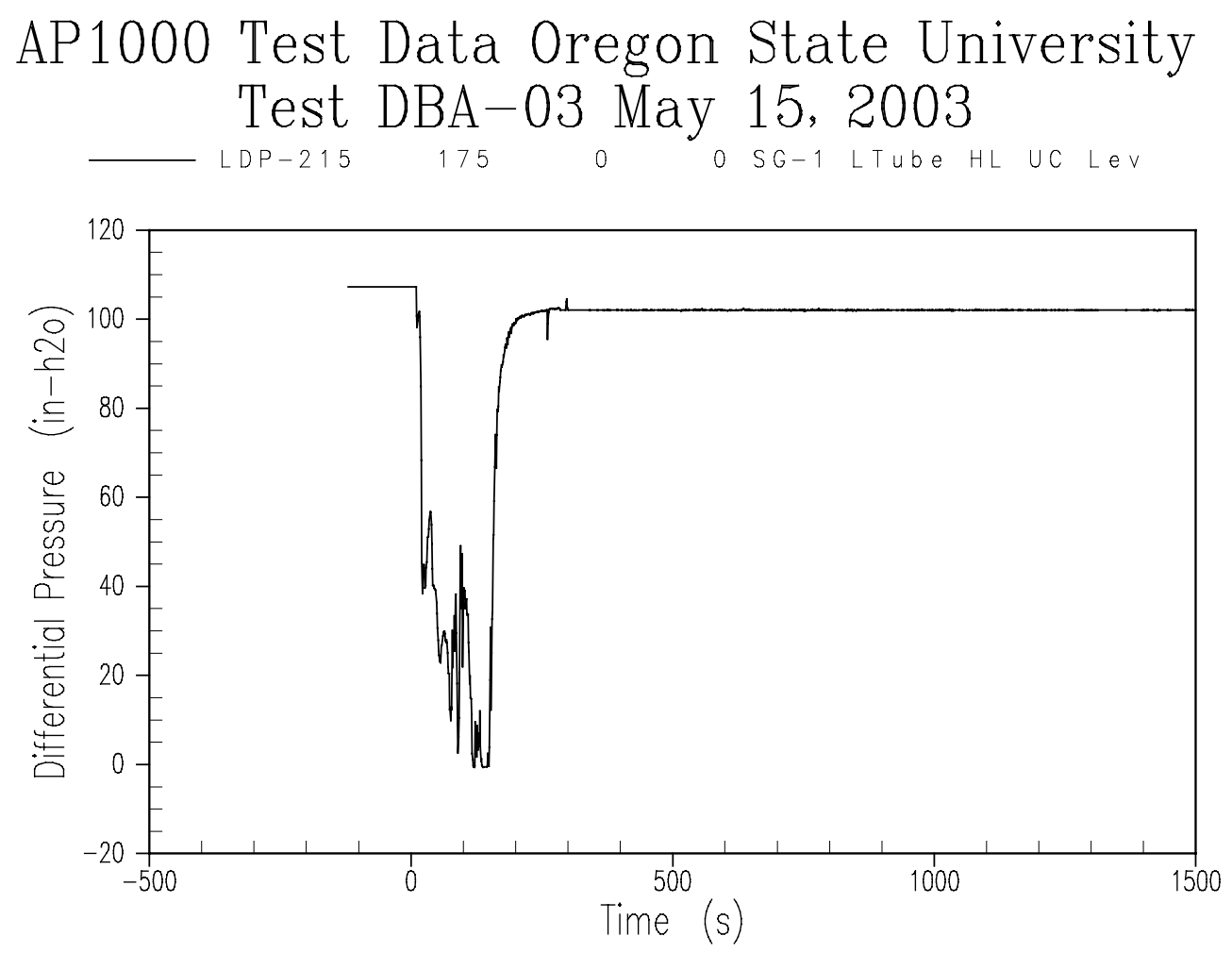

Figure A-14 Steam Generator 1 Tube Level 

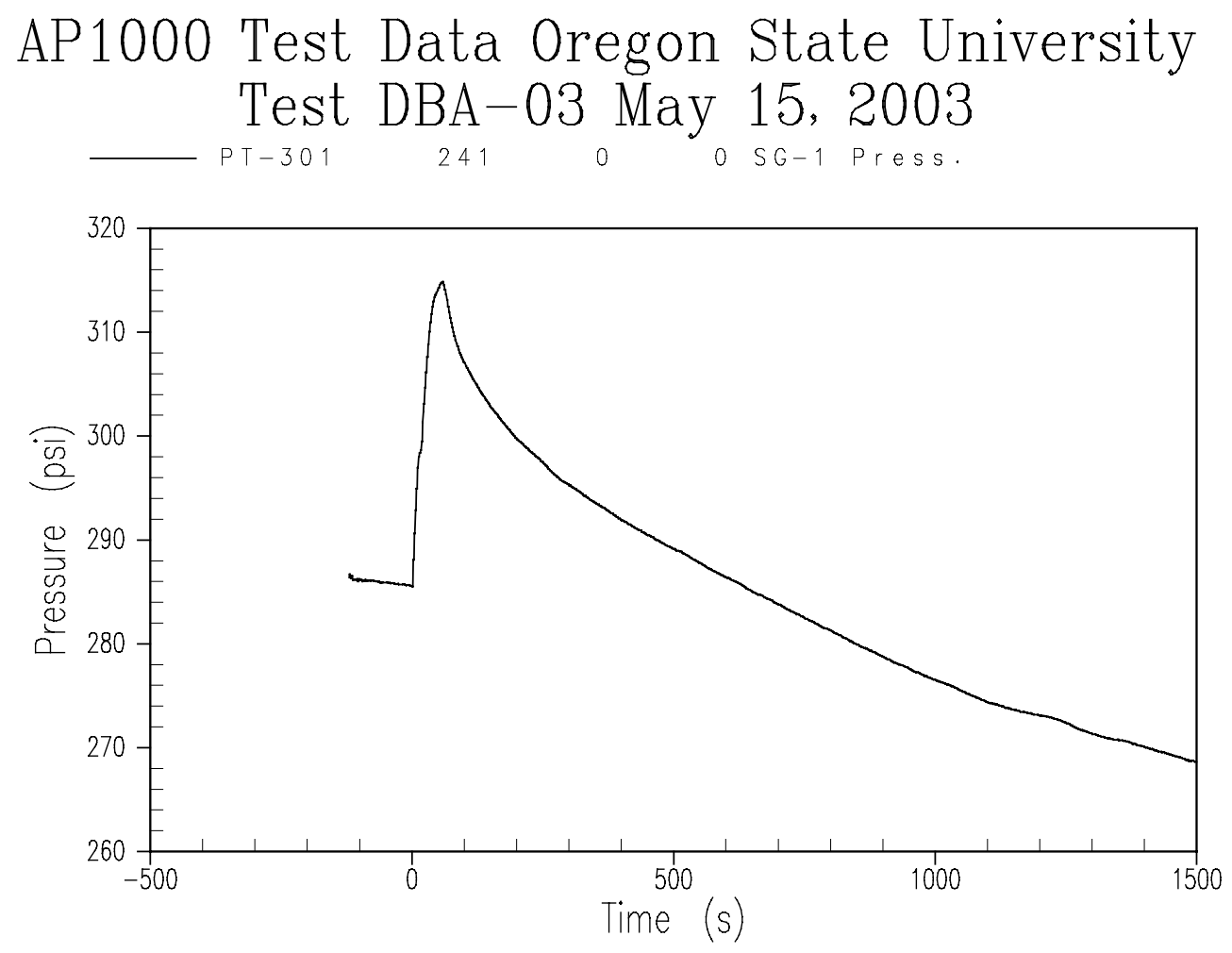

Figure A-15 Steam Generator 1 Secondary Pressure 

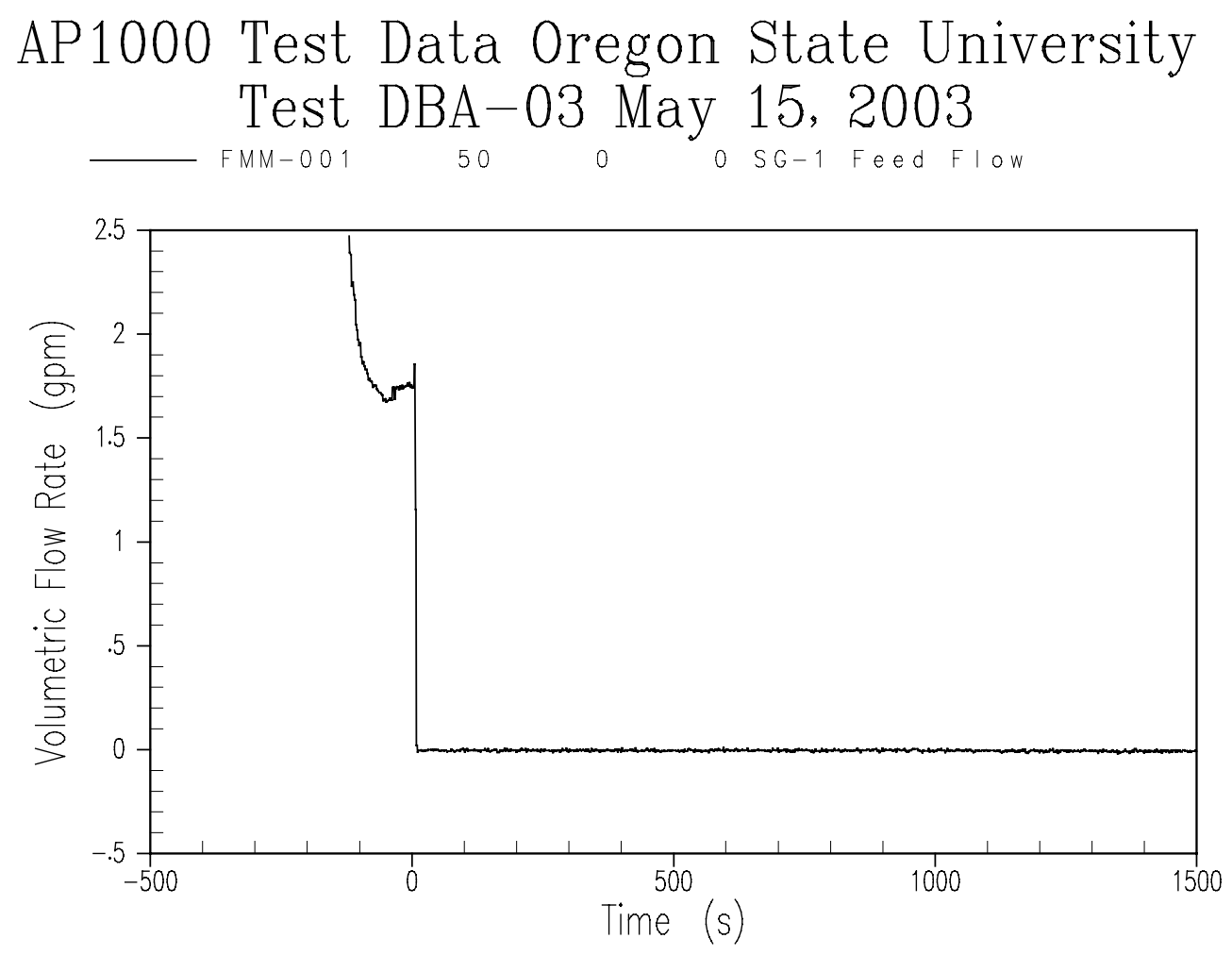

Figure A-16 Steam Generator 1 Feed Flow Rate 

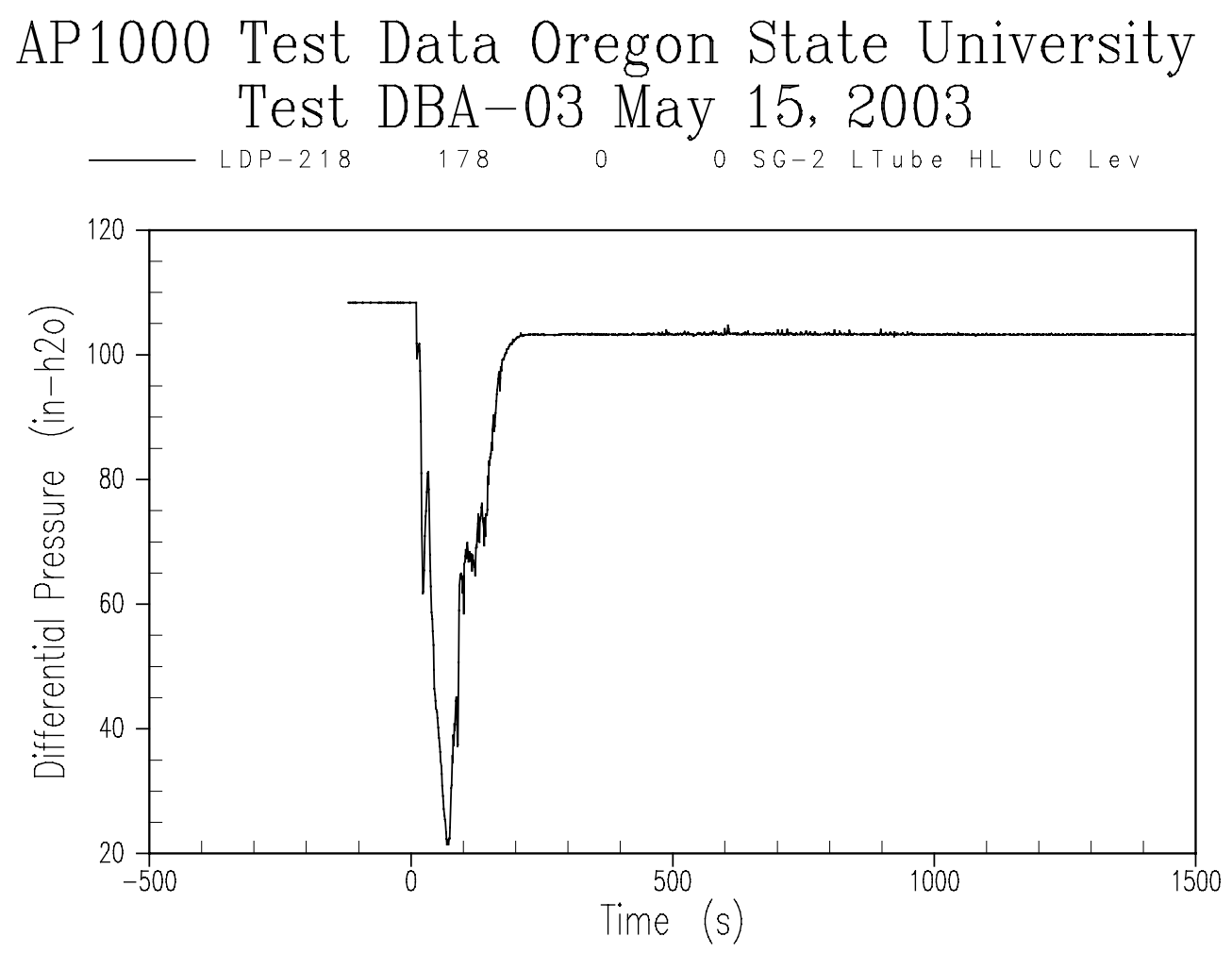

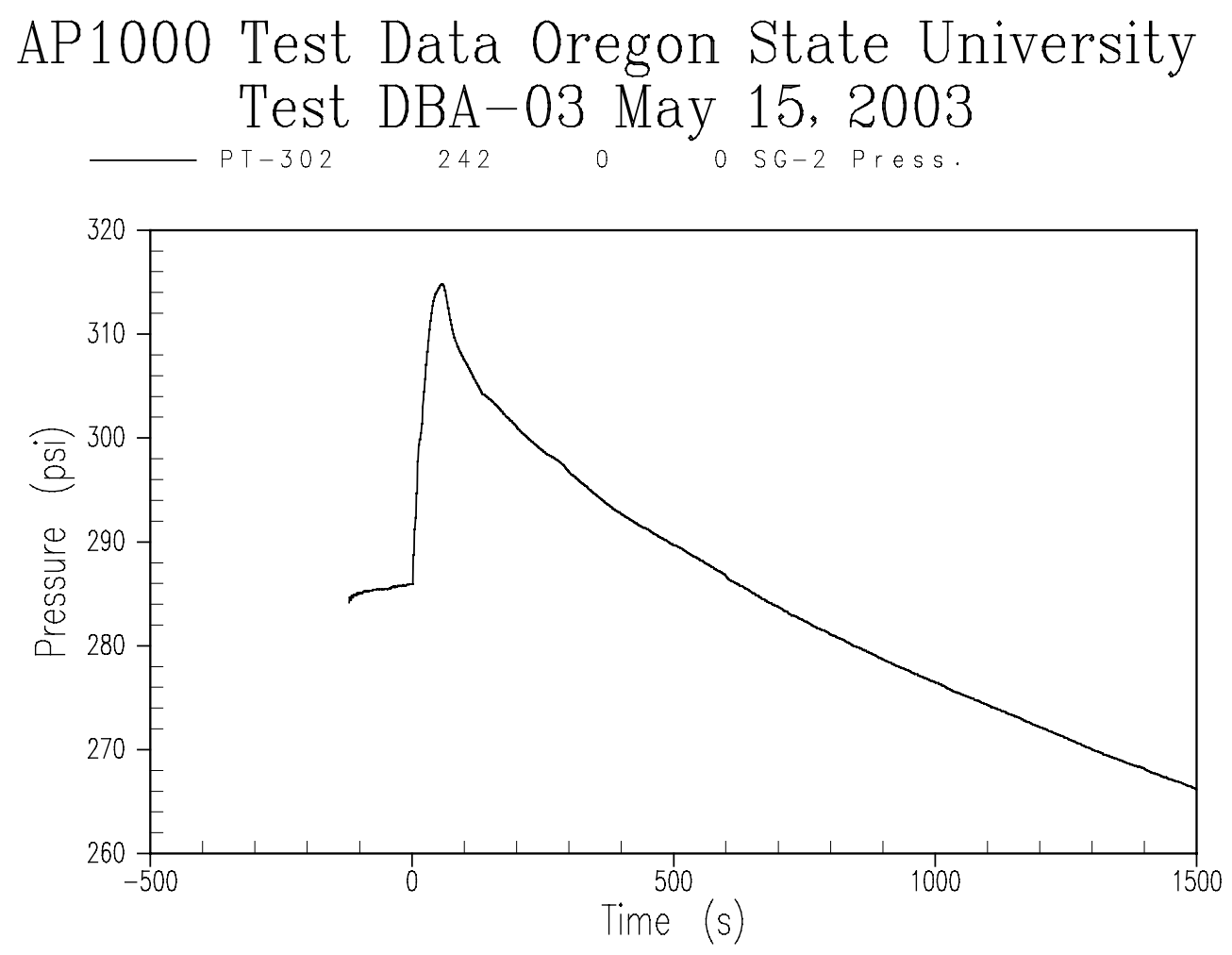

Figure A-18 Steam Generator 2 Secondary Pressure 

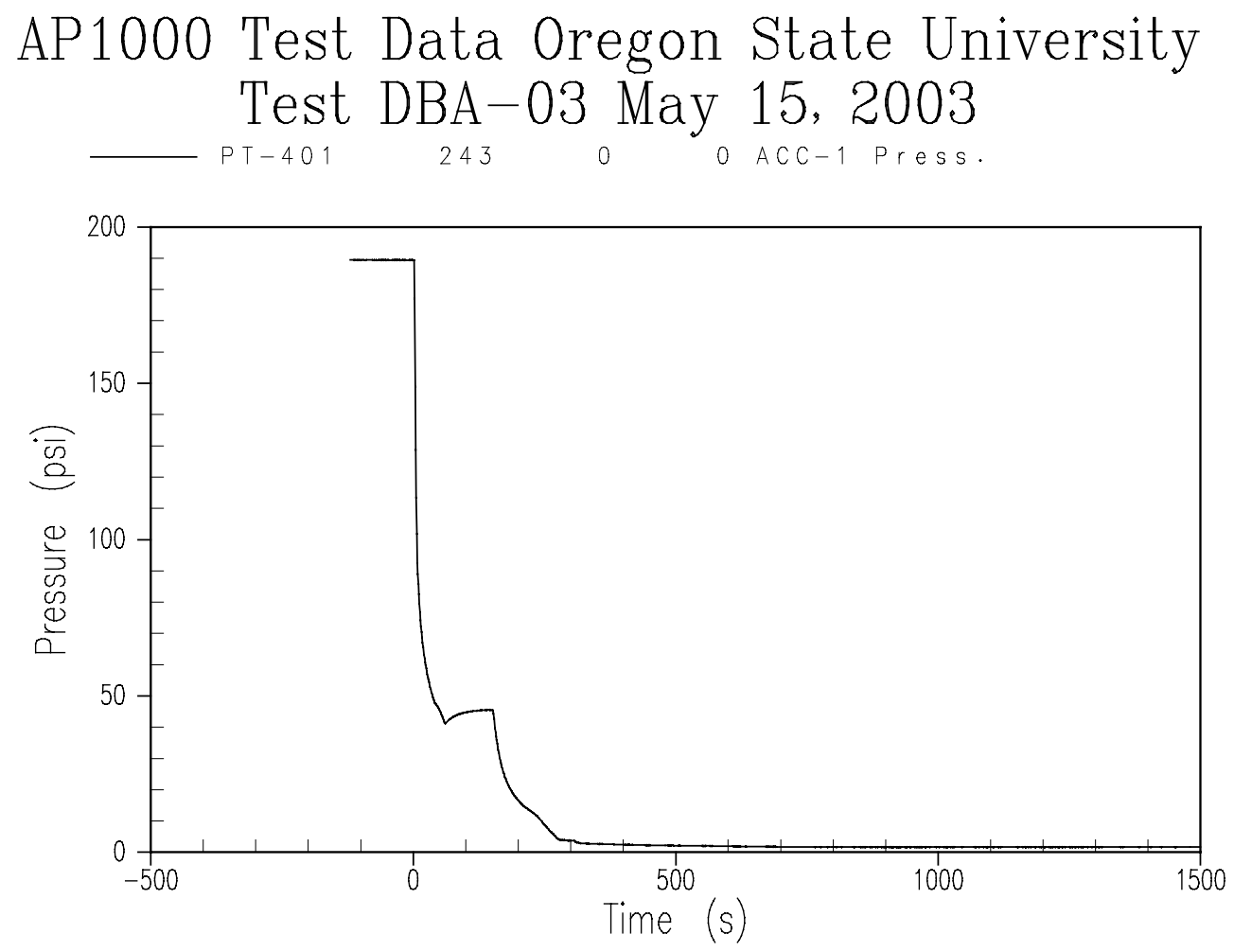

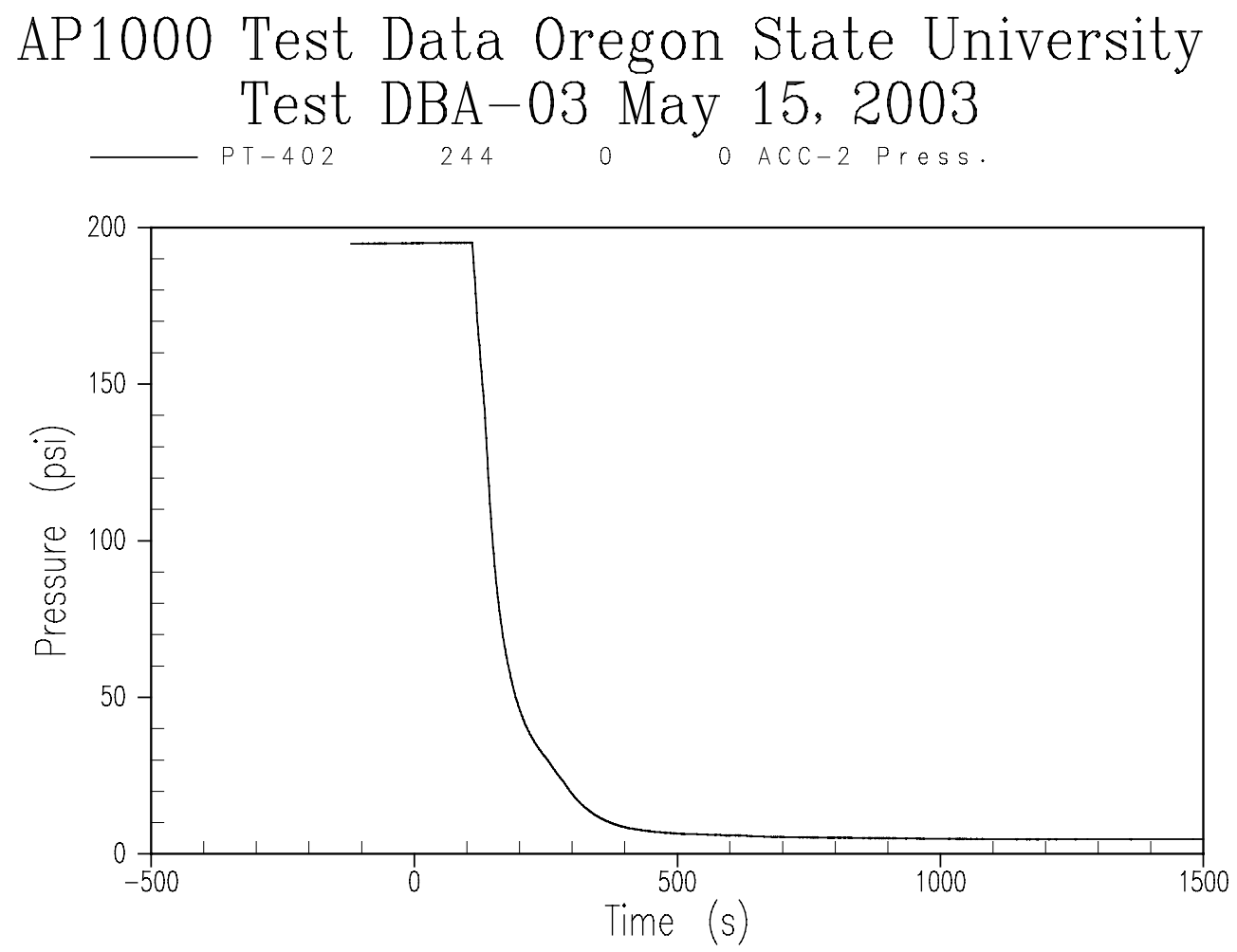

Figure A-20 Accumulator 2 Pressure 

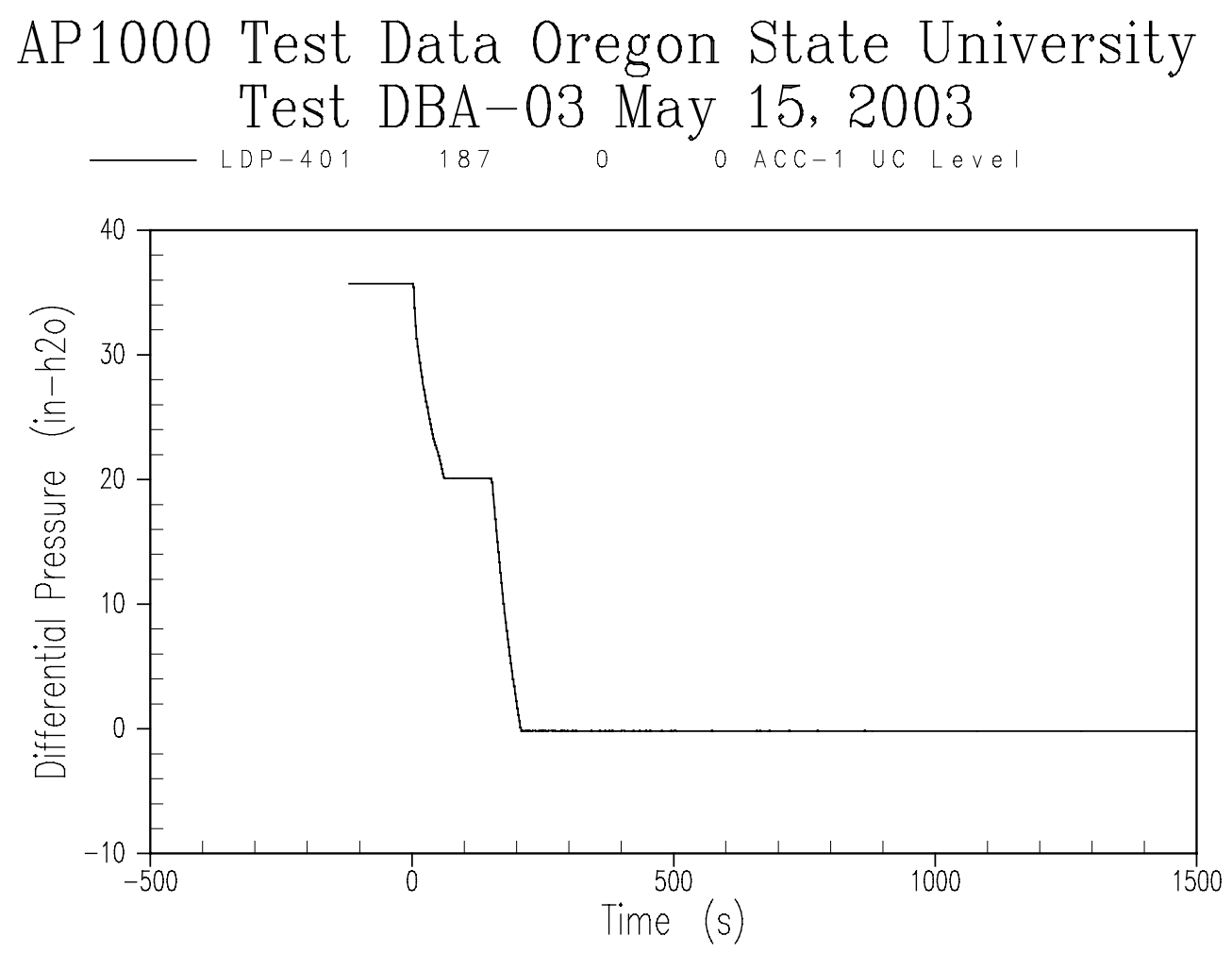

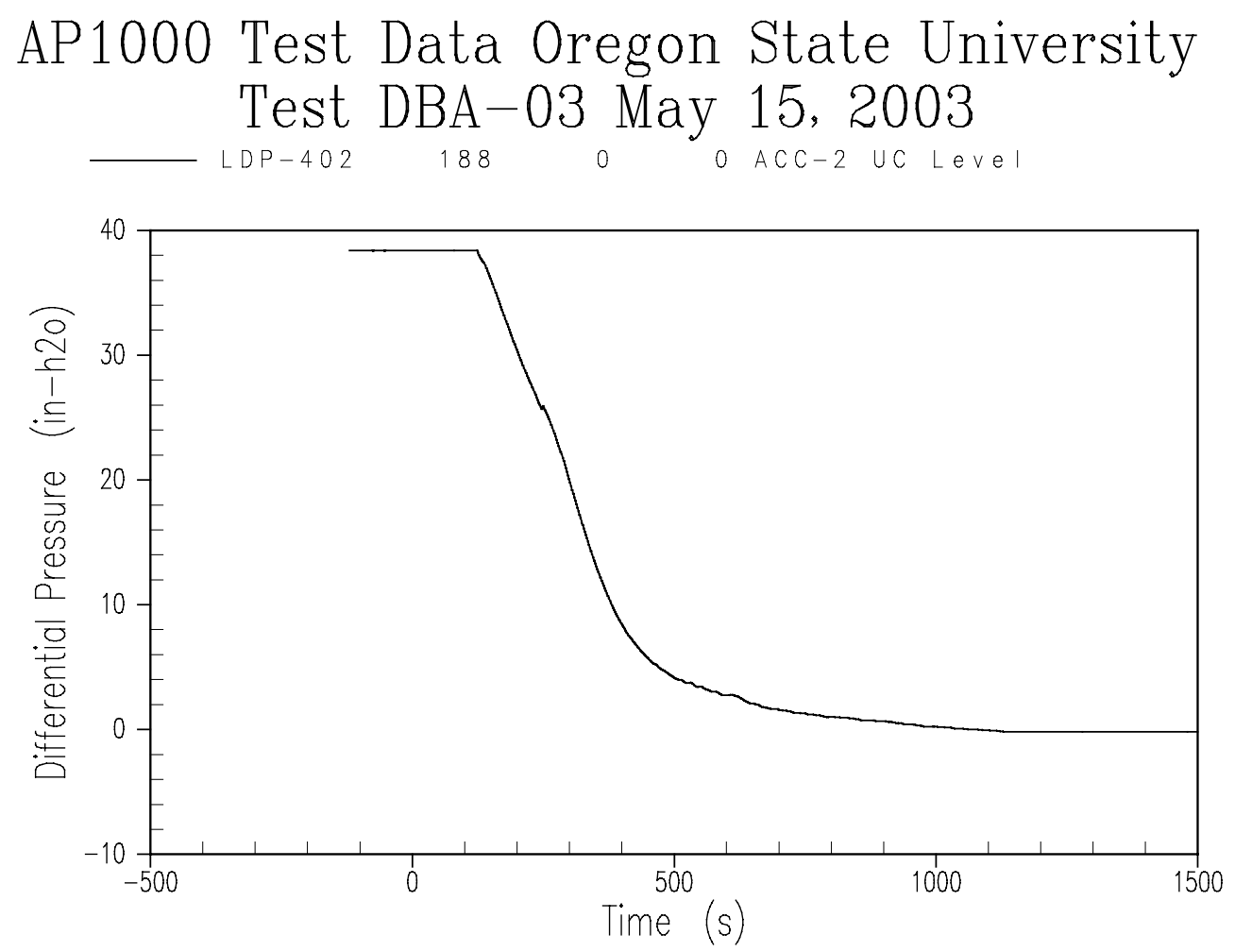

Figure A-22 Accumulator 2 Liquid Level 

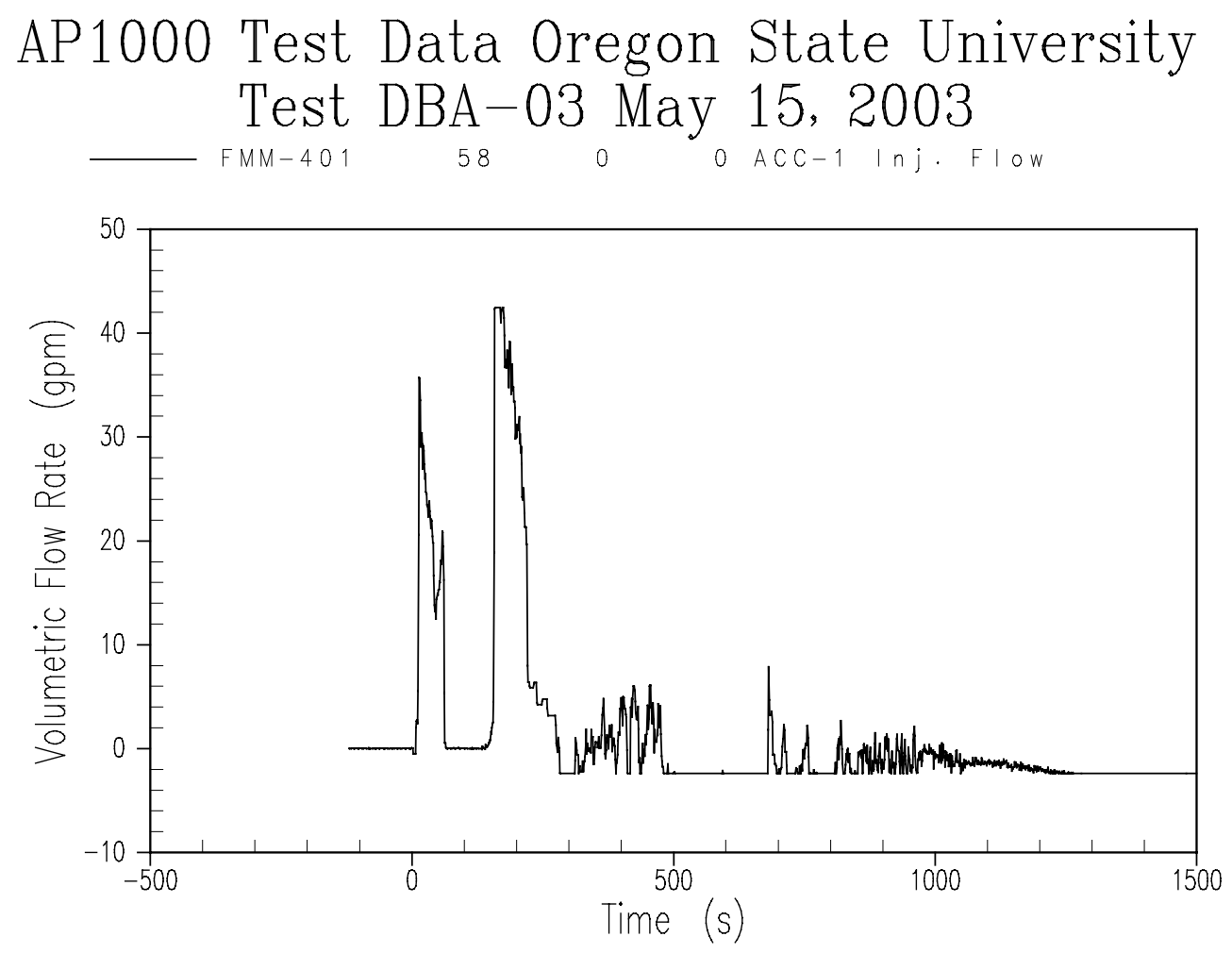

Figure A-23 Accumulator 1 Flow Rate 

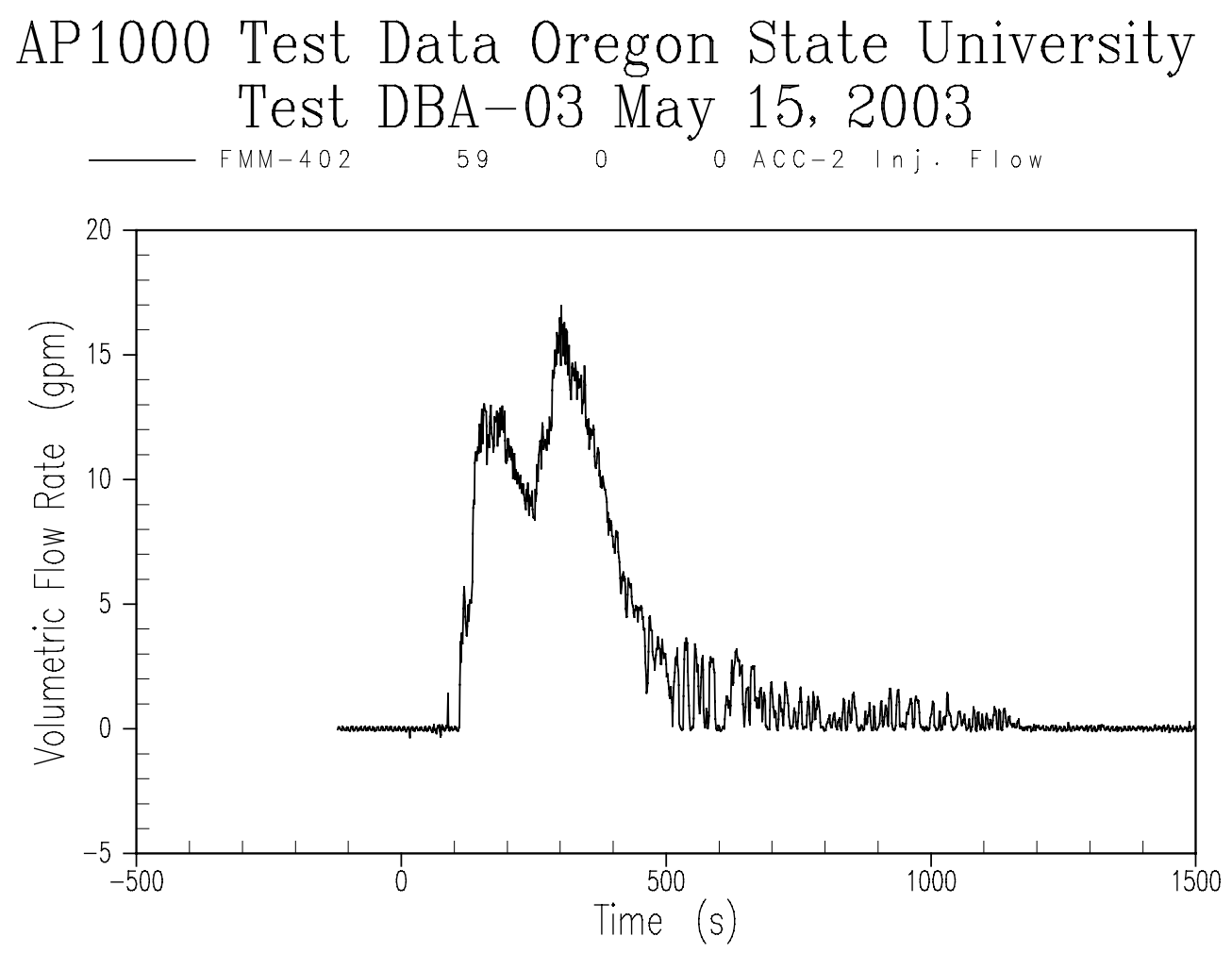

Figure A-24 Accumulator 2 Flow Rate 

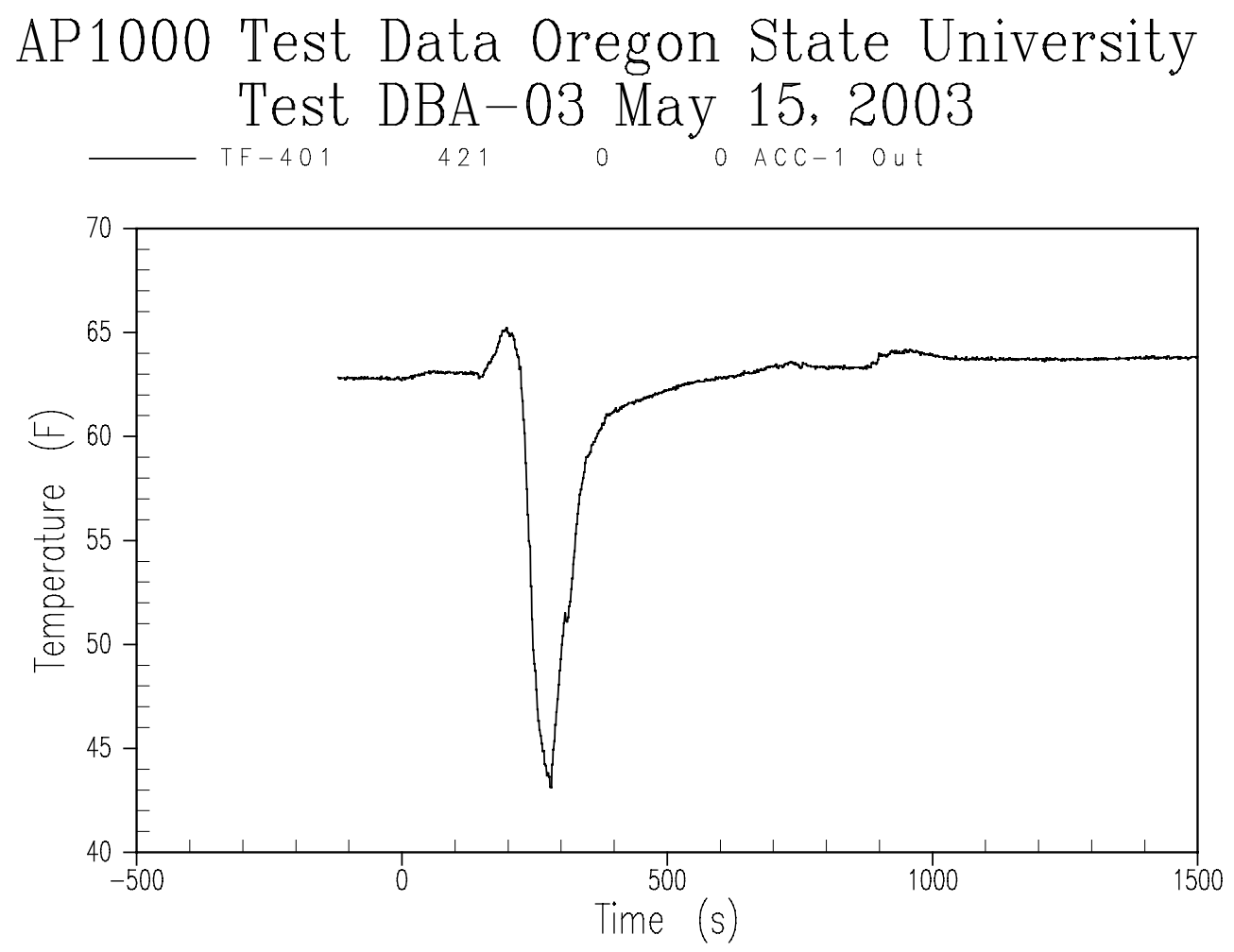

Figure A-25 Accumulator 1 Liquid Discharge Temperature 

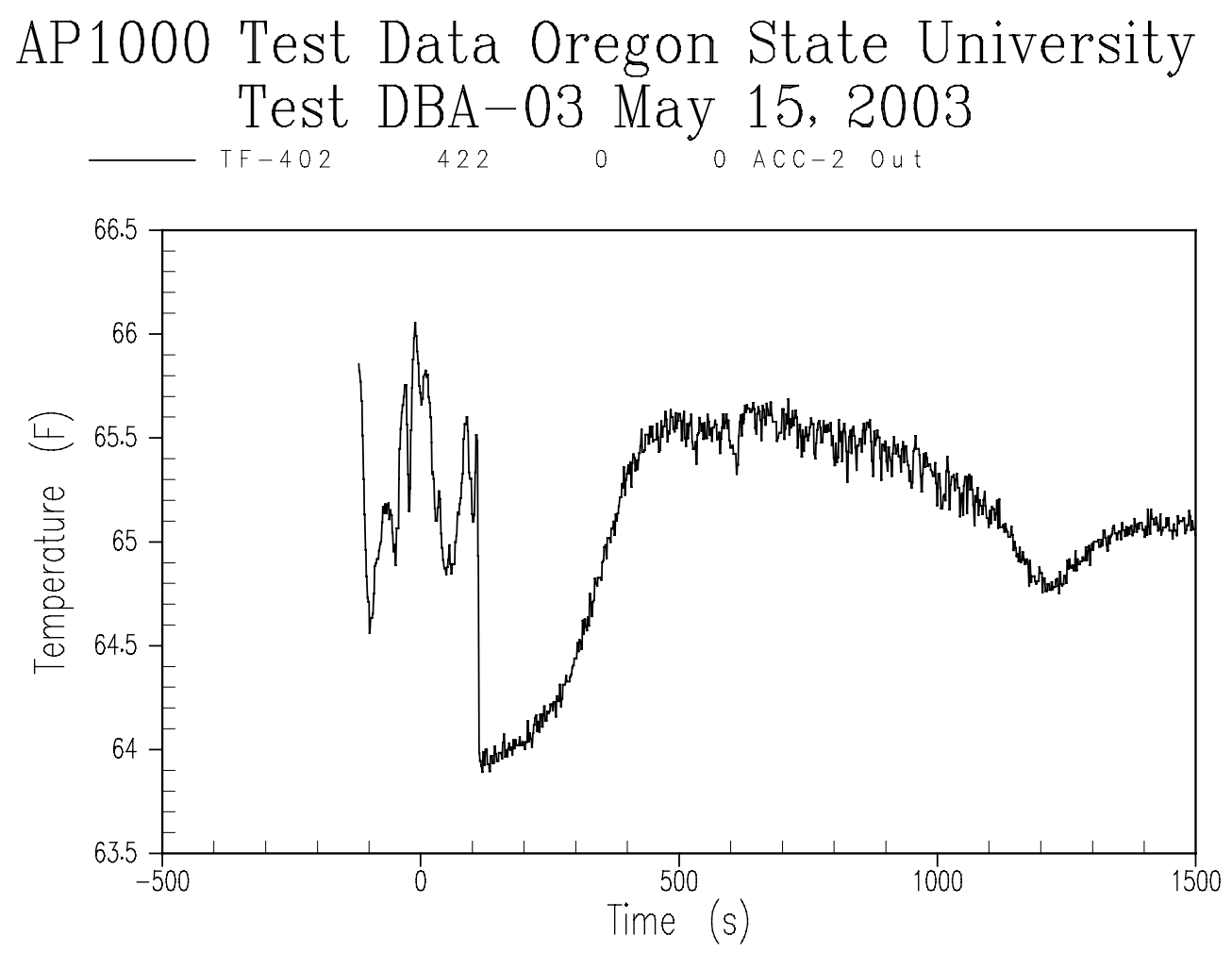

Figure A-26 Accumulator 2 Liquid Discharge Temperature 

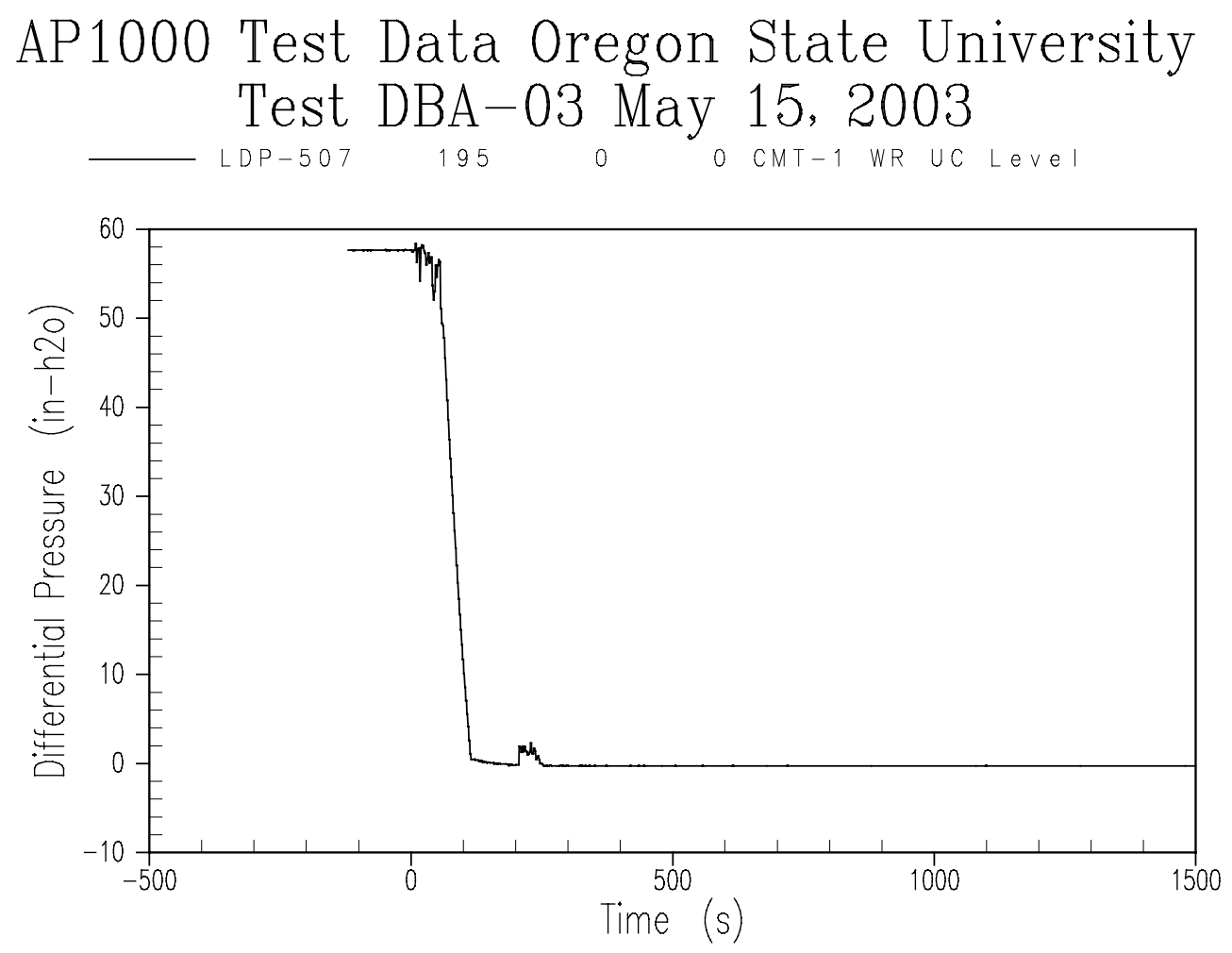

Figure A-27 Core Makeup Tank 1 Liquid Level 

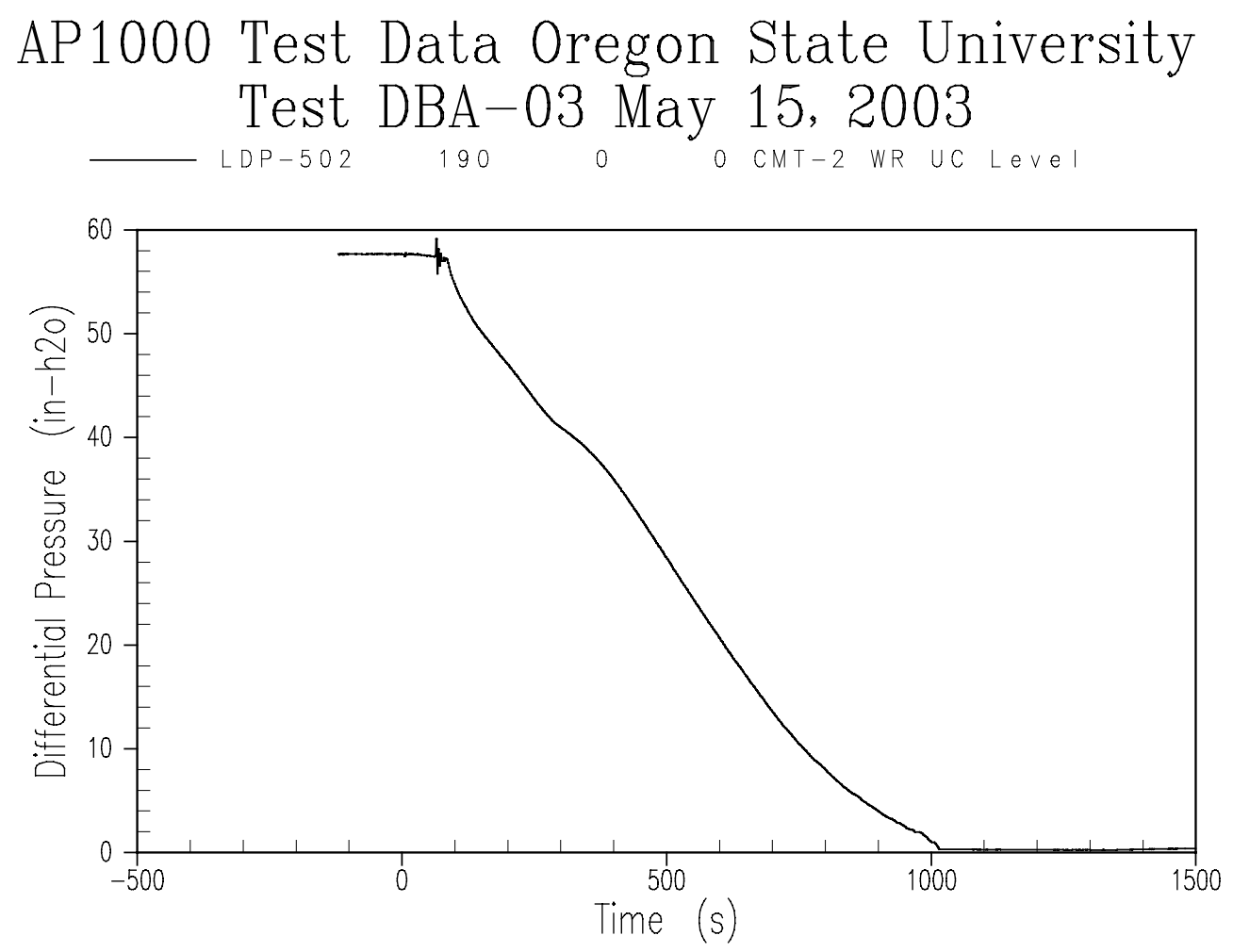

Figure A-28 Core Makeup Tank 2 Liquid Level 

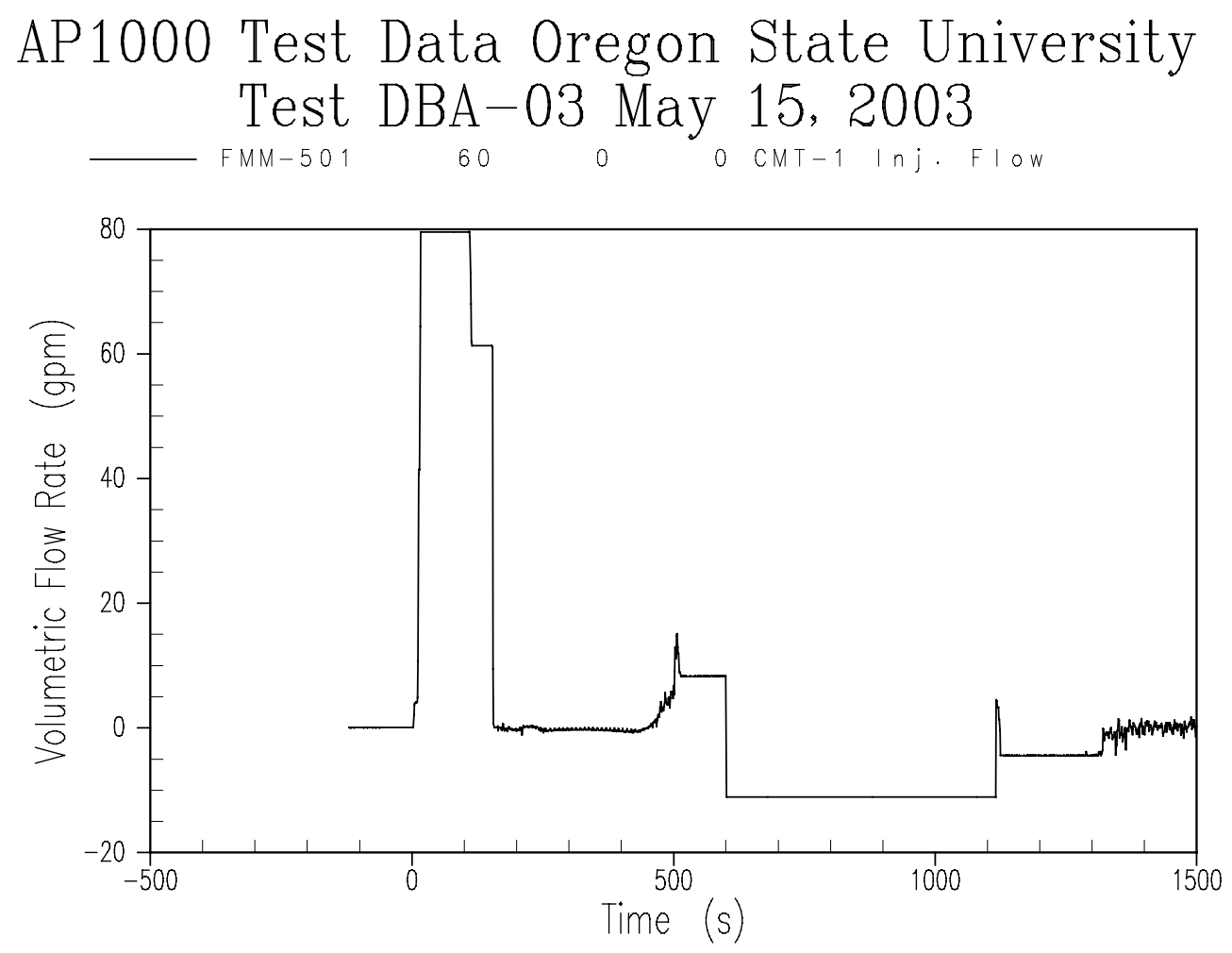

Figure A-29 Core Makeup Tank 1 Flow Rate 

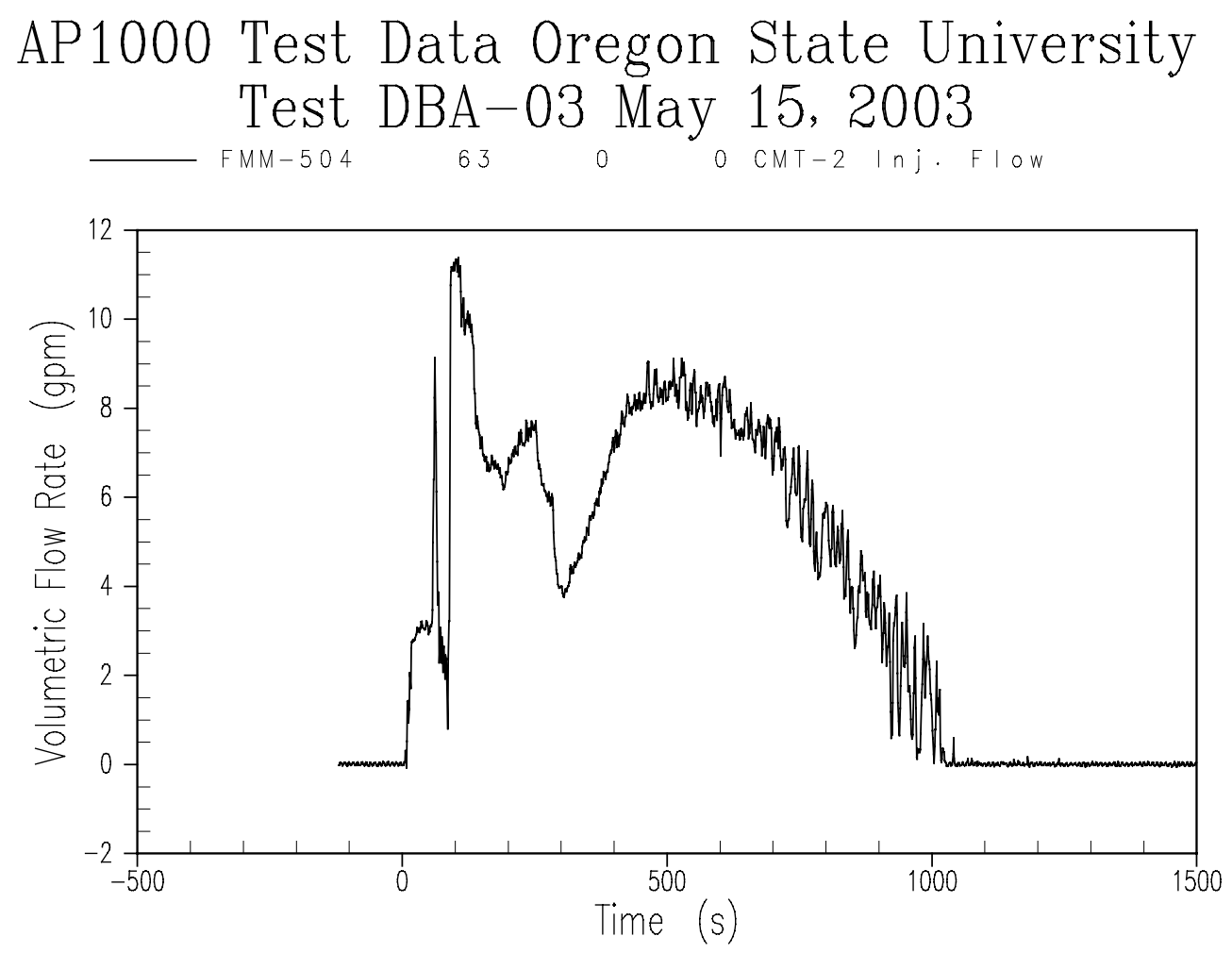

Figure A-30 Core Makeup Tank 2 Flow Rate 

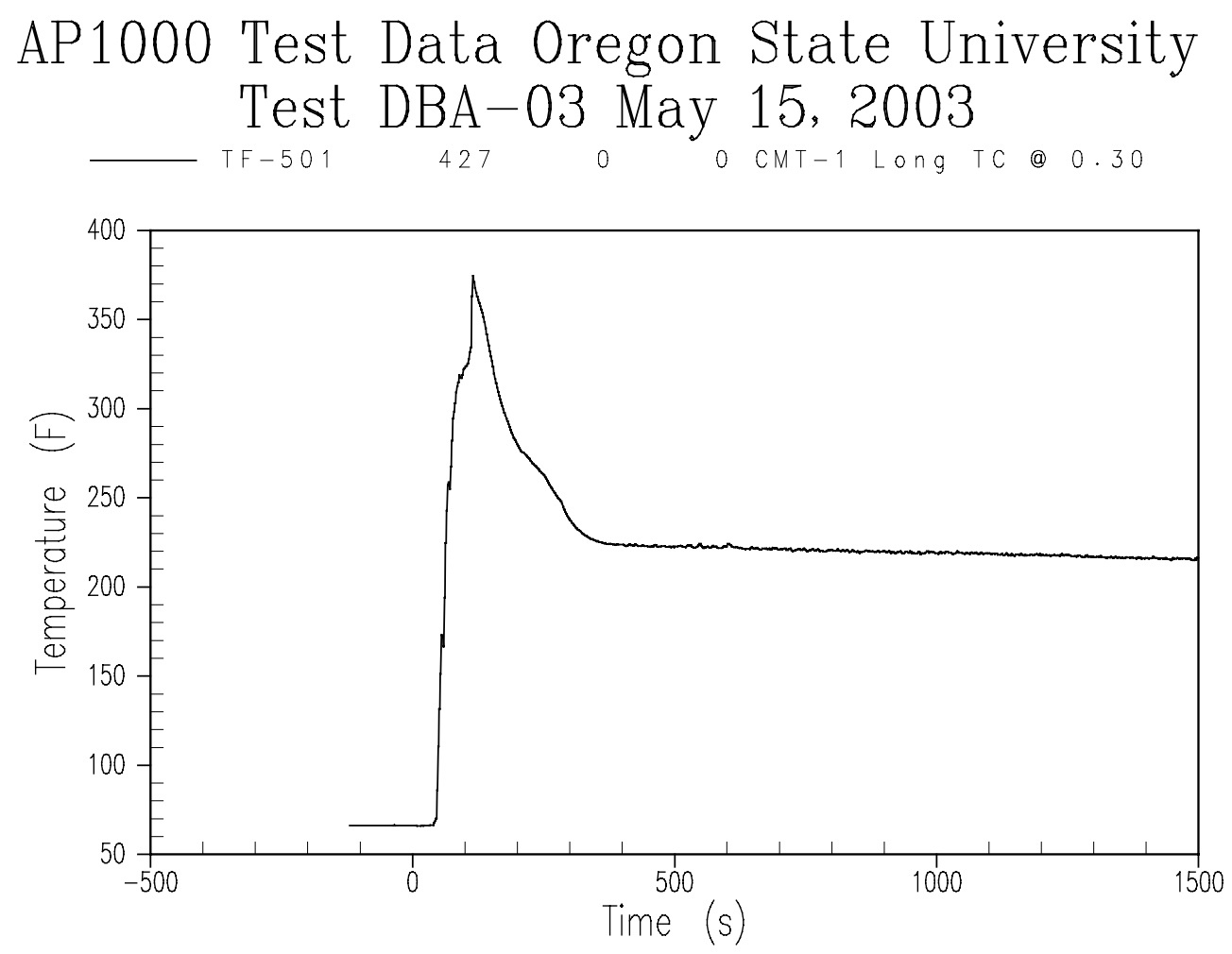

Figure A-31 Core Makeup Tank 1 Liquid Temperature - Bottom 

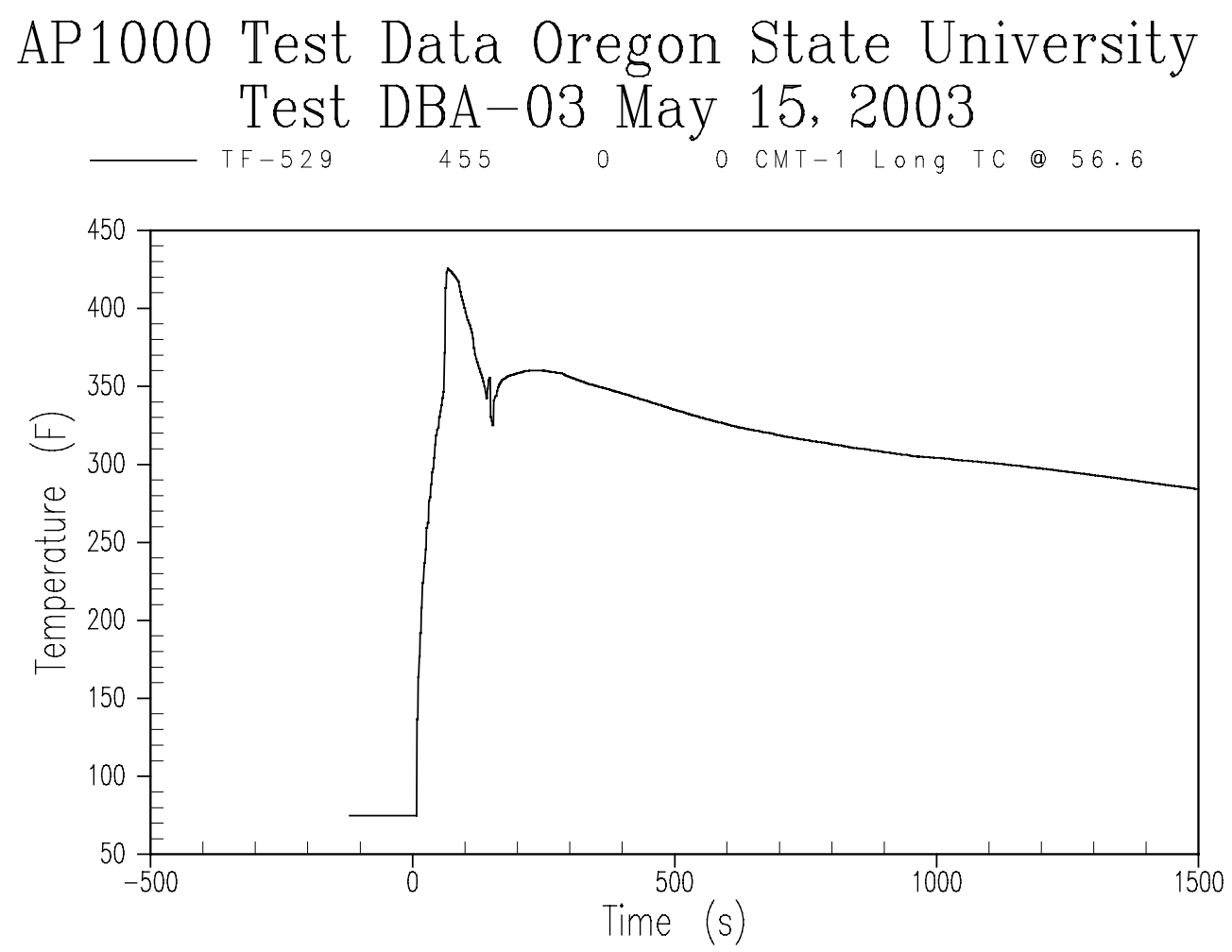

Figure A-32 Core Makeup Tank 1 Liquid Temperature - Top 

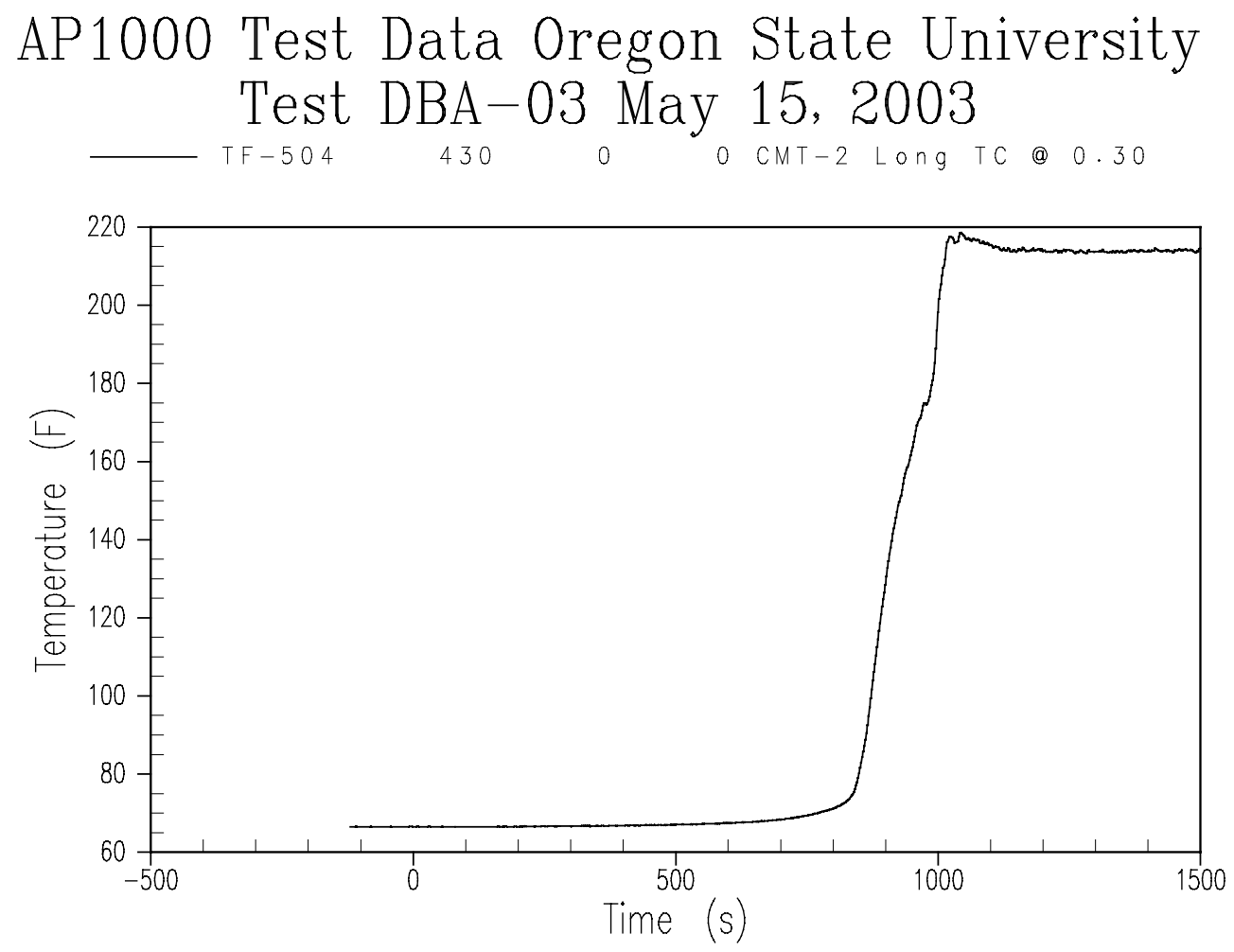

Figure A-33 Core Makeup Tank 2 Liquid Temperature - Bottom 

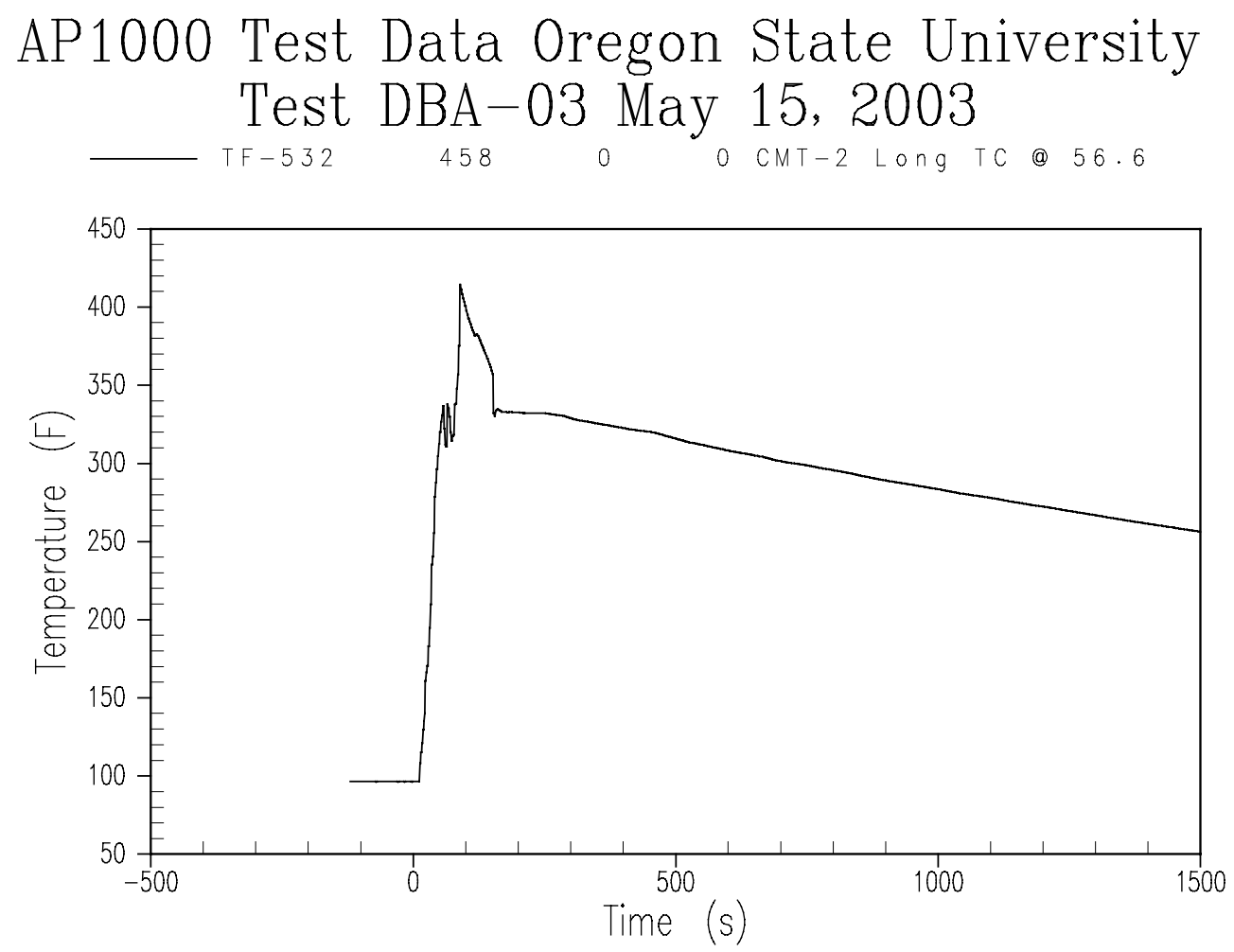

Figure A-34 Core Makeup Tank 2 Liquid Temperature - Top 

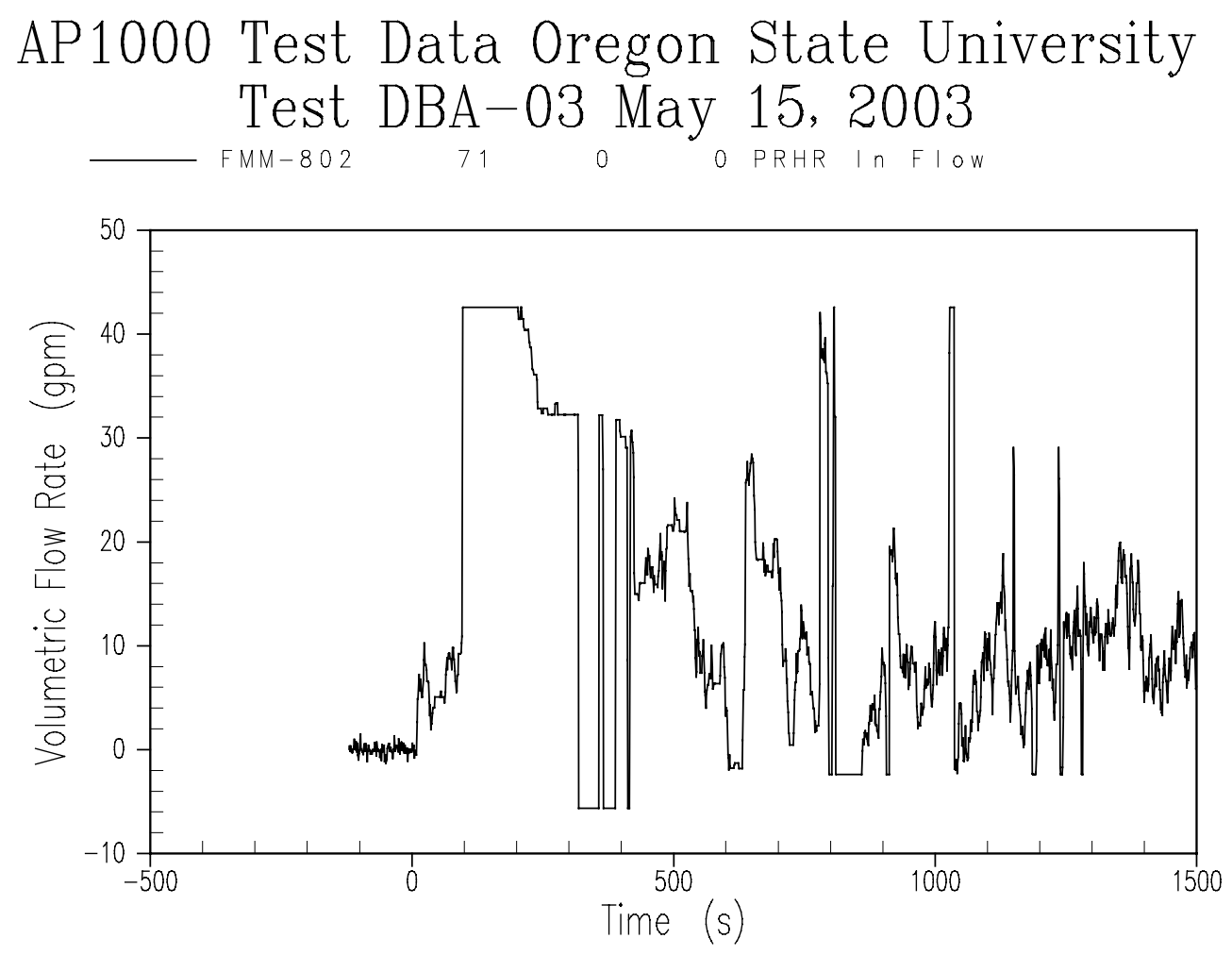

Figure A-35 Passive Residual Heat Removal Inlet Flow Rate 


\section{AP1000 Test Data Oregon State University Test DBA-03 May 15, 2003 \\ $\begin{array}{llll}L D P-802 & 213 & 0 & 0\end{array}$}

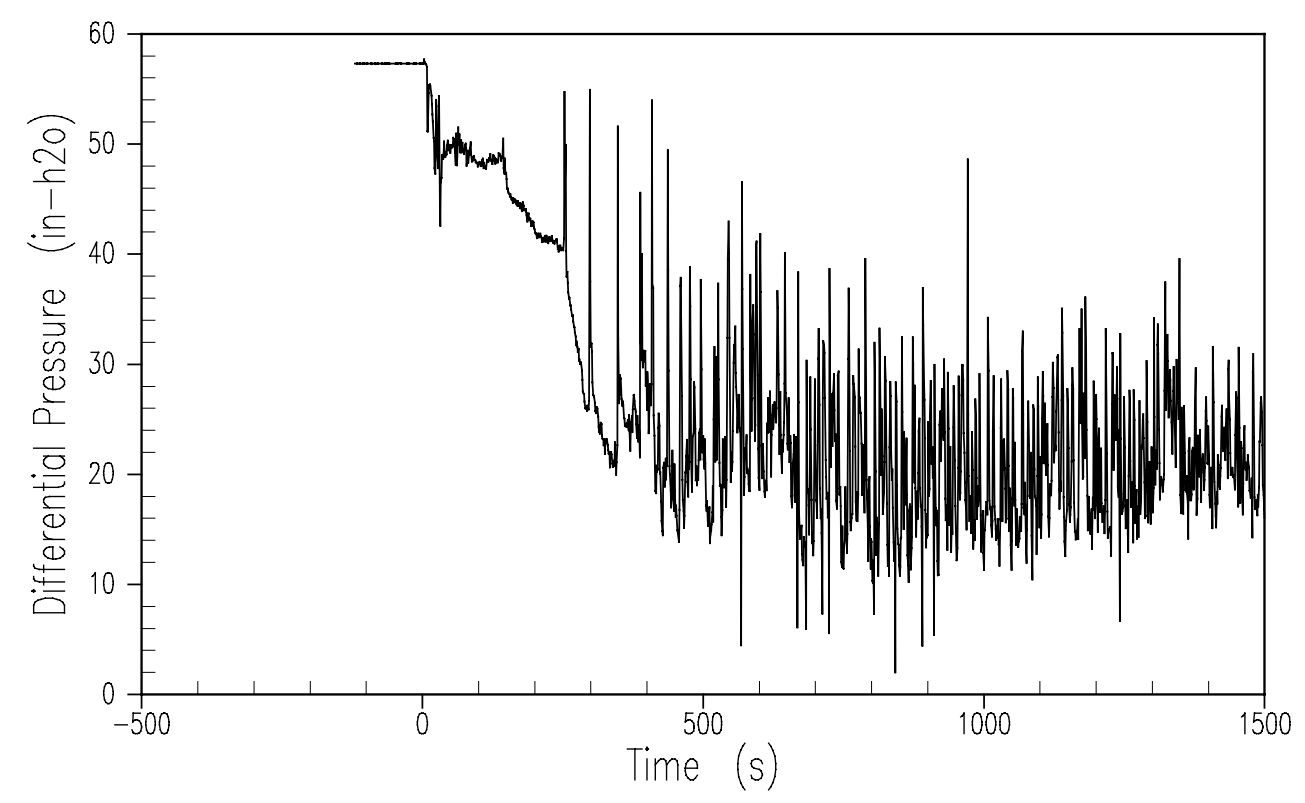

Figure A-36 Passive Residual Heat Removal Liquid Level 

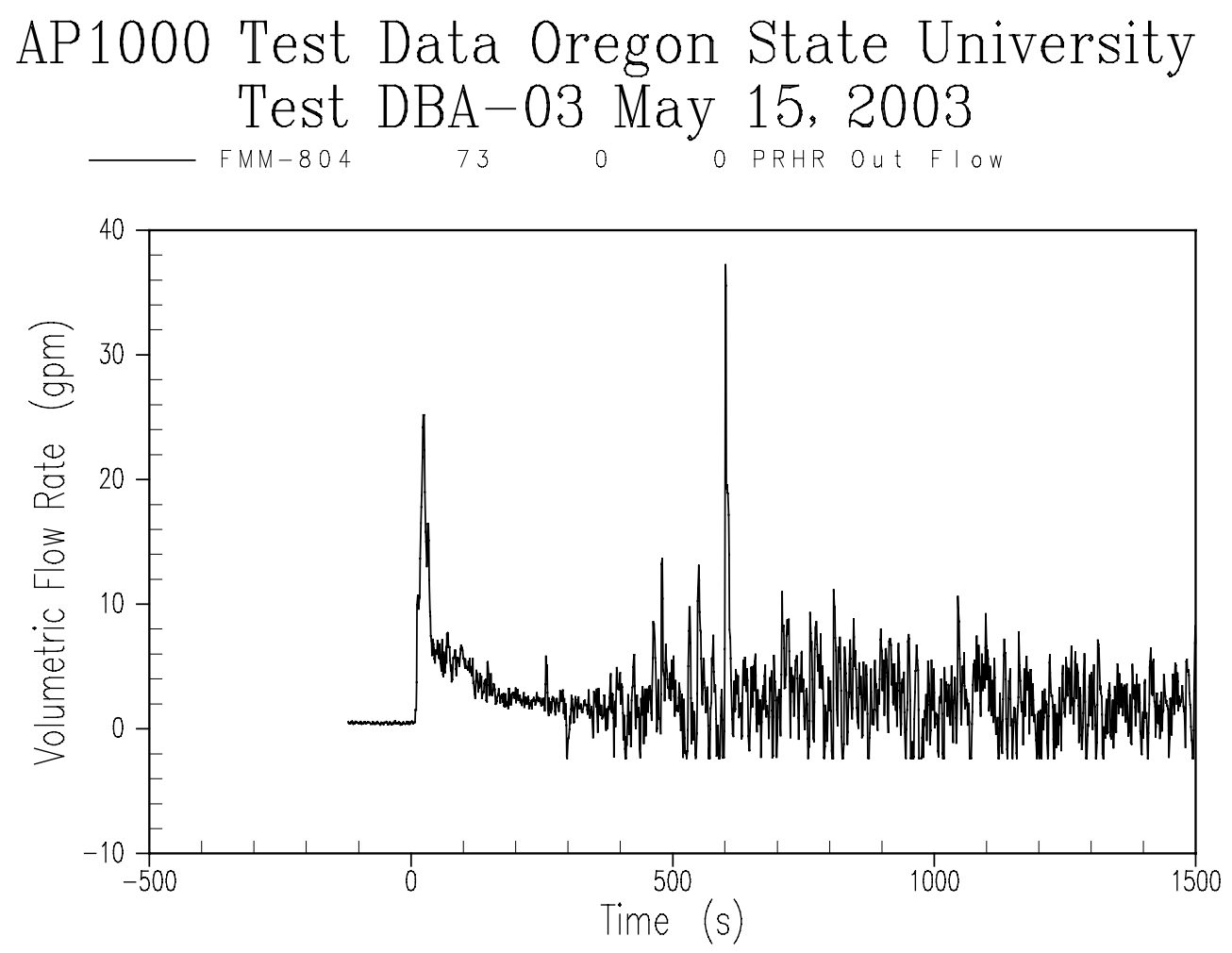

Figure A-37 Passive Residual Heat Removal Outlet Flow Rate 

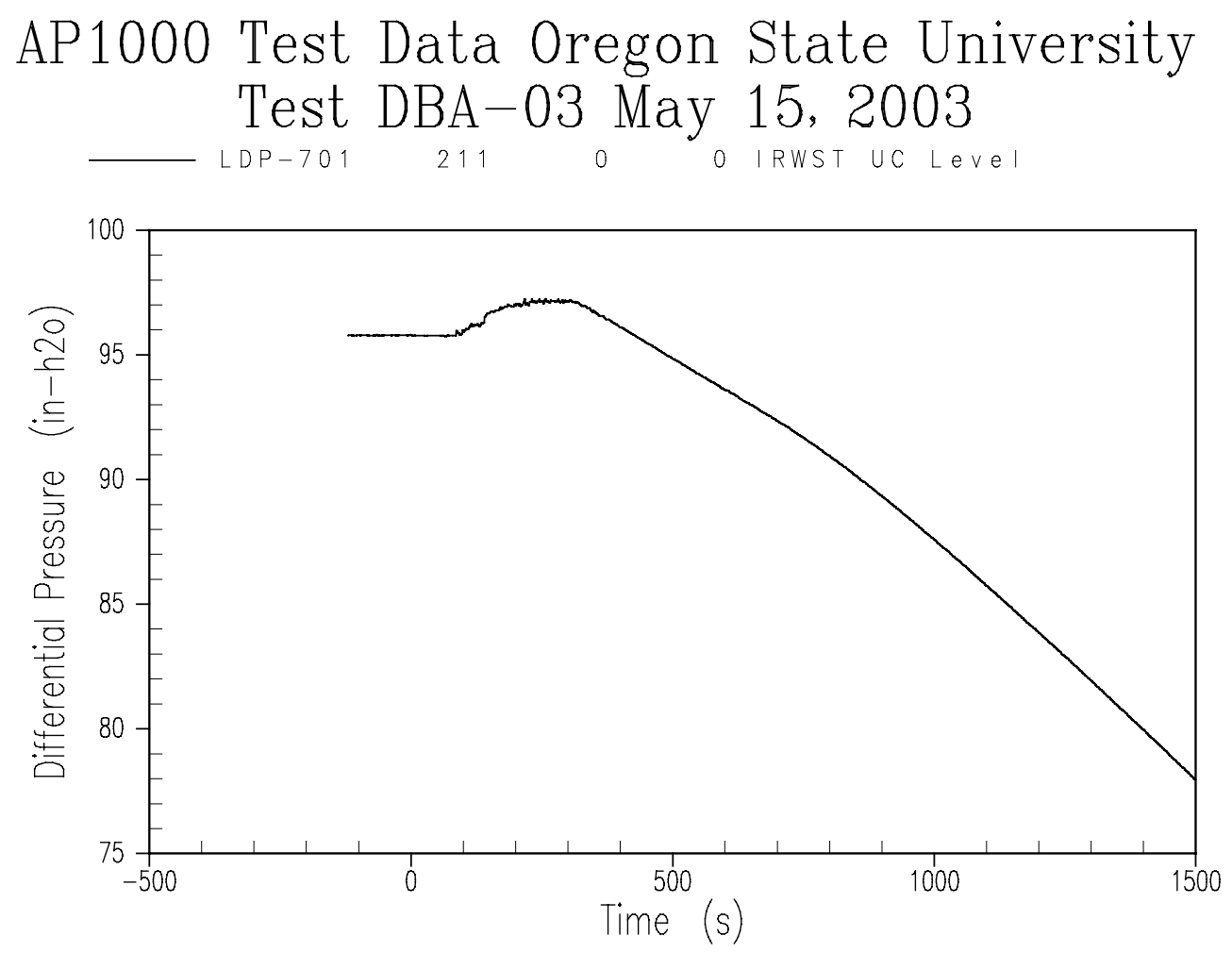

Figure A-38 IRWST Liquid Level 

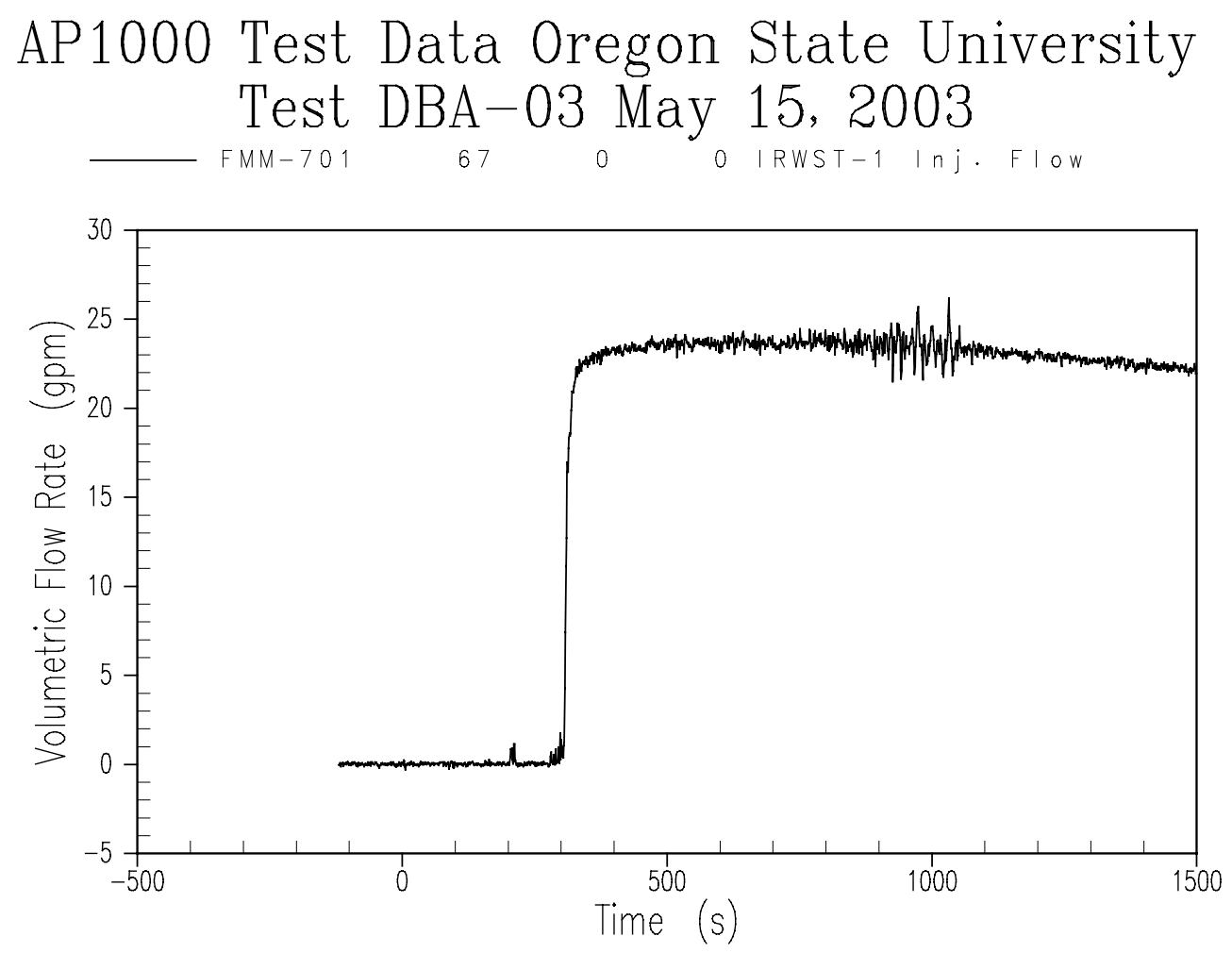

Figure A-39 IRWST Discharge Line 1 Flow Rate 

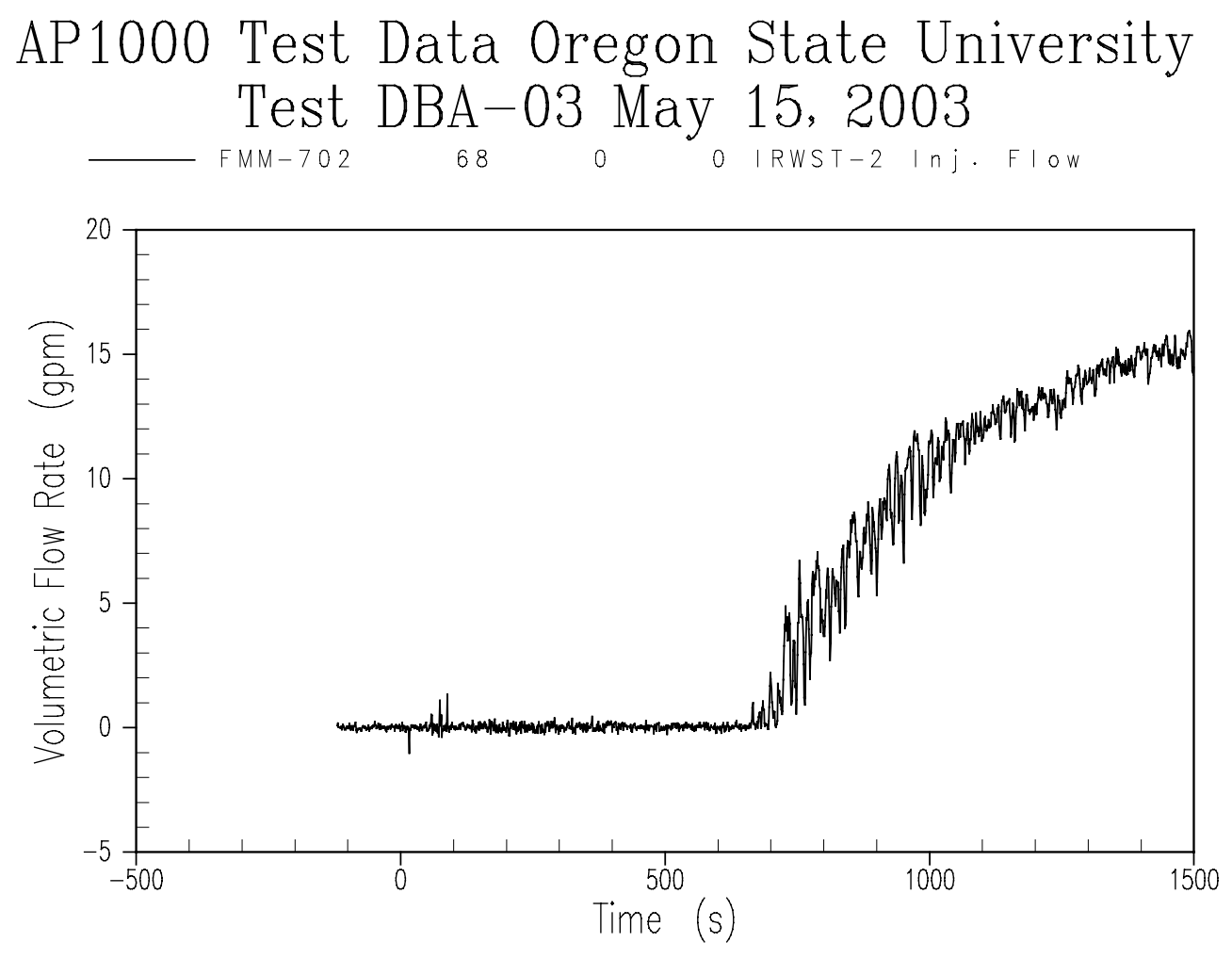

Figure A-40 IRWST Discharge Line 2 Flow Rate 

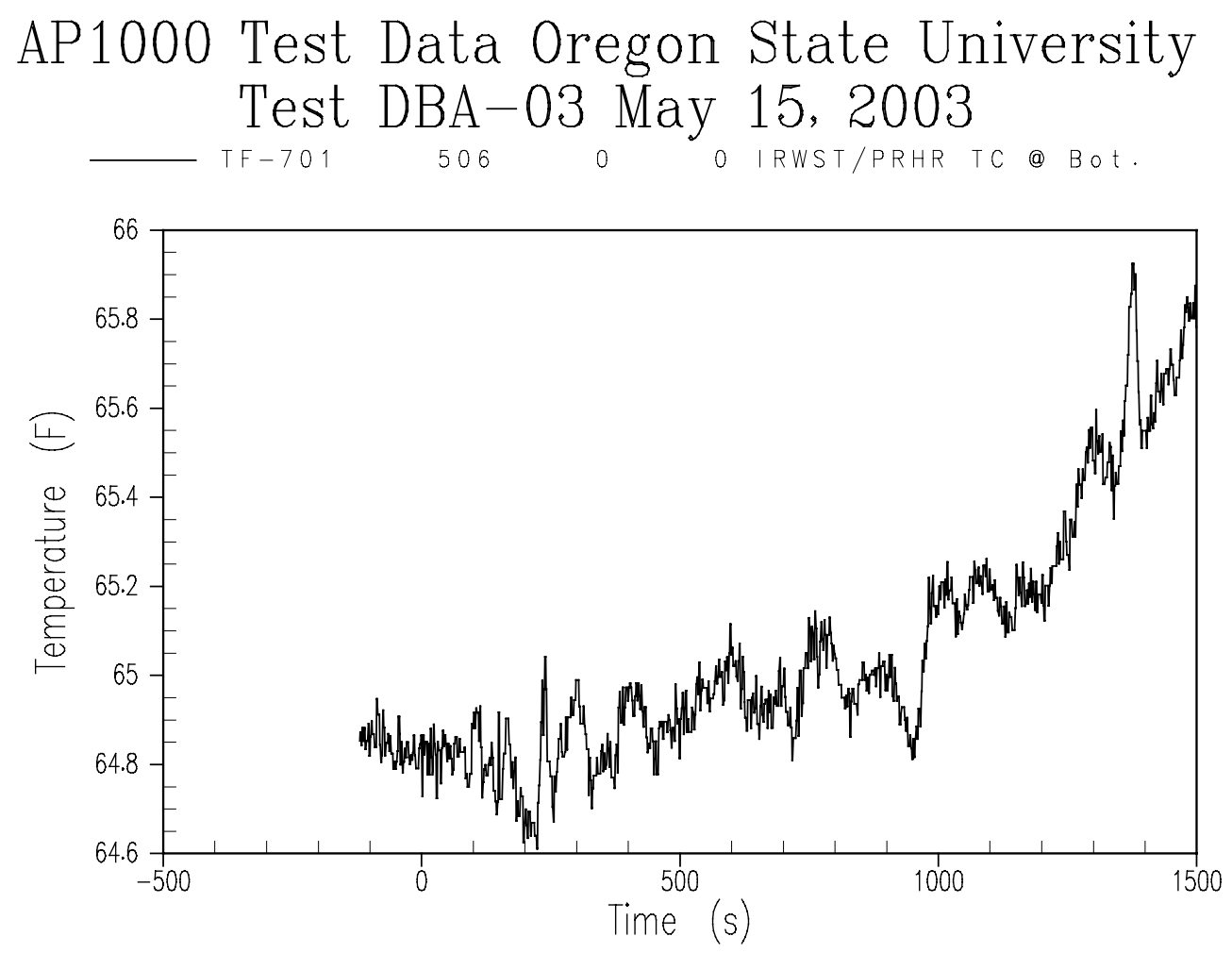

Figure A-41 IRWST Fluid Temperature 

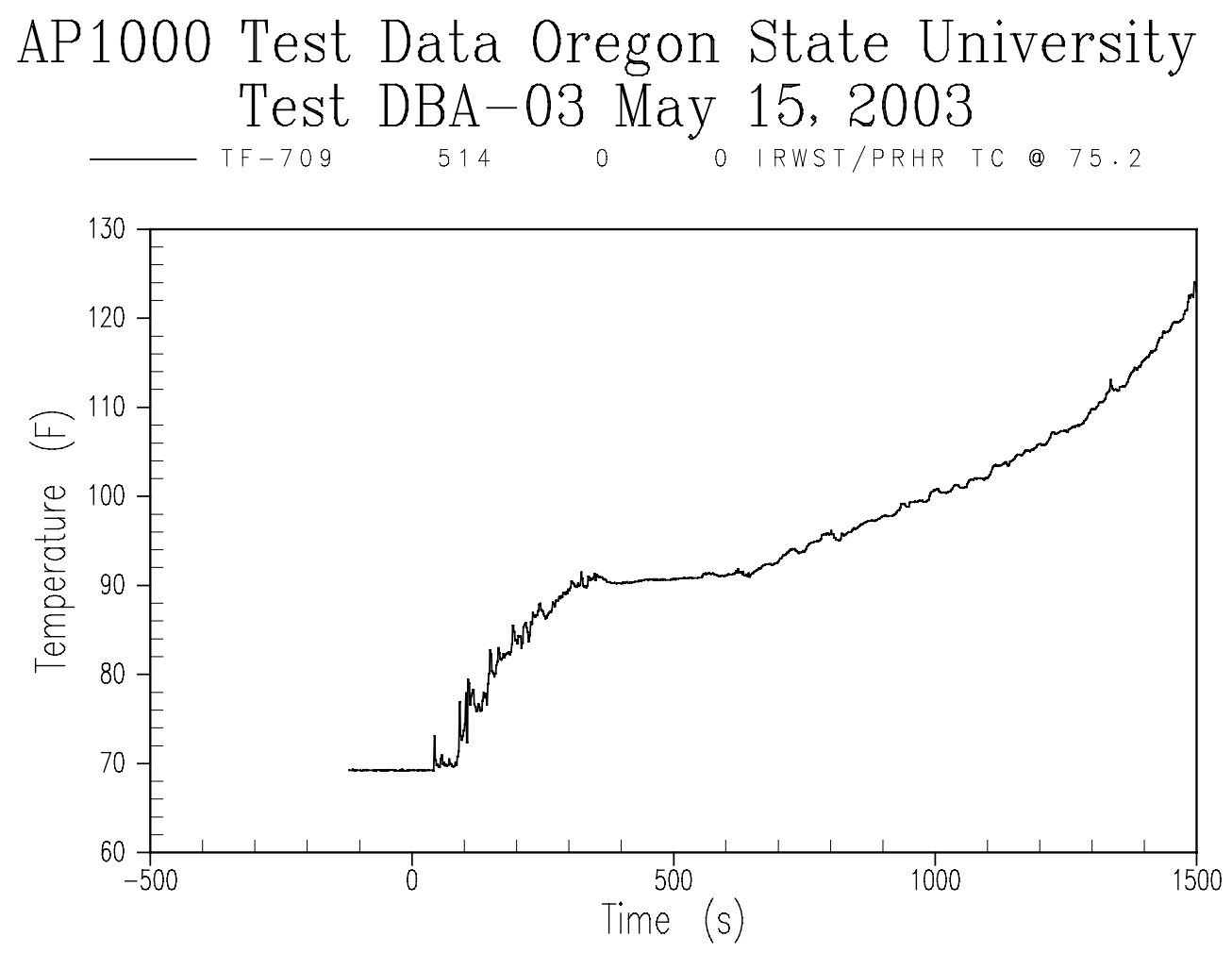

Figure A-42 IRWST Fluid Temperature 

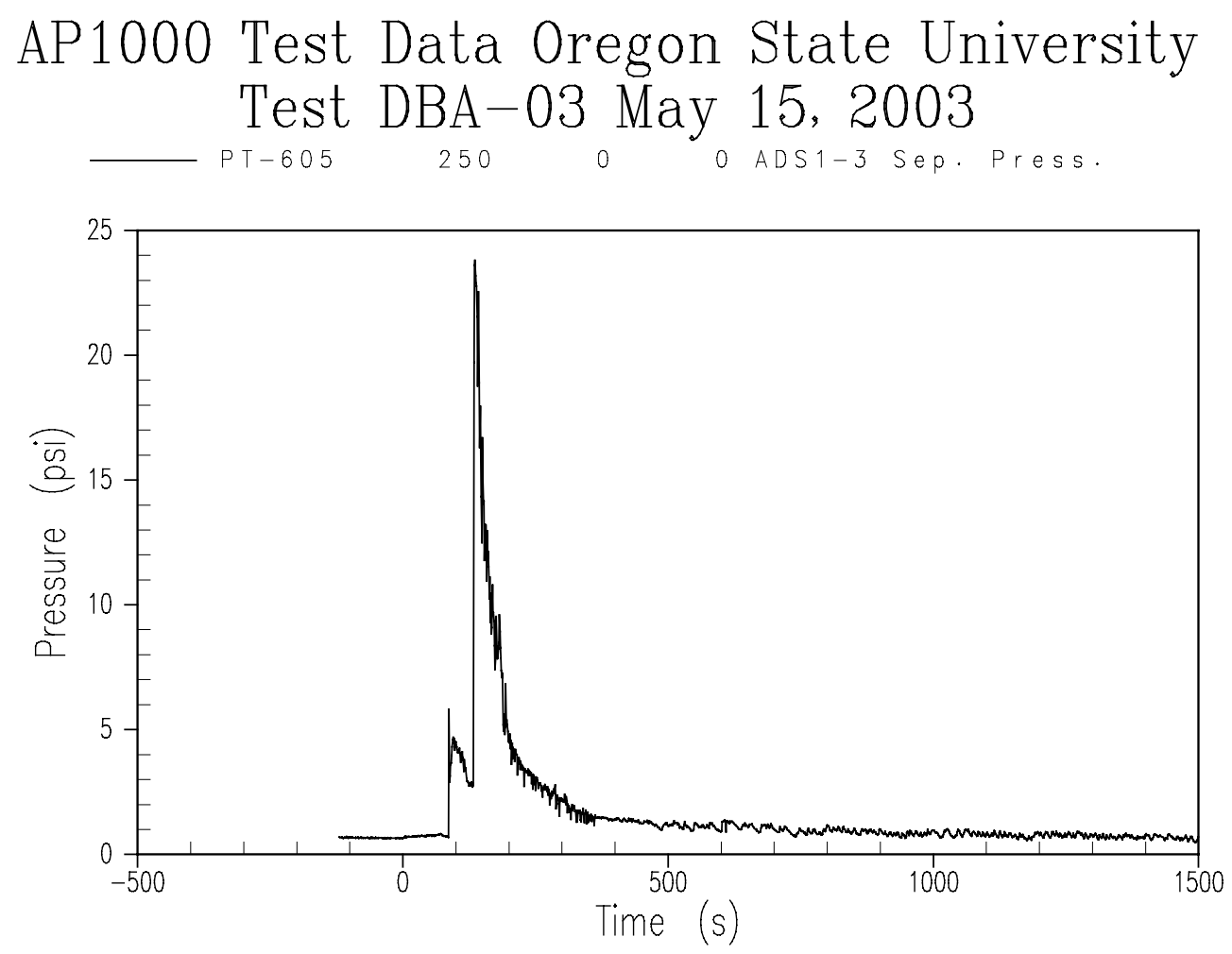

Figure A-43 ADS 1-3 Separator Pressure 

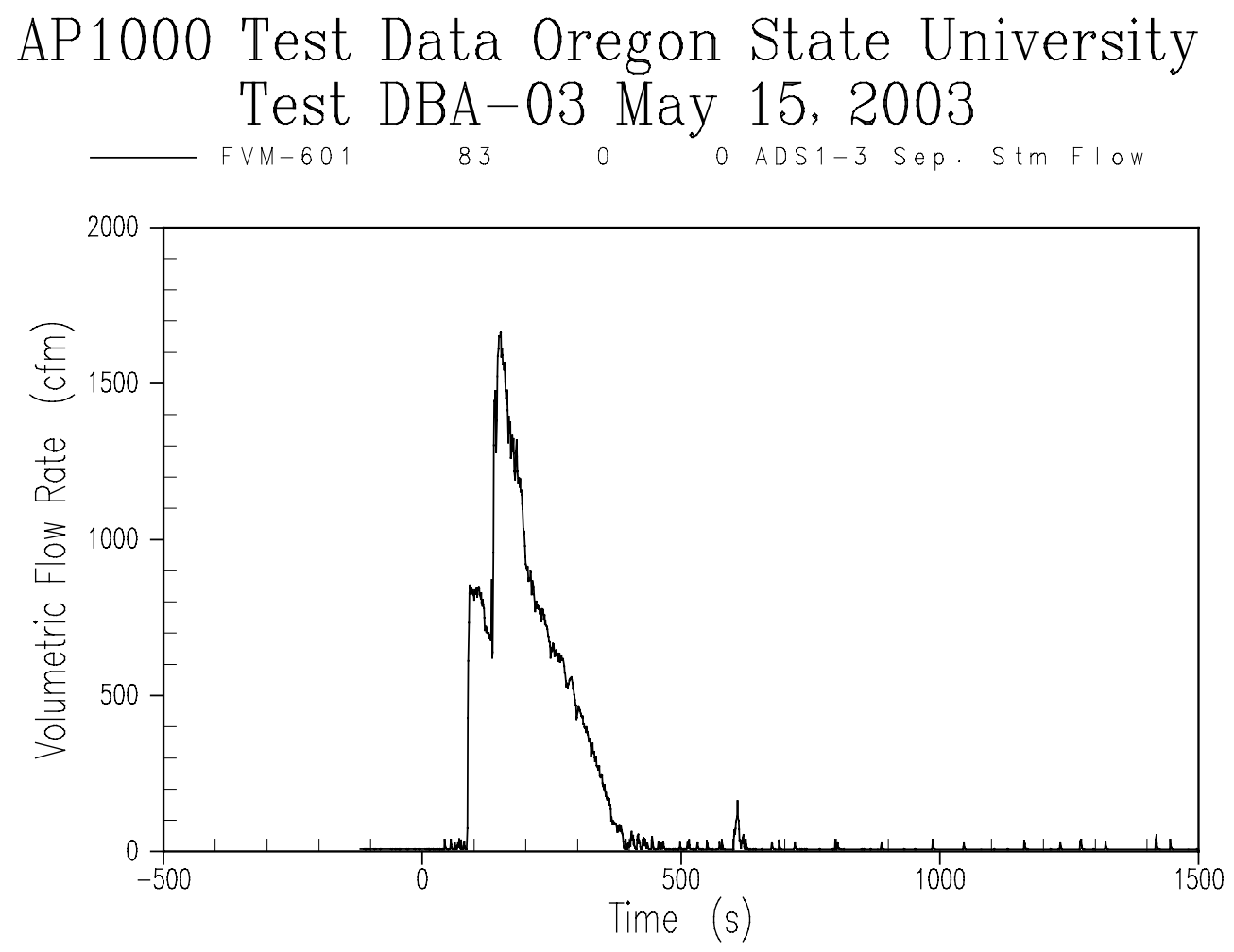

Figure A-44 ADS 1-3 Separator Steam Flow Rate 

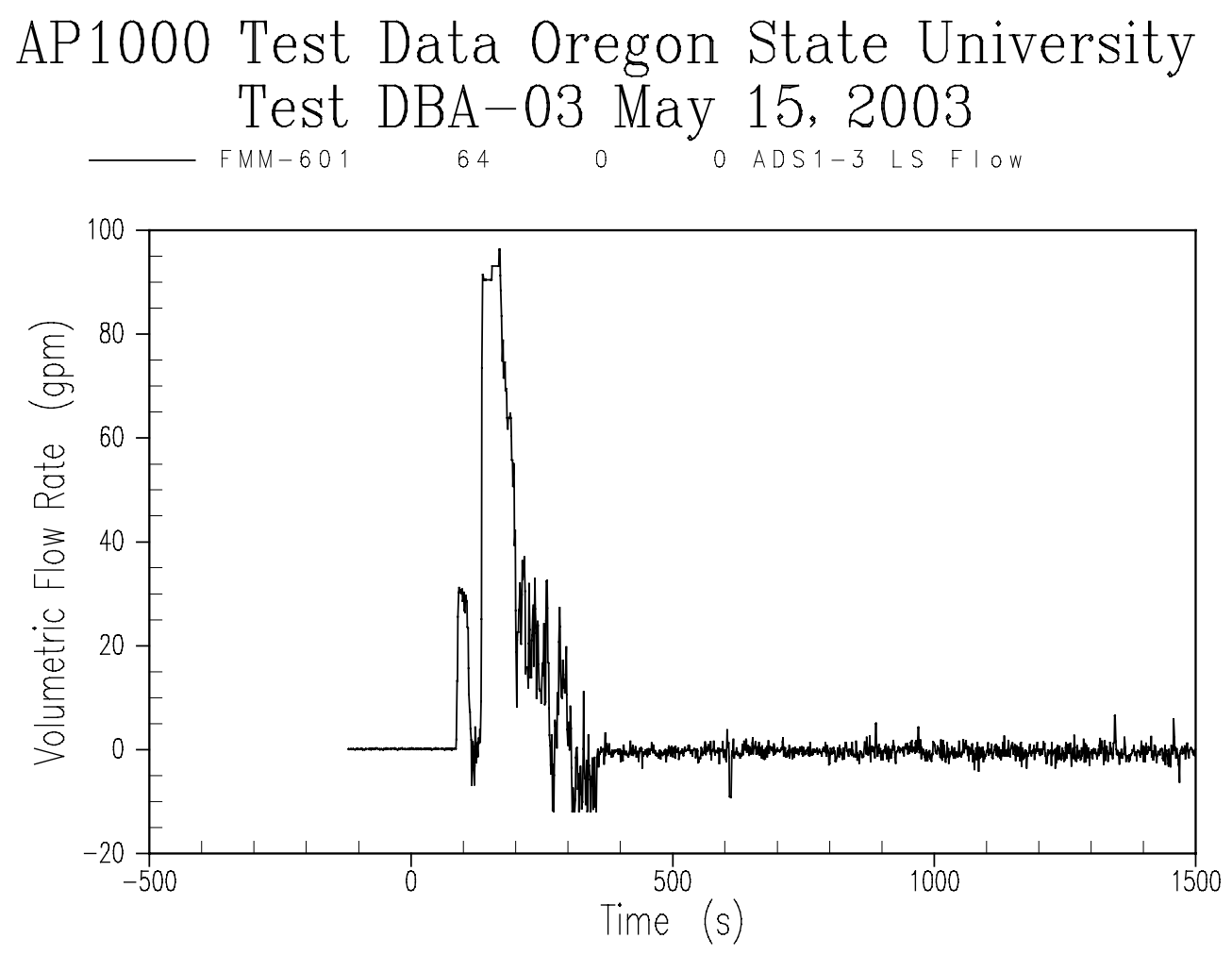

Figure A-45 ADS 1-3 Separator Liquid Flow Rate 

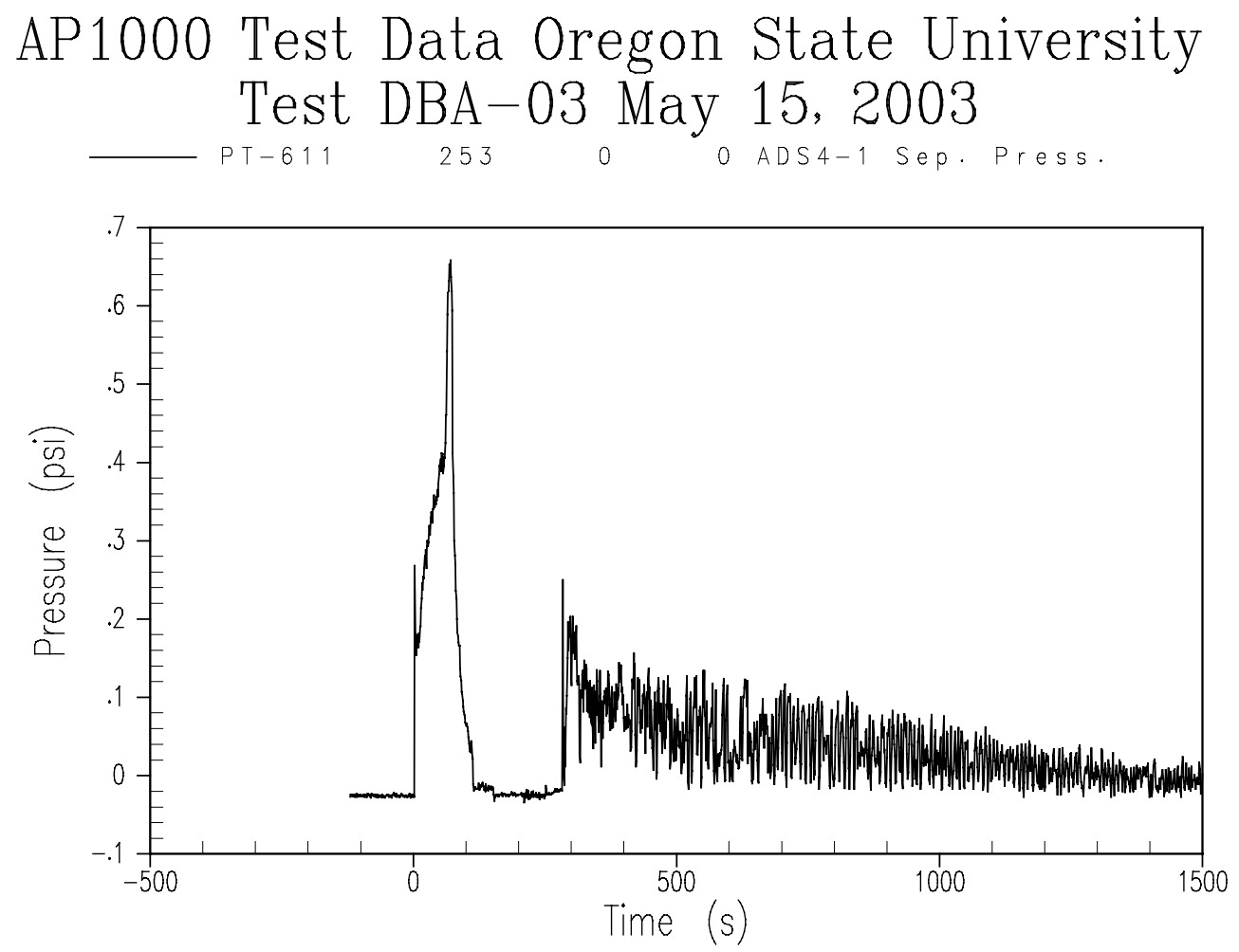

Figure A-46 ADS 4-1 Separator Pressure 

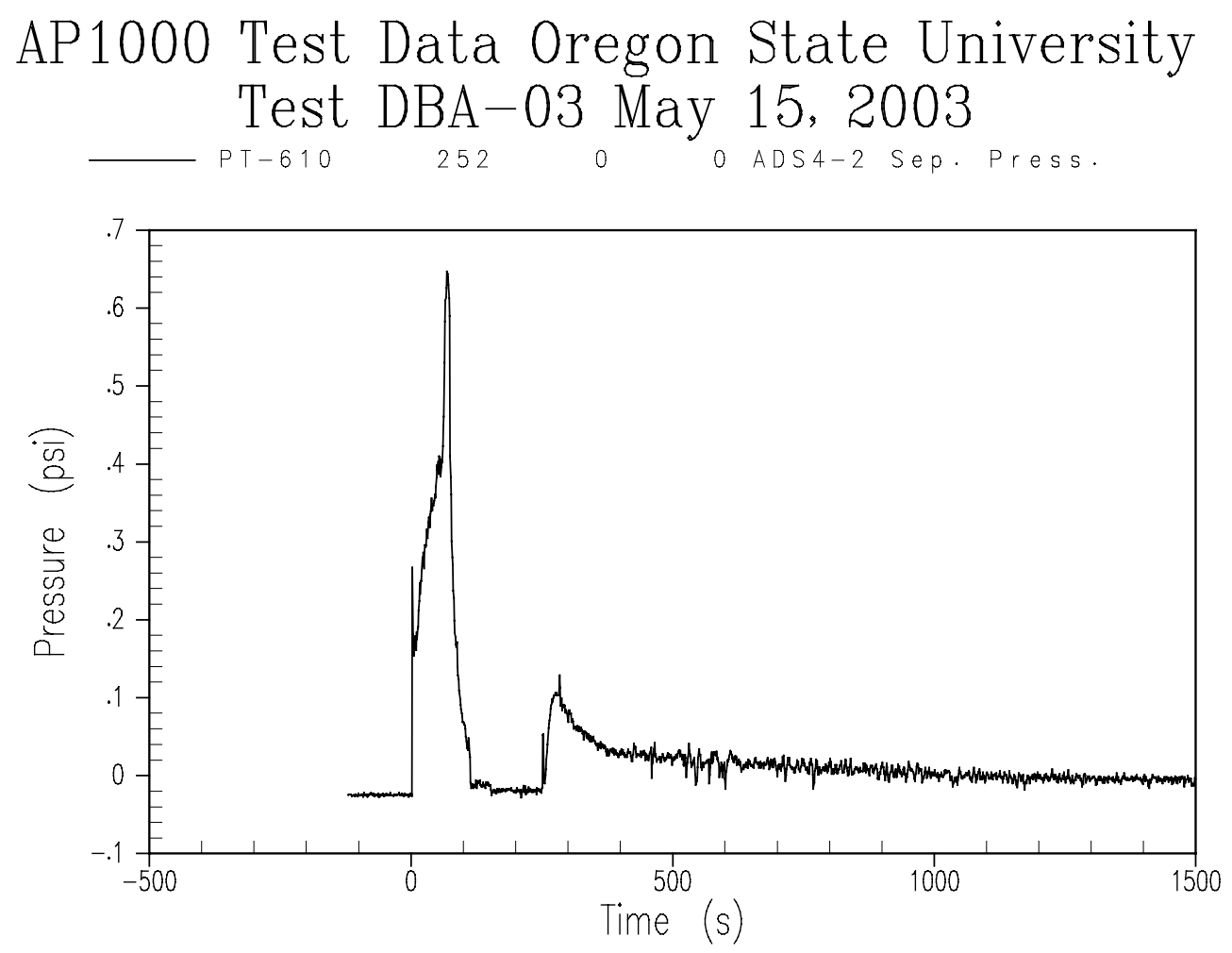

Figure A-47 ADS 4-2 Separator Pressure 

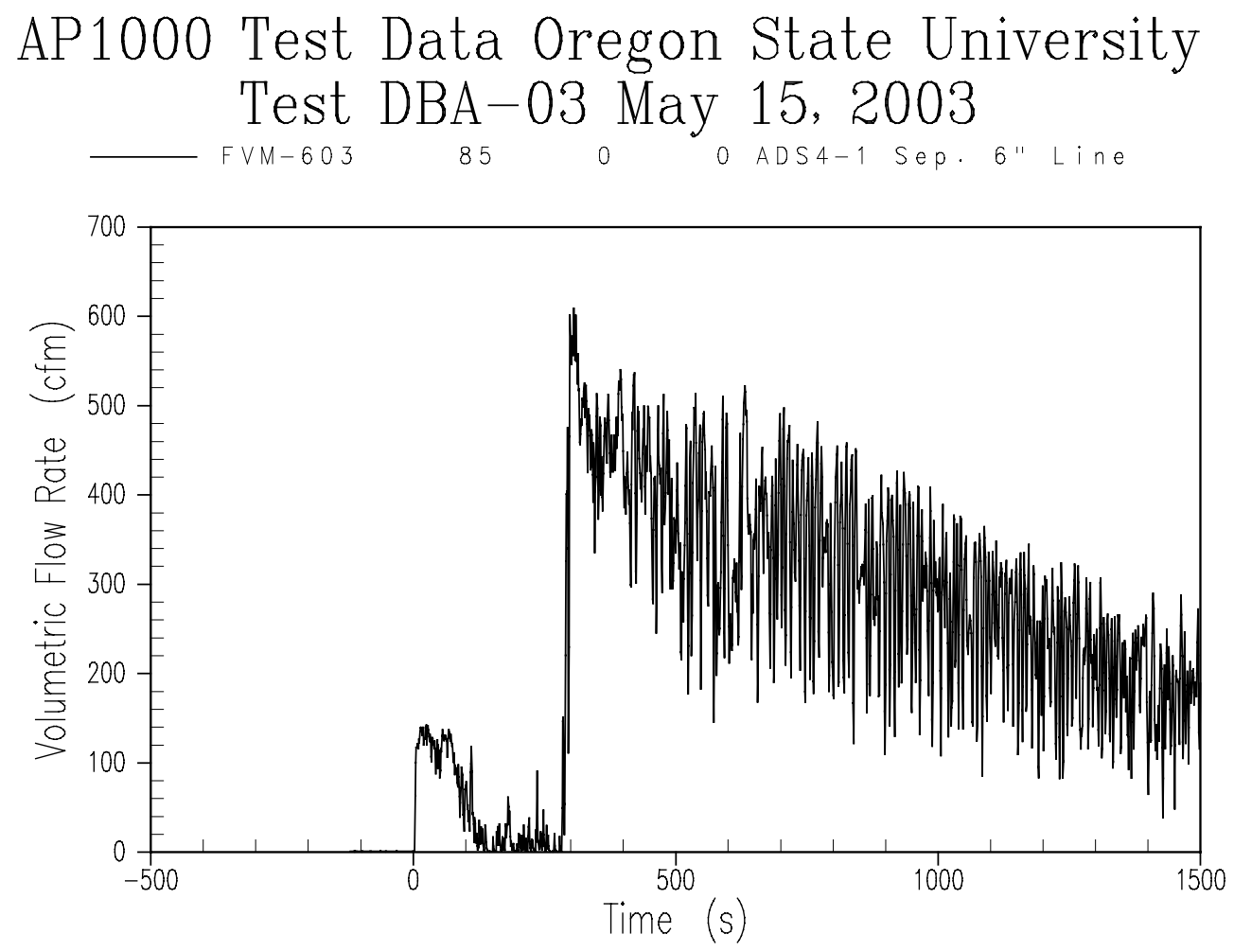

Figure A-48 ADS 4-1 Separator Steam Flow Rate 

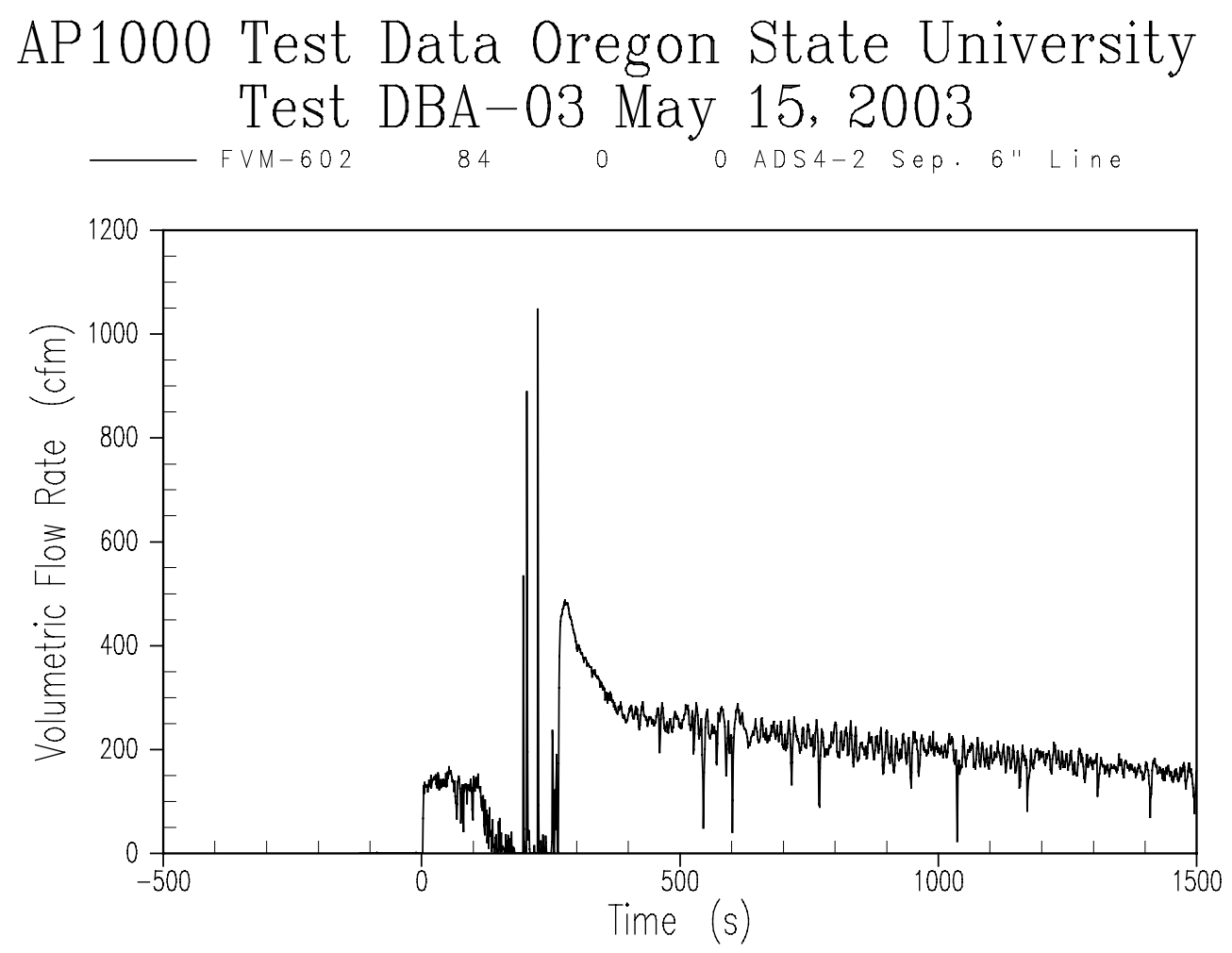

Figure A-49 ADS 4-2 Separator Steam Flow Rate 

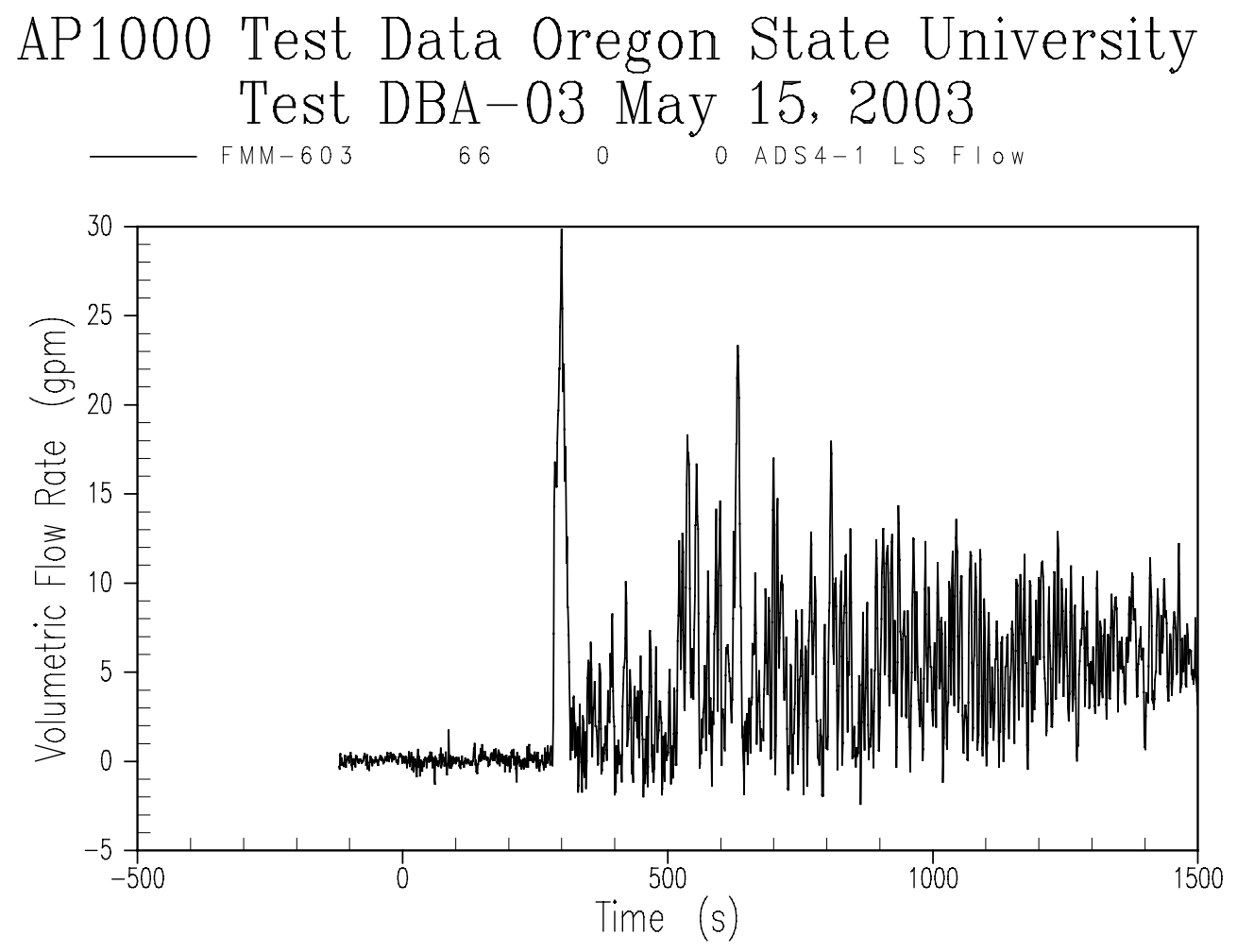

Figure A-50 ADS 4-1 Separator Liquid Flow Rate 

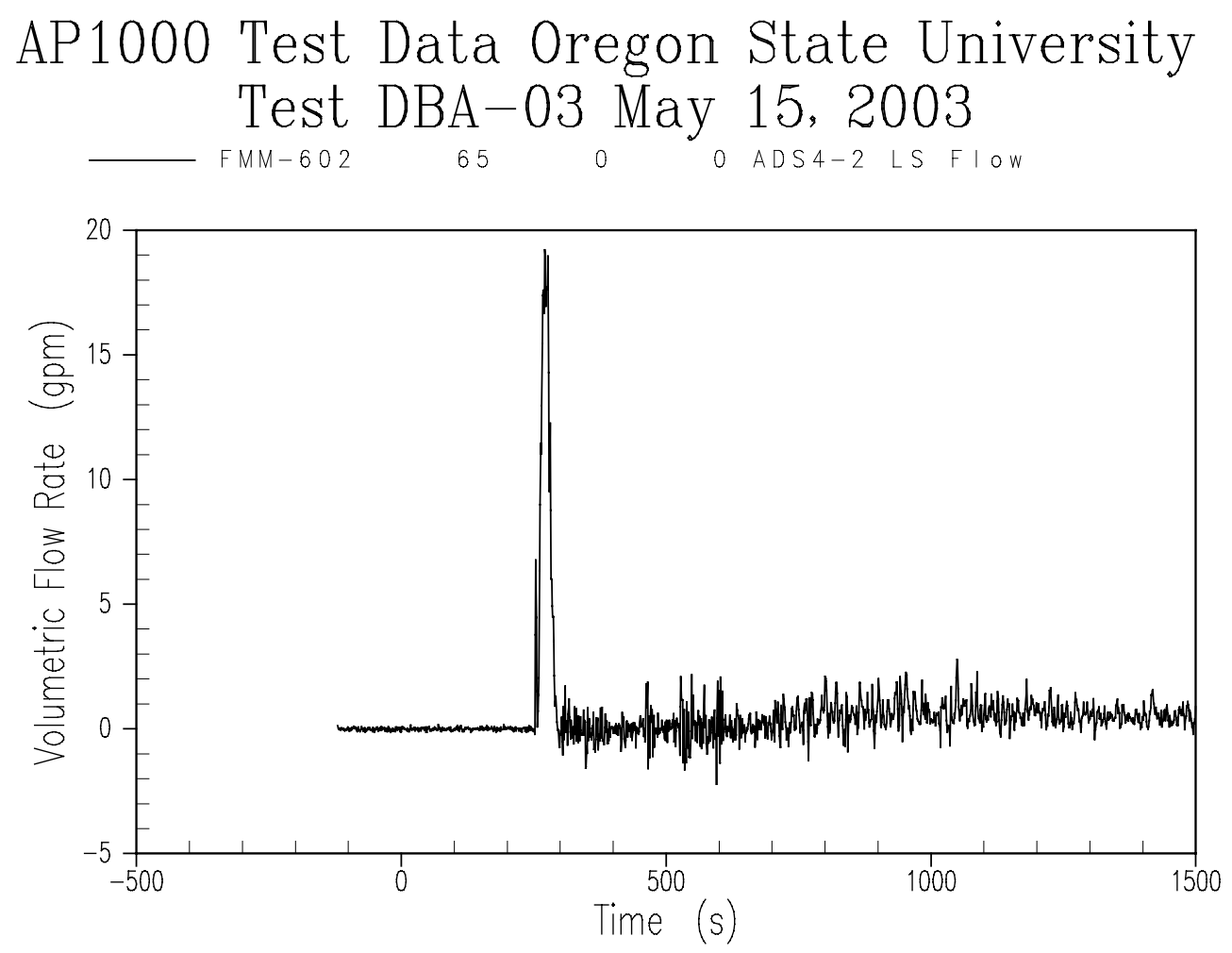

Figure A-51 ADS 4-2 Separator Liquid Flow Rate 

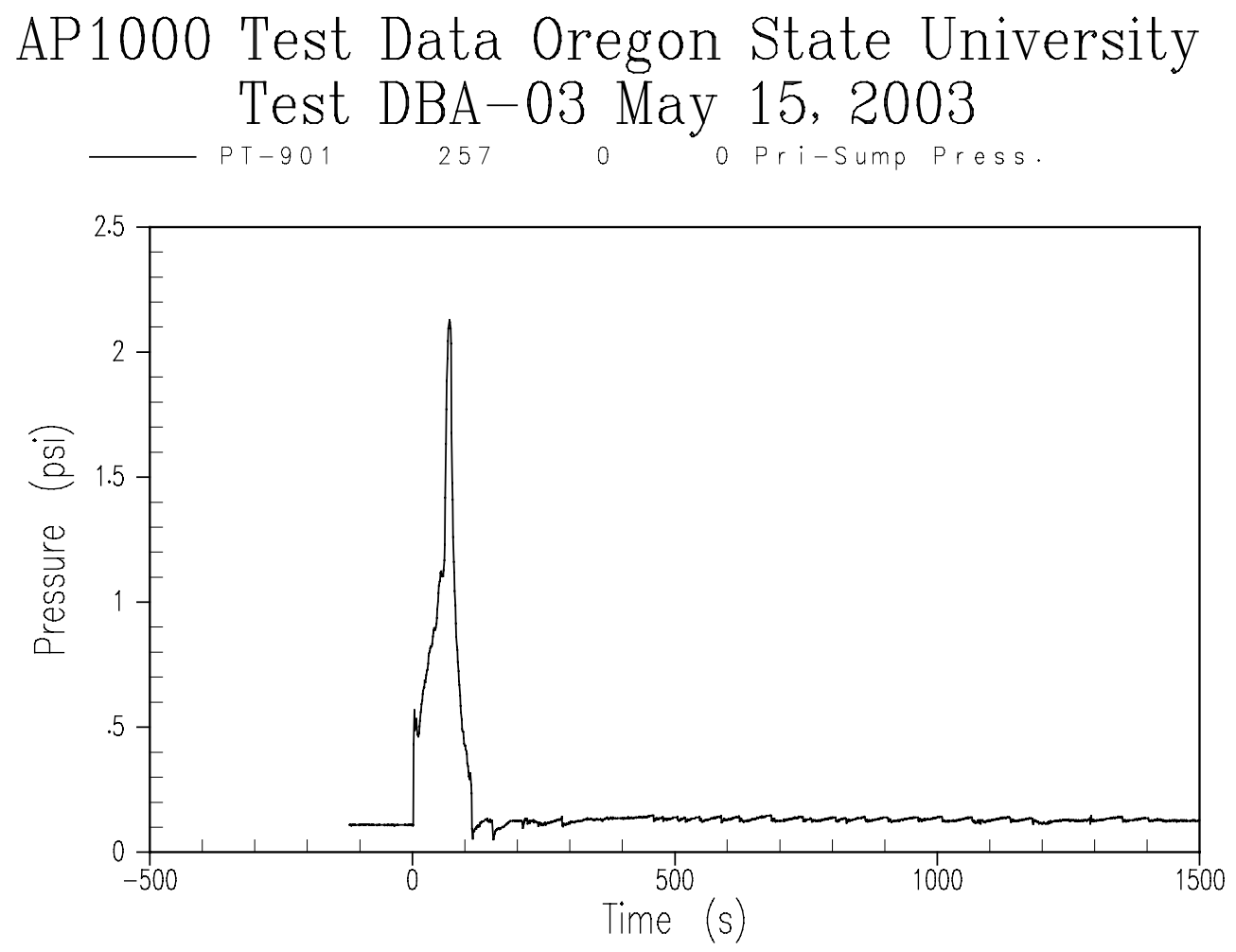

Figure A-52 Primary Sump Pressure 


\section{AP1000 Test Data Oregon State University Test DBA-03 May 15, 2003 \\ $\begin{array}{llll}L D P-901 & 214 & 0 & \text { o Pri-Sump UC Level }\end{array}$}

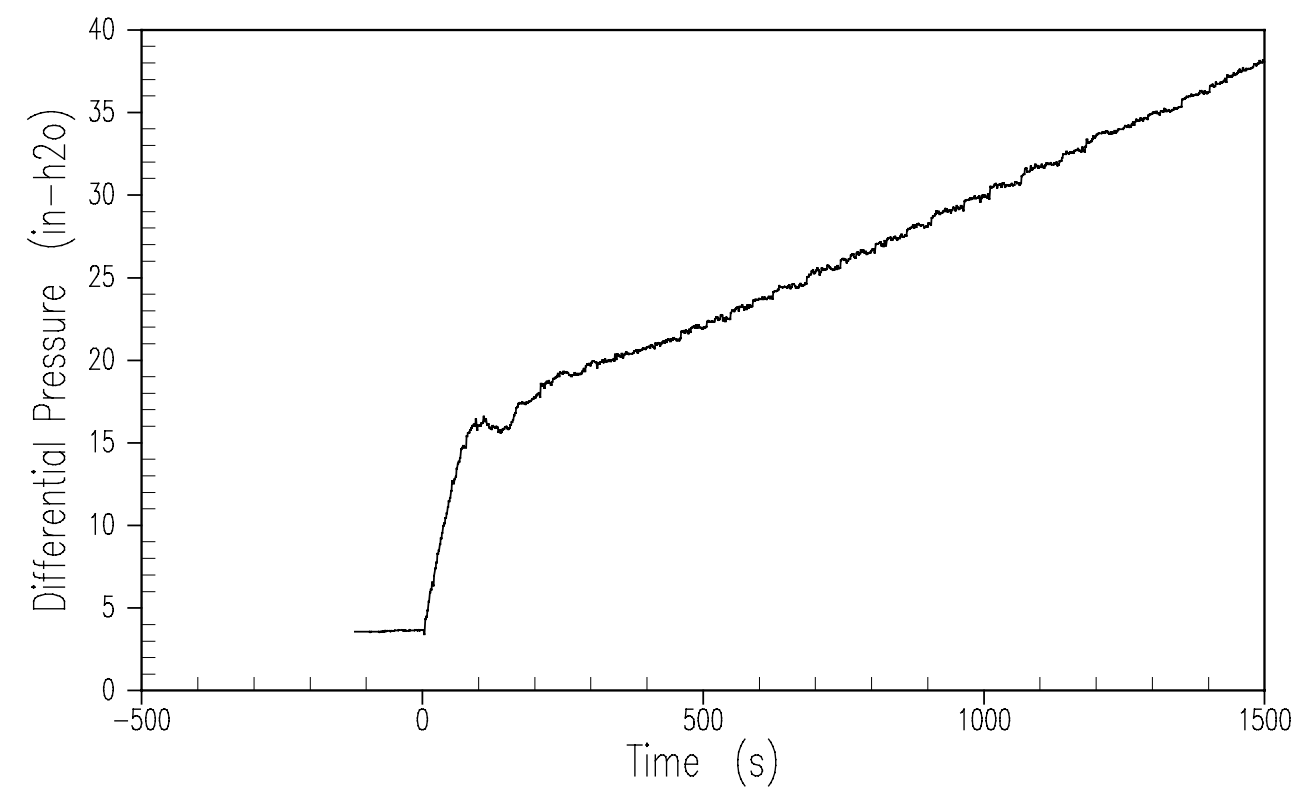

Figure A-53 Primary Sump Liquid Level 

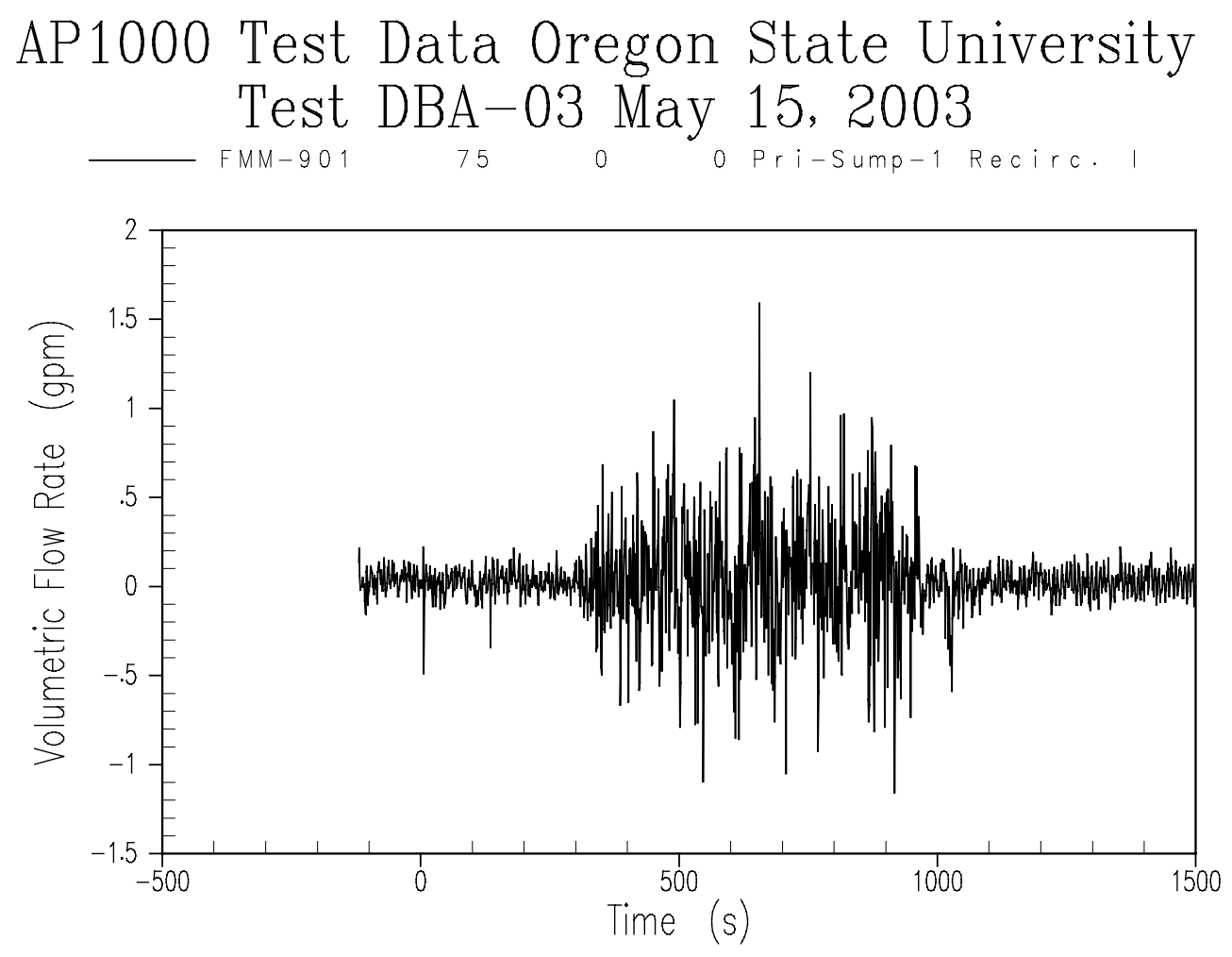

Figure A-54 Primary Sump Injection Flow Rate 


\section{AP1000 Test Data Oregon State University Test DBA-03 May 15, 2003 \\ $\begin{array}{lllll}\text { FMM-902 } & 76 & 0 & \text { o Pri-sump-2 Recirc. I }\end{array}$}

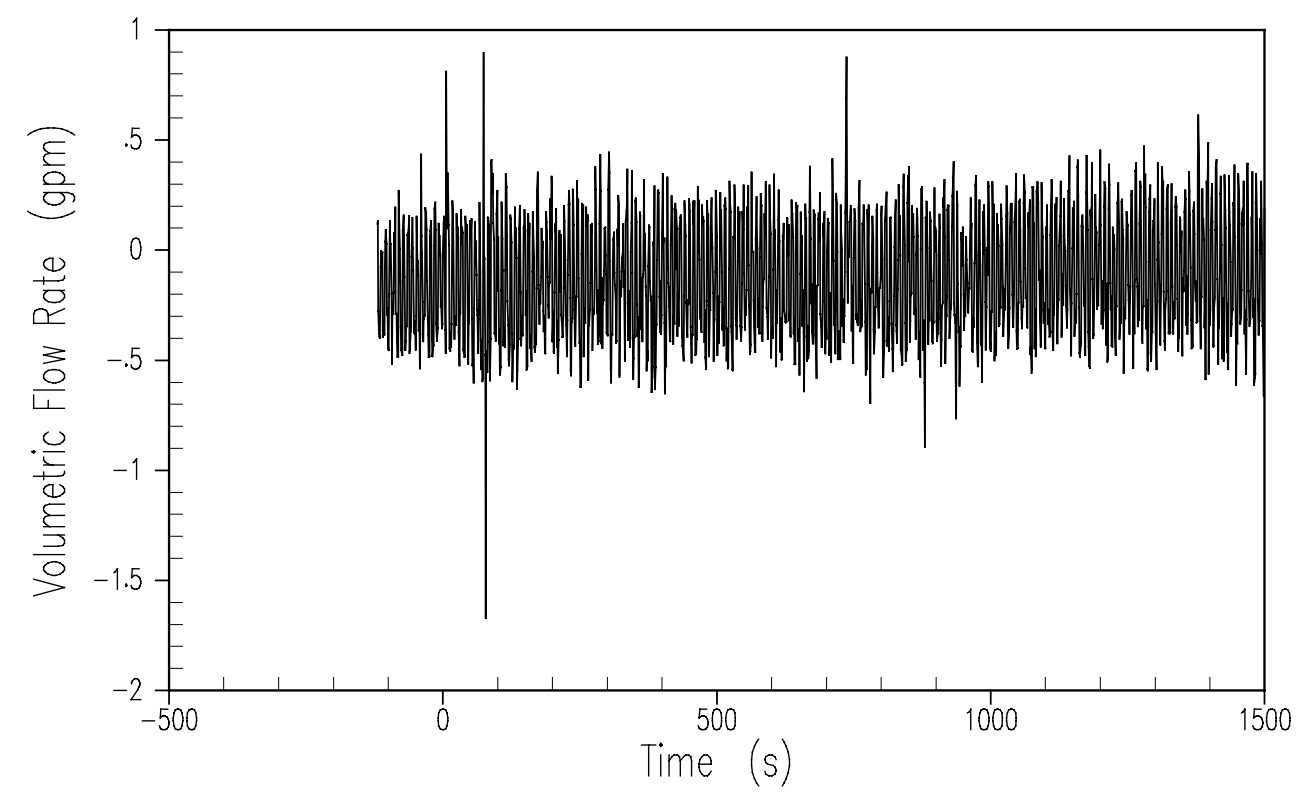

Figure A-55 Primary Sump Injection Flow Rate 

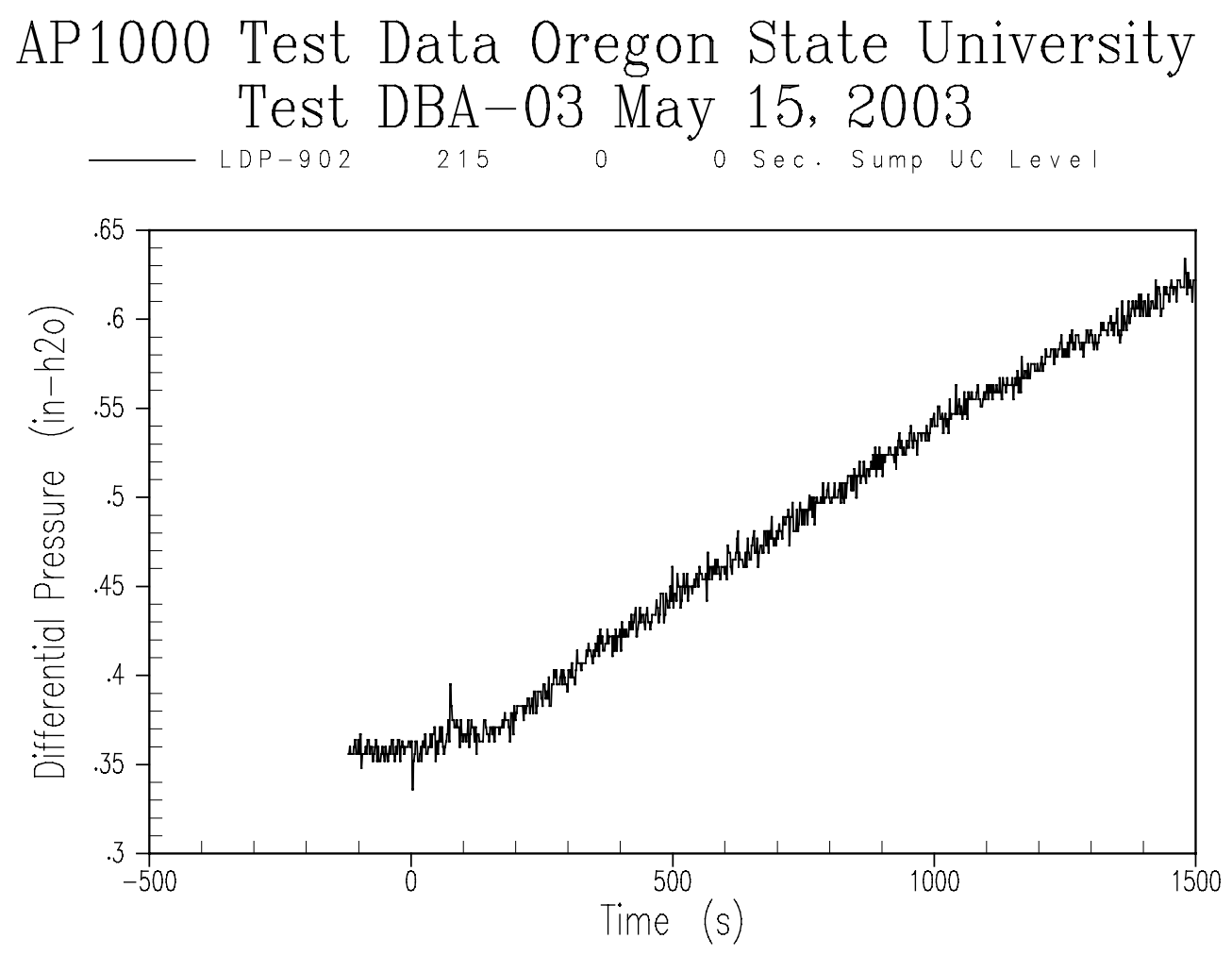

Figure A-56 Secondary Sump Liquid Level 

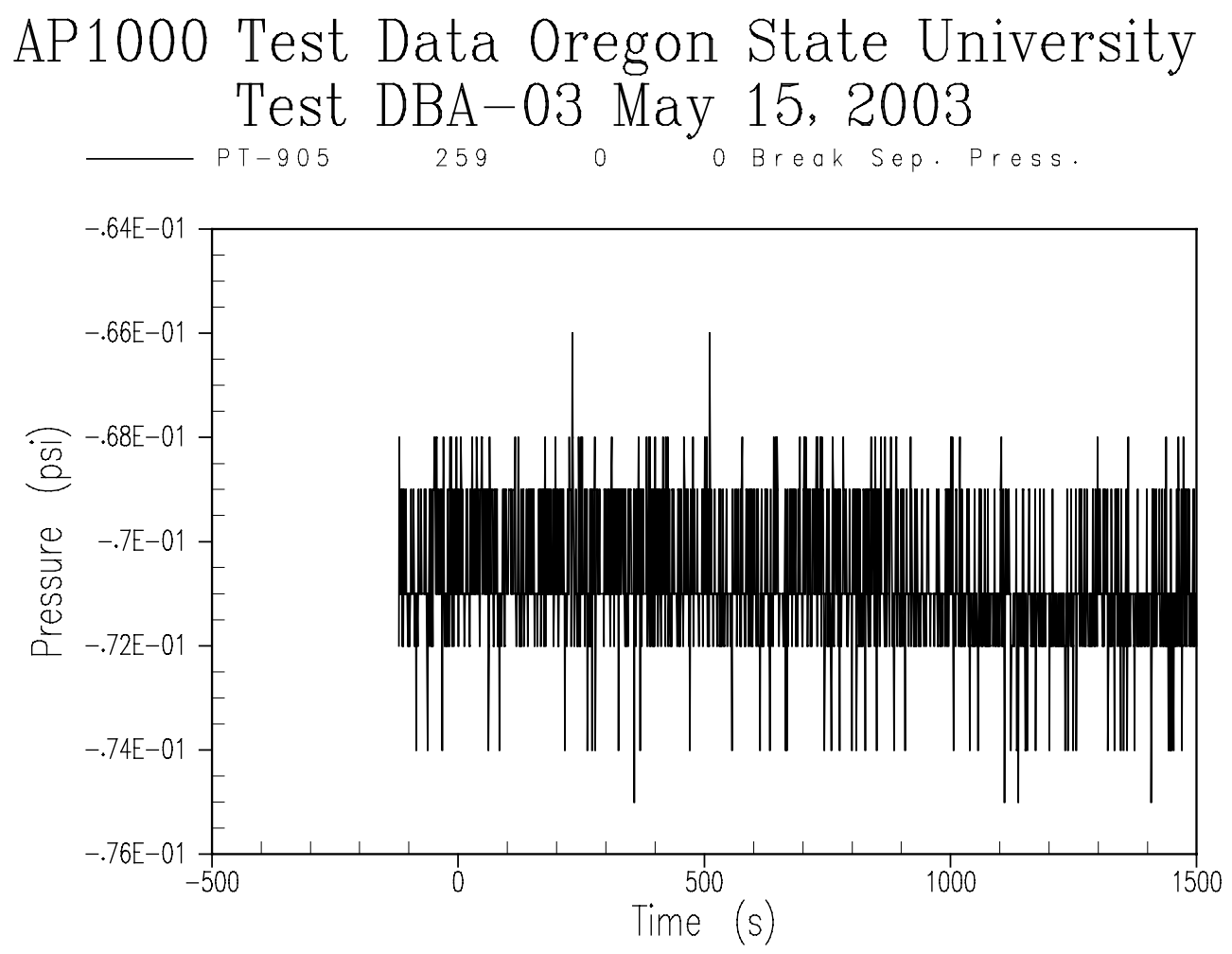

Figure A-57 Break Separator Pressure 

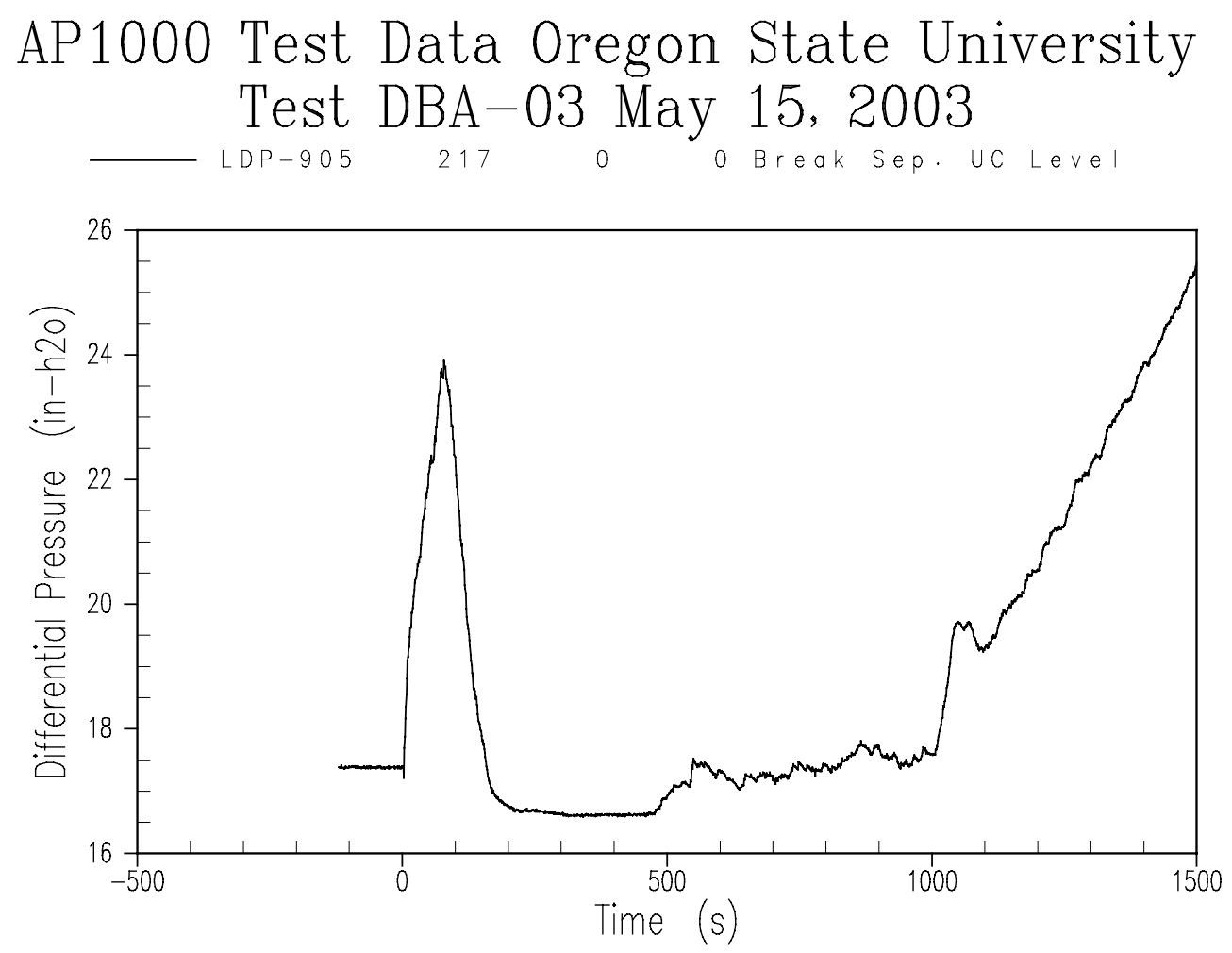

Figure A-58 Break Separator Liquid Level 

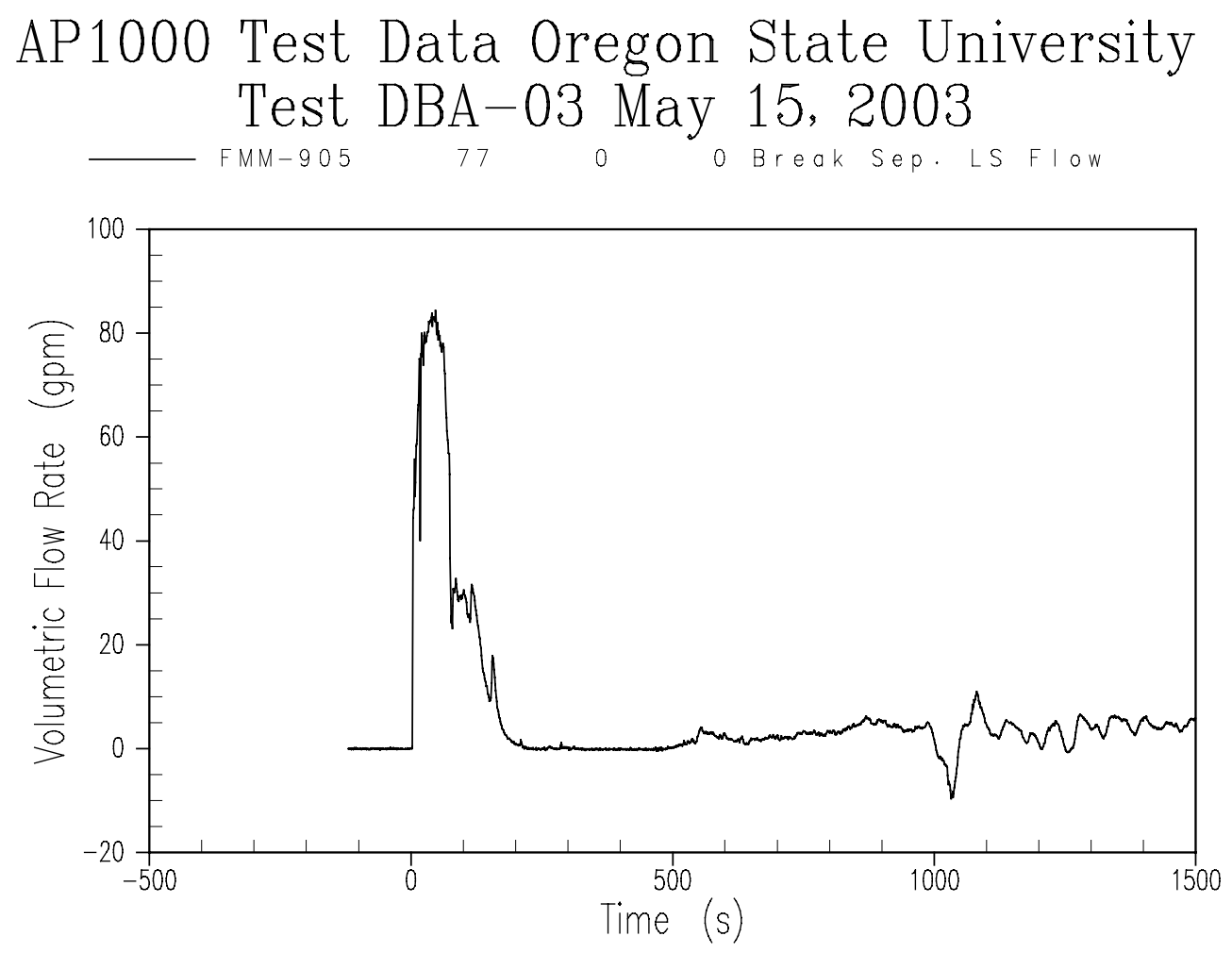

Figure A-59 Break Separator Flow to Primary Sump 

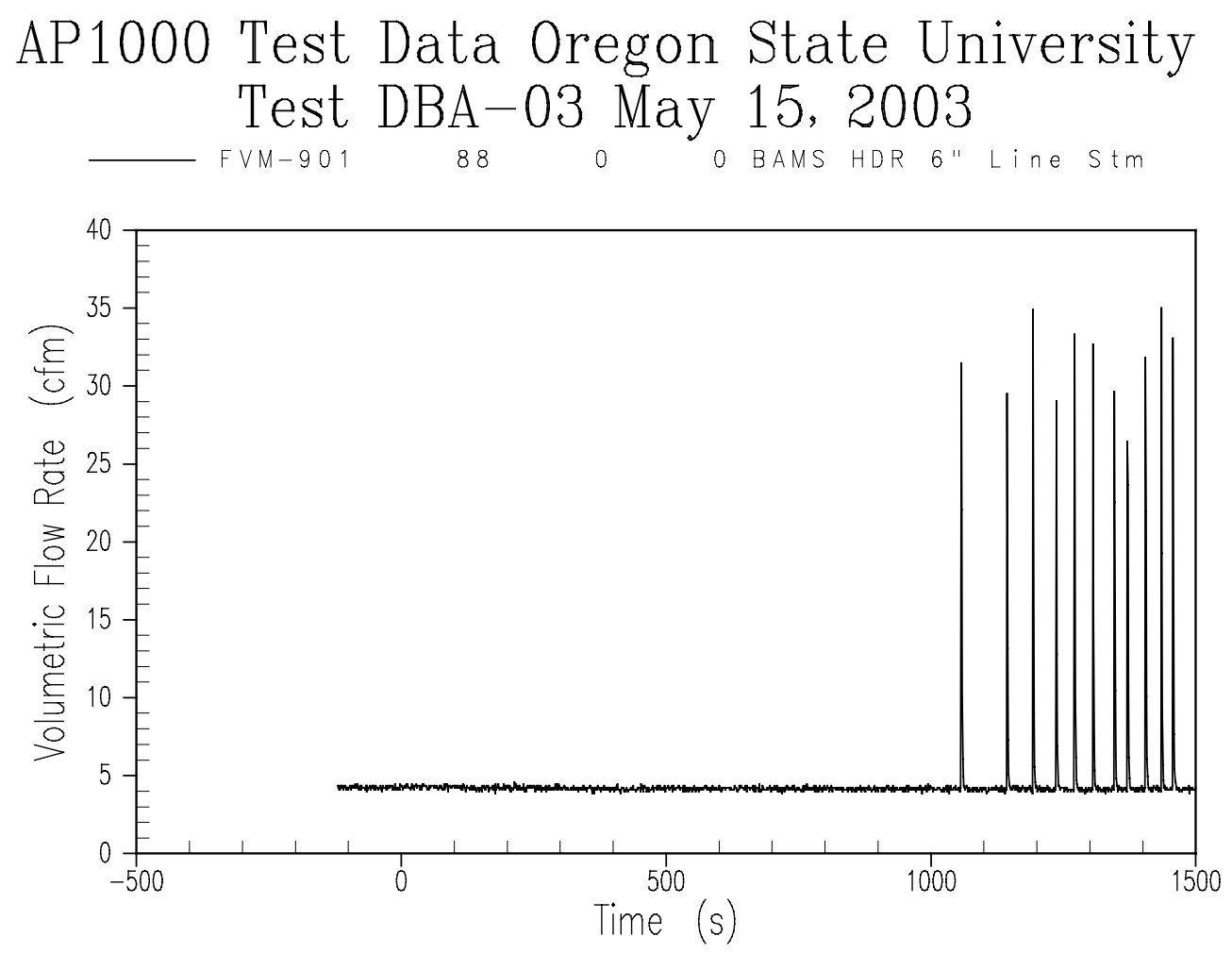

Figure A-60 BAMS Steam Flow Rate 

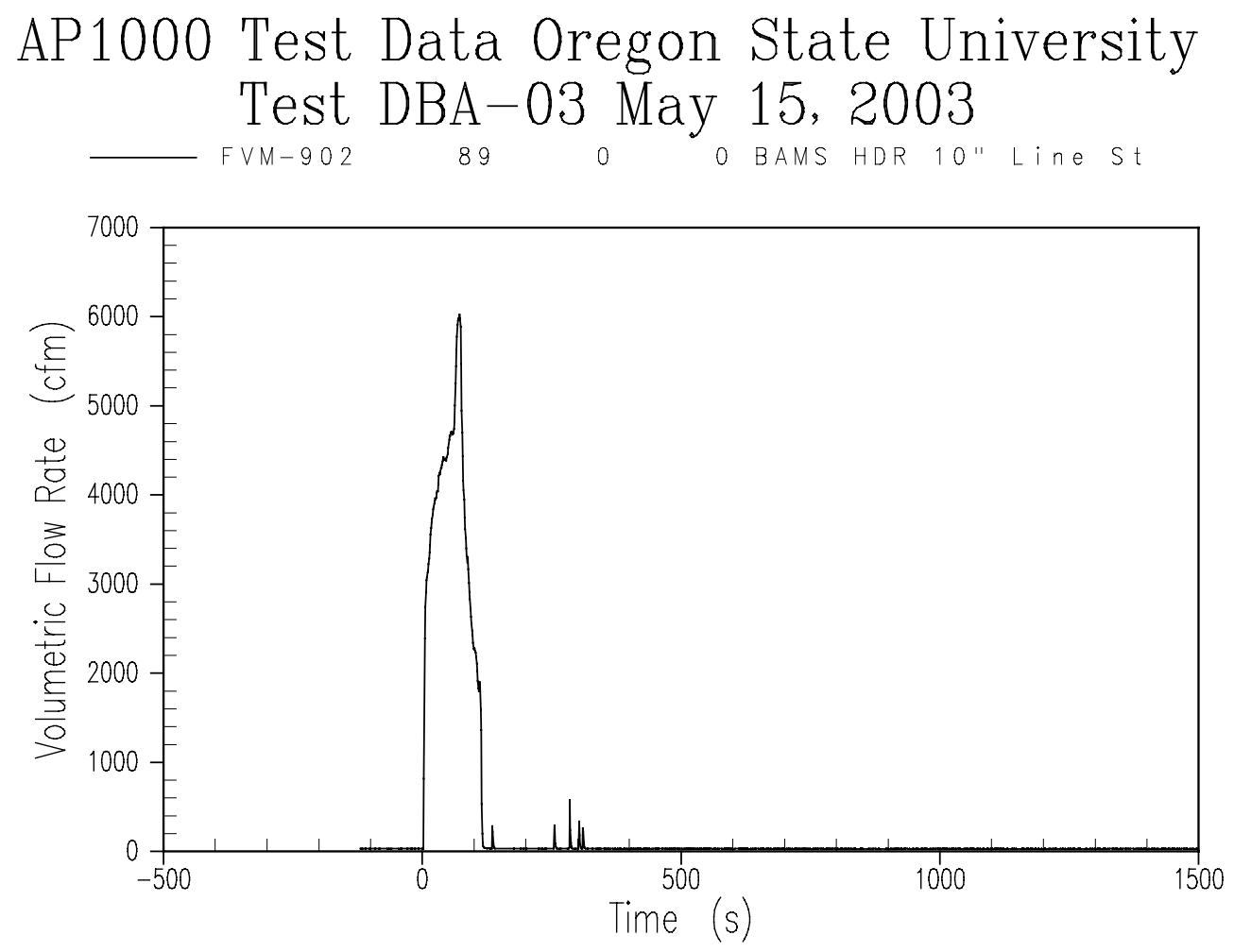

Figure A-61 BAMS Steam Flow Rate 

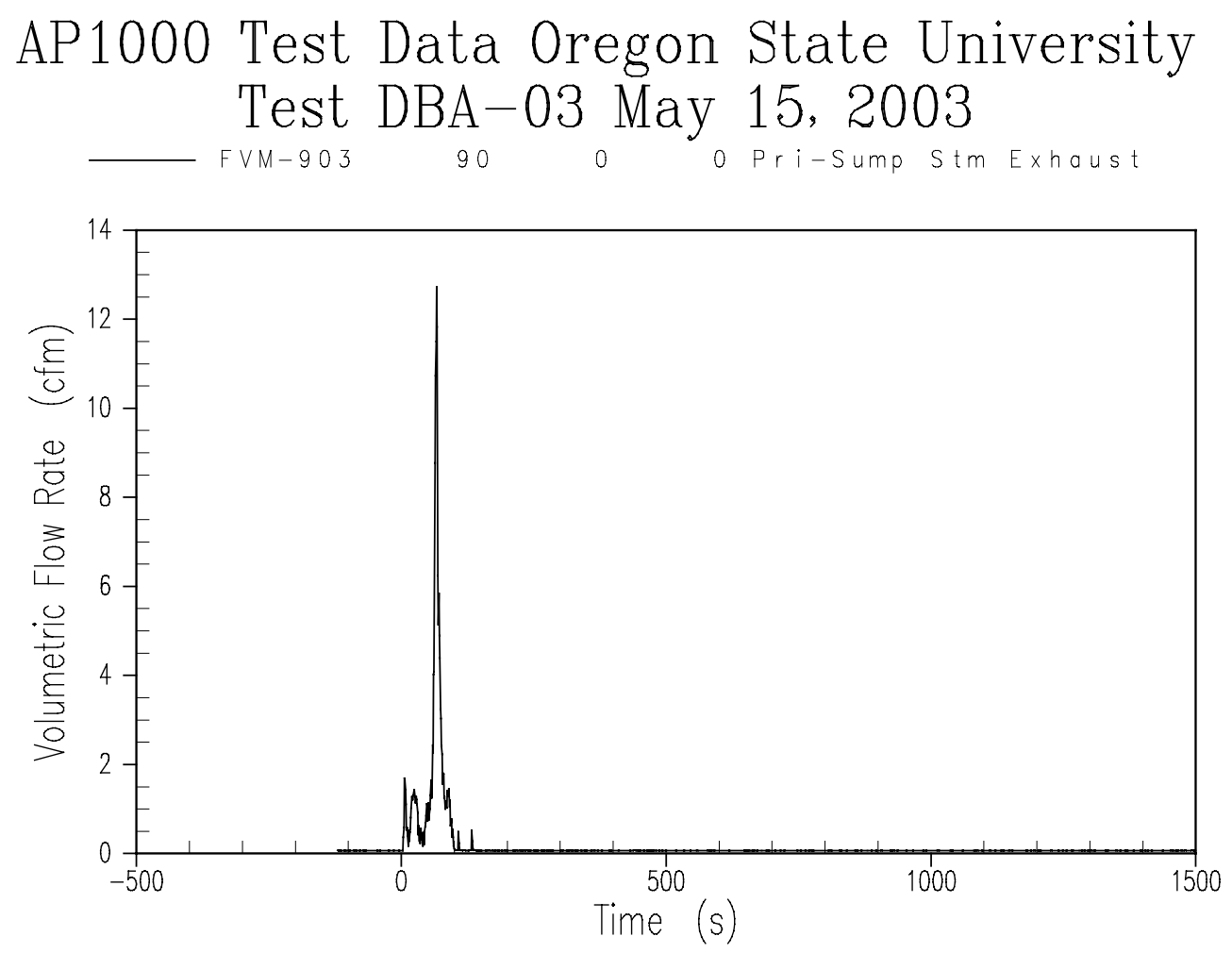

Figure A-62 BAMS/Primary Sump Steam Flow Rate 

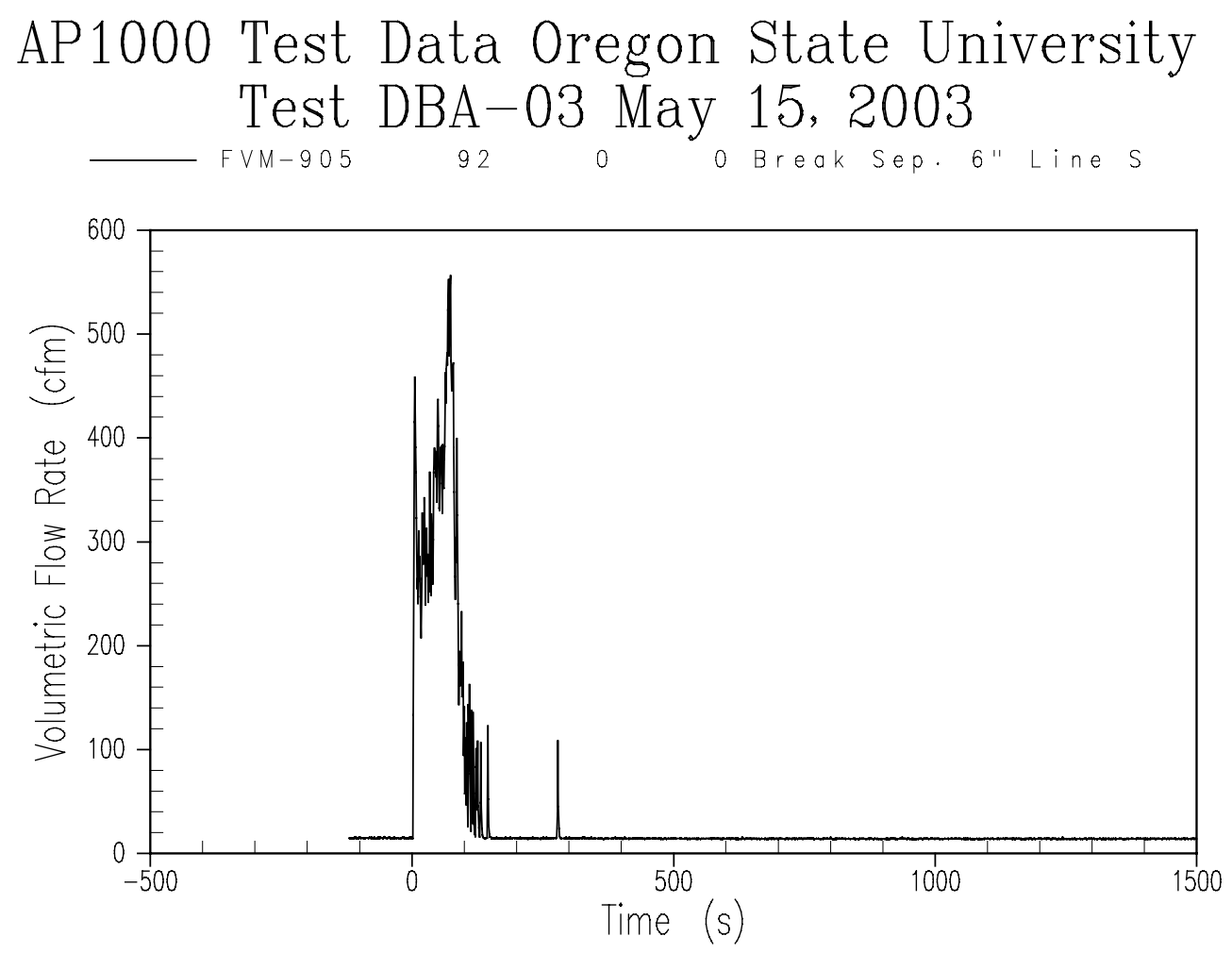

Figure A-63 BAMS/Separator Steam Flow Rate - 6-inch Pipe 

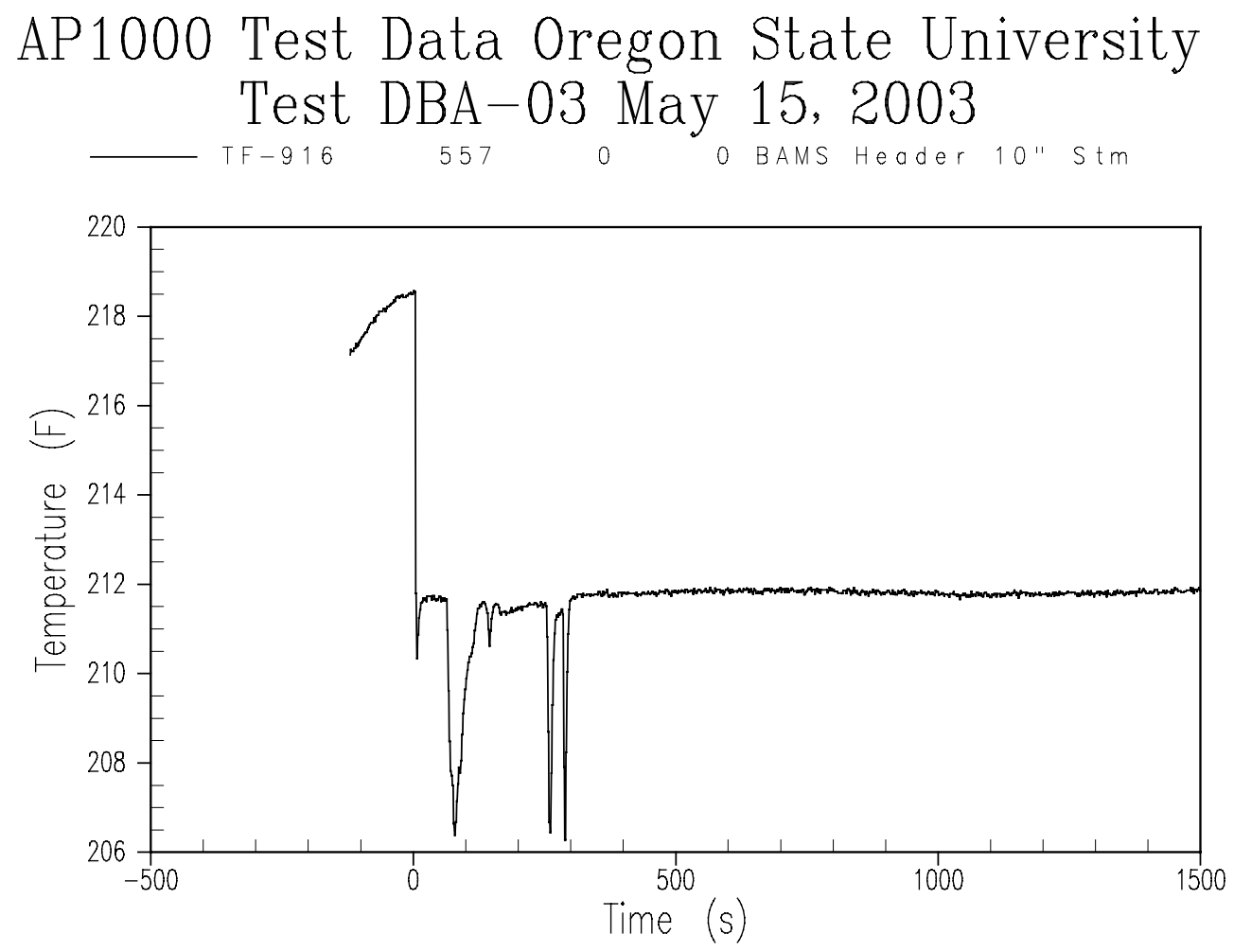

Figure A-64 BAMS/Exhaust Line Temperature 

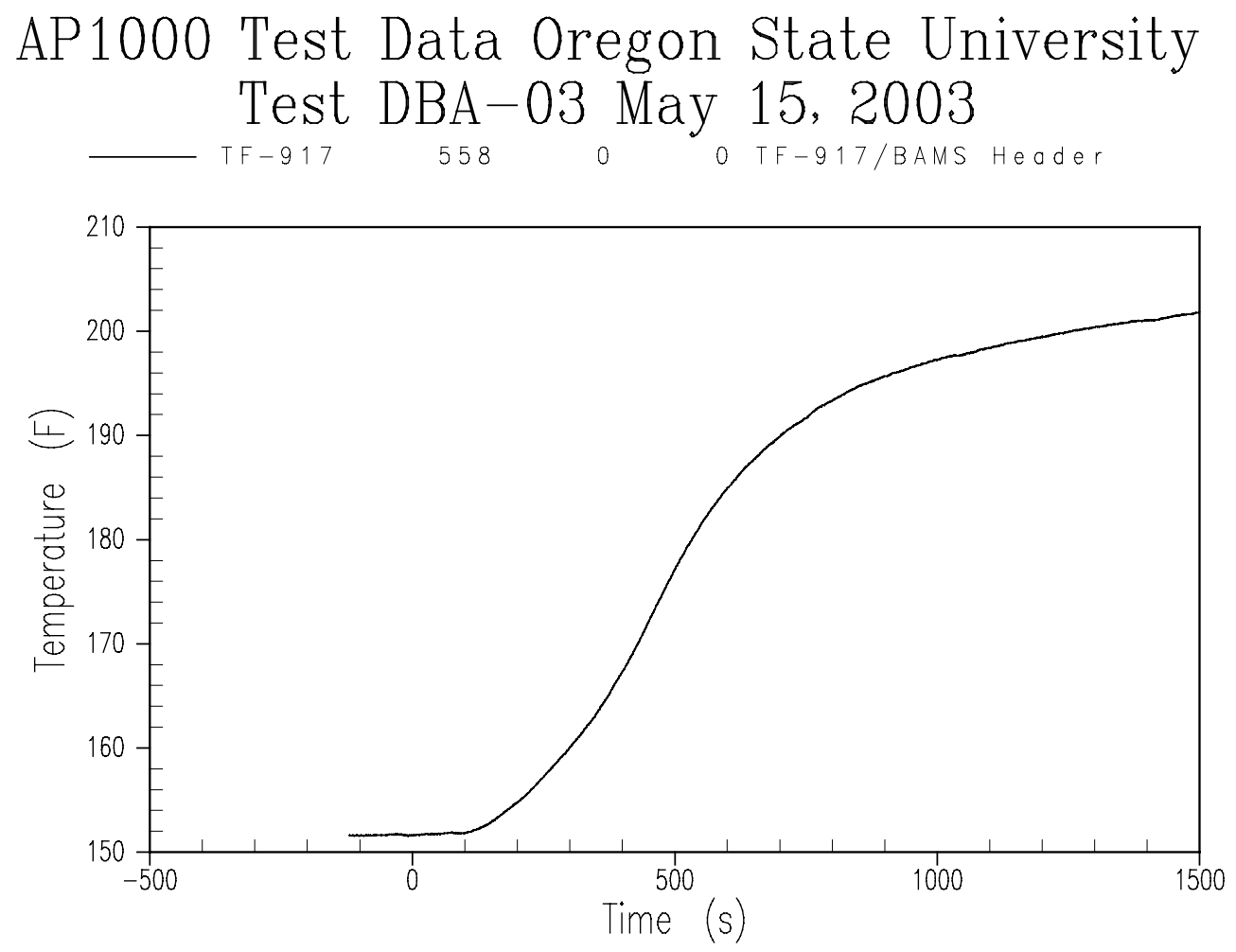

Figure A-65 BAMS/Exhaust Line Temperature 

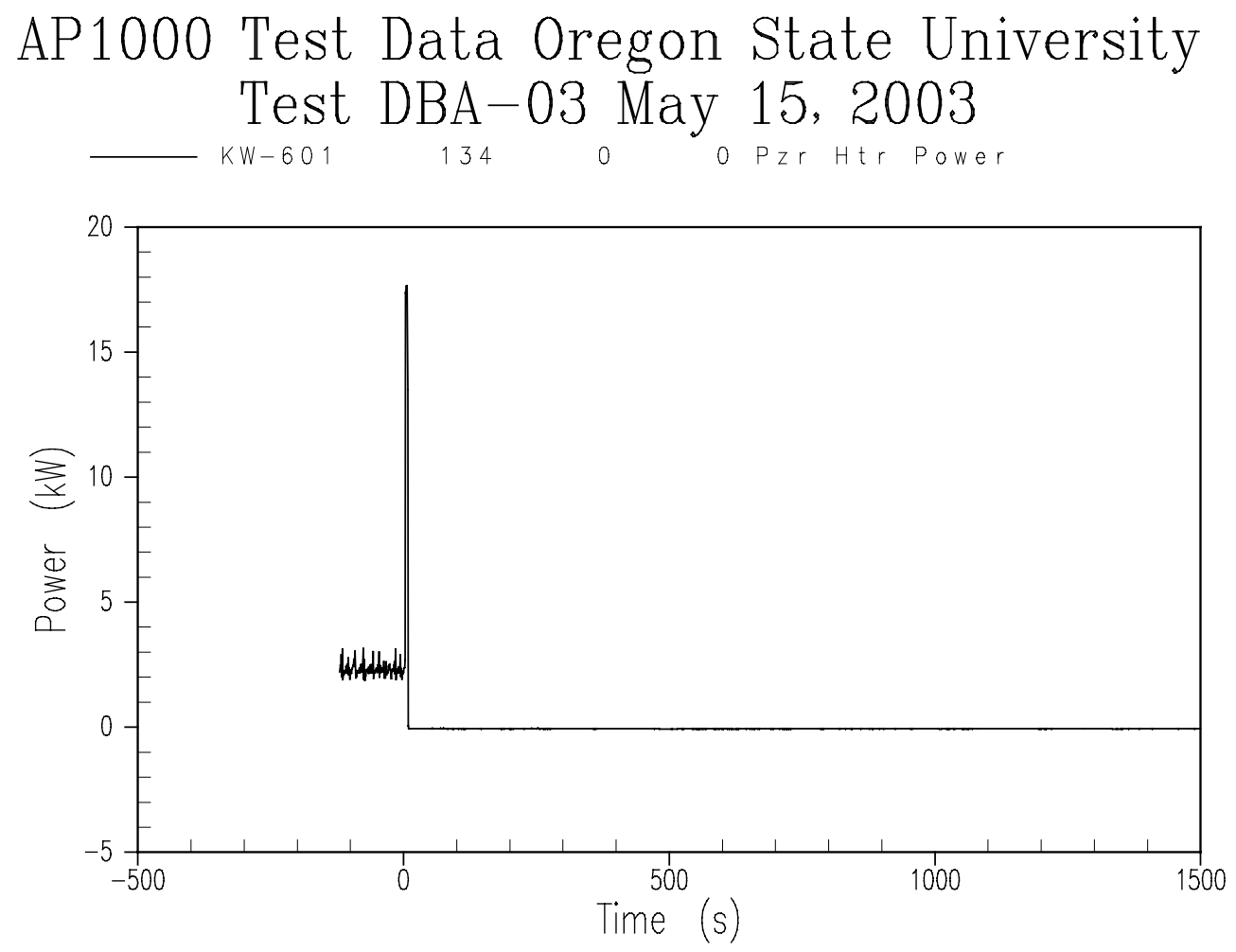

Figure A-66 Pressurizer Heater Input Power 

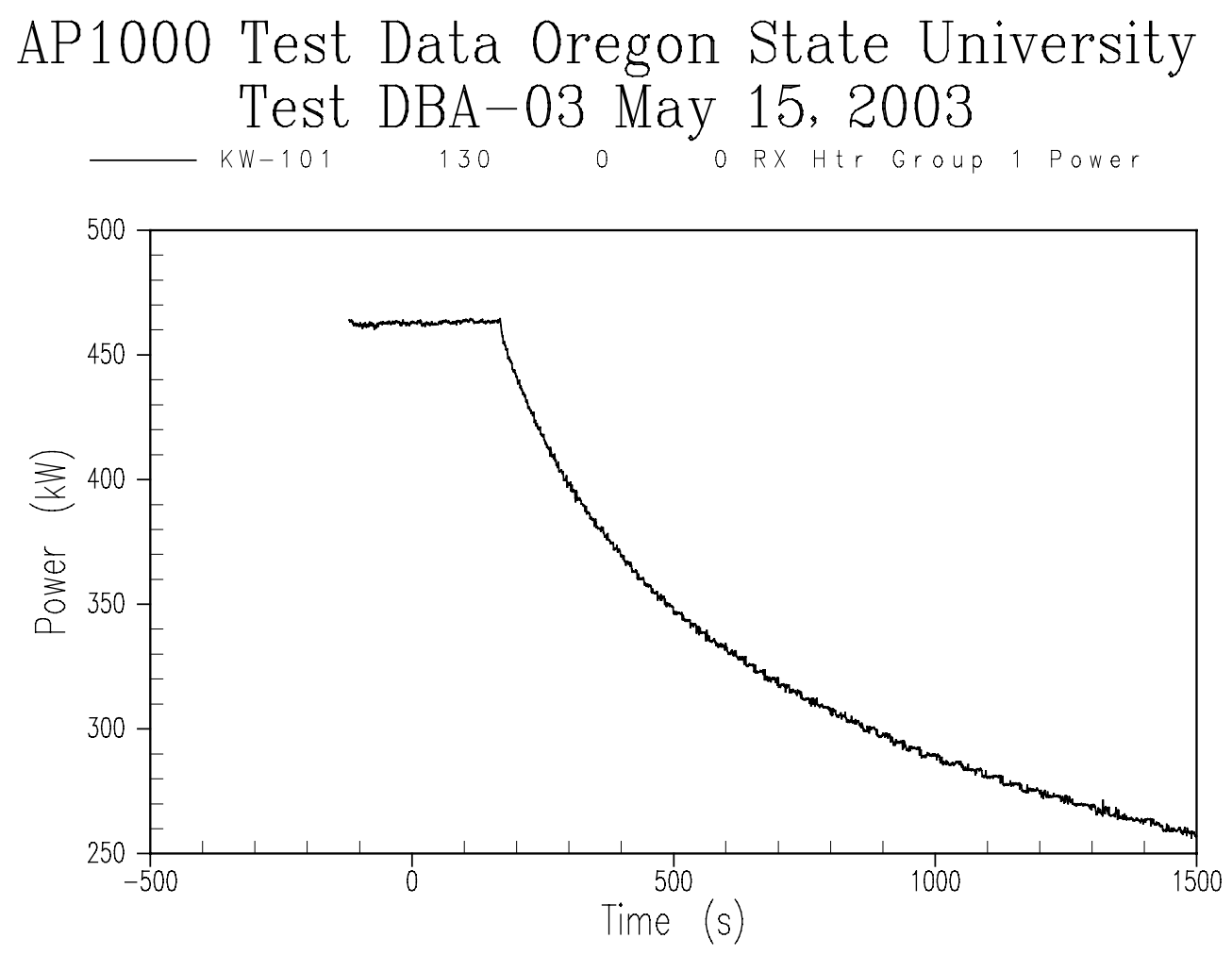

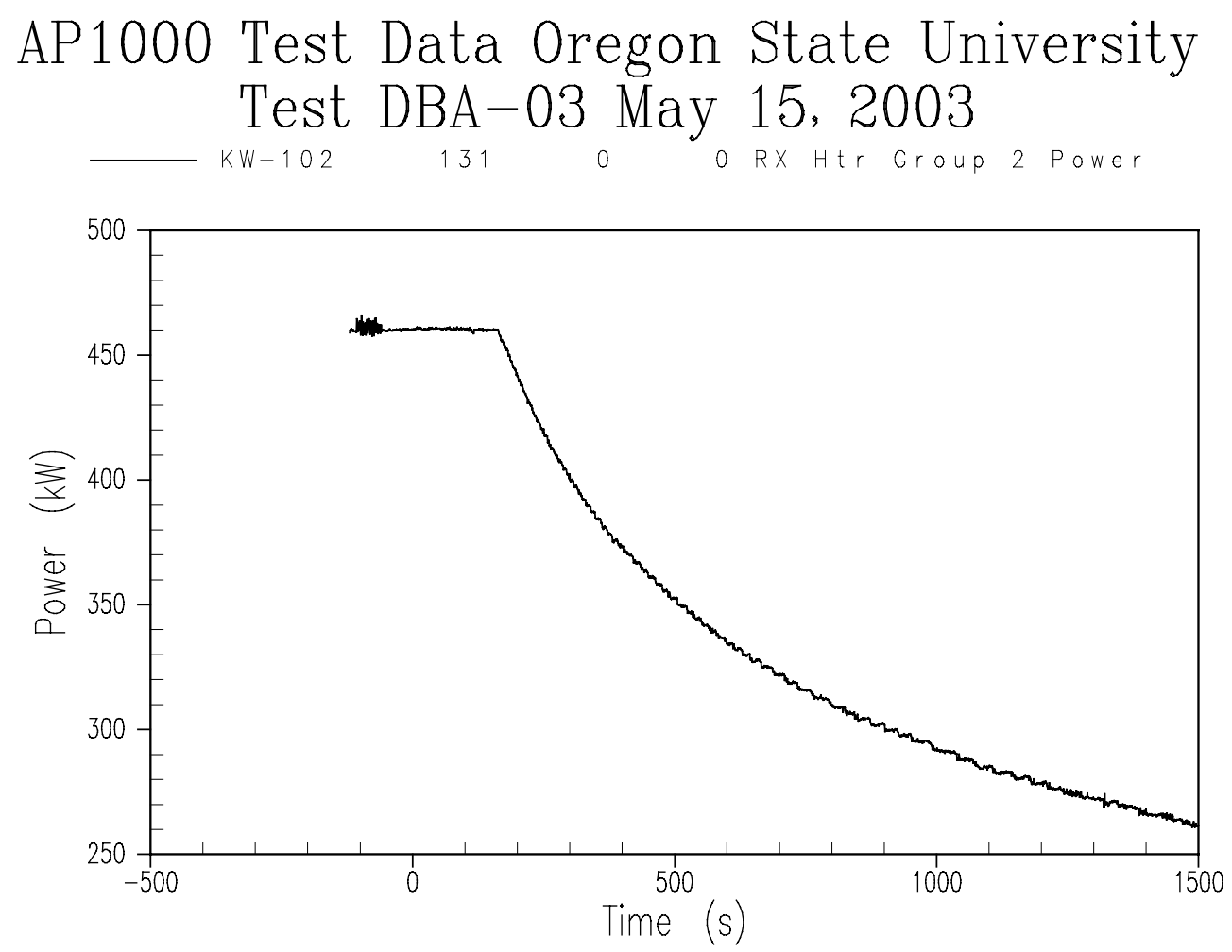

Figure A-68 Core Power Input Power 

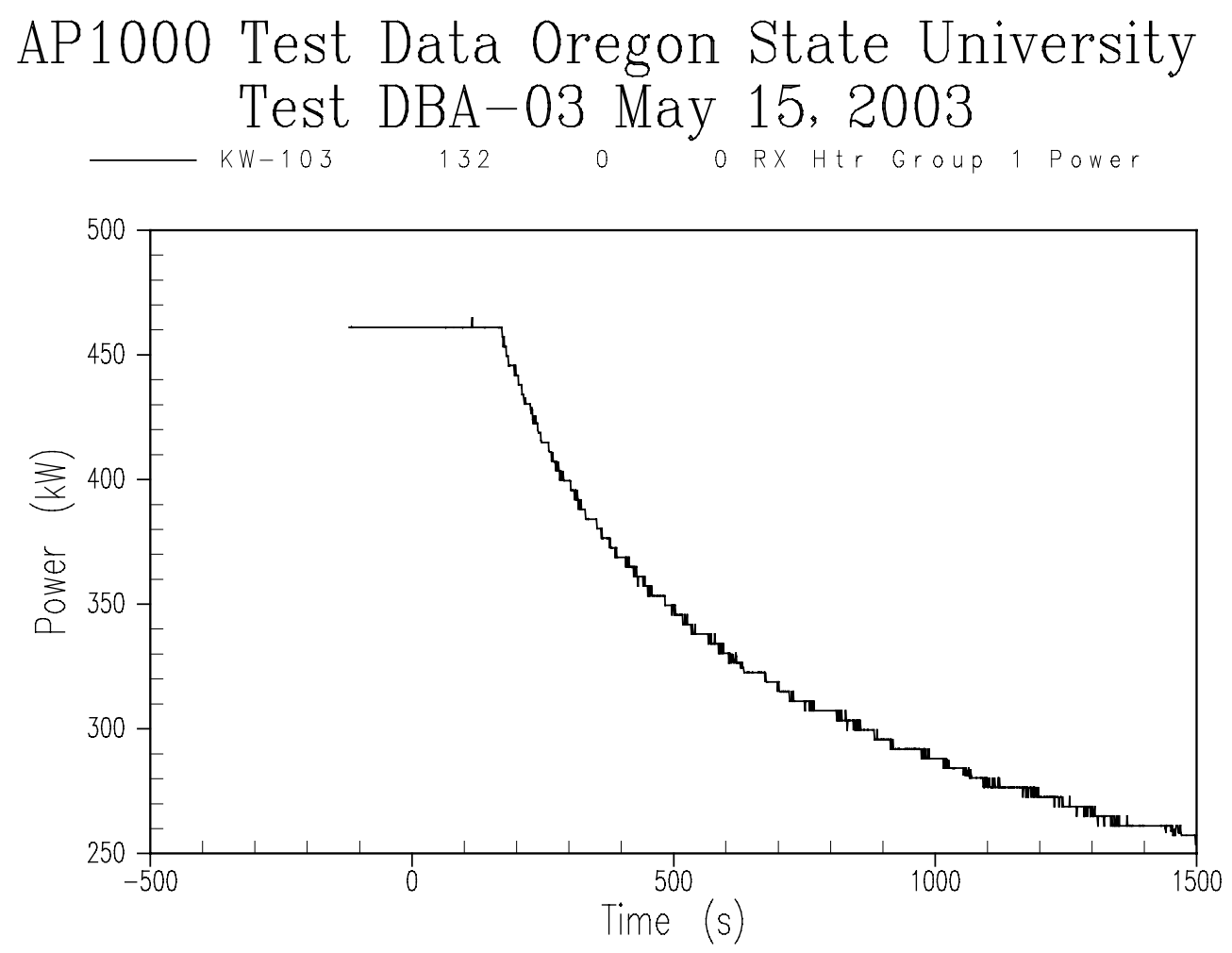

Figure A-69 Core Power Input Power 

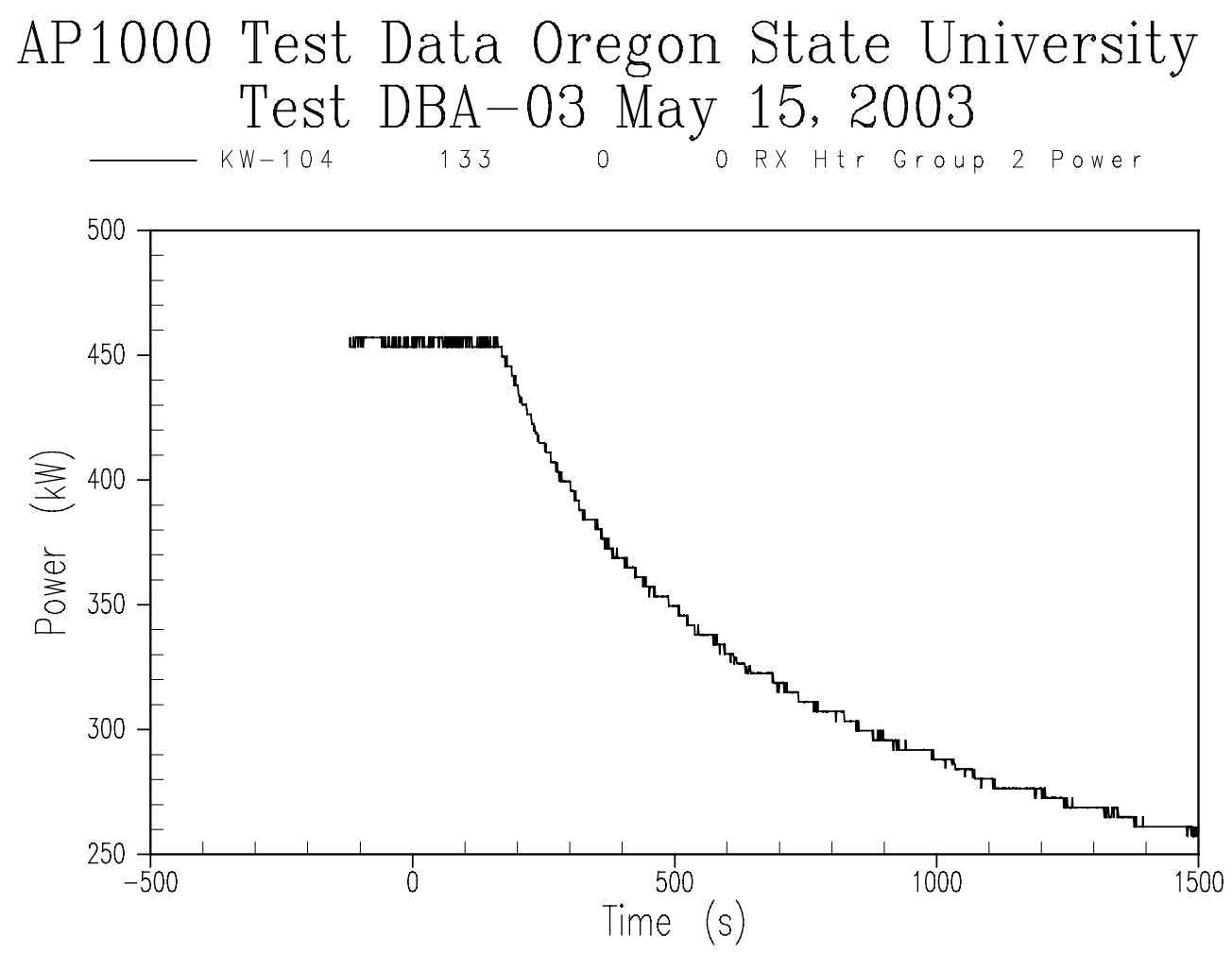

Figure A-70 Core Power Input Power 

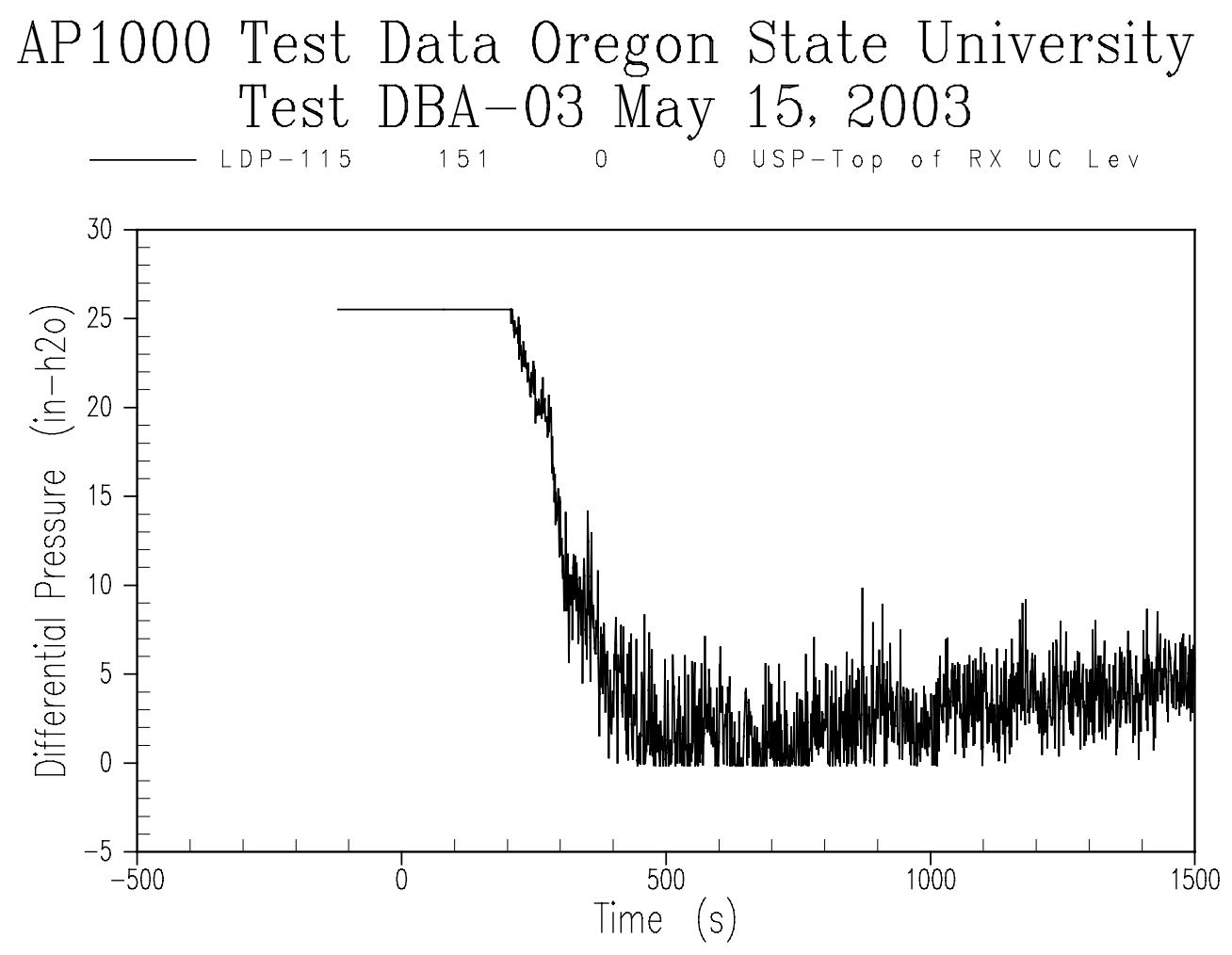

Figure A-71 Reactor Vessel Liquid Level Between Top of Vessel - Upper Support Plate 

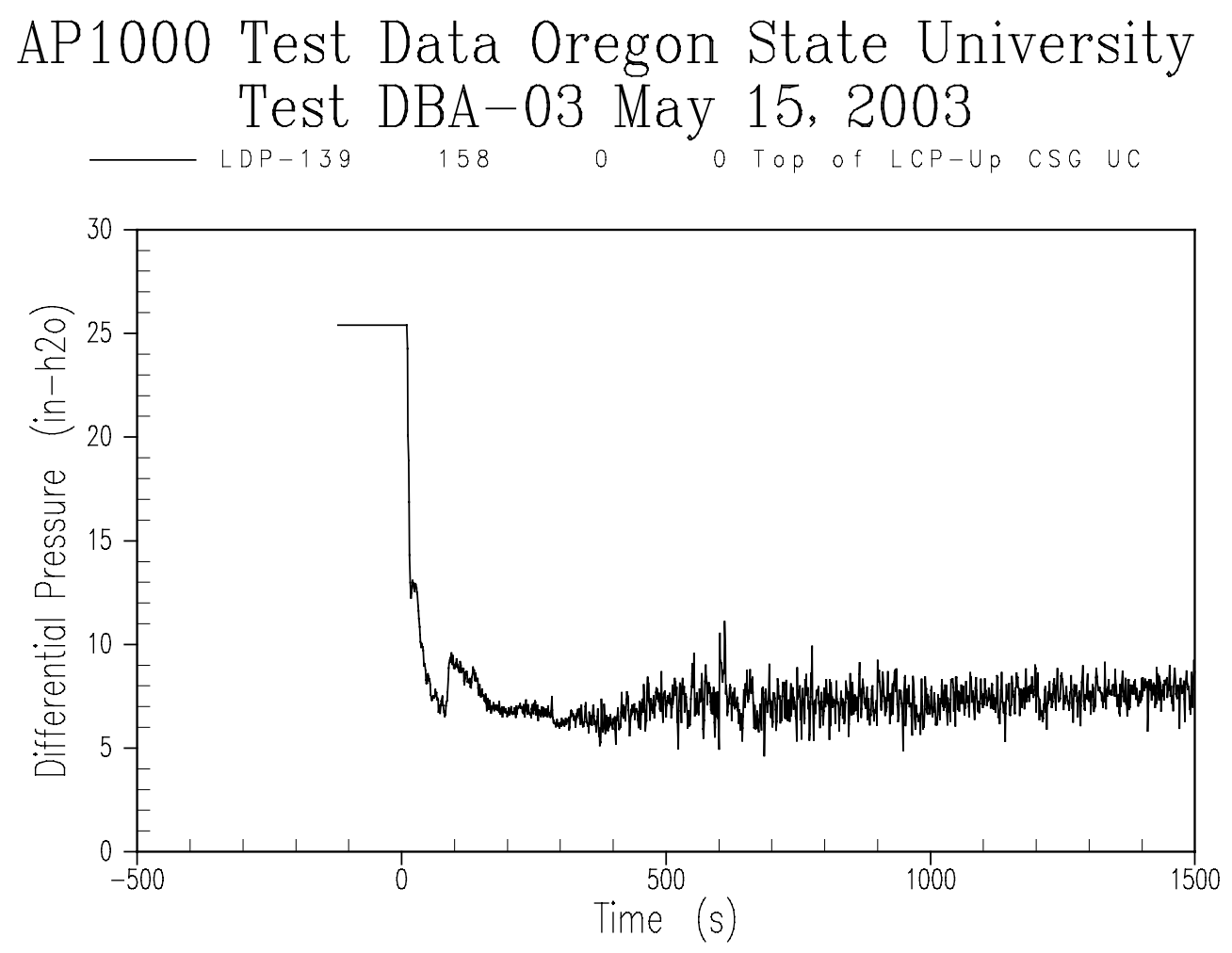

Figure A-72 Reactor Vessel Liquid Level Between Bottom of Upper Support Plate Upper Core Spacer Grid 

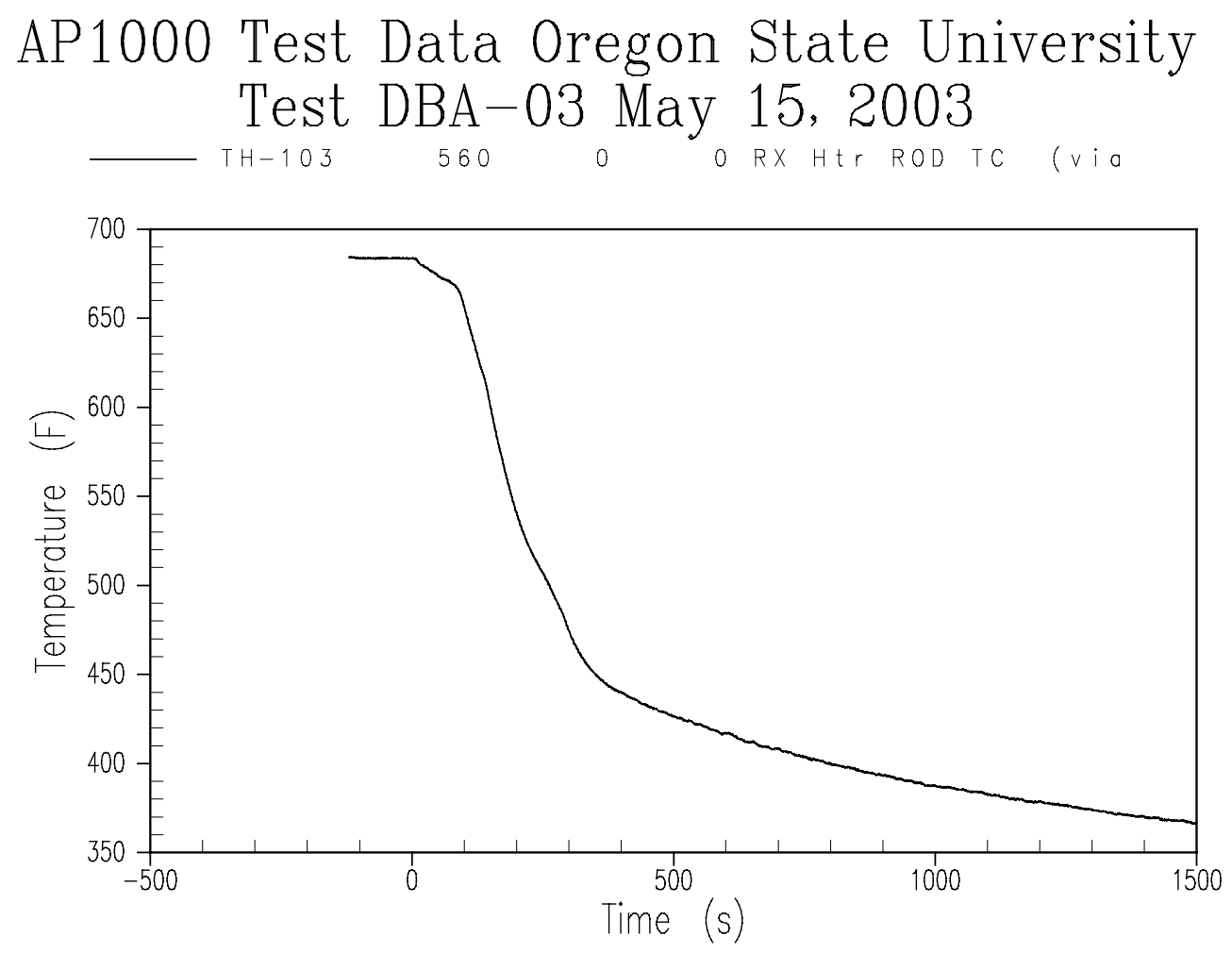

Figure A-73 Inner Core Thermocouple Measuring Heater Temperature 

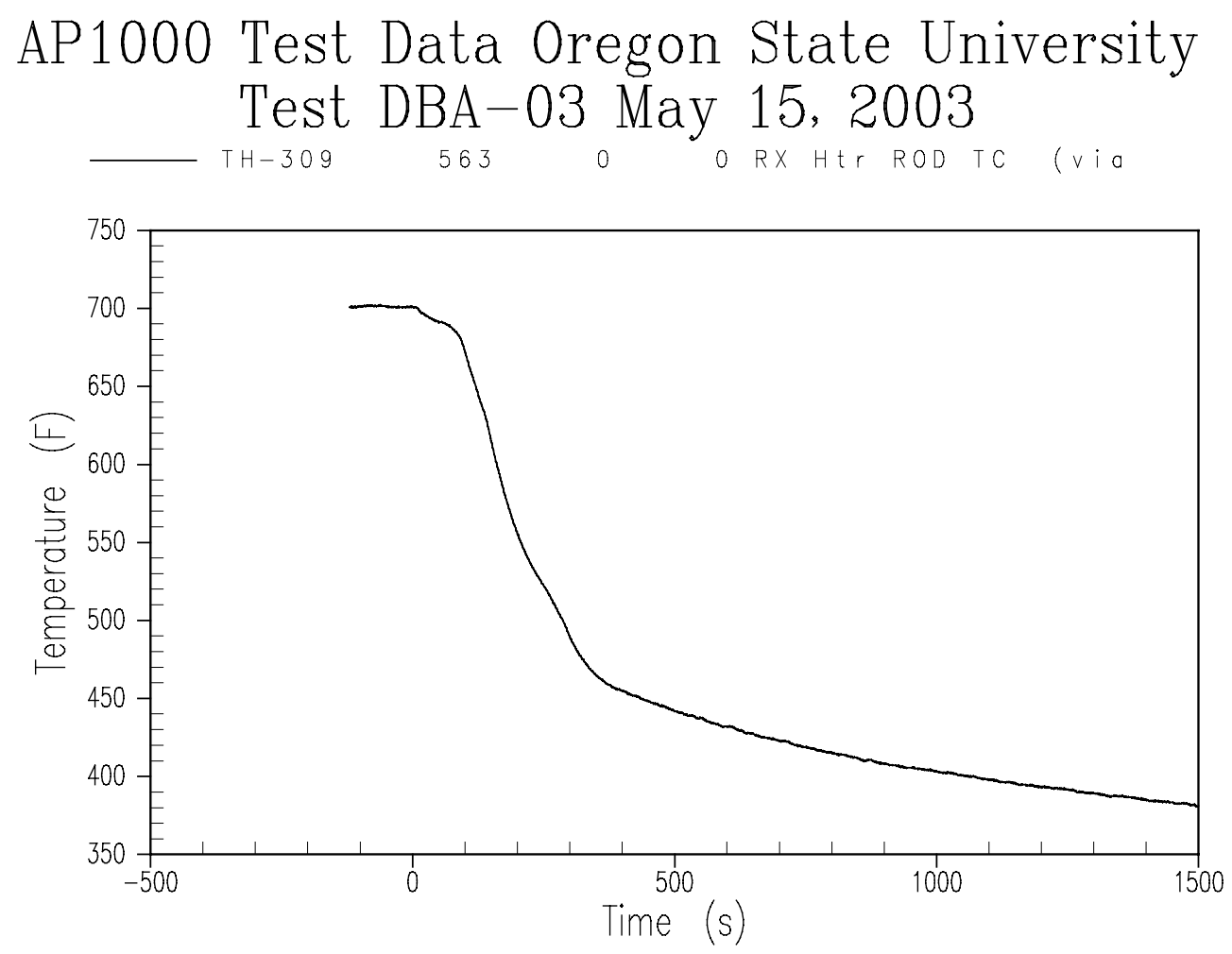

Figure A-74 Outer Core Thermocouple Measuring Heater Temperature 


\section{APPENDIX B}

TEST DATA 


\section{Appendix E -- OSU-AP1000-05}




\section{WESTINGHOUSE PROPRIETARY INFORMATION NOTICE}

This report contains information proprietary to Westinghouse Electric Company. A non-proprietary version of this report has also been prepared for distribution to the United States Nuclear Regulatory Commission (NRC).

In order to conform to the requirements of 10 CFR 2.790 of the Commission's regulations concerning the protection of proprietary information so submitted to the $\mathrm{NRC}$, the information which is proprietary in the proprietary version of this document is contained within brackets, and where the proprietary information has been deleted in the non-proprietary versions, only the brackets remain (the information that was contained within the brackets in the proprietary versions having been deleted). The justification for claiming the information so designated as proprietary is indicated in both versions by means of lower case letters (a) through (f) located as a superscript immediately following the brackets enclosing each item of information being identified as proprietary or in the margin opposite such information. These lower case letters refer to the types of information Westinghouse customarily holds in confidence identified in Sections (4)(ii)(a) through (4)(ii)(f) of the affidavit accompanying the transmittal of these documents pursuant to 10 CFR 2.790(b)(1).

\section{COPYRIGHT NOTICE}

This document bears an Oregon State University copyright notice. The NRC is permitted to make the number of copies of the information contained in these reports which are necessary for its internal use in connection with generic and plant-specific reviews and approvals as well as the issuance, denial, amendment, transfer, renewal, modification, suspension, revocation, or violation of a license, permit, order, or regulation subject to the requirements of $10 \mathrm{CFR} 2.790$ regarding restrictions on public disclosure to the extent such information has been identified as proprietary by Westinghouse, copyright protection notwithstanding. With respect to the non-proprietary versions of these reports, the NRC is permitted to make the number of copies beyond these necessary for its internal use which are necessary in order to have one copy available for public viewing in the appropriate docket files in the public document room in Washington, DC and in local public document rooms as may be required by NRC regulations if the number of copies submitted is insufficient for this purpose. Copies made by the NRC must include the copyright notice in all instances and the proprietary notice if the original was identified as proprietary. 


\title{
Oregon State University
}

Department of Nuclear Engineering

\section{Advanced Thermal Hydraulic RESEARCH LABORATORY}

\section{TEST ACCEPTANCE REPORT}

OSU-AP1000-05

\section{AP1000 2-INCH COLD LEG BREAK WITH 3 OF 4 ADS 4}

\author{
Revision 0
}




\title{
TEST ACCEPTANCE REPORT
}

\author{
OSU-AP1000-05
}

\section{AP1000 2-INCH COLD LEG BREAK WITH 3 OF 4 ADS 4}
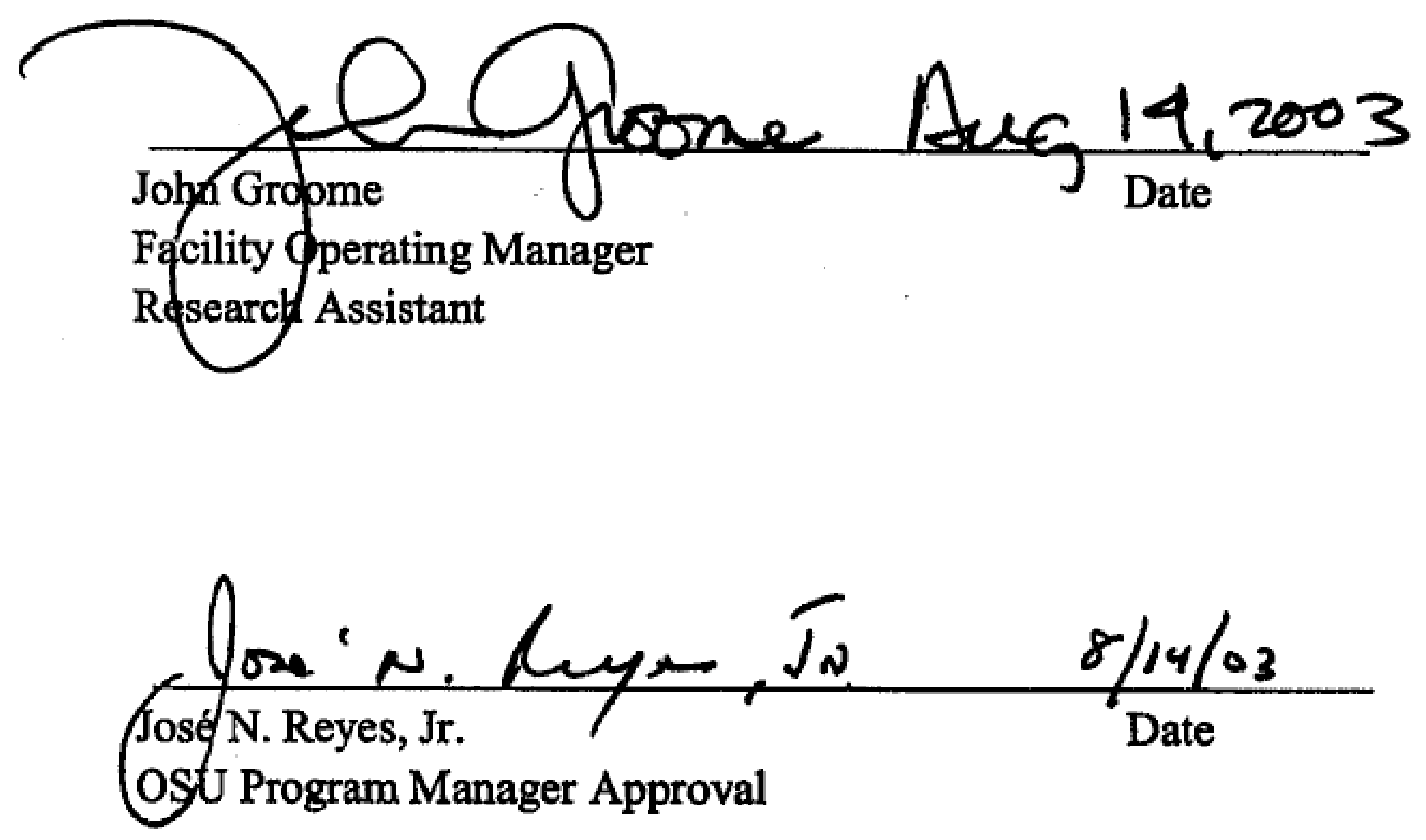

Oregon State University

116 Radiation Center

Corvallis, Oregon 97331

(C) 2003 Oregon State University

All Rights Reserved 


\section{TABLE OF CONTENTS}

$\underline{\text { Section }}$

Title

$\underline{\text { Page }}$

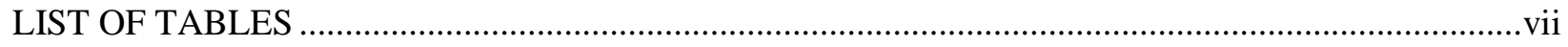

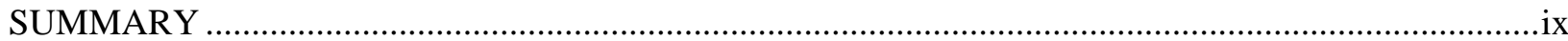

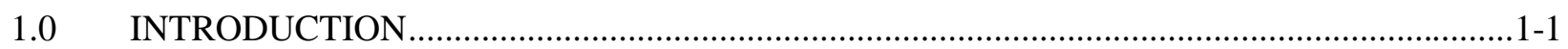

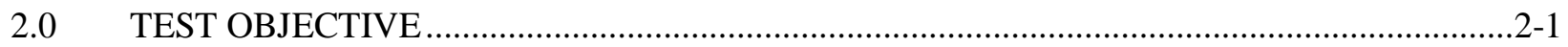

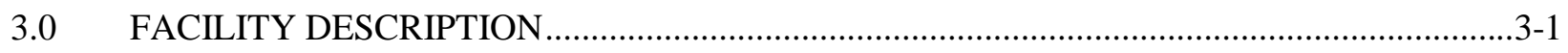

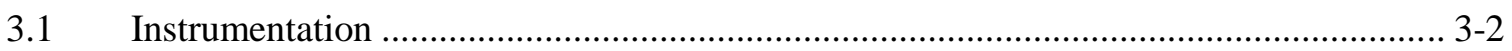

4.0 OSU TESTING PROGRAM MATRIX ...........................................................................

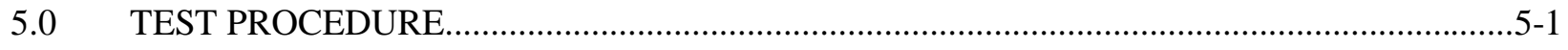

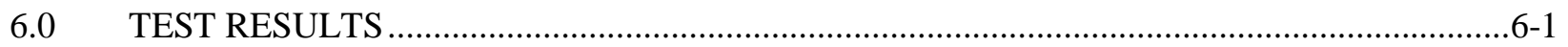

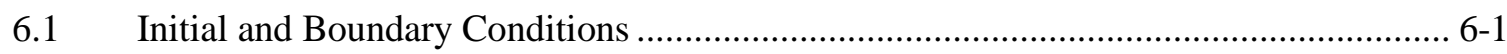

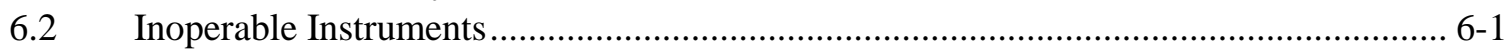

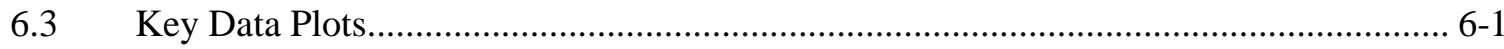

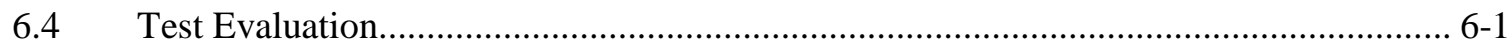

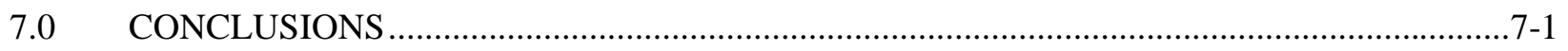

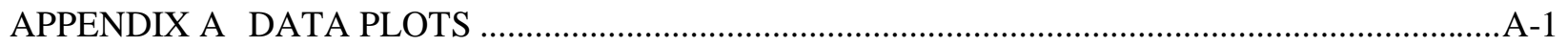

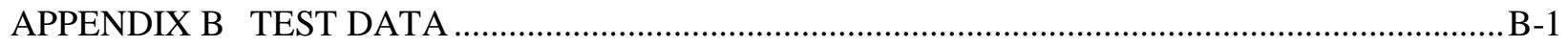




\section{LIST OF TABLES}

$\underline{\text { Table }}$

Table 4-1 OSU Test Matrix (Specified July 1, 2003).

Table 6-1 Actual Test Initial Conditions

$\underline{\text { Title }}$ $\underline{\text { Page }}$ 4-2 6-2

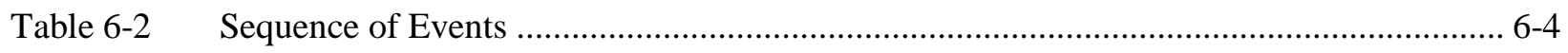

Table 6-3 Inoperable Instruments for DBA-04-D Test............................................................. 6-6

Table 6-4 Data Plots for QL Reports for DBA-04-D by Component ........................................... 6-7 


\section{SUMMARY}

This report covers the test DBA-04, 2-inch Cold Leg Break simulation loss-of-coolant accident (LOCA) performed on July 1, 2003. The objective of this test was to obtain thermal-hydraulic data for 2-inch Cold Leg Break simulation. The test performed met the specified conditions. The top of the heater bundle was always covered during this event. The test was performed for about 17,000 seconds. The transient continued through Automatic Depressurization System (ADS) actuation, core makeup tank (CMT), accumulator, incontainment refueling water storage tank (IRWST) injection, and sump recirculation injection.

This report presents the initial assessment of the test data collected. If this test is to be used by Westinghouse to support AP1000 Design Certification, additional validation of the use of this information will be documented separately. In the interim, the list of invalid data channels may change. 


\subsection{INTRODUCTION}

The Department of Nuclear Engineering at Oregon State University is performing a series of tests for the U.S. Department of Energy (DOE). These tests are being conducted in the Advanced Plant Experiment (APEX-1000) test facility, which is a reduced pressure and height model of the two-loop Westinghouse AP1000 pressurized water reactor. The purpose of the testing is to:

- evaluate the thermal-hydraulic performance of the passive safety systems of the full-scale AP1000, and

- to assess and validate the safety analyses techniques and computer codes used in predicting the transient system behavior.

The AP1000 Long-Term Cooling Test is a 1/4 height scale, low-pressure integral systems test simulating thermal-hydraulic phenomenon for the AP1000 passive safety systems for small-break loss-of-coolant accidents (LOCAs) and long-term cooling. It accurately models the details of the AP1000 geometry, including the primary system, the passive safety systems, and a part of the non-safety grade Chemical and Volume Control System, as well as a partial non-safety grade Normal Residual Removal System. The interconnecting pipe routings are also duplicated in the model.

The overall objective of the Long-Term Cooling Test program is to obtain test data at various modes of operation. The Oregon State University (OSU) experiments will examine the passive safety system response for the small-break and large-break LOCA transition into long-term cooling. (The list of the tests to be performed is provided in the attached OSU Test Matrix provided in Section 4.0.) The facility permits a range of small-break LOCAs to be simulated at different locations on the primary system, such as the cold leg, hot leg, core makeup tank (CMT) cold leg pressure balance line, and direct vessel injection line. The break orientation (top or bottom of the cold leg) may also be studied. Selected tests continue into the long-term cooling, post-accident mode in which the passive safety injection is from the reactor sump as well as the incontainment refueling water storage tank (IRWST). A large-break, post-accident, long-term cooling situation will also be simulated. 


\subsection{TEST OBJECTIVE}

The purpose of this test is to obtain thermal-hydraulic data for a 2-inch Cold Leg Break simulation. The break is located in cold leg \#3, which is a non-balance line cold leg. The data obtained from the test will be used to verify the AP1000 thermal-hydraulic computer codes for AP1000 phenomena such as gravity injection, natural convection, and post-accident long-term core cooling behavior.

The acceptance criteria for the OSU tests are as follows:

- Test initial conditions will be achieved within a specified tolerance.

- Set points will be achieved within an acceptable tolerance band.

- All instrumentation should be operational before the test.

- Any critical instruments not operating will be identified to the test engineer before the tests. These instruments must be operational before and during the test or exceptions should be approved.

- A zero check of LDPs, DPs, and FDPs will be performed. 


\subsection{FACILITY DESCRIPTION}

A detailed facility description for the OSU test facility is documented in Reference 1 . The OSU test facility has been specifically scaled, designed, and constructed to investigate the AP600 passive safety system behavior and to provide data for safety analysis computer code validation. The facility has been modified to model the AP1000.

The scaled test design accurately models the details of the AP1000 geometry, including the primary system and the pipe routings and layout for the passive safety systems. A detailed scaling report (Reference 2) was used to develop the test design modifications. The primary system consists of one hot leg and two cold legs with two active pumps and an active steam generator for each loop. Two CMTs are connected to one primary loop, and the pressurizer is connected to the other primary loop as in the AP1000 plant design. Gas-driven accumulators are connected to the DVI lines. The discharge lines from the CMT, and one-of-two IRWST and reactor sump lines are connected to each DVI line. The Automatic Depressurization System (ADS), consisting of stages 1, 2 and 3, simulates either one or two of the independent trains used in the AP1000. The two-phase flow from the ADS stages 1-to-3 is separated in a swirl-vane separator and the liquid and vapor flows are measured to obtain the total ADS flow rate. The separated flow streams are then recombined and discharged into the IRWST through a sparger, preserving the mass and energy flow into the IRWST. The injection from the reactor sump is also simulated. Note that the OSU facility models both AP1000 primary and secondary sumps. The primary sump collects the condensate return, the liquid break flow, and the liquid flow from the fourth-stage ADS; and will provide long-term injection to the reactor vessel. The secondary sump simulates the portions of containment that will remain dry during most events. This sump will collect water only when the primary sump reaches its overflow level, and provides no injection to the reactor vessel.

The time period for the experimental simulations includes not only the IRWST injection, but also the draining of the IRWST and the sump injection to simulate the long-term cooling of the AP1000. This simulation could be from several hours to a day. The time scale for the OSU test facility is one-half; that is, events occur in half the normal time. To model the long-term cooling aspects of the transient, the two-phase flow from the break is separated in a swirl-vane separator and the liquid and vapor portions of the total flow are measured. The liquid fraction of the flow is discharged to the reactor primary sump as in the AP1000 plant. The vapor is discharged to the atmosphere. The capability exists to return a portion of the equivalent liquid flow to the IRWST and primary sump to simulate the condensate return from the passive containment to the IRWST and primary sump. A similar approach is also used for the fourth-stage ADS valve on the hot leg. The two-phase flow is separated in a swirl-vane separator, the two streams are measured, and the liquid phase is discharged into the primary sump while the vapor flow is discharged to the atmosphere. Again, the capability exists to return a portion of the liquid equivalent added to the IRWST and primary sump. In addition, all other steam vents from the facility are measured (e.g., the IRWST vent), and a portion of the liquid equivalent may be added back to the facility. Note that not all of the steam discharge would be returned as liquid equivalent. A portion of the discharge would be removed to simulate the steam that is not available for recirculation because it provides containment pressurization. The IRWST and primary sump can be pressurized in the OSU facility to simulate the containment pressurization following a postulated LOCA.

A multi-tube passive residual heat removal (PRHR) heat exchanger is located in the IRWST. The heat exchanger uses the same C-tube design as the AP1000 and has two instrumented tubes to obtain wall heat 
fluxes during the tests. There are primary fluid thermocouples, wall thermocouples, and differential pressure drop measurements to determine when the heat exchanger begins to drain. The IRWST is also instrumented with strings of fluid thermocouples, to determine the degree of mixing within the tank and to assess the temperature of the coolant that is delivered to the test vessel.

The reactor vessel for the OSU tests includes a 0.914-meter (3-foot) heated core consisting of forty-eight 0.025 -meter (1-inch) diameter heater rods. The heater rods have a top skewed power shape. The $1000 \mathrm{~kW}$ of electrical power available at the OSU test site will be used to simulate decay heat. Wall thermocouples are swaged inside the heater rods to measure the heater rod wall temperature.

Thermocouple rods in the heater rod bundle measure the axial coolant temperature distribution. The scaled flow volume in the core is preserved as well as the flow volume in the test vessel upper plenum. There are simulated reactor internals in the upper plenum to preserve the flow area and to correctly scale the fluid volume. The reactor vessel includes an annular downcomer into which the four cold legs and the two DVI lines are connected. The hot legs penetrate the reactor annulus and connect with the loops. The AP1000 reactor vessel neutron reflector is simulated using a ceramic liner to reduce the metal heat release to the coolant.

There were no special/unique requirements for the test other than those specified in the Initial Conditions. The specified conditions were verified on the control board prior to test implementation.

\subsection{Instrumentation}

The instrumentation has been designed to calculate a transient mass and energy balance on the test facility. All two-phase flow streams exiting the facility are separated, and each component is measured separately as a single phase flow using conventional measurement devices such as magnetic flow meters and vortex flow meters. Note that magnetic flowmeters are not designed for two-phase flow and will indicate erratically. Also note, the vortex flowmeters are referenced to $212^{\circ} \mathrm{F}$ and the LDPs are referenced to $60^{\circ} \mathrm{F}$. All vertical components have differential pressure cells that act as level instruments to measure the transient mass change in the component. The hot and cold leg diameters are sufficiently large in the OSU test facility such that a narrow range differential pressure cell can be used to determine if the flow becomes stratified.

Single flow measurements are made of the CMT, accumulator, IRWST, and sump flows into the reactor vessel through the direct vessel injection lines.

Various types of instrumentation are provided in the test facility; for example, thermocouples for coolant and wall temperatures, flowmeters, pressure transducers, differential pressure transducers, and weigh tanks. 


\subsection{OSU TESTING PROGRAM MATRIX}

The test matrix for the OSU test facility is shown in Table 4-1. To satisfy the test objectives, several transients will be performed to provide data on the AP1000 passive safety system response for a range of break sizes, locations, orientations, and single failure assumptions. The break size orifices are scaled based on simulating a 1-inch, 2-inch, or 4-inch pipe break.

The designation for this test is DBA-04-D, which identifies the test as a design basis 2-inch Cold Leg Break. The test matrix may be adjusted for future tests based on results and insights gained with each test. 


\begin{tabular}{|c|c|c|}
\hline \multicolumn{3}{|c|}{$\begin{array}{l}\text { TABLE 4-1 } \\
\text { OSU TEST MATRIX (SPECIFIED JULY 1, 2003) }\end{array}$} \\
\hline Test Title & Break Location and Size & Single Failure Assumed \\
\hline DBA-01-D & $\begin{array}{l}\text { Double-ended DVI Line break with } \\
\text { continuation into long-term cooling }\end{array}$ & Fail 1 of 2 lines in 1 ADS- 4 train \\
\hline DBA-02-D & $\begin{array}{l}\text { Double-ended DVI Line break with } \\
\text { continuation into long-term cooling }\end{array}$ & $\begin{array}{l}\text { Fail } 1 \text { of } 2 \text { lines in } 1 \text { ADS- } 4 \text { train } \\
\text { (Adjusted ADS-4 Resistance) } \\
\text { Single failure on non-pressurizer side }\end{array}$ \\
\hline DBA-03-D & $\begin{array}{l}\text { Double-ended DVI Line break with } \\
\text { continuation into long-term cooling }\end{array}$ & $\begin{array}{l}\text { Fail } 1 \text { of } 2 \text { lines in } 1 \text { ADS- } 4 \text { train } \\
\text { Single failure on pressurizer side }\end{array}$ \\
\hline DBA-04-D & 2-inch Cold Leg Break (CL \#3) & $\begin{array}{l}\text { Fail } 1 \text { of } 2 \text { lines in } 1 \text { ADS- } 4 \text { train } \\
\text { Single failure on pressurizer side }\end{array}$ \\
\hline TR-02-D & $\begin{array}{l}\text { Transition Test ADS4 opening, } 125 \text { psig initial } \\
\text { pressure and decay power } 480 \mathrm{sec}\end{array}$ & $\begin{array}{l}\text { Fail } 1 \text { of } 2 \text { lines in } 1 \text { ADS-4 train } \\
\text { (No ADS 1-3) }\end{array}$ \\
\hline TR-03-D & $\begin{array}{l}\text { Transition Test ADS4 opening, } 85 \text { psig initial } \\
\text { pressure and decay power } 1800 \mathrm{sec}\end{array}$ & $\begin{array}{l}\text { Fail } 1 \text { of } 2 \text { lines in } 1 \text { ADS-4 train } \\
\text { (No ADS 1-3) }\end{array}$ \\
\hline TR-04-D & $\begin{array}{l}\text { Transition Test ADS4 opening, } 85 \text { psig initial } \\
\text { pressure and decay power } 480 \mathrm{sec}\end{array}$ & $\begin{array}{l}\text { No Failure of ADS-4 lines assumed } \\
\qquad \text { (No ADS 1-3) }\end{array}$ \\
\hline EN-01-D & $\begin{array}{l}\text { Entrainment Test with Revised Upper Internals, } \\
1000 \mathrm{~kW} \text { reactor power, } 14.7 \mathrm{psi}\end{array}$ & Fail 1 of 2 lines in 1 ADS- 4 train \\
\hline EN-01-D & $\begin{array}{l}\text { Entrainment Test with Revised Upper Internals, } \\
700 \mathrm{~kW} \text { reactor power, } 14.7 \mathrm{psi}\end{array}$ & Fail 1 of 2 lines in 1 ADS- 4 train \\
\hline PRA-01-D & PRA Test - DEDVI with no accumulators & No ADS-4 Failure assumed \\
\hline PRA-02-D & $\begin{array}{l}\text { PRA Test - 3-inch Hot Leg Break with no } \\
\text { CMTs }\end{array}$ & No ADS-4 Failure assumed \\
\hline
\end{tabular}




\subsection{TEST PROCEDURE}

The test was performed as per a written procedure. There were no special/unique requirements for the test other than those specified in the initial conditions in Table 6-1. The specified conditions were checked on the control board prior to test implementation.

The appropriate prerequisites were completed and initial conditions were satisfied. The required break simulation piping and break instrumentation were installed per P\&ID drawing OSU 600904, Rev. 1. A break spool insert simulating the 2-inch Cold Leg Break in AP1000 was installed in the break spool in cold leg \#3 which is a cold leg without a CMT balance line. Flow from the break is directed horizontally into the break separator. The 100-percent flow nozzle was installed in the ADS 4-2 (on the hot leg 2) and the 50-percent flow nozzle was installed in ADS 4-1 (on the hot leg 1). Flow nozzles that simulate full flow for ADS-1, ADS-2, and ADS-3 were installed. As per the AP600 tests, ADS-3 has been scaled for full flow from all three stages, and ADS-1 and ADS- 2 are closed when ADS-3 is opened.

Fill and vent was performed per APEX Operations Manual Procedure OP-B.2. Instruments were checked for required calibration.

With the break valve TS-202 closed, flow was used to warm up the bypass line by opening isolation valves RCS-901 and RCS-902. After the appropriate prerequisites were completed and the test facility achieved specified initial conditions, the CMT warm-up bypass line isolation valves RCS-901 and RCS-902 were closed to maintain the $<80^{\circ} \mathrm{F}$ condition at the top of the CMT \#1. With the CMT balance line valves (RCS-529 and RCS-530) placed in the open and automatic mode, both CMTs reached the reactor coolant system (RCS) pressure.

Once all other initial conditions were satisfied, a break through TS-202 was initiated. The transient continued through ADS actuation, and CMT, accumulator, IRWST injection, and sump injection. Per Westinghouse instructions, ADS 4-2 was actuated automatically according to the actuation logic, and ADS 4-1 was actuated manually after a 30-second delay. All other actions were automatic and required no operator action. 


\subsection{TEST RESULTS}

The test results for test DBA-04-D are provided in the following subsections.

\subsection{Initial and Boundary Conditions}

Table 6-1 provides a comparison of the specified and actual conditions for test DBA-04-D. The values in this table were averaged over approximately 2 minutes preceding the test. Test initial conditions were achieved for the steam generator pressure, pressurizer pressure, pressurizer level, steam generator 01 narrow-range level, and steam generator 02 narrow-range level. Test initial conditions for the hot leg temperature were found to be acceptable, and the results will not be adversely affected.

The actual power decay curves are provided in data plots in Appendix B. The measured maximum power was $913 \mathrm{~kW}$, which was less than the facility maximum power of $1000 \mathrm{~kW}$. The programmed decay heat curve was adjusted to account for this difference, and the differences between the actual and specified power decay are considered acceptable.

PT-501 and PT-502 pressure instruments indicate the pressure changes in the CMT-1 and CMT-2. CMT-1 (PT-501) and CMT-2 (PT-502) confirm that 1 minute after the test button was pushed, both CMTs reach RCS pressure.

The sequence of events is shown in Table 6-2. This table compares the actual sequence of events with the specified timing. As can be seen in this table, all the events occurred at or very near to when the event was planned.

\subsection{Inoperable Instruments}

Table 6-3 provides a list of the instrumentation channels considered inoperable for the DBA-04-D test.

\subsection{Key Data Plots}

Table 6-4 provides a list of the instrumentation channels sorted by component, and includes instrument number, units, and quick look plot number. The selection of channels was based on projecting an overall picture of the test results, which would then be examined by referring to the detailed data plots or tapes.

\subsection{Test Evaluation}

The following observations were made during the test:

1. The peak power before the test was initiated was $913 \mathrm{~kW}$. The decay heat curve was adjusted for this value.

2. The test was not terminated at stable sump injection. The sump level was decreased to determine the effect of sump injection flow and core cooling. Two decreased sump levels were tested for approximately 10 minutes to demonstrate injection and effective core cooling at lower containment flood-up levels. The test was terminated when the sump level was drained to the wall-to-wall flooding level in containment. 


\begin{tabular}{|c|c|c|c|}
\hline \multicolumn{4}{|c|}{$\begin{array}{c}\text { TABLE 6-1 } \\
\text { ACTUAL TEST INITIAL CONDITIONS }\end{array}$} \\
\hline Conditions & Instrument No. & Actual & Comment \\
\hline Pressurizer Pressure & PT-604 & 370 psig & \\
\hline $\begin{array}{l}\text { Hot Leg Temperature } \\
\quad \# 1\end{array}$ & $\begin{array}{l}\text { TF-141* } \\
\text { TF-205 } \\
\text { TF-143 }\end{array}$ & $\begin{array}{c}426.3^{\circ} \mathrm{F} \\
428^{\circ} \mathrm{F} \\
428.4^{\circ} \mathrm{F}\end{array}$ & \\
\hline $\begin{array}{l}\text { Hot Leg Temperature } \\
\quad \# 2\end{array}$ & $\begin{array}{l}\text { TF-140* } \\
\text { TF-206 } \\
\text { TF-142 }\end{array}$ & $\begin{array}{l}427.6^{\circ} \mathrm{F} \\
426.3^{\circ} \mathrm{F} \\
428.5^{\circ} \mathrm{F}\end{array}$ & \\
\hline $\begin{array}{l}\text { SG Press. } \\
\quad \# 1 \\
\# 2 \\
\text { Header }\end{array}$ & $\begin{array}{l}\text { PT-301* } \\
\text { PT-302* } \\
\text { PT-002 }\end{array}$ & $\begin{array}{c}285 \mathrm{psig} \\
287 \mathrm{psig} \\
245.5 \mathrm{psig}\end{array}$ & \\
\hline Pressurizer Level & $\begin{array}{l}\text { LDP-601 uncompensated } \\
\text { LDP-601 } \\
\text { Compensated by SC-608 }\end{array}$ & $\begin{array}{l}64.2 \text { inches } \\
77.1 \text { inches }\end{array}$ & $\begin{array}{l}440^{\circ} \mathrm{F} \text { used for density } \\
\text { compensation }\end{array}$ \\
\hline $\begin{array}{l}\text { SG Level } \\
\quad \# 1 \mathrm{NR}\end{array}$ & $\begin{array}{l}\text { LDP-303 uncompensated } \\
\text { LDP-303 compensated by } \\
\text { average of TF-305 and } \\
\text { TF-307 }\end{array}$ & $\begin{array}{l}21.1 \text { inches } \\
25.5 \text { inches }\end{array}$ & $\begin{array}{l}413^{\circ} \mathrm{F} \text { used for density } \\
\text { compensation }\end{array}$ \\
\hline $\begin{array}{l}\text { SG Level } \\
\# 2 \mathrm{NR}\end{array}$ & $\begin{array}{l}\text { LDP-304 uncompensated } \\
\text { LDP-304 compensated by } \\
\text { average of TF-306 and } \\
\text { TF-308 }\end{array}$ & $\begin{array}{l}20.8 \text { inches } \\
24.4 \text { inches }\end{array}$ & $\begin{array}{l}414^{\circ} \mathrm{F} \text { used for density } \\
\text { compensation }\end{array}$ \\
\hline IRWST Temperature & TF-701 & $77.4^{\circ} \mathrm{F}$ & Accepted $\left(<80^{\circ} \mathrm{F}\right)$ \\
\hline $\begin{array}{l}\text { CMT Temperature } \\
\text { \#1 } \\
\# 2\end{array}$ & $\begin{array}{l}\text { TF-511 } \\
\text { TF-514 }\end{array}$ & $\begin{array}{c}77^{\circ} \mathrm{F} \\
77.2^{\circ} \mathrm{F}\end{array}$ & Accepted $\left(<80^{\circ} \mathrm{F}\right)$ \\
\hline $\begin{array}{l}\text { Accumulator } \\
\text { Temperature } \\
\# 1 \\
\# 2\end{array}$ & $\begin{array}{l}\text { TF-401 } \\
\text { TF-402 }\end{array}$ & $\begin{array}{l}77.5^{\circ} \mathrm{F} \\
78.5^{\circ} \mathrm{F}\end{array}$ & Accepted $\left(<80^{\circ} \mathrm{F}\right)$ \\
\hline IRWST Level & LDP-701 & 93.3 inches & \\
\hline
\end{tabular}




\begin{tabular}{||c|c|c|c||}
\hline \hline \multicolumn{2}{|c||}{ TABLE 6-1 (Continued) } \\
\multicolumn{1}{||c||}{ ACTUAL TEST INITIAL CONDITIONS } \\
\hline \hline Conditions & Instrument No. & Actual & Comment \\
\hline Accumulator Level & & & \\
$\# 1$ & LDP-401 & 35.7 inches & \\
\hline Accumulator Pressure & LDP-402 & 36.7 inches & \\
$\# 1$ & & & \\
$\# 2$ & PT-401 & $188 \mathrm{psig}$ & \\
\hline CMT Level & PT-402 & $190.2 \mathrm{psig}$ & \\
$\# 1$ & & & \\
$\# 2$ & LDP-507 & $57.2 \mathrm{inches}$ & \\
\hline CMT Pressure & LDP-502 & $57.7 \mathrm{inches}$ & \\
$\# 1$ & & & \\
$\# 2$ & PT-501 & $374 \mathrm{psig}$ & \\
\hline \hline
\end{tabular}




\begin{tabular}{|c|c|c|}
\hline \multicolumn{3}{|c|}{$\begin{array}{c}\text { TABLE 6-2 } \\
\text { SEQUENCE OF EVENTS }\end{array}$} \\
\hline Event & Setpoint & Actual Time (sec) \\
\hline PB Depressed & N/A & -120 \\
\hline Break Valve(s) Open & 0 & 0 \\
\hline Feed Pump Trip & $3.6 \mathrm{sec}$ & 3 \\
\hline CMT01 Outlet Valve Open (RCS-535) & $6.1 \mathrm{sec}$ & 6 \\
\hline CMT02 Outlet Valve Open (RCS-536) & $6.1 \mathrm{sec}$ & 6 \\
\hline PRHR HX Outlet Vlv Open (RCS-804) & $6.1 \mathrm{sec}$ & 5 \\
\hline RCP \#1 Trip & $8.6 \mathrm{sec}$ & 8 \\
\hline RCP \#2 Trip & $8.6 \mathrm{sec}$ & 8 \\
\hline RCP \#3 Trip & $8.6 \mathrm{sec}$ & 8 \\
\hline RCP \#4 Trip & $8.6 \mathrm{sec}$ & 8 \\
\hline CMT \#1 Level Low (LDP-507) & 41 inches & 377 \\
\hline CMT \#2 Level Low (LDP-502) & 41 inches & 401 \\
\hline ADS \#1 Actuation (RCS-601) & CMT Level Low $+15 \mathrm{sec}$ & 391 \\
\hline ADS \#2 Actuation (RCS-602) & CMT Level Low $+62 \mathrm{sec}$ & 439 \\
\hline ADS \#3 Actuation (RCS-603) & CMT Level Low + $122 \mathrm{sec}$ & 499 \\
\hline Low Reactor Pressure (P-107) & 40 psig & 615 \\
\hline IRWST Valve Actuation (RCS-711) & $\begin{array}{l}\text { Low Reactor Pressure } \\
\qquad(<40 \mathrm{psig})\end{array}$ & 617 \\
\hline IRWST Valve Actuation (RCS-712) & $\begin{array}{l}\text { Low Reactor Pressure } \\
\qquad(<40 \mathrm{psig})\end{array}$ & 617 \\
\hline CMT \#1 Low Low Level (LDP-507) & 17.14 inches & 773 \\
\hline CMT \#2 Low Low Level (LDP-502) & 17.14 inches & 776 \\
\hline ADS 4-1 Actuation (RCS-615) & $\begin{array}{l}\text { CMT Low Low (17.14") and } \\
\text { CMT Low }\left(41^{\prime \prime}\right)+180 \mathrm{sec}\end{array}$ & 777 \\
\hline ADS 4-2 Actuation (RCS-616) & $\begin{array}{l}\text { CMT Low Low (17.14") and } \\
\text { CMT Low (41") + } 180 \mathrm{sec}\end{array}$ & 807 \\
\hline Sump Valve Actuation (CSS-909) & IRWST Level Low Low & 8579 \\
\hline
\end{tabular}




\begin{tabular}{|c|c|c|}
\hline \multicolumn{3}{|c|}{$\begin{array}{c}\text { TABLE 6-2 (Continued) } \\
\text { SEQUENCE OF EVENTS }\end{array}$} \\
\hline Event & Setpoint & Actual Time (sec) \\
\hline Sump Valve Actuation (CSS-910) & IRWST Level Low Low & 8579 \\
\hline $\begin{array}{l}\text { Accumulator Injection } \\
\# 1 \text { (FMM-401) }\end{array}$ & N/A & 439 \\
\hline $\begin{array}{l}\text { Accumulator Injection } \\
\text { \#2 (FMM-402) }\end{array}$ & N/A & 438 \\
\hline $\begin{array}{l}\text { IRWST Injection } \\
\text { DVI \#1 (FMM-701) }\end{array}$ & N/A & 1358 \\
\hline $\begin{array}{l}\text { IRWST Injection } \\
\text { DVI \#2 (FMM-702) }\end{array}$ & N/A & 1358 \\
\hline $\begin{array}{l}\text { Accumulator Empty } \\
\text { \#1 (LDP-401) }\end{array}$ & N/A & 915 down to 1.2 inch level \\
\hline $\begin{array}{l}\text { Accumulator Empty } \\
\text { \#2 (LDP-402) }\end{array}$ & N/A & 930 down to 0.1 inch level \\
\hline $\begin{array}{l}\text { CMT Empty } \\
\# 1 \text { (LDP-507) }\end{array}$ & N/A & 1060 down to 0.1 inch level \\
\hline $\begin{array}{l}\text { CMT Empty } \\
\text { \#2 (LDP-502) }\end{array}$ & N/A & 1060 \\
\hline Sump Injection DVI \# 1 (FMM-901) & N/A & 8581 \\
\hline Sump Injection DVI \# 2 (FMM-902) & N/A & 8580 \\
\hline
\end{tabular}




\begin{tabular}{|c|c|c|}
\hline \multicolumn{3}{|c|}{$\begin{array}{c}\text { TABLE 6-3 } \\
\text { INOPERABLE INSTRUMENTS FOR DBA-04-D TEST }\end{array}$} \\
\hline Instrument Number & Instrument Type & Inoperable Description \\
\hline $\begin{array}{l}\text { TW-202 } \\
\text { TW-204 } \\
\text { TW-205 } \\
\text { TW-206 } \\
\text { TW-209 } \\
\text { TW-803 } \\
\text { TW-804 }\end{array}$ & Thermocouple & Inoperative \\
\hline TH-603 & $\begin{array}{l}\text { Thermocouple measuring heater } \\
\text { temperature }\end{array}$ & Inoperative \\
\hline FMM-202 & Magnetic flow meter & Inoperative \\
\hline $\begin{array}{l}\text { TF-170 } \\
\text { TF-221 } \\
\text { TF-509 } \\
\text { TF-512 }\end{array}$ & $\begin{array}{l}\text { Thermocouple measuring fluid } \\
\text { temperature }\end{array}$ & Inoperative \\
\hline FVM-905 & Vortex flow meter & Erratic \\
\hline
\end{tabular}




\begin{tabular}{|c|c|c|c|c|}
\hline \multicolumn{5}{|c|}{$\begin{array}{l}\text { TABLE 6-4 } \\
\text { DATA PLOTS FOR QL REPORTS FOR DBA-04-D BY COMPONENT }\end{array}$} \\
\hline Component & Channel & Units & QL-Plot & Comment \\
\hline $\begin{array}{l}\text { Reactor Vessel } \\
\text { Pressure }\end{array}$ & PT-107 & psig & 44 & \\
\hline Reactor Vessel Level & LDP-127 & inch of $\mathrm{H}_{2} \mathrm{O}$ & 29 & \\
\hline $\begin{array}{l}\text { Reactor Vessel } \\
\text { Downcomer Level }\end{array}$ & LDP-140 & inch of $\mathrm{H}_{2} \mathrm{O}$ & 31 & \\
\hline $\begin{array}{l}\text { Cold Leg \#1 Fluid } \\
\text { Temperature }\end{array}$ & TF-107 & ${ }^{\circ} \mathrm{F}$ & 58 & \\
\hline $\begin{array}{l}\text { Cold Leg \#2 Fluid } \\
\text { Temperature }\end{array}$ & TF-108 & ${ }^{\circ} \mathrm{F}$ & 59 & \\
\hline $\begin{array}{l}\text { Cold Leg \#3 Fluid } \\
\text { Temperature }\end{array}$ & TF-103 & ${ }^{\circ} \mathrm{F}$ & 56 & \\
\hline $\begin{array}{l}\text { Cold Leg \#4 Fluid } \\
\text { Temperature }\end{array}$ & TF-104 & ${ }^{\circ} \mathrm{F}$ & 57 & \\
\hline $\begin{array}{l}\text { Reactor Vessel Fluid } \\
\text { Temp Upper Head }\end{array}$ & TF-120 & ${ }^{\circ} \mathrm{F}$ & 60 & \\
\hline $\begin{array}{l}\text { RCS Hot Leg \#1 } \\
\text { Temperature }\end{array}$ & TF-143 & ${ }^{\circ} \mathrm{F}$ & 62 & \\
\hline $\begin{array}{l}\text { RCS Hot Leg \#2 } \\
\text { Temperature }\end{array}$ & TF-142 & ${ }^{\circ} \mathrm{F}$ & 61 & \\
\hline Pressurizer Pressure & $\begin{array}{l}\text { PT-604 (WR) and PT-603 } \\
\text { (LP Indication) }\end{array}$ & psig & 49,50 & \\
\hline $\begin{array}{l}\text { Pressurizer Liquid } \\
\text { Level }\end{array}$ & LDP-601 & inch of $\mathrm{H}_{2} \mathrm{O}$ & 38 & $\begin{array}{l}\text { Sharp decrease followed } \\
\text { by rapid refill. }\end{array}$ \\
\hline SG \#1 Tube Level & LDP-215 & inch of $\mathrm{H}_{2} \mathrm{O}$ & 32 & \\
\hline $\begin{array}{l}\text { SG \#1 Secondary } \\
\text { Pressure }\end{array}$ & PT-301 & psig & 45 & \\
\hline SG \#1 Feed Flow Rate & FMM-001 & gpm & 1 & \\
\hline SG \#2 Tube Level & LDP-218 & inch of $\mathrm{H}_{2} \mathrm{O}$ & 33 & \\
\hline $\begin{array}{l}\text { SG \#2 Secondary } \\
\text { Pressure }\end{array}$ & PT-302 & psig & 46 & \\
\hline
\end{tabular}




\begin{tabular}{|c|c|c|c|c|}
\hline \multicolumn{5}{|c|}{$\begin{array}{l}\text { TABLE 6-4 (Continued) } \\
\text { DATA PLOTS FOR QL REPORTS FOR DBA-04-D BY COMPONENT }\end{array}$} \\
\hline Component & Channel & Units & QL-Plot & Comment \\
\hline $\begin{array}{l}\text { Accumulators \#1 and } \\
\text { \#2 Pressure }\end{array}$ & PT-401 and PT-402 & psig & 47,48 & \\
\hline $\begin{array}{l}\text { Accumulators \#1 and } \\
\text { \#2 Liquid Level }\end{array}$ & LDP-401 and LDP-402 & inch of $\mathrm{H}_{2} \mathrm{O}$ & 34,35 & \\
\hline $\begin{array}{l}\text { Accumulators \#1 and } \\
\text { \#2 Flow Rate }\end{array}$ & FMM-401 and FMM-402 & gpm & 2,3 & \\
\hline $\begin{array}{l}\text { Accumulators \#1 and } \\
\text { \#2 Liquid Discharge } \\
\text { Temperature }\end{array}$ & TF-401 and TF-402 & ${ }^{\circ} \mathrm{F}$ & 63,64 & \\
\hline $\begin{array}{l}\text { CMT \#1 and \#2 Liquid } \\
\text { Level }\end{array}$ & LDP-507 and LDP-502 & inch of $\mathrm{H}_{2} \mathrm{O}$ & 36,37 & \\
\hline $\begin{array}{l}\text { CMT \#1 and \#2 Flow } \\
\text { Rate }\end{array}$ & FMM-501 and FMM-504 & gpm & 4,5 & \\
\hline $\begin{array}{l}\text { CMT \#1 and \#2 Liquid } \\
\text { Temperature }\end{array}$ & $\begin{array}{l}\text { TF-501 and TF-529 and } \\
\text { TF-504 and TF-532 }\end{array}$ & ${ }^{\circ} \mathrm{F}$ & $65-68$ & \\
\hline PRHR Inlet Flow Rate & FMM-802 & gpm & 11 & \\
\hline PRHR Liquid Level & LDP-802 & inch of $\mathrm{H}_{2} \mathrm{O}$ & 40 & \\
\hline $\begin{array}{l}\text { PRHR Outlet Flow } \\
\text { Rate }\end{array}$ & FMM-804 & gpm & 12 & \\
\hline IRWST Liquid Level & LDP-701 & inch of $\mathrm{H}_{2} \mathrm{O}$ & 39 & \\
\hline $\begin{array}{l}\text { IRWST Discharge Line } \\
\# 1 \text { and \#2 Flow Rate }\end{array}$ & FMM-701 and FMM-702 & gpm & 9,10 & \\
\hline $\begin{array}{l}\text { IRWST Fluid } \\
\text { Temperature }\end{array}$ & TF-701 and TF-709 & ${ }^{\circ} \mathrm{F}$ & 69,70 & \\
\hline $\begin{array}{l}\text { ADS 1-3 Separator } \\
\text { Pressure }\end{array}$ & PT-605 & psig & 51 & \\
\hline $\begin{array}{l}\text { ADS 1-3 Separator } \\
\text { Steam Flow Rate }\end{array}$ & FVM-601 & $\mathrm{scfm}$ & 16 & \\
\hline $\begin{array}{l}\text { ADS 1-3 Separator } \\
\text { Liquid Flow Rate }\end{array}$ & FMM-601 & gpm & 6 & \\
\hline
\end{tabular}




\begin{tabular}{|c|c|c|c|c|}
\hline \multicolumn{5}{|c|}{$\begin{array}{l}\text { TABLE 6-4 (Continued) } \\
\text { DATA PLOTS FOR QL REPORTS FOR DBA-04-D BY COMPONENT }\end{array}$} \\
\hline Component & Channel & Units & QL-Plot & Comment \\
\hline $\begin{array}{l}\text { ADS } 4-1 \text { and } 4-2 \\
\text { Separator Pressure }\end{array}$ & PT-611 and PT-610 & psig & 52,53 & \\
\hline $\begin{array}{l}\text { ADS 4-1 Separator } \\
\text { Steam Flow Rate }\end{array}$ & FVM-603 & $\operatorname{scfm}$ & 18 & \\
\hline $\begin{array}{l}\text { ADS 4-2 Separator } \\
\text { Steam Flow Rate }\end{array}$ & FVM-602 & scfm & 17 & \\
\hline $\begin{array}{l}\text { ADS 4-1 Separator } \\
\text { Liquid Flow Rate }\end{array}$ & FMM-603 & gpm & 8 & \\
\hline $\begin{array}{l}\text { ADS 4-2 Separator } \\
\text { Liquid Flow Rate }\end{array}$ & FMM-602 & gpm & 7 & \\
\hline Primary Sump Pressure & PT-901 & psig & 54 & \\
\hline $\begin{array}{l}\text { Primary Sump Liquid } \\
\text { Level }\end{array}$ & LDP-901 & inch of $\mathrm{H}_{2} \mathrm{O}$ & 41 & \\
\hline $\begin{array}{l}\text { Primary Sump } \\
\text { Injection Flow Rate }\end{array}$ & FMM-901 and FMM-902 & gpm & 13,14 & \\
\hline $\begin{array}{l}\text { Secondary Sump } \\
\text { Liquid Level }\end{array}$ & LDP-902 & inch of $\mathrm{H}_{2} \mathrm{O}$ & 42 & \\
\hline $\begin{array}{l}\text { Break Separator } \\
\text { Pressure }\end{array}$ & PT-905 & psig & 55 & \\
\hline $\begin{array}{l}\text { Break Separator Liquid } \\
\text { Level }\end{array}$ & LDP-905 & inch of $\mathrm{H}_{2} \mathrm{O}$ & 43 & \\
\hline $\begin{array}{l}\text { Break Separator Flow } \\
\text { to Primary Sump }\end{array}$ & FMM-905 & gpm & 15 & \\
\hline $\begin{array}{l}\text { BAMS Steam Flow } \\
\text { Rate }\end{array}$ & FVM-901 & $\mathrm{scfm}$ & 19 & \\
\hline $\begin{array}{l}\text { BAMS Steam Flow } \\
\text { Rate }\end{array}$ & FVM-902 & $\mathrm{scfm}$ & 20 & \\
\hline $\begin{array}{l}\text { BAMS/Primary Sump } \\
\text { Steam Flow Rate }\end{array}$ & FVM-903 & $\mathrm{scfm}$ & 21 & \\
\hline
\end{tabular}




\begin{tabular}{|c|c|c|c|c|}
\hline \multicolumn{5}{|c|}{$\begin{array}{l}\text { TABLE 6-4 (Continued) } \\
\text { DATA PLOTS FOR QL REPORTS FOR DBA-04-D BY COMPONENT }\end{array}$} \\
\hline Component & Channel & Units & QL-Plot & Comment \\
\hline $\begin{array}{l}\text { BAMS/Separator } \\
\text { Steam Flow Rate } \\
-6 \text { inch Pipe }\end{array}$ & FVM-905 & $\mathrm{scfm}$ & 22 & \\
\hline $\begin{array}{l}\text { BAMS/Exhaust Line } \\
\text { Temp. }\end{array}$ & TF-916 and SC-917 & ${ }^{\circ} \mathrm{F}$ & 71,72 & \\
\hline $\begin{array}{l}\text { PZR Heater Input } \\
\text { Power }\end{array}$ & KW-601 & $\mathrm{kW}$ & 27 & \\
\hline $\begin{array}{l}\text { Core Power Input } \\
\text { Power }\end{array}$ & $\begin{array}{l}\text { KW-101, KW-102, } \\
\text { KW-103, and KW-104 }\end{array}$ & $\mathrm{kW}$ & $23-26$ & \\
\hline $\begin{array}{l}\text { Reactor Vessel Liquid } \\
\text { Level Between Top of } \\
\text { Vessel - Upper Support } \\
\text { Plate }\end{array}$ & LDP-115 & $\begin{array}{l}\text { inches of } \\
\text { water }\end{array}$ & 28 & \\
\hline $\begin{array}{l}\text { Reactor Vessel Liquid } \\
\text { Level Between bottom } \\
\text { of Upper Support Plate } \\
\text { - Upper Core Spacer } \\
\text { Grid }\end{array}$ & LDP-139 & $\begin{array}{l}\text { inches of } \\
\text { water }\end{array}$ & 30 & \\
\hline $\begin{array}{l}\text { Inner Core } \\
\text { Thermocouple } \\
\text { Measuring Heater } \\
\text { Temperature }\end{array}$ & TH-103-4 & ${ }^{\circ} \mathrm{F}$ & 73 & \\
\hline $\begin{array}{l}\text { Outer Core } \\
\text { Thermocouple } \\
\text { Measuring Heater } \\
\text { Temperature }\end{array}$ & ТH-309-4 & ${ }^{\circ} \mathrm{F}$ & 74 & \\
\hline
\end{tabular}




\subsection{CONCLUSIONS}

The DBA-04-D tests were successfully completed, and the data logged in the DAS. All critical instruments were found to operate properly with the exception of those noted in Section 6.4. The test was found to be acceptable. 
APPENDIX A

DATA PLOTS 

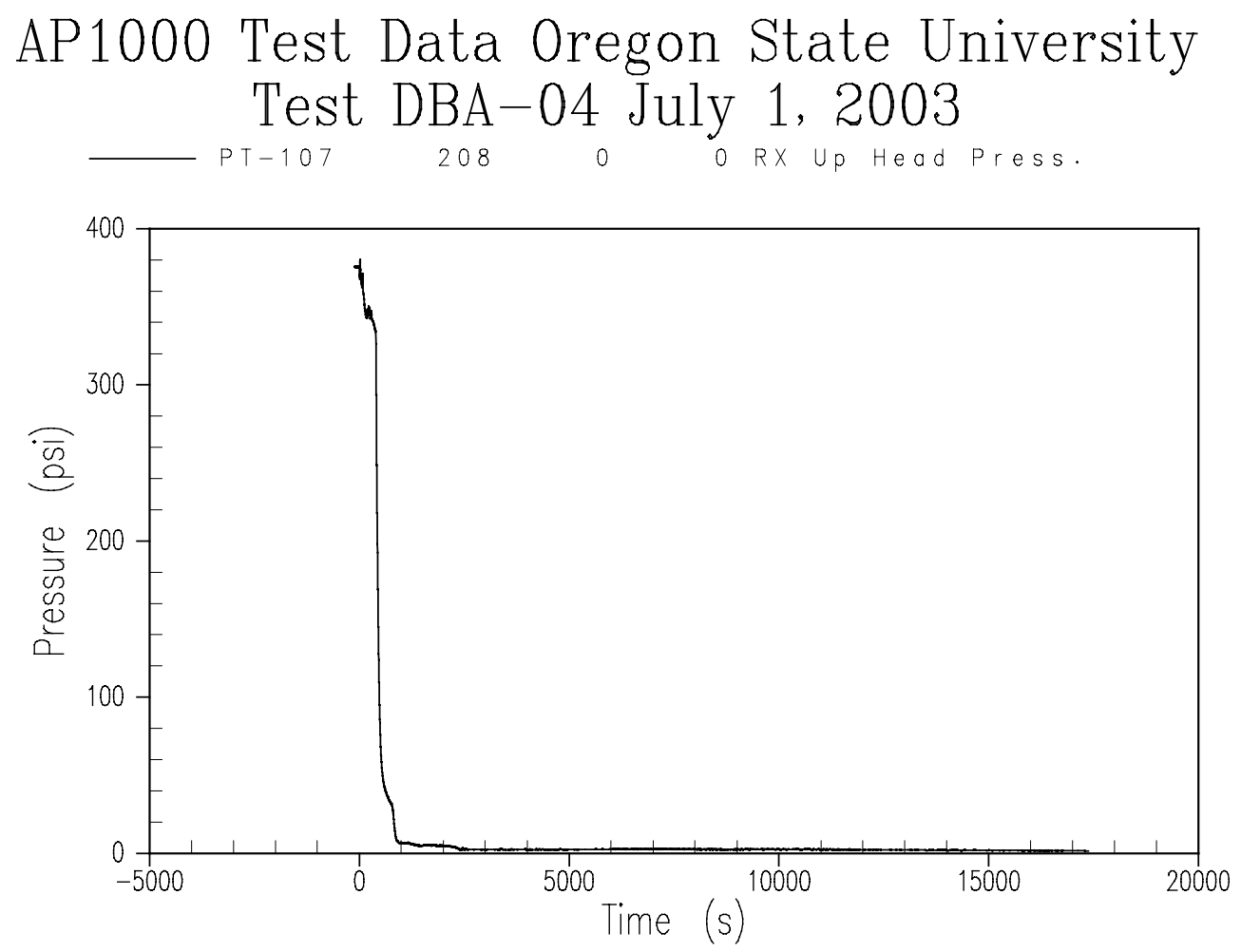

Figure A-1 Reactor Vessel Pressure 


\section{AP1000 Test Data Oregon State University Test DBA-04 July 1, 2003 \\ $\begin{array}{llll}\text { LDP-127 } & 136 & 0 & 0\end{array}$}

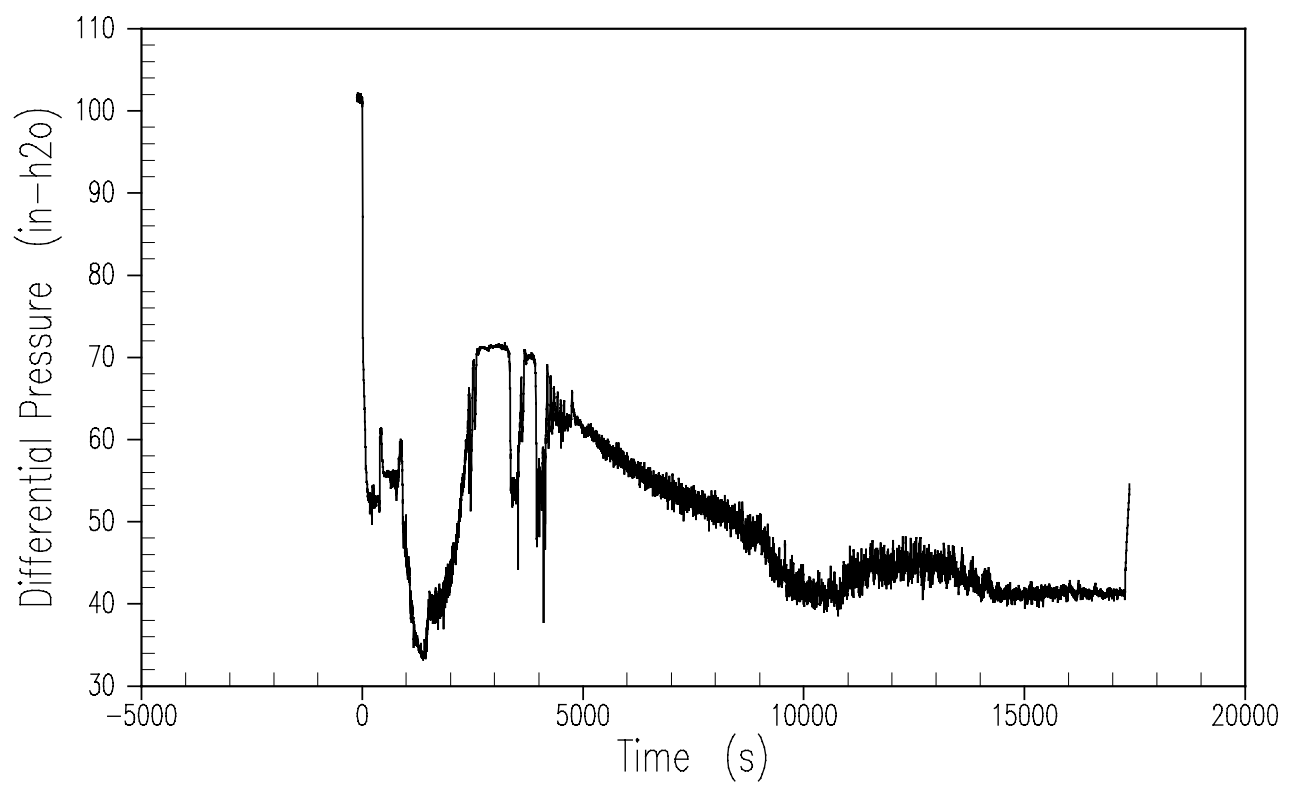

Figure A-2 Reactor Vessel Level 
AP1000 Test Data Oregon State University Test DBA-04 July 1, 2003

- LDP-140 $139 \quad$ o $\quad$ o Bot. of RX-Bot. of F

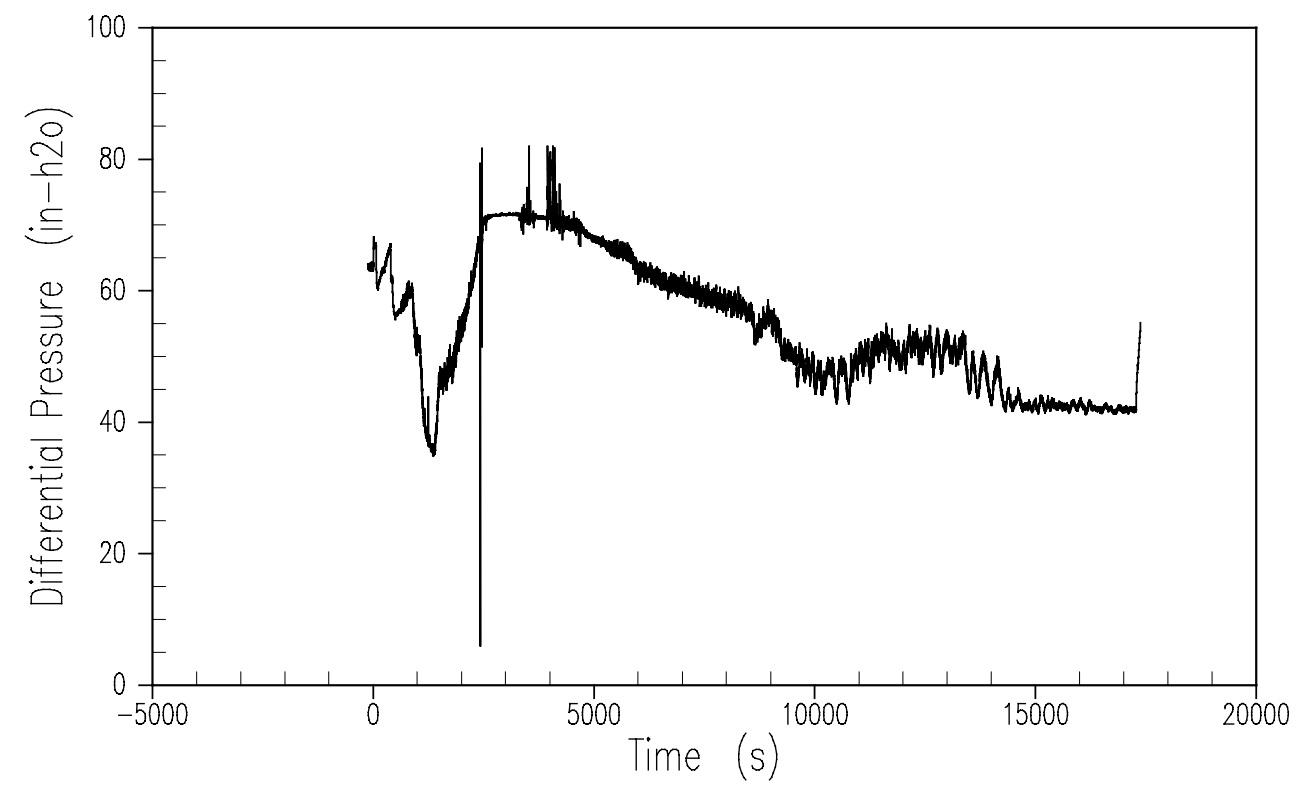

Figure A-3 Reactor Vessel Downcomer Level 

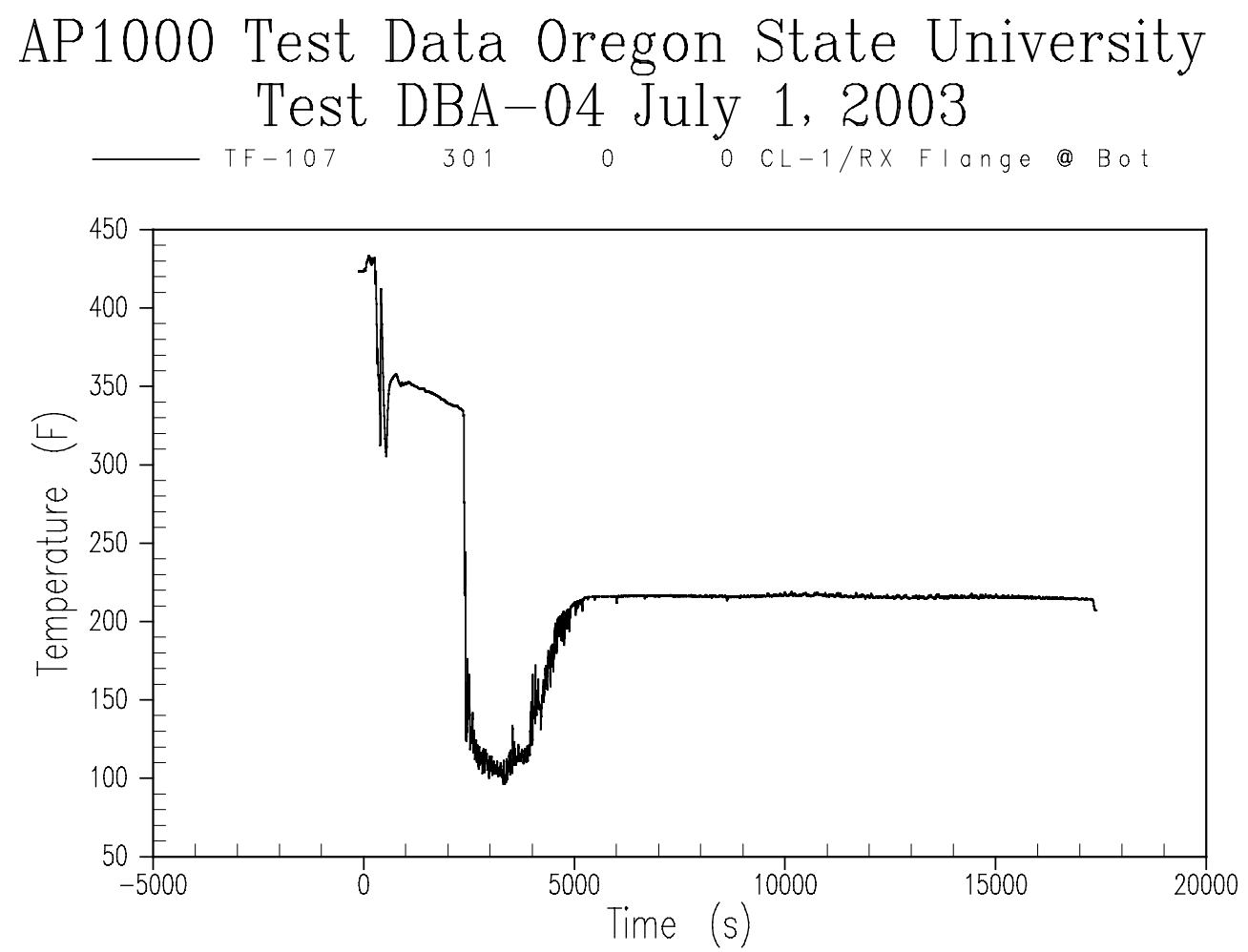

Figure A-4 Cold Leg 1 Fluid Temperature 

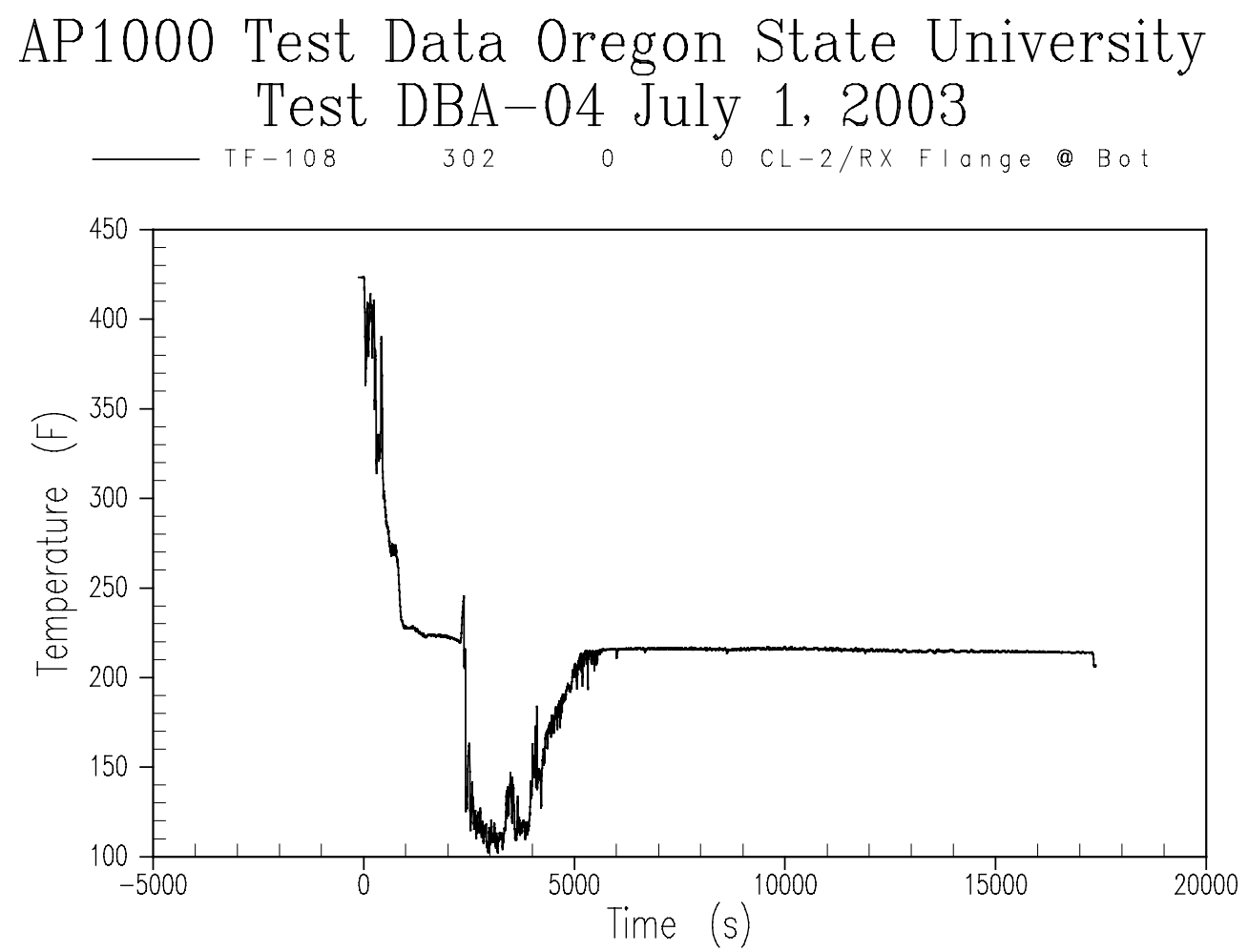

Figure A-5 Cold Leg 2 Fluid Temperature 

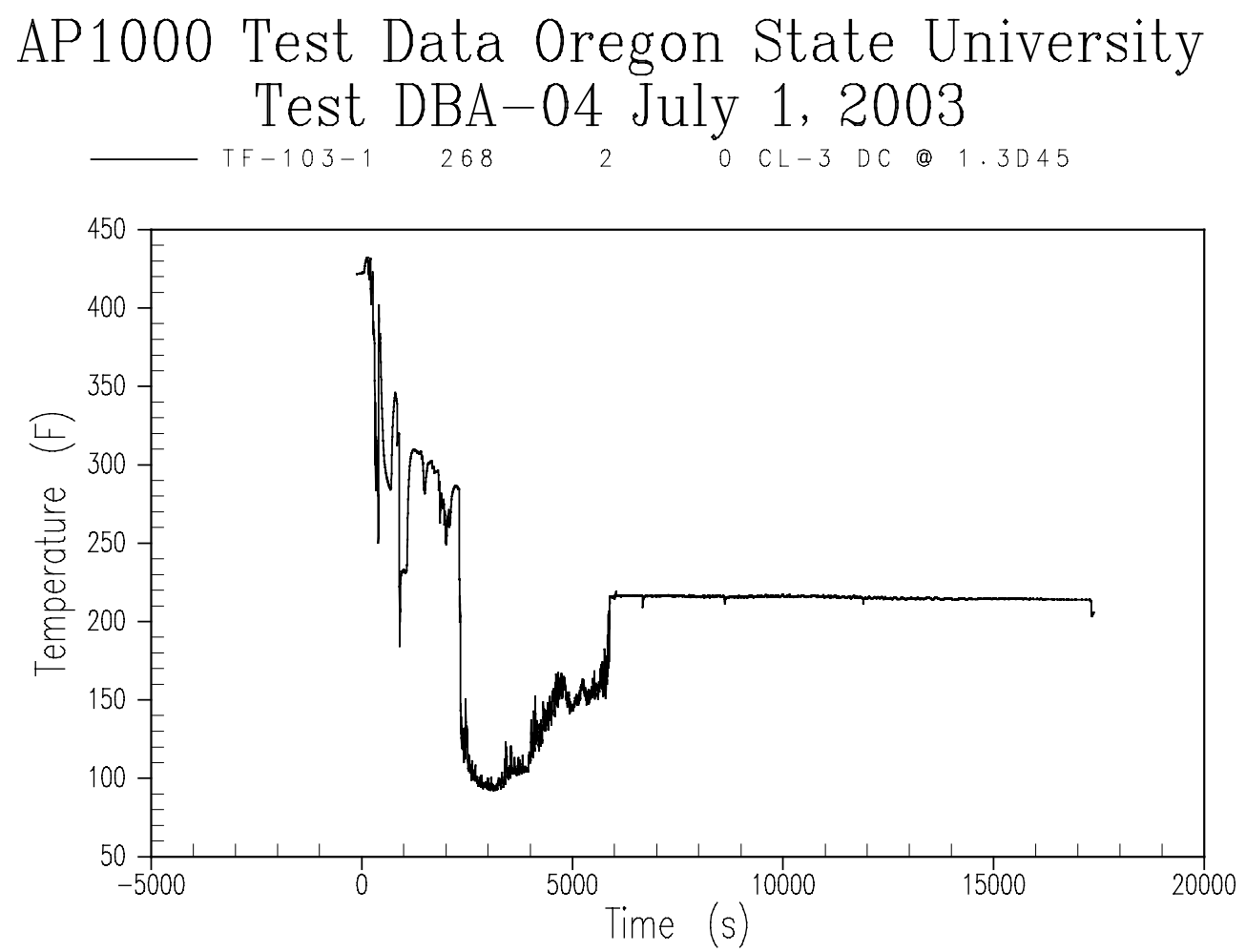

Figure A-6 Cold Leg 3 Fluid Temperature 

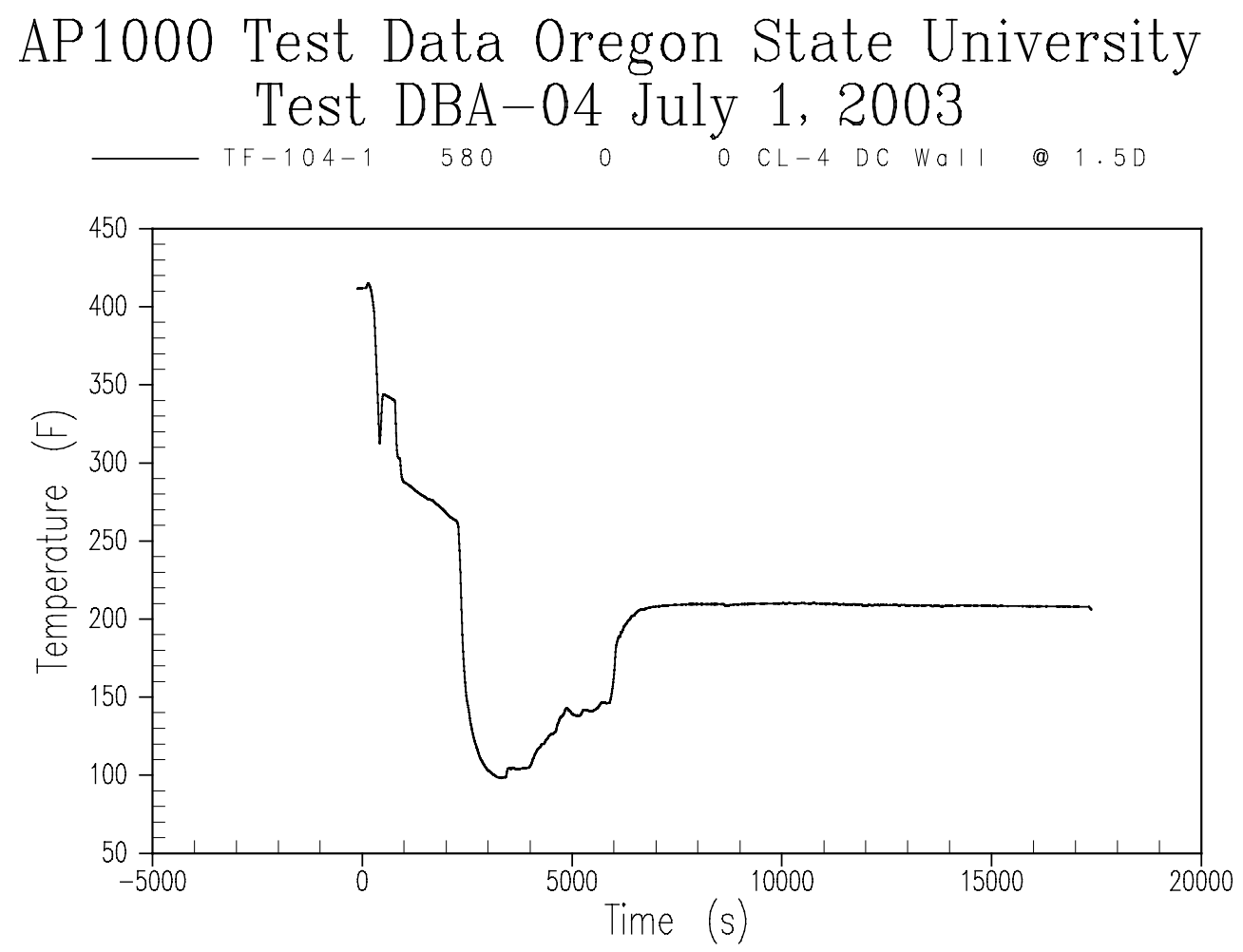

Figure A-7 Cold Leg 4 Fluid Temperature 

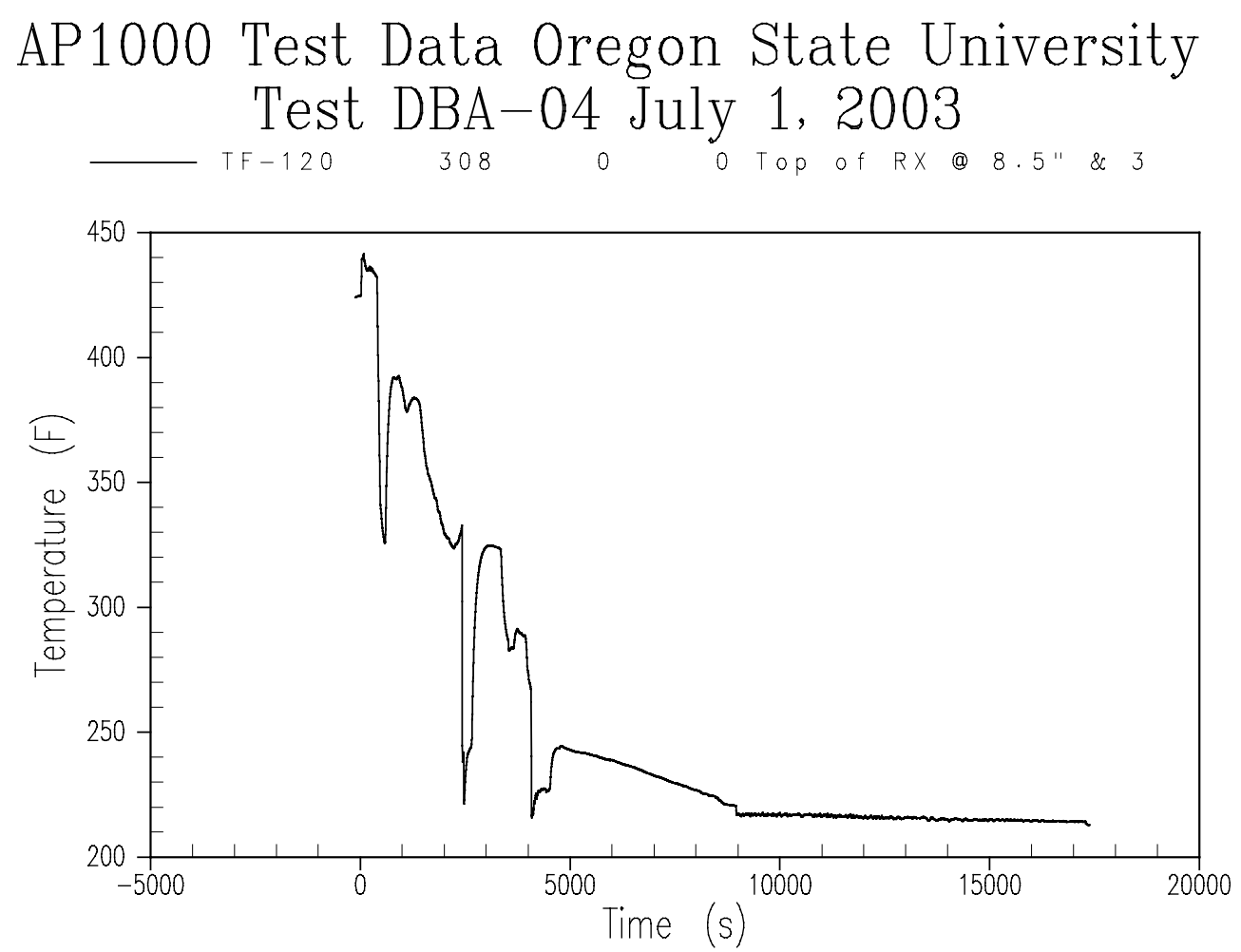

Figure A-8 Reactor Vessel Fluid Temperature Upper Head 

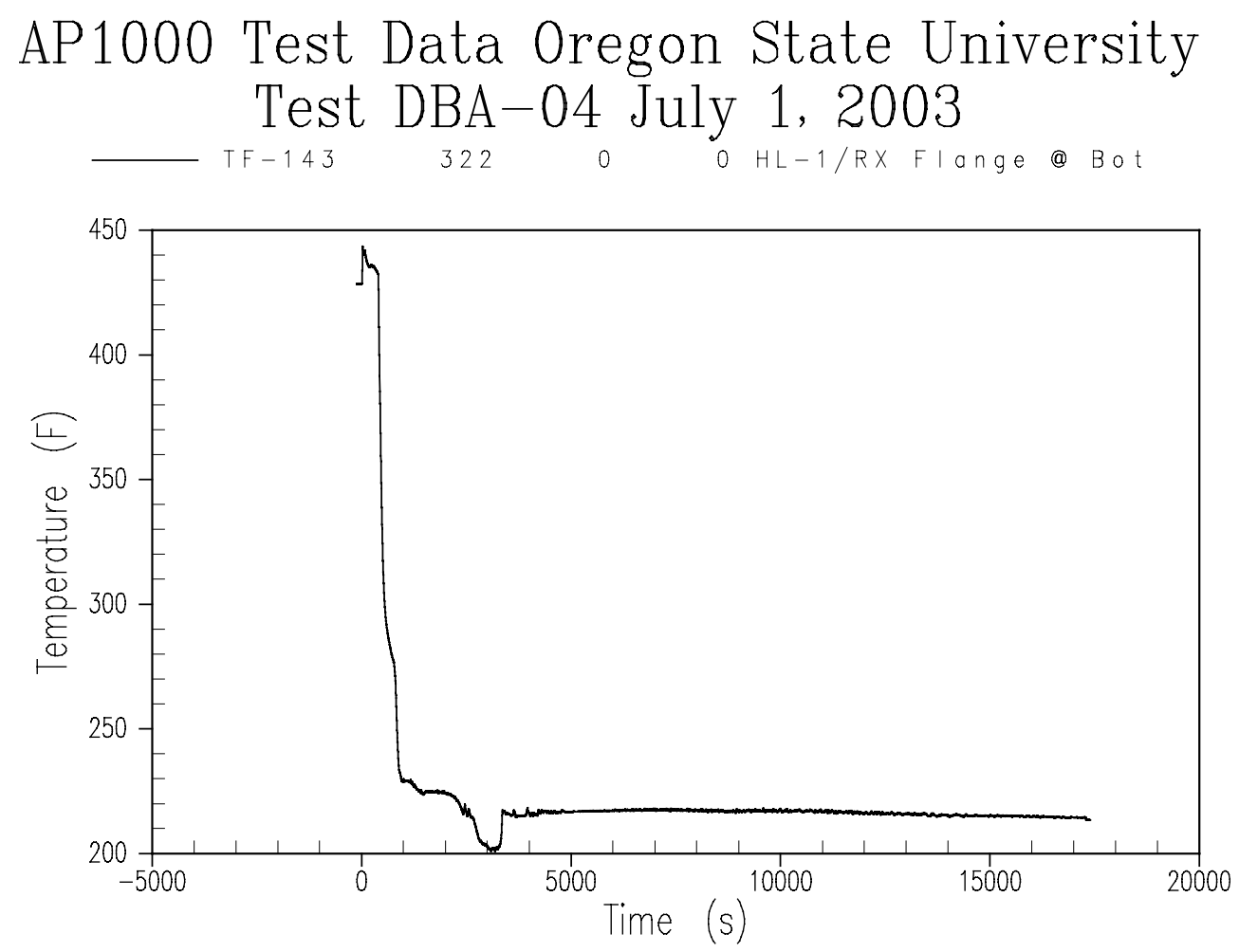

Figure A-9 Reactor Coolant System Hot Leg 1 Temperature 

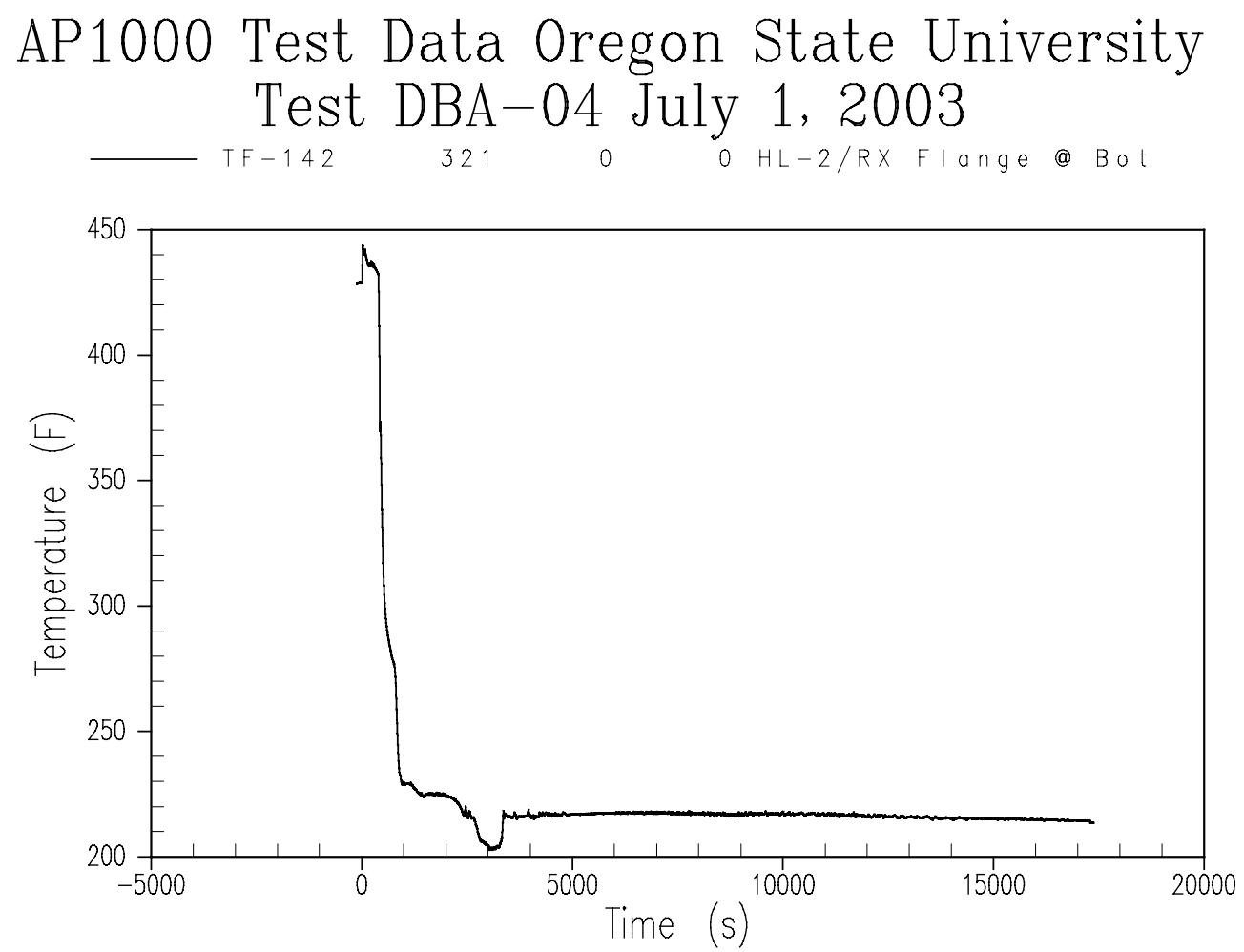

Figure A-10 Reactor Coolant System Hot Leg 2 Temperature 

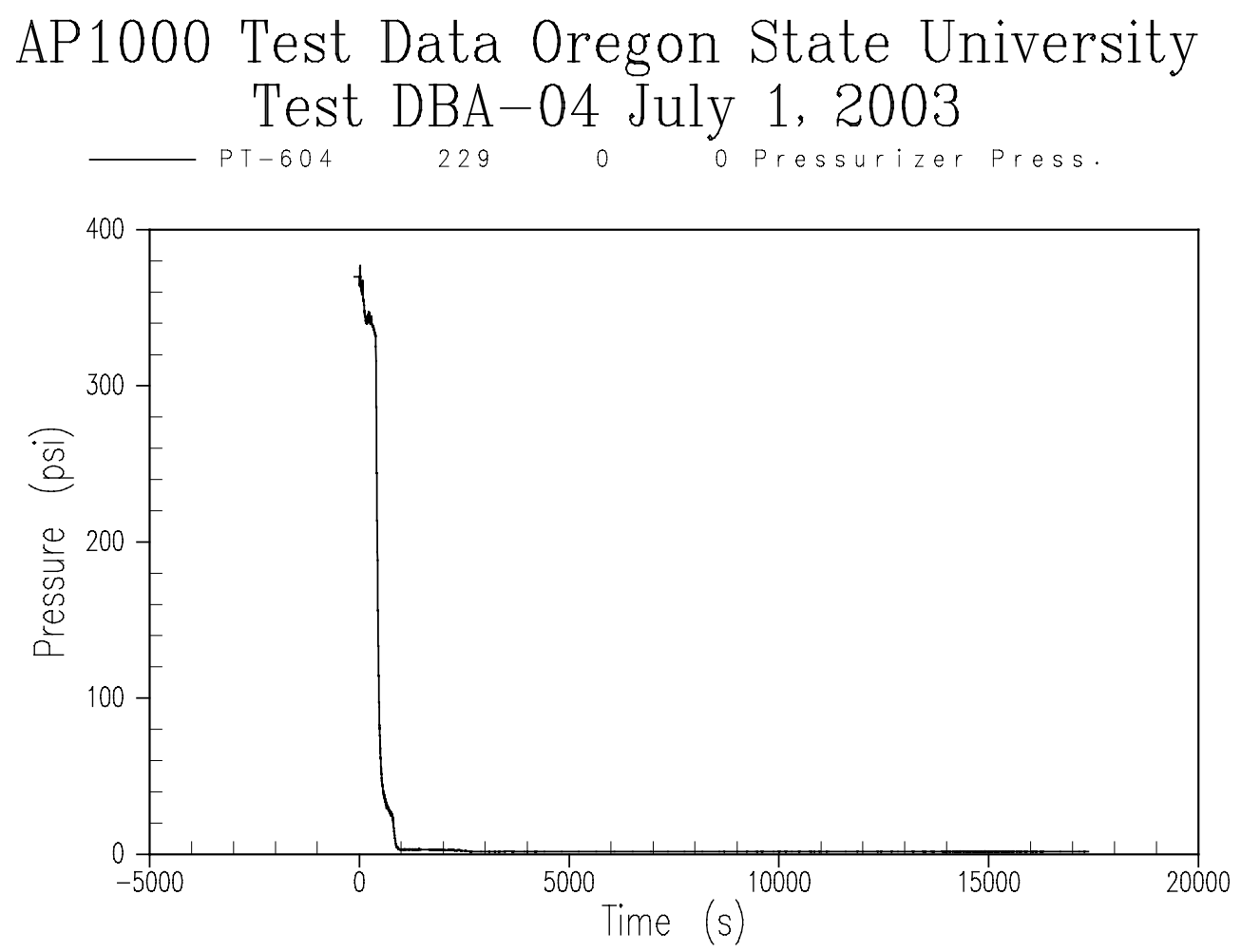

Figure A-11 Pressurizer Pressure - Wide Range 

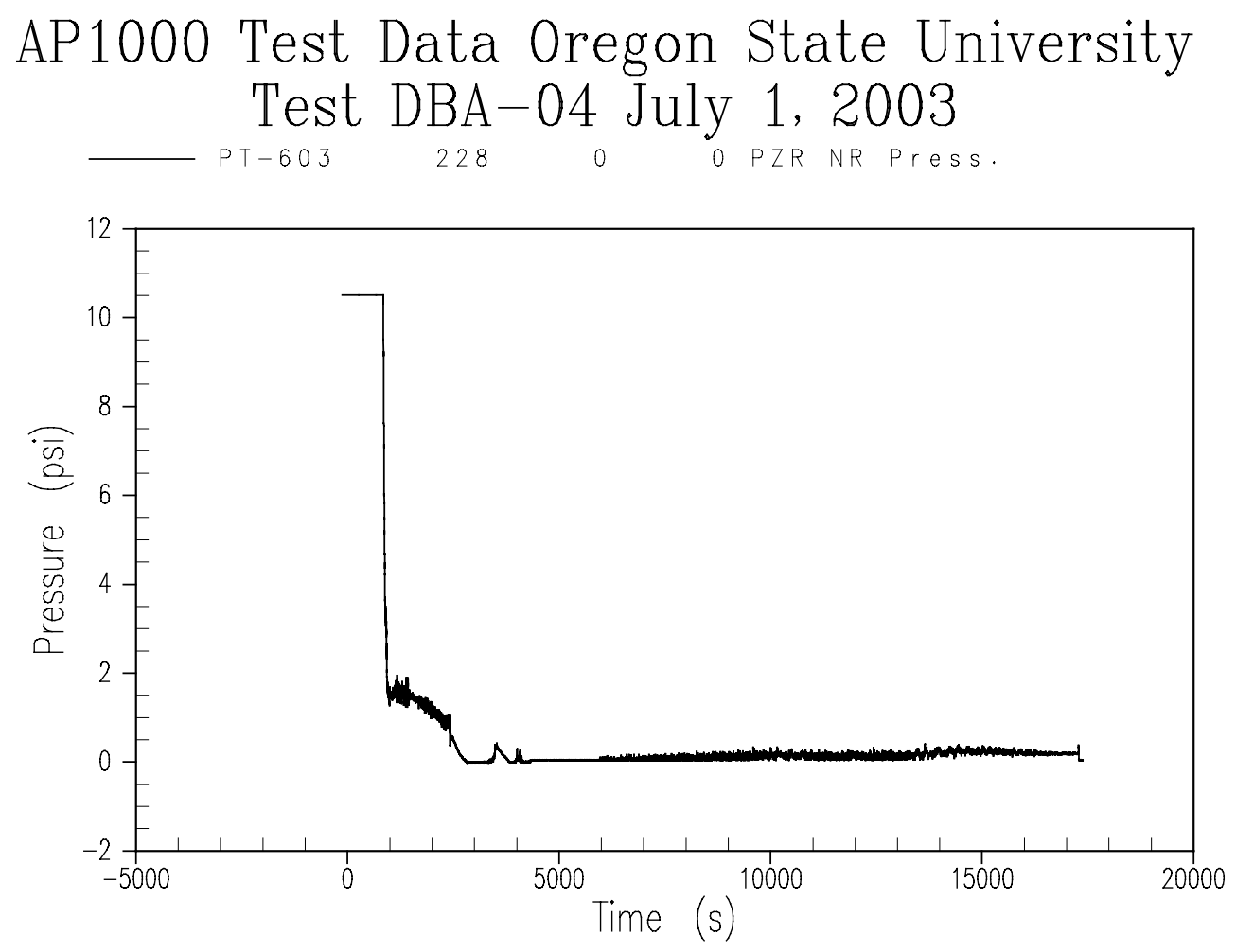

Figure A-12 Pressurizer Pressure - Narrow Range 


\section{AP1000 Test Data Oregon State University Test DBA-04 July 1, 2003 \\ $\begin{array}{llll}\text { LDP-601 } & 179 & 0 & 0\end{array}$}

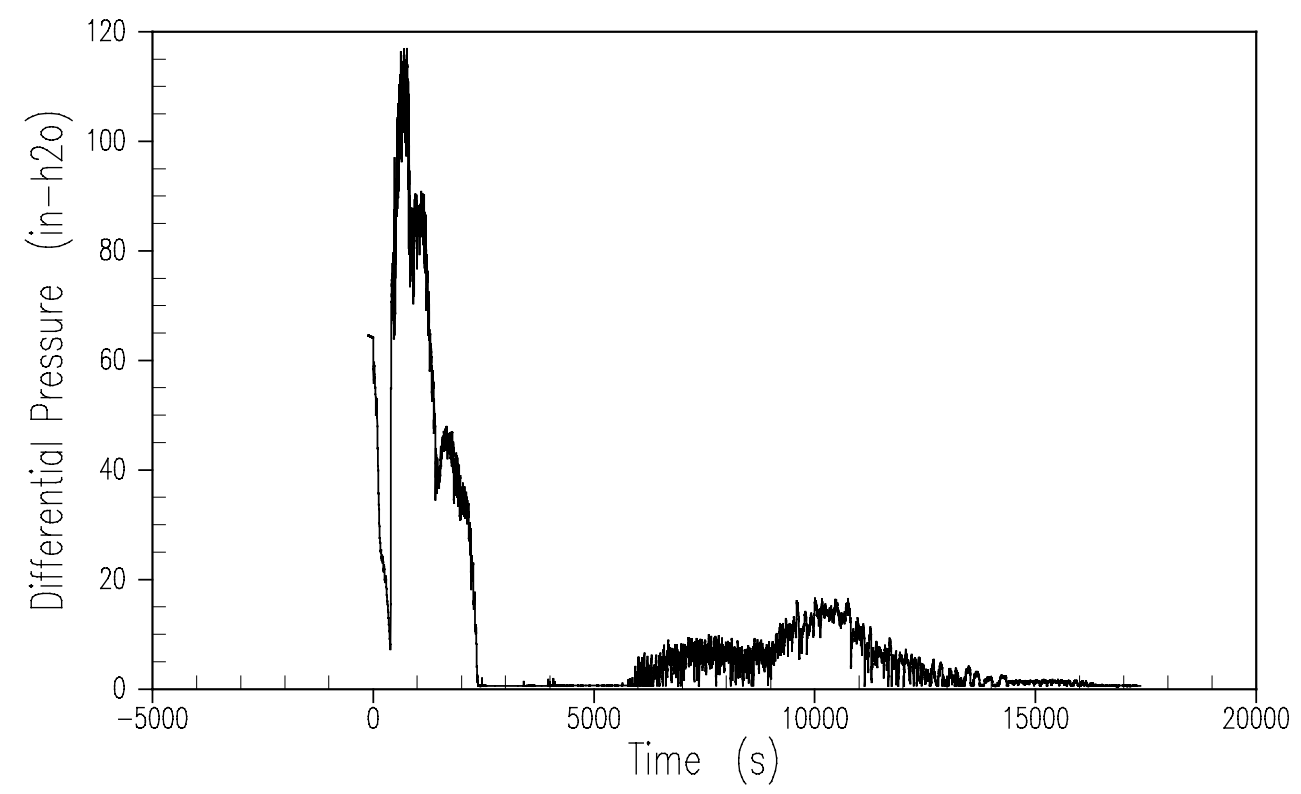

Figure A-13 Pressurizer Liquid Level 

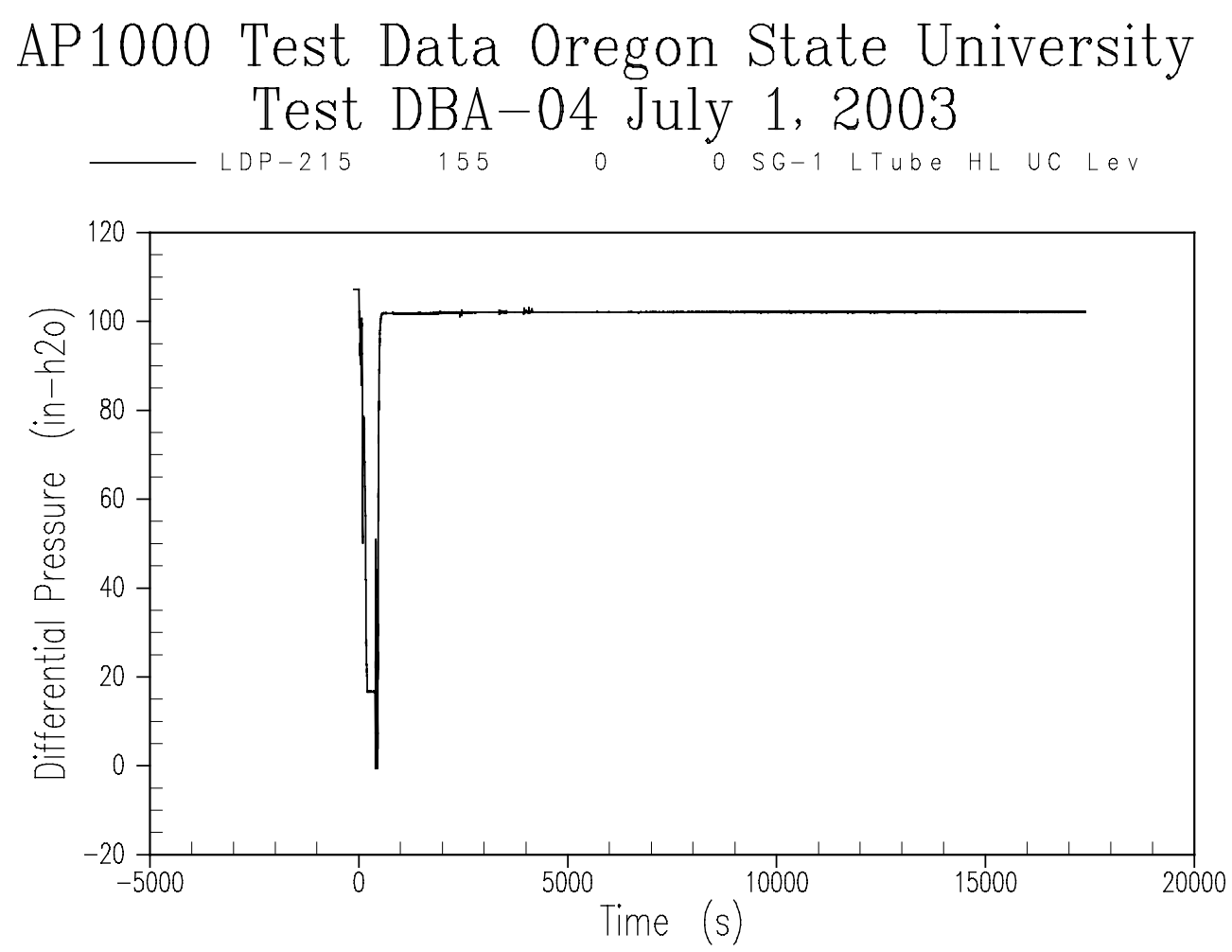

Figure A-14 Steam Generator 1 Tube Level 

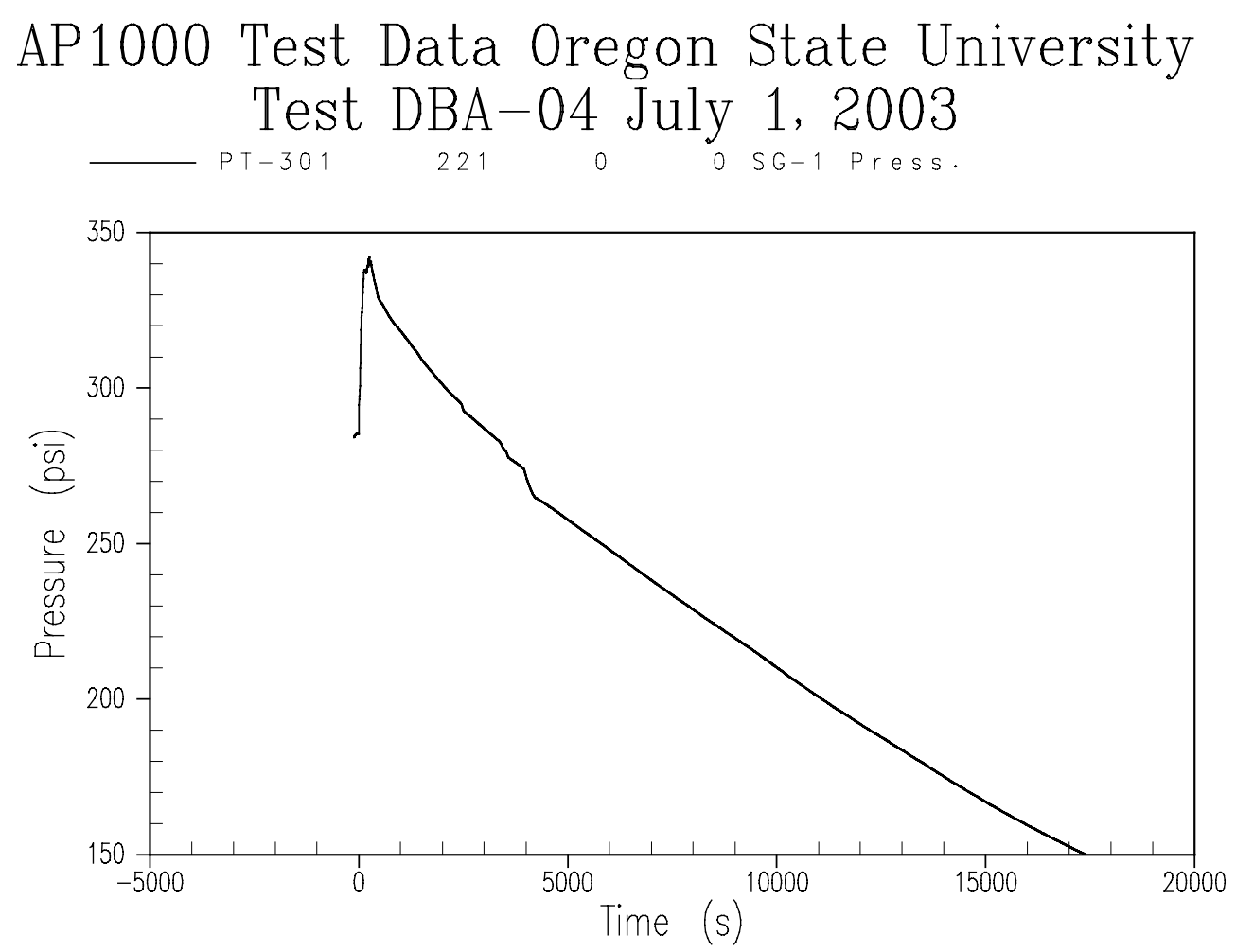

Figure A-15 Steam Generator 1 Secondary Pressure 
AP1000 Test Data Oregon State University Test DBA-04 July 1, 2003

$\begin{array}{llll}\text { FMM-001 } & 50 & 0 & \text { O SG-1 Feed FI OW }\end{array}$

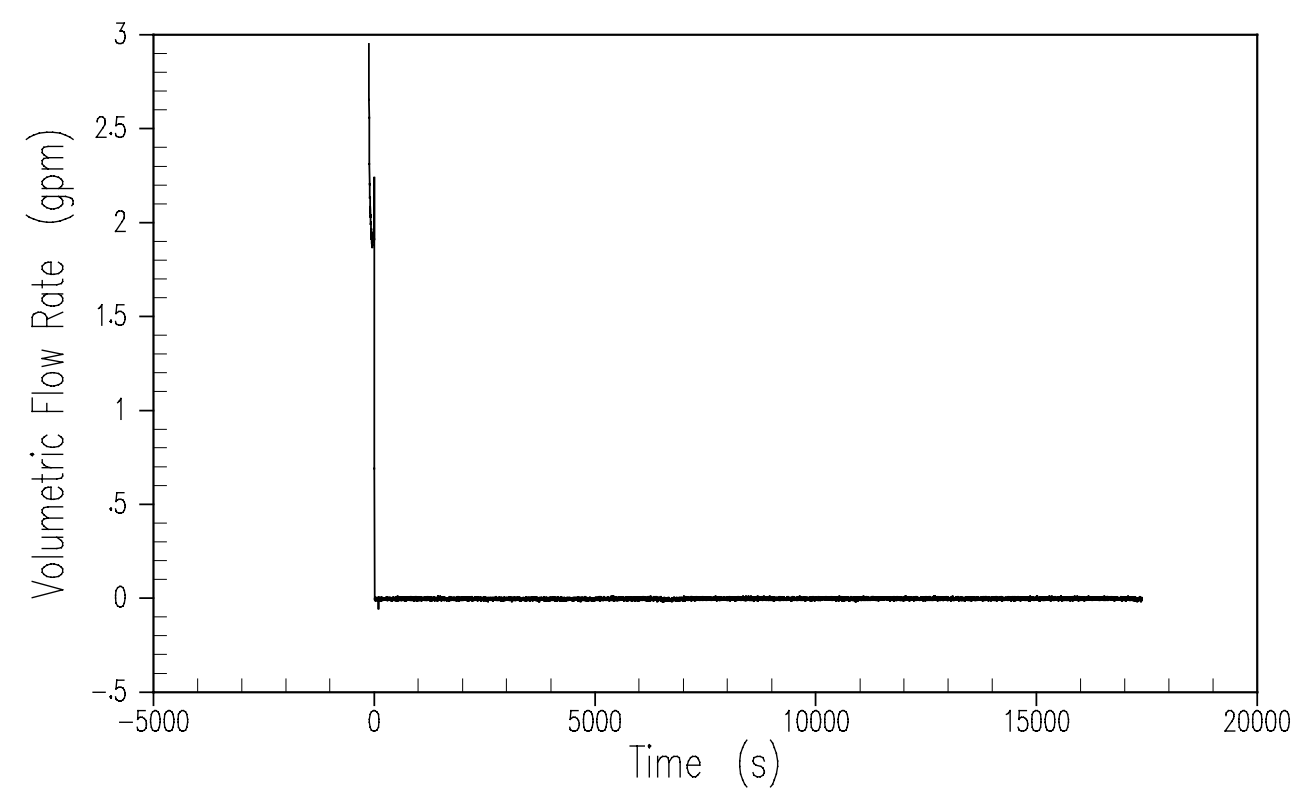

Figure A-16 Steam Generator 1 Feed Flow Rate 

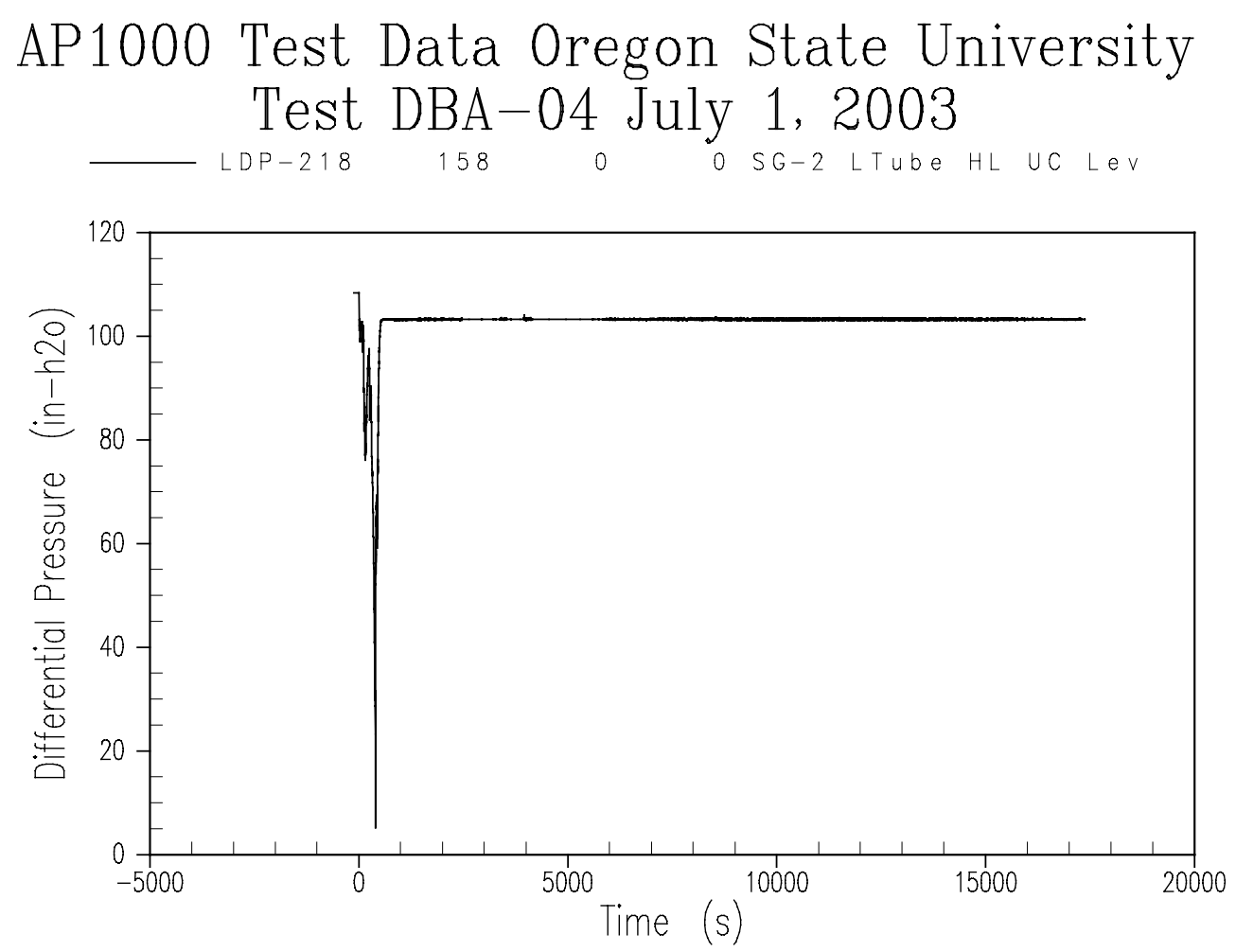

Figure A-17 Steam Generator 2 Tube Level 

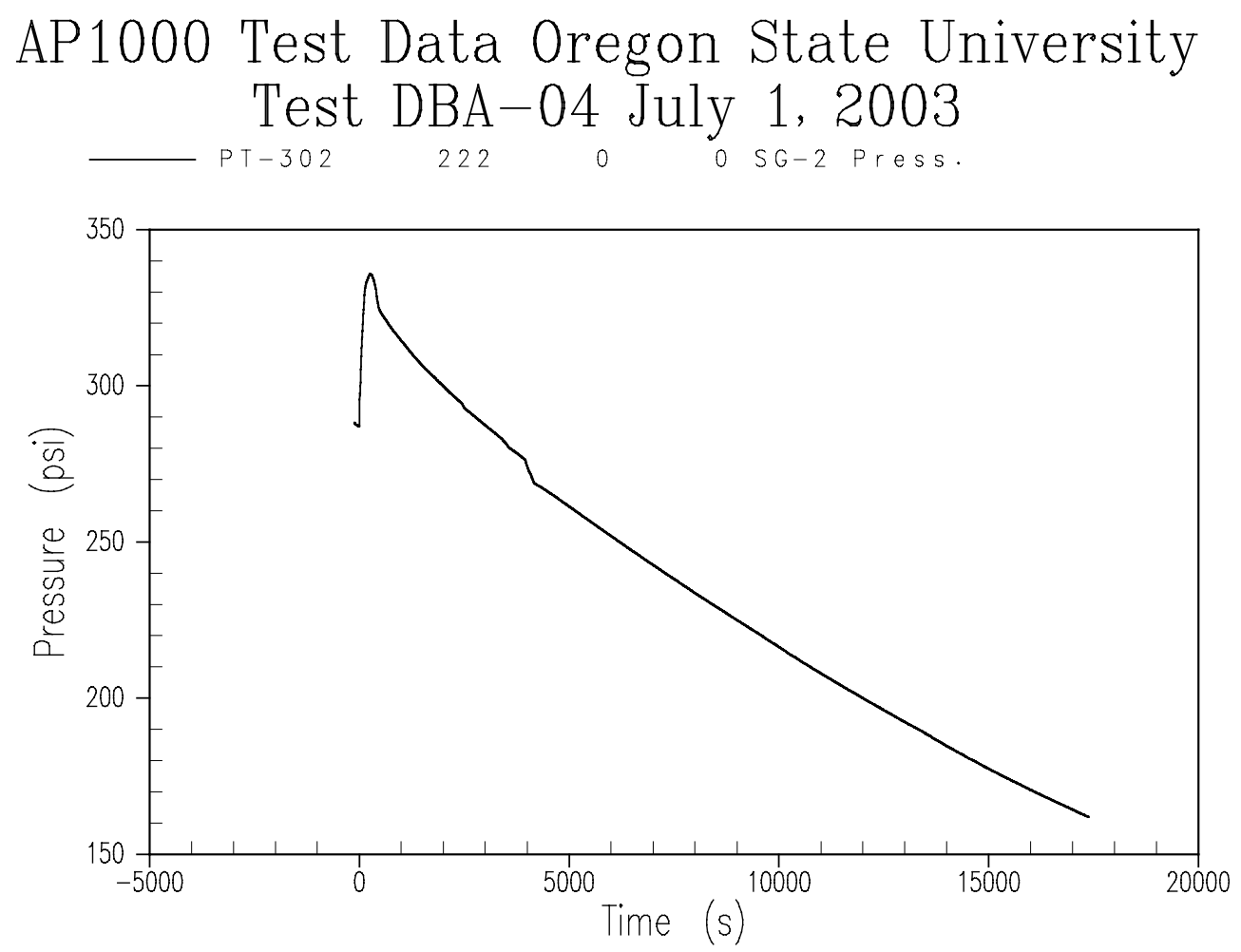

Figure A-18 Steam Generator 2 Secondary Pressure 


\section{AP1000 Test Data Oregon State University Test DBA-04 July 1, 2003}

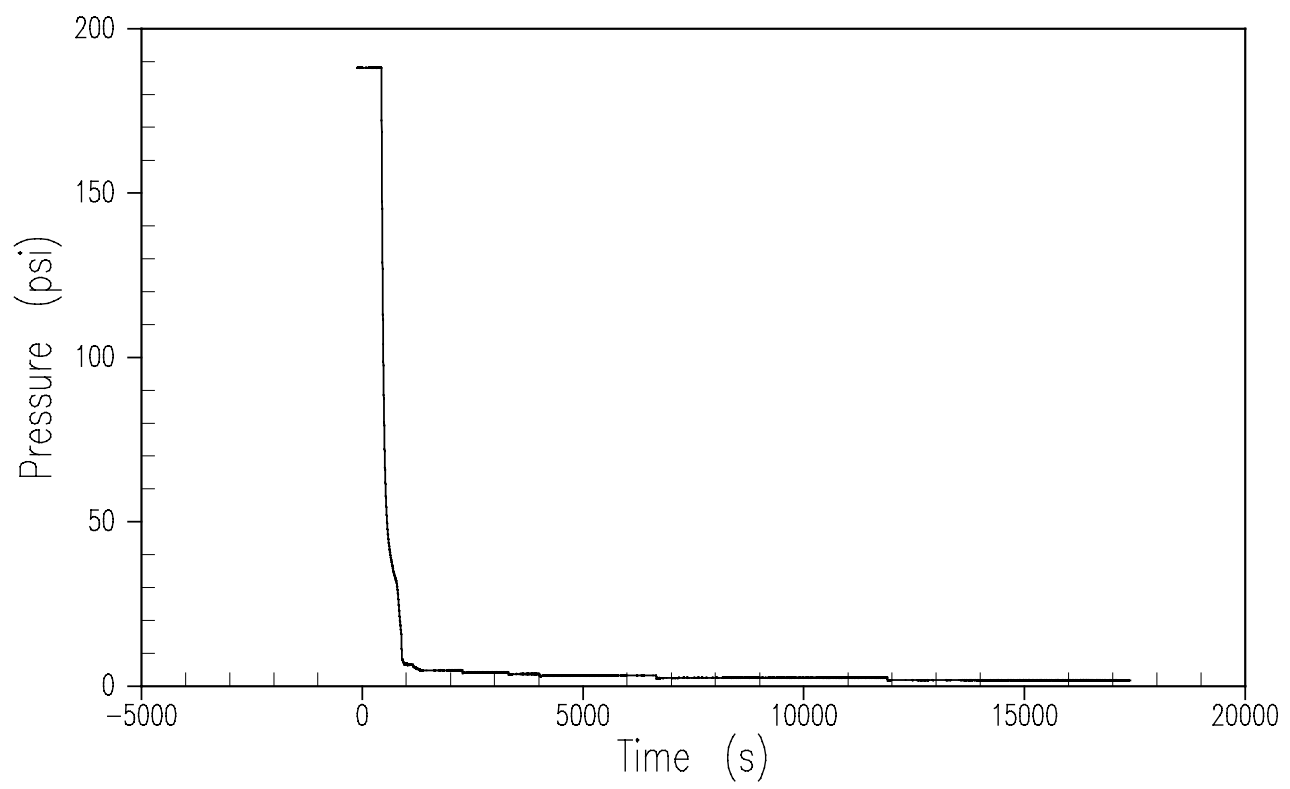

Figure A-19 Accumulator 1 Pressure 


\section{AP1000 Test Data Oregon State University Test DBA-04 July 1, 2003}

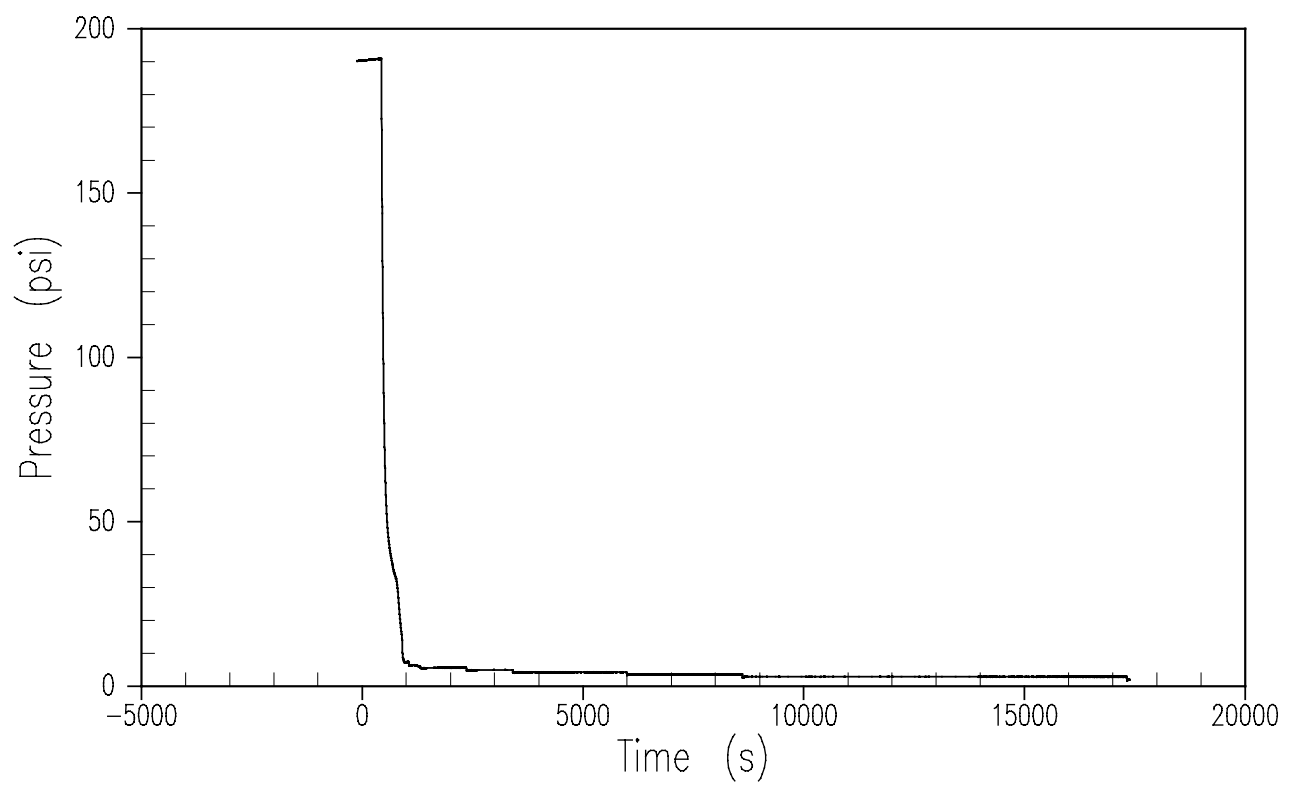

Figure A-20 Accumulator 2 Pressure 

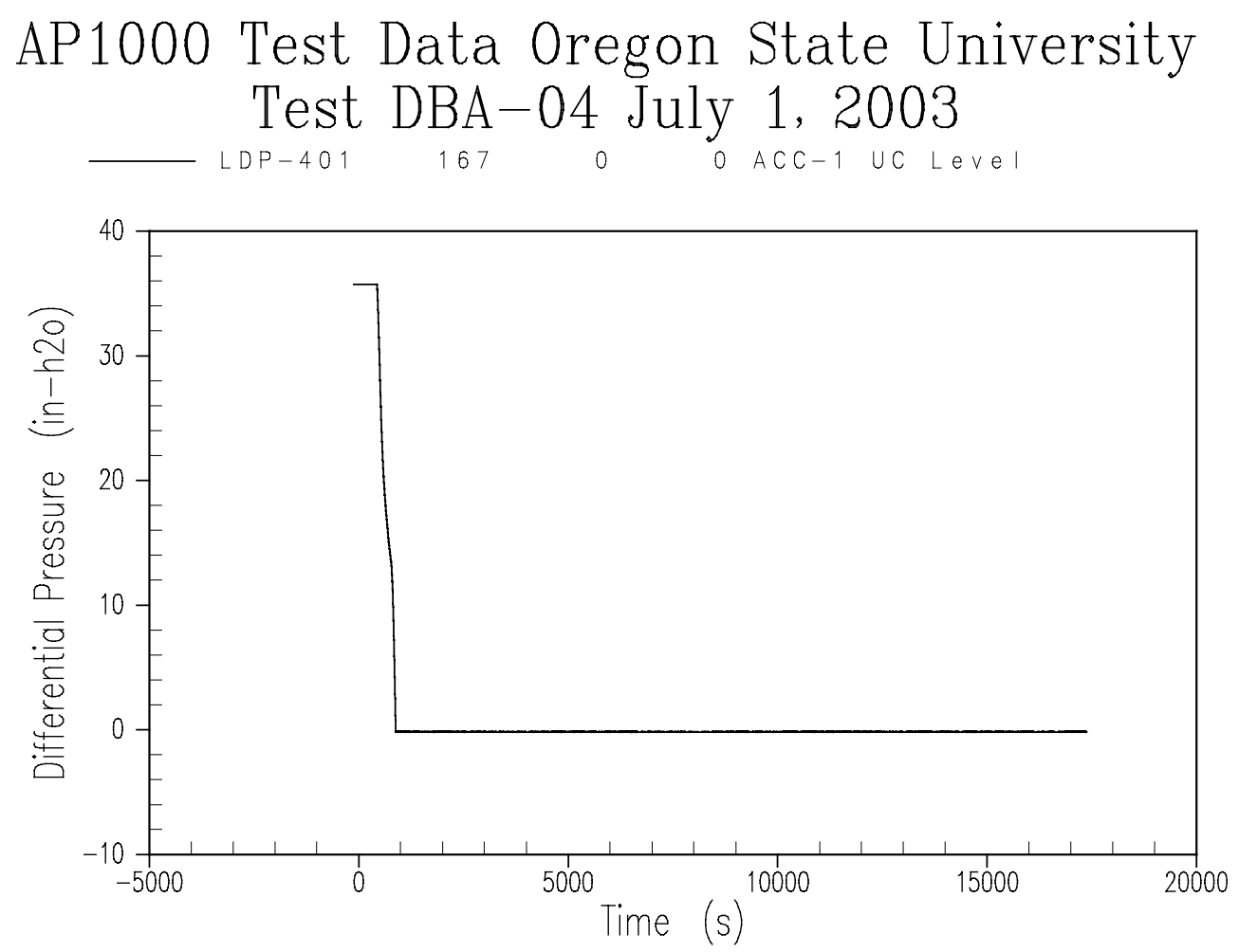

Figure A-21 Accumulator 1 Liquid Level 

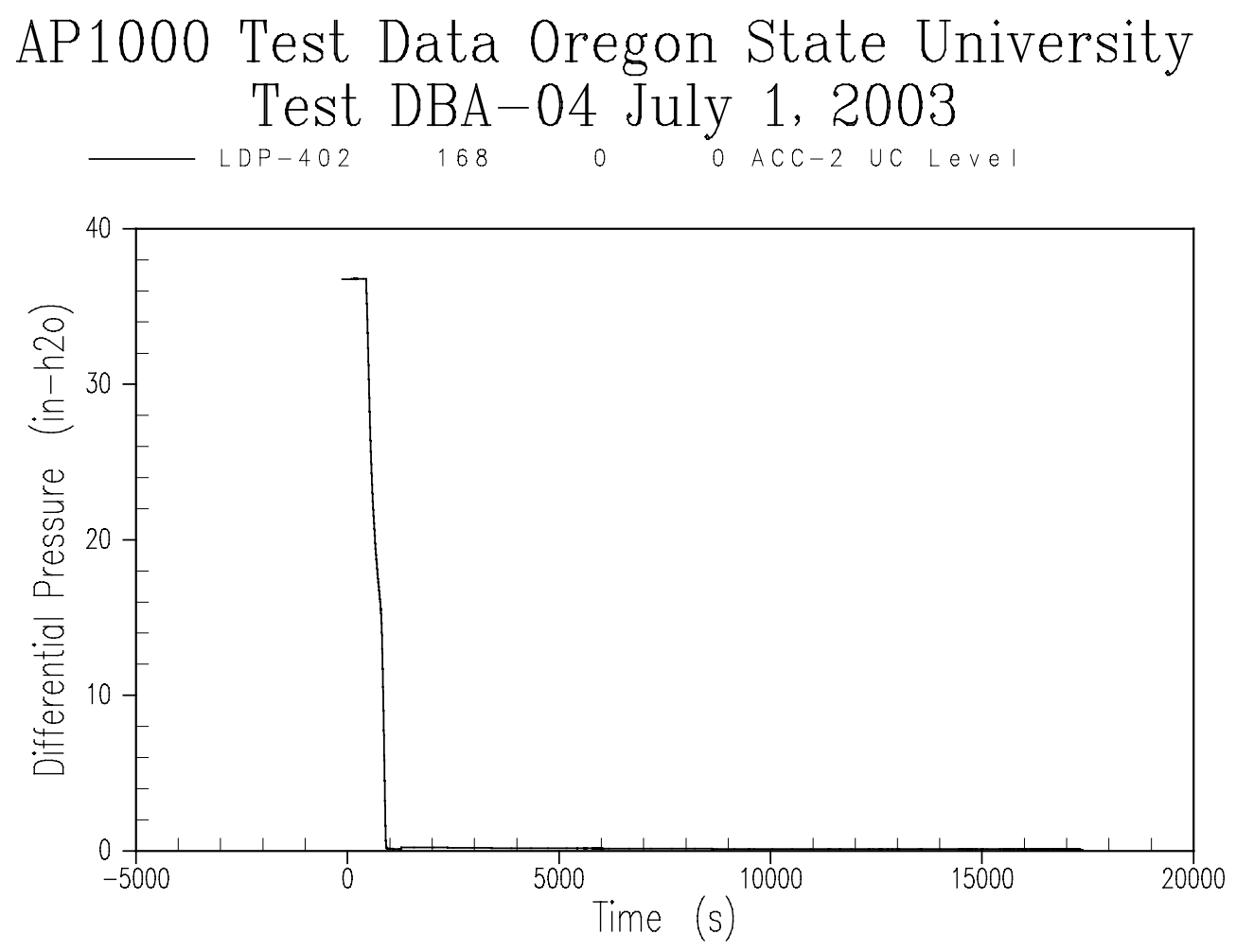

Figure A-22 Accumulator 2 Liquid Level 


\section{AP1000 Test Data Oregon State University Test DBA-04 July 1, 2003 \\ $\begin{array}{lllllll}F M M-401 & 58 & 0 & 0 & A C C-1 & \text { In j. F I OW }\end{array}$}

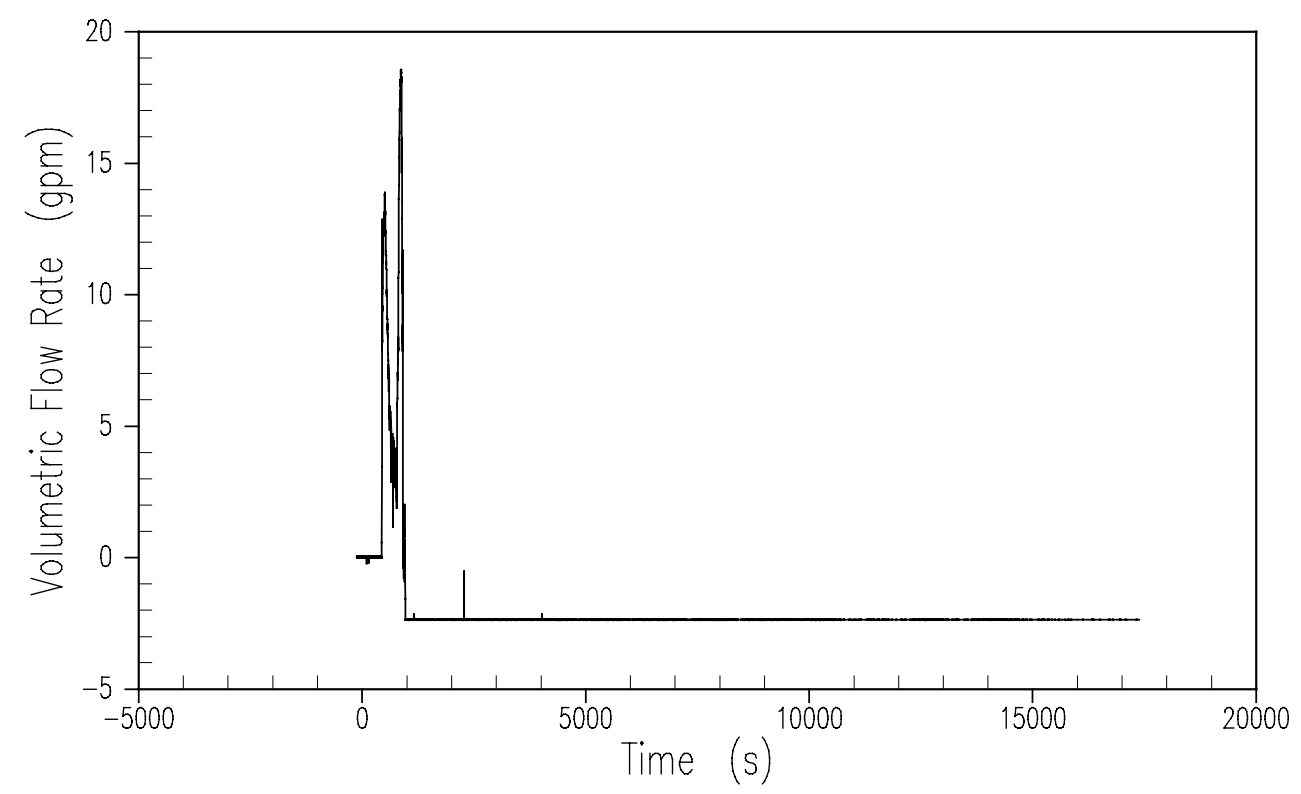

Figure A-23 Accumulator 1 Flow Rate 


\section{AP1000 Test Data Oregon State University Test DBA-04 July 1, 2003 \\ $\begin{array}{lllllll}F M M-402 & 59 & 0 & 0 & A C C-2 & \text { Inj. F I ow }\end{array}$}

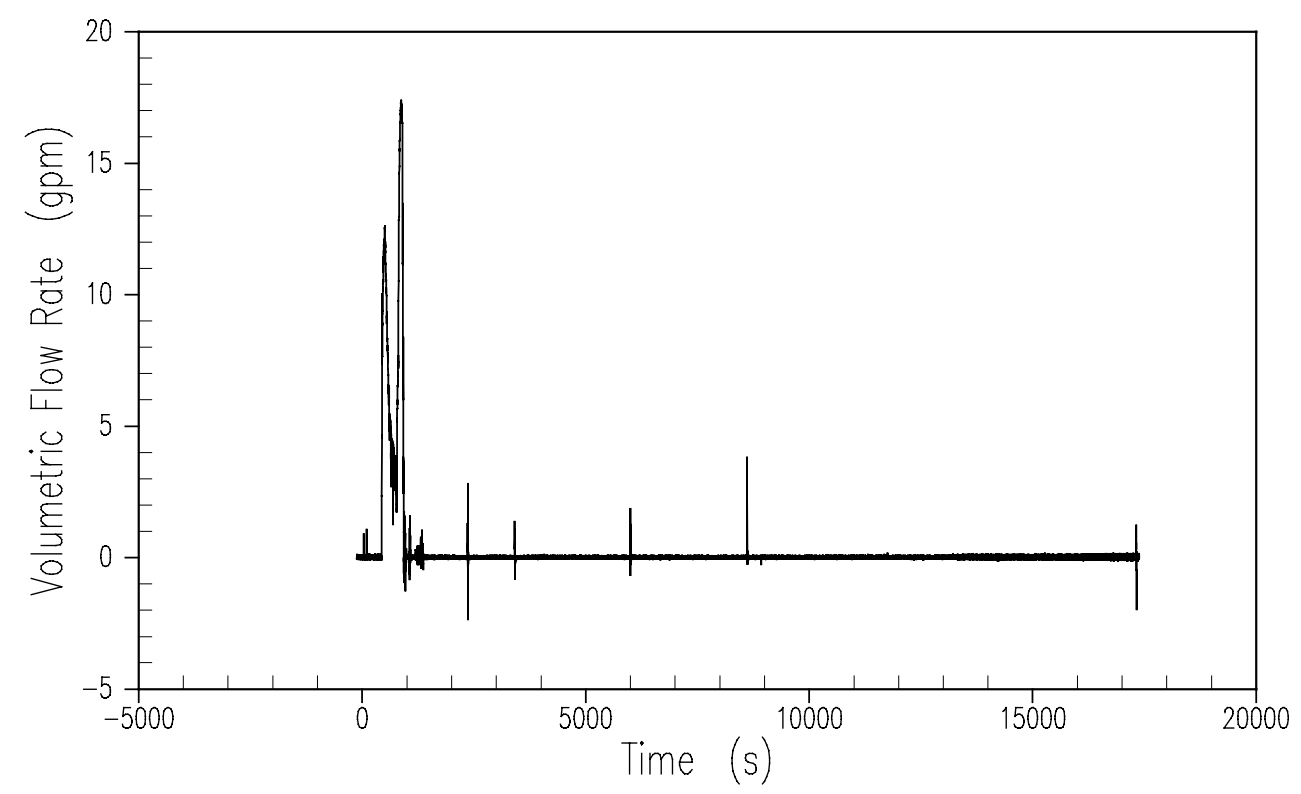

Figure A-24 Accumulator 2 Flow Rate 

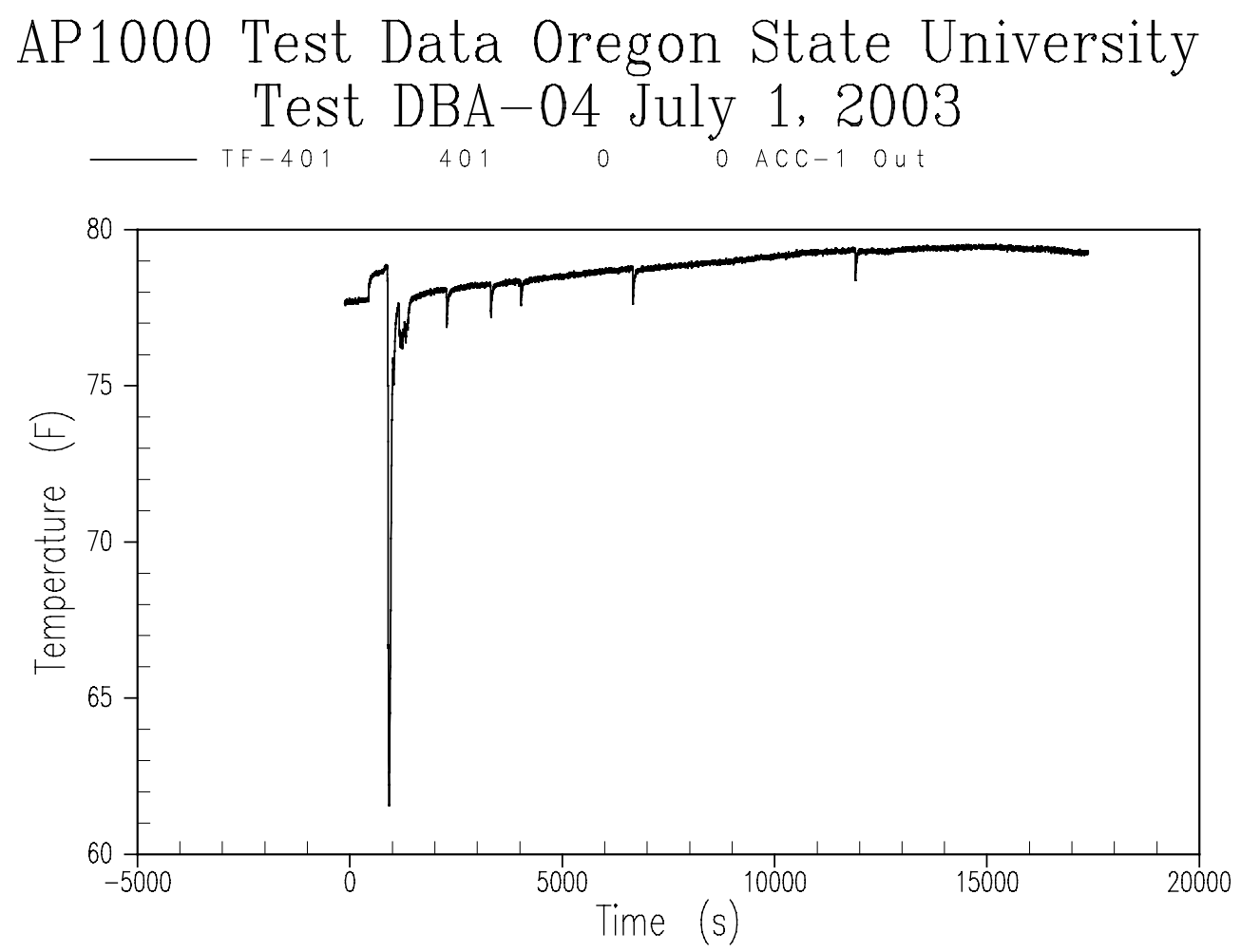

Figure A-25 Accumulator 1 Liquid Discharge Temperature 

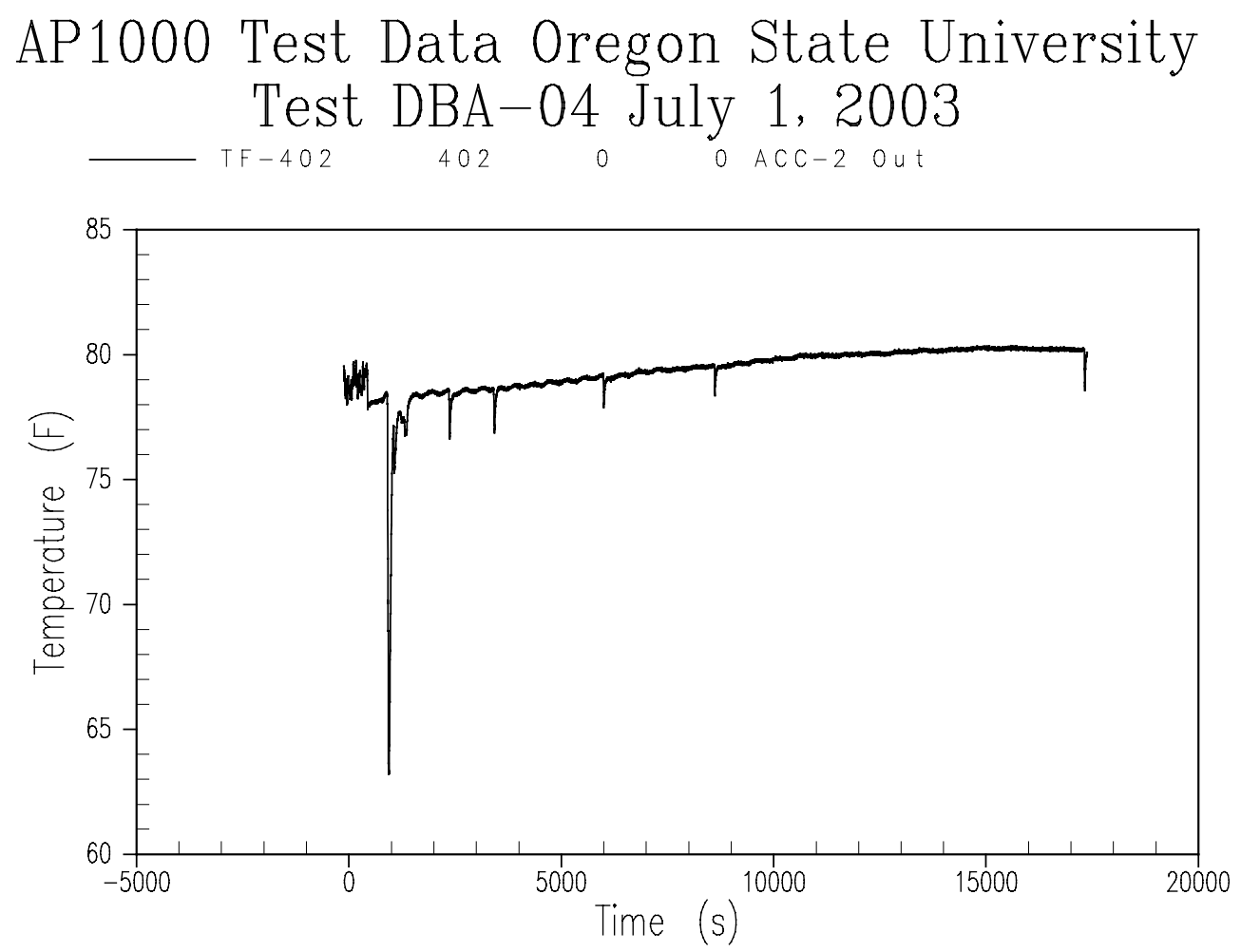

Figure A-26 Accumulator 2 Liquid Discharge Temperature 

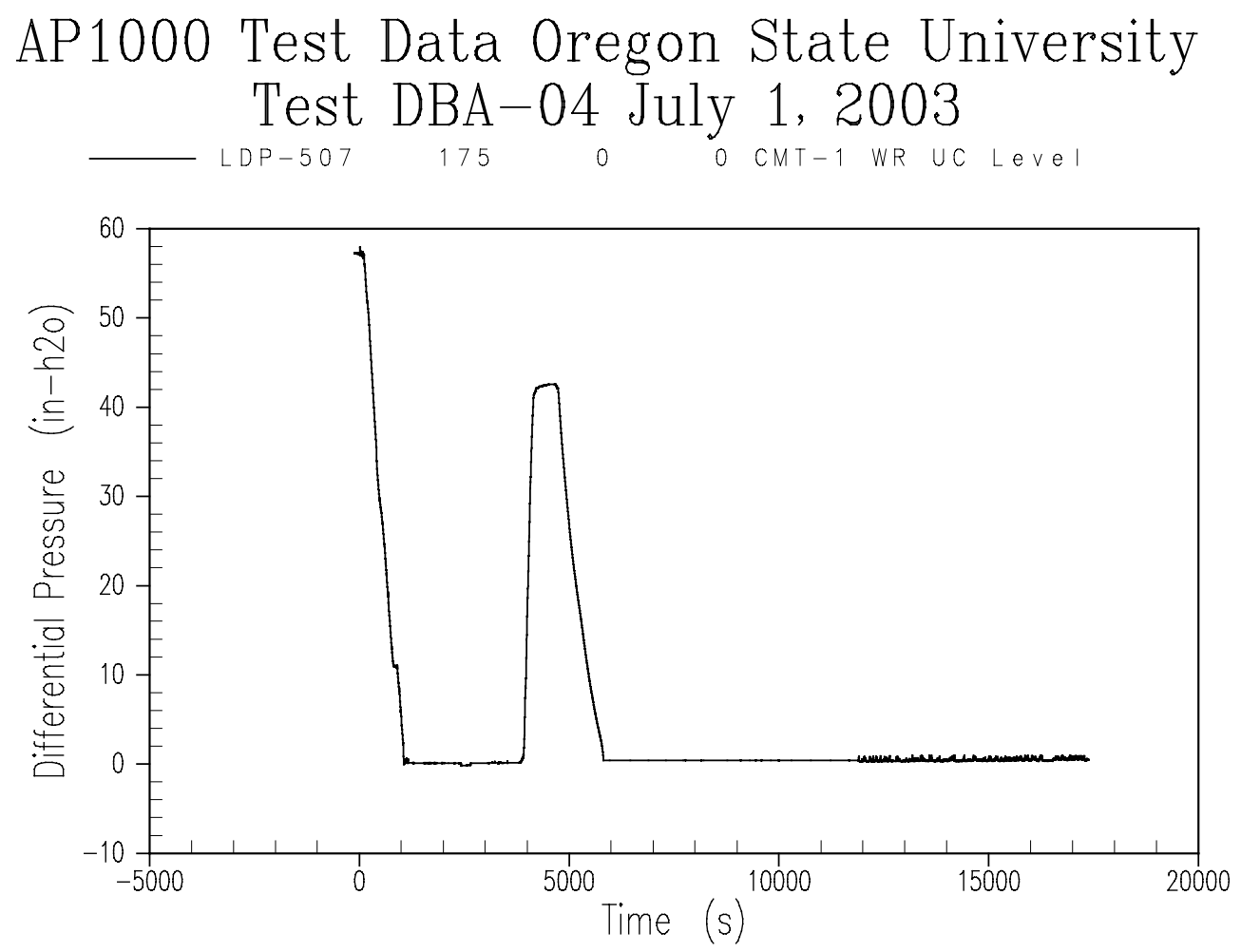

Figure A-27 Core Makeup Tank 1 Liquid Level 

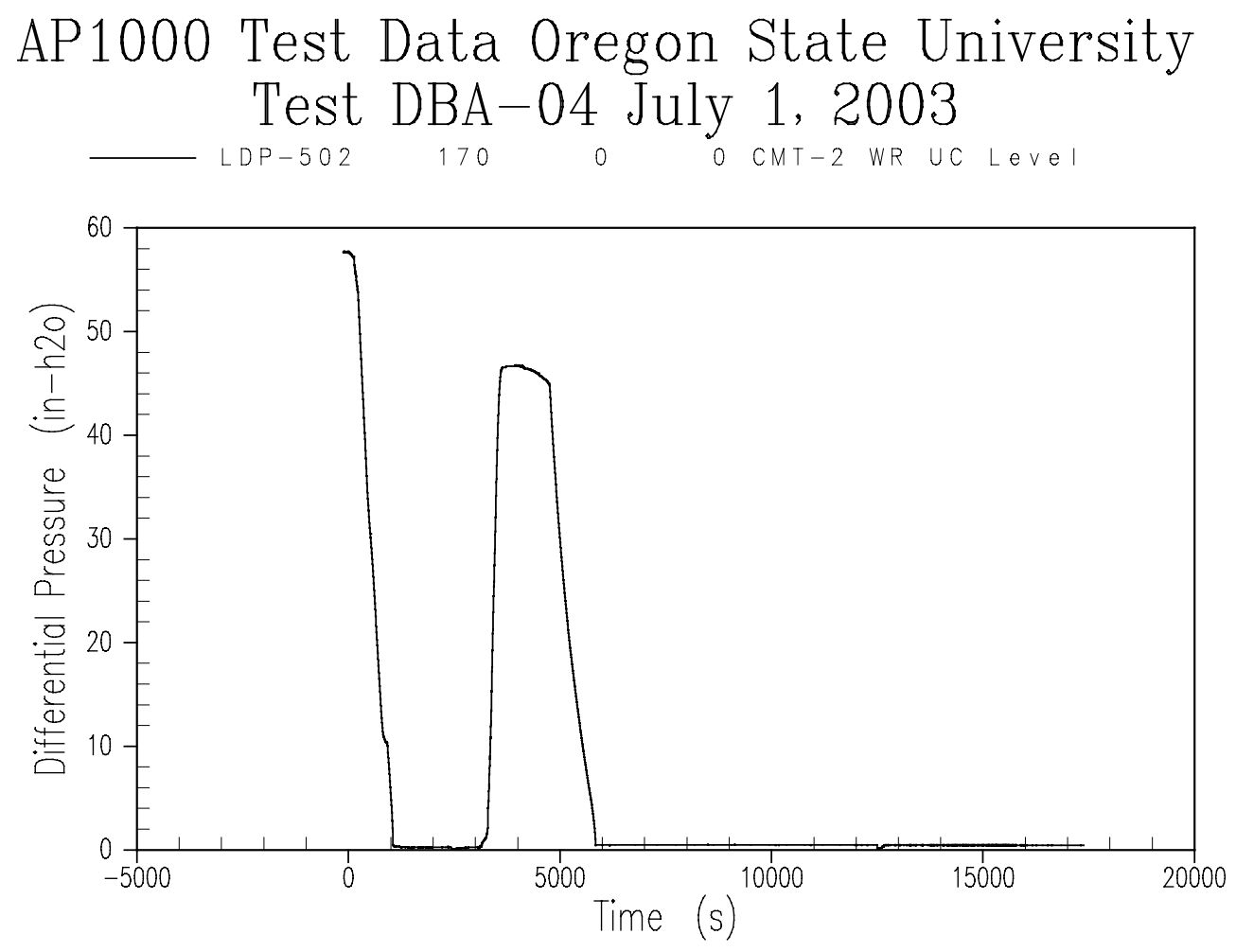

Figure A-28 Core Makeup Tank 2 Liquid Level 
AP1000 Test Data Oregon State University Test DBA-04 July 1, 2003 FMM-501 $60 \quad 0 \quad 0$ CMT-1 Inj. FIow

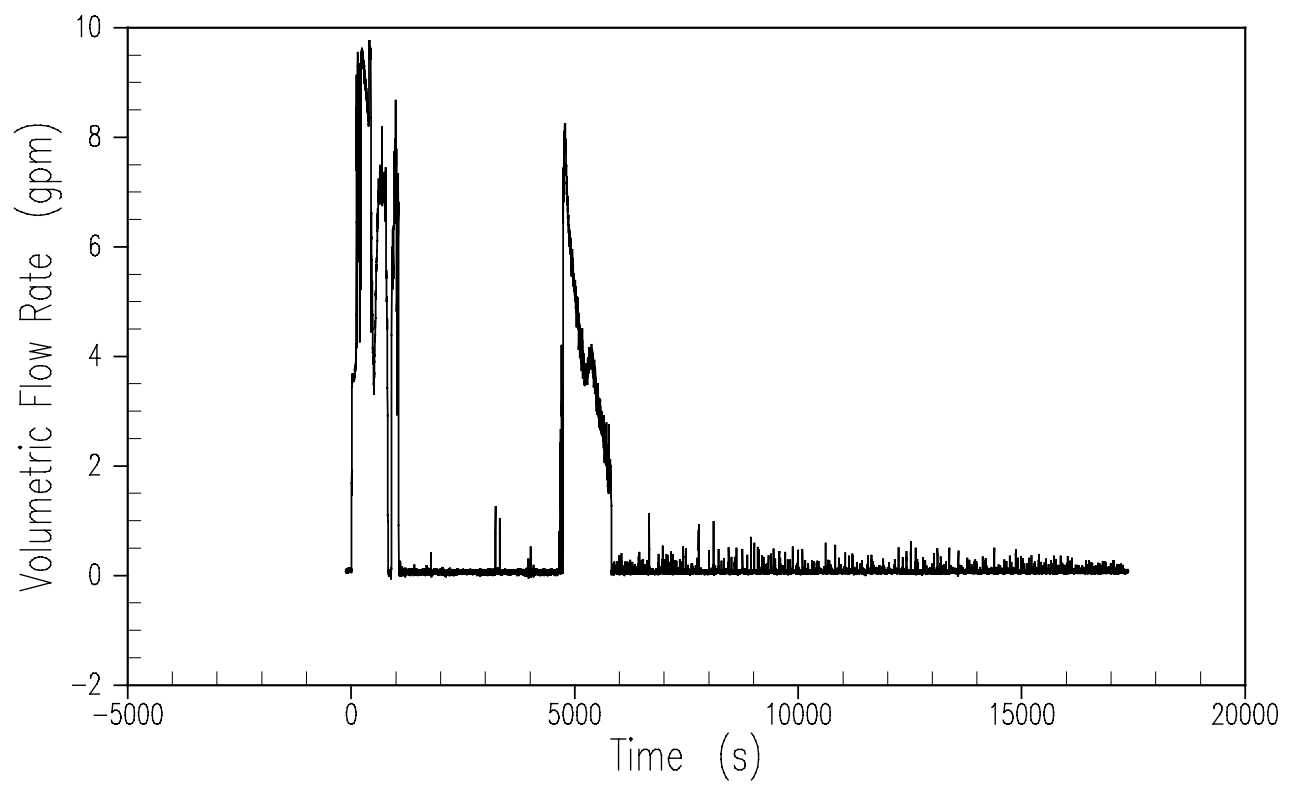

Figure A-29 Core Makeup Tank 1 Flow Rate 


\section{AP1000 Test Data Oregon State University Test DBA-04 July 1, 2003 $\begin{array}{llllllll}F M M-504 & 63 & 0 & 0 & C M T-2 & \text { Inj. F I ow }\end{array}$}

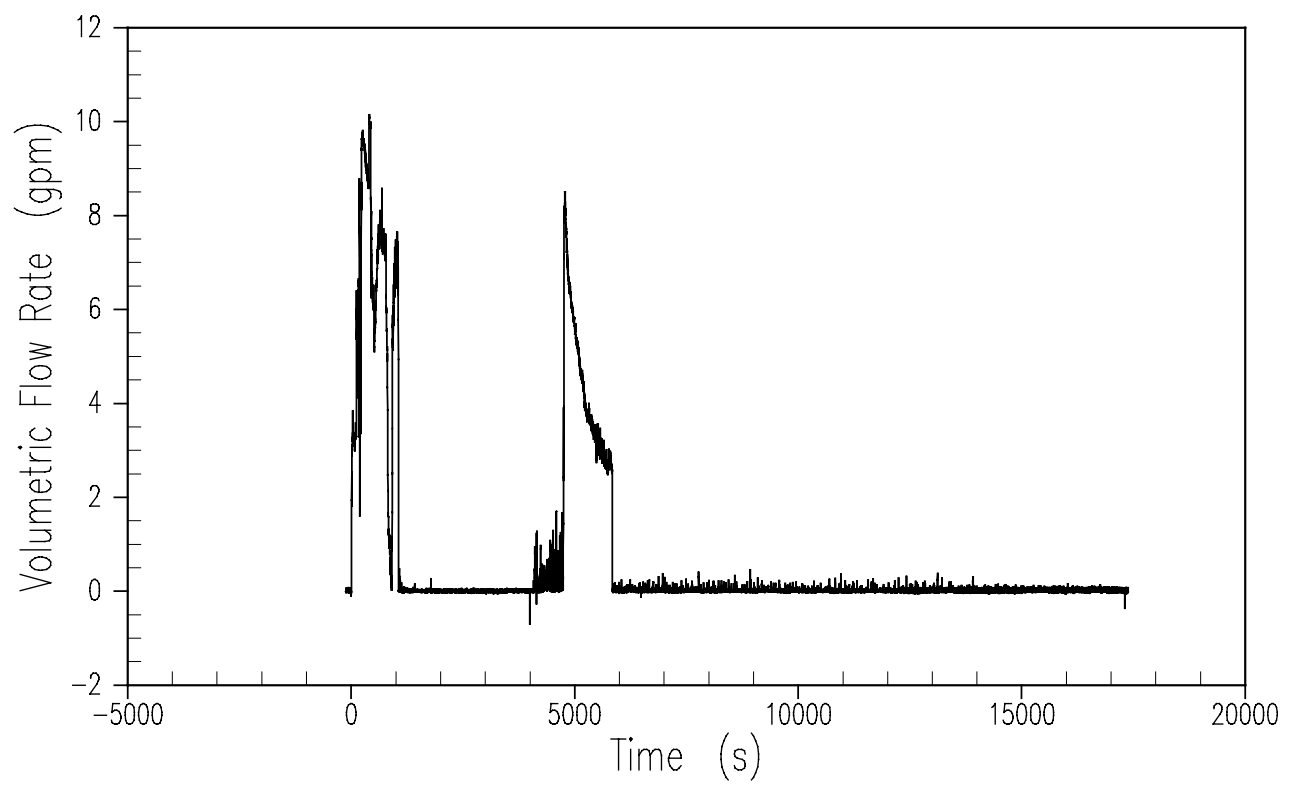

Figure A-30 Core Makeup Tank 2 Flow Rate 

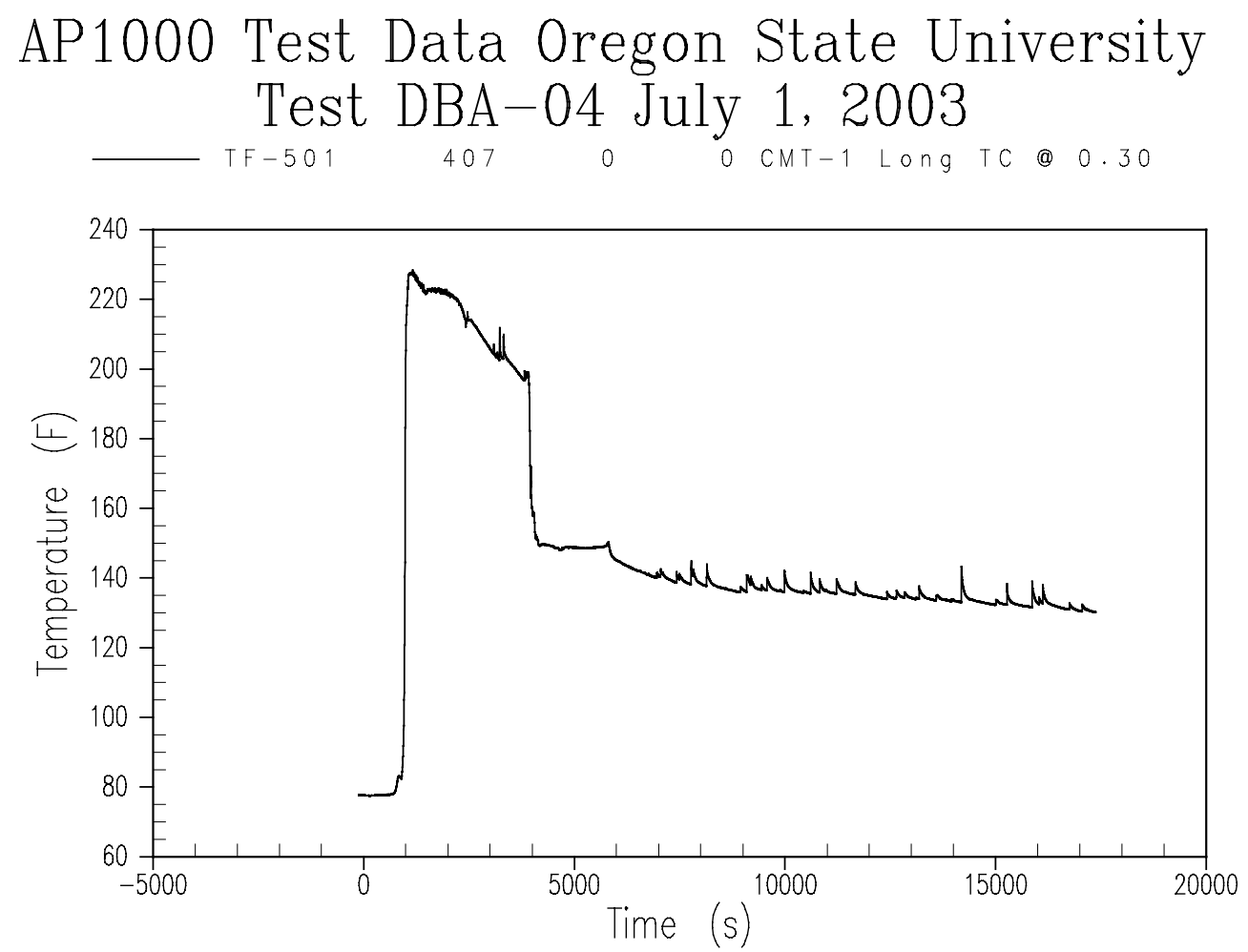

Figure A-31 Core Makeup Tank 1 Liquid Temperature - Bottom 

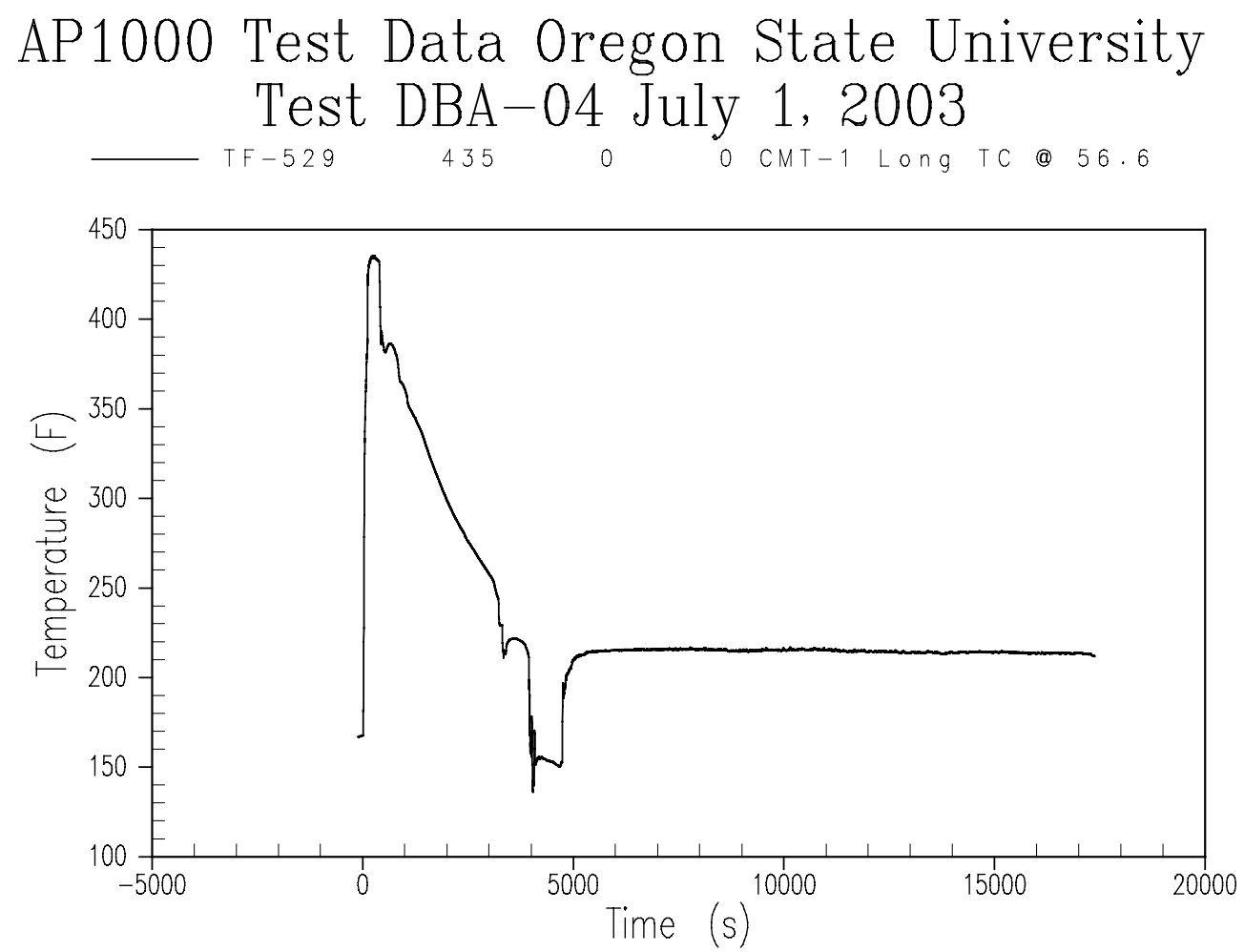

Figure A-32 Core Makeup Tank 1 Liquid Temperature - Top 

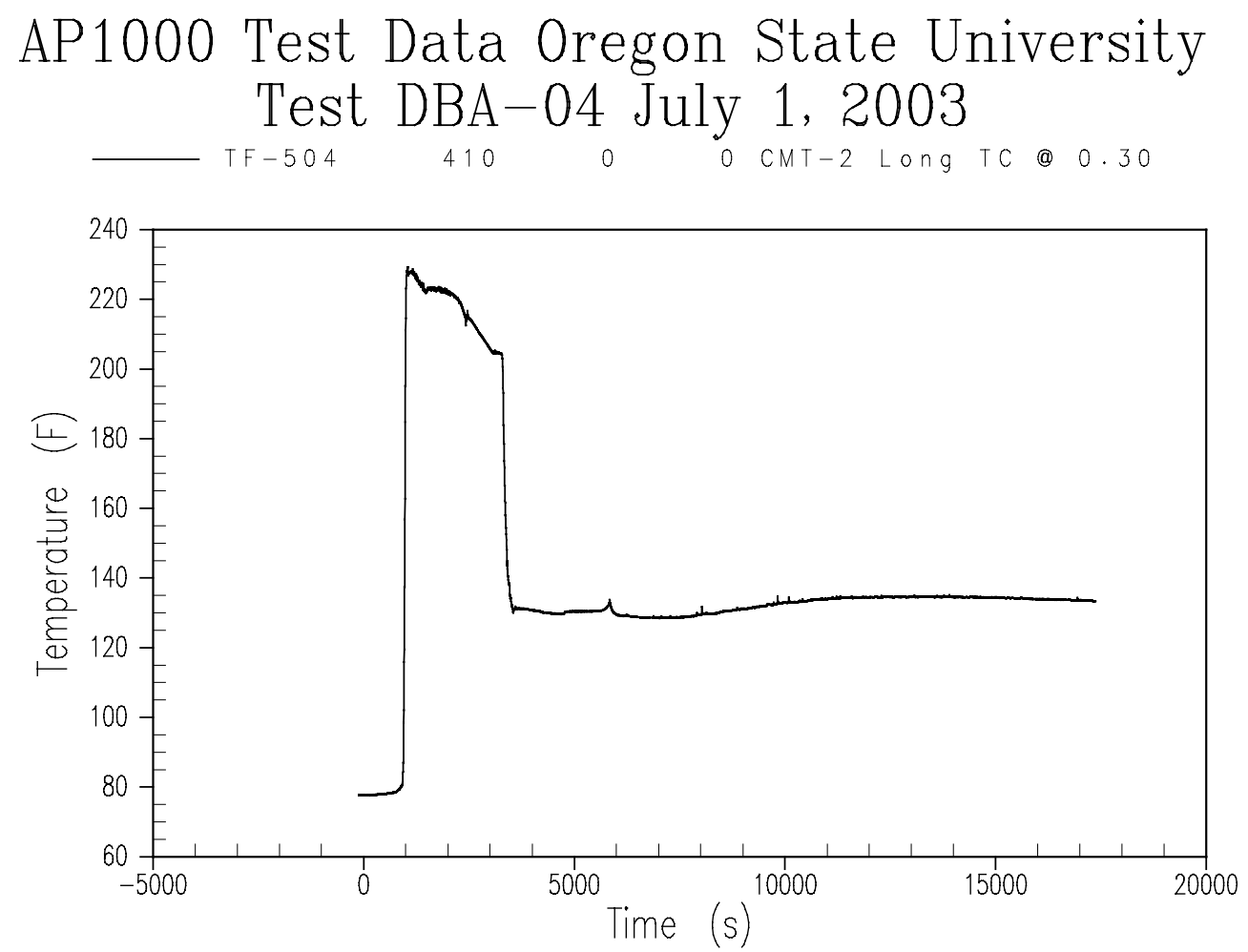

Figure A-33 Core Makeup Tank 2 Liquid Temperature - Bottom 

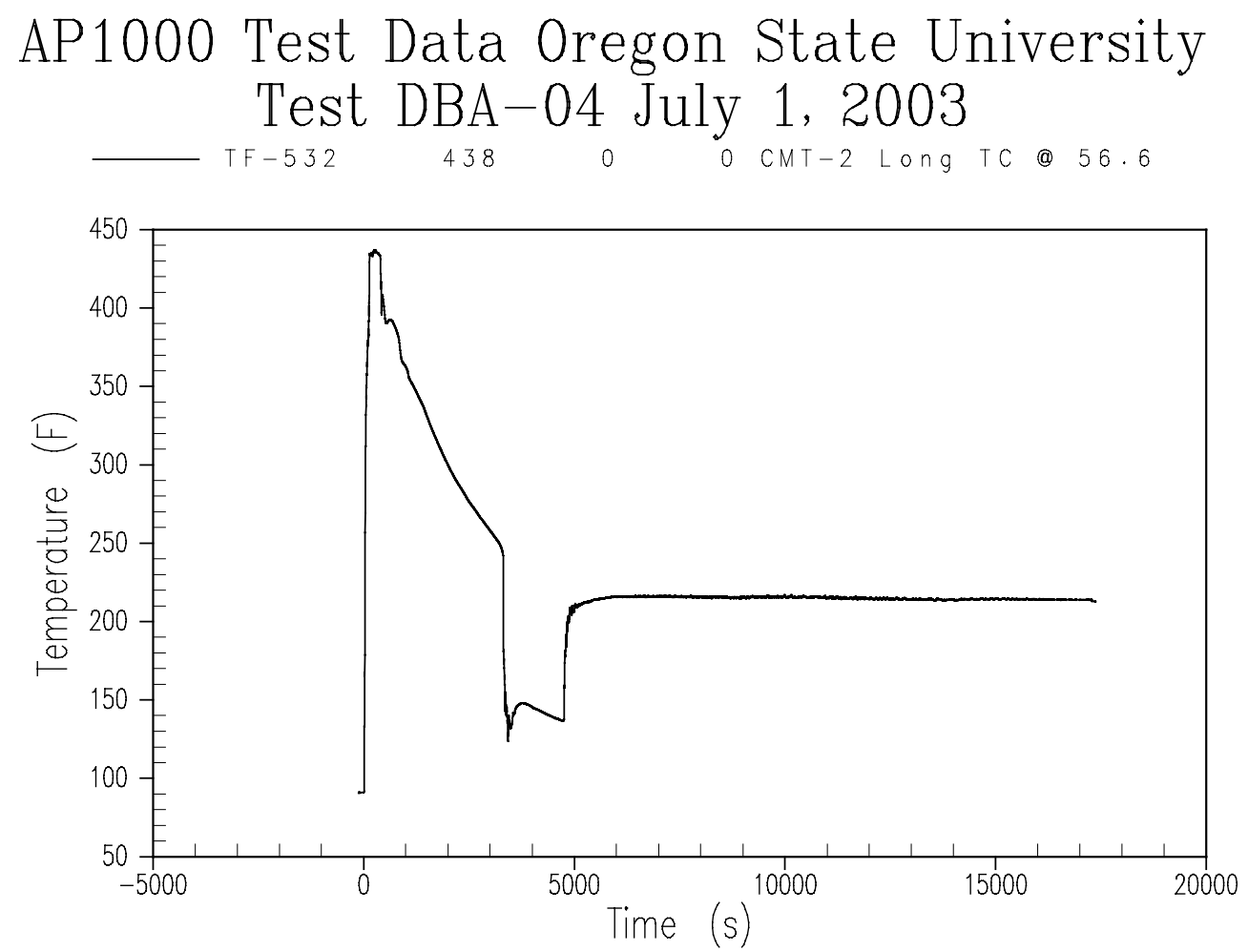

Figure A-34 Core Makeup Tank 2 Liquid Temperature - Top 

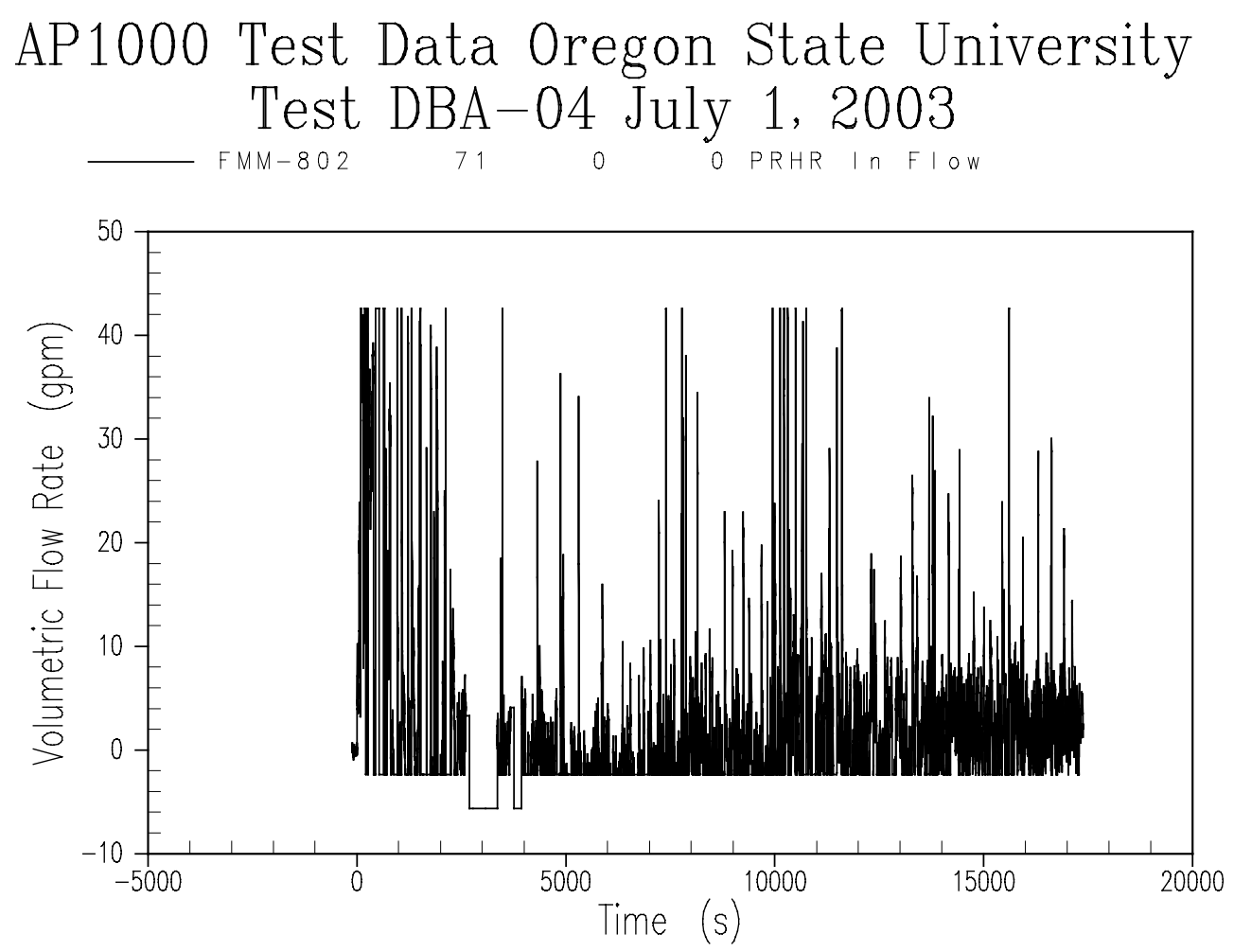

Figure A-35 Passive Residual Heat Removal Inlet Flow Rate 


\section{AP1000 Test Data Oregon State University Test DBA-04 July 1, 2003 \\ $\begin{array}{llll}L D P-802 & 193 & 0 & 0\end{array}$}

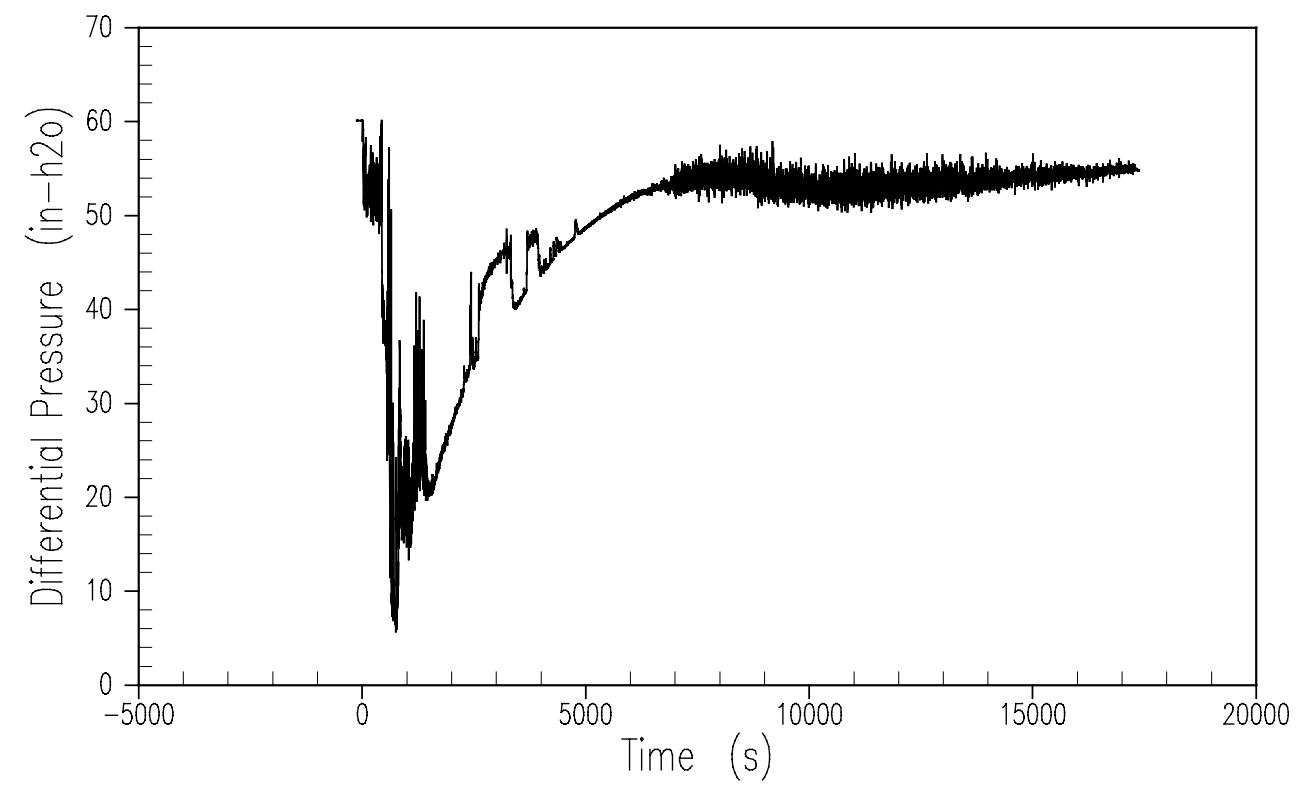

Figure A-36 Passive Residual Heat Removal Liquid Level 


\section{AP1000 Test Data Oregon State University Test DBA-04 July 1, 2003

$$
\text { FMM-804 } \quad 73 \quad 0 \text { PRHR Out FIow }
$$

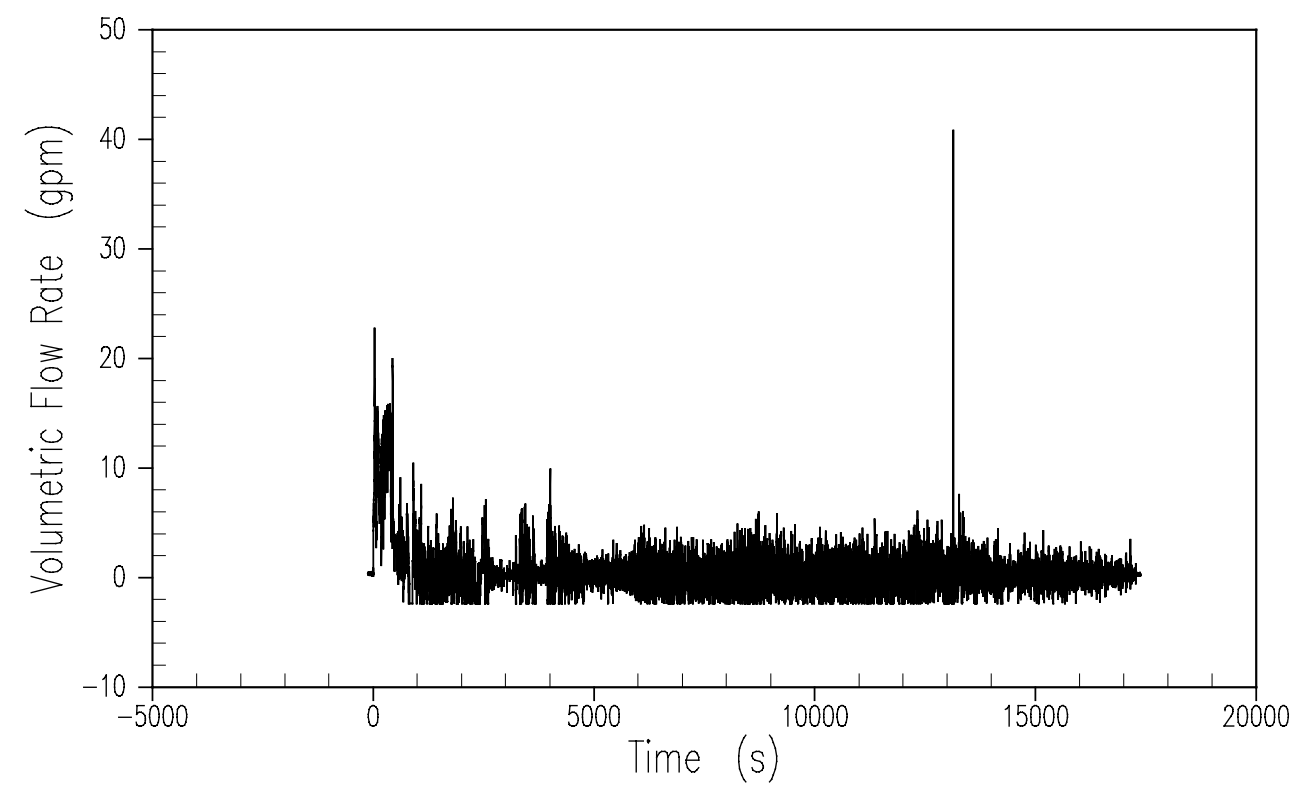

Figure A-37 Passive Residual Heat Removal Outlet Flow Rate 
AP1000 Test Data Oregon State University Test DBA-04 July 1, 2003

$\left[\begin{array}{llll|l}\text { LDP-701 } & 191 & 0 & 0 & \text { IRWST UC LeVe }\end{array}\right.$

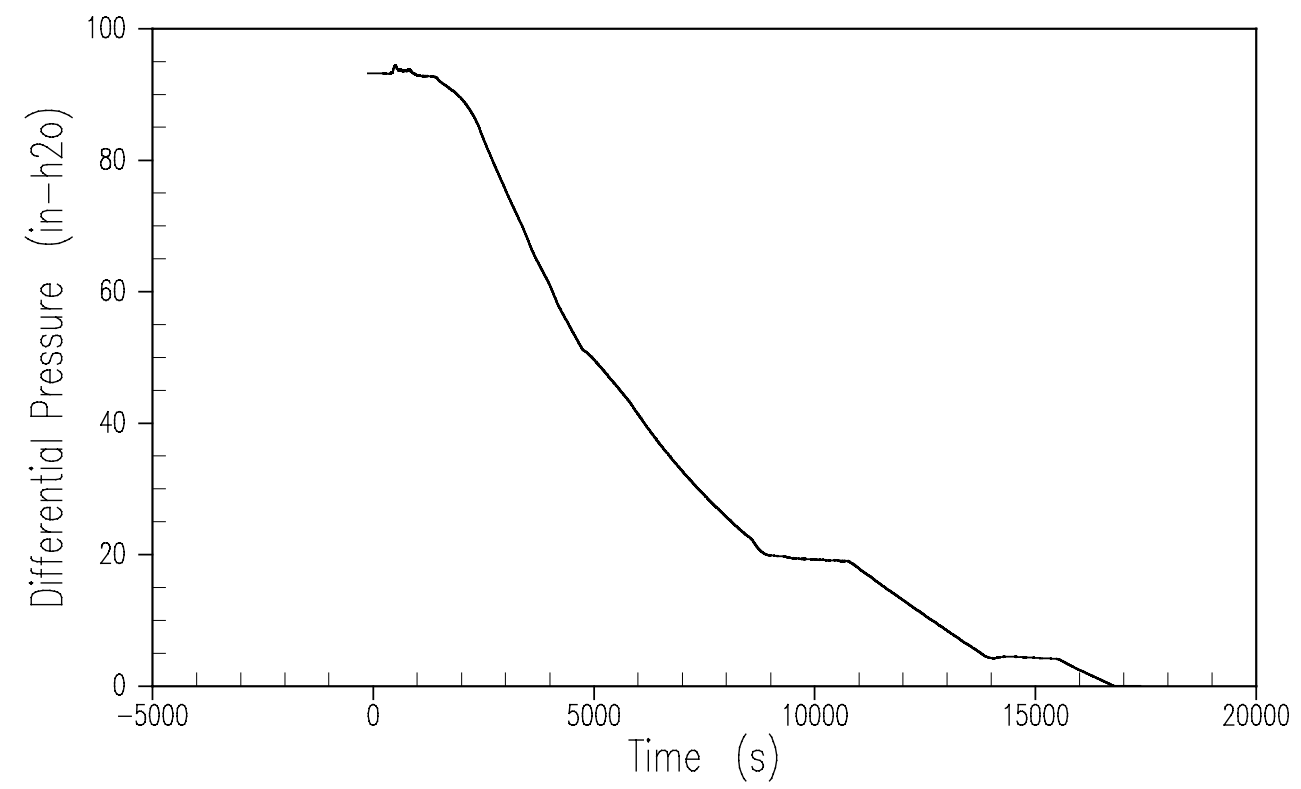

Figure A-38 IRWST Liquid Level 


\section{AP1000 Test Data Oregon State University Test DBA-04 July 1, 2003 \\ \begin{tabular}{llll|l|l|l|l|}
$F M M-701$ & 67 & 0 & 0 & IRWST-1 I I W
\end{tabular}}

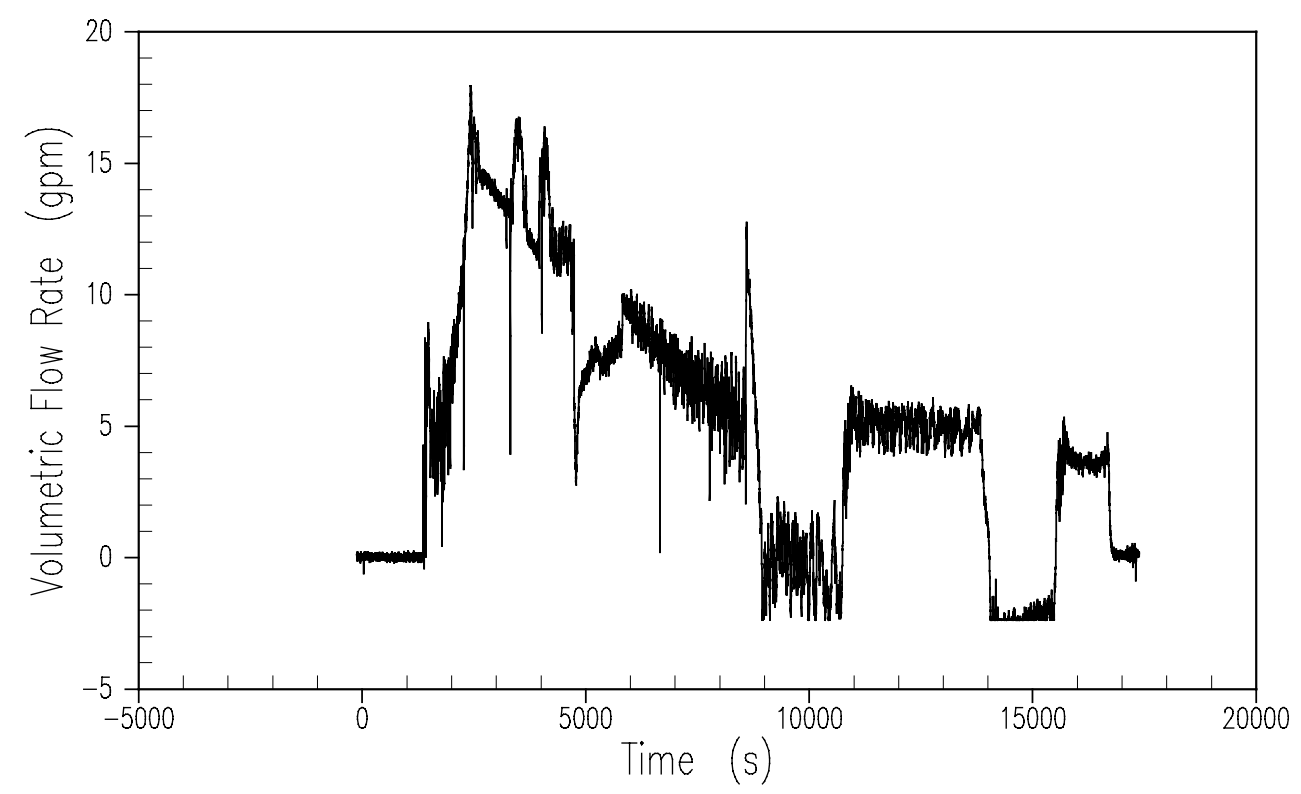

Figure A-39 IRWST Discharge Line 1 Flow Rate 


\section{AP1000 Test Data Oregon State University Test DBA-04 July 1, 2003 \\ \begin{tabular}{llll|l}
$F M M-702$ & 68 & 0 & 0 & IRWST-2 Inj. F I OW
\end{tabular}}

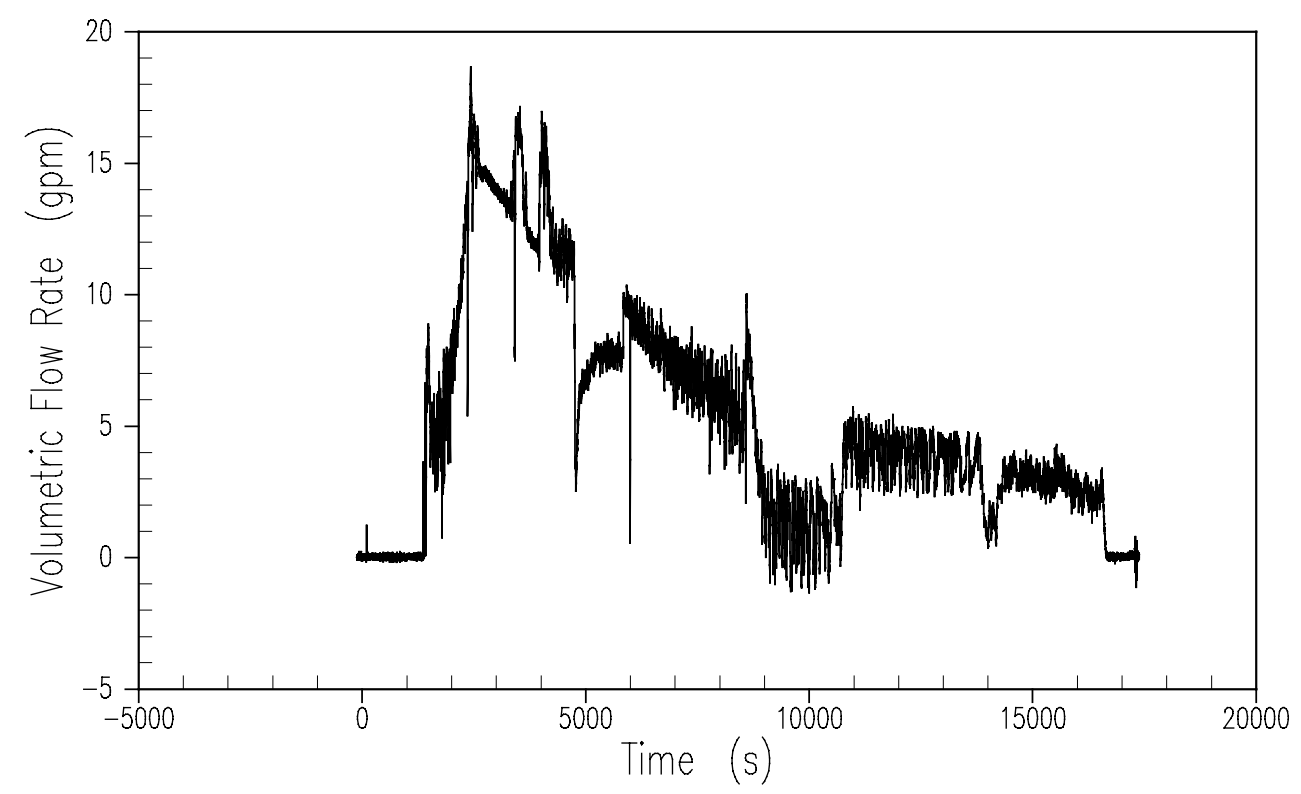

Figure A-40 IRWST Discharge Line 2 Flow Rate 
AP1000 Test Data Oregon State University Test DBA-04 July 1, 2003

$\begin{array}{llll}T F-701 & 486 & 0 & 0\end{array}$

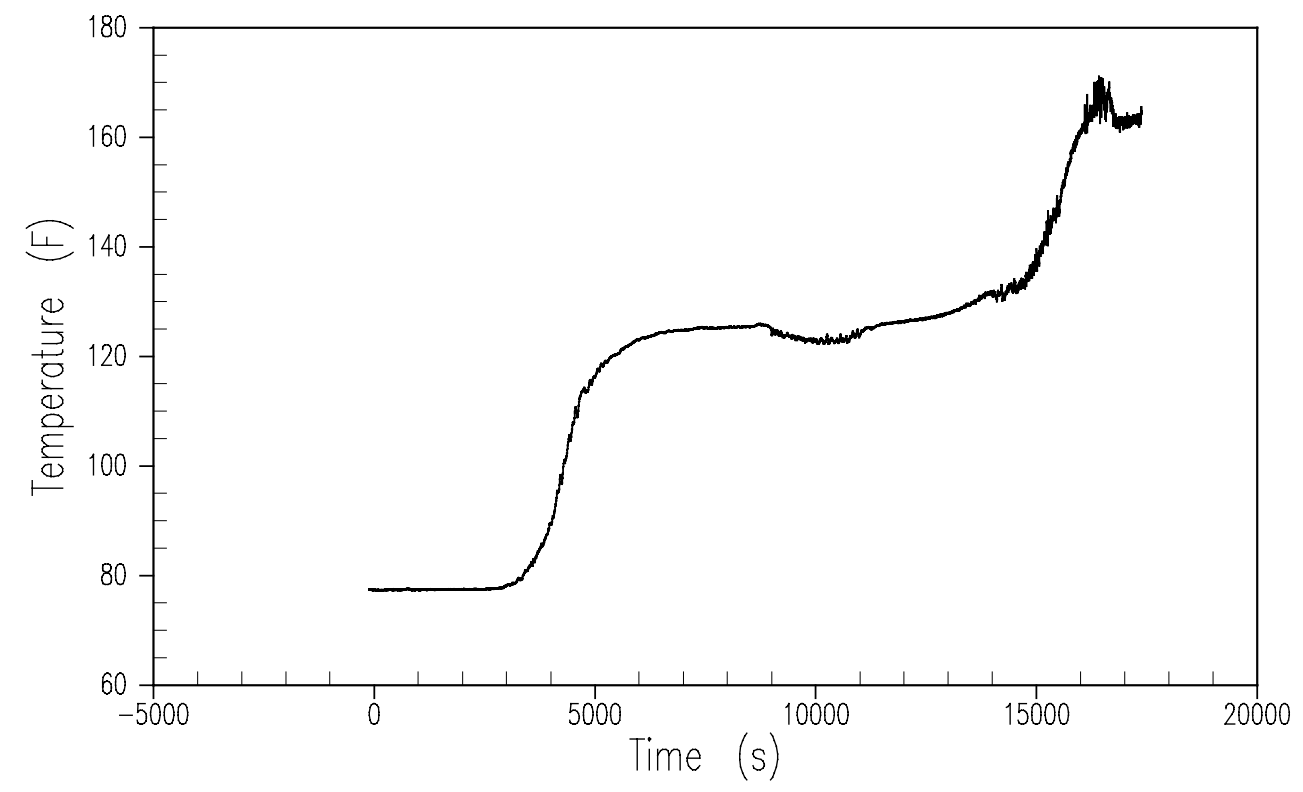

Figure A-41 IRWST Fluid Temperature - Bottom 

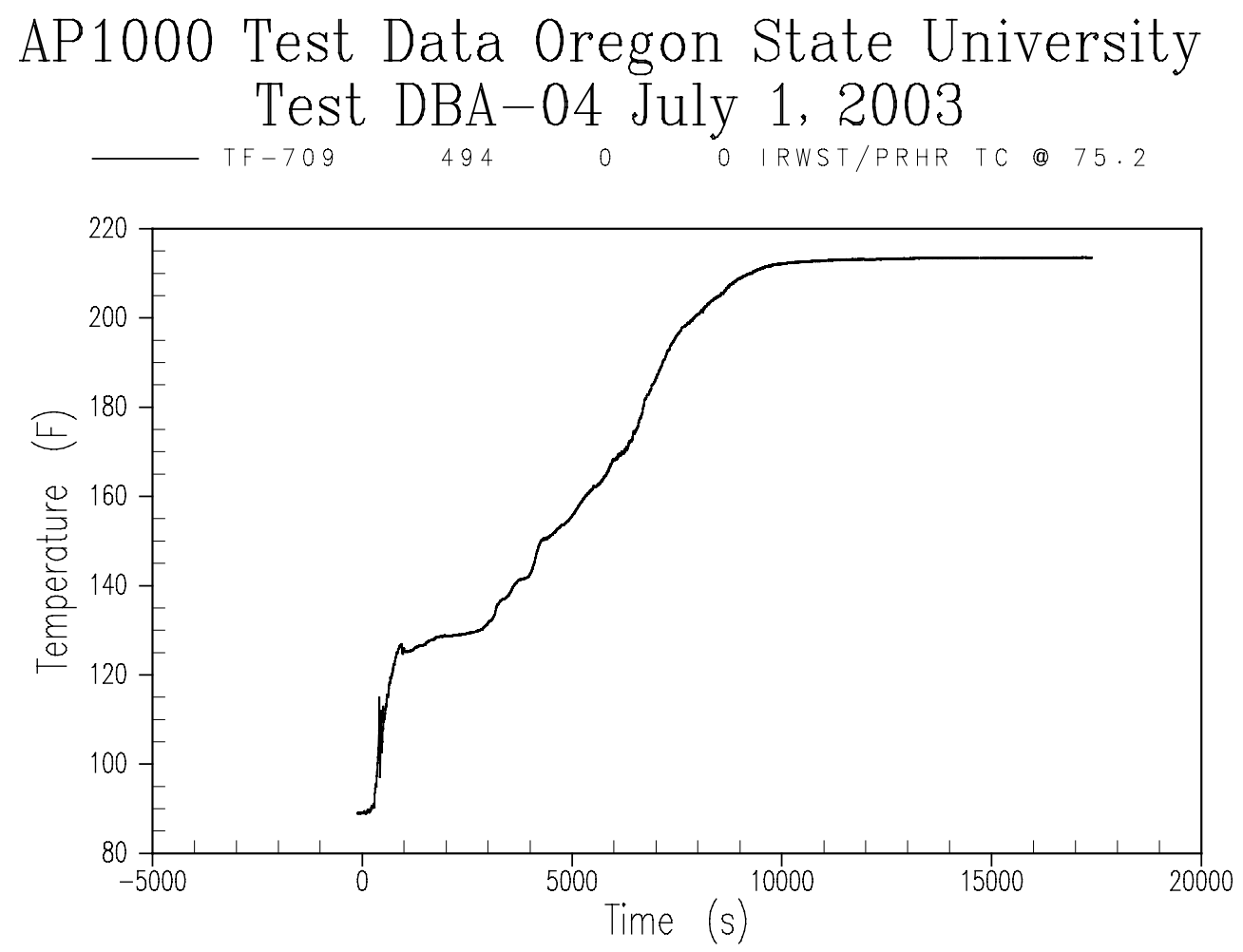

Figure A-42 IRWST Fluid Temperature - Top 

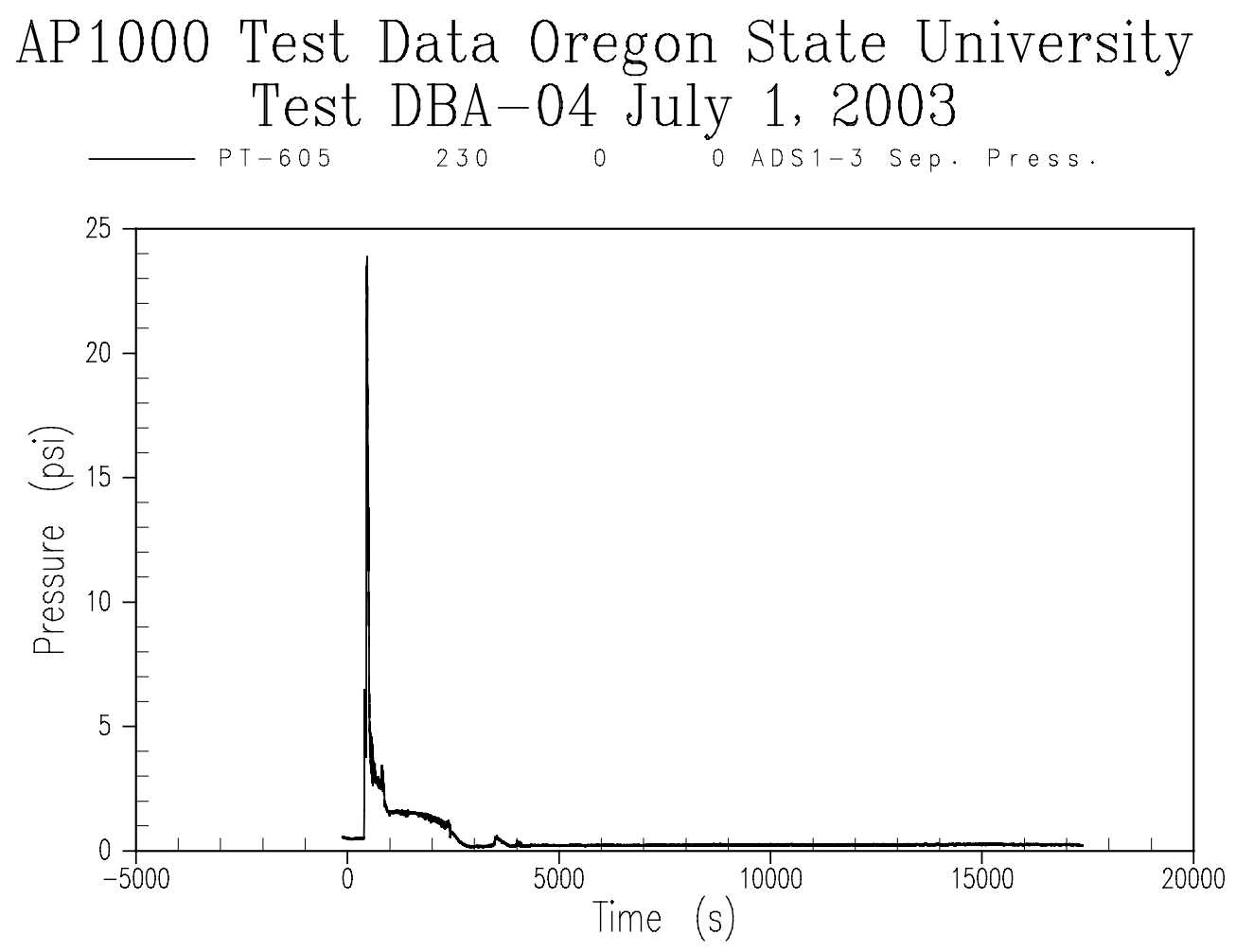

Figure A-43 ADS 1-3 Separator Pressure 

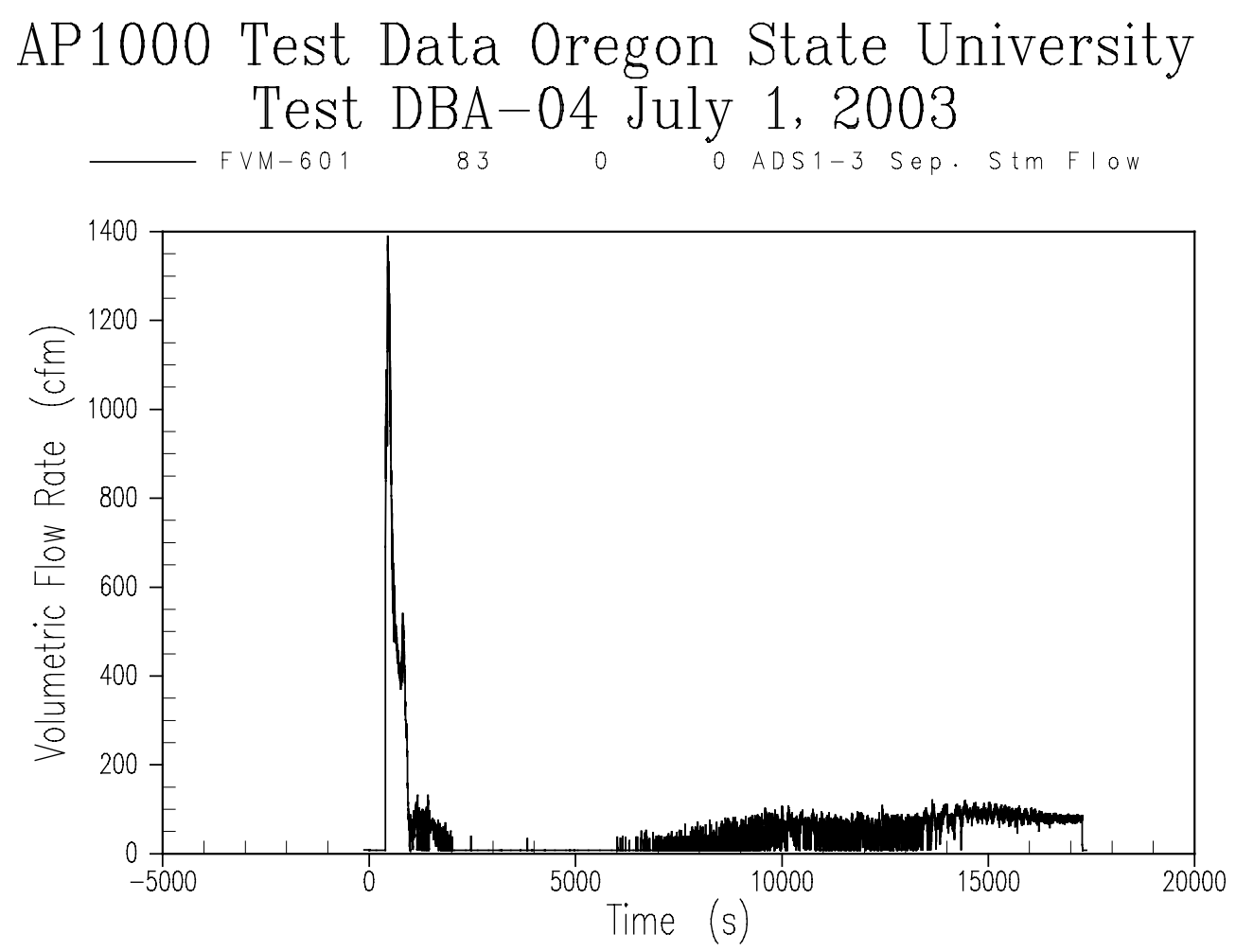

Figure A-44 ADS 1-3 Separator Steam Flow Rate 


\section{AP1000 Test Data Oregon State University Test DBA-04 July 1, 2003 \\ $\begin{array}{lllllll}\text { FMM-601 } & 64 & 0 & 0 & \text { ADS } 1-3 \\ \text { LS FIOW }\end{array}$}

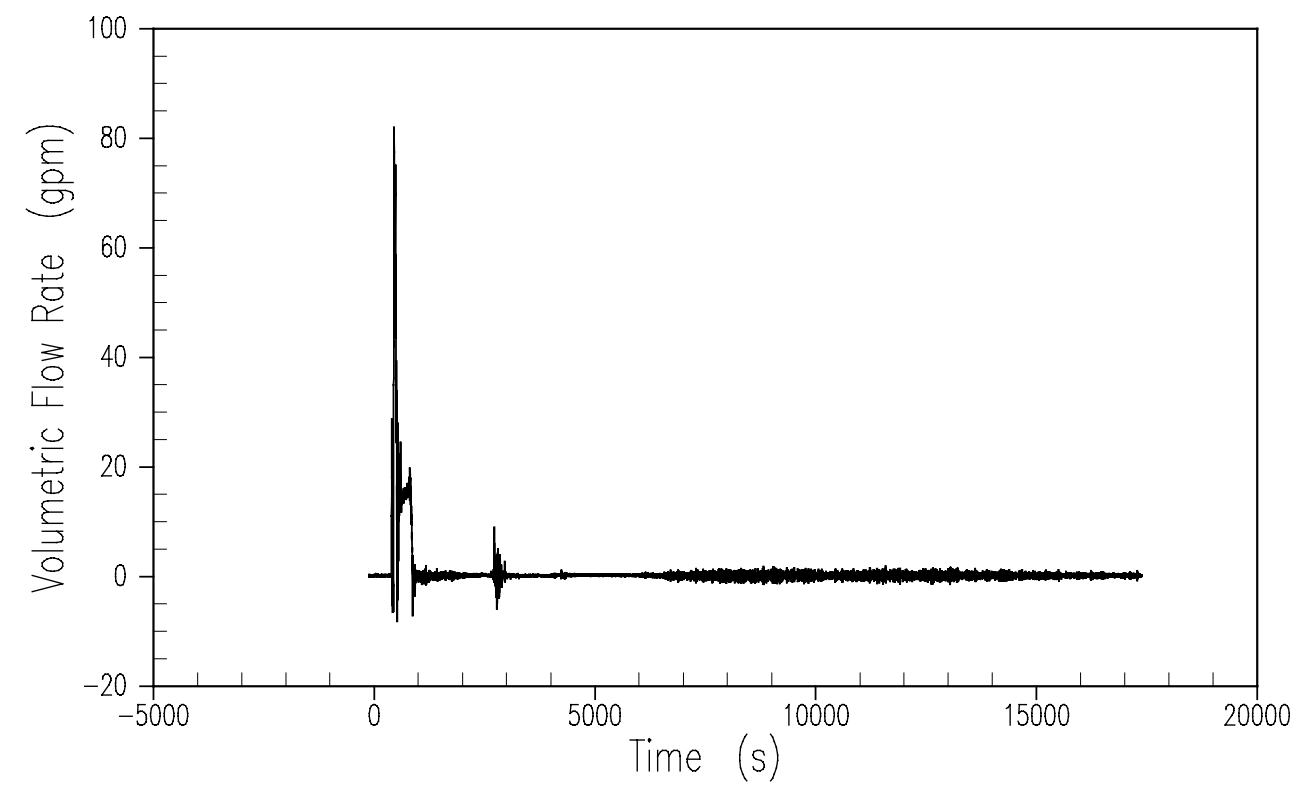

Figure A-45 ADS 1-3 Separator Liquid Flow Rate 
AP1000 Test Data Oregon State University Test DBA-04 July 1, 2003

$\begin{array}{lllll}P T-611 & 233 & 0 & \text { ADS4-1 Sep.Press. }\end{array}$

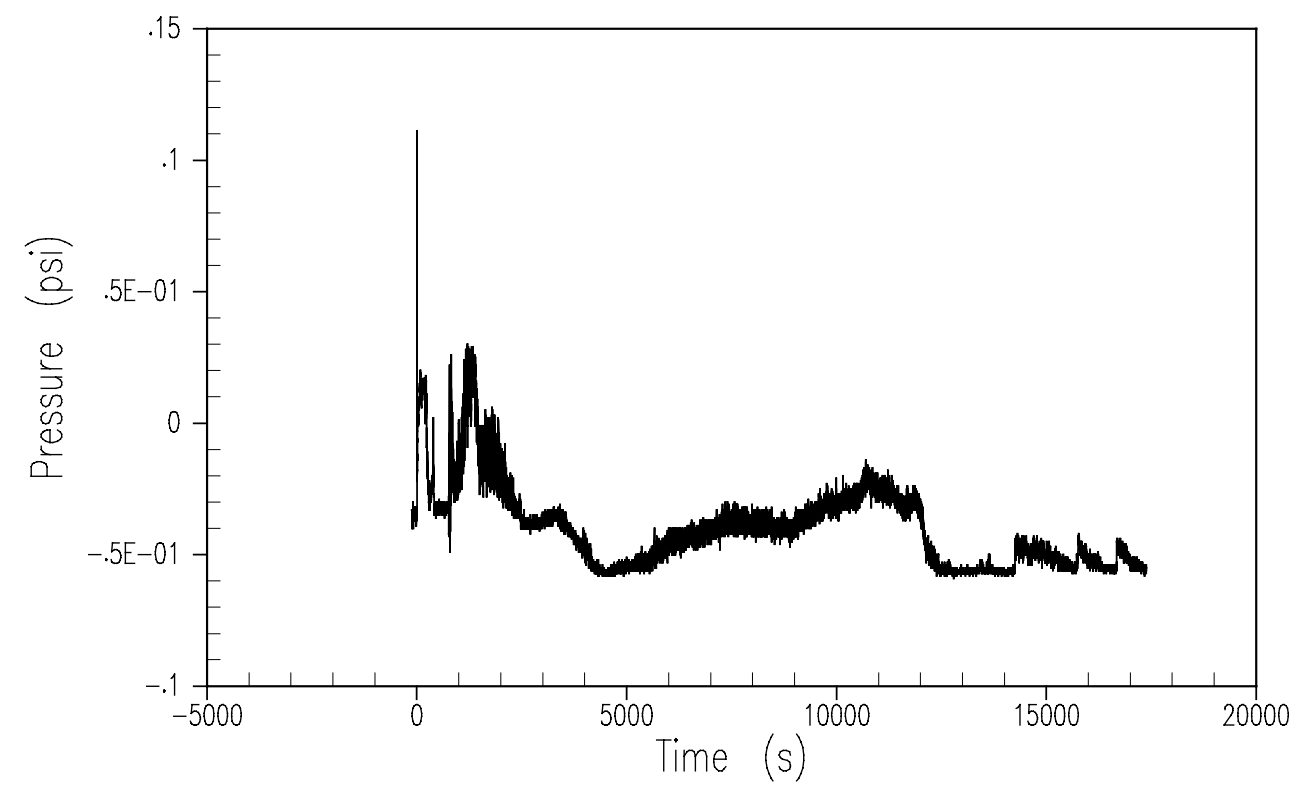

Figure A-46 ADS 4-1 Separator Pressure 

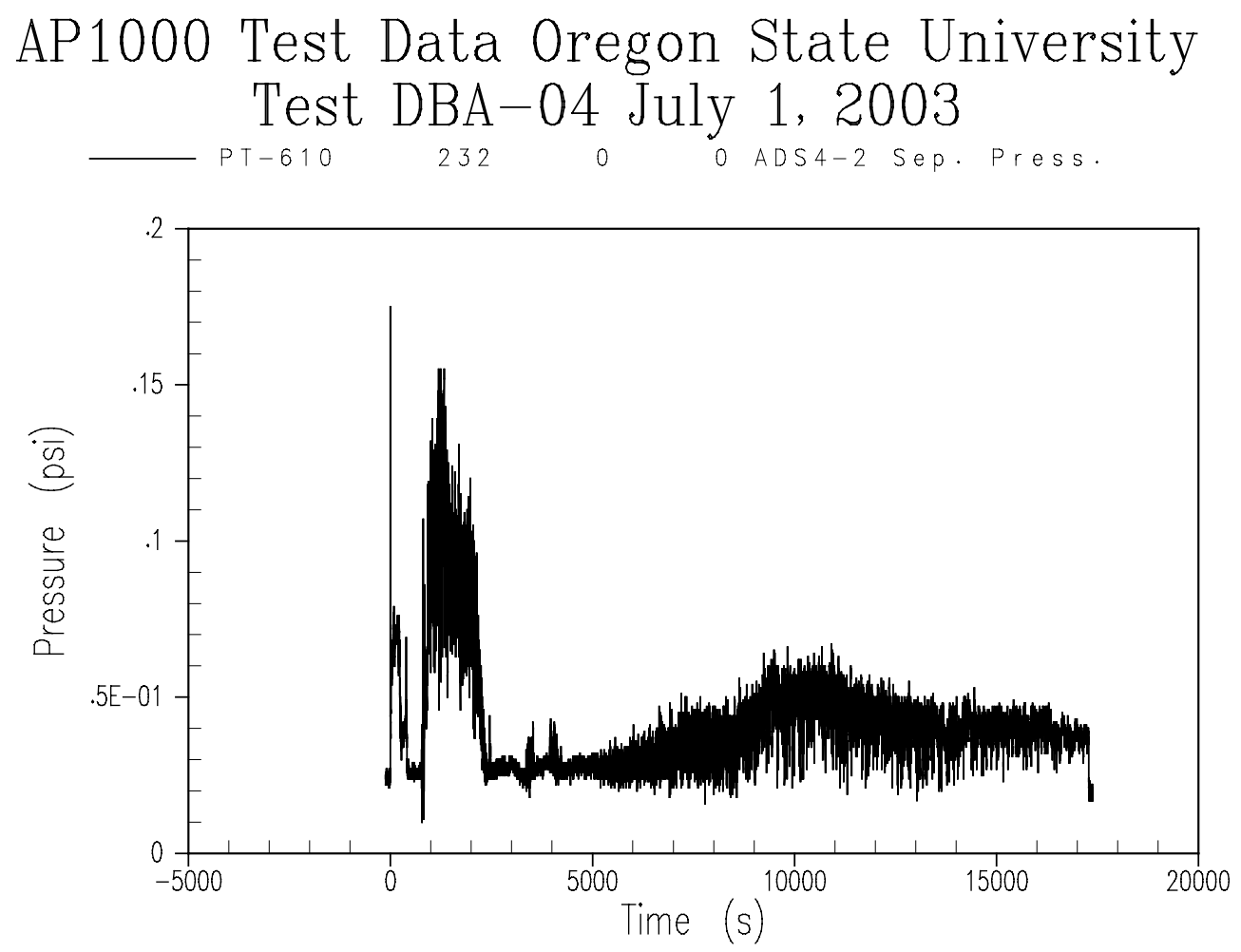

Figure A-47 ADS 4-2 Separator Pressure 


\section{AP1000 Test Data Oregon State University Test DBA-04 July 1, 2003 \\ FVM-603 $85 \quad 0 \quad 0$ ADS4-1 Sep. 6" Line}

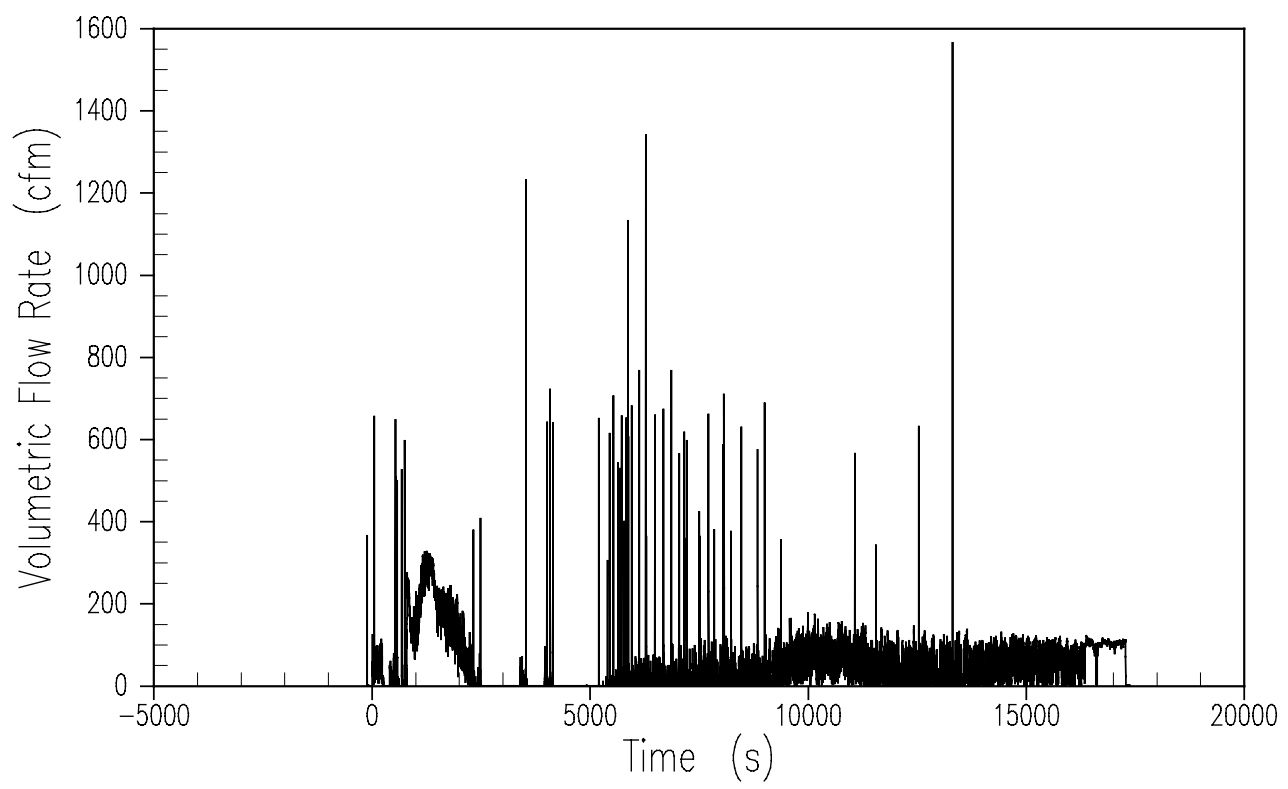

Figure A-48 ADS 4-1 Separator Steam Flow Rate 


\section{AP1000 Test Data Oregon State University Test DBA-04 July 1, 2003 \\ FVM-602 $84 \quad 0 \quad 0$ ADS 4-2 Sep. 6" Line}

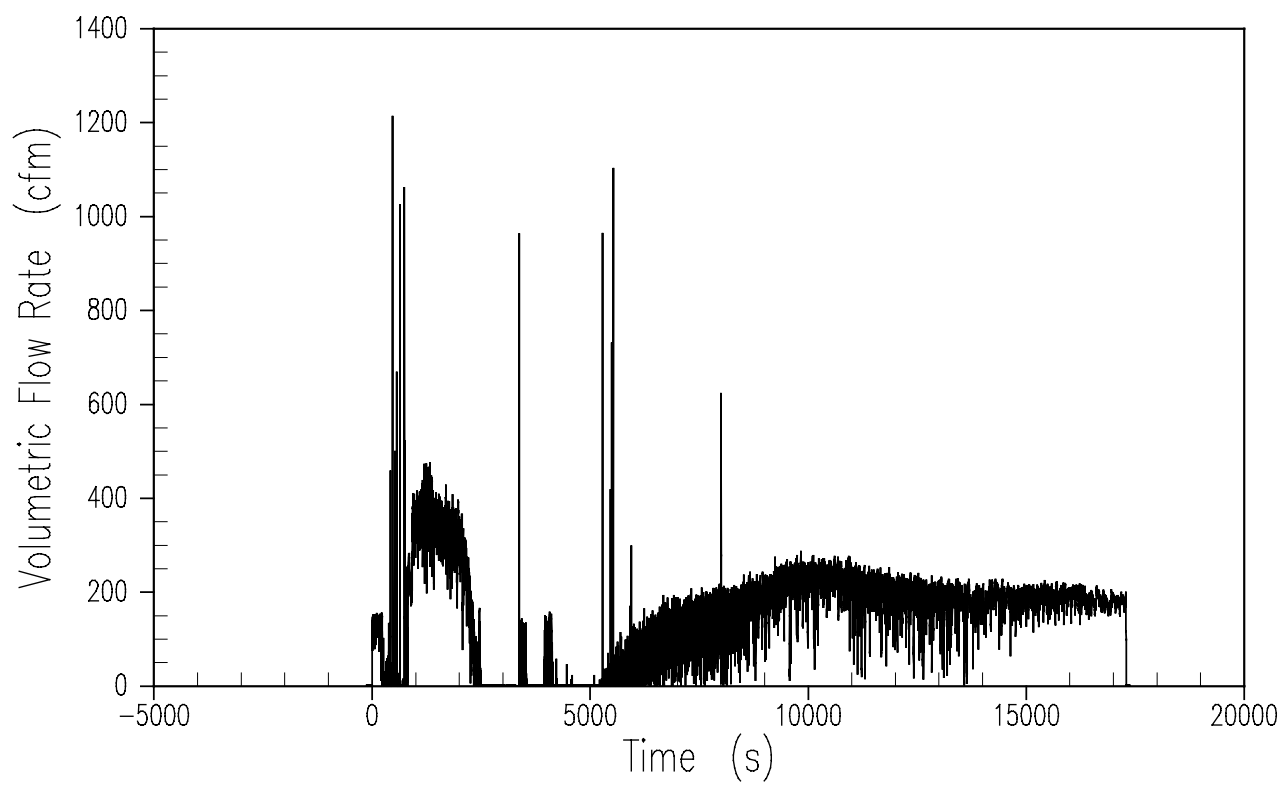

Figure A-49 ADS 4-2 Separator Steam Flow Rate 


\section{AP1000 Test Data Oregon State University Test DBA-04 July 1, 2003 \\ $\begin{array}{lllllll}F M M-603 & 66 & 0 & 0 & \text { ADS } 4-1 \\ \text { LS FIOW }\end{array}$}

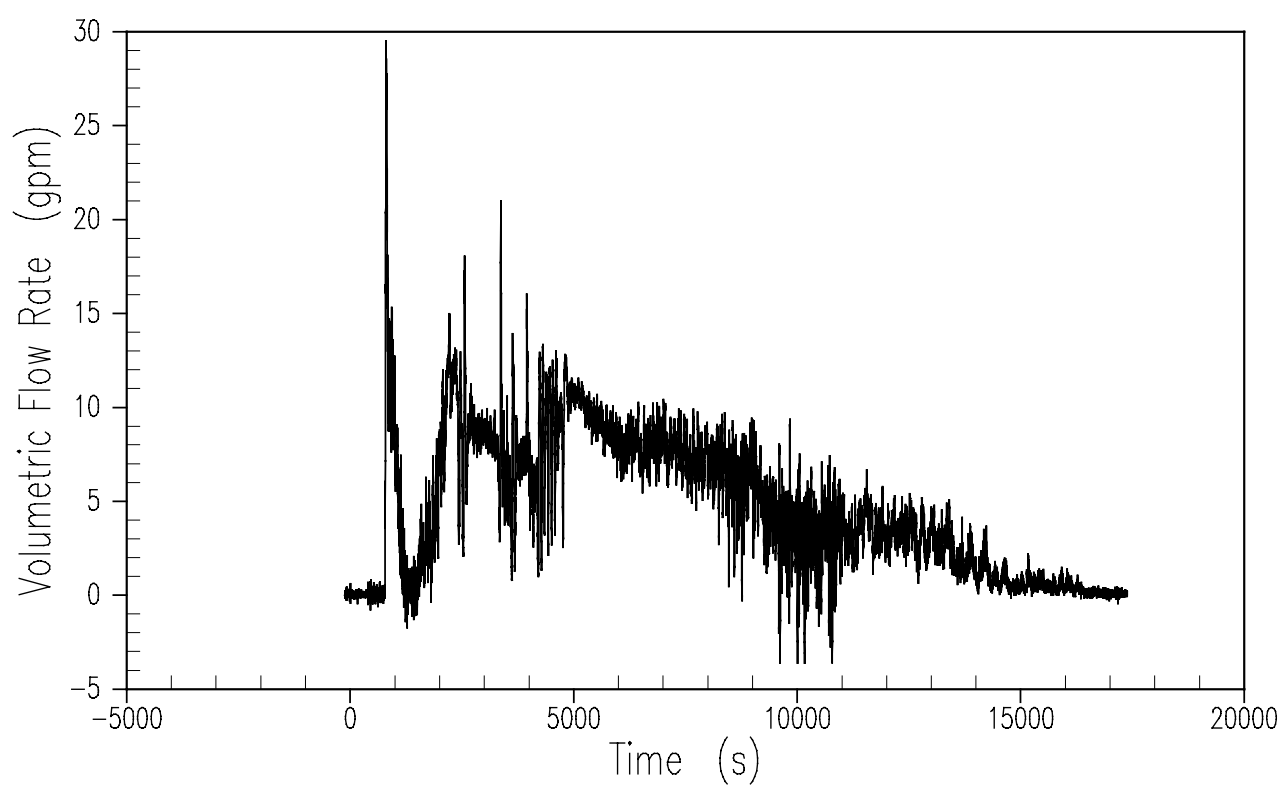

Figure A-50 ADS 4-1 Separator Liquid Flow Rate 

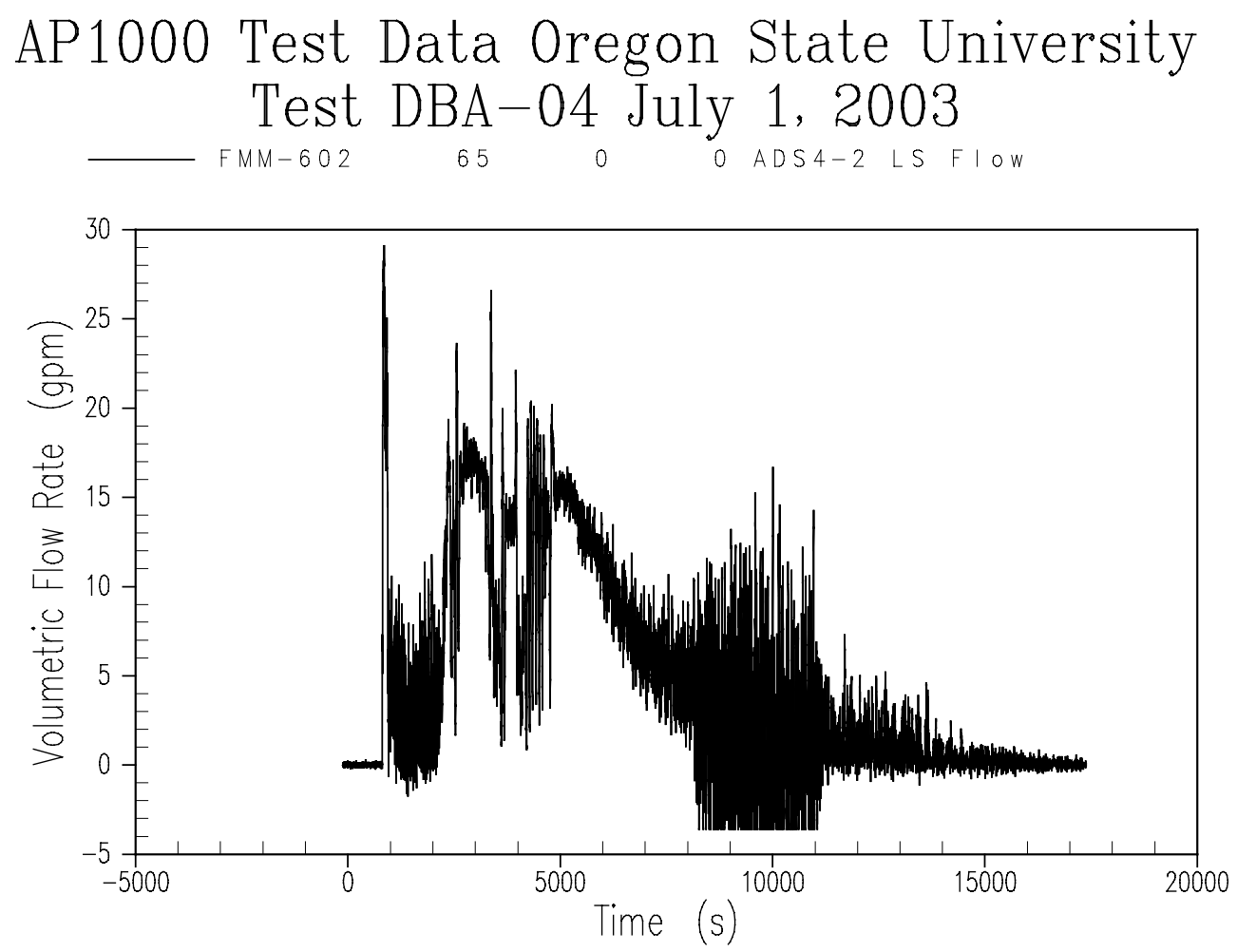

Figure A-51 ADS 4-2 Separator Liquid Flow Rate 


\section{AP1000 Test Data Oregon State University Test DBA-04 July 1, 2003 $\begin{array}{llll}P T-901 & 237 & 0 & 0\end{array}$}

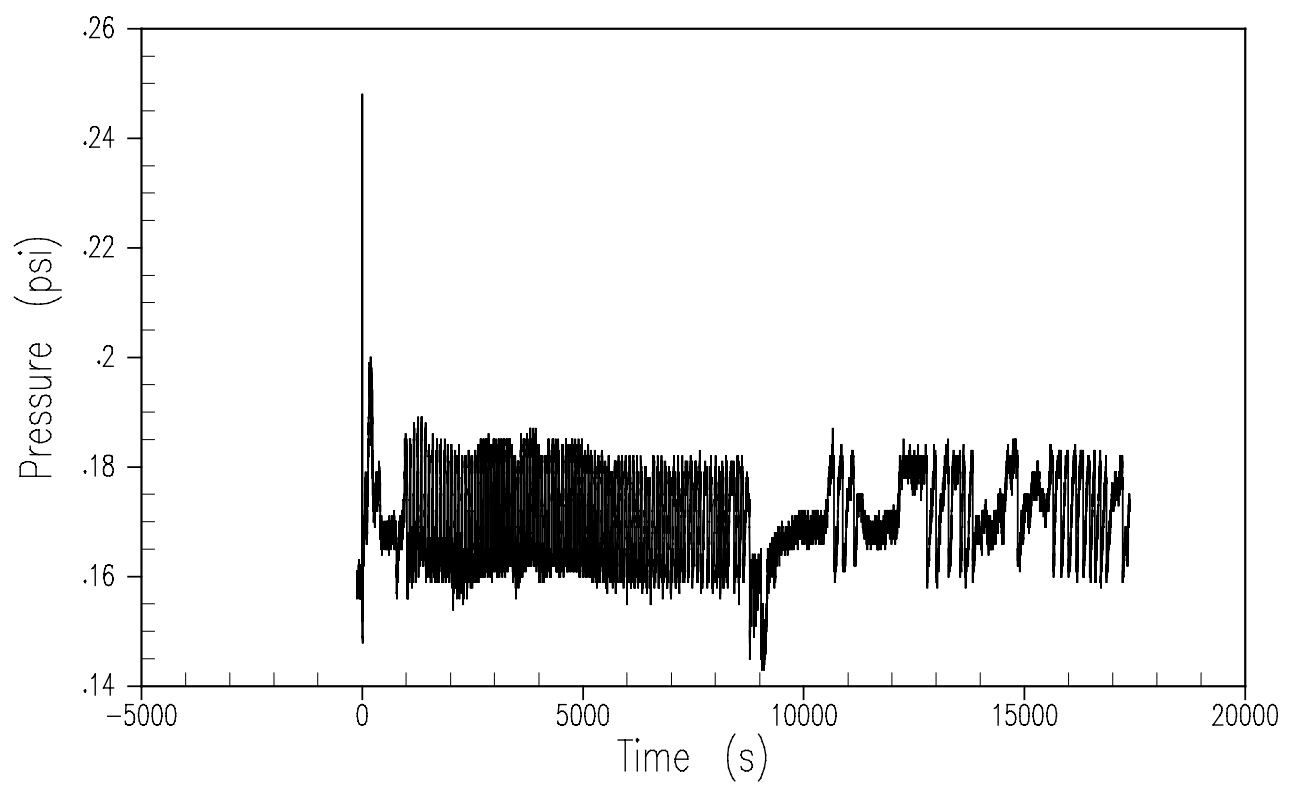

Figure A-52 Primary Sump Pressure 


\section{AP1000 Test Data Oregon State University Test DBA-04 July 1, 2003

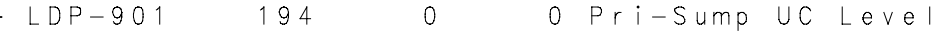

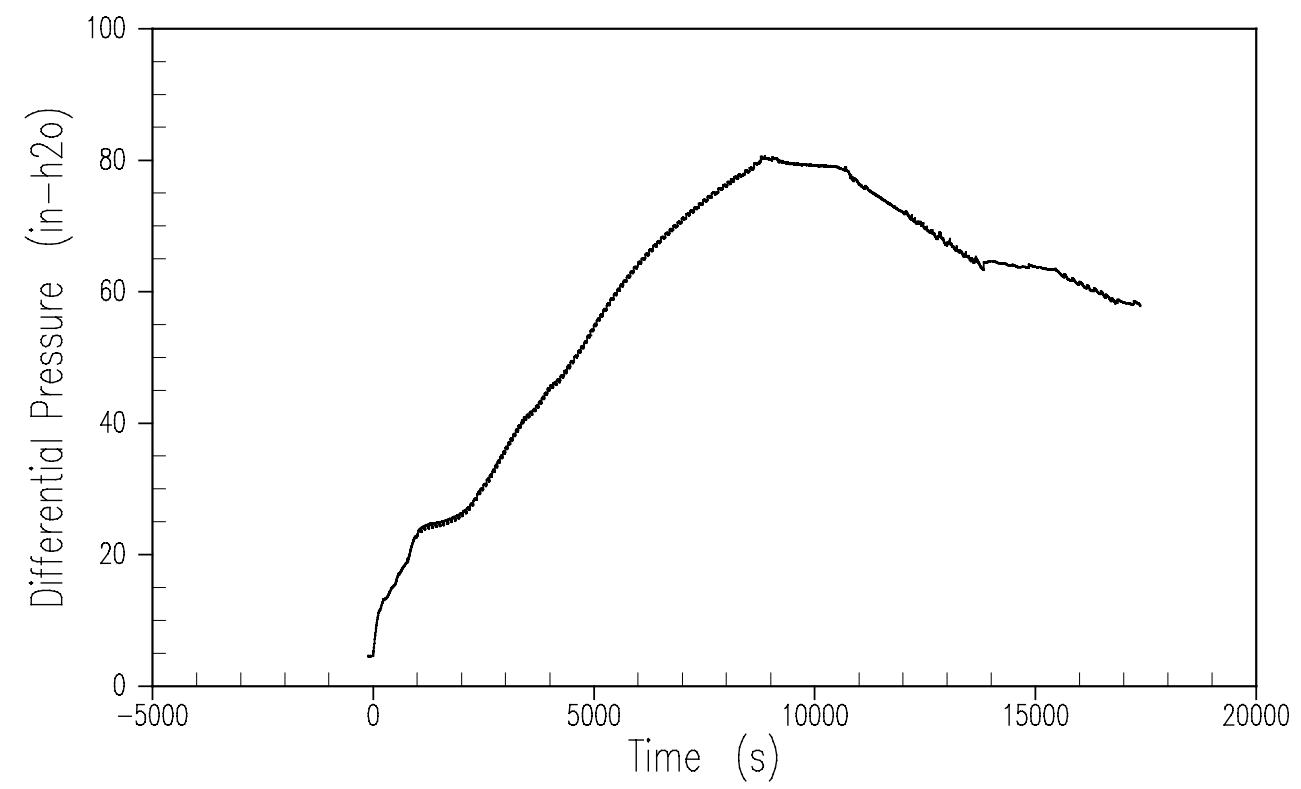

Figure A-53 Primary Sump Liquid Level 


\section{AP1000 Test Data Oregon State University Test DBA-04 July 1, 2003 \\ $\begin{array}{llll}F M M-901 & 75 & 0 & 0\end{array}$}

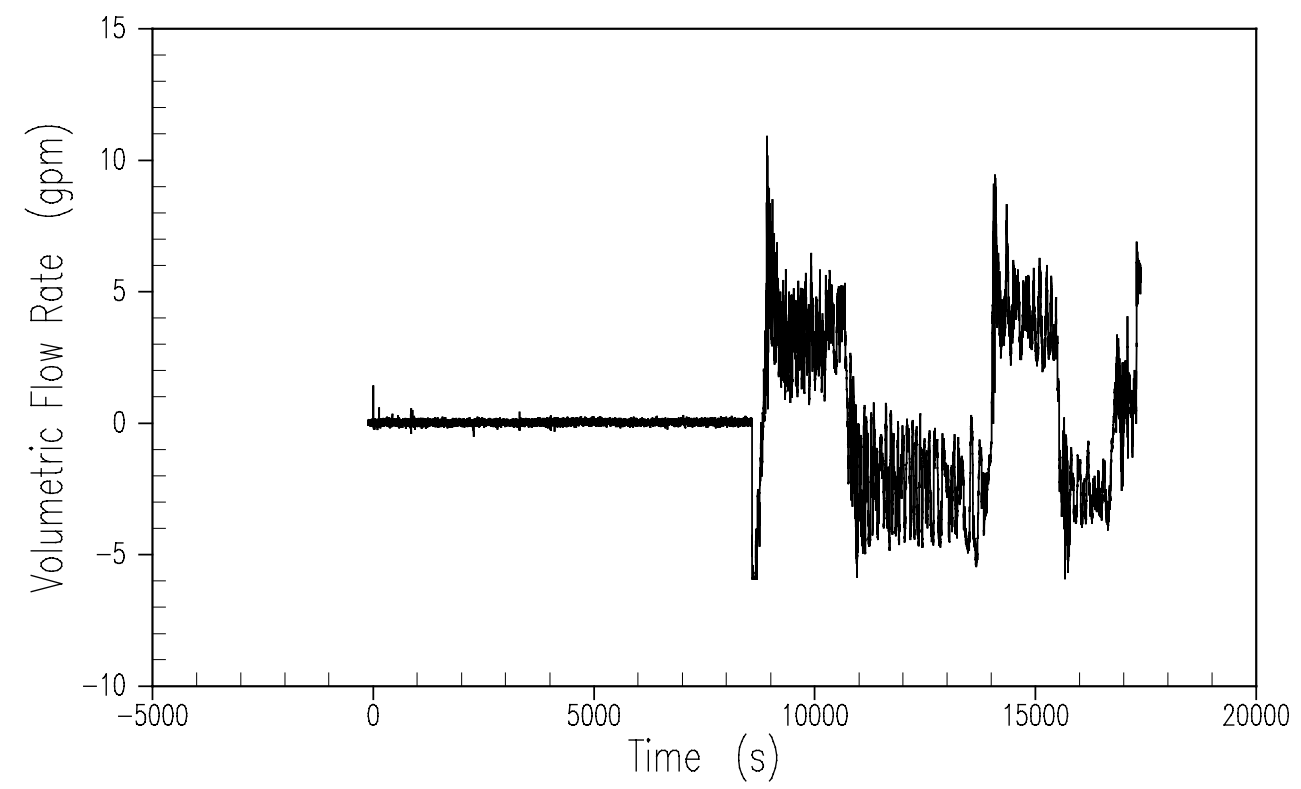

Figure A-54 Primary Sump 1 Injection Flow Rate 

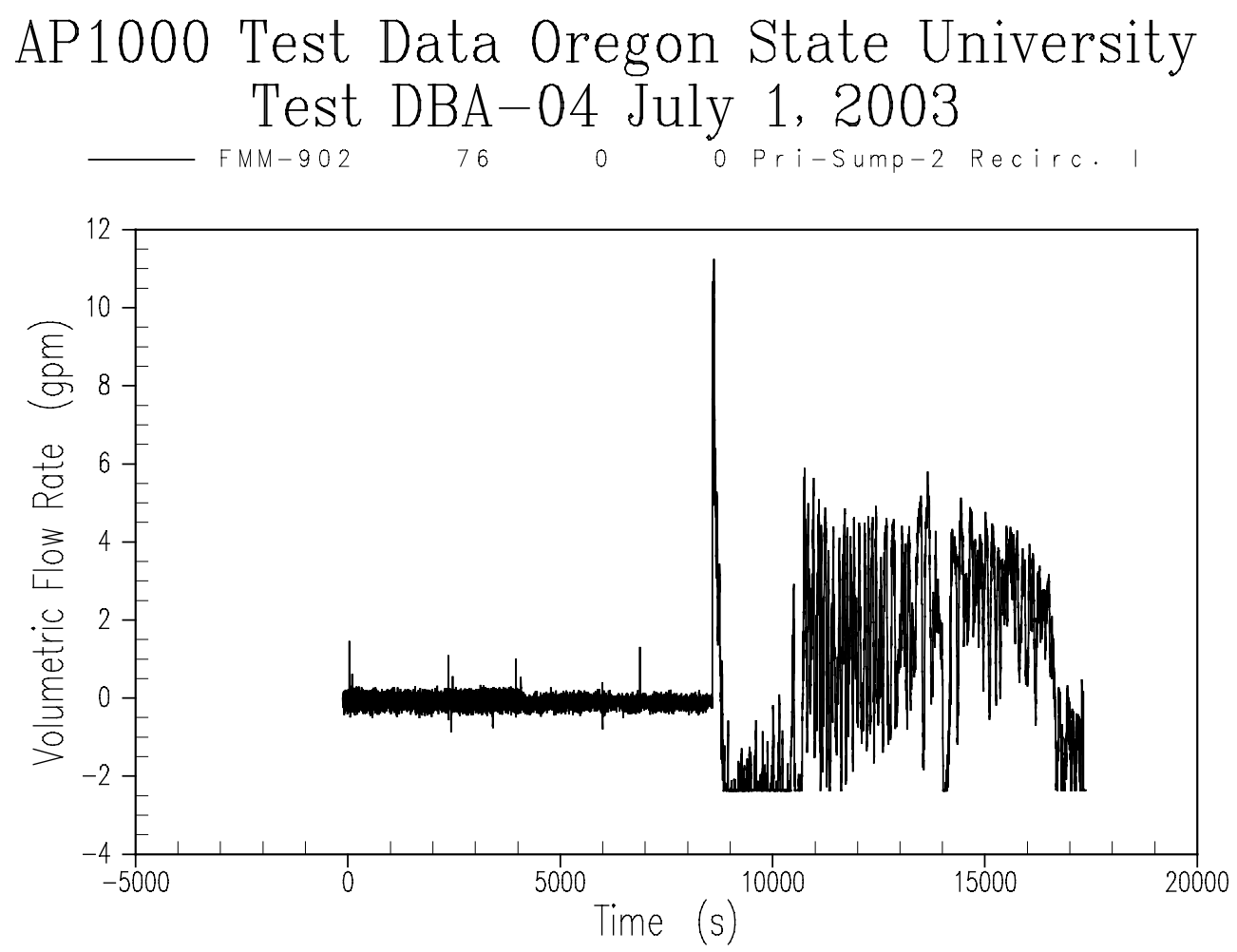

Figure A-55 Primary Sump 2 Injection Flow Rate 

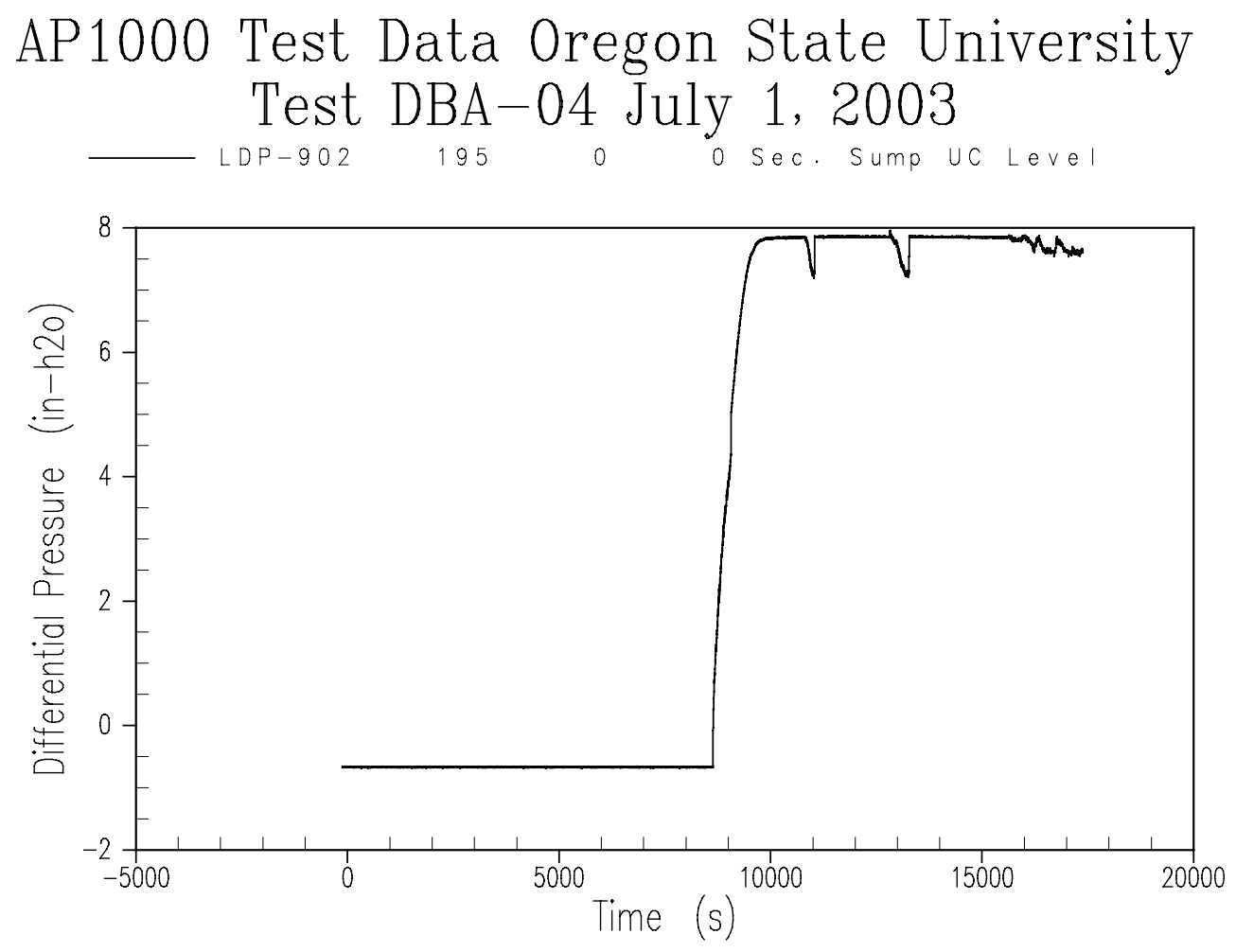

Figure A-56 Secondary Sump Liquid Level 


\section{AP1000 Test Data Oregon State University Test DBA-04 July 1, 2003 \\ $\begin{array}{llll}P T-905 & 239 & 0 & 0 \\ P r e a k & \text { Sep. Press. }\end{array}$}

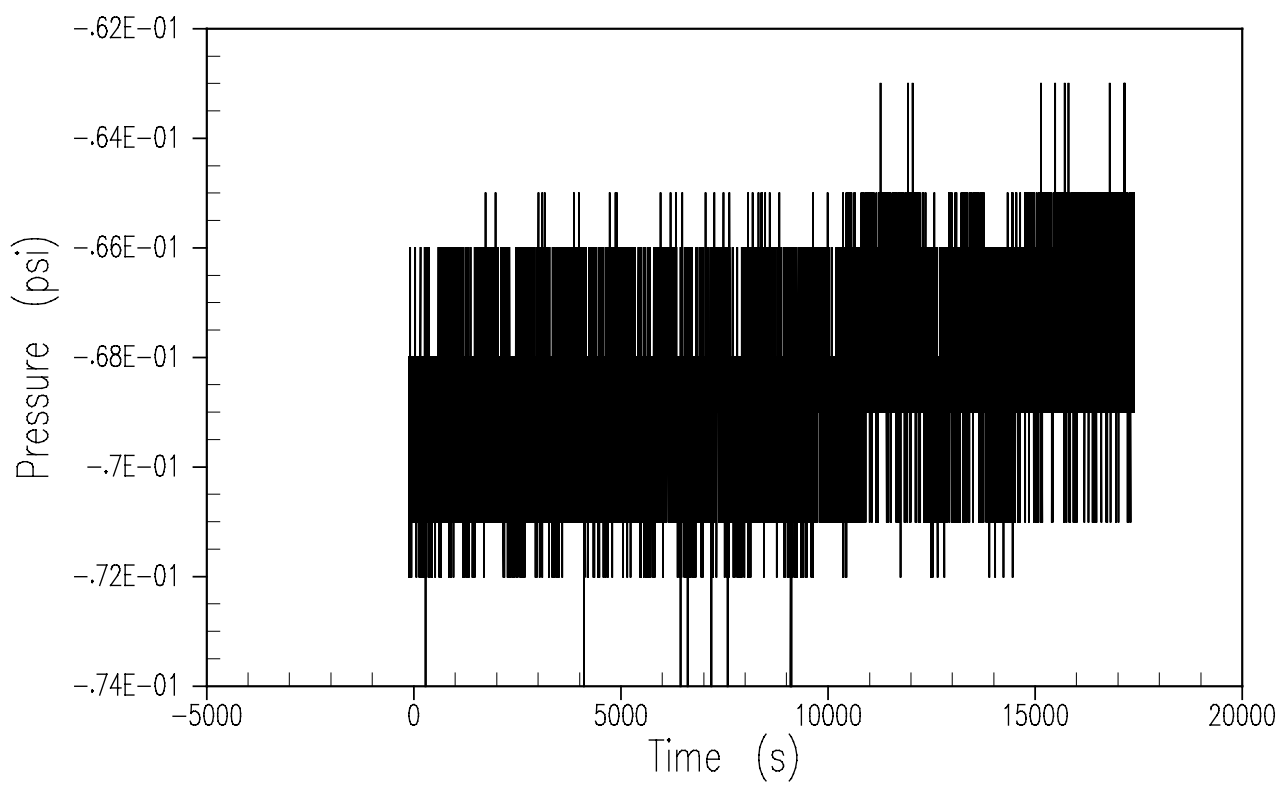

Figure A-57 Break Separator Pressure 
AP1000 Test Data Oregon State University Test DBA-04 July 1, 2003

— LDP-905 $197 \quad 0 \quad$ O Break Sep. UC Leve I

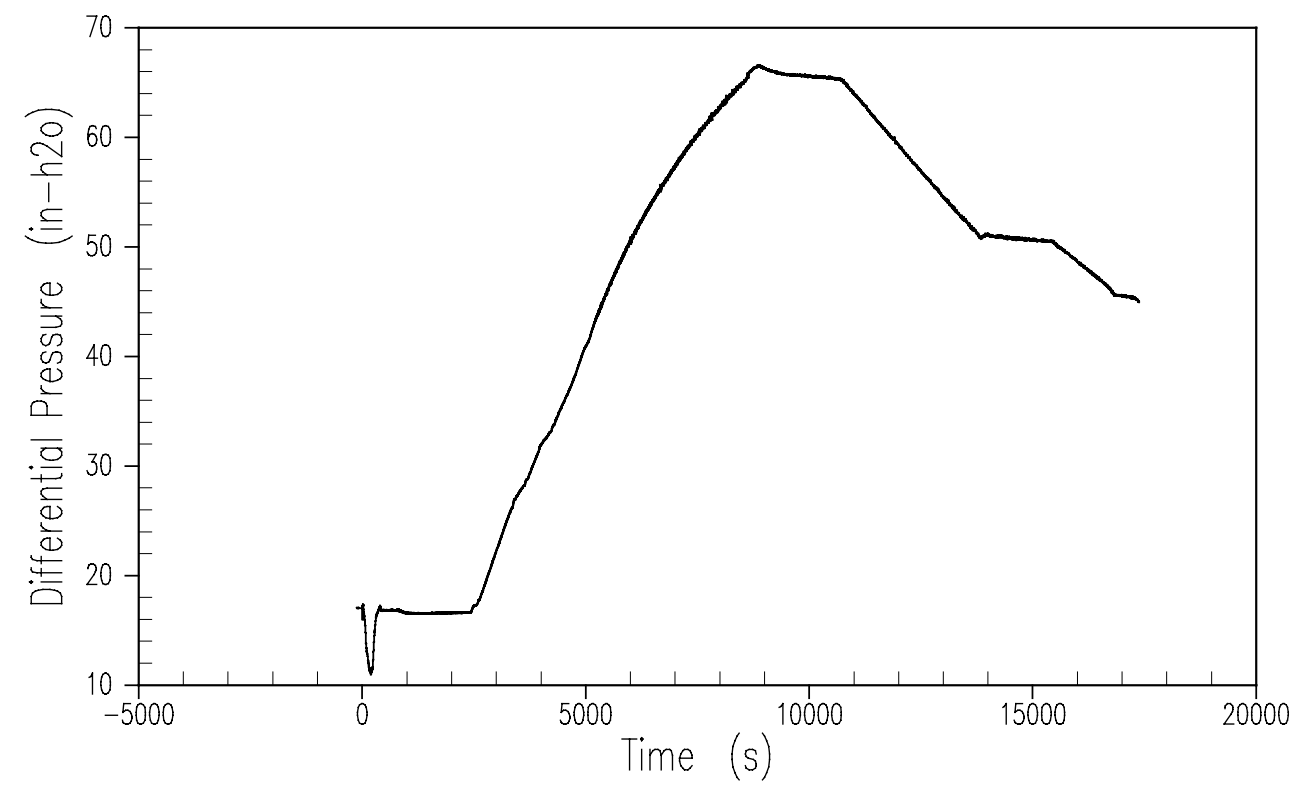

Figure A-58 Break Separator Liquid Level 


\section{AP1000 Test Data Oregon State University Test DBA-04 July 1, 2003

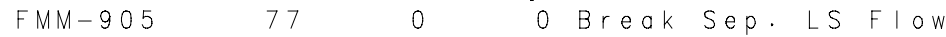

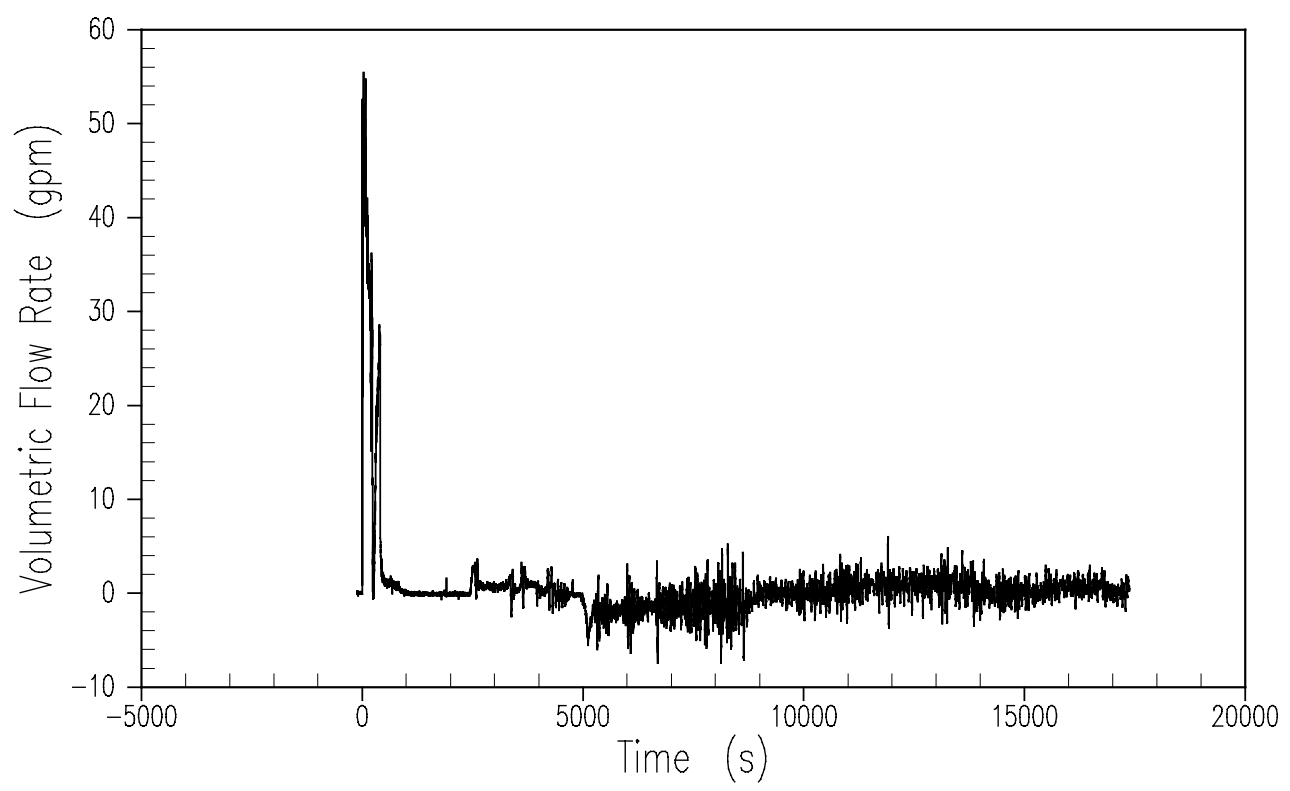

Figure A-59 Break Separator Flow to Primary Sump 

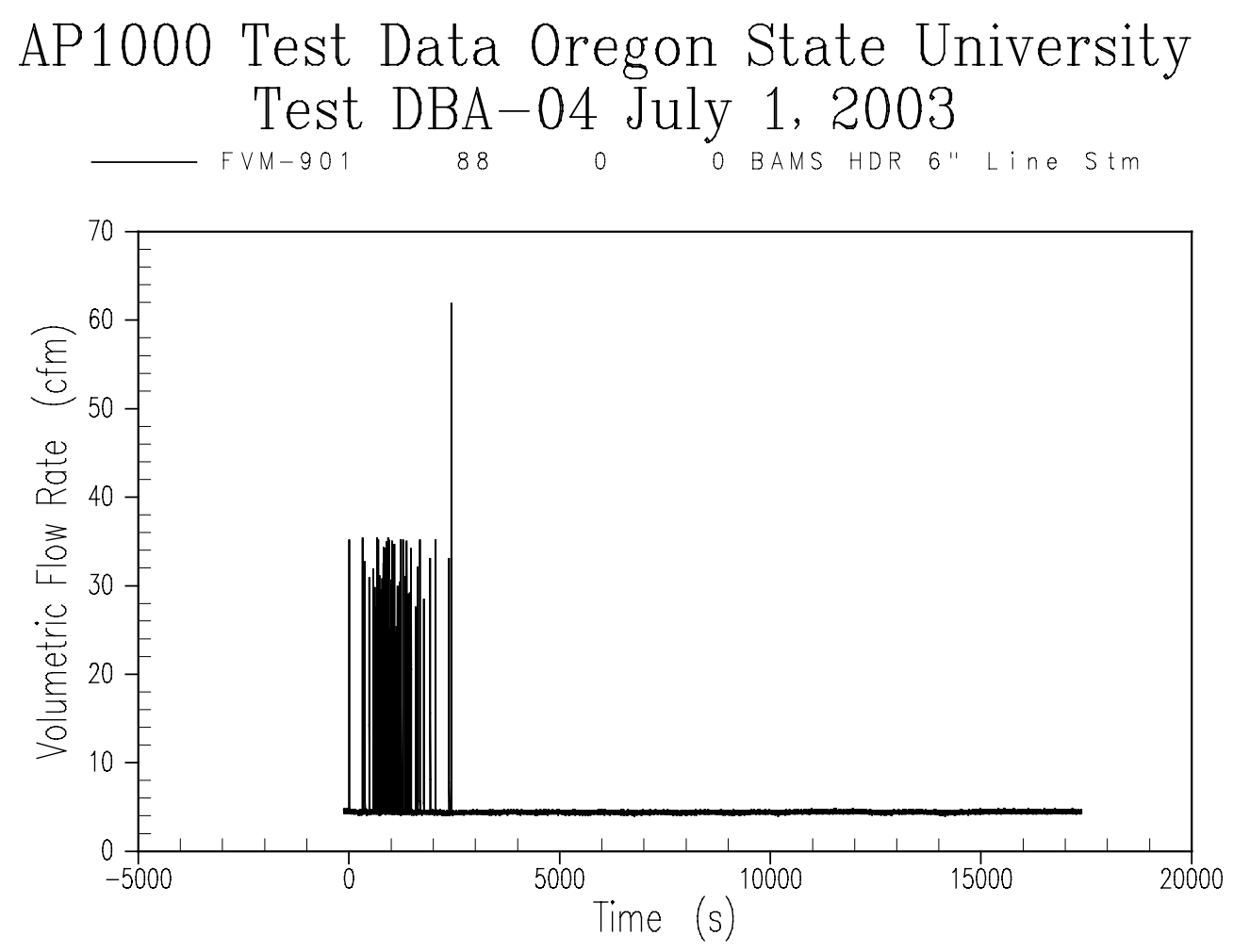

Figure A-60 BAMS Steam Flow Rate - 6-inch Line 

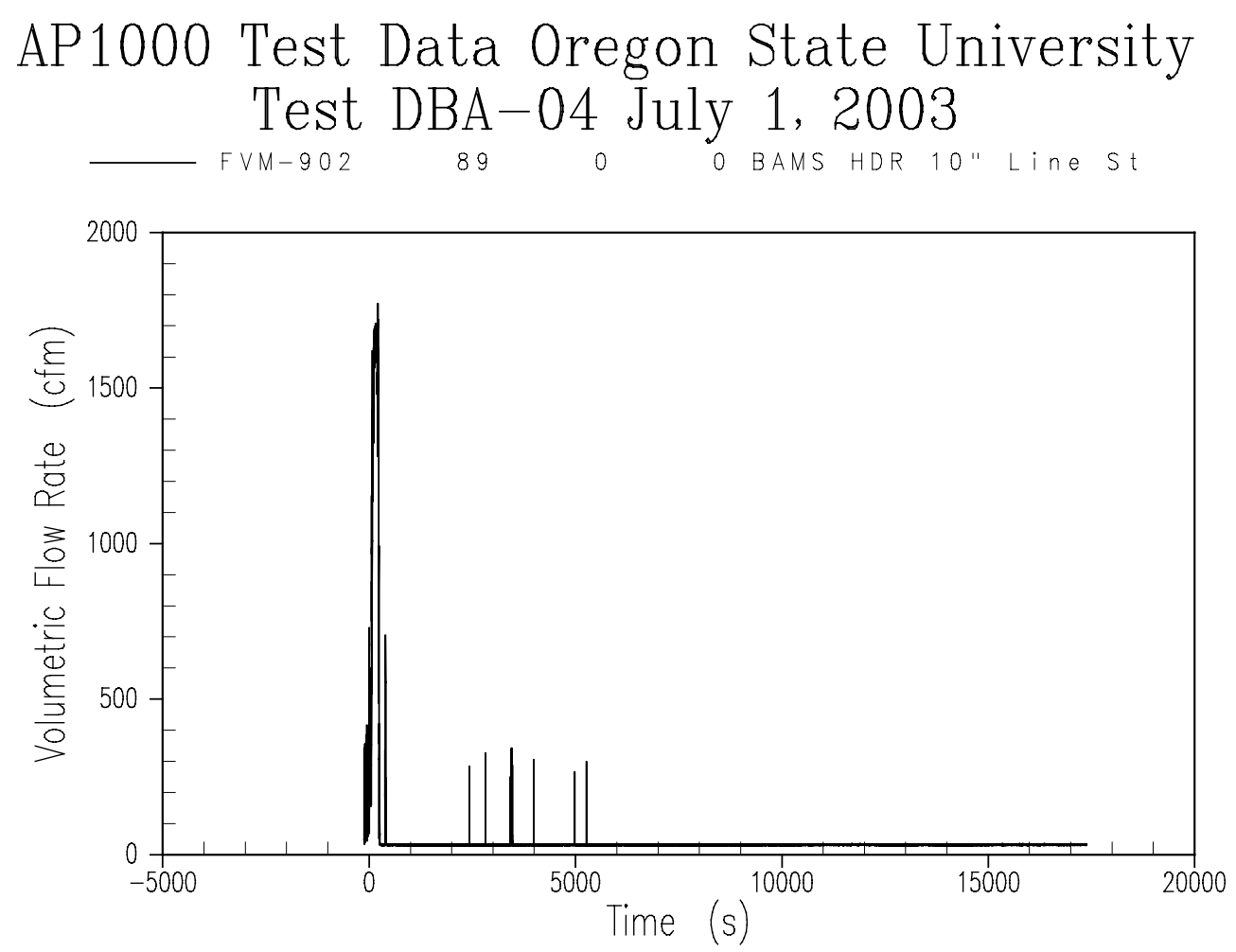

Figure A-61 BAMS Steam Flow Rate - 10-inch Line 
AP1000 Test Data Oregon State University Test DBA-04 July 1, 2003

$\begin{array}{llll}\text { FVM-903 } & 90 & 0 & \text { Pri-Sump Stm Exhaust }\end{array}$

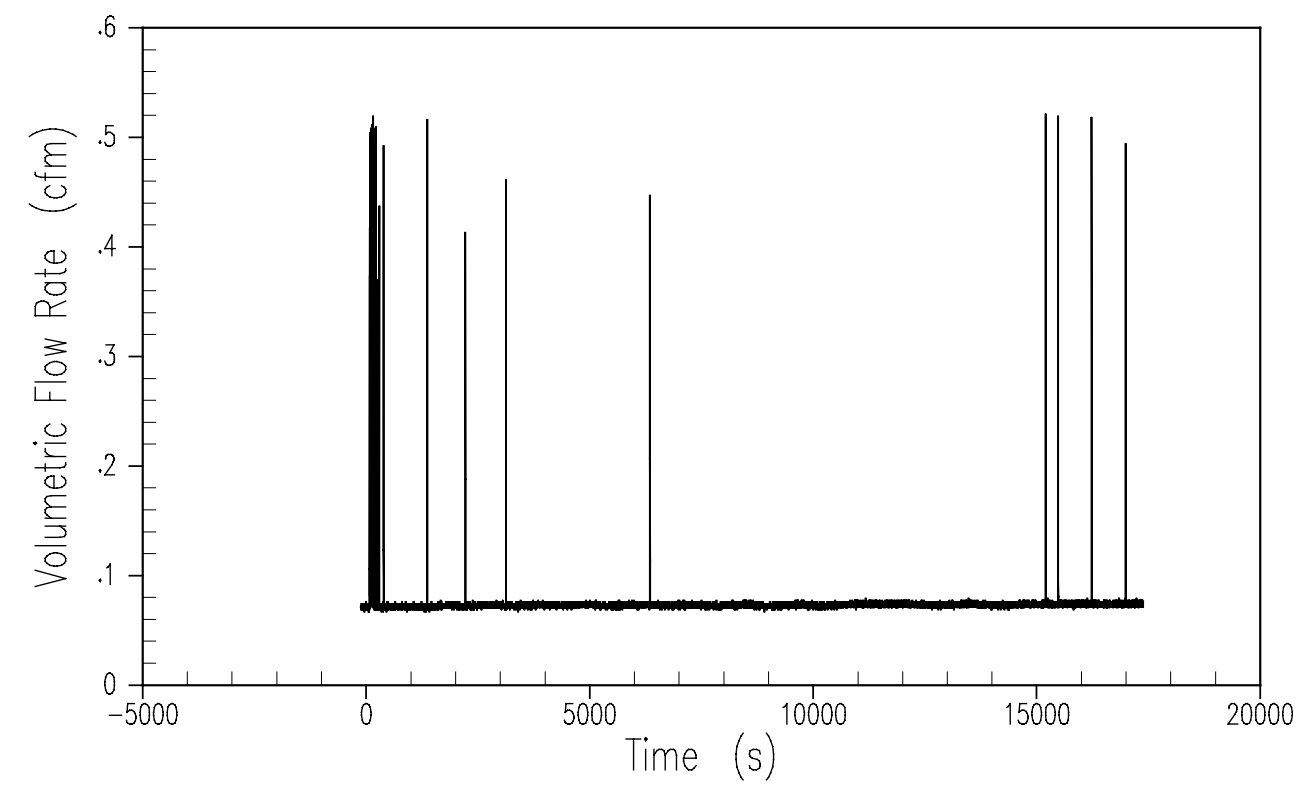

Figure A-62 BAMS/Primary Sump Steam Flow Rate 

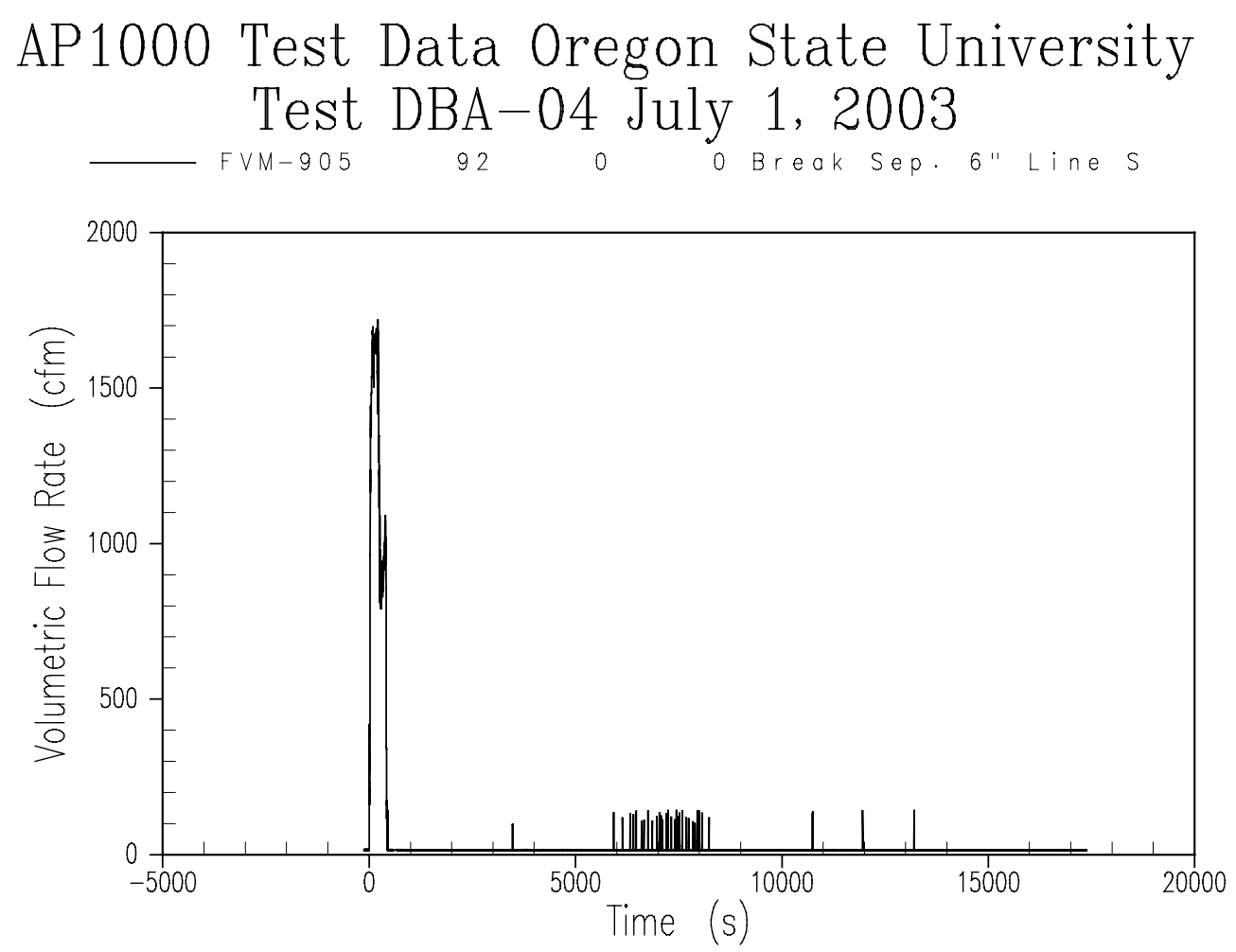

Figure A-63 BAMS/Separator Steam Flow Rate - 6-inch Pipe 
AP1000 Test Data Oregon State University Test DBA-04 July 1, 2003

$T F-916$

537

o BAMS Header $10^{\prime \prime}$ Stm

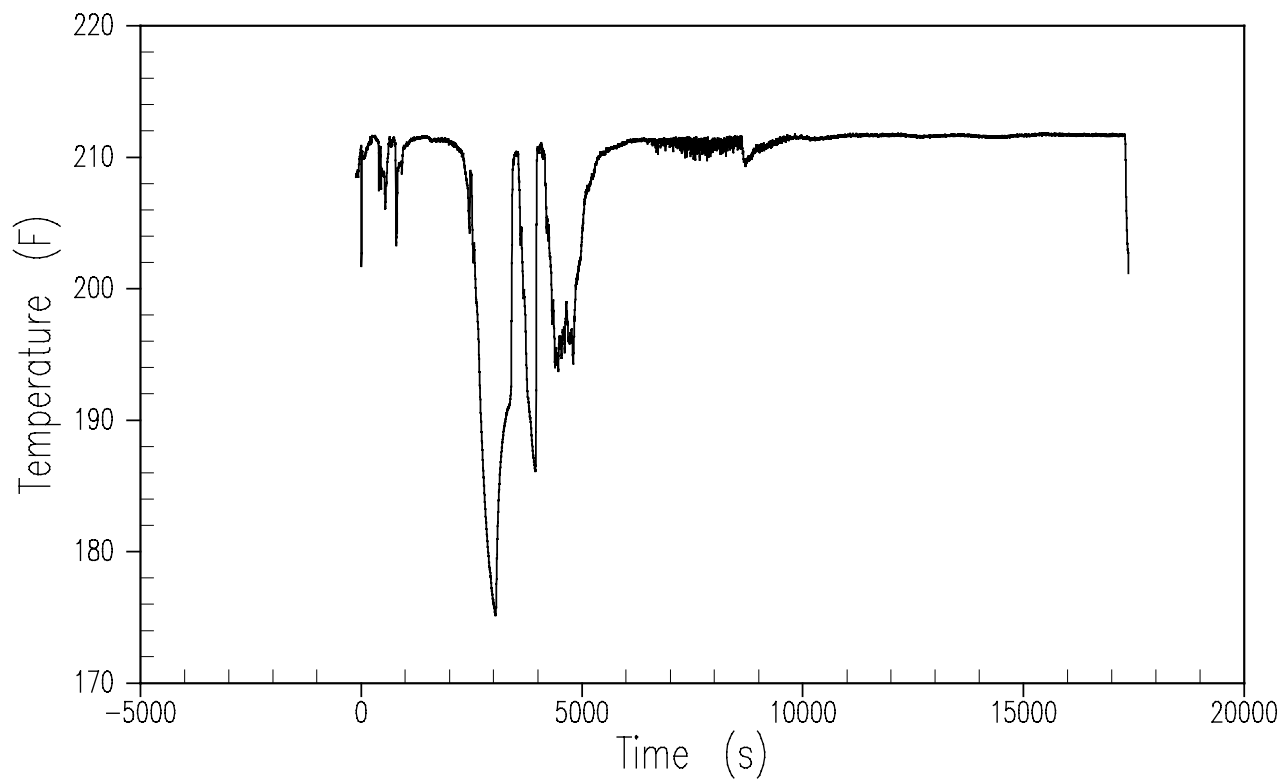

Figure A-64 BAMS/Exhaust Line Temperature - 10-inch Line 
AP1000 Test Data Oregon State University Test DBA-04 July 1, 2003

$\longrightarrow$ TF -917

$538 \quad 0 \quad 0 \quad T F-917 /$ BAMS Header

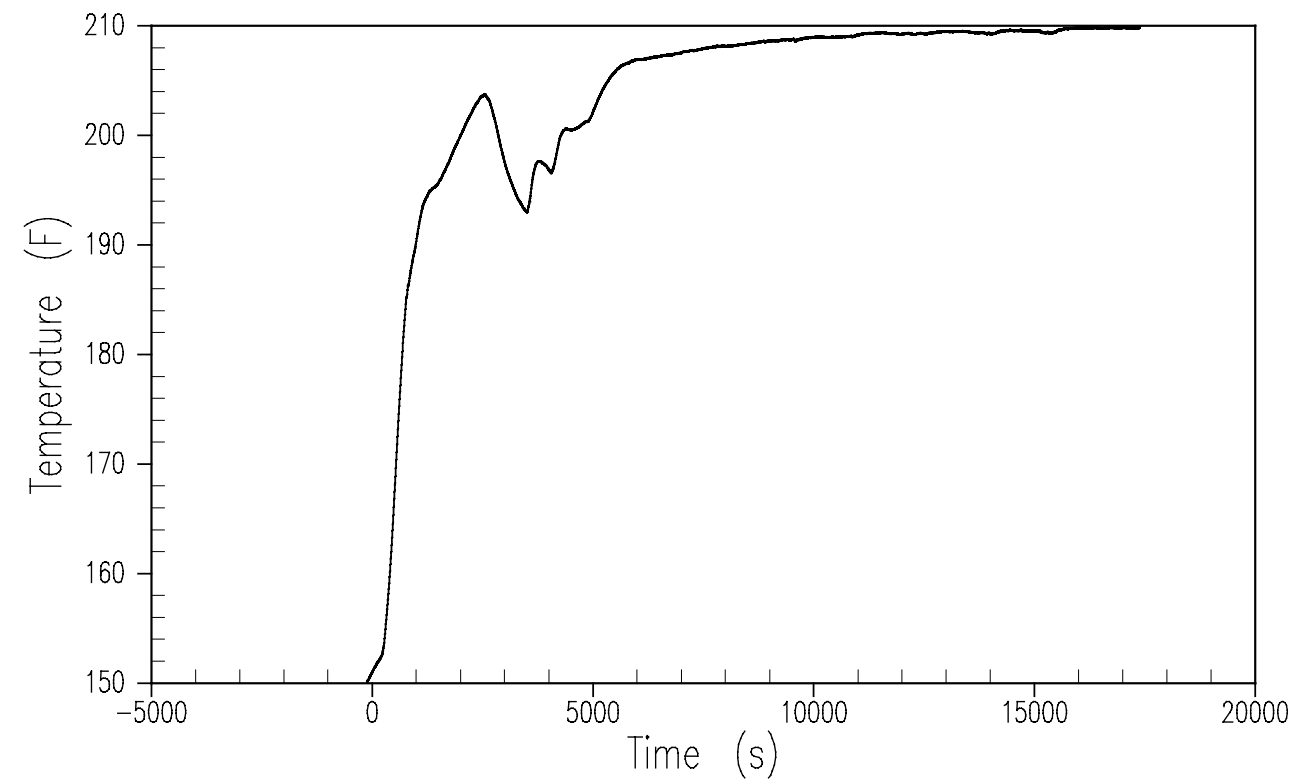

Figure A-65 BAMS/Exhaust Line Temperature - Header 

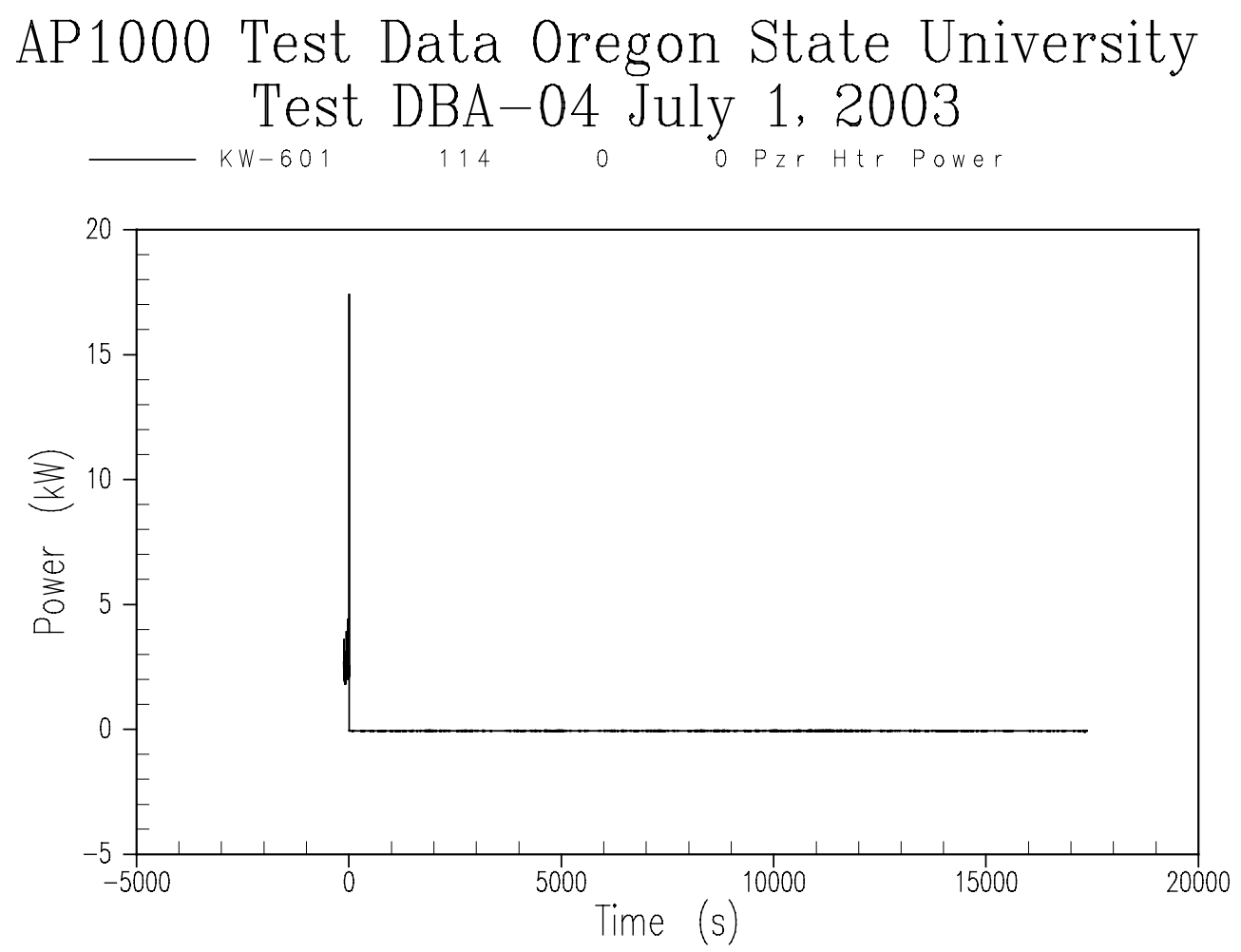

Figure A-66 Pressurizer Heater Input Power 

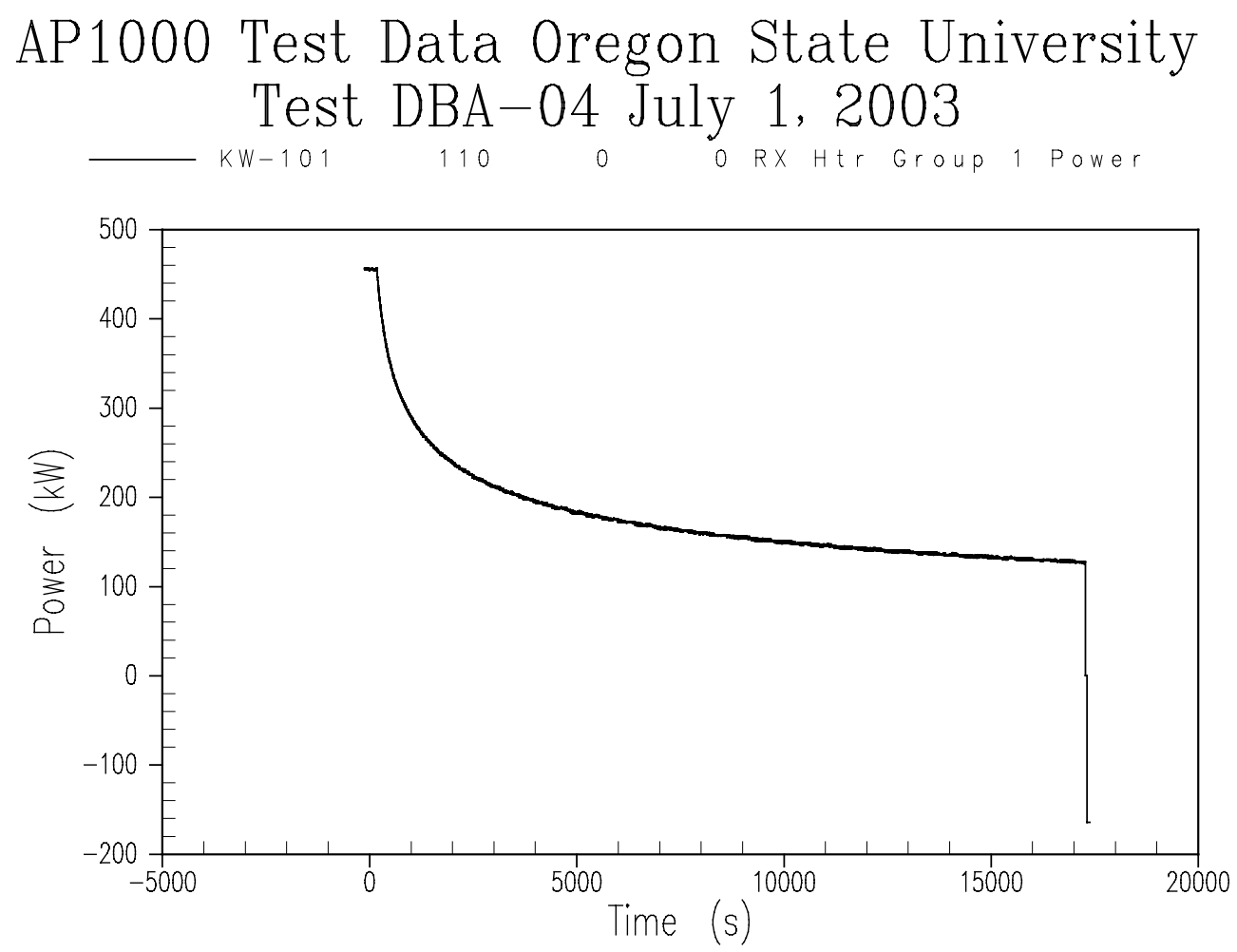

Figure A-67 Core Power Group 1 Input Power 

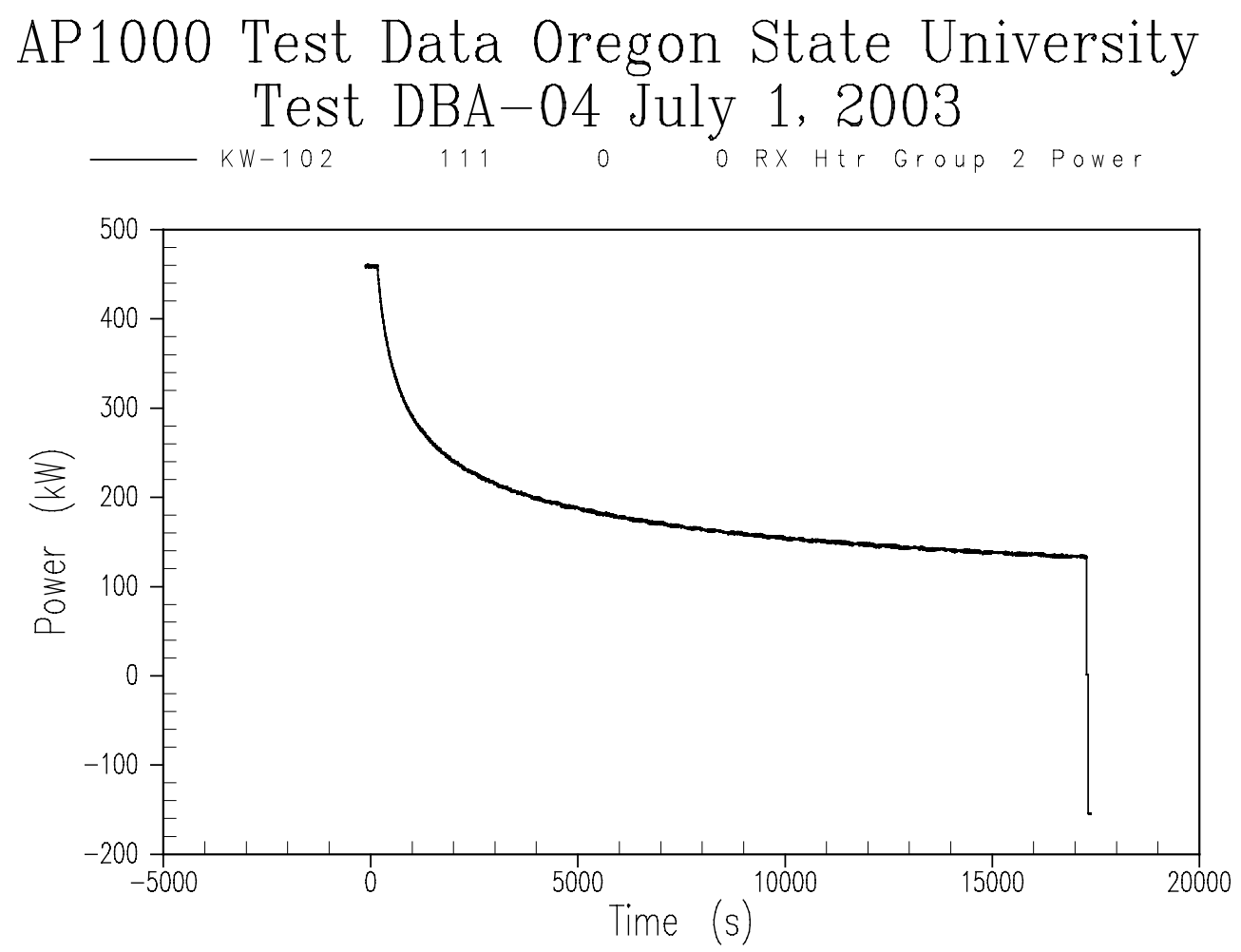

Figure A-68 Core Power Group 2 Input Power 

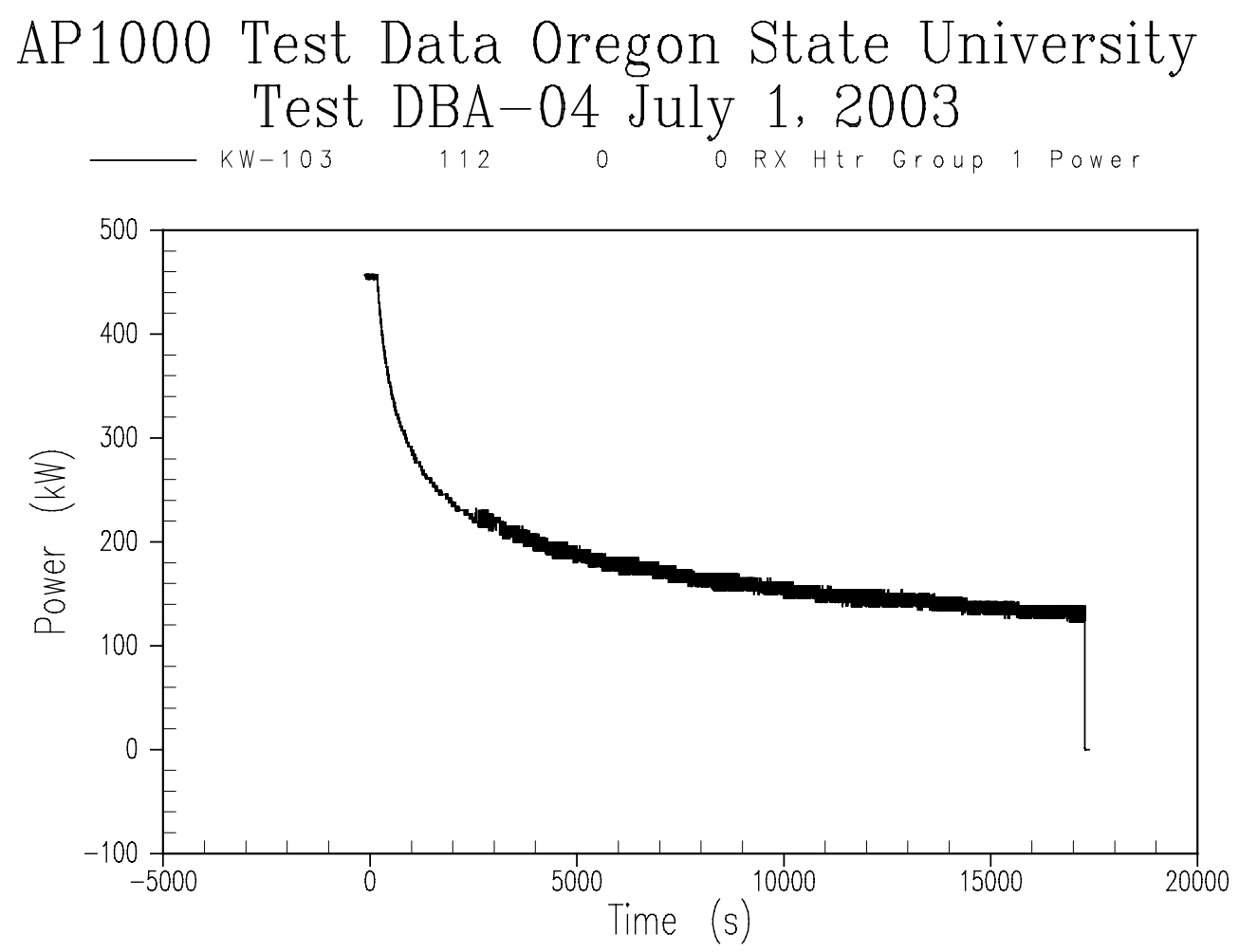

Figure A-69 Core Power Group 1 Alternate Input Power 

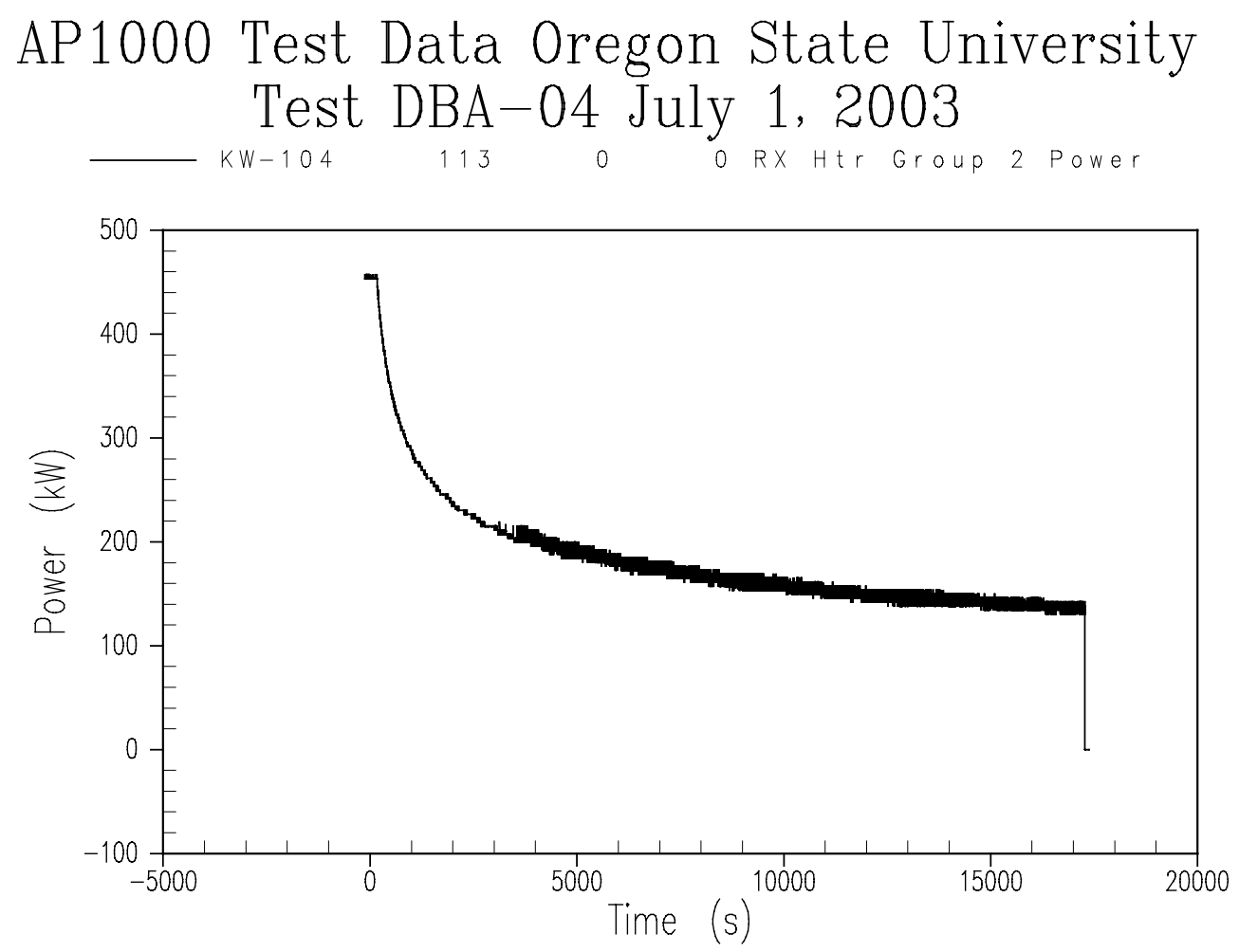

Figure A-70 Core Power Group 2 Alternate Input Power 

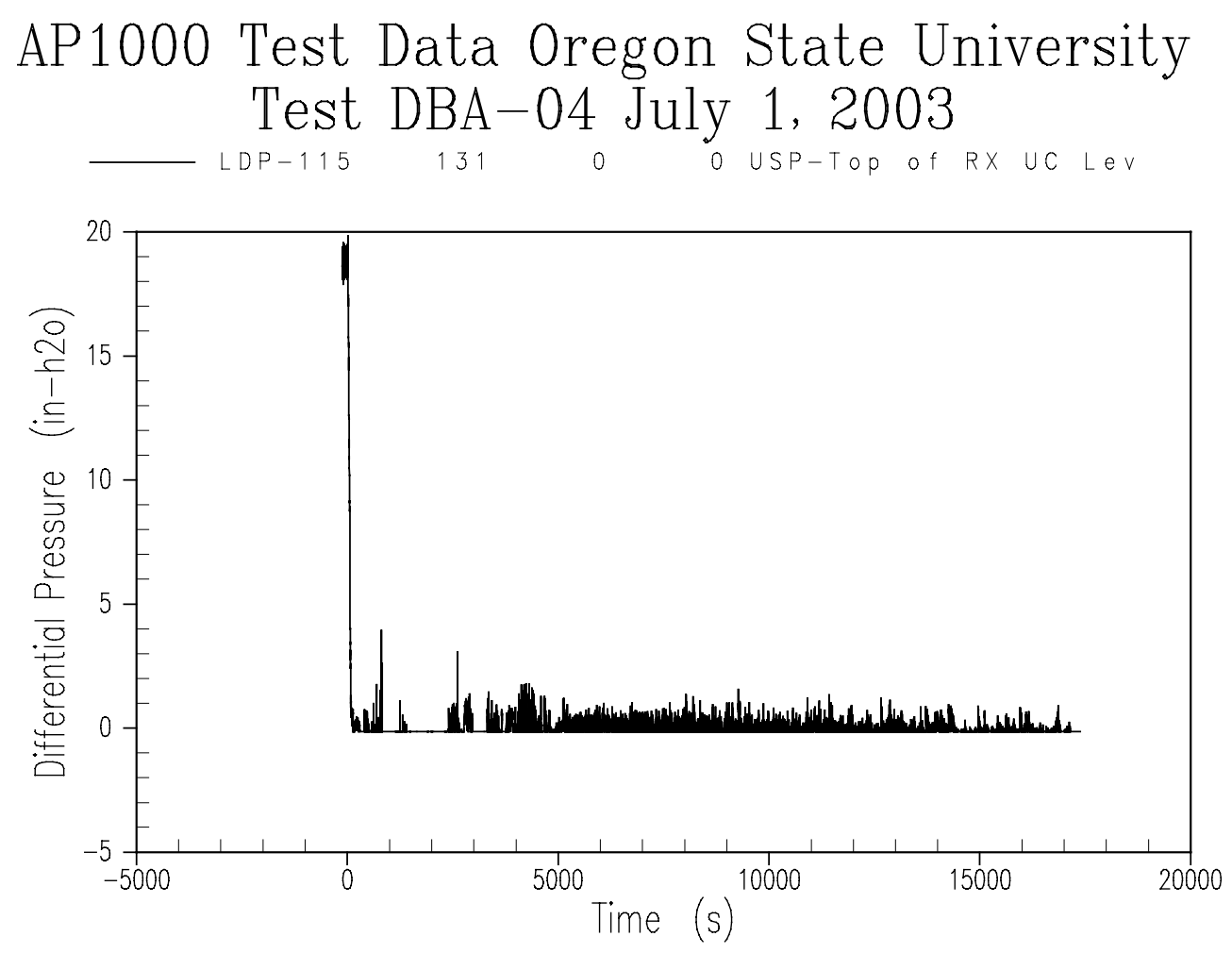

Figure A-71 Reactor Vessel Liquid Level Between Top of Vessel - Upper Support Plate 

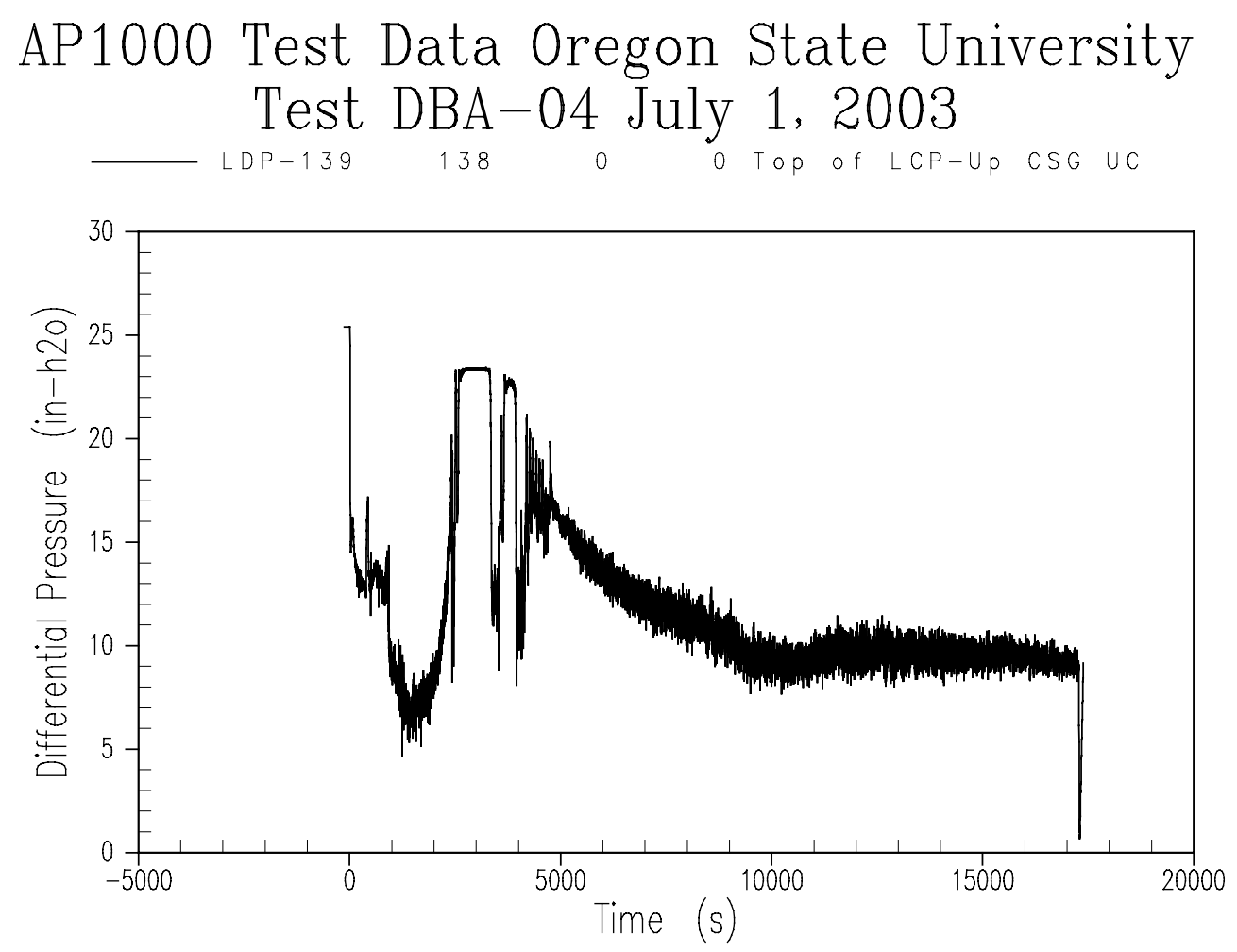

Figure A-72 Reactor Vessel Liquid Level Between Bottom of Upper Support Plate Upper Core Spacer Grid 

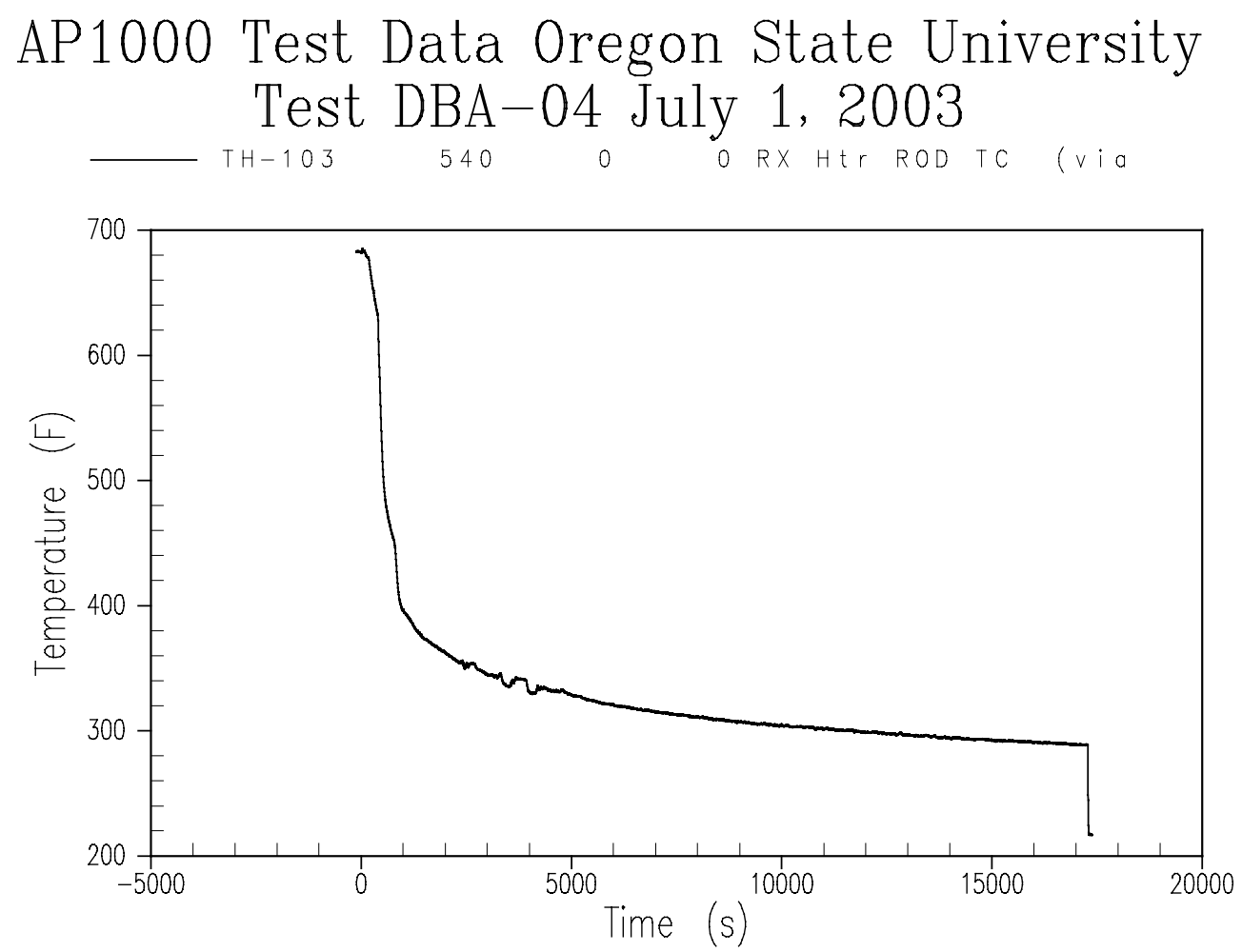

Figure A-73 Inner Core Thermocouple Measuring Heater Temperature 

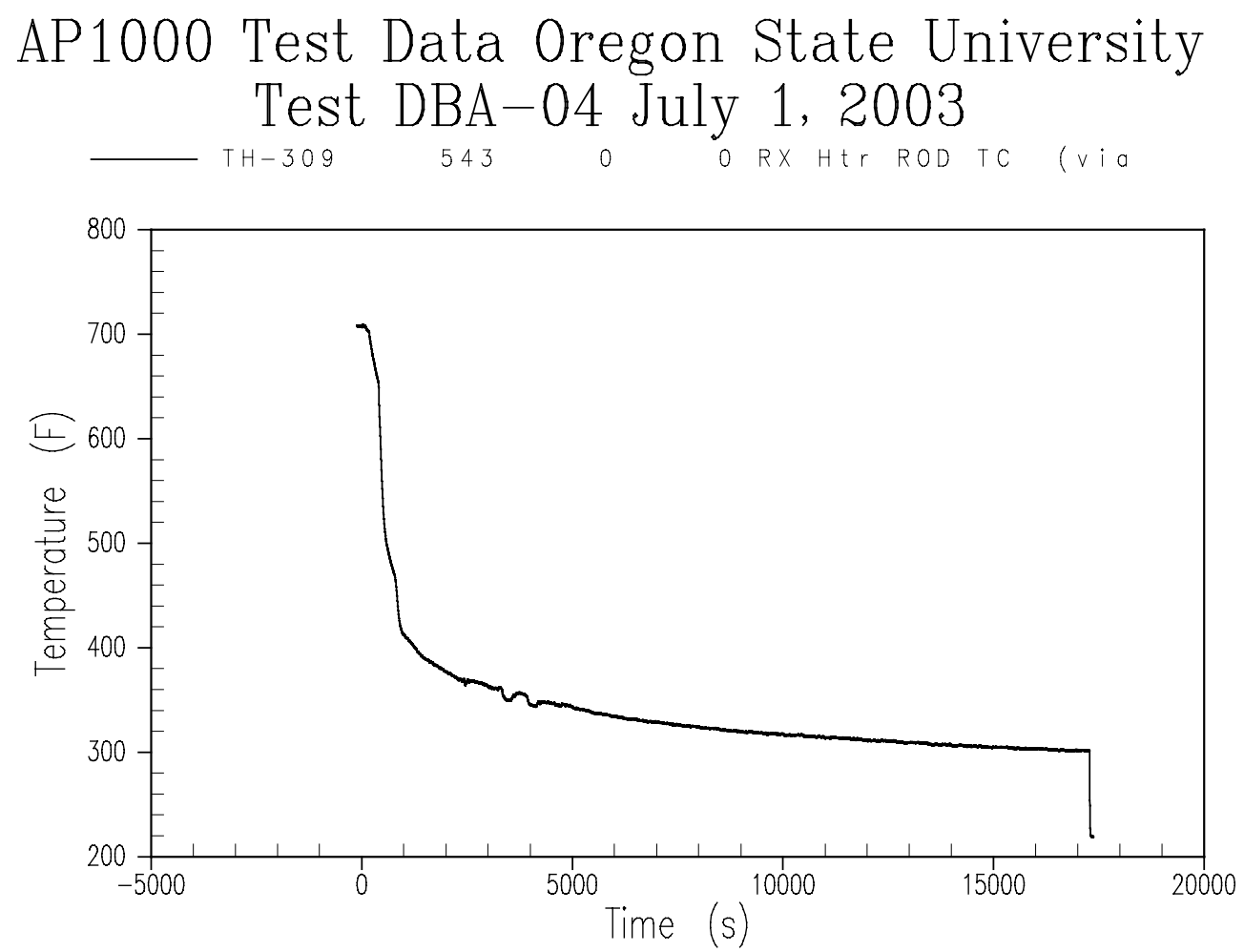

Figure A-74 Outer Core Thermocouple Measuring Heater Temperature 
APPENDIX B

TEST DATA 


\title{
Oregon State University
}

Department of Nuclear Engineering

\section{Advanced Thermal Hydraulic RESEARCH LABORATORY}

\section{TEST ACCEPTANCE REPORT}

\author{
OSU-AP1000-06
}

\section{AP1000 2-INCH COLD LEG BREAK WITH 3 OF 4 ADS 4 ADS Actuation at Plant-Prototypic Pressure}

Revision 0 


\title{
TEST ACCEPTANCE REPORT
}

\author{
OSU-AP1000-06
}

\section{AP1000 2-INCH COLD LEG BREAK WITH 3 OF 4 ADS 4 ADS Actuation at Plant-Prototypic Pressure}
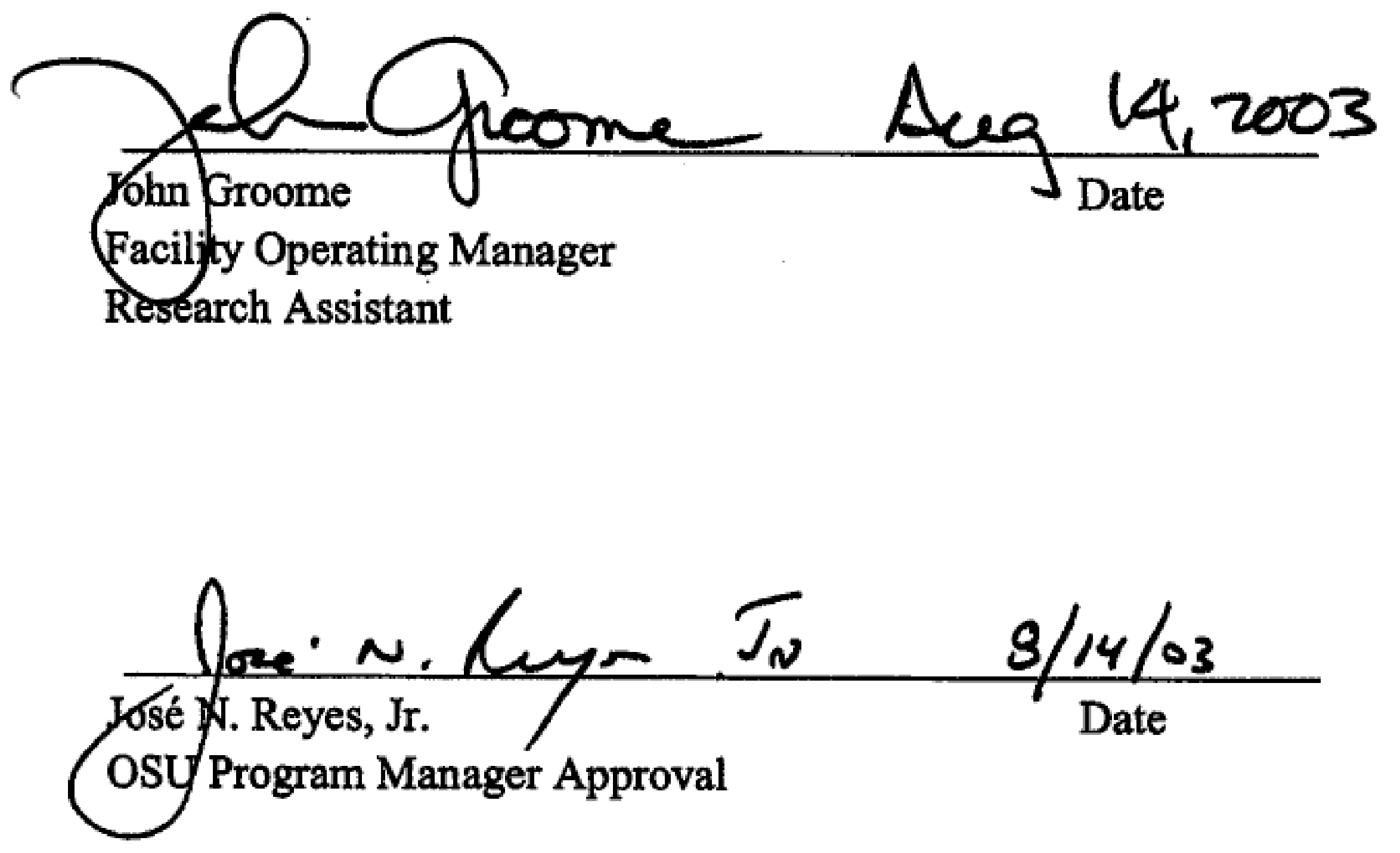

\author{
Oregon State University \\ 116 Radiation Center \\ Corvallis, Oregon 97331
}

(C) 2003 Oregon State University

All Rights Reserved 


\section{WESTINGHOUSE PROPRIETARY INFORMATION NOTICE}

This report contains information proprietary to Westinghouse Electric Company. A non-proprietary version of this report has also been prepared for distribution to the United States Nuclear Regulatory Commission (NRC).

In order to conform to the requirements of 10 CFR 2.790 of the Commission's regulations concerning the protection of proprietary information so submitted to the $\mathrm{NRC}$, the information which is proprietary in the proprietary version of this document is contained within brackets, and where the proprietary information has been deleted in the non-proprietary versions, only the brackets remain (the information that was contained within the brackets in the proprietary versions having been deleted). The justification for claiming the information so designated as proprietary is indicated in both versions by means of lower case letters (a) through (f) located as a superscript immediately following the brackets enclosing each item of information being identified as proprietary or in the margin opposite such information. These lower case letters refer to the types of information Westinghouse customarily holds in confidence identified in Sections (4)(ii)(a) through (4)(ii)(f) of the affidavit accompanying the transmittal of these documents pursuant to 10 CFR 2.790(b)(1).

\section{COPYRIGHT NOTICE}

This document bears an Oregon State University copyright notice. The NRC is permitted to make the number of copies of the information contained in these reports which are necessary for its internal use in connection with generic and plant-specific reviews and approvals as well as the issuance, denial, amendment, transfer, renewal, modification, suspension, revocation, or violation of a license, permit, order, or regulation subject to the requirements of $10 \mathrm{CFR} 2.790$ regarding restrictions on public disclosure to the extent such information has been identified as proprietary by Westinghouse, copyright protection notwithstanding. With respect to the non-proprietary versions of these reports, the NRC is permitted to make the number of copies beyond these necessary for its internal use which are necessary in order to have one copy available for public viewing in the appropriate docket files in the public document room in Washington, DC and in local public document rooms as may be required by NRC regulations if the number of copies submitted is insufficient for this purpose. Copies made by the NRC must include the copyright notice in all instances and the proprietary notice if the original was identified as proprietary. 


\section{TABLE OF CONTENTS}

$\underline{\text { Section }}$

$\underline{\text { Title }}$

$\underline{\text { Page }}$

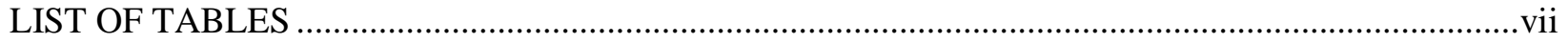

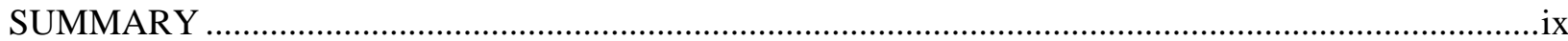

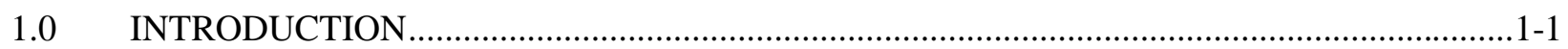

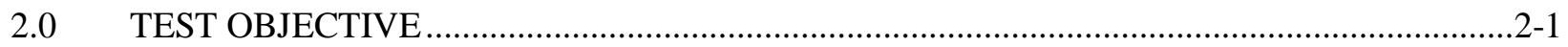

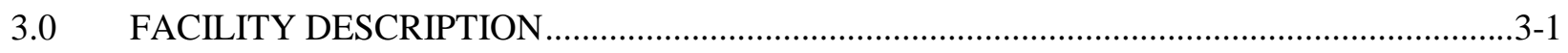

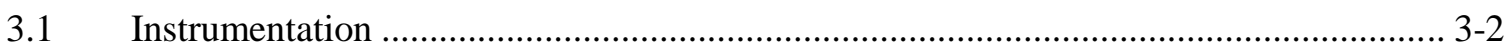

4.0 OSU TESTING PROGRAM MATRIX ...........................................................................

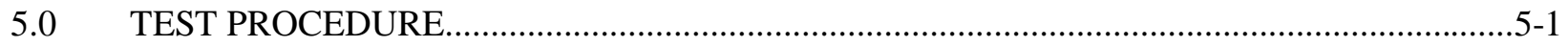

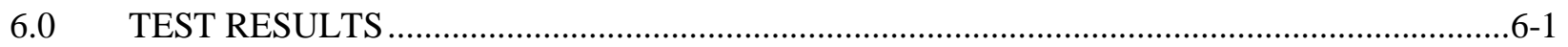

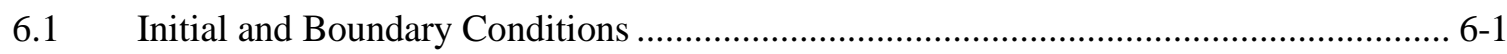

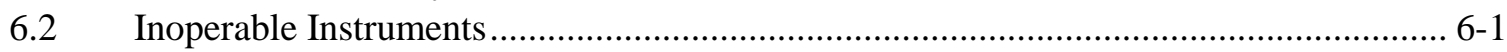

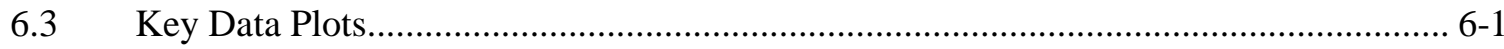

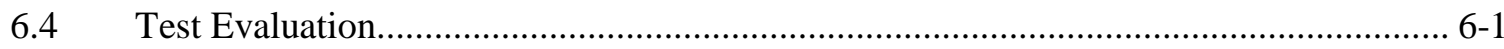

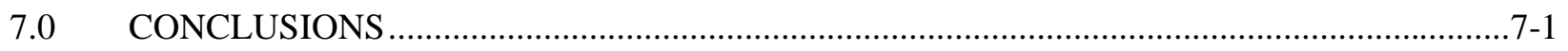

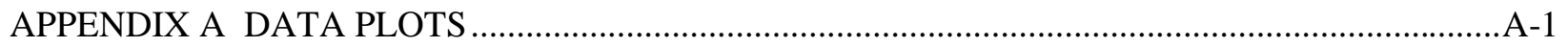

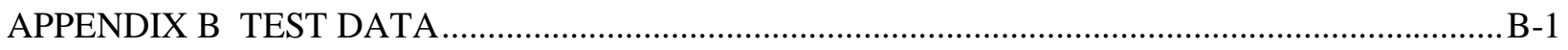




\section{LIST OF TABLES}

$\underline{\text { Table }}$

Table 4-1 OSU Test Matrix (Specified July 9, 2003).

Table 6-1 Actual Test Initial Conditions

$\underline{\text { Title }}$ $\underline{\text { Page }}$ 4-2 6-3

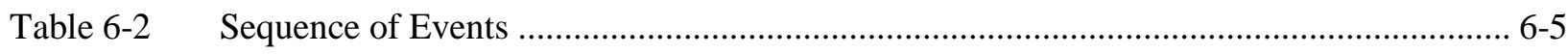

Table 6-3 Inoperable Instruments for T2R-02-D Test .............................................................. 6-7

Table 6-4 Data Plots for QL Reports for T2R-02-D by Component ........................................... 6-8 


\section{SUMMARY}

This report covers the test TR-02, 2-inch Cold Leg Break simulation loss-of-coolant accident (LOCA) performed on July 9, 2003. The objective of this test was to obtain thermal-hydraulic data for 2-inch Cold Leg Break simulation with ADS-4 actuation at plant-prototypic pressure conditions. The test performed met the specified conditions. The top of the heater bundle was always covered during this event. The test was performed for about 2200 seconds. The transient continued through Automatic Depressurization System (ADS) actuation, core makeup tank (CMT), accumulator, and incontainment refueling water storage tank (IRWST) injection.

This report presents the initial assessment of the test data collected. If this test is to be used by Westinghouse to support AP1000 Design Certification, additional validation of the use of this information will be documented separately. In the interim, the list of invalid data channels may change. 


\subsection{INTRODUCTION}

The Department of Nuclear Engineering at Oregon State University is performing a series of tests for the U.S. Department of Energy (DOE). These tests are being conducted in the Advanced Plant Experiment (APEX-1000) test facility, which is a reduced pressure and height model of the two-loop Westinghouse AP1000 pressurized water reactor. The purpose of the testing is to:

- evaluate the thermal-hydraulic performance of the passive safety systems of the full-scale AP1000, and

- to assess and validate the safety analyses techniques and computer codes used in predicting the transient system behavior.

The AP1000 Long-Term Cooling Test is a 1/4 height scale, low-pressure integral systems test simulating thermal-hydraulic phenomenon for the AP1000 passive safety systems for small-break loss-of-coolant accidents (LOCAs) and long-term cooling. It accurately models the details of the AP1000 geometry, including the primary system, the passive safety systems, and a part of the non-safety grade Chemical and Volume Control System, as well as a partial non-safety grade Normal Residual Removal System. The interconnecting pipe routings are also duplicated in the model.

The overall objective of the Long-Term Cooling Test program is to obtain test data at various modes of operation. The Oregon State University (OSU) experiments will examine the passive safety system response for the small-break and large-break LOCA transition into long-term cooling. (The list of the tests to be performed is provided in the attached OSU Test Matrix provided in Section 4.0.) The facility permits a range of small-break LOCAs to be simulated at different locations on the primary system, such as the cold leg, hot leg, core makeup tank (CMT) cold leg pressure balance line, and direct vessel injection line. The break orientation (top or bottom of the cold leg) may also be studied. Selected tests continue into the long-term cooling, post-accident mode in which the passive safety injection is from the reactor sump as well as the incontainment refueling water storage tank (IRWST). A large-break, post-accident, long-term cooling situation will also be simulated. 


\subsection{TEST OBJECTIVE}

The purpose of this test is to obtain thermal-hydraulic data for a 2-inch Cold Leg Break simulation with ADS-4 actuation at plant-prototypic pressure conditions. The break is located in cold leg \#3 which is a non-balance line cold leg. The data obtained from the test will be used to verify the AP1000 thermal-hydraulic computer codes for AP1000 phenomena such as gravity injection, natural convection, and post-accident long-term core cooling behavior.

The acceptance criteria for the OSU tests are as follows:

- Test initial conditions will be achieved within a specified tolerance.

- Set points will be achieved within an acceptable tolerance band.

- All instrumentation should be operational before the test.

- Any critical instruments not operating will be identified to the test engineer before the tests. These instruments must be operational before and during the test or exceptions should be approved.

- A zero check of LDPs, DPs, and FDPs will be performed. 


\subsection{FACILITY DESCRIPTION}

A detailed facility description for the OSU test facility is documented in Reference 1 . The OSU test facility has been specifically scaled, designed, and constructed to investigate the AP600 passive safety system behavior and to provide data for safety analysis computer code validation. The facility has been modified to model the AP1000.

The scaled test design accurately models the details of the AP1000 geometry, including the primary system and the pipe routings and layout for the passive safety systems. A detailed scaling report (Reference 2) was used to develop the test design modifications. The primary system consists of one hot leg and two cold legs with two active pumps and an active steam generator for each loop. Two CMTs are connected to one primary loop, and the pressurizer is connected to the other primary loop as in the AP1000 plant design. Gas-driven accumulators are connected to the DVI lines. The discharge lines from the CMT, and one-of-two IRWST and reactor sump lines are connected to each DVI line. The Automatic Depressurization System (ADS), consisting of stages 1, 2 and 3, simulates either one or two of the independent trains used in the AP1000. The two-phase flow from the ADS stages 1-to-3 is separated in a swirl-vane separator and the liquid and vapor flows are measured to obtain the total ADS flow rate. The separated flow streams are then recombined and discharged into the IRWST through a sparger, preserving the mass and energy flow into the IRWST. The injection from the reactor sump is also simulated. Note that the OSU facility models both AP1000 primary and secondary sumps. The primary sump collects the condensate return, the liquid break flow, and the liquid flow from the fourth-stage ADS; and will provide long-term injection to the reactor vessel. The secondary sump simulates the portions of containment that will remain dry during most events. This sump will collect water only when the primary sump reaches its overflow level, and provides no injection to the reactor vessel.

The time period for the experimental simulations includes not only the IRWST injection, but also the draining of the IRWST and the sump injection to simulate the long-term cooling of the AP1000. This simulation could be from several hours to a day. The time scale for the OSU test facility is one-half; that is, events occur in half the normal time. To model the long-term cooling aspects of the transient, the two-phase flow from the break is separated in a swirl-vane separator and the liquid and vapor portions of the total flow are measured. The liquid fraction of the flow is discharged to the reactor primary sump as in the AP1000 plant. The vapor is discharged to the atmosphere. The capability exists to return a portion of the equivalent liquid flow to the IRWST and primary sump to simulate the condensate return from the passive containment to the IRWST and primary sump. A similar approach is also used for the fourth-stage ADS valve on the hot leg. The two-phase flow is separated in a swirl-vane separator, the two streams are measured, and the liquid phase is discharged into the primary sump while the vapor flow is discharged to the atmosphere. Again, the capability exists to return a portion of the liquid equivalent added to the IRWST and primary sump. In addition, all other steam vents from the facility are measured (e.g., the IRWST vent), and a portion of the liquid equivalent may be added back to the facility. Note that not all of the steam discharge would be returned as liquid equivalent. A portion of the discharge would be removed to simulate the steam that is not available for recirculation because it provides containment pressurization. The IRWST and primary sump can be pressurized in the OSU facility to simulate the containment pressurization following a postulated LOCA.

A multi-tube passive residual heat removal (PRHR) heat exchanger is located in the IRWST. The heat exchanger uses the same C-tube design as the AP1000 and has two instrumented tubes to obtain wall heat 
fluxes during the tests. There are primary fluid thermocouples, wall thermocouples, and differential pressure drop measurements to determine when the heat exchanger begins to drain. The IRWST is also instrumented with strings of fluid thermocouples, to determine the degree of mixing within the tank and to assess the temperature of the coolant that is delivered to the test vessel.

The reactor vessel for the OSU tests includes a 0.914-meter (3-foot) heated core consisting of forty-eight 0.025-meter (1-inch) diameter heater rods. The heater rods have a top skewed power shape. The $1000 \mathrm{~kW}$ of electrical power available at the OSU test site will be used to simulate decay heat. Wall thermocouples are swaged inside the heater rods to measure the heater rod wall temperature.

Thermocouple rods in the heater rod bundle measure the axial coolant temperature distribution. The scaled flow volume in the core is preserved as well as the flow volume in the test vessel upper plenum. There are simulated reactor internals in the upper plenum to preserve the flow area and to correctly scale the fluid volume. The reactor vessel includes an annular downcomer into which the four cold legs and the two DVI lines are connected. The hot legs penetrate the reactor annulus and connect with the loops. The AP1000 reactor vessel neutron reflector is simulated using a ceramic liner to reduce the metal heat release to the coolant.

There were no special/unique requirements for the test other than those specified in the Initial Conditions. The specified conditions were verified on the control board prior to test implementation.

\subsection{Instrumentation}

The instrumentation has been designed to calculate a transient mass and energy balance on the test facility. All two-phase flow streams exiting the facility are separated, and each component is measured separately as a single phase flow using conventional measurement devices such as magnetic flow meters and vortex flow meters. Note that magnetic flowmeters are not designed for two-phase flow and will indicate erratically. Also note, the vortex flowmeters are referenced to $212^{\circ} \mathrm{F}$ and the LDPs are referenced to $60^{\circ} \mathrm{F}$. All vertical components have differential pressure cells that act as level instruments to measure the transient mass change in the component. The hot and cold leg diameters are sufficiently large in the OSU test facility such that a narrow range differential pressure cell can be used to determine if the flow becomes stratified.

Single flow measurements are made of the CMT, accumulator, IRWST, and sump flows into the reactor vessel through the direct vessel injection lines.

Various types of instrumentation are provided in the test facility; for example, thermocouples for coolant and wall temperatures, flowmeters, pressure transducers, differential pressure transducers, and weigh tanks. 


\subsection{OSU TESTING PROGRAM MATRIX}

The test matrix for the OSU test facility is shown in Table 4-1. To satisfy the test objectives, several transients will be performed to provide data on the AP1000 passive safety system response for a range of break sizes, locations, orientations, and single failure assumptions. The break size orifices are scaled based on simulating a 1-inch, 2-inch, or 4-inch pipe break.

The designation for this test is TR-02, which identifies the test as a design basis 2-inch Cold Leg Break with ADS-4 actuation at plant-prototypic pressure conditions. The test matrix may be adjusted for future tests based on results and insights gained with each test. 


\begin{tabular}{|c|c|c|}
\hline \multicolumn{3}{|c|}{$\begin{array}{l}\text { TABLE 4-1 } \\
\text { OSU TEST MATRIX (SPECIFIED JULY 9, 2003) }\end{array}$} \\
\hline Test Title & Break Location and Size & Single Failure Assumed \\
\hline DBA-01-D & $\begin{array}{l}\text { Double-ended DVI Line break with } \\
\text { continuation into long-term cooling }\end{array}$ & Fail 1 of 2 lines in 1 ADS- 4 train \\
\hline DBA-02-D & $\begin{array}{l}\text { Double-ended DVI Line break with } \\
\text { continuation into long-term cooling }\end{array}$ & $\begin{array}{l}\text { Fail } 1 \text { of } 2 \text { lines in } 1 \text { ADS-4 train } \\
\text { (Adjusted ADS-4 Resistance) } \\
\text { Single failure on non-pressurizer side }\end{array}$ \\
\hline DBA-03-D & $\begin{array}{l}\text { Double-ended DVI Line break with } \\
\text { continuation into long-term cooling }\end{array}$ & $\begin{array}{l}\text { Fail } 1 \text { of } 2 \text { lines in } 1 \text { ADS- } 4 \text { train } \\
\text { Single failure on pressurizer side }\end{array}$ \\
\hline DBA-04-D & 2-inch Cold Leg Break (CL \#3) & $\begin{array}{l}\text { Fail } 1 \text { of } 2 \text { lines in } 1 \text { ADS- } 4 \text { train } \\
\text { Single failure on pressurizer side }\end{array}$ \\
\hline TR-02-D & $\begin{array}{l}\text { Transition Test ADS } 4 \text { opening, } 125 \text { psig initial } \\
\text { pressure and decay power } 480 \mathrm{sec}\end{array}$ & $\begin{array}{l}\text { Fail } 1 \text { of } 2 \text { lines in } 1 \text { ADS-4 train } \\
\text { (No ADS 1-3) }\end{array}$ \\
\hline TR-03-D & $\begin{array}{l}\text { Transition Test ADS4 opening, } 85 \text { psig initial } \\
\text { pressure and decay power } 1800 \mathrm{sec}\end{array}$ & $\begin{array}{l}\text { Fail } 1 \text { of } 2 \text { lines in } 1 \text { ADS-4 train } \\
\text { (No ADS 1-3) }\end{array}$ \\
\hline TR-04-D & $\begin{array}{l}\text { Transition Test ADS4 opening, } 85 \text { psig initial } \\
\text { pressure and decay power } 480 \mathrm{sec}\end{array}$ & $\begin{array}{l}\text { No Failure of ADS-4 lines assumed } \\
\text { (No ADS 1-3) }\end{array}$ \\
\hline EN-01-D & $\begin{array}{l}\text { Entrainment Test with Revised Upper Internals, } \\
1000 \mathrm{~kW} \text { reactor power, } 14.7 \mathrm{psi}\end{array}$ & Fail 1 of 2 lines in 1 ADS- 4 train \\
\hline EN-01-D & $\begin{array}{l}\text { Entrainment Test with Revised Upper Internals, } \\
700 \mathrm{~kW} \text { reactor power, } 14.7 \mathrm{psi}\end{array}$ & Fail 1 of 2 lines in 1 ADS- 4 train \\
\hline PRA-01-D & PRA Test - DEDVI with no accumulators & No ADS-4 Failure assumed \\
\hline PRA-02-D & $\begin{array}{l}\text { PRA Test - 3-inch Hot Leg Break with no } \\
\text { CMTs }\end{array}$ & No ADS-4 Failure assumed \\
\hline
\end{tabular}




\subsection{TEST PROCEDURE}

The test was performed as per a written procedure. There were no special/unique requirements for the test other than those specified in the initial conditions in Table 6-1. The specified conditions were checked on the control board prior to test implementation.

The appropriate prerequisites were completed and initial conditions were satisfied. The required break simulation piping and break instrumentation were installed per P\&ID drawing OSU 600904, Rev. 1. A break spool insert simulating the 2-inch Cold Leg Break in AP1000 was installed in the break spool in cold leg \#3 which is a cold leg without a CMT balance line. Flow from the break is directed horizontally into the break separator. The 100-percent flow nozzle was installed in the ADS 4-2 (on the hot leg 2) and the 50-percent flow nozzle was installed in ADS 4-1 (on the hot leg 1). Flow nozzles that simulate full flow for ADS-1, ADS-2, and ADS-3 were installed. As per the AP600 tests, ADS-3 has been scaled for full flow from all three stages, and ADS-1 and ADS- 2 are closed when ADS-3 is opened.

Fill and vent was performed per APEX Operations Manual Procedure OP-B.2. Instruments were checked for required calibration.

With the break valve TS-202 closed, flow was used to warm up the bypass line by opening isolation valves RCS-901 and RCS-902. After the appropriate prerequisites were completed and the test facility achieved specified initial conditions, the CMT warm-up bypass line isolation valves RCS-901 and RCS-902 were closed to maintain the $<80^{\circ} \mathrm{F}$ condition at the top of the CMT- 1 . With the CMT balance line valves (RCS-529 and RCS-530) placed in the open and automatic mode, both CMTs reached the reactor coolant system (RCS) pressure.

Once all other initial conditions were satisfied, a break through TS-202 was initiated. The transient continued through ADS actuation. After ADS-1 was actuated, the RCS pressure was monitored until it reached approximately $100 \mathrm{psia}$. At this point a number of operator actions were taken:

1. ADS4-1 was manually actuated, and ADS4-2 was actuated approximately 30 seconds later

2. Accumulators were isolated for the remainder of the test

3. The heater rod power controller was adjusted to simulate decay heat levels at 800 seconds (determined from test DBA-04 as the properly scaled time of ADS-4 actuation)

4. The level of the CMTs was recorded. The CMTs were allowed to drain an additional 20 inches, and then were isolated to simulate the inventory at ADS-4 actuation level.

Since the IRWST was not pressurized to simulate full-height, the IRWST cut-in for full-pressure conditions could not be simulated. Instead, the test continued to depressurize until the lower IRWST cut-in pressure was reached, and stable IRWST injection was established. The test was terminated at this time. IRWST actuation was automatic and required no operator action. 


\subsection{TEST RESULTS}

The test results for test TR-02-D are provided in the following subsections.

\subsection{Initial and Boundary Conditions}

Table 6-1 provides a comparison of the specified and actual conditions for test TR-02-D. The values in this table were averaged over approximately 2 minutes preceding the test. Test initial conditions were achieved for the steam generator pressure, pressurizer pressure, pressurizer level, steam generator 01 narrow-range level, and steam generator 02 narrow-range level. Test initial conditions for the hot leg temperature were found to be acceptable, and the results will not be adversely affected.

The actual power decay curves are provided in data plots in Appendix B. The measured maximum power was $905 \mathrm{~kW}$ which was less than the facility maximum power of $1000 \mathrm{~kW}$. The programmed decay heat curve was adjusted to account for this difference, and the differences between the actual and specified power decay are considered acceptable.

PT-501 and PT-502 pressure instruments indicate the pressure changes in the CMT-1 and CMT-2. CMT-1 (PT-501) and CMT-2 (PT-502) confirm that 1 minute after the test button was pushed, both CMTs reach RCS pressure.

The sequence of events is shown in Table 6-2. This table compares the actual sequence of events with the specified timing. As can be seen in this table, all the events occurred at or very near to when the event was planned.

\subsection{Inoperable Instruments}

Table 6-3 provides a list of the instrumentation channels considered inoperable for the DBA-04-D test.

\subsection{Key Data Plots}

Table 6-4 provides a list of the instrumentation channels sorted by component, and includes instrument number, units, and quick look plot number. The selection of channels was based on projecting an overall picture of the test results, which would then be examined by referring to the detailed data plots or tapes.

\subsection{Test Evaluation}

The following observations were made during the test:

1. The peak power before the test was initiated was $905 \mathrm{~kW}$. The decay heat curve was adjusted for this value.

2. As was discussed in Section 5.0, the test required significant operator action to simulate ADS-4 actuation at plant-prototypic pressure. These actions were performed properly, and ADS-4 was actuated when the RCS pressure reached approximately 100 psia. 
3. The test procedure called for the test to be terminated when the RCS pressure reached the plant-prototypic IRWST cut-in pressure of 28 psia. This pressure was reached with no core uncovery observed. In addition, the pressure eventually decreased to the scaled IRWST cut-in pressure of 18 psia and stable IRWST injection was established with no core uncovery observed. 


\begin{tabular}{|c|c|c|c|}
\hline \multicolumn{4}{|c|}{$\begin{array}{c}\text { TABLE 6-1 } \\
\text { ACTUAL TEST INITIAL CONDITIONS }\end{array}$} \\
\hline Conditions & Instrument No. & Actual & Comment \\
\hline Pressurizer Pressure & PT-604 & 370 psig & \\
\hline $\begin{array}{l}\text { Hot Leg Temperature } \\
\quad \# 1\end{array}$ & $\begin{array}{l}\text { TF-141* } \\
\text { TF-205 } \\
\text { TF-143 }\end{array}$ & $\begin{array}{c}426^{\circ} \mathrm{F} \\
427.8^{\circ} \mathrm{F} \\
428.3^{\circ} \mathrm{F}\end{array}$ & \\
\hline $\begin{array}{l}\text { Hot Leg Temperature } \\
\quad \# 2\end{array}$ & $\begin{array}{l}\text { TF-140* } \\
\text { TF-206 } \\
\text { TF-142 }\end{array}$ & $\begin{array}{l}427.8^{\circ} \mathrm{F} \\
426.3^{\circ} \mathrm{F} \\
428.4^{\circ} \mathrm{F}\end{array}$ & \\
\hline $\begin{array}{l}\text { SG Pressure } \\
\# 1 \\
\# 2 \\
\text { Header }\end{array}$ & $\begin{array}{l}\text { PT-301* } \\
\text { PT-302* } \\
\text { PT-002 }\end{array}$ & $\begin{array}{l}286.6 \mathrm{psig} \\
284.4 \mathrm{psig} \\
268.3 \mathrm{psig}\end{array}$ & \\
\hline PZR Level & $\begin{array}{l}\text { LDP-601 uncompensated } \\
\text { LDP-601 } \\
\text { Compensated by SC-608 }\end{array}$ & $\begin{array}{l}65.1 \text { inches } \\
77.1 \text { inches }\end{array}$ & $\begin{array}{l}440^{\circ} \mathrm{F} \text { used for density } \\
\text { compensation }\end{array}$ \\
\hline $\begin{array}{l}\text { SG Level } \\
\text { \#1 NR }\end{array}$ & $\begin{array}{l}\text { LDP-303 uncompensated } \\
\text { LDP-303 compensated by } \\
\text { average of TF-305 and } \\
\text { TF-307 }\end{array}$ & $\begin{array}{l}20.1 \text { inches } \\
24.3 \text { inches }\end{array}$ & $\begin{array}{l}413^{\circ} \mathrm{F} \text { used for density } \\
\text { compensation }\end{array}$ \\
\hline $\begin{array}{l}\text { SG Level } \\
\# 2 \mathrm{NR}\end{array}$ & $\begin{array}{l}\text { LDP-304 uncompensated } \\
\text { LDP-304 compensated by } \\
\text { average of TF-306 and } \\
\text { TF-308 }\end{array}$ & $\begin{array}{l}20.8 \text { inches } \\
25 \text { inches }\end{array}$ & $\begin{array}{l}414^{\circ} \mathrm{F} \text { used for density } \\
\text { compensation }\end{array}$ \\
\hline IRWST Temperature & TF-701 & $72^{\circ} \mathrm{F}$ & Accepted $\left(<80^{\circ} \mathrm{F}\right)$ \\
\hline $\begin{array}{l}\text { CMT Temperature } \\
\text { \#1 } \\
\# 2\end{array}$ & $\begin{array}{l}\text { TF-529 } \\
\text { TF-532 }\end{array}$ & $\begin{array}{c}72^{\circ} \mathrm{F} \\
75.2^{\circ} \mathrm{F}\end{array}$ & Accepted $\left(<80^{\circ} \mathrm{F}\right)$ \\
\hline $\begin{array}{l}\text { Accumulator } \\
\text { Temperature } \\
\# 1 \\
\# 2\end{array}$ & $\begin{array}{l}\text { TF-403 } \\
\text { TF-404 }\end{array}$ & $\begin{array}{l}73.3^{\circ} \mathrm{F} \\
75.2^{\circ} \mathrm{F}\end{array}$ & Accepted $\left(<80^{\circ} \mathrm{F}\right)$ \\
\hline IRWST Level & LDP-701 & 91.9 inches & \\
\hline
\end{tabular}




\begin{tabular}{||c|c|c|c||}
\hline \hline \multicolumn{2}{|c||}{ TABLE 6-1 (Continued) } \\
\multicolumn{1}{||c||}{ ACTUAL TEST INITIAL CONDITIONS } \\
\hline \hline Conditions & Instrument No. & Actual & Comment \\
\hline Accumulator Level & & & \\
$\# 1$ & LDP-401 & 35.8 inches & \\
\hline Accumulator Pressure & LDP-402 & 36.8 inches & \\
$\# 1$ & & & \\
$\# 2$ & PT-401 & $228.6 \mathrm{psig}$ & \\
\hline CMT Level & PT-402 & $233.9 \mathrm{psig}$ & \\
$\# 1$ & & & \\
$\# 2$ & LDP-507 & $57.6 \mathrm{inches}$ & \\
\hline CMT Pressure & LDP-502 & $57.7 \mathrm{inches}$ & \\
$\# 1$ & & & \\
$\# 2$ & PT-501 & $374 \mathrm{psig}$ & \\
\hline \hline
\end{tabular}




\begin{tabular}{|c|c|c|}
\hline \multicolumn{3}{|c|}{$\begin{array}{c}\text { TABLE 6-2 } \\
\text { SEQUENCE OF EVENTS }\end{array}$} \\
\hline Event & Setpoint & Actual Time (sec) \\
\hline PB Depressed & N/A & -120 \\
\hline Break Valve(s) Open & 0 & 0 \\
\hline Feed Pump Trip & $0 \mathrm{sec}$ & 0 \\
\hline CMT01 Outlet Valve Open (RCS-535) & $6.1 \mathrm{sec}$ & 3 \\
\hline CMT02 Outlet Valve Open (RCS-536) & $6.1 \mathrm{sec}$ & 3 \\
\hline PRHR HX Outlet Valve Open (RCS-804) & $6.1 \mathrm{sec}$ & 3 \\
\hline RCP \#1 Trip & $4.6 \mathrm{sec}$ & 5 \\
\hline RCP \#2 Trip & $4.6 \mathrm{sec}$ & 6 \\
\hline RCP \#3 Trip & $4.6 \mathrm{sec}$ & 6 \\
\hline RCP \#4 Trip & $4.6 \mathrm{sec}$ & 6 \\
\hline CMT \#1 Level Low (LDP-507) & 41 inches & 359 \\
\hline CMT \#2 Level Low (LDP-502) & 41 inches & 397 \\
\hline ADS \#1 Actuation (RCS-601) & CMT Level Low $+15 \mathrm{sec}$ & 375 \\
\hline ADS \#2 Actuation (RCS-602) & CMT Level Low $+62 \mathrm{sec}$ & 421 \\
\hline ADS \#3 Actuation (RCS-603) & CMT Level Low + $122 \mathrm{sec}$ & 481 \\
\hline Low Reactor Pressure (P-107) & 40 psig & 515 \\
\hline IRWST Valve Actuation (RCS-711) & $\begin{array}{l}\text { Low Reactor Pressure } \\
\qquad(<40 \mathrm{psig})\end{array}$ & 517 \\
\hline IRWST Valve Actuation (RCS-712) & $\begin{array}{l}\text { Low Reactor Pressure } \\
\qquad(<40 \mathrm{psig})\end{array}$ & 517 \\
\hline ADS 4-1 Actuation (RCS-615) & $\begin{array}{l}\text { CMT Low Low (17.14") and } \\
\text { CMT Low }\left(41^{\prime \prime}\right)+180 \mathrm{sec}\end{array}$ & 474 \\
\hline ADS 4-2 Actuation (RCS-616) & $\begin{array}{l}\text { CMT Low Low (17.14") and } \\
\text { CMT Low }\left(41^{\prime \prime}\right)+180 \mathrm{sec}\end{array}$ & 499 \\
\hline $\begin{array}{l}\text { Accumulator Injection } \\
\# 1 \text { (FMM-401) }\end{array}$ & N/A & 412 \\
\hline $\begin{array}{l}\text { Accumulator Injection } \\
\text { \#2 (FMM-402) }\end{array}$ & N/A & 410 \\
\hline
\end{tabular}




\begin{tabular}{|c|c|c|}
\hline \multicolumn{3}{|c|}{$\begin{array}{l}\text { TABLE 6-2 (Continued) } \\
\text { SEQUENCE OF EVENTS }\end{array}$} \\
\hline Event & Setpoint & Actual Time (sec) \\
\hline $\begin{array}{l}\text { IRWST Injection } \\
\text { DVI \#1 (FMM-701) }\end{array}$ & N/A & 875 \\
\hline $\begin{array}{l}\text { IRWST Injection } \\
\text { DVI \#2 (FMM-702) }\end{array}$ & N/A & 872 \\
\hline $\begin{array}{l}\text { CMT Empty } \\
\quad \# 1 \text { (LDP-507) }\end{array}$ & N/A & 584 down to 21 inch level \\
\hline $\begin{array}{l}\text { CMT Empty } \\
\text { \#2 (LDP-502) }\end{array}$ & N/A & 581 down to 22.75 inch level \\
\hline
\end{tabular}




\begin{tabular}{|c|c|c|}
\hline \multicolumn{3}{|c|}{$\begin{array}{c}\text { TABLE 6-3 } \\
\text { INOPERABLE INSTRUMENTS FOR TR-02-D TEST }\end{array}$} \\
\hline Instrument Number & Instrument Type & Inoperable Description \\
\hline $\begin{array}{l}\text { TW-202 } \\
\text { TW-204 } \\
\text { TW-205 } \\
\text { TW-206 } \\
\text { TW-209 } \\
\text { TW-803 } \\
\text { TW-804 }\end{array}$ & Thermocouple & Inoperative \\
\hline TH-603 & $\begin{array}{l}\text { Thermocouple measuring heater } \\
\text { temperature }\end{array}$ & Inoperative \\
\hline FMM-202 & Magnetic flow meter & Inoperative \\
\hline $\begin{array}{l}\text { TF-170 } \\
\text { TF-221 } \\
\text { TF-509 } \\
\text { TF-512 }\end{array}$ & $\begin{array}{l}\text { Thermocouple measuring fluid } \\
\text { temperature }\end{array}$ & Inoperative \\
\hline FVM-905 & Vortex flow meter & Erratic \\
\hline
\end{tabular}




\begin{tabular}{|c|c|c|c|c|}
\hline \multicolumn{5}{|c|}{$\begin{array}{l}\text { TABLE 6-4 } \\
\text { DATA PLOTS FOR QL REPORTS FOR TR-02-D BY COMPONENT }\end{array}$} \\
\hline Component & Channel & Units & QL-Plot & Comment \\
\hline $\begin{array}{l}\text { Reactor Vessel } \\
\text { Pressure }\end{array}$ & PT-107 & psig & 44 & \\
\hline Reactor Vessel Level & LDP-127 & inch of $\mathrm{H}_{2} \mathrm{O}$ & 29 & \\
\hline $\begin{array}{l}\text { Reactor Vessel } \\
\text { Downcomer Level }\end{array}$ & LDP-140 & inch of $\mathrm{H}_{2} \mathrm{O}$ & 31 & \\
\hline $\begin{array}{l}\text { Cold Leg \#1 Fluid } \\
\text { Temperature }\end{array}$ & TF-107 & ${ }^{\circ} \mathrm{F}$ & 58 & \\
\hline $\begin{array}{l}\text { Cold Leg \#2 Fluid } \\
\text { Temperature }\end{array}$ & TF-108 & ${ }^{\circ} \mathrm{F}$ & 59 & \\
\hline $\begin{array}{l}\text { Cold Leg \#3 Fluid } \\
\text { Temperature }\end{array}$ & TF-103 & ${ }^{\circ} \mathrm{F}$ & 56 & \\
\hline $\begin{array}{l}\text { Cold Leg \#4 Fluid } \\
\text { Temperature }\end{array}$ & TF-104 & ${ }^{\circ} \mathrm{F}$ & 57 & \\
\hline $\begin{array}{l}\text { Reactor Vessel Fluid } \\
\text { Temp Upper Head }\end{array}$ & TF-120 & ${ }^{\circ} \mathrm{F}$ & 60 & \\
\hline $\begin{array}{l}\text { RCS Hot Leg \#1 } \\
\text { Temperature }\end{array}$ & TF-143 & ${ }^{\circ} \mathrm{F}$ & 62 & \\
\hline $\begin{array}{l}\text { RCS Hot Leg \#2 } \\
\text { Temperature }\end{array}$ & TF-142 & ${ }^{\circ} \mathrm{F}$ & 61 & \\
\hline Pressurizer Pressure & $\begin{array}{l}\text { PT-604 (WR) and PT-603 } \\
\text { (LP Indication) }\end{array}$ & psig & 49,50 & \\
\hline $\begin{array}{l}\text { Pressurizer Liquid } \\
\text { Level }\end{array}$ & LDP-601 & inch of $\mathrm{H}_{2} \mathrm{O}$ & 38 & $\begin{array}{l}\text { Sharp decrease followed } \\
\text { by rapid refill. }\end{array}$ \\
\hline SG \#1 Tube Level & LDP-215 & inch of $\mathrm{H}_{2} \mathrm{O}$ & 32 & \\
\hline $\begin{array}{l}\text { SG \#1 Secondary } \\
\text { Pressure }\end{array}$ & PT-301 & psig & 45 & \\
\hline SG \#1 Feed Flow Rate & FMM-001 & gpm & 1 & \\
\hline SG \#2 Tube Level & LDP-218 & inch of $\mathrm{H}_{2} \mathrm{O}$ & 33 & \\
\hline $\begin{array}{l}\text { SG \#2 Secondary } \\
\text { Pressure }\end{array}$ & PT-302 & psig & 46 & \\
\hline
\end{tabular}




\begin{tabular}{|c|c|c|c|c|}
\hline \multicolumn{5}{|c|}{$\begin{array}{l}\text { TABLE 6-4 (Continued) } \\
\text { DATA PLOTS FOR QL REPORTS FOR TR-02-D BY COMPONENT }\end{array}$} \\
\hline Component & Channel & Units & QL-Plot & Comment \\
\hline $\begin{array}{l}\text { Accumulators \#1 and } \\
\text { \#2 Pressure }\end{array}$ & PT-401 and PT-402 & psig & 47,48 & \\
\hline $\begin{array}{l}\text { Accumulators \#1 and } \\
\text { \#2 Liquid Level }\end{array}$ & LDP-401 and LDP-402 & inch of $\mathrm{H}_{2} \mathrm{O}$ & 34,35 & \\
\hline $\begin{array}{l}\text { Accumulators \#1 and } \\
\text { \#2 Flow Rate }\end{array}$ & FMM-401 and FMM-402 & gpm & 2,3 & \\
\hline $\begin{array}{l}\text { Accumulators \#1 and } \\
\text { \#2 Liquid Discharge } \\
\text { Temperature }\end{array}$ & TF-401 and TF-402 & ${ }^{\circ} \mathrm{F}$ & 63,64 & \\
\hline $\begin{array}{l}\text { CMT \#1 and \#2 Liquid } \\
\text { Level }\end{array}$ & LDP-507 and LDP-502 & inch of $\mathrm{H}_{2} \mathrm{O}$ & 36,37 & \\
\hline $\begin{array}{l}\text { CMT \#1 and \#2 Flow } \\
\text { Rate }\end{array}$ & FMM-501 and FMM-504 & gpm & 4,5 & \\
\hline $\begin{array}{l}\text { CMT \#1 and \#2 Liquid } \\
\text { Temperature }\end{array}$ & $\begin{array}{l}\text { TF-501 and TF-529 and } \\
\text { TF-504 and TF-532 }\end{array}$ & ${ }^{\circ} \mathrm{F}$ & $65-68$ & \\
\hline PRHR Inlet Flow Rate & FMM-802 & gpm & 11 & \\
\hline PRHR Liquid Level & LDP-802 & inch of $\mathrm{H}_{2} \mathrm{O}$ & 40 & \\
\hline $\begin{array}{l}\text { PRHR Outlet Flow } \\
\text { Rate }\end{array}$ & FMM-804 & gpm & 12 & \\
\hline IRWST Liquid Level & LDP-701 & inch of $\mathrm{H}_{2} \mathrm{O}$ & 39 & \\
\hline $\begin{array}{l}\text { IRWST Discharge Line } \\
\# 1 \text { and \#2 Flow Rate }\end{array}$ & FMM-701 and FMM-702 & gpm & 9,10 & \\
\hline $\begin{array}{l}\text { IRWST Fluid } \\
\text { Temperature }\end{array}$ & TF-701 and TF-709 & ${ }^{\circ} \mathrm{F}$ & 69,70 & \\
\hline $\begin{array}{l}\text { ADS 1-3 Separator } \\
\text { Pressure }\end{array}$ & PT-605 & psig & 51 & \\
\hline $\begin{array}{l}\text { ADS 1-3 Separator } \\
\text { Steam Flow Rate }\end{array}$ & FVM-601 & $\mathrm{scfm}$ & 16 & \\
\hline $\begin{array}{l}\text { ADS 1-3 Separator } \\
\text { Liquid Flow Rate }\end{array}$ & FMM-601 & gpm & 6 & \\
\hline
\end{tabular}




\begin{tabular}{|c|c|c|c|c|}
\hline \multicolumn{5}{|c|}{$\begin{array}{c}\text { TABLE 6-4 (Continued) } \\
\text { DATA PLOTS FOR QL REPORTS FOR TR-02-D BY COMPONENT }\end{array}$} \\
\hline Component & Channel & Units & QL-Plot & Comment \\
\hline $\begin{array}{l}\text { ADS 4-1 and ADS 4-2 } \\
\text { Separator Pressure }\end{array}$ & PT-611 and PT-610 & psig & 52,53 & \\
\hline $\begin{array}{l}\text { ADS 4-1 Separator } \\
\text { Steam Flow Rate }\end{array}$ & FVM-603 & $\operatorname{scfm}$ & 18 & \\
\hline $\begin{array}{l}\text { ADS 4-2 Separator } \\
\text { Steam Flow Rate }\end{array}$ & FVM-602 & scfm & 17 & \\
\hline $\begin{array}{l}\text { ADS 4-1 Separator } \\
\text { Liquid Flow Rate }\end{array}$ & FMM-603 & gpm & 8 & \\
\hline $\begin{array}{l}\text { ADS 4-2 Separator } \\
\text { Liquid Flow Rate }\end{array}$ & FMM-602 & gpm & 7 & \\
\hline Primary Sump Pressure & PT-901 & psig & 54 & \\
\hline $\begin{array}{l}\text { Primary Sump Liquid } \\
\text { Level }\end{array}$ & LDP-901 & inch of $\mathrm{H}_{2} \mathrm{O}$ & 41 & \\
\hline $\begin{array}{l}\text { Primary Sump } \\
\text { Injection Flow Rate }\end{array}$ & FMM-901 and FMM-902 & gpm & 13,14 & \\
\hline $\begin{array}{l}\text { Secondary Sump } \\
\text { Liquid Level }\end{array}$ & LDP-902 & inch of $\mathrm{H}_{2} \mathrm{O}$ & 42 & \\
\hline $\begin{array}{l}\text { Break Separator } \\
\text { Pressure }\end{array}$ & PT-905 & psig & 55 & \\
\hline $\begin{array}{l}\text { Break Separator Liquid } \\
\text { Level }\end{array}$ & LDP-905 & inch of $\mathrm{H}_{2} \mathrm{O}$ & 43 & \\
\hline $\begin{array}{l}\text { Break Separator Flow } \\
\text { to Primary Sump }\end{array}$ & FMM-905 & gpm & 15 & \\
\hline $\begin{array}{l}\text { BAMS Steam Flow } \\
\text { Rate }\end{array}$ & FVM-901 & $\mathrm{scfm}$ & 19 & \\
\hline $\begin{array}{l}\text { BAMS Steam Flow } \\
\text { Rate }\end{array}$ & FVM-902 & $\mathrm{scfm}$ & 20 & \\
\hline $\begin{array}{l}\text { BAMS/Primary Sump } \\
\text { Steam Flow Rate }\end{array}$ & FVM-903 & $\mathrm{scfm}$ & 21 & \\
\hline
\end{tabular}




\begin{tabular}{|c|c|c|c|c|}
\hline \multicolumn{5}{|c|}{$\begin{array}{c}\text { TABLE 6-4 (Continued) } \\
\text { DATA PLOTS FOR QL REPORTS FOR TR-02-D BY COMPONENT }\end{array}$} \\
\hline Component & Channel & Units & QL-Plot & Comment \\
\hline $\begin{array}{l}\text { BAMS/Separator } \\
\text { Steam Flow Rate - } \\
6 \text { inch Pipe }\end{array}$ & FVM-905 & $\mathrm{scfm}$ & 22 & \\
\hline $\begin{array}{l}\text { BAMS/Exhaust Line } \\
\text { Temperature }\end{array}$ & TF-916 and SC-917 & ${ }^{\circ} \mathrm{F}$ & 71,72 & \\
\hline $\begin{array}{l}\text { PZR Heater Input } \\
\text { Power }\end{array}$ & KW-601 & $\mathrm{kW}$ & 27 & \\
\hline $\begin{array}{l}\text { Core Power Input } \\
\text { Power }\end{array}$ & $\begin{array}{l}\text { KW-101, KW-102, } \\
\text { KW-103, and KW-104 }\end{array}$ & $\mathrm{kW}$ & $23-26$ & \\
\hline $\begin{array}{l}\text { Reactor Vessel Liquid } \\
\text { Level Between Top of } \\
\text { Vessel - Upper Support } \\
\text { Plate }\end{array}$ & LDP-115 & $\begin{array}{l}\text { inches of } \\
\text { water }\end{array}$ & 28 & \\
\hline $\begin{array}{l}\text { Reactor Vessel Liquid } \\
\text { Level Between Bottom } \\
\text { of Upper Support } \\
\text { Plate - Upper Core } \\
\text { Spacer Grid }\end{array}$ & LDP-139 & $\begin{array}{l}\text { inches of } \\
\text { water }\end{array}$ & 30 & \\
\hline $\begin{array}{l}\text { Inner Core } \\
\text { Thermocouple } \\
\text { Measuring Heater } \\
\text { Temperature }\end{array}$ & TH-103-4 & ${ }^{\circ} \mathrm{F}$ & 73 & \\
\hline $\begin{array}{l}\text { Outer Core } \\
\text { Thermocouple } \\
\text { Measuring Heater } \\
\text { Temperature }\end{array}$ & ТH-309-4 & ${ }^{\circ} \mathrm{F}$ & 74 & \\
\hline
\end{tabular}




\subsection{CONCLUSIONS}

The TR-02-D test was successfully completed, and the data logged in the DAS. All critical instruments were found to operate properly with the exception of those noted in Section 6.4. The test was found to be acceptable. 
APPENDIX A

DATA PLOTS 


\section{AP1000 Test Data Oregon State University Test TR-02 July 9, 2003 $\begin{array}{llll}\text { PT-107 } & 208 & 0 & 0\end{array}$}

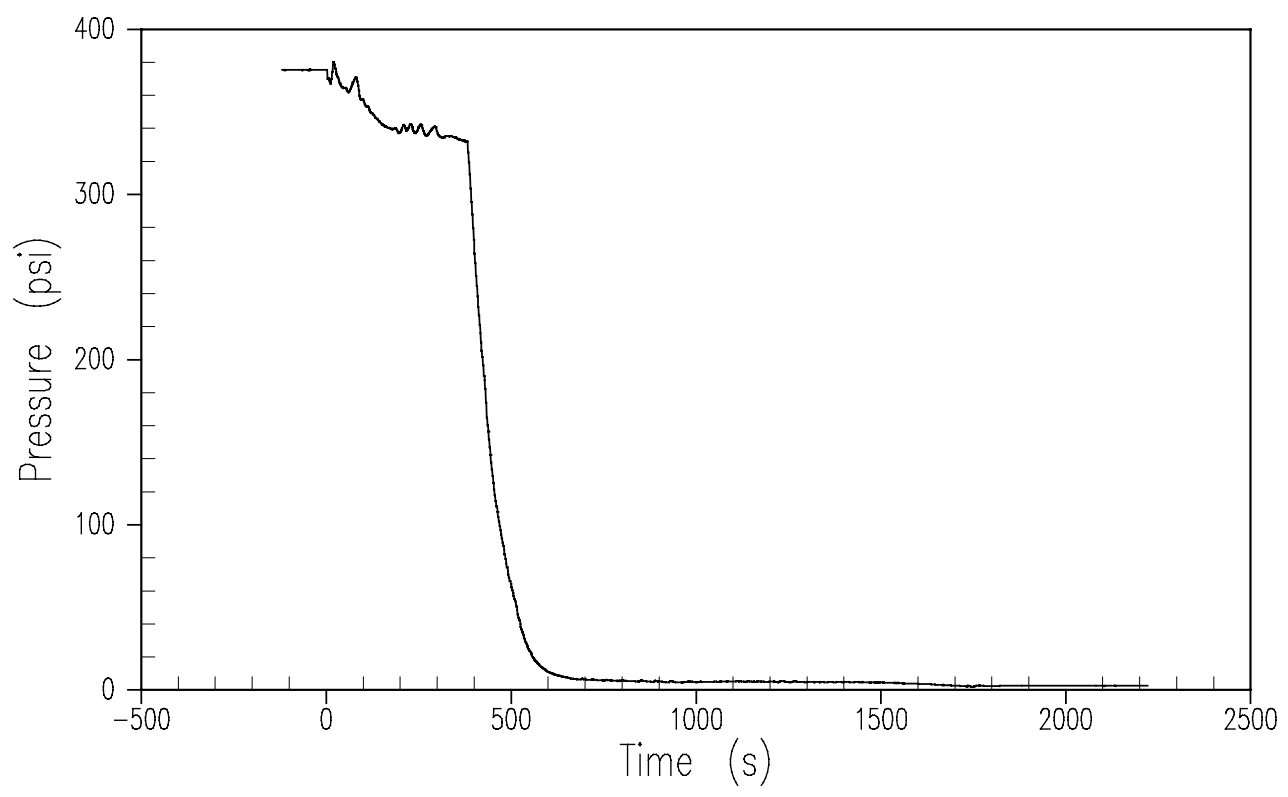




\section{AP1000 Test Data Oregon State University Test TR-02 July 9, 2003 \\ $\begin{array}{llll}L D P-127 & 136 & 0 & 0\end{array}$}

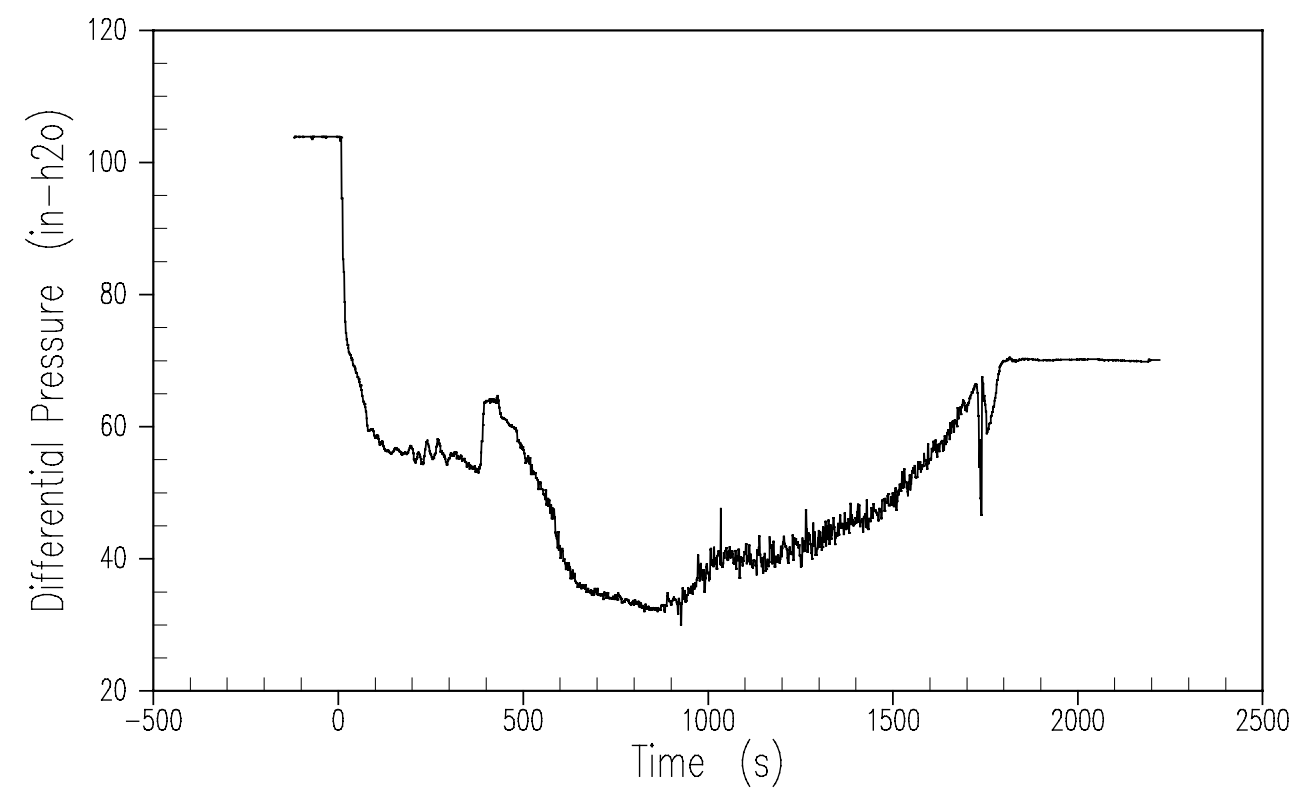

Figure A-2 Reactor Vessel Level 

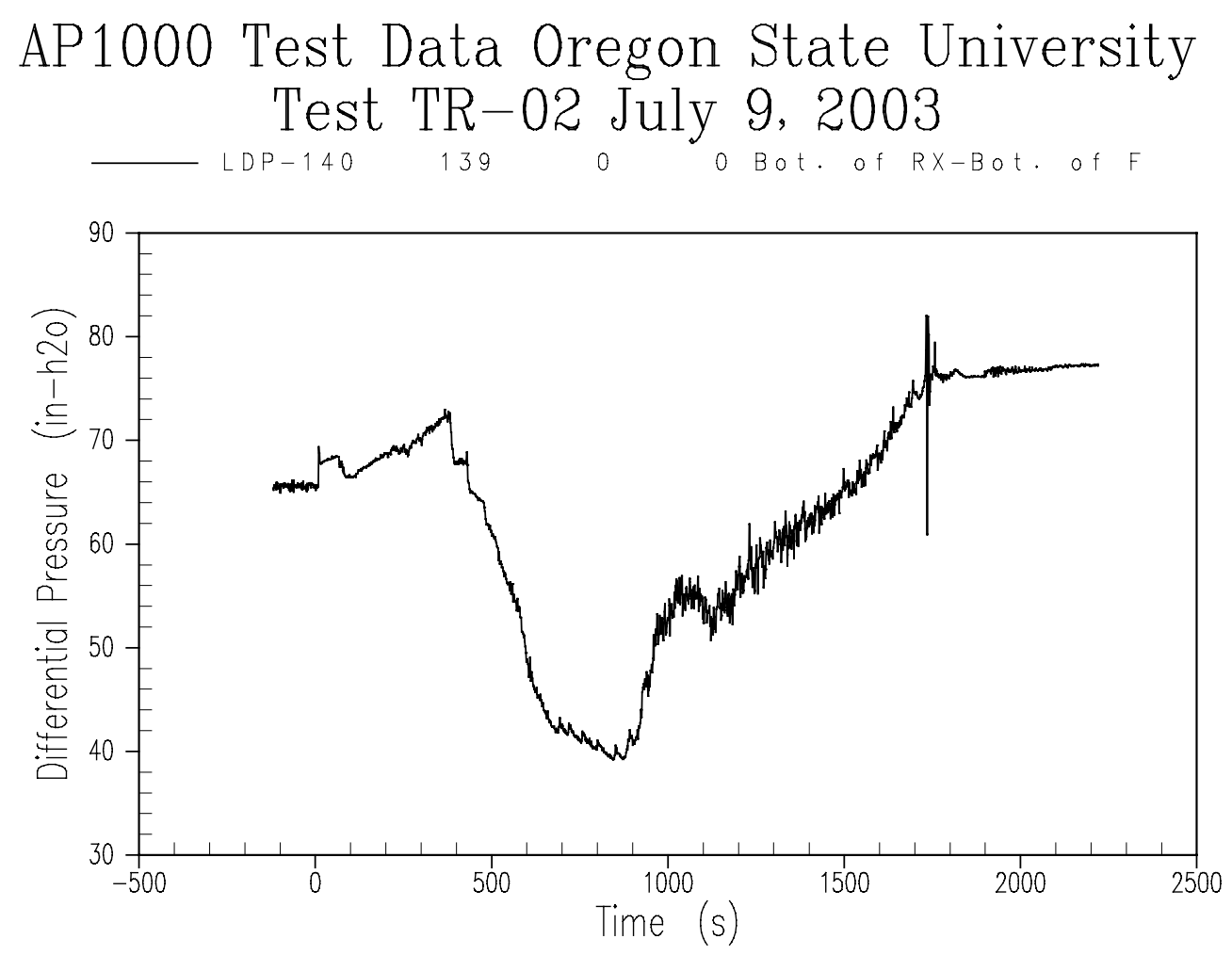

Figure A-3 Reactor Vessel Downcomer Level 

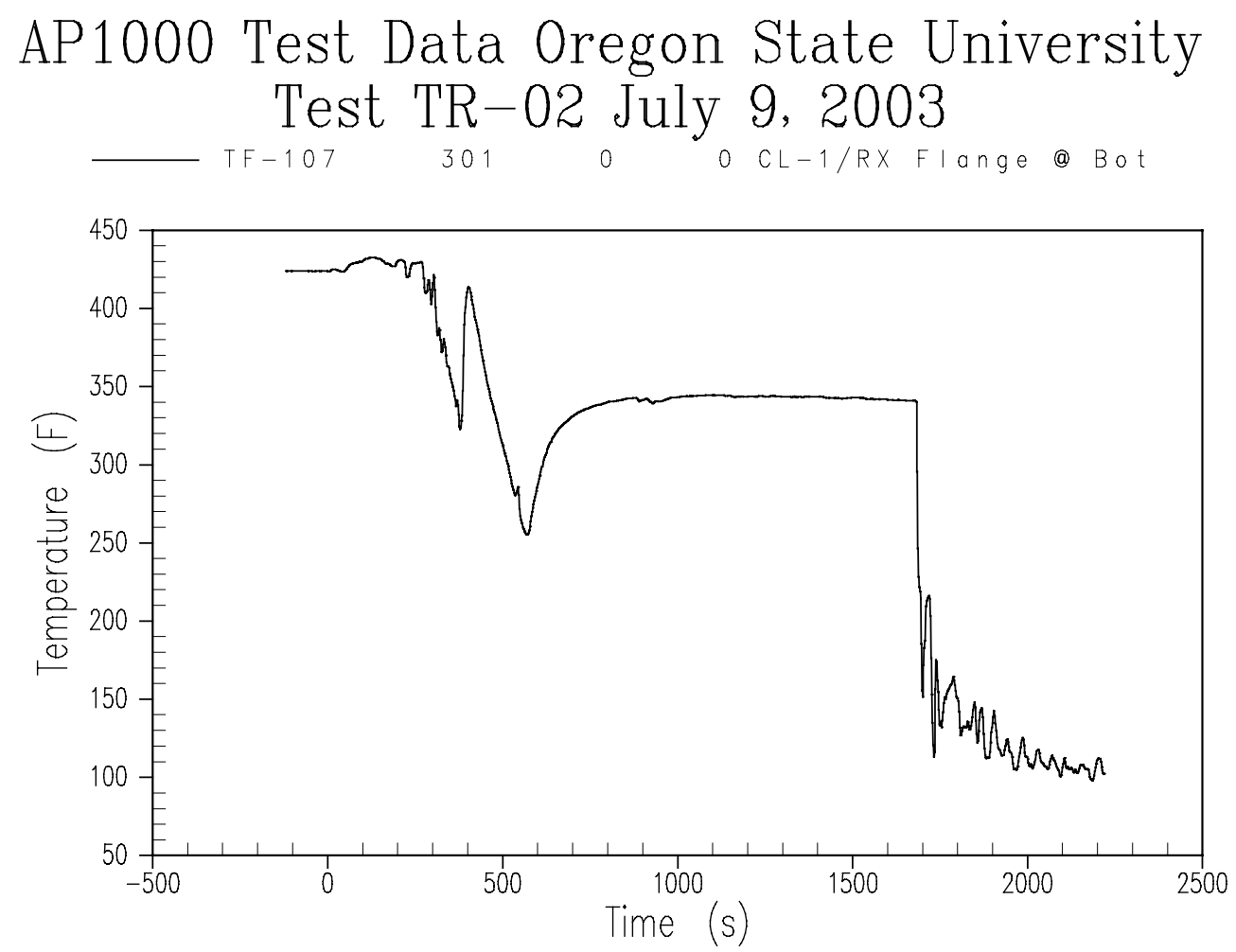

Figure A-4 Cold Leg 1 Fluid Temperature 

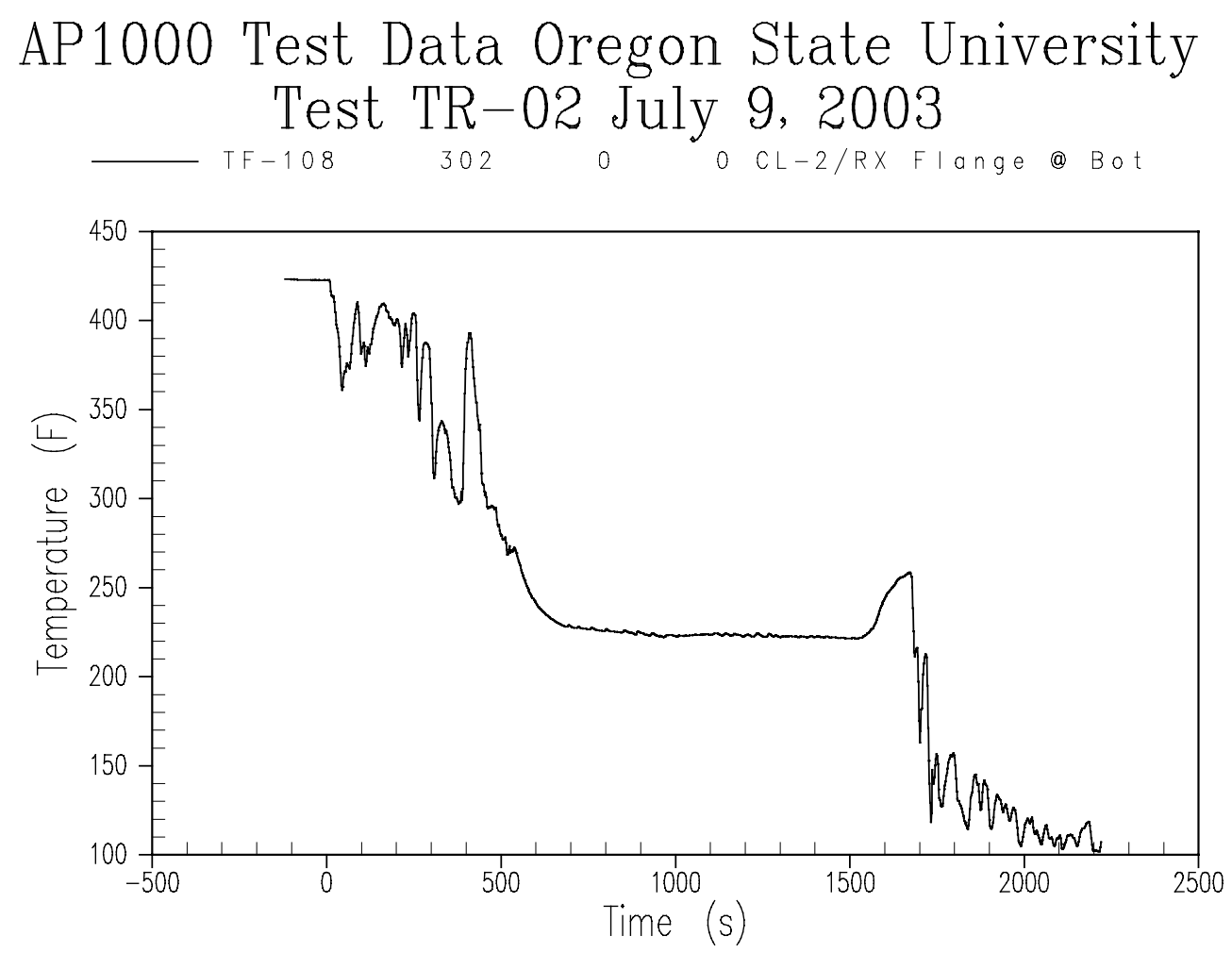

Figure A-5 Cold Leg 2 Fluid Temperature 

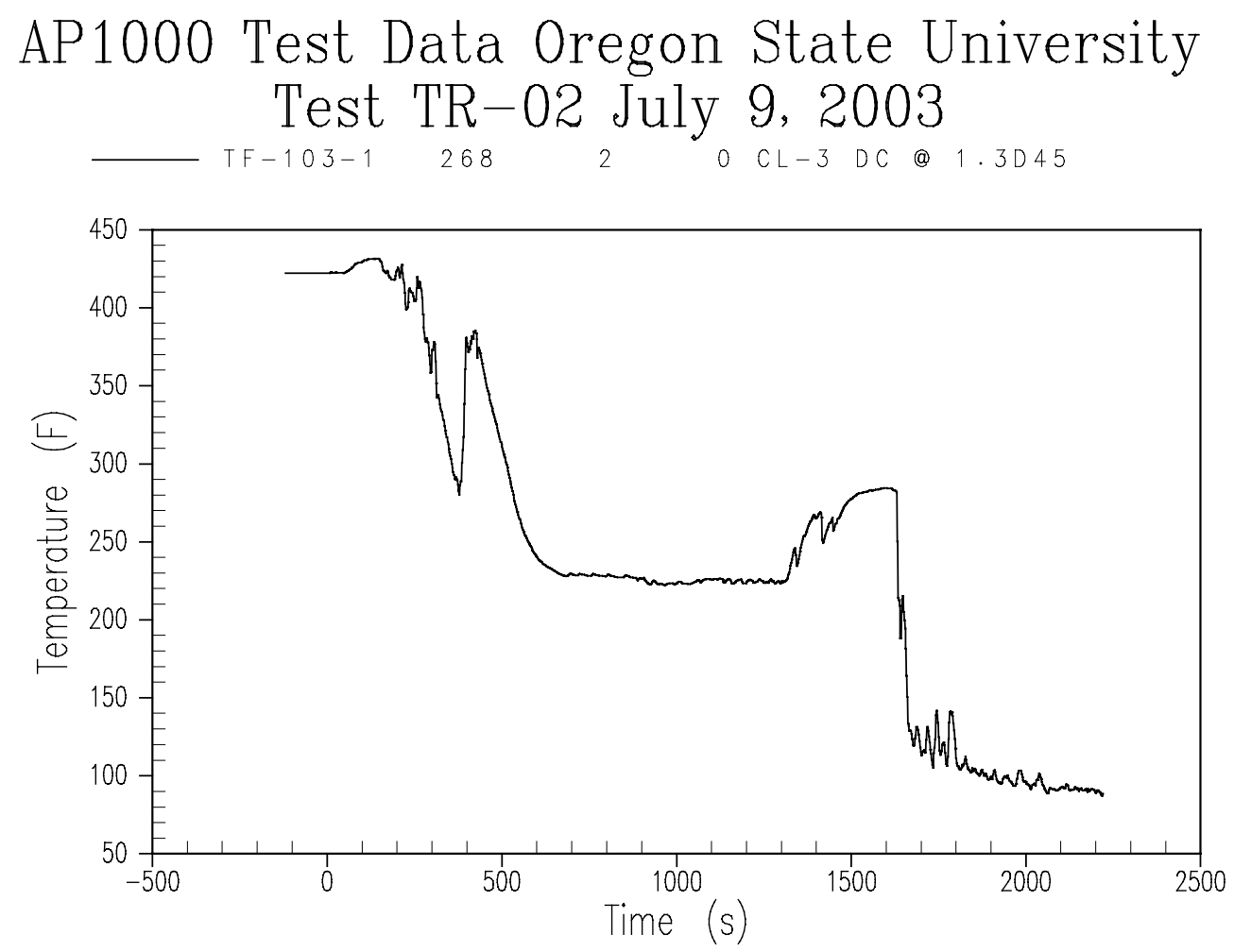

Figure A-6 Cold Leg 3 Fluid Temperature 

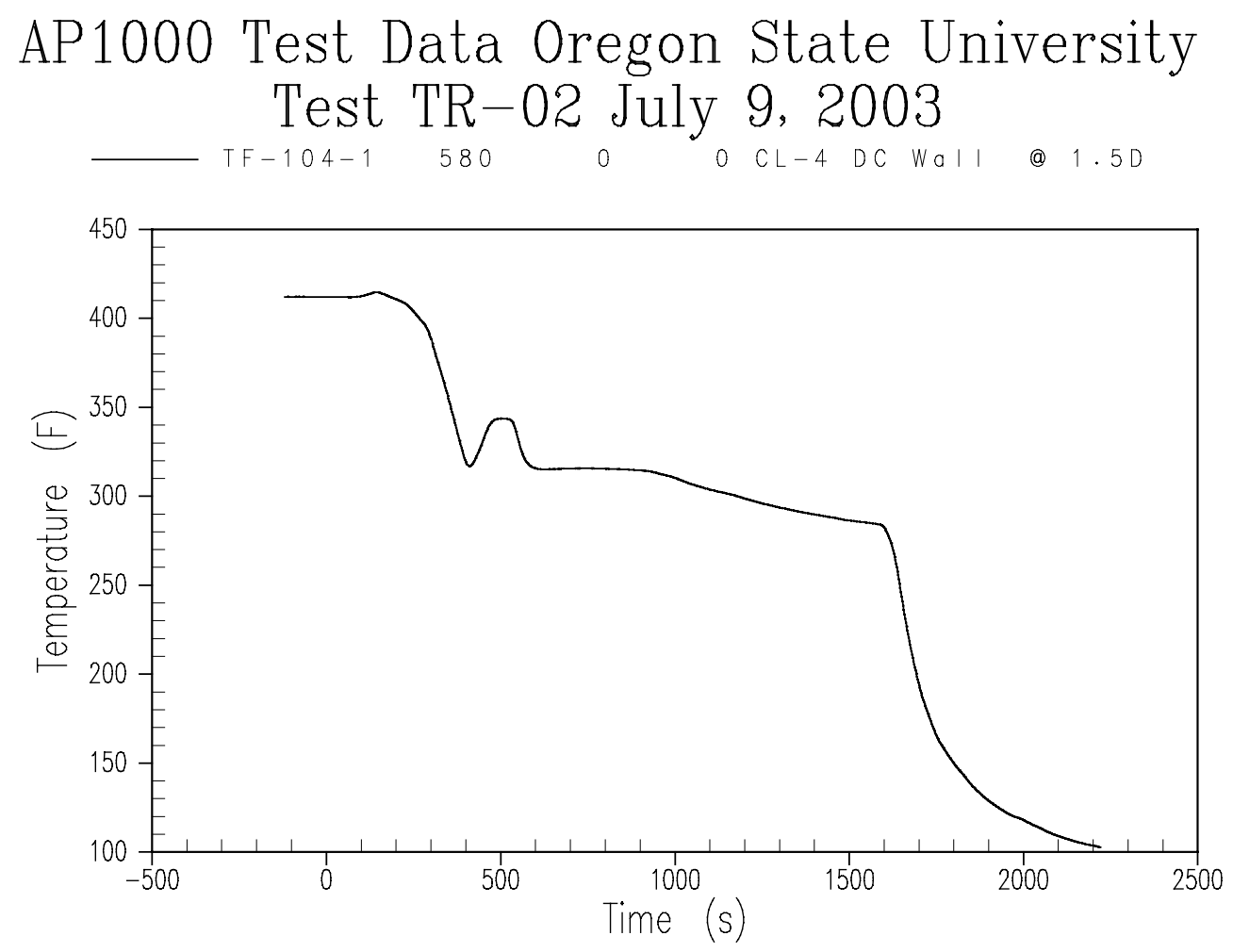

Figure A-7 Cold Leg 4 Fluid Temperature 

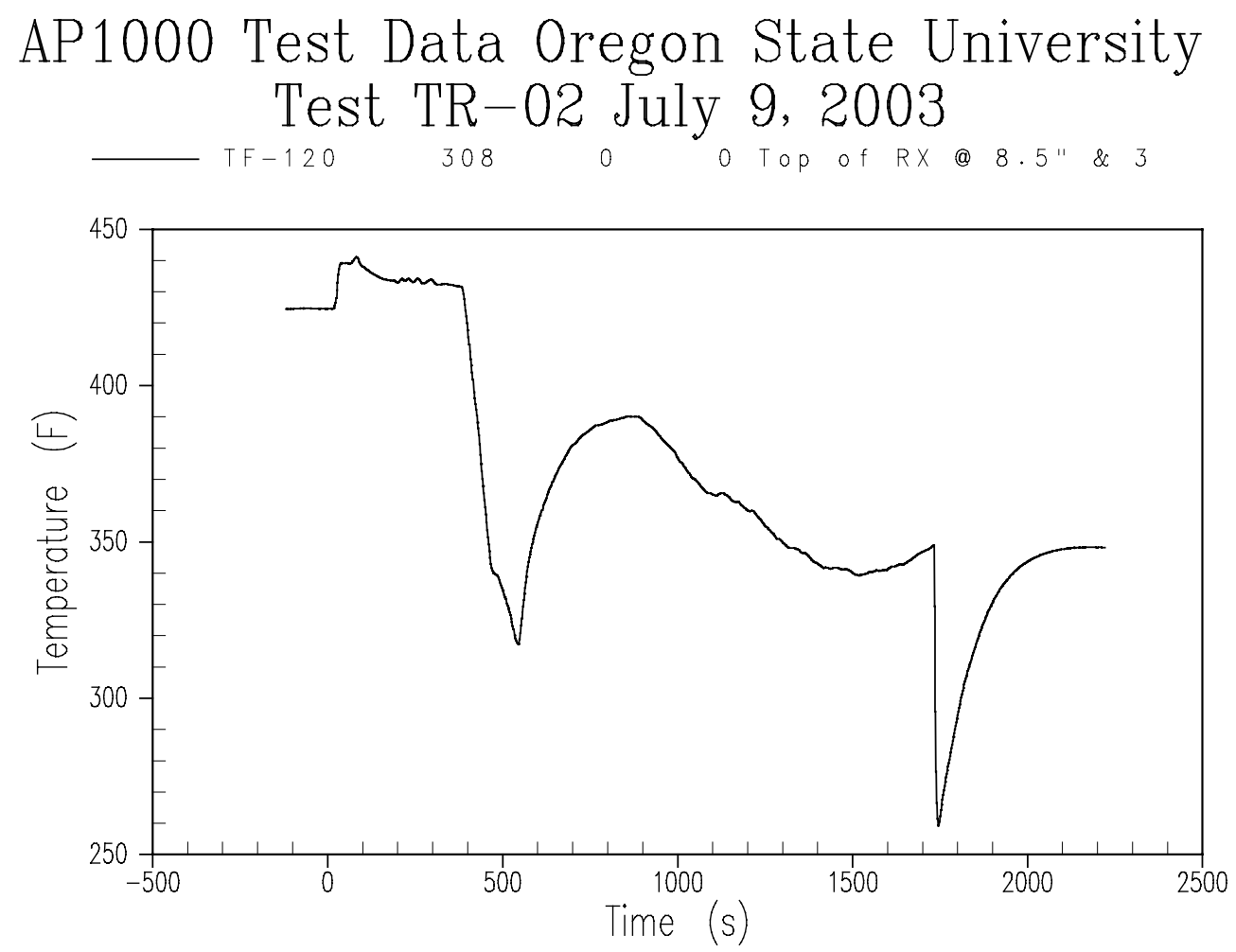

Figure A-8 Reactor Vessel Fluid Temperature Upper Head 

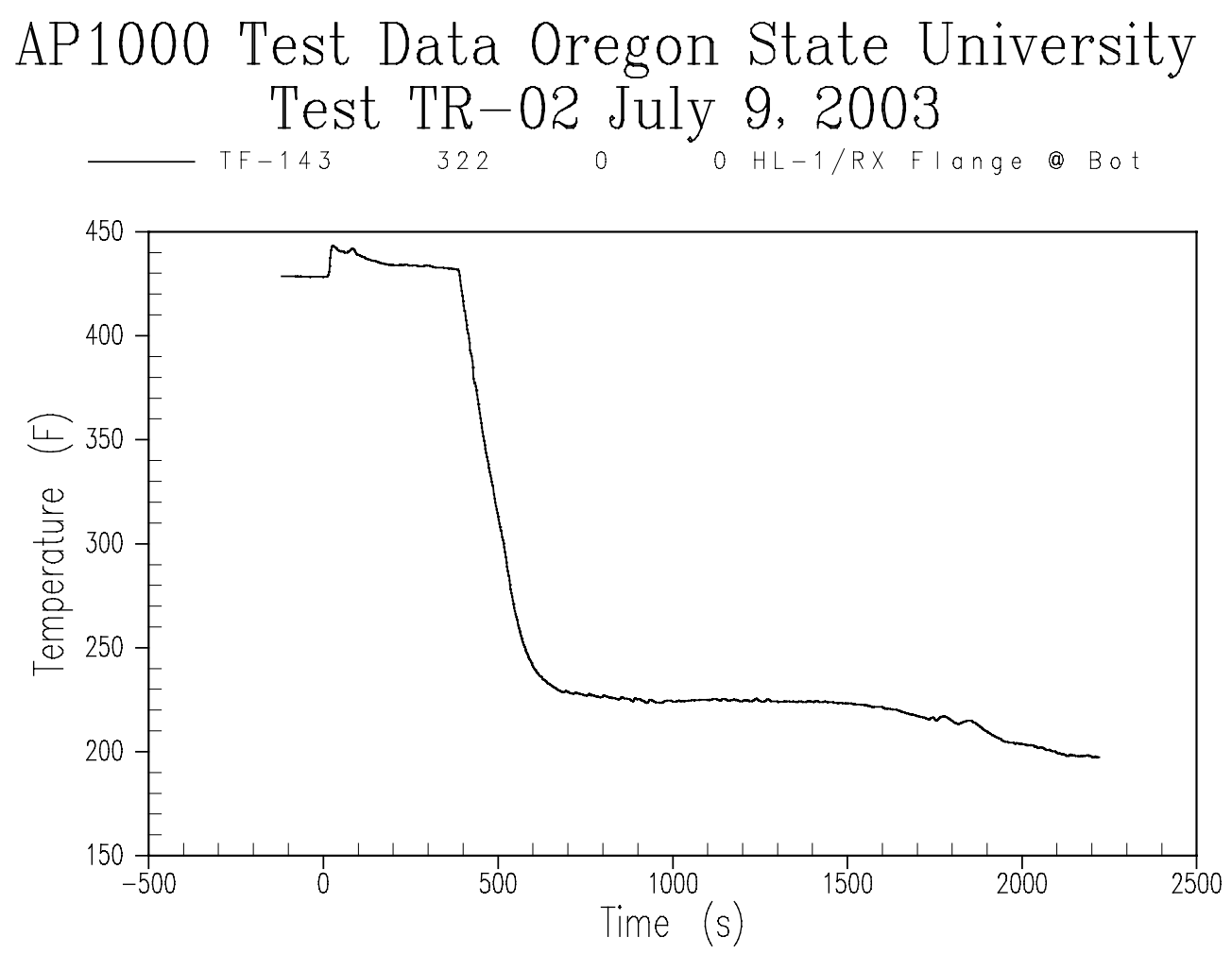

Figure A-9 Reactor Coolant System Hot Leg 1 Temperature 

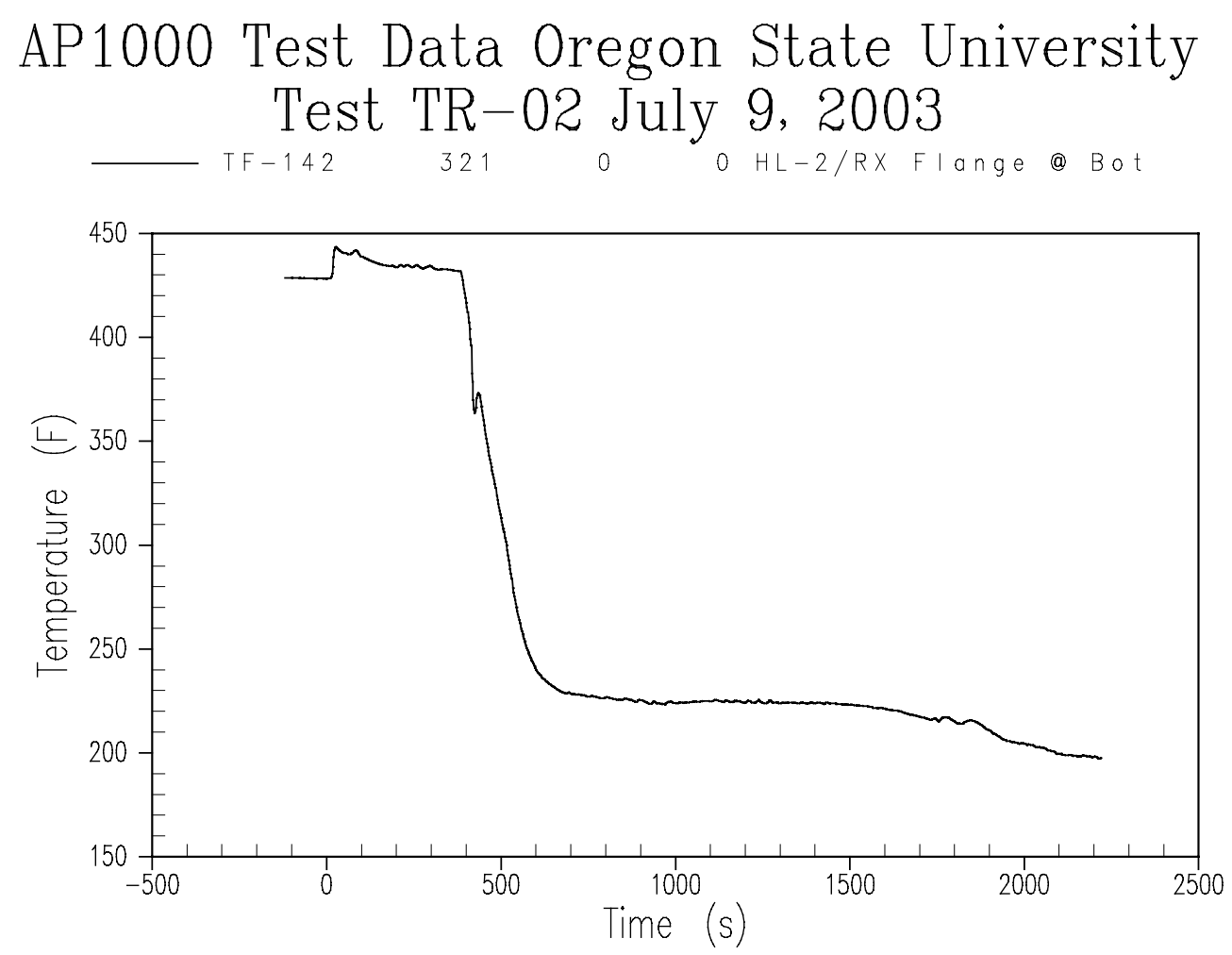

Figure A-10 Reactor Coolant System Hot Leg 2 Temperature 


\section{AP1000 Test Data Oregon State University Test TR-02 July 9, 2003 \\ $\begin{array}{llll}\mathrm{PT}-604 & 229 & 0 & \text { o pressurizer Press. }\end{array}$}

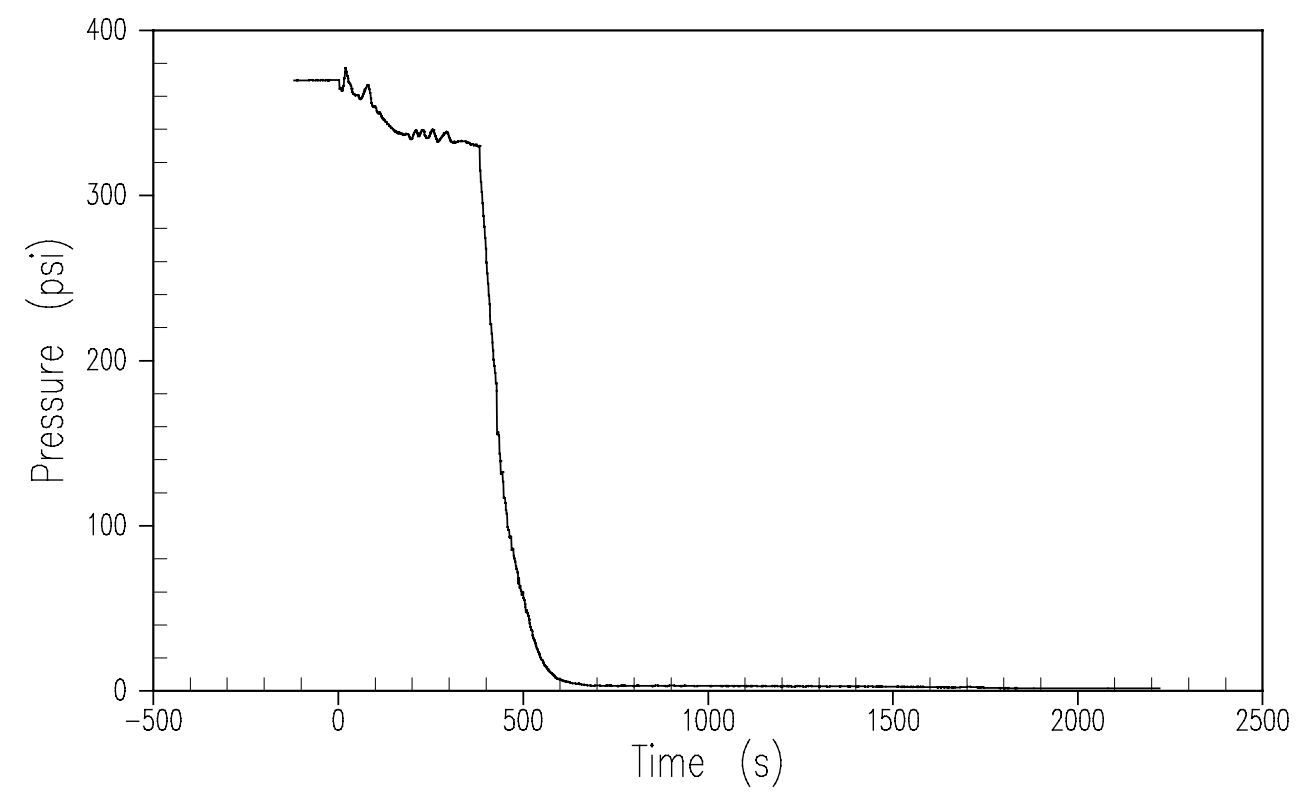

Figure A-11 Pressurizer Pressure - Wide Range 


\section{AP1000 Test Data Oregon State University Test TR-02 July 9, 2003
PT -603
228
o PZR NR Press.

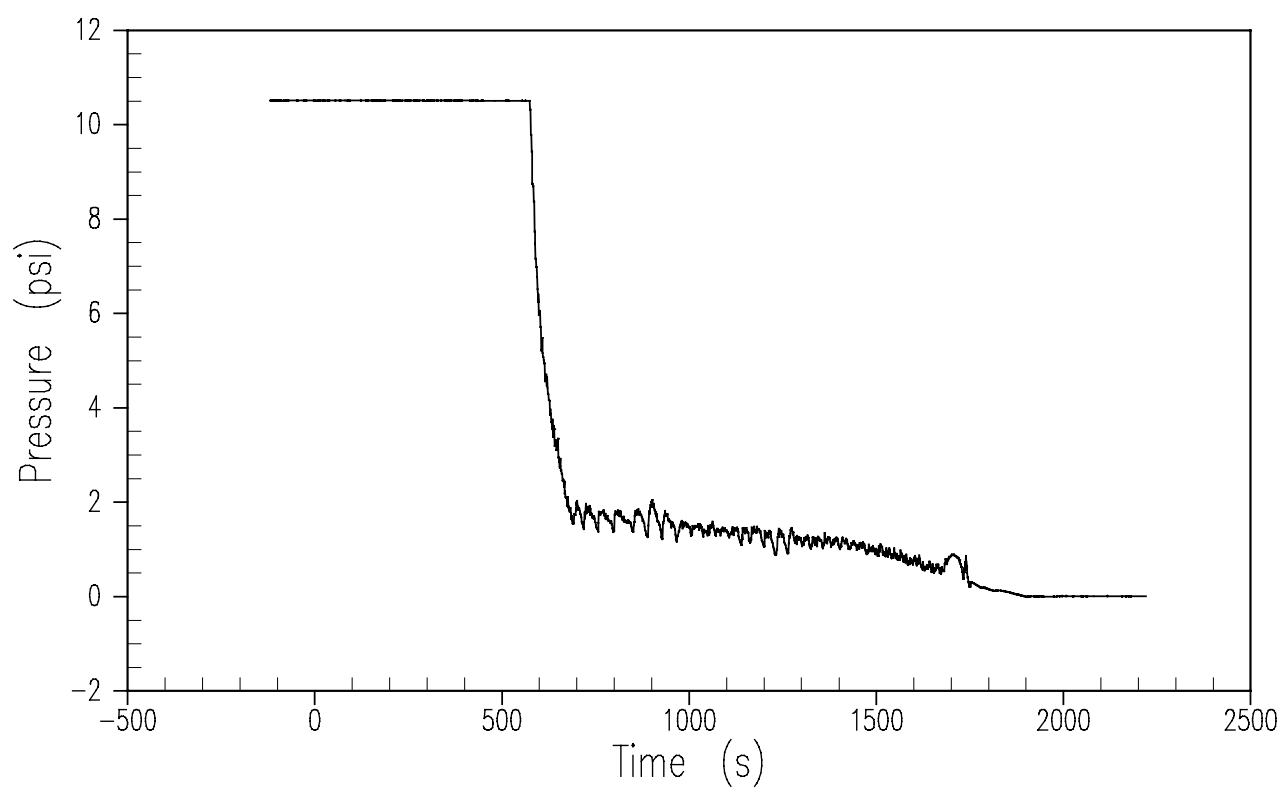

Figure A-12 Pressurizer Pressure - Narrow Range 


\section{AP1000 Test Data Oregon State University Test TR-02 July 9, 2003 \\ $\begin{array}{lllll}L D P-601 & 179 & 0 & 0 & P Z R\end{array}$ WR UC Level}

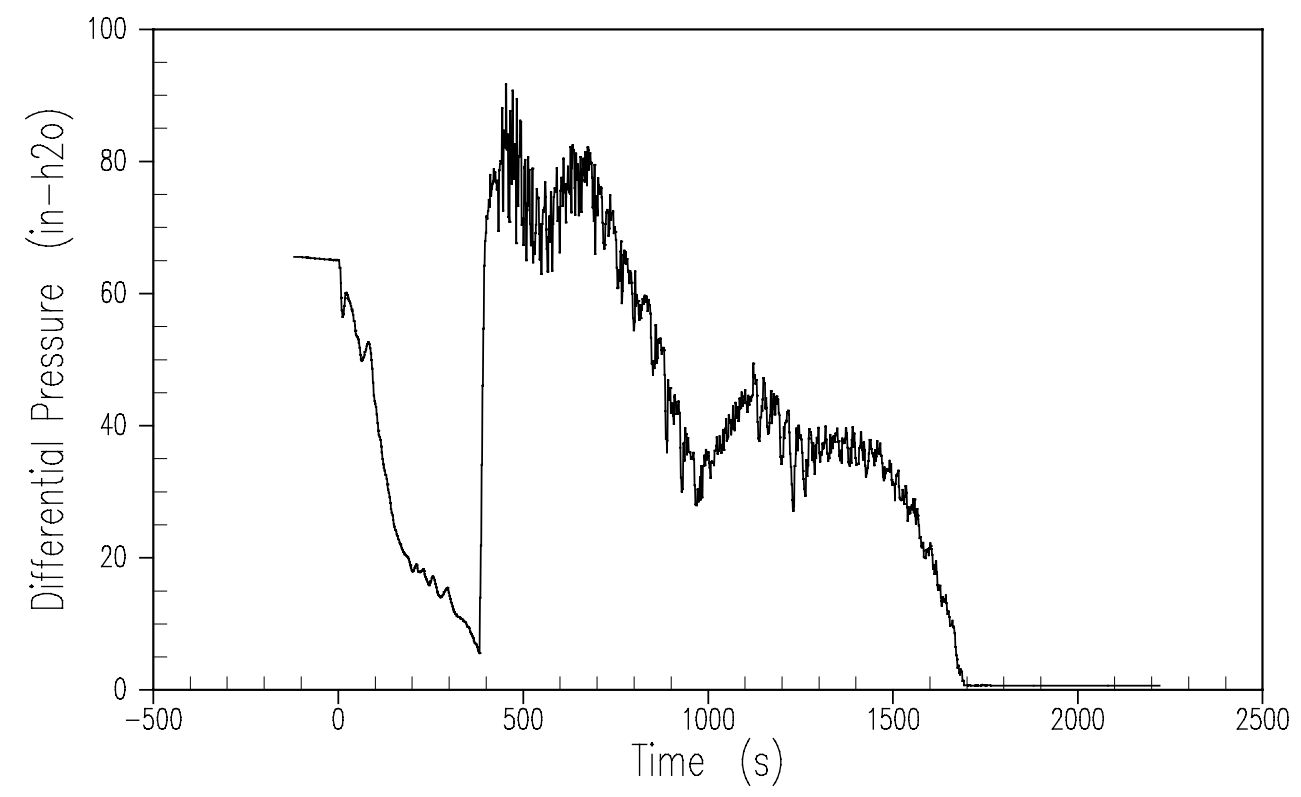

Figure A-13 Pressurizer Liquid Level 


\section{AP1000 Test Data Oregon State University Test TR-02 July 9, 2003 \\ $\begin{array}{llllll}L D P-215 & 155 & 0 & \text { O SG-1 LTube HL UC LeV }\end{array}$}

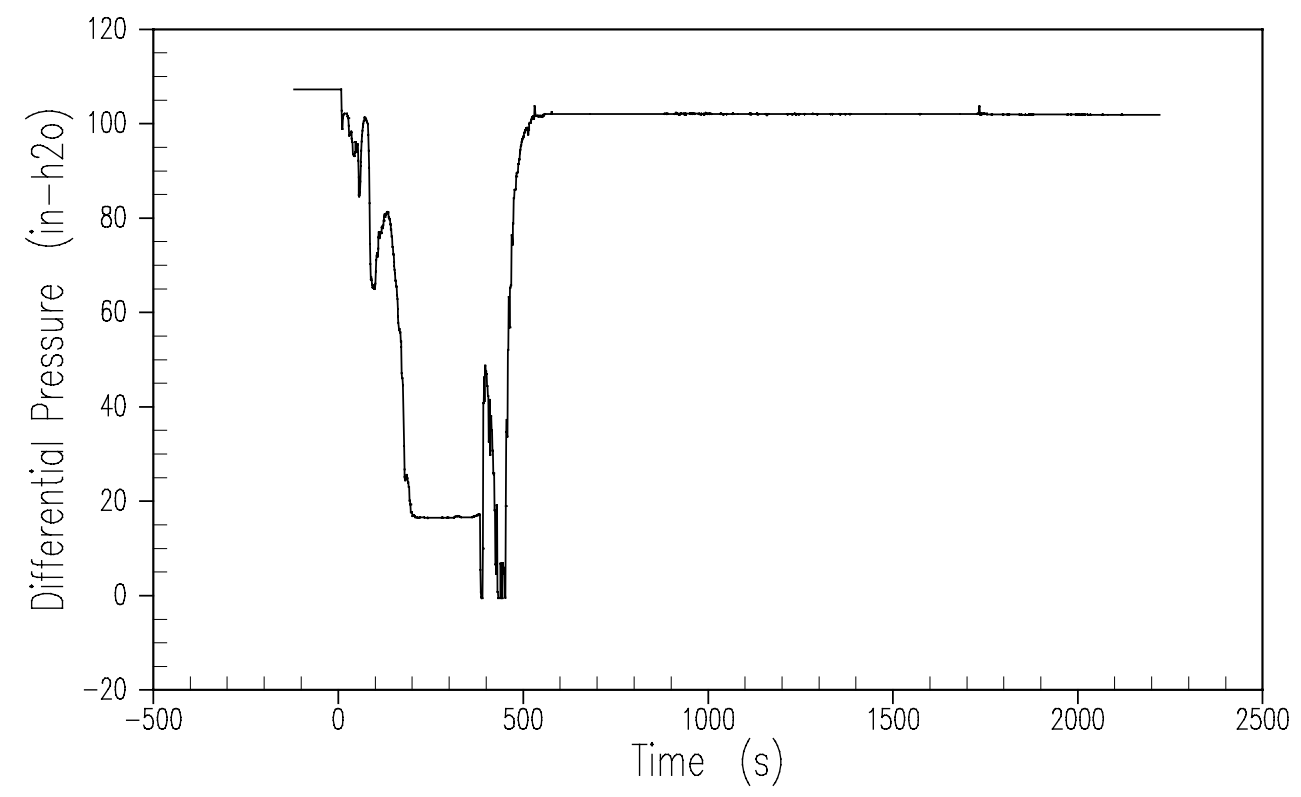




\section{AP1000 Test Data Oregon State University Test TR-02 July 9, 2003
221
o SG-1 Press. \\ $P T-301$}

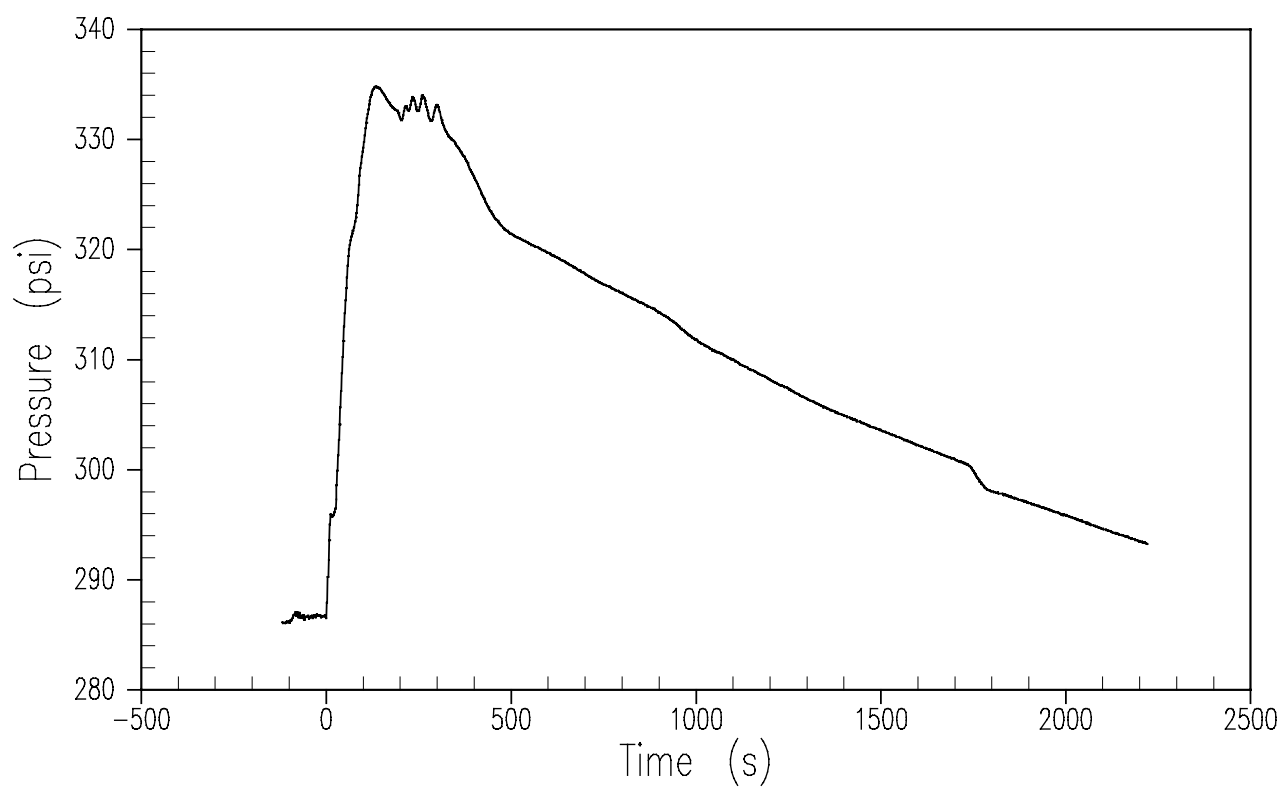

Figure A-15 Steam Generator 1 Secondary Pressure 

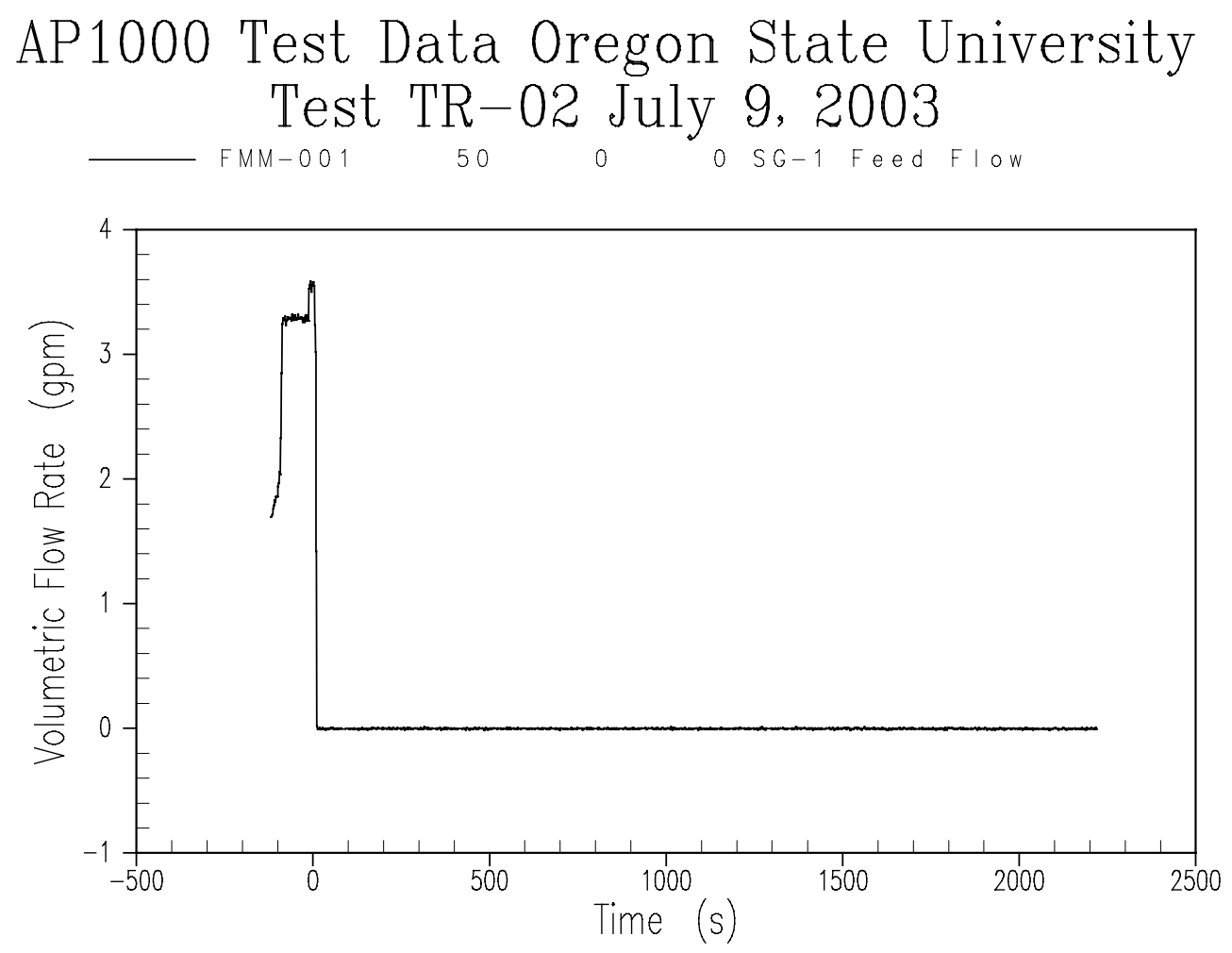

Figure A-16 Steam Generator 1 Feed Flow Rate 

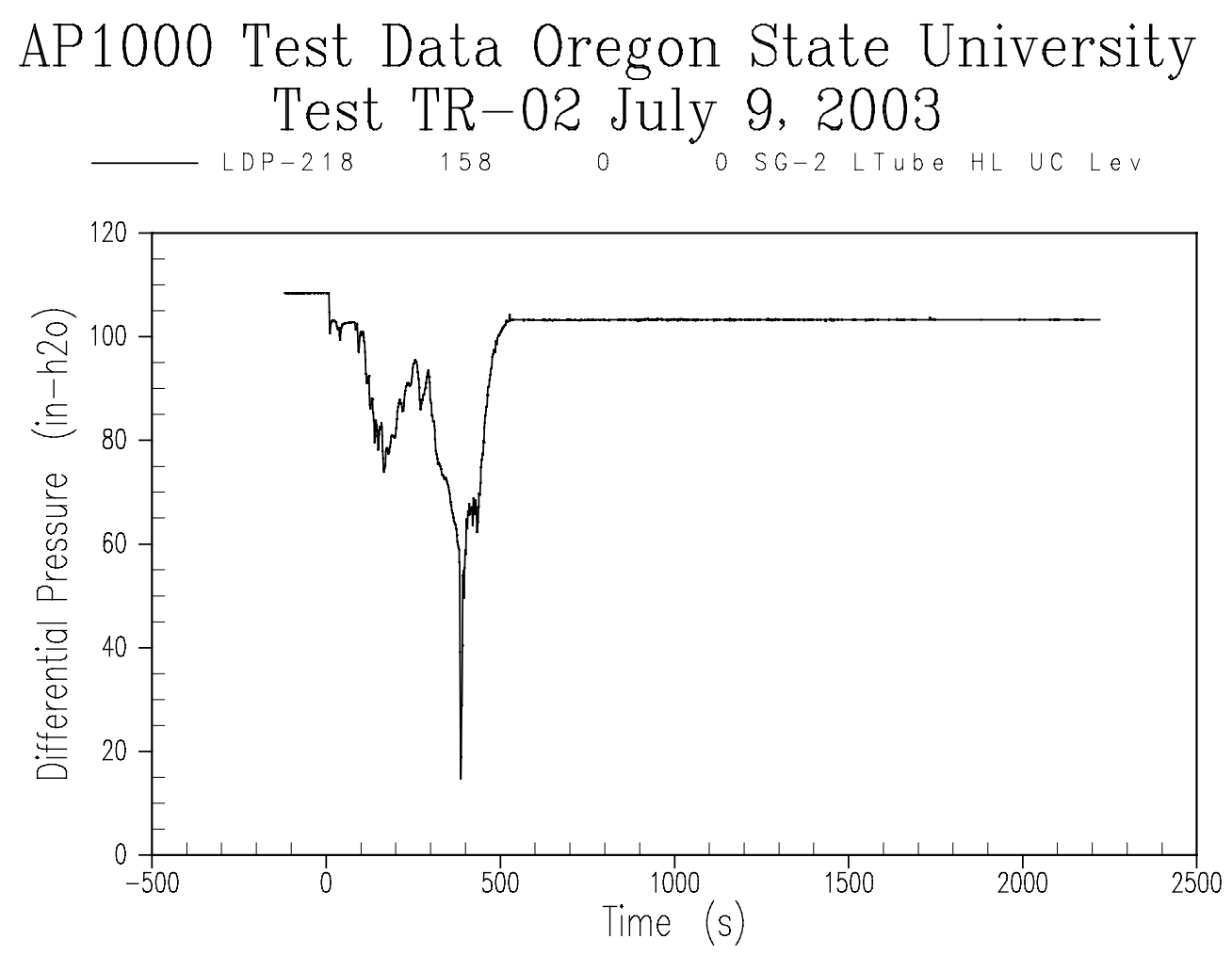

Figure A-17 Steam Generator 2 Tube Level 


\section{AP1000 Test Data Oregon State University Test TR-02 July 9, 2003
$P T-302$
222
o SG-2 Press.

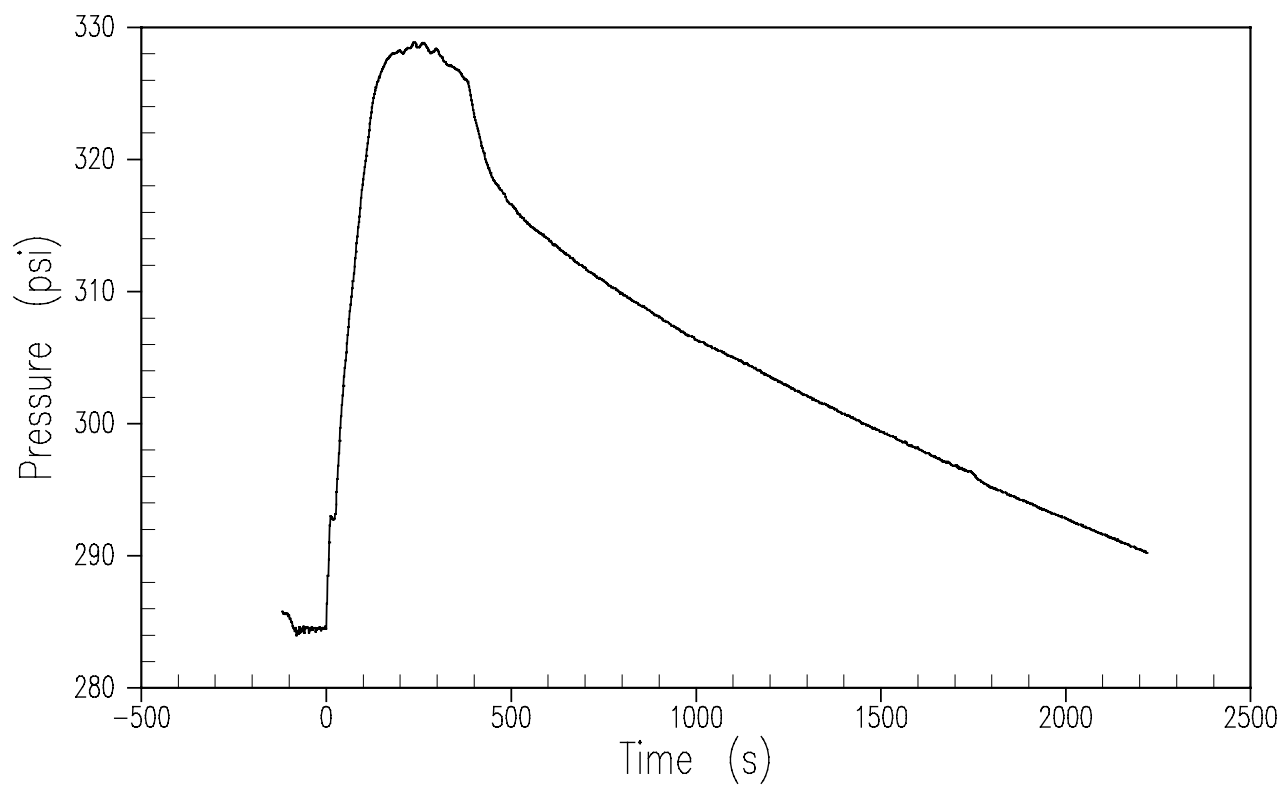

Figure A-18 Steam Generator 2 Secondary Pressure 


\section{AP1000 Test Data Oregon State University Test TR-02 July 9, 2003
$P T-401$
223
o ACC-1 Press.

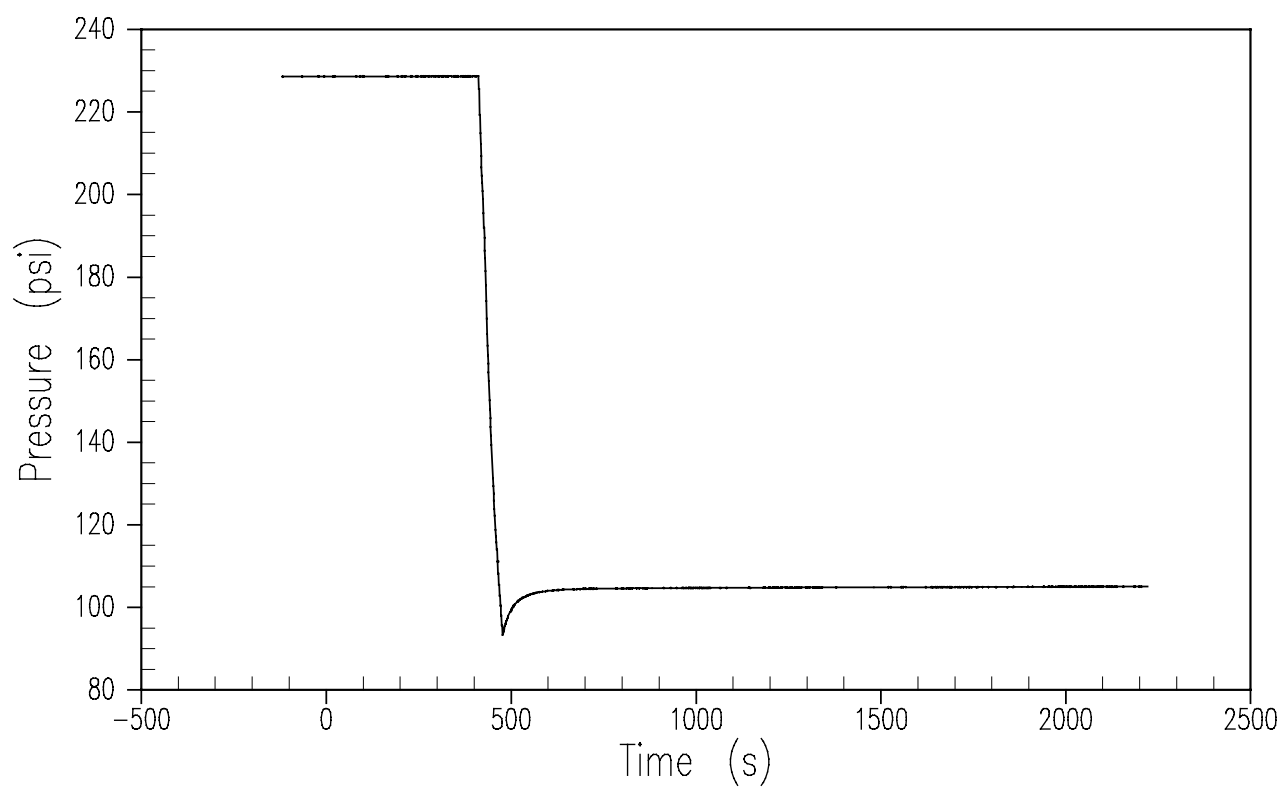

Figure A-19 Accumulator 1 Pressure 


\section{AP1000 Test Data Oregon State University Test TR-02 July 9, 2003
$P T-402$
224
o ACC-2 Press.

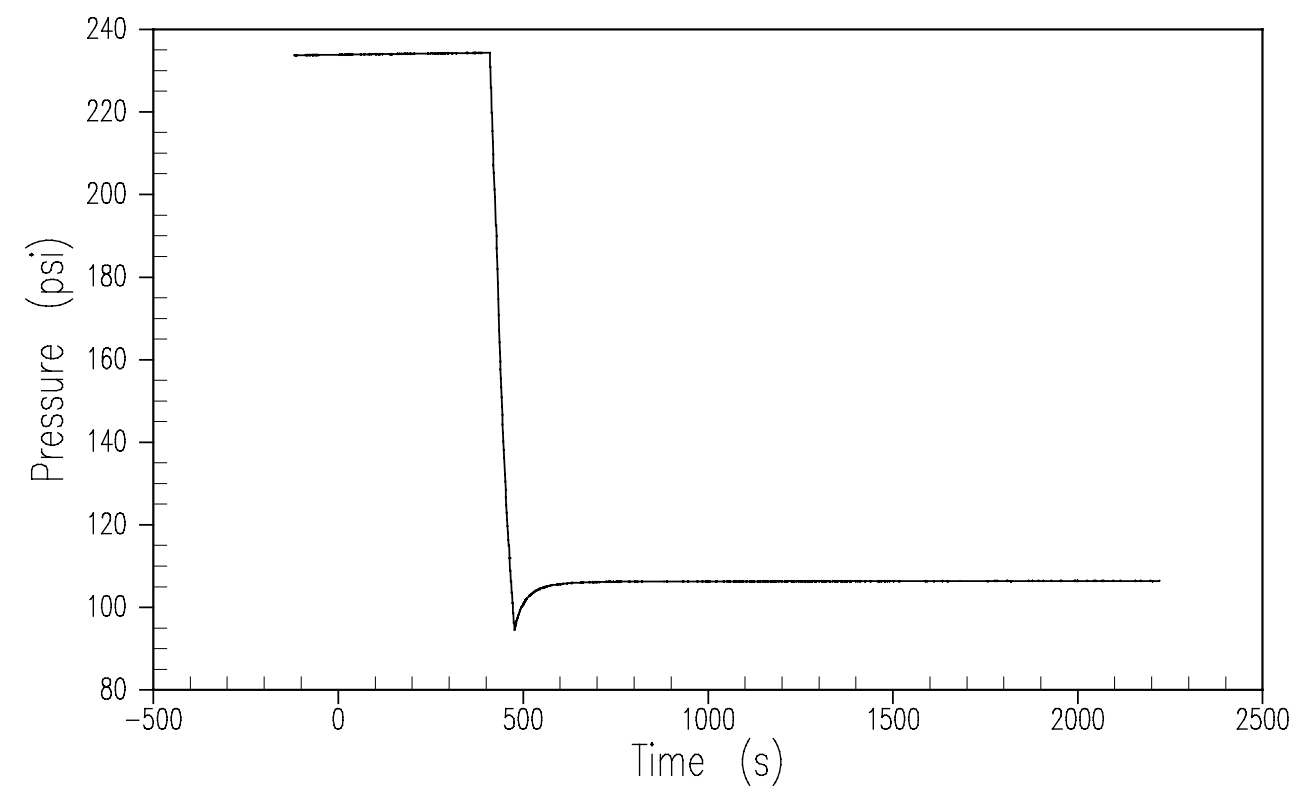

Figure A-20 Accumulator 2 Pressure 


\section{AP1000 Test Data Oregon State University Test TR-02 July 9, 2003
167
o ACC-1 UC Leve। \\ $L D P-401$}

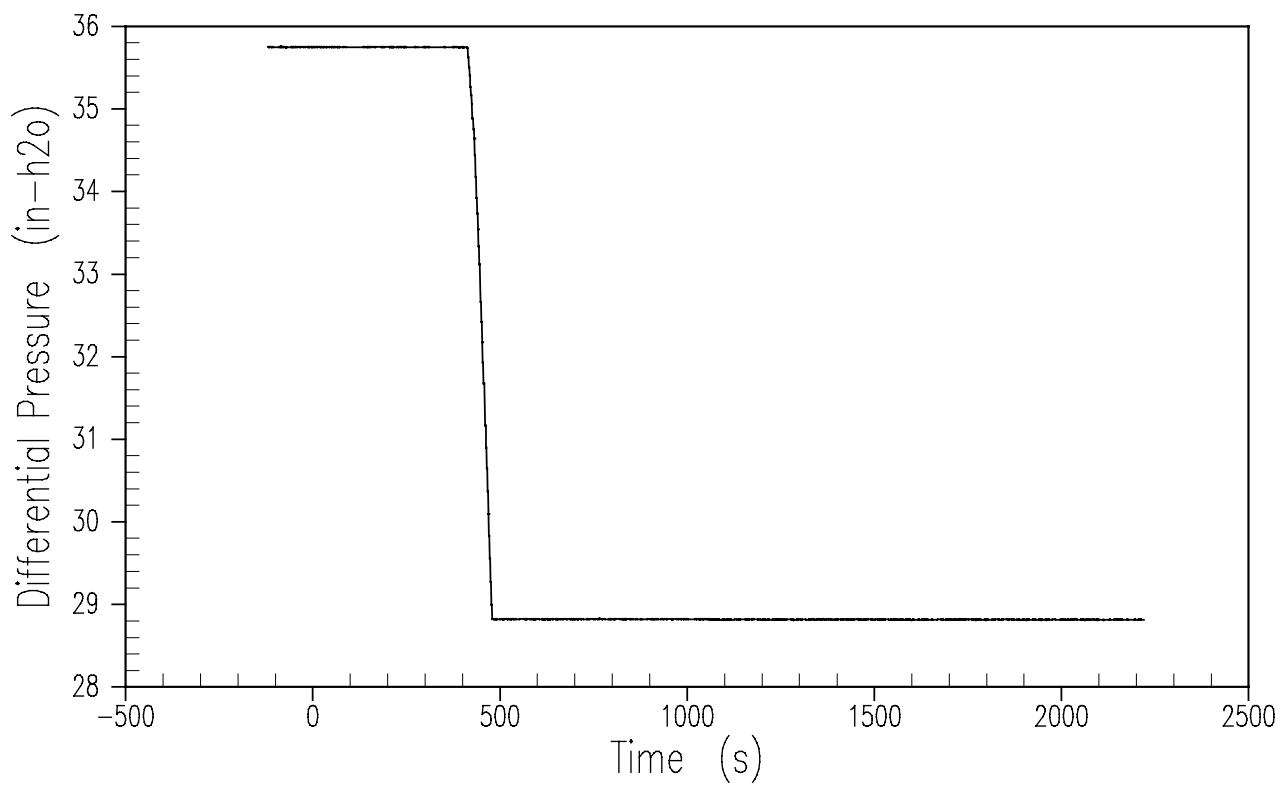

Figure A-21 Accumulator 1 Liquid Level 


\section{AP1000 Test Data Oregon State University Test TR-02 July 9, 2003
LDP- 402
168
0
o ACC-2 UC Level

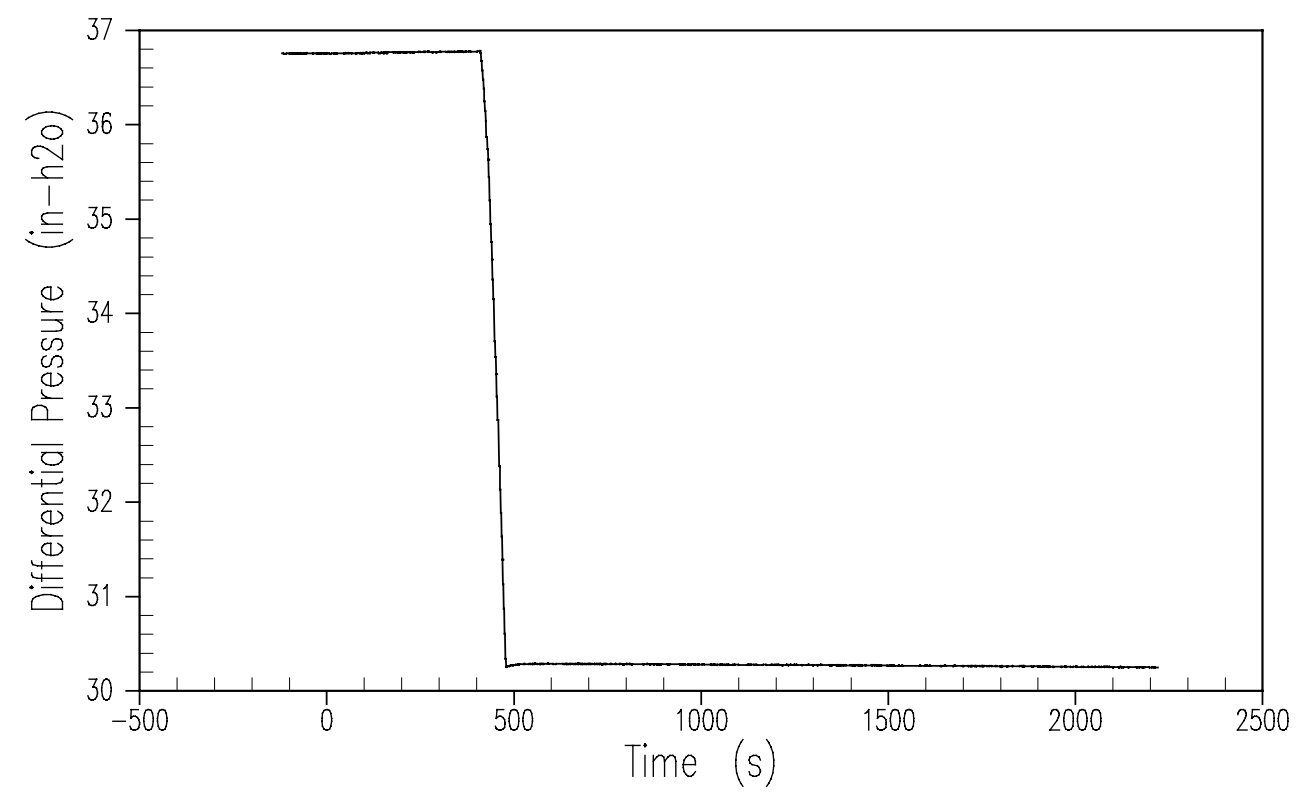

Figure A-22 Accumulator 2 Liquid Level 

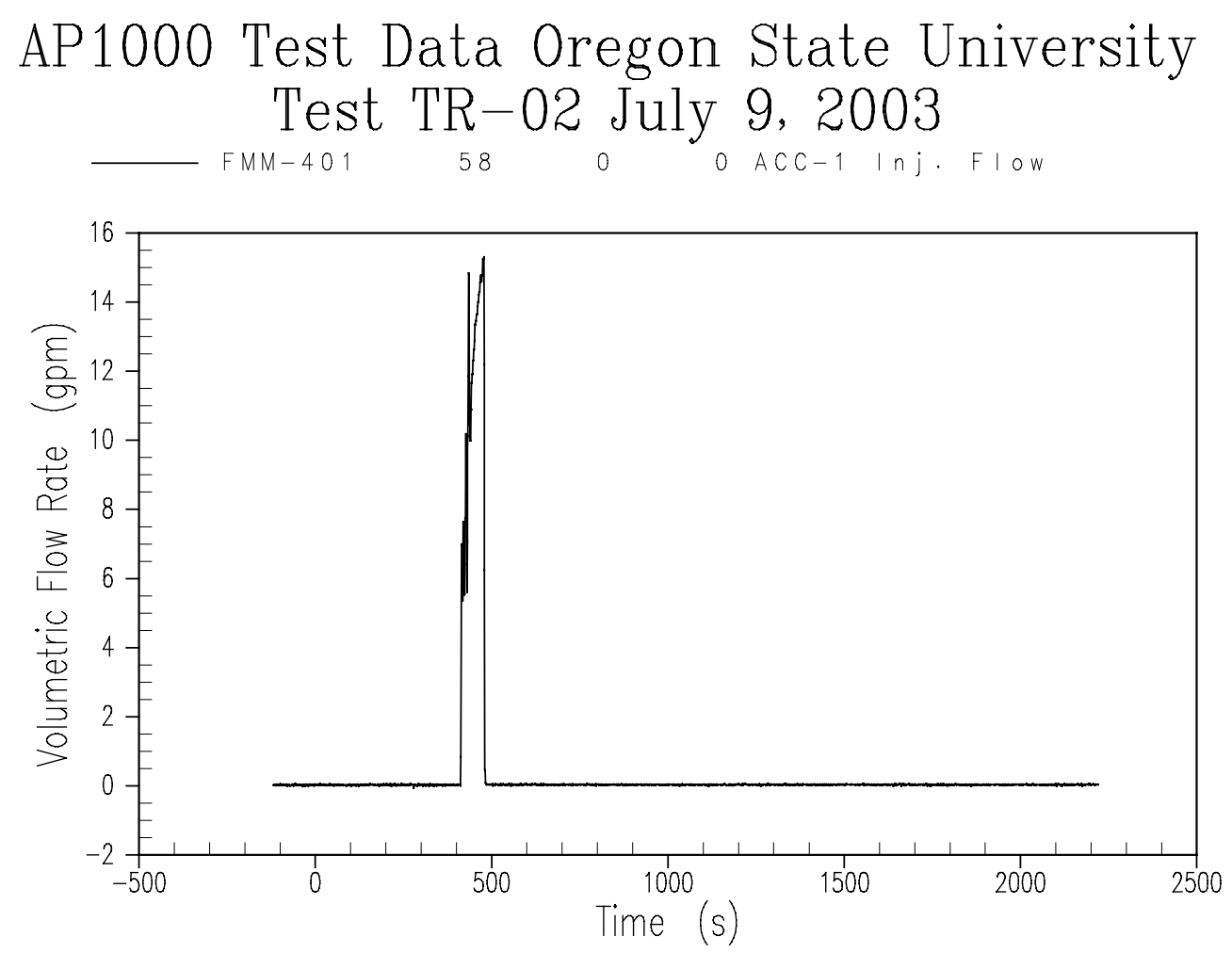

Figure A-23 Accumulator 1 Flow Rate 

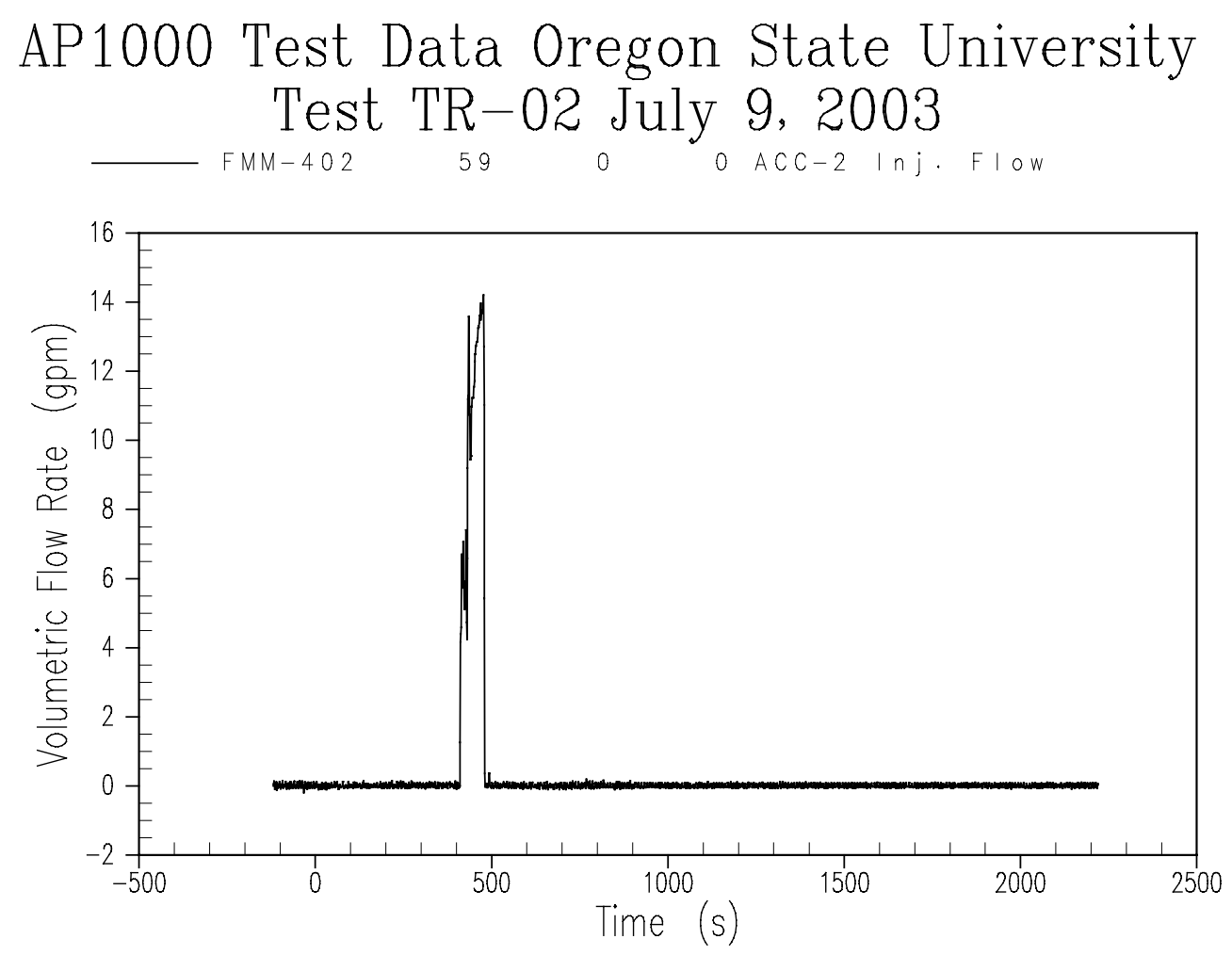

Figure A-24 Accumulator 2 Flow Rate 


\section{AP1000 Test Data Oregon State University Test TR-02 July 9, 2003 $\begin{array}{llllllll}T F-401 & 401 & 0 & 0 & A C C-1 & 0 u t\end{array}$}

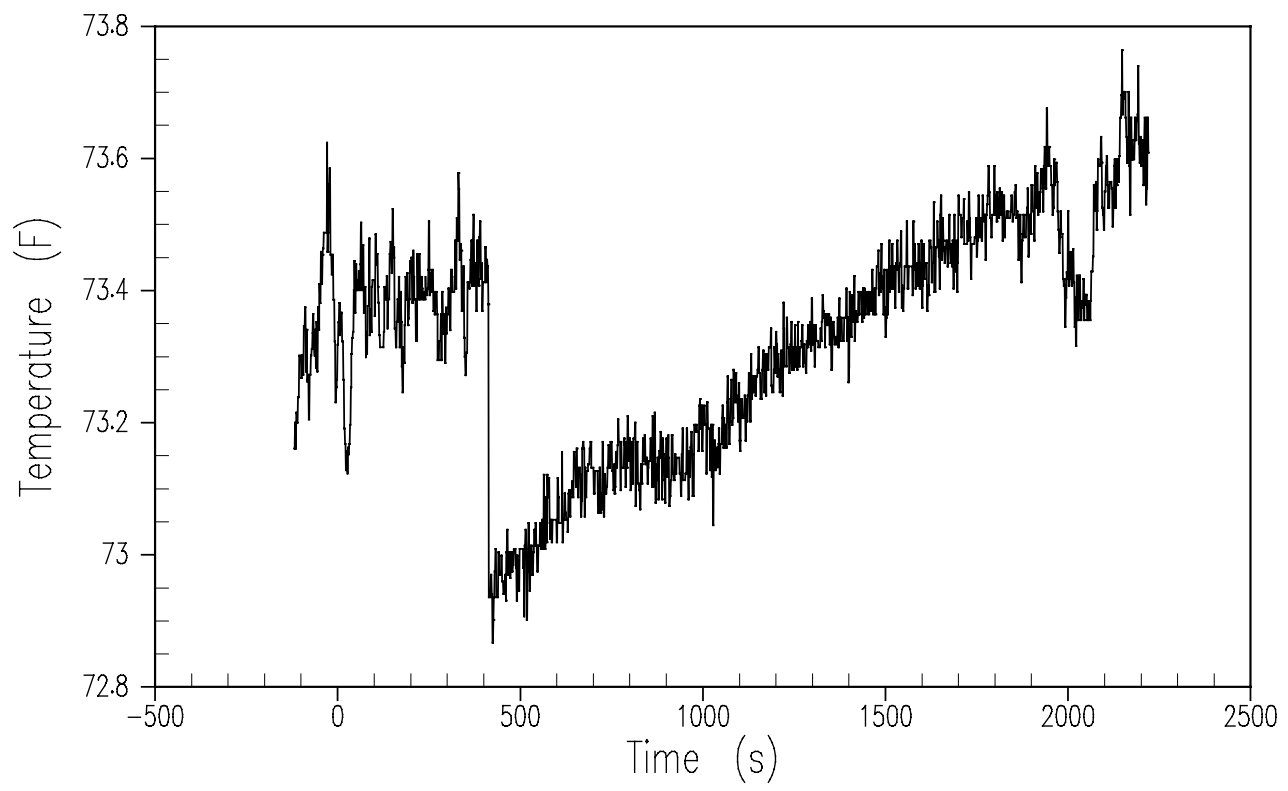

Figure A-25 Accumulator 1 Liquid Discharge Temperature 


\section{AP1000 Test Data Oregon State University Test TR-02 July 9, 2003

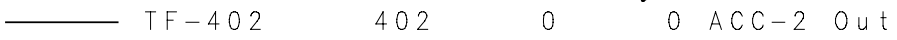

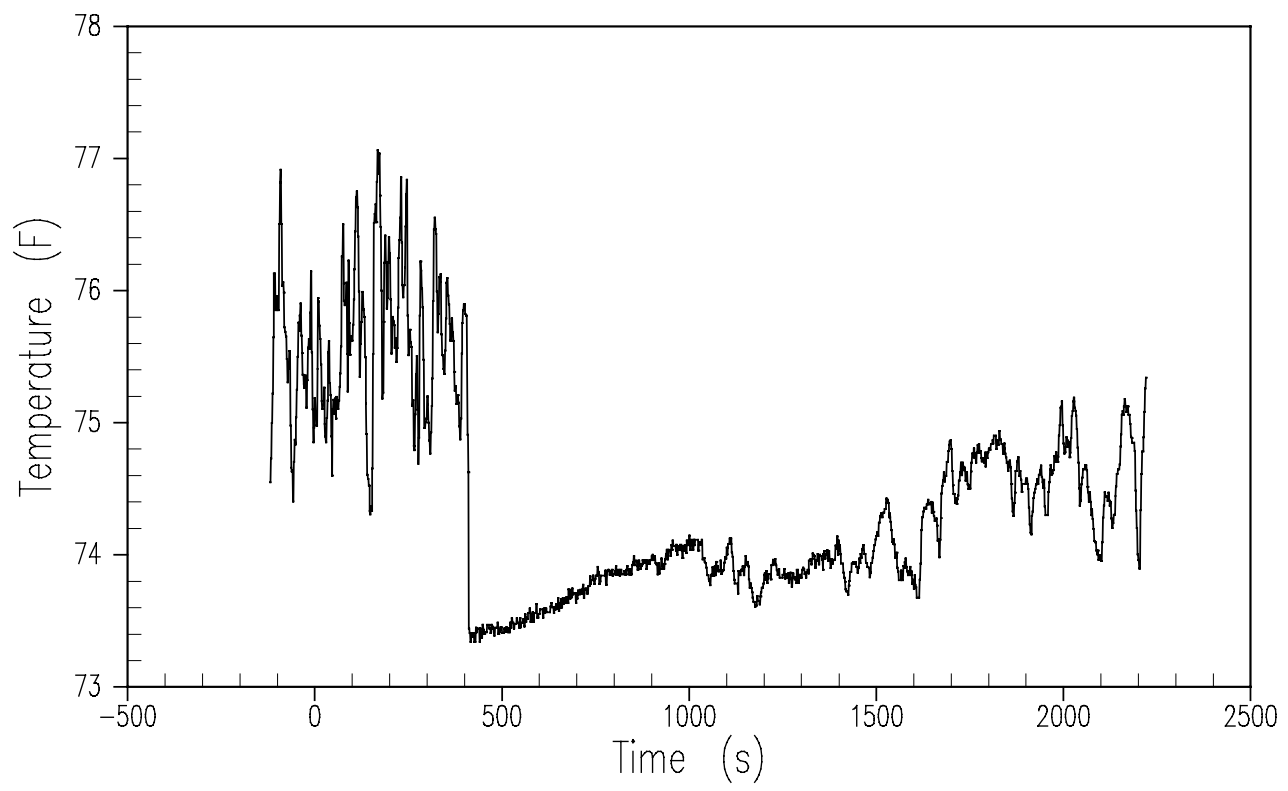

Figure A-26 Accumulator 2 Liquid Discharge Temperature 


\section{AP1000 Test Data Oregon State University Test TR-02 July 9, 2003
LDP -507
175
o CMT-1 WR UC Level

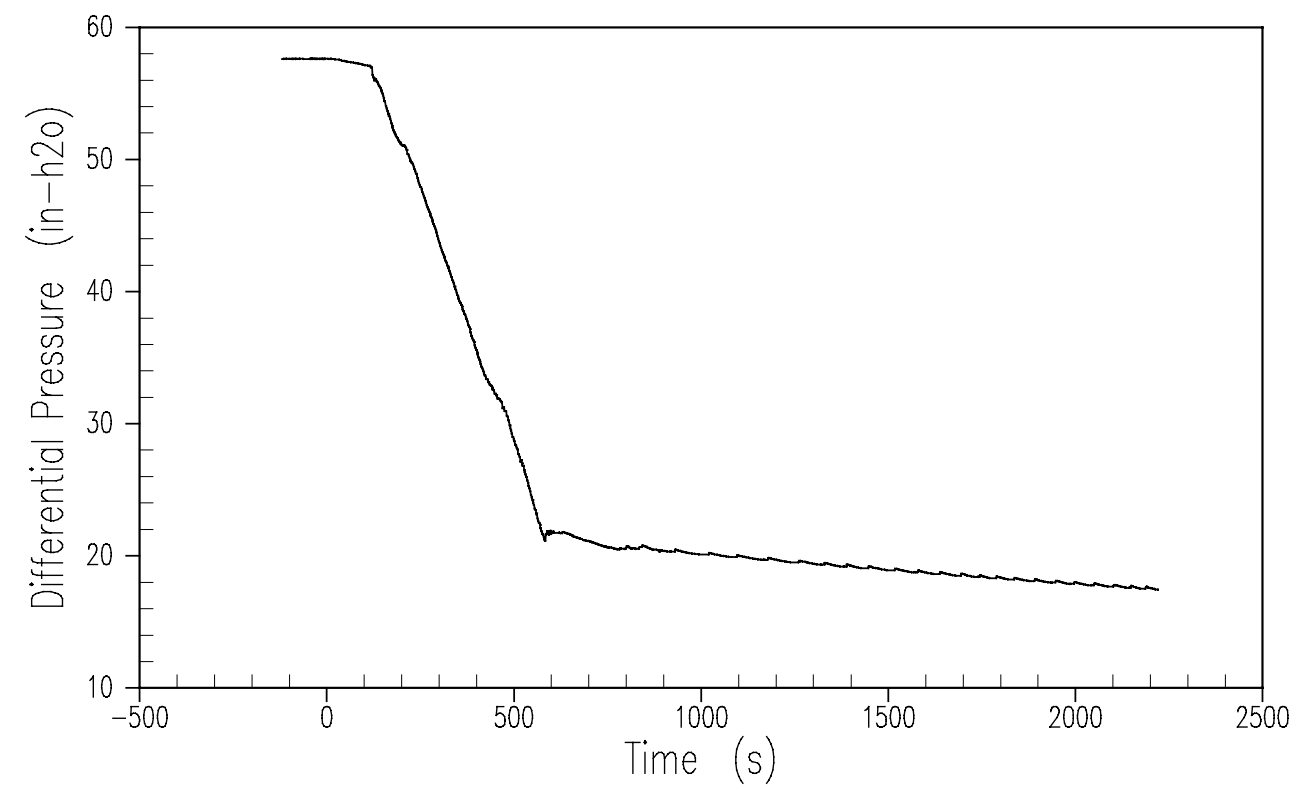

Figure A-27 Core Makeup Tank 1 Liquid Level 


\section{AP1000 Test Data Oregon State University Test TR-02 July 9, 2003

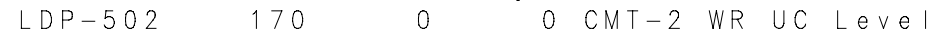

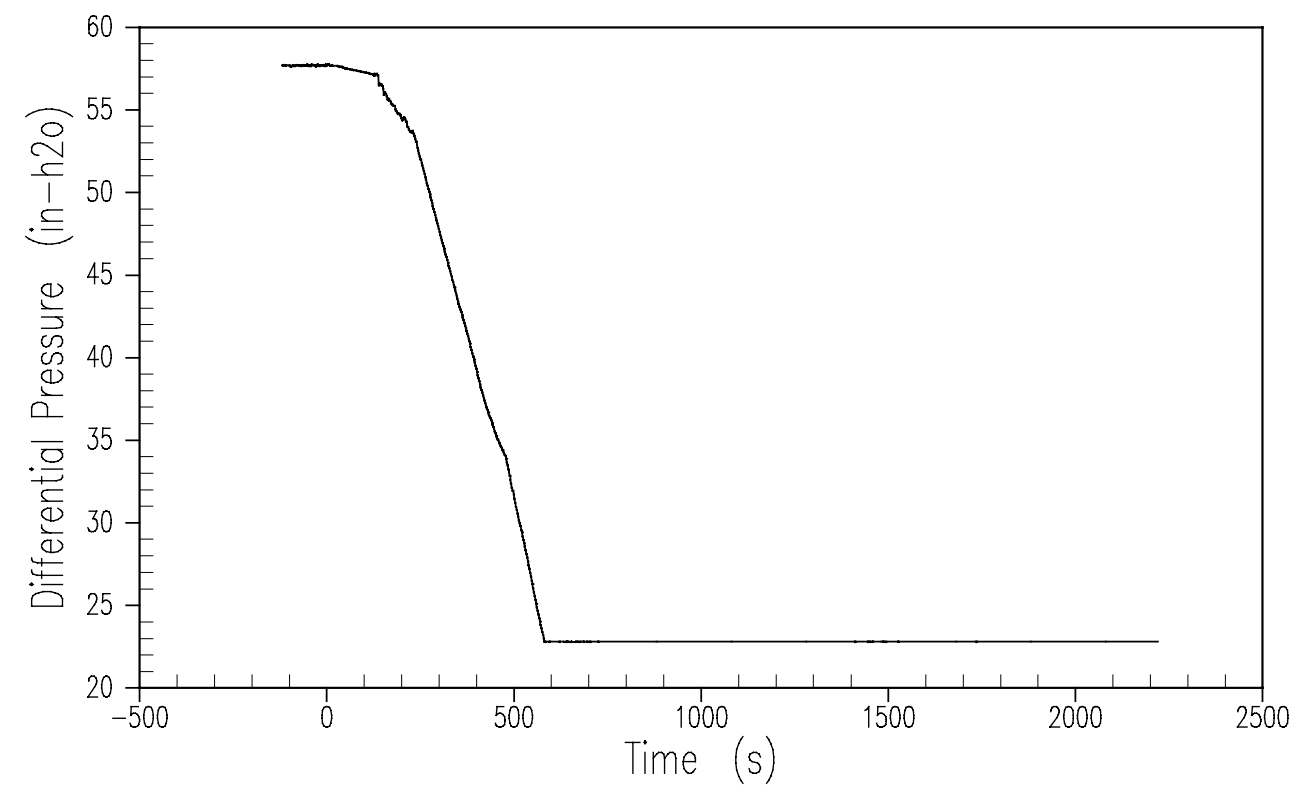

Figure A-28 Core Makeup Tank 2 Liquid Level 

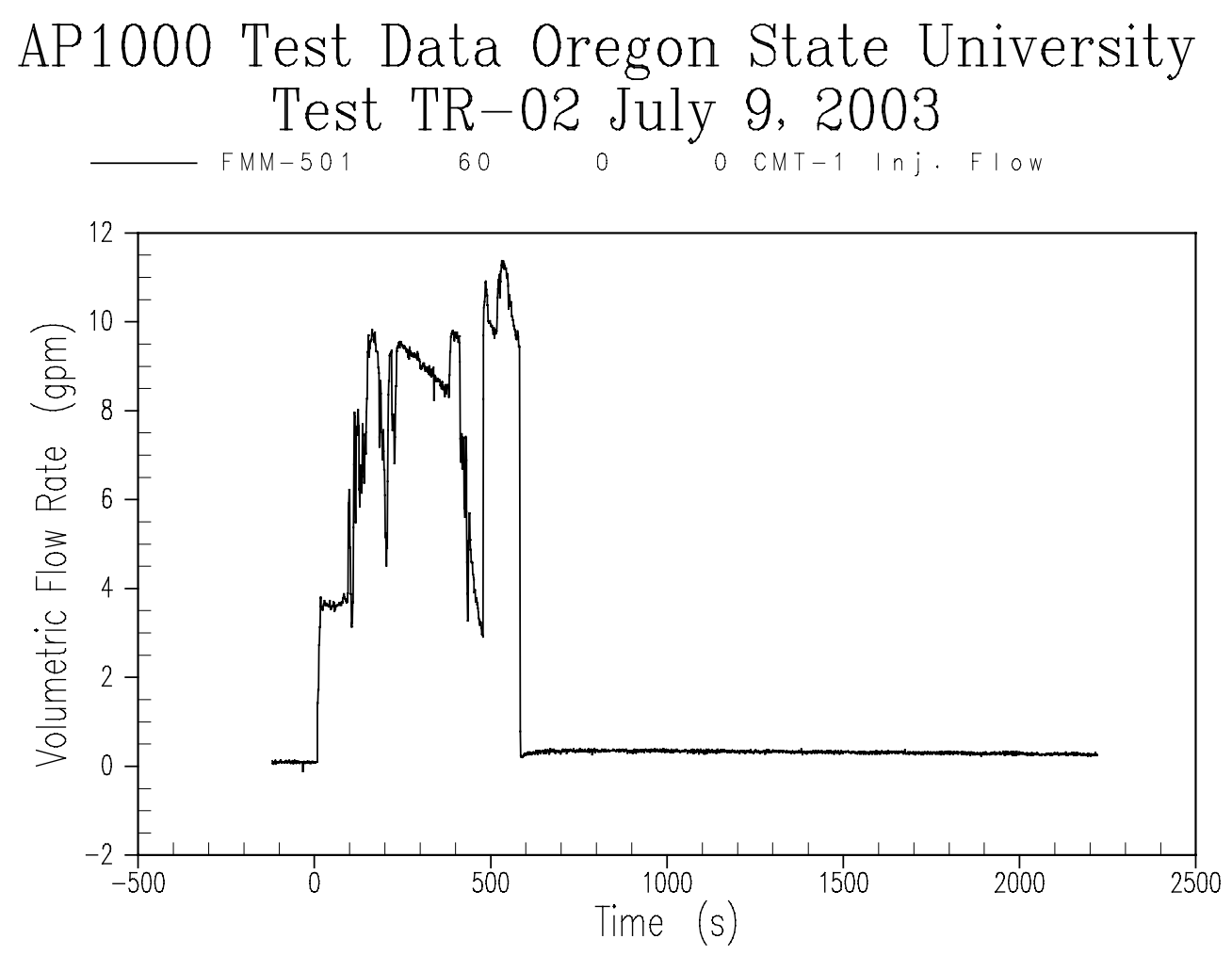

Figure A-29 Core Makeup Tank 1 Flow Rate 

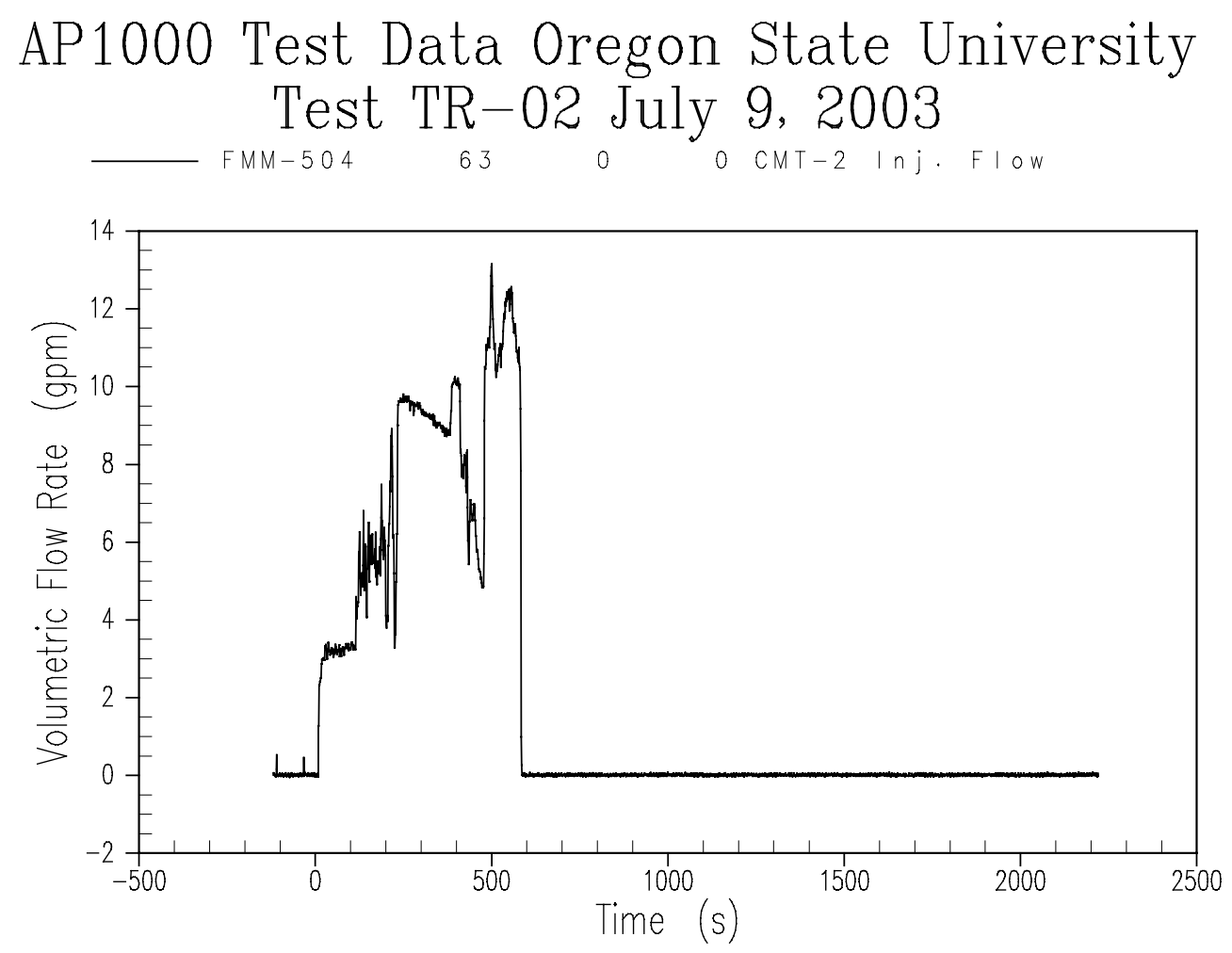

Figure A-30 Core Makeup Tank 2 Flow Rate 

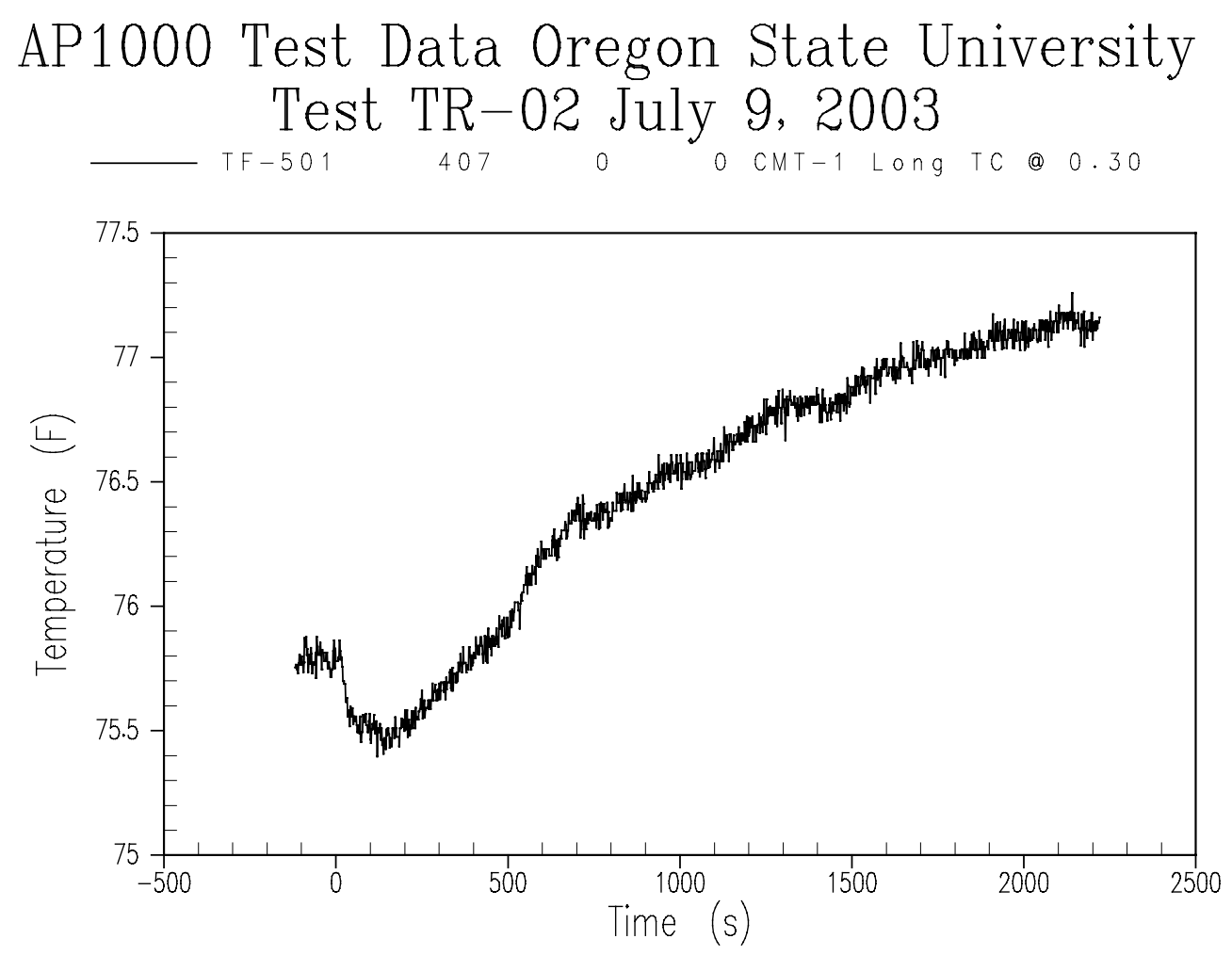

Figure A-31 Core Makeup Tank 1 Liquid Temperature - Bottom 

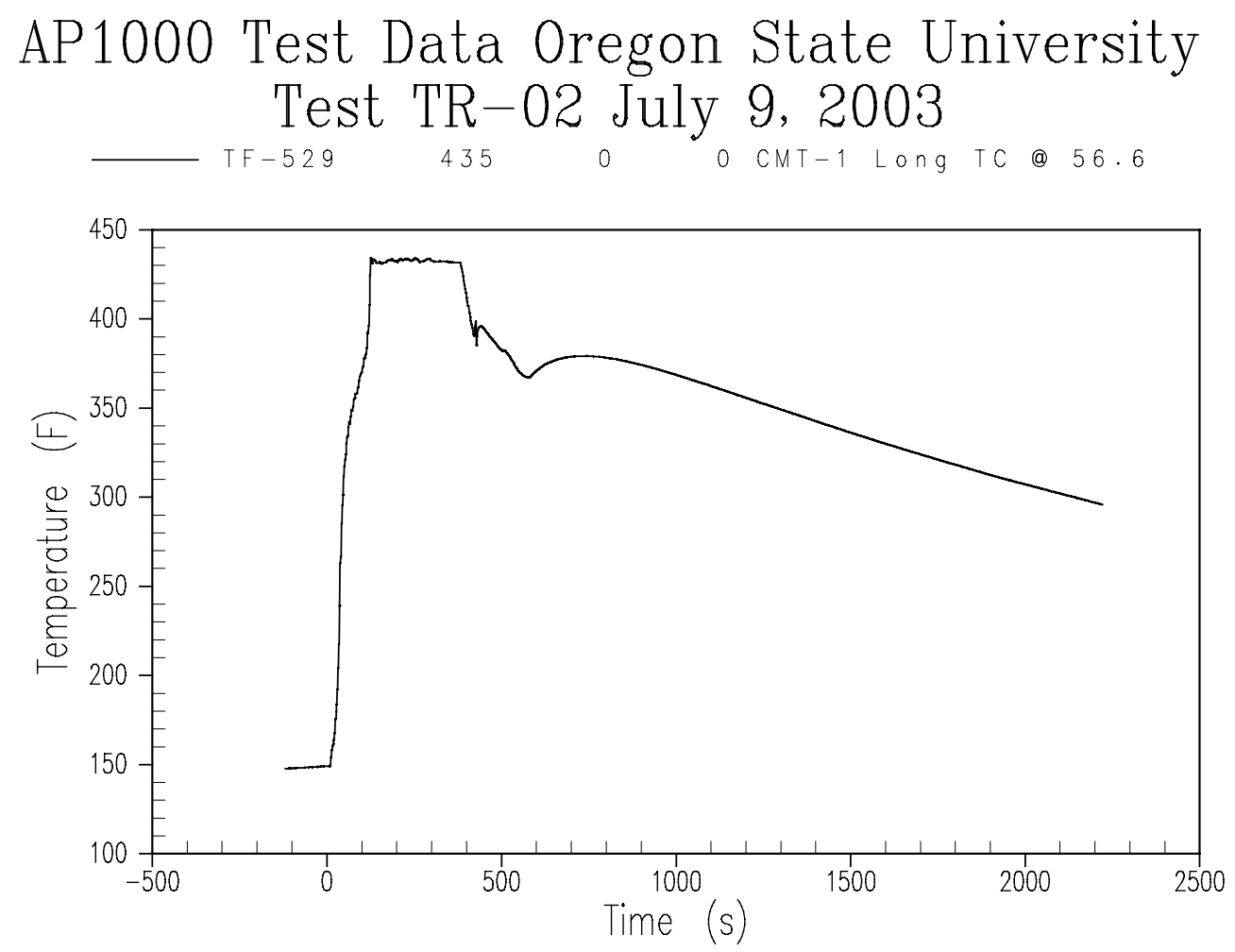

Figure A-32 Core Makeup Tank 1 Liquid Temperature - Top 

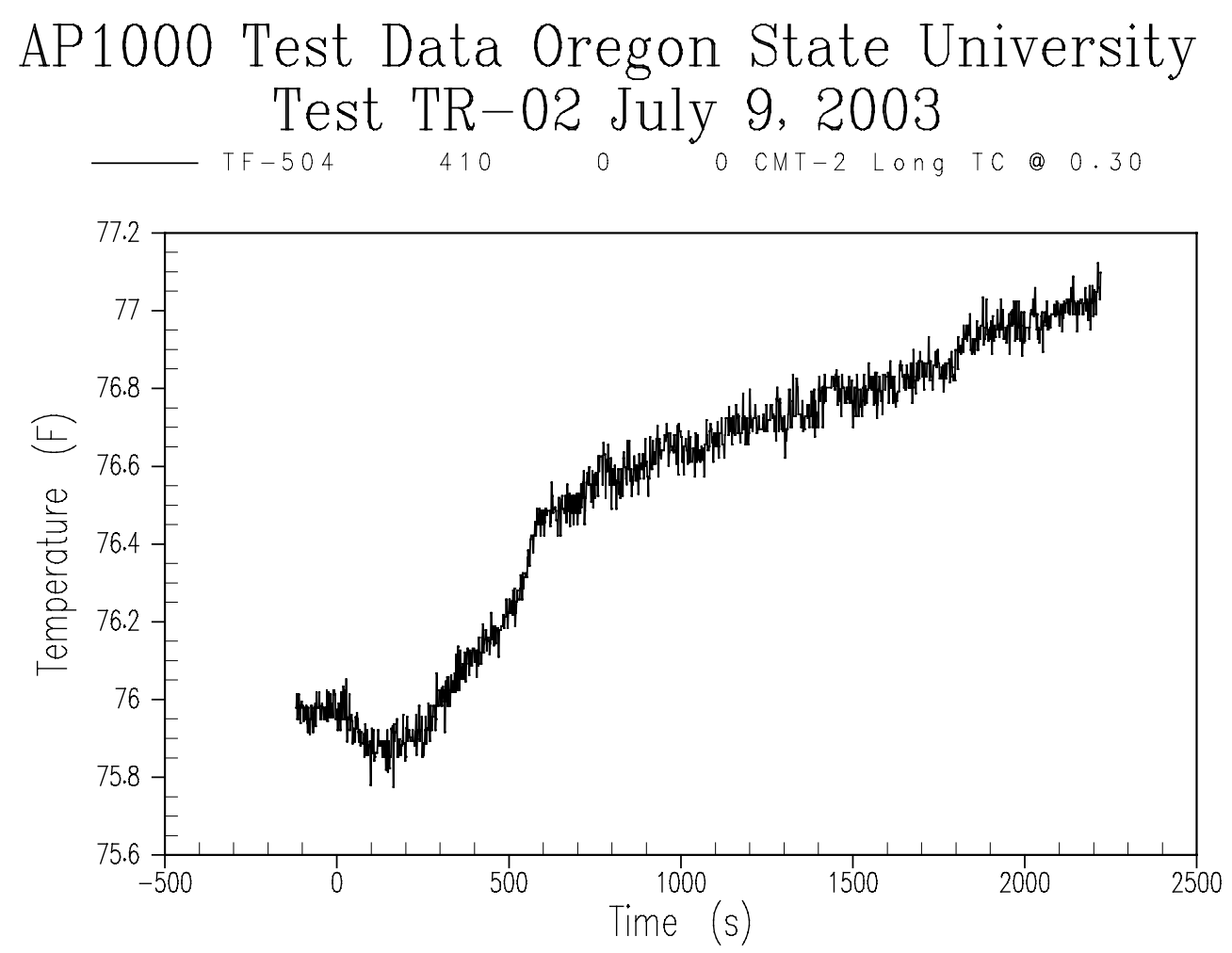

Figure A-33 Core Makeup Tank 2 Liquid Temperature - Bottom 

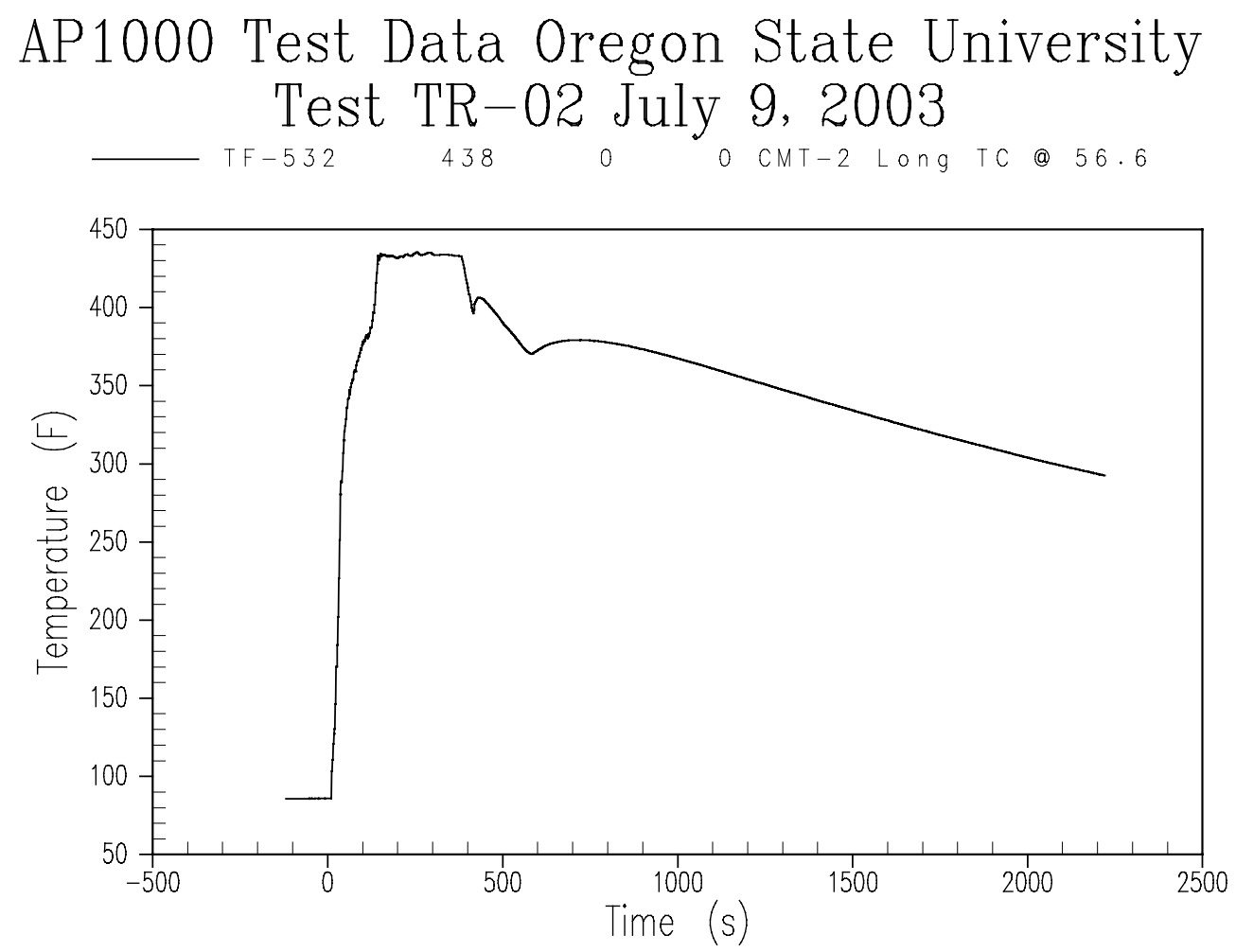

Figure A-34 Core Makeup Tank 2 Liquid Temperature - Top 


\section{AP1000 Test Data Oregon State University Test TR-02 July 9, 2003 $\begin{array}{lllllll}\text { FMM-802 } & 71 & 0 & 0 & \text { PRHR In F IOW }\end{array}$}

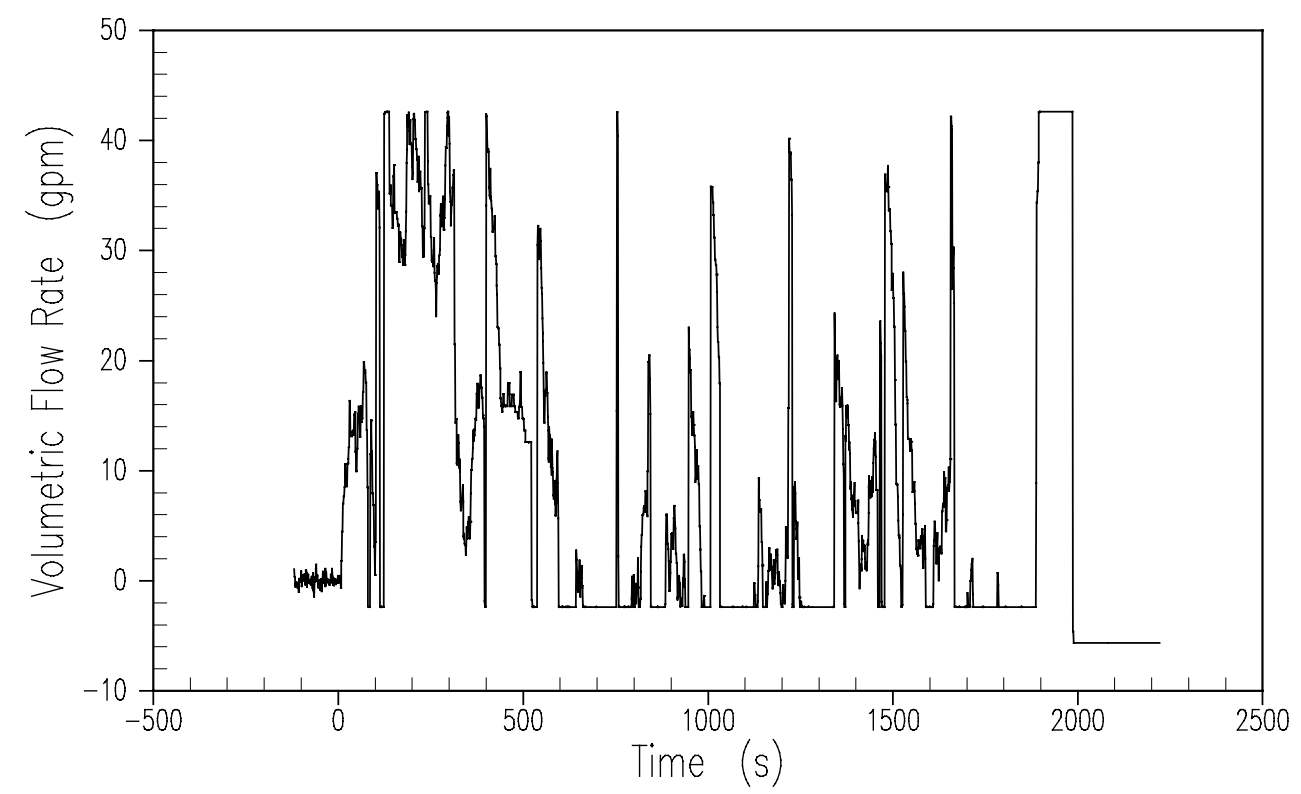

Figure A-35 Passive Residual Heat Removal Inlet Flow Rate 


\section{AP1000 Test Data Oregon State University Test TR-02 July 9, 2003 \\ — LDP-802 $193 \quad 0 \quad$ O PRHR WR UC Level}

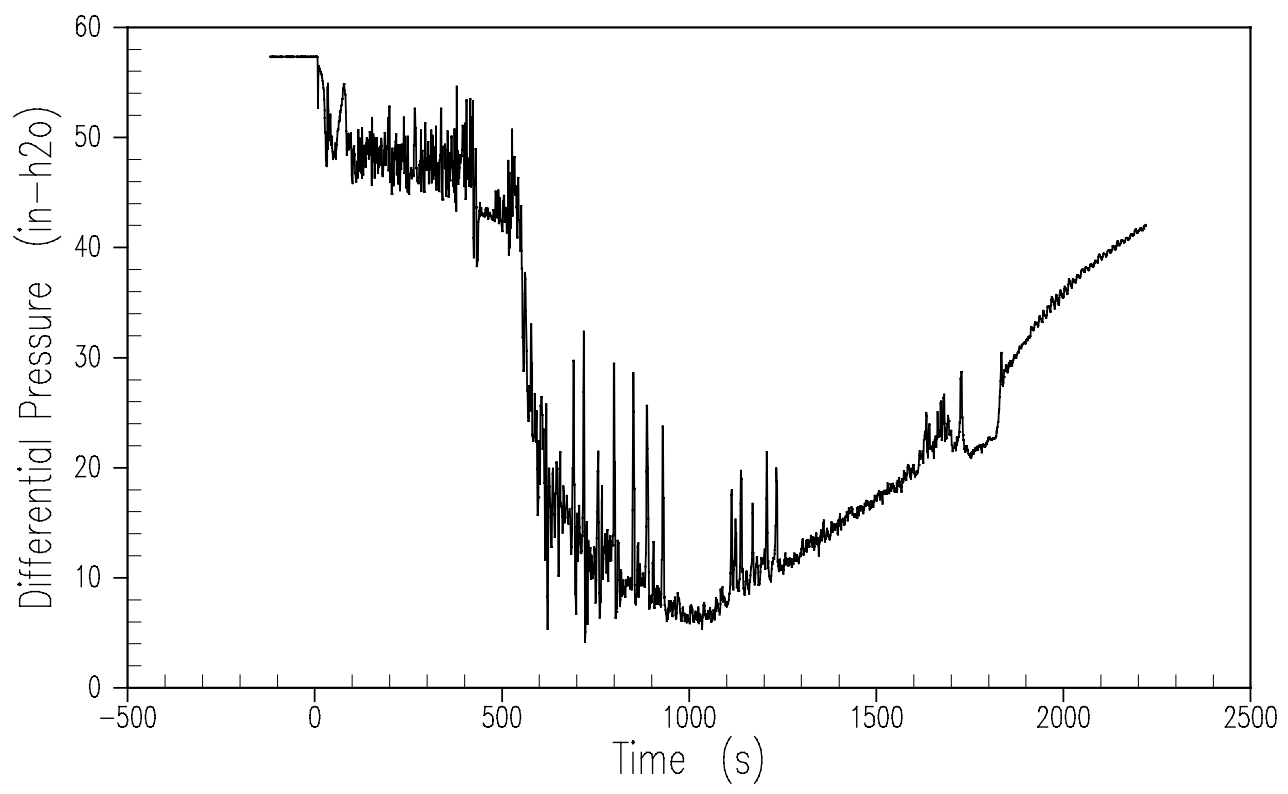

Figure A-36 Passive Residual Heat Removal Liquid Level 


\section{AP1000 Test Data Oregon State University Test TR-02 July 9, 2003 \\ $\begin{array}{llllll}F M M-804 & 73 & 0 & \text { O PRHR Out FIOW }\end{array}$}

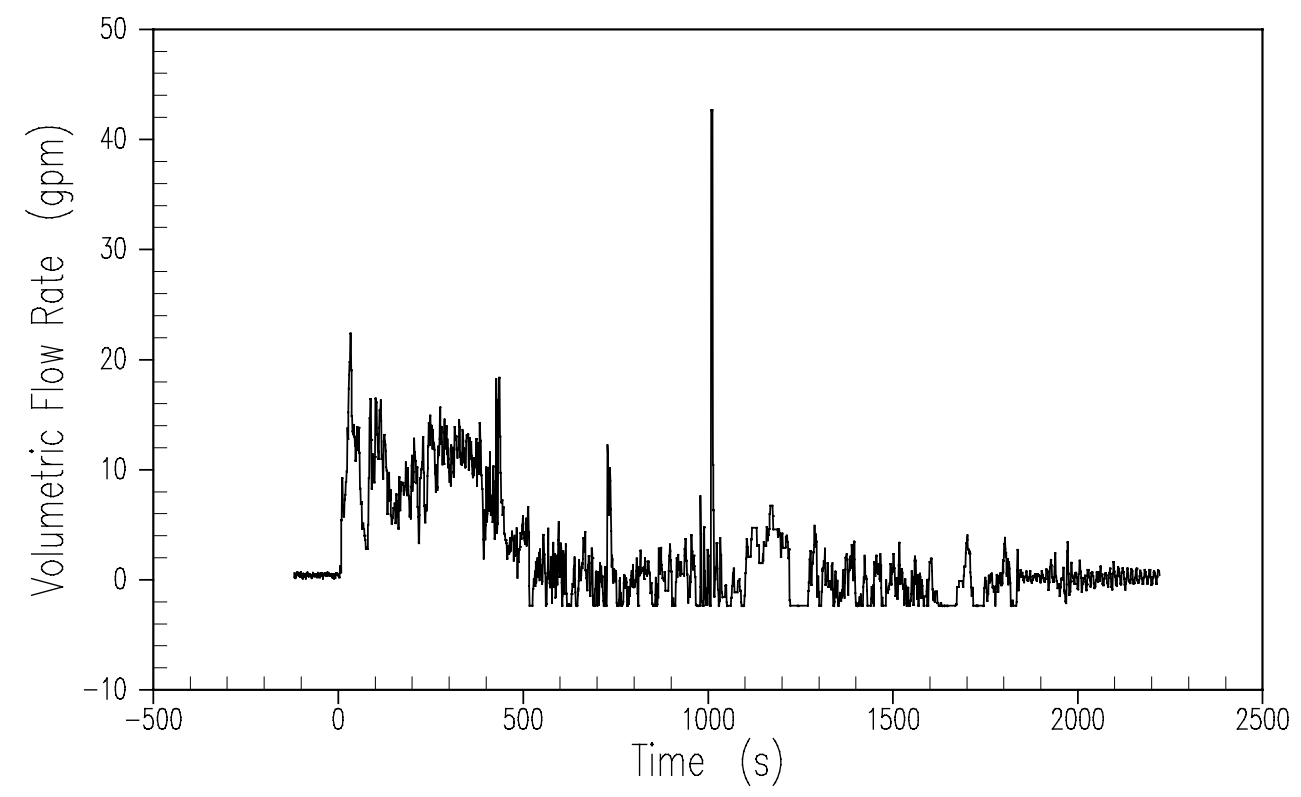

Figure A-37 Passive Residual Heat Removal Outlet Flow Rate 

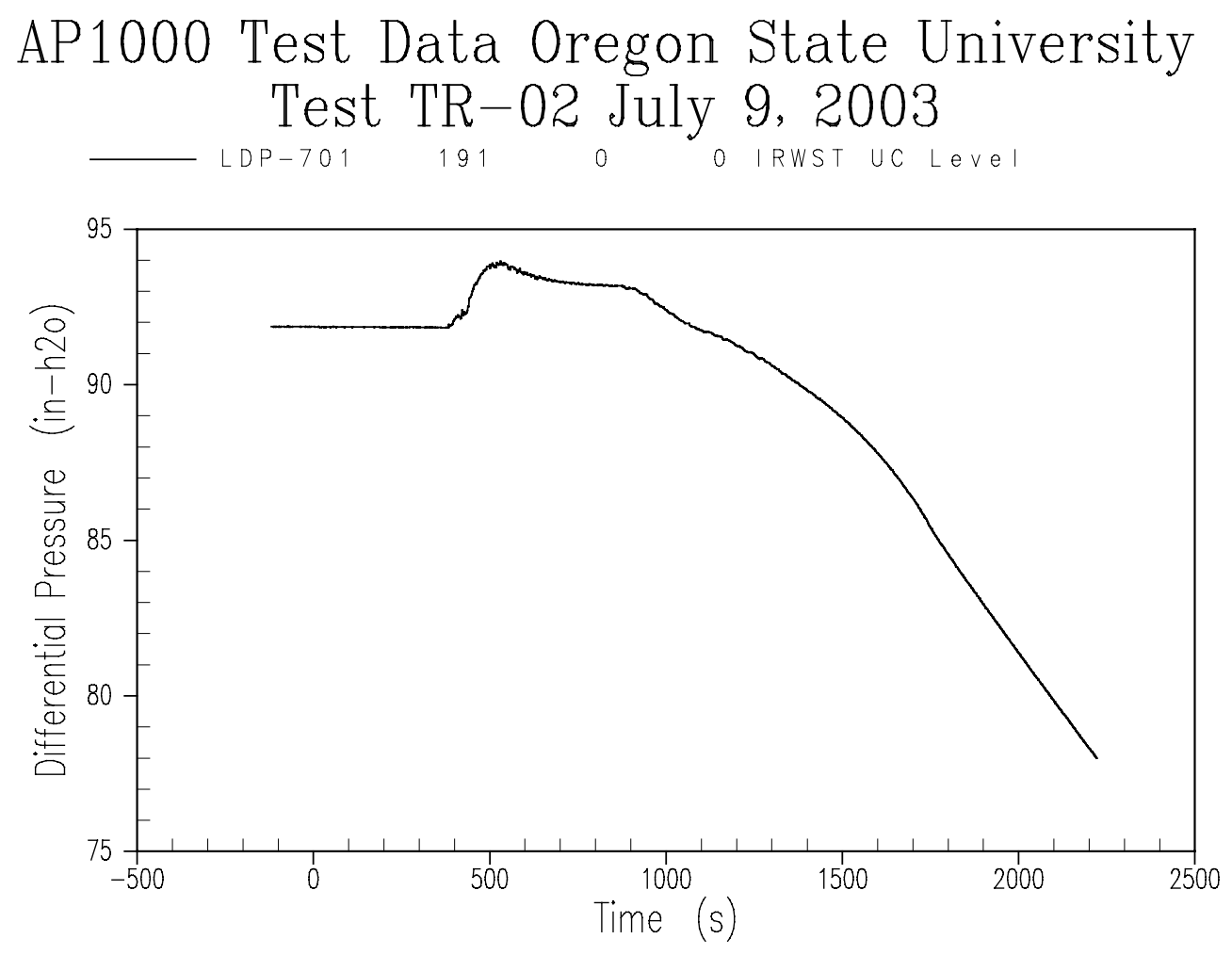

Figure A-38 IRWST Liquid Level 

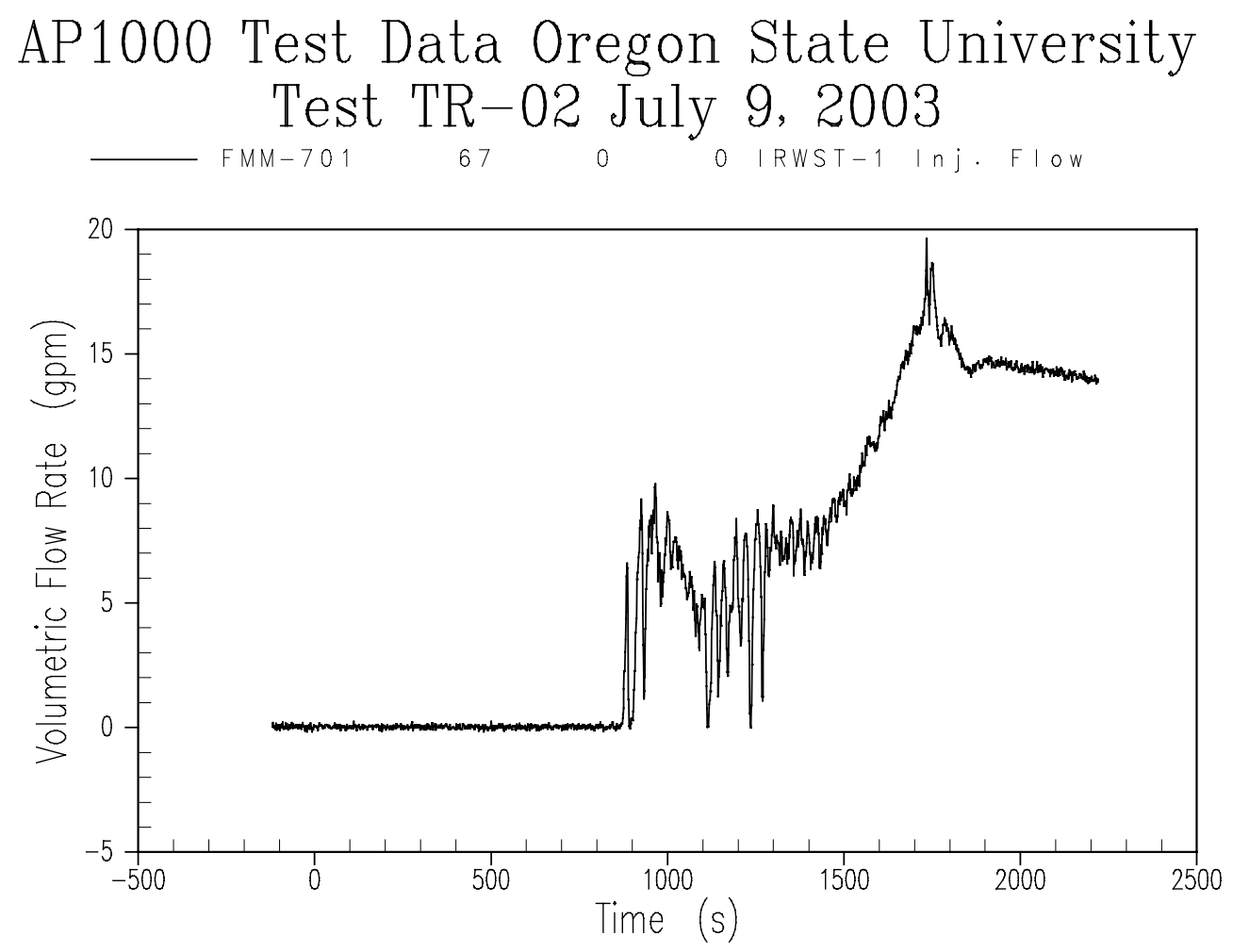

Figure A-39 IRWST Discharge Line 1 Flow Rate 


\section{AP1000 Test Data Oregon State University Test TR-02 July 9, 2003
$\mathrm{FMM}-702$
68
o I RWST-2 Inj. FI OW

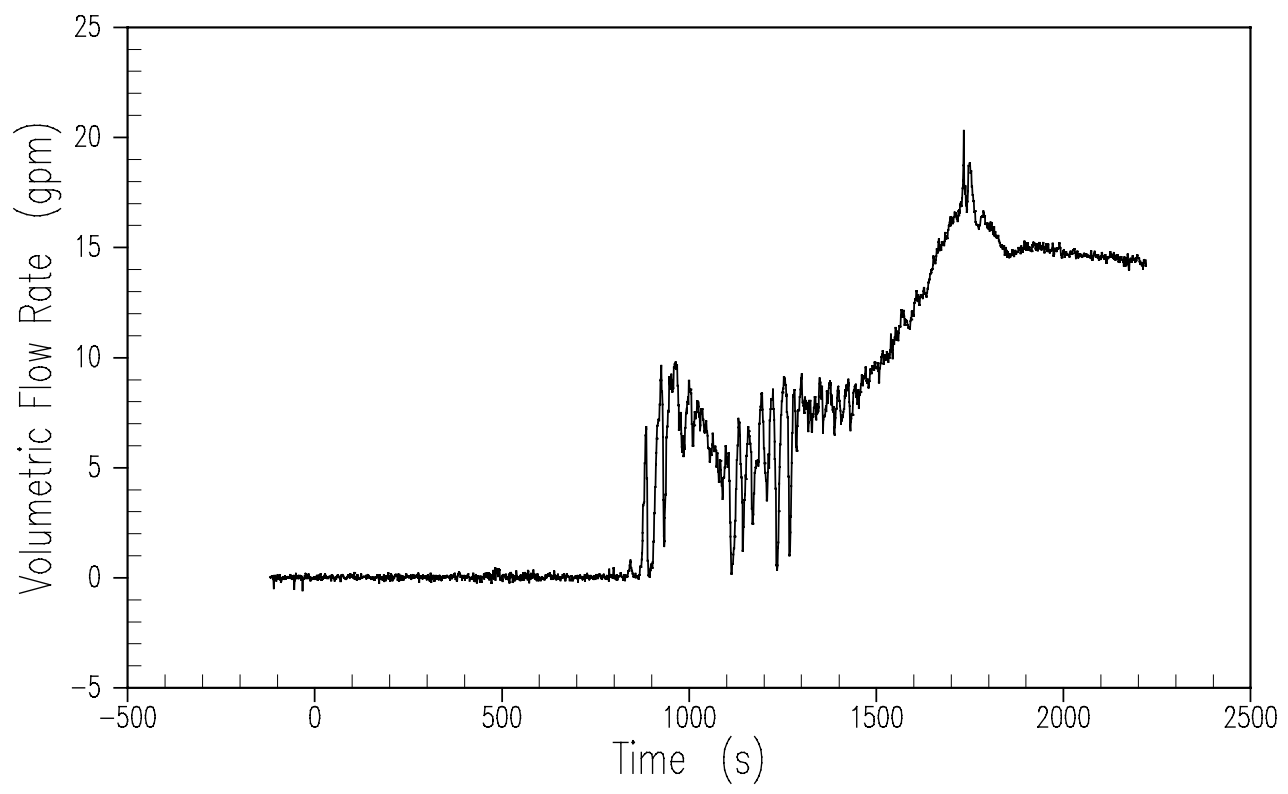

Figure A-40 IRWST Discharge Line 2 Flow Rate 

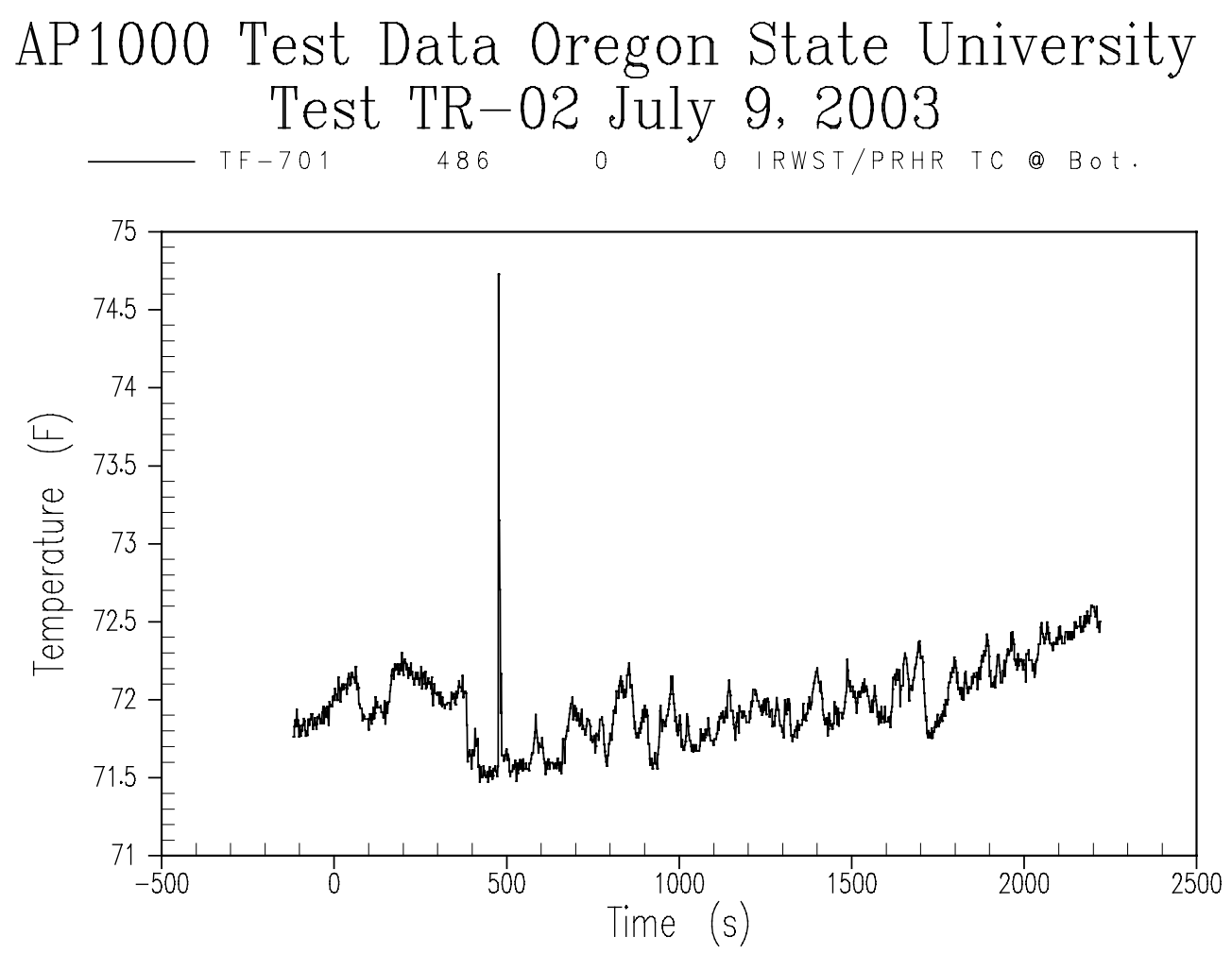

Figure A-41 IRWST Fluid Temperature - Bottom 


\section{AP1000 Test Data Oregon State University Test TR-02 July 9, 2003 \\ $\begin{array}{lllllll}T F-709 & 494 & 0 & 0 & \text { IRWST/PRHR TC @ } & 75.2\end{array}$}

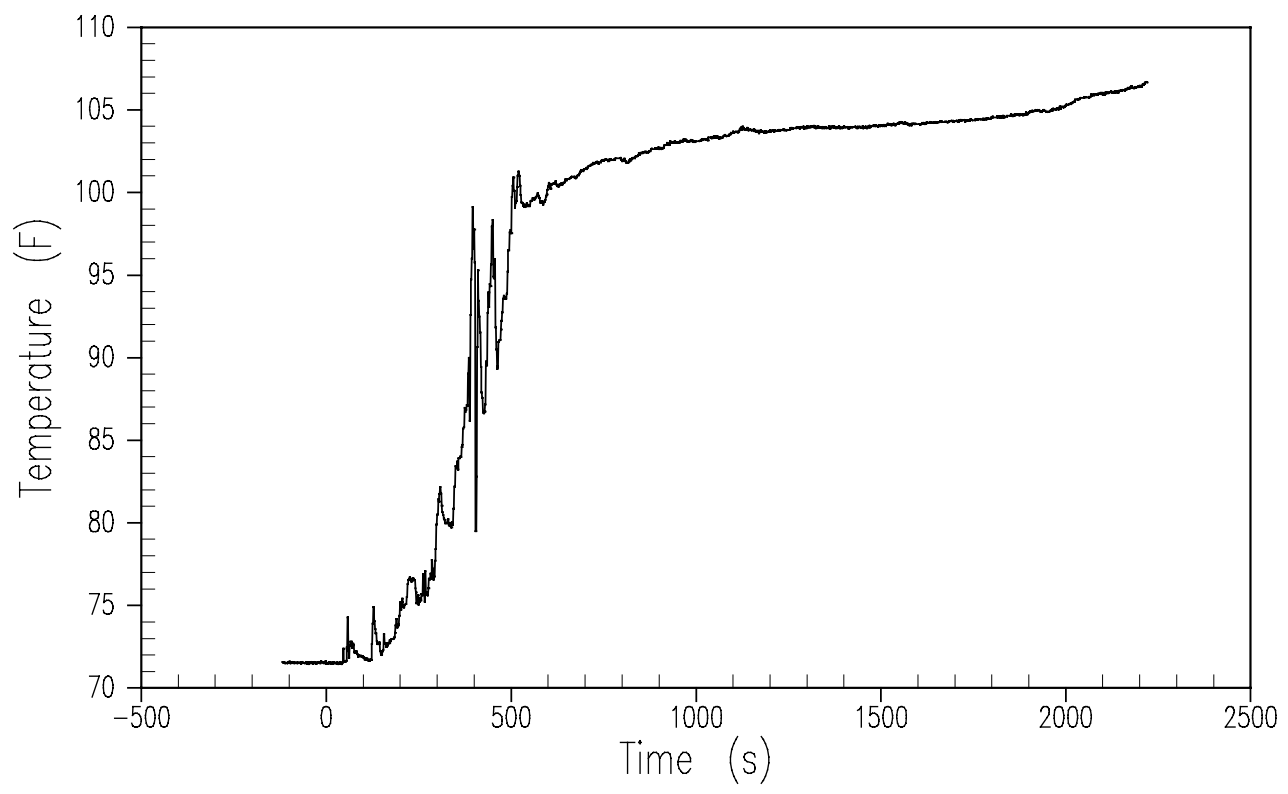

Figure A-42 IRWST Fluid Temperature - Top 


\section{AP1000 Test Data Oregon State University Test TR-02 July 9, 2003
$P T-605$
230
o ADS1-3 sep. Press.

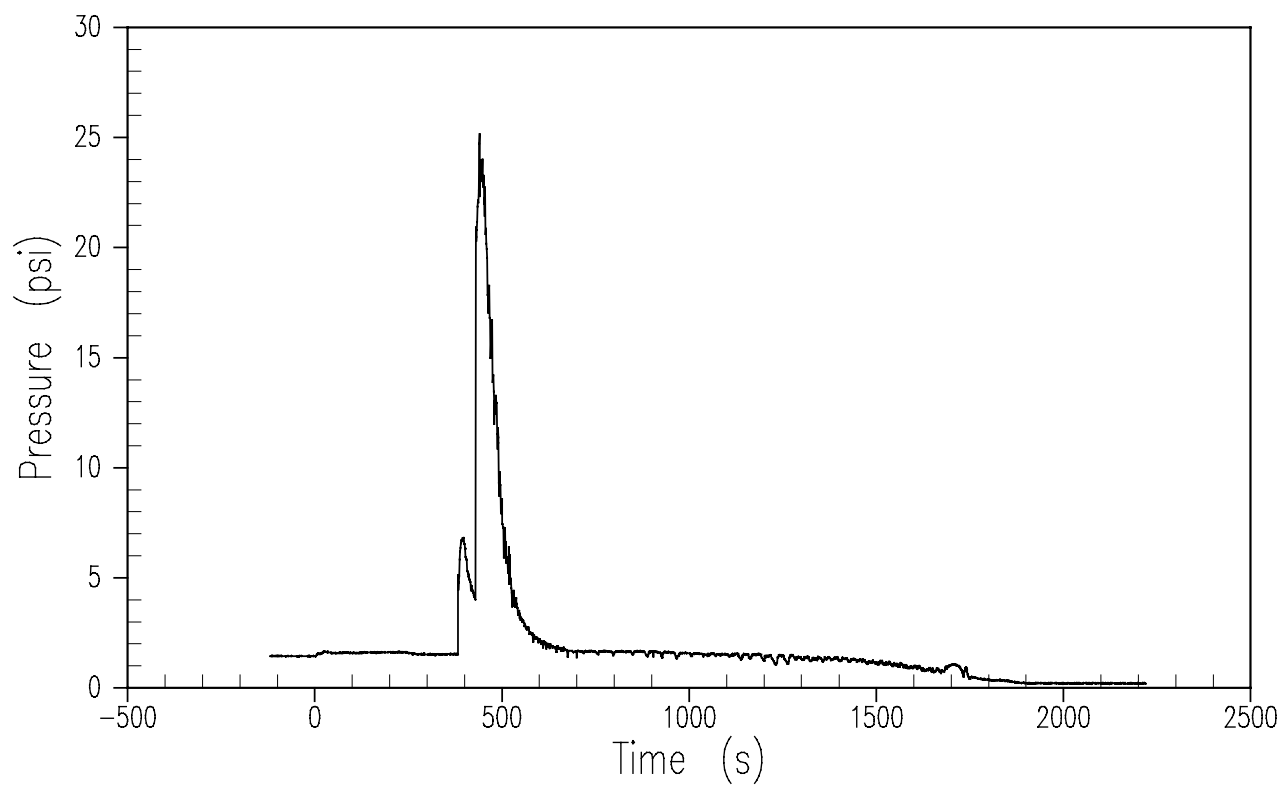

Figure A-43 ADS 1-3 Separator Pressure 

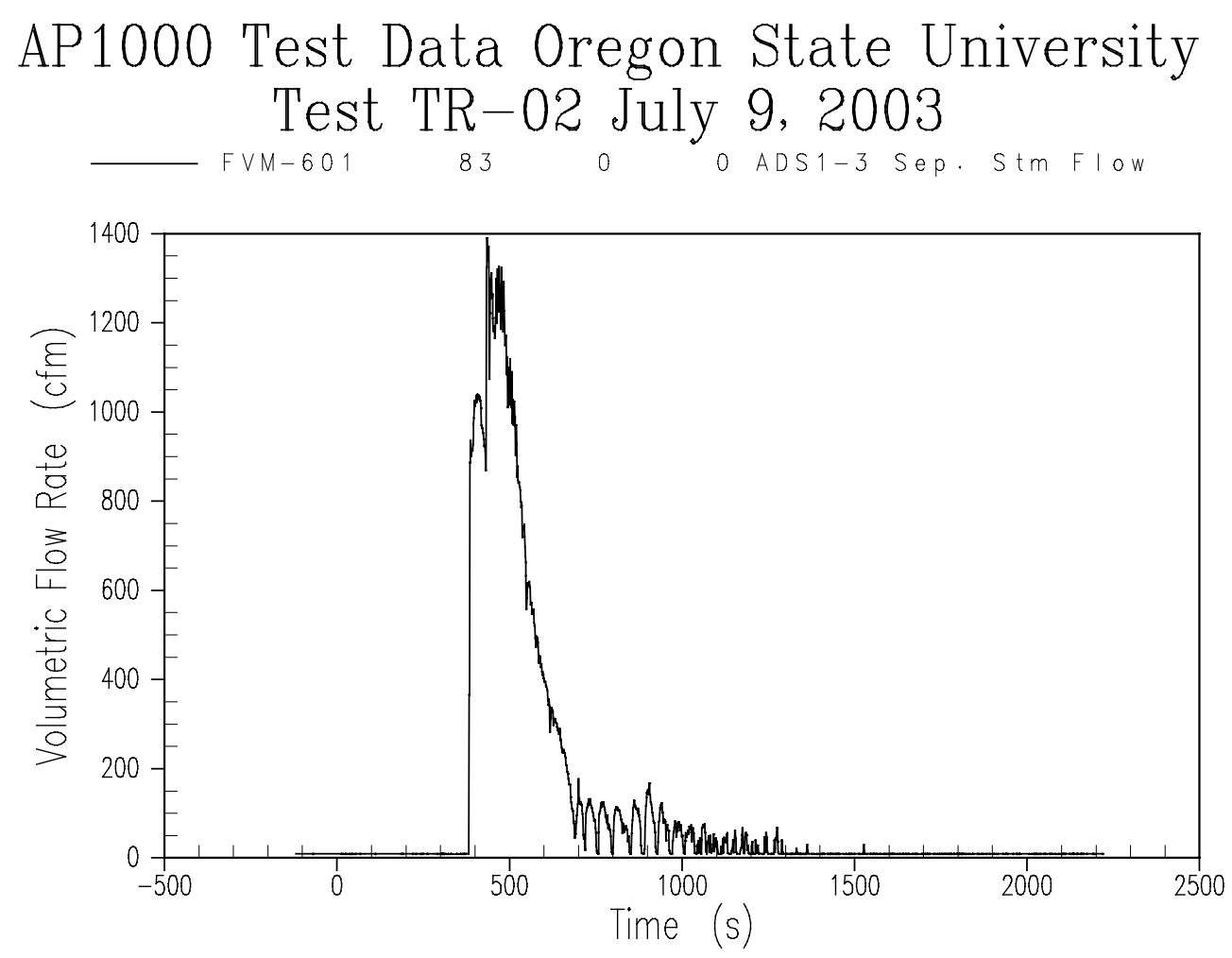

Figure A-44 ADS 1-3 Separator Steam Flow Rate 


\section{AP1000 Test Data Oregon State University Test TR-02 July 9, 2003 \\ $\begin{array}{llllll}F M M-601 & 64 & 0 & 0 & A D S 1-3 & \text { LS FIOW }\end{array}$}

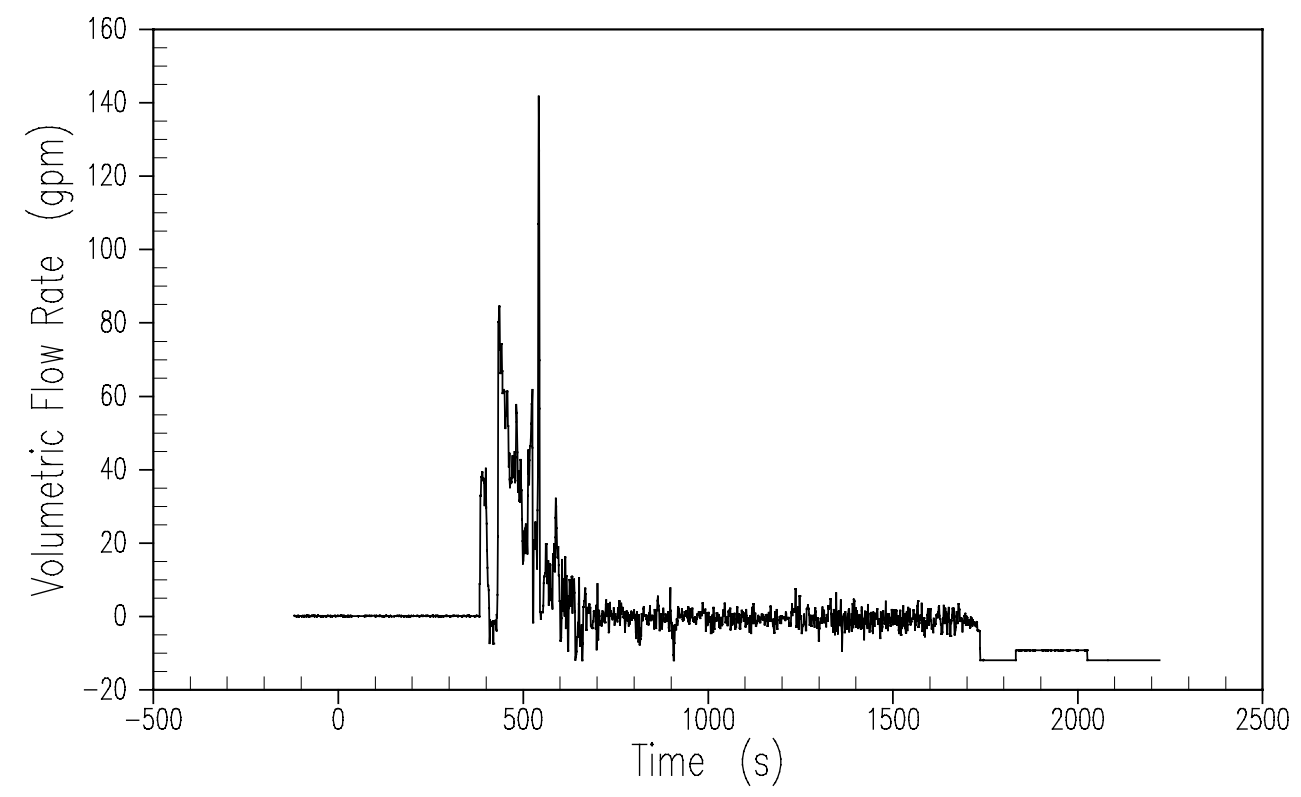

Figure A-45 ADS 1-3 Separator Liquid Flow Rate 

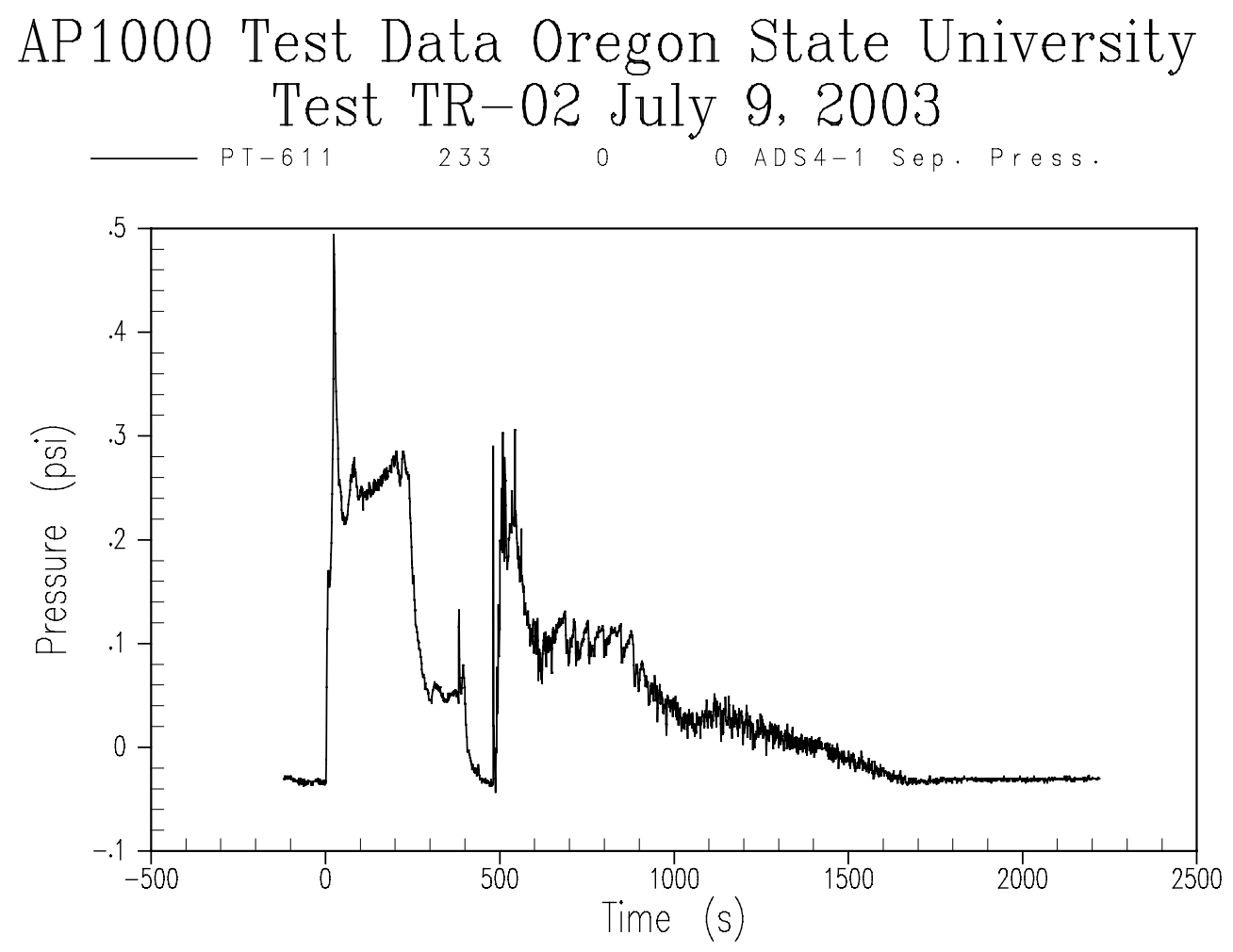

Figure A-46 ADS 4-1 Separator Pressure 

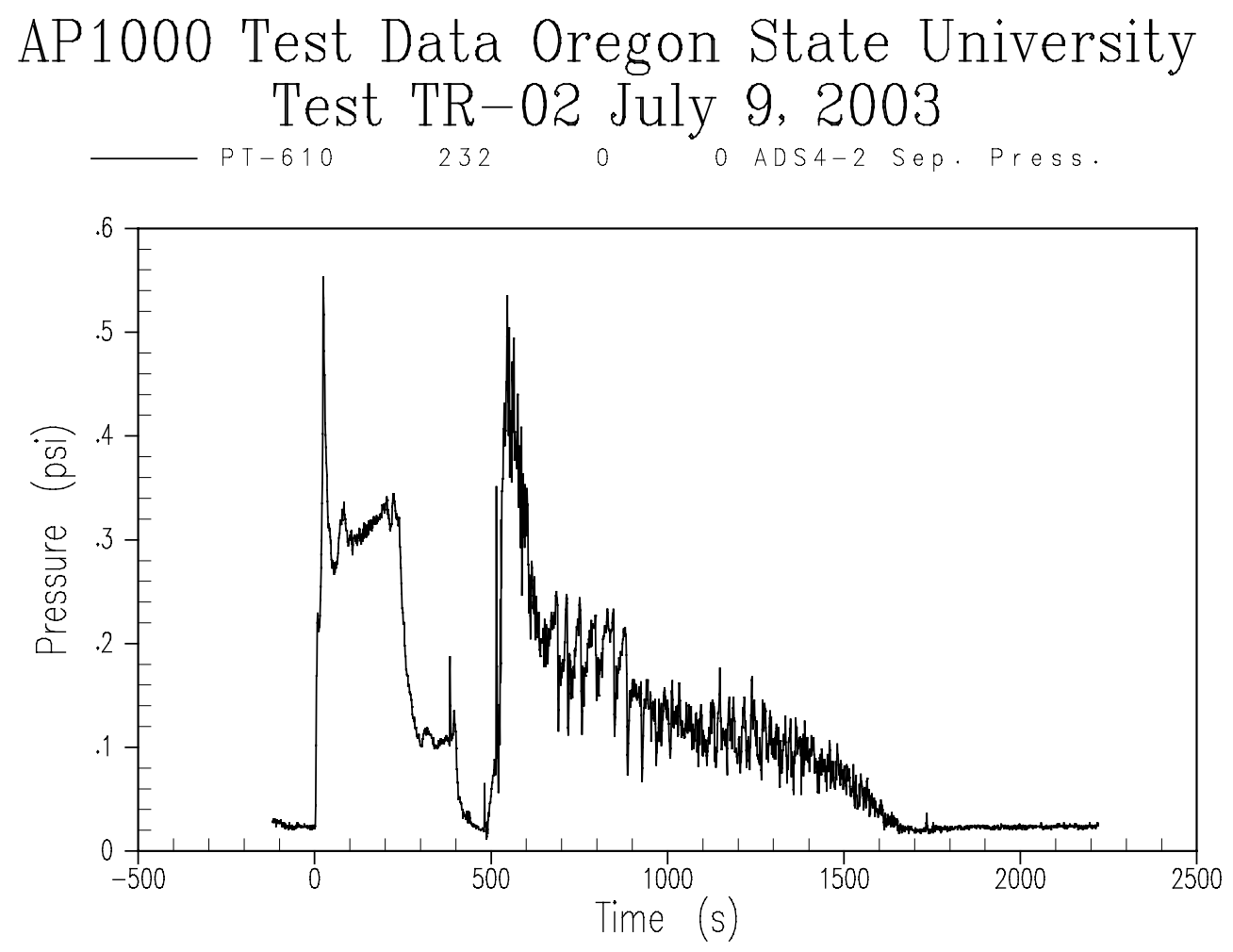

Figure A-47 ADS 4-2 Separator Pressure 


\section{AP1000 Test Data Oregon State University Test TR-02 July 9, 2003 \\ FVM-603 $85 \quad 0 \quad 0$ ADS4-1 Sep. 6" Line}

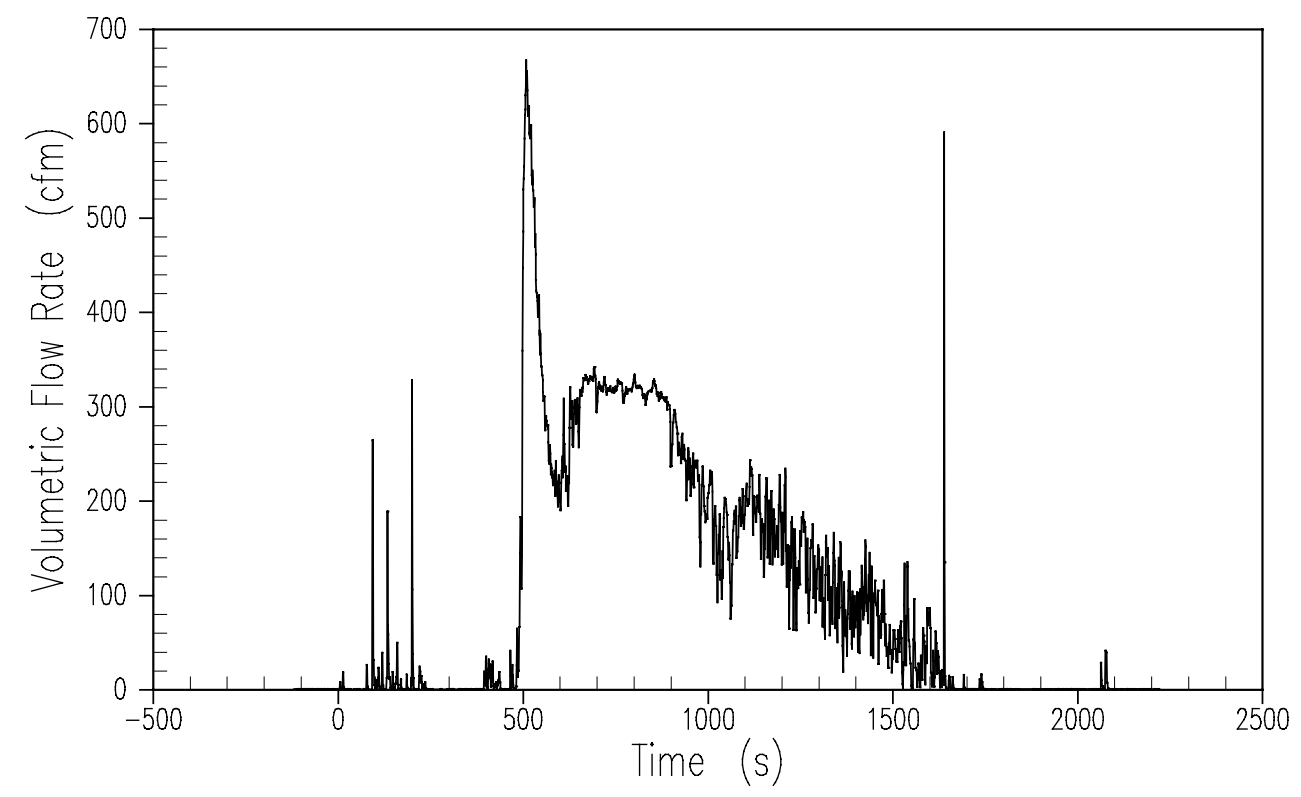

Figure A-48 ADS 4-1 Separator Steam Flow Rate 


\section{AP1000 Test Data Oregon State University Test TR-02 July 9, 2003 \\ FVM-602 $84 \quad 0 \quad$ O ADS4-2 Sep. 6" Line}

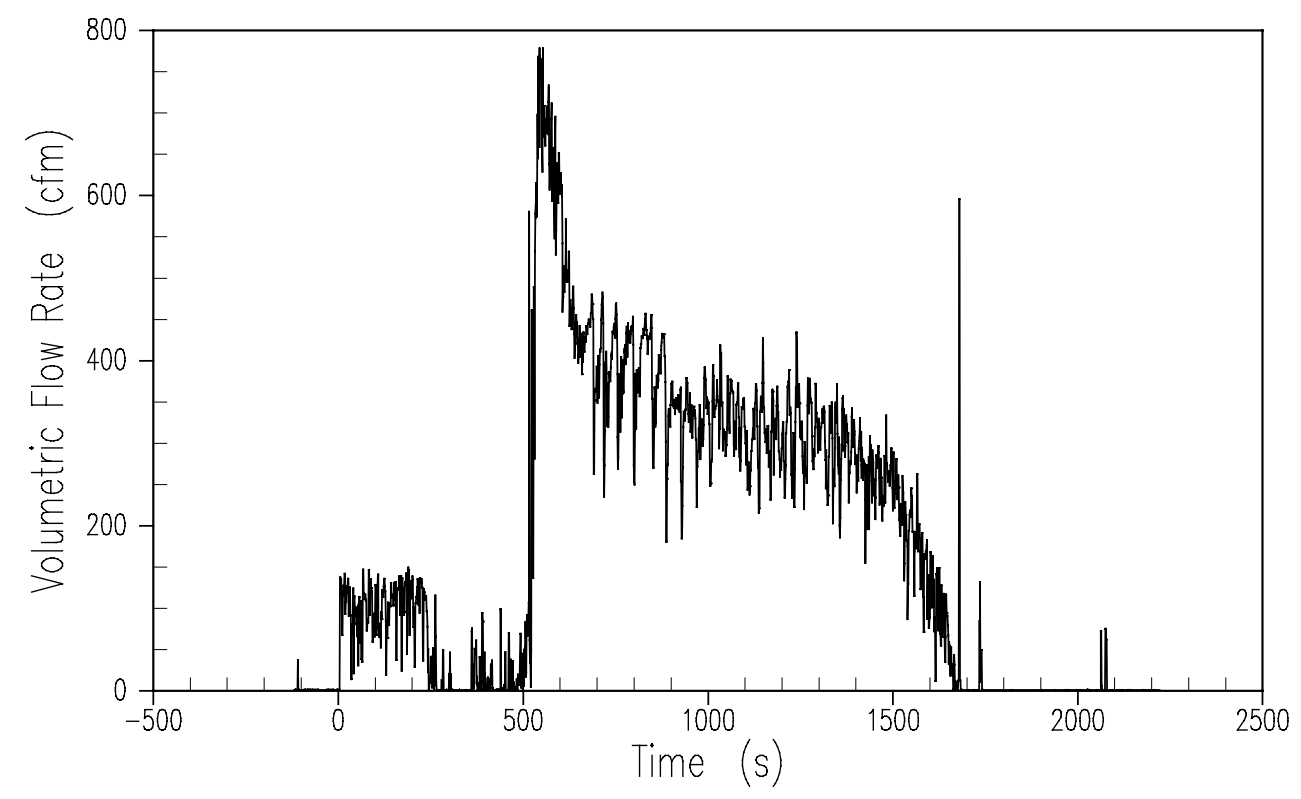

Figure A-49 ADS 4-2 Separator Steam Flow Rate 


\section{AP1000 Test Data Oregon State University Test TR-02 July 9, 2003

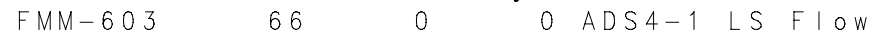

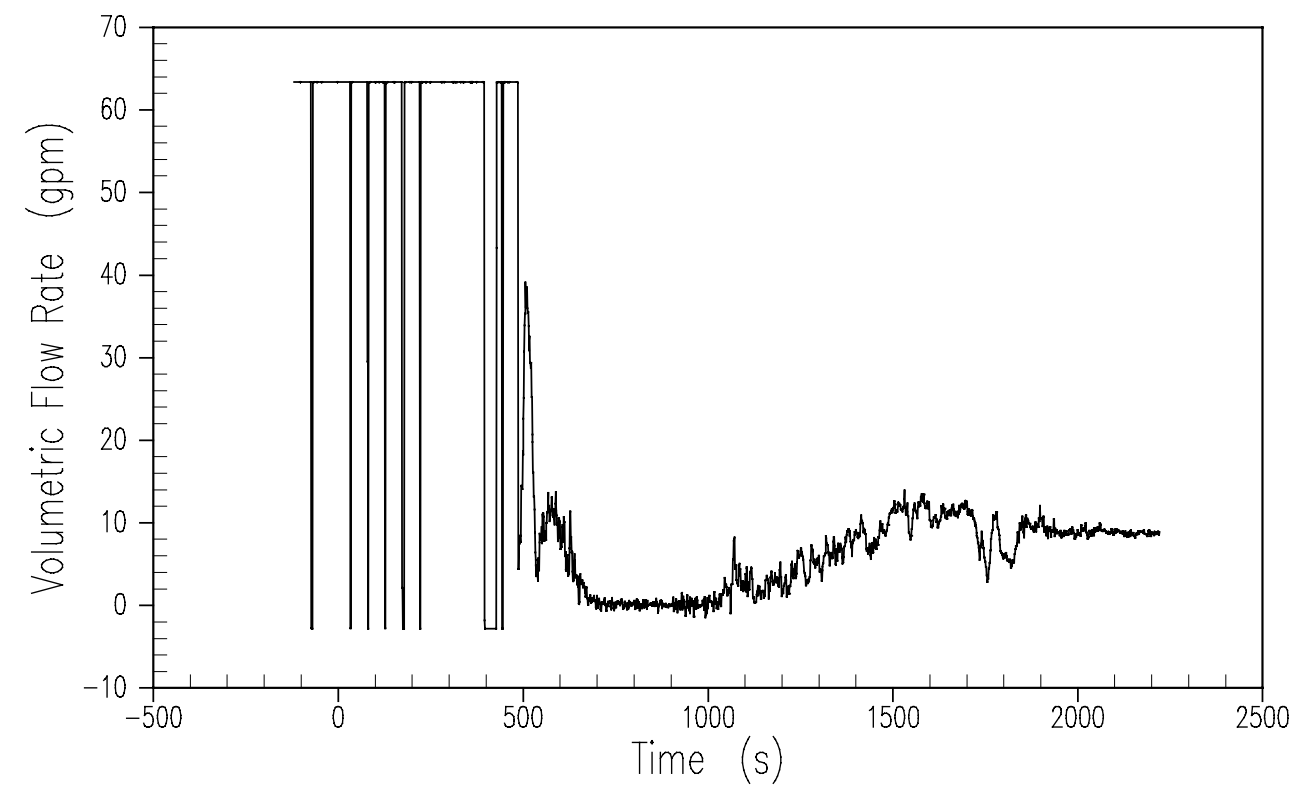

Figure A-50 ADS 4-1 Separator Liquid Flow Rate 


\section{AP1000 Test Data Oregon State University Test TR-02 July 9, 2003 \\ $\begin{array}{llllll}F M M-602 & 65 & 0 & 0 & A D S 4-2 & \text { LS FIOW }\end{array}$}

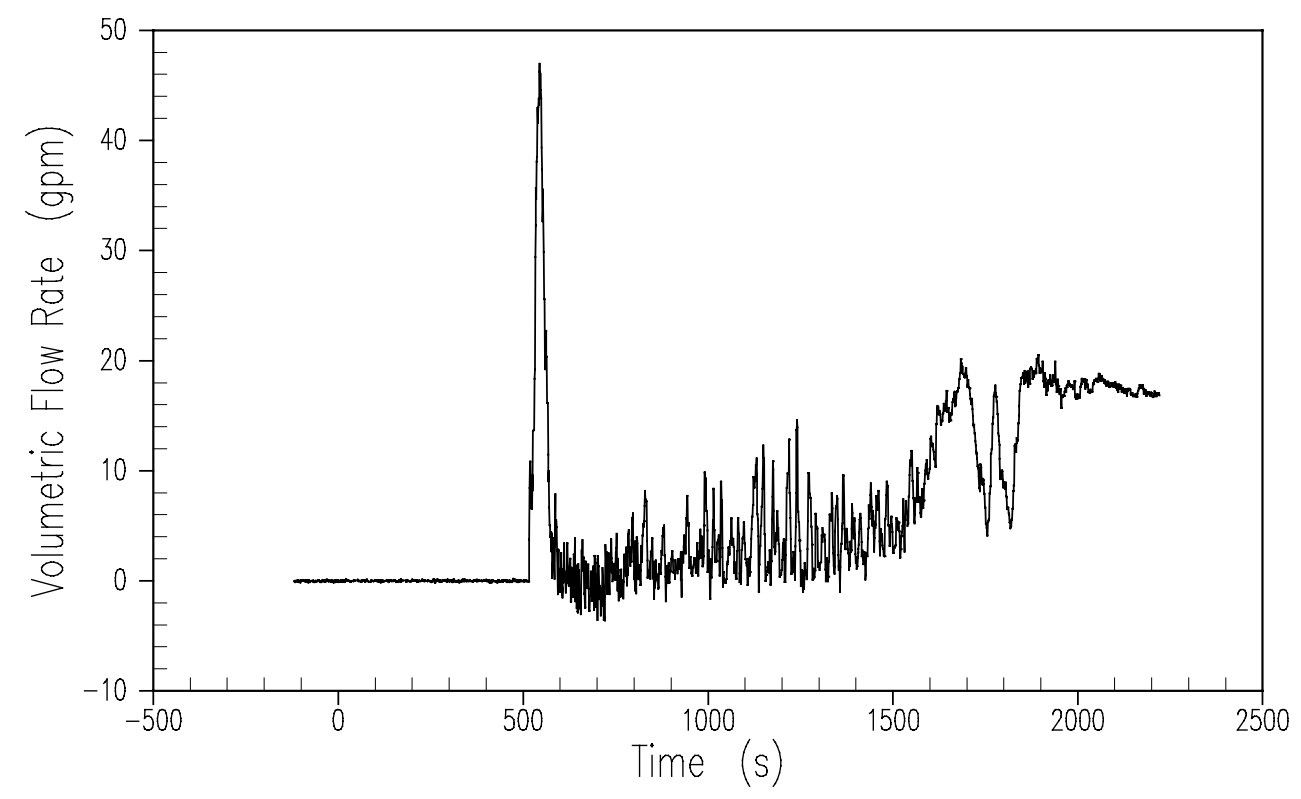

Figure A-51 ADS 4-2 Separator Liquid Flow Rate 

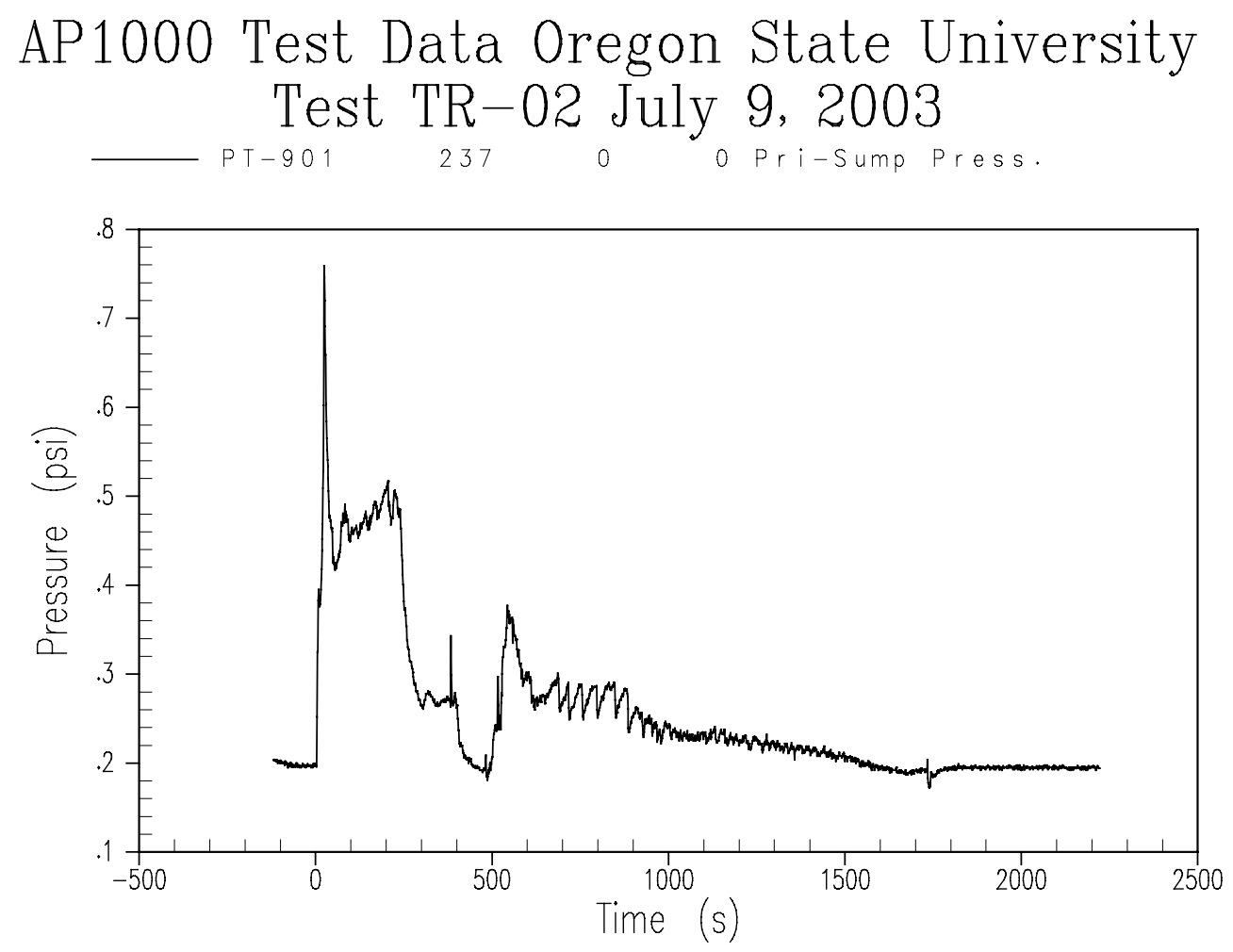

Figure A-52 Primary Sump Pressure 

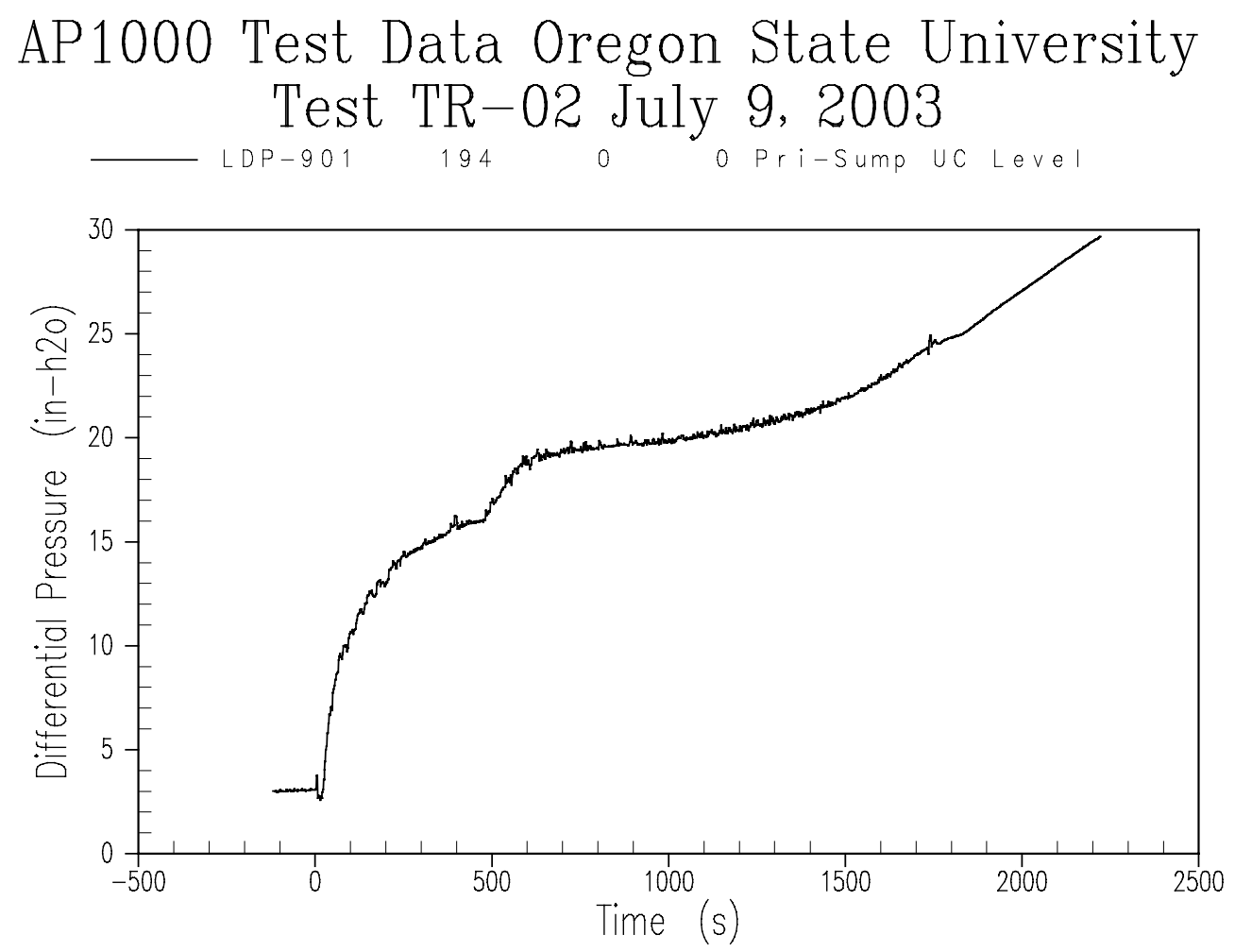

Figure A-53 Primary Sump Liquid Level 


\section{AP1000 Test Data Oregon State University Test TR-02 July 9, 2003 \\ FMM-901 $75 \quad 0 \quad 0$ Pri-Sump-1 Recirc. I}

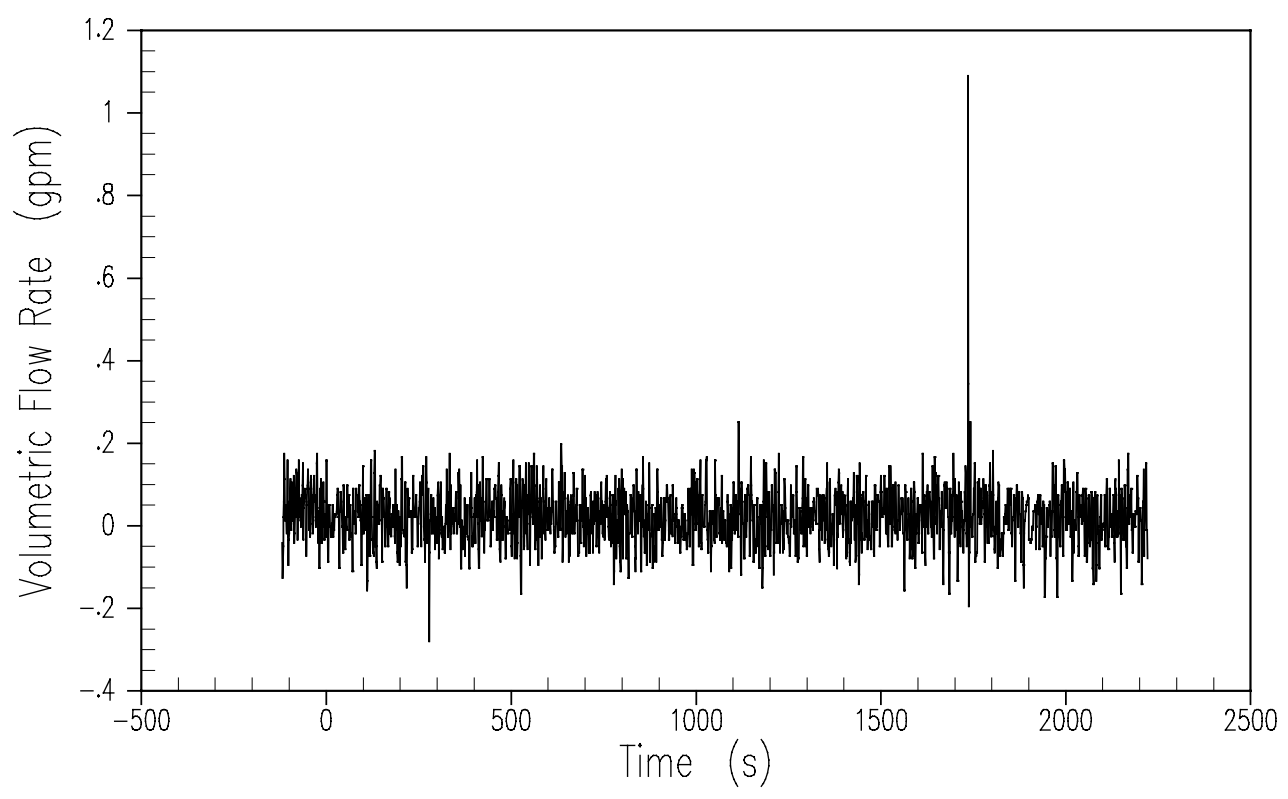

Figure A-54 Primary Sump 1 Injection Flow Rate 


\section{AP1000 Test Data Oregon State University Test TR-02 July 9, 2003 \\ FMM-902 \\ 76 \\ o Pri-Sump-2 Recirc. I}

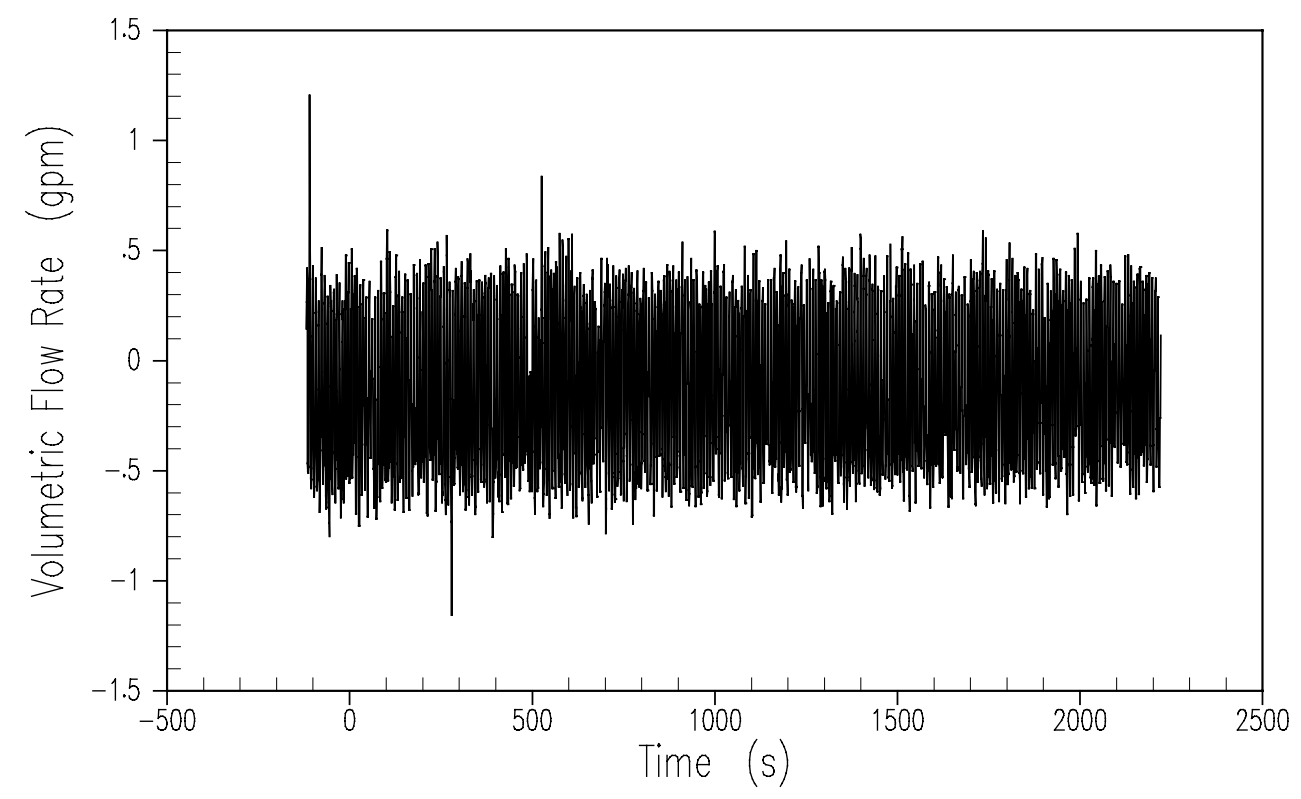

Figure A-55 Primary Sump 2 Injection Flow Rate 


\section{AP1000 Test Data Oregon State University Test TR-02 July 9, 2003}

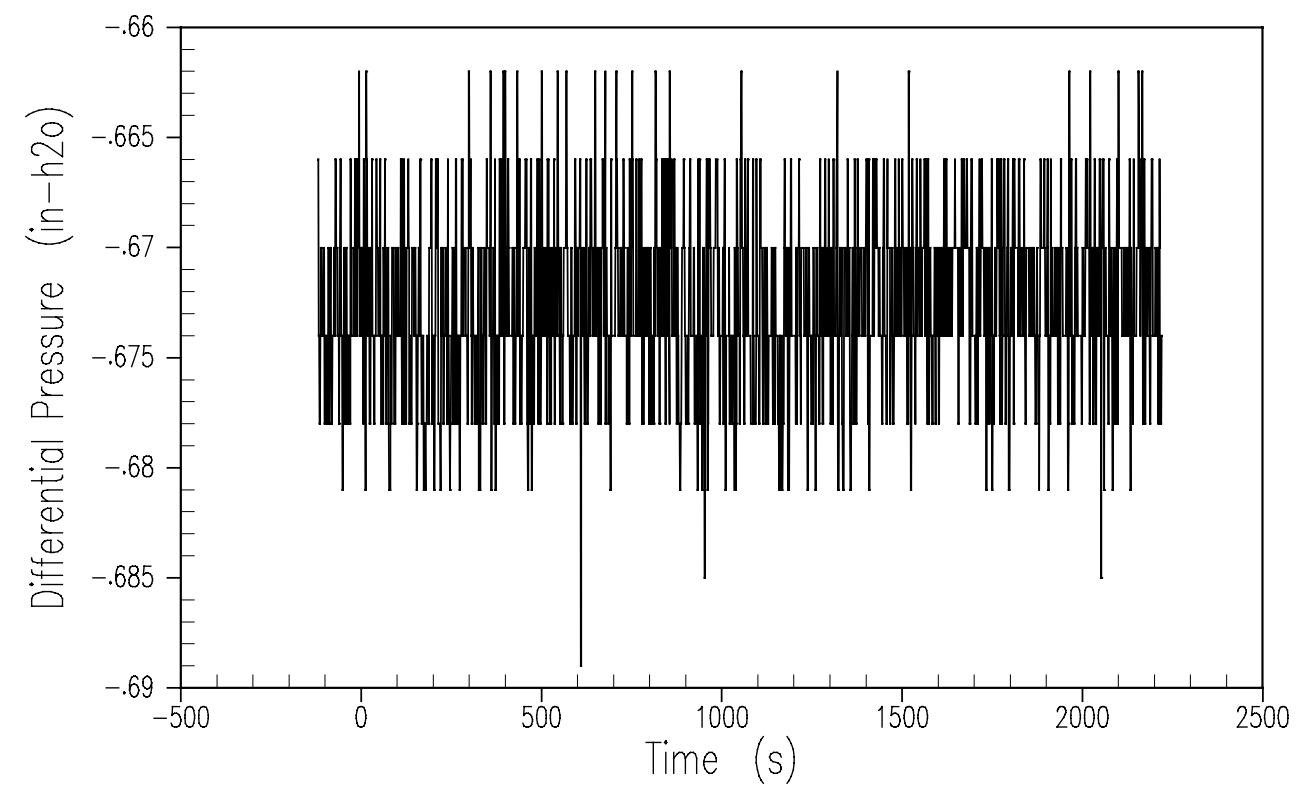

Figure A-56 Secondary Sump Liquid Level 


\section{AP1000 Test Data Oregon State University Test TR-02 July 9, 2003
$\mathrm{PT}-905$
239
o Break Sep. Press.

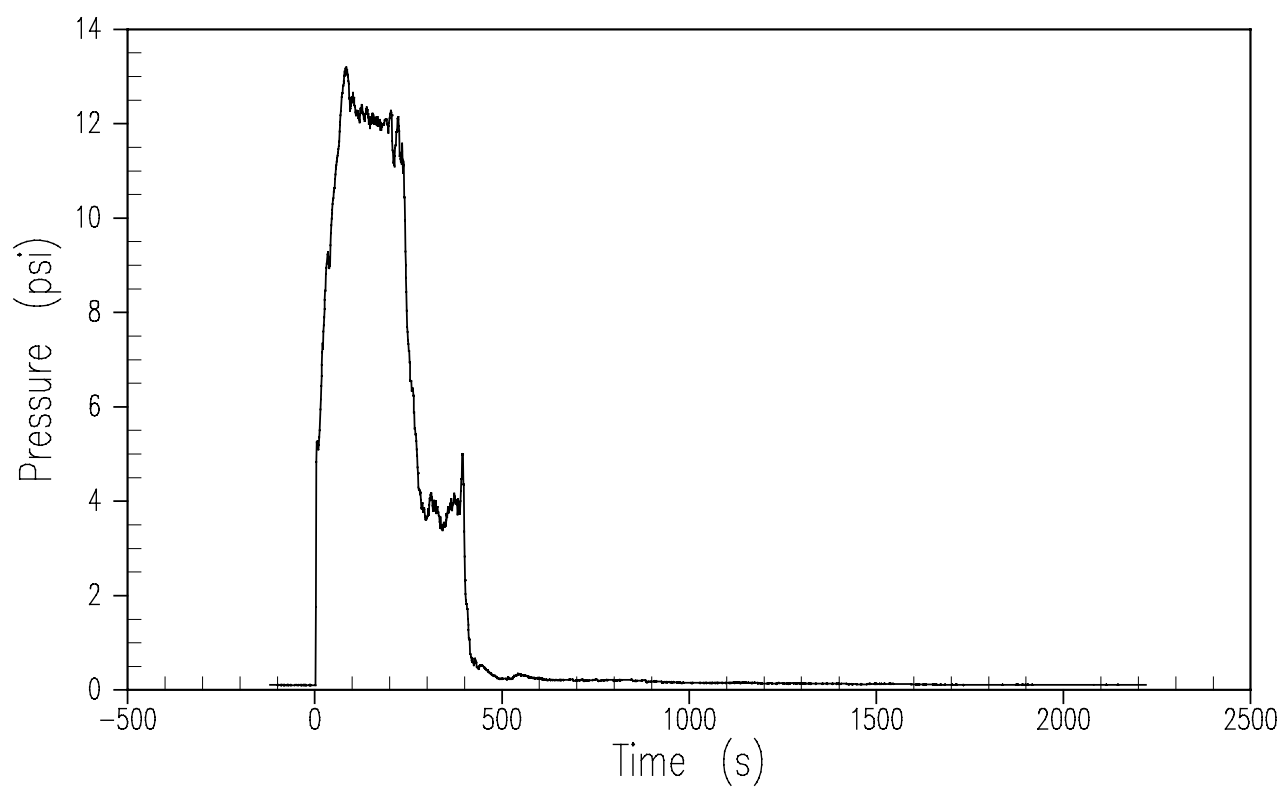

Figure A-57 Break Separator Pressure 

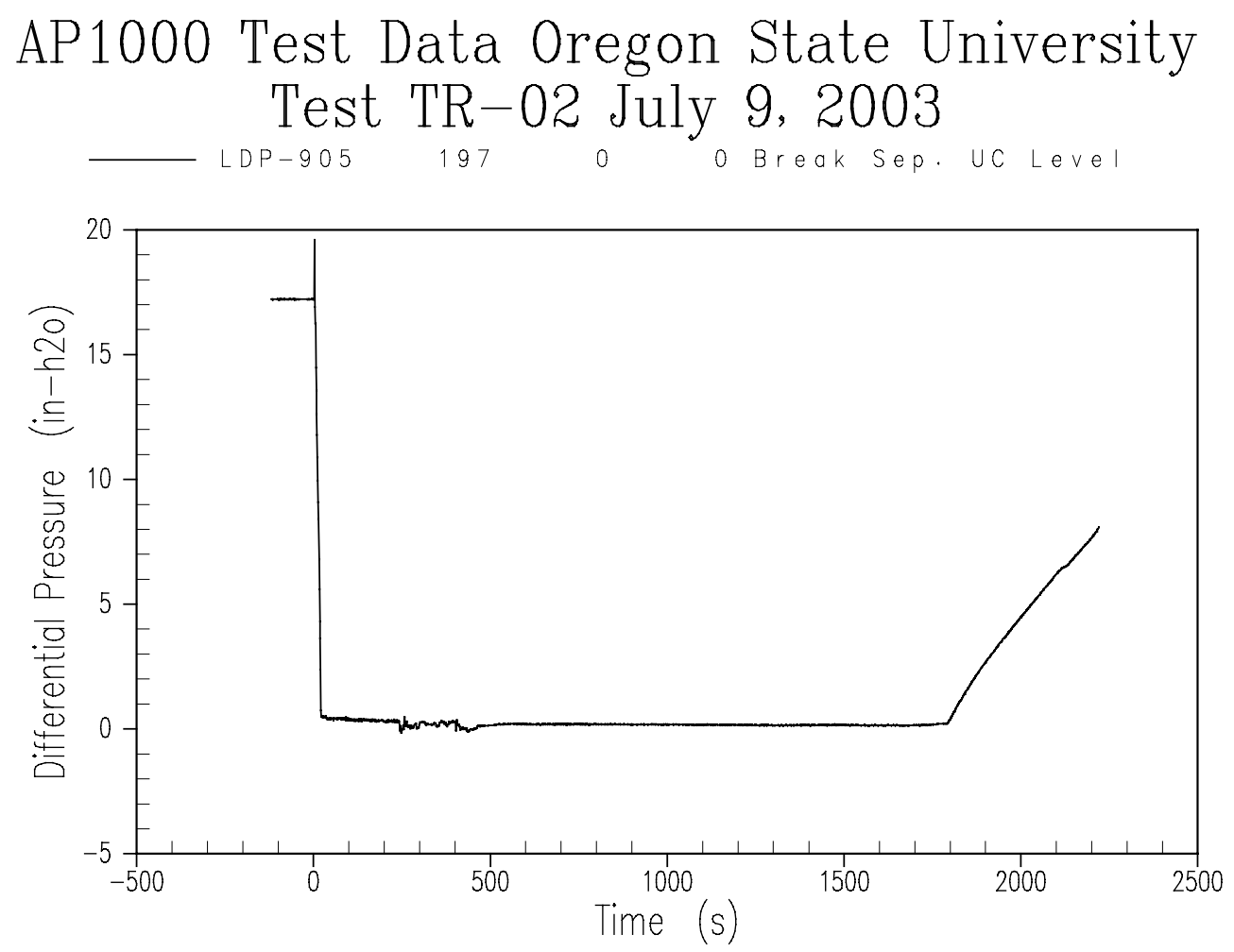

Figure A-58 Break Separator Liquid Level 

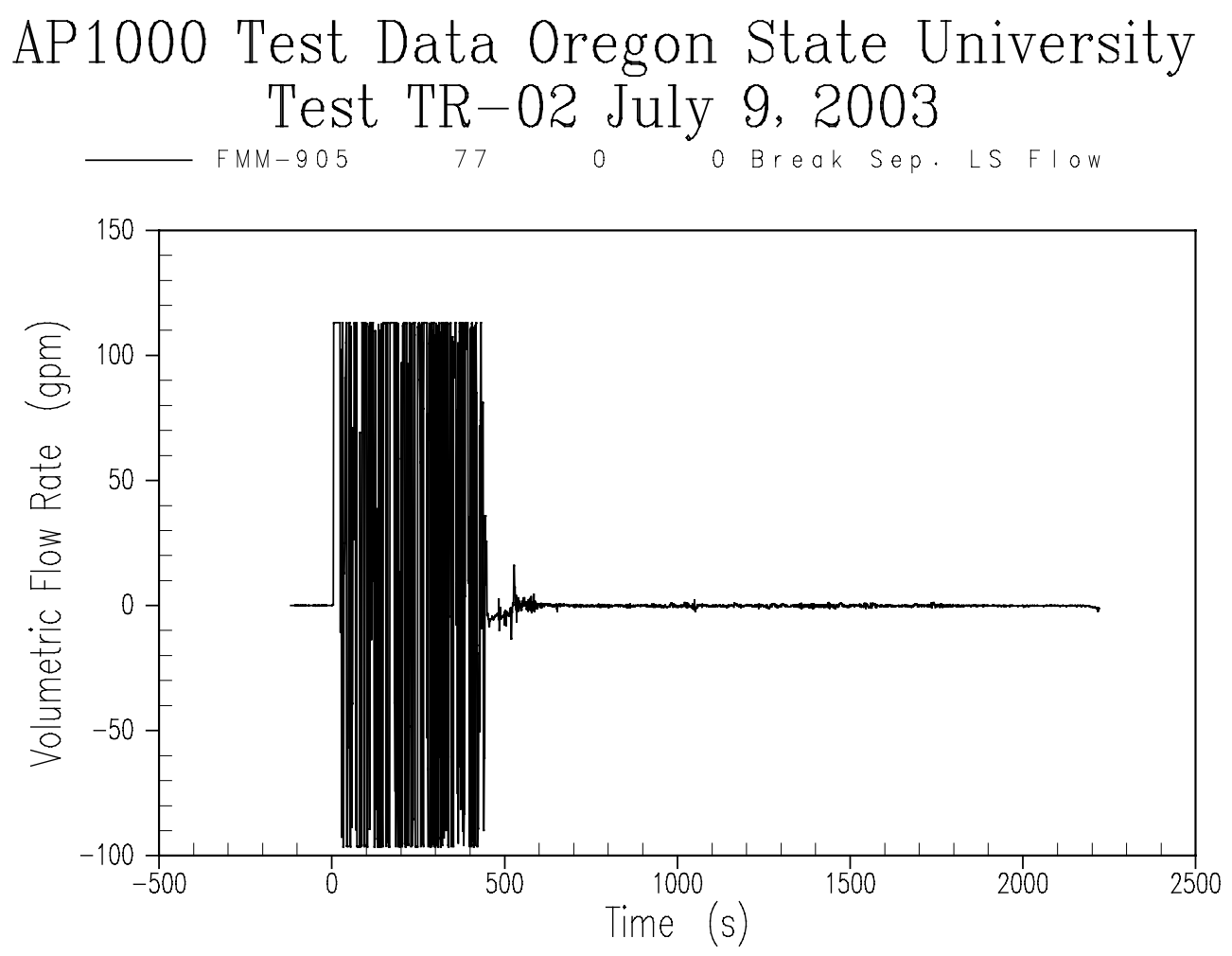

Figure A-59 Break Separator Flow to Primary Sump 


\section{AP1000 Test Data Oregon State University Test TR-02 July 9, 2003 \\ FVM-901 $88 \quad 0 \quad 0$ BAMS HDR 6" Line Stm}

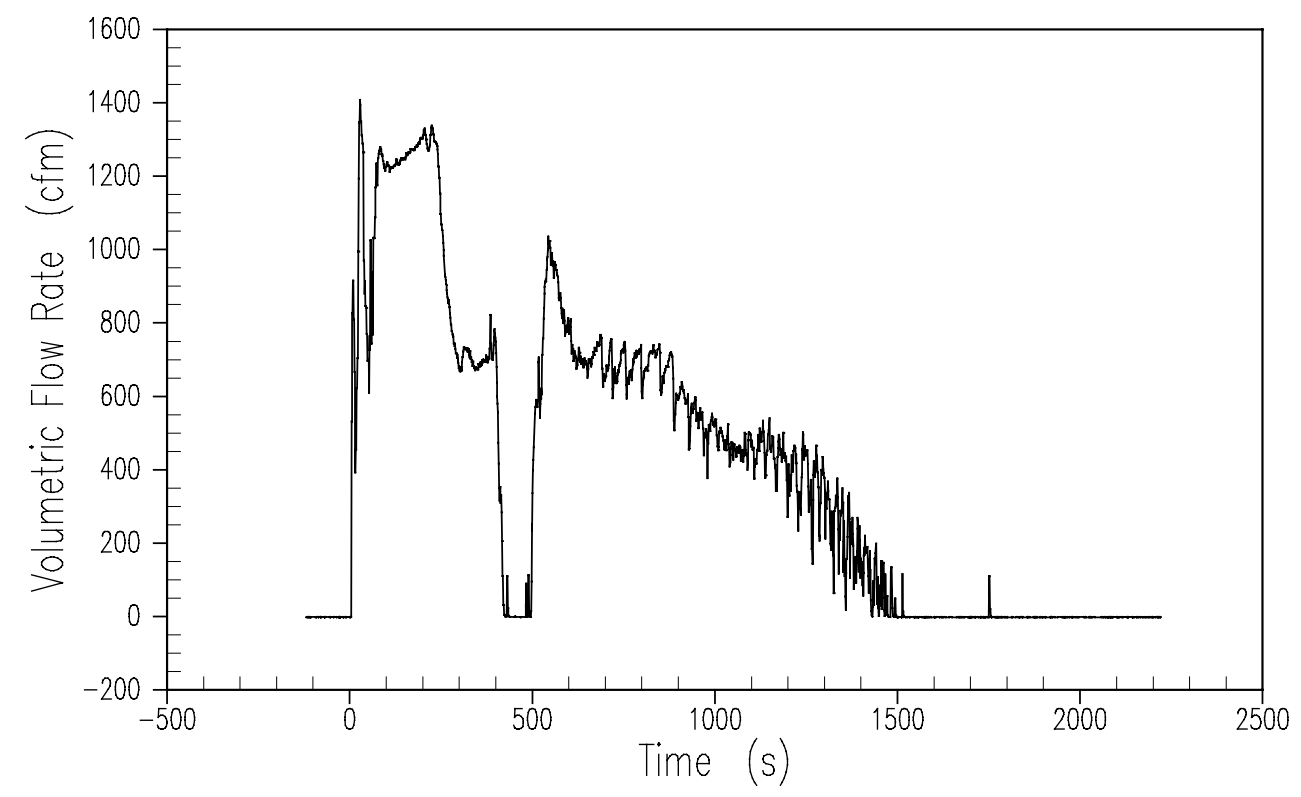

Figure A-60 BAMS Steam Flow Rate - 6-inch Line 


\section{AP1000 Test Data Oregon State University Test TR-02 July 9, 2003 \\ FVM-902 $89 \quad 0 \quad 0$ BAMS HDR $10^{\prime \prime}$ Line St}

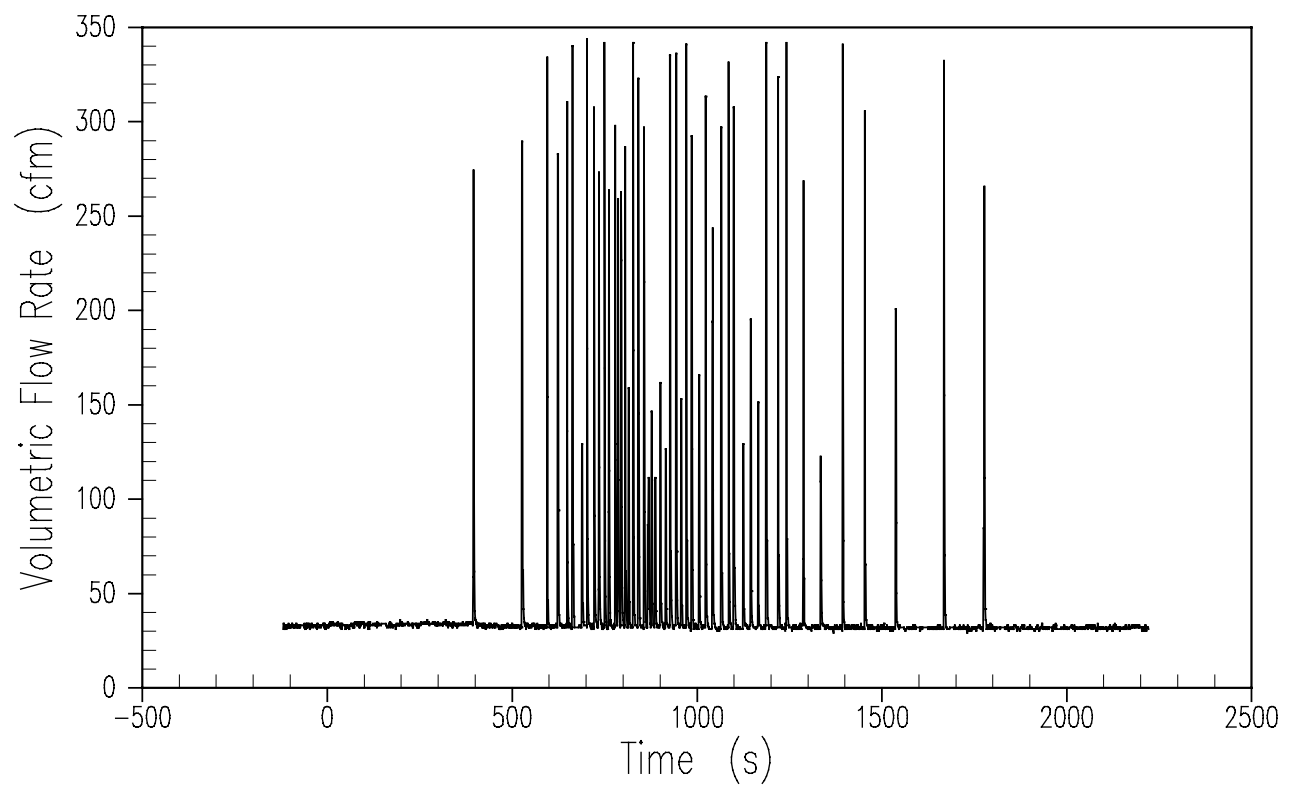

Figure A-61 BAMS Steam Flow Rate - 10-inch Line 

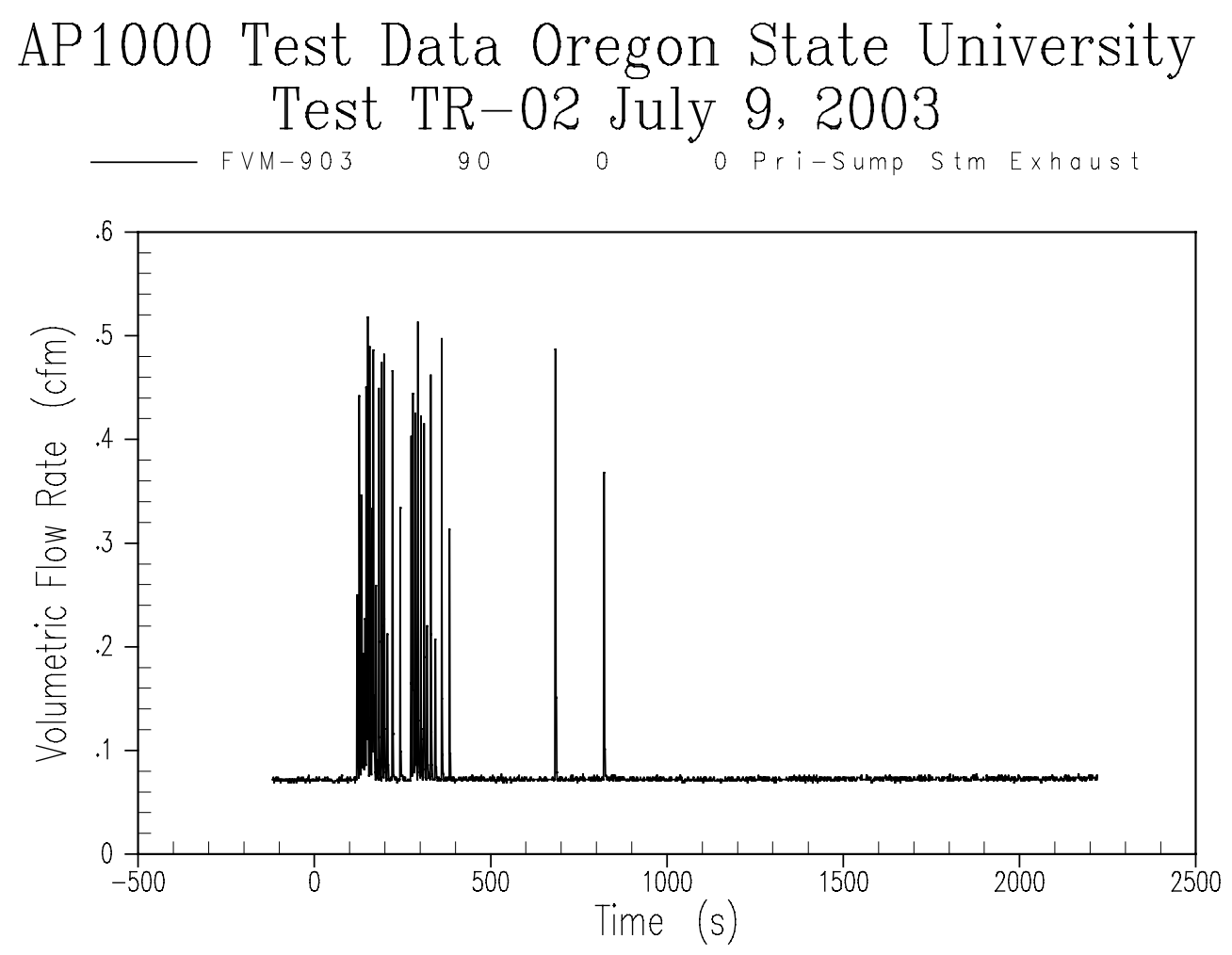

Figure A-62 BAMS/Primary Sump Steam Flow Rate 

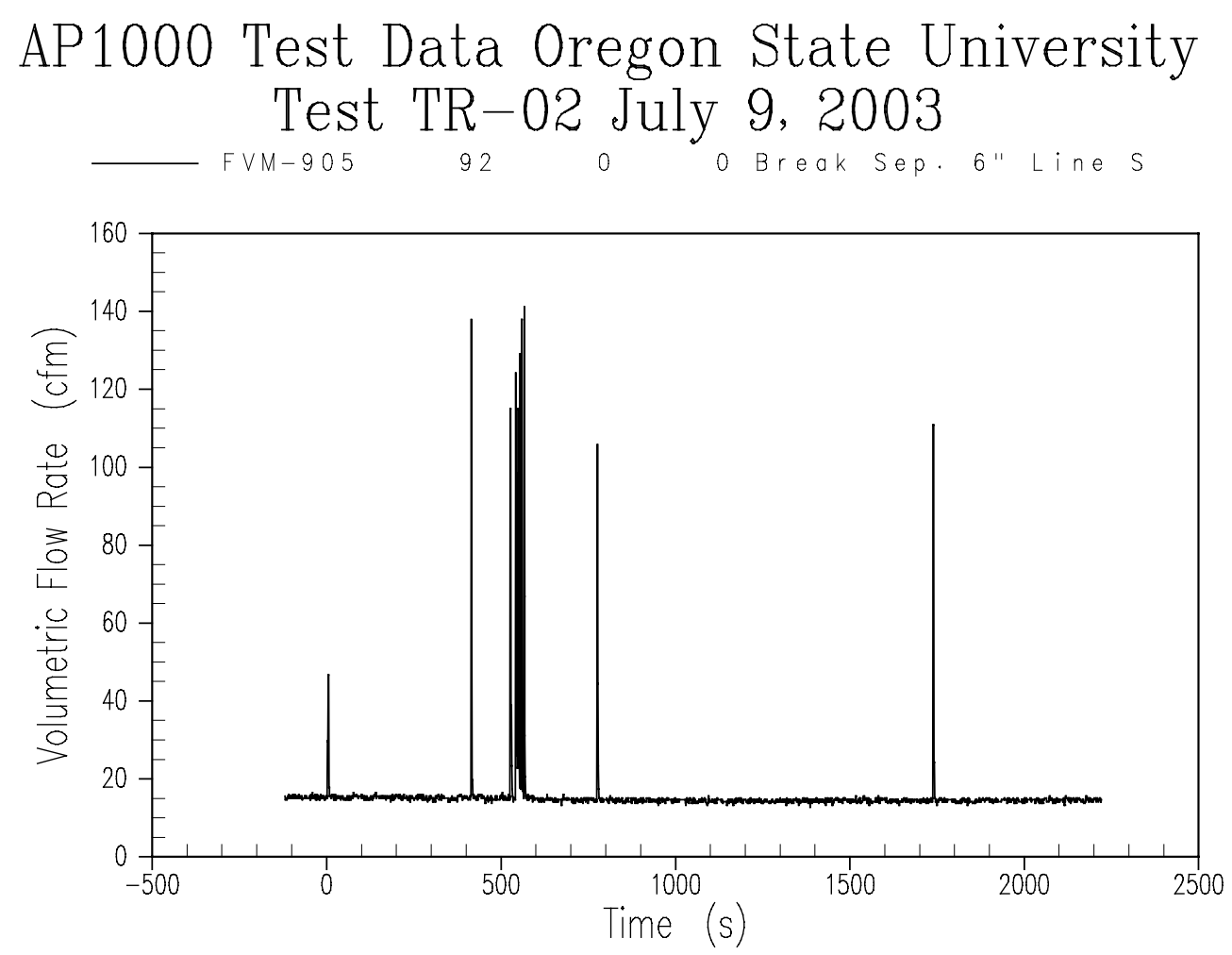

Figure A-63 BAMS/Separator Steam Flow Rate - 6-inch Pipe 

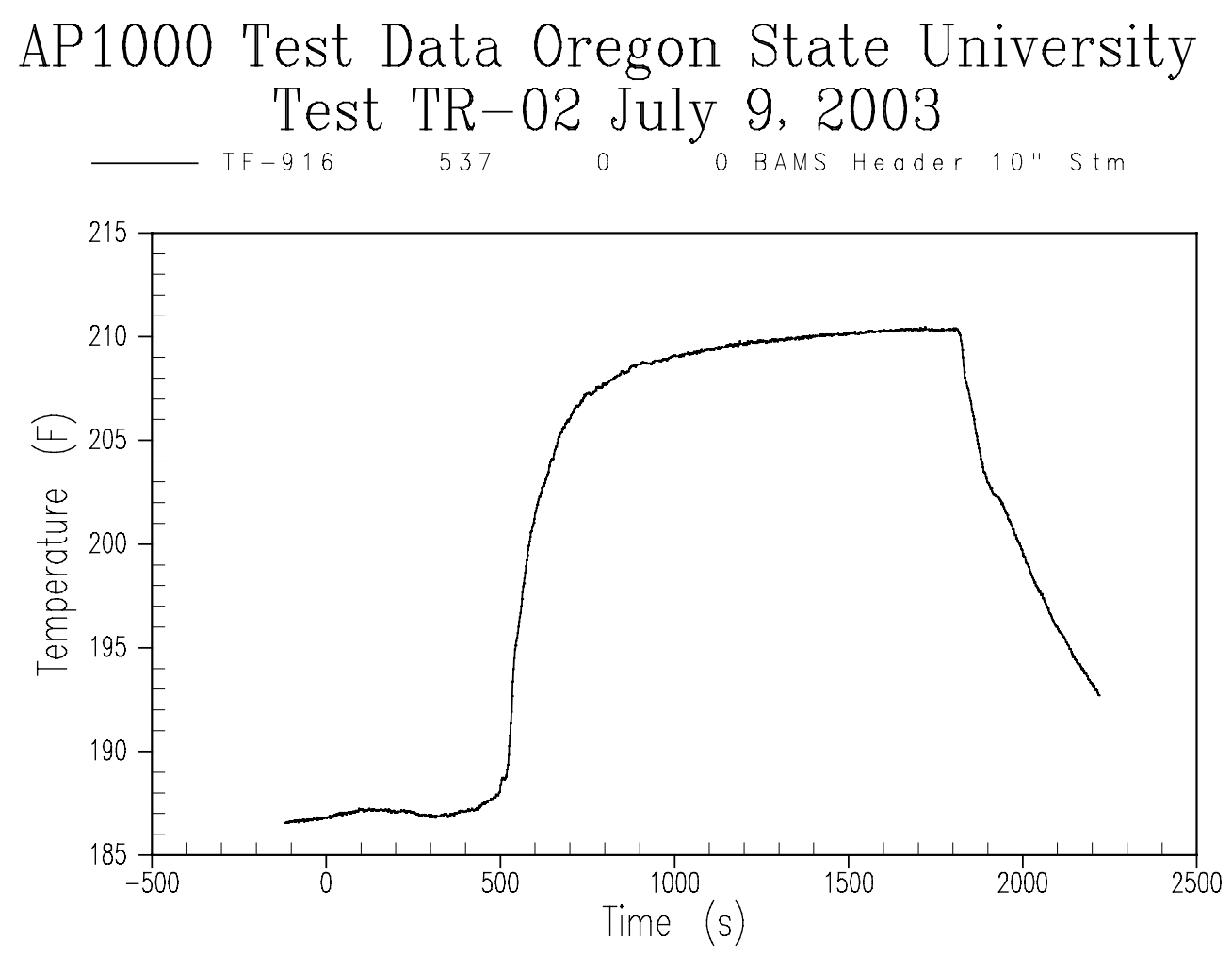

Figure A-64 BAMS/Exhaust Line Temperature - 10-inch Line 


\section{AP1000 Test Data Oregon State University Test TR-02 July 9, 2003 \\ $\begin{array}{lllll}T F-917 & 538 & 0 & 0 & T F-917 / B A M S \\ H e a d e r\end{array}$}

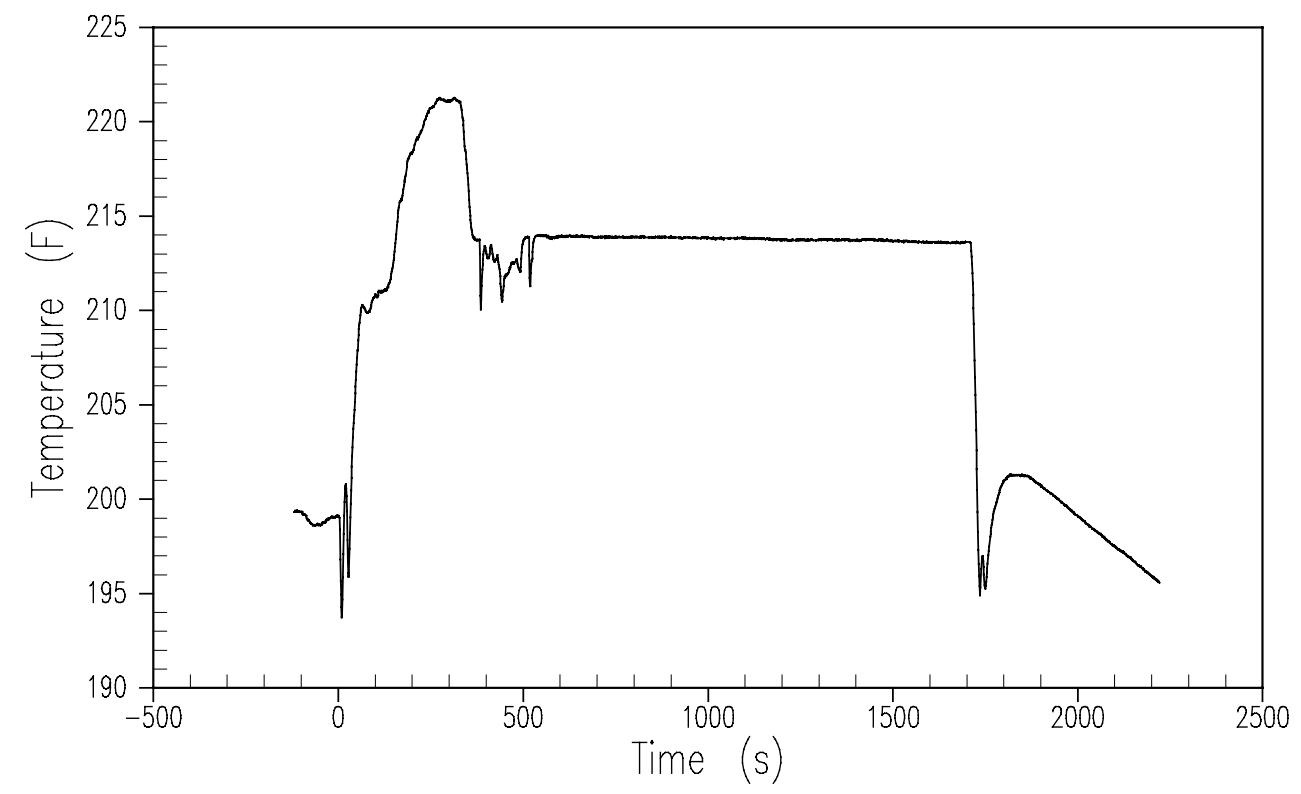

Figure A-65 BAMS/Exhaust Line Temperature - Header 


\section{AP1000 Test Data Oregon State University Test TR-02 July 9, 2003 \\ $\begin{array}{llllll}K W-601 & 114 & 0 & \text { P Pr Htr Power }\end{array}$}

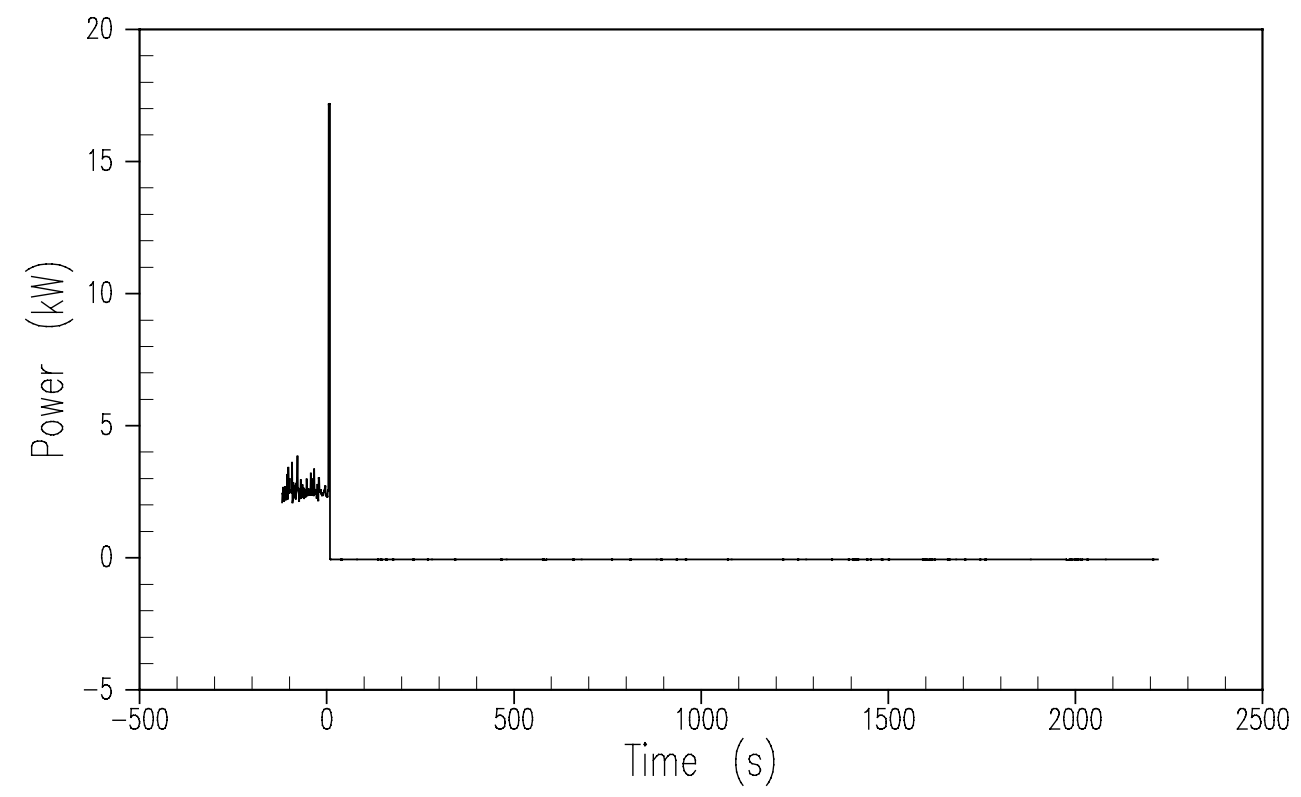

Figure A-66 Pressurizer Heater Input Power 

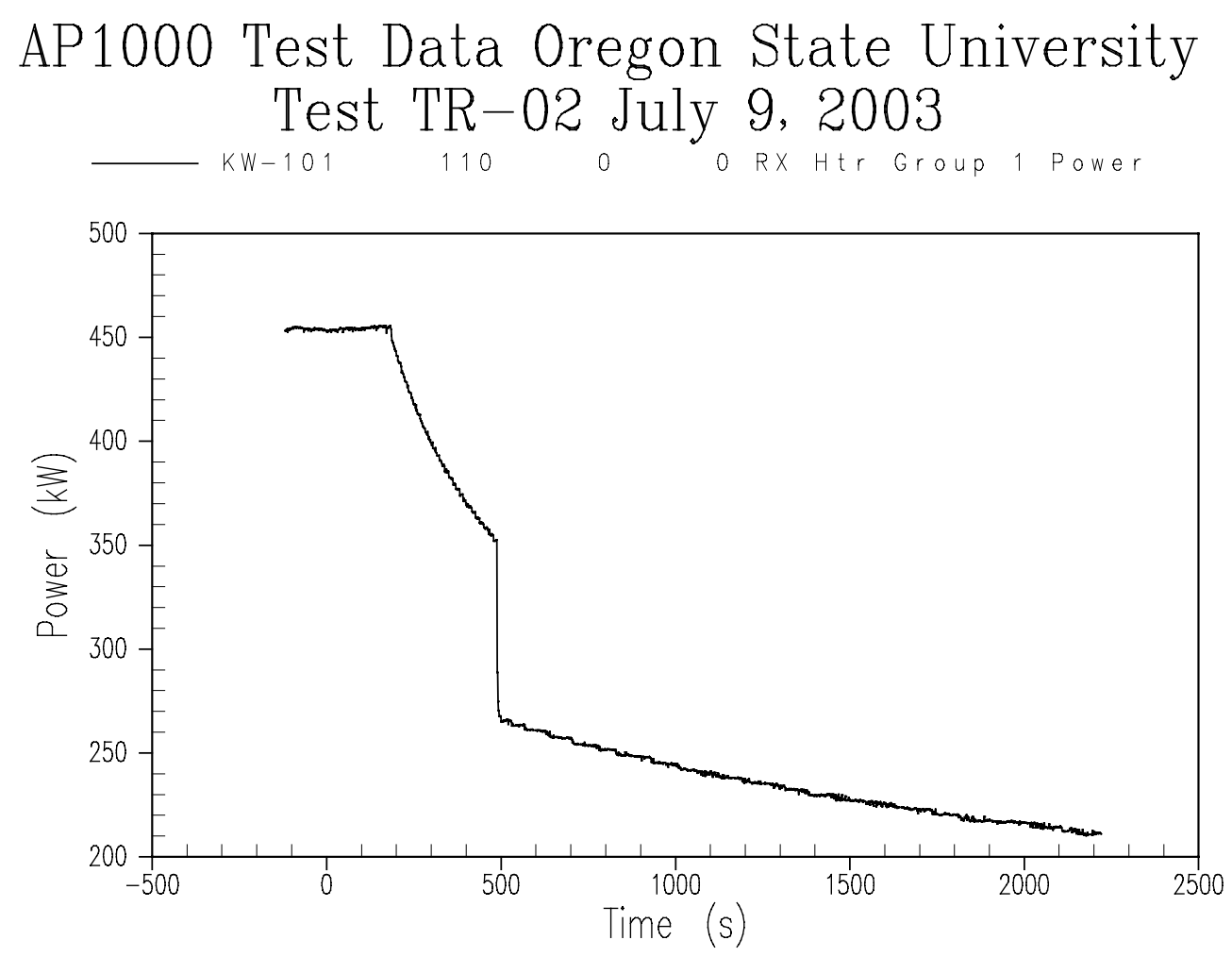

Figure A-67 Core Power Group 1 Input Power 

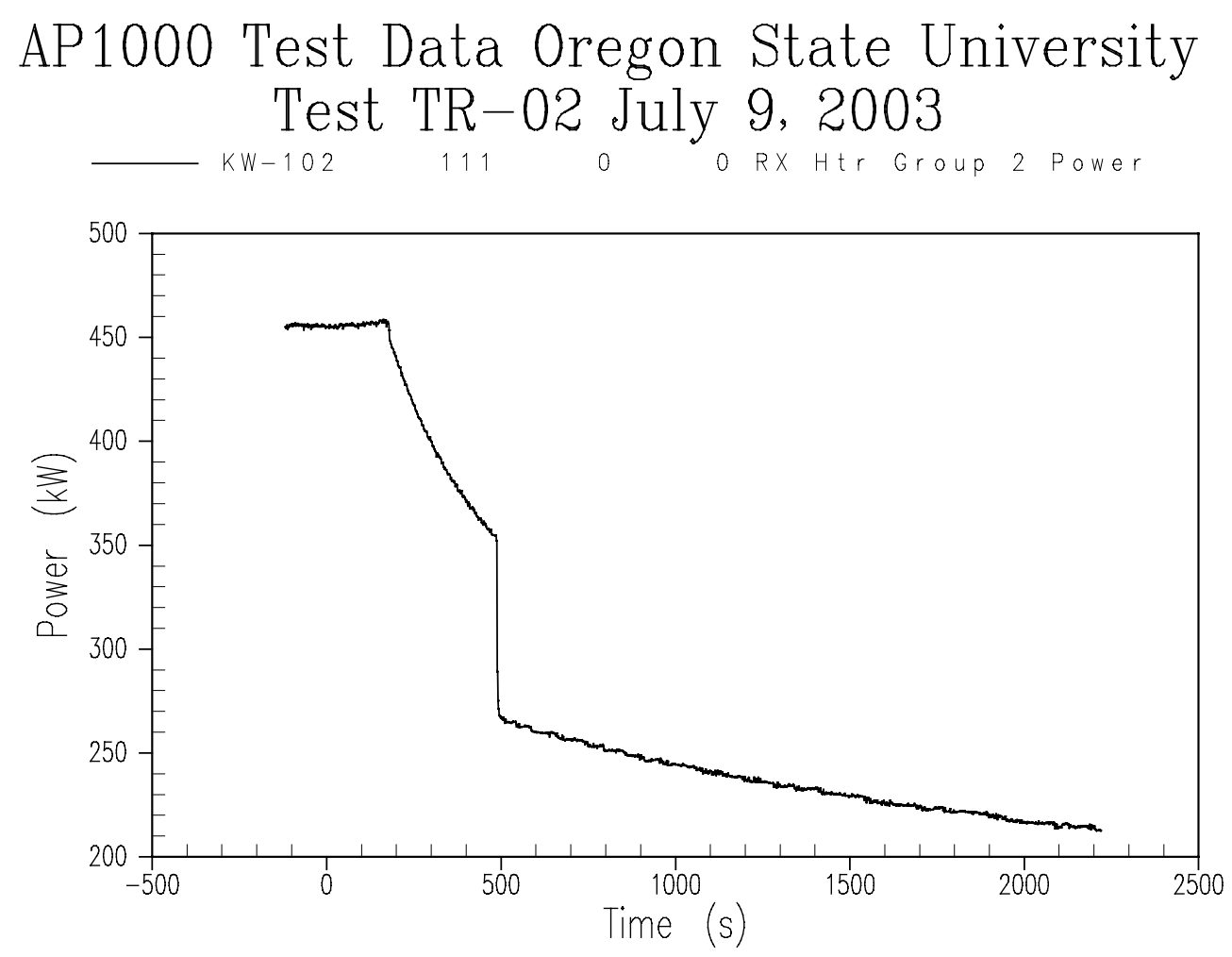

Figure A-68 Core Power Group 2 Input Power 

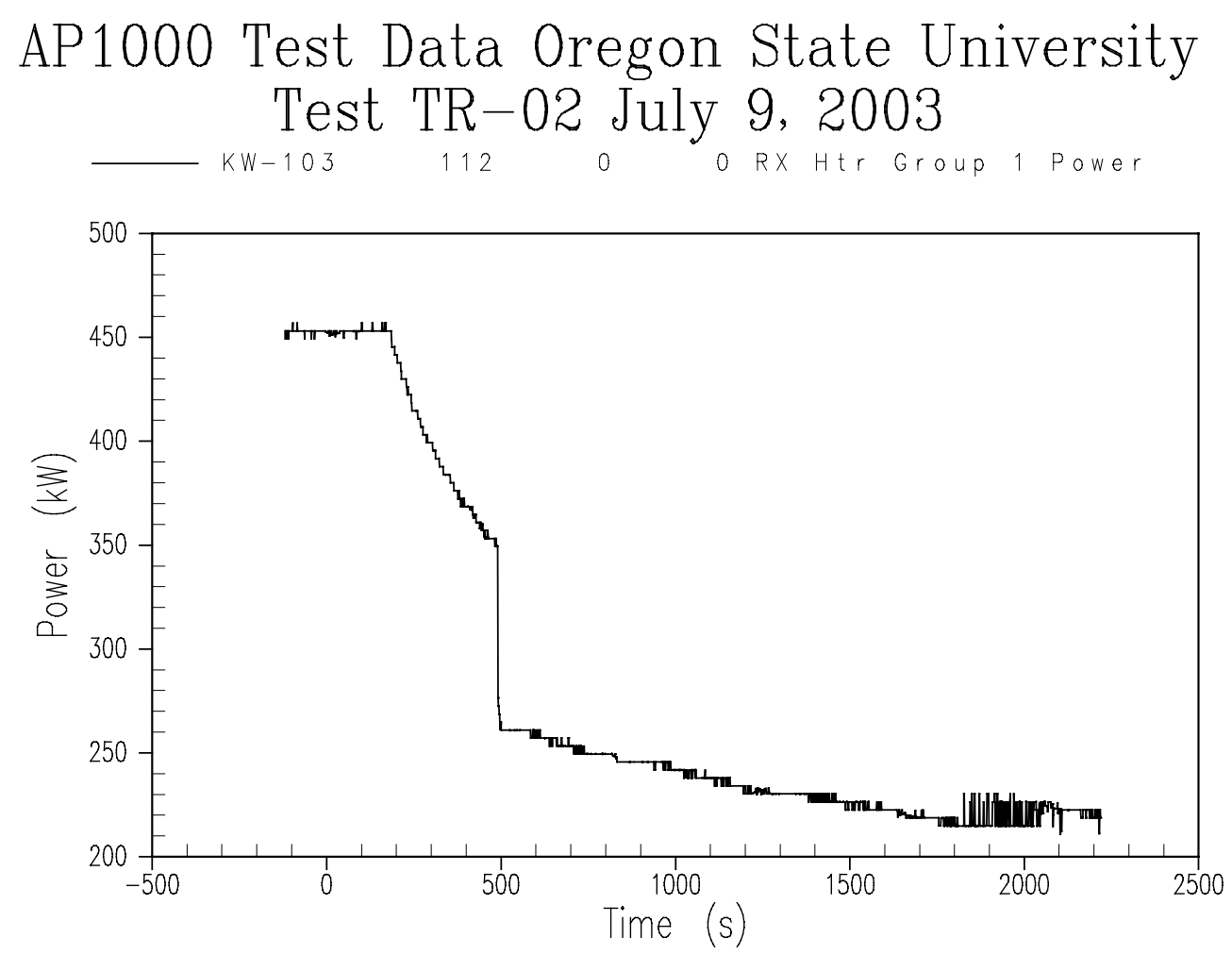

Figure A-69 Core Power Group 1 Alternate Input Power 

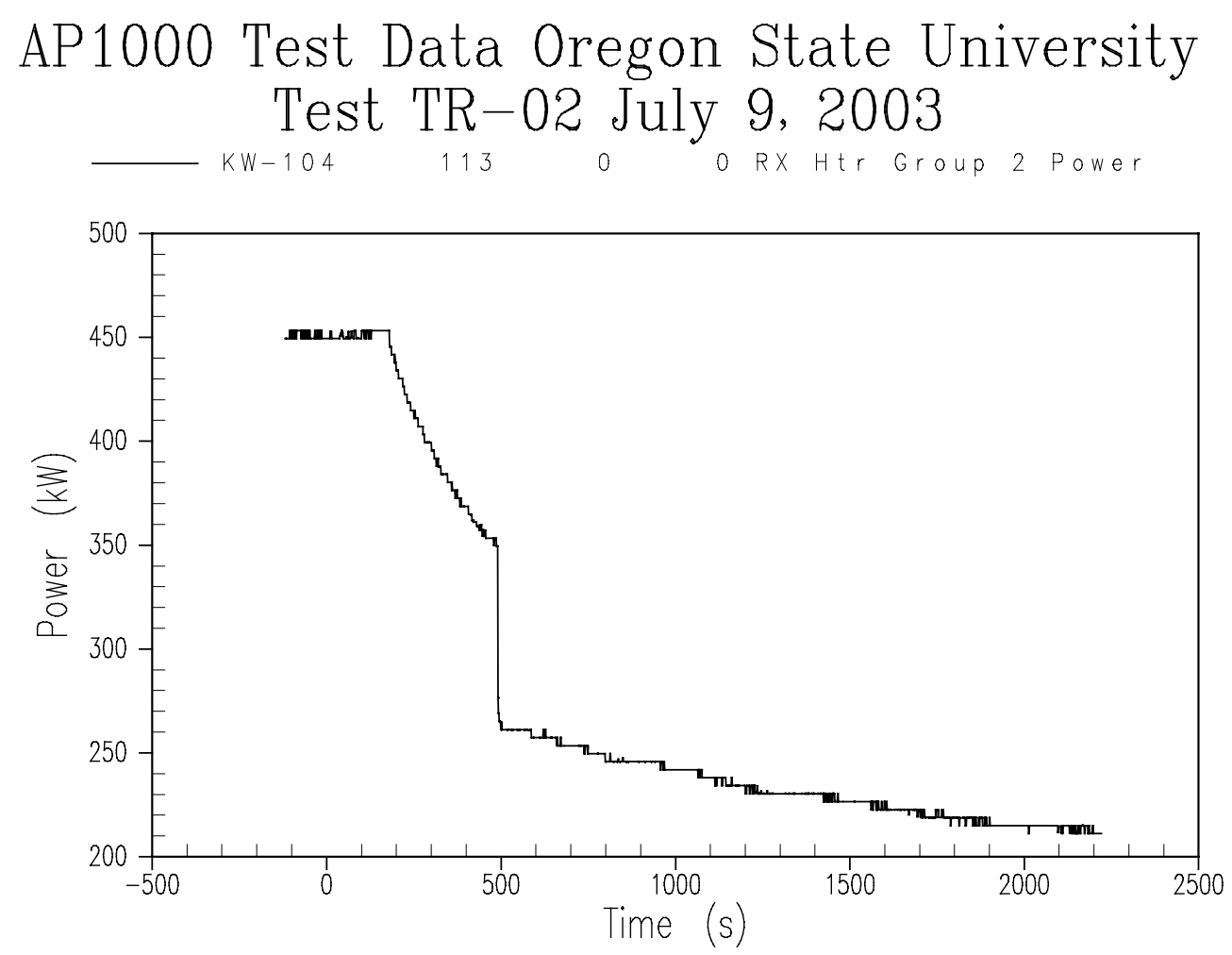

Figure A-70 Core Power Group 2 Alternate Input Power 

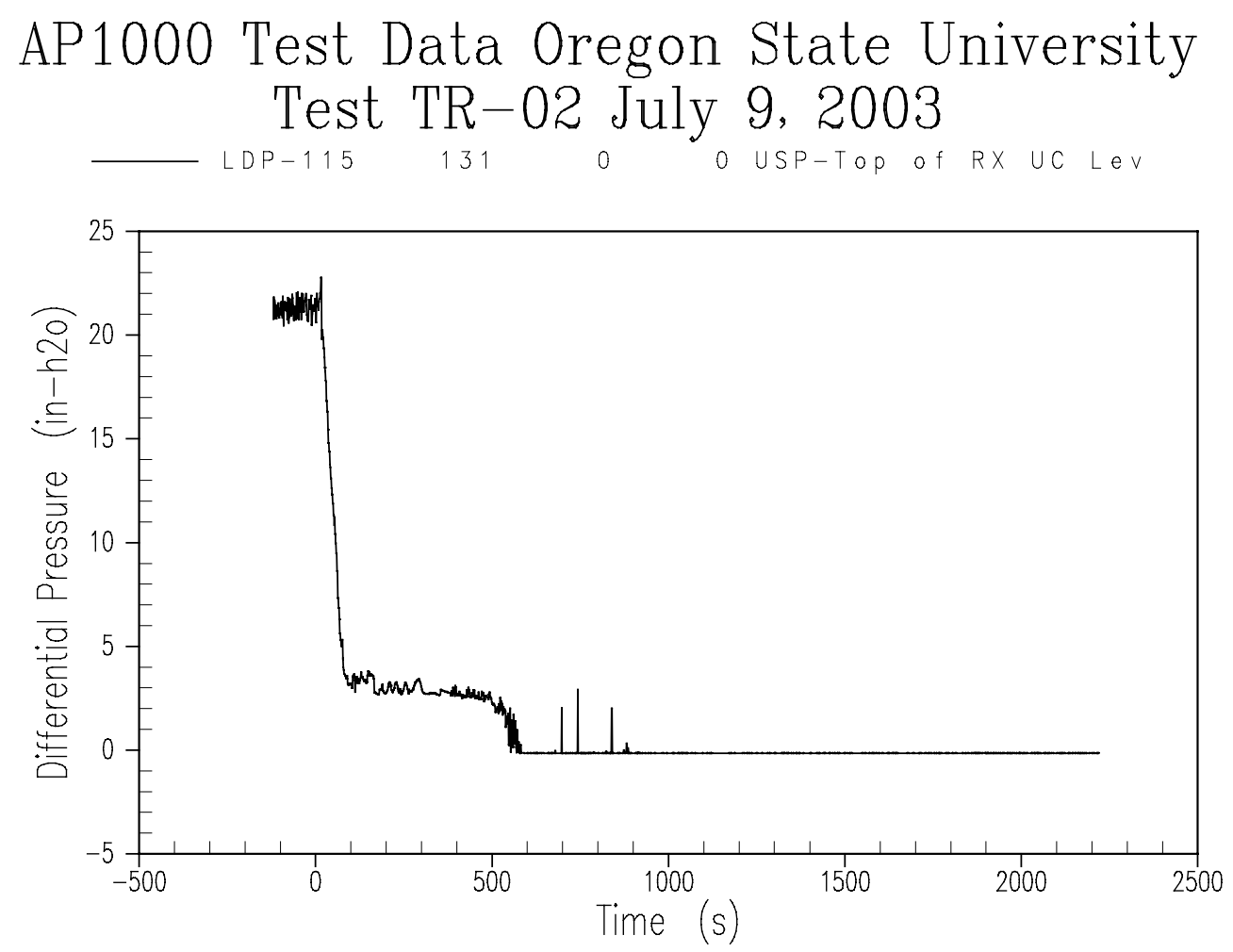

Figure A-71 Reactor Vessel Liquid Level Between Top of Vessel - Upper Support Plate 

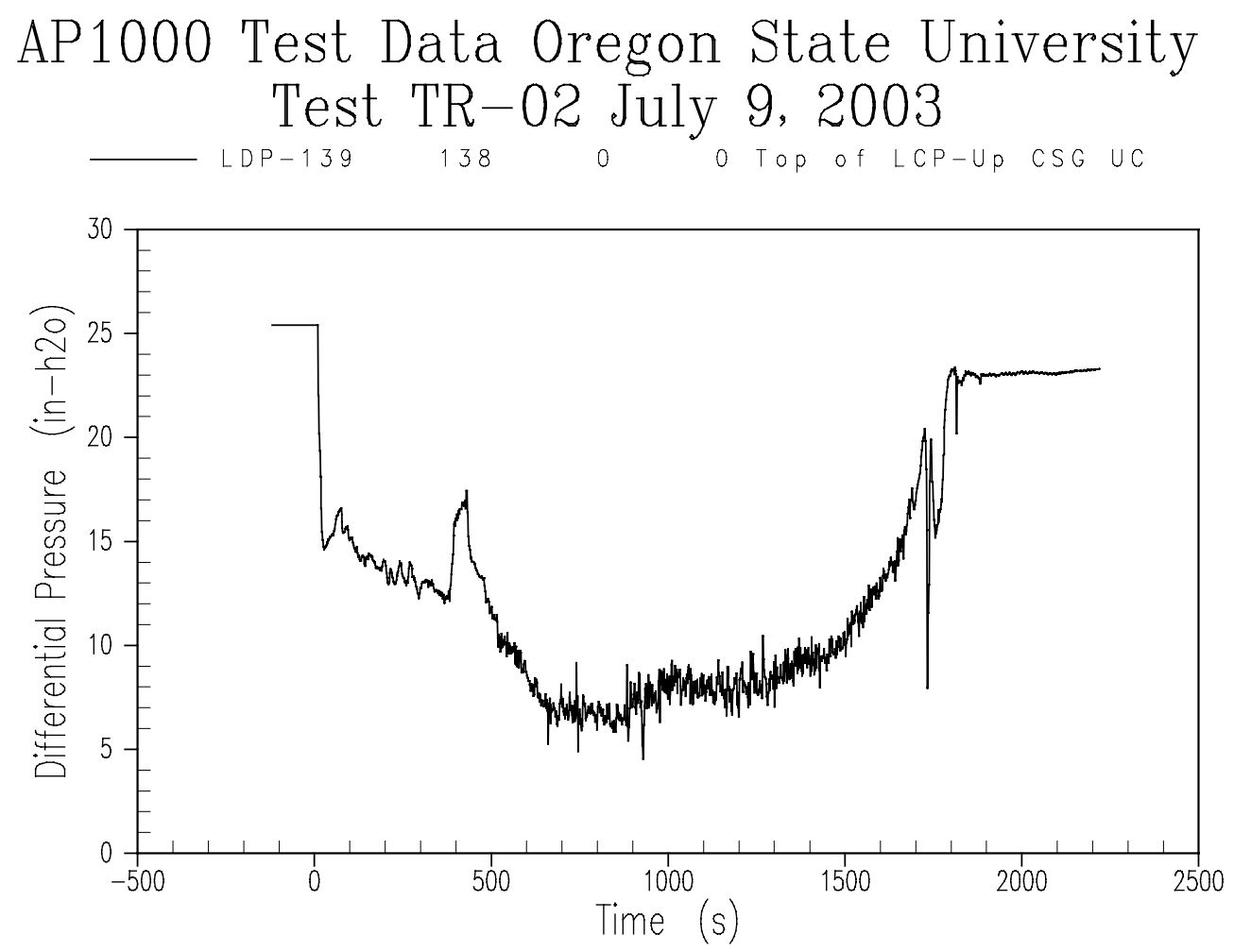

Figure A-72 Reactor Vessel Liquid Level Between Bottom of Upper Support Plate Upper Core Spacer Grid 

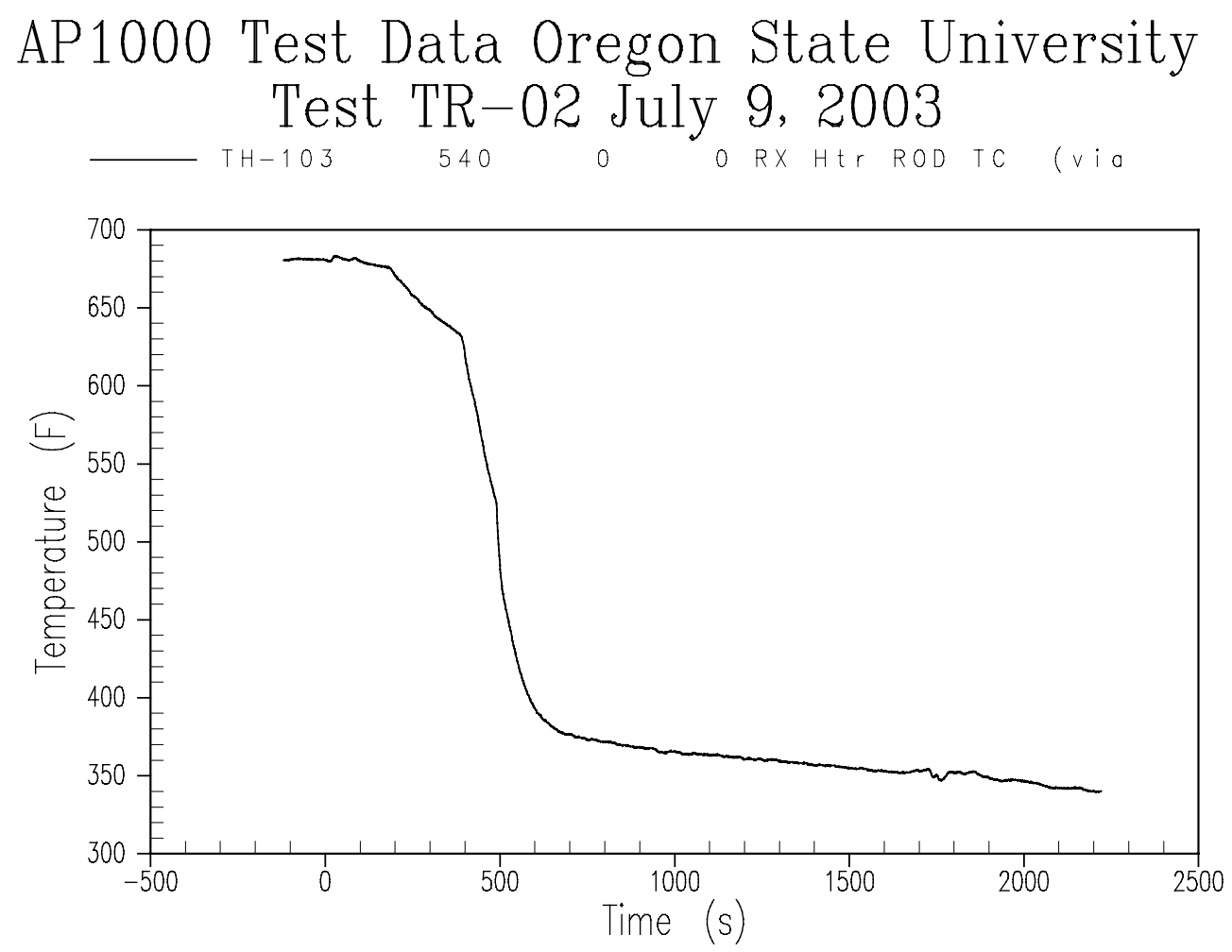

Figure A-73 Inner Core Thermocouple Measuring Heater Temperature 

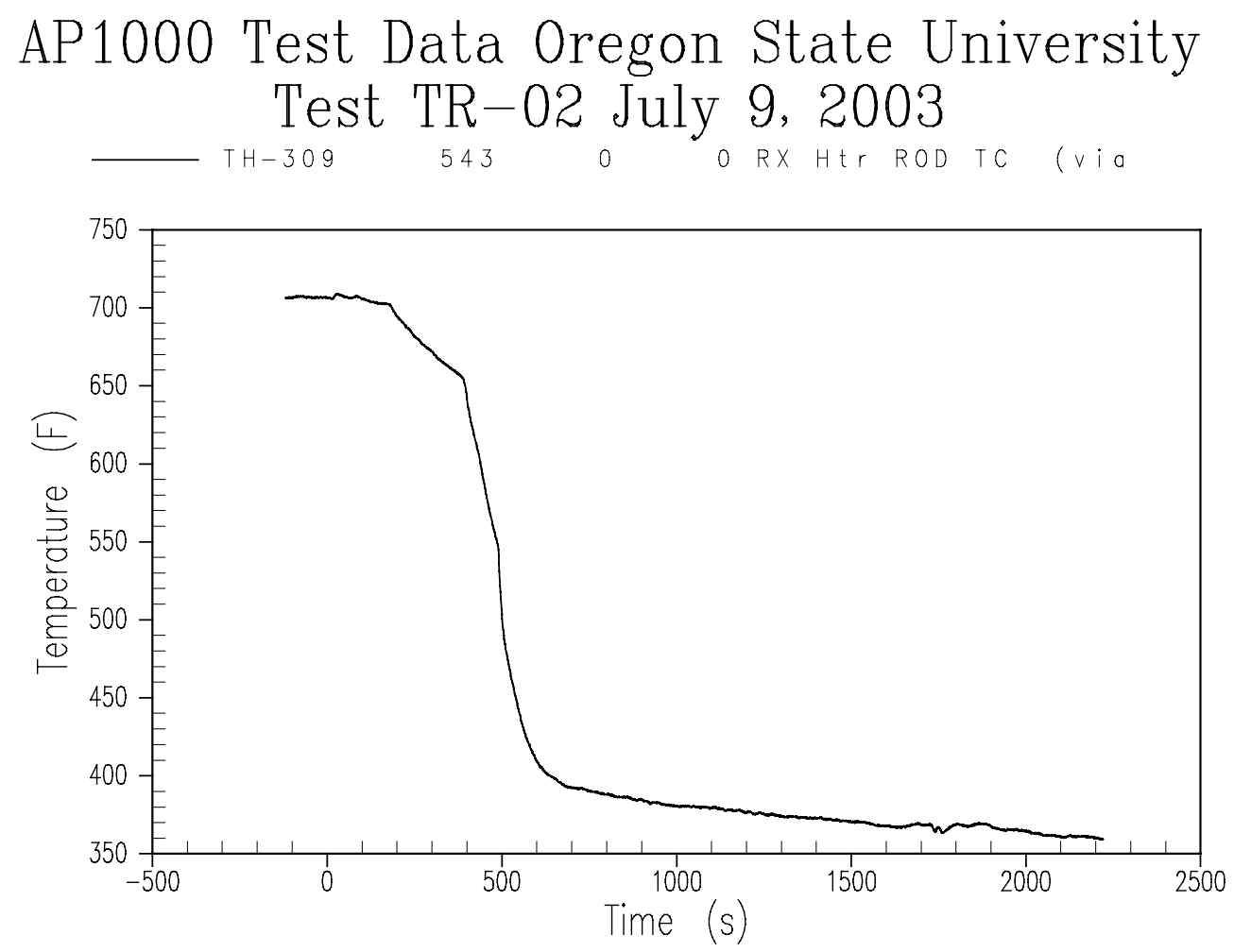

Figure A-74 Outer Core Thermocouple Measuring Heater Temperature 


\section{APPENDIX B}

TEST DATA 
Appendix G -- OSU-AP1000-07 


\title{
Oregon State University
}

Department of Nuclear Engineering

\author{
AdVANCED THERMAL HYdRAuliC \\ RESEARCH LABORATORY
}

TEST SUMMARY REPORT

OSU-AP1000-07

ADS-01-D

AP1000 ADS-4 Characterization - Low POWER

Revision 0 


\title{
TEST SUMMARY REPORT
}

\author{
OSU-AP1000-07
}

ADS-01-D

\section{AP1000 ADS-4 ChARACTERIZATION - LOW POWER}
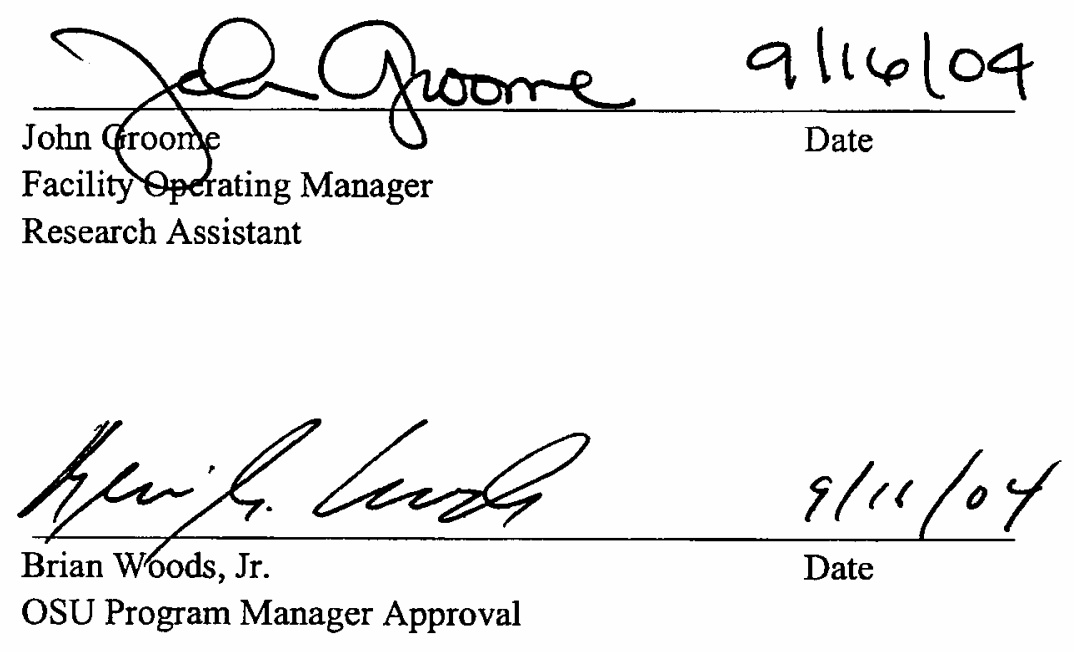

Oregon State University 116 Radiation Center

Corvallis, Oregon 97331

(C) 2004 Oregon State University

All Rights Reserved 


\section{WESTINGHOUSE PROPRIETARY INFORMATION NOTICE}

This report contains information proprietary to Westinghouse Electric Company. A non-proprietary version of this report has also been prepared for distribution to the United States Nuclear Regulatory Commission (NRC).

In order to conform to the requirements of 10 CFR 2.790 of the Commission's regulations concerning the protection of proprietary information so submitted to the NRC, the information which is proprietary in the proprietary version of this document is contained within brackets, and where the proprietary information has been deleted in the non-proprietary versions, only the brackets remain (the information that was contained within the brackets in the proprietary versions having been deleted). The justification for claiming the information so designated as proprietary is indicated in both versions by means of lower case letters (a) through (f) located as a superscript immediately following the brackets enclosing each item of information being identified as proprietary or in the margin opposite such information. These lower case letters refer to the types of information Westinghouse customarily holds in confidence identified in Sections (4)(ii)(a) through (4)(ii)(f) of the affidavit accompanying the transmittal of these documents pursuant to 10 CFR $2.790(b)(1)$.

\section{COPYRIGHT NOTICE}

This document bears an Oregon State University copyright notice. The NRC is permitted to make the number of copies of the information contained in these reports which are necessary for its internal use in connection with generic and plant-specific reviews and approvals as well as the issuance, denial, amendment, transfer, renewal, modification, suspension, revocation, or violation of a license, permit, order, or regulation subject to the requirements of 10 CFR 2.790 regarding restrictions on public disclosure to the extent such information has been identified as proprietary by Westinghouse, copyright protection notwithstanding. With respect to the non-proprietary versions of these reports, the NRC is permitted to make the number of copies beyond these necessary for its internal use which are necessary in order to have one copy available for public viewing in the appropriate docket files in the public document room in Washington, DC and in local public document rooms as may be required by NRC regulations if the number of copies submitted is insufficient for this purpose. Copies made by the NRC must include the copyright notice in all instances and the proprietary notice if the original was identified as proprietary. 
TABLE OF CONTENTS

$\underline{\text { Section }}$

Title

$\underline{\text { Page }}$

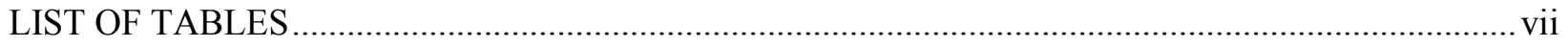

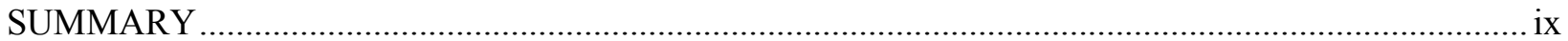

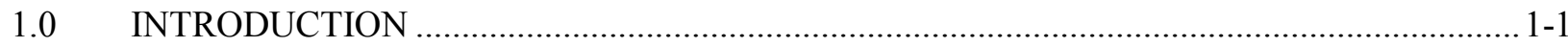

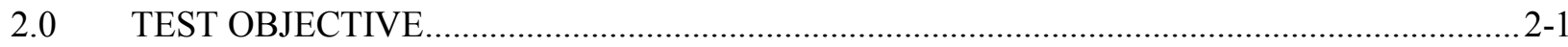

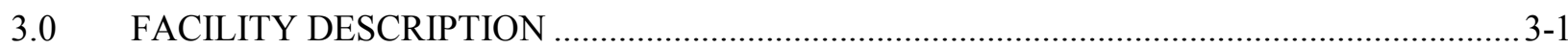

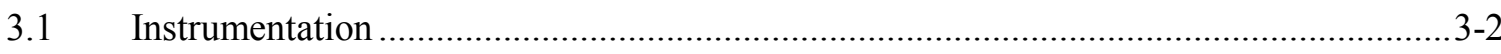

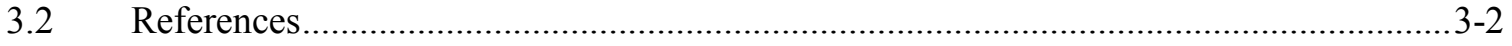

4.0 OREGON STATE UNIVERSITY TESTING PROGRAM MATRIX .......................................4-1

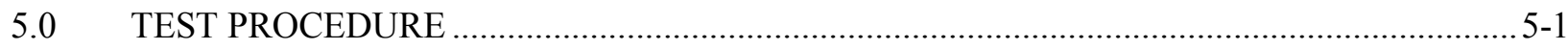

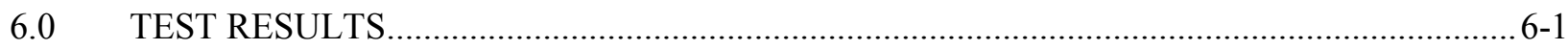

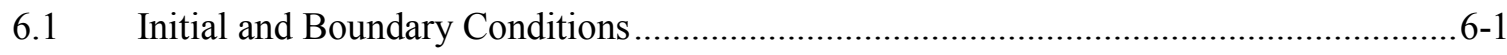

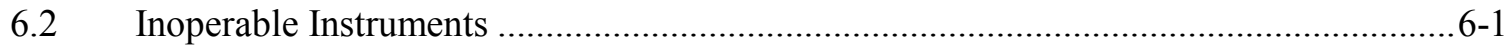

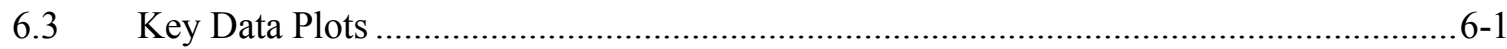

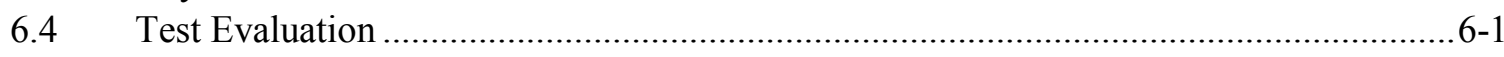

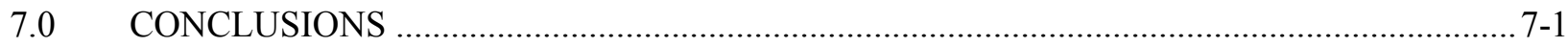

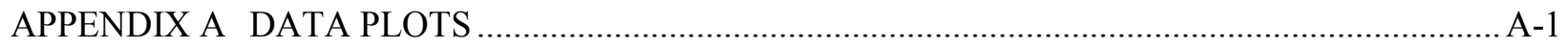

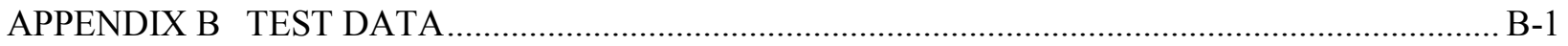




\section{LIST OF TABLES}

$\underline{\text { Table }}$

Table 4-1

Table 6-1

Table 6-2

Table 6-3 $\underline{\text { Title }}$

Page

OSU Test Matrix (as specified December 10, 2003) 4-2

Actual Test Initial Conditions.

6-2

Inoperable Instruments for ADS-01-D Test...............................................................6-3

Data Plots for QL Reports for ADS-01-D by Component. $6-4$ 


\section{SUMMARY}

This report covers the test ADS-01-D, Automatic Depressurization System 4 (ADS-4) characterization at low-power conditions, performed on December 10, 2003. This test has been designated OSU-AP1000-07 by the Advanced Plant Experiment (APEX). The objective of this test was to determine the steady-state two-phase flow and pressure drop characteristics of the ADS-4 lines at low-power/low-flow conditions typical of long-term cooling. The ADS-4 line instrumentation was upgraded to include three differential pressure cells on each line. The test was conducted with the Reactor Coolant System drained down to the midpoint of the hot leg and the pressurizer empty. Gravity injection from the in-containment refueling water storage tank was used to provide injection flow, and the core power was set to a constant value. The facility was run in this configuration for 15 to 30 minutes as liquid and vapor flows from the ADS-4 lines were measured along with the pressure drop distribution along the lines. This process was repeated by changing the core power, which resulted in different void fractions in the upper plenum and changed the gravity injection flow. Six power levels were tested ranging from $360 \mathrm{~kW}$ to $20 \mathrm{~kW}$. In addition, three power levels ( $20 \mathrm{~kW}$ to $80 \mathrm{~kW}$ ) were tested with the sump drained to simulate reduced gravity flow at degraded sump conditions.

This report presents the initial assessment of the test data collected. If this test is to be used by Westinghouse to support AP1000 Design Certification, additional validation of the use of this information will be documented separately. In the interim, the list of invalid data channels may change. 


\subsection{INTRODUCTION}

The Department of Nuclear Engineering at Oregon State University (OSU) is performing a series of tests for the U.S. Department of Energy. These tests are being conducted in the Advanced Plant Experiment (APEX-1000) test facility, which is a reduced pressure and height model of the two-loop Westinghouse AP1000 pressurized water reactor. The purpose of the testing is to:

- evaluate the thermal-hydraulic performance of the passive safety systems of the full-scale AP1000, and

- assess and validate the safety analyses techniques and computer codes used in predicting the transient system behavior.

The AP1000 Long-Term Cooling Test is a 1/4 height scale, low-pressure integral systems test simulating thermal-hydraulic phenomenon for the AP1000 passive safety systems for small-break loss-of-coolant accidents (LOCAs) and long-term cooling. It accurately models the details of the AP1000 geometry, including the primary system, the passive safety systems, and a part of the non-safety grade Chemical and Volume Control System, as well as a partial non-safety grade Normal Residual Heat Removal System. The interconnecting pipe routings are also duplicated in the model.

The overall objective of the Long-Term Cooling Test program is to obtain test data at various modes of operation. The OSU experiments will examine the passive safety system response for the small-break and large-break LOCA transition into long-term cooling. (The list of the tests to be performed is in the OSU Test Matrix provided in Section 4.0.) The facility permits a range of small-break LOCAs to be simulated at different locations on the primary system, such as the cold leg, hot leg, core makeup tank (CMT) cold leg pressure balance line, and direct vessel injection (DVI) line. The break orientation (top or bottom of the cold leg) may also be studied. Selected tests continue into the long-term cooling, post-accident mode in which the passive safety injection is from the reactor sump as well as the in-containment refueling water storage tank (IRWST). 


\subsection{TEST OBJECTIVE}

The purpose of this test is to repeat the previous test ADS-01-D, which was to obtain thermal-hydraulic data for the Automatic Depressurization System 4 (ADS-4) lines under steady-state conditions typical of long-term cooling. The data obtained from the test will be used to verify the AP1000 thermal-hydraulic computer codes for AP1000 phenomena, such as gravity injection, natural convection, and post-accident long-term core cooling behavior.

The acceptance criteria for the OSU tests are as follows:

- Test initial conditions will be achieved within a specified tolerance.

- Setpoints will be achieved within an acceptable tolerance band.

- All instrumentation should be operational before the test.

- Any critical instruments not operating will be identified to the test engineer before the tests. These instruments must be operational before and during the test or exceptions should be approved.

- A zero check of LDPs, DPs, and FDPs will be performed. 


\subsection{FACILITY DESCRIPTION}

A detailed facility description for the OSU test facility is documented in Reference 3-1. The OSU test facility has been specifically scaled, designed, and constructed to investigate the AP600 passive safety system behavior and to provide data for safety analysis computer code validation. The facility has been modified to model the AP1000.

The scaled test design accurately models the details of the AP1000 geometry, including the primary system and the pipe routings and layout for the passive safety systems. A detailed scaling report (Reference 3-2) was used to develop the test design modifications. The primary system consists of one hot leg and two cold legs with two active pumps and an active steam generator for each loop. Two CMTs are connected to one primary loop, and the pressurizer is connected to the other primary loop as in the AP1000 plant design. Gas-driven accumulators are connected to the DVI lines. The discharge lines from the CMT, and one-of-two IRWST and reactor sump lines are connected to each DVI line. The ADS consisting of stages 1,2, and 3 - simulates either one or two of the independent trains used in the AP1000. The two-phase flow from the ADS stages 1-to-3 is separated in a swirl-vane separator, and the liquid and vapor flows are measured to obtain the total ADS flow rate. The separated flow streams are then recombined and discharged into the IRWST through a sparger, preserving the mass and energy flow into the IRWST. The injection from the reactor sump is also simulated. Note that the OSU facility models both AP1000 primary and secondary sumps. The primary sump collects the condensate return, the liquid break flow, and the liquid flow from the fourth-stage ADS; and will provide long-term injection to the reactor vessel. The secondary sump simulates the portions of containment that will remain dry during most events. This sump collects water only when the primary sump reaches its overflow level, and provides no injection to the reactor vessel.

The time period for the experimental simulations includes not only the IRWST injection, but also the draining of the IRWST and the sump injection to simulate the long-term cooling of the AP1000. This simulation could be from several hours to a day. The time scale for the OSU test facility is one-half; that is, events occur in half the normal time. To model the long-term cooling aspects of the transient, the two-phase flow from the break is separated in a swirl-vane separator and the liquid and vapor portions of the total flow are measured. The liquid fraction of the flow is discharged to the reactor primary sump as in the AP1000 plant. The vapor is discharged to the atmosphere. The capability exists to return a portion of the equivalent liquid flow to the IRWST and primary sump to simulate the condensate return from the passive containment to the IRWST and primary sump. A similar approach is also used for the fourth-stage ADS valve on the hot leg. The two-phase flow is separated in a swirl-vane separator, the two streams are measured, and the liquid phase is discharged into the primary sump while the vapor flow is discharged to the atmosphere. Again, the capability exists to return a portion of the liquid equivalent added to the IRWST and primary sump. In addition, all other steam vents from the facility are measured (for example, the IRWST vent), and a portion of the liquid equivalent may be added back to the facility. Note that not all of the steam discharge would be returned as liquid equivalent. A portion of the discharge would be removed to simulate the steam that is not available for recirculation because it provides containment pressurization. The IRWST and primary sump can be pressurized in the OSU facility to simulate the containment pressurization following a postulated LOCA.

A multi-tube passive residual heat removal (PRHR) heat exchanger is located in the IRWST. The heat exchanger uses the same C-tube design as the AP1000 and has two instrumented tubes to obtain wall heat 
fluxes during the tests. There are primary fluid thermocouples, wall thermocouples, and differential pressure drop measurements to determine when the heat exchanger begins to drain. The IRWST is also instrumented with strings of fluid thermocouples to determine the degree of mixing within the tank and to assess the temperature of the coolant that is delivered to the test vessel.

The reactor vessel for the OSU tests includes a 0.914-meter (3-foot) heated core consisting of forty-eight 0.025 -meter (1-inch) diameter heater rods. The heater rods have a top skewed power shape. The $1000 \mathrm{~kW}$ of electrical power available at the OSU test site will be used to simulate decay heat. Wall thermocouples are swaged inside the heater rods to measure the heater rod wall temperature.

Thermocouple rods in the heater rod bundle measure the axial coolant temperature distribution. The scaled flow volume in the core is preserved as well as the flow volume in the test vessel upper plenum. There are simulated reactor internals in the upper plenum to preserve the flow area and to correctly scale the fluid volume. The reactor vessel includes an annular downcomer into which the four cold legs and the two DVI lines are connected. The hot legs penetrate the reactor annulus and connect with the loops. The AP1000 reactor vessel neutron reflector is simulated using a ceramic liner to reduce the metal heat release to the coolant.

There were no special/unique requirements for the test other than those specified in the Initial Conditions. The specified conditions were verified on the control board prior to test implementation.

\subsection{Instrumentation}

The instrumentation has been designed to calculate a transient mass and energy balance on the test facility. All two-phase flow streams exiting the facility are separated, and each component is measured separately as a single-phase flow using conventional measurement devices, such as magnetic flow meters and vortex flow meters. Note that magnetic flow meters are not designed for two-phase flow and will indicate erratically. Also, the vortex flow meters are referenced to $212^{\circ} \mathrm{F}$ and the LDPs are referenced to $60^{\circ} \mathrm{F}$. All vertical components have differential pressure cells that act as level instruments to measure the transient mass change in the component. The hot and cold leg diameters are sufficiently large in the OSU test facility so that a narrow-range differential pressure cell can be used to determine if the flow becomes stratified.

Single flow measurements are made of the CMT, accumulator, IRWST, and sump flows into the reactor vessel through the DVI lines.

Various types of instrumentation are provided in the test facility; for example, thermocouples for coolant and wall temperatures, flow meters, pressure transducers, differential pressure transducers, and weigh tanks.

\subsection{References}

3-1 APP-LTCT-T2R-005, OSU APEX-1000 Test Facility Description Report

3-2 APP-LTCT-T2R-004, Scaling Assessment for the Design of the OSU APEX-1000 Test Facility 


\subsection{OREGON STATE UNIVERSITY TESTING PROGRAM MATRIX}

The test matrix for the OSU test facility is shown in Table 4-1. To satisfy the test objectives, several transients will be performed to provide data on the AP1000 passive safety system response for a range of break sizes, locations, orientations, and single failure assumptions. The break size orifices are scaled based on simulating a 0.5 -inch, 1-inch, 2 -inch, or larger pipe break.

The designation for this test is ADS-01-D, which identifies the test as an ADS-4 characterization test. The test matrix may be adjusted for future tests based on results and insights gained with each test. 


\begin{tabular}{|c|c|c|}
\hline \multicolumn{3}{|c|}{$\begin{array}{c}\text { TABLE 4-1 } \\
\text { OSU TEST MATRIX (AS SPECIFIED DECEMBER 10, 2003) }\end{array}$} \\
\hline Test Title & Break Location and Size & Single Failure Assumed \\
\hline DBA-01-D & $\begin{array}{l}\text { Double-ended DVI Line break with } \\
\text { continuation into long-term cooling }\end{array}$ & Fail 1 of 2 lines in one ADS- 4 train \\
\hline DBA-02-D & $\begin{array}{l}\text { Double-ended DVI Line break with } \\
\text { continuation into long-term cooling }\end{array}$ & $\begin{array}{l}\text { Fail } 1 \text { of } 2 \text { lines in one ADS- } 4 \text { train } \\
\text { (Adjusted ADS-4 Resistance) }\end{array}$ \\
\hline DBA-03-D & $\begin{array}{l}\text { Double-ended DVI Line break with } \\
\text { continuation into long-term cooling }\end{array}$ & $\begin{array}{l}\text { Fail } 1 \text { of } 2 \text { lines in one ADS- } 4 \text { train } \\
\text { (Failure location sensitivity) }\end{array}$ \\
\hline TR-01-D & $\begin{array}{l}\text { Transition Test ADS- } 4 \text { opening, } 85 \text { psig initial } \\
\text { pressure and decay power } 480 \mathrm{sec}\end{array}$ & $\begin{array}{l}\text { Fail } 1 \text { of } 2 \text { lines in one ADS-4 train } \\
\text { (No ADS 1-3) }\end{array}$ \\
\hline TR-02-D & $\begin{array}{l}\text { Transition Test ADS- } 4 \text { opening, } 100 \text { psig initial } \\
\text { pressure with prototypic plant conditions }\end{array}$ & $\begin{array}{l}\text { Fail } 1 \text { of } 2 \text { lines in one ADS-4 train } \\
\text { (No ADS 1-3) }\end{array}$ \\
\hline DBA-04-D & 2-inch Cold Leg Break & $\begin{array}{l}\text { Fail } 1 \text { of } 2 \text { lines in one ADS- } 4 \text { train } \\
\text { (Failure on non-pressurizer hot leg) }\end{array}$ \\
\hline ADS-01-D & $\begin{array}{l}\text { ADS-4 Two-Phase Characterization Test at } \\
\text { low-power conditions (steady-state points) }\end{array}$ & $\begin{array}{l}\text { Various ADS-4 line configurations } \\
\qquad \text { (No ADS 1-3) }\end{array}$ \\
\hline ADS-02-D & $\begin{array}{l}\text { ADS-4 Two-Phase Characterization Test at } \\
\text { high-power conditions (steady-state points) }\end{array}$ & $\begin{array}{l}\text { Various ADS-4 line configurations } \\
\qquad \text { (No ADS 1-3) }\end{array}$ \\
\hline DBA-03R-D & Repeat of Test DBA-03-D & $\begin{array}{l}\text { Fail } 1 \text { of } 2 \text { lines in one ADS- } 4 \text { train } \\
\text { (Failure on pressurizer hot leg) }\end{array}$ \\
\hline DBA-05-D & Inadvertent ADS-1 Actuation & $\begin{array}{l}\text { Fail } 1 \text { of } 2 \text { lines in one ADS- } 4 \text { train } \\
\text { (Failure on non-pressurizer hot leg) }\end{array}$ \\
\hline DBA-06-D & 0.5-inch Cold Leg Break & $\begin{array}{l}\text { Fail } 1 \text { of } 2 \text { lines in one ADS- } 4 \text { train } \\
\text { (Failure on non-pressurizer hot leg) }\end{array}$ \\
\hline
\end{tabular}




\subsection{TEST PROCEDURE}

The test was performed per a written procedure. There were no special/unique requirements for the test other than those specified in the initial conditions in Table 6-1. The specified conditions were checked on the control board before test implementation.

The appropriate prerequisites were completed and initial conditions were satisfied.

Fill and vent was performed per APEX Operations Manual Procedure OP-B.2. Instruments were checked for required calibration.

Since the CMTs and accumulators are not used in this test, normal procedures to ensure proper initial conditions were not used.

Once all other initial conditions were satisfied, ADS-4 was actuated and the IRWST isolation valves were opened. Once steady-state was established (typically 2000 seconds), the power was reduced and another steady-state point was tested. This was repeated for six power levels ranging from $360 \mathrm{~kW}$ to $20 \mathrm{~kW}$. Three additional steady-state test points were obtained by draining the sump to simulated degraded sump conditions consistent with wall-to-wall floodup in the AP1000. 


\subsection{TEST RESULTS}

The test results for test ADS-01-D are provided in the following subsections.

\subsection{Initial and Boundary Conditions}

Table 6-1 provides a comparison of the specified and actual conditions for test ADS-01-D. The values in this table were averaged over approximately 2 minutes preceding the test.

\subsection{Inoperable Instruments}

Table 6-2 lists the instrumentation channels considered inoperable for the ADS-01-D test.

\subsection{Key Data Plots}

Table 6-3 lists the instrumentation channels sorted by component and includes the instrument number and units. The selection of channels was based on projecting an overall picture of the test results, which would then be examined by referring to the detailed data plots or tapes.

\subsection{Test Evaluation}

The following observations were made during the test:

1. Steady-state ADS-4 flows were observed after approximately 2000 seconds at each core power level.

2. The two-phase mixture level in the upper plenum remained at or above the hot leg for all power levels tested.

3. Significant liquid flow was observed from the ADS-4 for all power levels tested. 


\begin{tabular}{||l|c|c|l||}
\hline \multicolumn{5}{|c||}{ TCBLE 6-1 } \\
\hline \multicolumn{1}{||c|}{ Conditions } & Instrument No. & Actual & \multicolumn{1}{c||}{ Comment } \\
\hline Upper Head Pressure & PT-107 & $2 \mathrm{psig}$ & \\
\hline Hot Leg Temperature \#1 & TF-141* & $216^{\circ} \mathrm{F}$ & \\
\hline Hot Leg Temperature \#2 & TF-140* & $216^{\circ} \mathrm{F}$ & $\begin{array}{l}\text { Assumed to be heated } \\
\text { post-ADS }\end{array}$ \\
\hline IRWST Temperature & TF-701 & $205^{\circ} \mathrm{F}$ & $\begin{array}{l}\text { Representative of level at } \\
\text { time of sump switchover }\end{array}$ \\
\hline IRWST Level & LDP-701 & 18 inches &
\end{tabular}

* These instruments are used to establish initial conditions at the test site. 


\begin{tabular}{|c|c|c|}
\hline \multicolumn{3}{|c|}{$\begin{array}{c}\text { TABLE 6-2 } \\
\text { INOPERABLE INSTRUMENTS FOR ADS-01-D TEST }\end{array}$} \\
\hline Instrument Number & Instrument Type & Inoperable Description \\
\hline $\begin{array}{l}\text { TW-202 } \\
\text { TW-204 } \\
\text { TW-205 } \\
\text { TW-206 } \\
\text { TW-209 } \\
\text { TW-803 } \\
\text { TW-804 }\end{array}$ & Thermocouple & Inoperative \\
\hline TH-603 & $\begin{array}{l}\text { Thermocouple measuring heater } \\
\text { temperature }\end{array}$ & Inoperative \\
\hline FMM-202 & Magnetic flow meter & Inoperative \\
\hline $\begin{array}{l}\text { TF-170 } \\
\text { TF-221 } \\
\text { TF-509 } \\
\text { TF-512 }\end{array}$ & $\begin{array}{l}\text { Thermocouple measuring fluid } \\
\text { temperature }\end{array}$ & Inoperative \\
\hline FVM-905 & Vortex flow meter & Erratic \\
\hline
\end{tabular}




\begin{tabular}{|c|c|c|c|}
\hline \multicolumn{4}{|c|}{$\begin{array}{c}\text { TABLE 6-3 } \\
\text { DATA PLOTS FOR QL REPORTS FOR ADS-01-D BY COMPONENT }\end{array}$} \\
\hline Component & Channel & Units & Comment \\
\hline Reactor Vessel Pressure & PT-107 & psig & Nearly atmospheric \\
\hline Reactor Vessel Level & LDP-127 & inch of $\mathrm{H}_{2} \mathrm{O}$ & $\begin{array}{l}\text { Collapsed level follows } \\
\text { reactor power }\end{array}$ \\
\hline $\begin{array}{l}\text { Reactor Vessel Downcomer } \\
\text { Level }\end{array}$ & LDP-140 & inch of $\mathrm{H}_{2} \mathrm{O}$ & \\
\hline $\begin{array}{l}\text { Reactor Two-Phase Mixture } \\
\text { Level }\end{array}$ & LT-120 & inches & $\begin{array}{l}\text { Level always at or above } \\
\text { hot leg }\end{array}$ \\
\hline $\begin{array}{l}\text { Reactor Vessel Fluid } \\
\text { Temperature Upper Head }\end{array}$ & TF-120 & ${ }^{\circ} \mathrm{F}$ & Saturated \\
\hline IRWST Liquid Level & LDP-701 & inch of $\mathrm{H}_{2} \mathrm{O}$ & \\
\hline $\begin{array}{l}\text { IRWST Discharge Line \#1 } \\
\text { and \#2 Flow Rate }\end{array}$ & FMM-701 and FMM-702 & gpm & \\
\hline IRWST Fluid Temperature & TF-701 and TF-709 & ${ }^{\circ} \mathrm{F}$ & IRWST heated \\
\hline $\begin{array}{l}\text { ADS 4-1 and 4-2 Separator } \\
\text { Pressure }\end{array}$ & PT-611 and PT-610 & psig & \\
\hline $\begin{array}{l}\text { ADS 4-1 Separator Steam } \\
\text { Flow Rate }\end{array}$ & FVM-603 & $\operatorname{scfm}$ & \\
\hline $\begin{array}{l}\text { ADS 4-2 Separator Steam } \\
\text { Flow Rate }\end{array}$ & FVM-602 & $\operatorname{scfm}$ & \\
\hline $\begin{array}{l}\text { ADS 4-1 Separator Liquid } \\
\text { Flow Rate }\end{array}$ & FMM-603 & gpm & \\
\hline $\begin{array}{l}\text { ADS 4-2 Separator Liquid } \\
\text { Flow Rate }\end{array}$ & FMM-602 & gpm & \\
\hline Primary Sump Liquid Level & LDP-901 & inch of $\mathrm{H}_{2} \mathrm{O}$ & \\
\hline $\begin{array}{l}\text { Primary Sump Injection Flow } \\
\text { Rate }\end{array}$ & FMM-901 and FMM-902 & gpm & \\
\hline Core Power Input Power & $\begin{array}{l}\text { KW-101, KW-102, } \\
\text { KW-103, and KW-104 }\end{array}$ & $\mathrm{kW}$ & Step changes \\
\hline
\end{tabular}




\subsection{CONCLUSIONS}

The ADS-01-D tests were successfully completed, and the data was logged in the DAS. All critical instruments were found to operate properly with the exception of those noted in Section 6.4. The test was acceptable. 
APPENDIX A

DATA PLOTS 


\section{AP1000 Test Data Oregon State University Test ADS-01 December 10,2003

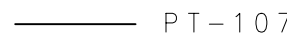 \\ 196 \\ o RX Up Head Press.}

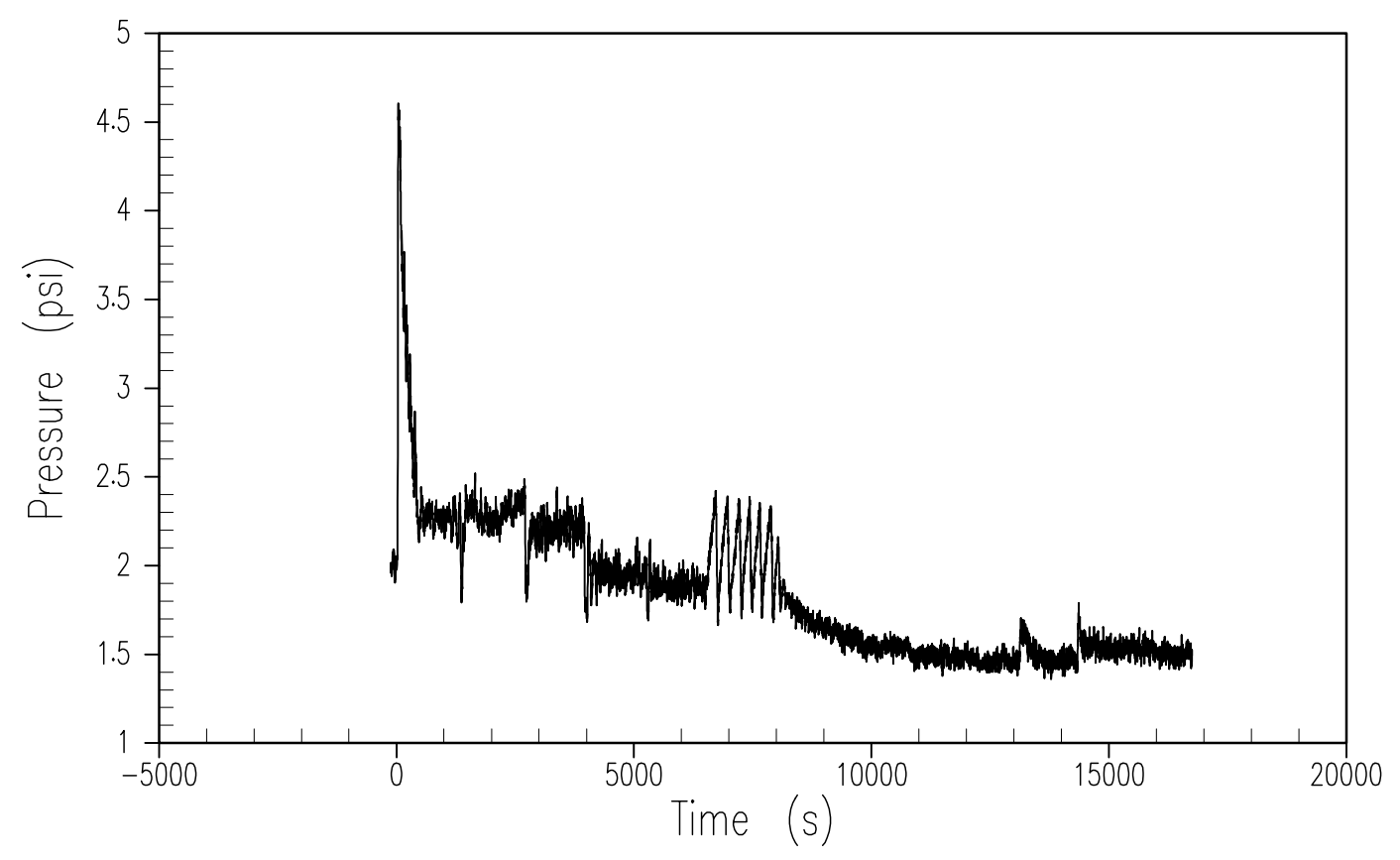

Figure A-1 Reactor Vessel Pressure 


\section{AP1000 Test Data Oregon State University Test ADS-01 December 10,2003

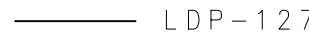 \\ 126 \\ 0 \\ o Reactor WR Leve}

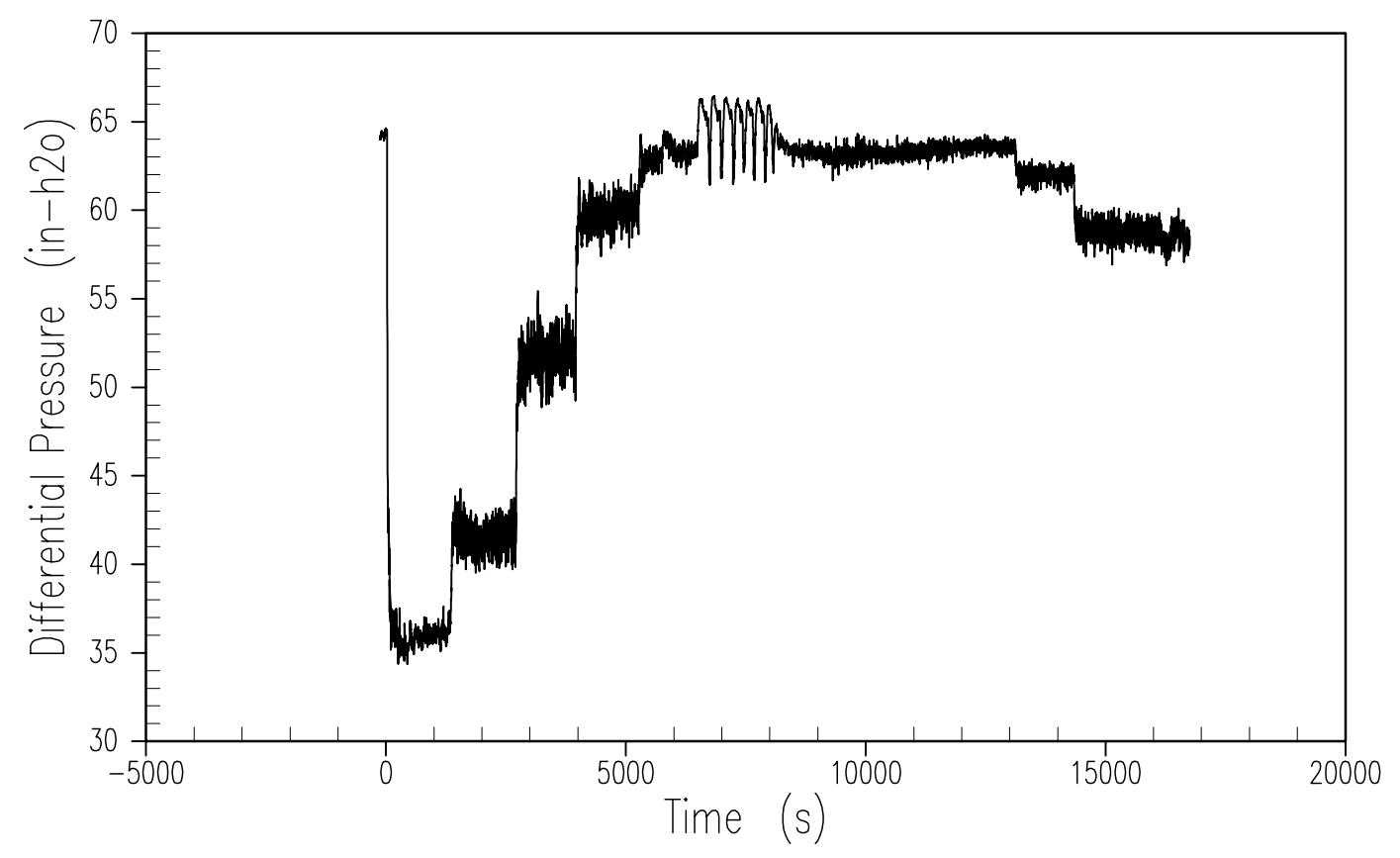

Figure A-2 Reactor Vessel Level 

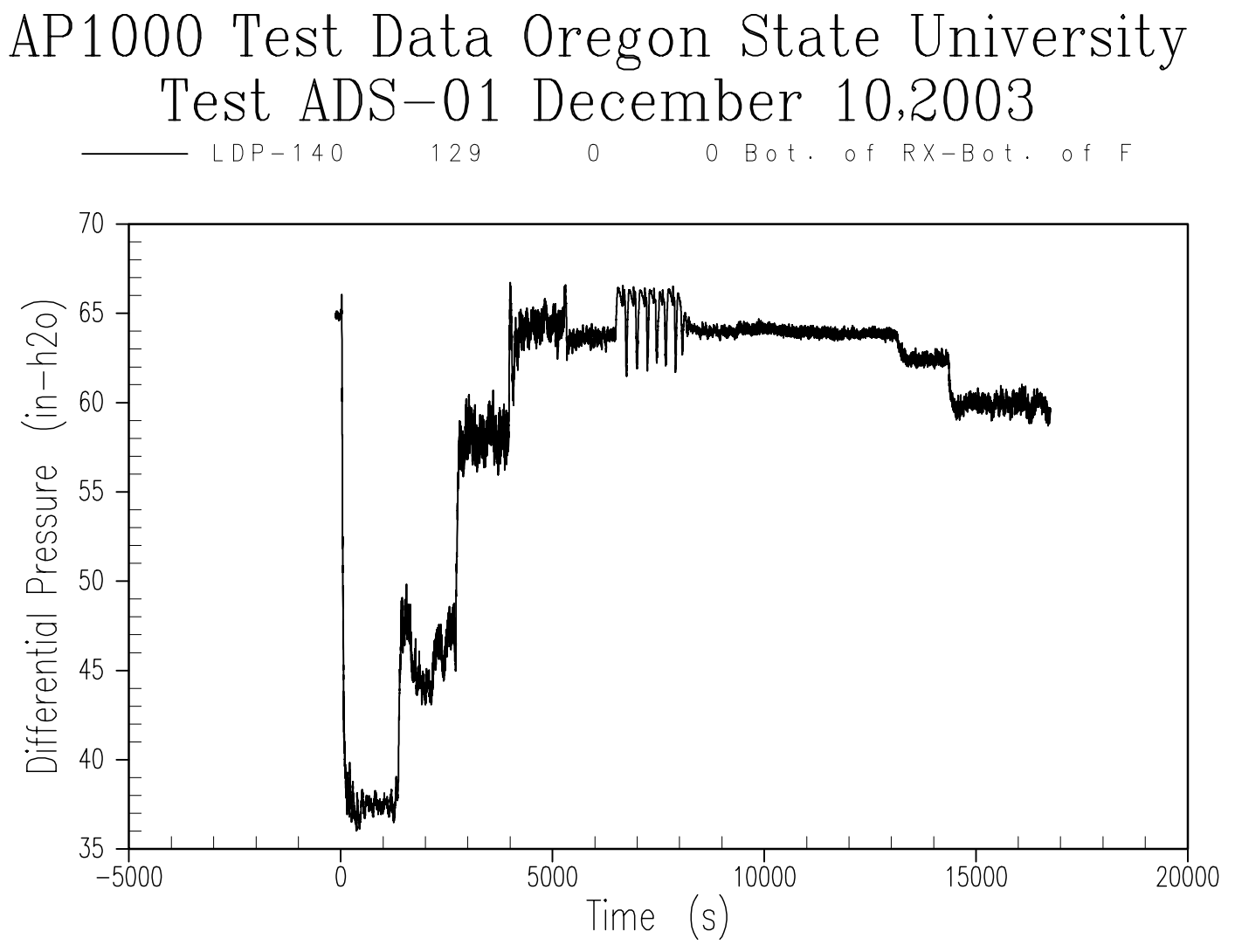

Figure A-3 Reactor Vessel Downcomer Level 

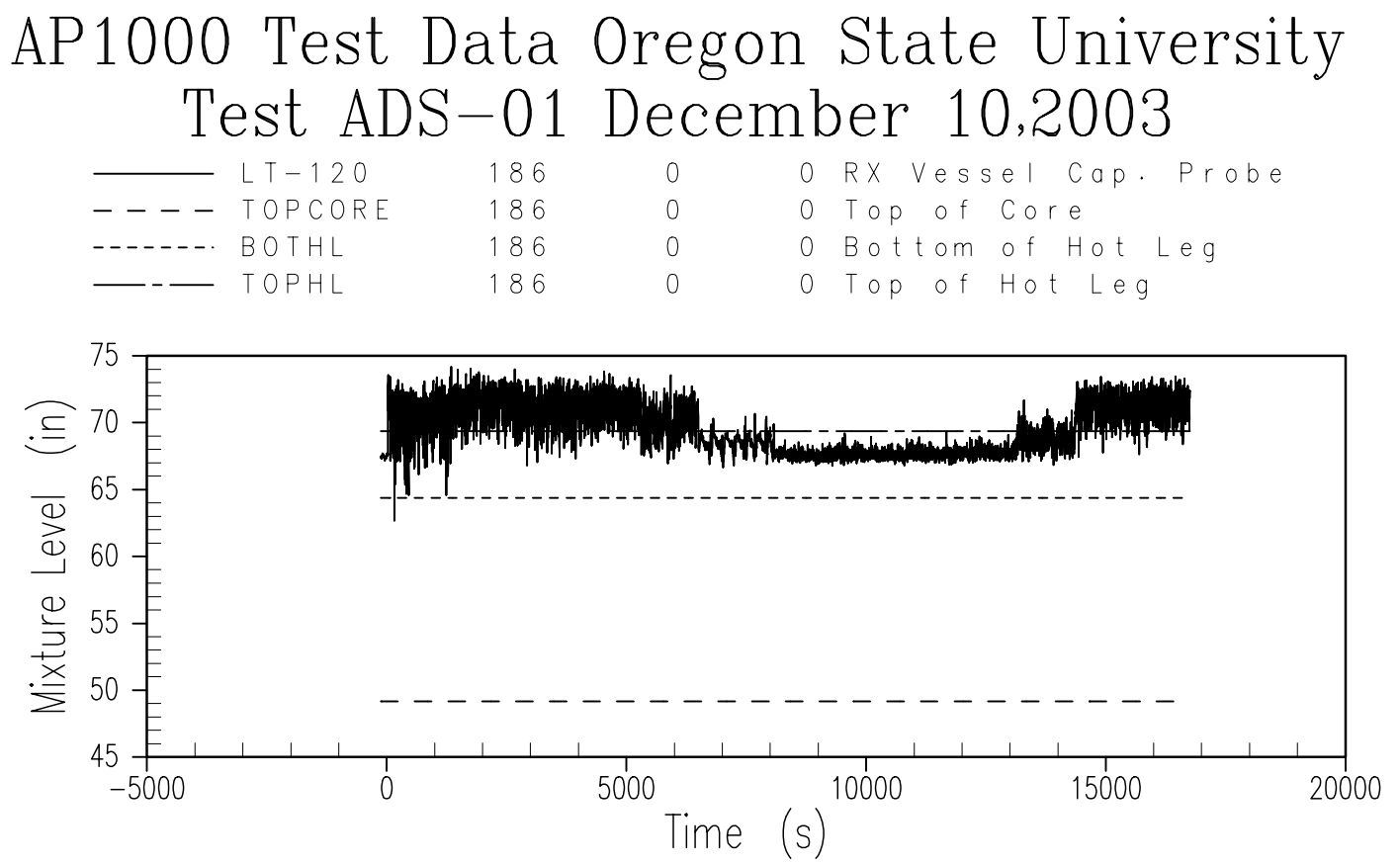

Figure A-4 Two-Phase Mixture Level in Upper Plenum 

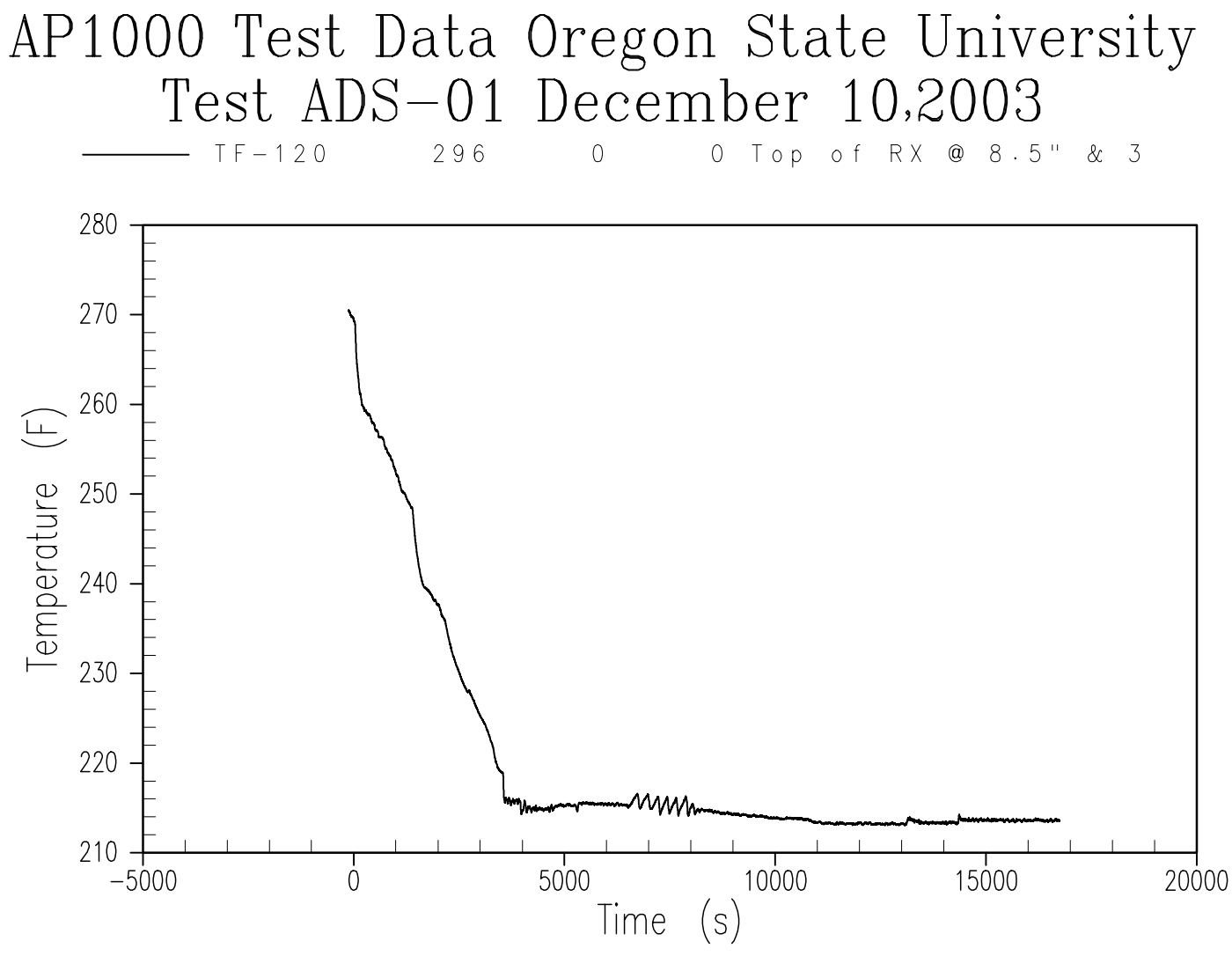

Figure A-5 Upper Head Temperature 


\section{AP1000 Test Data Oregon State University Test ADS-01 December 10,2003

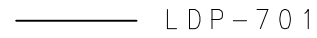 \\ 179 \\ 0 \\ o IRWST UC LeveI}

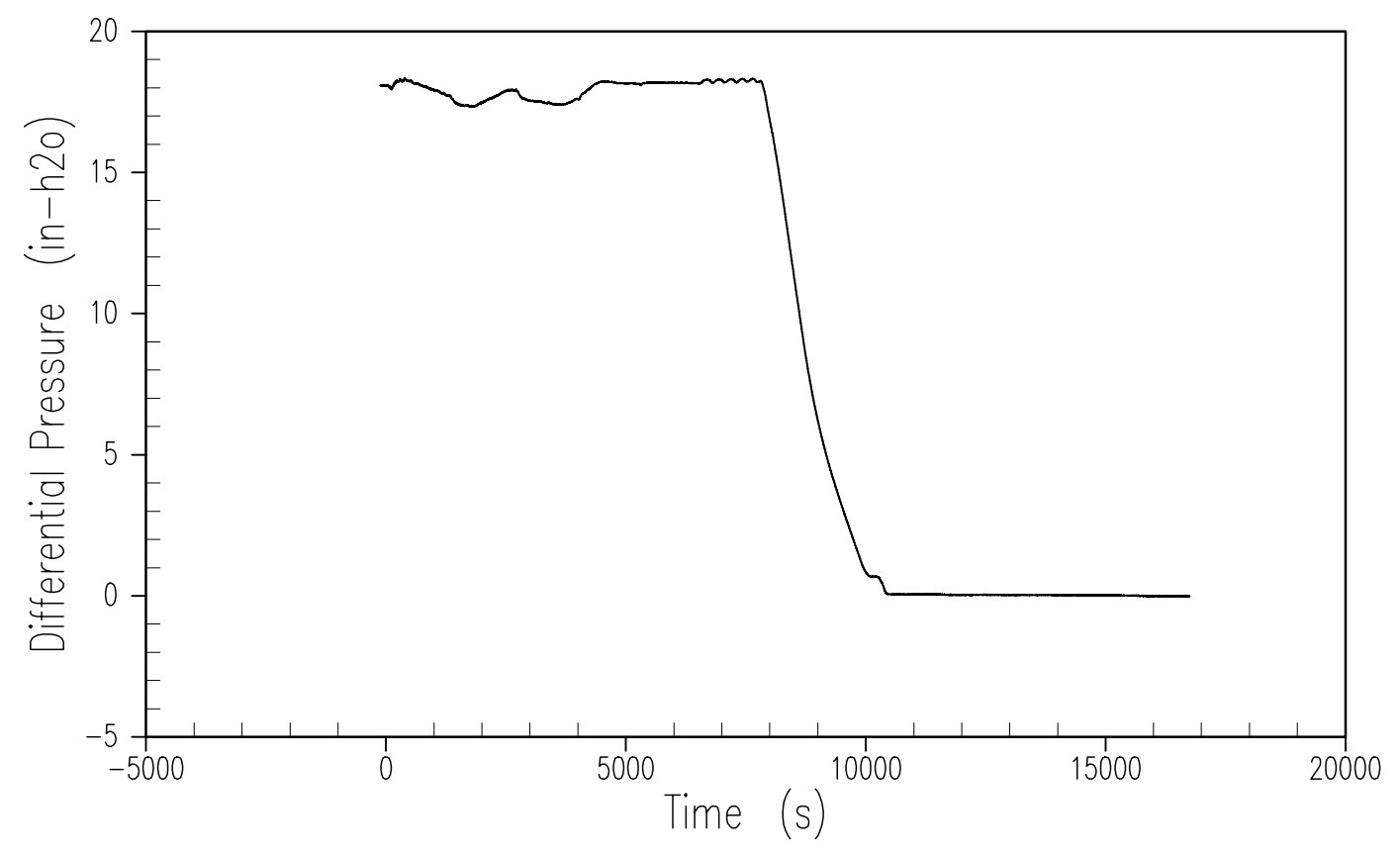

Figure A-6 IRWST Liquid Level 


\section{AP1000 Test Data Oregon State University Test ADS-01 December 10,2003
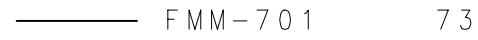 \\ 0 \\ o I RWST-1 Inj. F I OW}

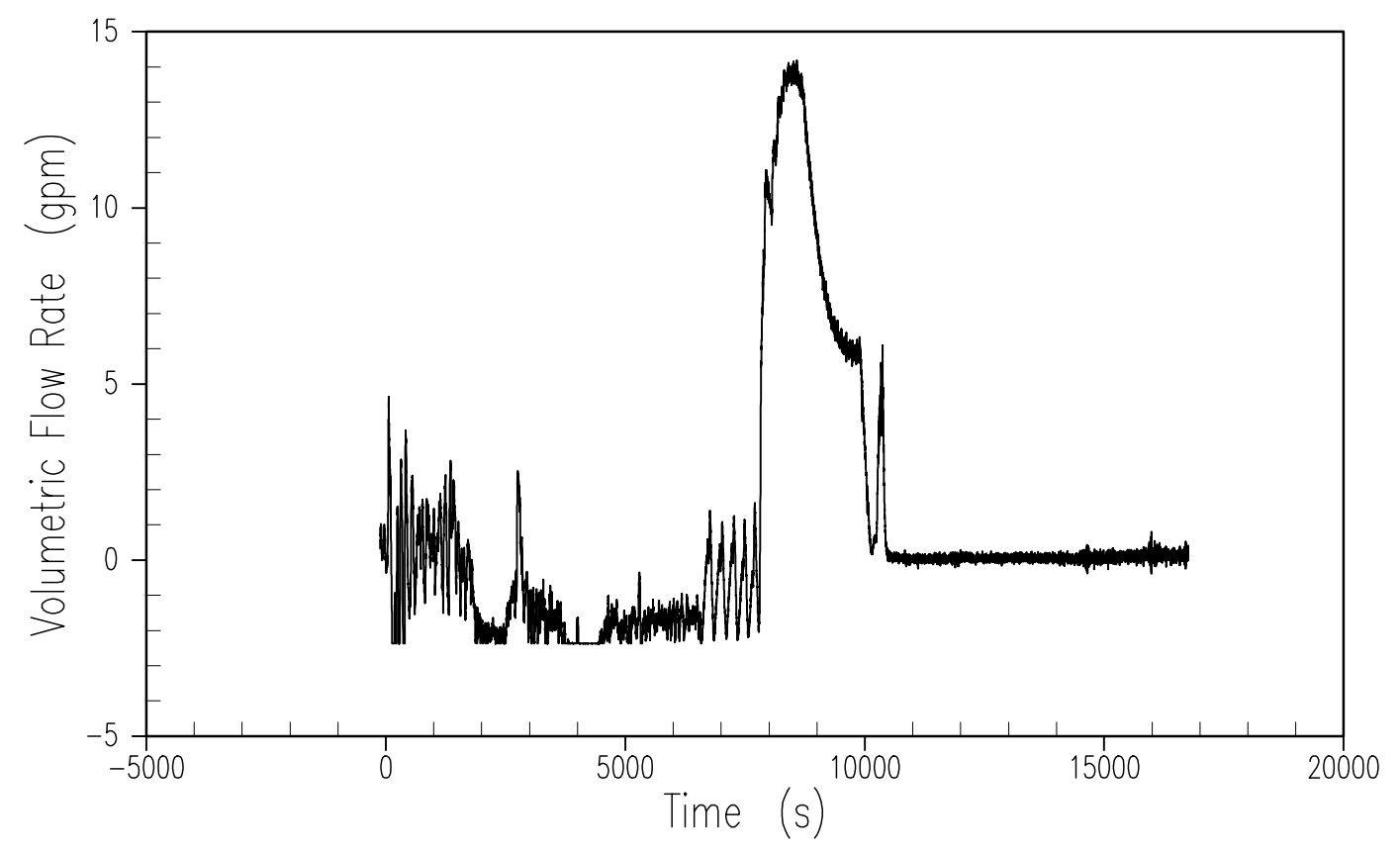

Figure A-7 IRWST Discharge Line 1 Flow Rate 


\section{AP1000 Test Data Oregon State University Test ADS-01 December 10,2003

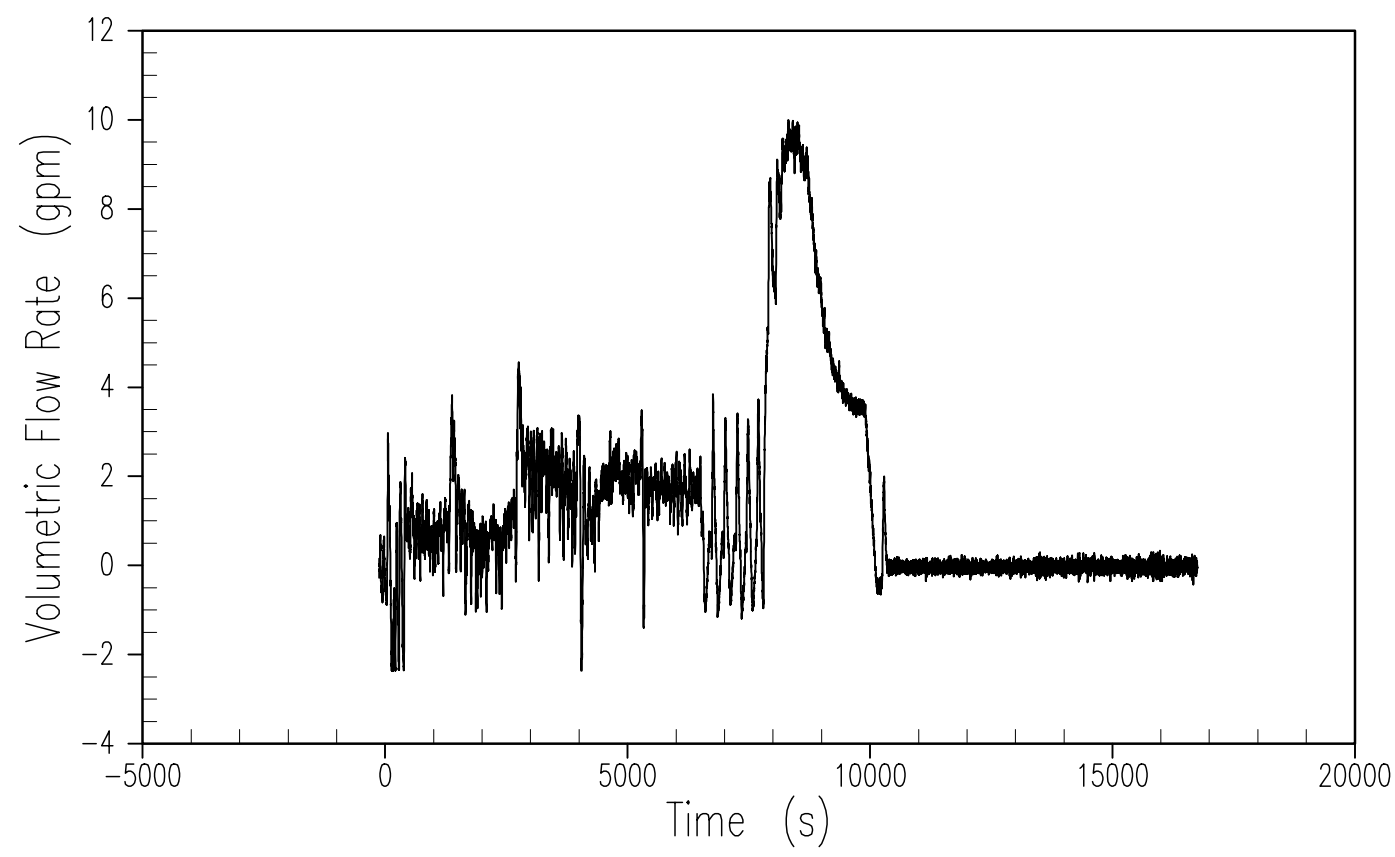

Figure A-8 IRWST Discharge Line 2 Flow Rate 

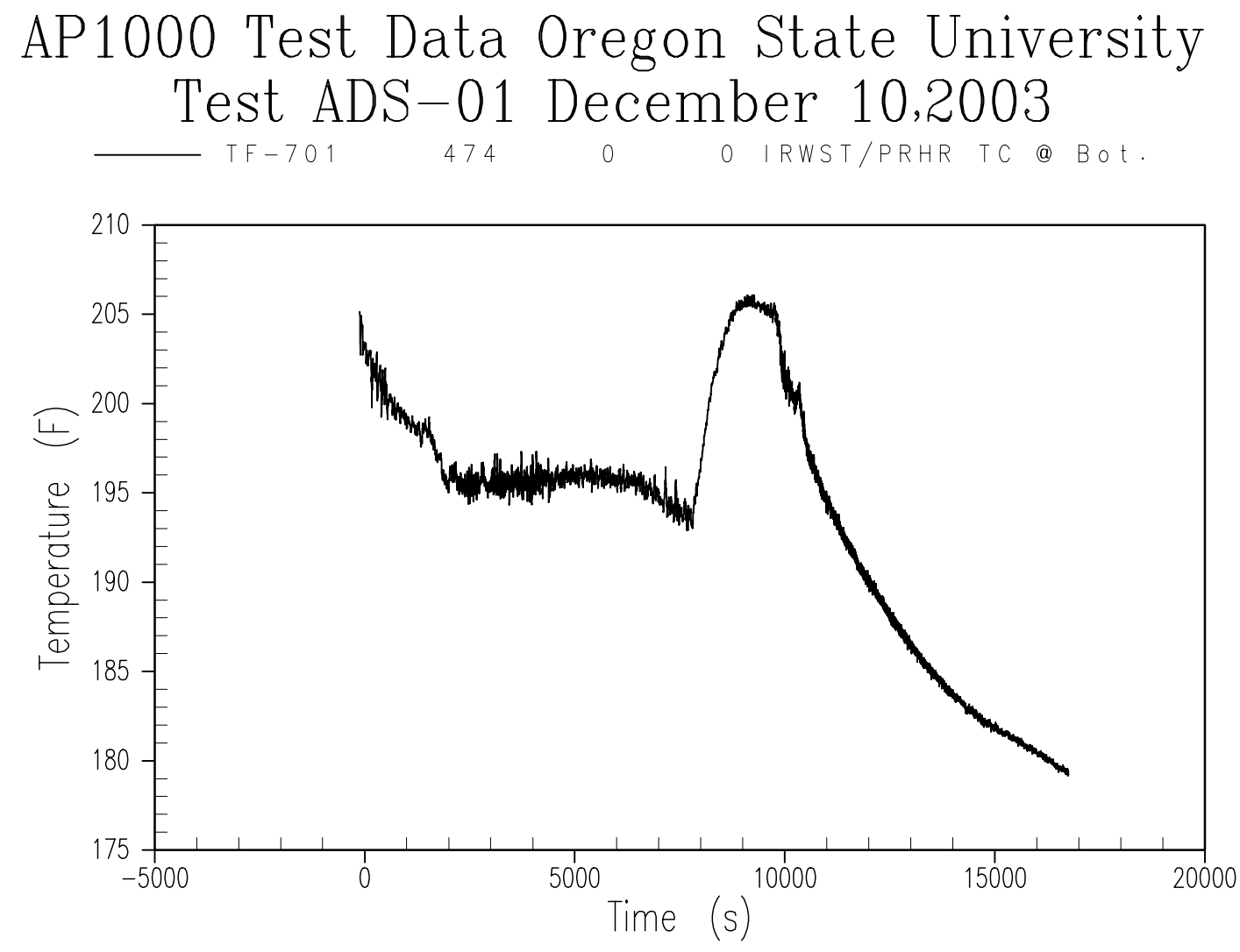

Figure A-9 IRWST Fluid Temperature - Bottom 

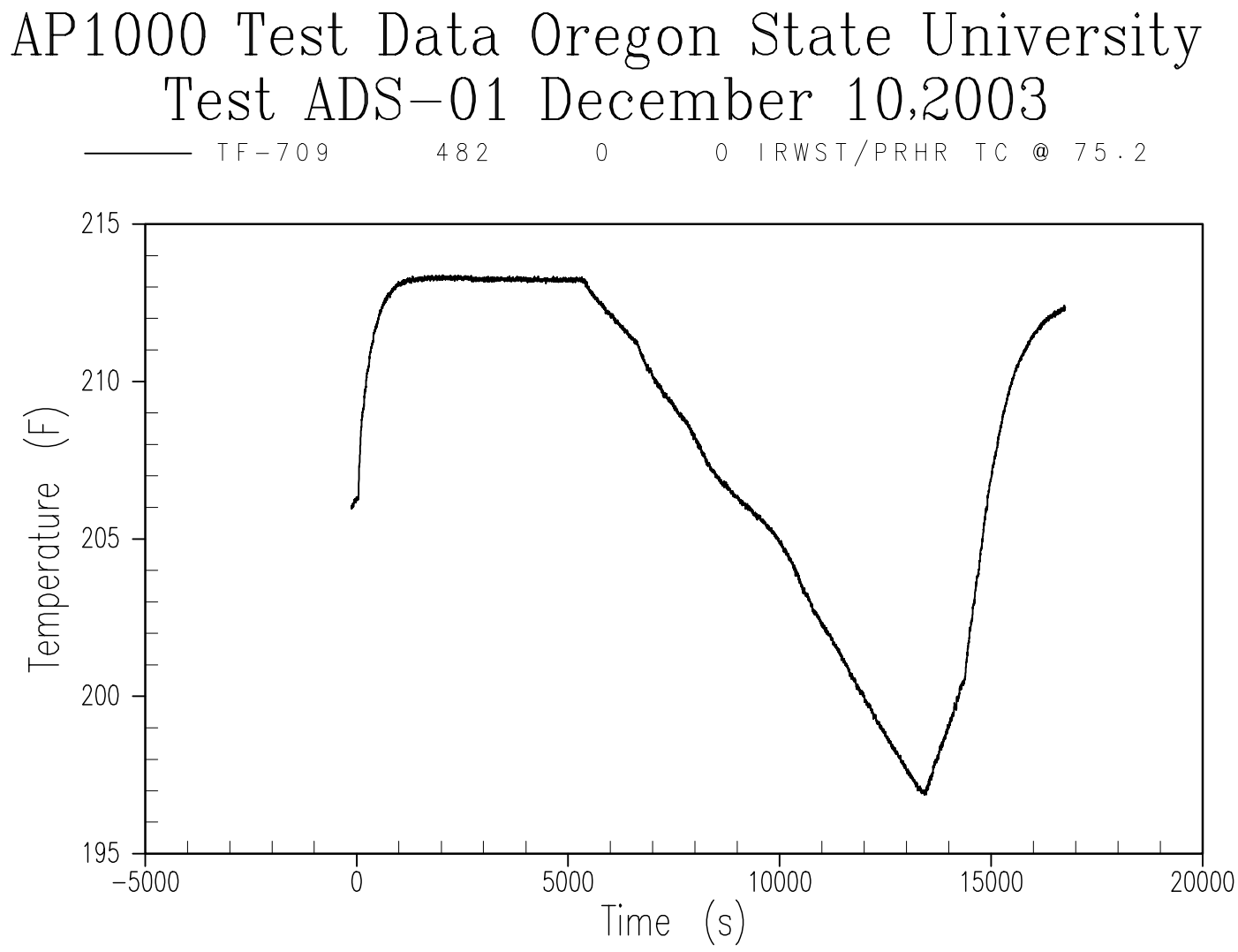

Figure A-10 IRWST Fluid Temperature - Top 

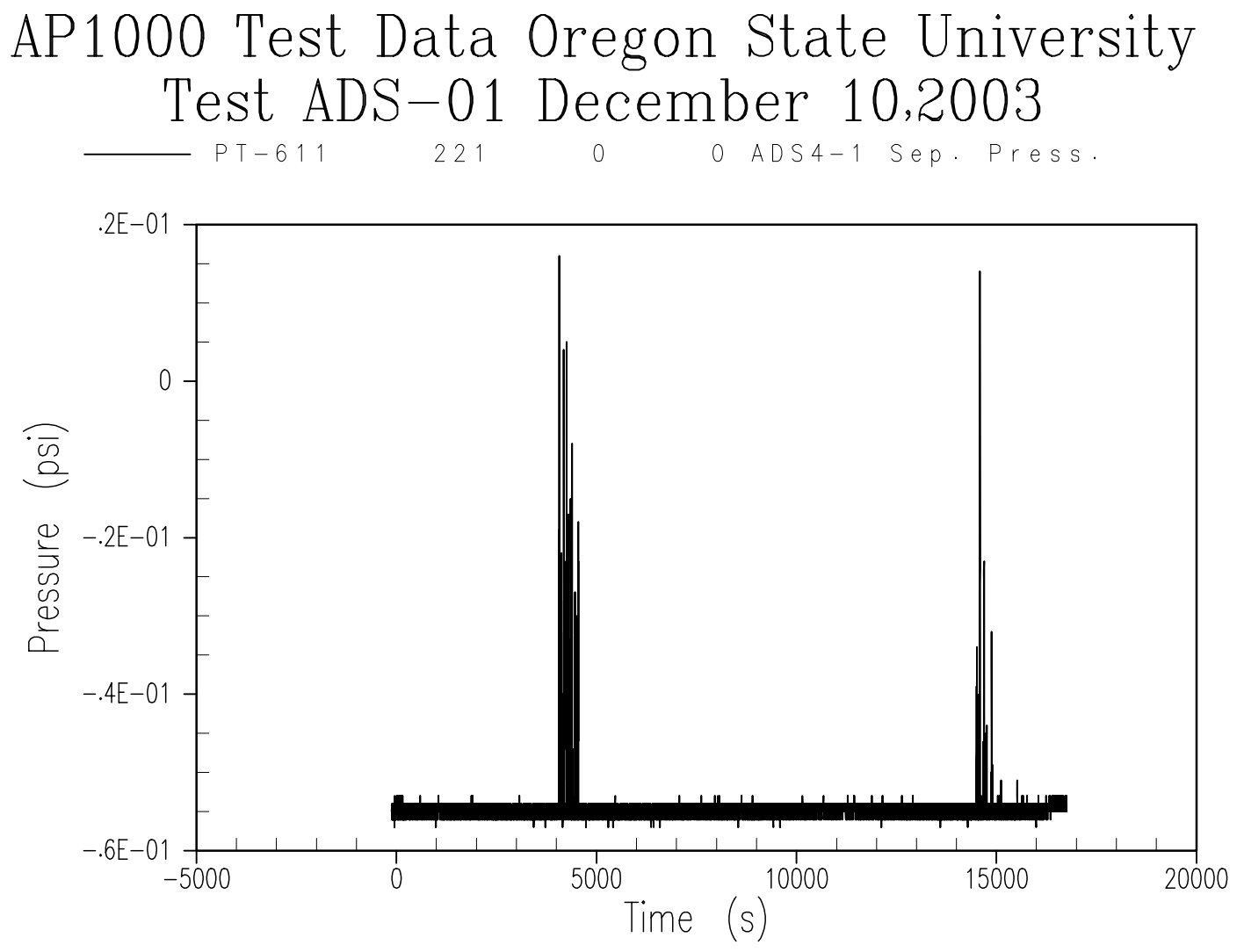

Figure A-11 ADS 4-1 Separator Pressure 


\section{AP1000 Test Data Oregon State University Test ADS-01 December 10,2003 \\ $\longrightarrow P T-610$

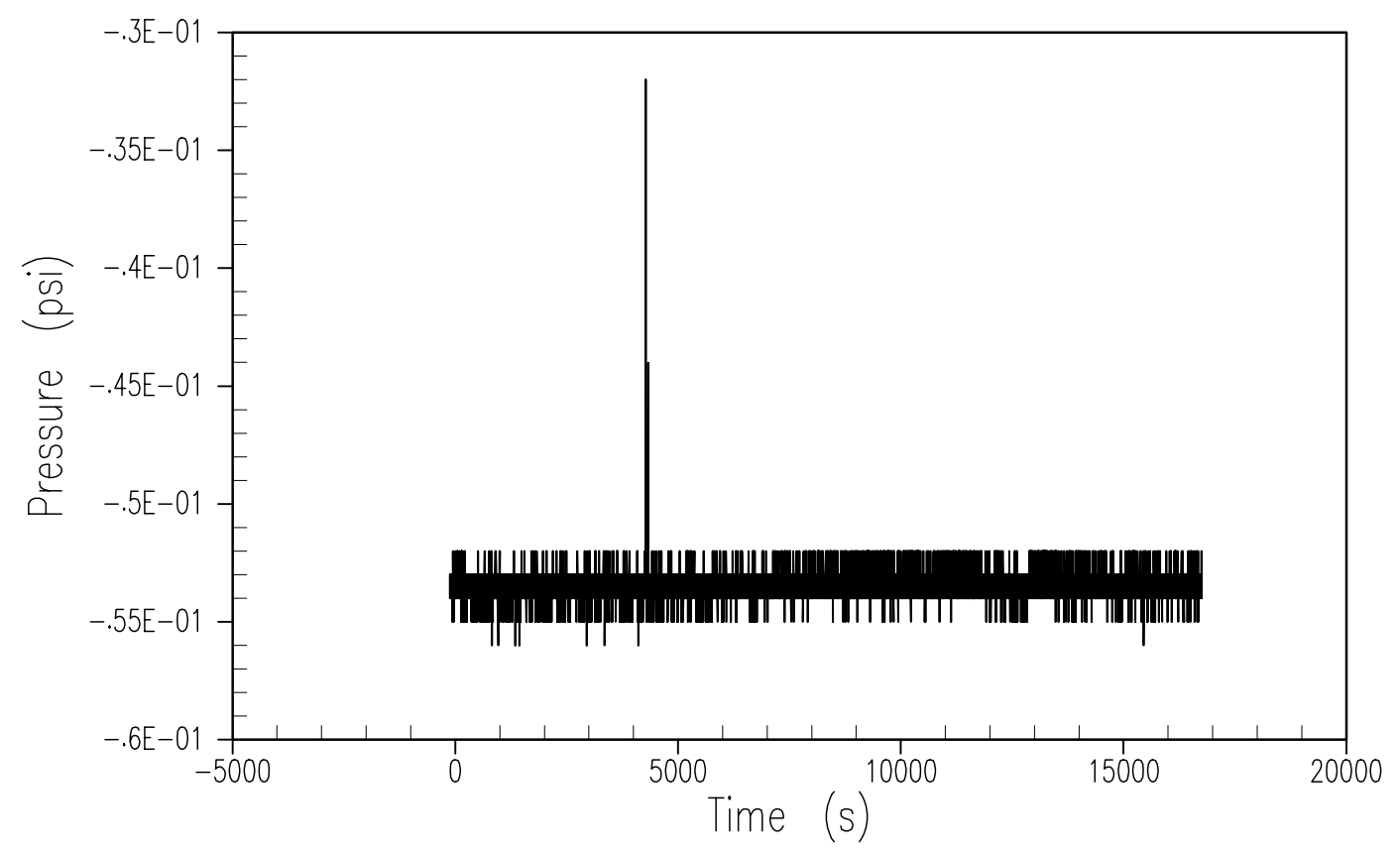

Figure A-12 ADS 4-2 Separator Pressure 

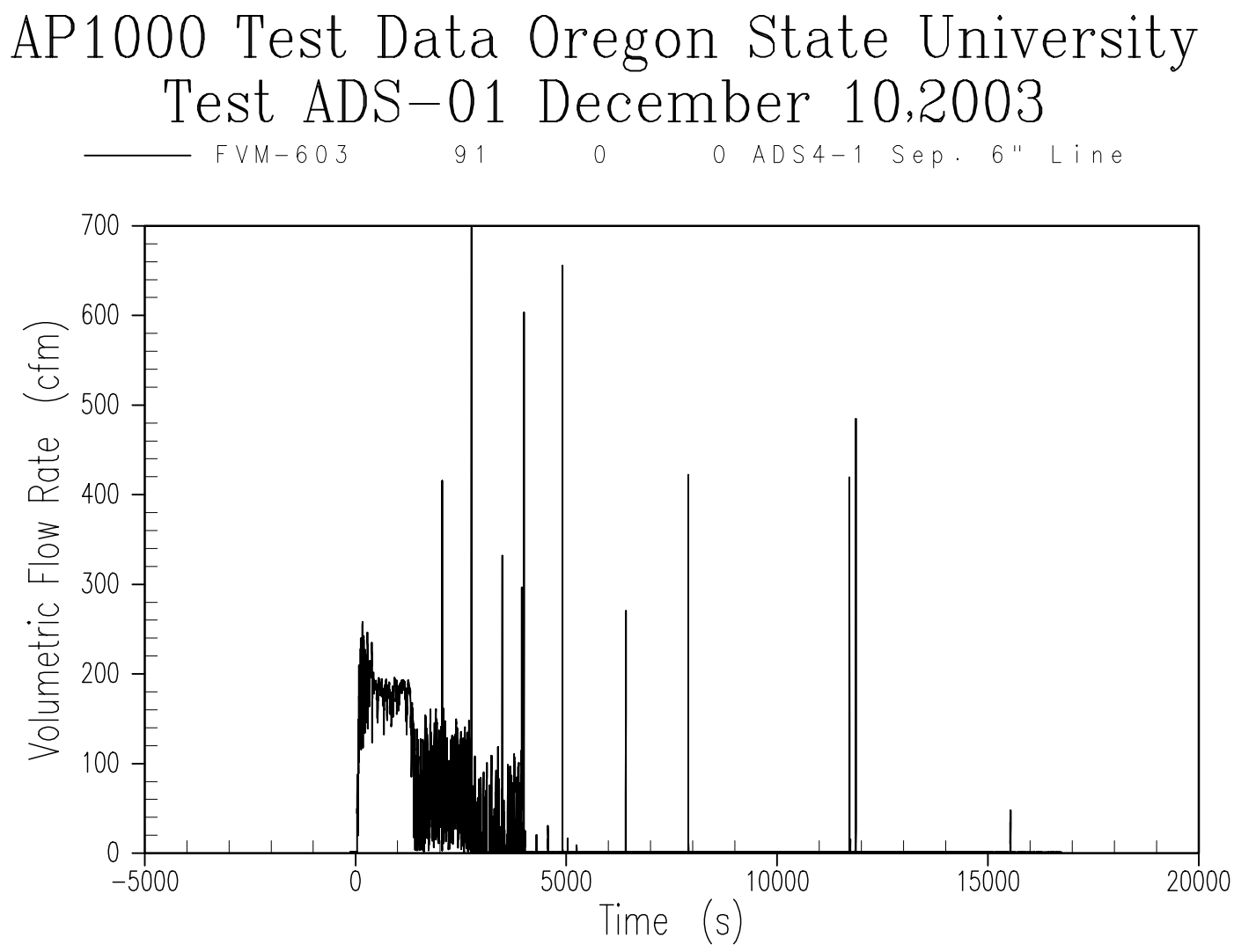

Figure A-13 ADS 4-1 Separator Steam Flow Rate 

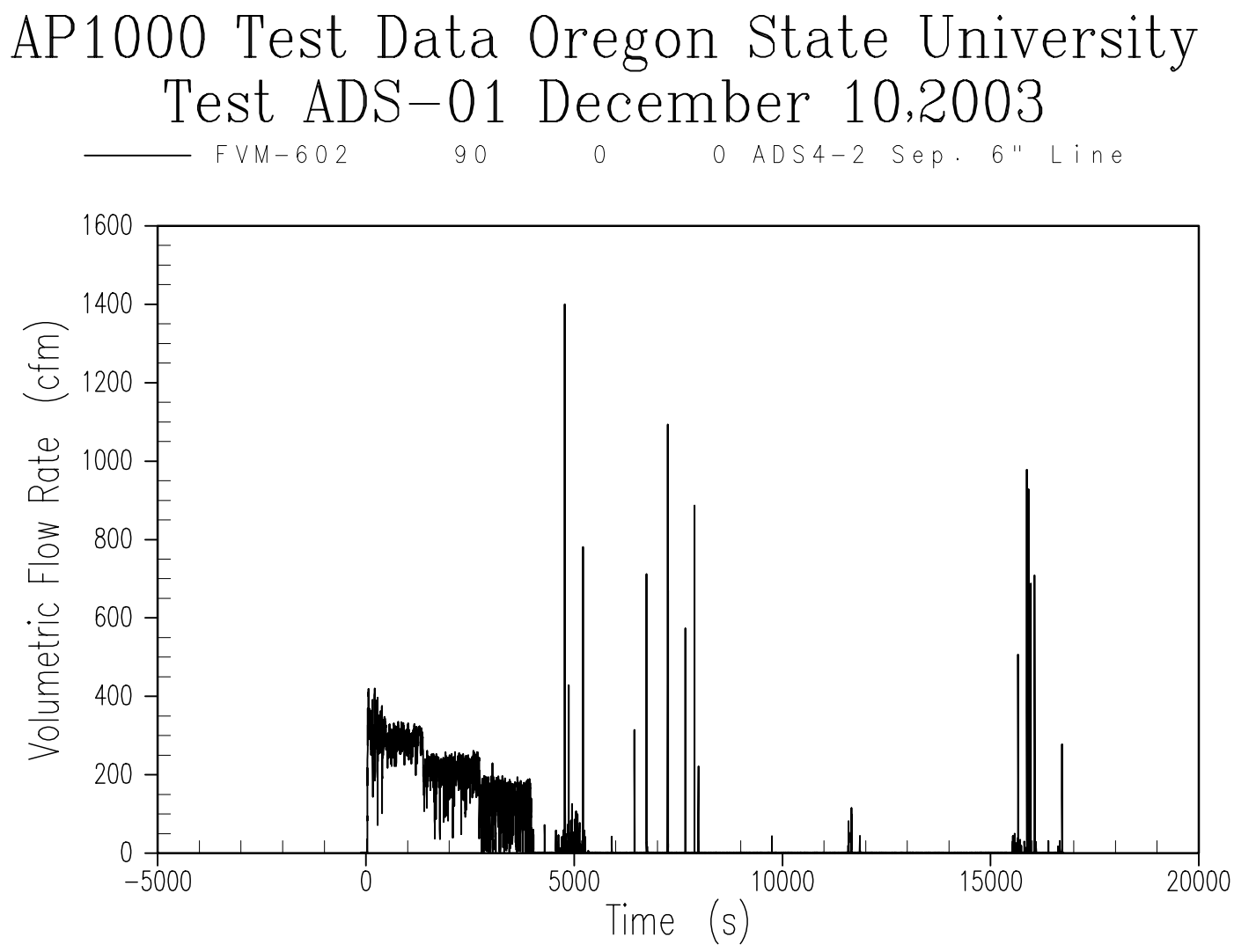

Figure A-14 ADS 4-2 Separator Steam Flow Rate 


\section{AP1000 Test Data Oregon State University Test ADS-01 December 10,2003

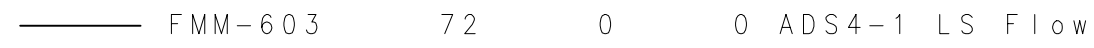

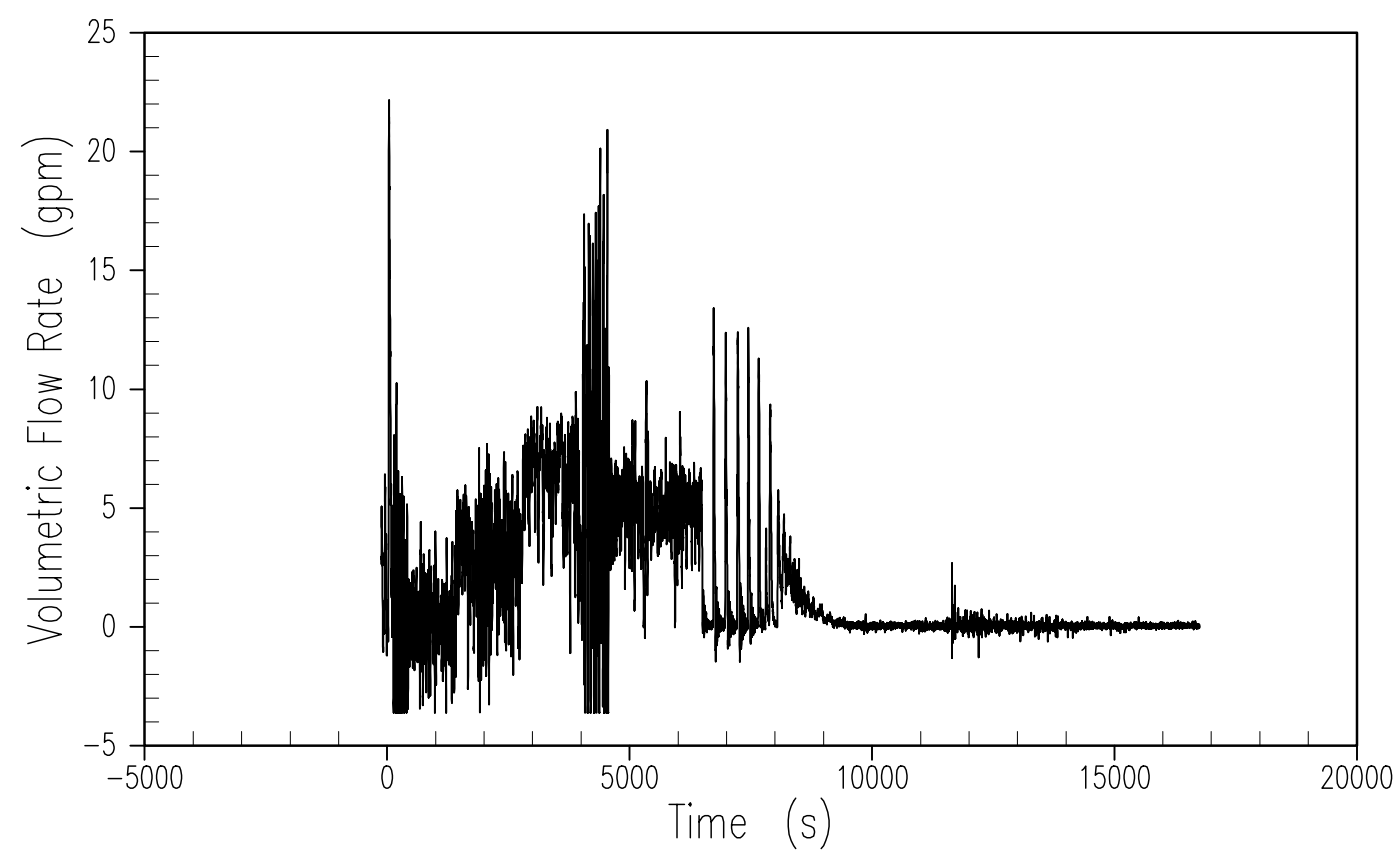

Figure A-15 ADS 4-1 Separator Liquid Flow Rate 


\section{AP1000 Test Data Oregon State University Test ADS-01 December 10,2003 $\longrightarrow$ FMM-602 71 \\ 0 \\ 0 ADS4-2 LS FIOW}

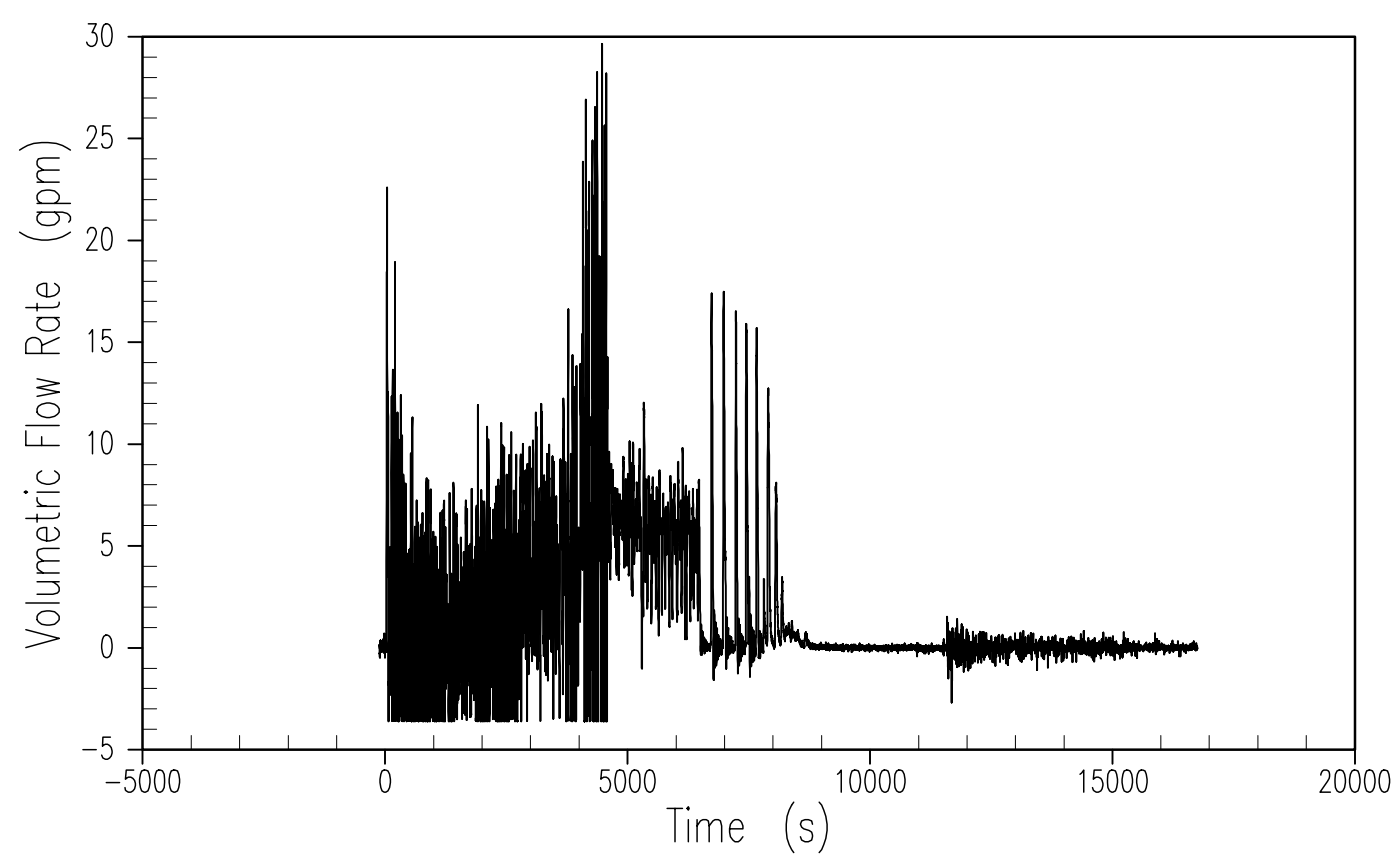

Figure A-16 ADS 4-2 Separator Liquid Flow Rate 

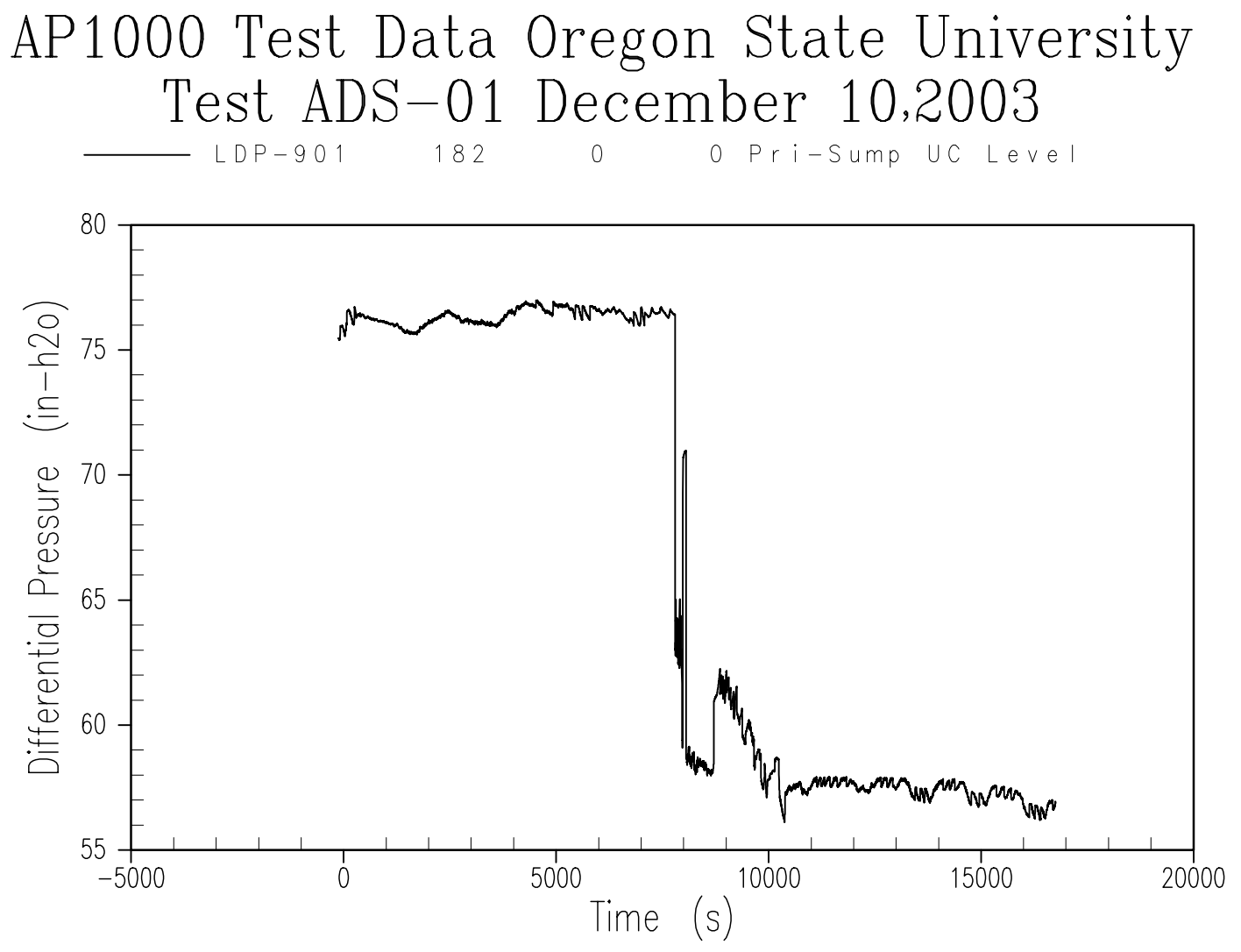

Figure A-17 Primary Sump Liquid Level 

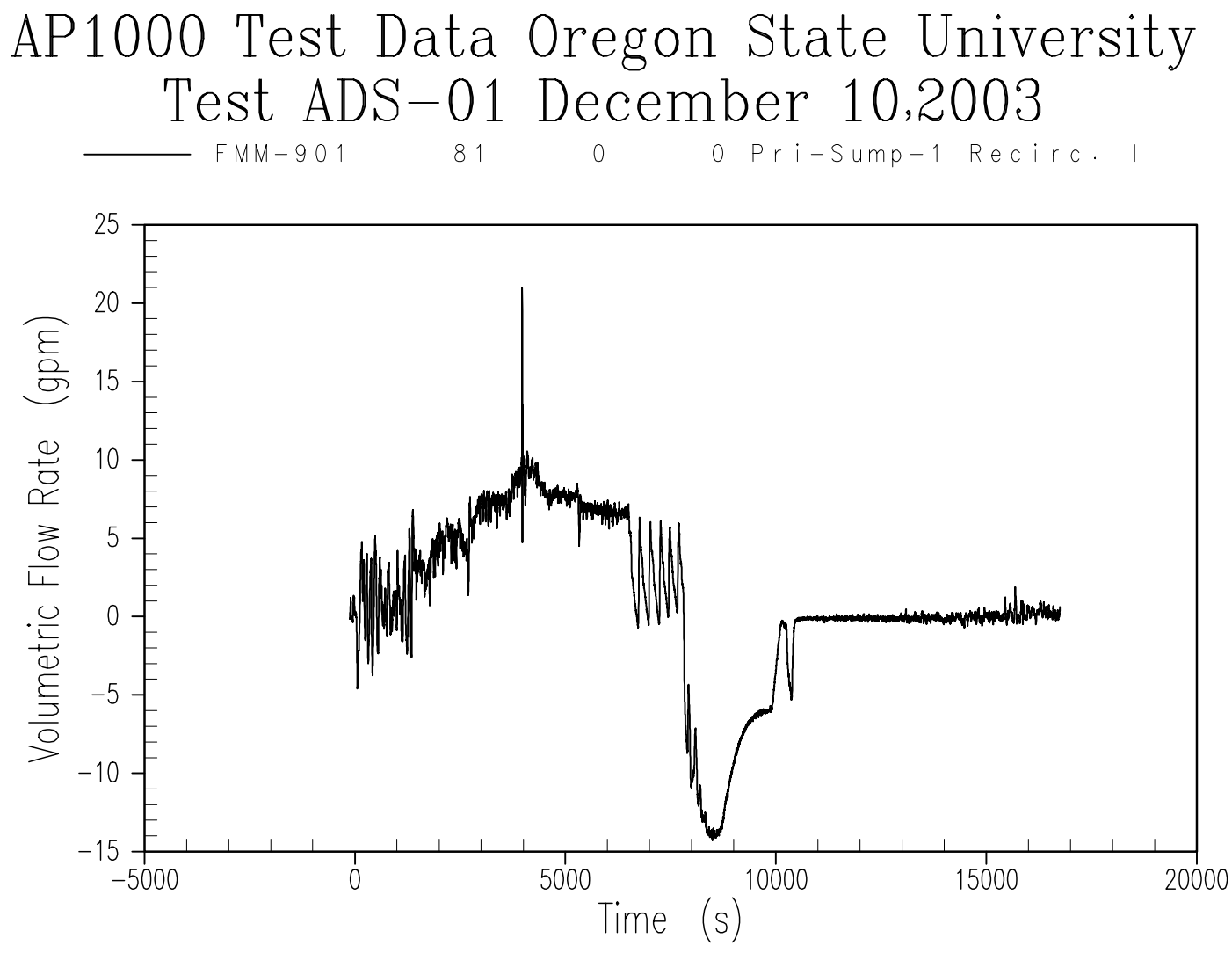

Figure A-18 Primary Sump 1 Injection Flow Rate 


\section{AP1000 Test Data Oregon State University Test ADS-01 December 10,2003}

$\longrightarrow$ FMM-902

$$
82 \quad 0
$$

o Pri-Sump-2 Recirc. I

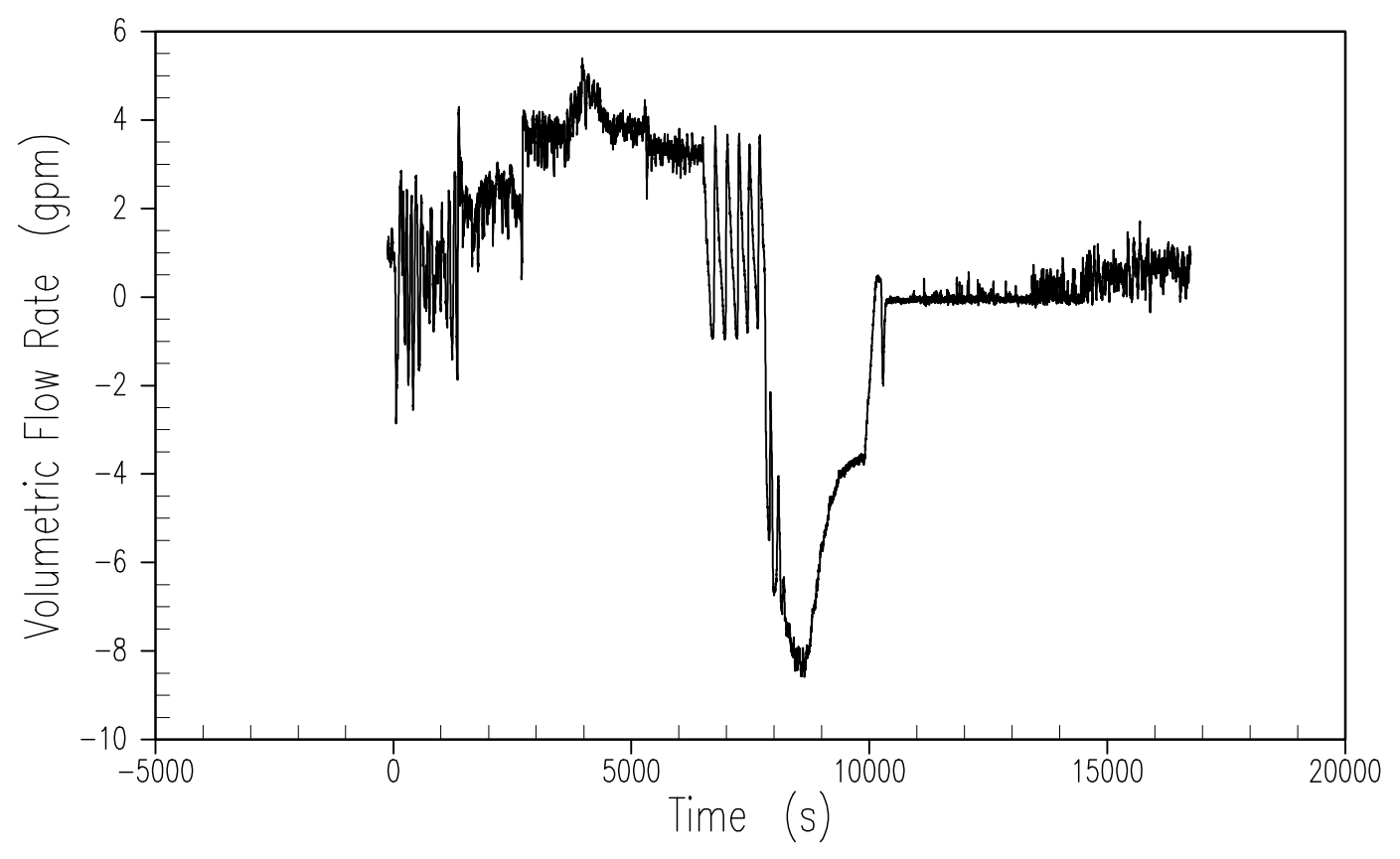

Figure A-19 Primary Sump 2 Injection Flow Rate 

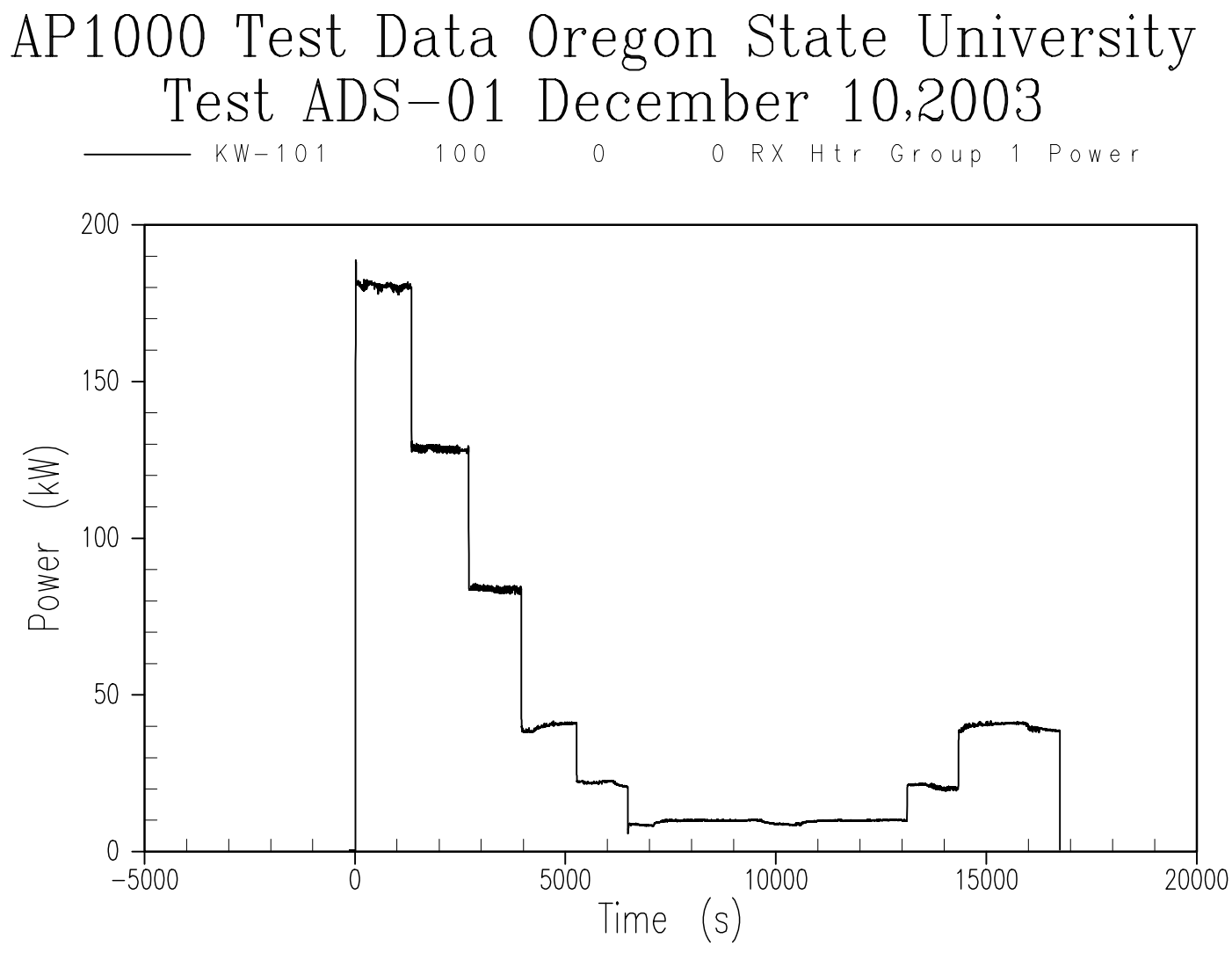

Figure A-20 Core Power Group 1 Input Power 

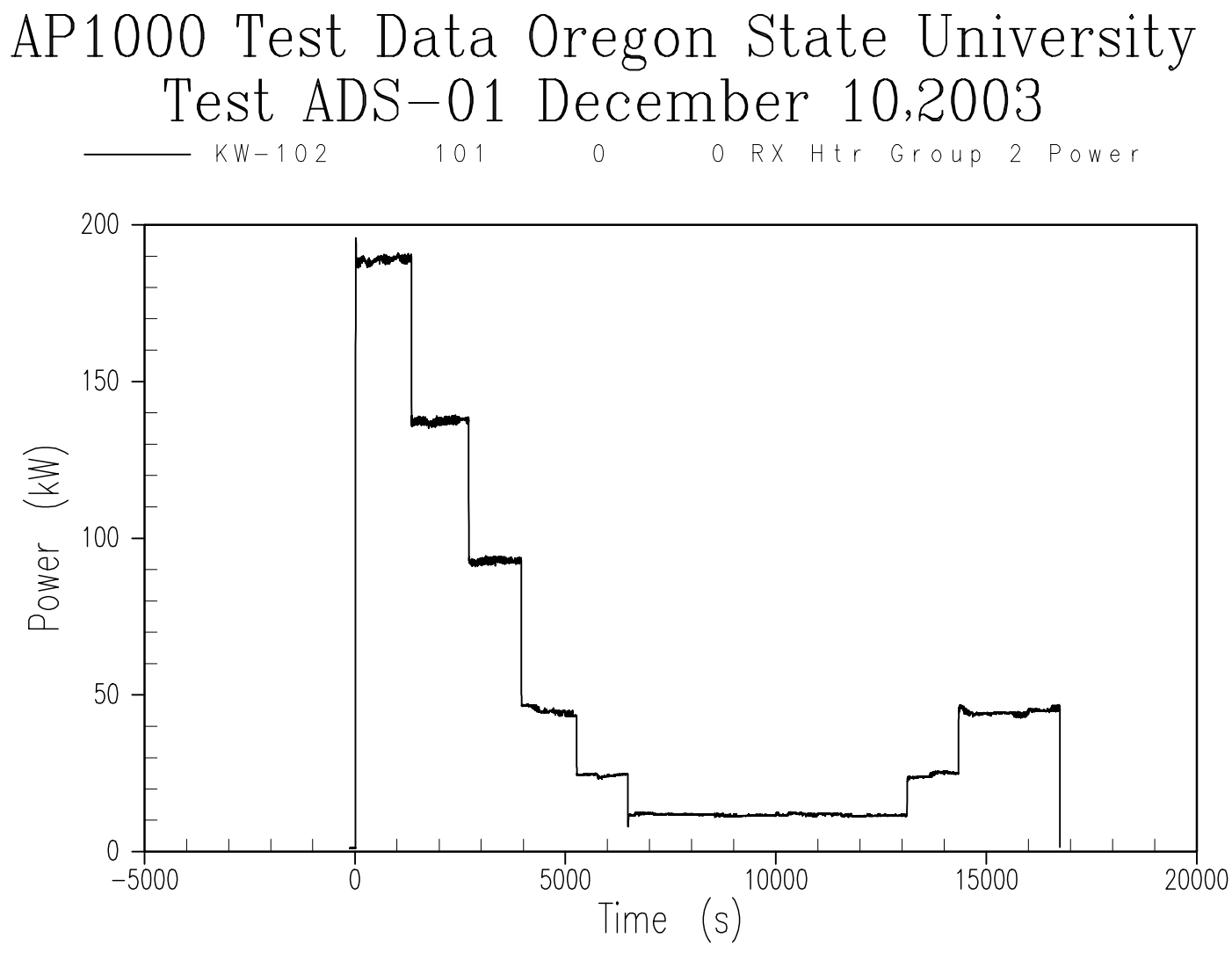

Figure A-21 Core Power Group 2 Input Power 

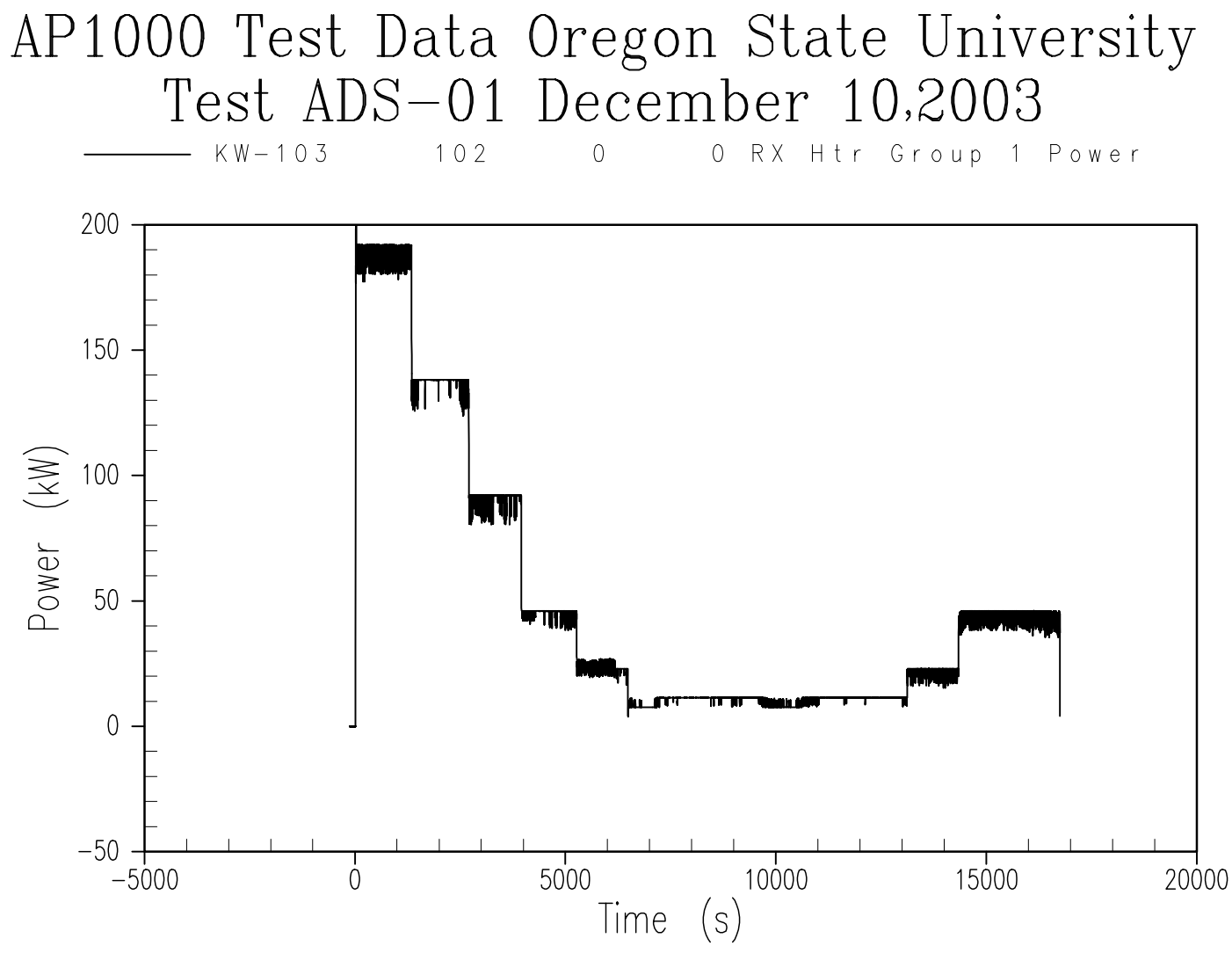

Figure A-22 Core Power Group 1 Alternate Input Power 

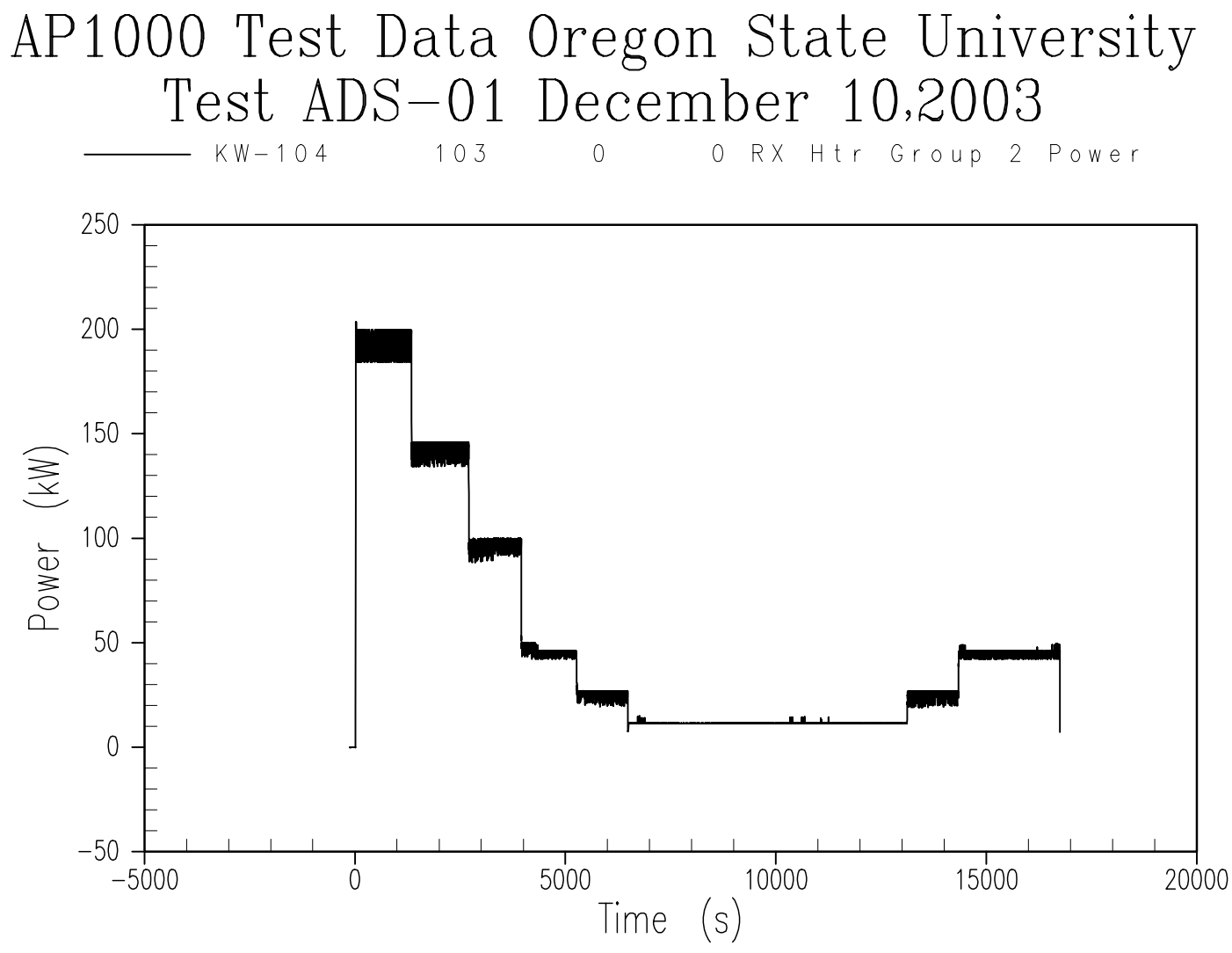

Figure A-23 Core Power Group 2 Alternate Input Power 
APPENDIX B

TEST DATA 


\title{
Oregon State University
}

Department of Nuclear Engineering

\author{
AdVANCED THERMAL HYdRAuliC \\ RESEARCH LABORATORY
}

\section{TEST SUMMARY REPORT}

OSU-AP1000-08

ADS-02-D

AP1000 ADS-4 CharaCterization - High POWER

Revision 0 


\title{
TEST SUMMARY REPORT
}

\author{
OSU-AP1000-08
}

ADS-02-D

AP1000 ADS-4 Characterization - High POWER

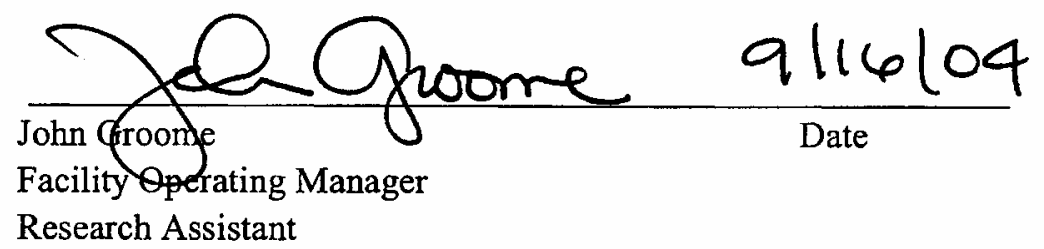

Research Assistant

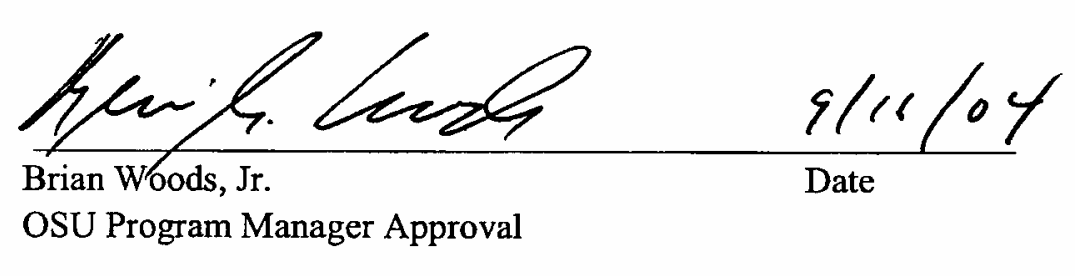

Oregon State University 116 Radiation Center

Corvallis, Oregon 97331

(C) 2004 Oregon State University

All Rights Reserved 


\section{WESTINGHOUSE PROPRIETARY INFORMATION NOTICE}

This report contains information proprietary to Westinghouse Electric Company. A non-proprietary version of this report has also been prepared for distribution to the United States Nuclear Regulatory Commission (NRC).

In order to conform to the requirements of 10 CFR 2.790 of the Commission's regulations concerning the protection of proprietary information so submitted to the $\mathrm{NRC}$, the information which is proprietary in the proprietary version of this document is contained within brackets, and where the proprietary information has been deleted in the non-proprietary versions, only the brackets remain (the information that was contained within the brackets in the proprietary versions having been deleted). The justification for claiming the information so designated as proprietary is indicated in both versions by means of lower case letters (a) through (f) located as a superscript immediately following the brackets enclosing each item of information being identified as proprietary or in the margin opposite such information. These lower case letters refer to the types of information Westinghouse customarily holds in confidence identified in Sections (4)(ii)(a) through (4)(ii)(f) of the affidavit accompanying the transmittal of these documents pursuant to 10 CFR $2.790(b)(1)$.

\section{COPYRIGHT NOTICE}

This document bears an Oregon State University copyright notice. The NRC is permitted to make the number of copies of the information contained in these reports which are necessary for its internal use in connection with generic and plant-specific reviews and approvals as well as the issuance, denial, amendment, transfer, renewal, modification, suspension, revocation, or violation of a license, permit, order, or regulation subject to the requirements of 10 CFR 2.790 regarding restrictions on public disclosure to the extent such information has been identified as proprietary by Westinghouse, copyright protection notwithstanding. With respect to the non-proprietary versions of these reports, the NRC is permitted to make the number of copies beyond these necessary for its internal use which are necessary in order to have one copy available for public viewing in the appropriate docket files in the public document room in Washington, DC and in local public document rooms as may be required by NRC regulations if the number of copies submitted is insufficient for this purpose. Copies made by the NRC must include the copyright notice in all instances and the proprietary notice if the original was identified as proprietary. 
TABLE OF CONTENTS

$\underline{\text { Section }}$

Title

Page

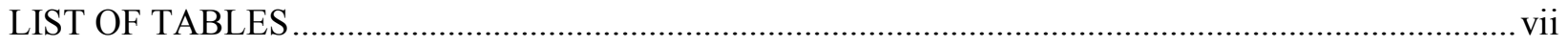

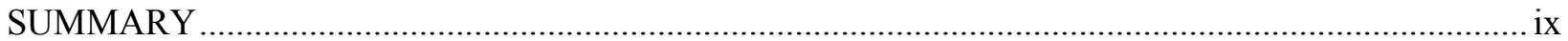

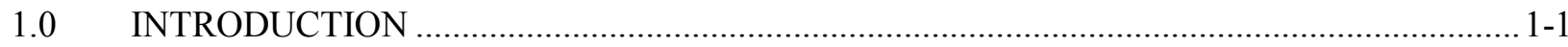

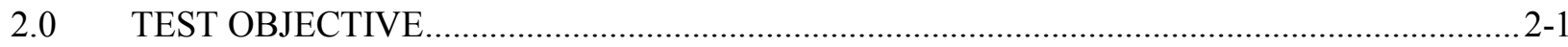

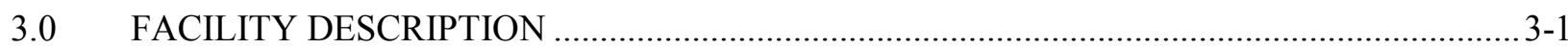

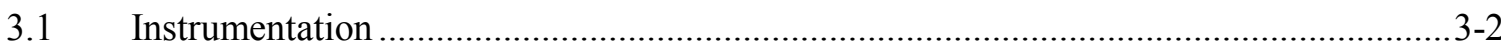

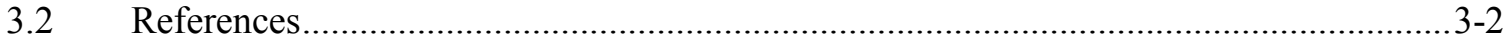

4.0 OREGON STATE UNIVERSITY TESTING PROGRAM MATRIX .......................................4-1

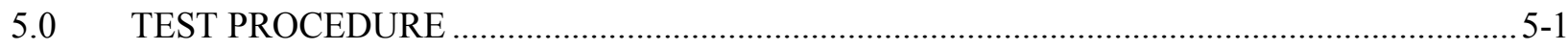

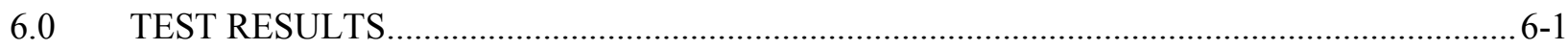

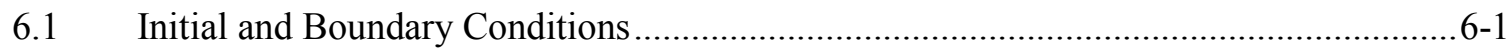

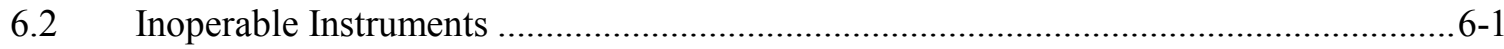

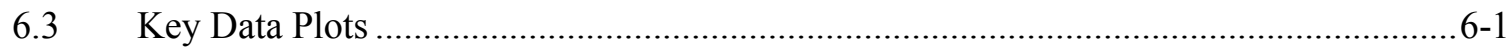

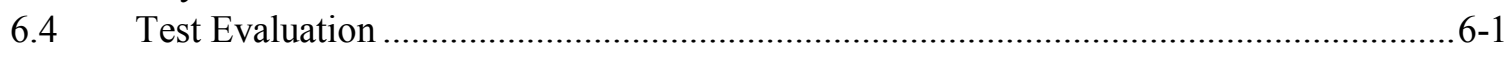

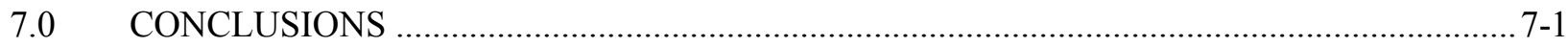

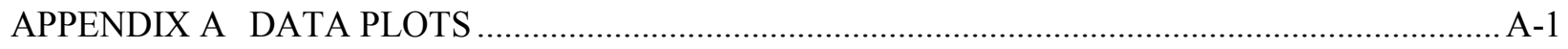

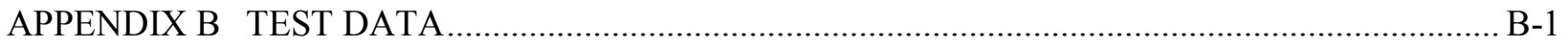




\section{LIST OF TABLES}

$\underline{\text { Table }}$

Table 4-1

Table 6-1

Table 6-2

Table 6-3 $\underline{\text { Title }}$

Page

OSU Test Matrix (as specified April 2, 2004) 4-2

Actual Test Initial Conditions... $6-2$

Inoperable Instruments for ADS-02-D Test...............................................................6-3

Data Plots for QL Reports for ADS-02-D by Component...... 6-4 


\section{SUMMARY}

This report covers the test ADS-02-D, Automatic Depressurization System 4 (ADS-4) characterization at high-power conditions, performed on April 2, 2004. This test has been designated OSU-AP1000-08 by the Advanced Plant Experiment (APEX). The objective of this test was to determine the steady-state two-phase flow and pressure drop characteristics of the ADS-4 lines at high-power conditions typical of the time period between ADS-4 actuation and in-containment refueling water storage tank injection. The ADS-4 line instrumentation was upgraded to include three differential pressure cells on each line. The test was conducted with the Reactor Coolant System drained down to the midpoint of the hot leg and the pressurizer empty. Core makeup flow was established at a constant rate using the Chemical and Volume Control System pump with a range of 0 to $7.5 \mathrm{gpm}$. The facility was run in this configuration for 15 to 30 minutes as liquid and vapor flows from the ADS-4 lines were measured along with the pressure drop distribution along the lines. This process was repeated by changing the Chemical and Volume Control System flow rate at a given power level. Three power levels were tested ranging from $430 \mathrm{~kW}$ to $60 \mathrm{~kW}$. In this way several injection flow/power steady-state conditions were established and the data recorded. For all cases, the two-phase mixture level was observed to be at or above the bottom of the hot leg, and liquid entrainment was measured in the ADS-4 lines. In addition, three steady-state points were established with the two-phase mixture level below the bottom of the hot leg. For these cases, little or no entrainment was observed.

This report presents the initial assessment of the test data collected. If this test is to be used by Westinghouse to support AP1000 Design Certification, additional validation of the use of this information will be documented separately. In the interim, the list of invalid data channels may change. 


\subsection{INTRODUCTION}

The Department of Nuclear Engineering at Oregon State University (OSU) is performing a series of tests for the U.S. Department of Energy. These tests are being conducted in the Advanced Plant Experiment (APEX-1000) test facility, which is a reduced pressure and height model of the two-loop Westinghouse AP1000 pressurized water reactor. The purpose of the testing is to:

- evaluate the thermal-hydraulic performance of the passive safety systems of the full-scale AP1000, and

- assess and validate the safety analyses techniques and computer codes used in predicting the transient system behavior.

The AP1000 Long-Term Cooling Test is a 1/4 height scale, low-pressure integral systems test simulating thermal-hydraulic phenomenon for the AP1000 passive safety systems for small-break loss-of-coolant accidents (LOCAs) and long-term cooling. It accurately models the details of the AP1000 geometry, including the primary system, the passive safety systems, and a part of the non-safety grade Chemical and Volume Control System, as well as a partial non-safety grade Normal Residual Heat Removal System. The interconnecting pipe routings are also duplicated in the model.

The overall objective of the Long-Term Cooling Test program is to obtain test data at various modes of operation. The OSU experiments will examine the passive safety system response for the small-break and large-break LOCA transition into long-term cooling. (The list of the tests to be performed is in the OSU Test Matrix provided in Section 4.0.) The facility permits a range of small-break LOCAs to be simulated at different locations on the primary system, such as the cold leg, hot leg, core makeup tank (CMT) cold leg pressure balance line, and direct vessel injection (DVI) line. The break orientation (top or bottom of the cold leg) may also be studied. Selected tests continue into the long-term cooling, post-accident mode in which the passive safety injection is from the reactor sump as well as the in-containment refueling water storage tank (IRWST). 


\subsection{TEST OBJECTIVE}

The purpose of this test is to repeat the previous test ADS-02-D, which was to obtain thermal-hydraulic data for the Automatic Depressurization System 4 (ADS-4) lines under steady-state conditions typical of the time period ranging from ADS-4 actuation to IRWST injection. The data obtained from the test will be used to verify the AP1000 thermal-hydraulic computer codes for AP1000 phenomena, such as gravity injection, natural convection, and post-accident long-term core cooling behavior.

The acceptance criteria for the OSU tests are as follows:

- Test initial conditions will be achieved within a specified tolerance.

- Setpoints will be achieved within an acceptable tolerance band.

- All instrumentation should be operational before the test.

- Any critical instruments not operating will be identified to the test engineer before the tests. These instruments must be operational before and during the test or exceptions should be approved.

- A zero check of LDPs, DPs, and FDPs will be performed. 


\subsection{FACILITY DESCRIPTION}

A detailed facility description for the OSU test facility is documented in Reference 3-1. The OSU test facility has been specifically scaled, designed, and constructed to investigate the AP600 passive safety system behavior and to provide data for safety analysis computer code validation. The facility has been modified to model the AP1000.

The scaled test design accurately models the details of the AP1000 geometry, including the primary system and the pipe routings and layout for the passive safety systems. A detailed scaling report (Reference 3-2) was used to develop the test design modifications. The primary system consists of one hot leg and two cold legs with two active pumps and an active steam generator for each loop. Two CMTs are connected to one primary loop, and the pressurizer is connected to the other primary loop as in the AP1000 plant design. Gas-driven accumulators are connected to the DVI lines. The discharge lines from the CMT, and one-of-two IRWST and reactor sump lines are connected to each DVI line. The ADS consisting of stages 1,2 , and 3 - simulates either one or two of the independent trains used in the AP1000. The two-phase flow from the ADS stages 1-to-3 is separated in a swirl-vane separator, and the liquid and vapor flows are measured to obtain the total ADS flow rate. The separated flow streams are then recombined and discharged into the IRWST through a sparger, preserving the mass and energy flow into the IRWST. The injection from the reactor sump is also simulated. Note that the OSU facility models both AP1000 primary and secondary sumps. The primary sump collects the condensate return, the liquid break flow, and the liquid flow from the fourth-stage ADS; and will provide long-term injection to the reactor vessel. The secondary sump simulates the portions of containment that will remain dry during most events. This sump collects water only when the primary sump reaches its overflow level, and provides no injection to the reactor vessel.

The time period for the experimental simulations includes not only the IRWST injection, but also the draining of the IRWST and the sump injection to simulate the long-term cooling of the AP1000. This simulation could be from several hours to a day. The time scale for the OSU test facility is one-half; that is, events occur in half the normal time. To model the long-term cooling aspects of the transient, the two-phase flow from the break is separated in a swirl-vane separator and the liquid and vapor portions of the total flow are measured. The liquid fraction of the flow is discharged to the reactor primary sump as in the AP1000 plant. The vapor is discharged to the atmosphere. The capability exists to return a portion of the equivalent liquid flow to the IRWST and primary sump to simulate the condensate return from the passive containment to the IRWST and primary sump. A similar approach is also used for the fourth-stage ADS valve on the hot leg. The two-phase flow is separated in a swirl-vane separator, the two streams are measured, and the liquid phase is discharged into the primary sump while the vapor flow is discharged to the atmosphere. Again, the capability exists to return a portion of the liquid equivalent added to the IRWST and primary sump. In addition, all other steam vents from the facility are measured (for example, the IRWST vent), and a portion of the liquid equivalent may be added back to the facility. Note that not all of the steam discharge would be returned as liquid equivalent. A portion of the discharge would be removed to simulate the steam that is not available for recirculation because it provides containment pressurization. The IRWST and primary sump can be pressurized in the OSU facility to simulate the containment pressurization following a postulated LOCA.

A multi-tube passive residual heat removal heat exchanger is located in the IRWST. The heat exchanger uses the same C-tube design as the AP1000 and has two instrumented tubes to obtain wall heat fluxes 
during the tests. There are primary fluid thermocouples, wall thermocouples, and differential pressure drop measurements to determine when the heat exchanger begins to drain. The IRWST is also instrumented with strings of fluid thermocouples to determine the degree of mixing within the tank and to assess the temperature of the coolant that is delivered to the test vessel.

The reactor vessel for the OSU tests includes a 0.914-meter (3-foot) heated core consisting of forty-eight 0.025 -meter (1-inch) diameter heater rods. The heater rods have a top skewed power shape. The $1000 \mathrm{~kW}$ of electrical power available at the OSU test site will be used to simulate decay heat. Wall thermocouples are swaged inside the heater rods to measure the heater rod wall temperature.

Thermocouple rods in the heater rod bundle measure the axial coolant temperature distribution. The scaled flow volume in the core is preserved as well as the flow volume in the test vessel upper plenum. There are simulated reactor internals in the upper plenum to preserve the flow area and to correctly scale the fluid volume. The reactor vessel includes an annular downcomer into which the four cold legs and the two DVI lines are connected. The hot legs penetrate the reactor annulus and connect with the loops. The AP1000 reactor vessel neutron reflector is simulated using a ceramic liner to reduce the metal heat release to the coolant.

There were no special/unique requirements for the test other than those specified in the Initial Conditions. The specified conditions were verified on the control board prior to test implementation.

\subsection{Instrumentation}

The instrumentation has been designed to calculate a transient mass and energy balance on the test facility. All two-phase flow streams exiting the facility are separated, and each component is measured separately as a single-phase flow using conventional measurement devices, such as magnetic flow meters and vortex flow meters. Note that magnetic flow meters are not designed for two-phase flow and will indicate erratically. Also, the vortex flow meters are referenced to $212^{\circ} \mathrm{F}$ and the LDPs are referenced to $60^{\circ} \mathrm{F}$. All vertical components have differential pressure cells that act as level instruments to measure the transient mass change in the component. The hot and cold leg diameters are sufficiently large in the OSU test facility so that a narrow-range differential pressure cell can be used to determine if the flow becomes stratified.

Single flow measurements are made of the CMT, accumulator, IRWST, and sump flows into the reactor vessel through the DVI lines.

Various types of instrumentation are provided in the test facility; for example, thermocouples for coolant and wall temperatures, flow meters, pressure transducers, differential pressure transducers, and weigh tanks.

\subsection{References}

3-1 APP-LTCT-T2R-005, OSU APEX-1000 Test Facility Description Report

3-2 APP-LTCT-T2R-004, Scaling Assessment for the Design of the OSU APEX-1000 Test Facility 


\subsection{OREGON STATE UNIVERSITY TESTING PROGRAM MATRIX}

The test matrix for the OSU test facility is shown in Table 4-1. To satisfy the test objectives, several transients will be performed to provide data on the AP1000 passive safety system response for a range of break sizes, locations, orientations, and single failure assumptions. The break size orifices are scaled based on simulating a 0.5 -inch, 1-inch, 2 -inch, or larger pipe break.

The designation for this test is ADS-02-D, which identifies the test as an ADS-4 characterization test. The test matrix may be adjusted for future tests based on results and insights gained with each test. 


\begin{tabular}{|c|c|c|}
\hline \multicolumn{3}{|c|}{$\begin{array}{c}\text { TABLE 4-1 } \\
\text { OSU TEST MATRIX (AS SPECIFIED APRIL 2, 2004) }\end{array}$} \\
\hline Test Title & Break Location and Size & Single Failure Assumed \\
\hline DBA-01-D & $\begin{array}{l}\text { Double-ended DVI Line break with } \\
\text { continuation into long-term cooling }\end{array}$ & Fail 1 of 2 lines in one ADS- 4 train \\
\hline DBA-02-D & $\begin{array}{l}\text { Double-ended DVI Line break with } \\
\text { continuation into long-term cooling }\end{array}$ & $\begin{array}{l}\text { Fail } 1 \text { of } 2 \text { lines in one ADS- } 4 \text { train } \\
\text { (Adjusted ADS-4 Resistance) }\end{array}$ \\
\hline DBA-03-D & $\begin{array}{l}\text { Double-ended DVI Line break with } \\
\text { continuation into long-term cooling }\end{array}$ & $\begin{array}{l}\text { Fail } 1 \text { of } 2 \text { lines in one ADS- } 4 \text { train } \\
\text { (Failure location sensitivity) }\end{array}$ \\
\hline TR-01-D & $\begin{array}{l}\text { Transition Test ADS- } 4 \text { opening, } 85 \text { psig initial } \\
\text { pressure and decay power } 480 \mathrm{sec}\end{array}$ & $\begin{array}{l}\text { Fail } 1 \text { of } 2 \text { lines in one ADS-4 train } \\
\text { (No ADS 1-3) }\end{array}$ \\
\hline TR-02-D & $\begin{array}{l}\text { Transition Test ADS-4 opening, } 100 \text { psig initial } \\
\text { pressure with prototypic plant conditions }\end{array}$ & $\begin{array}{l}\text { Fail } 1 \text { of } 2 \text { lines in one ADS-4 train } \\
\text { (No ADS 1-3) }\end{array}$ \\
\hline DBA-04-D & 2-inch Cold Leg Break & $\begin{array}{l}\text { Fail } 1 \text { of } 2 \text { lines in one ADS- } 4 \text { train } \\
\text { (Failure on non-pressurizer hot leg) }\end{array}$ \\
\hline ADS-01-D & $\begin{array}{l}\text { ADS-4 Two-Phase Characterization Test at } \\
\text { low-power conditions (steady-state points) }\end{array}$ & $\begin{array}{l}\text { Various ADS-4 line configurations } \\
\text { (No ADS 1-3) }\end{array}$ \\
\hline ADS-02-D & $\begin{array}{l}\text { ADS-4 Two-Phase Characterization Test at } \\
\text { high-power conditions (steady-state points) }\end{array}$ & $\begin{array}{l}\text { Various ADS-4 line configurations } \\
\text { (No ADS 1-3) }\end{array}$ \\
\hline DBA-03R-D & Repeat of Test DBA-03-D & $\begin{array}{l}\text { Fail } 1 \text { of } 2 \text { lines in one ADS- } 4 \text { train } \\
\text { (Failure on pressurizer hot leg) }\end{array}$ \\
\hline DBA-05-D & Inadvertent ADS-1 Actuation & $\begin{array}{l}\text { Fail } 1 \text { of } 2 \text { lines in one ADS- } 4 \text { train } \\
\text { (Failure on non-pressurizer hot leg) }\end{array}$ \\
\hline DBA-06-D & 0.5-inch Cold Leg Break & $\begin{array}{l}\text { Fail } 1 \text { of } 2 \text { lines in one ADS-4 train } \\
\text { (Failure on non-pressurizer hot leg) }\end{array}$ \\
\hline
\end{tabular}




\subsection{TEST PROCEDURE}

The test was performed per a written procedure. There were no special/unique requirements for the test other than those specified in the initial conditions in Table 6-1. The specified conditions were checked on the control board before test implementation.

The appropriate prerequisites were completed and initial conditions were satisfied.

Fill and vent was performed per APEX Operations Manual Procedure OP-B.2. Instruments were checked for required calibration.

Since the CMTs and accumulators are not used in this test, normal procedures to ensure proper initial conditions were not used.

Once all other initial conditions were satisfied, ADS-4 was actuated. The reactor power was set to a constant value, and the Chemical and Volume Control System pump controller was used to set the injection flow to a constant value. Once steady-state was established (typically 2000 seconds), the flow was changed and another steady-state point was tested. This was repeated for five injection flows per power level to simulate core exit quality in the range of 0 to 100 percent. After five different flow rates were tested, the core power was increased and the process was repeated for $60 \mathrm{~kW}, 150 \mathrm{~kW}$, and $430 \mathrm{~kW}$. After the fifth point was completed at the high-power level, the flow was reduced below the core steaming rate and the two-phase mixture level fell below the hot leg elevation. Two additional points were examined at the reduced mixture level to determine the effect on liquid entrainment. 


\subsection{TEST RESULTS}

The test results for test ADS-02-D are provided in the following subsections.

\subsection{Initial and Boundary Conditions}

Table 6-1 provides a comparison of the specified and actual conditions for test ADS-02-D. The values in this table were averaged over approximately 2 minutes preceding the test.

\subsection{Inoperable Instruments}

Table 6-2 lists the instrumentation channels considered inoperable for the ADS-02-D test.

\subsection{Key Data Plots}

Table 6-3 lists the instrumentation channels sorted by component and includes the instrument number and units. The selection of channels was based on projecting an overall picture of the test results, which would then be examined by referring to the detailed data plots or tapes.

\subsection{Test Evaluation}

The following observations were made during the test:

1. Steady-state ADS-4 flows were observed after approximately 2000 seconds at each core power/injection flow level.

2. The two-phase mixture level in the upper plenum remained at or above the hot leg for all power/flow conditions tested except when the flow was reduced below the steaming rate and the mixture level fell into the upper plenum.

3. Liquid entrainment was observed from the ADS-4 for the cases where the injection flow was greater than the steaming rate and the two-phase mixture level was at or above the bottom of the hot leg for all power/flow conditions tested.

4. Liquid entrainment approached zero when the injection flow was equal to the core steaming rate or the two-phase mixture level was below the bottom of the hot leg. 


\begin{tabular}{||c|c|c|c||}
\hline \multicolumn{5}{|c||}{ TABLE 6-1 } \\
\hline \multicolumn{1}{||c|}{ Conditions } & Instrument No. & Actual & Comment \\
\hline Upper Head Pressure & PT-107 & $2 \mathrm{psig}$ & \\
\hline Hot Leg Temperature \#1 & TF-141* & $216^{\circ} \mathrm{F}$ & \\
\hline Hot Leg Temperature \#2 & TF-140* & $216^{\circ} \mathrm{F}$ & \\
\hline IRWST Temperature & TF-701 & $68.5^{\circ} \mathrm{F}$ & \\
\hline
\end{tabular}

* These instruments are used to establish initial conditions at the test site. 


\begin{tabular}{|c|c|c|}
\hline \multicolumn{3}{|c|}{$\begin{array}{c}\text { TABLE 6-2 } \\
\text { INOPERABLE INSTRUMENTS FOR ADS-02-D TEST }\end{array}$} \\
\hline Instrument Number & Instrument Type & Inoperable Description \\
\hline $\begin{array}{l}\text { TW-202 } \\
\text { TW-204 } \\
\text { TW-205 } \\
\text { TW-206 } \\
\text { TW-209 } \\
\text { TW-803 } \\
\text { TW-804 }\end{array}$ & Thermocouple & Inoperative \\
\hline TH-603 & $\begin{array}{l}\text { Thermocouple measuring heater } \\
\text { temperature }\end{array}$ & Inoperative \\
\hline FMM-202 & Magnetic flow meter & Inoperative \\
\hline $\begin{array}{l}\text { TF-170 } \\
\text { TF-221 } \\
\text { TF-509 } \\
\text { TF-512 }\end{array}$ & $\begin{array}{l}\text { Thermocouple measuring fluid } \\
\text { temperature }\end{array}$ & Inoperative \\
\hline FVM-905 & Vortex flow meter & Erratic \\
\hline
\end{tabular}




\begin{tabular}{|c|c|c|c|}
\hline \multicolumn{4}{|c|}{$\begin{array}{c}\text { TABLE 6-3 } \\
\text { DATA PLOTS FOR QL REPORTS FOR ADS-02-D BY COMPONENT }\end{array}$} \\
\hline Component & Channel & Units & Comment \\
\hline Reactor Vessel Pressure & PT-107 & psig & Nearly atmospheric \\
\hline Reactor Vessel Level & LDP-127 & inch of $\mathrm{H}_{2} \mathrm{O}$ & $\begin{array}{l}\text { Collapsed level follows } \\
\text { reactor power }\end{array}$ \\
\hline $\begin{array}{l}\text { Reactor Vessel Downcomer } \\
\text { Level }\end{array}$ & LDP-140 & inch of $\mathrm{H}_{2} \mathrm{O}$ & \\
\hline $\begin{array}{l}\text { Reactor Two-Phase Mixture } \\
\text { Level }\end{array}$ & LT-120 & inches & $\begin{array}{l}\text { Level always at or above } \\
\text { hot leg }\end{array}$ \\
\hline $\begin{array}{l}\text { Reactor Vessel Fluid } \\
\text { Temperature Upper Head }\end{array}$ & TF-120 & ${ }^{\circ} \mathrm{F}$ & Saturated \\
\hline $\begin{array}{l}\text { Chemical and Volume Control } \\
\text { System Pump Flow }\end{array}$ & FMM-801 & gpm & $\begin{array}{l}\text { Step changes to specify } \\
\text { injection flow }\end{array}$ \\
\hline IRWST Fluid Temperature & TF-701 and TF-709 & ${ }^{\circ} \mathrm{F}$ & \\
\hline $\begin{array}{l}\text { ADS 4-1 Separator Steam } \\
\text { Flow Rate }\end{array}$ & FVM-603 & scfm & \\
\hline $\begin{array}{l}\text { ADS 4-2 Separator Steam } \\
\text { Flow Rate }\end{array}$ & FVM-602 & scfm & \\
\hline $\begin{array}{l}\text { ADS 4-1 Separator Liquid } \\
\text { Flow Rate }\end{array}$ & FMM-603 & gpm & \\
\hline $\begin{array}{l}\text { ADS 4-2 Separator Liquid } \\
\text { Flow Rate }\end{array}$ & FMM-602 & gpm & \\
\hline Core Power Input Power & $\begin{array}{l}\text { KW-101, KW-102, } \\
\text { KW-103, and KW-104 }\end{array}$ & $\mathrm{kW}$ & Step changes \\
\hline
\end{tabular}




\subsection{CONCLUSIONS}

The ADS-02-D tests were successfully completed, and the data was logged in the DAS. All critical instruments were found to operate properly with the exception of those noted in Section 6.4. The test was acceptable. 
APPENDIX A

DATA PLOTS 

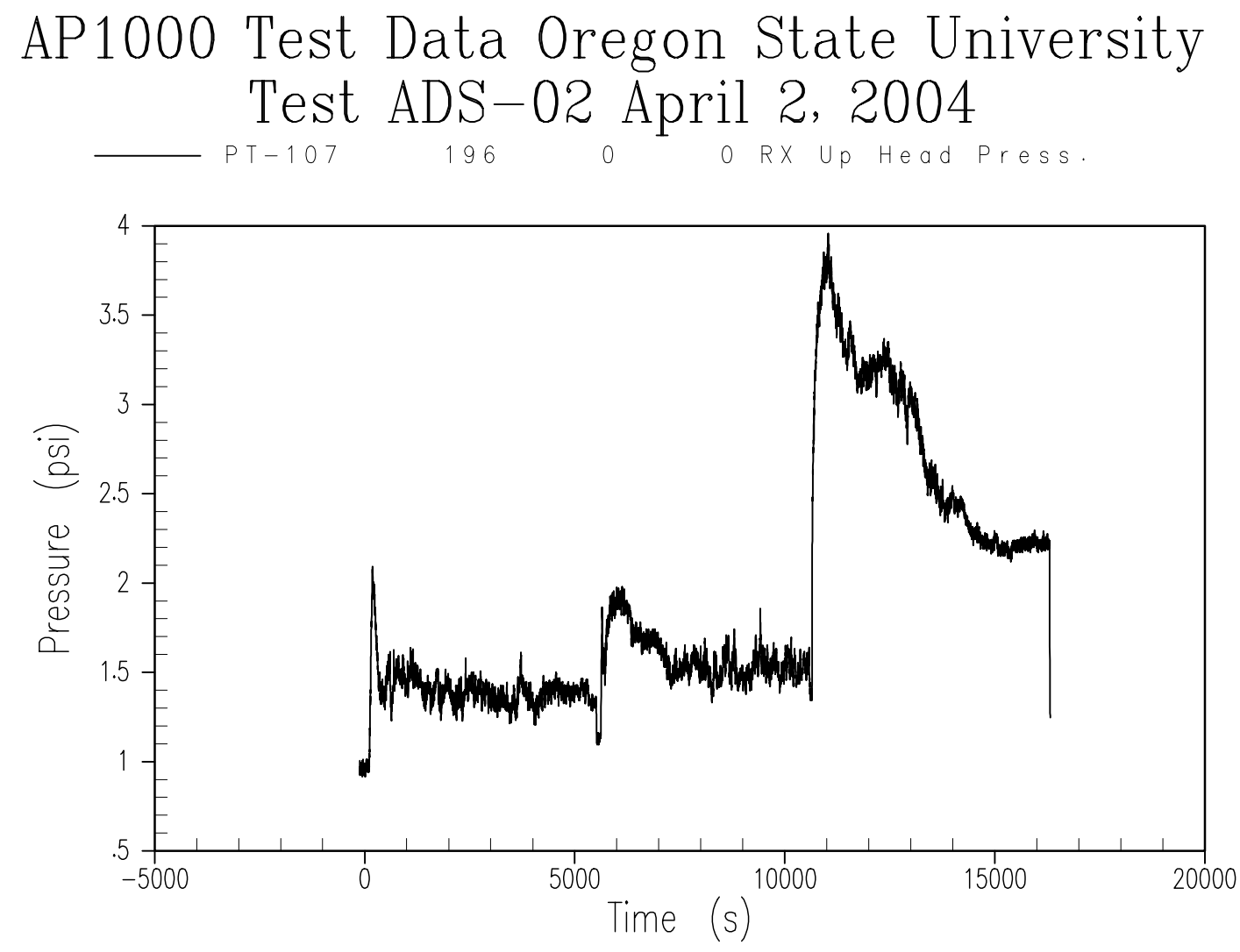

Figure A-1 Reactor Vessel Pressure 

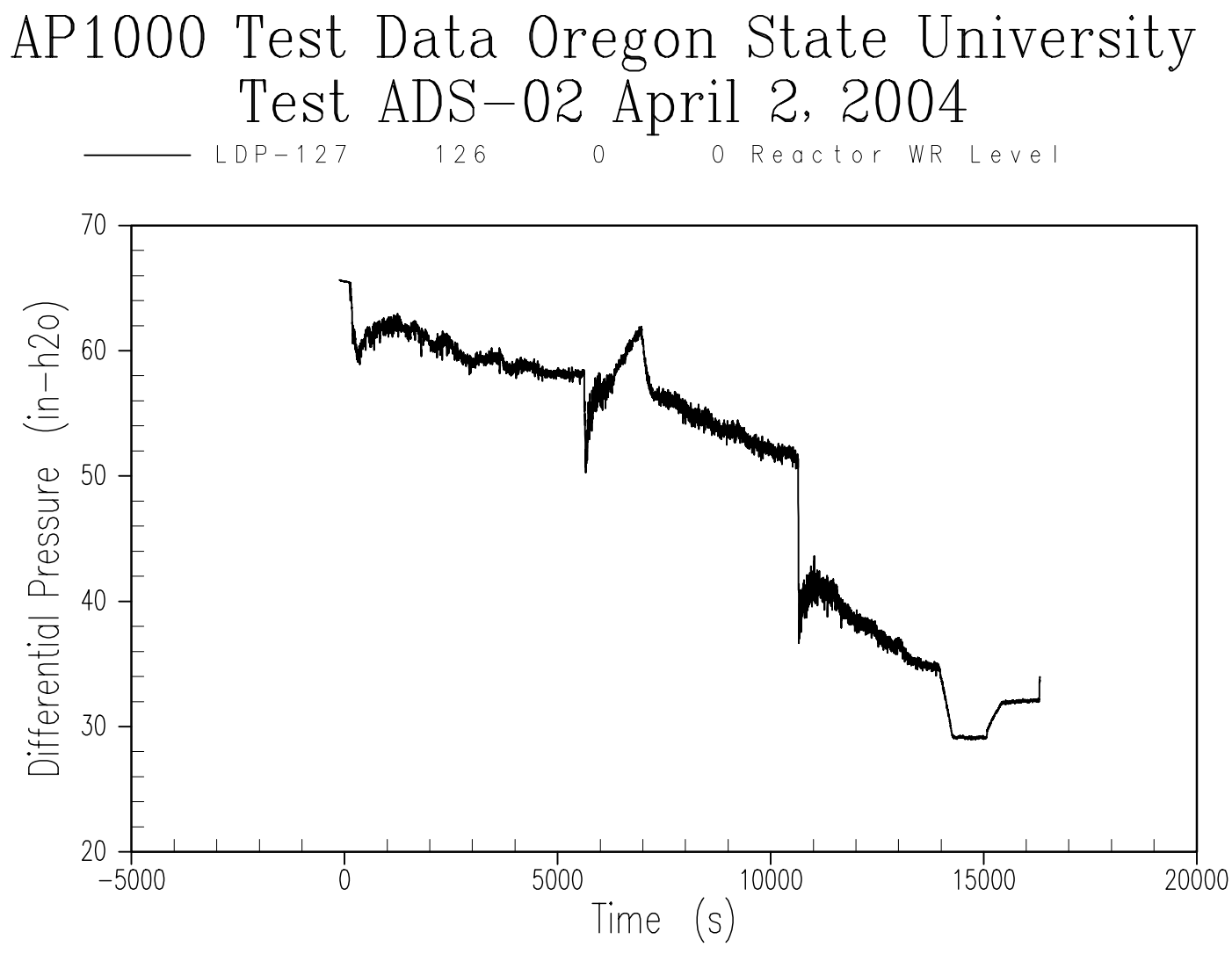

Figure A-2 Reactor Vessel Level 

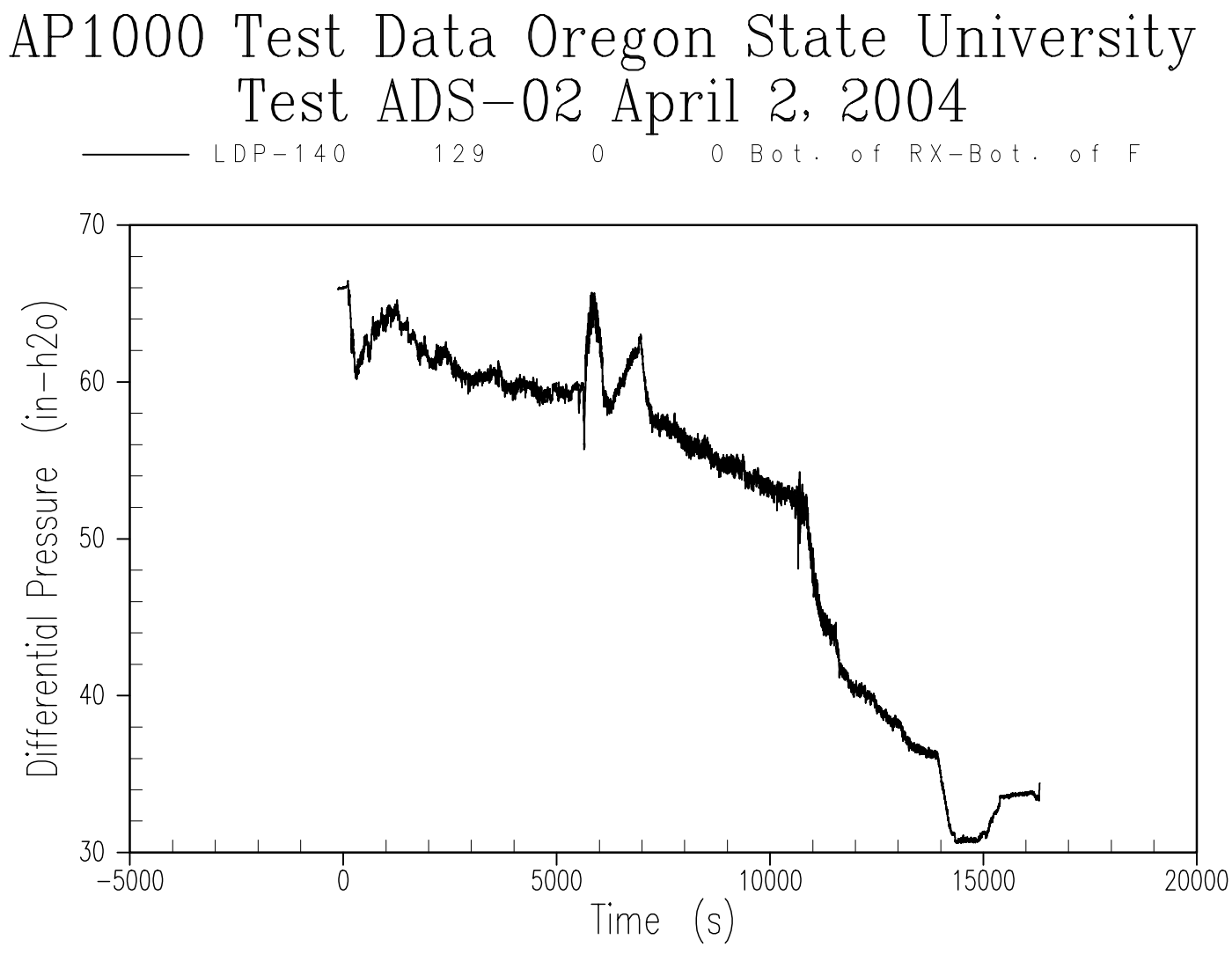

Figure A-3 Reactor Vessel Downcomer Level 

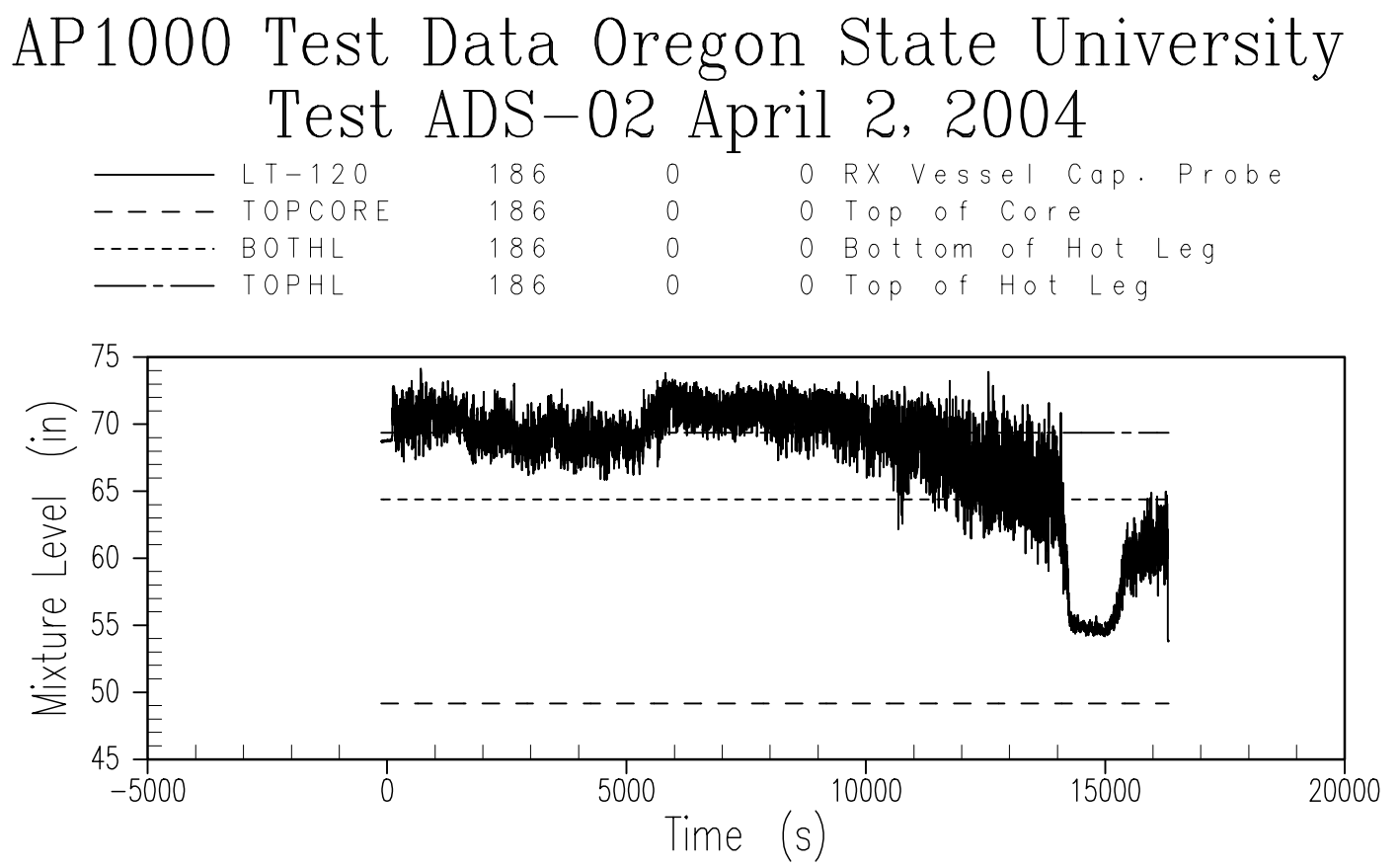

Figure A-4 Two-Phase Mixture Level in Upper Plenum 

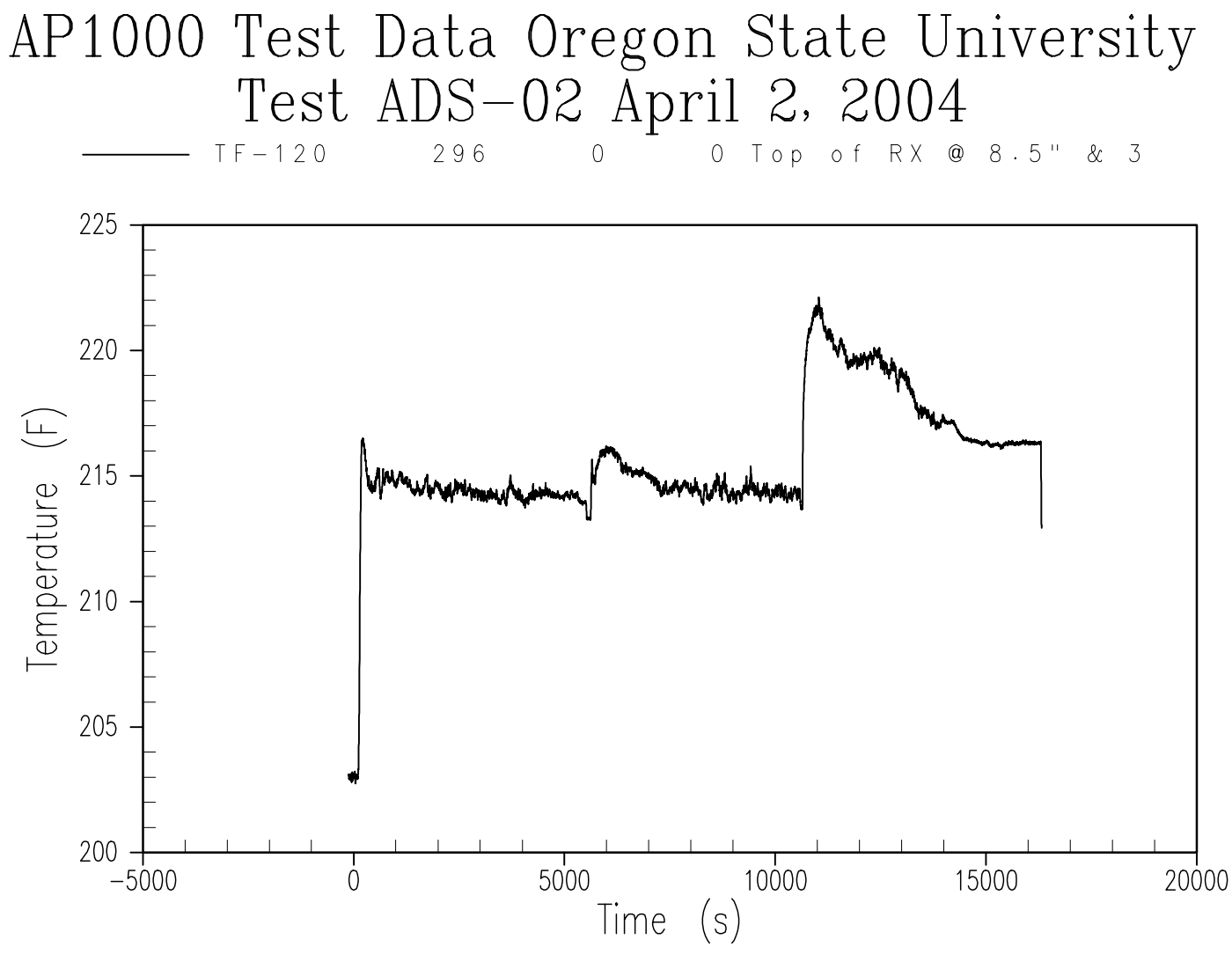

Figure A-5 Upper Head Temperature 

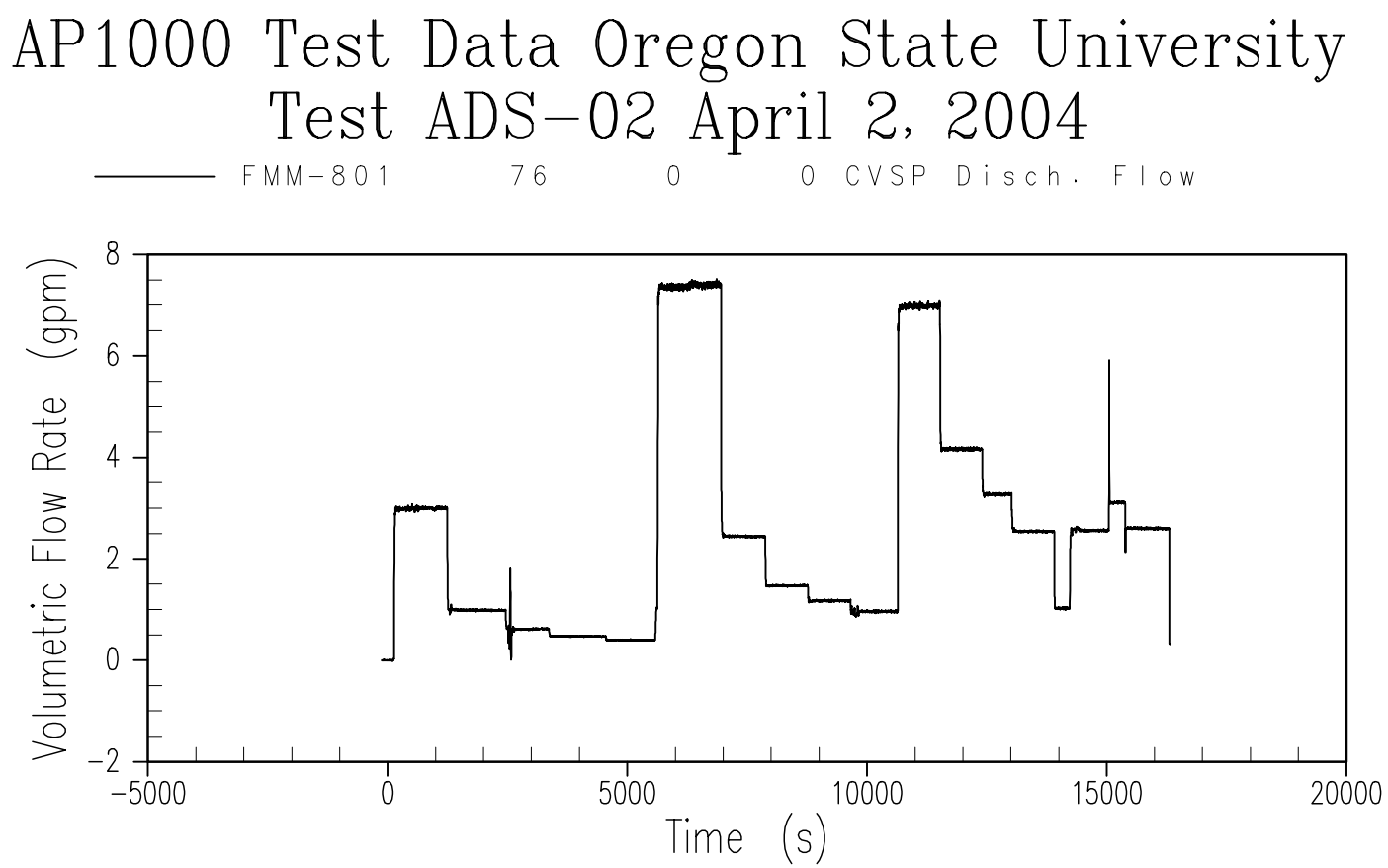

Figure A-6 Chemical and Volume Control System Injection Flow 

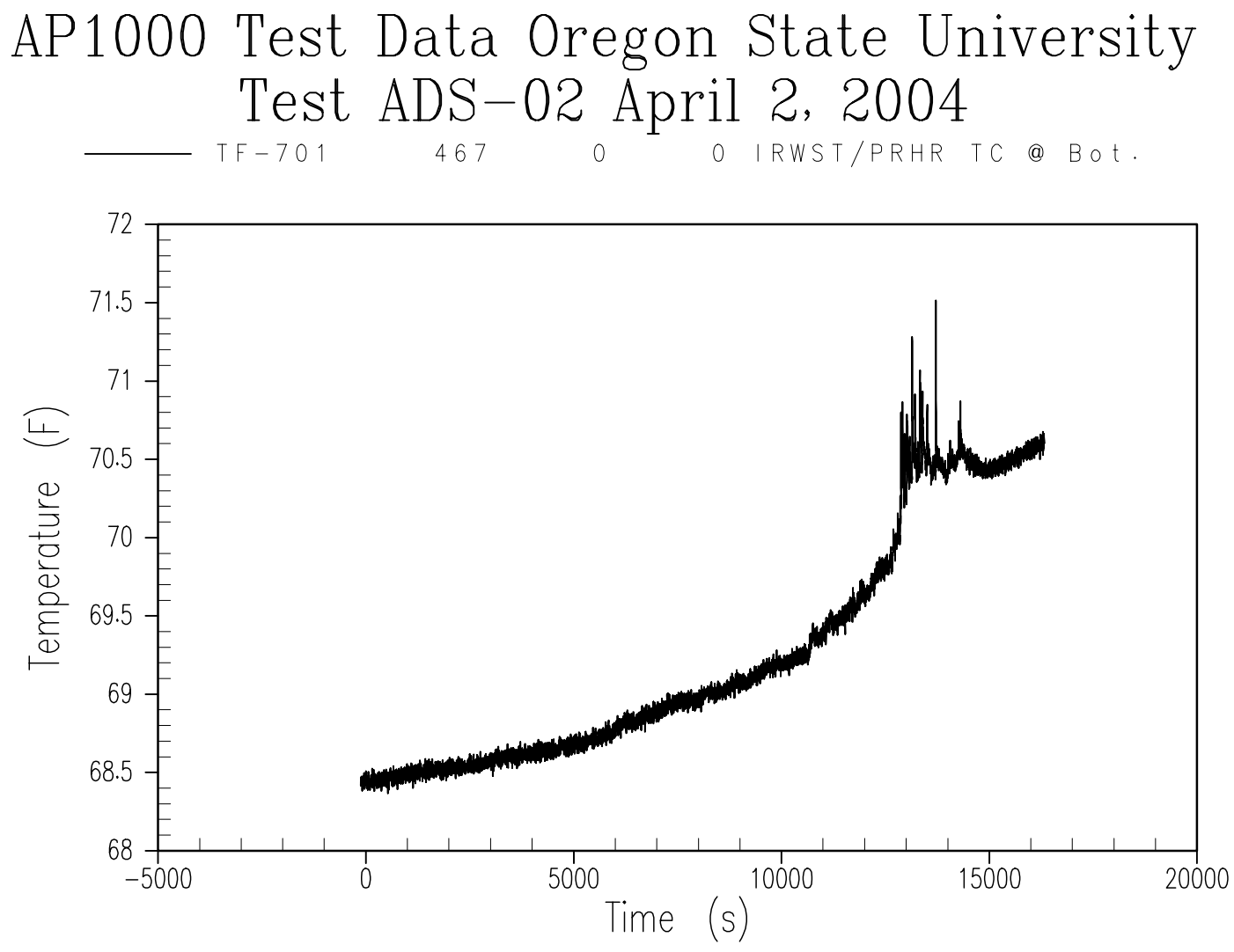

Figure A-7 IRWST Fluid Temperature - Bottom 

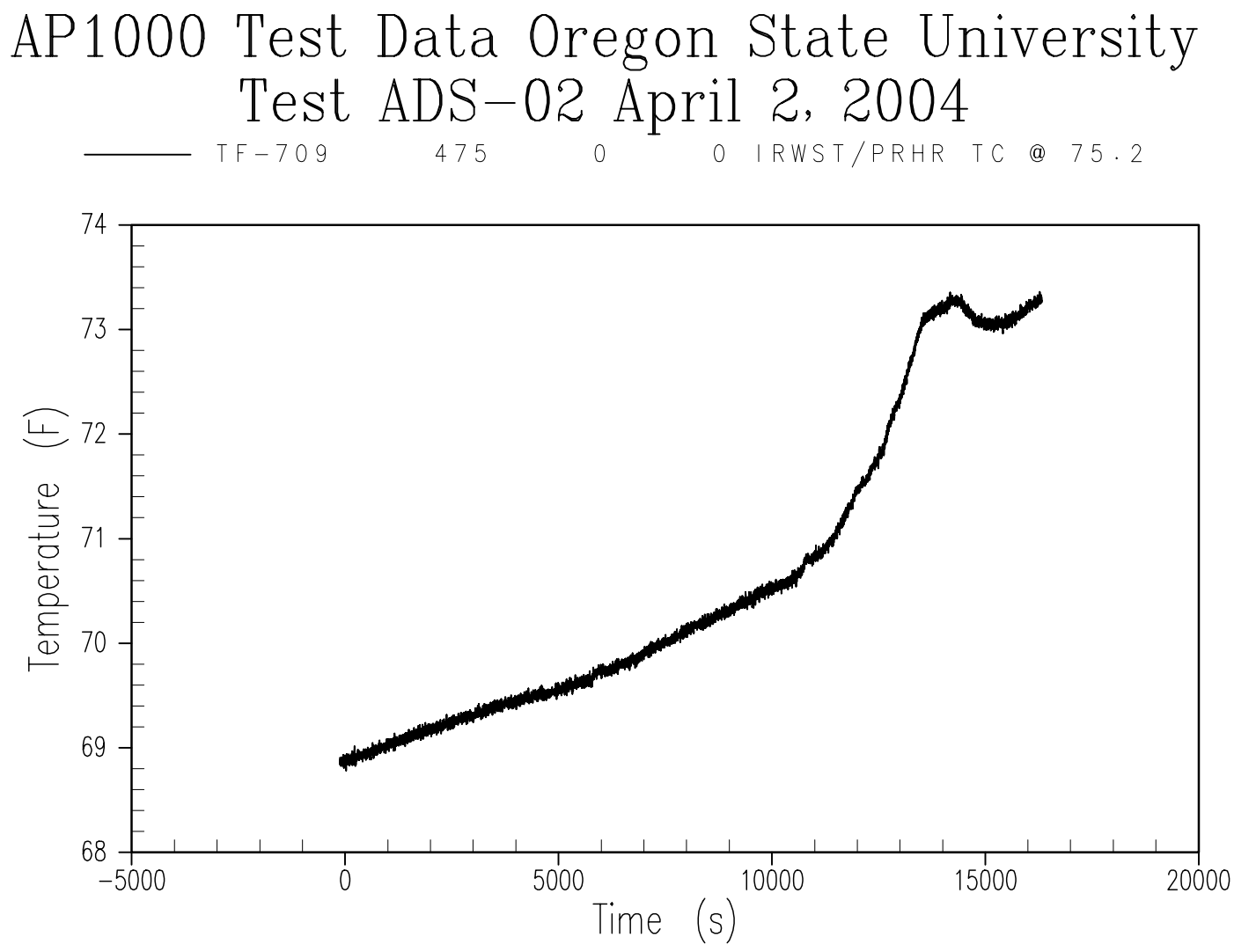

Figure A-8 IRWST Fluid Temperature - Top 

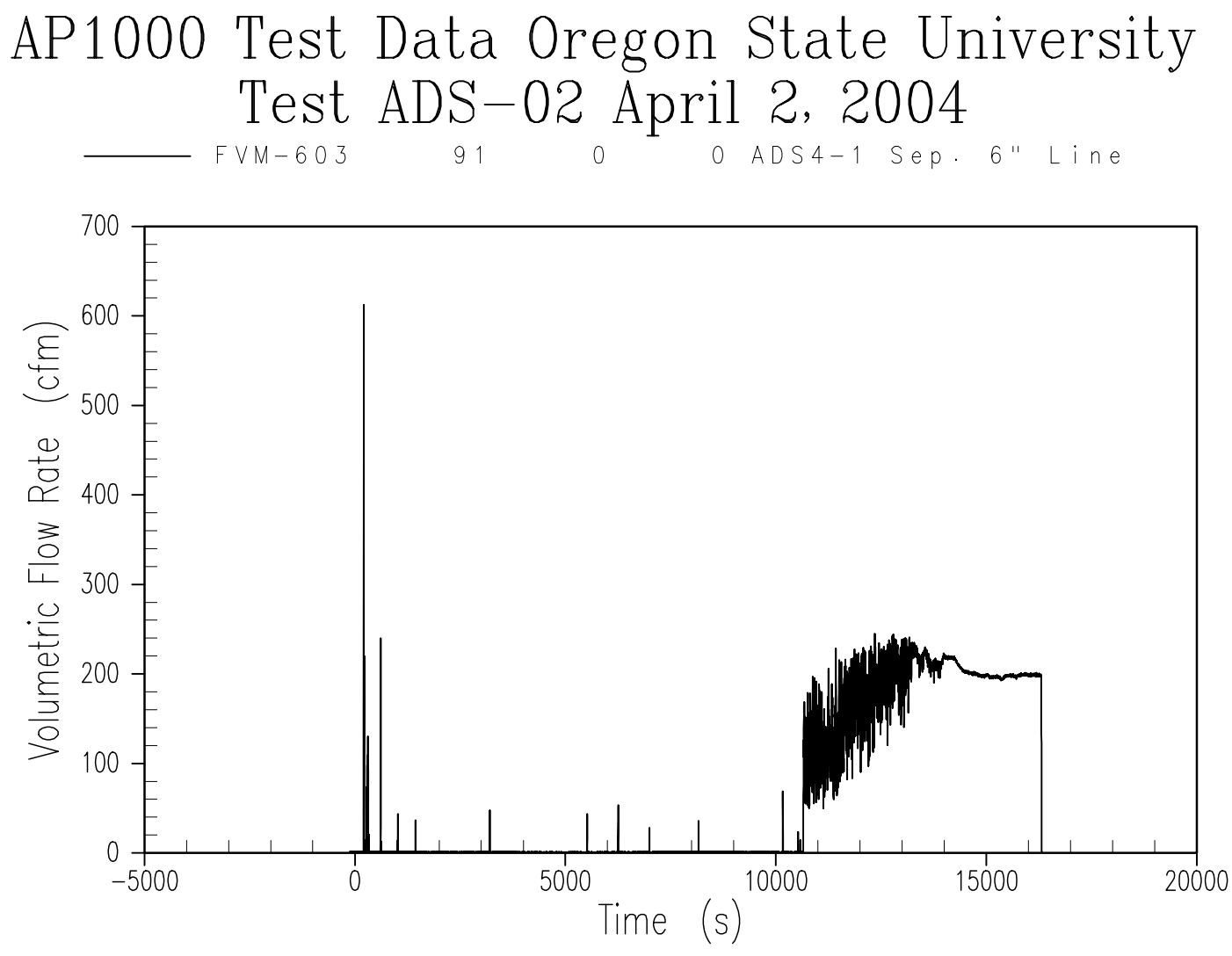

Figure A-9 ADS 4-1 Separator Steam Flow Rate 

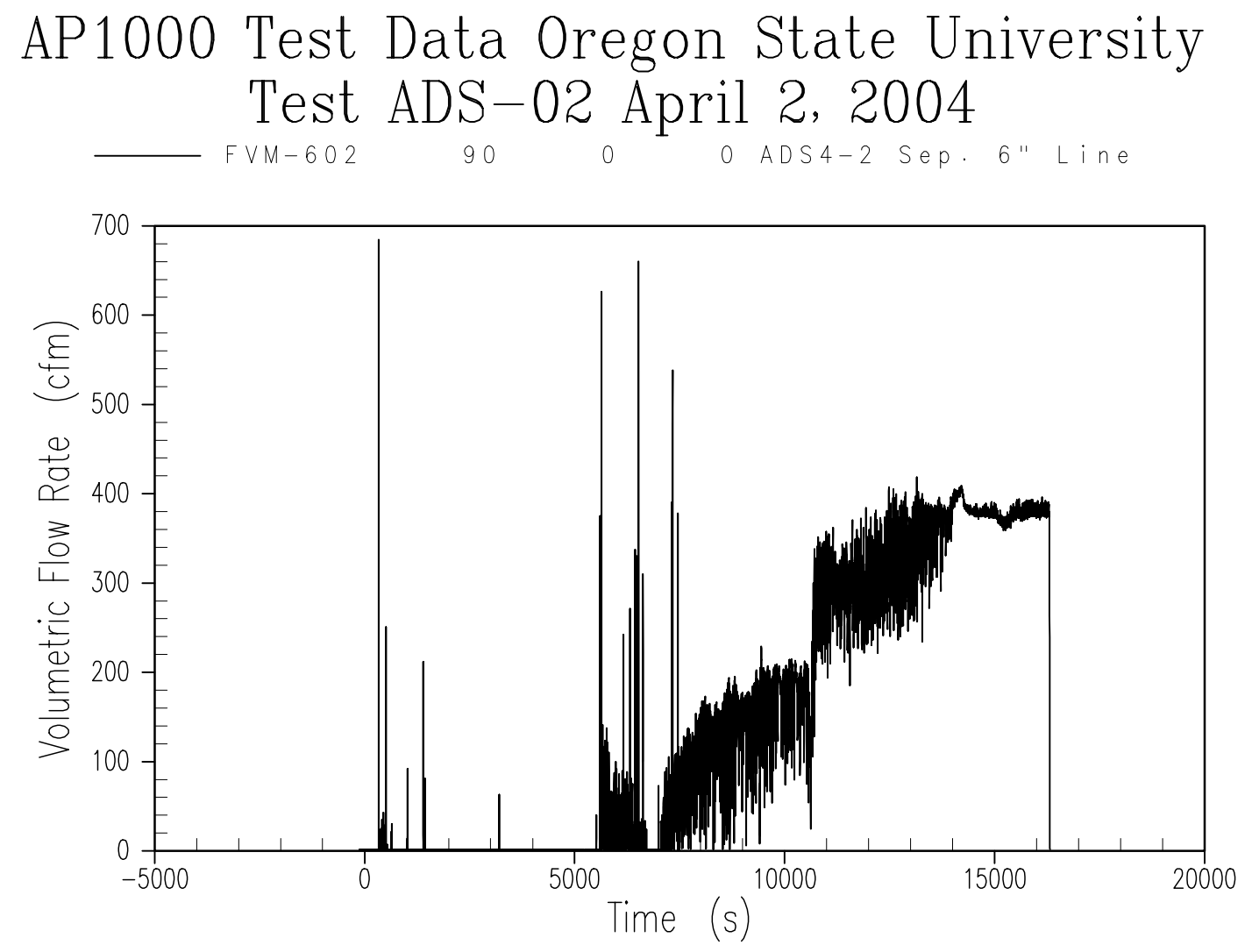

Figure A-10 ADS 4-2 Separator Steam Flow Rate 

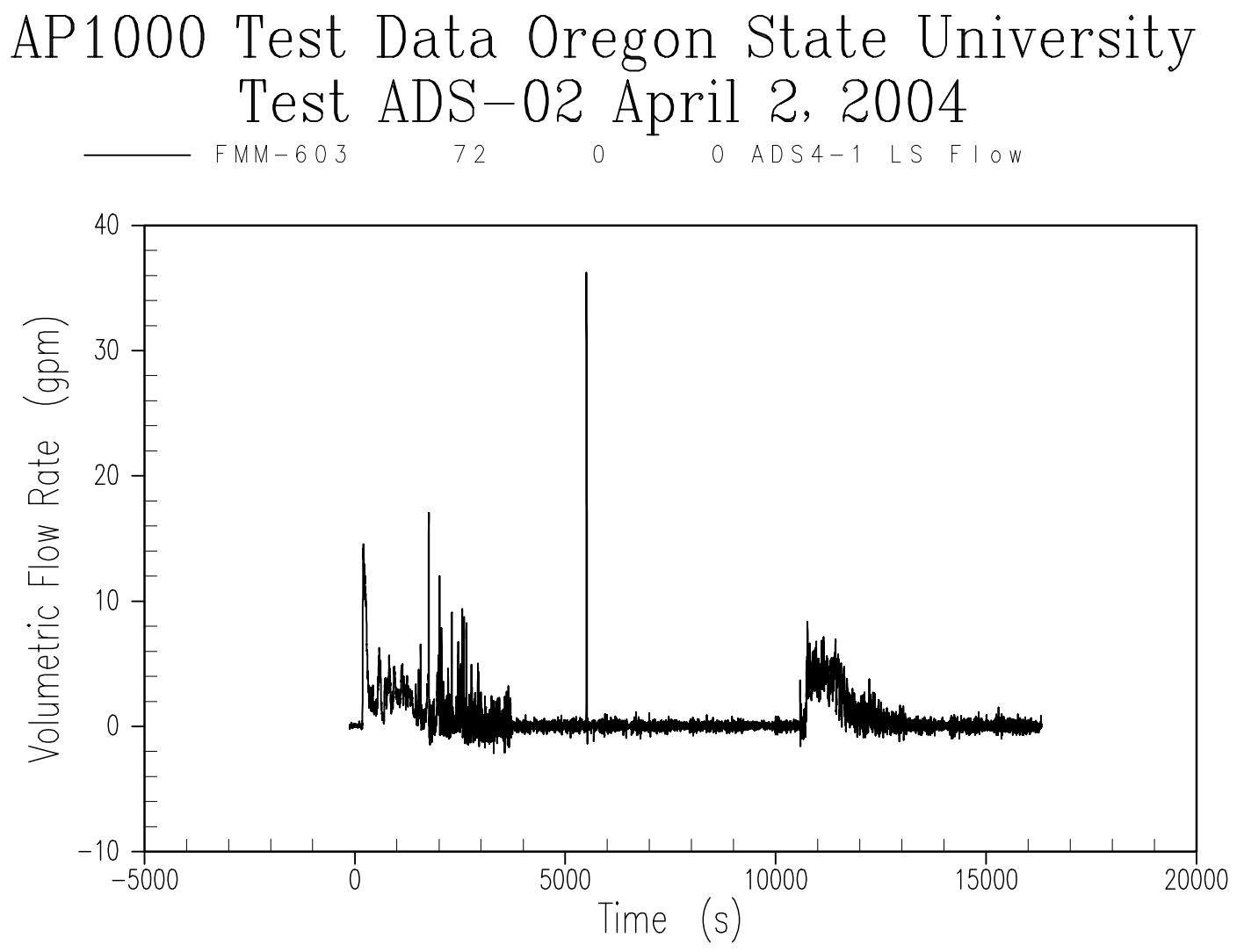

Figure A-11 ADS 4-1 Separator Liquid Flow Rate 


\section{AP1000 Test Data Oregon State University Test ADS-02 April 2, 2004

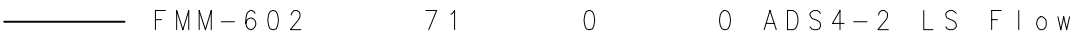

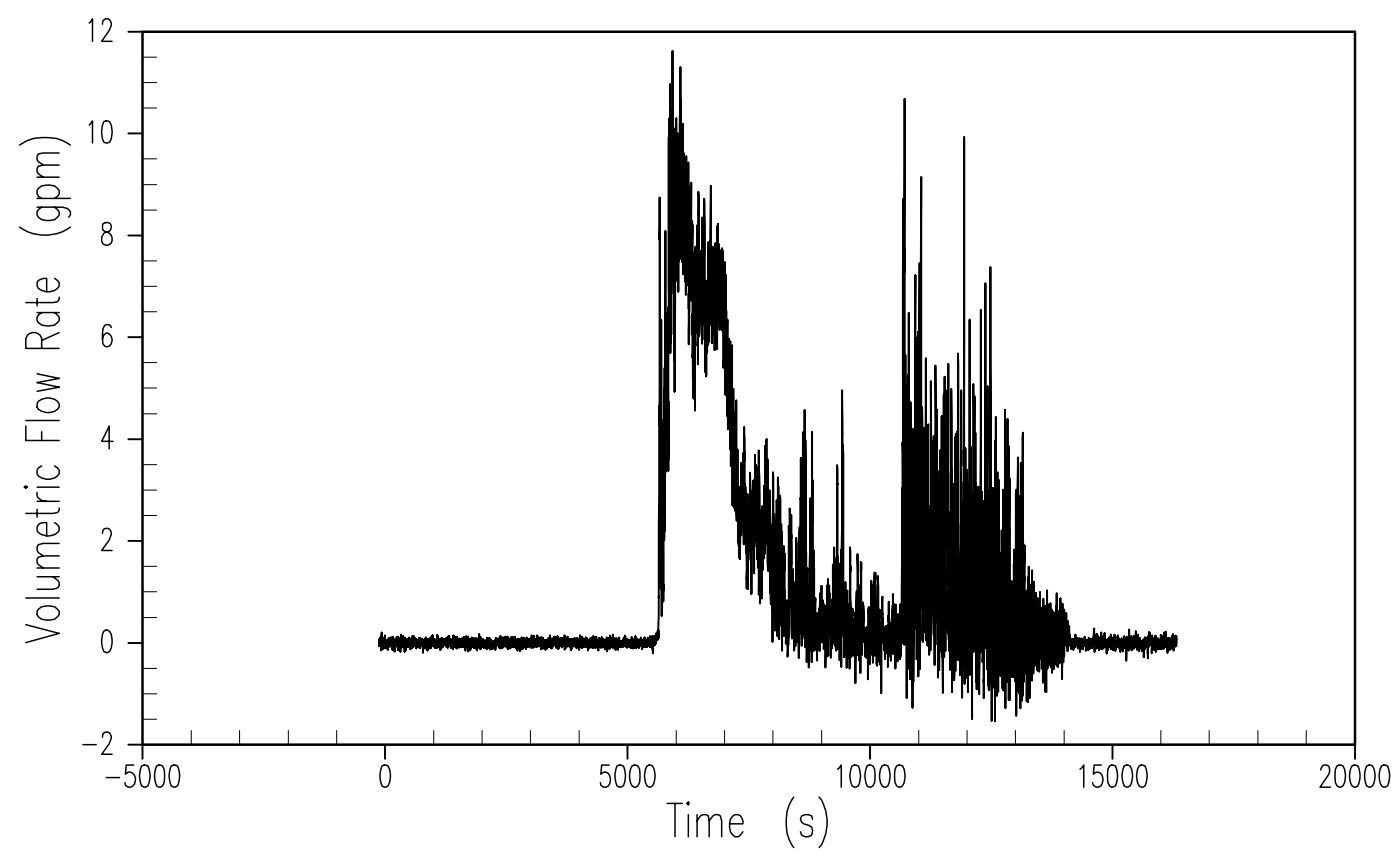

Figure A-12 ADS 4-2 Separator Liquid Flow Rate 

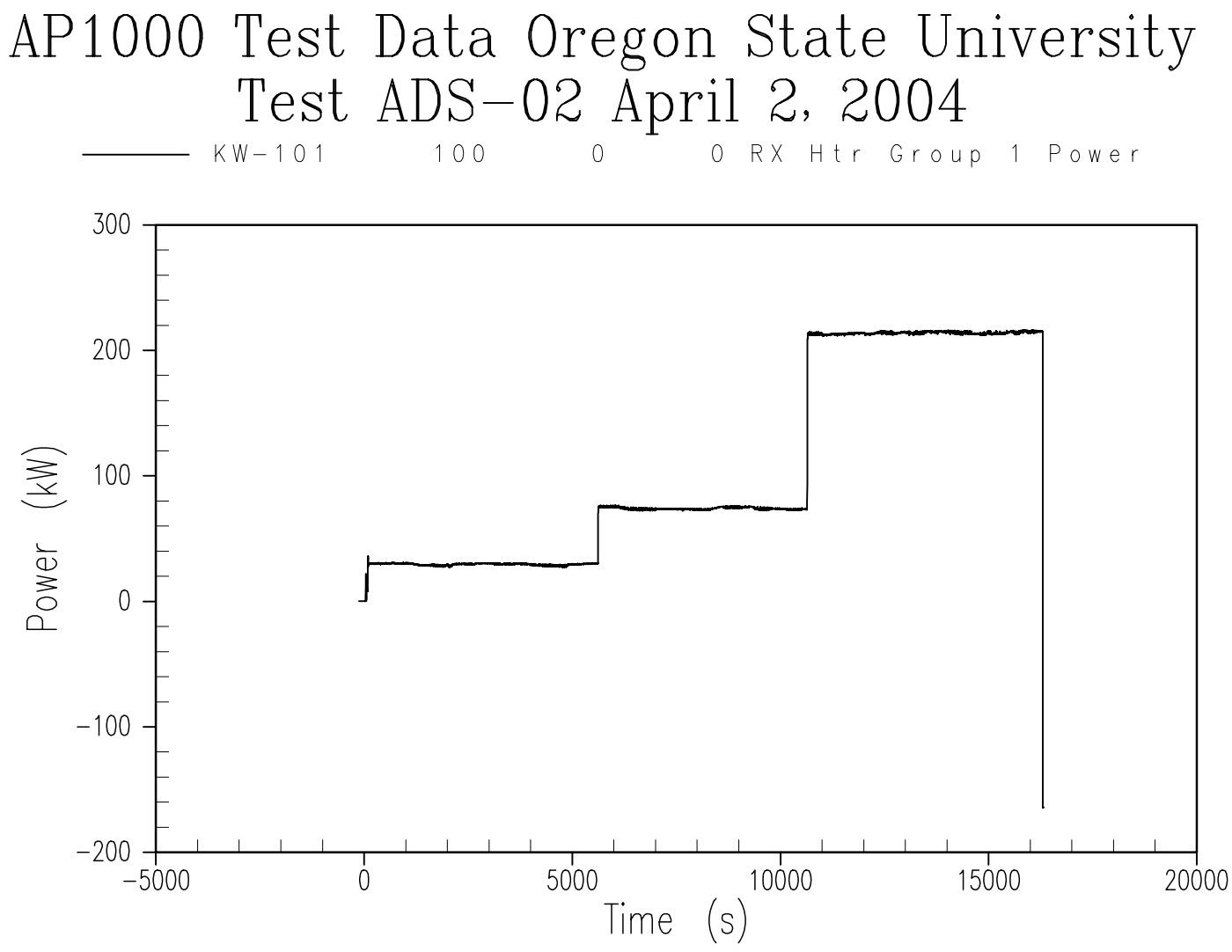

Figure A-13 Core Power Group 1 Input Power 

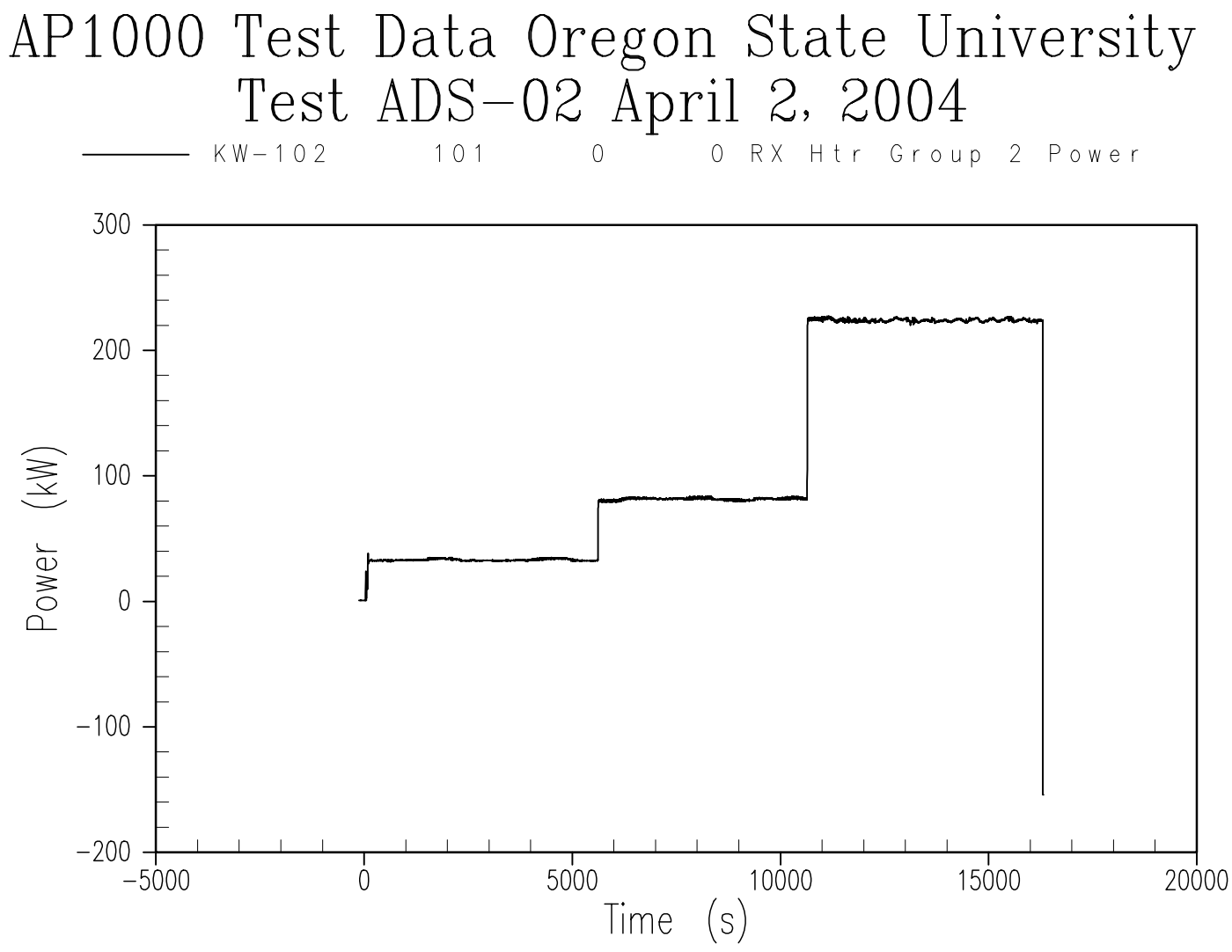

Figure A-14 Core Power Group 2 Input Power 

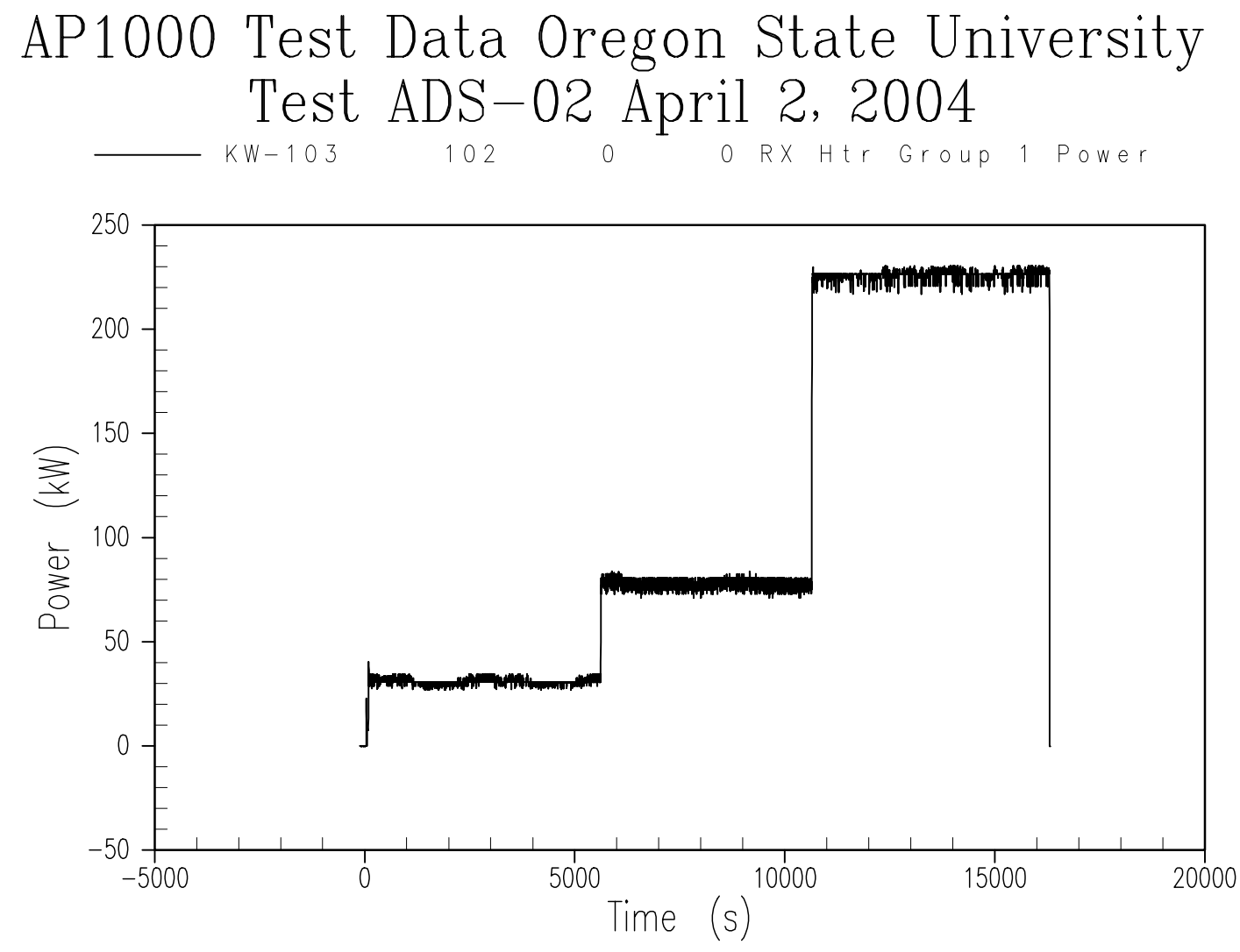

Figure A-15 Core Power Group 1 Alternate Input Power 

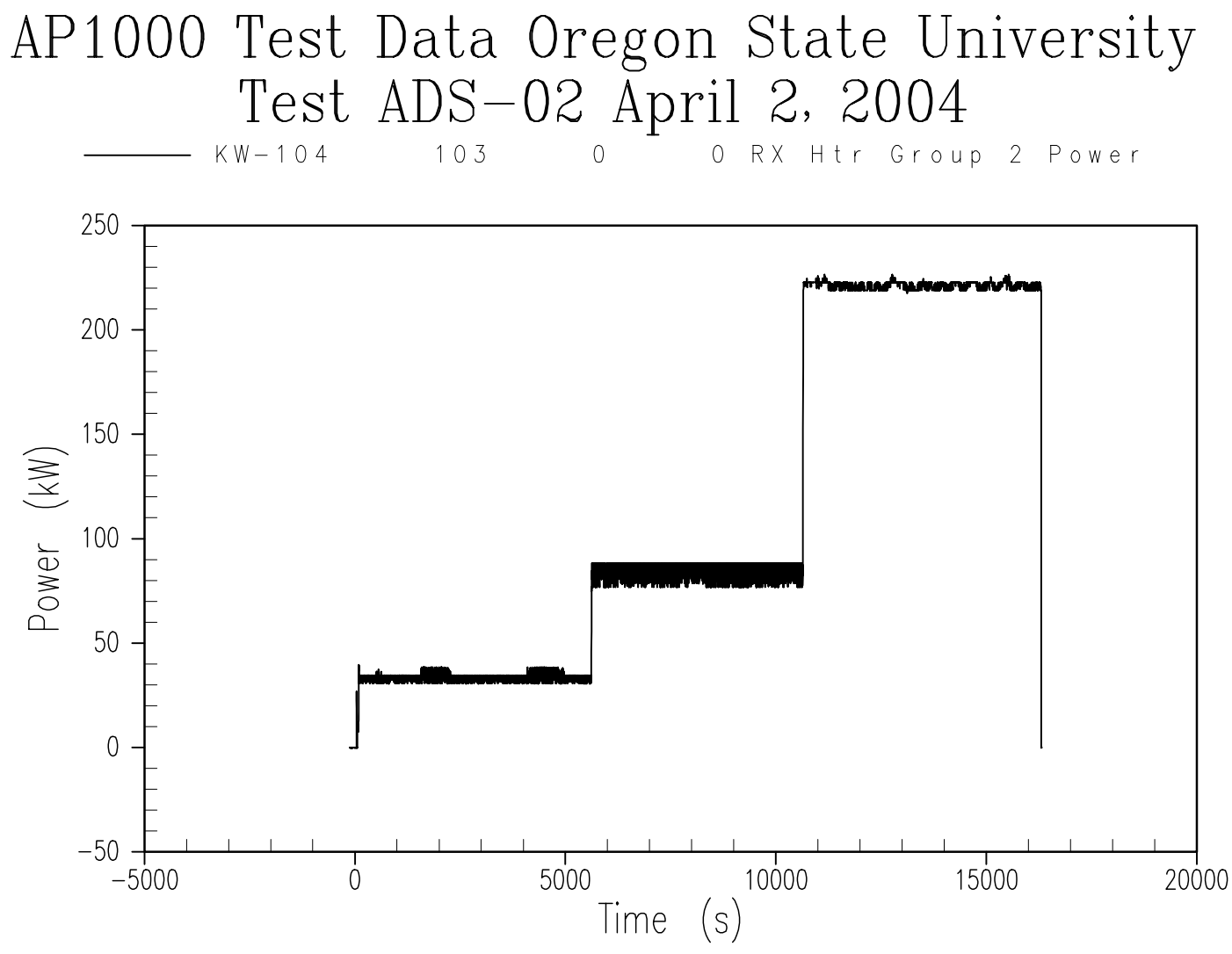

Figure A-16 Core Power Group 2 Alternate Input Power 
APPENDIX B

TEST DATA 


\title{
Oregon State University
}

Department of Nuclear Engineering

\author{
AdVANCED THERMAL HYdRAuliC \\ RESEARCH LABORATORY
}

\section{TEST SUMMARY REPORT}

OSU-AP1000-09

\section{DBA-03R-D}

\section{AP1000 DOUBLE-ENDED DVI WITH 3 OF 4 ADS-4}

Revision 0 


\title{
TEST SUMMARY REPORT
}

\author{
OSU-AP1000-09
}

DBA-03R-D

\section{AP1000 DOUBLE-ENDED DVI WITH 3 OF 4 ADS-4}
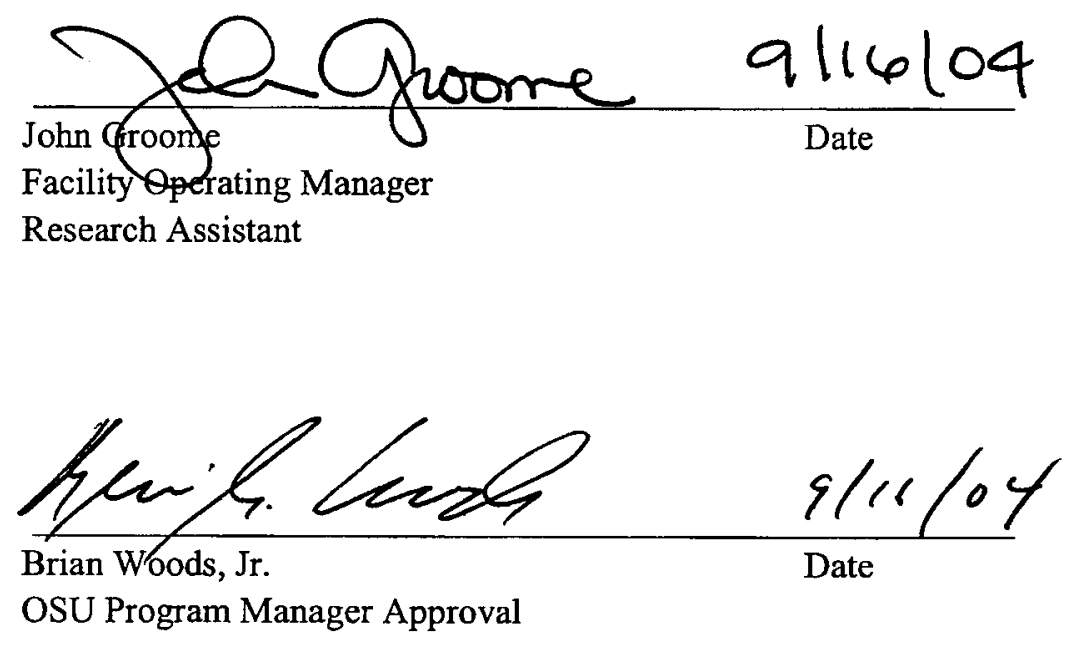

Oregon State University 116 Radiation Center

Corvallis, Oregon 97331

(C) 2004 Oregon State University

All Rights Reserved 


\section{WESTINGHOUSE PROPRIETARY INFORMATION NOTICE}

This report contains information proprietary to Westinghouse Electric Company. A non-proprietary version of this report has also been prepared for distribution to the United States Nuclear Regulatory Commission (NRC).

In order to conform to the requirements of 10 CFR 2.790 of the Commission's regulations concerning the protection of proprietary information so submitted to the NRC, the information which is proprietary in the proprietary version of this document is contained within brackets, and where the proprietary information has been deleted in the non-proprietary versions, only the brackets remain (the information that was contained within the brackets in the proprietary versions having been deleted). The justification for claiming the information so designated as proprietary is indicated in both versions by means of lower case letters (a) through (f) located as a superscript immediately following the brackets enclosing each item of information being identified as proprietary or in the margin opposite such information. These lower case letters refer to the types of information Westinghouse customarily holds in confidence identified in Sections (4)(ii)(a) through (4)(ii)(f) of the affidavit accompanying the transmittal of these documents pursuant to 10 CFR 2.790(b)(1).

\section{COPYRIGHT NOTICE}

This document bears an Oregon State University copyright notice. The NRC is permitted to make the number of copies of the information contained in these reports which are necessary for its internal use in connection with generic and plant-specific reviews and approvals as well as the issuance, denial, amendment, transfer, renewal, modification, suspension, revocation, or violation of a license, permit, order, or regulation subject to the requirements of 10 CFR 2.790 regarding restrictions on public disclosure to the extent such information has been identified as proprietary by Westinghouse, copyright protection notwithstanding. With respect to the non-proprietary versions of these reports, the NRC is permitted to make the number of copies beyond these necessary for its internal use which are necessary in order to have one copy available for public viewing in the appropriate docket files in the public document room in Washington, DC and in local public document rooms as may be required by NRC regulations if the number of copies submitted is insufficient for this purpose. Copies made by the NRC must include the copyright notice in all instances and the proprietary notice if the original was identified as proprietary. 
TABLE OF CONTENTS

$\underline{\text { Section }}$

Title

Page

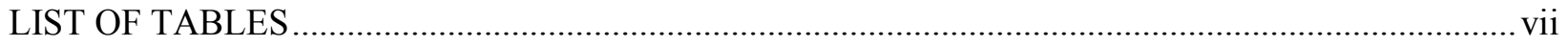

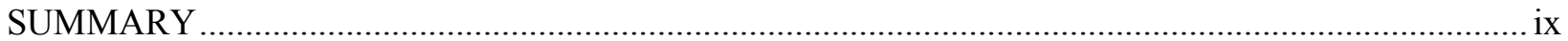

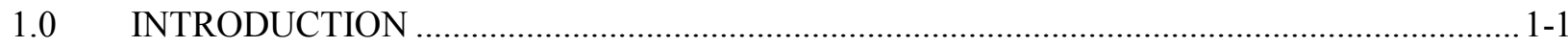

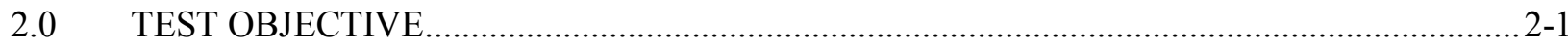

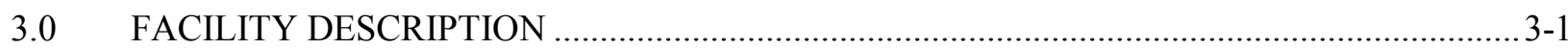

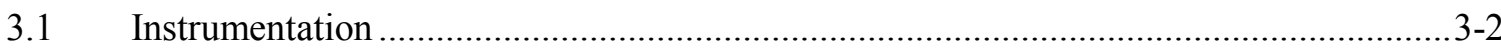

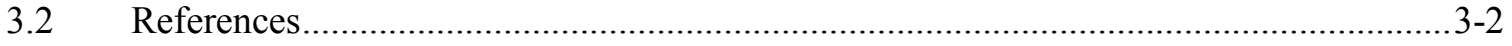

4.0 OREGON STATE UNIVERSITY TESTING PROGRAM MATRIX ..................................... $4-1$

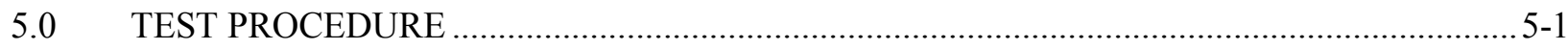

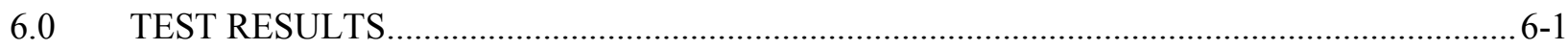

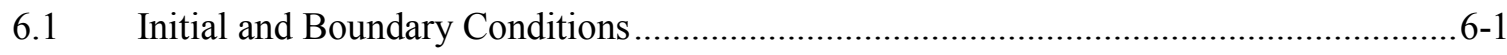

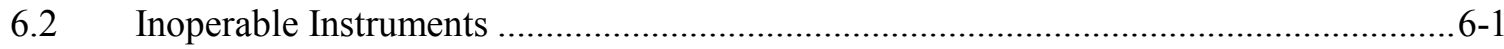

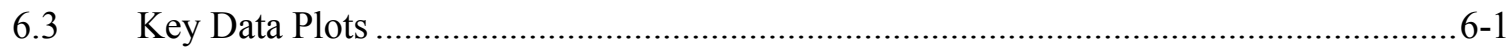

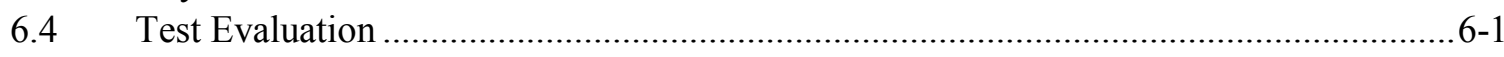

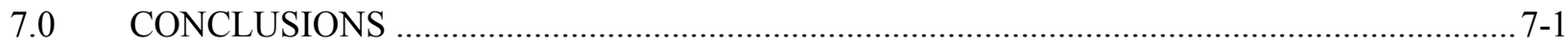

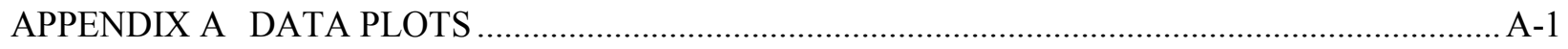

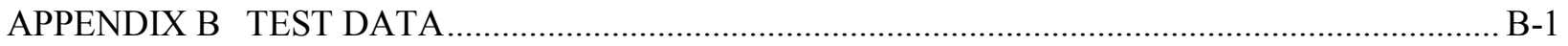




\section{LIST OF TABLES}

$\underline{\text { Table }}$

Table 4-1

Table 6-1

Table 6-2

Table 6-3

Table 6-4 $\underline{\text { Title }}$

Page

OSU Test Matrix (as specified April 19, 2004) $4-2$

Actual Test Initial Conditions. $6-2$

Sequence of Events..... 6-4

Inoperable Instruments for DBA-03R-D Test ............................................................6-6

Data Plots for QL Reports for DBA-03R-D by Component.. 6-7 


\section{SUMMARY}

This report covers the test DBA-03R-D, double-ended direct vessel injection (DVI) line break simulation loss-of-coolant accident, performed on April 15, 2004. This test has been designated OSU-AP1000-09 by the Advanced Plant Experiment (APEX). The objective of this test was to repeat a previous test, whose purpose was to obtain thermal-hydraulic data for a double-ended DVI line break simulation. The test performed met the specified conditions. The top of the heater bundle was always covered during this event. The test was performed for about 2,800 seconds. The transient continued through Automatic Depressurization System actuation and achieved stable in-containment refueling water storage tank injection. The test is a repeat of DBA-03 (OSU-AP1000-04) and is being performed to demonstrate the repeatability of the test facility.

This report presents the initial assessment of the test data collected. If this test is to be used by Westinghouse to support AP1000 Design Certification, additional validation of the use of this information will be documented separately. In the interim, the list of invalid data channels may change. 


\subsection{INTRODUCTION}

The Department of Nuclear Engineering at Oregon State University (OSU) is performing a series of tests for the U.S. Department of Energy. These tests are being conducted in the Advanced Plant Experiment (APEX-1000) test facility, which is a reduced pressure and height model of the two-loop Westinghouse AP1000 pressurized water reactor. The purpose of the testing is to:

- evaluate the thermal-hydraulic performance of the passive safety systems of the full-scale AP1000, and

- assess and validate the safety analyses techniques and computer codes used in predicting the transient system behavior.

The AP1000 Long-Term Cooling Test is a 1/4 height scale, low-pressure integral systems test simulating thermal-hydraulic phenomenon for the AP1000 passive safety systems for small-break loss-of-coolant accidents (LOCAs) and long-term cooling. It accurately models the details of the AP1000 geometry, including the primary system, the passive safety systems, and a part of the non-safety grade Chemical and Volume Control System, as well as a partial non-safety grade Normal Residual Heat Removal System. The interconnecting pipe routings are also duplicated in the model.

The overall objective of the Long-Term Cooling Test program is to obtain test data at various modes of operation. The OSU experiments will examine the passive safety system response for the small-break and large-break LOCA transition into long-term cooling. (The list of the tests to be performed is in the OSU Test Matrix provided in Section 4.0.) The facility permits a range of small-break LOCAs to be simulated at different locations on the primary system, such as the cold leg, hot leg, core makeup tank (CMT) cold leg pressure balance line, and direct vessel injection (DVI) line. The break orientation (top or bottom of the cold leg) may also be studied. Selected tests continue into the long-term cooling, post-accident mode in which the passive safety injection is from the reactor sump as well as the in-containment refueling water storage tank (IRWST). 


\subsection{TEST OBJECTIVE}

The purpose of this test is to repeat the previous test DBA-03R-D, which was to obtain thermal-hydraulic data for a double-ended DVI line break simulation. The break is located in the DVI-1 line close to the DVI-1 nozzle as it enters the reactor vessel. The assumed single failure for this test is one of four Automatic Depressurization System 4 (ADS-4) valves. The single failure location is on the nonpressurizer hot leg. The data obtained from the test will be used to verify the AP1000 thermal-hydraulic computer codes for AP1000 phenomena, such as gravity injection, natural convection, and post-accident long-term core cooling behavior.

The acceptance criteria for the OSU tests are as follows:

- Test initial conditions will be achieved within a specified tolerance.

- Setpoints will be achieved within an acceptable tolerance band.

- All instrumentation should be operational before the test.

- Any critical instruments not operating will be identified to the test engineer before the tests. These instruments must be operational before and during the test or exceptions should be approved.

- A zero check of LDPs, DPs, and FDPs will be performed.

In addition, the top-most level instrument in the reactor vessel was found to give an inconsistent reading for test DBA-03R-D. It is expected that this test will yield more consistent results. 


\subsection{FACILITY DESCRIPTION}

A detailed facility description for the OSU test facility is documented in Reference 3-1. The OSU test facility has been specifically scaled, designed, and constructed to investigate the AP600 passive safety system behavior and to provide data for safety analysis computer code validation. The facility has been modified to model the AP1000.

The scaled test design accurately models the details of the AP1000 geometry, including the primary system and the pipe routings and layout for the passive safety systems. A detailed scaling report (Reference 3-2) was used to develop the test design modifications. The primary system consists of one hot leg and two cold legs with two active pumps and an active steam generator for each loop. Two CMTs are connected to one primary loop, and the pressurizer is connected to the other primary loop as in the AP1000 plant design. Gas-driven accumulators are connected to the DVI lines. The discharge lines from the CMT, and one-of-two IRWST and reactor sump lines are connected to each DVI line. The ADS consisting of stages 1,2 , and 3 - simulates either one or two of the independent trains used in the AP1000. The two-phase flow from the ADS stages 1-to-3 is separated in a swirl-vane separator, and the liquid and vapor flows are measured to obtain the total ADS flow rate. The separated flow streams are then recombined and discharged into the IRWST through a sparger, preserving the mass and energy flow into the IRWST. The injection from the reactor sump is also simulated. Note that the OSU facility models both AP1000 primary and secondary sumps. The primary sump collects the condensate return, the liquid break flow, and the liquid flow from the fourth-stage ADS; and will provide long-term injection to the reactor vessel. The secondary sump simulates the portions of containment that will remain dry during most events. This sump collects water only when the primary sump reaches its overflow level, and provides no injection to the reactor vessel.

The time period for the experimental simulations includes not only the IRWST injection, but also the draining of the IRWST and the sump injection to simulate the long-term cooling of the AP1000. This simulation could be from several hours to a day. The time scale for the OSU test facility is one-half; that is, events occur in half the normal time. To model the long-term cooling aspects of the transient, the two-phase flow from the break is separated in a swirl-vane separator and the liquid and vapor portions of the total flow are measured. The liquid fraction of the flow is discharged to the reactor primary sump as in the AP1000 plant. The vapor is discharged to the atmosphere. The capability exists to return a portion of the equivalent liquid flow to the IRWST and primary sump to simulate the condensate return from the passive containment to the IRWST and primary sump. A similar approach is also used for the fourth-stage ADS valve on the hot leg. The two-phase flow is separated in a swirl-vane separator, the two streams are measured, and the liquid phase is discharged into the primary sump while the vapor flow is discharged to the atmosphere. Again, the capability exists to return a portion of the liquid equivalent added to the IRWST and primary sump. In addition, all other steam vents from the facility are measured (for example, the IRWST vent), and a portion of the liquid equivalent may be added back to the facility. Note that not all of the steam discharge would be returned as liquid equivalent. A portion of the discharge would be removed to simulate the steam that is not available for recirculation because it provides containment pressurization. The IRWST and primary sump can be pressurized in the OSU facility to simulate the containment pressurization following a postulated LOCA.

A multi-tube passive residual heat removal (PRHR) heat exchanger is located in the IRWST. The heat exchanger uses the same C-tube design as the AP1000 and has two instrumented tubes to obtain wall heat 
fluxes during the tests. There are primary fluid thermocouples, wall thermocouples, and differential pressure drop measurements to determine when the heat exchanger begins to drain. The IRWST is also instrumented with strings of fluid thermocouples to determine the degree of mixing within the tank and to assess the temperature of the coolant that is delivered to the test vessel.

The reactor vessel for the OSU tests includes a 0.914-meter (3-foot) heated core consisting of forty-eight 0.025 -meter (1-inch) diameter heater rods. The heater rods have a top skewed power shape. The $1000 \mathrm{~kW}$ of electrical power available at the OSU test site will be used to simulate decay heat. Wall thermocouples are swaged inside the heater rods to measure the heater rod wall temperature.

Thermocouple rods in the heater rod bundle measure the axial coolant temperature distribution. The scaled flow volume in the core is preserved as well as the flow volume in the test vessel upper plenum. There are simulated reactor internals in the upper plenum to preserve the flow area and to correctly scale the fluid volume. The reactor vessel includes an annular downcomer into which the four cold legs and the two DVI lines are connected. The hot legs penetrate the reactor annulus and connect with the loops. The AP1000 reactor vessel neutron reflector is simulated using a ceramic liner to reduce the metal heat release to the coolant.

There were no special/unique requirements for the test other than those specified in the Initial Conditions. The specified conditions were verified on the control board prior to test implementation.

\subsection{Instrumentation}

The instrumentation has been designed to calculate a transient mass and energy balance on the test facility. All two-phase flow streams exiting the facility are separated, and each component is measured separately as a single-phase flow using conventional measurement devices, such as magnetic flow meters and vortex flow meters. Note that magnetic flow meters are not designed for two-phase flow and will indicate erratically. Also, the vortex flow meters are referenced to $212^{\circ} \mathrm{F}$ and the LDPs are referenced to $60^{\circ} \mathrm{F}$. All vertical components have differential pressure cells that act as level instruments to measure the transient mass change in the component. The hot and cold leg diameters are sufficiently large in the OSU test facility so that a narrow-range differential pressure cell can be used to determine if the flow becomes stratified.

Single flow measurements are made of the CMT, accumulator, IRWST, and sump flows into the reactor vessel through the DVI lines.

Various types of instrumentation are provided in the test facility; for example, thermocouples for coolant and wall temperatures, flow meters, pressure transducers, differential pressure transducers, and weigh tanks.

\subsection{References}

3-1 APP-LTCT-T2R-005, OSU APEX-1000 Test Facility Description Report

3-2 APP-LTCT-T2R-004, Scaling Assessment for the Design of the OSU APEX-1000 Test Facility 


\subsection{OREGON STATE UNIVERSITY TESTING PROGRAM MATRIX}

The test matrix for the OSU test facility is shown in Table 4-1. To satisfy the test objectives, several transients will be performed to provide data on the AP1000 passive safety system response for a range of break sizes, locations, orientations, and single failure assumptions. The break size orifices are scaled based on simulating a 0.5 -inch, 1-inch, 2-inch, or larger pipe break.

The designation for this test is DBA-03R-D, which identifies the test as a design basis double-ended DVI line break. The test matrix may be adjusted for future tests based on results and insights gained with each test. 


\begin{tabular}{|c|c|c|}
\hline \multicolumn{3}{|c|}{$\begin{array}{c}\text { TABLE 4-1 } \\
\text { OSU TEST MATRIX (AS SPECIFIED APRIL 19, 2004) }\end{array}$} \\
\hline Test Title & Break Location and Size & Single Failure Assumed \\
\hline DBA-01-D & $\begin{array}{l}\text { Double-ended DVI Line break with } \\
\text { continuation into long-term cooling }\end{array}$ & Fail 1 of 2 lines in one ADS- 4 train \\
\hline DBA-02-D & $\begin{array}{l}\text { Double-ended DVI Line break with } \\
\text { continuation into long-term cooling }\end{array}$ & $\begin{array}{l}\text { Fail } 1 \text { of } 2 \text { lines in one ADS- } 4 \text { train } \\
\text { (Adjusted ADS-4 Resistance) }\end{array}$ \\
\hline DBA-03-D & $\begin{array}{l}\text { Double-ended DVI Line break with } \\
\text { continuation into long-term cooling }\end{array}$ & $\begin{array}{l}\text { Fail } 1 \text { of } 2 \text { lines in one ADS- } 4 \text { train } \\
\text { (Failure location sensitivity) }\end{array}$ \\
\hline TR-01-D & $\begin{array}{l}\text { Transition Test ADS-4 opening, } 85 \text { psig initial } \\
\text { pressure and decay power } 480 \mathrm{sec}\end{array}$ & $\begin{array}{l}\text { Fail } 1 \text { of } 2 \text { lines in one ADS-4 train } \\
\qquad(\text { No ADS 1-3) }\end{array}$ \\
\hline TR-02-D & $\begin{array}{l}\text { Transition Test ADS-4 opening, } 100 \text { psig initial } \\
\text { pressure with prototypic plant conditions }\end{array}$ & $\begin{array}{l}\text { Fail } 1 \text { of } 2 \text { lines in one ADS-4 train } \\
\qquad(\text { No ADS 1-3) }\end{array}$ \\
\hline DBA-04-D & 2-inch Cold Leg Break & $\begin{array}{l}\text { Fail } 1 \text { of } 2 \text { lines in one ADS- } 4 \text { train } \\
\text { (Failure on non-pressurizer hot leg) }\end{array}$ \\
\hline ADS-01-D & $\begin{array}{l}\text { ADS-4 Two-Phase Characterization Test at } \\
\text { low-power conditions (steady-state points) }\end{array}$ & $\begin{array}{l}\text { Various ADS-4 line configurations } \\
\qquad \text { (No ADS 1-3) }\end{array}$ \\
\hline ADS-02-D & $\begin{array}{l}\text { ADS-4 Two-Phase Characterization Test at } \\
\text { high-power conditions (steady-state points) }\end{array}$ & $\begin{array}{l}\text { Various ADS-4 line configurations } \\
\qquad \text { (No ADS 1-3) }\end{array}$ \\
\hline DBA-03R-D & Repeat of Test DBA-03-D & $\begin{array}{l}\text { Fail } 1 \text { of } 2 \text { lines in one ADS- } 4 \text { train } \\
\text { (Failure on pressurizer hot leg) }\end{array}$ \\
\hline DBA-05-D & Inadvertent ADS-1 Actuation & $\begin{array}{l}\text { Fail } 1 \text { of } 2 \text { lines in one ADS- } 4 \text { train } \\
\text { (Failure on non-pressurizer hot leg) }\end{array}$ \\
\hline DBA-06-D & 0.5-inch Cold Leg Break & $\begin{array}{l}\text { Fail } 1 \text { of } 2 \text { lines in one ADS- } 4 \text { train } \\
\text { (Failure on non-pressurizer hot leg) }\end{array}$ \\
\hline
\end{tabular}




\subsection{TEST PROCEDURE}

The test was performed per a written procedure. There were no special/unique requirements for the test other than those specified in the initial conditions in Table 6-1. The specified conditions were checked on the control board before test implementation.

The appropriate prerequisites were completed and initial conditions were satisfied. The required break simulation piping and break instrumentation were installed per P\&ID drawing OSU 600904, Rev. 1. A break spool insert simulating the double-ended break in AP1000 was installed in the break spool in DVI horizontal line. DVI-1 nozzle flow from the reactor is directed horizontally into the break separator. The combined flow of DVI line (from CMT-1, accumulator 1, primary sump, and IRWST) is connected horizontally to the primary sump tank. The 100-percent flow nozzle was installed in ADS 4-1 (on hot leg 1), and the 50-percent flow nozzle was installed in ADS 4-2 (on hot leg 2). Flow nozzles that simulate full flow for ADS-1, ADS-2, and ADS-3 were installed. As per the AP600 tests, ADS-3 has been scaled for full flow from all three stages, and ADS-1 and ADS-2 are closed when ADS-3 is opened.

Fill and vent was performed per APEX Operations Manual Procedure OP-B.2. Instruments were checked for required calibration.

With the break valves TS-202 and TS-203 closed, flow was used to warm up the bypass line by opening isolation valves RCS-901 and RCS-902. After the appropriate prerequisites were completed and the test facility achieved specified initial conditions, the CMT warmup bypass line isolation valves RCS-901 and RCS-902 were closed to maintain the $<80^{\circ} \mathrm{F}$ condition at the top of the CMT-1. With the CMT balance line valves (RCS-529 and RCS-530) placed in the open and automatic mode, both CMTs reached the Reactor Coolant System pressure.

Once all other initial conditions were satisfied, a break through TS-202 and TS-203 was initiated. The test continued through ADS actuation; and CMT, accumulator, IRWST, and sump injection. Per Westinghouse instructions, ADS 4-2 was actuated automatically according to the actuation logic and ADS 4-1 was actuated manually after a 30-second delay. All other actions were automatic and required no operator action. 


\subsection{TEST RESULTS}

The test results for test DBA-03R-D are provided in the following subsections.

\subsection{Initial and Boundary Conditions}

Table 6-1 provides a comparison of the specified and actual conditions for test DBA-03R-D. The values in this table were averaged over approximately 2 minutes preceding the test. Test initial conditions were achieved for the steam generator pressure, pressurizer pressure, pressurizer level, steam generator 01 narrow-range level, and steam generator 02 narrow-range level. Test initial conditions for the hot leg temperature were found to be acceptable, and the results will not be adversely affected.

The actual power decay curves are provided in data plots in Appendix B. The measured maximum power was $863 \mathrm{~kW}$, which was less than the facility maximum power of $1000 \mathrm{~kW}$. The programmed decay heat curve was adjusted to account for this difference, and the differences between the actual and specified power decay are considered acceptable.

PT-501 and PT-502 pressure instruments indicate the pressure changes in the CMT-1 and CMT-2. CMT-1 (PT-501) and CMT-2 (PT-502) confirm that 1 minute after the test button was pushed, both CMTs reach Reactor Coolant System pressure.

The sequence of events is shown in Table 6-2. This table compares the actual sequence of events with the specified timing. As can be seen in this table, all the events occurred at or very near to when the event was planned.

\subsection{Inoperable Instruments}

Table 6-3 lists the instrumentation channels considered inoperable for the DBA-03R-D test.

\subsection{Key Data Plots}

Table 6-4 lists the instrumentation channels sorted by component and includes the instrument number, units, and quick look (QL) plot number. The selection of channels was based on projecting an overall picture of the test results, which would then be examined by referring to the detailed data plots or tapes.

\subsection{Test Evaluation}

The following observations were made during the test:

1. The peak power before the test was initiated was $925 \mathrm{~kW}$. The decay heat curve was adjusted for this value. 


\begin{tabular}{|c|c|c|c|}
\hline \multicolumn{4}{|c|}{$\begin{array}{c}\text { TABLE 6-1 } \\
\text { ACTUAL TEST INITIAL CONDITIONS }\end{array}$} \\
\hline Conditions & Instrument No. & Actual & Comment \\
\hline Pressurizer Pressure & PT-604 & 370 psig & \\
\hline Hot Leg Temperature \#1 & $\begin{array}{l}\text { TF-141* } \\
\text { TF-205 } \\
\text { TF-143 }\end{array}$ & $\begin{array}{l}426^{\circ} \mathrm{F} \\
428^{\circ} \mathrm{F} \\
429^{\circ} \mathrm{F}\end{array}$ & \\
\hline Hot Leg Temperature \#2 & $\begin{array}{l}\text { TF-140* } \\
\text { TF-206 } \\
\text { TF-142 }\end{array}$ & $\begin{array}{l}428^{\circ} \mathrm{F} \\
427^{\circ} \mathrm{F} \\
429^{\circ} \mathrm{F}\end{array}$ & \\
\hline $\begin{array}{l}\text { Steam Generator Pressure } \\
\quad \# 1 \\
\# 2 \\
\text { Header }\end{array}$ & $\begin{array}{l}\text { PT-301* } \\
\text { PT-302* } \\
\text { PT-002 }\end{array}$ & $\begin{array}{l}286 \text { psig } \\
286 \text { psig } \\
251 \text { psig }\end{array}$ & \\
\hline Pressurizer Level & $\begin{array}{l}\text { LDP-601 uncompensated } \\
\text { LDP-601 } \\
\text { Compensated by SC-608 }\end{array}$ & $\begin{array}{l}68.02 \text { inches } \\
81.9 \text { inches }\end{array}$ & $\begin{array}{l}440^{\circ} \mathrm{F} \text { used for density } \\
\text { compensation }\end{array}$ \\
\hline $\begin{array}{l}\text { Steam Generator Level } \\
\# 1 \mathrm{NR}\end{array}$ & $\begin{array}{l}\text { LDP-303 uncompensated } \\
\text { LDP-303 compensated by } \\
\text { average of TF-305 and } \\
\text { TF-307 }\end{array}$ & $\begin{array}{l}21.4 \text { inches } \\
25.8 \text { inches }\end{array}$ & $\begin{array}{l}413^{\circ} \mathrm{F} \text { used for density } \\
\text { compensation }\end{array}$ \\
\hline $\begin{array}{l}\text { Steam Generator Level } \\
\# 2 \mathrm{NR}\end{array}$ & $\begin{array}{l}\text { LDP-304 uncompensated } \\
\text { LDP-304 compensated by } \\
\text { average of TF-306 and } \\
\text { TF-308 }\end{array}$ & $\begin{array}{l}21.6 \text { inches } \\
25.3 \text { inches }\end{array}$ & $\begin{array}{l}414^{\circ} \mathrm{F} \text { used for density } \\
\text { compensation }\end{array}$ \\
\hline IRWST Temperature & TF-701 & $62.4^{\circ} \mathrm{F}$ & Accepted $\left(<80^{\circ} \mathrm{F}\right)$ \\
\hline $\begin{array}{l}\text { CMT Temperature } \\
\text { \#1 } \\
\# 2\end{array}$ & $\begin{array}{l}\text { TF-513 } \\
\text { TF-516 }\end{array}$ & $\begin{array}{l}72.1^{\circ} \mathrm{F} \\
71.5^{\circ} \mathrm{F}\end{array}$ & Accepted $\left(<80^{\circ} \mathrm{F}\right)$ \\
\hline $\begin{array}{l}\text { Accumulator } \\
\text { Temperature } \\
\# 1 \\
\# 2\end{array}$ & $\begin{array}{l}\text { TF-403 } \\
\text { TF-404 }\end{array}$ & $\begin{array}{l}74.5^{\circ} \mathrm{F} \\
74.2^{\circ} \mathrm{F}\end{array}$ & Accepted $\left(<80^{\circ} \mathrm{F}\right)$ \\
\hline IRWST Level & LDP-701 & 92.4 inches & \\
\hline
\end{tabular}




\begin{tabular}{||c|c|c|c||}
\hline \multicolumn{5}{|c||}{ TABLE 6-1 (Continued) } \\
ACTUAL TEST INITIAL CONDITIONS \\
\hline \hline Conditions & Instrument No. & Actual & Comment \\
\hline Accumulator Level & & & \\
$\# 1$ & LDP-401 & 36.3 inches & \\
$\# 2$ & LDP-402 & 36.8 inches & \\
\hline Accumulator Pressure & & & \\
$\# 1$ & PT-401 & $187.1 \mathrm{psig}$ & \\
$\# 2$ & PT-402 & $189.1 \mathrm{psig}$ & \\
\hline CMT Level & & & \\
$\# 1$ & LDP-507 & 57.1 inches & \\
$\# 2$ & LDP-502 & $57.6 \mathrm{inches}$ & \\
\hline CMT Pressure & PT-501 & $373.8 \mathrm{psig}$ & \\
$\# 1$ & PT-502 & $374.8 \mathrm{psig}$ & \\
$\# 2$ & \multicolumn{2}{||}{} \\
\hline
\end{tabular}

* These instruments are used to establish initial conditions at the test site. 


\begin{tabular}{|c|c|c|}
\hline \multicolumn{3}{|c|}{$\begin{array}{c}\text { TABLE 6-2 } \\
\text { SEQUENCE OF EVENTS }\end{array}$} \\
\hline Event & Setpoint & Actual Time (sec) \\
\hline PB Depressed & $\mathrm{N} / \mathrm{A}$ & -120 \\
\hline Break Valve(s) Open & 0 & 0 \\
\hline Feed Pump Trip & $3.6 \mathrm{sec}$ & 3 \\
\hline CMT \#1 Outlet Valve Open (RCS-535) & $6.1 \mathrm{sec}$ & 6 \\
\hline CMT \#2 Outlet Valve Open (RCS-536) & $6.1 \mathrm{sec}$ & 6 \\
\hline PRHR HX Outlet Valve Open (RCS-804) & $6.1 \mathrm{sec}$ & 6 \\
\hline Reactor Coolant Pump \#1 Trip & $8.6 \mathrm{sec}$ & 8 \\
\hline Reactor Coolant Pump \#2 Trip & $8.6 \mathrm{sec}$ & 8 \\
\hline Reactor Coolant Pump \#3 Trip & $8.6 \mathrm{sec}$ & 8 \\
\hline Reactor Coolant Pump \#4 Trip & $8.6 \mathrm{sec}$ & 8 \\
\hline CMT \#1 Level Low (LDP-507) & 41 inches & 69 \\
\hline CMT \#2 Level Low (LDP-502) & 41 inches & 394 \\
\hline ADS \#1 Actuation (RCS-601) & CMT Level Low $+15 \mathrm{sec}$ & 87 \\
\hline ADS \#2 Actuation (RCS-602) & CMT Level Low $+62 \mathrm{sec}$ & 134 \\
\hline ADS \#3 Actuation (RCS-603) & CMT Level Low $+122 \mathrm{sec}$ & 194 \\
\hline Low Reactor Pressure (P-107) & $40 \mathrm{psig}$ & 206 \\
\hline IRWST Valve Actuation (RCS-711) & $\begin{array}{l}\text { Low Reactor Pressure } \\
\qquad(<40 \text { psig })\end{array}$ & 205 \\
\hline IRWST Valve Actuation (RCS-712) & $\begin{array}{l}\text { Low Reactor Pressure } \\
\qquad(<40 \text { psig })\end{array}$ & 205 \\
\hline CMT \#1 Low Low Level (LDP-507) & 17.14 inches & 93 \\
\hline CMT \#2 Low Low Level (LDP-502) & 17.14 inches & 694 \\
\hline ADS 4-1 Actuation (RCS-615) & $\begin{array}{l}\text { CMT Low Low (17.14") and } \\
\text { CMT Low }\left(41^{\prime \prime}\right)+180 \mathrm{sec}\end{array}$ & 285 \\
\hline ADS 4-2 Actuation (RCS-616) & $\begin{array}{l}\text { CMT Low Low (17.14") and } \\
\text { CMT Low (41") + } 180 \mathrm{sec}\end{array}$ & 253 \\
\hline Sump Valve Actuation (CSS-909) & IRWST Level Low Low & 5585 \\
\hline Sump Valve Actuation (CSS-910) & IRWST Level Low Low & 5585 \\
\hline
\end{tabular}




\begin{tabular}{|c|c|c|}
\hline \multicolumn{3}{|c|}{$\begin{array}{c}\text { TABLE 6-2 (Continued) } \\
\text { SEQUENCE OF EVENTS }\end{array}$} \\
\hline Event & Setpoint & Actual Time (sec) \\
\hline $\begin{array}{l}\text { Accumulator Injection } \\
\text { \#1 (FMM-401) }\end{array}$ & N/A & 0 \\
\hline $\begin{array}{l}\text { Accumulator Injection } \\
\text { \#2 (FMM-402) }\end{array}$ & N/A & 112 \\
\hline $\begin{array}{l}\text { IRWST Injection } \\
\text { DVI \#1 (FMM-701) }\end{array}$ & N/A & 313 \\
\hline $\begin{array}{l}\text { IRWST Injection } \\
\text { DVI \#2 (FMM-702) }\end{array}$ & N/A & 860 \\
\hline $\begin{array}{l}\text { Accumulator Empty } \\
\text { \#1 (LDP-401) }\end{array}$ & N/A & 205 down to 1.2 inch level \\
\hline $\begin{array}{l}\text { Accumulator Empty } \\
\text { \#2 (LDP-402) }\end{array}$ & N/A & 397 down to 0.1 inch level \\
\hline $\begin{array}{l}\text { CMT Empty } \\
\# 1 \text { (LDP-507) }\end{array}$ & $\mathrm{N} / \mathrm{A}$ & 137 down to 0.1 inch level \\
\hline $\begin{array}{l}\text { CMT Empty } \\
\text { \#2 (LDP-502) }\end{array}$ & N/A & 985 \\
\hline Sump Injection DVI \#1 (FMM-901) & N/A & 5585 \\
\hline Sump Injection DVI \#2 (FMM-902) & $\mathrm{N} / \mathrm{A}$ & 5585 \\
\hline
\end{tabular}




\begin{tabular}{||c|l|l||}
\hline \multicolumn{3}{|c||}{ TABLE 6-3 } \\
\hline \multicolumn{1}{|c||}{ INOPERABLE INSTRUMENTS FOR DBA-03R-D TEST } \\
\hline \hline Instrument Number & \multicolumn{1}{|c||}{ Instrument Type } & \multicolumn{1}{c|}{ Inoperable Description } \\
\hline $\begin{array}{l}\text { TW-202 } \\
\text { TW-204 }\end{array}$ & Thermocouple & Inoperative \\
TW-205 & & \\
TW-206 & & \\
TW-209 & & \\
TW-803 & & Inoperative \\
\hline TW-804 & & \\
\hline TH-603 & Thermocouple measuring heater & Inoperative \\
\hline FMM-202 & temperature & Inoperative \\
\hline TF-170 & Magnetic flow meter & \\
TF-221 & temperature & \\
TF-509 & & Erratic \\
\hline TF-512 & & Vortex flow meter \\
\hline FVM-905 &
\end{tabular}




\begin{tabular}{|c|c|c|c|c|}
\hline \multicolumn{5}{|c|}{$\begin{array}{l}\text { TABLE 6-4 } \\
\text { DATA PLOTS FOR QL REPORTS FOR DBA-03R-D BY COMPONENT }\end{array}$} \\
\hline Component & Channel & Units & QL-Plot & Comment \\
\hline $\begin{array}{l}\text { Reactor Vessel } \\
\text { Pressure }\end{array}$ & PT-107 & psig & 44 & \\
\hline Reactor Vessel Level & LDP-127 & inch of $\mathrm{H}_{2} \mathrm{O}$ & 29 & \\
\hline $\begin{array}{l}\text { Reactor Vessel } \\
\text { Downcomer Level }\end{array}$ & LDP-140 & inch of $\mathrm{H}_{2} \mathrm{O}$ & 31 & \\
\hline $\begin{array}{l}\text { Cold Leg \#1 Fluid } \\
\text { Temperature }\end{array}$ & TF-107 & ${ }^{\circ} \mathrm{F}$ & 58 & \\
\hline $\begin{array}{l}\text { Cold Leg \#2 Fluid } \\
\text { Temperature }\end{array}$ & TF-108 & ${ }^{\circ} \mathrm{F}$ & 59 & \\
\hline $\begin{array}{l}\text { Cold Leg \#3 Fluid } \\
\text { Temperature }\end{array}$ & TF-103 & ${ }^{\circ} \mathrm{F}$ & 56 & \\
\hline $\begin{array}{l}\text { Cold Leg \#4 Fluid } \\
\text { Temperature }\end{array}$ & TF-104 & ${ }^{\circ} \mathrm{F}$ & 57 & \\
\hline $\begin{array}{l}\text { Reactor Vessel Fluid } \\
\text { Temperature Upper } \\
\text { Head }\end{array}$ & $\mathrm{TF}-120$ & ${ }^{\circ} \mathrm{F}$ & 60 & \\
\hline $\begin{array}{l}\text { Reactor Coolant } \\
\text { System Hot Leg \#1 } \\
\text { Temperature }\end{array}$ & TF-143 & ${ }^{\circ} \mathrm{F}$ & 62 & \\
\hline $\begin{array}{l}\text { Reactor Coolant } \\
\text { System Hot Leg \#2 } \\
\text { Temperature }\end{array}$ & TF-142 & ${ }^{\circ} \mathrm{F}$ & 61 & \\
\hline Pressurizer Pressure & $\begin{array}{l}\text { PT-604 (WR) and PT-603 } \\
\text { (LP Indication) }\end{array}$ & psig & 49,50 & \\
\hline $\begin{array}{l}\text { Pressurizer Liquid } \\
\text { Level }\end{array}$ & LDP-601 & inch of $\mathrm{H}_{2} \mathrm{O}$ & 38 & $\begin{array}{l}\text { Sharp decrease followed } \\
\text { by rapid refill }\end{array}$ \\
\hline $\begin{array}{l}\text { Steam Generator \#1 } \\
\text { Tube Level }\end{array}$ & LDP-215 & inch of $\mathrm{H}_{2} \mathrm{O}$ & 32 & \\
\hline $\begin{array}{l}\text { Steam Generator \#1 } \\
\text { Secondary Pressure }\end{array}$ & PT-301 & psig & 45 & \\
\hline
\end{tabular}




\begin{tabular}{|c|c|c|c|c|}
\hline \multicolumn{5}{|c|}{$\begin{array}{l}\text { TABLE 6-4 (Continued) } \\
\text { DATA PLOTS FOR QL REPORTS FOR DBA-03R-D BY COMPONENT }\end{array}$} \\
\hline Component & Channel & Units & QL-Plot & Comment \\
\hline $\begin{array}{l}\text { Steam Generator \#1 } \\
\text { Feed Flow Rate }\end{array}$ & FMM-001 & gpm & 1 & \\
\hline $\begin{array}{l}\text { Steam Generator \#2 } \\
\text { Tube Level }\end{array}$ & LDP-218 & inch of $\mathrm{H}_{2} \mathrm{O}$ & 33 & \\
\hline $\begin{array}{l}\text { Steam Generator \#2 } \\
\text { Secondary Pressure }\end{array}$ & PT-302 & psig & 46 & \\
\hline $\begin{array}{l}\text { Accumulators \#1 and } \\
\# 2 \text { Pressure }\end{array}$ & PT-401 and PT-402 & psig & 47,48 & \\
\hline $\begin{array}{l}\text { Accumulators \#1 and } \\
\# 2 \text { Liquid Level }\end{array}$ & LDP-401 and LDP-402 & inch of $\mathrm{H}_{2} \mathrm{O}$ & 34,35 & \\
\hline $\begin{array}{l}\text { Accumulators \#1 and } \\
\text { \#2 Flow Rate }\end{array}$ & FMM-401 and FMM-402 & gpm & 2,3 & \\
\hline $\begin{array}{l}\text { Accumulators \#1 and } \\
\text { \#2 Liquid Discharge } \\
\text { Temperature }\end{array}$ & TF-401 and TF-402 & ${ }^{\circ} \mathrm{F}$ & 63,64 & \\
\hline $\begin{array}{l}\text { CMT \#1 and \#2 Liquid } \\
\text { Level }\end{array}$ & LDP-507 and LDP-502 & inch of $\mathrm{H}_{2} \mathrm{O}$ & 36,37 & \\
\hline $\begin{array}{l}\text { CMT \#1 and \#2 Flow } \\
\text { Rate }\end{array}$ & FMM-501 and FMM-504 & gpm & 4,5 & \\
\hline $\begin{array}{l}\text { CMT \#1 and \#2 Liquid } \\
\text { Temperature }\end{array}$ & $\begin{array}{l}\text { TF-501 and TF-529; and } \\
\text { TF-504 and TF-532 }\end{array}$ & ${ }^{\circ} \mathrm{F}$ & $65-68$ & \\
\hline PRHR Inlet Flow Rate & FMM-802 & gpm & 11 & \\
\hline PRHR Liquid Level & LDP-802 & inch of $\mathrm{H}_{2} \mathrm{O}$ & 40 & \\
\hline $\begin{array}{l}\text { PRHR Outlet Flow } \\
\text { Rate }\end{array}$ & FMM-804 & gpm & 12 & \\
\hline IRWST Liquid Level & LDP-701 & inch of $\mathrm{H}_{2} \mathrm{O}$ & 39 & \\
\hline $\begin{array}{l}\text { IRWST Discharge Line } \\
\# 1 \text { and \#2 Flow Rate }\end{array}$ & FMM-701 and FMM-702 & gpm & 9,10 & \\
\hline $\begin{array}{l}\text { IRWST Fluid } \\
\text { Temperature }\end{array}$ & TF-701 and TF-709 & ${ }^{\circ} \mathrm{F}$ & 69,70 & \\
\hline $\begin{array}{l}\text { ADS 1-3 Separator } \\
\text { Pressure }\end{array}$ & PT-605 & psig & 51 & \\
\hline
\end{tabular}




\begin{tabular}{|c|c|c|c|c|}
\hline \multicolumn{5}{|c|}{$\begin{array}{l}\text { TABLE 6-4 (Continued) } \\
\text { DATA PLOTS FOR QL REPORTS FOR DBA-03R-D BY COMPONENT }\end{array}$} \\
\hline Component & Channel & Units & QL-Plot & Comment \\
\hline $\begin{array}{l}\text { ADS 1-3 Separator } \\
\text { Steam Flow Rate }\end{array}$ & FVM-601 & $\operatorname{scfm}$ & 16 & \\
\hline $\begin{array}{l}\text { ADS 1-3 Separator } \\
\text { Liquid Flow Rate }\end{array}$ & FMM-601 & gpm & 6 & \\
\hline $\begin{array}{l}\text { ADS-4 Separator \#1 } \\
\text { and \#2 Pressure }\end{array}$ & PT-611 and PT-610 & psig & 52,53 & \\
\hline $\begin{array}{l}\text { ADS 4-1 Separator } \\
\text { Steam Flow Rate }\end{array}$ & FVM-603 & $\mathrm{scfm}$ & 18 & \\
\hline $\begin{array}{l}\text { ADS 4-2 Separator } \\
\text { Steam Flow Rate }\end{array}$ & FVM-602 & $\mathrm{scfm}$ & 17 & \\
\hline $\begin{array}{l}\text { ADS 4-1 Separator } \\
\text { Liquid Flow Rate }\end{array}$ & FMM-603 & gpm & 8 & \\
\hline $\begin{array}{l}\text { ADS 4-2 Separator } \\
\text { Liquid Flow Rate }\end{array}$ & FMM-602 & gpm & 7 & \\
\hline Primary Sump Pressure & PT-901 & psig & 54 & \\
\hline $\begin{array}{l}\text { Primary Sump Liquid } \\
\text { Level }\end{array}$ & LDP-901 & inch of $\mathrm{H}_{2} \mathrm{O}$ & 41 & \\
\hline $\begin{array}{l}\text { Primary Sump } \\
\text { Injection Flow Rate }\end{array}$ & FMM-901 and FMM-902 & gpm & 13,14 & \\
\hline $\begin{array}{l}\text { Secondary Sump } \\
\text { Liquid Level }\end{array}$ & LDP-902 & inch of $\mathrm{H}_{2} \mathrm{O}$ & 42 & \\
\hline $\begin{array}{l}\text { Break Separator } \\
\text { Pressure }\end{array}$ & PT-905 & psig & 55 & \\
\hline $\begin{array}{l}\text { Break Separator Liquid } \\
\text { Level }\end{array}$ & LDP-905 & inch of $\mathrm{H}_{2} \mathrm{O}$ & 43 & \\
\hline $\begin{array}{l}\text { Break Separator Flow } \\
\text { to Primary Sump }\end{array}$ & FMM-905 & gpm & 15 & \\
\hline $\begin{array}{l}\text { BAMS Steam Flow } \\
\text { Rate }\end{array}$ & FVM-901 & $\mathrm{scfm}$ & 19 & \\
\hline $\begin{array}{l}\text { BAMS Steam Flow } \\
\text { Rate }\end{array}$ & FVM-902 & $\mathrm{scfm}$ & 20 & \\
\hline
\end{tabular}




\begin{tabular}{|c|c|c|c|c|}
\hline \multicolumn{5}{|c|}{$\begin{array}{l}\text { TABLE 6-4 (Continued) } \\
\text { DATA PLOTS FOR QL REPORTS FOR DBA-03R-D BY COMPONENT }\end{array}$} \\
\hline Component & Channel & Units & QL-Plot & Comment \\
\hline $\begin{array}{l}\text { BAMS/Primary Sump } \\
\text { Steam Flow Rate }\end{array}$ & FVM-903 & $\operatorname{scfm}$ & 21 & \\
\hline $\begin{array}{l}\text { BAMS/Separator } \\
\text { Steam Flow Rate- } \\
\text { 6-inch Pipe }\end{array}$ & FVM-905 & $\operatorname{scfm}$ & 22 & \\
\hline $\begin{array}{l}\text { BAMS/Exhaust Line } \\
\text { Temperature }\end{array}$ & TF-916 and SC-917 & ${ }^{\circ} \mathrm{F}$ & 71,72 & \\
\hline $\begin{array}{l}\text { Pressurizer Heater } \\
\text { Input Power }\end{array}$ & KW-601 & $\mathrm{kW}$ & 27 & \\
\hline $\begin{array}{l}\text { Core Power Input } \\
\text { Power }\end{array}$ & $\begin{array}{l}\text { KW-101, KW-102, } \\
\text { KW-103, and KW-104 }\end{array}$ & $\mathrm{kW}$ & $23-26$ & \\
\hline $\begin{array}{l}\text { Reactor Vessel Liquid } \\
\text { Level Between Top of } \\
\text { Vessel - Upper Support } \\
\text { Plate }\end{array}$ & LDP-115 & inch of $\mathrm{H}_{2} \mathrm{O}$ & 28 & \\
\hline $\begin{array}{l}\text { Reactor Vessel Liquid } \\
\text { Level Between Bottom } \\
\text { of Upper Support } \\
\text { Plate - Upper Core } \\
\text { Spacer Grid }\end{array}$ & LDP-139 & inch of $\mathrm{H}_{2} \mathrm{O}$ & 30 & \\
\hline $\begin{array}{l}\text { Inner Core } \\
\text { Thermocouple } \\
\text { Measuring Heater } \\
\text { Temperature }\end{array}$ & TH-103-4 & ${ }^{\circ} \mathrm{F}$ & 73 & \\
\hline $\begin{array}{l}\text { Outer Core } \\
\text { Thermocouple } \\
\text { Measuring Heater } \\
\text { Temperature }\end{array}$ & TH-309-4 & ${ }^{\circ} \mathrm{F}$ & 74 & \\
\hline
\end{tabular}




\subsection{CONCLUSIONS}

The DBA-03R-D test was successfully completed, and the data was logged in the DAS. All critical instruments were found to operate properly with the exception of those noted in Section 6.4. The test was acceptable. 
APPENDIX A

DATA PLOTS 

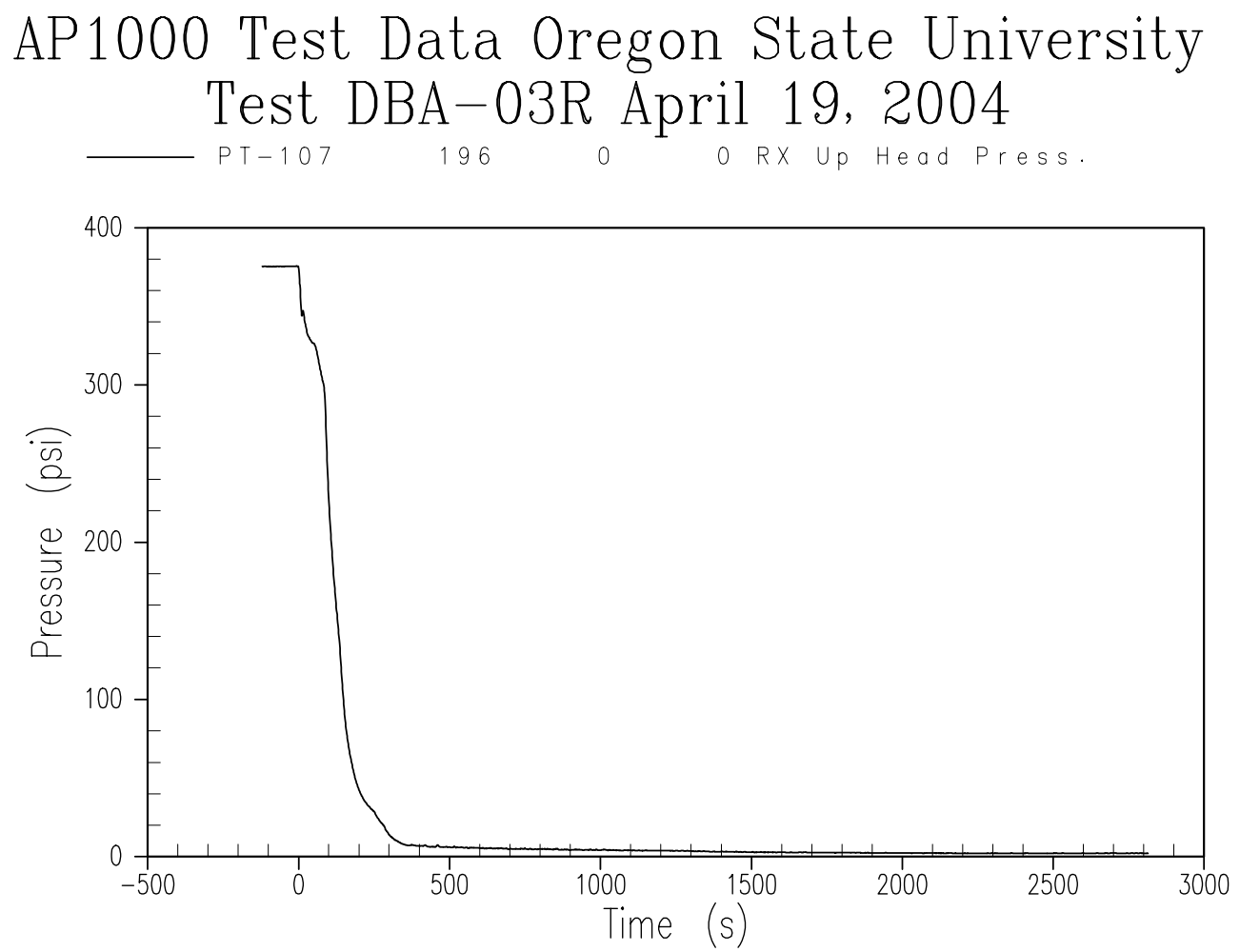

Figure A-1 Reactor Vessel Pressure 

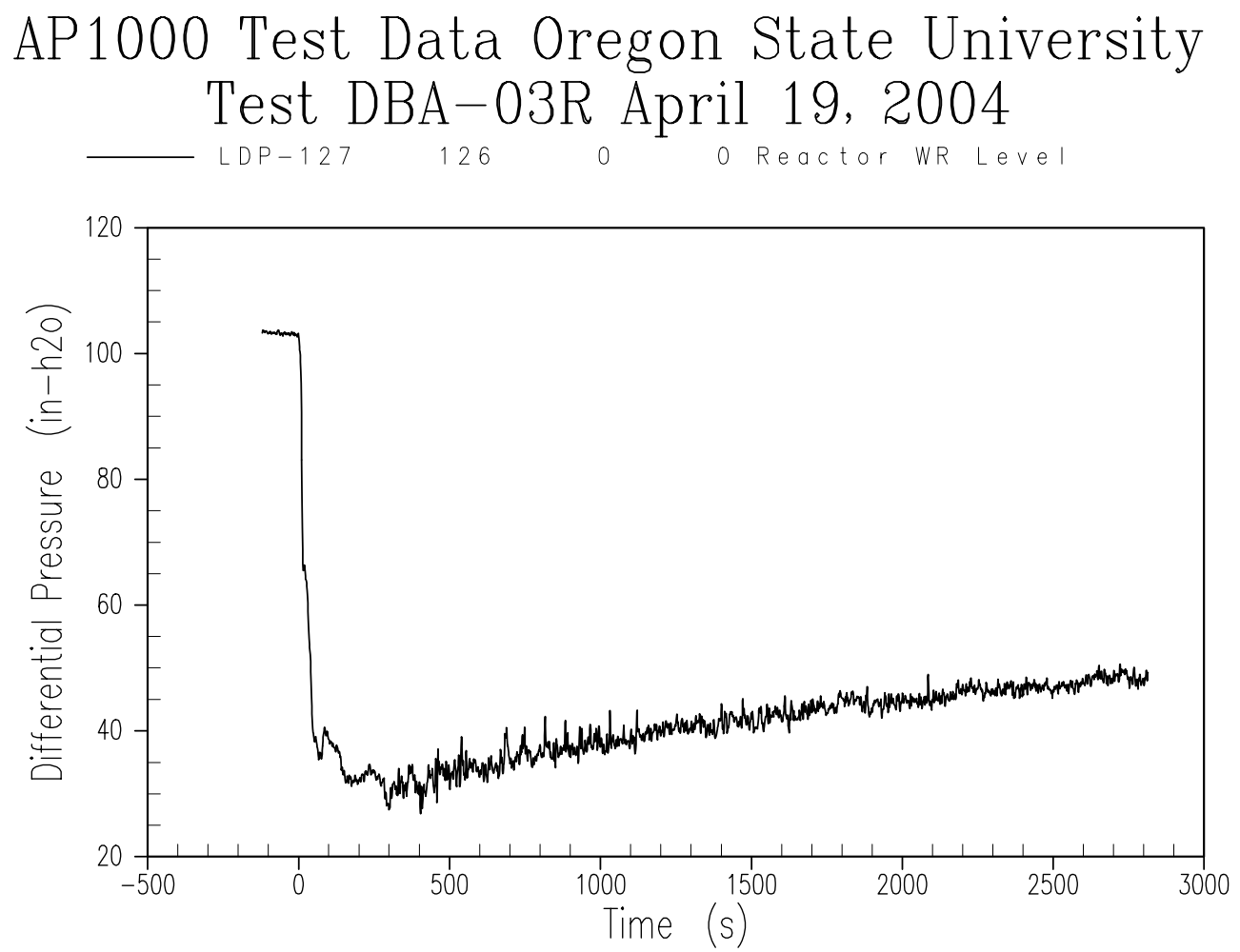

Figure A-2 Reactor Vessel Level 

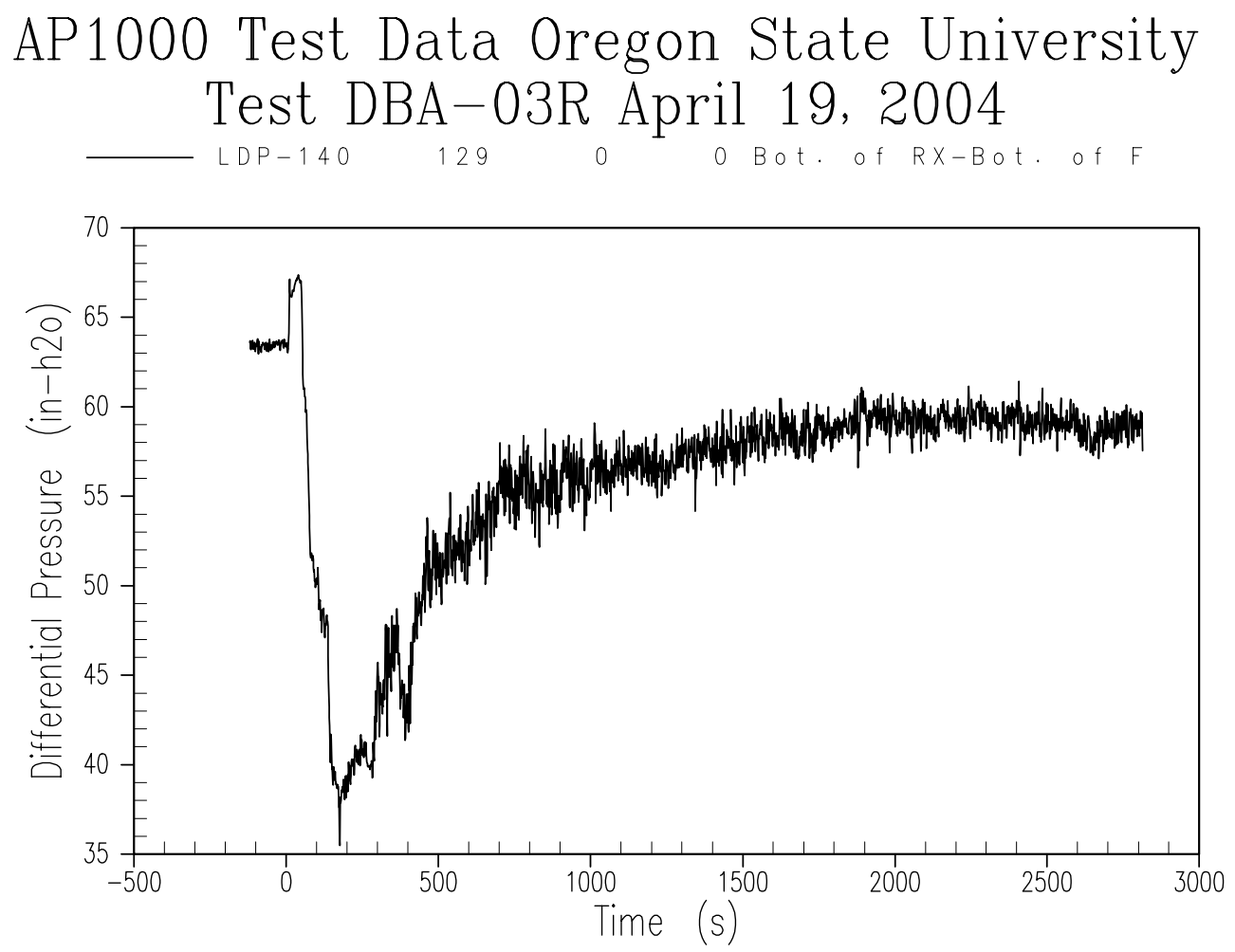

Figure A-3 Reactor Vessel Downcomer Level 
AP1000 Test Data Oregon State University Test DBA-03R April 19, 2004
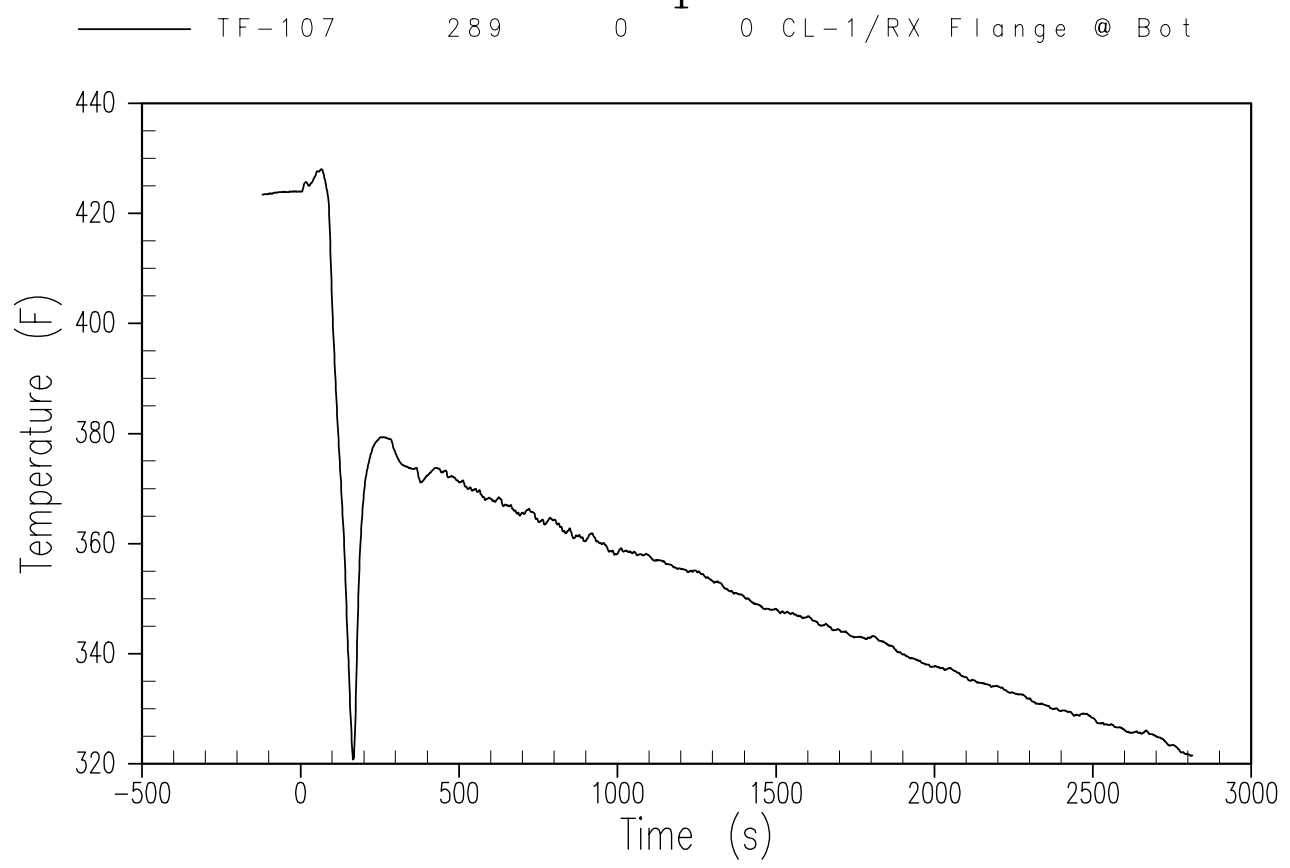

Figure A-4 Cold Leg 1 Fluid Temperature 
AP1000 Test Data Oregon State University Test DBA-03R April 19, 2004

$-T F-108$

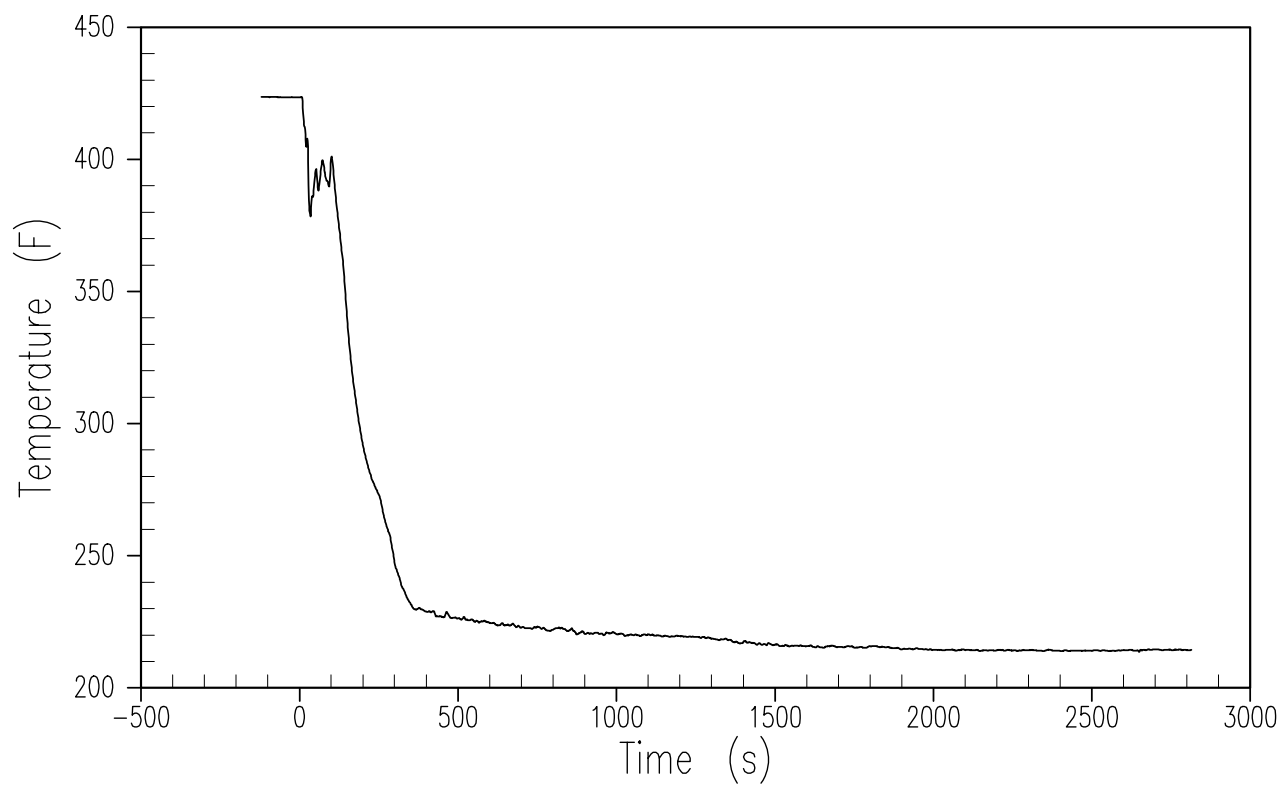

Figure A-5 Cold Leg 2 Fluid Temperature 

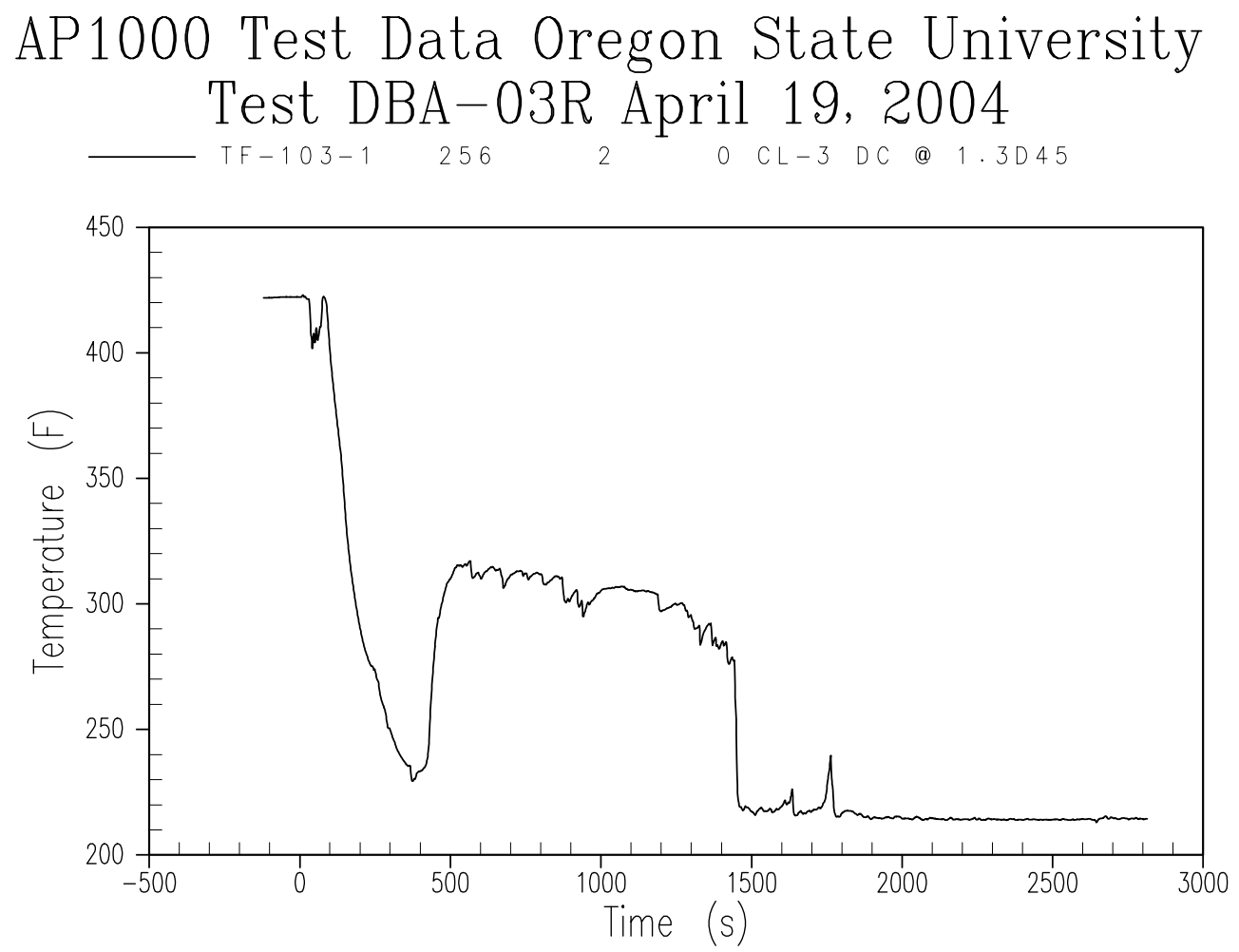

Figure A-6 Cold Leg 3 Fluid Temperature 
AP1000 Test Data Oregon State University Test DBA-03R April 19, 2004
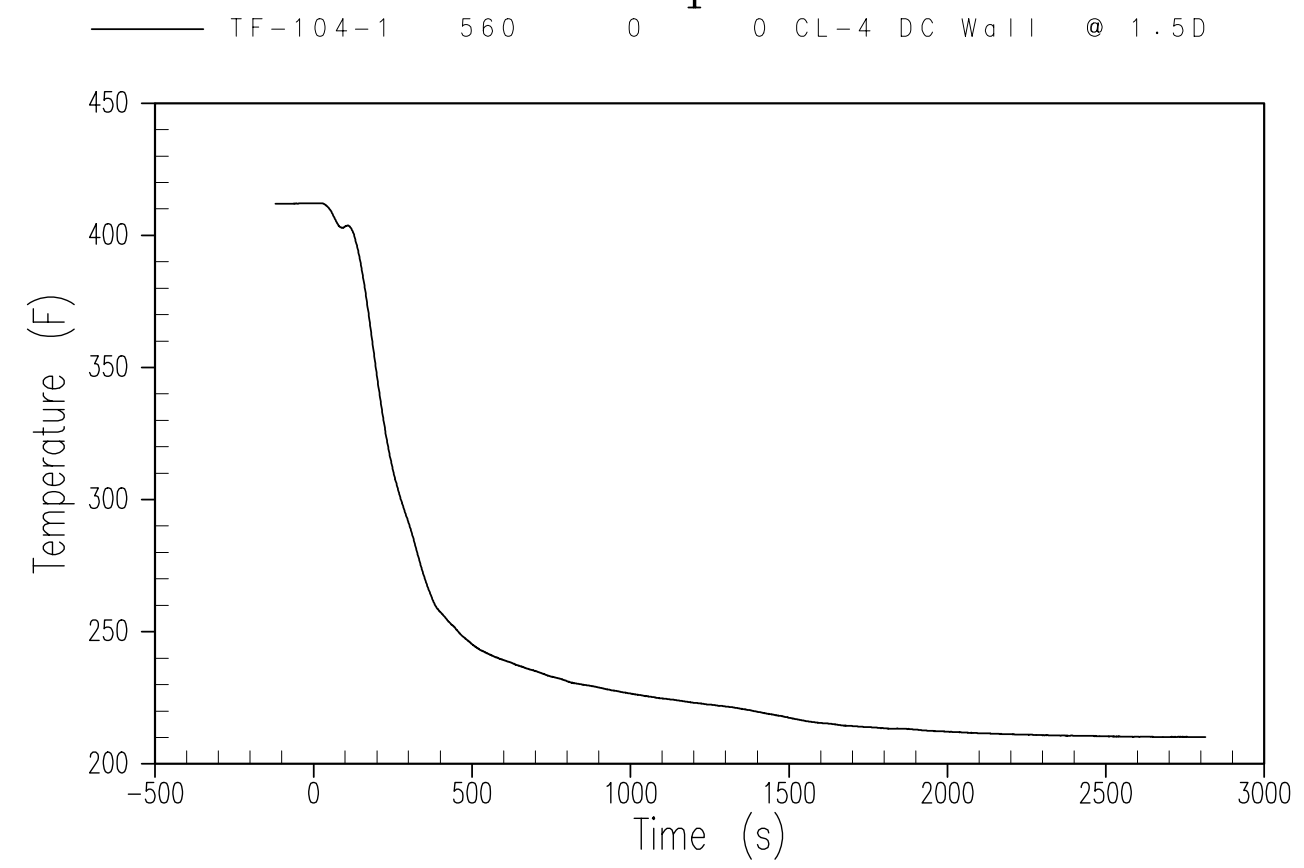

Figure A-7 Cold Leg 4 Fluid Temperature 

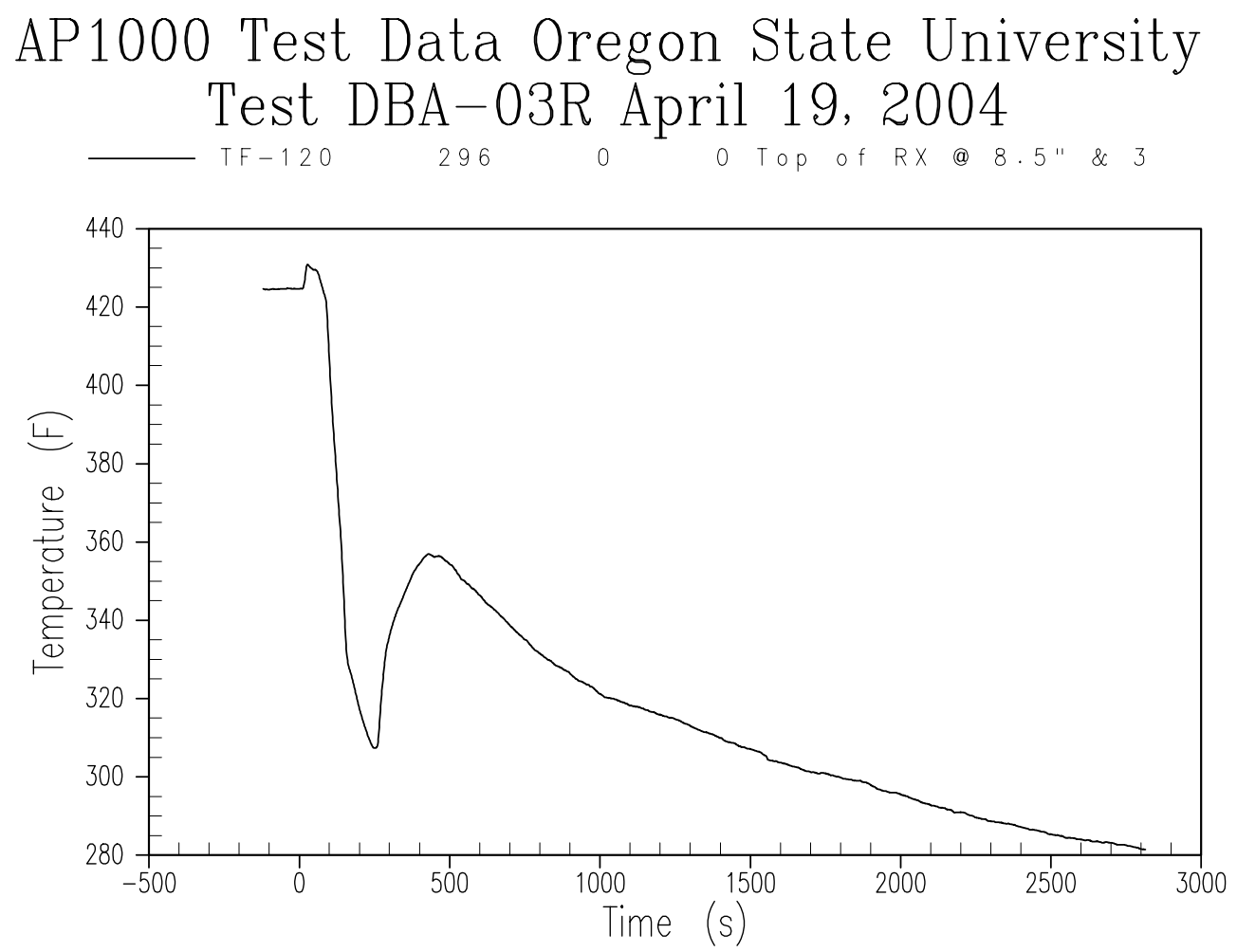

Figure A-8 Reactor Vessel Fluid Temperature Upper Head 
AP1000 Test Data Oregon State University Test DBA-03R April 19, 2004

$-T F-143$

310

0

$0 H L-1 / R X F I a n g e @ B o t$

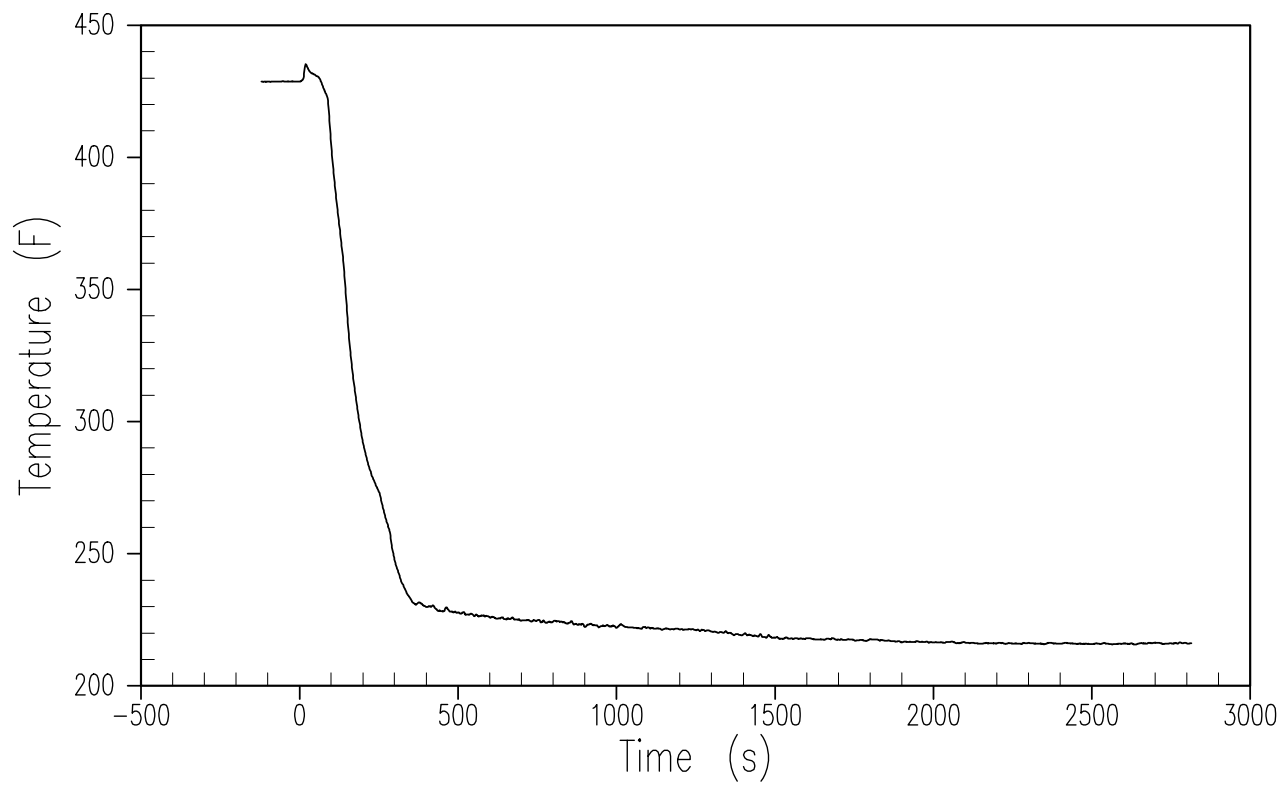

Figure A-9 Reactor Coolant System Hot Leg 1 Temperature 
AP1000 Test Data Oregon State University Test DBA-03R April 19, 2004

- TF-142

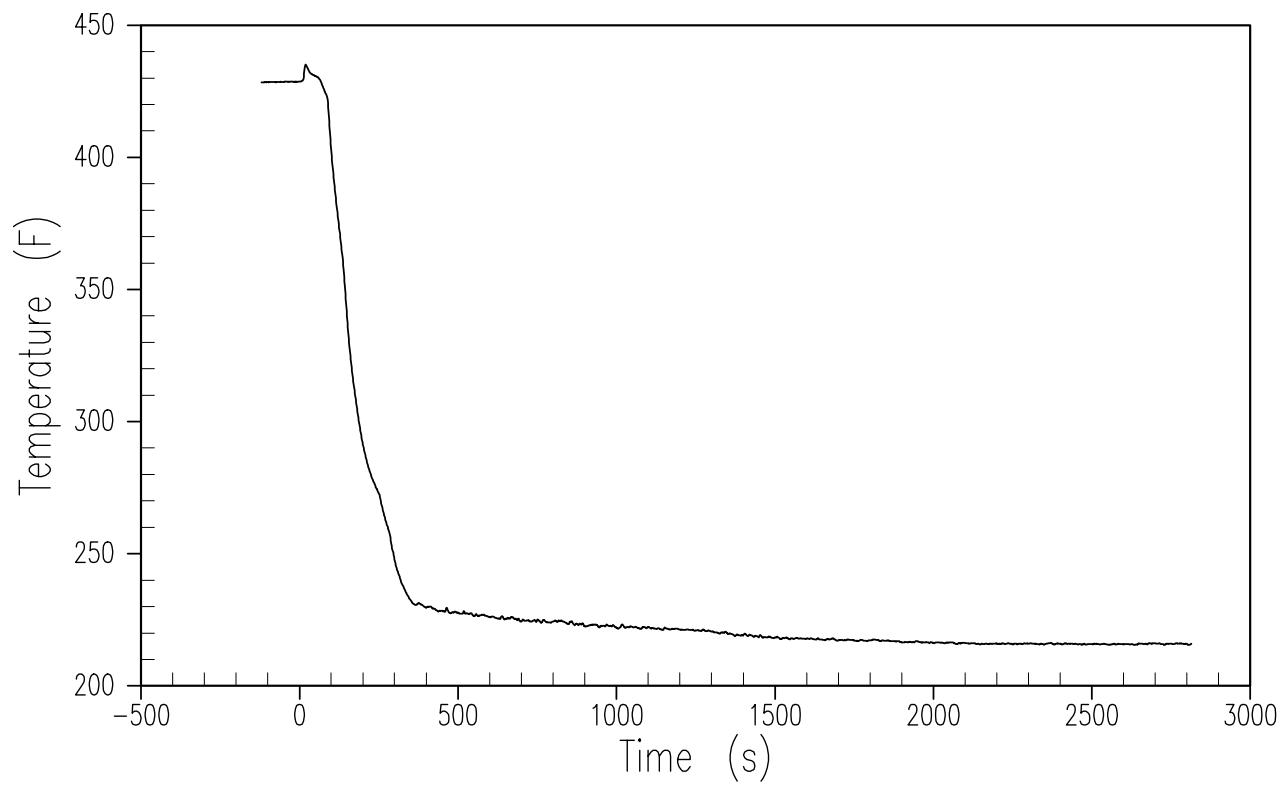

Figure A-10 Reactor Coolant System Hot Leg 2 Temperature 

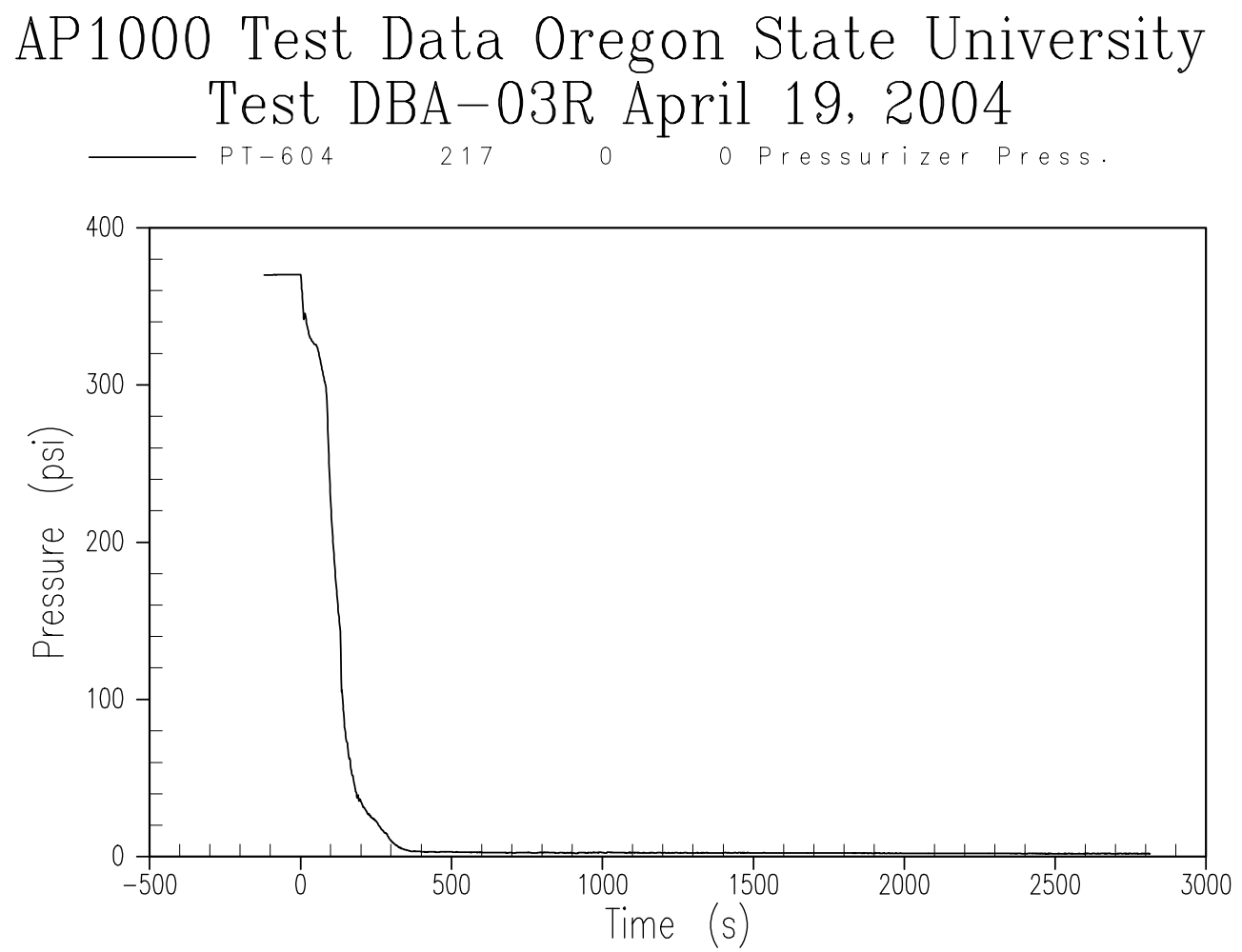

Figure A-11 Pressurizer Pressure - Wide Range 

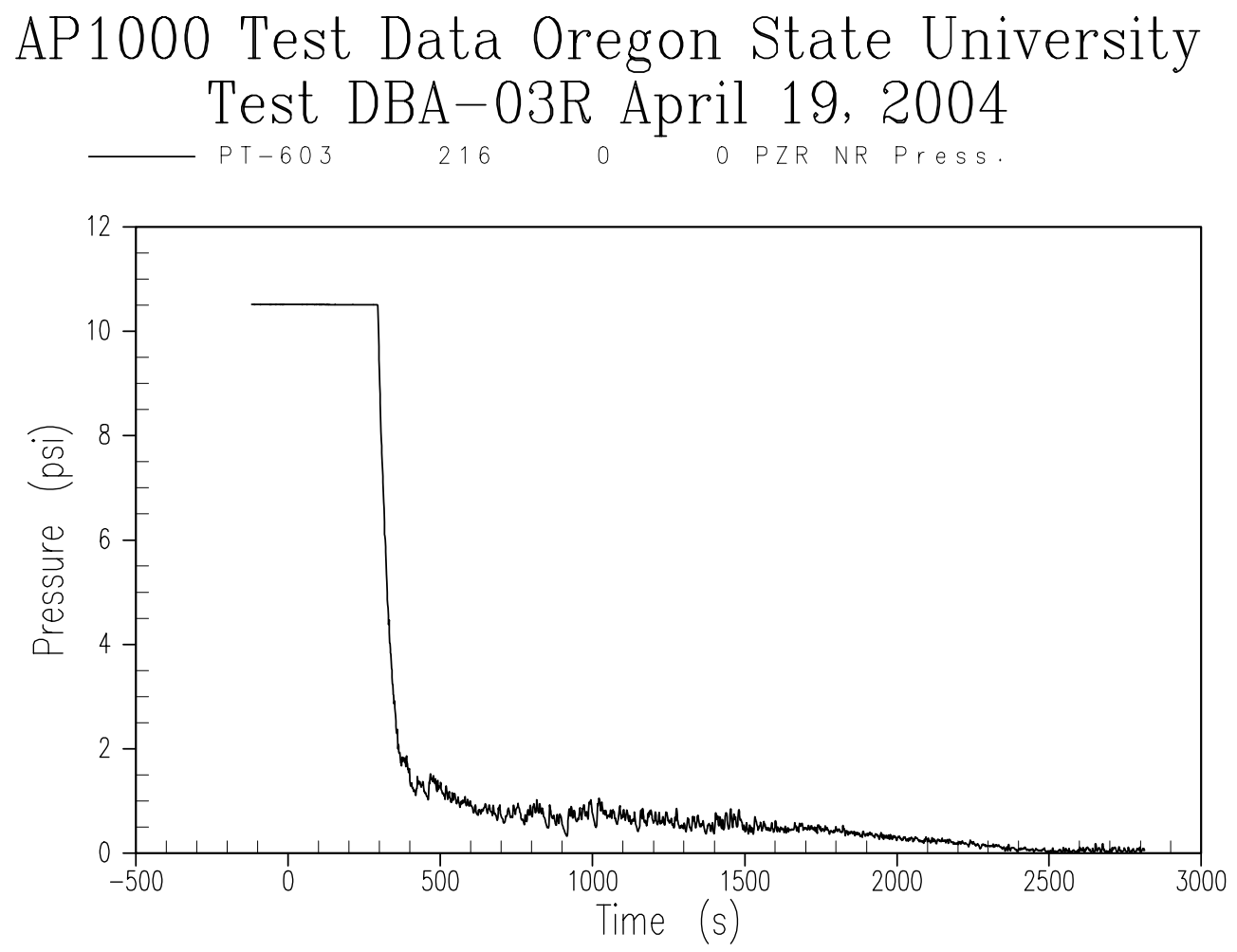

Figure A-12 Pressurizer Pressure - Narrow Range 

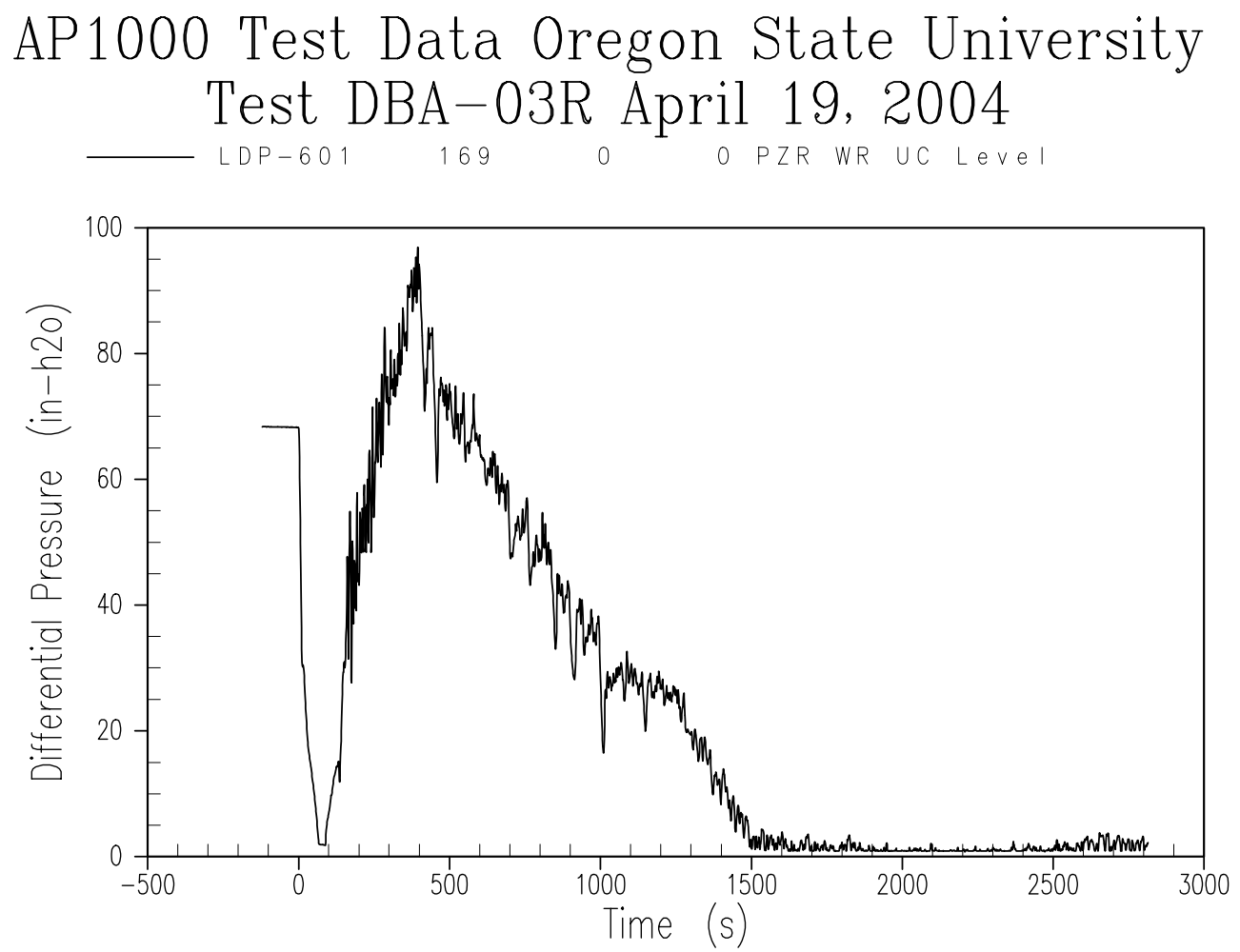

Figure A-13 Pressurizer Liquid Level 

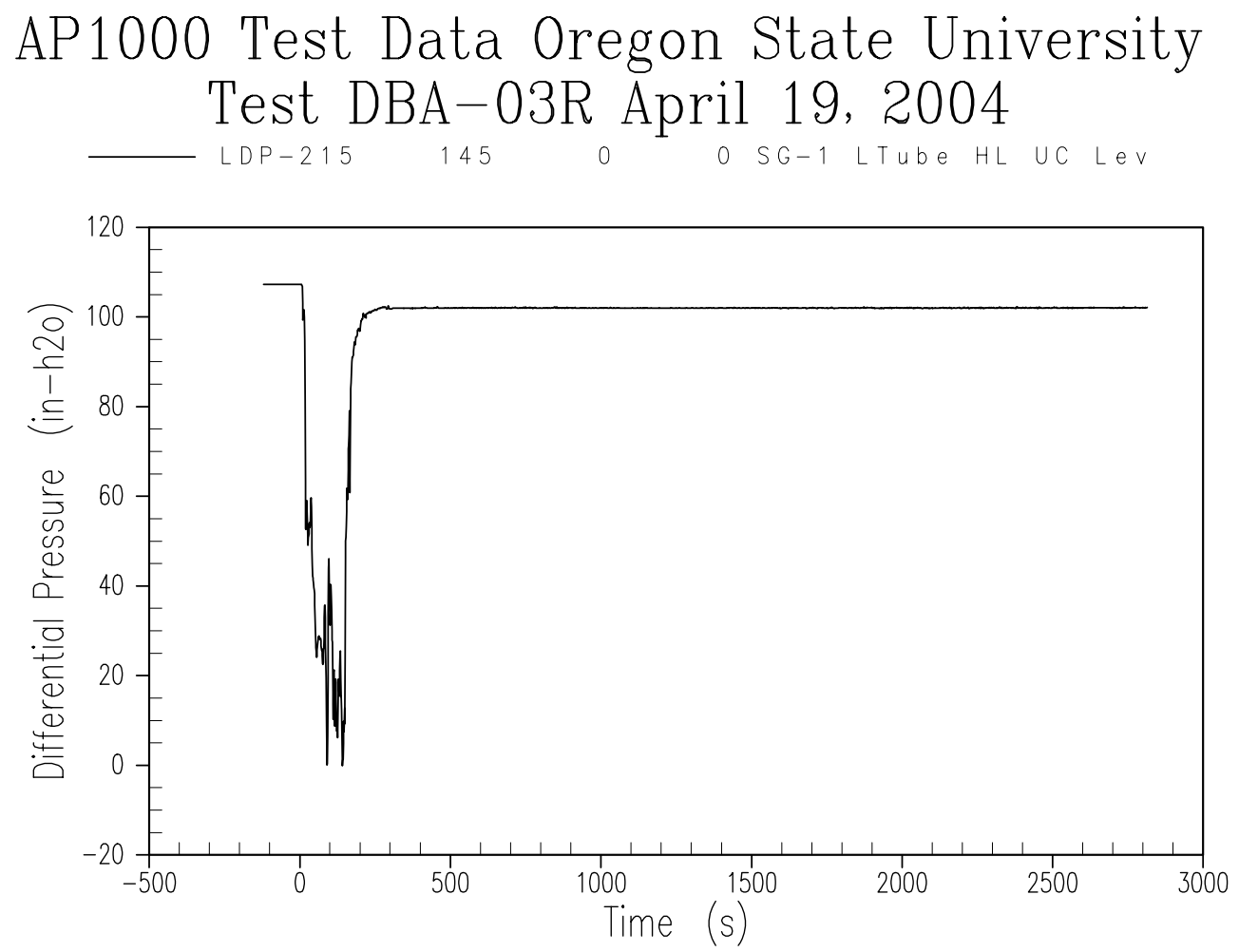

Figure A-14 Steam Generator 1 Tube Level 

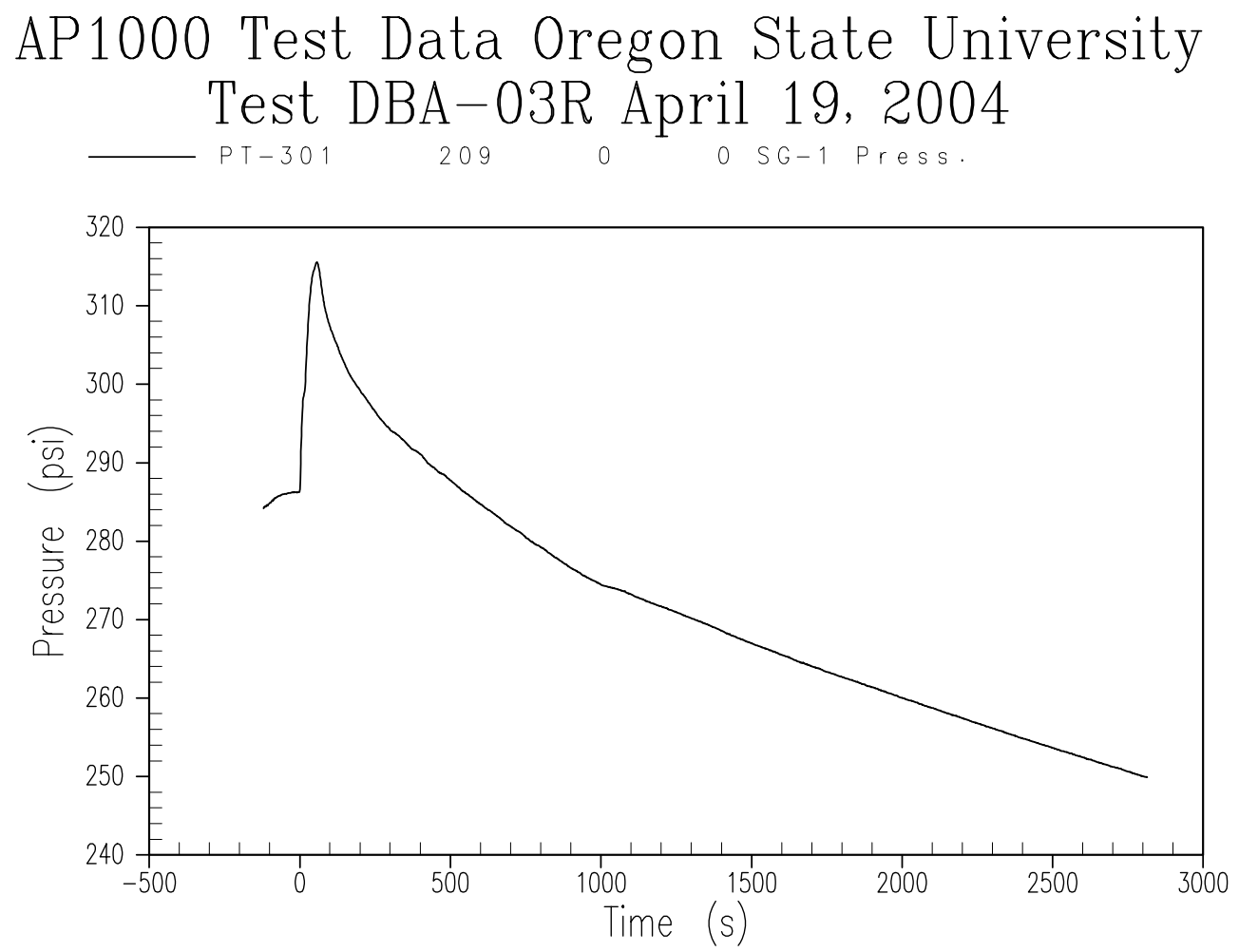

Figure A-15 Steam Generator 1 Secondary Pressure 

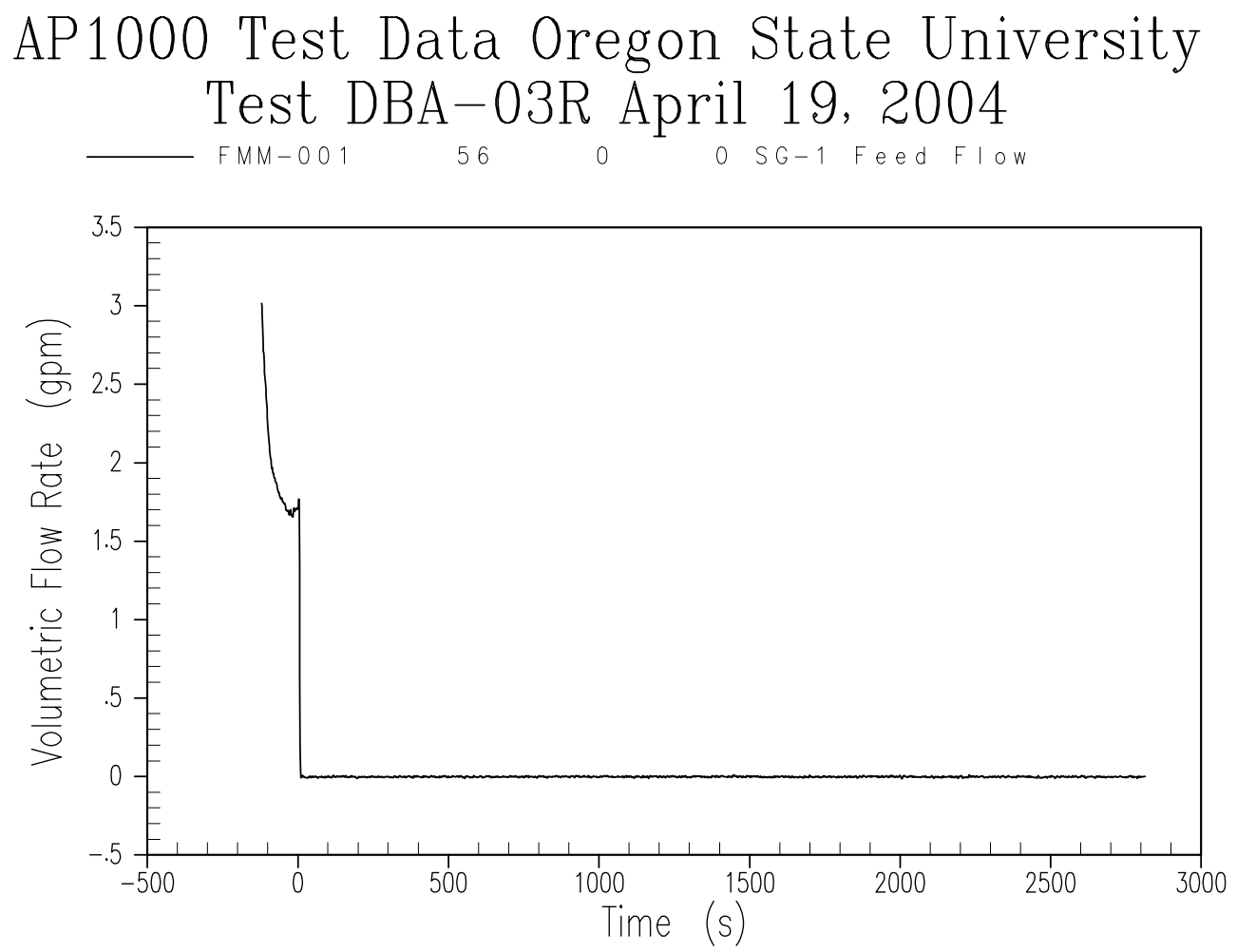

Figure A-16 Steam Generator 1 Feed Flow Rate 

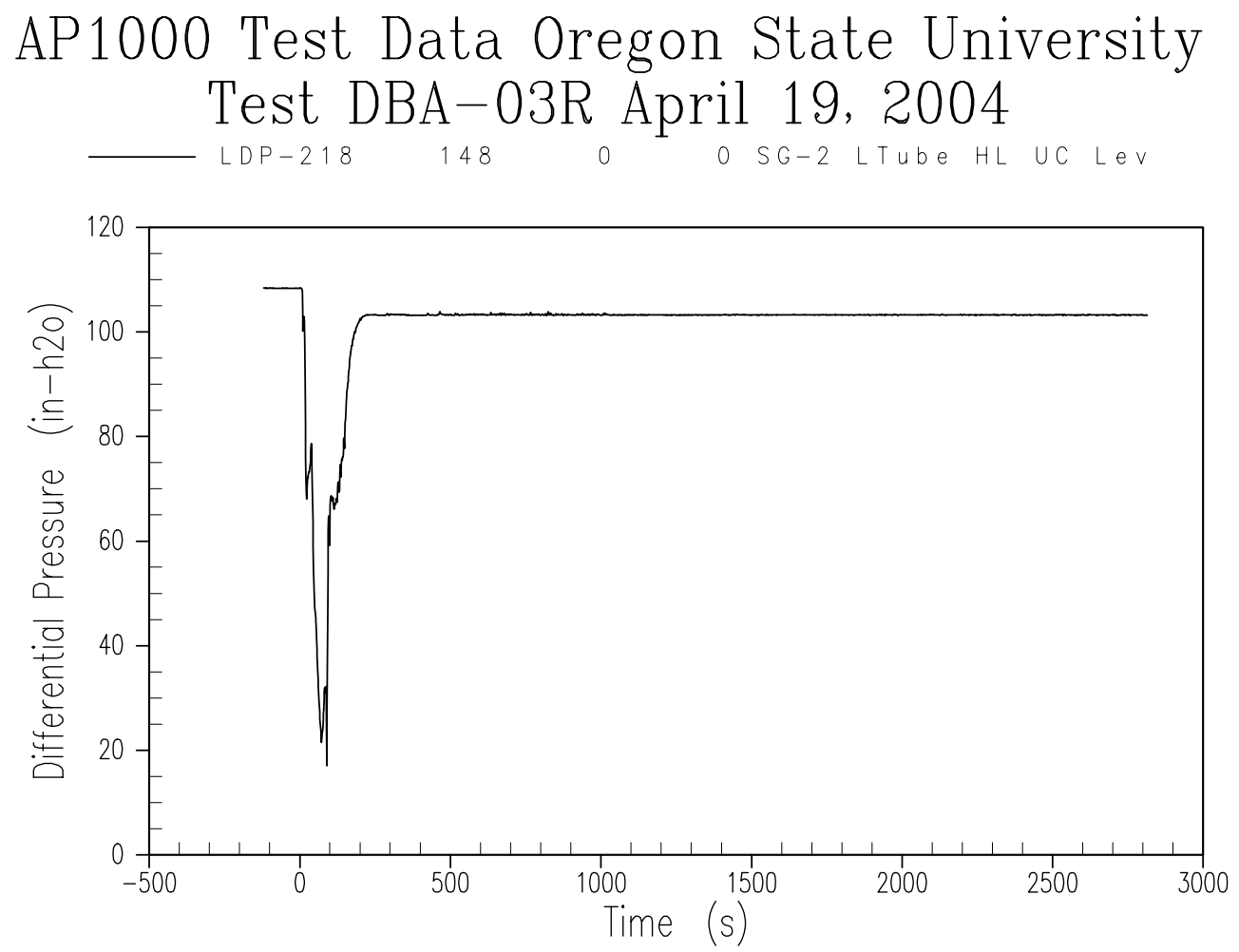

Figure A-17 Steam Generator 2 Tube Level 

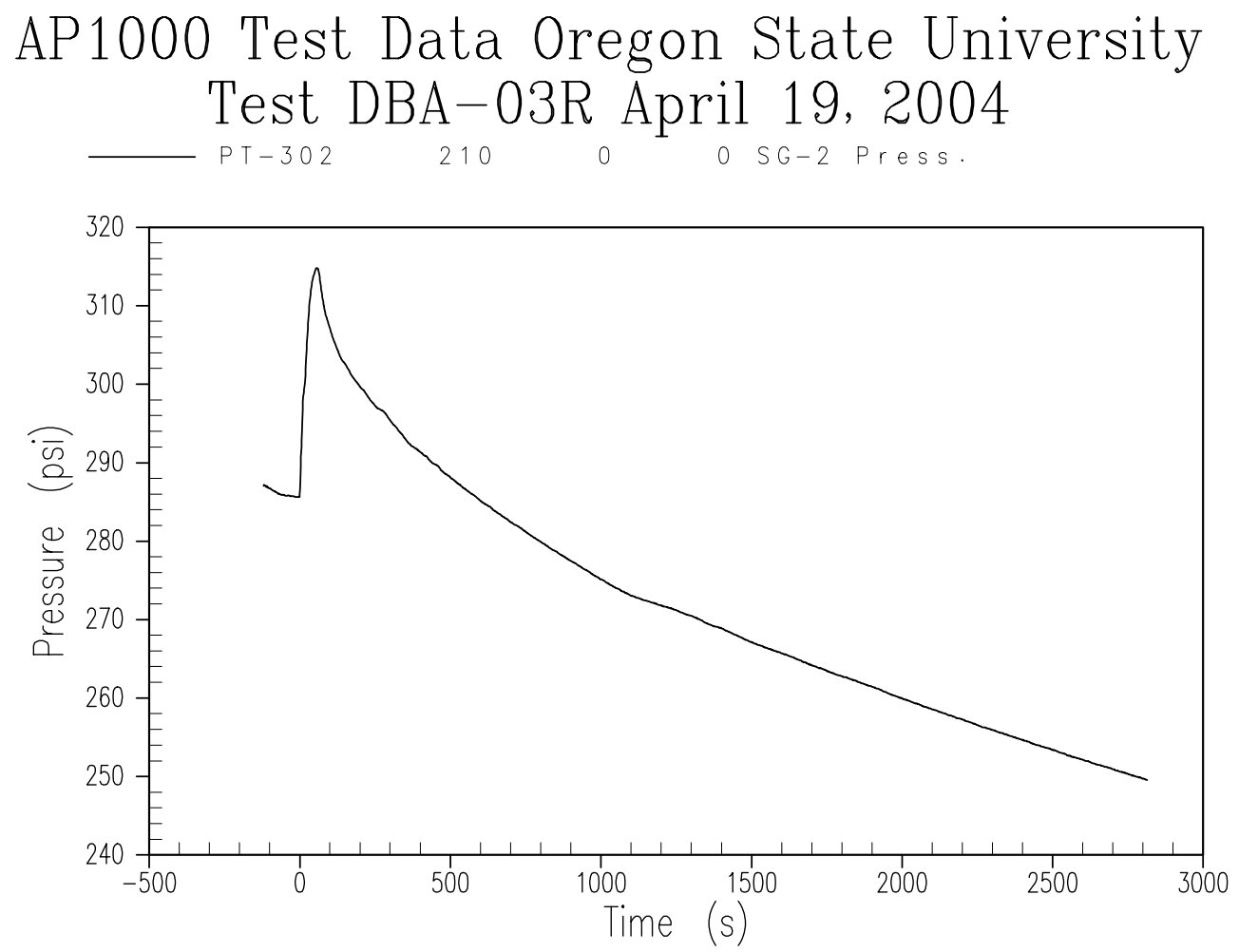

Figure A-18 Steam Generator 2 Secondary Pressure 

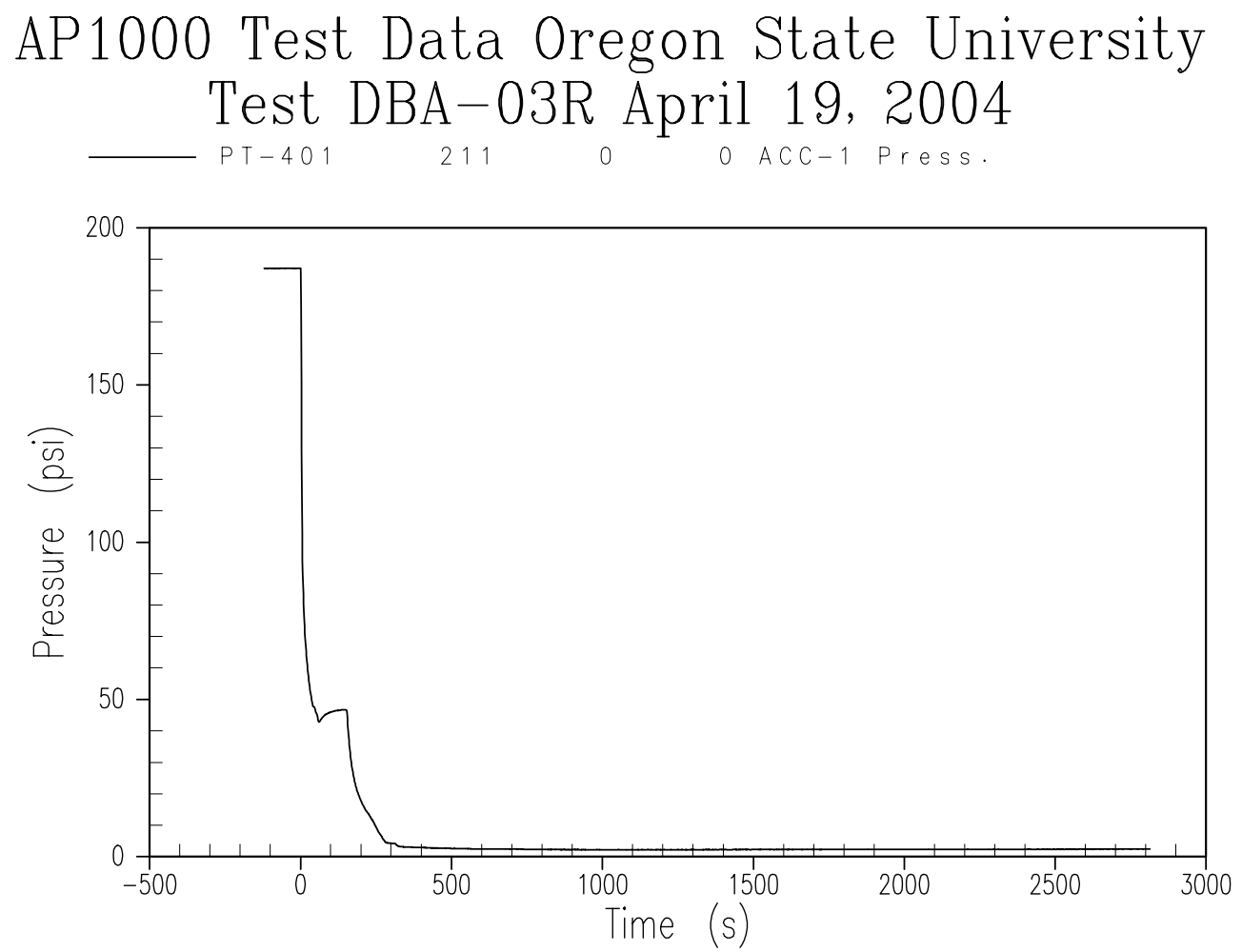

Figure A-19 Accumulator 1 Pressure 

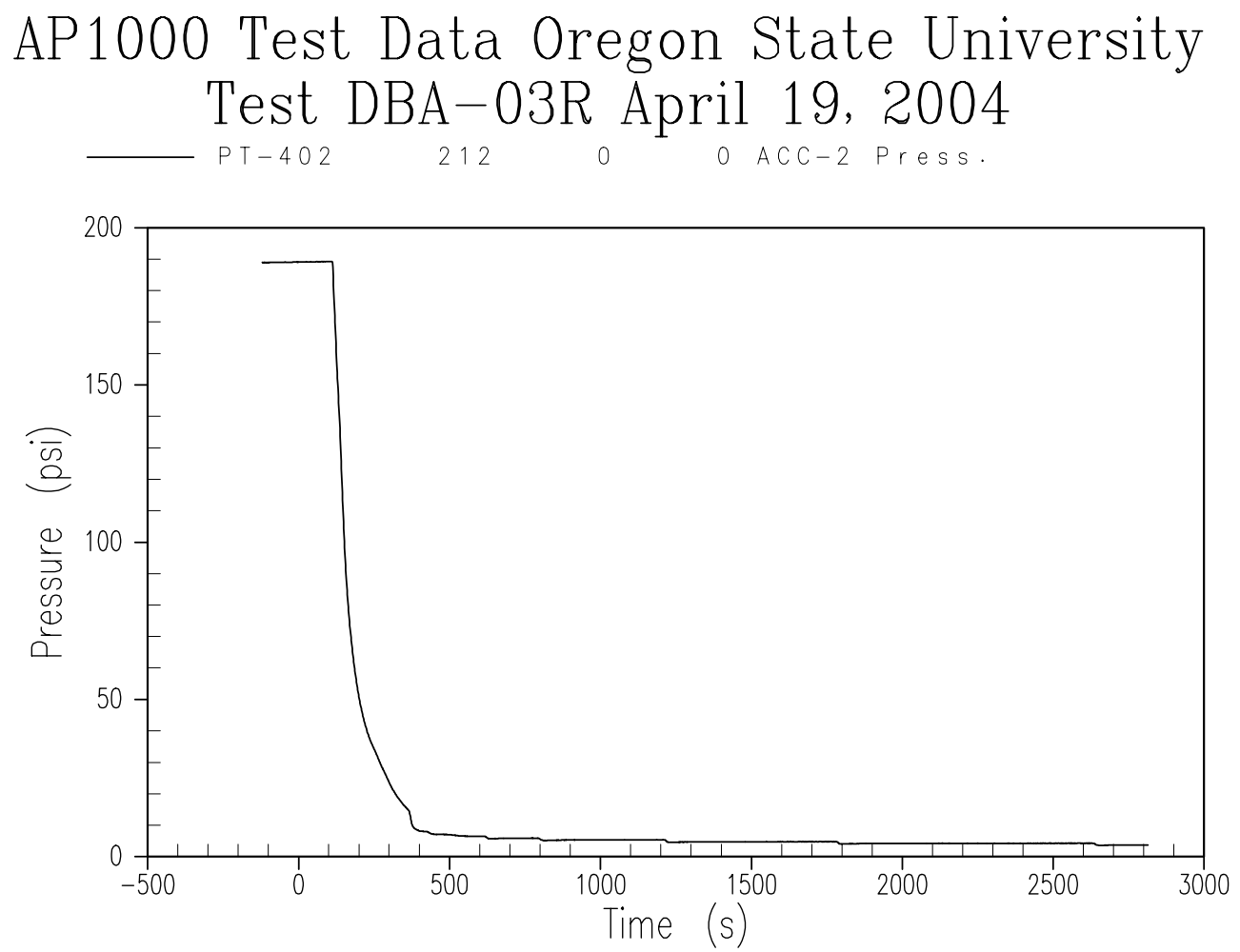

Figure A-20 Accumulator 2 Pressure 

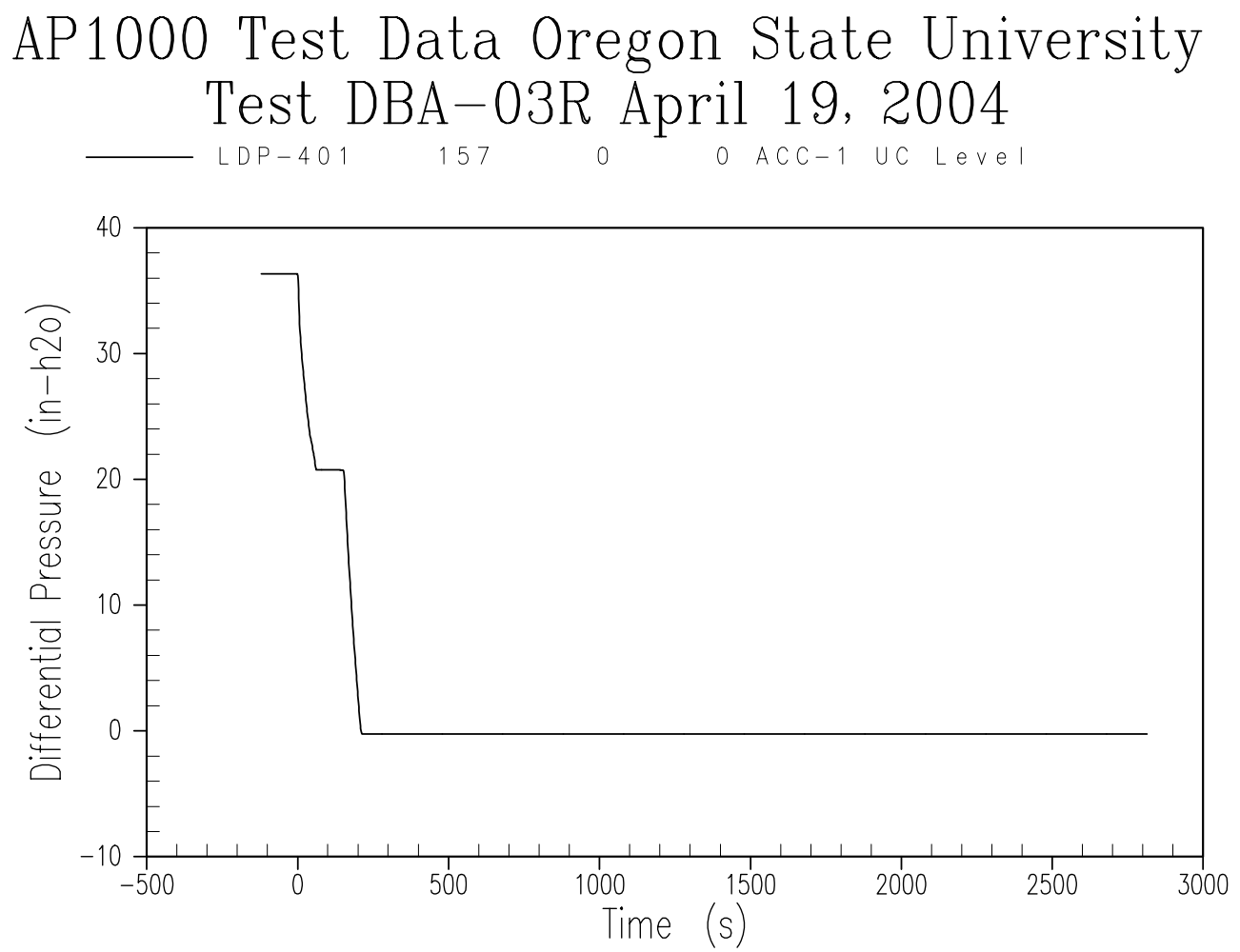

Figure A-21 Accumulator 1 Liquid Level 

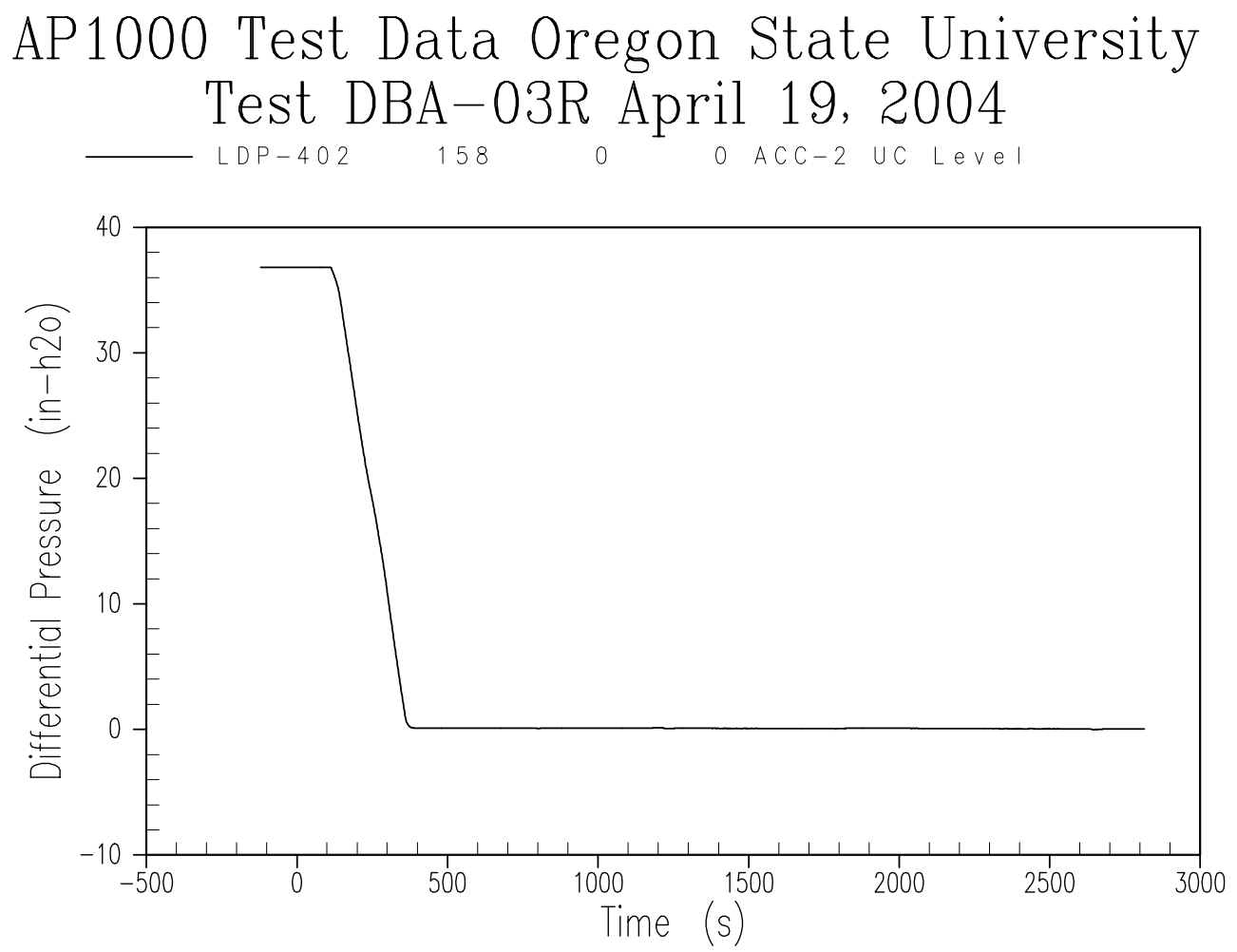

Figure A-22 Accumulator 2 Liquid Level 

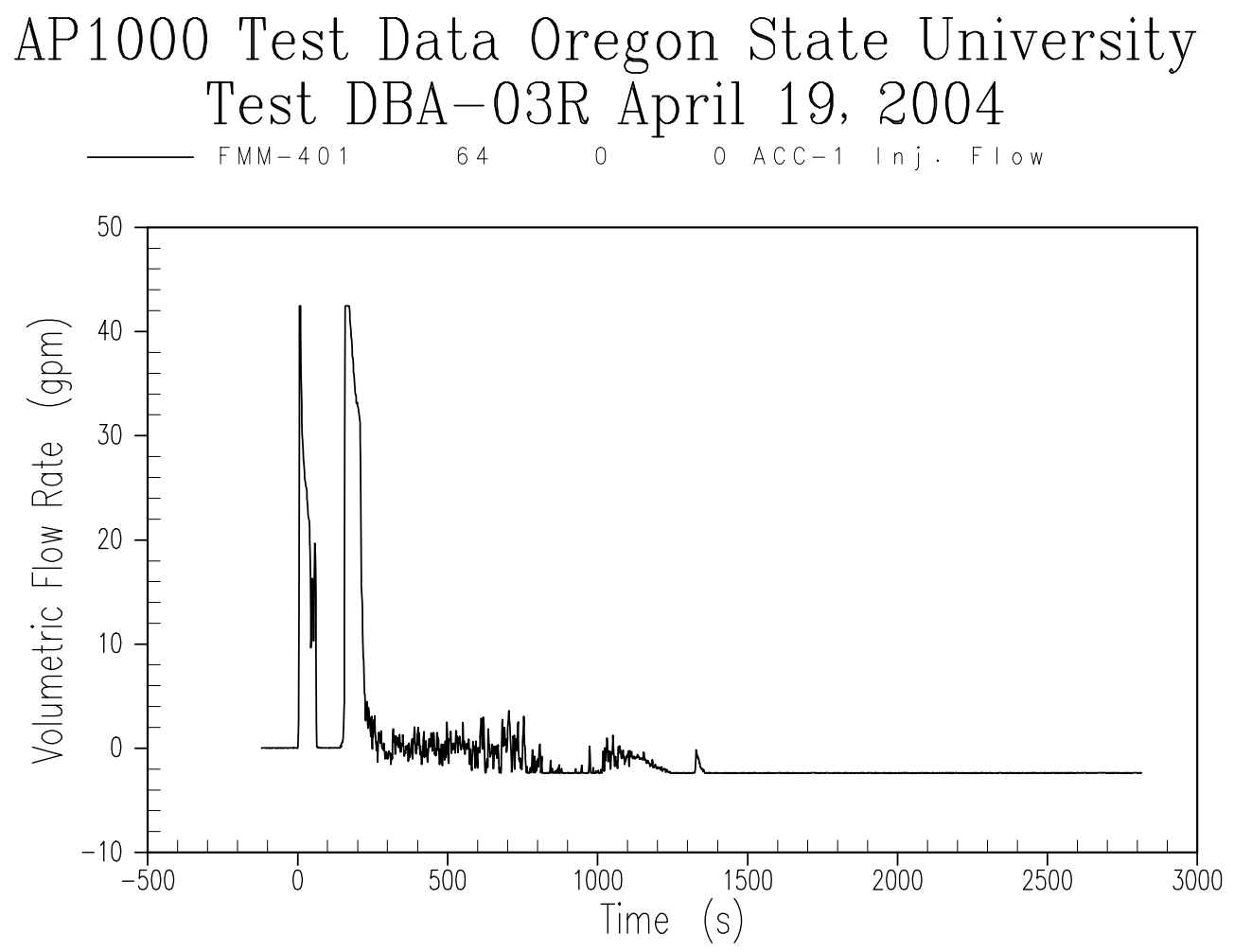

Figure A-23 Accumulator 1 Flow Rate 

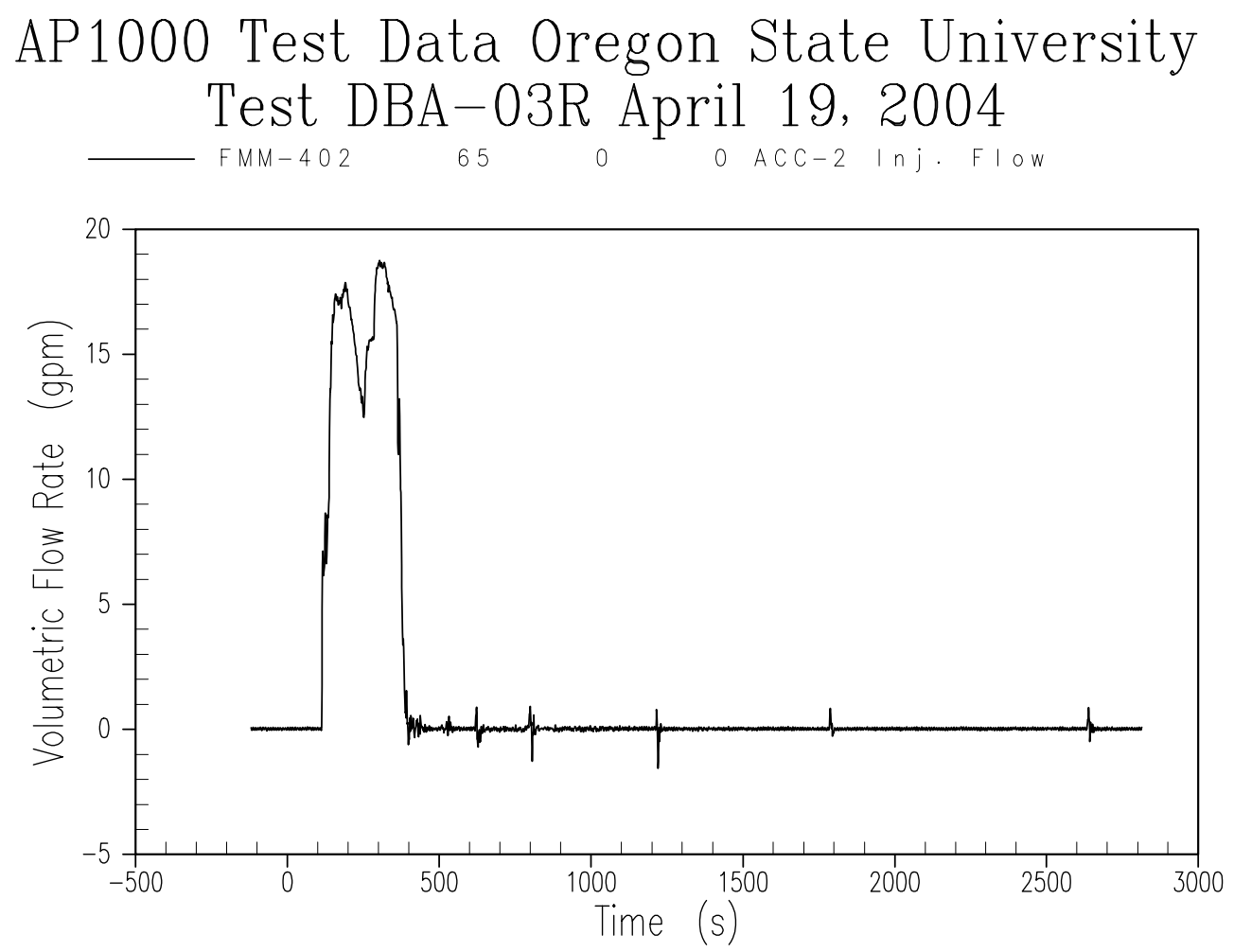

Figure A-24 Accumulator 2 Flow Rate 

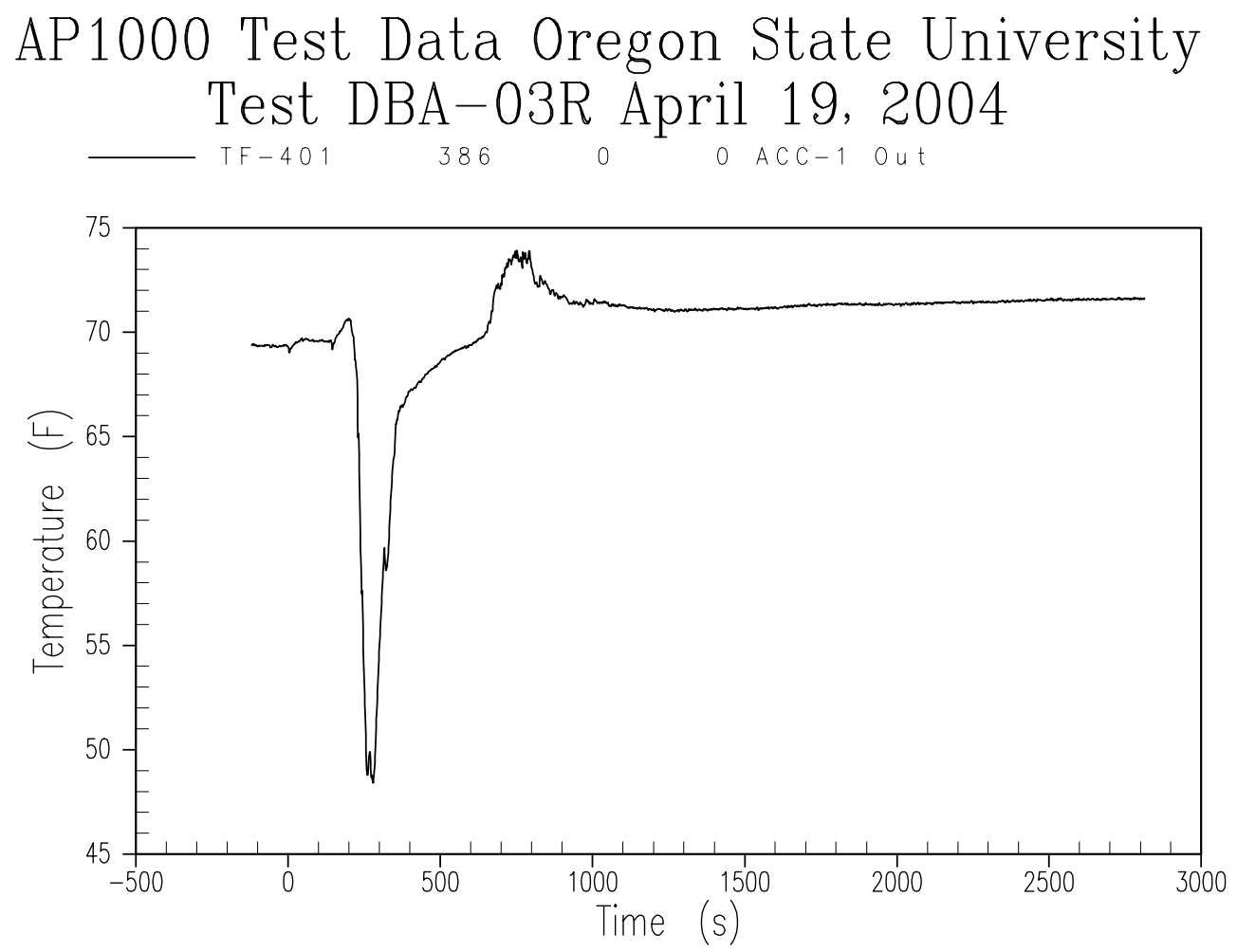

Figure A-25 Accumulator 1 Liquid Discharge Temperature 

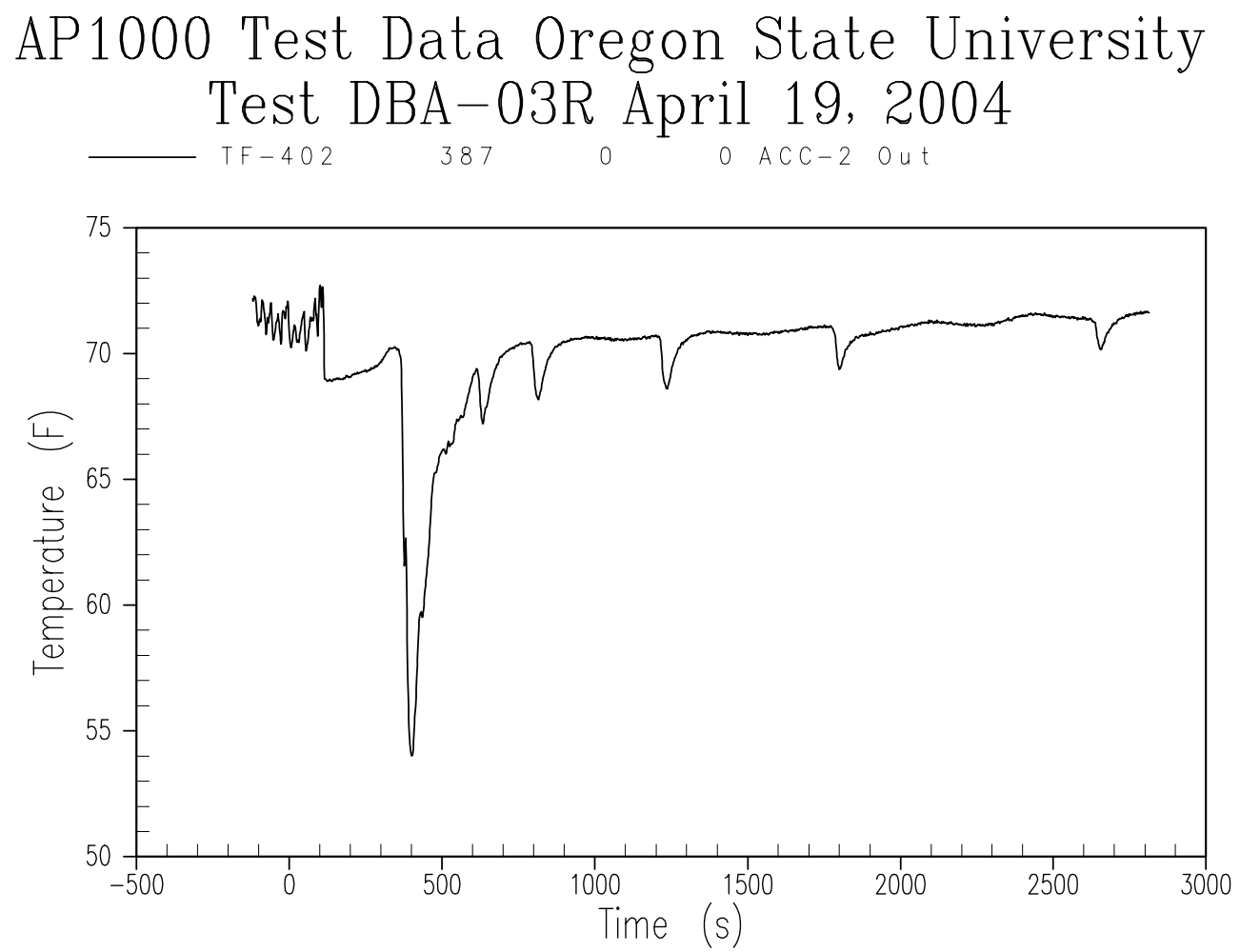

Figure A-26 Accumulator 2 Liquid Discharge Temperature 

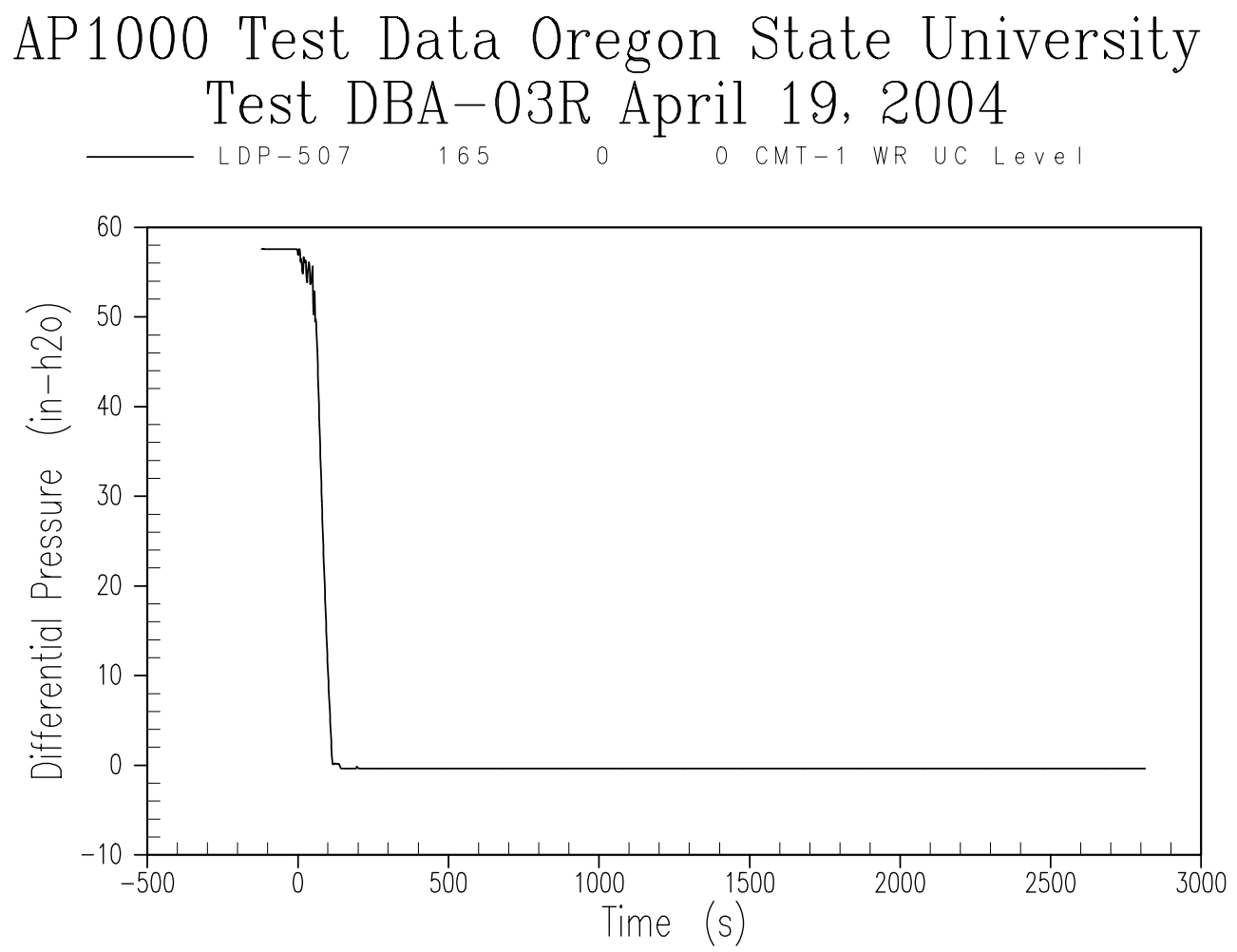

Figure A-27 Core Makeup Tank 1 Liquid Level 

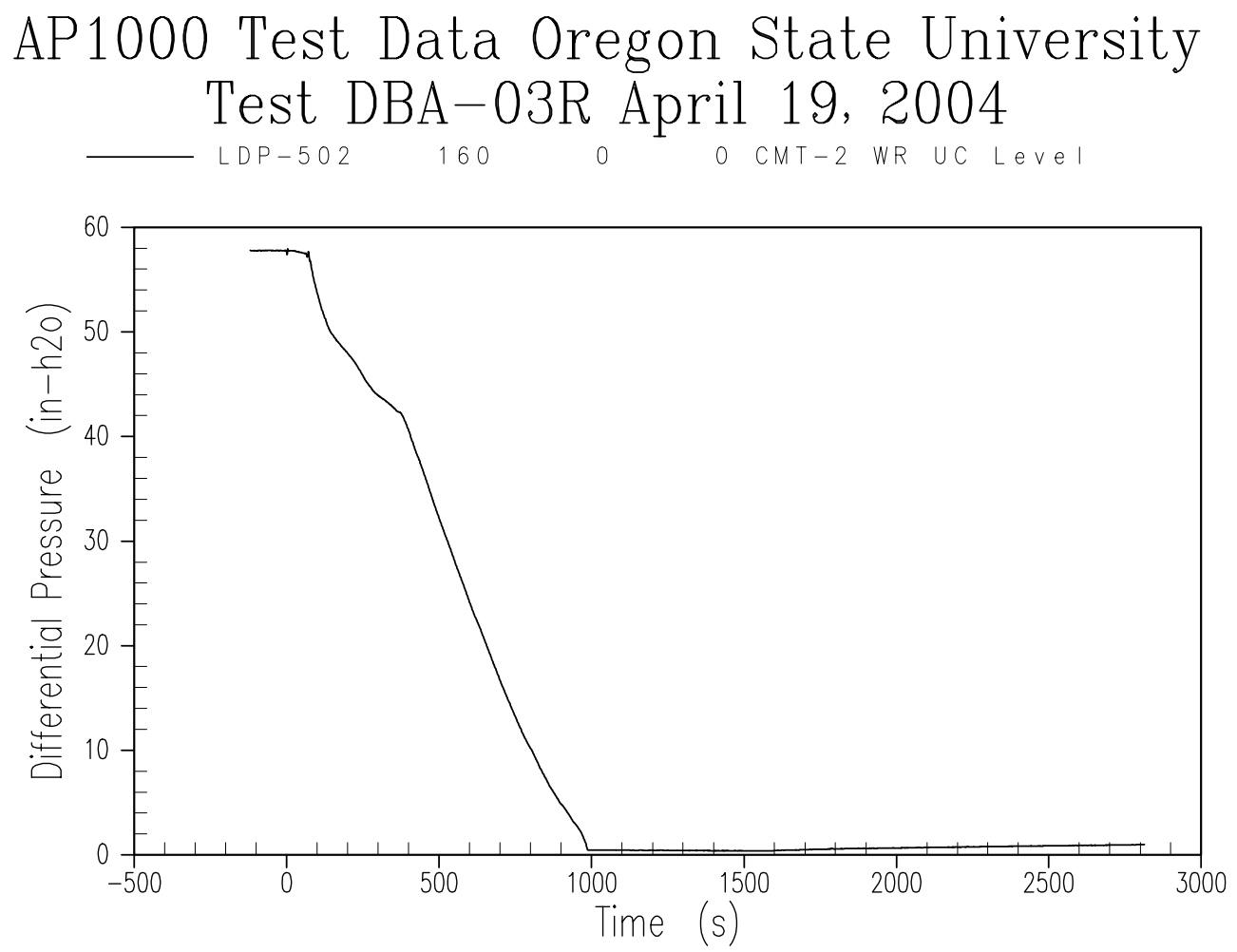

Figure A-28 Core Makeup Tank 2 Liquid Level 
AP1000 Test Data Oregon State University Test DBA-03R April 19, 2004
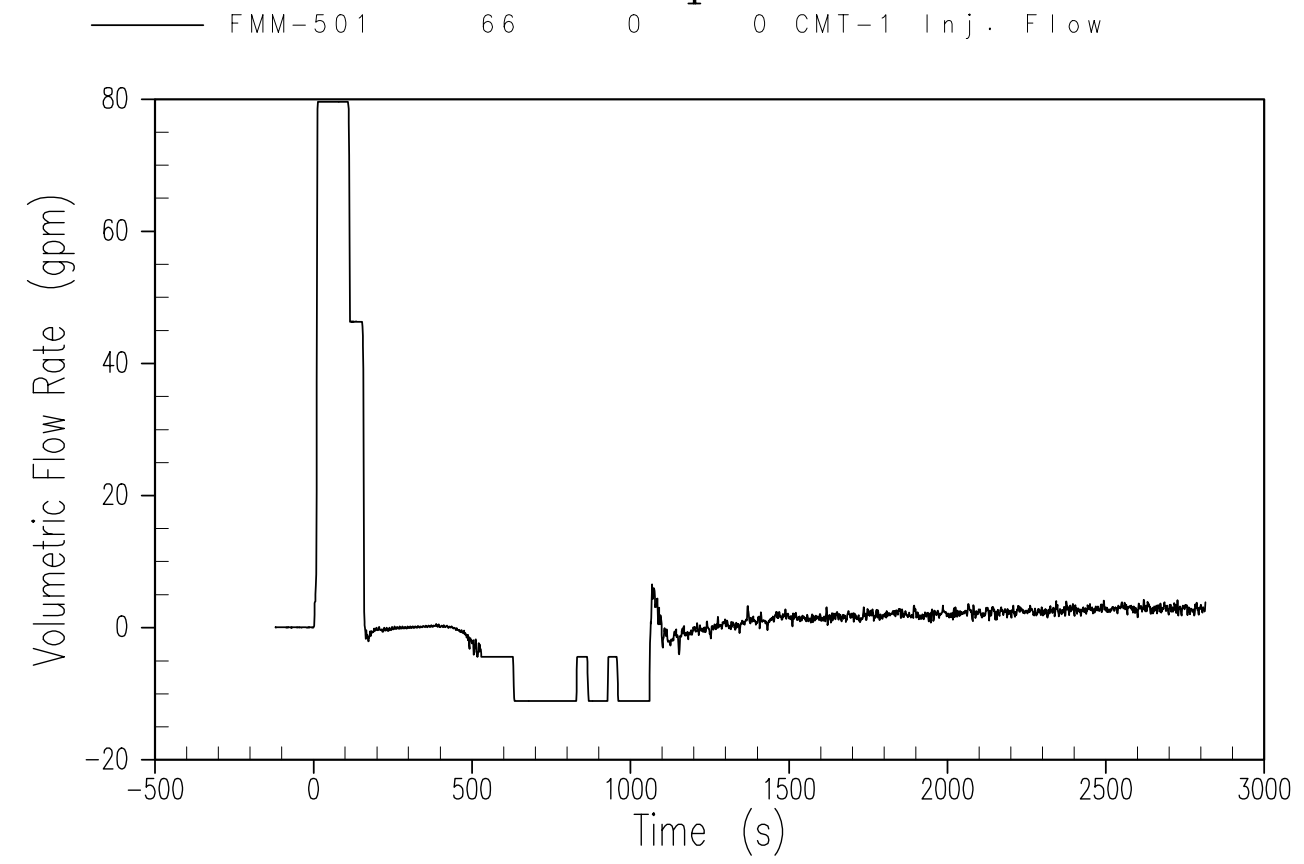

Figure A-29 Core Makeup Tank 1 Flow Rate 

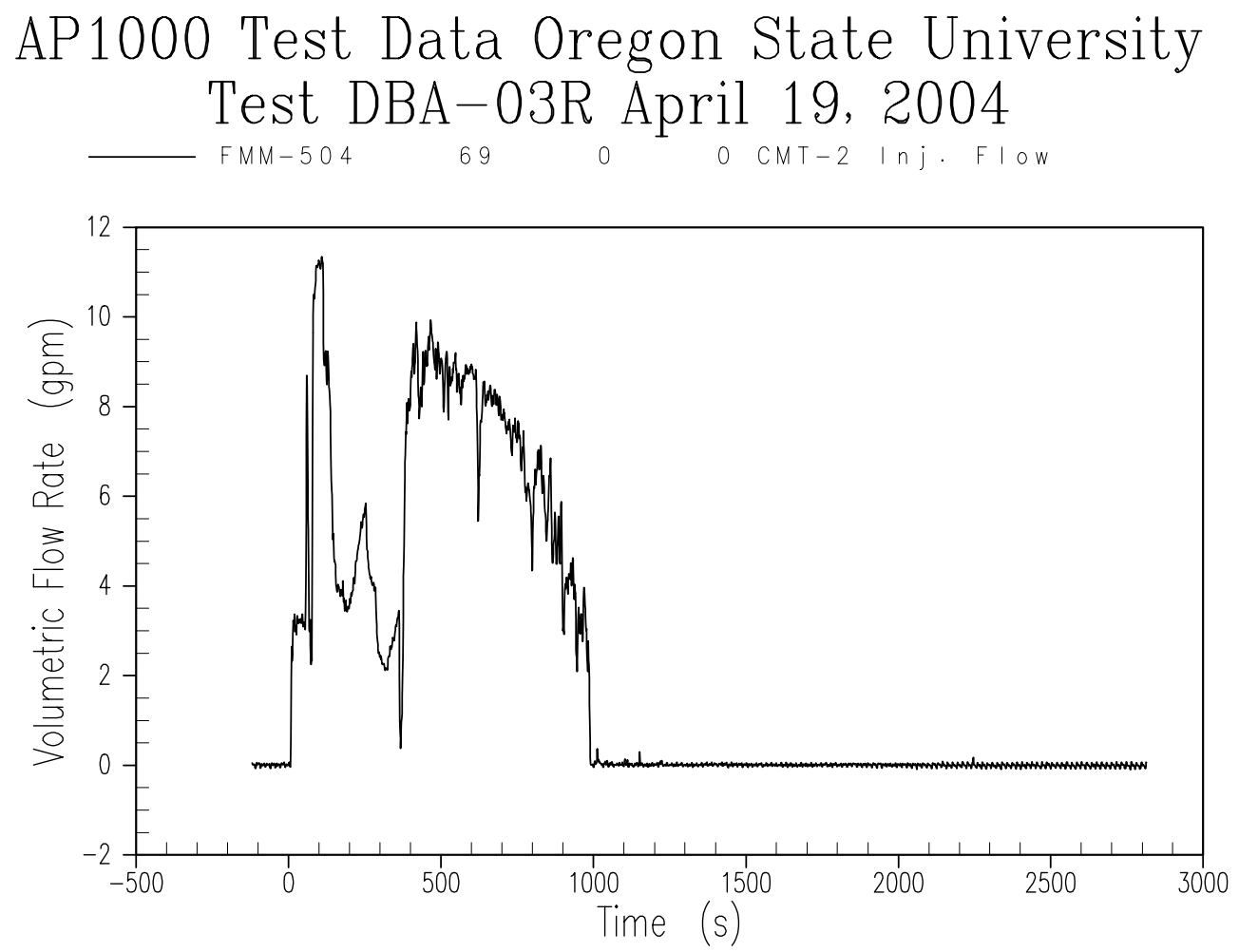

Figure A-30 Core Makeup Tank 2 Flow Rate 
AP1000 Test Data Oregon State University Test DBA-03R April 19, 2004
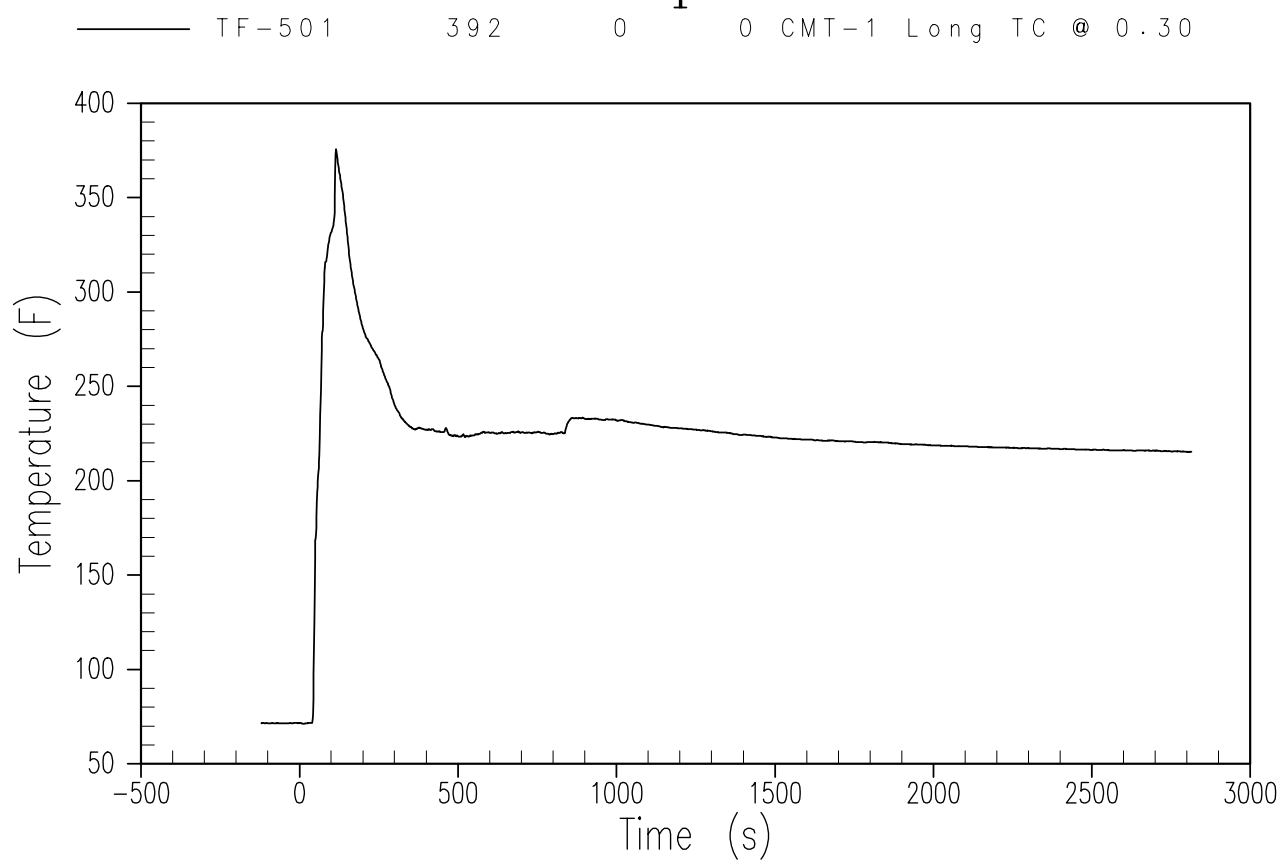

Figure A-31 Core Makeup Tank 1 Liquid Temperature - Bottom 
AP1000 Test Data Oregon State University Test DBA-03R April 19, 2004

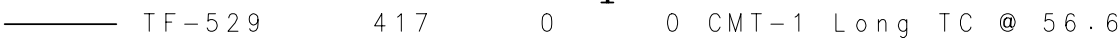

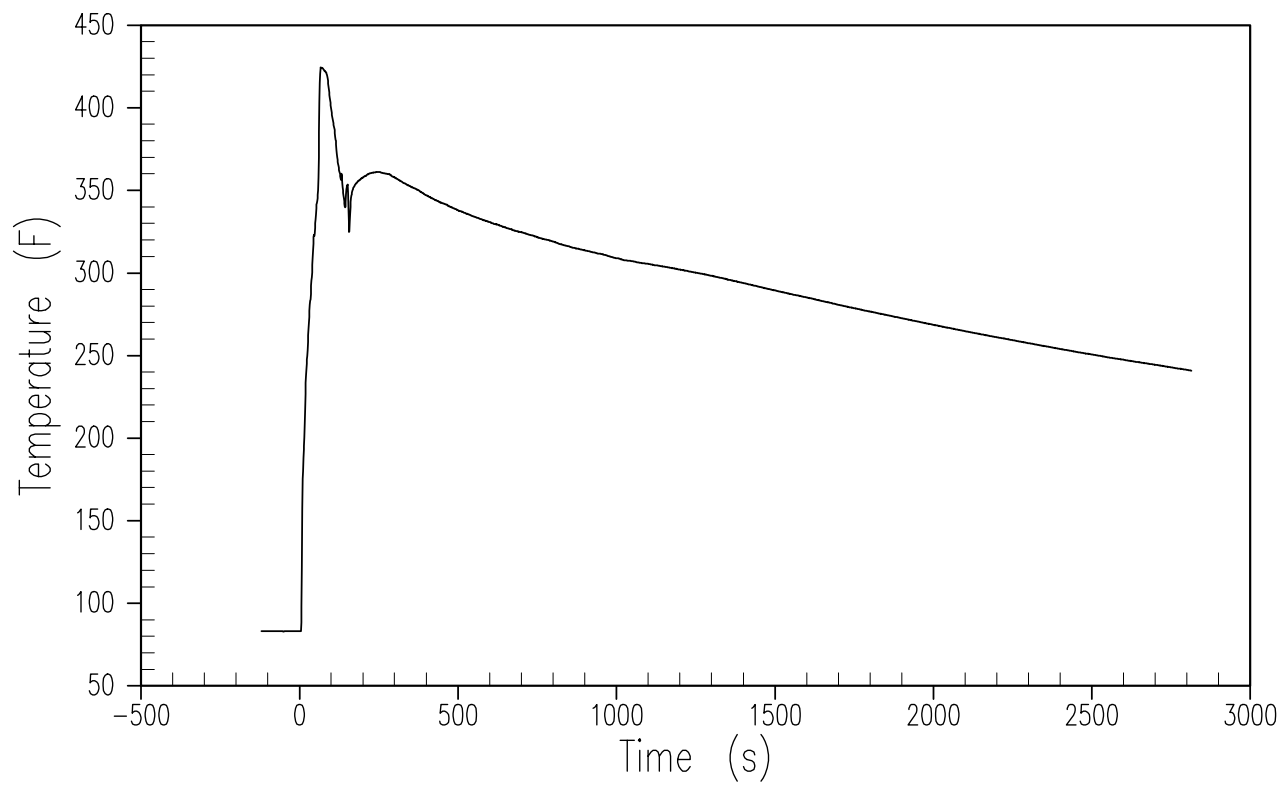

Figure A-32 Core Makeup Tank 1 Liquid Temperature - Top 
AP1000 Test Data Oregon State University Test DBA-03R April 19, 2004
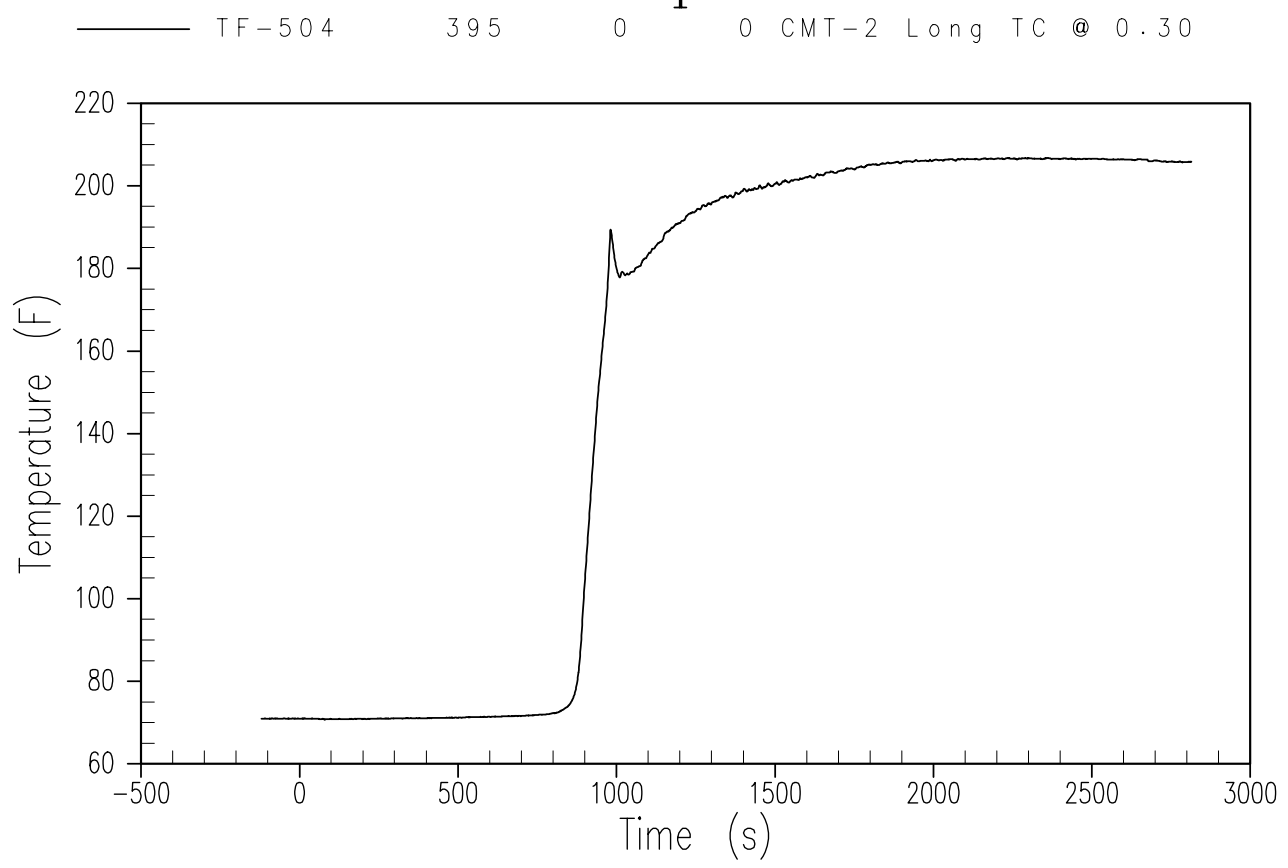

Figure A-33 Core Makeup Tank 2 Liquid Temperature - Bottom 
AP1000 Test Data Oregon State University Test DBA-03R April 19, 2004
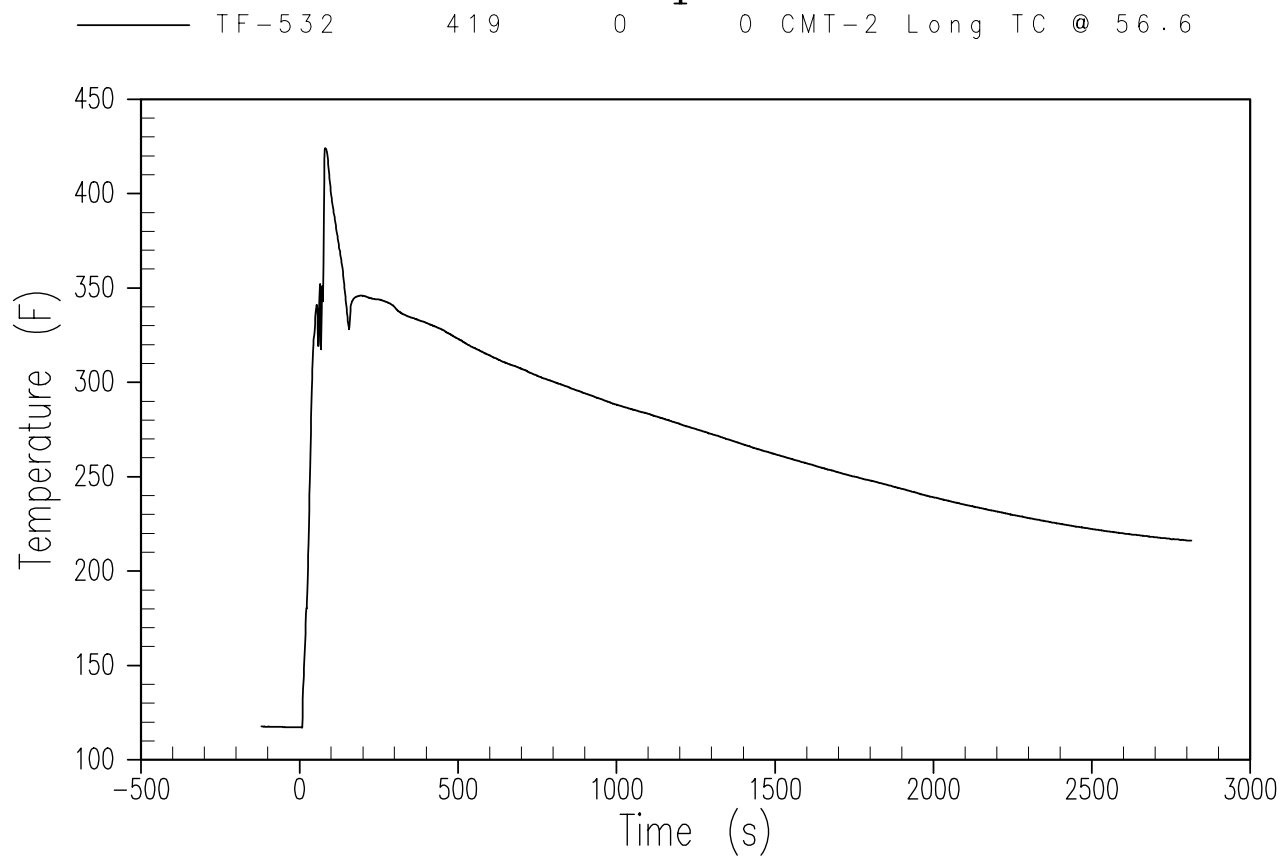

Figure A-34 Core Makeup Tank 2 Liquid Temperature - Top 


\section{AP1000 Test Data Oregon State University Test DBA-03R April 19, 2004

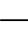

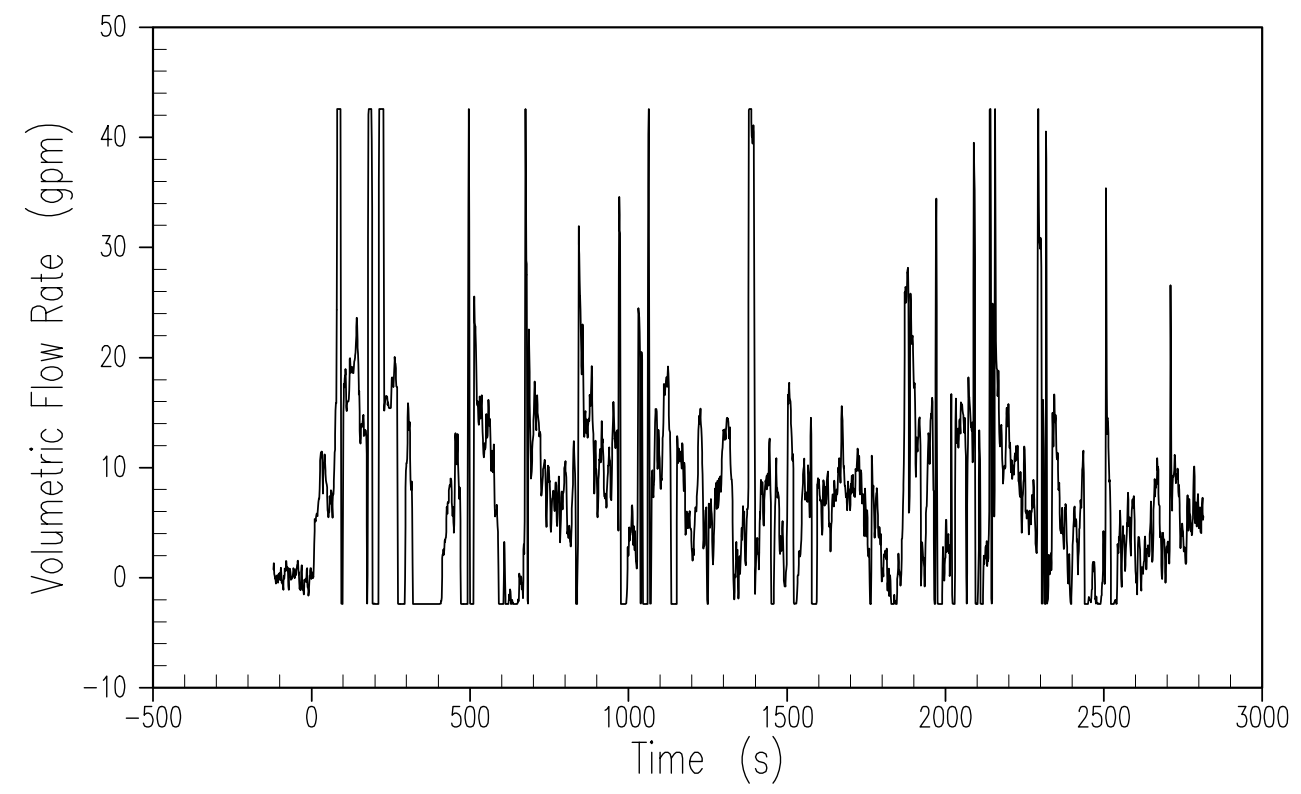

Figure A-35 Passive Residual Heat Removal Inlet Flow Rate 


\section{AP1000 Test Data Oregon State University Test DBA-03R April 19, 2004

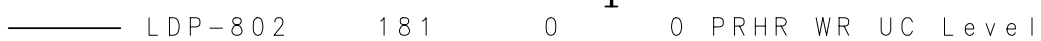

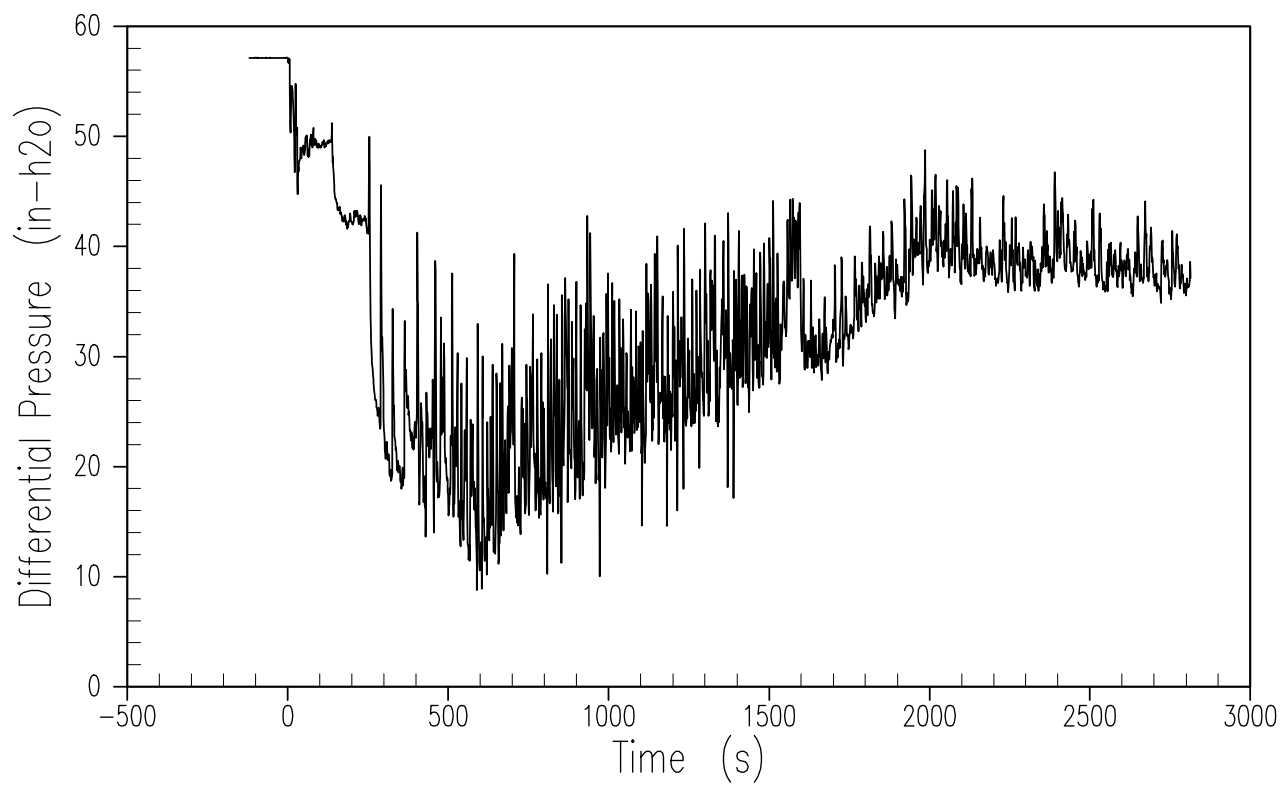

Figure A-36 Passive Residual Heat Removal Liquid Level 


\section{AP1000 Test Data Oregon State University Test DBA-03R April 19, 2004}

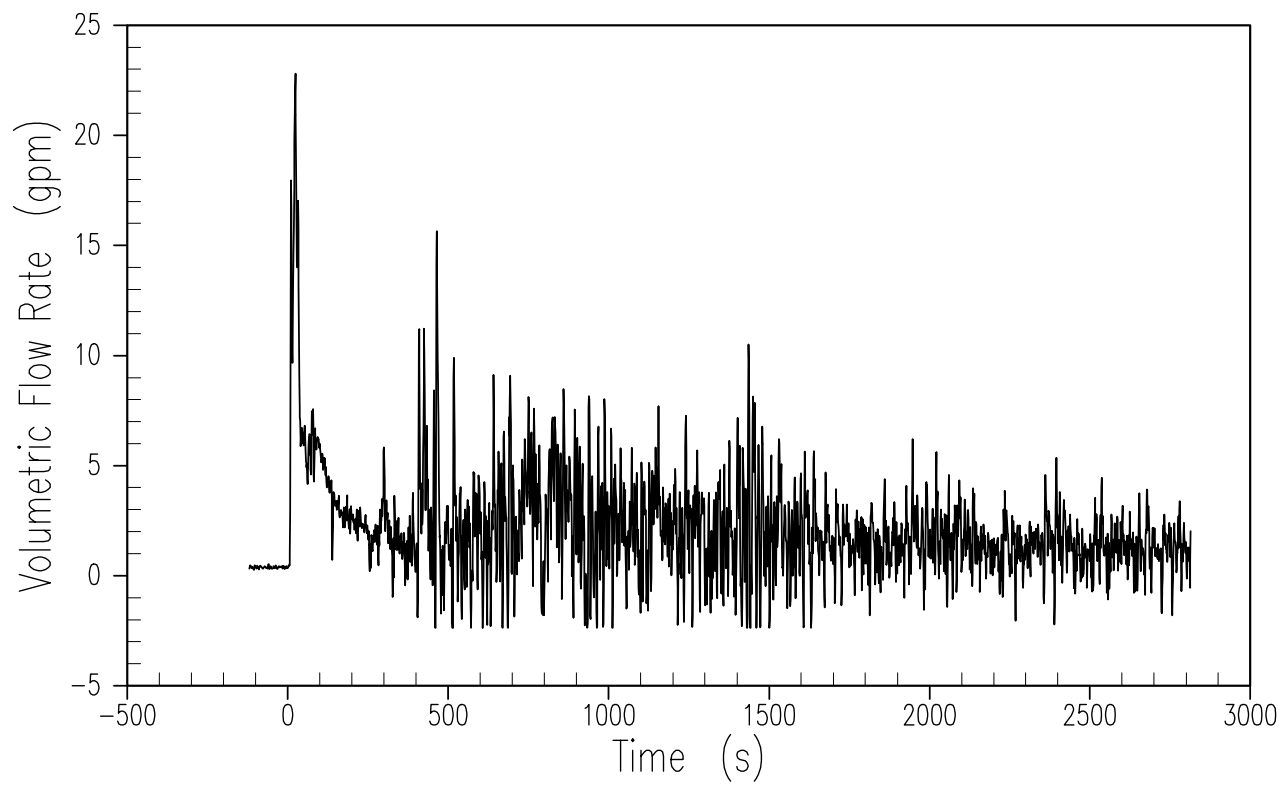

Figure A-37 Passive Residual Heat Removal Outlet Flow Rate 

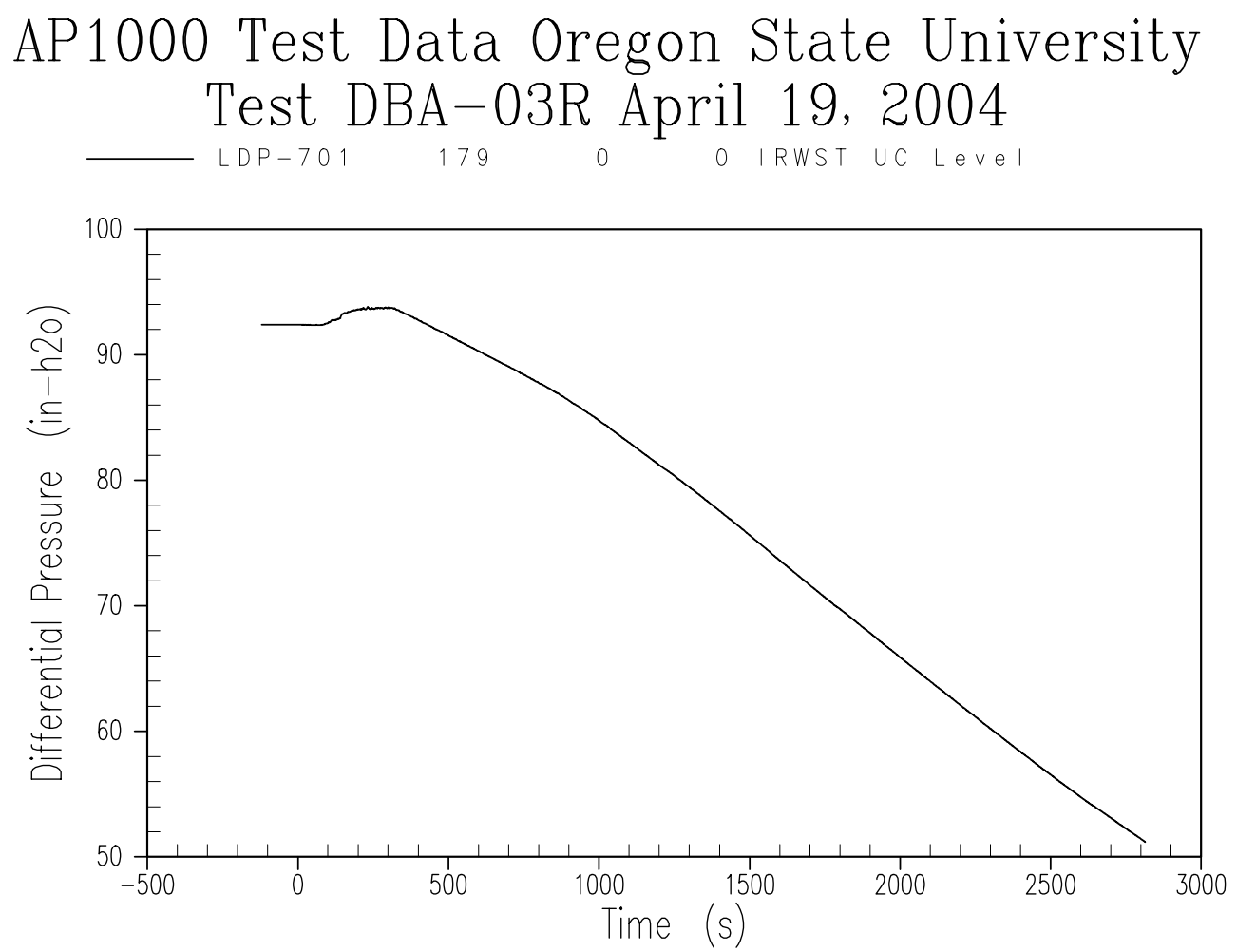

Figure A-38 IRWST Liquid Level 

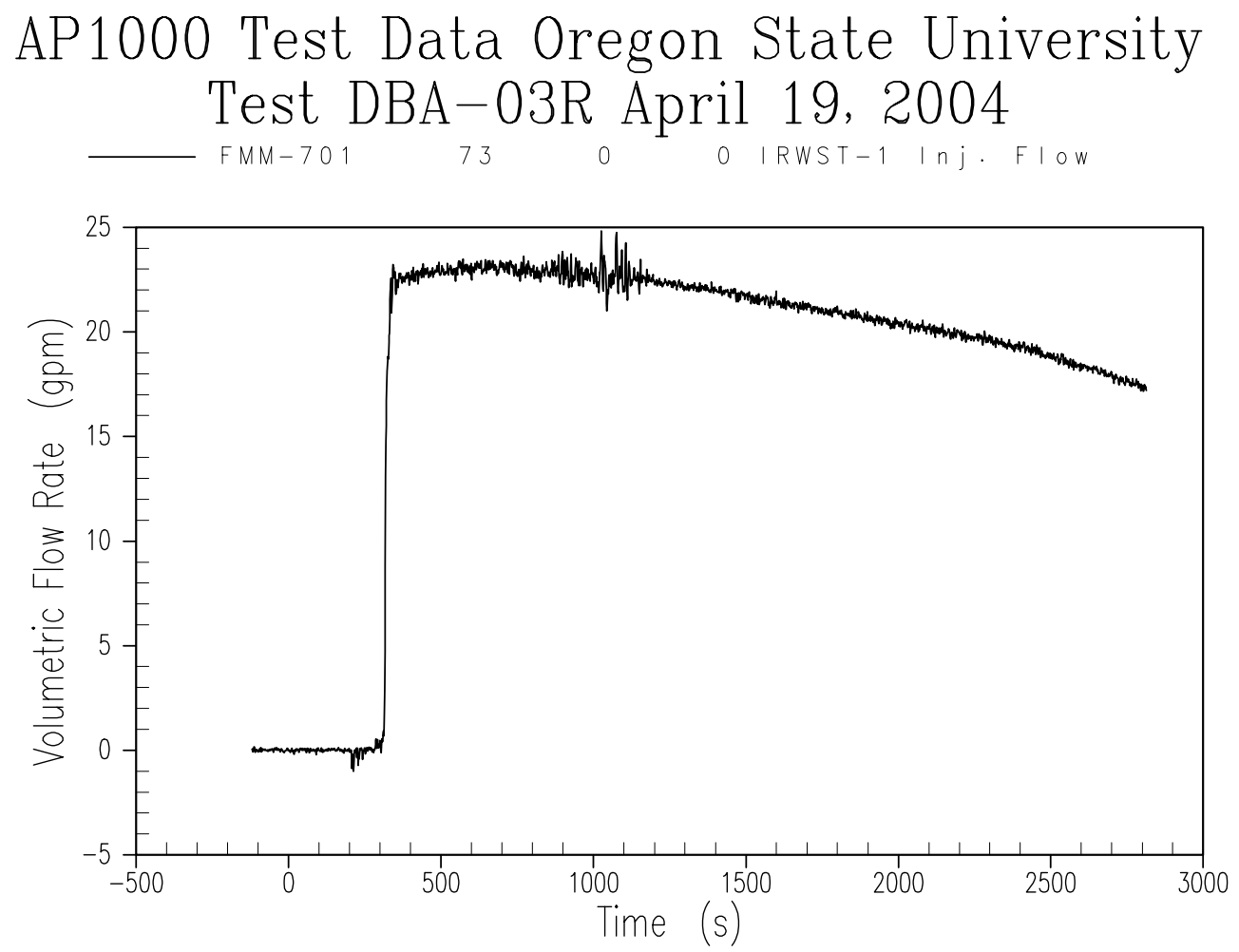

Figure A-39 IRWST Discharge Line 1 Flow Rate 

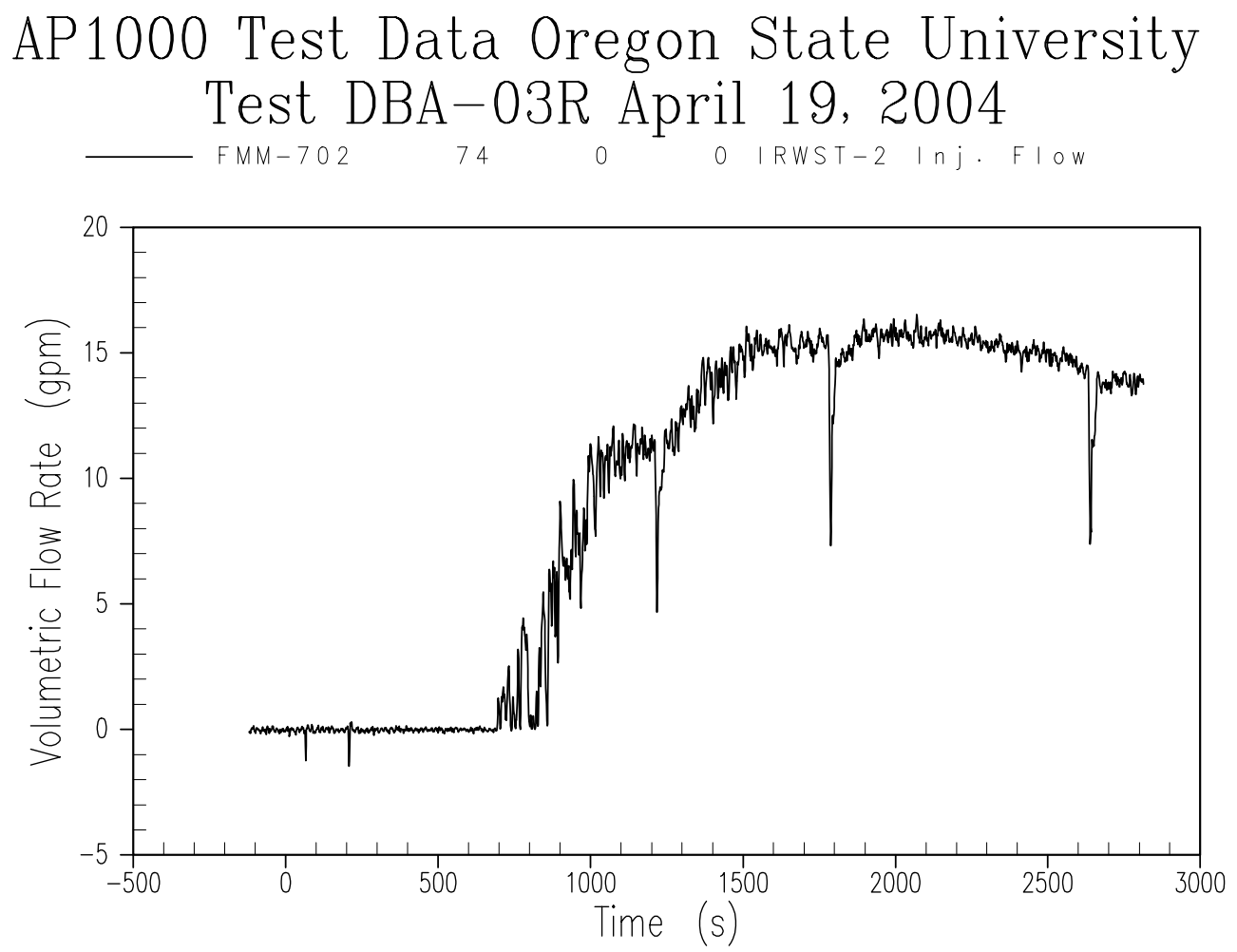

Figure A-40 IRWST Discharge Line 2 Flow Rate 

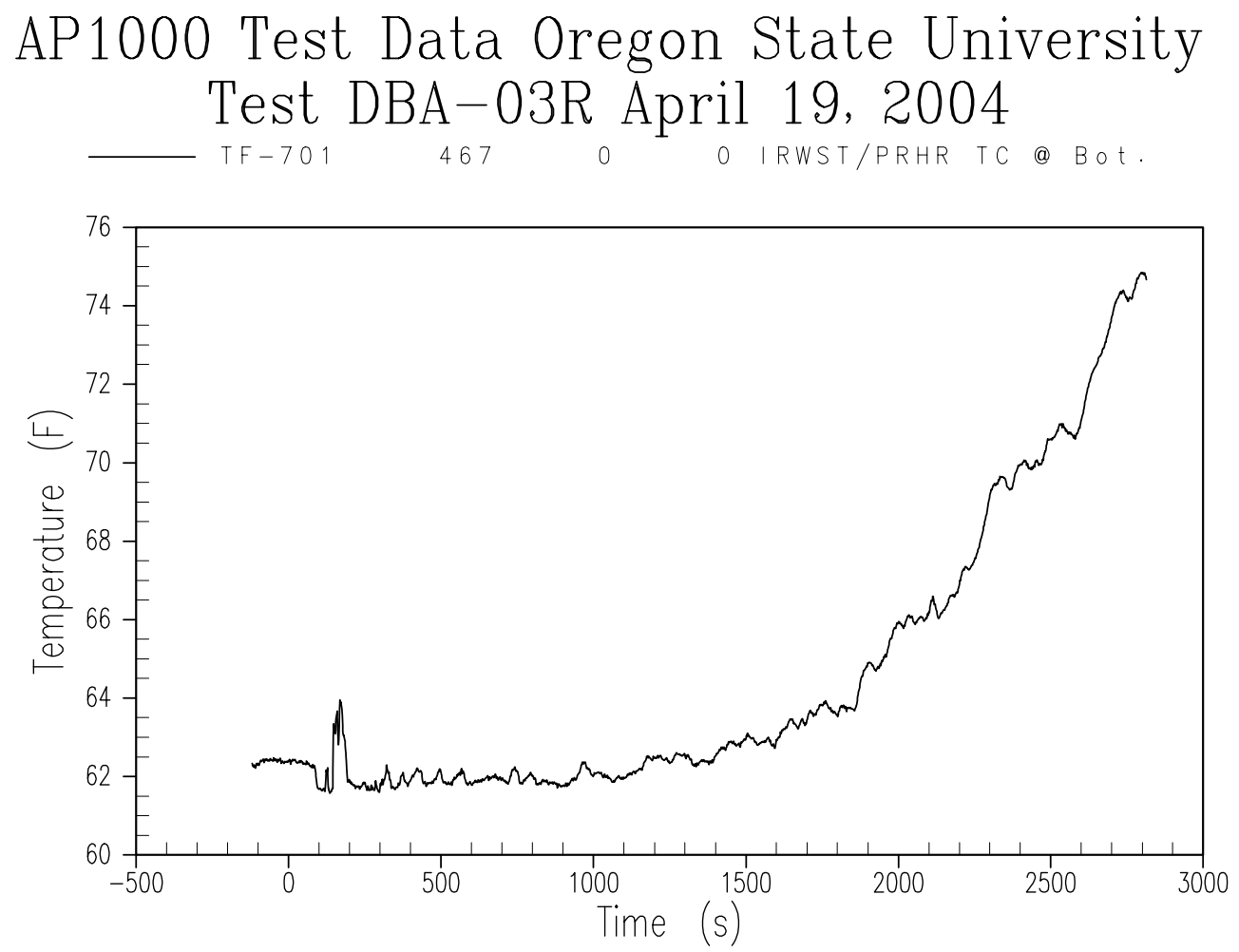

Figure A-41 IRWST Fluid Temperature - Bottom 
AP1000 Test Data Oregon State University Test DBA-03R April 19, 2004
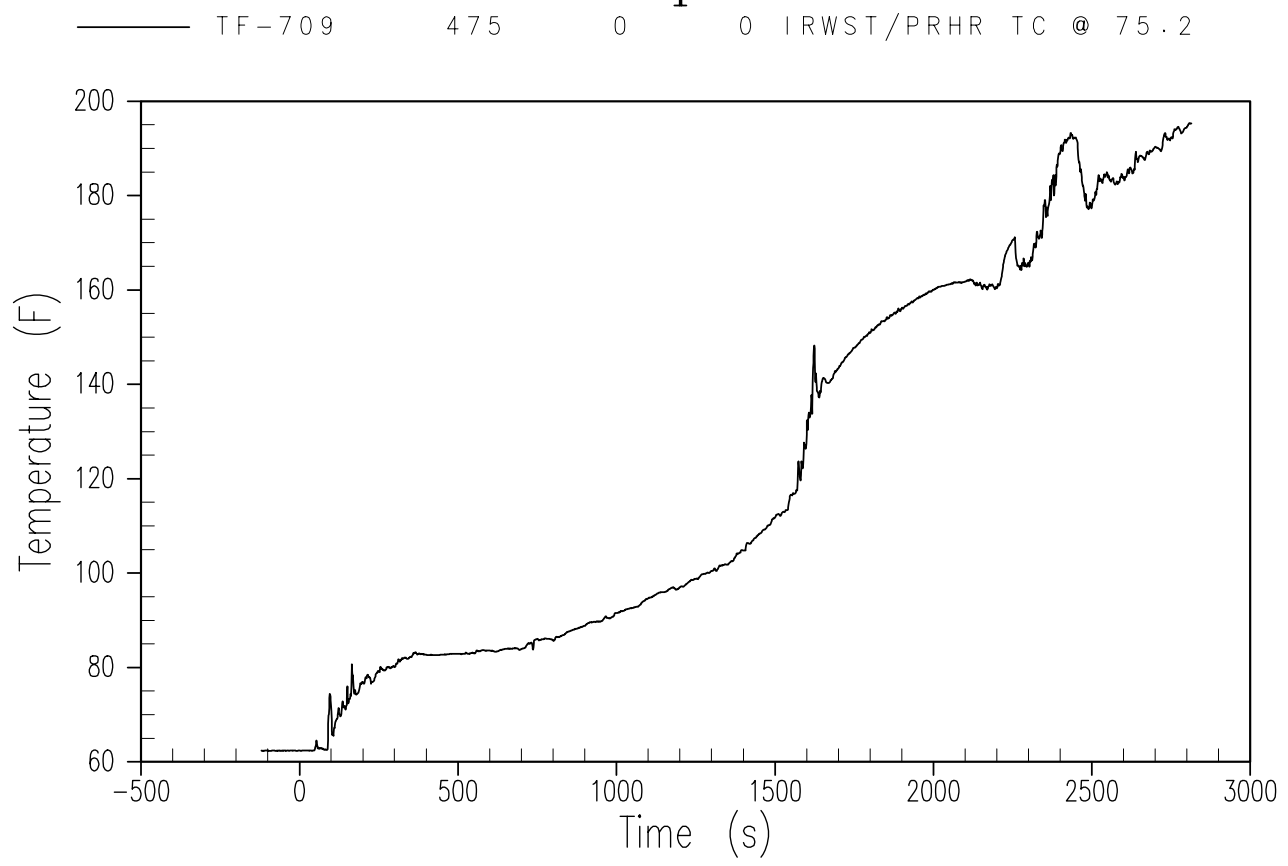

Figure A-42 IRWST Fluid Temperature - Top 

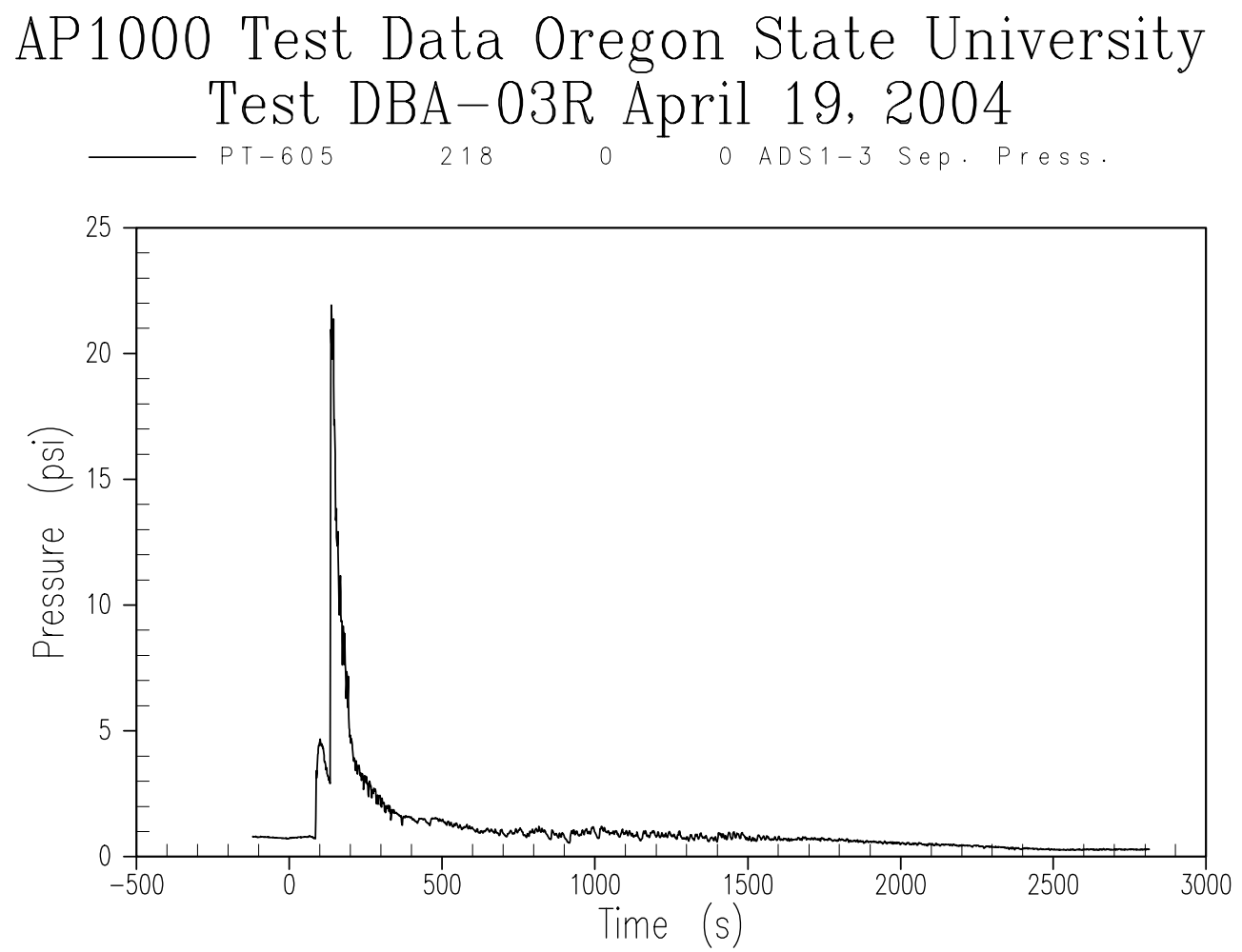

Figure A-43 ADS 1-3 Separator Pressure 

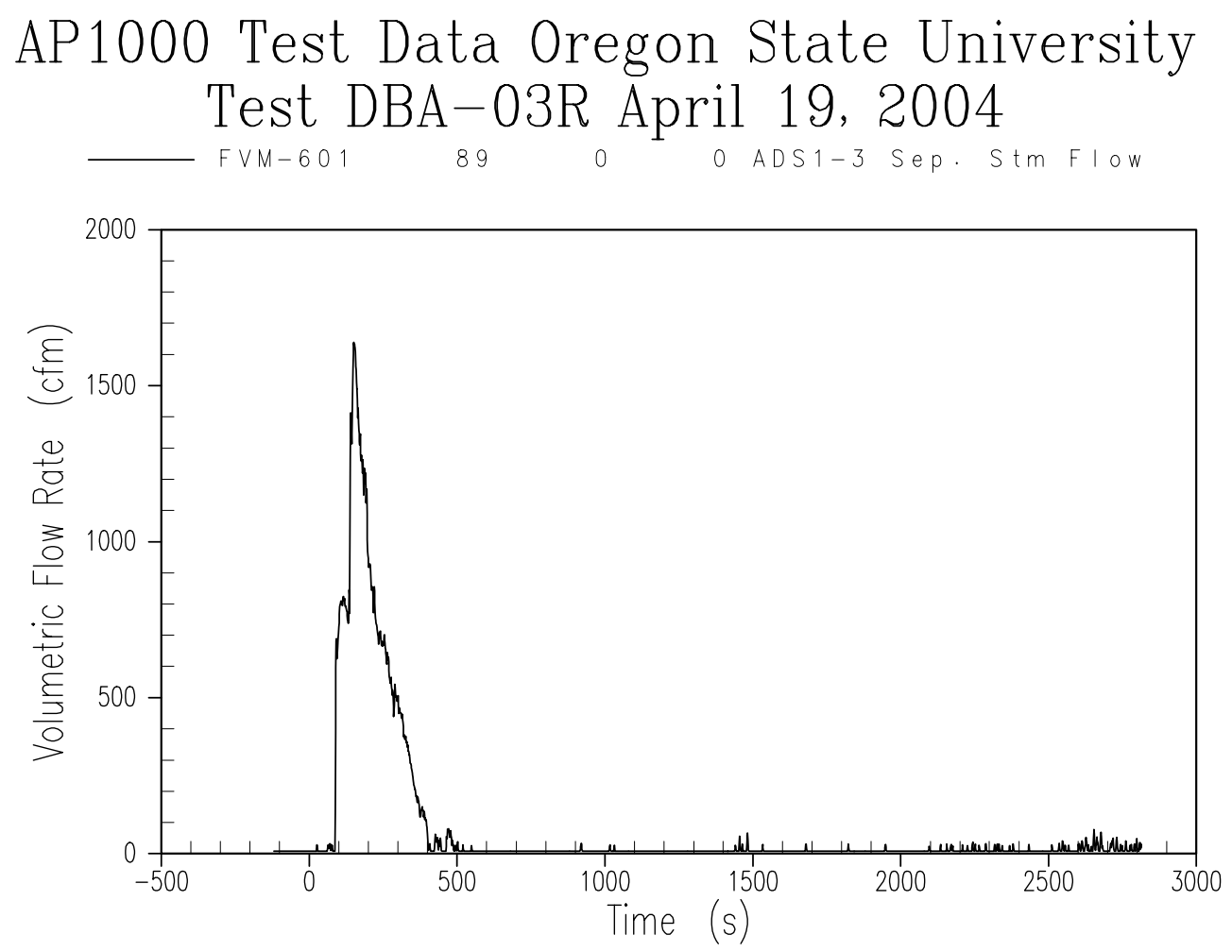

Figure A-44 ADS 1-3 Separator Steam Flow Rate 

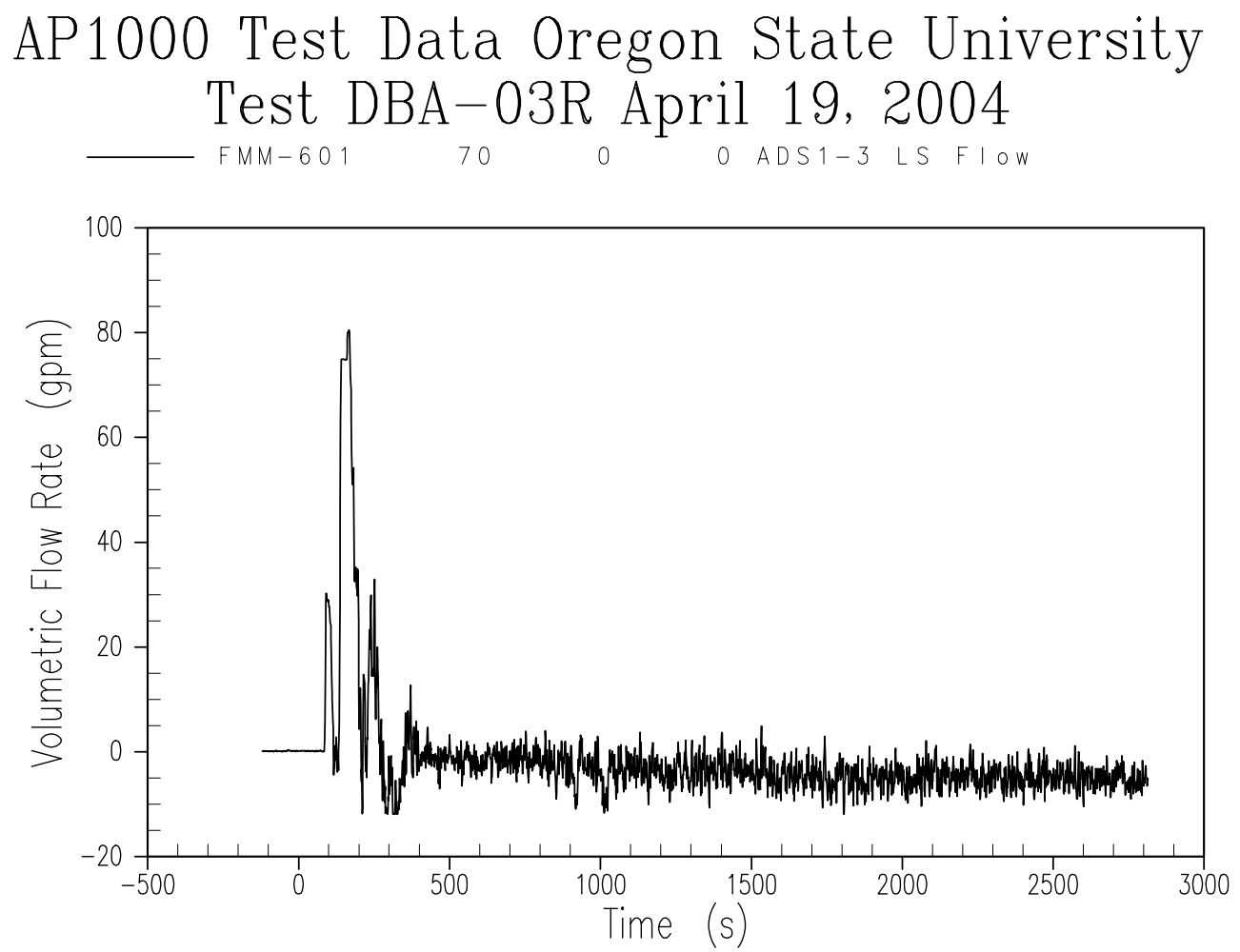

Figure A-45 ADS 1-3 Separator Liquid Flow Rate 
AP1000 Test Data Oregon State University Test DBA-03R April 19, 2004

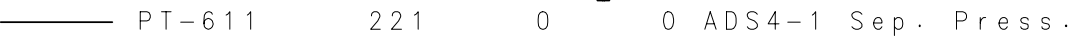

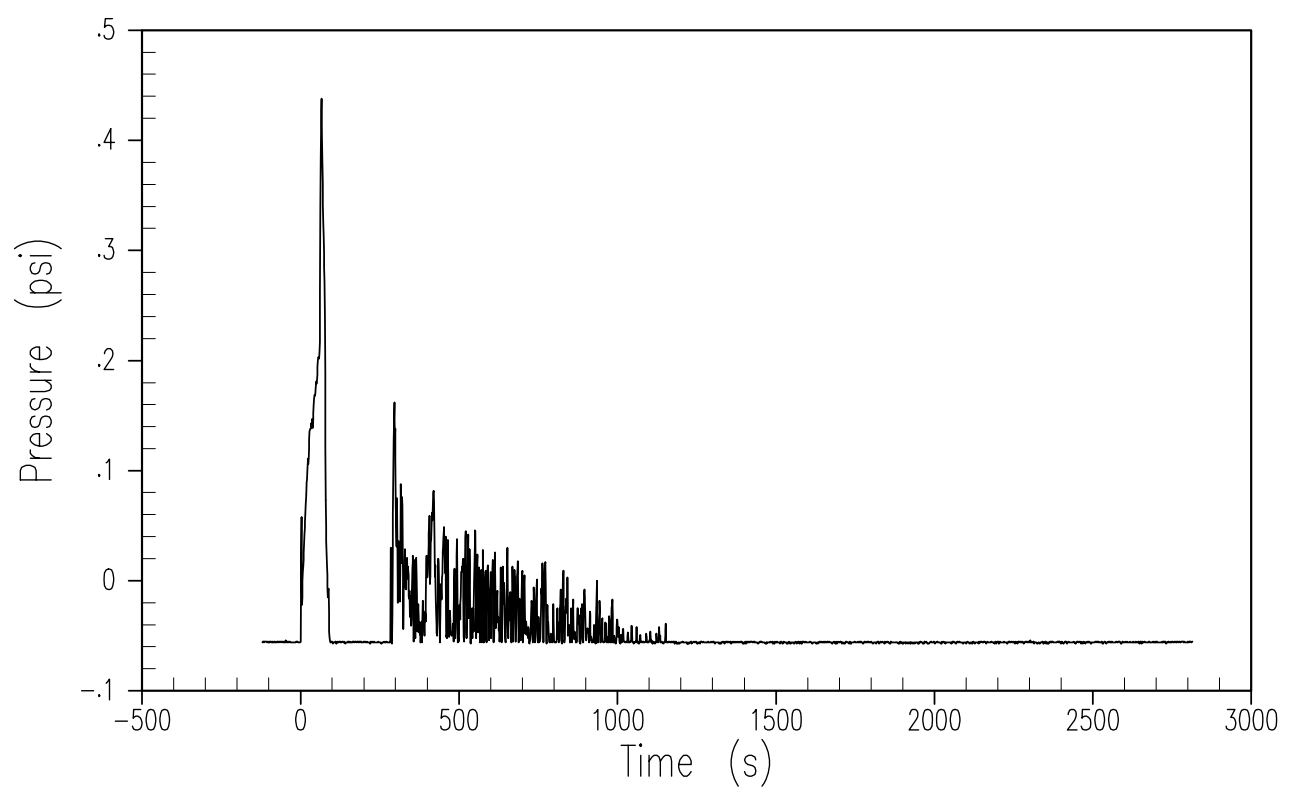

Figure A-46 ADS 4-1 Separator Pressure 
AP1000 Test Data Oregon State University Test DBA-03R April 19, 2004

$\longrightarrow P T-610$

220

0

o ADs4-2 sep. Press.

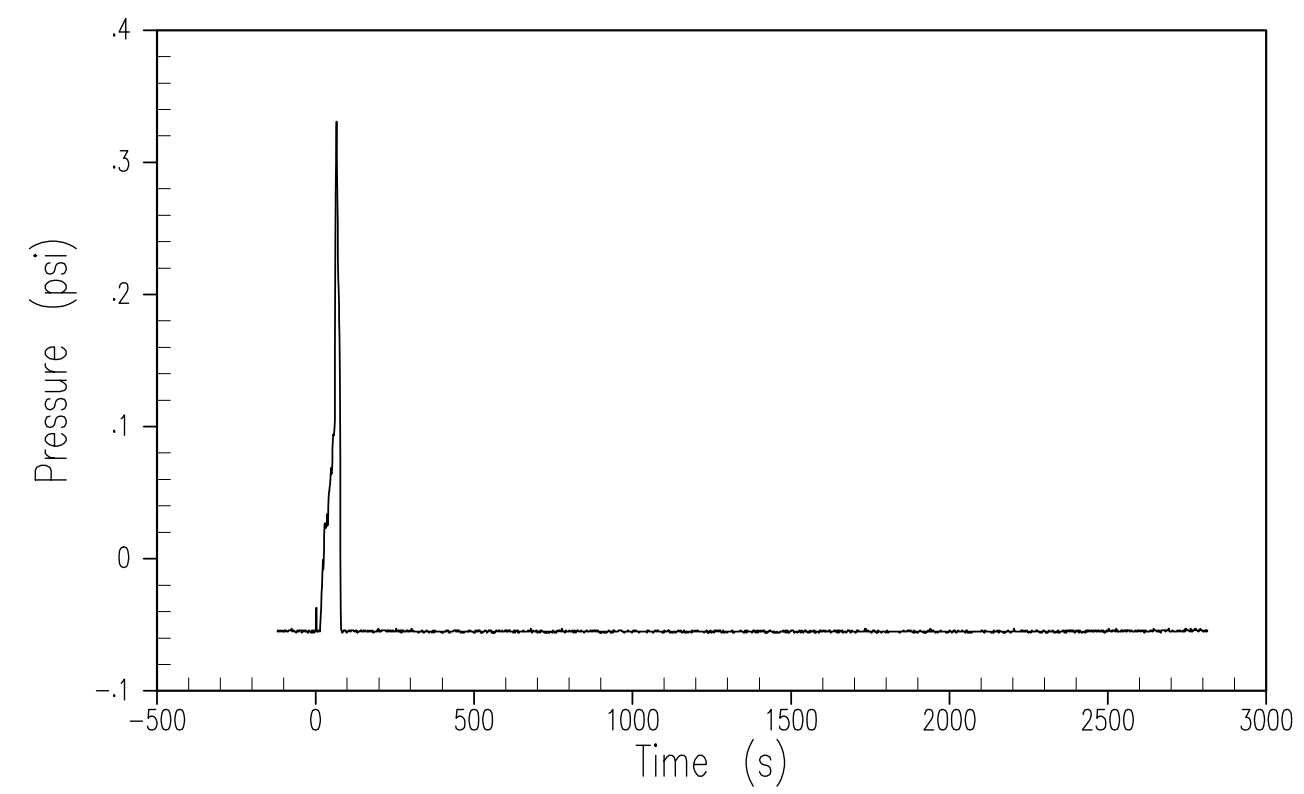

Figure A-47 ADS 4-2 Separator Pressure 


\section{AP1000 Test Data Oregon State University Test DBA-03R April 19, 2004 \\ - FVM-603 $91 \quad 0$ o ADS4-1 Sep. 6"Line}

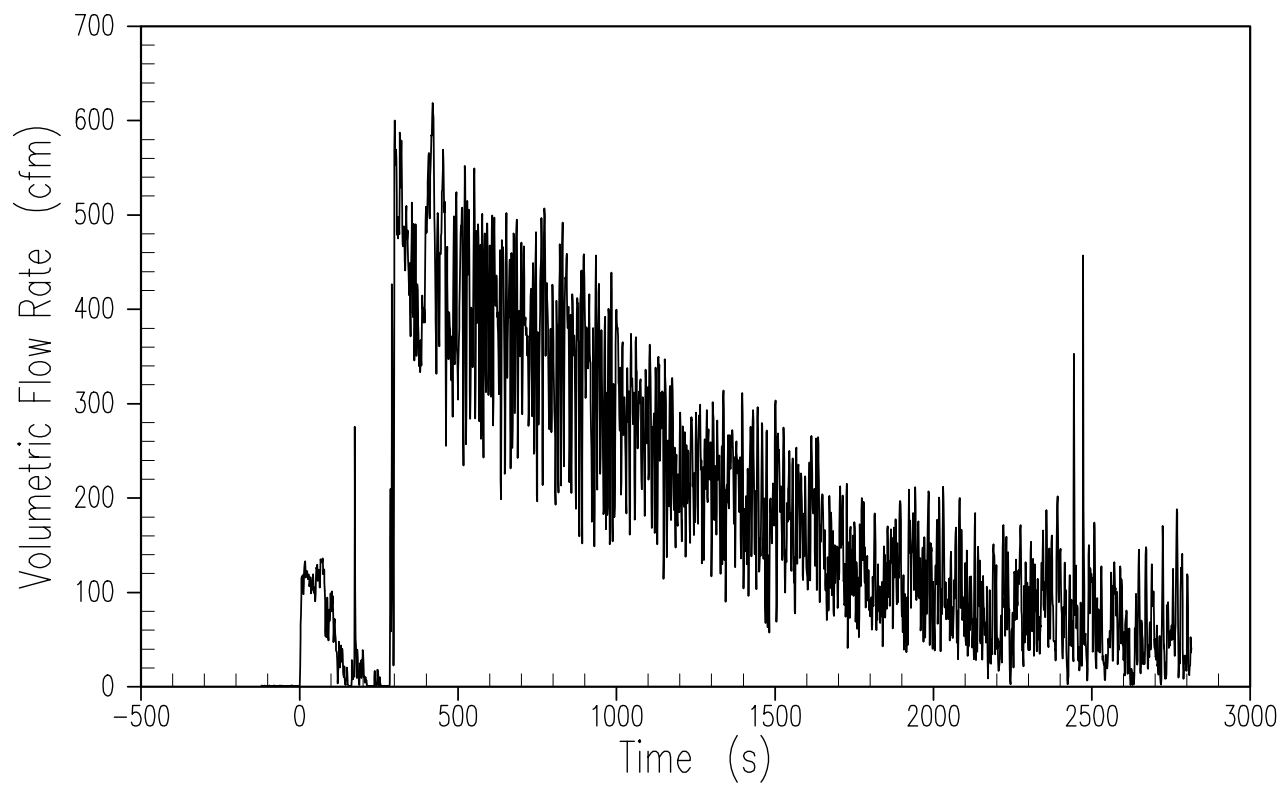

Figure A-48 ADS 4-1 Separator Steam Flow Rate 


\section{AP1000 Test Data Oregon State University Test DBA-03R April 19, 2004

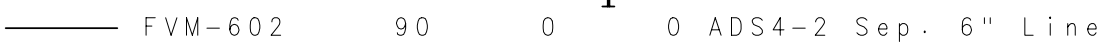

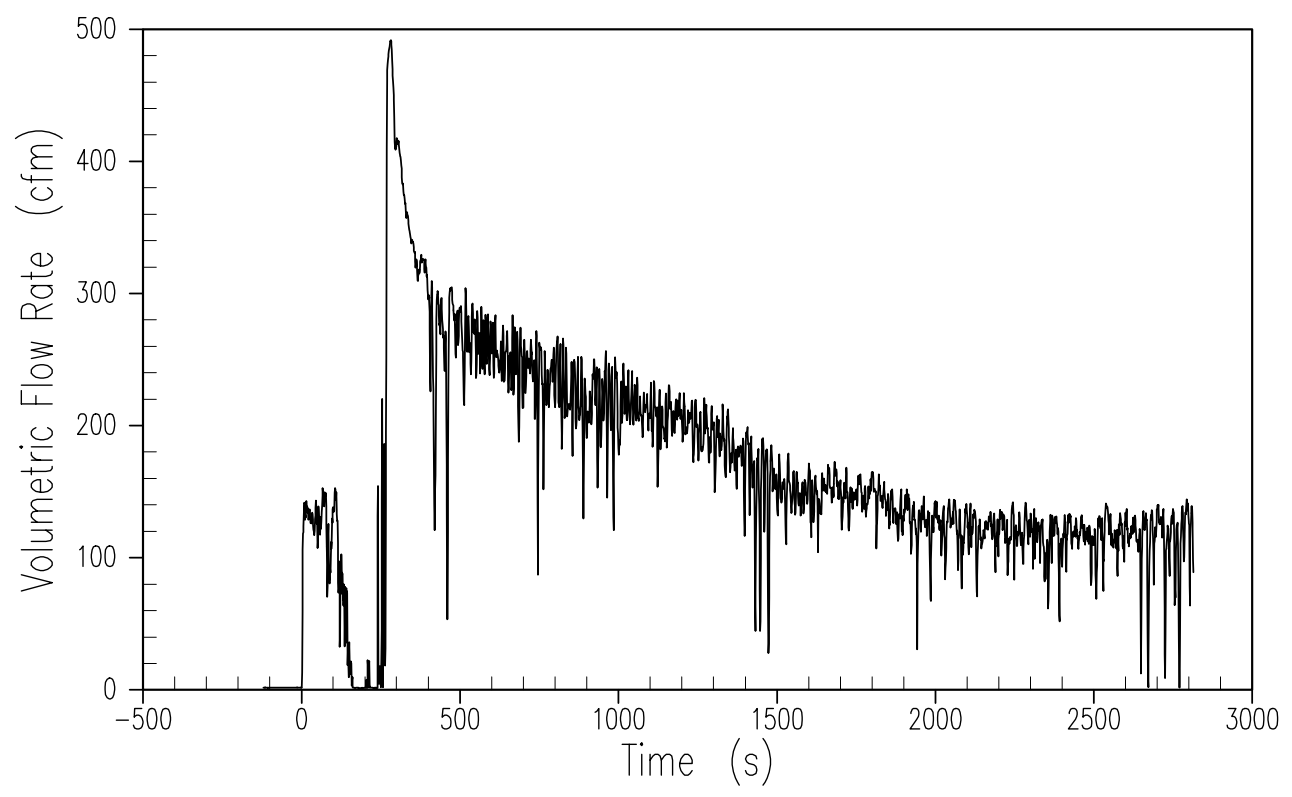

Figure A-49 ADS 4-2 Separator Steam Flow Rate 


\section{AP1000 Test Data Oregon State University Test DBA-03R April 19, 2004

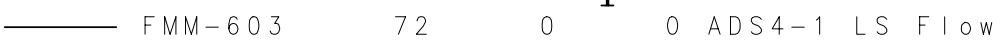

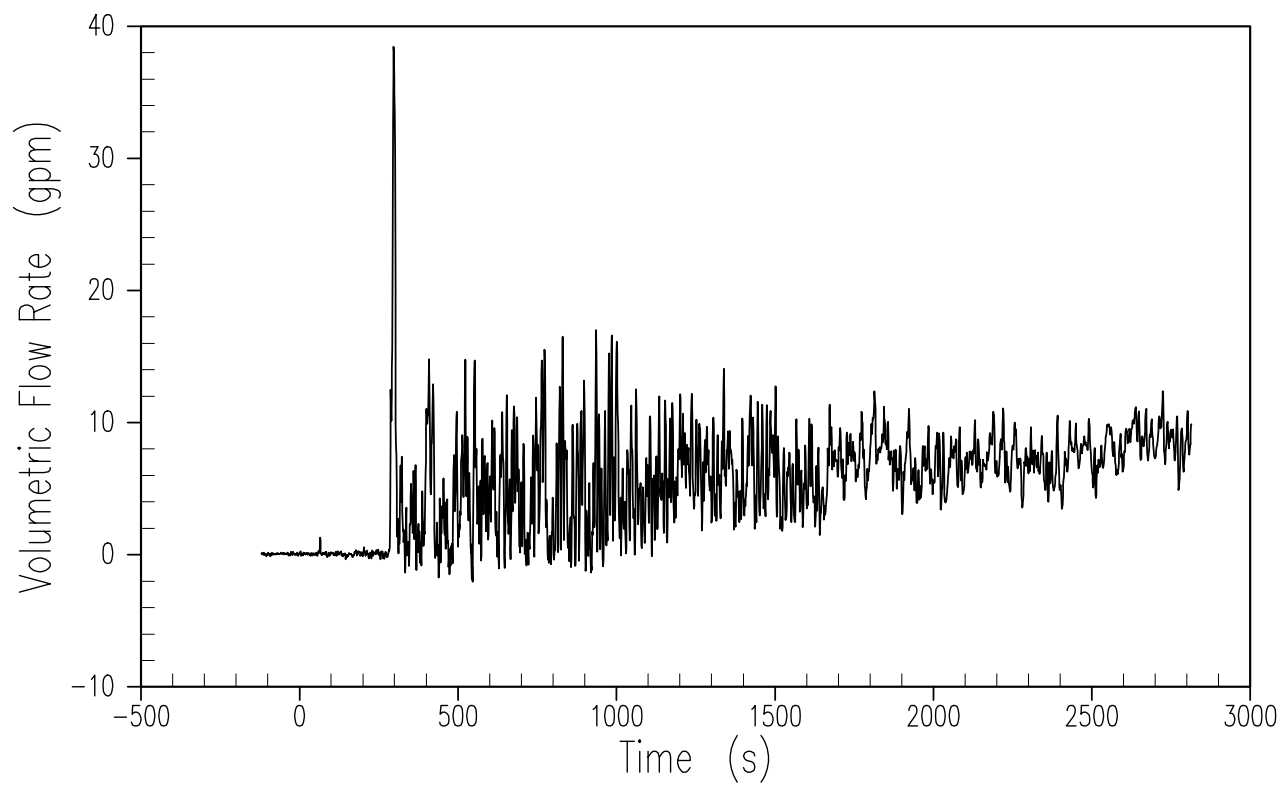

Figure A-50 ADS 4-1 Separator Liquid Flow Rate 

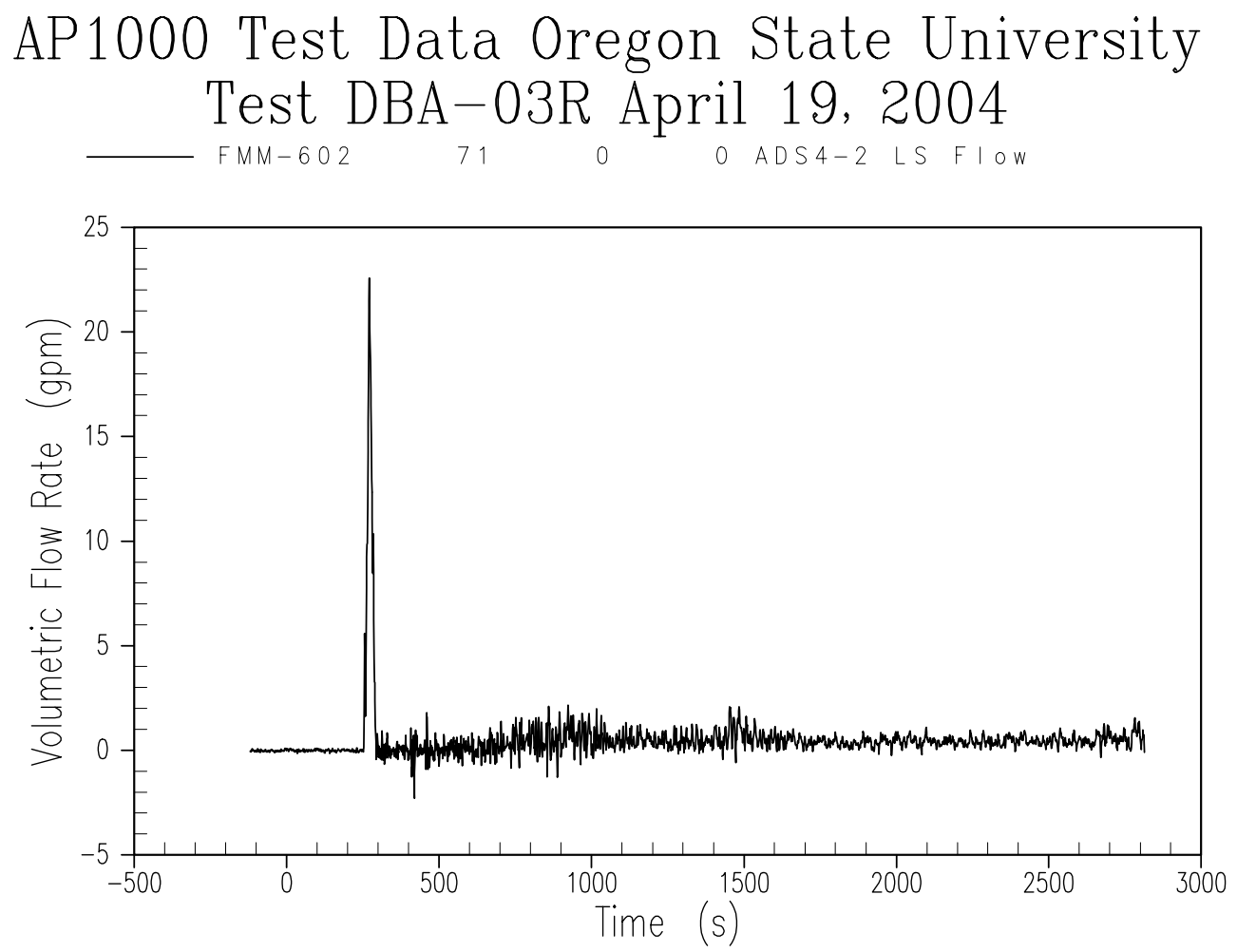

Figure A-51 ADS 4-2 Separator Liquid Flow Rate 

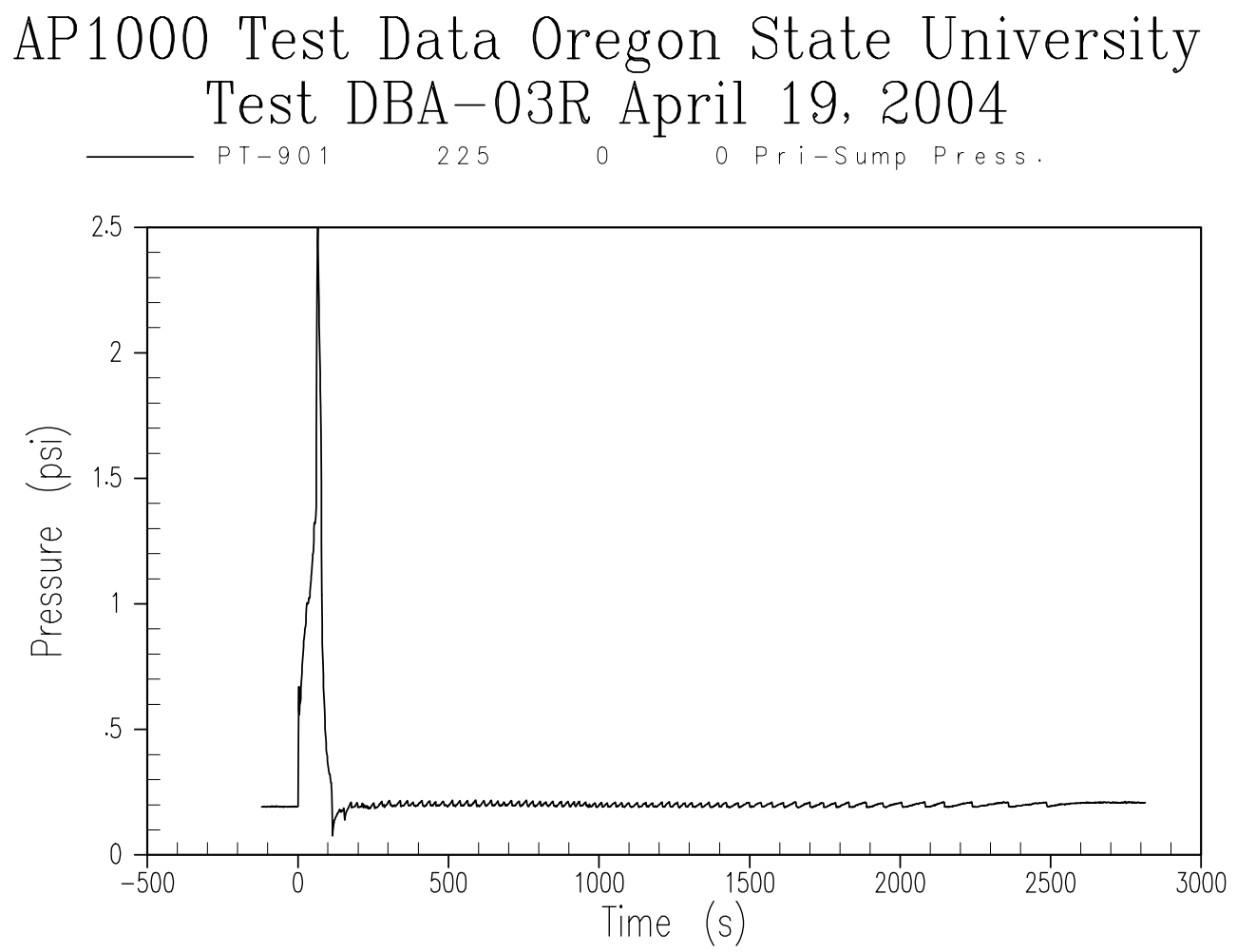

Figure A-52 Primary Sump Pressure 

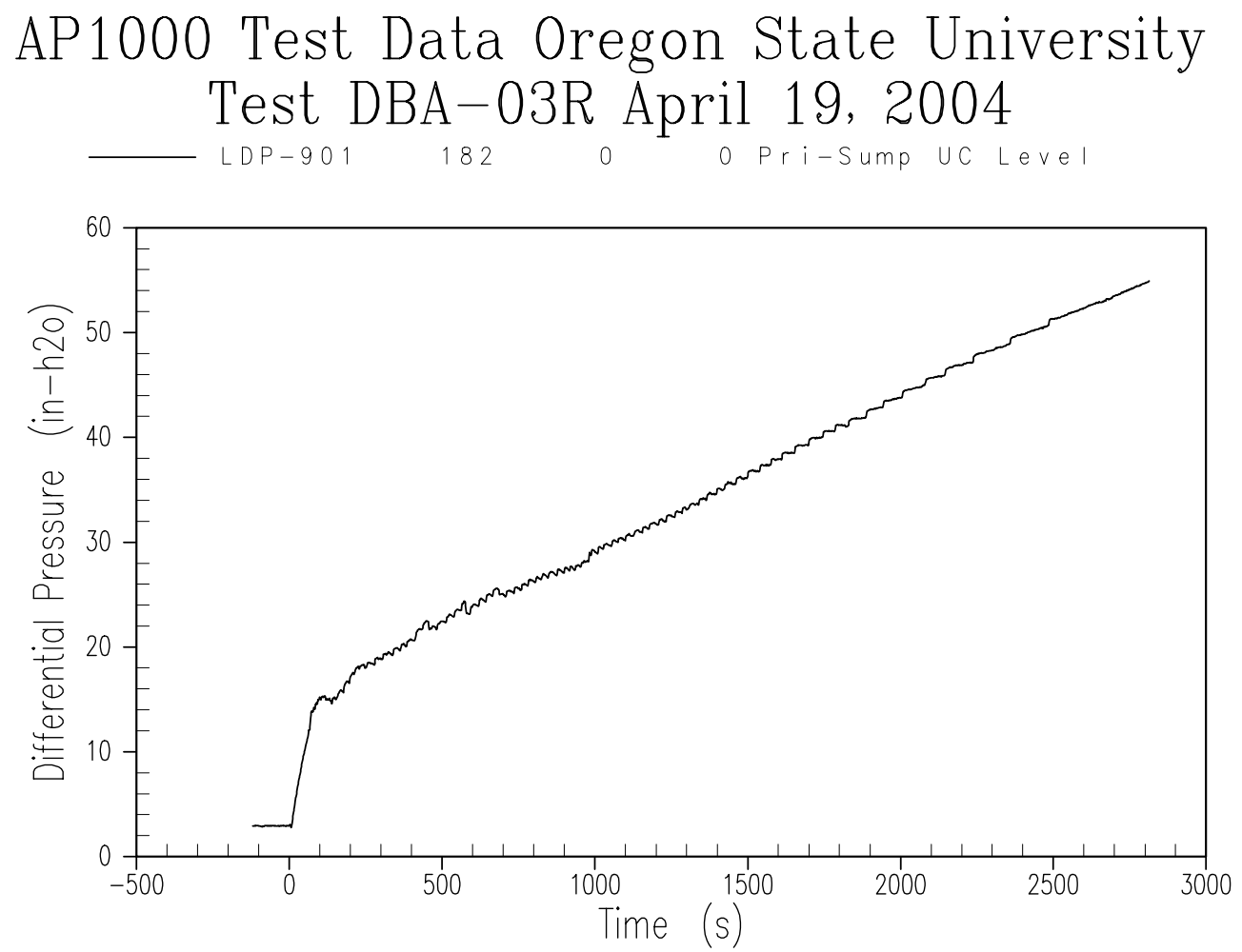

Figure A-53 Primary Sump Liquid Level 

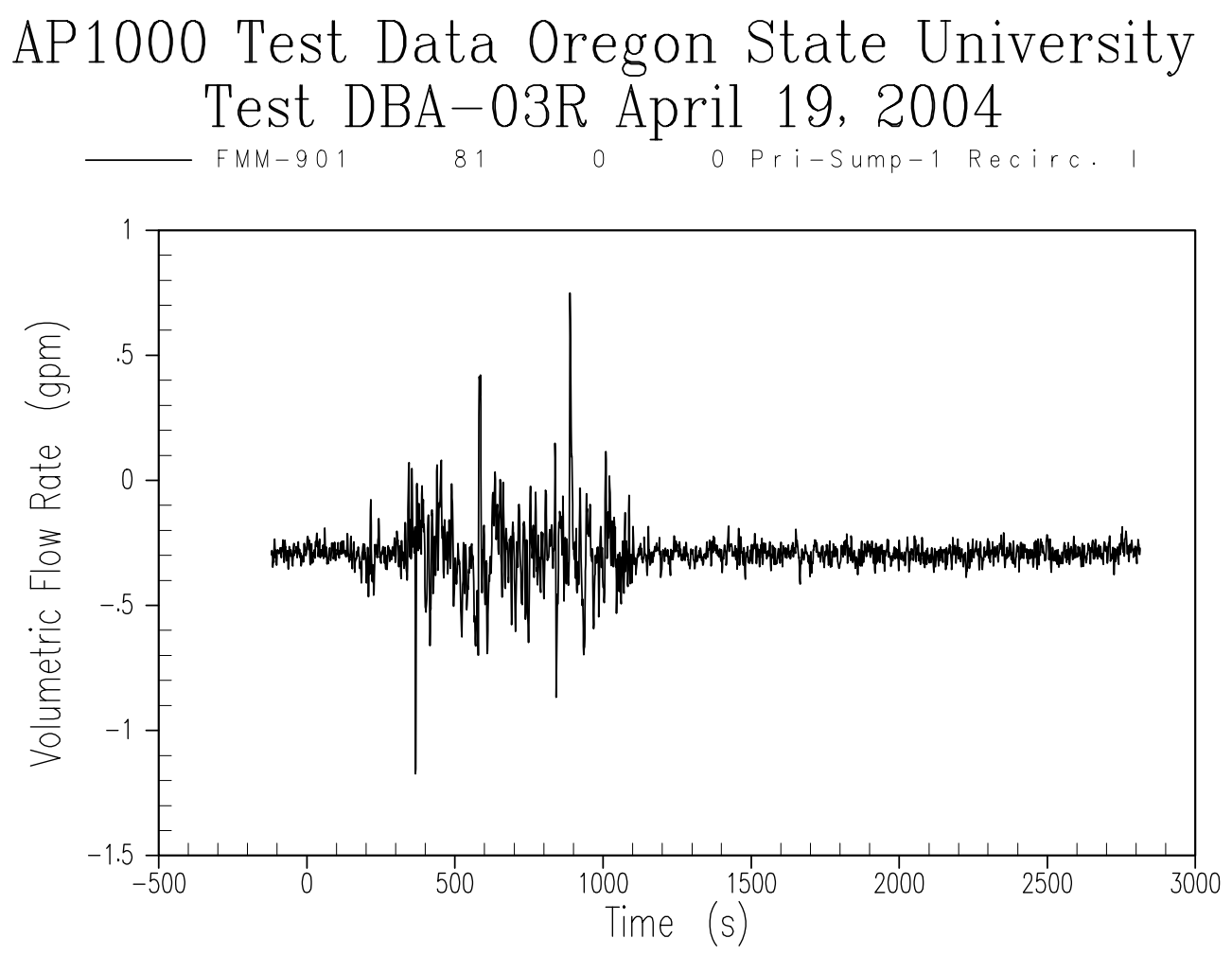

Figure A-54 Primary Sump 1 Injection Flow Rate 


\section{AP1000 Test Data Oregon State University Test DBA-03R April 19, 2004 \\ - FMM -902 \\ $82 \quad 0$ \\ o Pri-Sump-2 Recirc.}

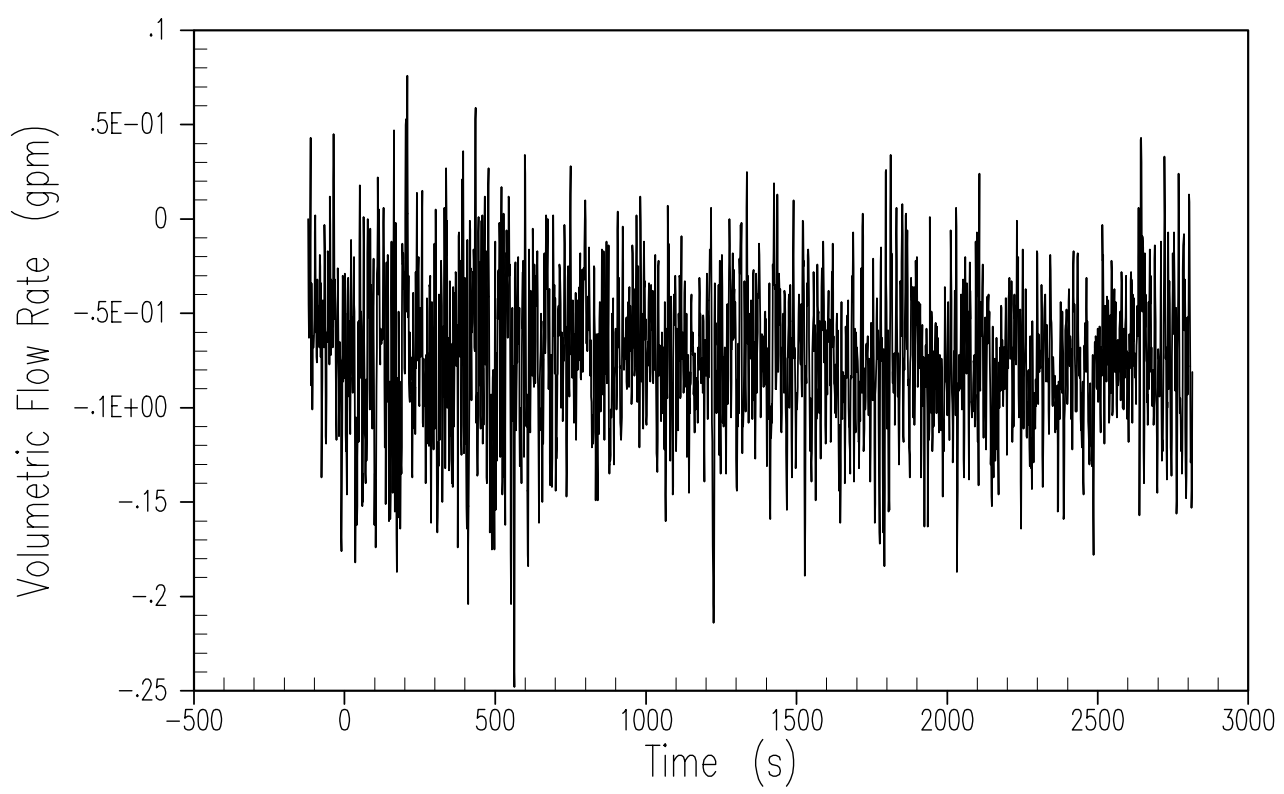

Figure A-55 Primary Sump 2 Injection Flow Rate 

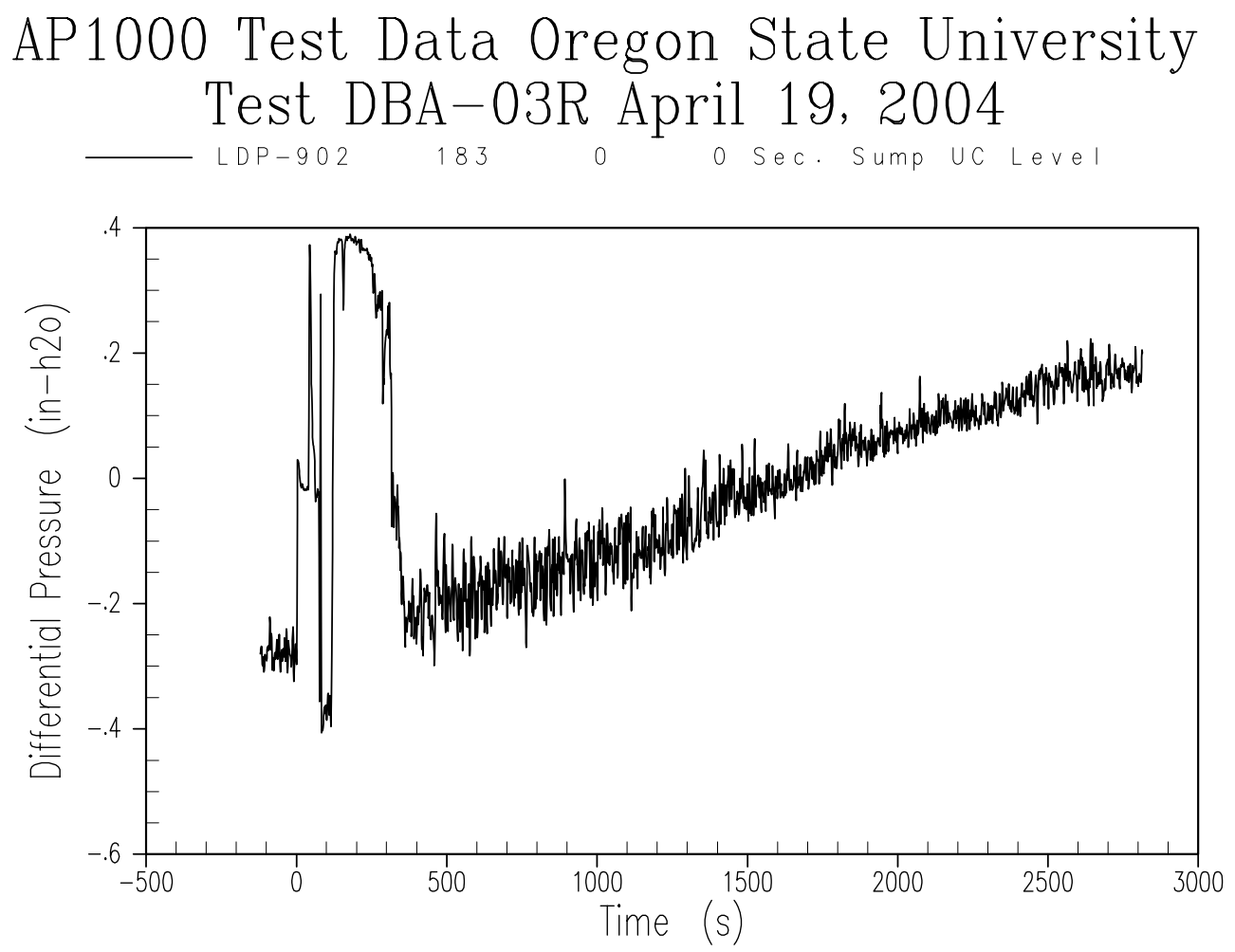

Figure A-56 Secondary Sump Liquid Level 
AP1000 Test Data Oregon State University Test DBA-03R April 19, 2004

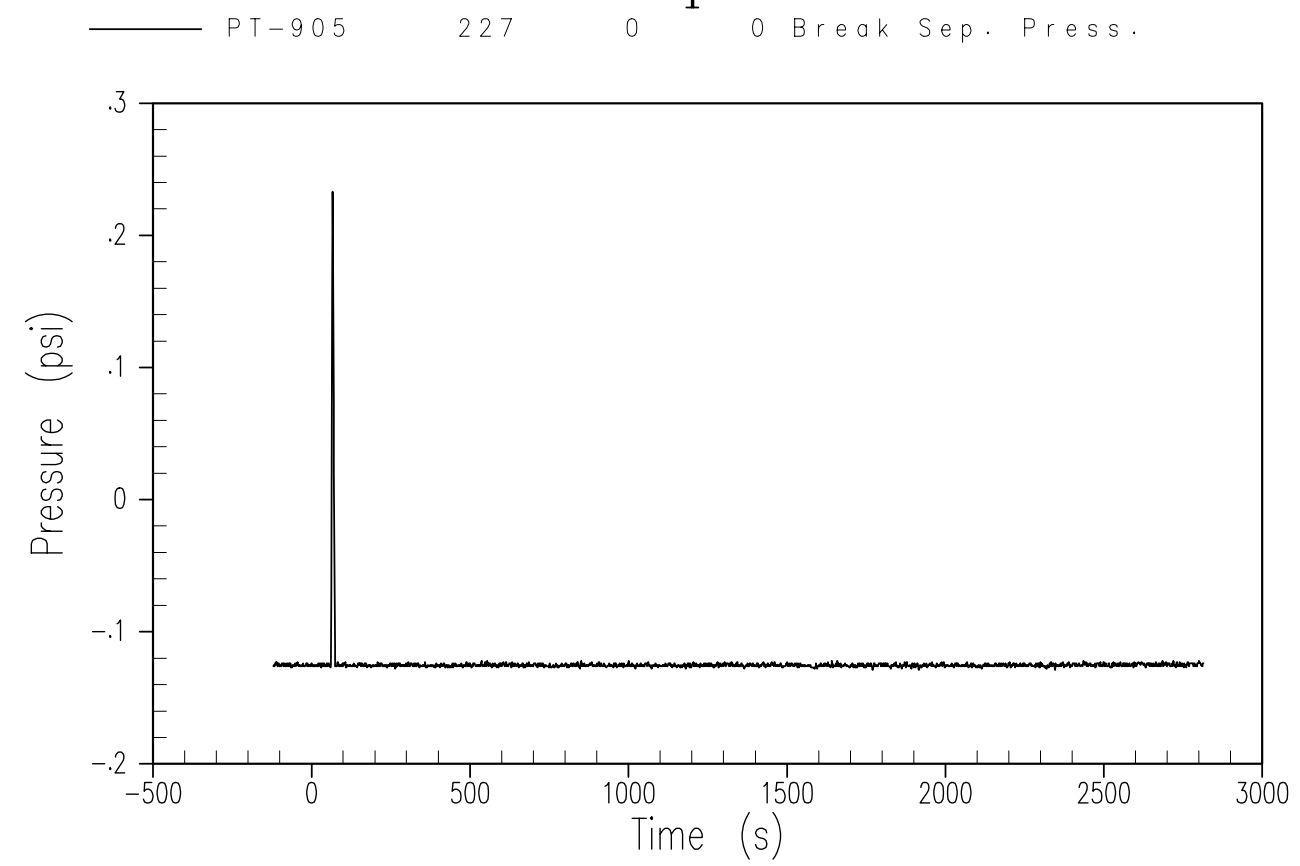

Figure A-57 Break Separator Pressure 

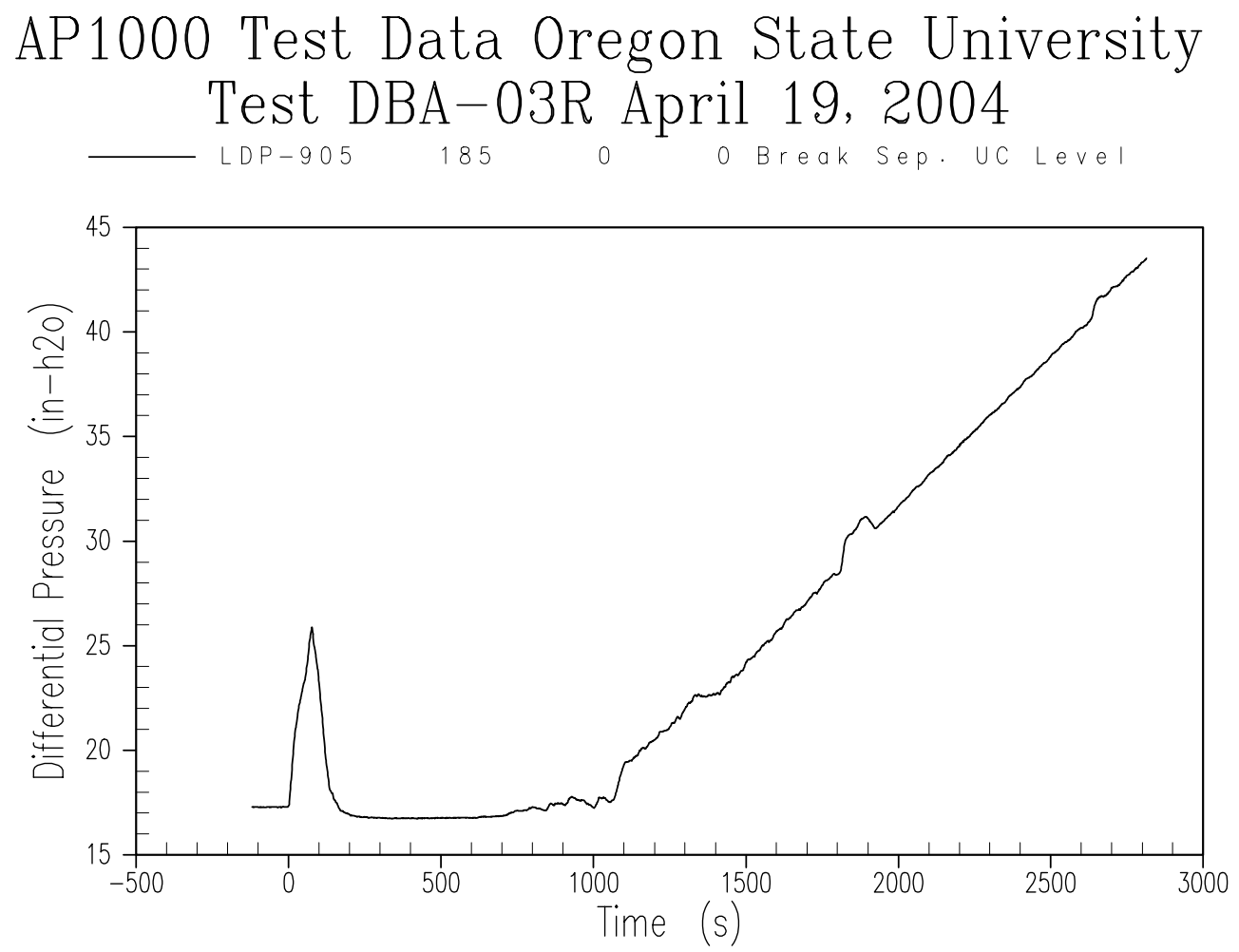

Figure A-58 Break Separator Liquid Level 

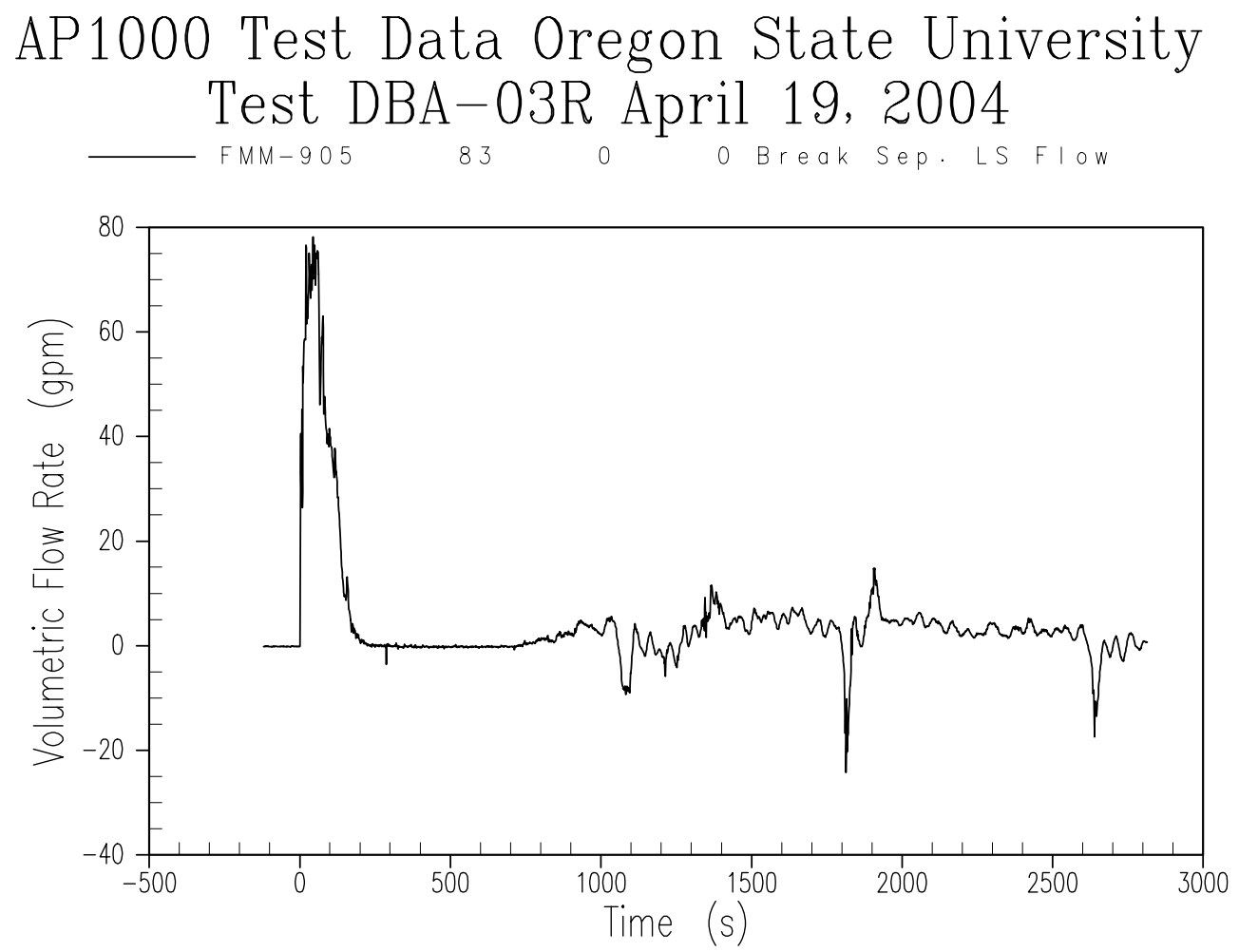

Figure A-59 Break Separator Flow to Primary Sump 

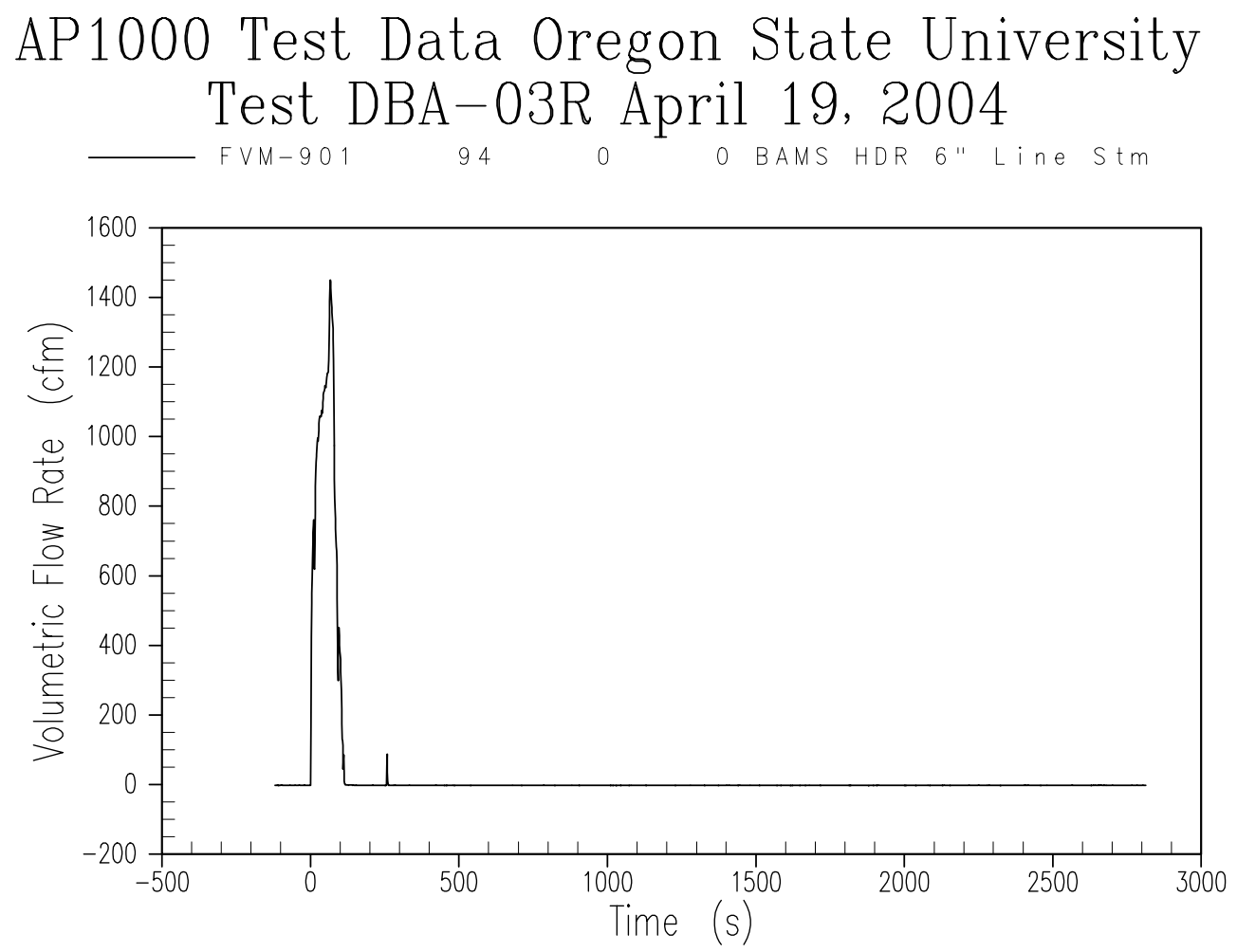

Figure A-60 BAMS Steam Flow Rate - 6-inch Line 
AP1000 Test Data Oregon State University Test DBA-03R April 19, 2004

FVM-902

$95 \quad 0$

O BAMS HDR $10^{\prime \prime}$ Line $\mathrm{St}$

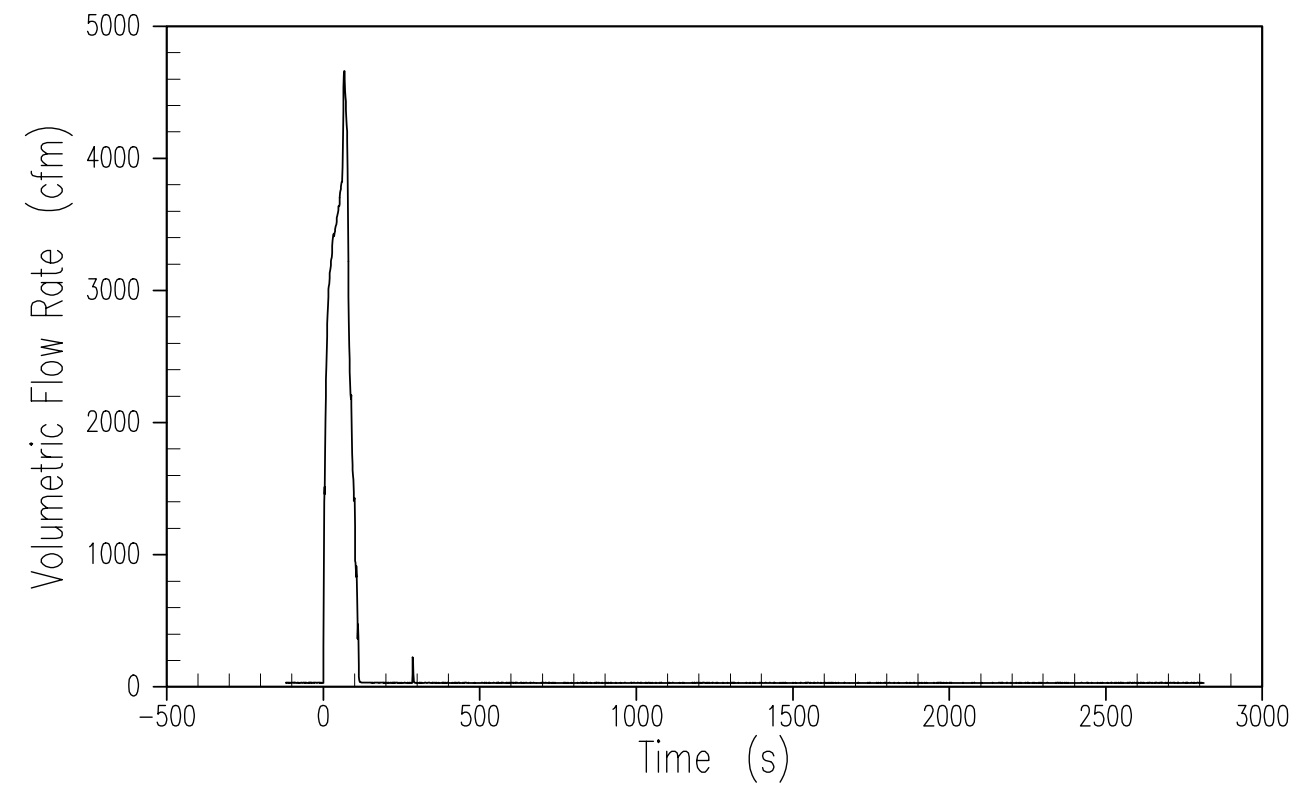

Figure A-61 BAMS Steam Flow Rate - 10-inch Line 

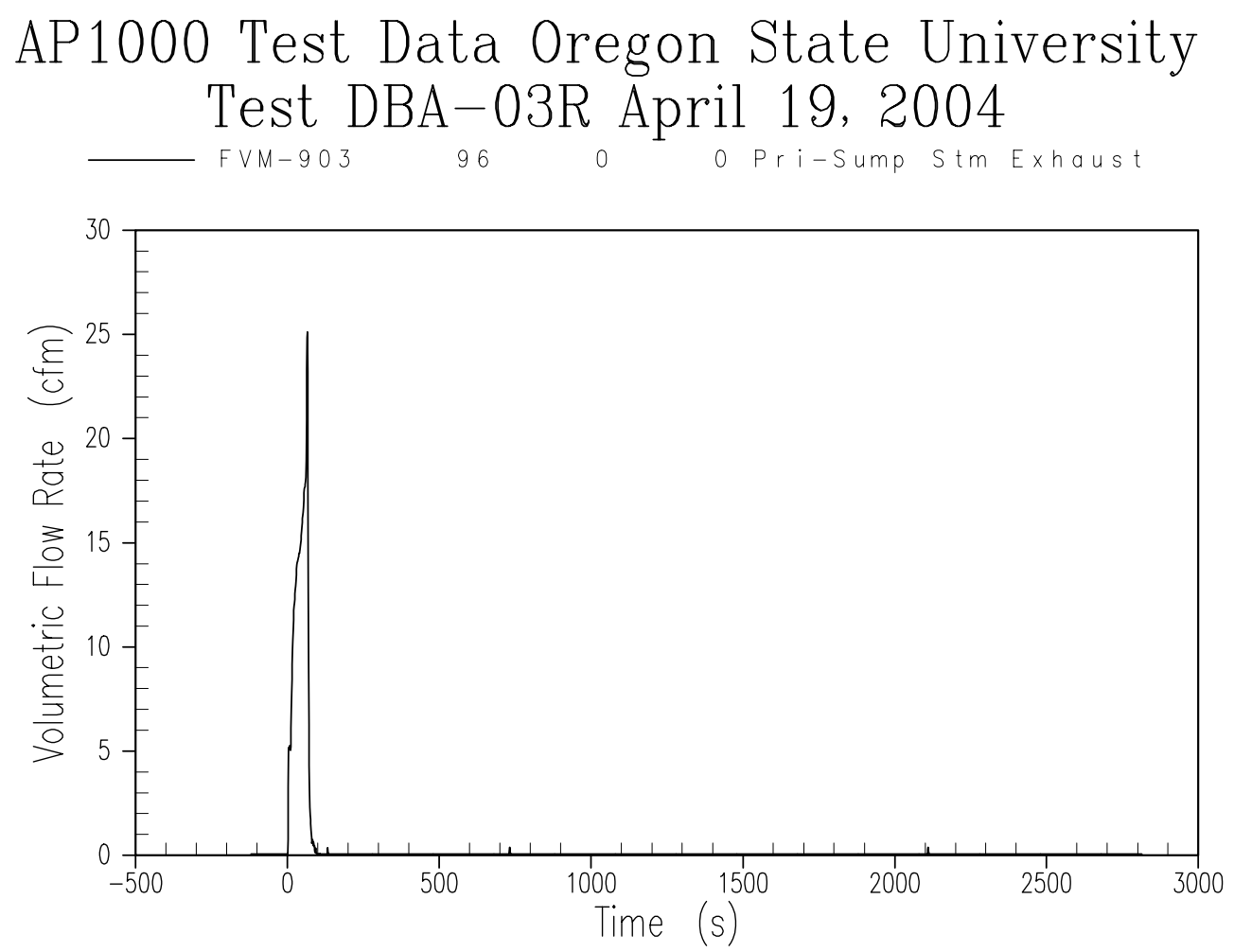

Figure A-62 BAMS/Primary Sump Steam Flow Rate 
AP1000 Test Data Oregon State University Test DBA-03R April 19, 2004

FVM-905

98

0

o Break Sep. 6" Line S

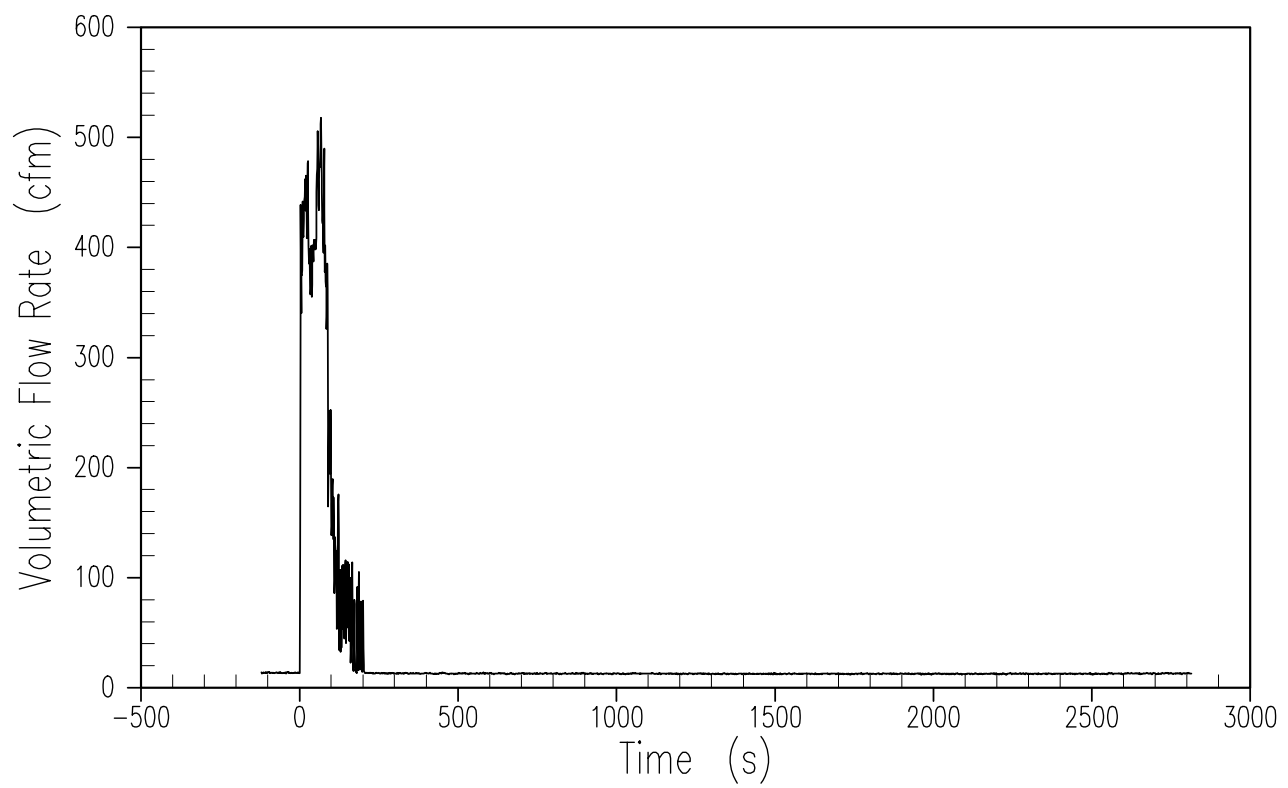

Figure A-63 BAMS/Separator Steam Flow Rate - 6-inch Pipe 
AP1000 Test Data Oregon State University Test DBA-03R April 19, 2004
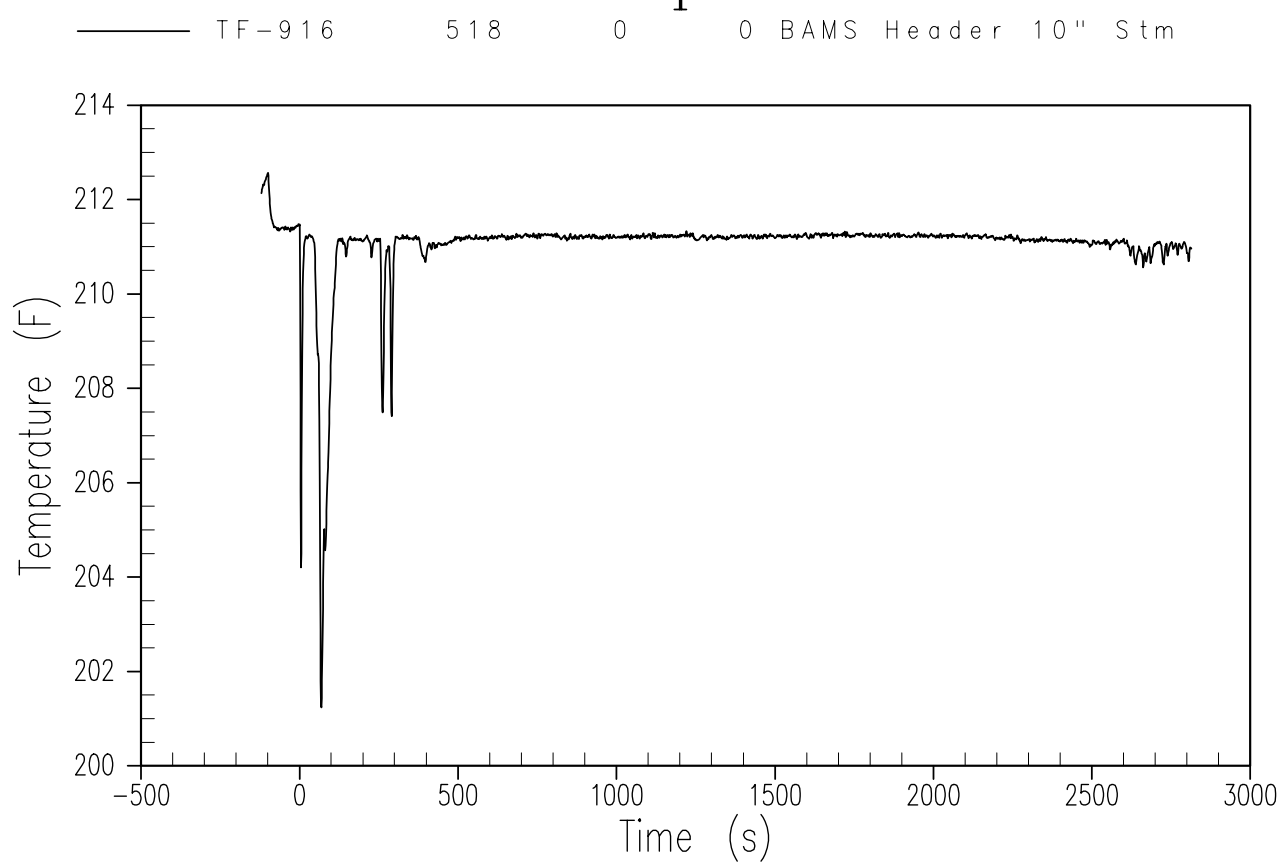

Figure A-64 BAMS/Exhaust Line Temperature - 10-inch Line 
AP1000 Test Data Oregon State University Test DBA-03R April 19, 2004
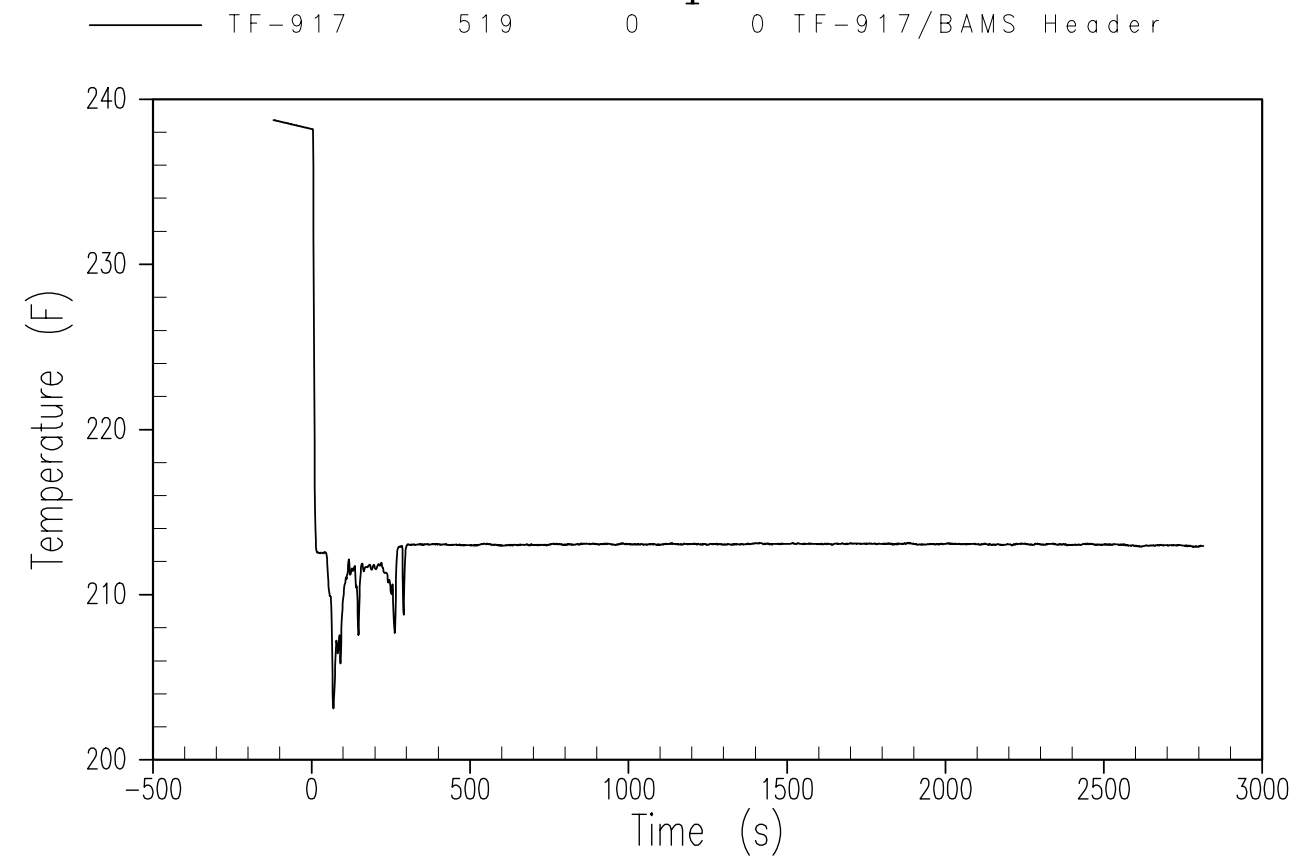

Figure A-65 BAMS/Exhaust Line Temperature - Header 

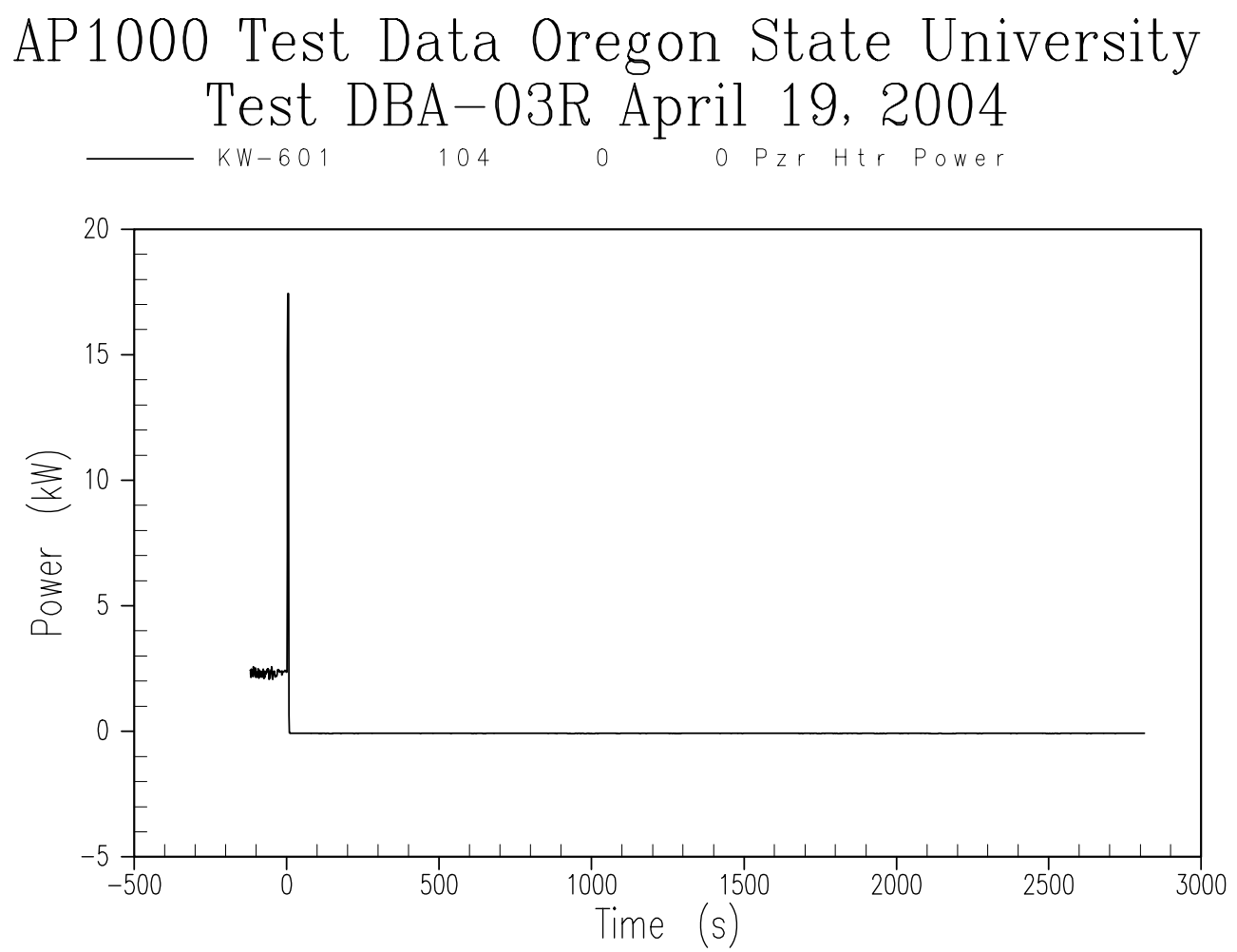

Figure A-66 Pressurizer Heater Input Power 

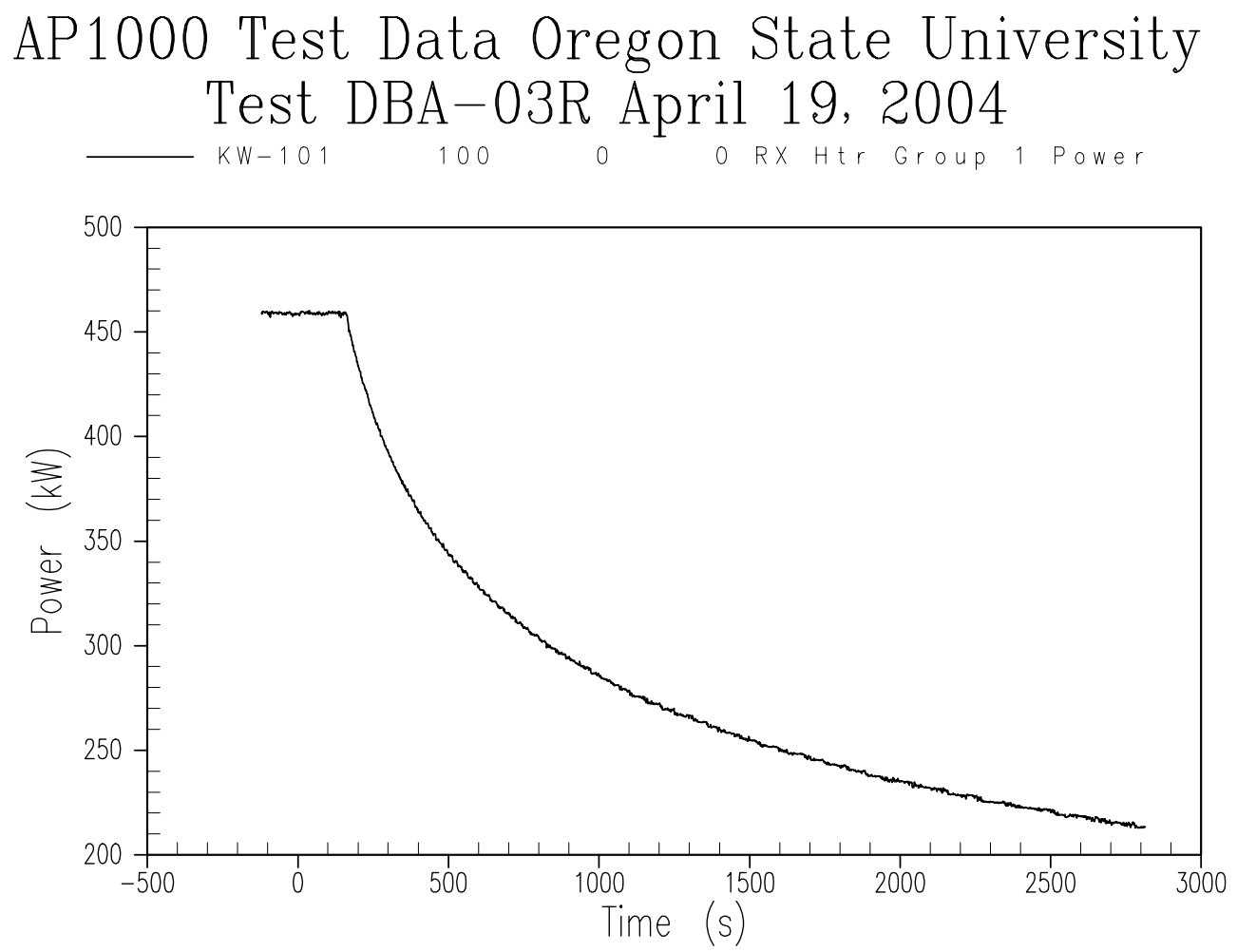

Figure A-67 Core Power Group 1 Input Power 

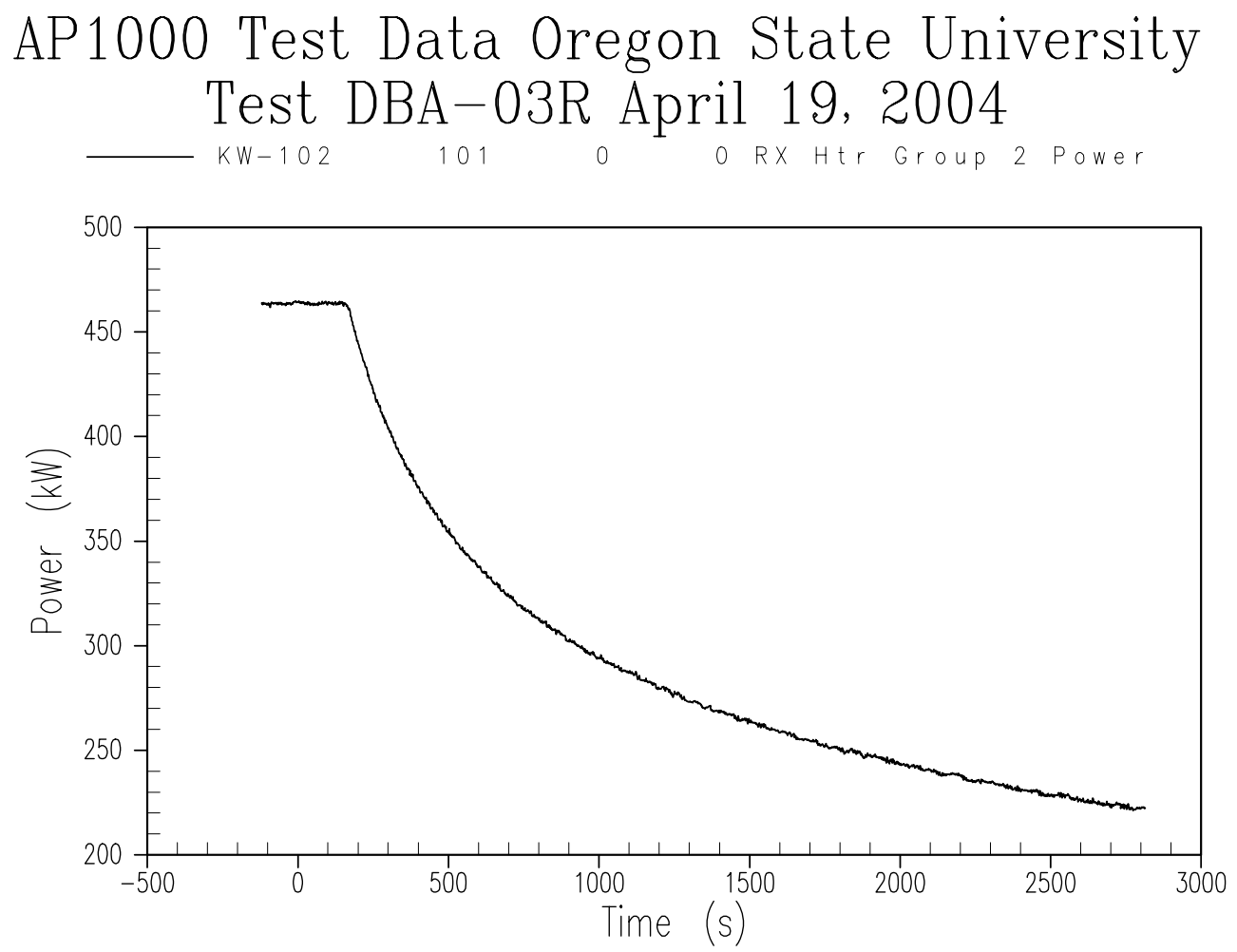

Figure A-68 Core Power Group 2 Input Power 

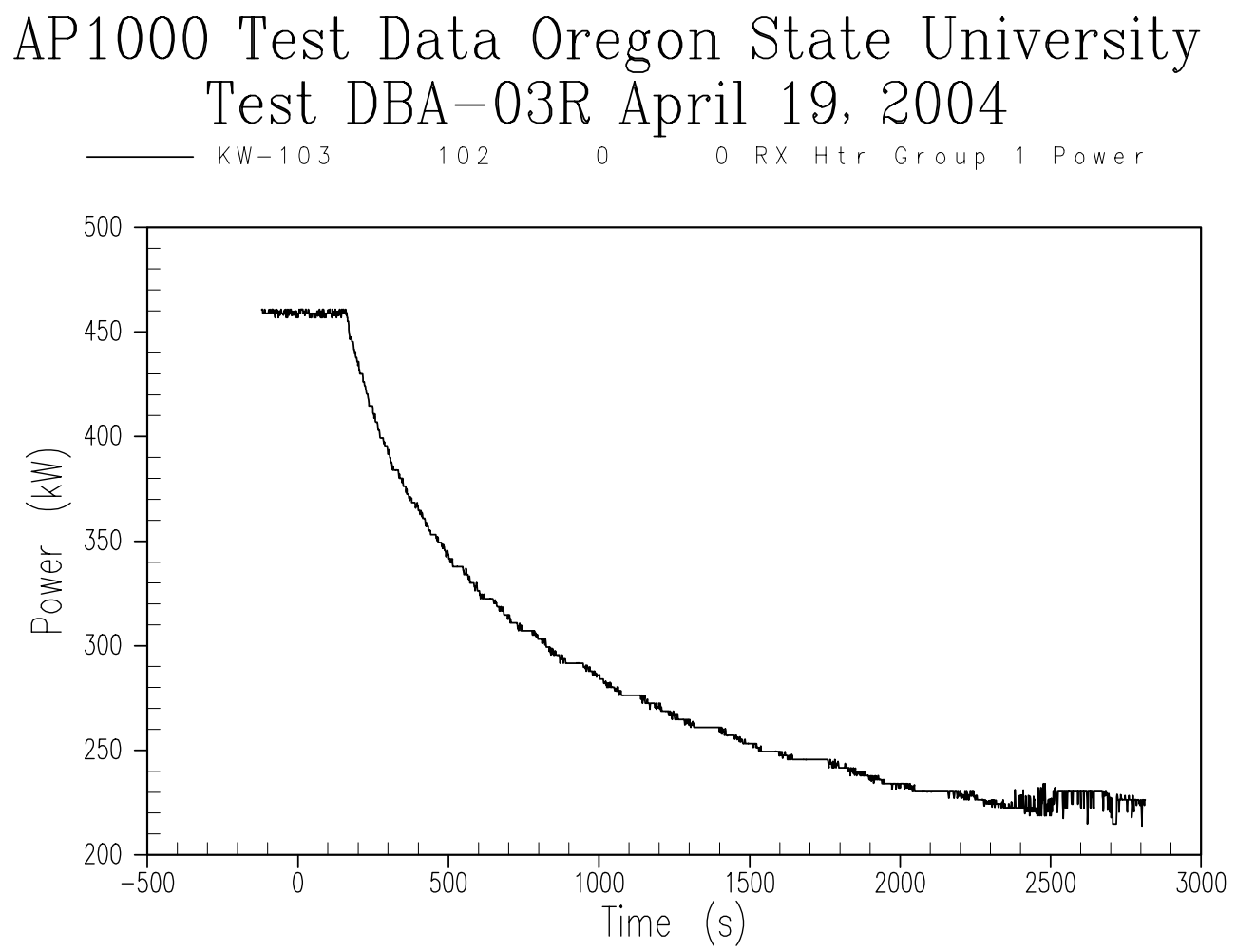

Figure A-69 Core Power Group 1 Alternate Input Power 

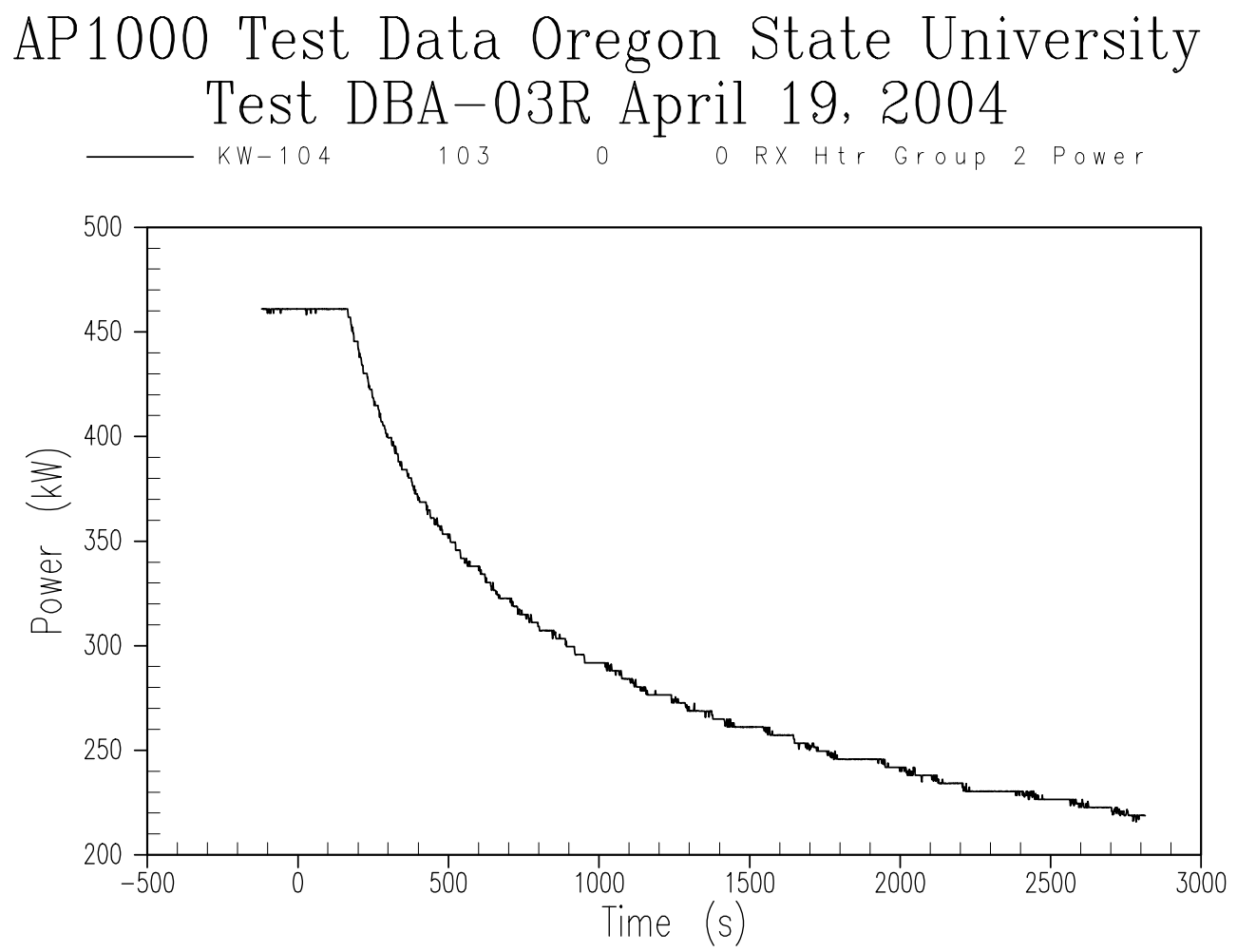

Figure A-70 Core Power Group 2 Alternate Input Power 

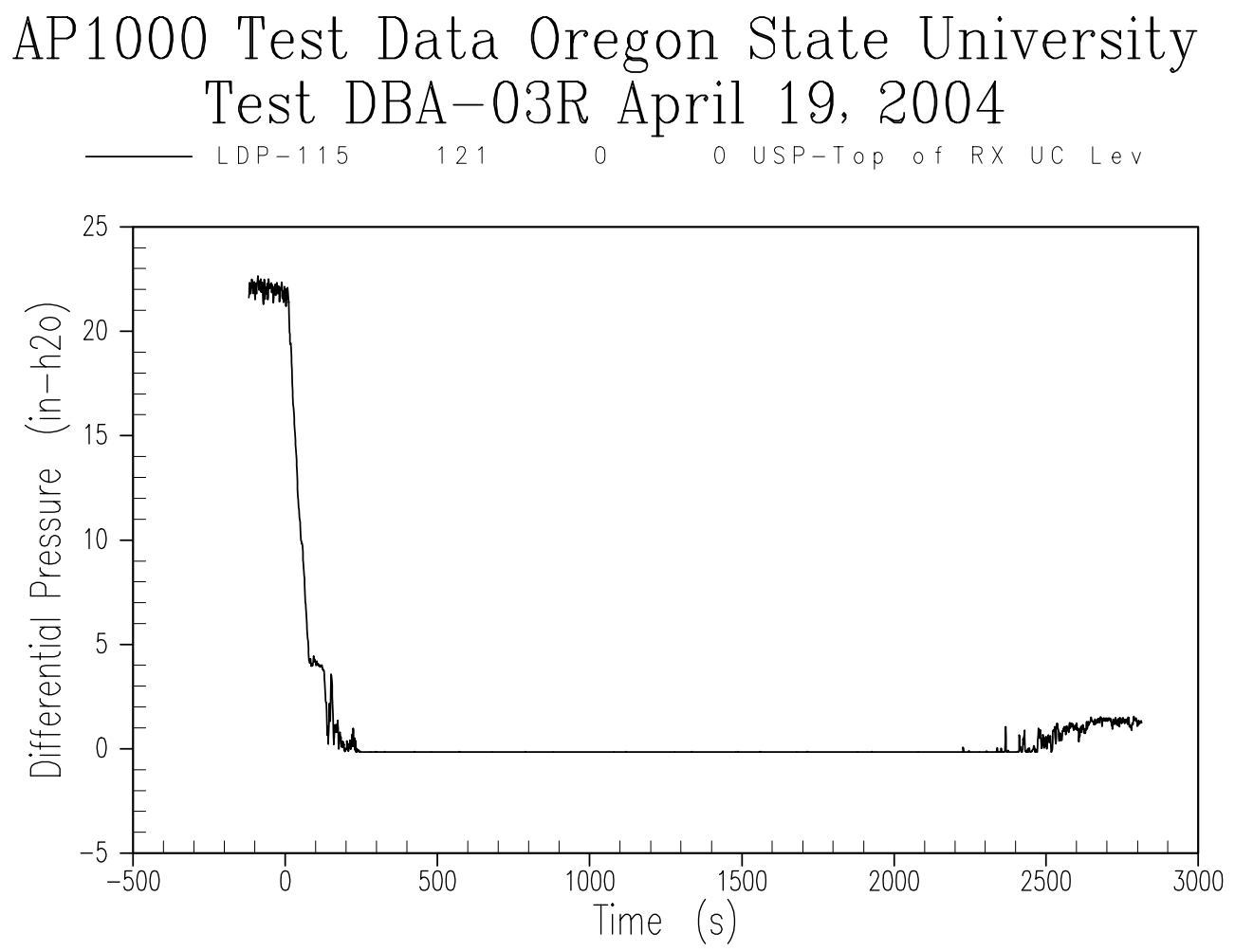

Figure A-71 Reactor Vessel Liquid Level Between Top of Vessel - Upper Support Plate 

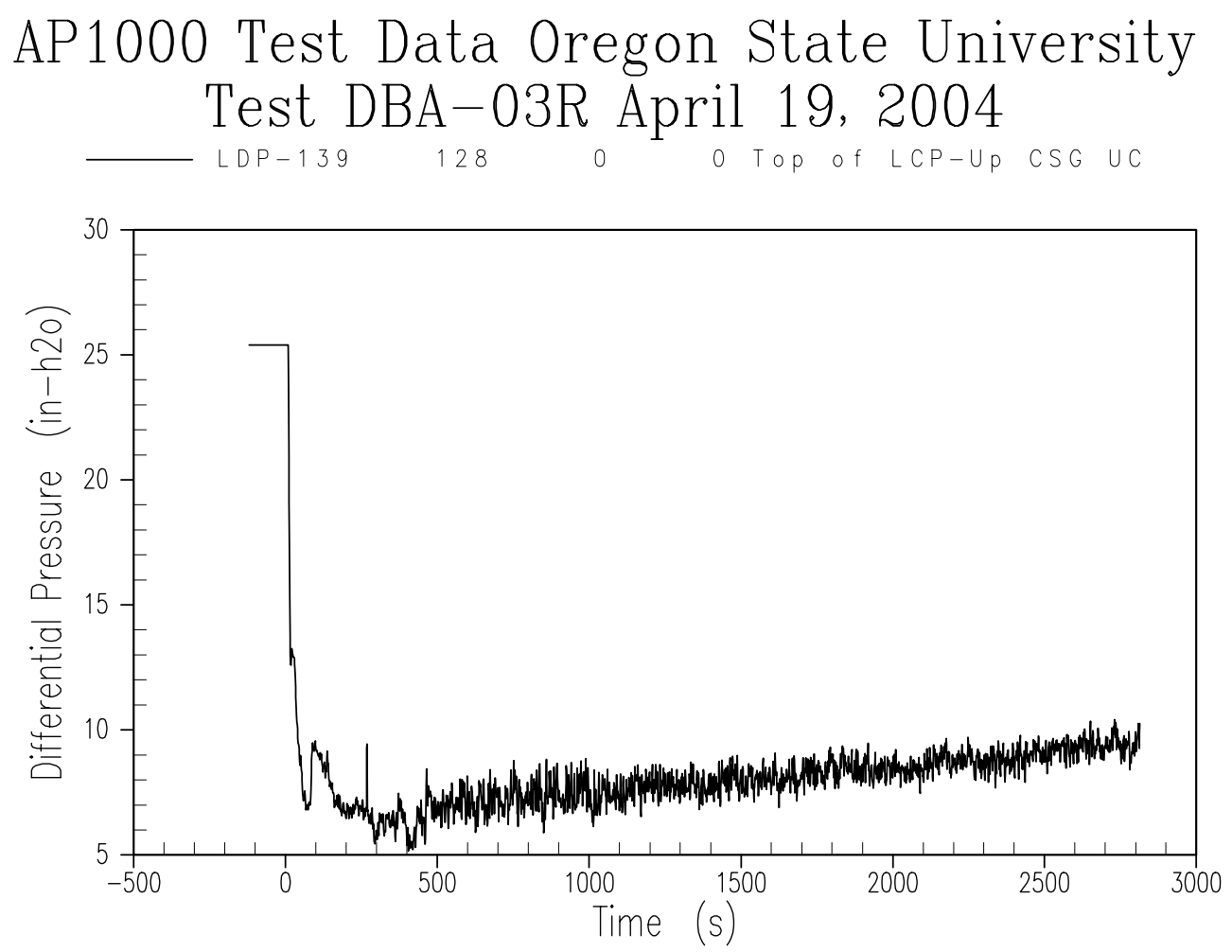

Figure A-72 Reactor Vessel Liquid Level Between Bottom of Upper Support Plate Upper Core Spacer Grid 

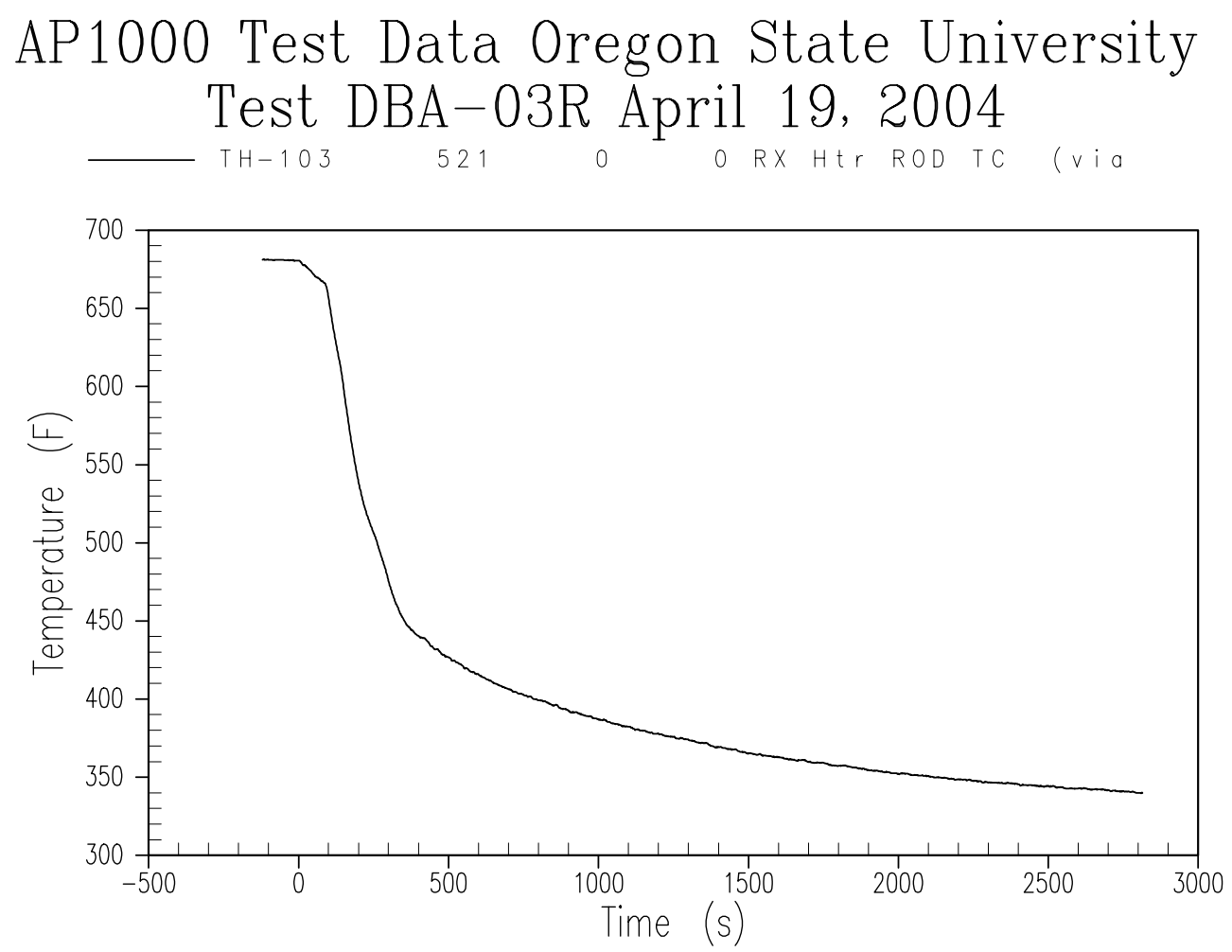

Figure A-73 Inner Core Thermocouple Measuring Heater Temperature 

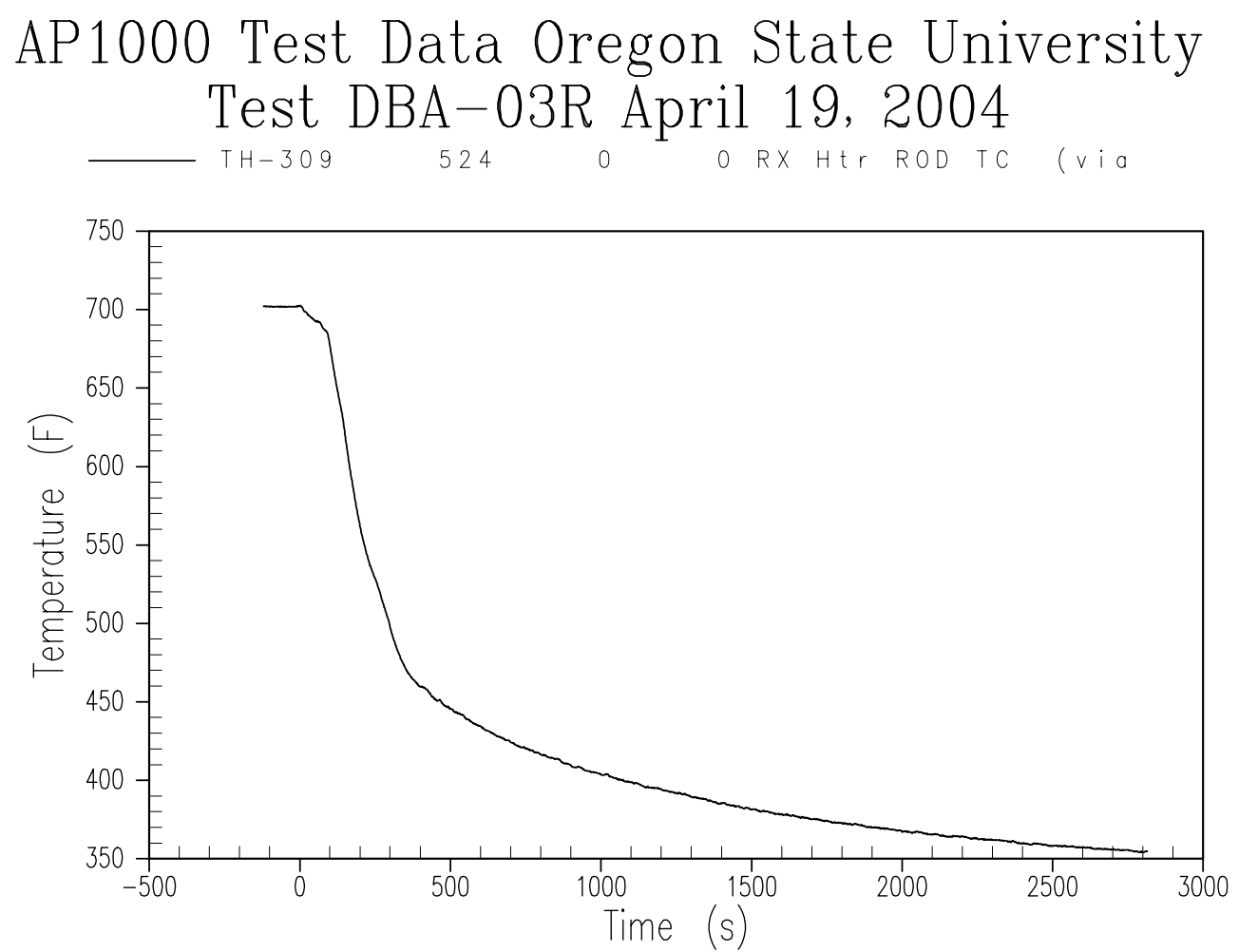

Figure A-74 Outer Core Thermocouple Measuring Heater Temperature 
APPENDIX B

TEST DATA 


\title{
Oregon State University
}

Department of Nuclear Engineering

\author{
Advanced Thermal Hydraulic \\ RESEARCH LABORATORY
}

\section{TEST SUMMARY REPORT}

OSU-AP1000-10

DBA-05-D

\section{AP1000 INADVERTENT ADS-1 ACTUATION WITH 3 OF 4 ADS-4}

Revision 0 


\title{
TEST SUMMARY REPORT
}

\author{
OSU-AP1000-10
}

DBA-05-D

\section{AP1000 InADVERTENT ADS-1 ACTUATION WITH 3 OF 4 ADS-4}

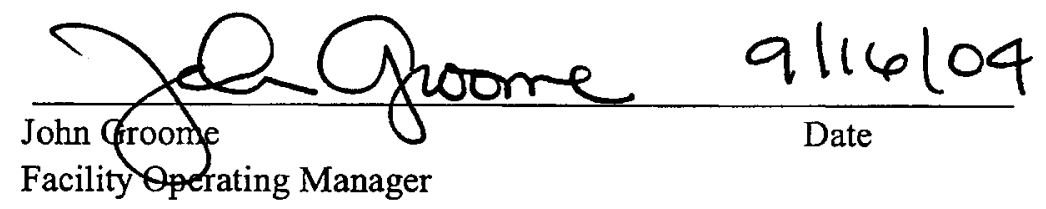

Research Assistant

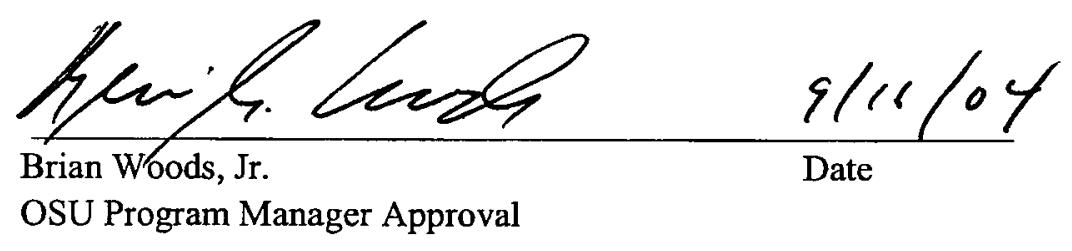

Oregon State University 116 Radiation Center

Corvallis, Oregon 97331

(C) 2004 Oregon State University

All Rights Reserved 


\section{WESTINGHOUSE PROPRIETARY INFORMATION NOTICE}

This report contains information proprietary to Westinghouse Electric Company. A non-proprietary version of this report has also been prepared for distribution to the United States Nuclear Regulatory Commission (NRC).

In order to conform to the requirements of 10 CFR 2.790 of the Commission's regulations concerning the protection of proprietary information so submitted to the NRC, the information which is proprietary in the proprietary version of this document is contained within brackets, and where the proprietary information has been deleted in the non-proprietary versions, only the brackets remain (the information that was contained within the brackets in the proprietary versions having been deleted). The justification for claiming the information so designated as proprietary is indicated in both versions by means of lower case letters (a) through (f) located as a superscript immediately following the brackets enclosing each item of information being identified as proprietary or in the margin opposite such information. These lower case letters refer to the types of information Westinghouse customarily holds in confidence identified in Sections (4)(ii)(a) through (4)(ii)(f) of the affidavit accompanying the transmittal of these documents pursuant to 10 CFR $2.790(b)(1)$.

\section{COPYRIGHT NOTICE}

This document bears an Oregon State University copyright notice. The NRC is permitted to make the number of copies of the information contained in these reports which are necessary for its internal use in connection with generic and plant-specific reviews and approvals as well as the issuance, denial, amendment, transfer, renewal, modification, suspension, revocation, or violation of a license, permit, order, or regulation subject to the requirements of 10 CFR 2.790 regarding restrictions on public disclosure to the extent such information has been identified as proprietary by Westinghouse, copyright protection notwithstanding. With respect to the non-proprietary versions of these reports, the NRC is permitted to make the number of copies beyond these necessary for its internal use which are necessary in order to have one copy available for public viewing in the appropriate docket files in the public document room in Washington, DC and in local public document rooms as may be required by NRC regulations if the number of copies submitted is insufficient for this purpose. Copies made by the NRC must include the copyright notice in all instances and the proprietary notice if the original was identified as proprietary. 
TABLE OF CONTENTS

$\underline{\text { Section }}$

Title

$\underline{\text { Page }}$

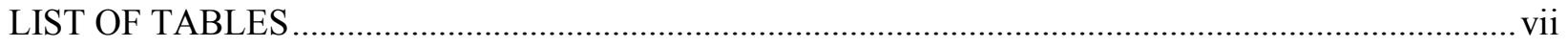

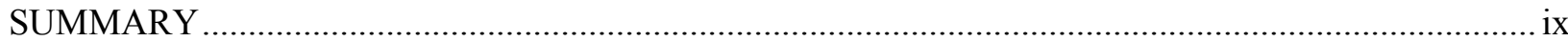

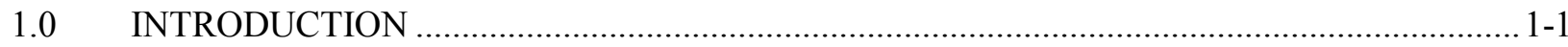

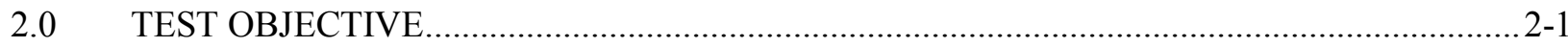

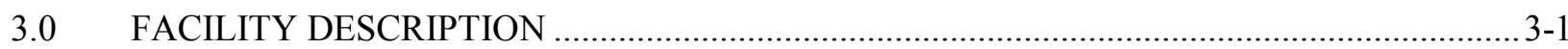

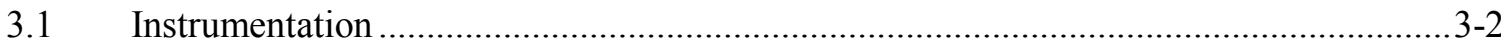

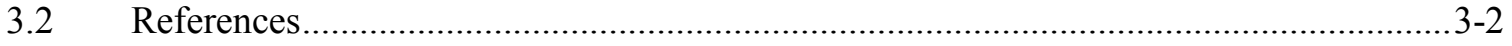

4.0 OREGON STATE UNIVERSITY TESTING PROGRAM MATRIX .......................................4-1

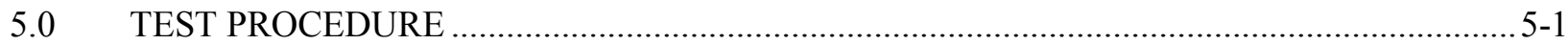

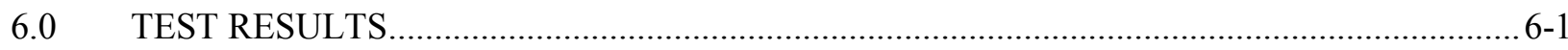

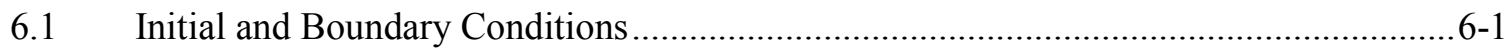

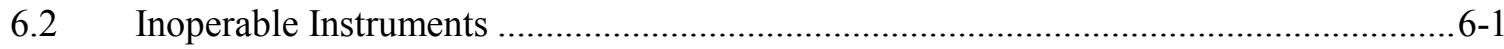

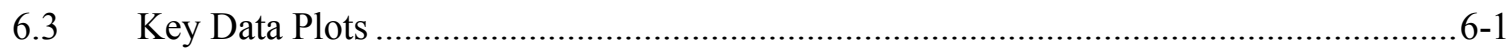

6.4 Test Evaluation ............................................................................................... $6-1$

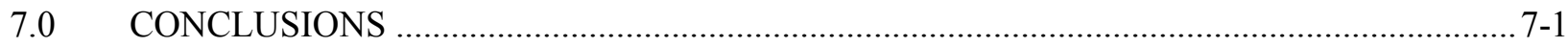

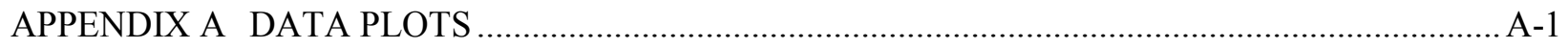

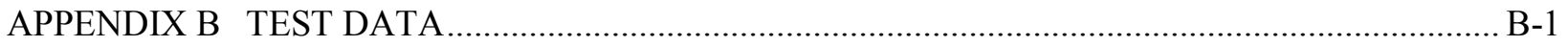




\section{LIST OF TABLES}

$\underline{\text { Table }}$

Table 4-1 OSU Test Matrix (as specified May 13, 2004)

Table 6-1 Actual Test Initial Conditions.

$\underline{\text { Title }}$

Page

Table 6-2

Table 6-3

Table 6-4
Sequence of Events

Inoperable Instruments for DBA-05-D Test

Data Plots for QL Reports for DBA-05-D by Component
$6-2$

4-2

$6-4$ $.6-6$ 6-7 


\section{SUMMARY}

This report covers the test DBA-05-D, inadvertent actuation of Automatic Depressurization System 1 (ADS-1) simulation loss-of-coolant accident, performed on May 13, 2004. This test has been designated OSU-AP1000-10 by the Advanced Plant Experiment (APEX). The objective of this test was to repeat a previous test, whose purpose was to obtain thermal-hydraulic data for an inadvertent ADS-1 simulation. The test performed met the specified conditions. The top of the heater bundle was always covered during this event. The test was performed for about 11,000 seconds. The transient continued through ADS actuation and achieved stable in-containment refueling water storage tank injection.

This report presents the initial assessment of the test data collected. If this test is to be used by Westinghouse to support AP1000 Design Certification, additional validation of the use of this information will be documented separately. In the interim, the list of invalid data channels may change. 


\subsection{INTRODUCTION}

The Department of Nuclear Engineering at Oregon State University (OSU) is performing a series of tests for the U.S. Department of Energy. These tests are being conducted in the Advanced Plant Experiment (APEX-1000) test facility, which is a reduced pressure and height model of the two-loop Westinghouse AP1000 pressurized water reactor. The purpose of the testing is to:

- evaluate the thermal-hydraulic performance of the passive safety systems of the full-scale AP1000, and

- assess and validate the safety analyses techniques and computer codes used in predicting the transient system behavior.

The AP1000 Long-Term Cooling Test is a 1/4 height scale, low-pressure integral systems test simulating thermal-hydraulic phenomenon for the AP1000 passive safety systems for small-break loss-of-coolant accidents (LOCAs) and long-term cooling. It accurately models the details of the AP1000 geometry, including the primary system, the passive safety systems, and a part of the non-safety grade Chemical and Volume Control System, as well as a partial non-safety grade Normal Residual Heat Removal System. The interconnecting pipe routings are also duplicated in the model.

The overall objective of the Long-Term Cooling Test program is to obtain test data at various modes of operation. The OSU experiments will examine the passive safety system response for the small-break and large-break LOCA transition into long-term cooling. (The list of the tests to be performed is in the OSU Test Matrix provided in Section 4.0.) The facility permits a range of small-break LOCAs to be simulated at different locations on the primary system, such as the cold leg, hot leg, core makeup tank (CMT) cold leg pressure balance line, and direct vessel injection (DVI) line. The break orientation (top or bottom of the cold leg) may also be studied. Selected tests continue into the long-term cooling, post-accident mode in which the passive safety injection is from the reactor sump as well as the in-containment refueling water storage tank (IRWST). 


\subsection{TEST OBJECTIVE}

The purpose of this test is to simulate an inadvertent actuation of the Automatic Depressurization System (ADS) causing the ADS-1 valve to open and subsequent ADS valves to open in sequence. There is no pipe break simulated in this test. The assumed single failure for this test is one of four ADS-4 valves. The single failure location is on the non-pressurizer hot leg. The data obtained from the test will be used to verify the AP1000 thermal-hydraulic computer codes for AP1000 phenomena, such as gravity injection, natural convection, and post-accident long-term core cooling behavior.

The acceptance criteria for the OSU tests are as follows:

- Test initial conditions will be achieved within a specified tolerance.

- Setpoints will be achieved within an acceptable tolerance band.

- All instrumentation should be operational before the test.

- Any critical instruments not operating will be identified to the test engineer before the tests. These instruments must be operational before and during the test or exceptions should be approved.

- A zero check of LDPs, DPs, and FDPs will be performed. 


\subsection{FACILITY DESCRIPTION}

A detailed facility description for the OSU test facility is documented in Reference 3-1. The OSU test facility has been specifically scaled, designed, and constructed to investigate the AP600 passive safety system behavior and to provide data for safety analysis computer code validation. The facility has been modified to model the AP1000.

The scaled test design accurately models the details of the AP1000 geometry, including the primary system and the pipe routings and layout for the passive safety systems. A detailed scaling report (Reference 3-2) was used to develop the test design modifications. The primary system consists of one hot leg and two cold legs with two active pumps and an active steam generator for each loop. Two CMTs are connected to one primary loop, and the pressurizer is connected to the other primary loop as in the AP1000 plant design. Gas-driven accumulators are connected to the DVI lines. The discharge lines from the CMT, and one-of-two IRWST and reactor sump lines are connected to each DVI line. The ADS consisting of stages 1,2 , and 3 - simulates either one or two of the independent trains used in the AP1000. The two-phase flow from the ADS stages 1-to-3 is separated in a swirl-vane separator, and the liquid and vapor flows are measured to obtain the total ADS flow rate. The separated flow streams are then recombined and discharged into the IRWST through a sparger, preserving the mass and energy flow into the IRWST. The injection from the reactor sump is also simulated. Note that the OSU facility models both AP1000 primary and secondary sumps. The primary sump collects the condensate return, the liquid break flow, and the liquid flow from the fourth-stage ADS; and will provide long-term injection to the reactor vessel. The secondary sump simulates the portions of containment that will remain dry during most events. This sump collects water only when the primary sump reaches its overflow level, and provides no injection to the reactor vessel.

The time period for the experimental simulations includes not only the IRWST injection, but also the draining of the IRWST and the sump injection to simulate the long-term cooling of the AP1000. This simulation could be from several hours to a day. The time scale for the OSU test facility is one-half; that is, events occur in half the normal time. To model the long-term cooling aspects of the transient, the two-phase flow from the break is separated in a swirl-vane separator and the liquid and vapor portions of the total flow are measured. The liquid fraction of the flow is discharged to the reactor primary sump as in the AP1000 plant. The vapor is discharged to the atmosphere. The capability exists to return a portion of the equivalent liquid flow to the IRWST and primary sump to simulate the condensate return from the passive containment to the IRWST and primary sump. A similar approach is also used for the fourth-stage ADS valve on the hot leg. The two-phase flow is separated in a swirl-vane separator, the two streams are measured, and the liquid phase is discharged into the primary sump while the vapor flow is discharged to the atmosphere. Again, the capability exists to return a portion of the liquid equivalent added to the IRWST and primary sump. In addition, all other steam vents from the facility are measured (for example, the IRWST vent), and a portion of the liquid equivalent may be added back to the facility. Note that not all of the steam discharge would be returned as liquid equivalent. A portion of the discharge would be removed to simulate the steam that is not available for recirculation because it provides containment pressurization. The IRWST and primary sump can be pressurized in the OSU facility to simulate the containment pressurization following a postulated LOCA.

A multi-tube passive residual heat removal (PRHR) heat exchanger is located in the IRWST. The heat exchanger uses the same C-tube design as the AP1000 and has two instrumented tubes to obtain wall heat 
fluxes during the tests. There are primary fluid thermocouples, wall thermocouples, and differential pressure drop measurements to determine when the heat exchanger begins to drain. The IRWST is also instrumented with strings of fluid thermocouples to determine the degree of mixing within the tank and to assess the temperature of the coolant that is delivered to the test vessel.

The reactor vessel for the OSU tests includes a 0.914-meter (3-foot) heated core consisting of forty-eight 0.025 -meter (1-inch) diameter heater rods. The heater rods have a top skewed power shape. The $1000 \mathrm{~kW}$ of electrical power available at the OSU test site will be used to simulate decay heat. Wall thermocouples are swaged inside the heater rods to measure the heater rod wall temperature.

Thermocouple rods in the heater rod bundle measure the axial coolant temperature distribution. The scaled flow volume in the core is preserved as well as the flow volume in the test vessel upper plenum. There are simulated reactor internals in the upper plenum to preserve the flow area and to correctly scale the fluid volume. The reactor vessel includes an annular downcomer into which the four cold legs and the two DVI lines are connected. The hot legs penetrate the reactor annulus and connect with the loops. The AP1000 reactor vessel neutron reflector is simulated using a ceramic liner to reduce the metal heat release to the coolant.

There were no special/unique requirements for the test other than those specified in the Initial Conditions. The specified conditions were verified on the control board prior to test implementation.

\subsection{Instrumentation}

The instrumentation has been designed to calculate a transient mass and energy balance on the test facility. All two-phase flow streams exiting the facility are separated, and each component is measured separately as a single-phase flow using conventional measurement devices, such as magnetic flow meters and vortex flow meters. Note that magnetic flow meters are not designed for two-phase flow and will indicate erratically. Also, the vortex flow meters are referenced to $212^{\circ} \mathrm{F}$ and the LDPs are referenced to $60^{\circ} \mathrm{F}$. All vertical components have differential pressure cells that act as level instruments to measure the transient mass change in the component. The hot and cold leg diameters are sufficiently large in the OSU test facility so that a narrow-range differential pressure cell can be used to determine if the flow becomes stratified.

Single flow measurements are made of the CMT, accumulator, IRWST, and sump flows into the reactor vessel through the DVI lines.

Various types of instrumentation are provided in the test facility; for example, thermocouples for coolant and wall temperatures, flow meters, pressure transducers, differential pressure transducers, and weigh tanks.

\subsection{References}

3-1 APP-LTCT-T2R-005, OSU APEX-1000 Test Facility Description Report

3-2 APP-LTCT-T2R-004, Scaling Assessment for the Design of the OSU APEX-1000 Test Facility 


\subsection{OREGON STATE UNIVERSITY TESTING PROGRAM MATRIX}

The test matrix for the OSU test facility is shown in Table 4-1. To satisfy the test objectives, several transients will be performed to provide data on the AP1000 passive safety system response for a range of break sizes, locations, orientations, and single failure assumptions. The break size orifices are scaled based on simulating a 0.5 -inch, 1-inch, 2-inch, or larger pipe break.

The designation for this test is DBA-05-D, which identifies the test as a design basis inadvertent ADS actuation. The test matrix may be adjusted for future tests based on results and insights gained with each test. 


\begin{tabular}{|c|c|c|}
\hline \multicolumn{3}{|c|}{$\begin{array}{c}\text { TABLE 4-1 } \\
\text { OSU TEST MATRIX (AS SPECIFIED MAY 13, 2004) }\end{array}$} \\
\hline Test Title & Break Location and Size & Single Failure Assumed \\
\hline DBA-01-D & $\begin{array}{l}\text { Double-ended DVI Line break with } \\
\text { continuation into long-term cooling }\end{array}$ & Fail 1 of 2 lines in one ADS- 4 train \\
\hline DBA-02-D & $\begin{array}{l}\text { Double-ended DVI Line break with } \\
\text { continuation into long-term cooling }\end{array}$ & $\begin{array}{l}\text { Fail } 1 \text { of } 2 \text { lines in one ADS- } 4 \text { train } \\
\text { (Adjusted ADS-4 Resistance) }\end{array}$ \\
\hline DBA-03-D & $\begin{array}{l}\text { Double-ended DVI Line break with } \\
\text { continuation into long-term cooling }\end{array}$ & $\begin{array}{l}\text { Fail } 1 \text { of } 2 \text { lines in one ADS- } 4 \text { train } \\
\text { (Failure location sensitivity) }\end{array}$ \\
\hline TR-01-D & $\begin{array}{l}\text { Transition Test ADS-4 opening, } 85 \text { psig initial } \\
\text { pressure and decay power } 480 \mathrm{sec}\end{array}$ & $\begin{array}{l}\text { Fail } 1 \text { of } 2 \text { lines in one ADS-4 train } \\
\qquad(\text { No ADS 1-3) }\end{array}$ \\
\hline TR-02-D & $\begin{array}{l}\text { Transition Test ADS-4 opening, } 100 \text { psig initial } \\
\text { pressure with prototypic plant conditions }\end{array}$ & $\begin{array}{l}\text { Fail } 1 \text { of } 2 \text { lines in one ADS-4 train } \\
\qquad(\text { No ADS 1-3) }\end{array}$ \\
\hline DBA-04-D & 2-inch Cold Leg Break & $\begin{array}{l}\text { Fail } 1 \text { of } 2 \text { lines in one ADS- } 4 \text { train } \\
\text { (Failure on non-pressurizer hot leg) }\end{array}$ \\
\hline ADS-01-D & $\begin{array}{l}\text { ADS-4 Two-Phase Characterization Test at } \\
\text { low-power conditions (steady-state points) }\end{array}$ & $\begin{array}{l}\text { Various ADS-4 line configurations } \\
\qquad \text { (No ADS 1-3) }\end{array}$ \\
\hline ADS-02-D & $\begin{array}{l}\text { ADS-4 Two-Phase Characterization Test at } \\
\text { high-power conditions (steady-state points) }\end{array}$ & $\begin{array}{l}\text { Various ADS-4 line configurations } \\
\qquad \text { (No ADS 1-3) }\end{array}$ \\
\hline DBA-03R-D & Repeat of Test DBA-03-D & $\begin{array}{l}\text { Fail } 1 \text { of } 2 \text { lines in one ADS- } 4 \text { train } \\
\text { (Failure on pressurizer hot leg) }\end{array}$ \\
\hline DBA-05-D & Inadvertent ADS-1 Actuation & $\begin{array}{l}\text { Fail } 1 \text { of } 2 \text { lines in one ADS- } 4 \text { train } \\
\text { (Failure on non-pressurizer hot leg) }\end{array}$ \\
\hline DBA-06-D & 0.5-inch Cold Leg Break & $\begin{array}{l}\text { Fail } 1 \text { of } 2 \text { lines in one ADS- } 4 \text { train } \\
\text { (Failure on non-pressurizer hot leg) }\end{array}$ \\
\hline
\end{tabular}




\subsection{TEST PROCEDURE}

The test was performed per a written procedure. There were no special/unique requirements for the test other than those specified in the initial conditions in Table 6-1. The specified conditions were checked on the control board before test implementation.

The appropriate prerequisites were completed and initial conditions were satisfied. The required break simulation piping and break instrumentation were installed per P\&ID drawing OSU 600904, Rev. 1. The 100-percent flow nozzle was installed in ADS 4-2 (on hot leg 1), and the 50-percent flow nozzle was installed in ADS 4-1 (on hot leg 2). Flow nozzles that simulate full flow for ADS-1, ADS-2, and ADS-3 were installed. As per the AP600 tests, ADS-3 has been scaled for full flow from all three stages, and ADS- 1 and ADS-2 are closed when ADS-3 is opened.

Fill and vent was performed per APEX Operations Manual Procedure OP-B.2. Instruments were checked for required calibration.

With the break valves TS-202 and TS-203 closed, flow was used to warm up the bypass line by opening isolation valves RCS-901 and RCS-902. After the appropriate prerequisites were completed and the test facility achieved specified initial conditions, the CMT warmup bypass line isolation valves RCS-901 and RCS-902 were closed to maintain the $<80^{\circ} \mathrm{F}$ condition at the top of the CMT-1. With the CMT balance line valves (RCS-529 and RCS-530) placed in the open and automatic mode, both CMTs reached the Reactor Coolant System pressure.

Once all other initial conditions were satisfied, a break through TS-202 and TS-203 was initiated. The test continued through ADS actuation; and CMT, accumulator, IRWST, and sump injection. Per Westinghouse instructions, ADS 4-1 was actuated automatically according to the actuation logic and ADS 4-2 was actuated manually after a 30-second delay. All other actions were automatic and required no operator action. 


\subsection{TEST RESULTS}

The test results for test DBA-05-D are provided in the following subsections.

\subsection{Initial and Boundary Conditions}

Table 6-1 provides a comparison of the specified and actual conditions for test DBA-05-D. The values in this table were averaged over approximately 2 minutes preceding the test. Test initial conditions were achieved for the steam generator pressure, pressurizer pressure, pressurizer level, steam generator 01 narrow-range level, and steam generator 02 narrow-range level. Test initial conditions for the hot leg temperature were found to be acceptable, and the results will not be adversely affected.

The actual power decay curves are provided in data plots in Appendix B. The measured maximum power was $863 \mathrm{~kW}$, which was less than the facility maximum power of $1000 \mathrm{~kW}$. The programmed decay heat curve was adjusted to account for this difference, and the differences between the actual and specified power decay are considered acceptable.

PT-501 and PT-502 pressure instruments indicate the pressure changes in the CMT-1 and CMT-2. CMT-1 (PT-501) and CMT-2 (PT-502) confirm that 1 minute after the test button was pushed, both CMTs reach Reactor Coolant System pressure.

The sequence of events is shown in Table 6-2. This table compares the actual sequence of events with the specified timing. As can be seen in this table, all the events occurred at or very near to when the event was planned.

\subsection{Inoperable Instruments}

Table 6-3 lists the instrumentation channels considered inoperable for the DBA-05-D test.

\subsection{Key Data Plots}

Table 6-4 lists the instrumentation channels sorted by component and includes the instrument number, units, and quick look (QL) plot number. The selection of channels was based on projecting an overall picture of the test results, which would then be examined by referring to the detailed data plots or tapes.

\subsection{Test Evaluation}

The following observations were made during the test:

1. The peak power before the test was initiated was $933 \mathrm{~kW}$. The decay heat curve was adjusted for this value. 


\begin{tabular}{|c|c|c|c|}
\hline \multicolumn{4}{|c|}{$\begin{array}{c}\text { TABLE 6-1 } \\
\text { ACTUAL TEST INITIAL CONDITIONS }\end{array}$} \\
\hline Conditions & Instrument No. & Actual & Comment \\
\hline Pressurizer Pressure & PT-604 & 370 psig & \\
\hline Hot Leg Temperature \#1 & $\begin{array}{l}\text { TF-141* } \\
\text { TF-205 } \\
\text { TF-143 }\end{array}$ & $\begin{array}{l}426^{\circ} \mathrm{F} \\
428^{\circ} \mathrm{F} \\
428^{\circ} \mathrm{F}\end{array}$ & \\
\hline Hot Leg Temperature \#2 & $\begin{array}{l}\text { TF-140* } \\
\text { TF-206 } \\
\text { TF-142 }\end{array}$ & $\begin{array}{l}428^{\circ} \mathrm{F} \\
426^{\circ} \mathrm{F} \\
428^{\circ} \mathrm{F}\end{array}$ & \\
\hline $\begin{array}{l}\text { Steam Generator Pressure } \\
\quad \# 1 \\
\# 2 \\
\text { Header }\end{array}$ & $\begin{array}{l}\text { PT-301* } \\
\text { PT-302* } \\
\text { PT-002 }\end{array}$ & $\begin{array}{l}286 \text { psig } \\
284 \text { psig } \\
258 \text { psig }\end{array}$ & \\
\hline Pressurizer Level & $\begin{array}{l}\text { LDP-601 uncompensated } \\
\text { LDP-601 } \\
\text { Compensated by SC-608 }\end{array}$ & $\begin{array}{l}70.7 \text { inches } \\
85 \text { inches }\end{array}$ & $\begin{array}{l}440^{\circ} \mathrm{F} \text { used for density } \\
\text { compensation }\end{array}$ \\
\hline $\begin{array}{l}\text { Steam Generator Level } \\
\# 1 \mathrm{NR}\end{array}$ & $\begin{array}{l}\text { LDP-303 uncompensated } \\
\text { LDP-303 compensated by } \\
\text { average of TF-305 and } \\
\text { TF-307 }\end{array}$ & $\begin{array}{l}20.8 \text { inches } \\
25.1 \text { inches }\end{array}$ & $\begin{array}{l}413^{\circ} \mathrm{F} \text { used for density } \\
\text { compensation }\end{array}$ \\
\hline $\begin{array}{l}\text { Steam Generator Level } \\
\# 2 \mathrm{NR}\end{array}$ & $\begin{array}{l}\text { LDP-304 uncompensated } \\
\text { LDP-304 compensated by } \\
\text { average of TF-306 and } \\
\text { TF-308 }\end{array}$ & $\begin{array}{l}21.4 \text { inches } \\
25.1 \text { inches }\end{array}$ & $\begin{array}{l}414^{\circ} \mathrm{F} \text { used for density } \\
\text { compensation }\end{array}$ \\
\hline IRWST Temperature & TF-701 & $71^{\circ} \mathrm{F}$ & Accepted $\left(<80^{\circ} \mathrm{F}\right)$ \\
\hline $\begin{array}{l}\text { CMT Temperature } \\
\text { \#1 } \\
\# 2\end{array}$ & $\begin{array}{l}\text { TF-513 } \\
\text { TF-516 }\end{array}$ & $\begin{array}{l}74.1^{\circ} \mathrm{F} \\
73.6^{\circ} \mathrm{F}\end{array}$ & Accepted $\left(<80^{\circ} \mathrm{F}\right)$ \\
\hline $\begin{array}{l}\text { Accumulator } \\
\text { Temperature } \\
\# 1 \\
\# 2\end{array}$ & $\begin{array}{l}\text { TF-403 } \\
\text { TF-404 }\end{array}$ & $\begin{array}{l}74.6^{\circ} \mathrm{F} \\
75^{\circ} \mathrm{F}\end{array}$ & Accepted $\left(<80^{\circ} \mathrm{F}\right)$ \\
\hline IRWST Level & LDP-701 & 92.2 inches & \\
\hline
\end{tabular}




\begin{tabular}{||c|c|c|c||}
\hline \multicolumn{5}{|c||}{ TABLE 6-1 (Continued) } \\
ACTUAL TEST INITIAL CONDITIONS \\
\hline \hline Conditions & Instrument No. & Actual & Comment \\
\hline Accumulator Level & & & \\
$\# 1$ & LDP-401 & 36.3 inches & \\
$\# 2$ & LDP-402 & 36.9 inches & \\
\hline Accumulator Pressure & & & \\
$\# 1$ & PT-401 & $188.1 \mathrm{psig}$ & \\
$\# 2$ & PT-402 & $193.8 \mathrm{psig}$ & \\
\hline CMT Level & & & \\
$\# 1$ & LDP-507 & 57.5 inches & \\
$\# 2$ & LDP-502 & 57.8 inches & \\
\hline CMT Pressure & PT-501 & $374.2 \mathrm{psig}$ & \\
$\# 1$ & PT-502 & $375.4 \mathrm{psig}$ & \\
$\# 2$ & \multicolumn{2}{||}{} \\
\hline
\end{tabular}

* These instruments are used to establish initial conditions at the test site. 


\begin{tabular}{|c|c|c|}
\hline \multicolumn{3}{|c|}{$\begin{array}{c}\text { TABLE 6-2 } \\
\text { SEQUENCE OF EVENTS }\end{array}$} \\
\hline Event & Setpoint & Actual Time (sec) \\
\hline PB Depressed & $\mathrm{N} / \mathrm{A}$ & -120 \\
\hline Break Valve(s) Open & 0 & 0 \\
\hline Feed Pump Trip & $3.6 \mathrm{sec}$ & 3 \\
\hline CMT \#1 Outlet Valve Open (RCS-535) & $6.1 \mathrm{sec}$ & 5 \\
\hline CMT \#2 Outlet Valve Open (RCS-536) & $6.1 \mathrm{sec}$ & 20 \\
\hline PRHR HX Outlet Valve Open (RCS-804) & $6.1 \mathrm{sec}$ & 0 \\
\hline Reactor Coolant Pump \#1 Trip & $8.6 \mathrm{sec}$ & 8 \\
\hline Reactor Coolant Pump \#2 Trip & $8.6 \mathrm{sec}$ & 8 \\
\hline Reactor Coolant Pump \#3 Trip & $8.6 \mathrm{sec}$ & 8 \\
\hline Reactor Coolant Pump \#4 Trip & $8.6 \mathrm{sec}$ & 8 \\
\hline CMT \#1 Level Low (LDP-507) & 41 inches & 275 \\
\hline CMT \#2 Level Low (LDP-502) & 41 inches & 268 \\
\hline ADS \#1 Actuation (RCS-601) & CMT Level Low $+15 \mathrm{sec}$ & 0 \\
\hline ADS \#2 Actuation (RCS-602) & CMT Level Low $+62 \mathrm{sec}$ & 47 \\
\hline ADS \#3 Actuation (RCS-603) & CMT Level Low $+122 \mathrm{sec}$ & 107 \\
\hline Low Reactor Pressure (P-107) & $40 \mathrm{psig}$ & 442 \\
\hline IRWST Valve Actuation (RCS-711) & $\begin{array}{l}\text { Low Reactor Pressure } \\
\qquad(<40 \text { psig })\end{array}$ & 428 \\
\hline IRWST Valve Actuation (RCS-712) & $\begin{array}{l}\text { Low Reactor Pressure } \\
\qquad(<40 \text { psig })\end{array}$ & 428 \\
\hline CMT \#1 Low Low Level (LDP-507) & 17.14 inches & 610 \\
\hline CMT \#2 Low Low Level (LDP-502) & 17.14 inches & 586 \\
\hline ADS 4-1 Actuation (RCS-615) & $\begin{array}{l}\text { CMT Low Low (17.14") and } \\
\text { CMT Low }\left(41^{\prime \prime}\right)+180 \mathrm{sec}\end{array}$ & 650 \\
\hline ADS 4-2 Actuation (RCS-616) & $\begin{array}{l}\text { CMT Low Low (17.14") and } \\
\text { CMT Low (41") + } 180 \mathrm{sec}\end{array}$ & 682 \\
\hline Sump Valve Actuation (CSS-909) & IRWST Level Low Low & 9404 \\
\hline Sump Valve Actuation (CSS-910) & IRWST Level Low Low & 9404 \\
\hline
\end{tabular}




\begin{tabular}{|c|c|c|}
\hline \multicolumn{3}{|c|}{$\begin{array}{c}\text { TABLE 6-2 (Continued) } \\
\text { SEQUENCE OF EVENTS }\end{array}$} \\
\hline Event & Setpoint & Actual Time (sec) \\
\hline $\begin{array}{l}\text { Accumulator Injection } \\
\text { \#1 (FMM-401) }\end{array}$ & N/A & 0 \\
\hline $\begin{array}{l}\text { Accumulator Injection } \\
\text { \#2 (FMM-402) }\end{array}$ & N/A & 123 \\
\hline $\begin{array}{l}\text { IRWST Injection } \\
\text { DVI \#1 (FMM-701) }\end{array}$ & N/A & 1235 \\
\hline $\begin{array}{l}\text { IRWST Injection } \\
\text { DVI \#2 (FMM-702) }\end{array}$ & N/A & 1235 \\
\hline $\begin{array}{l}\text { Accumulator Empty } \\
\text { \#1 (LDP-401) }\end{array}$ & N/A & 750 down to 1.2 inch level \\
\hline $\begin{array}{l}\text { Accumulator Empty } \\
\text { \#2 (LDP-402) }\end{array}$ & N/A & 830 down to 0.1 inch level \\
\hline $\begin{array}{l}\text { CMT Empty } \\
\# 1 \text { (LDP-507) }\end{array}$ & $\mathrm{N} / \mathrm{A}$ & 960 down to 0.1 inch level \\
\hline $\begin{array}{l}\text { CMT Empty } \\
\text { \#2 (LDP-502) }\end{array}$ & N/A & 920 \\
\hline Sump Injection DVI \#1 (FMM-901) & N/A & 9404 \\
\hline Sump Injection DVI \#2 (FMM-902) & $\mathrm{N} / \mathrm{A}$ & 9404 \\
\hline
\end{tabular}




\begin{tabular}{|c|c|c|}
\hline \multicolumn{3}{|c|}{$\begin{array}{c}\text { TABLE 6-3 } \\
\text { INOPERABLE INSTRUMENTS FOR DBA-05-D TEST }\end{array}$} \\
\hline Instrument Number & Instrument Type & Inoperable Description \\
\hline $\begin{array}{l}\text { TW-202 } \\
\text { TW-204 } \\
\text { TW-205 } \\
\text { TW-206 } \\
\text { TW-209 } \\
\text { TW-803 } \\
\text { TW-804 }\end{array}$ & Thermocouple & Inoperative \\
\hline TH-603 & $\begin{array}{l}\text { Thermocouple measuring heater } \\
\text { temperature }\end{array}$ & Inoperative \\
\hline FMM-202 & Magnetic flow meter & Inoperative \\
\hline $\begin{array}{l}\text { TF-170 } \\
\text { TF-221 } \\
\text { TF-509 } \\
\text { TF-512 }\end{array}$ & $\begin{array}{l}\text { Thermocouple measuring fluid } \\
\text { temperature }\end{array}$ & Inoperative \\
\hline FVM-905 & Vortex flow meter & Erratic \\
\hline
\end{tabular}




\begin{tabular}{|c|c|c|c|c|}
\hline \multicolumn{5}{|c|}{$\begin{array}{l}\text { TABLE 6-4 } \\
\text { DATA PLOTS FOR QL REPORTS FOR DBA-05-D BY COMPONENT }\end{array}$} \\
\hline Component & Channel & Units & QL-Plot & Comment \\
\hline $\begin{array}{l}\text { Reactor Vessel } \\
\text { Pressure }\end{array}$ & PT-107 & psig & 1 & \\
\hline Reactor Vessel Level & LDP-127 & inch of $\mathrm{H}_{2} \mathrm{O}$ & 2 & \\
\hline $\begin{array}{l}\text { Reactor Vessel } \\
\text { Downcomer Level }\end{array}$ & LDP-140 & inch of $\mathrm{H}_{2} \mathrm{O}$ & 3 & \\
\hline $\begin{array}{l}\text { Cold Leg \#1 Fluid } \\
\text { Temperature }\end{array}$ & TF-107 & ${ }^{\circ} \mathrm{F}$ & 4 & \\
\hline $\begin{array}{l}\text { Cold Leg \#2 Fluid } \\
\text { Temperature }\end{array}$ & TF-108 & ${ }^{\circ} \mathrm{F}$ & 5 & \\
\hline $\begin{array}{l}\text { Cold Leg \#3 Fluid } \\
\text { Temperature }\end{array}$ & TF-103 & ${ }^{\circ} \mathrm{F}$ & 6 & \\
\hline $\begin{array}{l}\text { Cold Leg \#4 Fluid } \\
\text { Temperature }\end{array}$ & TF-104 & ${ }^{\circ} \mathrm{F}$ & 7 & \\
\hline $\begin{array}{l}\text { Reactor Vessel Fluid } \\
\text { Temperature Upper } \\
\text { Head }\end{array}$ & TF-120 & ${ }^{\circ} \mathrm{F}$ & 8 & \\
\hline $\begin{array}{l}\text { Reactor Coolant } \\
\text { System Hot Leg \#1 } \\
\text { Temperature }\end{array}$ & TF-143 & ${ }^{\circ} \mathrm{F}$ & 9 & \\
\hline $\begin{array}{l}\text { Reactor Coolant } \\
\text { System Hot Leg \#2 } \\
\text { Temperature }\end{array}$ & TF-142 & ${ }^{\circ} \mathrm{F}$ & 10 & \\
\hline Pressurizer Pressure & $\begin{array}{l}\text { PT-604 (WR) and PT-603 } \\
\text { (LP Indication) }\end{array}$ & psig & 11,12 & \\
\hline $\begin{array}{l}\text { Pressurizer Liquid } \\
\text { Level }\end{array}$ & LDP-601 & inch of $\mathrm{H}_{2} \mathrm{O}$ & 13 & $\begin{array}{l}\text { Sharp decrease followed } \\
\text { by rapid refill }\end{array}$ \\
\hline $\begin{array}{l}\text { Steam Generator \#1 } \\
\text { Tube Level }\end{array}$ & LDP-215 & inch of $\mathrm{H}_{2} \mathrm{O}$ & 14 & \\
\hline $\begin{array}{l}\text { Steam Generator \#1 } \\
\text { Secondary Pressure }\end{array}$ & PT-301 & psig & 15 & \\
\hline
\end{tabular}




\begin{tabular}{|c|c|c|c|c|}
\hline \multicolumn{5}{|c|}{$\begin{array}{l}\text { TABLE 6-4 (Continued) } \\
\text { DATA PLOTS FOR QL REPORTS FOR DBA-05-D BY COMPONENT }\end{array}$} \\
\hline Component & Channel & Units & QL-Plot & Comment \\
\hline $\begin{array}{l}\text { Steam Generator \#1 } \\
\text { Feed Flow Rate }\end{array}$ & FMM-001 & gpm & 16 & \\
\hline $\begin{array}{l}\text { Steam Generator \#2 } \\
\text { Tube Level }\end{array}$ & LDP-218 & inch of $\mathrm{H}_{2} \mathrm{O}$ & 17 & \\
\hline $\begin{array}{l}\text { Steam Generator \#2 } \\
\text { Secondary Pressure }\end{array}$ & PT-302 & psig & 18 & \\
\hline $\begin{array}{l}\text { Accumulators \#1 and } \\
\# 2 \text { Pressure }\end{array}$ & PT-401 and PT-402 & psig & 19,20 & \\
\hline $\begin{array}{l}\text { Accumulators \#1 and } \\
\# 2 \text { Liquid Level }\end{array}$ & LDP-401 and LDP-402 & inch of $\mathrm{H}_{2} \mathrm{O}$ & 21,22 & \\
\hline $\begin{array}{l}\text { Accumulators \#1 and } \\
\text { \#2 Flow Rate }\end{array}$ & FMM-401 and FMM-402 & gpm & 23,24 & \\
\hline $\begin{array}{l}\text { Accumulators \#1 and } \\
\text { \#2 Liquid Discharge } \\
\text { Temperature }\end{array}$ & TF-401 and TF-402 & ${ }^{\circ} \mathrm{F}$ & 25,26 & \\
\hline $\begin{array}{l}\text { CMT \#1 and \#2 Liquid } \\
\text { Level }\end{array}$ & LDP-507 and LDP-502 & inch of $\mathrm{H}_{2} \mathrm{O}$ & 27,28 & \\
\hline $\begin{array}{l}\text { CMT \#1 and \#2 Flow } \\
\text { Rate }\end{array}$ & FMM-501 and FMM-504 & gpm & 29,30 & \\
\hline $\begin{array}{l}\text { CMT \#1 and \#2 Liquid } \\
\text { Temperature }\end{array}$ & $\begin{array}{l}\mathrm{TF}-501 \text { and TF-529; and } \\
\text { TF-504 and TF-532 }\end{array}$ & ${ }^{\circ} \mathrm{F}$ & $31-34$ & \\
\hline PRHR Inlet Flow Rate & FMM-802 & gpm & 35 & \\
\hline PRHR Liquid Level & LDP-802 & inch of $\mathrm{H}_{2} \mathrm{O}$ & 36 & \\
\hline $\begin{array}{l}\text { PRHR Outlet Flow } \\
\text { Rate }\end{array}$ & FMM-804 & gpm & 37 & \\
\hline IRWST Liquid Level & LDP-701 & inch of $\mathrm{H}_{2} \mathrm{O}$ & 38 & \\
\hline $\begin{array}{l}\text { IRWST Discharge Line } \\
\# 1 \text { and \#2 Flow Rate }\end{array}$ & FMM-701 and FMM-702 & gpm & 39,40 & \\
\hline $\begin{array}{l}\text { IRWST Fluid } \\
\text { Temperature }\end{array}$ & TF-701 and TF-709 & ${ }^{\circ} \mathrm{F}$ & 41,42 & \\
\hline $\begin{array}{l}\text { ADS 1-3 Separator } \\
\text { Pressure }\end{array}$ & PT-605 & psig & 43 & \\
\hline
\end{tabular}




\begin{tabular}{|c|c|c|c|c|}
\hline \multicolumn{5}{|c|}{$\begin{array}{l}\text { TABLE 6-4 (Continued) } \\
\text { DATA PLOTS FOR QL REPORTS FOR DBA-05-D BY COMPONENT }\end{array}$} \\
\hline Component & Channel & Units & QL-Plot & Comment \\
\hline $\begin{array}{l}\text { ADS 1-3 Separator } \\
\text { Steam Flow Rate }\end{array}$ & FVM-601 & $\operatorname{scfm}$ & 44 & \\
\hline $\begin{array}{l}\text { ADS 1-3 Separator } \\
\text { Liquid Flow Rate }\end{array}$ & FMM-601 & gpm & 45 & \\
\hline $\begin{array}{l}\text { ADS-4 Separator \#1 } \\
\text { and \#2 Pressure }\end{array}$ & PT-611 and PT-610 & psig & 46,47 & \\
\hline $\begin{array}{l}\text { ADS 4-1 Separator } \\
\text { Steam Flow Rate }\end{array}$ & FVM-603 & $\mathrm{scfm}$ & 48 & \\
\hline $\begin{array}{l}\text { ADS 4-2 Separator } \\
\text { Steam Flow Rate }\end{array}$ & FVM-602 & scfm & 49 & \\
\hline $\begin{array}{l}\text { ADS 4-1 Separator } \\
\text { Liquid Flow Rate }\end{array}$ & FMM-603 & gpm & 50 & \\
\hline $\begin{array}{l}\text { ADS 4-2 Separator } \\
\text { Liquid Flow Rate }\end{array}$ & FMM-602 & gpm & 51 & \\
\hline Primary Sump Pressure & PT-901 & psig & 52 & \\
\hline $\begin{array}{l}\text { Primary Sump Liquid } \\
\text { Level }\end{array}$ & LDP-901 & inch of $\mathrm{H}_{2} \mathrm{O}$ & 53 & \\
\hline $\begin{array}{l}\text { Primary Sump } \\
\text { Injection Flow Rate }\end{array}$ & FMM-901 and FMM-902 & gpm & 54,55 & \\
\hline $\begin{array}{l}\text { Secondary Sump } \\
\text { Liquid Level }\end{array}$ & LDP-902 & inch of $\mathrm{H}_{2} \mathrm{O}$ & 56 & \\
\hline $\begin{array}{l}\text { Break Separator } \\
\text { Pressure }\end{array}$ & PT-905 & psig & 57 & \\
\hline $\begin{array}{l}\text { Break Separator Liquid } \\
\text { Level }\end{array}$ & LDP-905 & inch of $\mathrm{H}_{2} \mathrm{O}$ & 58 & \\
\hline $\begin{array}{l}\text { Break Separator Flow } \\
\text { to Primary Sump }\end{array}$ & FMM-905 & gpm & 59 & \\
\hline $\begin{array}{l}\text { BAMS Steam Flow } \\
\text { Rate }\end{array}$ & FVM-901 & $\mathrm{scfm}$ & 60 & \\
\hline $\begin{array}{l}\text { BAMS Steam Flow } \\
\text { Rate }\end{array}$ & FVM-902 & $\mathrm{scfm}$ & 61 & \\
\hline
\end{tabular}


TABLE 6-4 (Continued)

DATA PLOTS FOR QL REPORTS FOR DBA-05-D BY COMPONENT

\begin{tabular}{|c|c|c|c|c|}
\hline Component & Channel & Units & QL-Plot & Comment \\
\hline $\begin{array}{l}\text { BAMS/Primary Sump } \\
\text { Steam Flow Rate }\end{array}$ & FVM-903 & $\mathrm{scfm}$ & 62 & \\
\hline $\begin{array}{l}\text { BAMS/Separator } \\
\text { Steam Flow Rate - } \\
\text { 6-inch Pipe }\end{array}$ & FVM-905 & $\operatorname{scfm}$ & 63 & \\
\hline $\begin{array}{l}\text { BAMS/Exhaust Line } \\
\text { Temperature }\end{array}$ & TF-916 and SC-917 & ${ }^{\circ} \mathrm{F}$ & 64,65 & \\
\hline $\begin{array}{l}\text { Pressurizer Heater } \\
\text { Input Power }\end{array}$ & KW-601 & $\mathrm{kW}$ & 66 & \\
\hline $\begin{array}{l}\text { Core Power Input } \\
\text { Power }\end{array}$ & $\begin{array}{c}\text { KW-101, KW-102, } \\
\text { KW-103, and KW-104 }\end{array}$ & $\mathrm{kW}$ & $67-70$ & \\
\hline $\begin{array}{l}\text { Reactor Vessel Liquid } \\
\text { Level Between Top of } \\
\text { Vessel - Upper Support } \\
\text { Plate }\end{array}$ & LDP-115 & inch of $\mathrm{H}_{2} \mathrm{O}$ & 71 & \\
\hline $\begin{array}{l}\text { Reactor Vessel Liquid } \\
\text { Level Between Bottom } \\
\text { of Upper Support } \\
\text { Plate - Upper Core } \\
\text { Spacer Grid }\end{array}$ & LDP-139 & inch of $\mathrm{H}_{2} \mathrm{O}$ & 72 & \\
\hline $\begin{array}{l}\text { Inner Core } \\
\text { Thermocouple } \\
\text { Measuring Heater } \\
\text { Temperature }\end{array}$ & TH-103-4 & ${ }^{\circ} \mathrm{F}$ & 73 & \\
\hline $\begin{array}{l}\text { Outer Core } \\
\text { Thermocouple } \\
\text { Measuring Heater } \\
\text { Temperature }\end{array}$ & TH-309-4 & ${ }^{\circ} \mathrm{F}$ & 74 & \\
\hline
\end{tabular}




\subsection{CONCLUSIONS}

The DBA-05-D test was successfully completed, and the data was logged in the DAS. All critical instruments were found to operate properly with the exception of those noted in Section 6.4. The test was acceptable. 
APPENDIX A

DATA PLOTS 

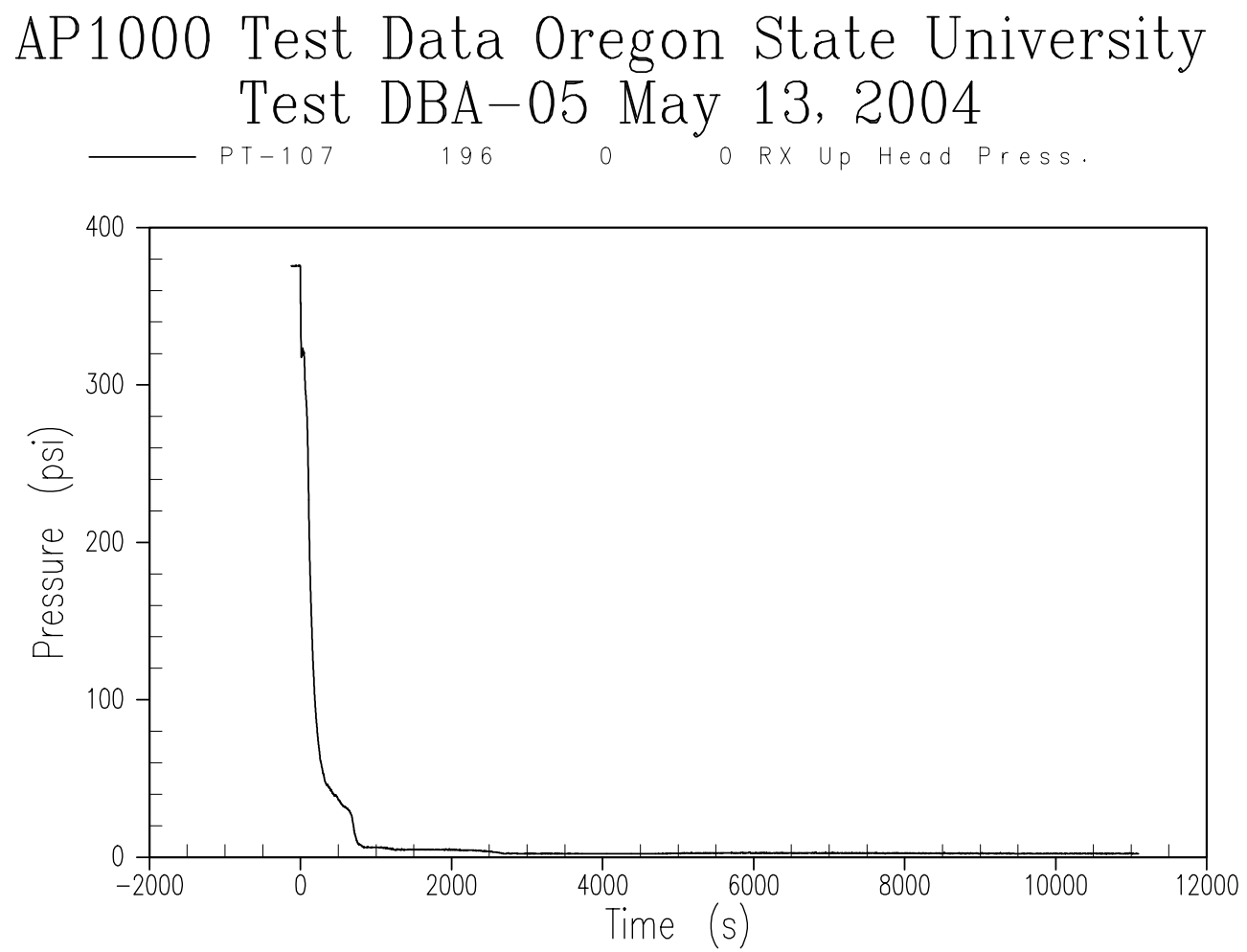

Figure A-1 Reactor Vessel Pressure 

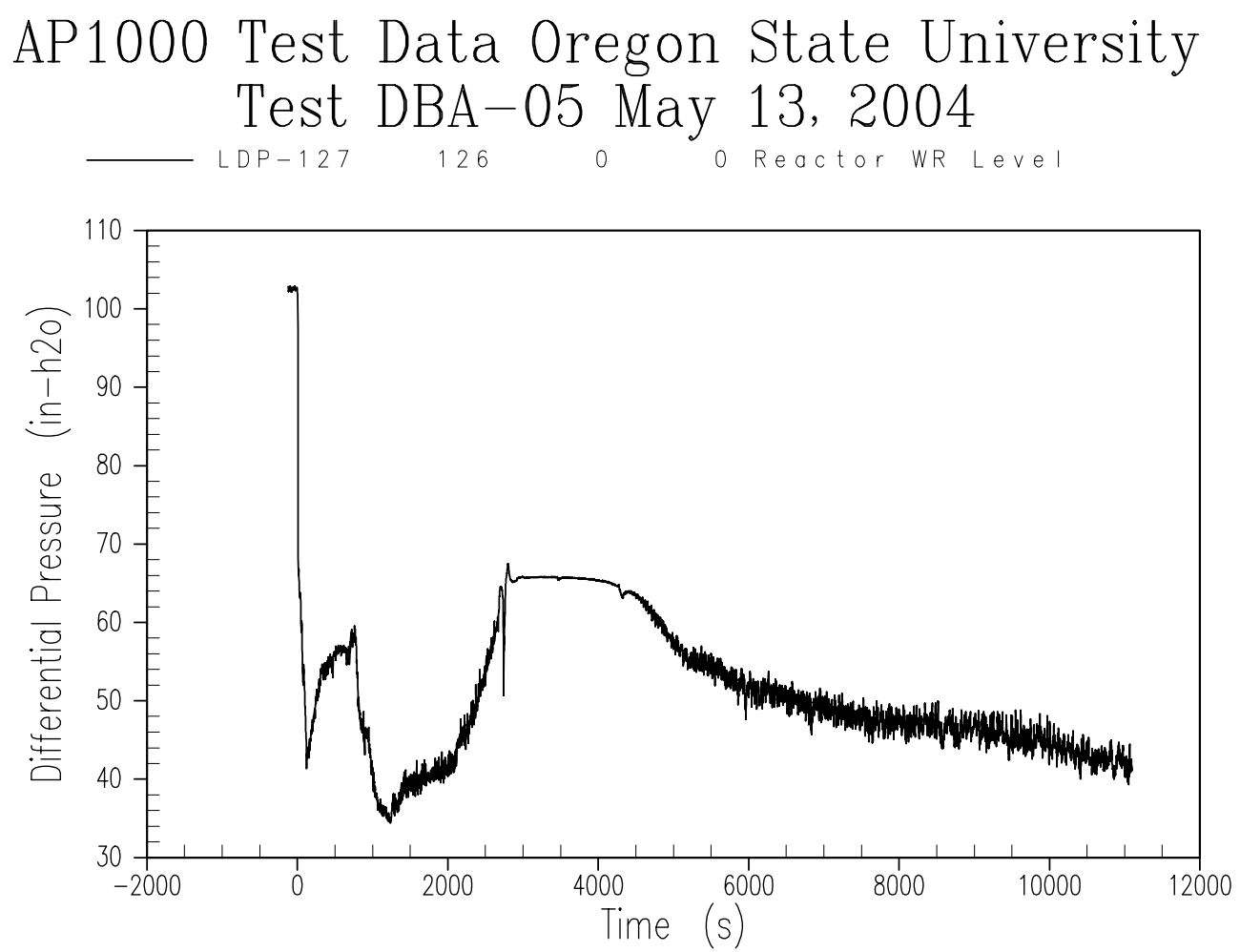

Figure A-2 Reactor Vessel Level 

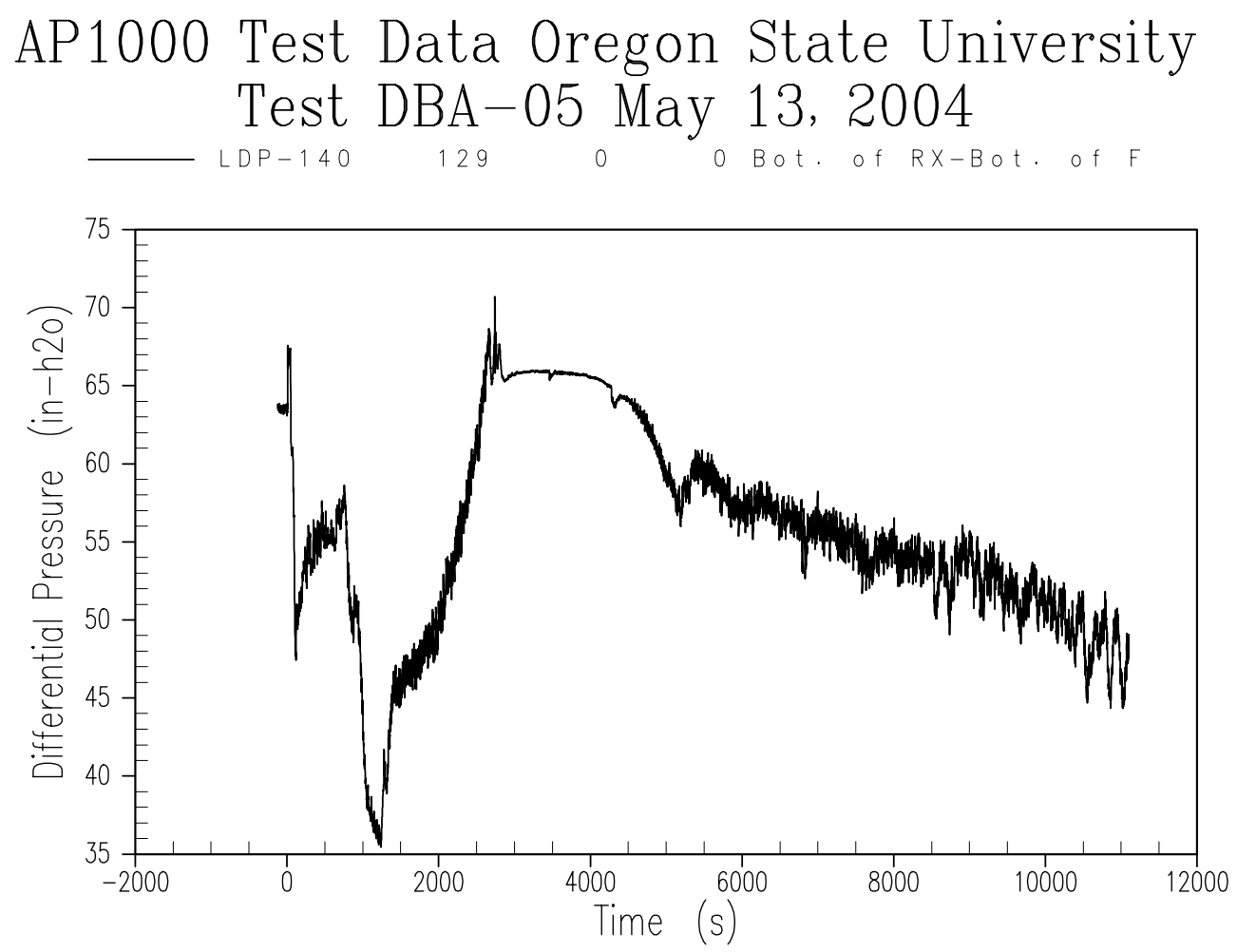

Figure A-3 Reactor Vessel Downcomer Level 
AP1000 Test Data Oregon State University Test DBA-05 May 13, 2004

TF-107 289

o $C L-1 / R X$ Flange (a) Bot

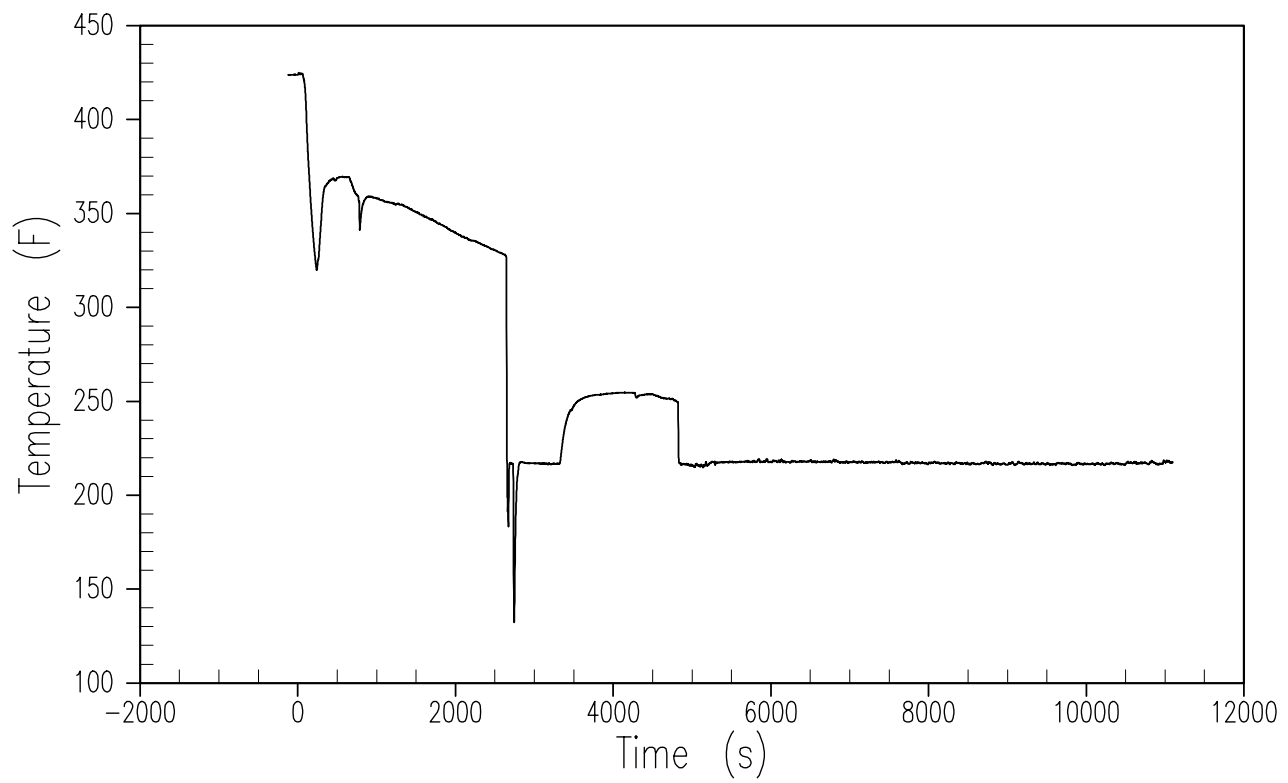

Figure A-4 Cold Leg 1 Fluid Temperature 
AP1000 Test Data Oregon State University Test DBA-05 May 13, 2004

TF $-108 \quad 290$

o $C L-2 / R X$ Flange (a) Bot

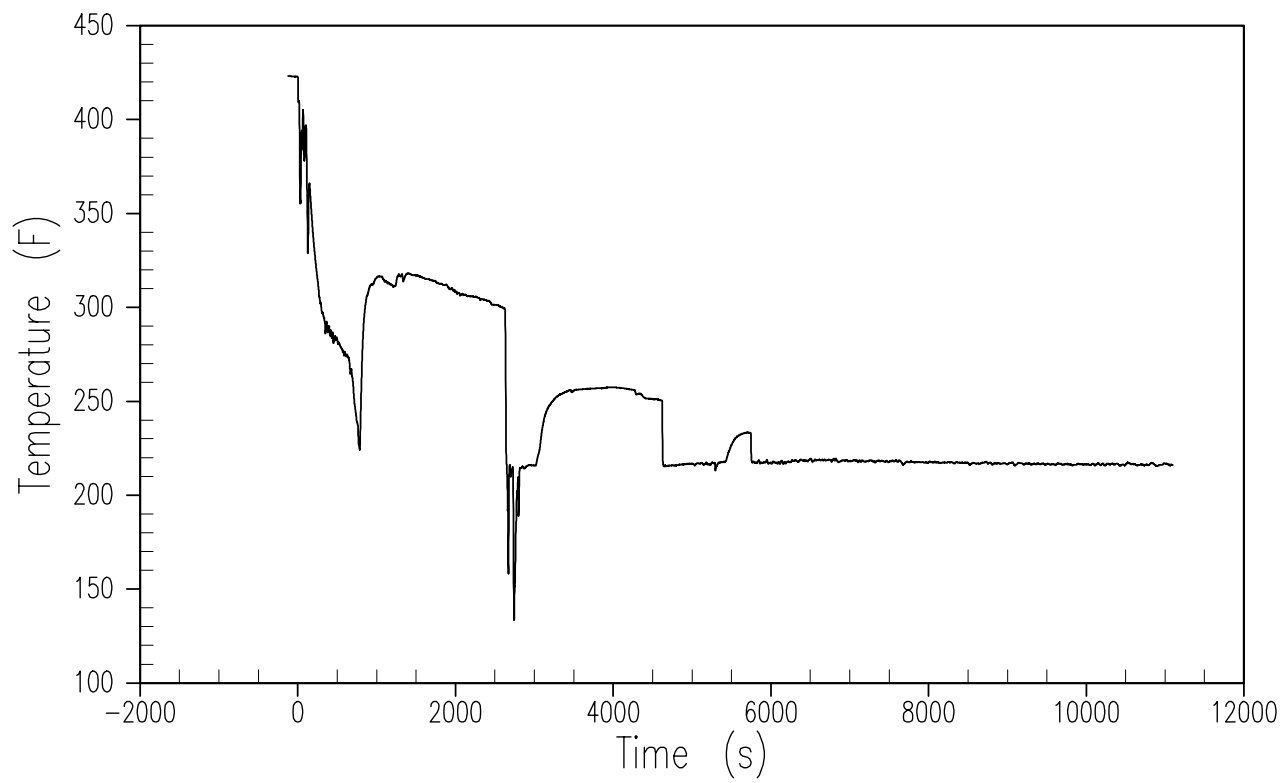

Figure A-5 Cold Leg 2 Fluid Temperature 
AP1000 Test Data Oregon State University Test DBA-05 May 13, 2004

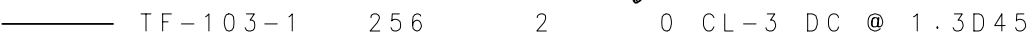

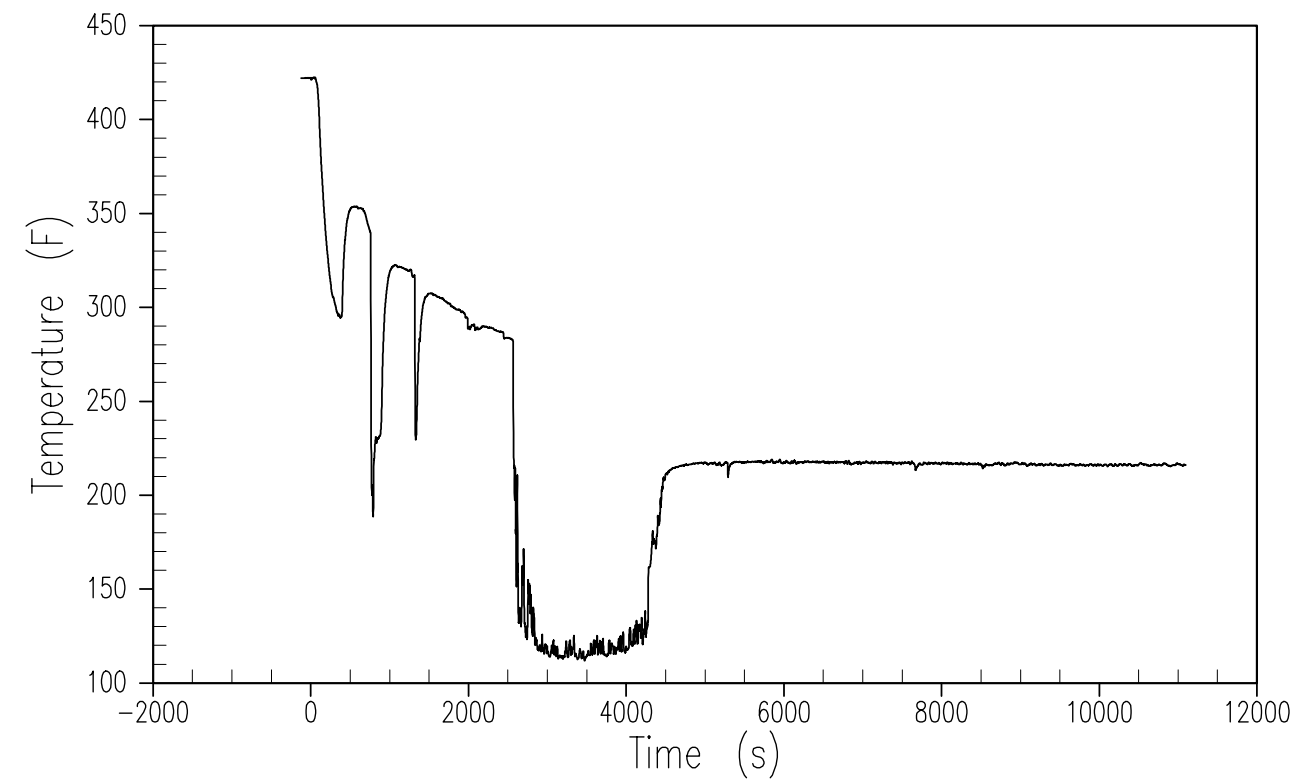

Figure A-6 Cold Leg 3 Fluid Temperature 
AP1000 Test Data Oregon State University Test DBA-05 May 13, 2004

- TF-104-1 560

(a) 1.50

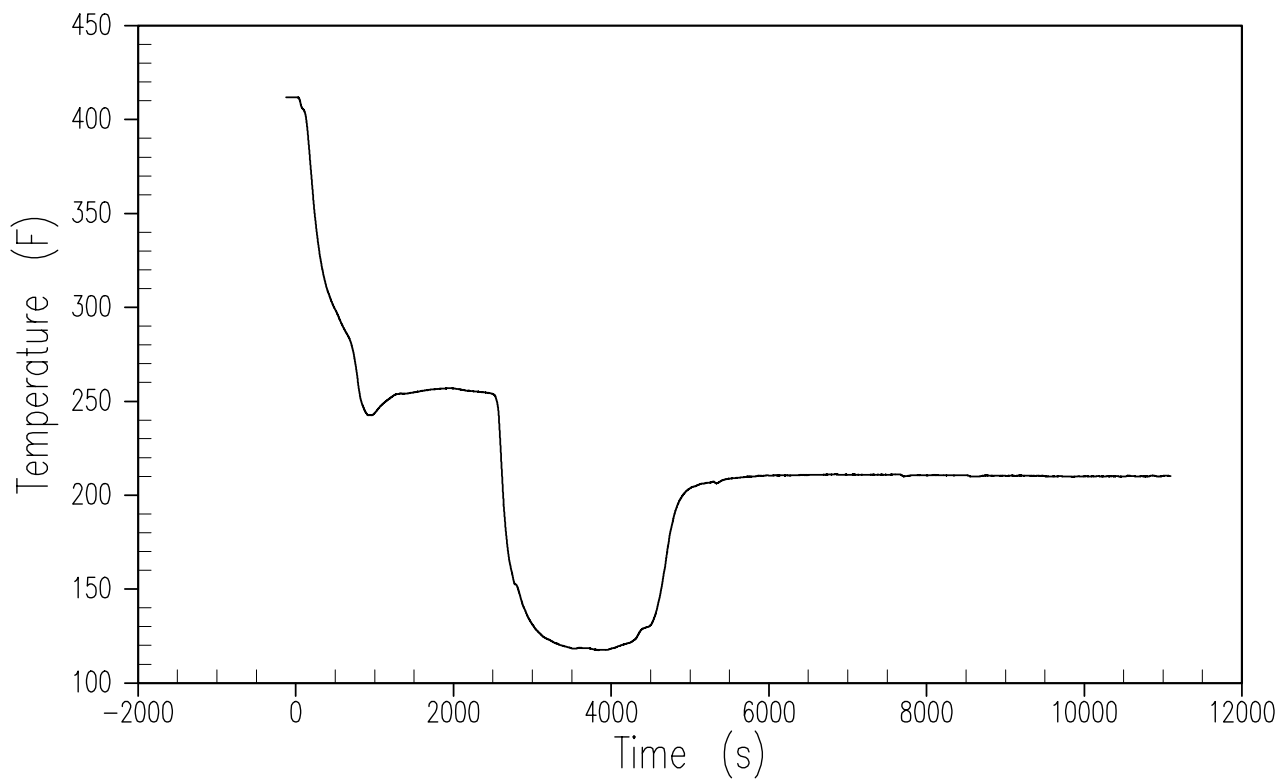

Figure A-7 Cold Leg 4 Fluid Temperature 

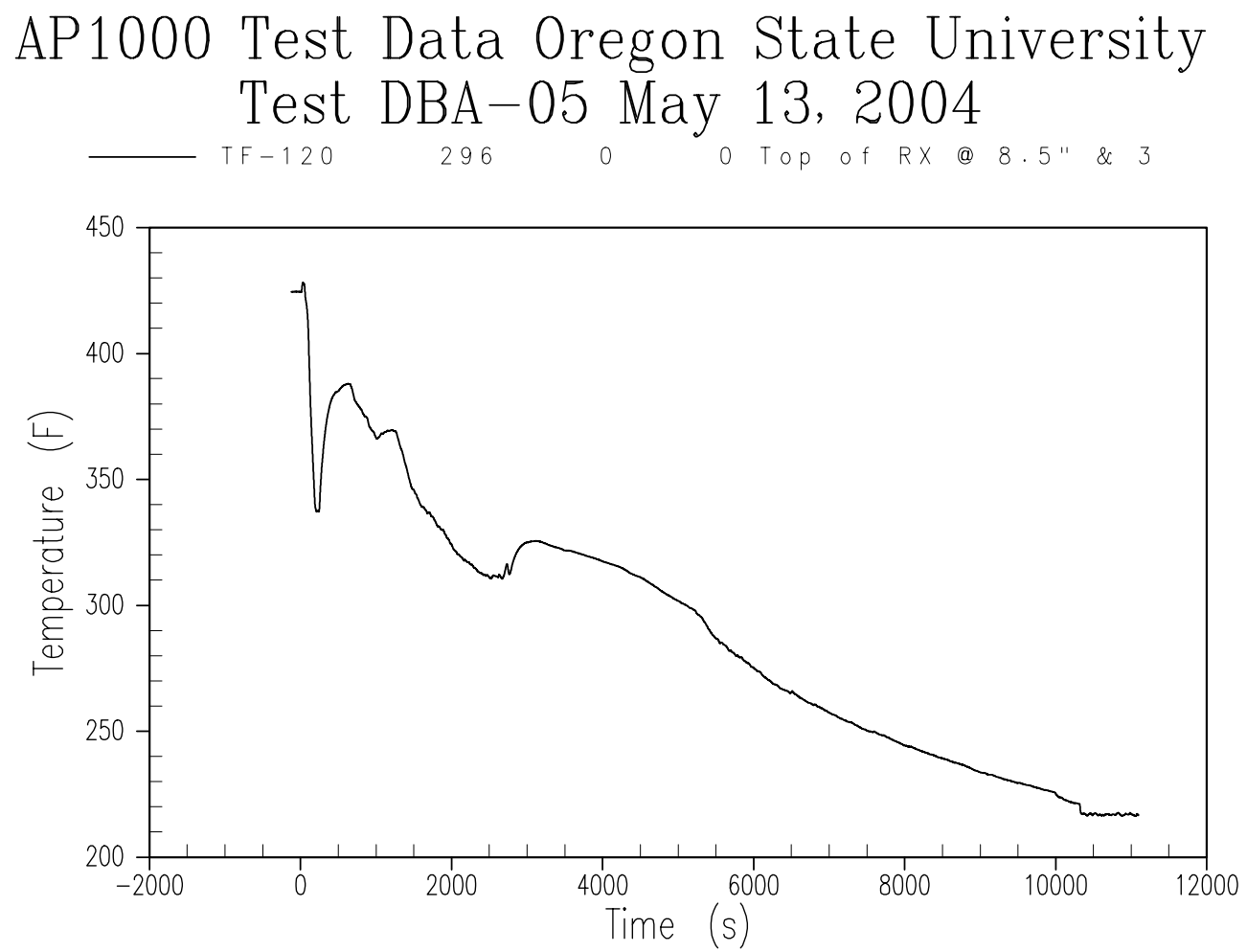

Figure A-8 Reactor Vessel Fluid Temperature Upper Head 
AP1000 Test Data Oregon State University Test DBA-05 May 13, 2004

$\longrightarrow$ TF -143

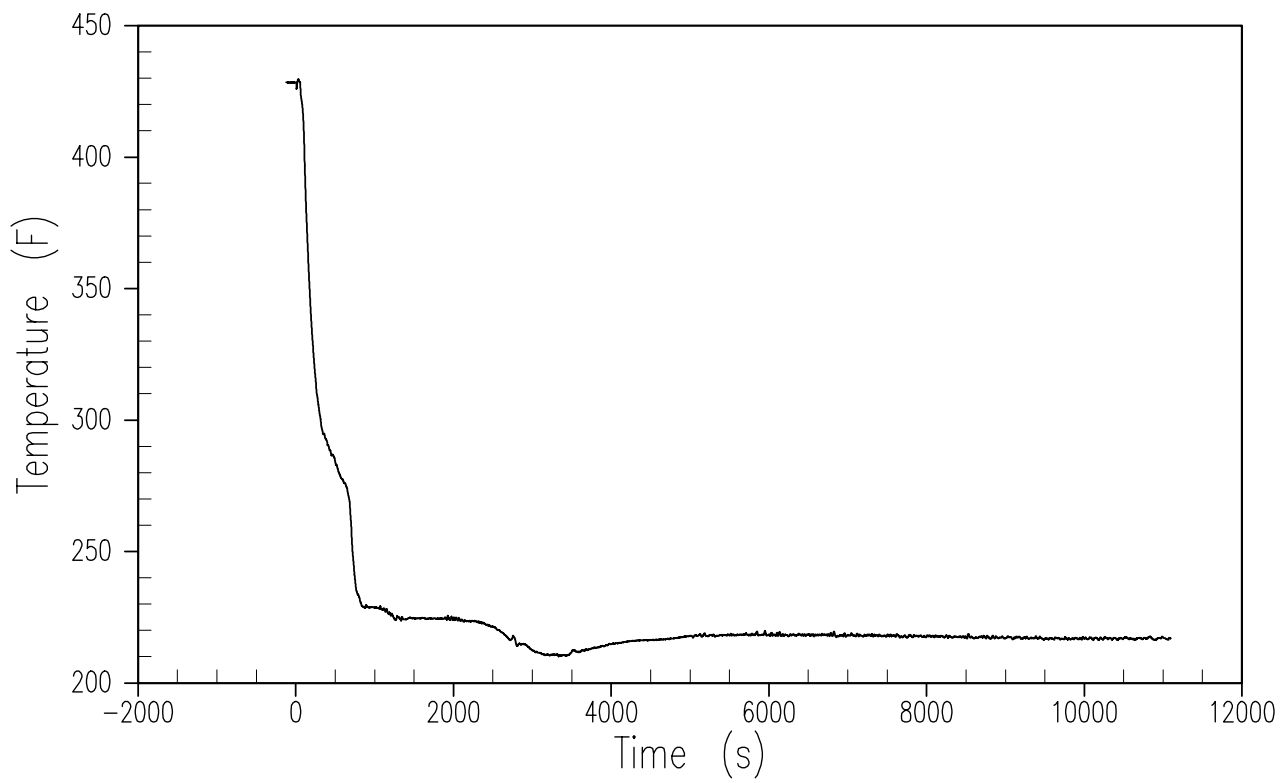

Figure A-9 Reactor Coolant System Hot Leg 1 Temperature 
AP1000 Test Data Oregon State University Test DBA-05 May 13, 2004

$\longrightarrow$ TF -142

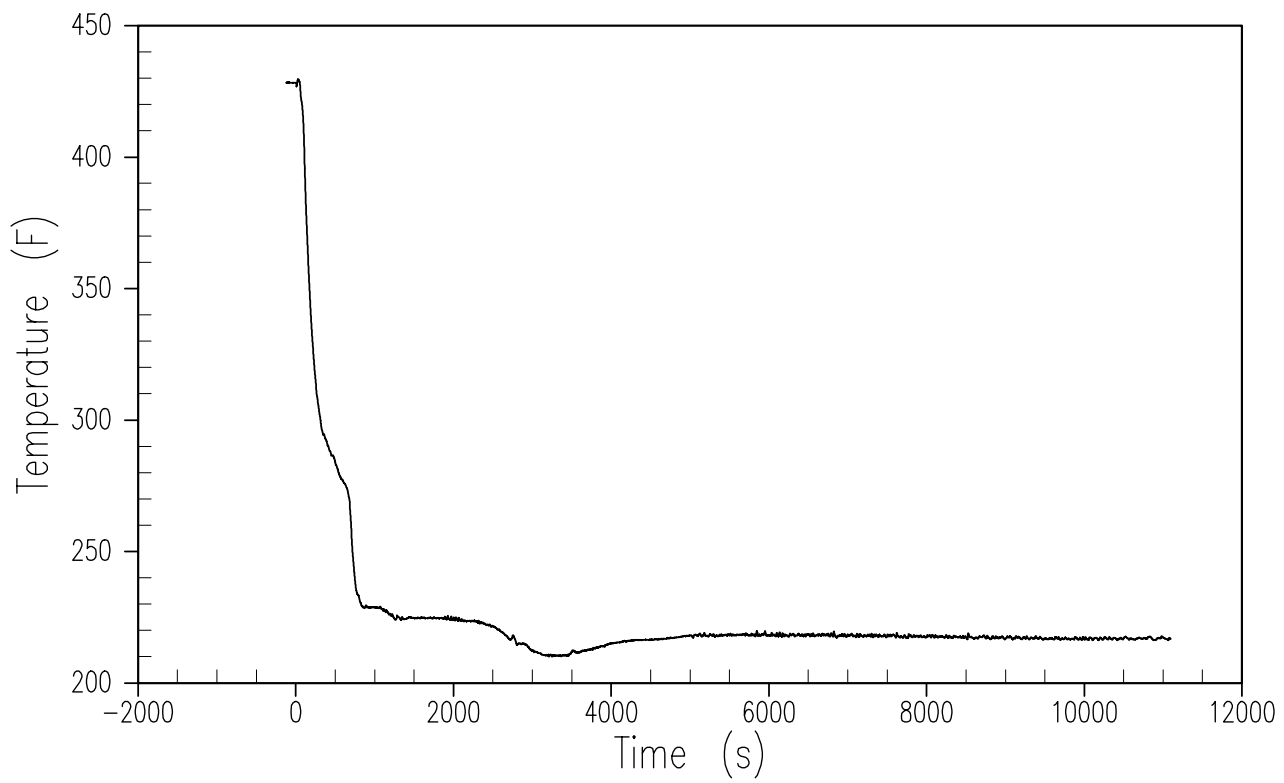

Figure A-10 Reactor Coolant System Hot Leg 2 Temperature 

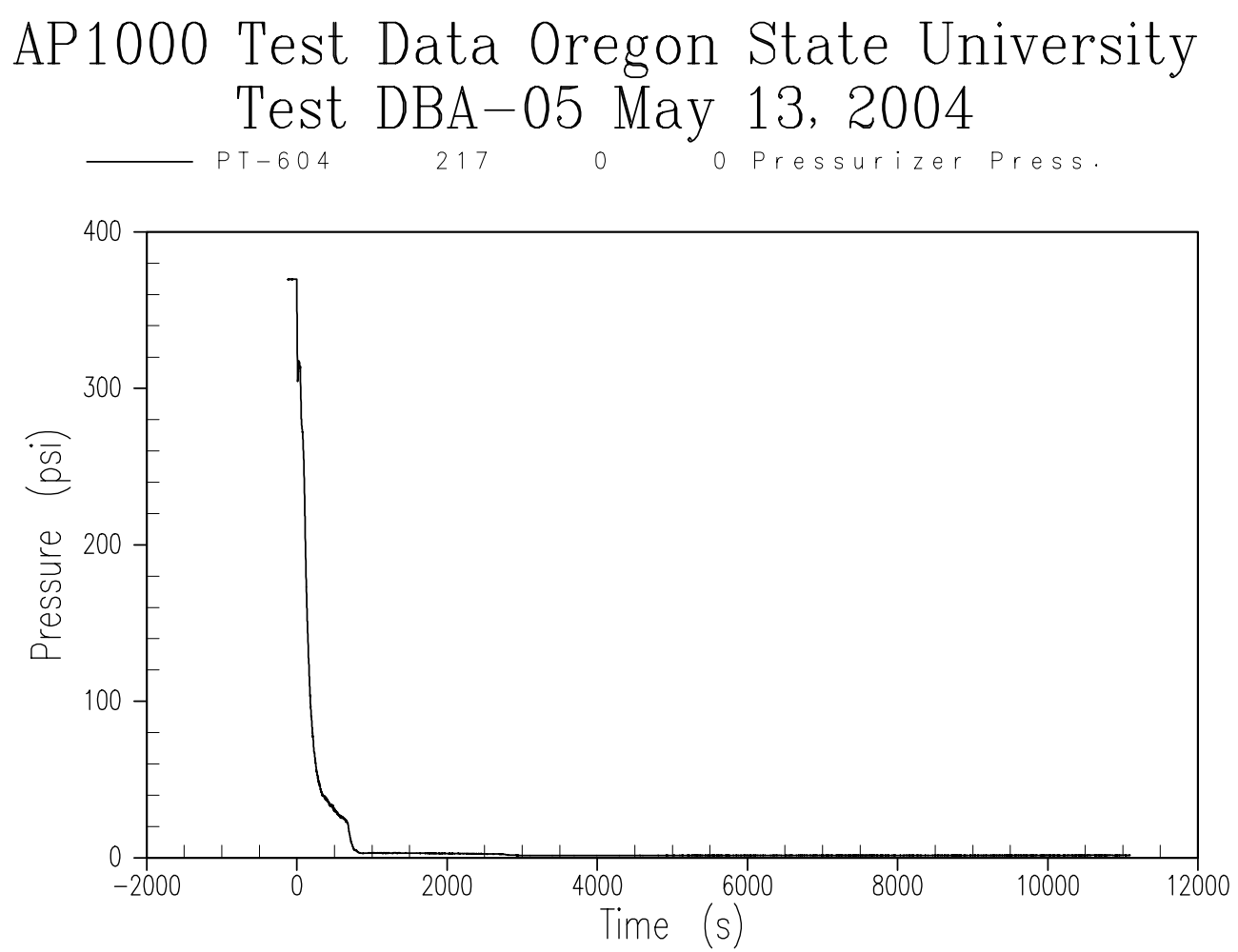

Figure A-11 Pressurizer Pressure - Wide Range 

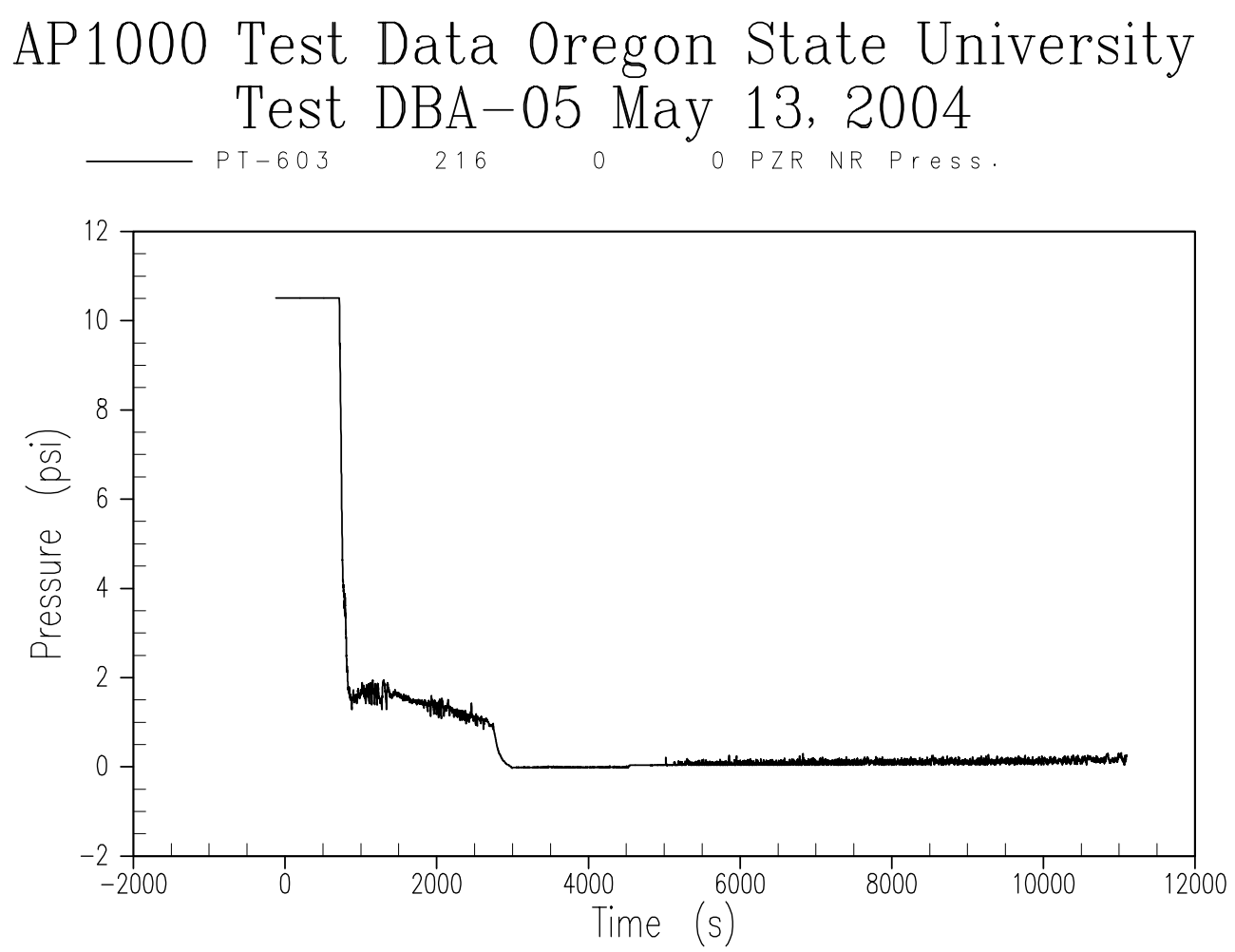

Figure A-12 Pressurizer Pressure - Narrow Range 

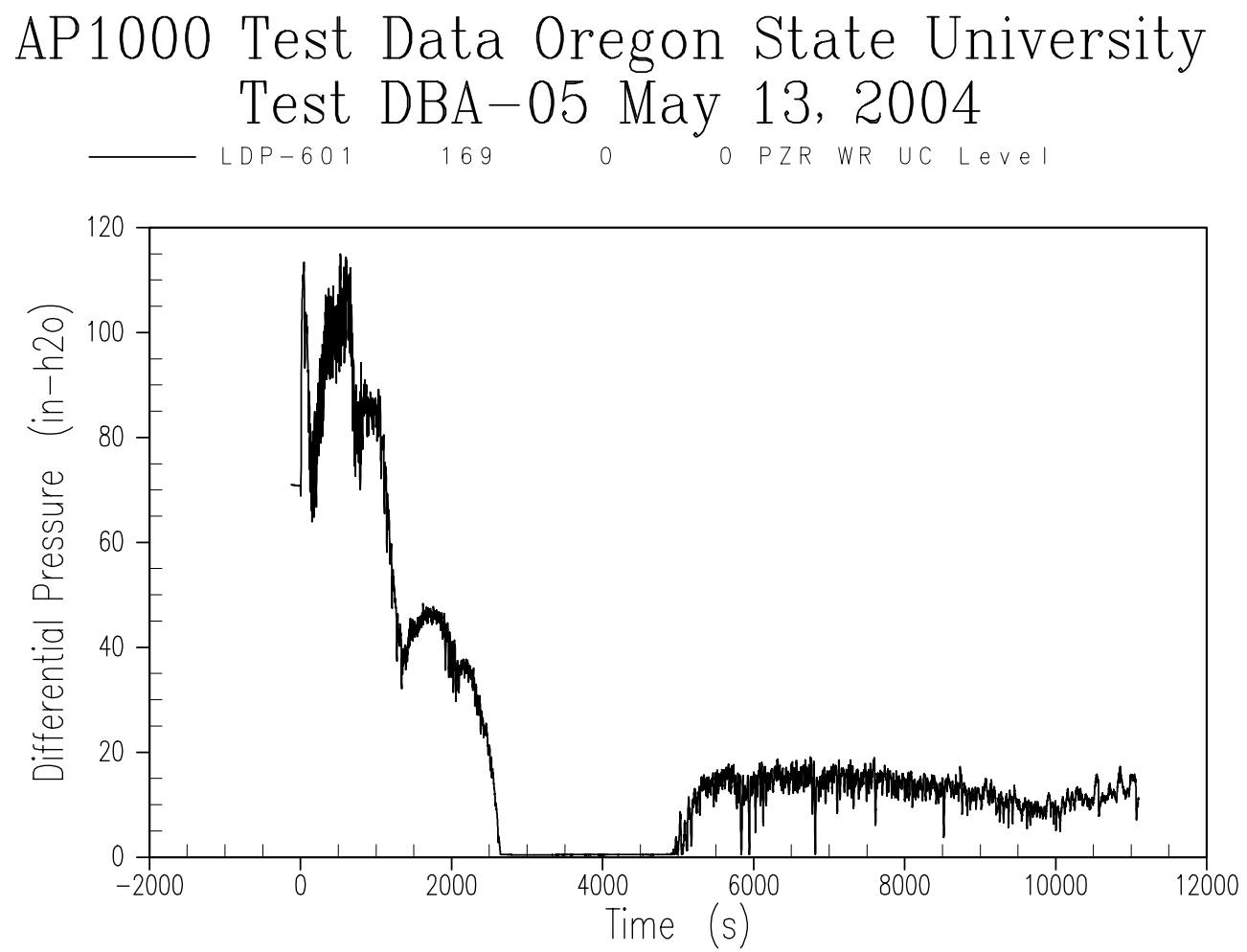

Figure A-13 Pressurizer Liquid Level 
AP1000 Test Data Oregon State University Test DBA-05 May 13, 2004

$\longrightarrow$ LDP $-215 \quad 145$

o SG-1 LTube HL UC Lev

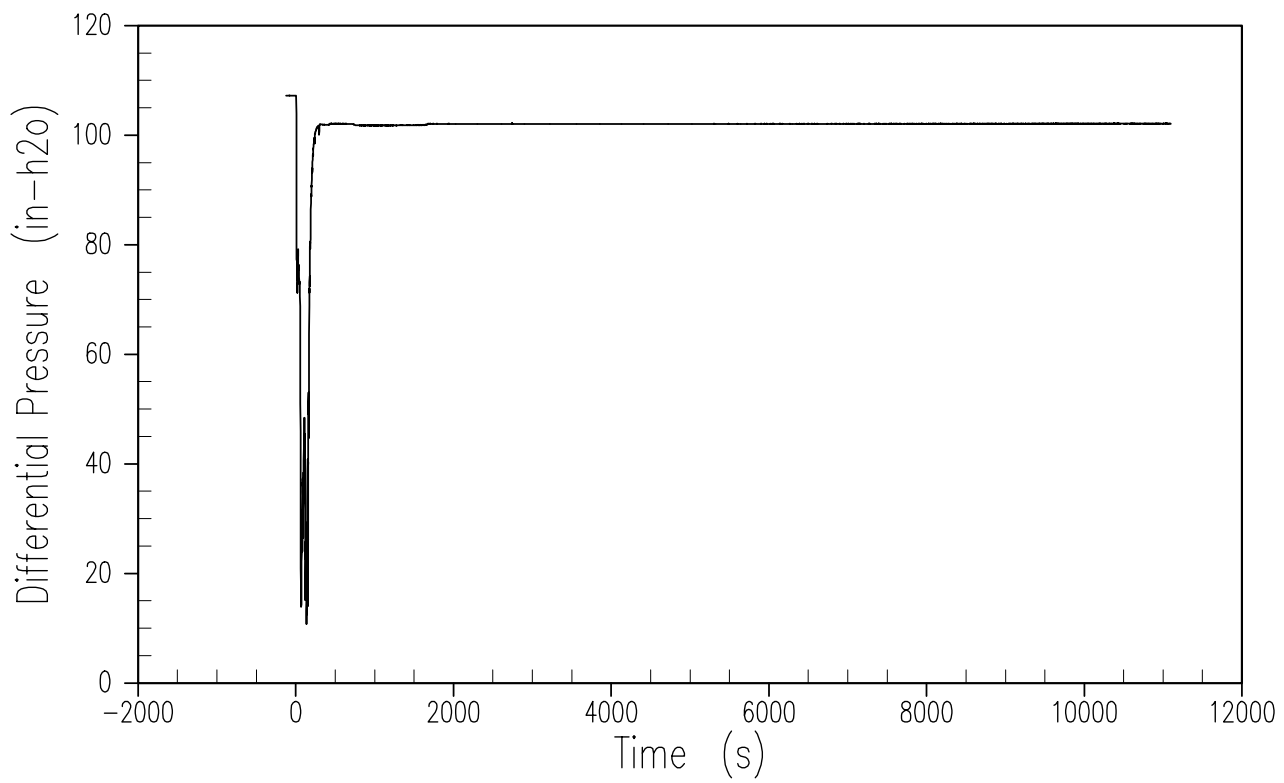

Figure A-14 Steam Generator 1 Tube Level 

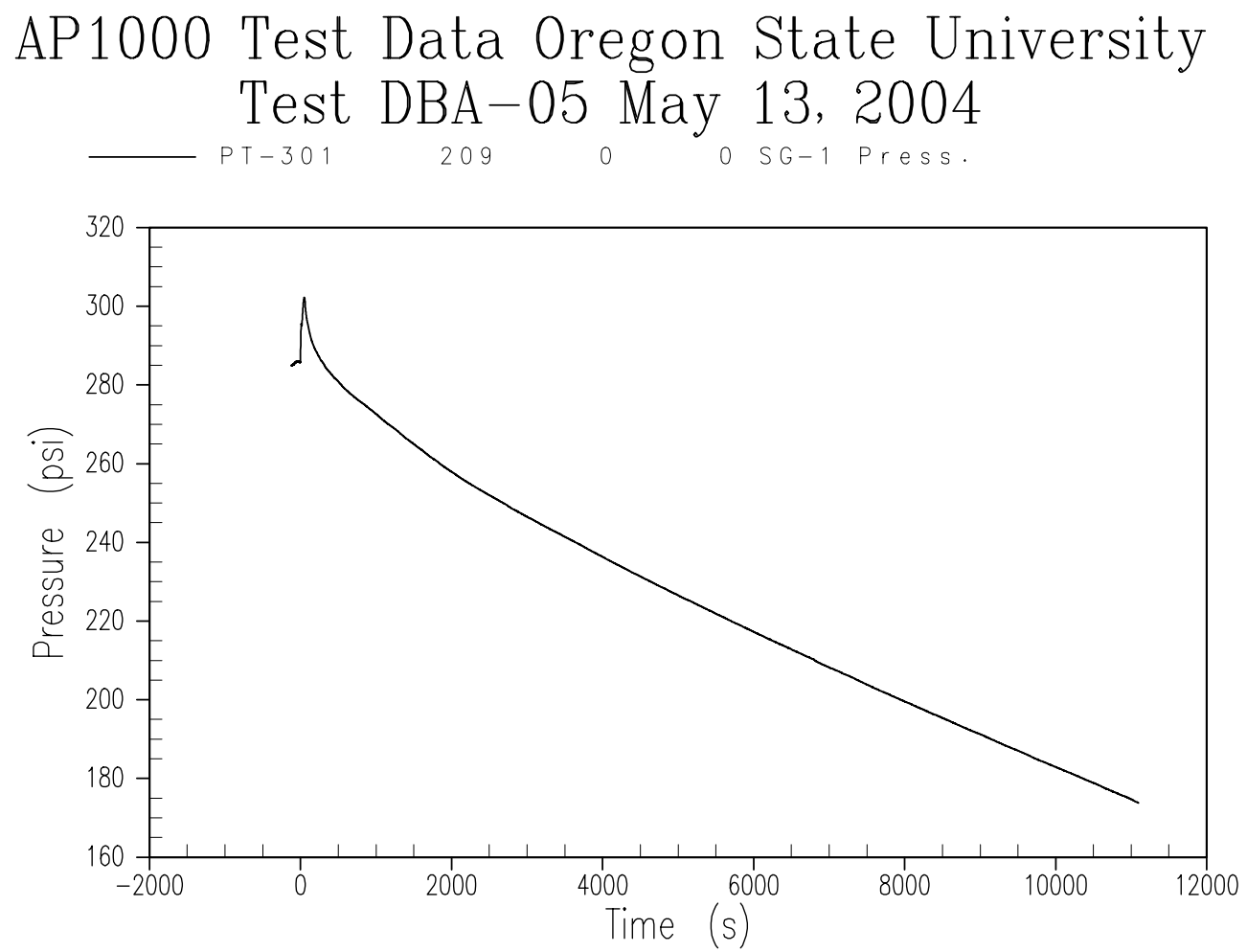

Figure A-15 Steam Generator 1 Secondary Pressure 

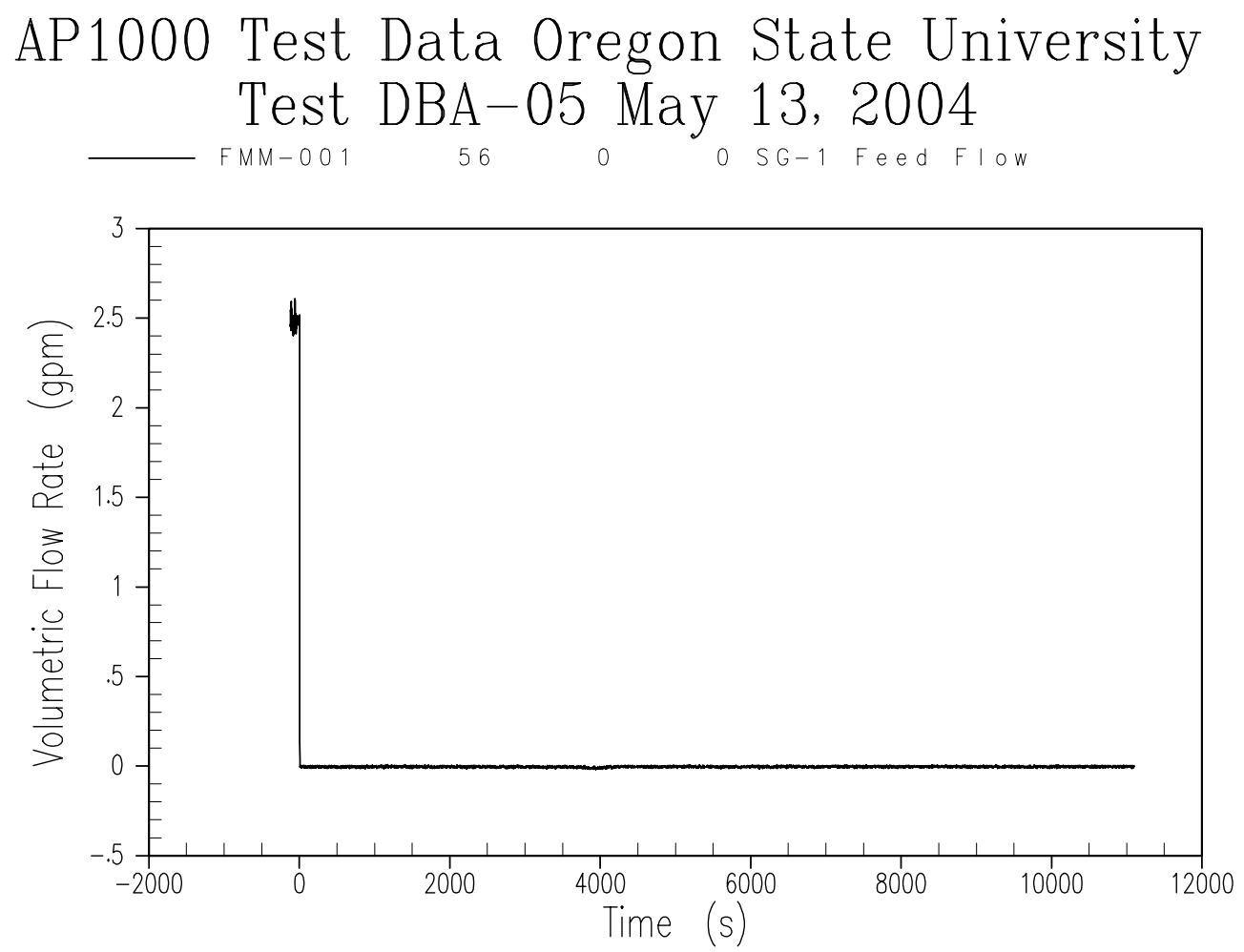

Figure A-16 Steam Generator 1 Feed Flow Rate 
AP1000 Test Data Oregon State University Test DBA-05 May 13, 2004

$\longrightarrow$ LDP $-218 \quad 148$

o SG-2 LTube HL UC Lev

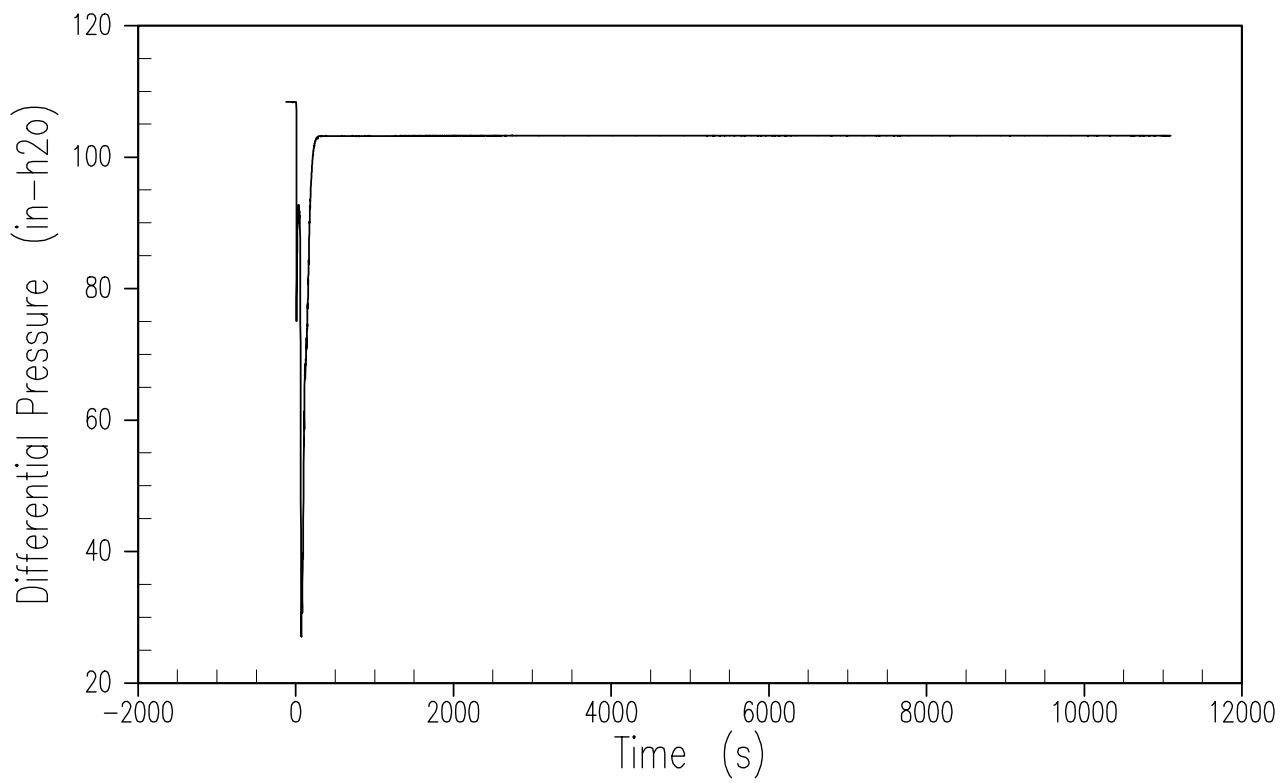

Figure A-17 Steam Generator 2 Tube Level 

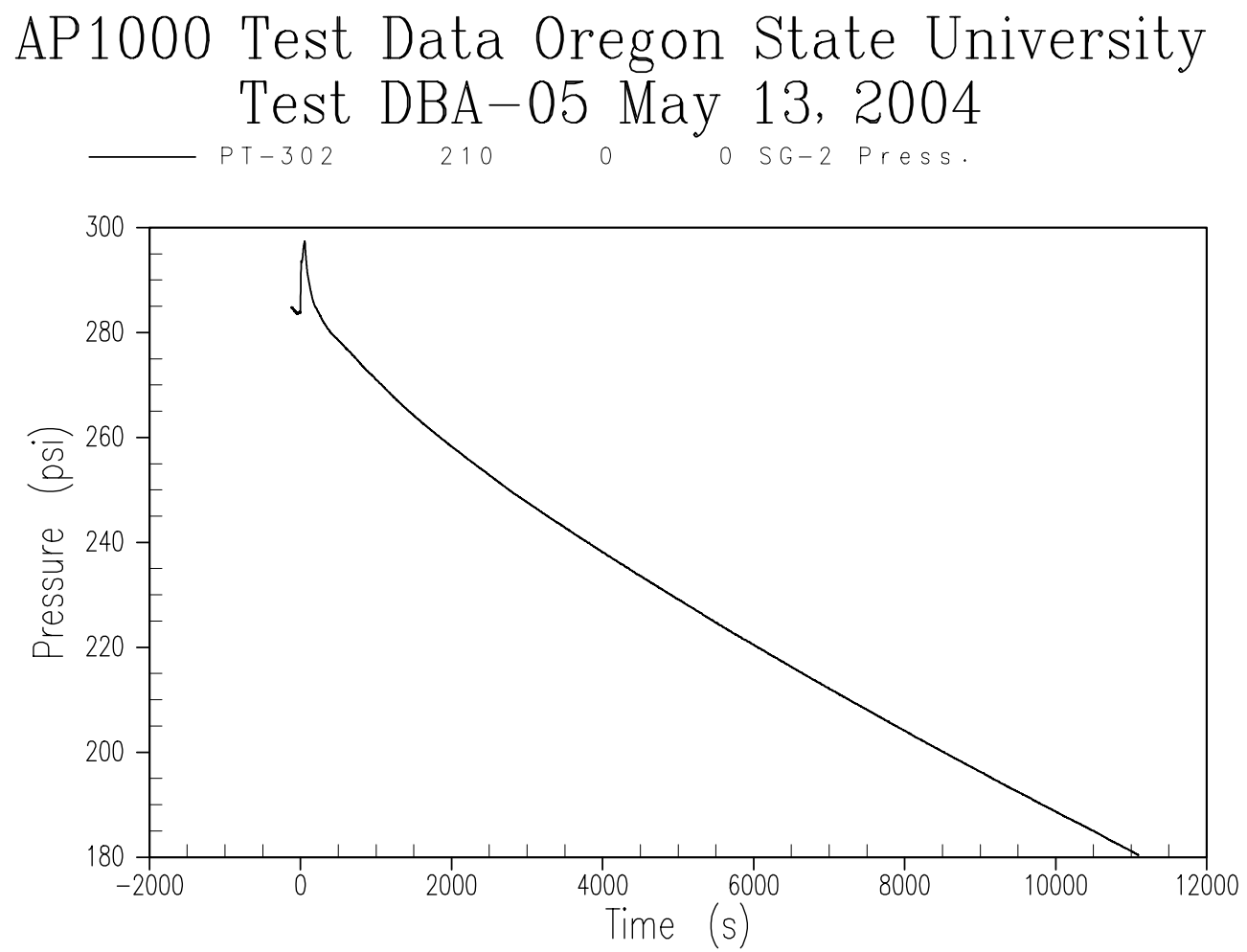

Figure A-18 Steam Generator 2 Secondary Pressure 

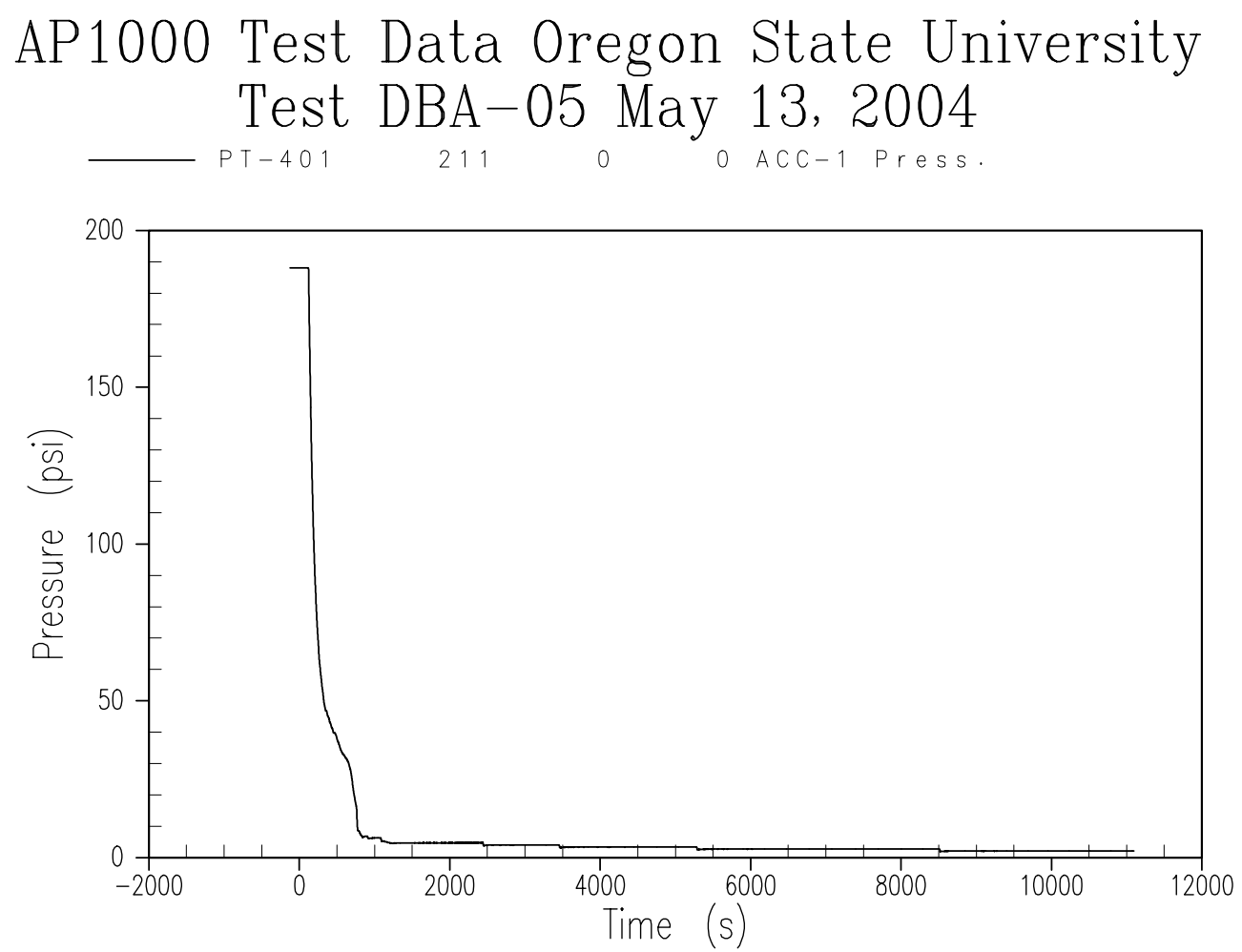

Figure A-19 Accumulator 1 Pressure 

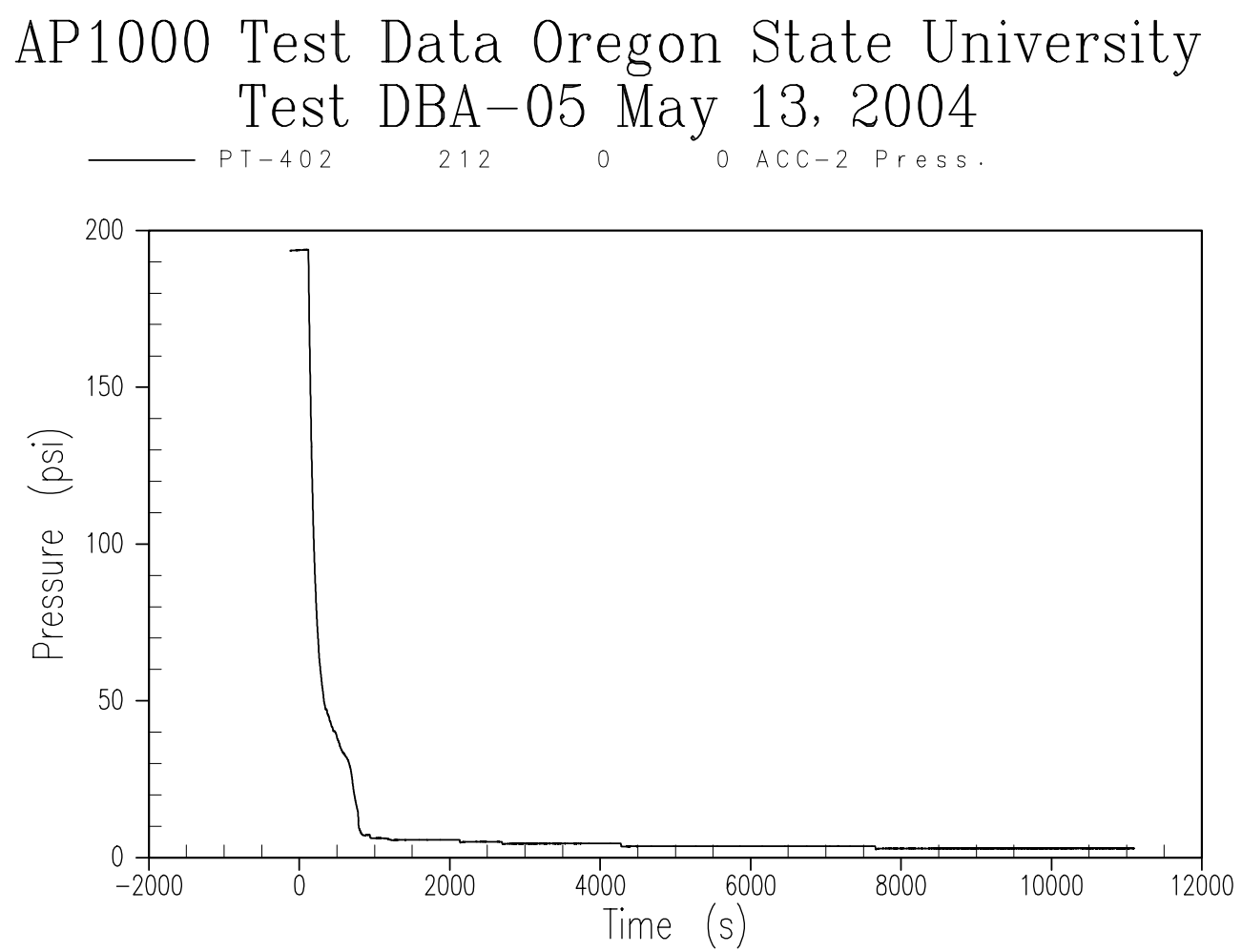

Figure A-20 Accumulator 2 Pressure 

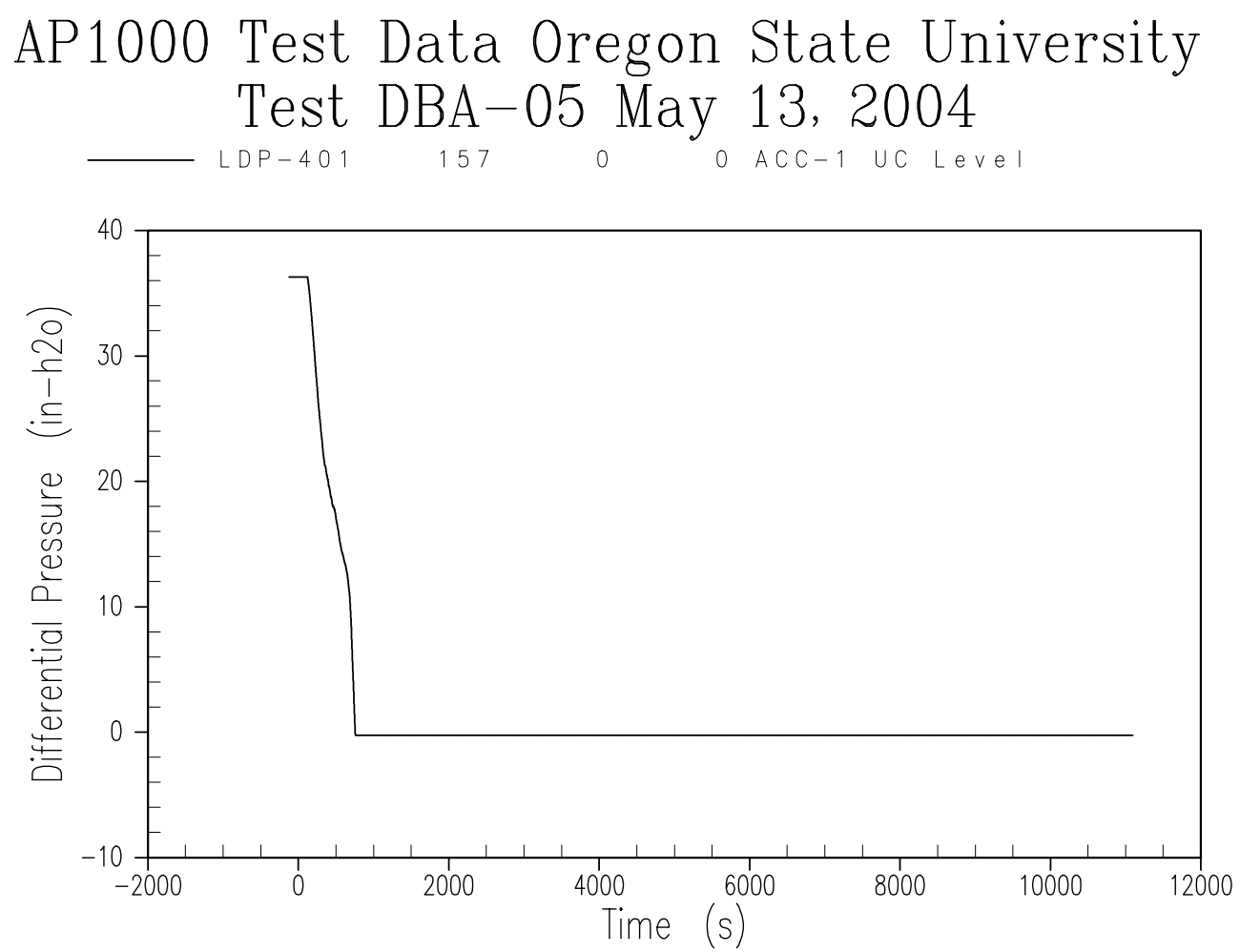

Figure A-21 Accumulator 1 Liquid Level 

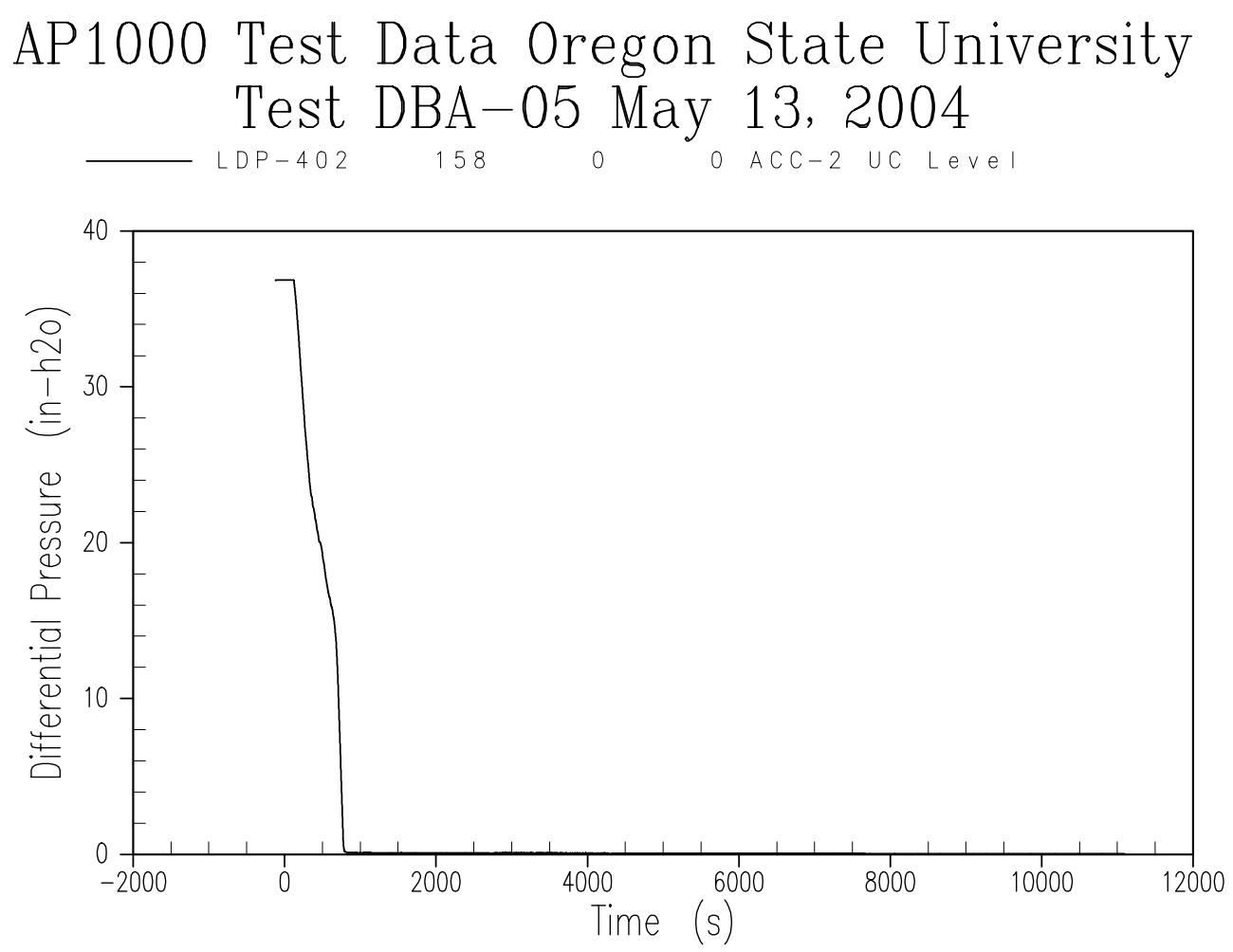

Figure A-22 Accumulator 2 Liquid Level 

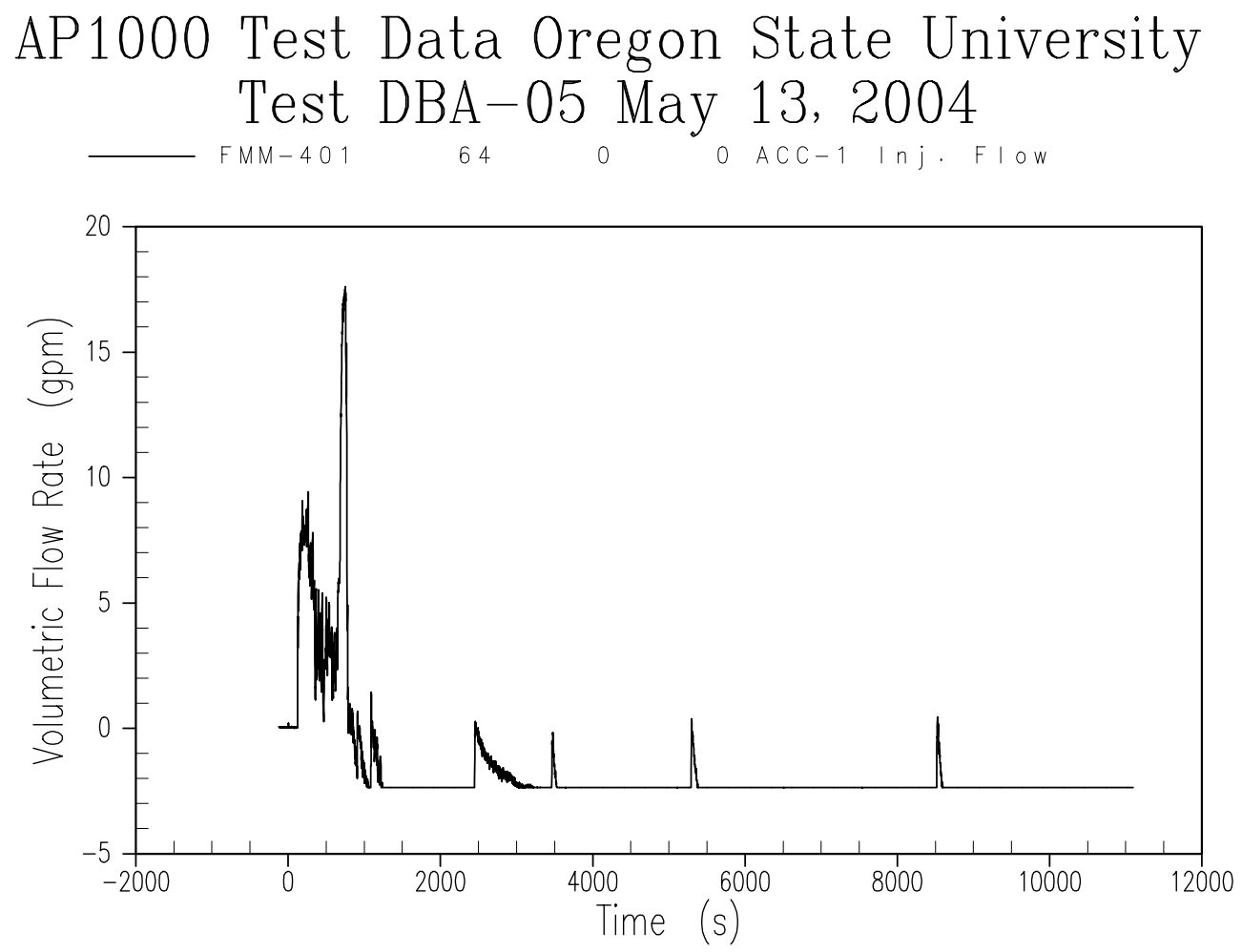

Figure A-23 Accumulator 1 Flow Rate 

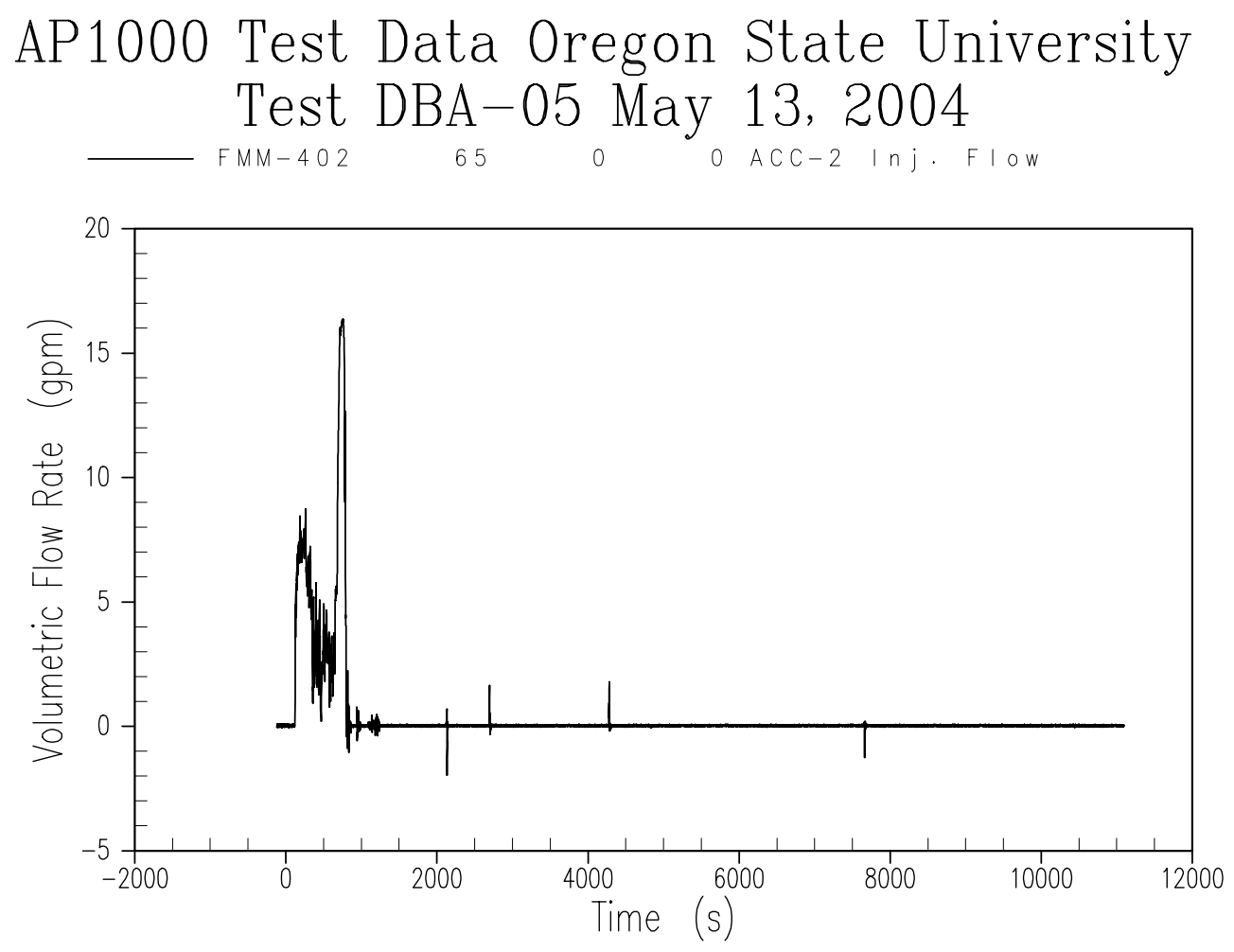

Figure A-24 Accumulator 2 Flow Rate 

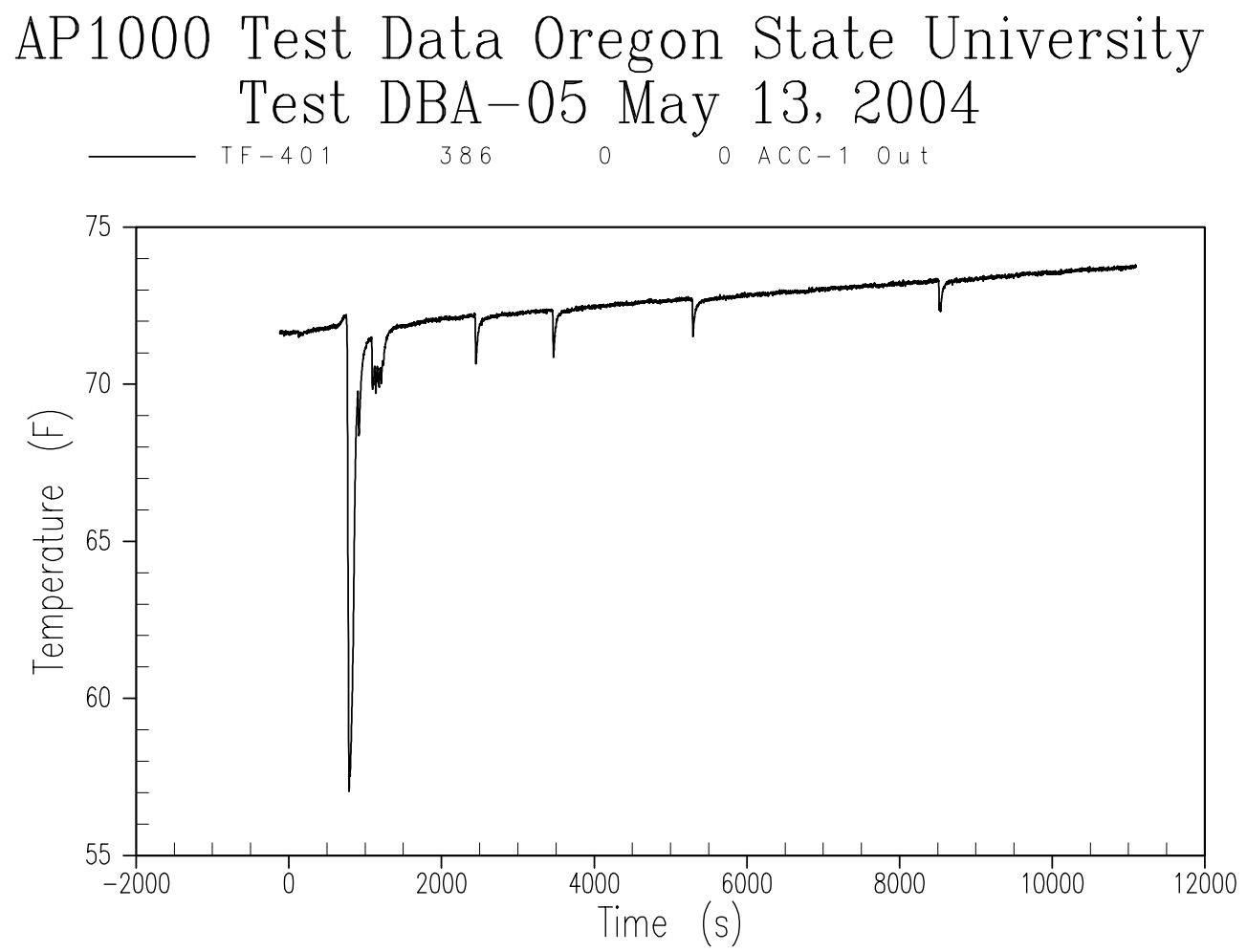

Figure A-25 Accumulator 1 Liquid Discharge Temperature 

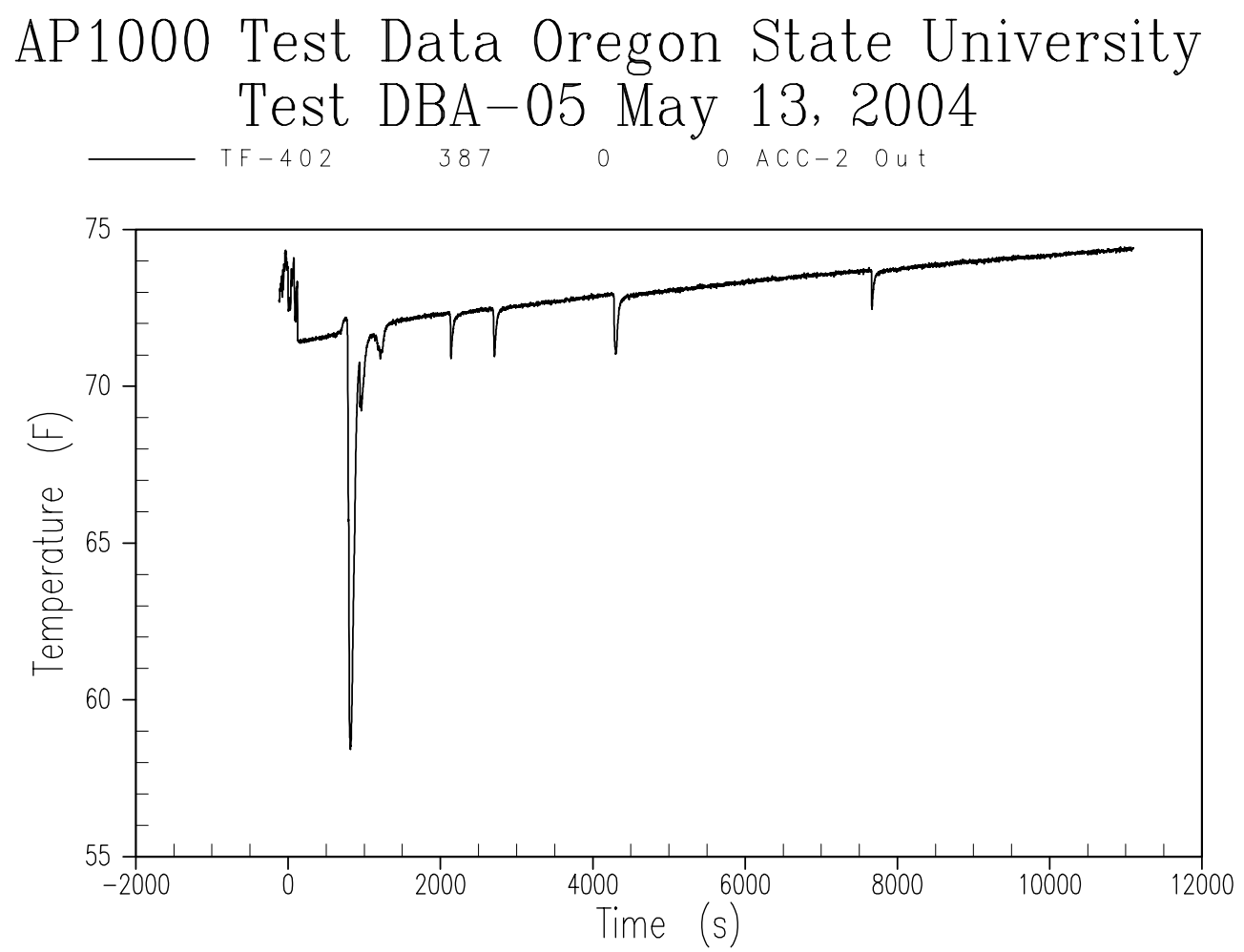

Figure A-26 Accumulator 2 Liquid Discharge Temperature 
AP1000 Test Data Oregon State University Test DBA-05 May 13, 2004

\section{$\longrightarrow$}

LDP-507 165

o CMT-1 WR UC Level

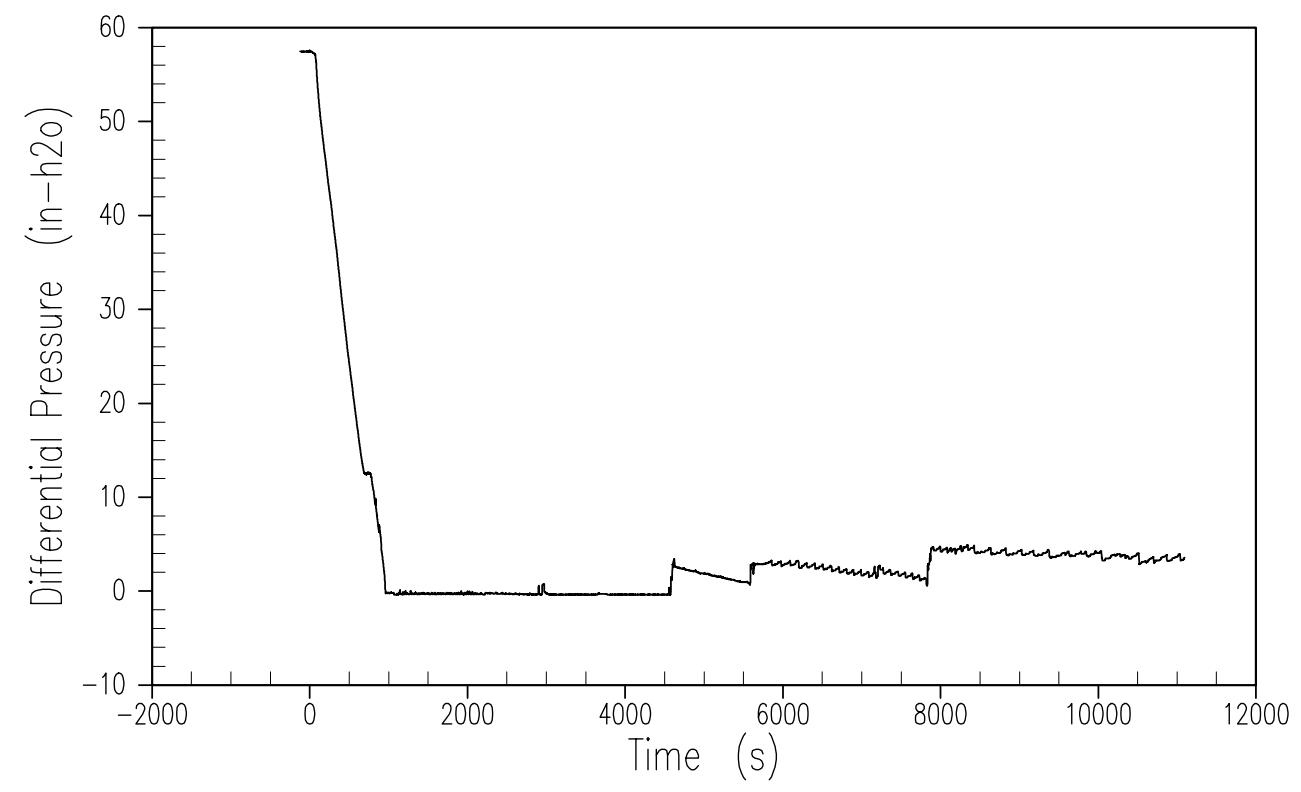

Figure A-27 Core Makeup Tank 1 Liquid Level 
AP1000 Test Data Oregon State University Test DBA-05 May 13, 2004

$\longrightarrow$ LDP-502 160

o CMT-2 WR UC Level

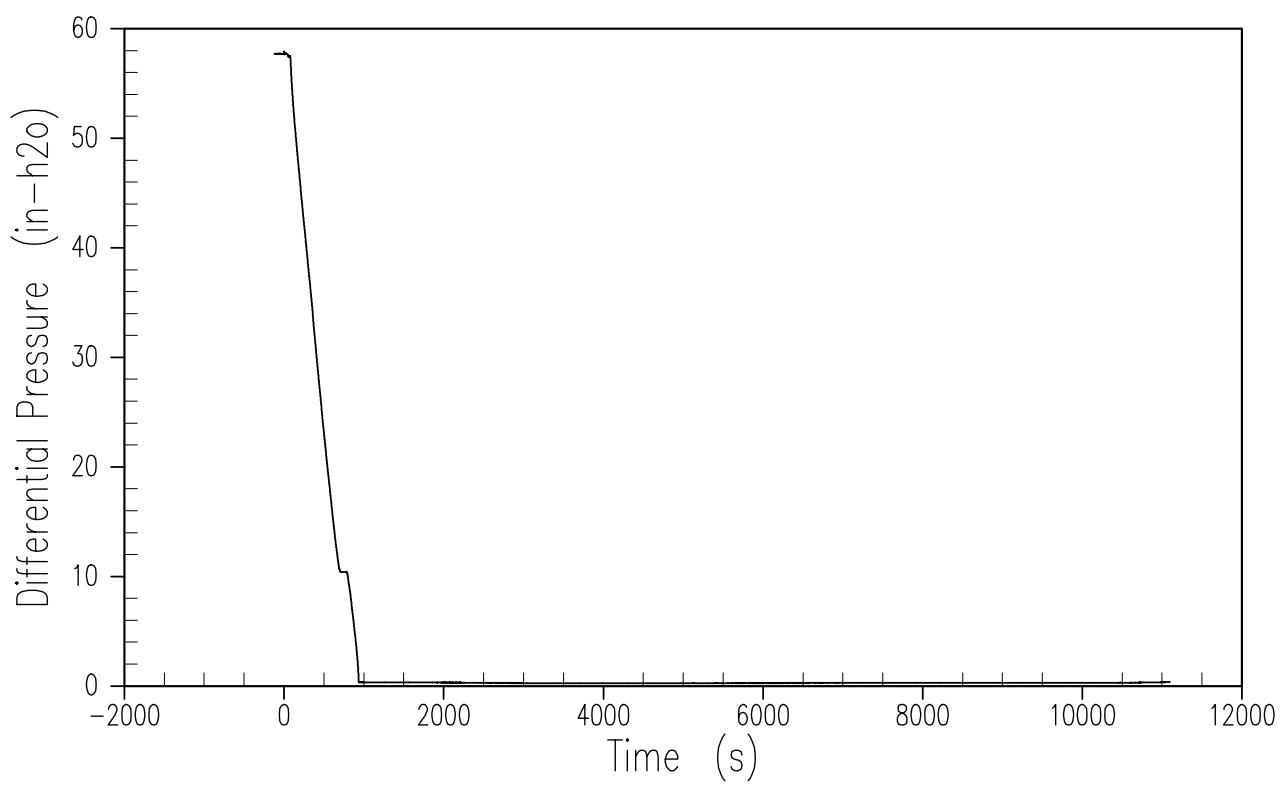

Figure A-28 Core Makeup Tank 2 Liquid Level 

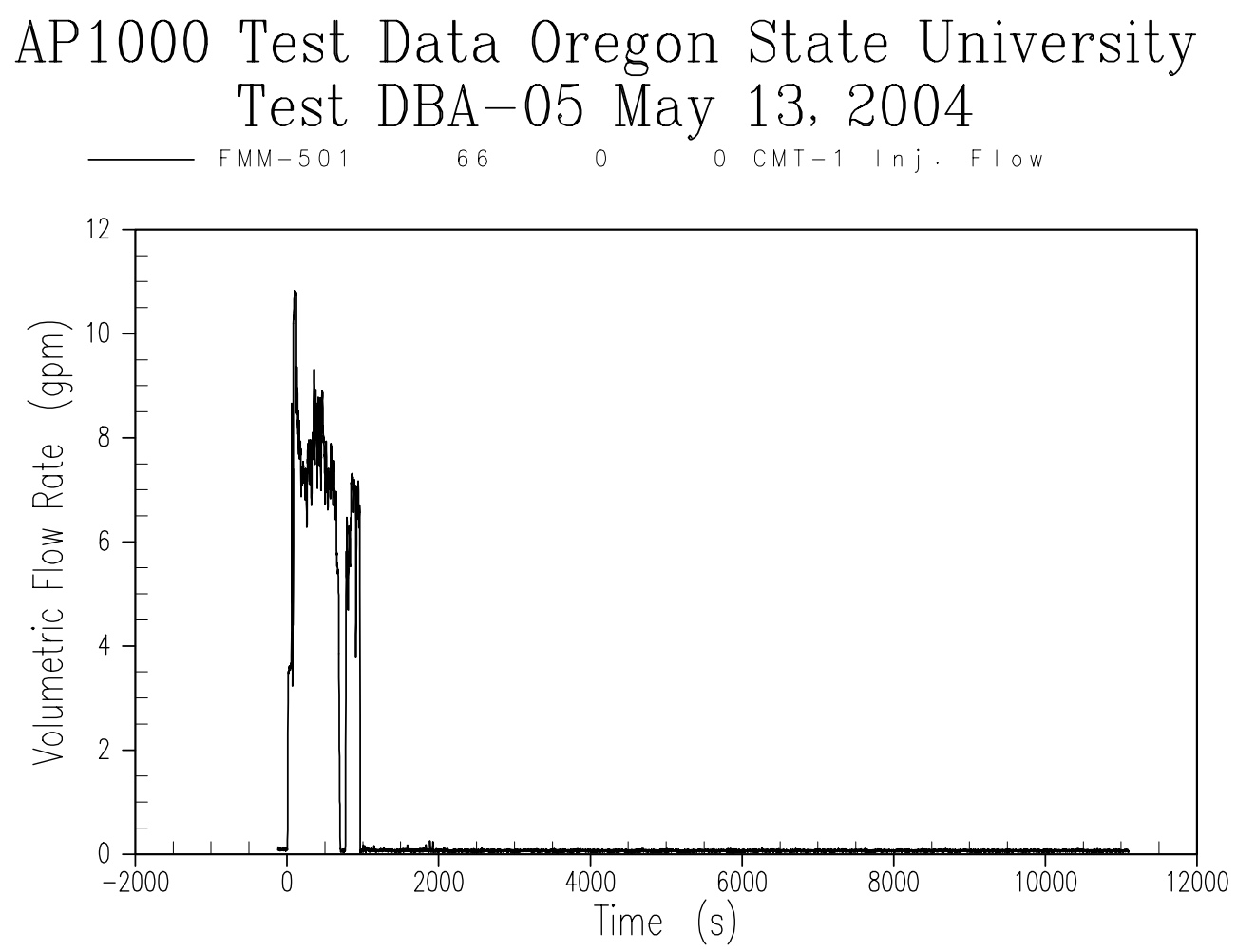

Figure A-29 Core Makeup Tank 1 Flow Rate 

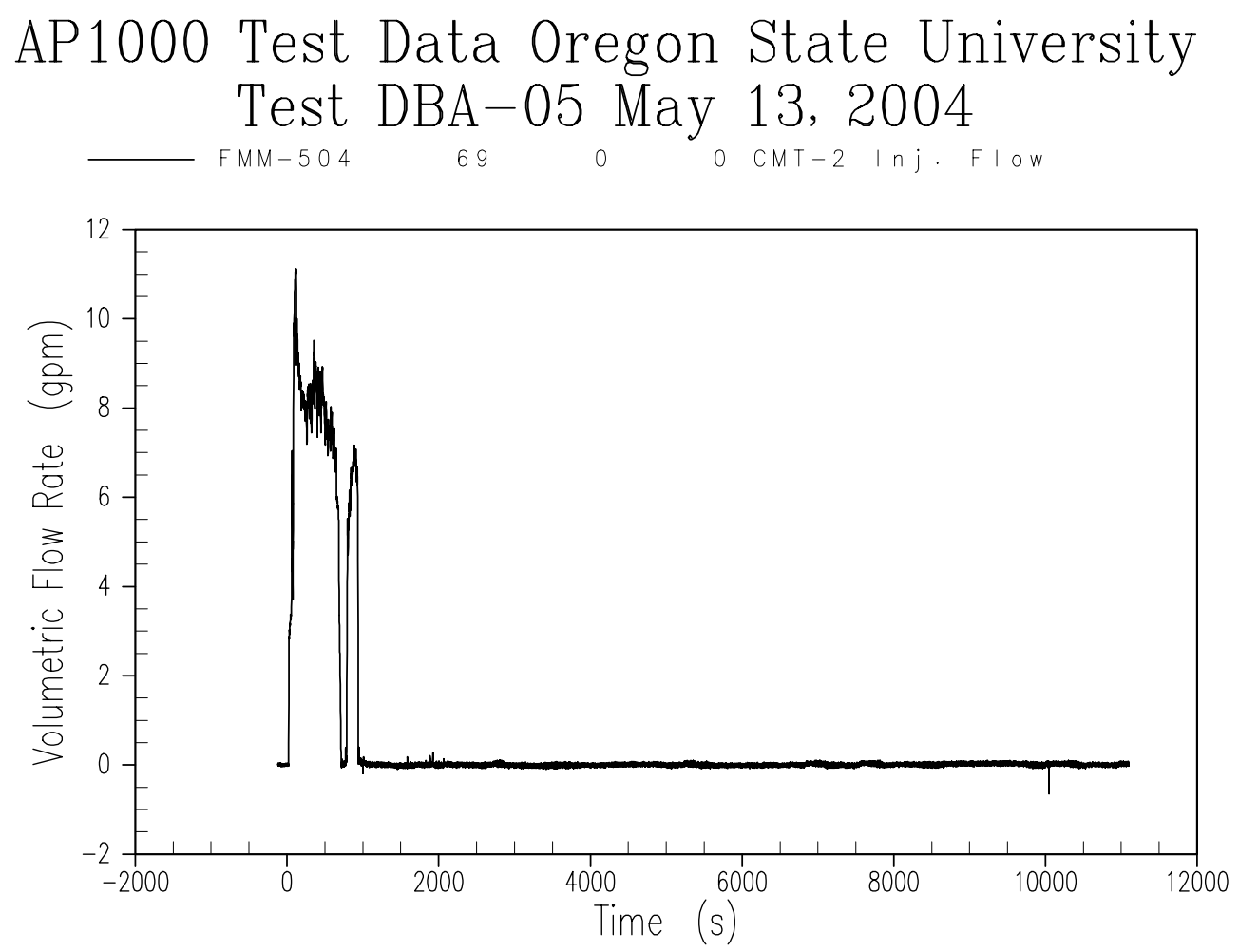

Figure A-30 Core Makeup Tank 2 Flow Rate 
AP1000 Test Data Oregon State University Test DBA-05 May 13, 2004

- TF-501 392

o CMT-1 LONg TC @ 0.30

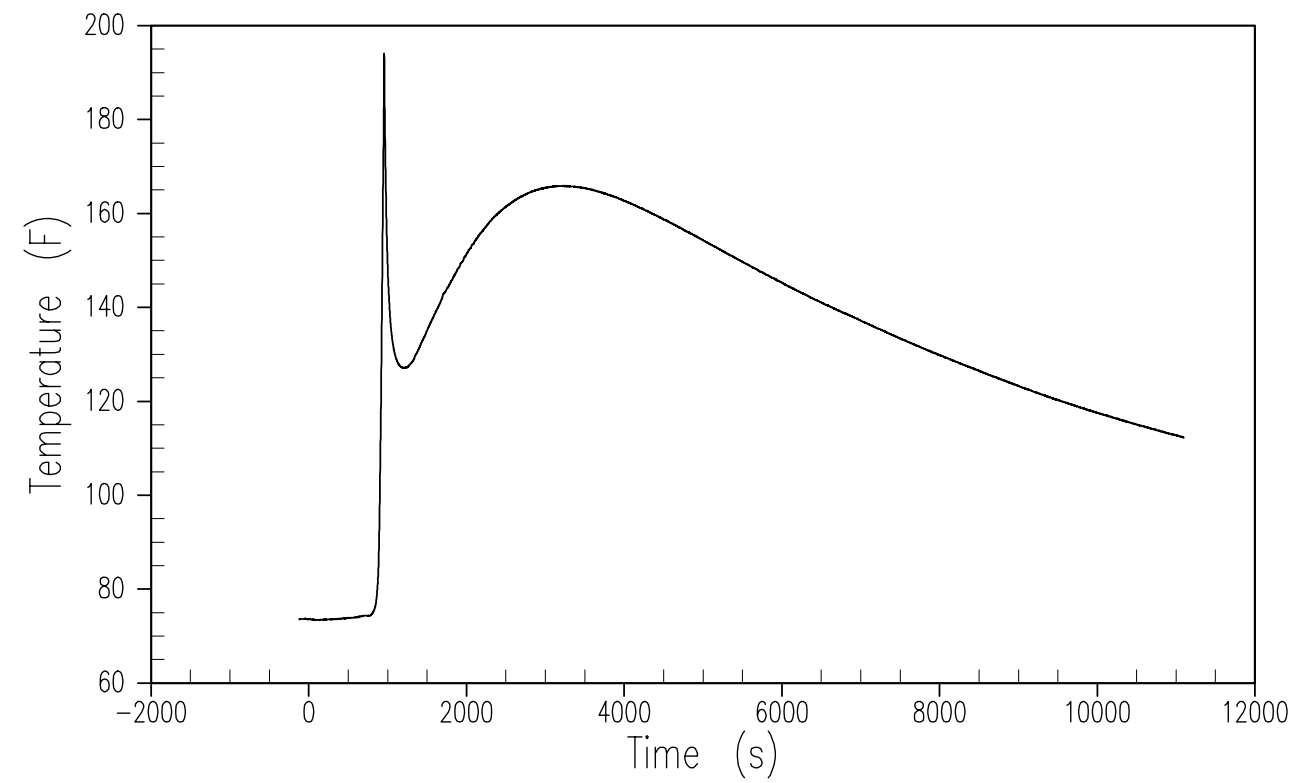

Figure A-31 Core Makeup Tank 1 Liquid Temperature - Bottom 
AP1000 Test Data Oregon State University Test DBA-05 May 13, 2004

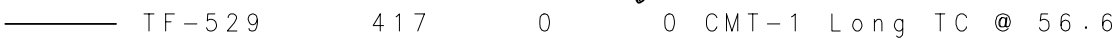

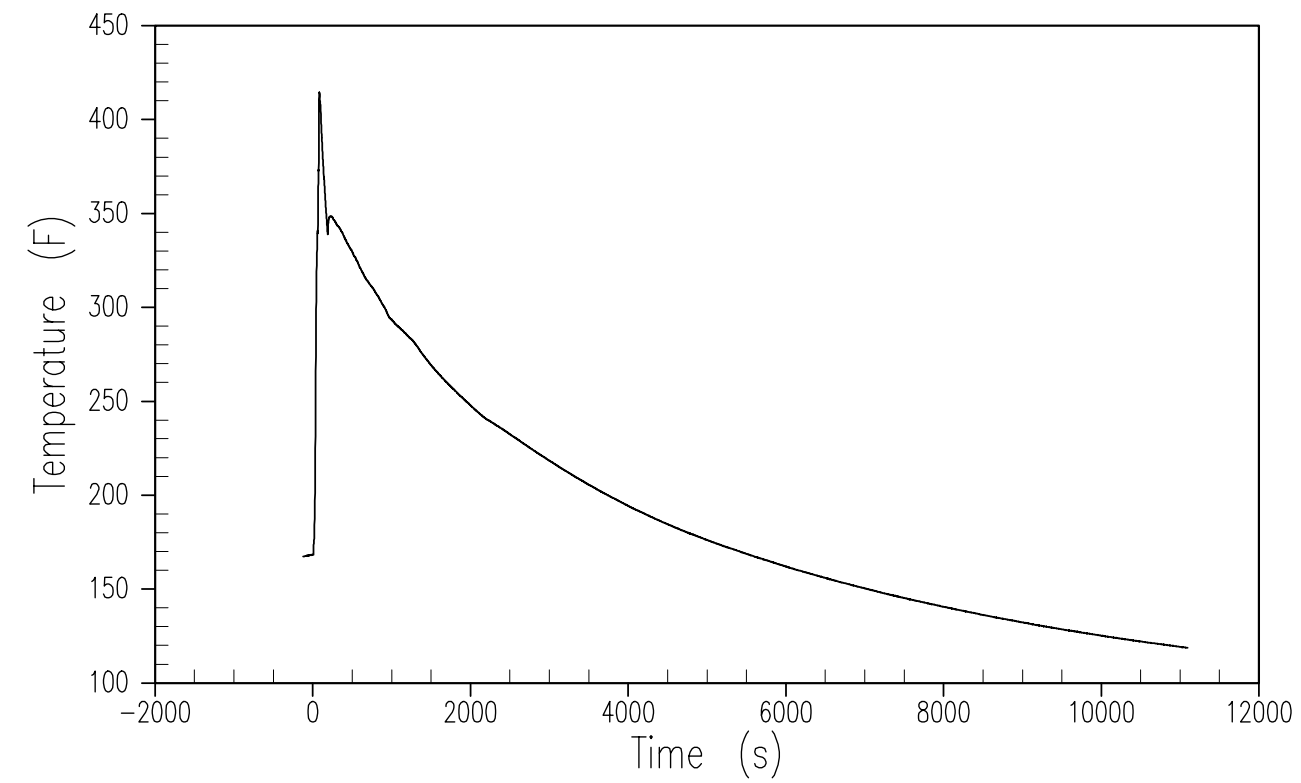

Figure A-32 Core Makeup Tank 1 Liquid Temperature - Top 
AP1000 Test Data Oregon State University Test DBA-05 May 13, 2004

T TF-50

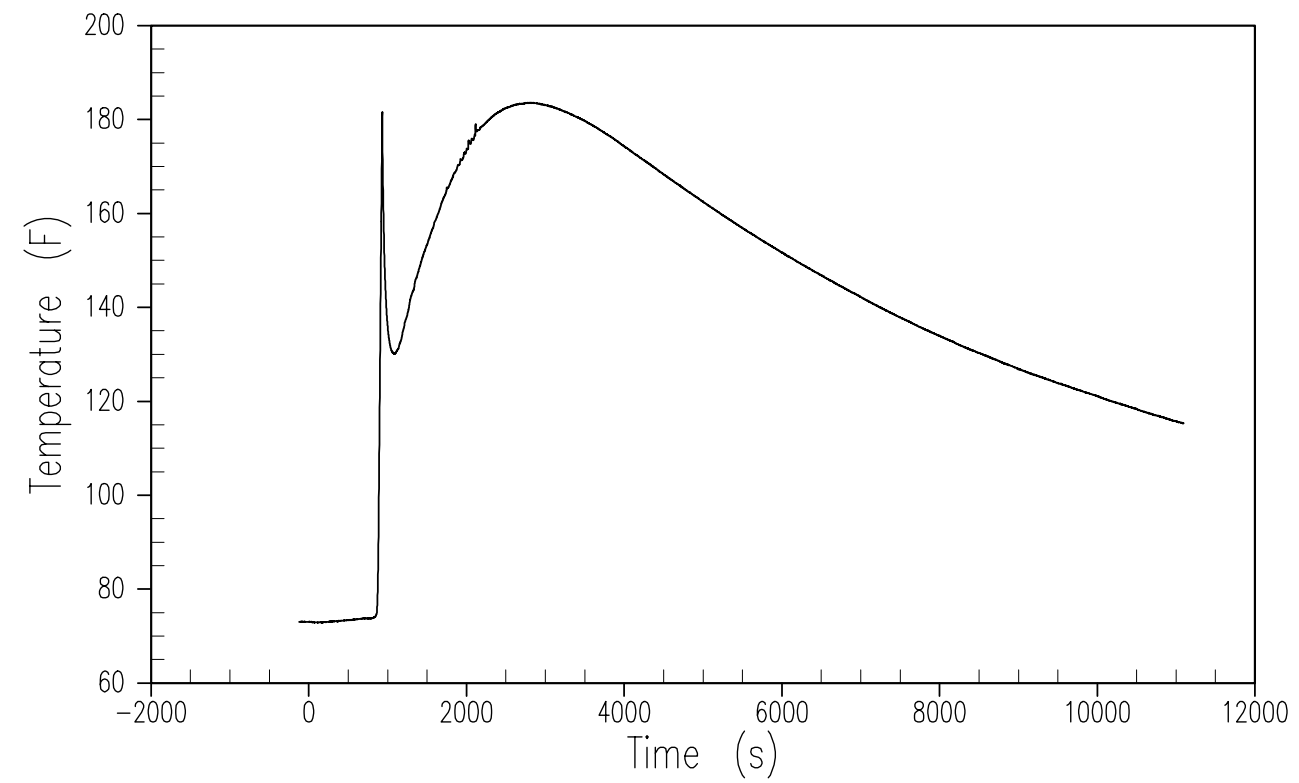

Figure A-33 Core Makeup Tank 2 Liquid Temperature - Bottom 
AP1000 Test Data Oregon State University Test DBA-05 May 13, 2004

$\longrightarrow$ TF-532 419

o CMT-2 Long TC @ 56.6

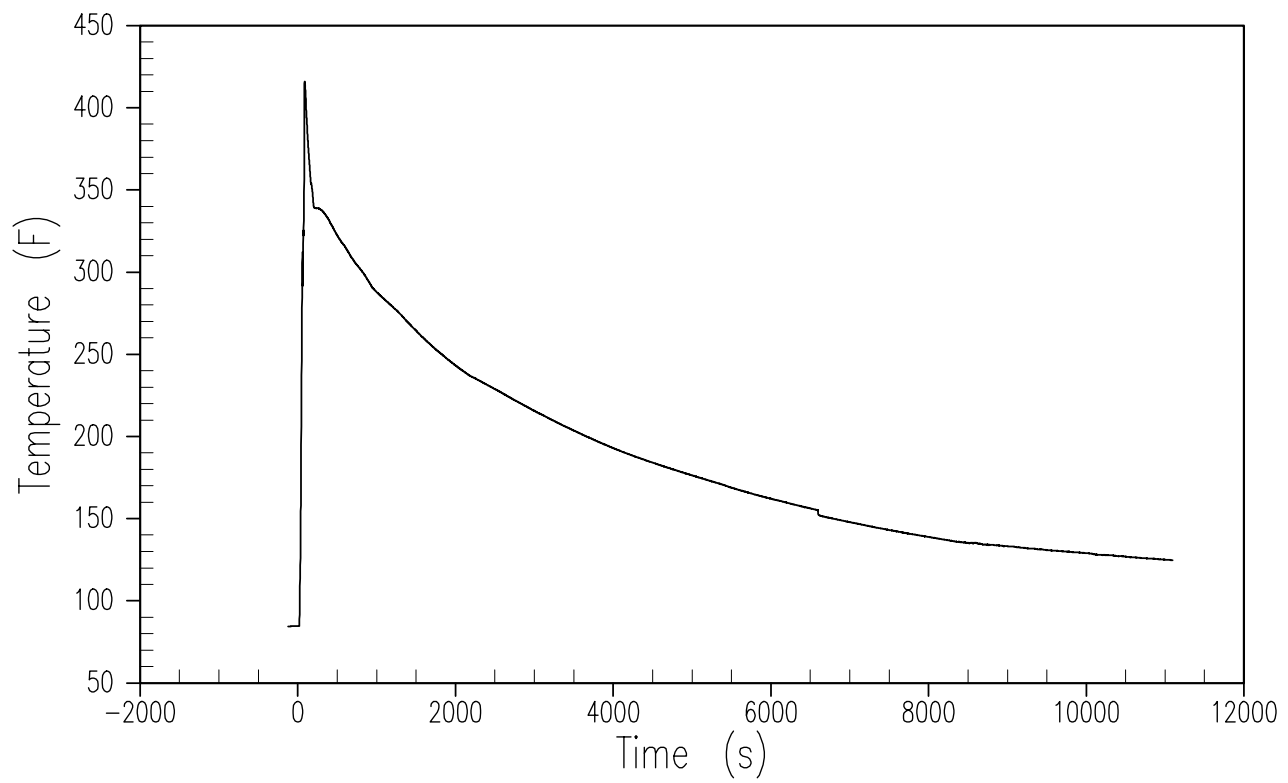

Figure A-34 Core Makeup Tank 2 Liquid Temperature - Top 


\section{AP1000 Test Data Oregon State University Test DBA-05 May 13, 2004}

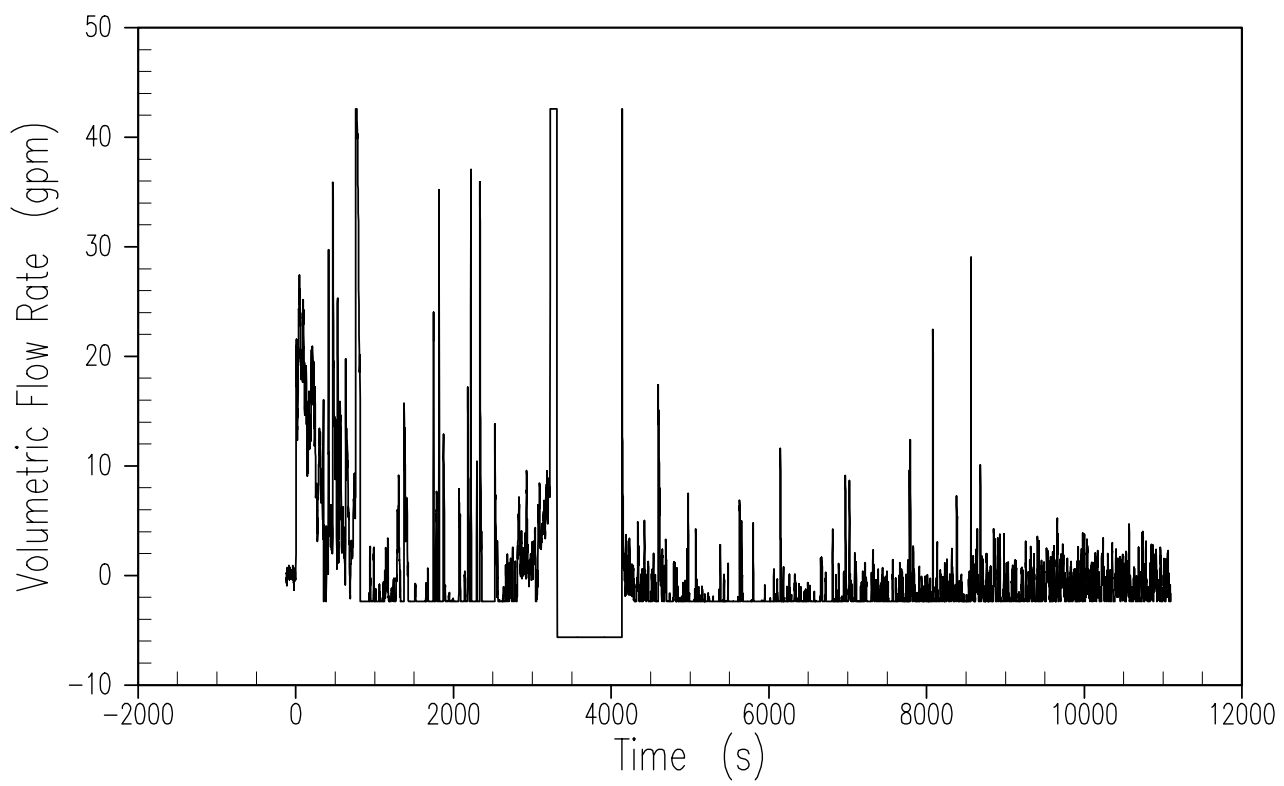

Figure A-35 Passive Residual Heat Removal Inlet Flow Rate 

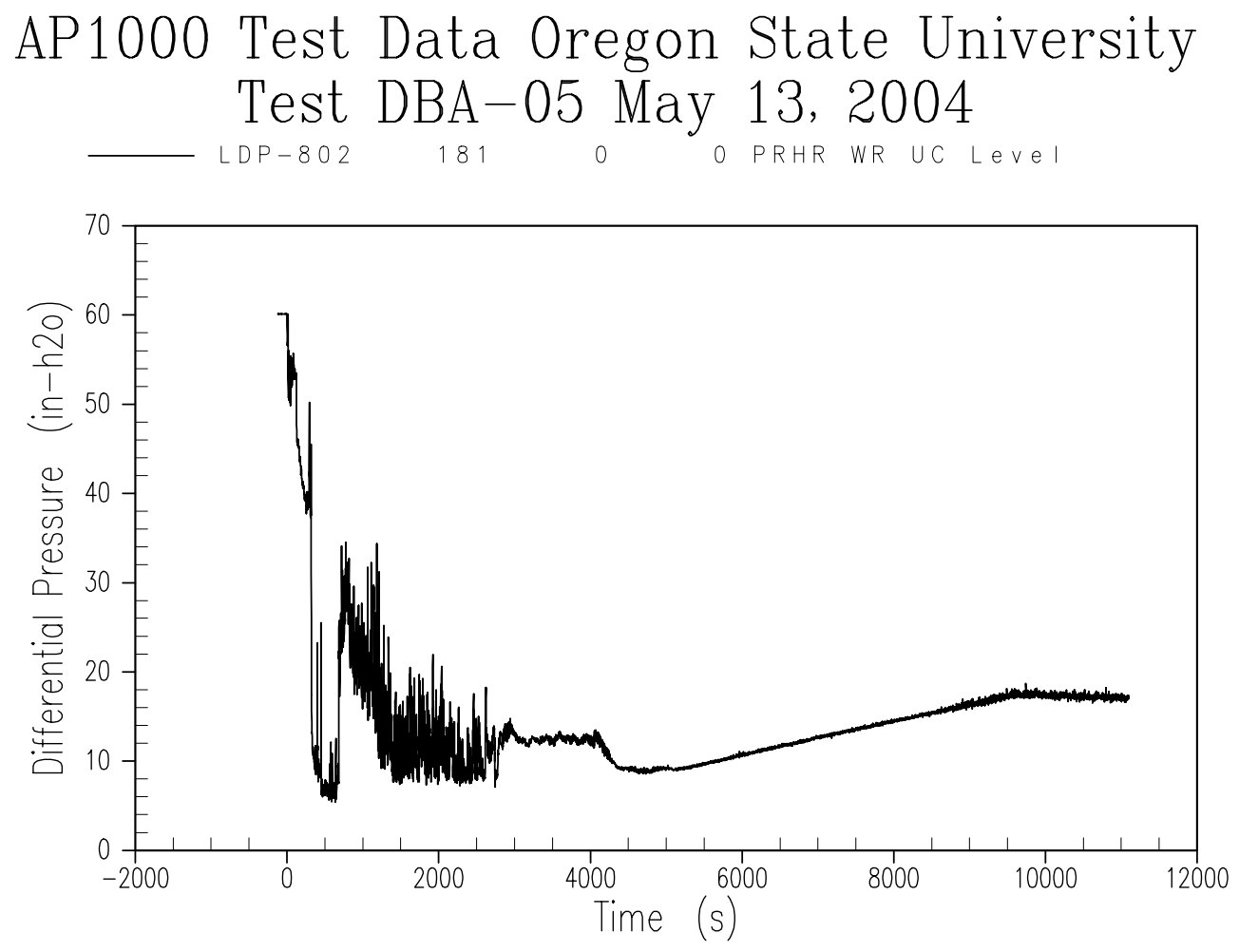

Figure A-36 Passive Residual Heat Removal Liquid Level 

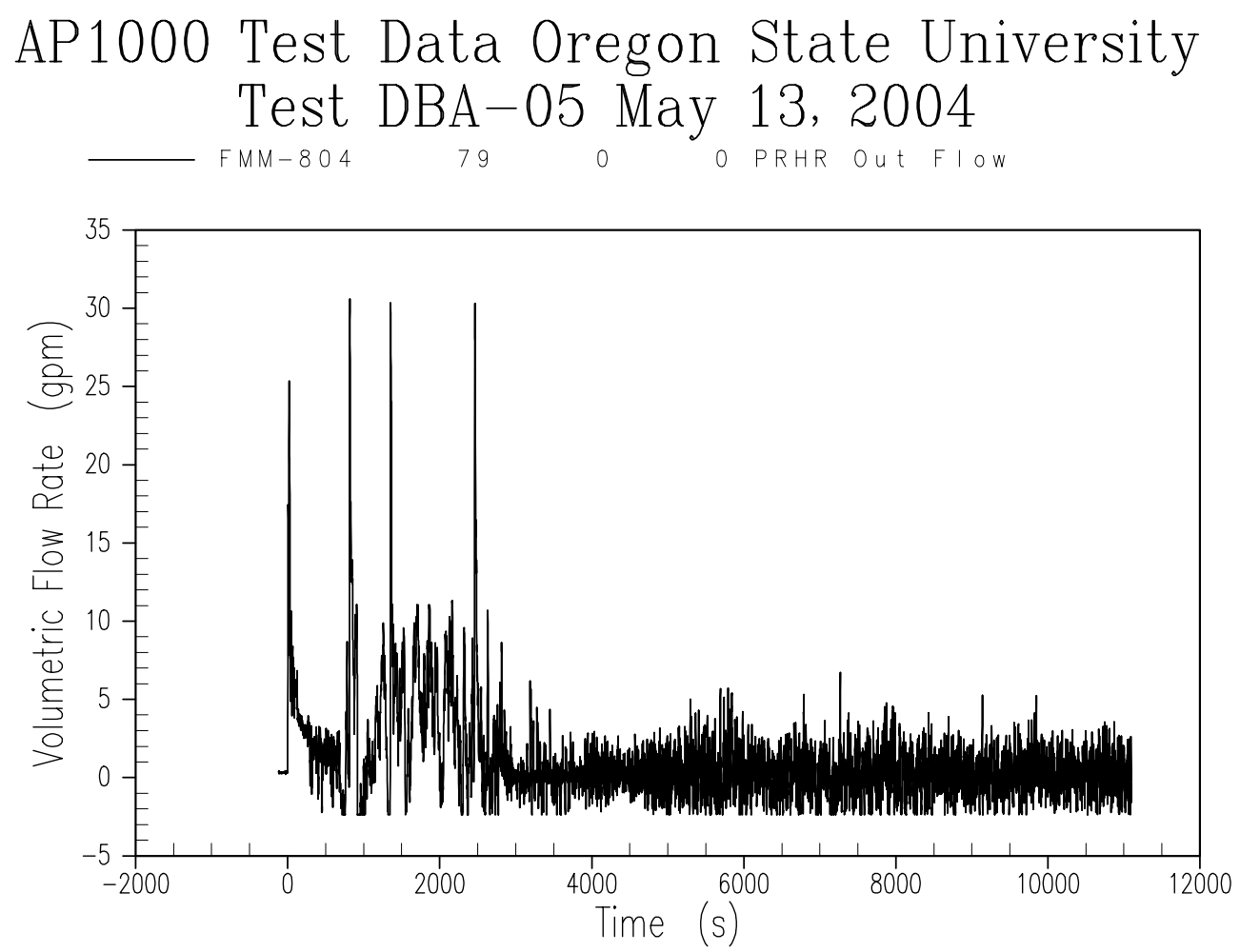

Figure A-37 Passive Residual Heat Removal Outlet Flow Rate 

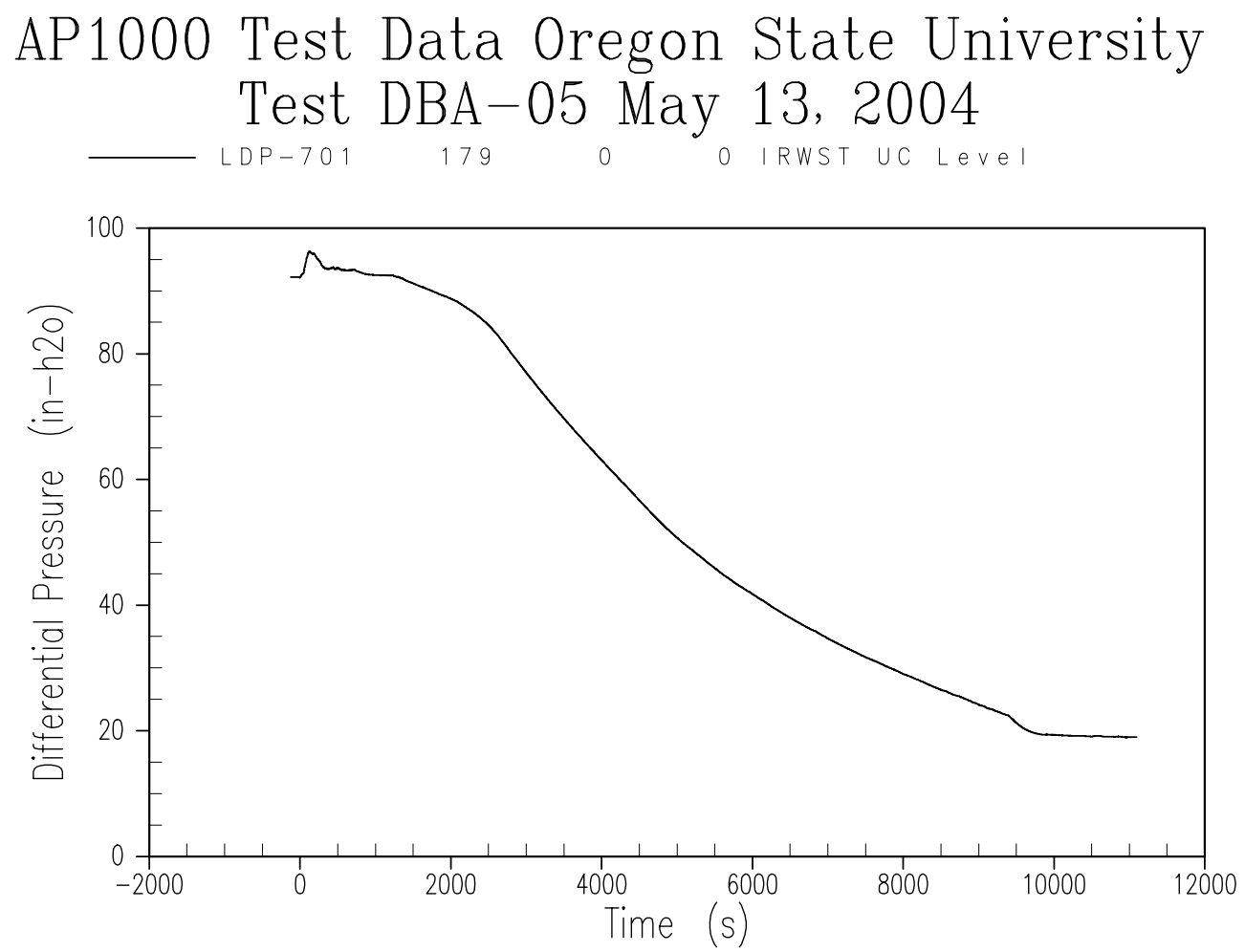

Figure A-38 IRWST Liquid Level 

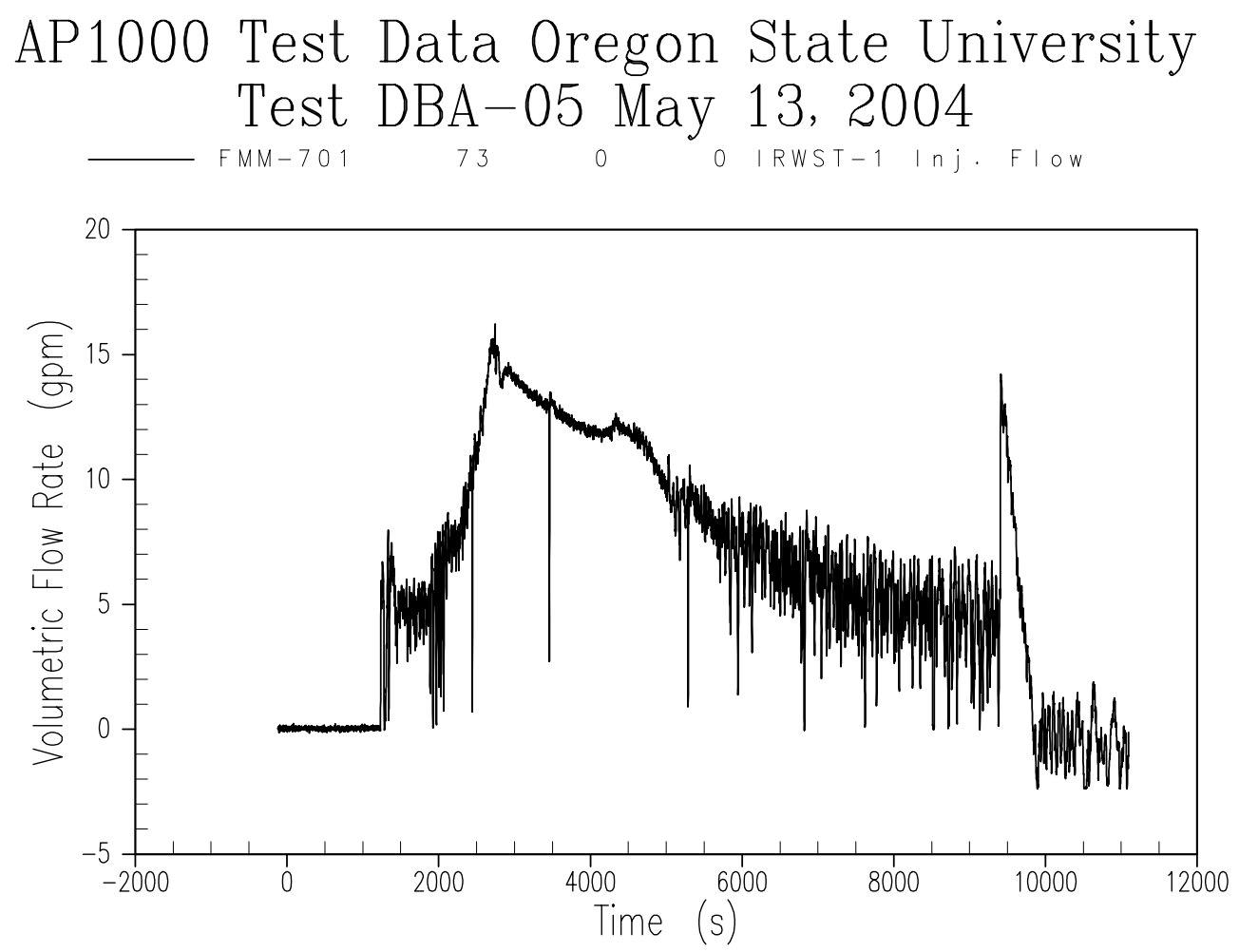

Figure A-39 IRWST Discharge Line 1 Flow Rate 

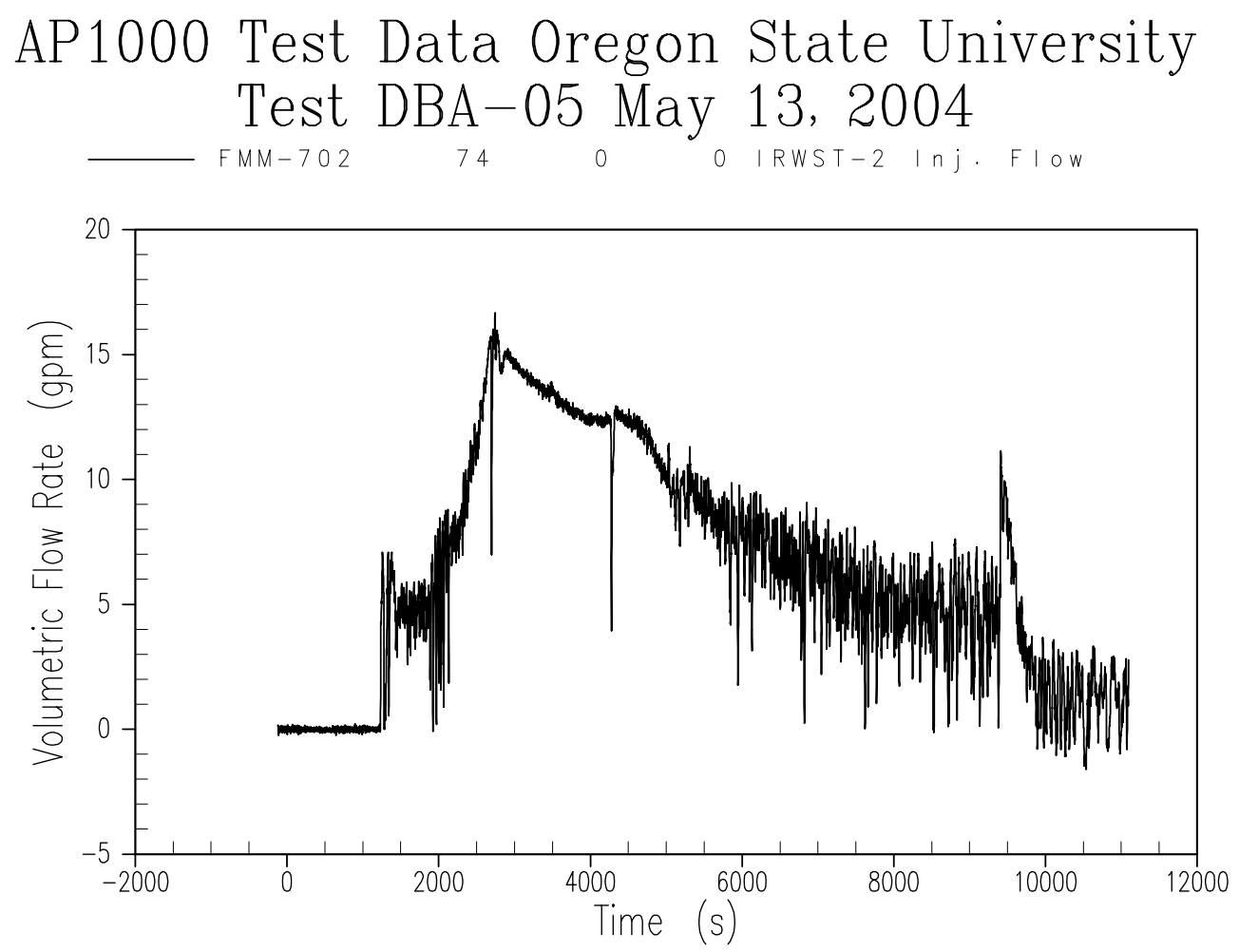

Figure A-40 IRWST Discharge Line 2 Flow Rate 
AP1000 Test Data Oregon State University Test DBA-05 May 13, 2004

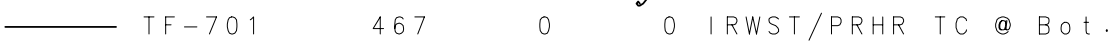

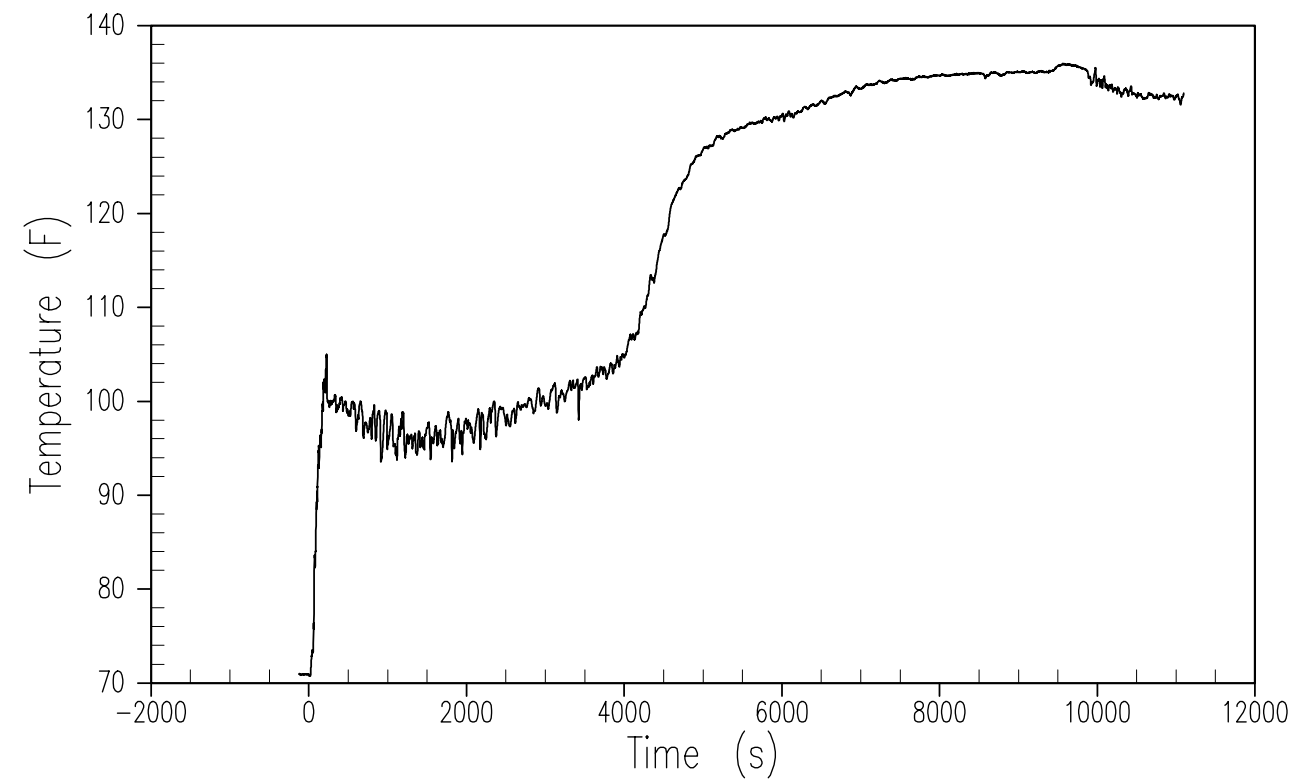

Figure A-41 IRWST Fluid Temperature - Bottom 
AP1000 Test Data Oregon State University Test DBA-05 May 13, 2004

- TF-70

475

O IRWST/PRHR TC @ 75.2

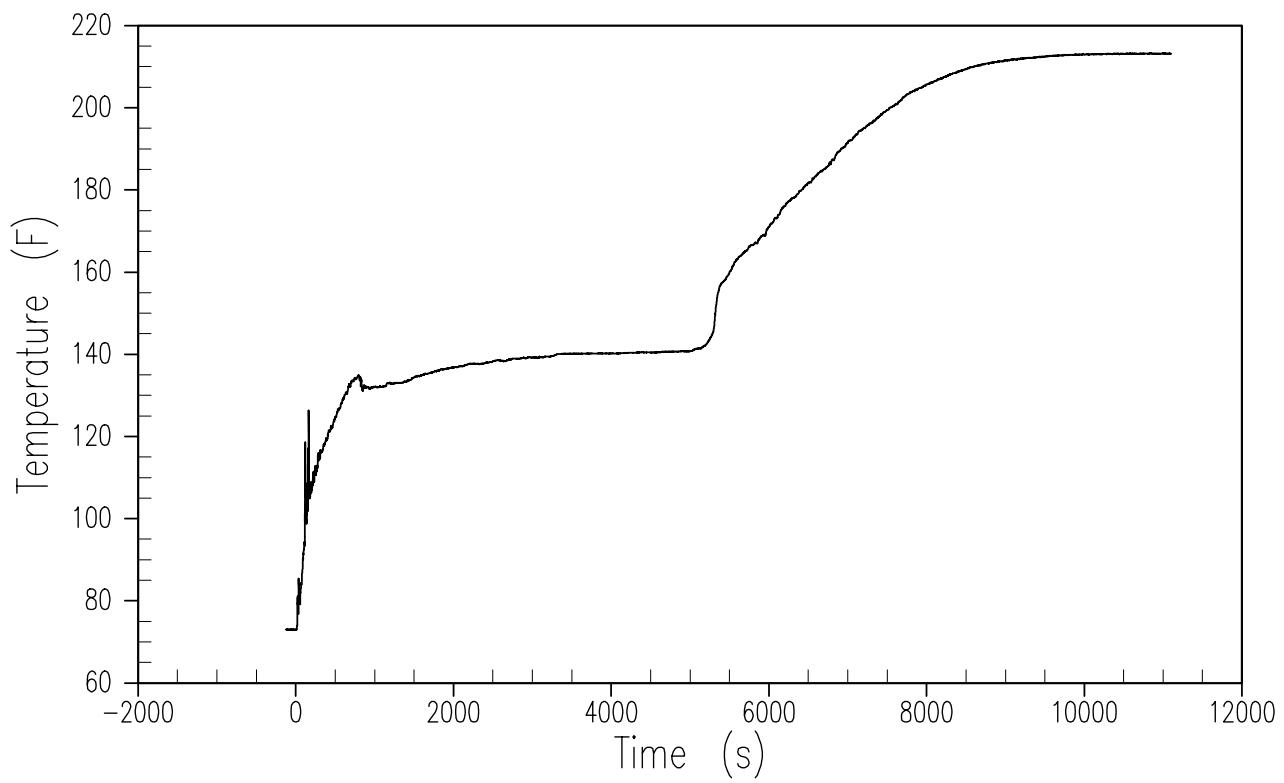

Figure A-42 IRWST Fluid Temperature - Top 

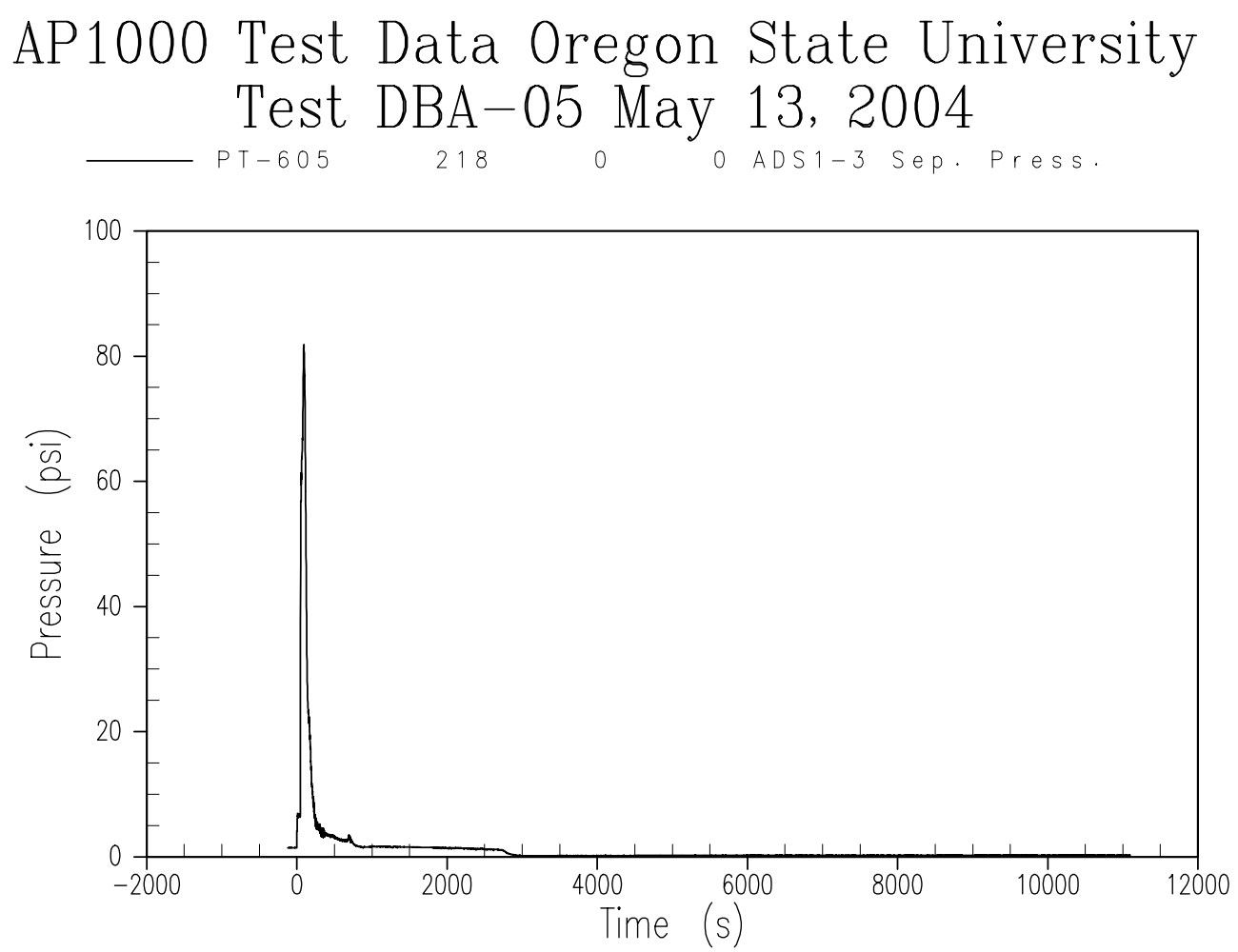

Figure A-43 ADS 1-3 Separator Pressure 

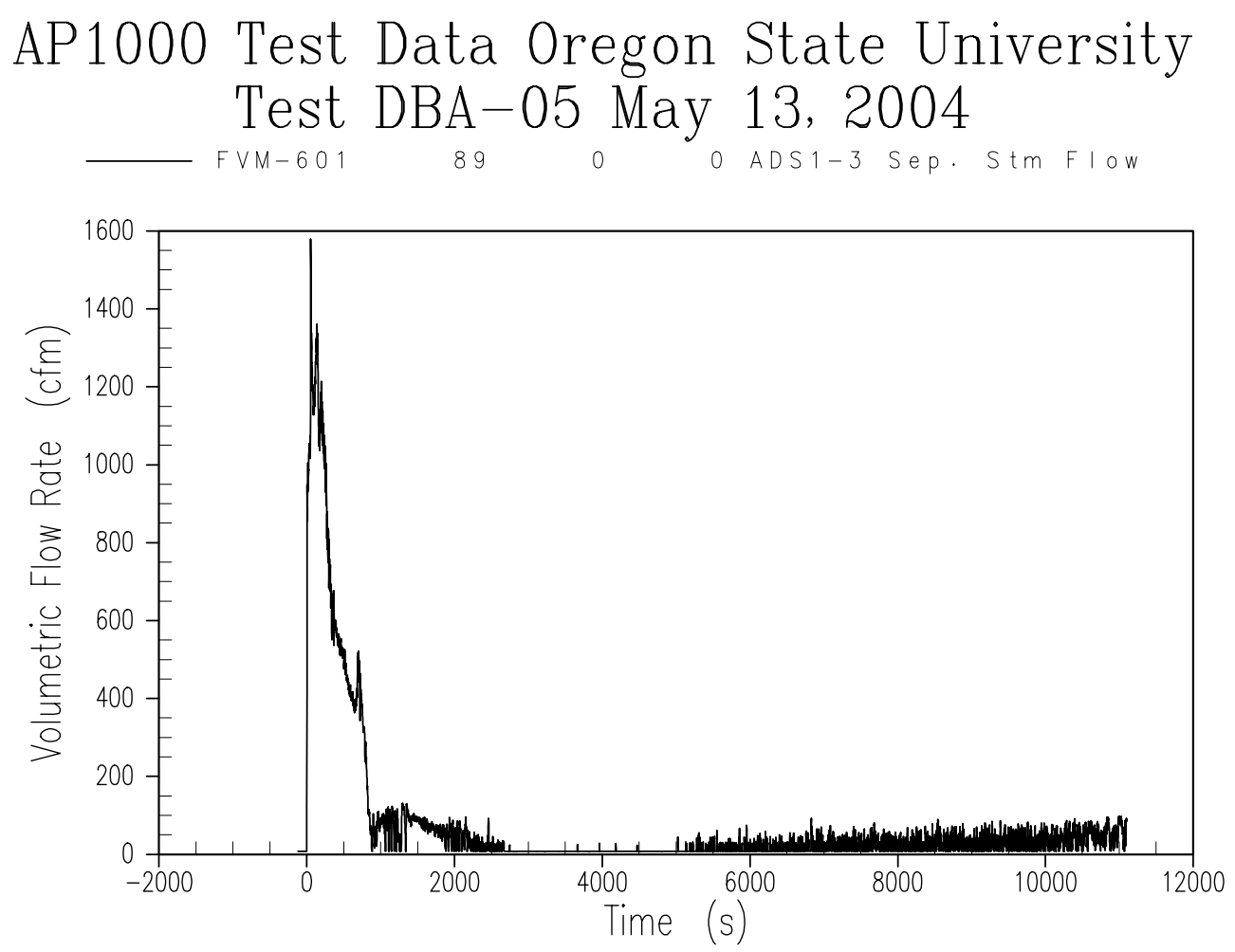

Figure A-44 ADS 1-3 Separator Steam Flow Rate 

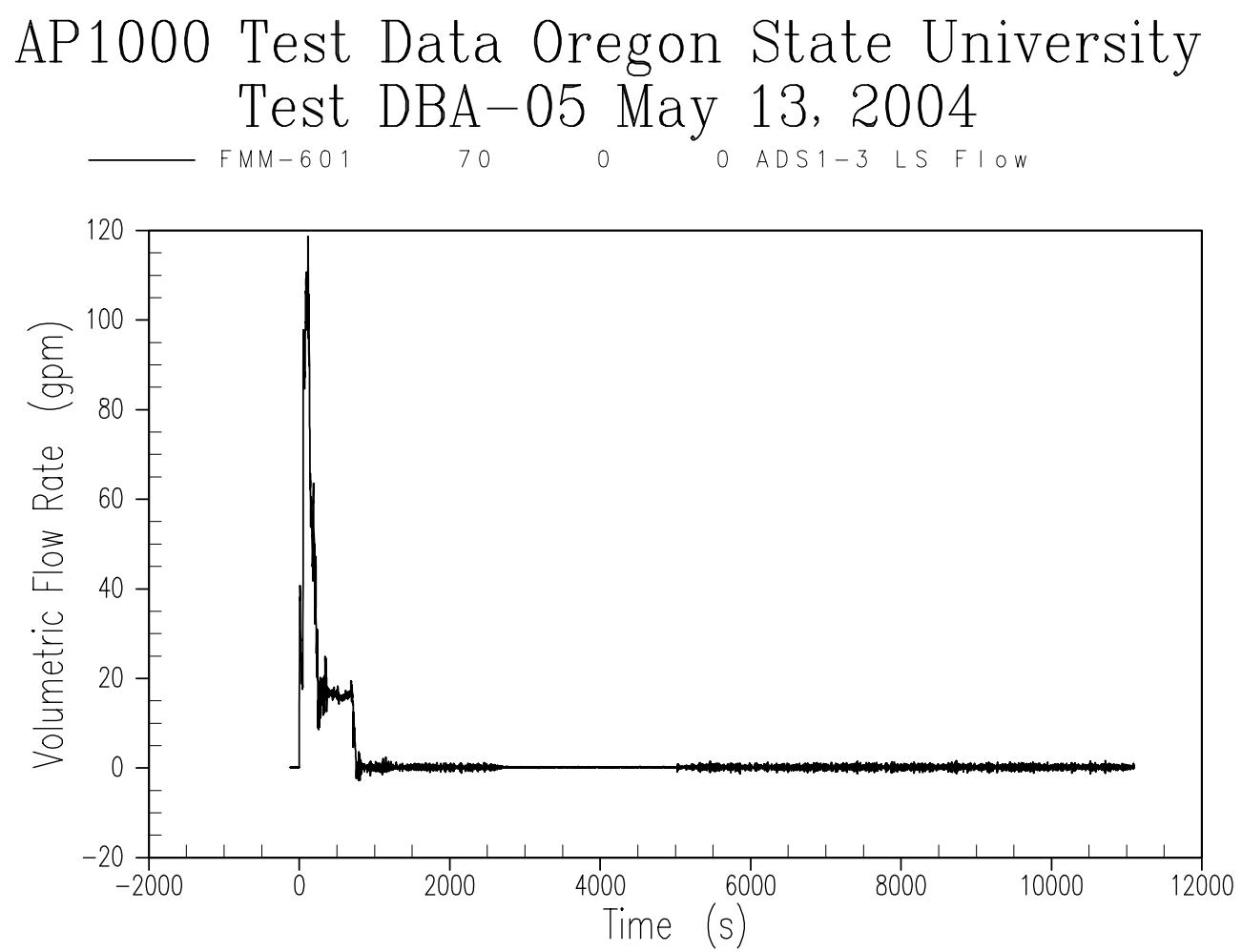

Figure A-45 ADS 1-3 Separator Liquid Flow Rate 

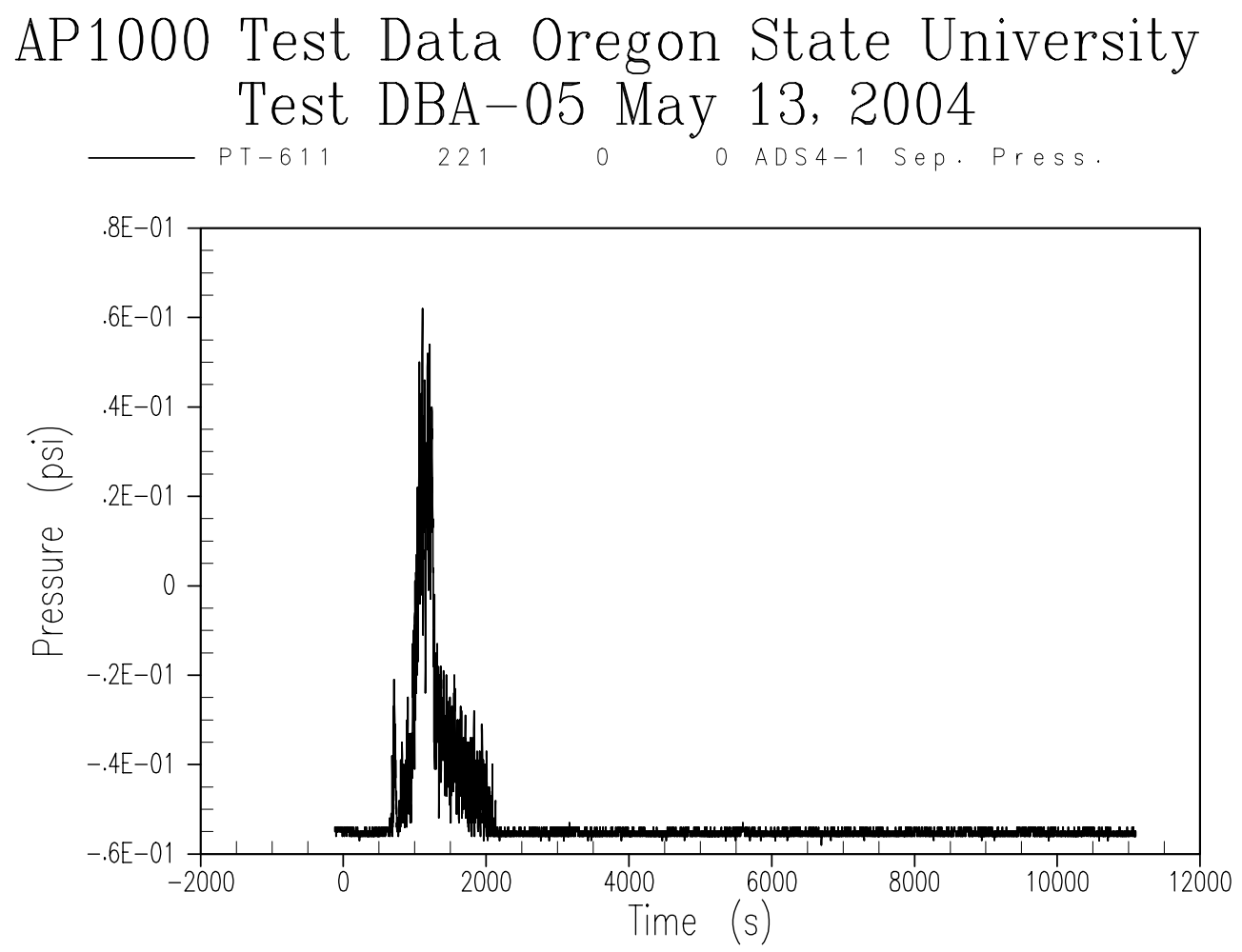

Figure A-46 ADS 4-1 Separator Pressure 

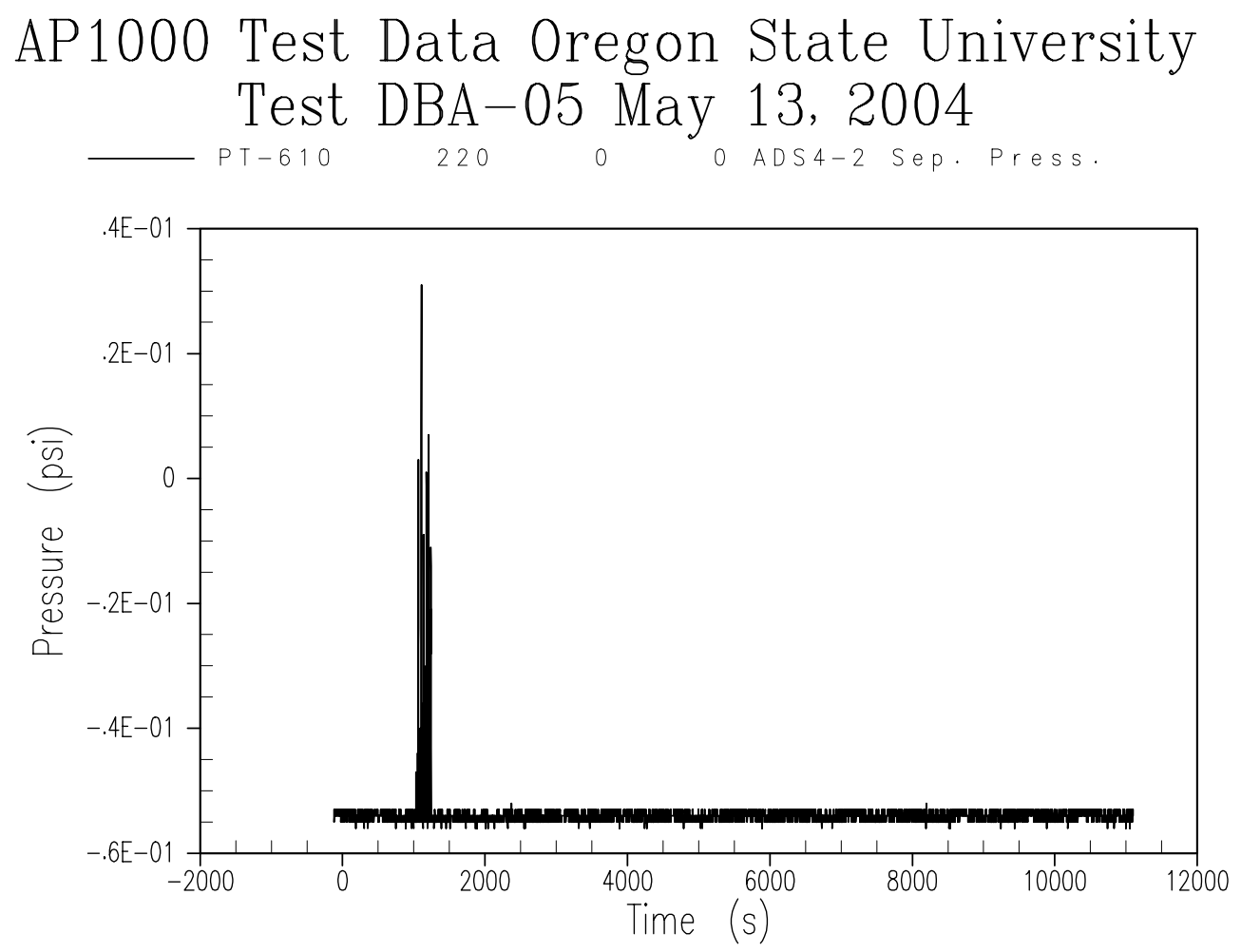

Figure A-47 ADS 4-2 Separator Pressure 


\section{AP1000 Test Data Oregon State University Test DBA-05 May 13, 2004 \\ $\begin{array}{cccccc}\text { FVM-603 } & 91 & 0 & \text { ADS } 4-1 & \text { Sep. 6" Line }\end{array}$}

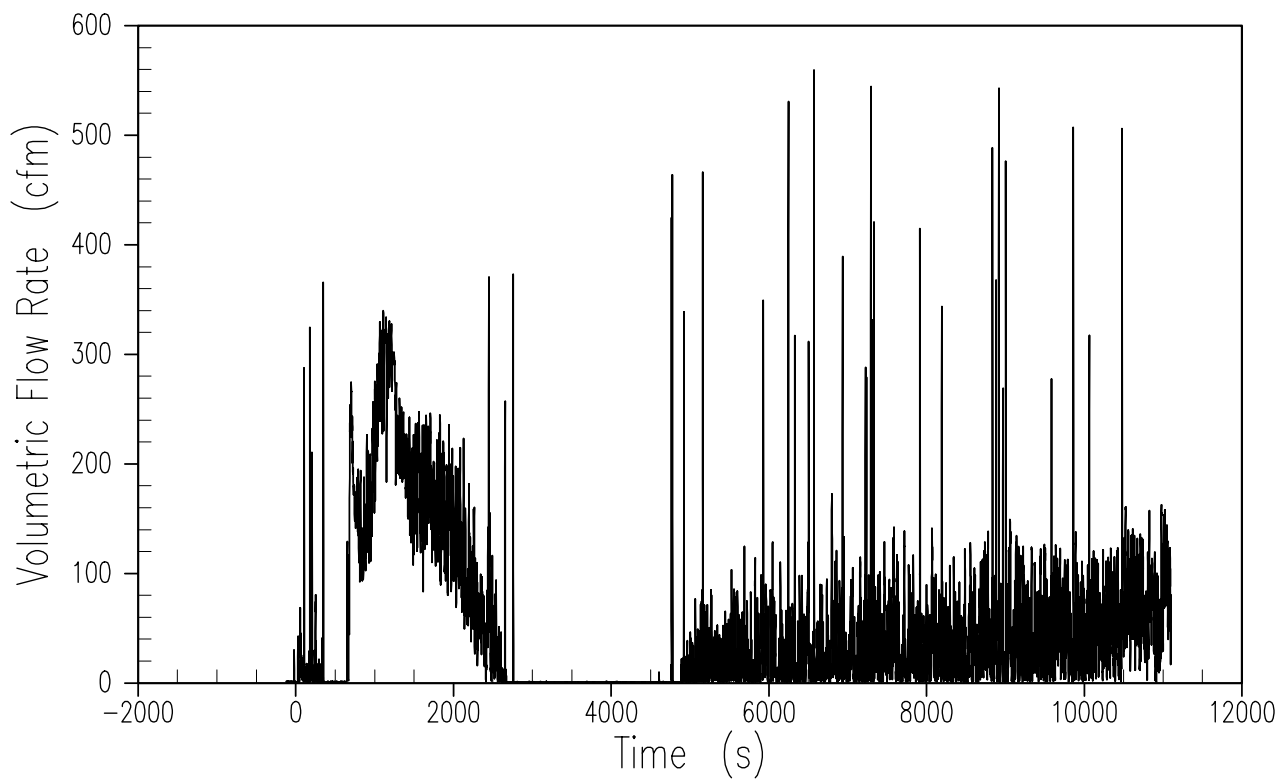

Figure A-48 ADS 4-1 Separator Steam Flow Rate 


\section{AP1000 Test Data Oregon State University Test DBA-05 May 13, 2004 \\ - FVM-602 $90 \quad 0 \quad$ o ADS4-2 Sep. 6" Line}

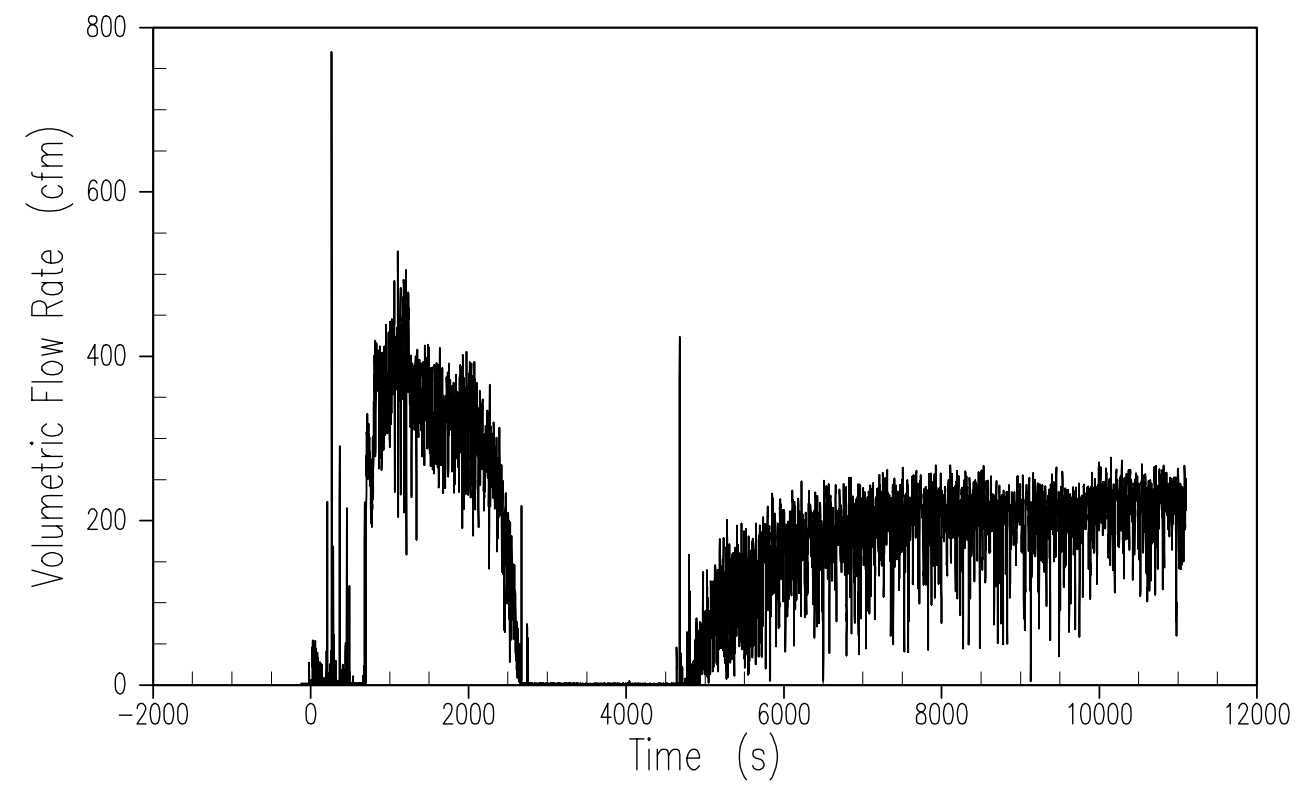

Figure A-49 ADS 4-2 Separator Steam Flow Rate 


\section{AP1000 Test Data Oregon State University Test DBA-05 May 13, 2004 \\ $\begin{array}{llllll}\text { FMM-603 } & 72 & 0 & \text { O ADS 4-1 LS FIOW }\end{array}$}

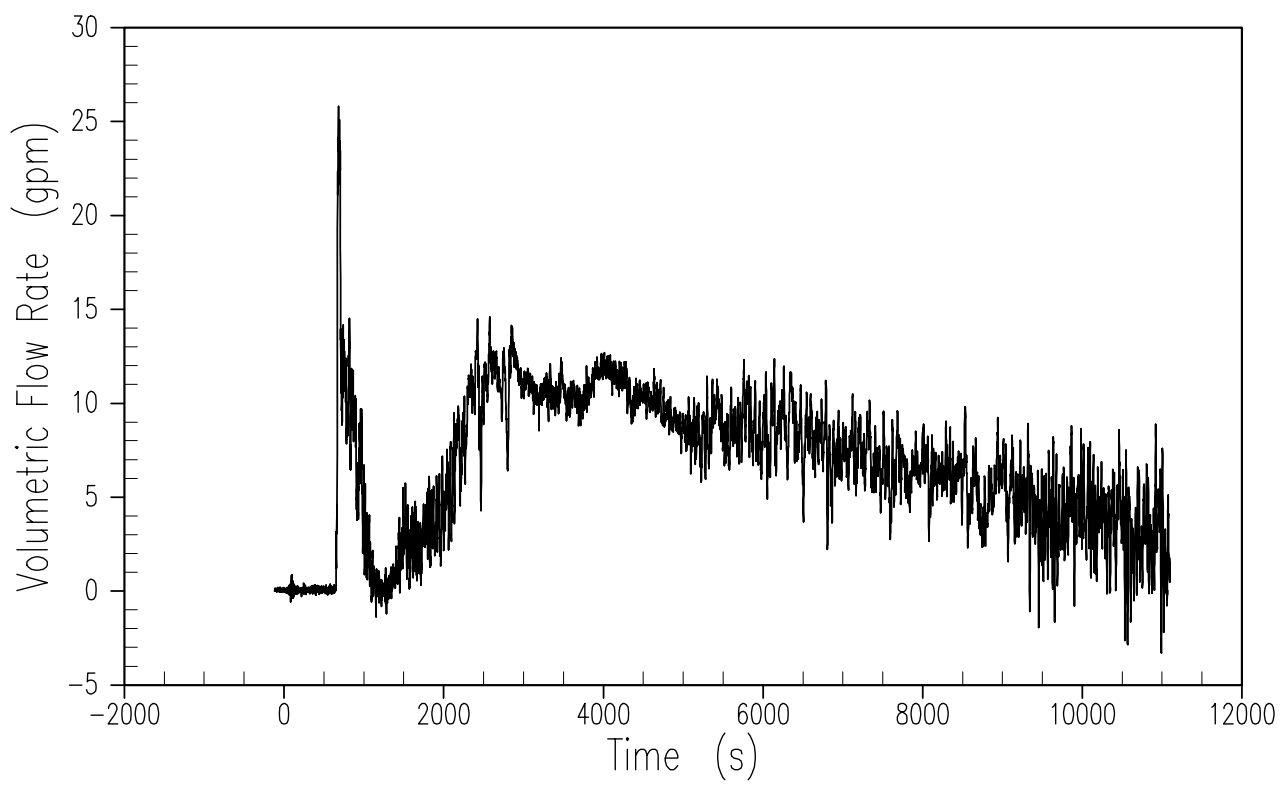

Figure A-50 ADS 4-1 Separator Liquid Flow Rate 

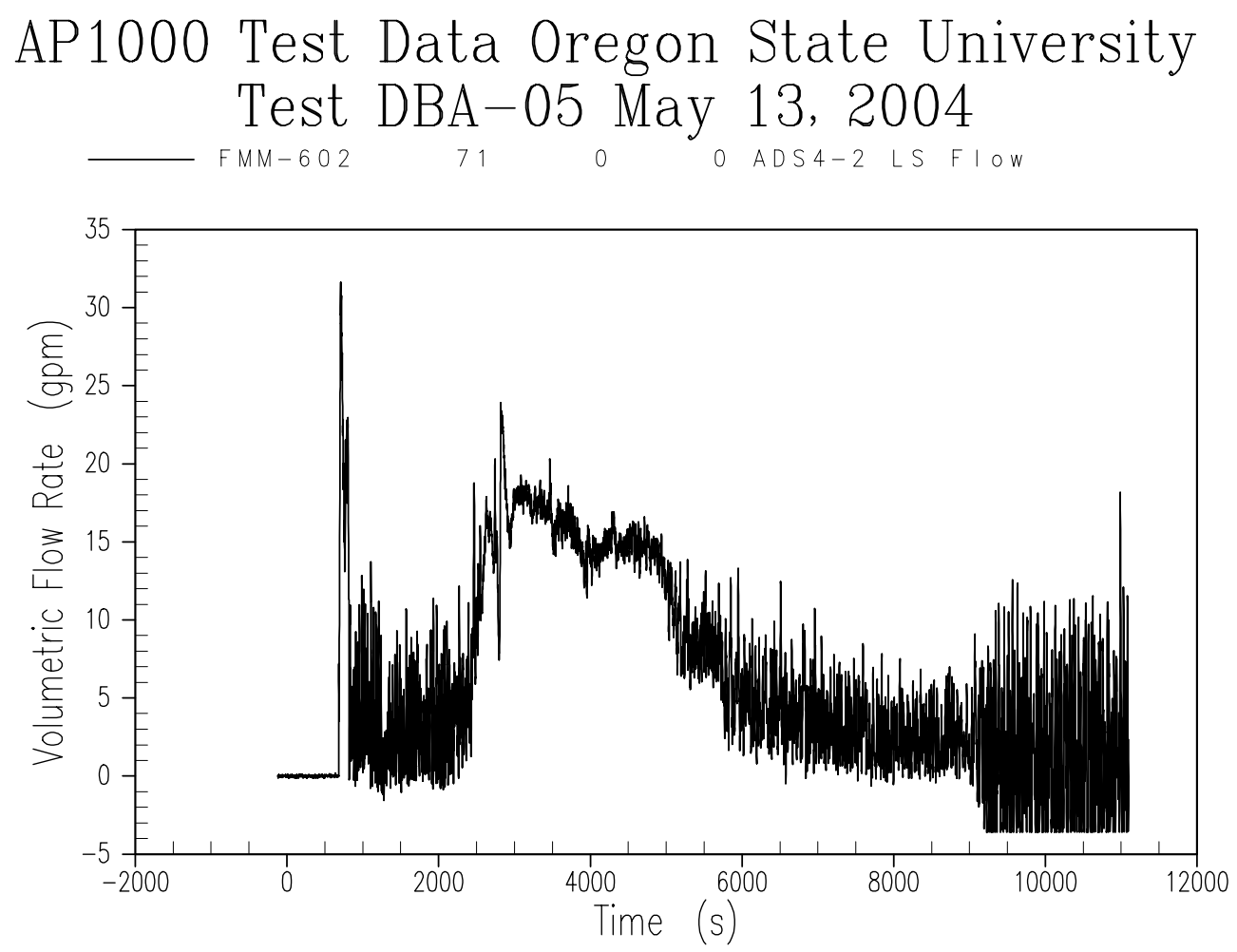

Figure A-51 ADS 4-2 Separator Liquid Flow Rate 


\section{AP1000 Test Data Oregon State University Test DBA-05 May 13, 2004 \\ — PT-901 $225 \quad 00$ o Pri-sump press.}

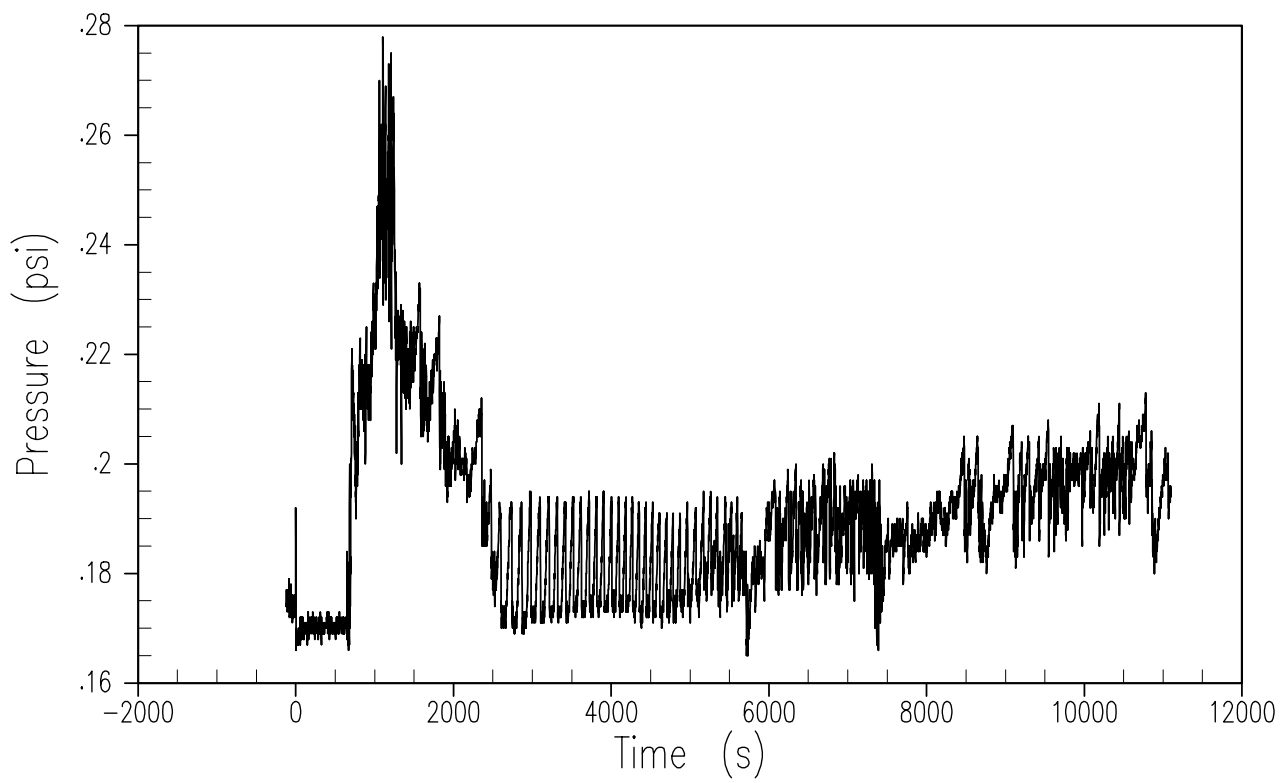

Figure A-52 Primary Sump Pressure 

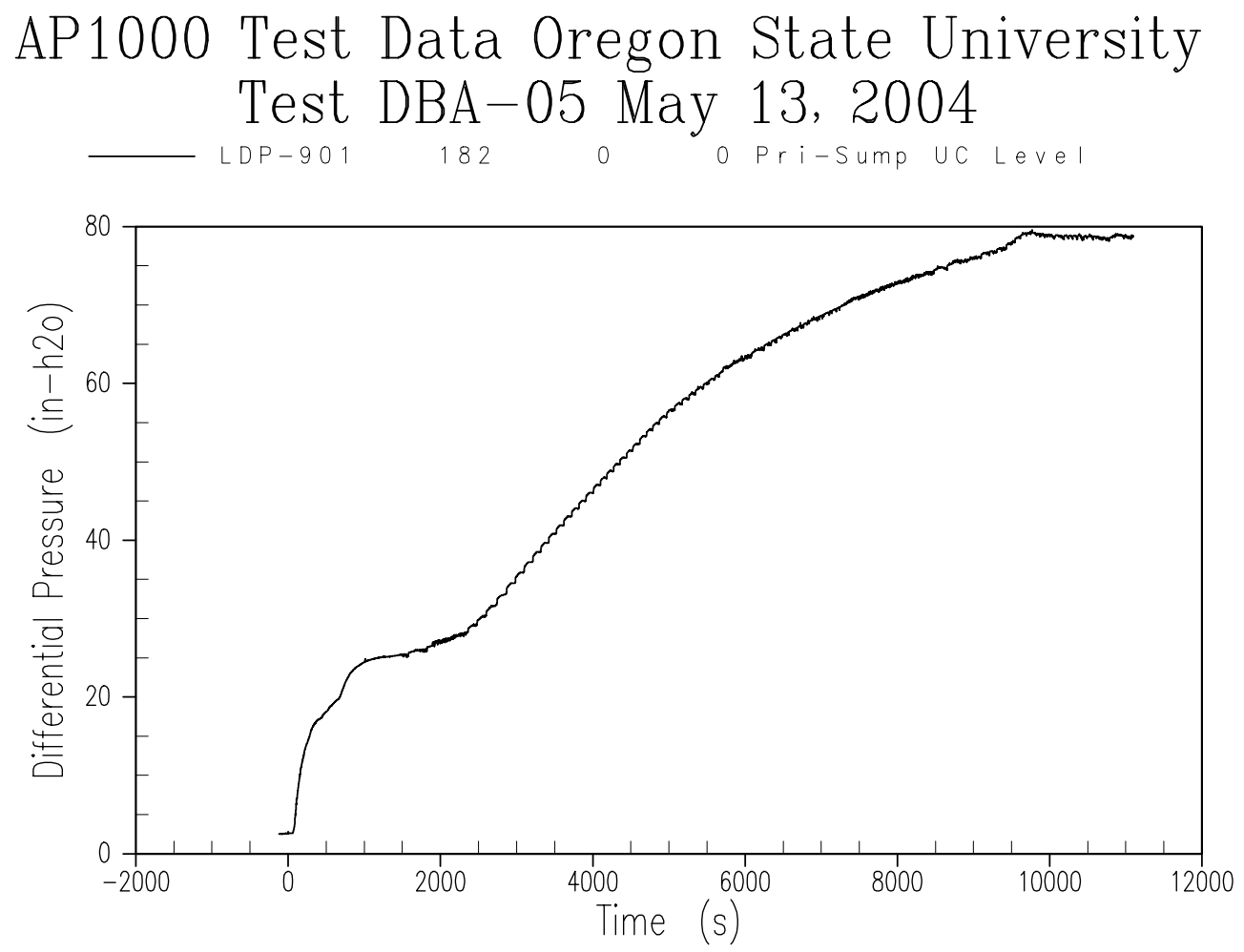

Figure A-53 Primary Sump Liquid Level 

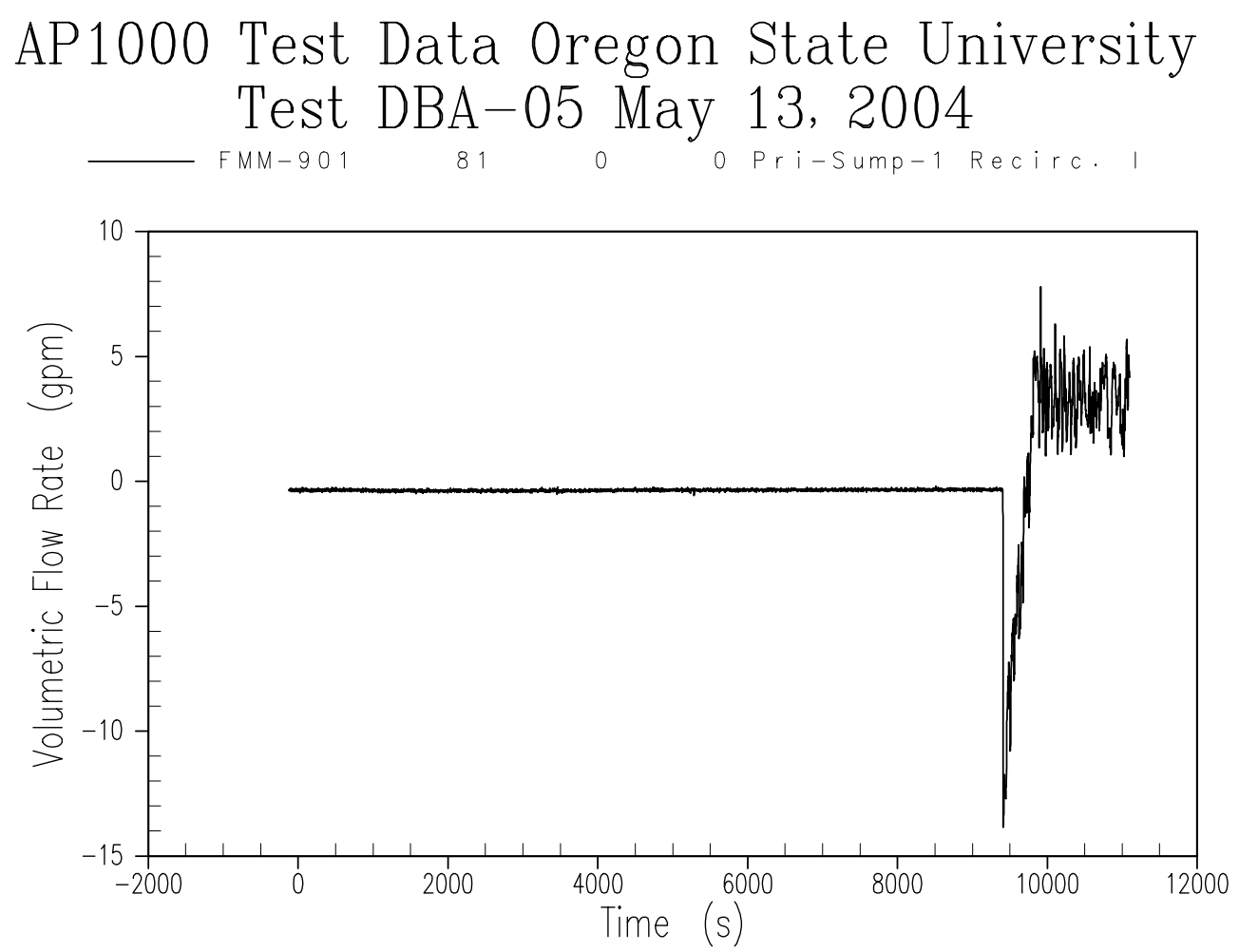

Figure A-54 Primary Sump 1 Injection Flow Rate 

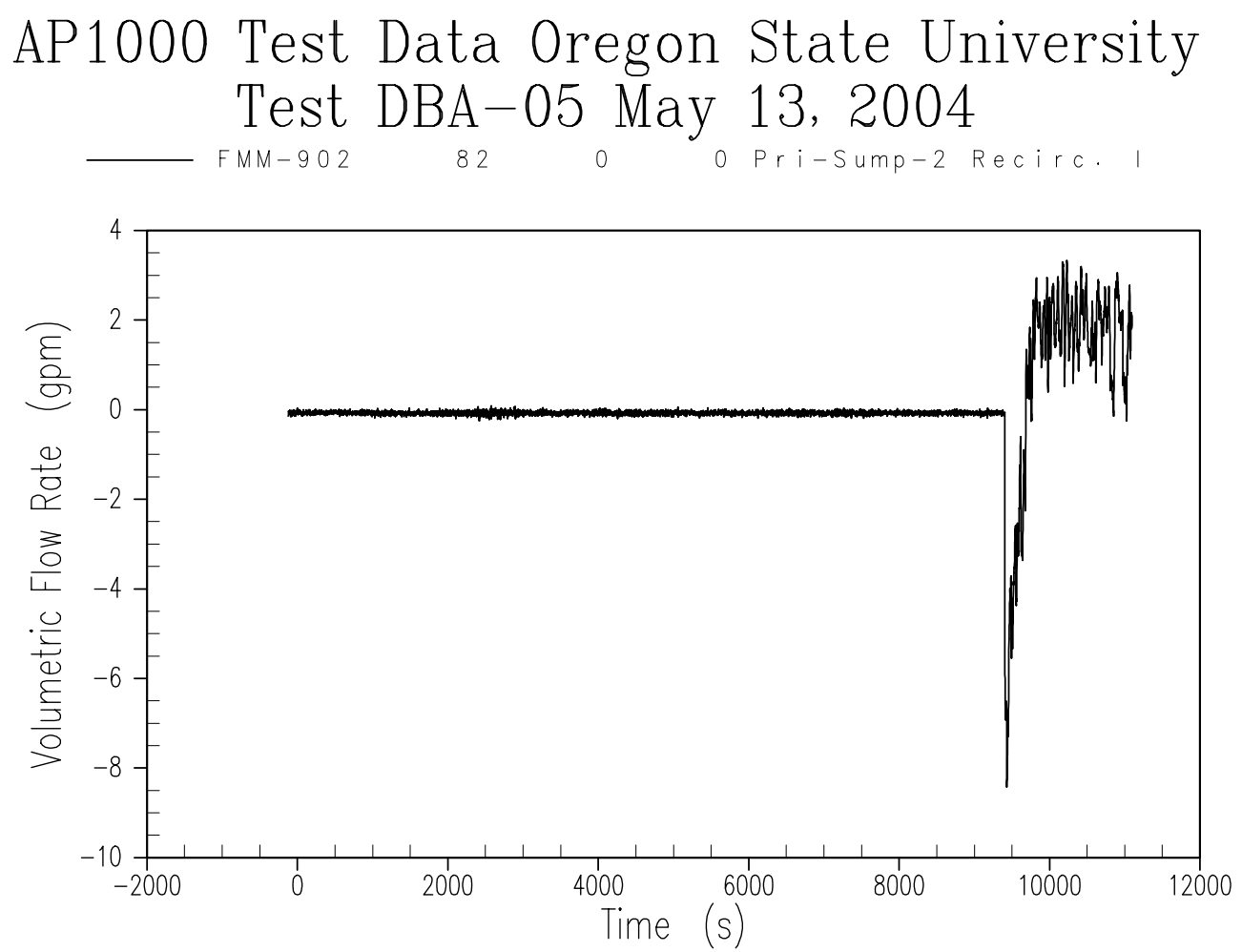

Figure A-55 Primary Sump 2 Injection Flow Rate 

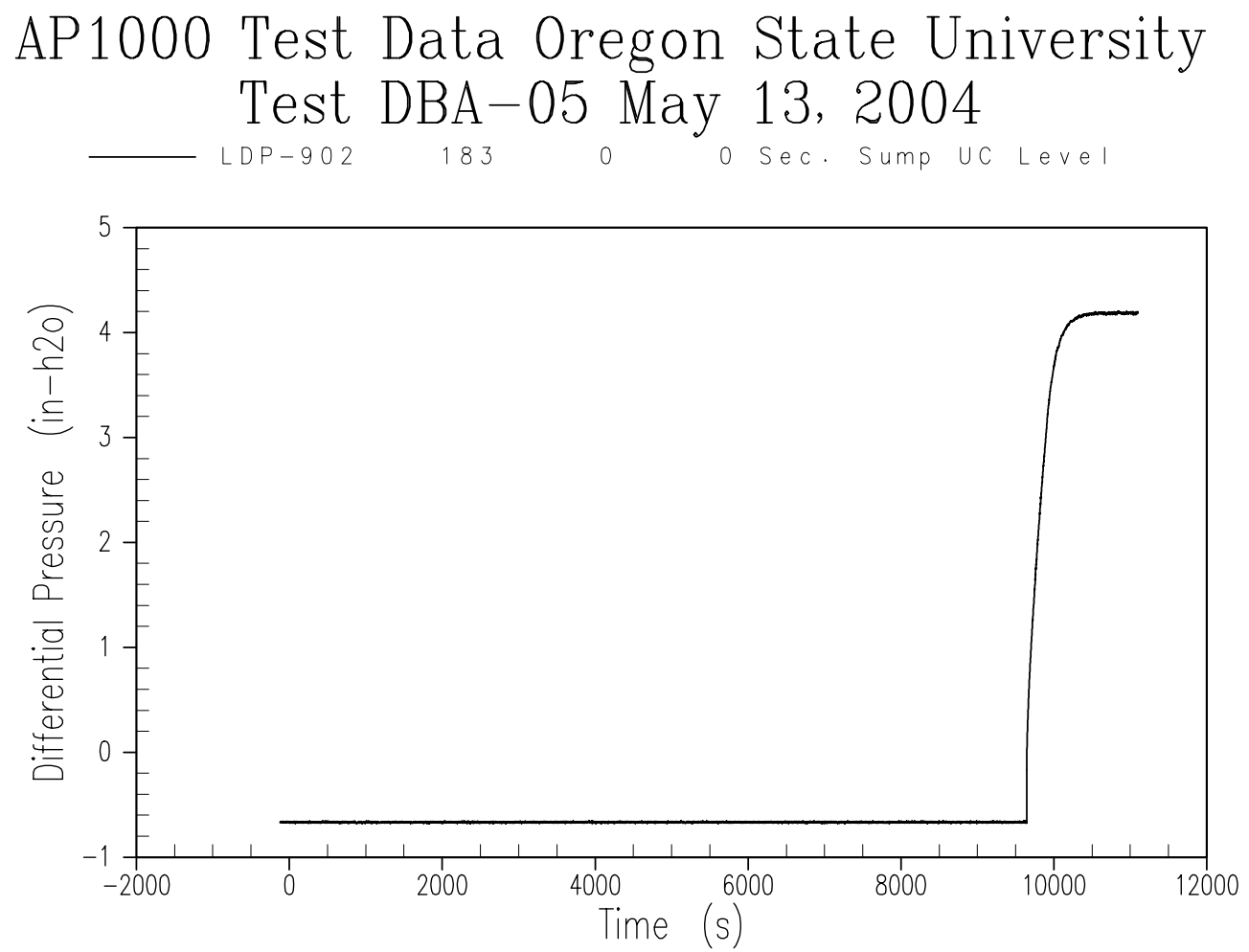

Figure A-56 Secondary Sump Liquid Level 

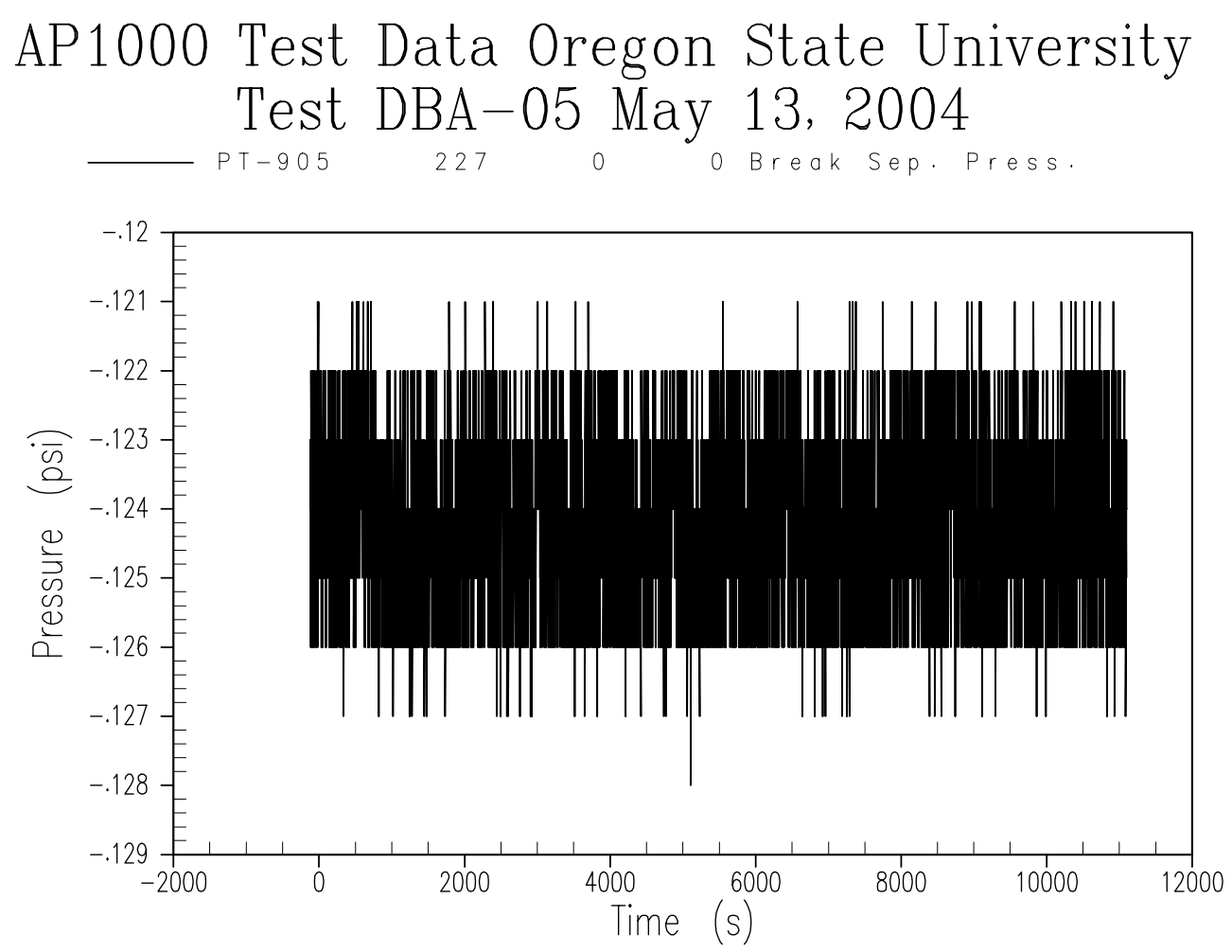

Figure A-57 Break Separator Pressure 

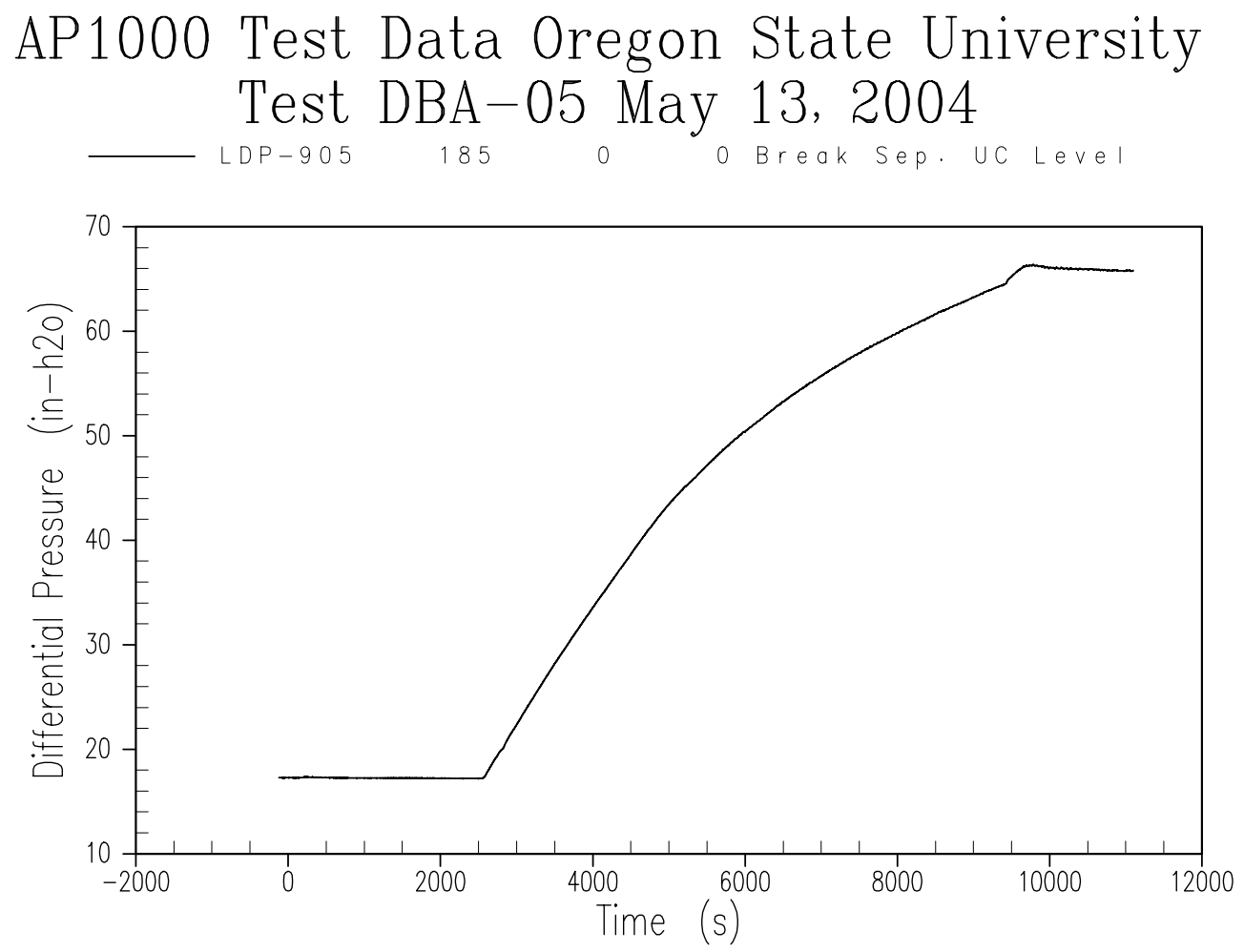

Figure A-58 Break Separator Liquid Level 

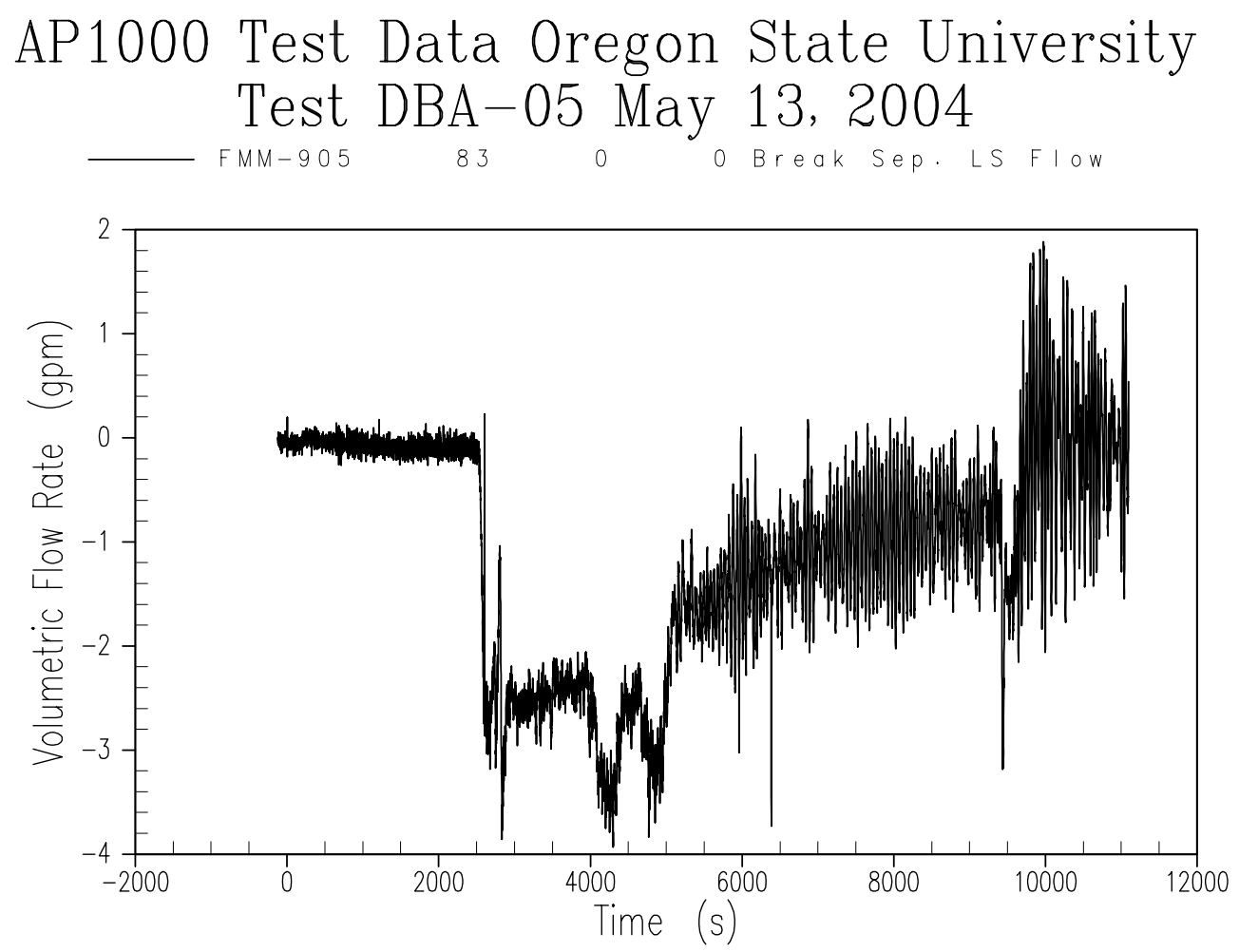

Figure A-59 Break Separator Flow to Primary Sump 

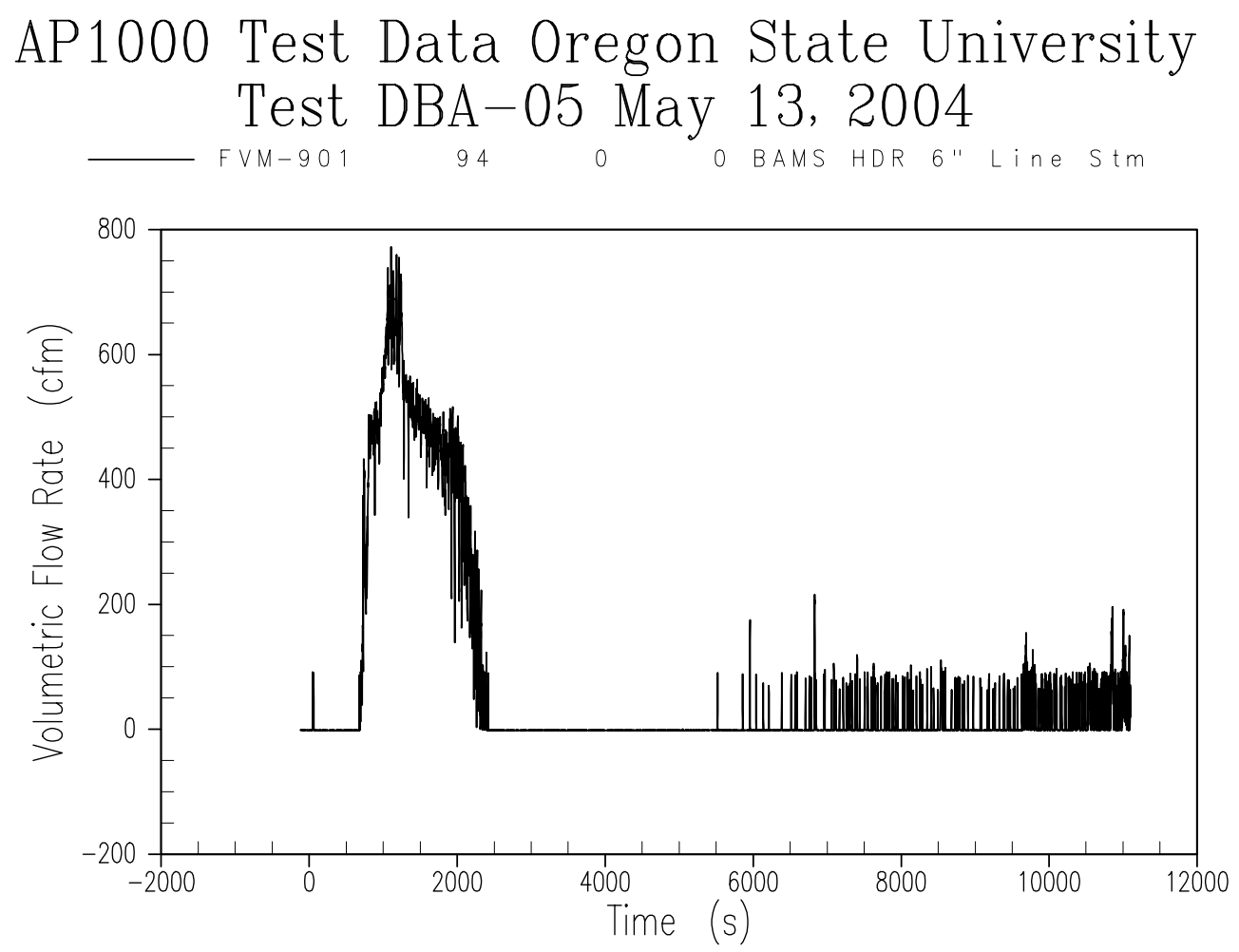

Figure A-60 BAMS Steam Flow Rate - 6-inch Line 


\section{AP1000 Test Data Oregon State University Test DBA-05 May 13, 2004

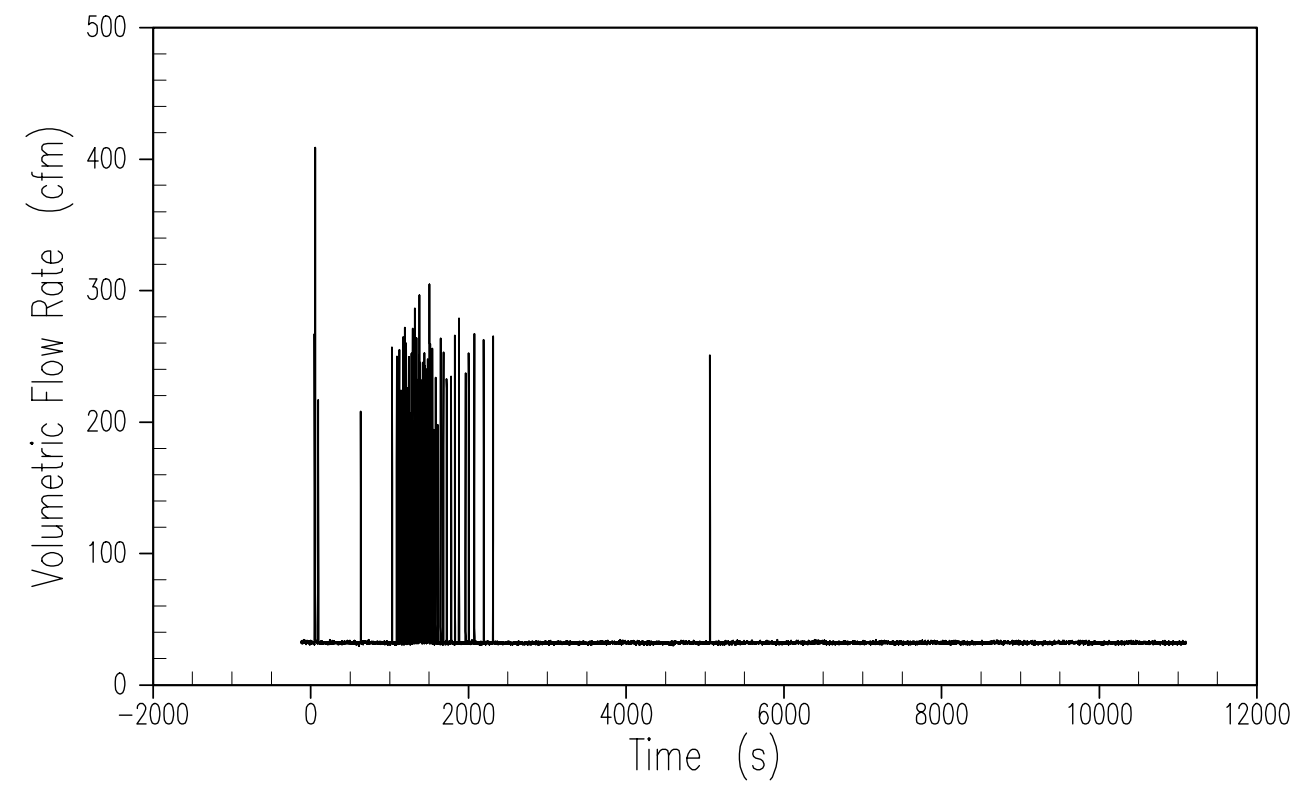

Figure A-61 BAMS Steam Flow Rate - 10-inch Line 

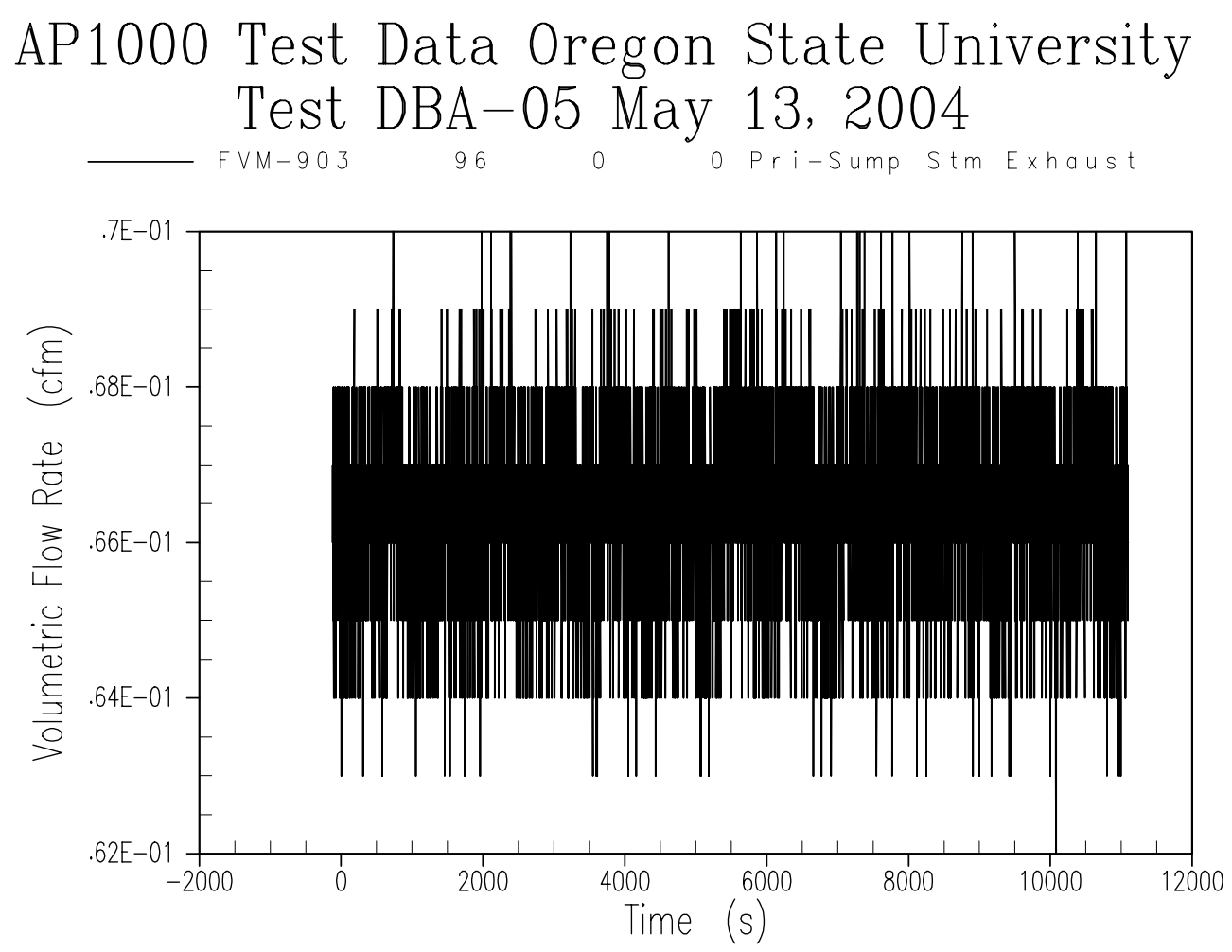

Figure A-62 BAMS/Primary Sump Steam Flow Rate 

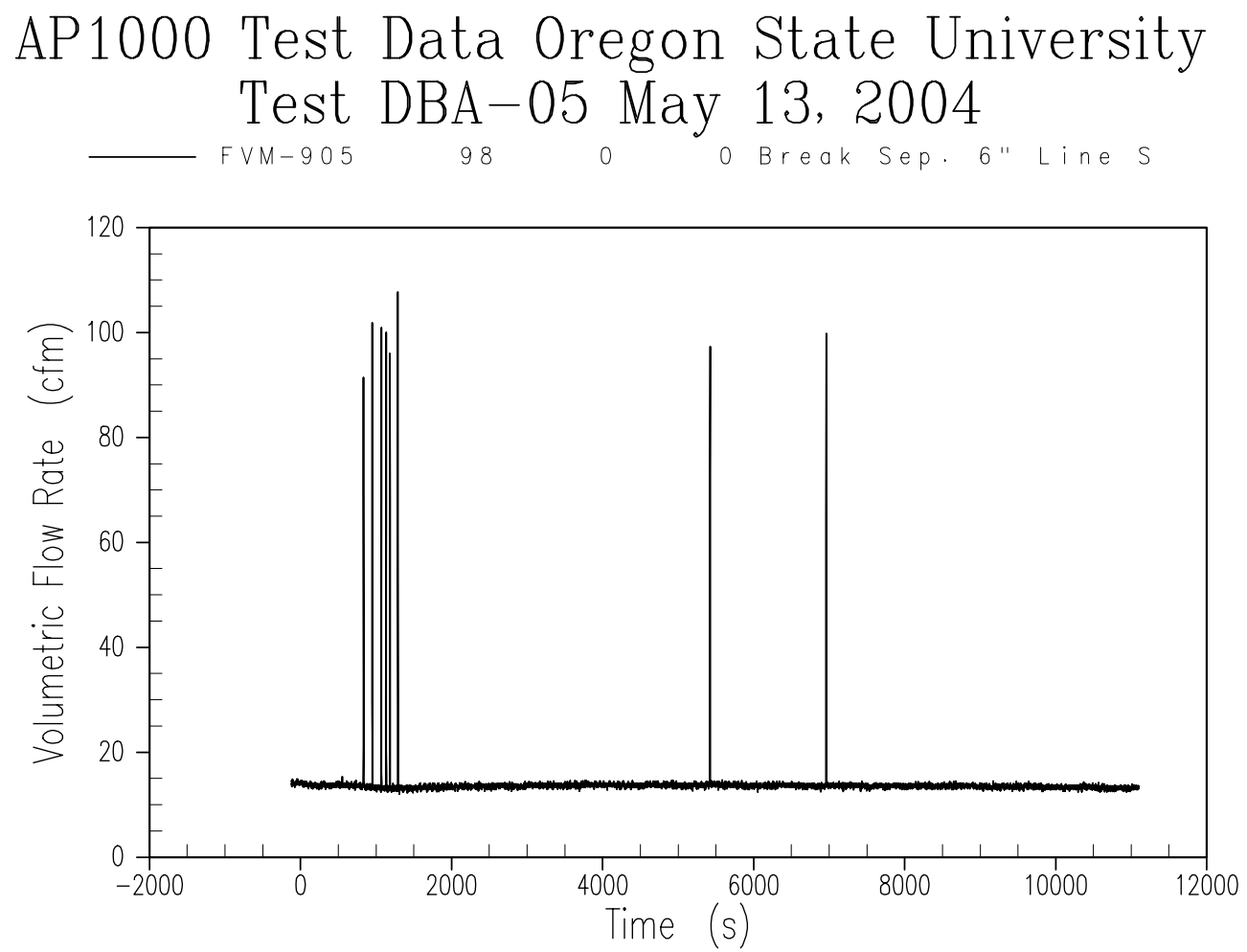

Figure A-63 BAMS/Separator Steam Flow Rate - 6-inch Pipe 
AP1000 Test Data Oregon State University Test DBA-05 May 13, 2004

- TF-916 $518 \quad 0 \quad 0$ BAMS Header 10 " Stm

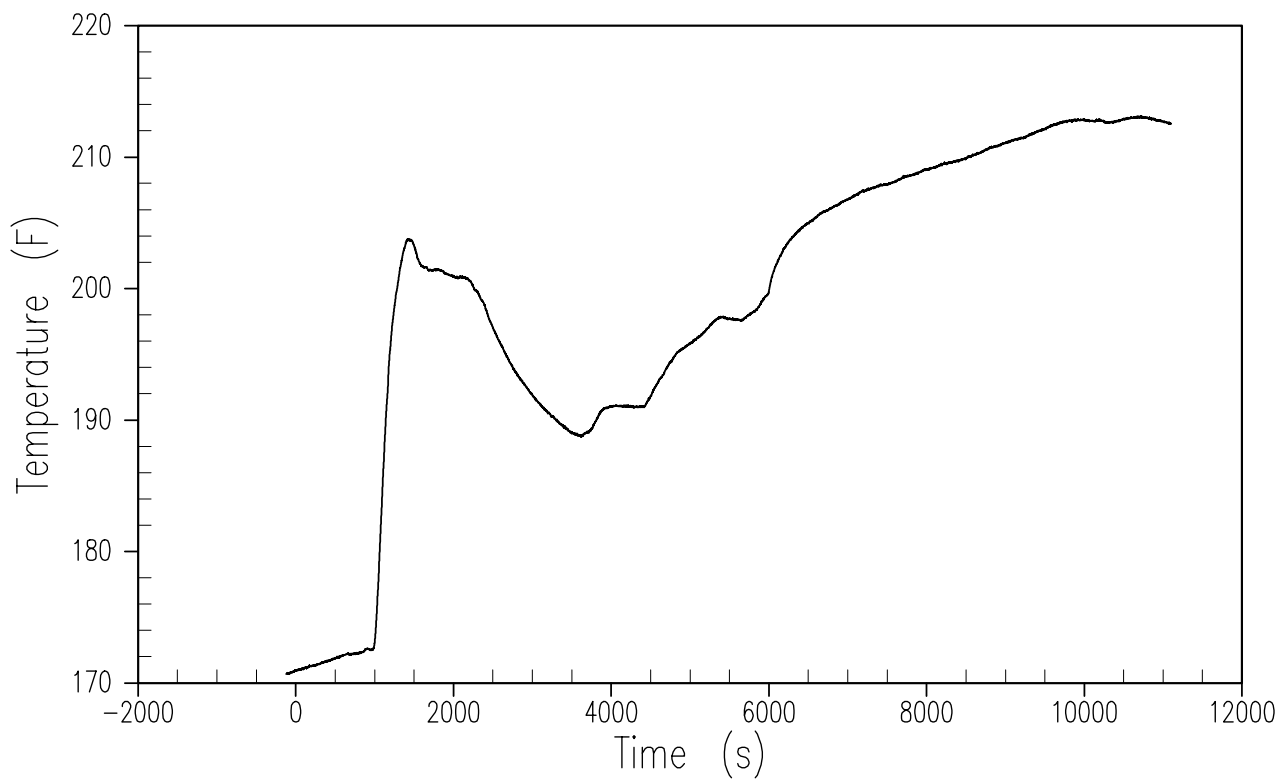

Figure A-64 BAMS/Exhaust Line Temperature - 10-inch Line 

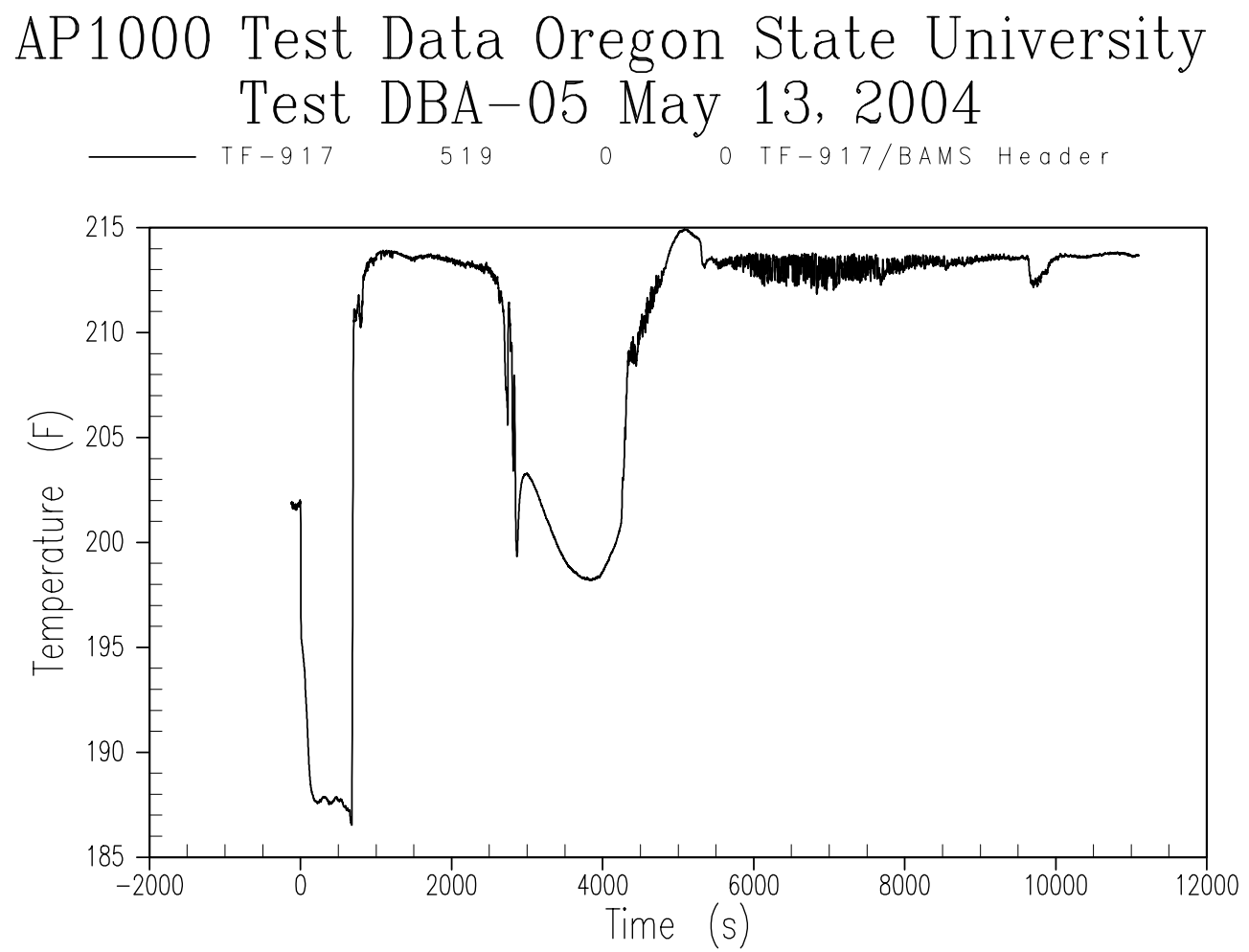

Figure A-65 BAMS/Exhaust Line Temperature - Header 

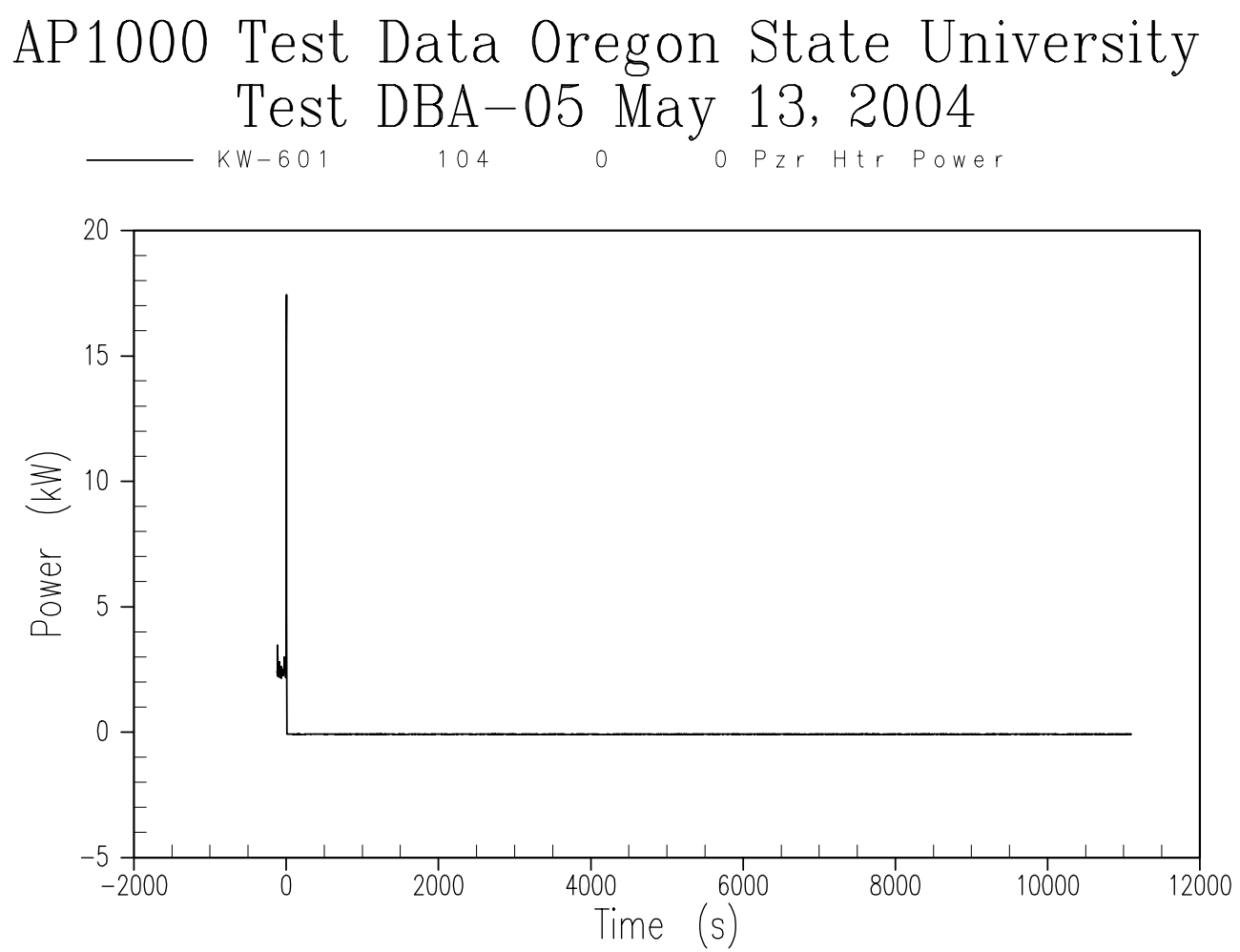

Figure A-66 Pressurizer Heater Input Power 

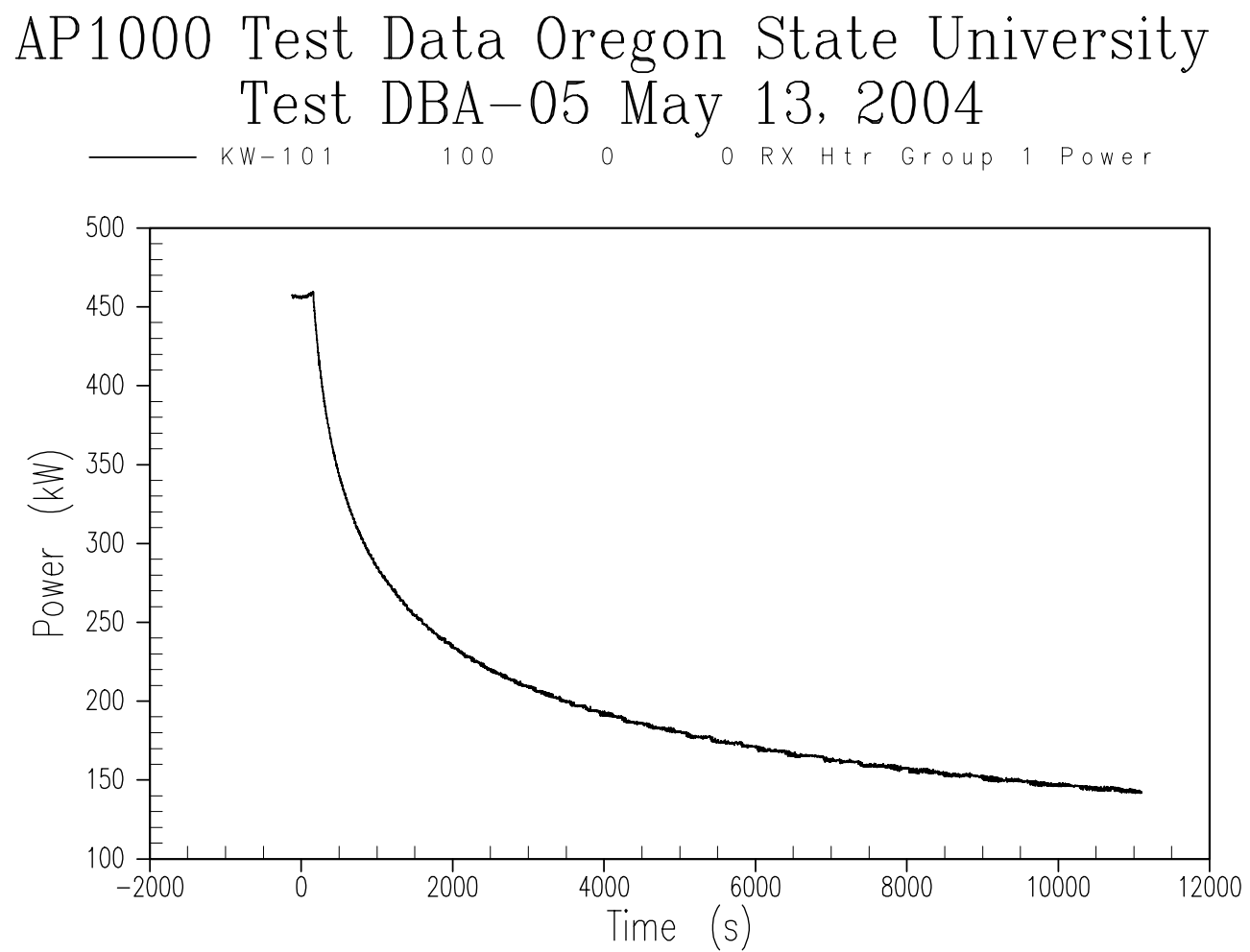

Figure A-67 Core Power Group 1 Input Power 

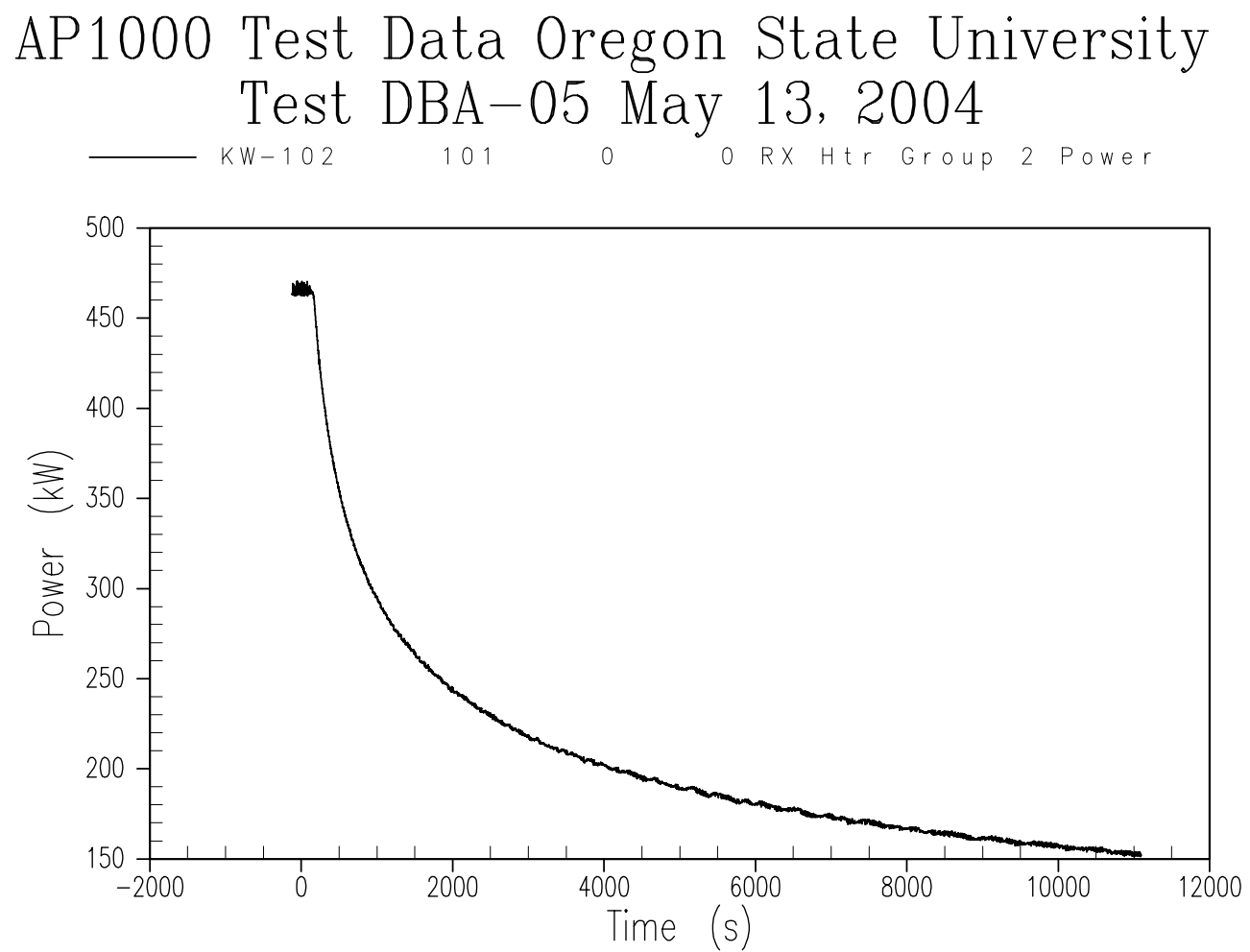

Figure A-68 Core Power Group 2 Input Power 

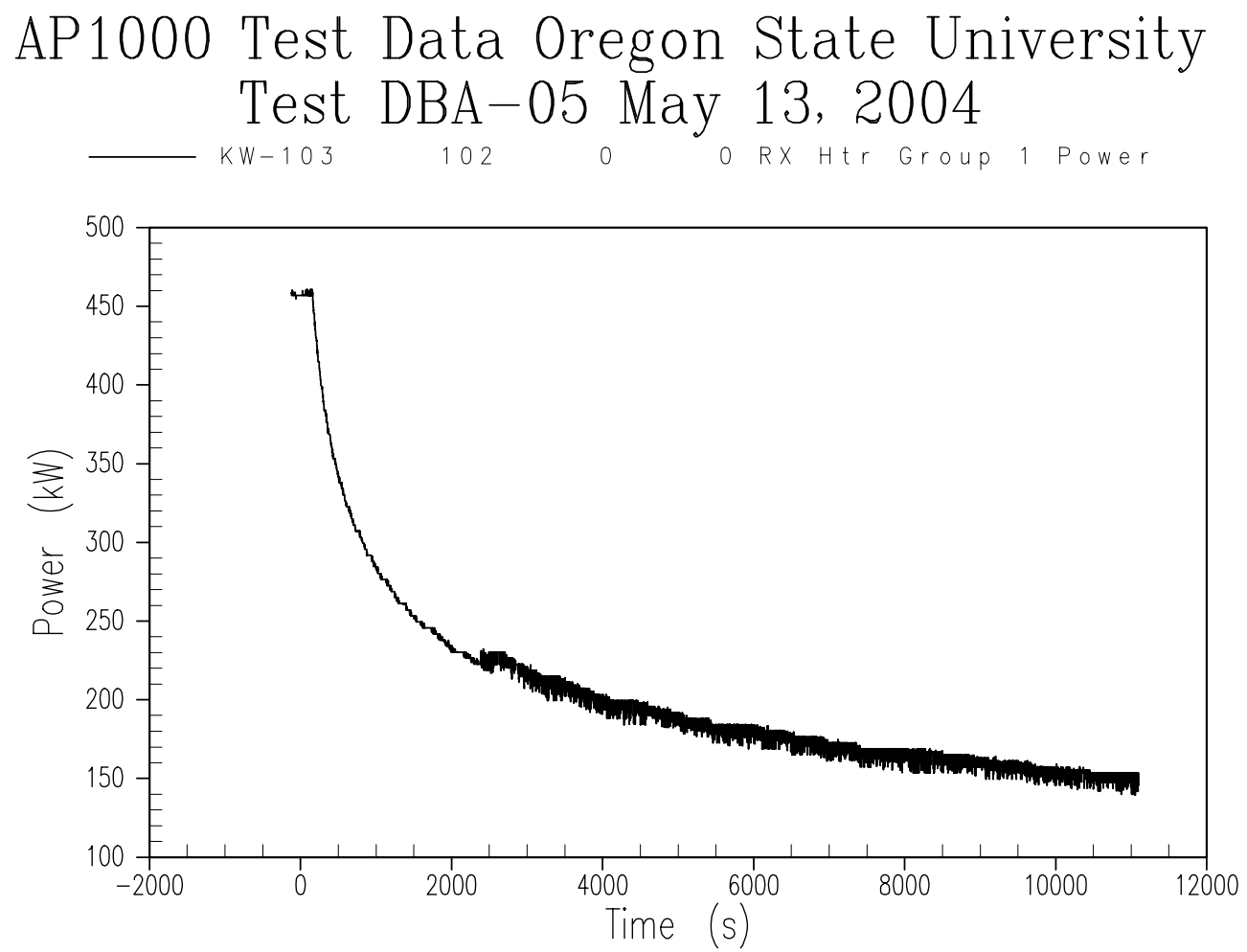

Figure A-69 Core Power Group 1 Alternate Input Power 

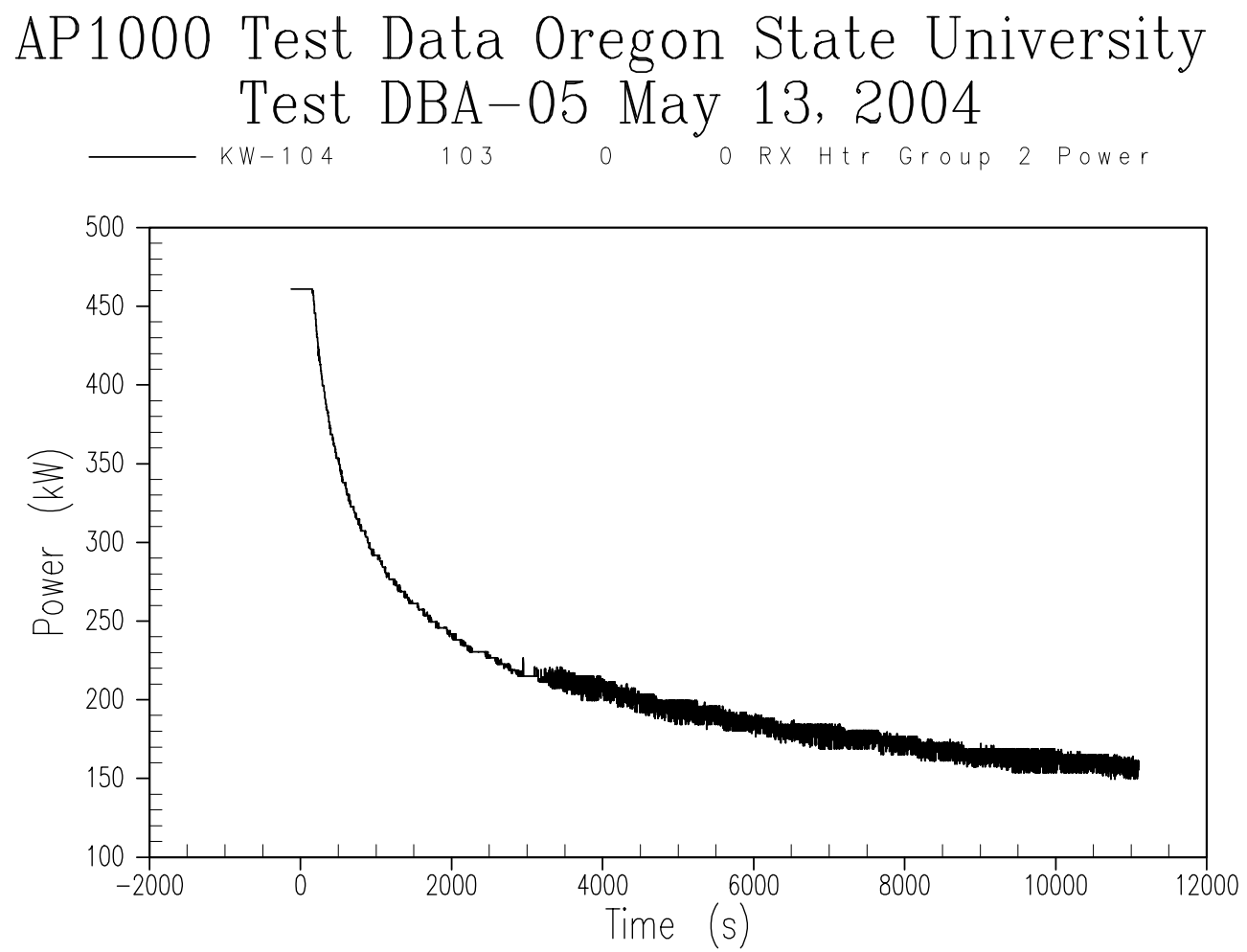

Figure A-70 Core Power Group 2 Alternate Input Power 

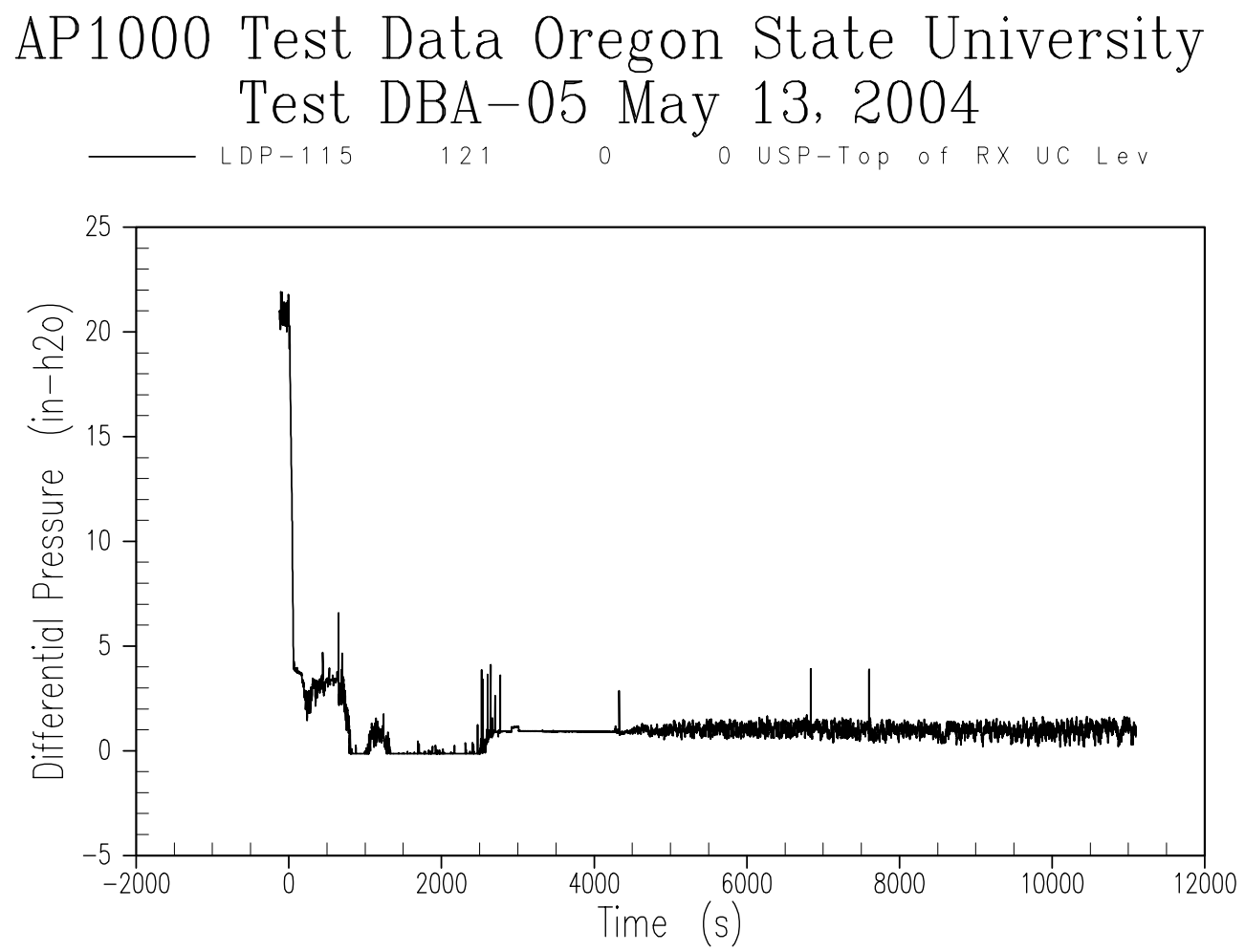

Figure A-71 Reactor Vessel Liquid Level Between Top of Vessel - Upper Support Plate 

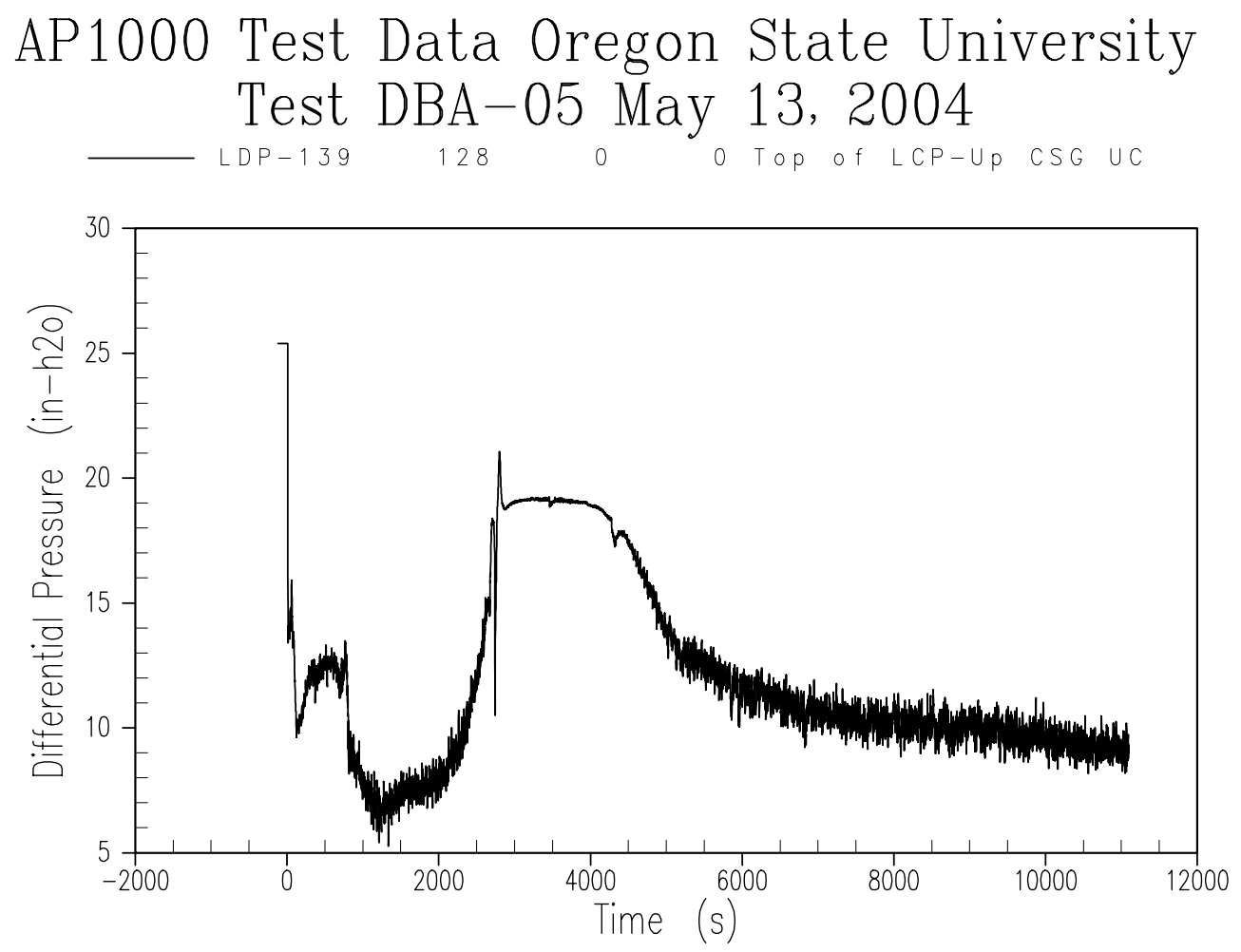

Figure A-72 Reactor Vessel Liquid Level Between Bottom of Upper Support Plate Upper Core Spacer Grid 

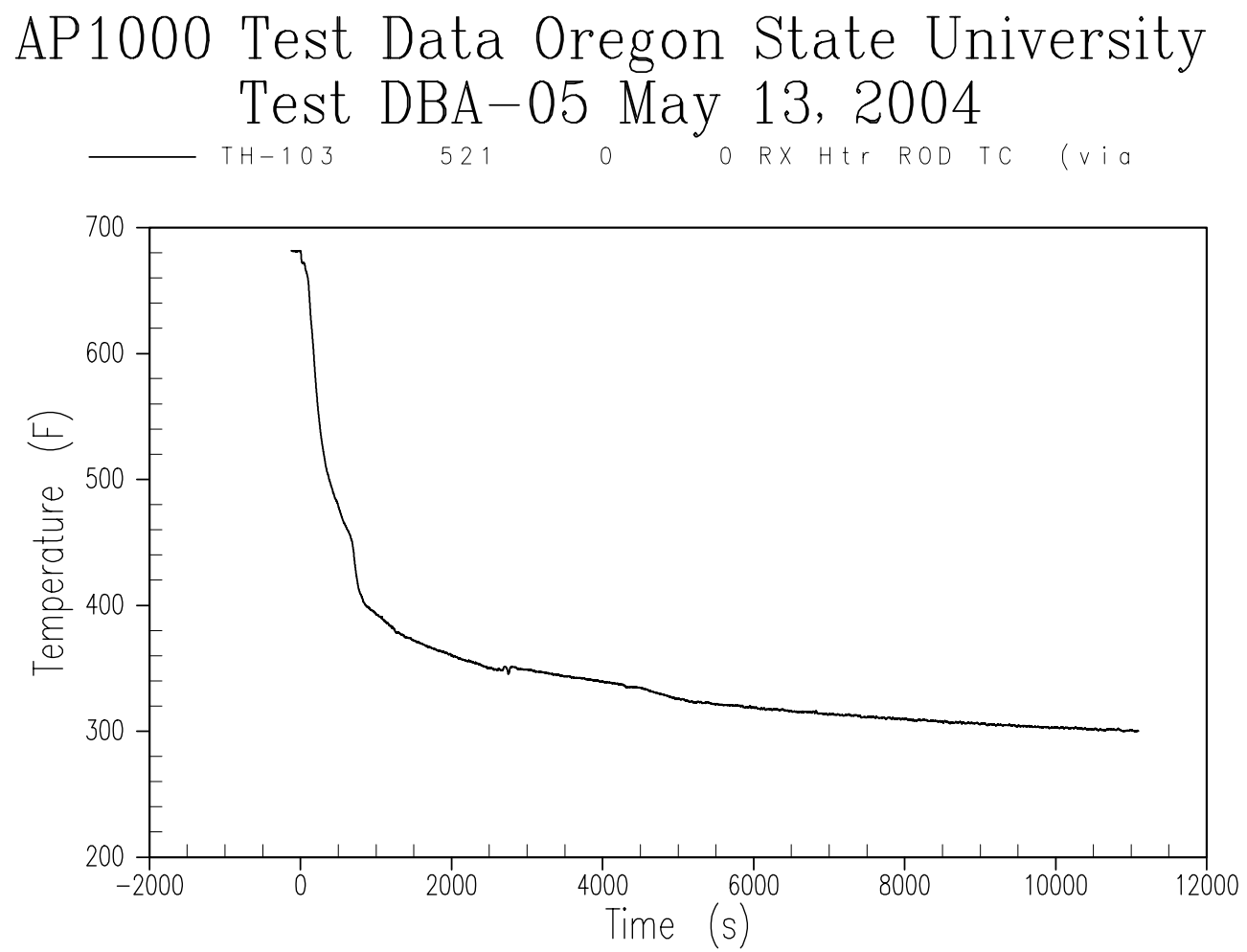

Figure A-73 Inner Core Thermocouple Measuring Heater Temperature 

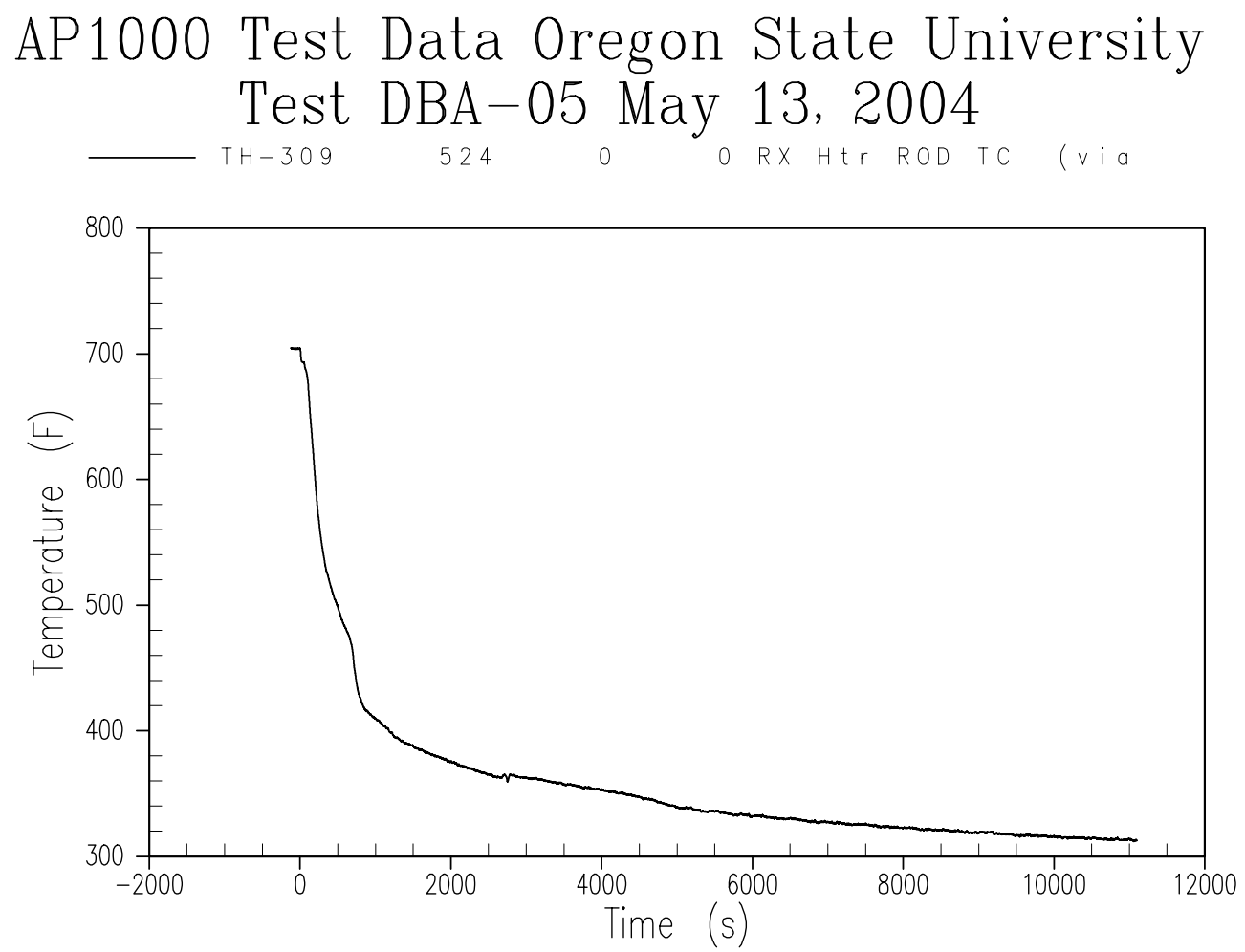

Figure A-74 Outer Core Thermocouple Measuring Heater Temperature 


\section{APPENDIX B}

TEST DATA 


\title{
Oregon State University
}

Department of Nuclear Engineering

\author{
AdVANCED THERMAL HYdRAuliC \\ RESEARCH LABORATORY
}

TEST SUMMARY REPORT

OSU-AP1000-11

DBA-06-D

AP1000 0.5-INCH COLD LEG BREAK WITH 3 OF 4 ADS-4

Revision 0 


\title{
TEST SUMMARY REPORT
}

\author{
OSU-AP1000-11
}

DBA-06-D

\section{AP1000 0.5-INCH COLD LEG BREAK WITH 3 OF 4 ADS-4}
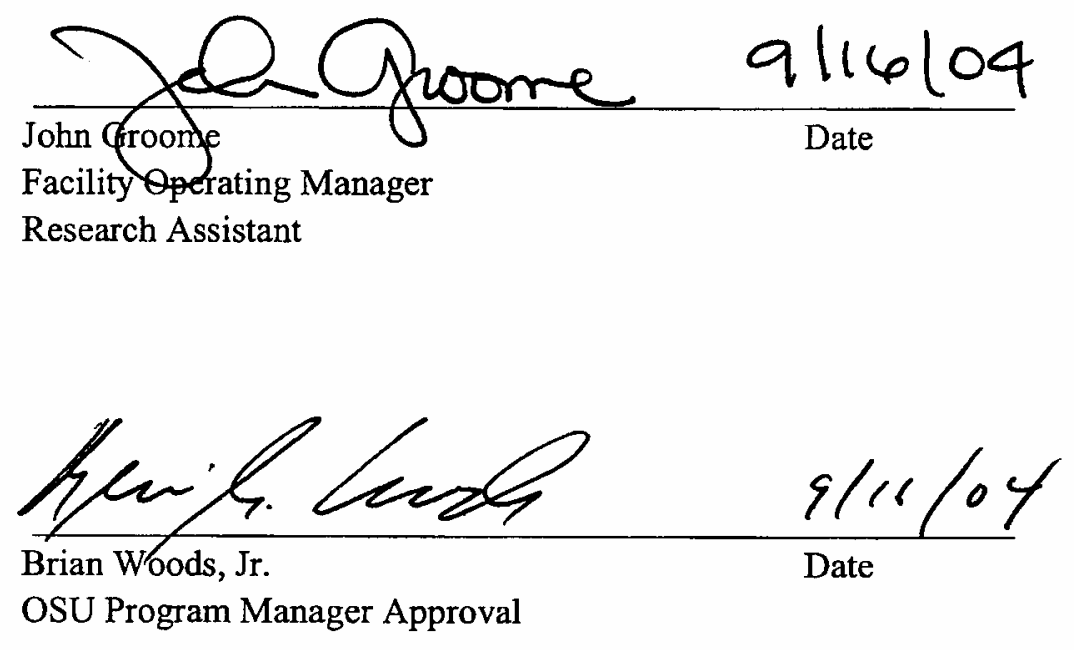

Oregon State University 116 Radiation Center

Corvallis, Oregon 97331

(C) 2004 Oregon State University

All Rights Reserved 


\section{WESTINGHOUSE PROPRIETARY INFORMATION NOTICE}

This report contains information proprietary to Westinghouse Electric Company. A non-proprietary version of this report has also been prepared for distribution to the United States Nuclear Regulatory Commission (NRC).

In order to conform to the requirements of 10 CFR 2.790 of the Commission's regulations concerning the protection of proprietary information so submitted to the NRC, the information which is proprietary in the proprietary version of this document is contained within brackets, and where the proprietary information has been deleted in the non-proprietary versions, only the brackets remain (the information that was contained within the brackets in the proprietary versions having been deleted). The justification for claiming the information so designated as proprietary is indicated in both versions by means of lower case letters (a) through (f) located as a superscript immediately following the brackets enclosing each item of information being identified as proprietary or in the margin opposite such information. These lower case letters refer to the types of information Westinghouse customarily holds in confidence identified in Sections (4)(ii)(a) through (4)(ii)(f) of the affidavit accompanying the transmittal of these documents pursuant to 10 CFR $2.790(b)(1)$.

\section{COPYRIGHT NOTICE}

This document bears an Oregon State University copyright notice. The NRC is permitted to make the number of copies of the information contained in these reports which are necessary for its internal use in connection with generic and plant-specific reviews and approvals as well as the issuance, denial, amendment, transfer, renewal, modification, suspension, revocation, or violation of a license, permit, order, or regulation subject to the requirements of 10 CFR 2.790 regarding restrictions on public disclosure to the extent such information has been identified as proprietary by Westinghouse, copyright protection notwithstanding. With respect to the non-proprietary versions of these reports, the NRC is permitted to make the number of copies beyond these necessary for its internal use which are necessary in order to have one copy available for public viewing in the appropriate docket files in the public document room in Washington, DC and in local public document rooms as may be required by NRC regulations if the number of copies submitted is insufficient for this purpose. Copies made by the NRC must include the copyright notice in all instances and the proprietary notice if the original was identified as proprietary. 
TABLE OF CONTENTS

$\underline{\text { Section }}$

Title

Page

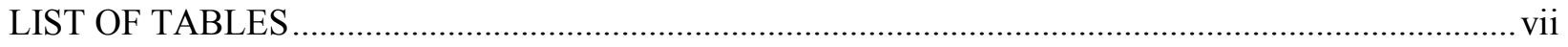

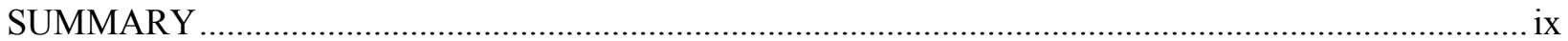

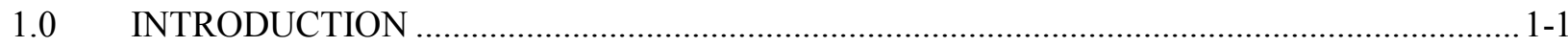

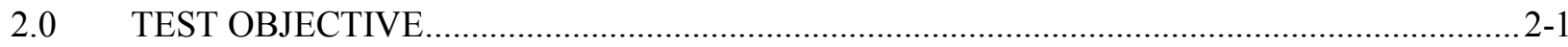

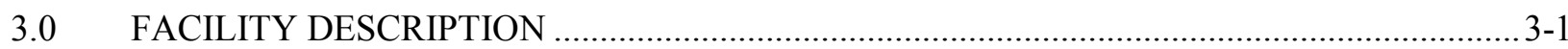

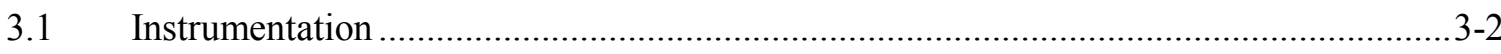

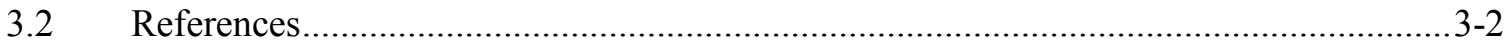

4.0 OREGON STATE UNIVERSITY TESTING PROGRAM MATRIX .......................................4-1

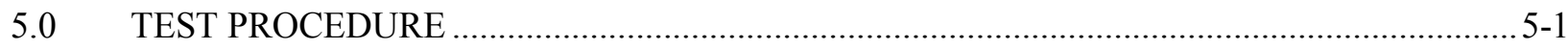

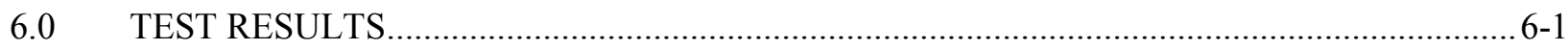

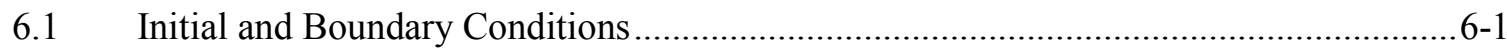

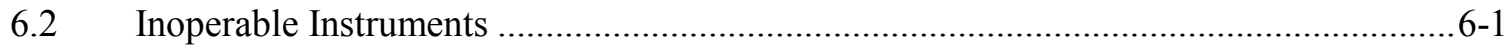

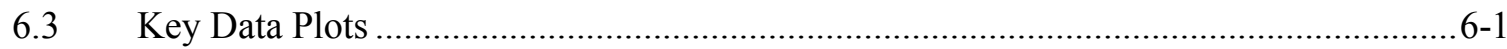

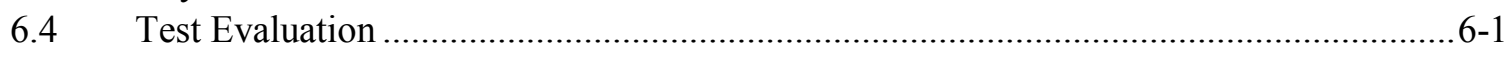

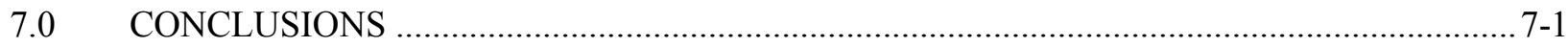

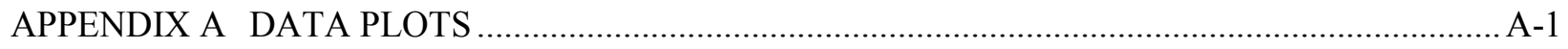

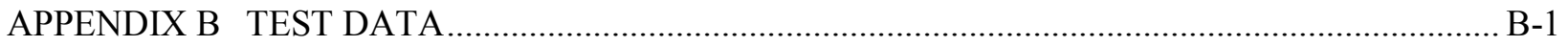




\section{LIST OF TABLES}

$\underline{\text { Table }}$

Table 4-1

Table 6-1

Table 6-2

Table 6-3

Table 6-4 $\underline{\text { Title }}$

Page

OSU Test Matrix (as specified June 3, 2004) $4-2$

Actual Test Initial Conditions..... $6-2$

Sequence of Events 6-4

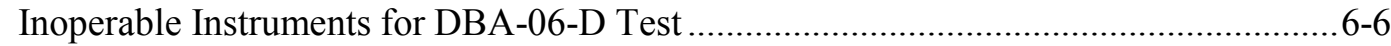

Data Plots for QL Reports for DBA-06-D by Component 6-7 


\section{SUMMARY}

This report covers the test DBA-06-D, 0.5-inch cold leg break simulation loss-of-coolant accident, performed on June 3, 2004. This test has been designated OSU-AP1000-10 by the Advanced Plant Experiment (APEX). The objective of this test was to obtain thermal-hydraulic data for a 0.5 -inch cold leg break simulation. The test performed met the specified conditions. The top of the heater bundle was always covered during this event. The test was performed for about 15,750 seconds. The transient continued through Automatic Depressurization System actuation and achieved stable in-containment refueling water storage tank injection.

This report presents the initial assessment of the test data collected. If this test is to be used by Westinghouse to support AP1000 Design Certification, additional validation of the use of this information will be documented separately. In the interim, the list of invalid data channels may change. 


\subsection{INTRODUCTION}

The Department of Nuclear Engineering at Oregon State University (OSU) is performing a series of tests for the U.S. Department of Energy. These tests are being conducted in the Advanced Plant Experiment (APEX-1000) test facility, which is a reduced pressure and height model of the two-loop Westinghouse AP1000 pressurized water reactor. The purpose of the testing is to:

- evaluate the thermal-hydraulic performance of the passive safety systems of the full-scale AP1000, and

- assess and validate the safety analyses techniques and computer codes used in predicting the transient system behavior.

The AP1000 Long-Term Cooling Test is a 1/4 height scale, low-pressure integral systems test simulating thermal-hydraulic phenomenon for the AP1000 passive safety systems for small-break loss-of-coolant accidents (LOCAs) and long-term cooling. It accurately models the details of the AP1000 geometry, including the primary system, the passive safety systems, and a part of the non-safety grade Chemical and Volume Control System, as well as a partial non-safety grade Normal Residual Heat Removal System. The interconnecting pipe routings are also duplicated in the model.

The overall objective of the Long-Term Cooling Test program is to obtain test data at various modes of operation. The OSU experiments will examine the passive safety system response for the small-break and large-break LOCA transition into long-term cooling. (The list of the tests to be performed is in the OSU Test Matrix provided in Section 4.0.) The facility permits a range of small-break LOCAs to be simulated at different locations on the primary system, such as the cold leg, hot leg, core makeup tank (CMT) cold leg pressure balance line, and direct vessel injection (DVI) line. The break orientation (top or bottom of the cold leg) may also be studied. Selected tests continue into the long-term cooling, post-accident mode in which the passive safety injection is from the reactor sump as well as the in-containment refueling water storage tank (IRWST). 


\subsection{TEST OBJECTIVE}

The purpose of this test is to simulate a 0.5 -inch cold leg break LOCA. The assumed single failure for this test is one of four Automatic Depressurization System 4 (ADS-4) valves. The single failure location is on the non-pressurizer hot leg. The data obtained from the test will be used to verify the AP1000 thermal-hydraulic computer codes for AP1000 phenomena, such as gravity injection, natural convection, and post-accident long-term core cooling behavior.

The acceptance criteria for the OSU tests are as follows:

- Test initial conditions will be achieved within a specified tolerance.

- Setpoints will be achieved within an acceptable tolerance band.

- All instrumentation should be operational before the test.

- Any critical instruments not operating will be identified to the test engineer before the tests. These instruments must be operational before and during the test or exceptions should be approved.

- A zero check of LDPs, DPs, and FDPs will be performed. 


\subsection{FACILITY DESCRIPTION}

A detailed facility description for the OSU test facility is documented in Reference 3-1. The OSU test facility has been specifically scaled, designed, and constructed to investigate the AP600 passive safety system behavior and to provide data for safety analysis computer code validation. The facility has been modified to model the AP1000.

The scaled test design accurately models the details of the AP1000 geometry, including the primary system and the pipe routings and layout for the passive safety systems. A detailed scaling report (Reference 3-2) was used to develop the test design modifications. The primary system consists of one hot leg and two cold legs with two active pumps and an active steam generator for each loop. Two CMTs are connected to one primary loop, and the pressurizer is connected to the other primary loop as in the AP1000 plant design. Gas-driven accumulators are connected to the DVI lines. The discharge lines from the CMT, and one-of-two IRWST and reactor sump lines are connected to each DVI line. The ADS consisting of stages 1,2 , and 3 - simulates either one or two of the independent trains used in the AP1000. The two-phase flow from the ADS stages 1-to-3 is separated in a swirl-vane separator, and the liquid and vapor flows are measured to obtain the total ADS flow rate. The separated flow streams are then recombined and discharged into the IRWST through a sparger, preserving the mass and energy flow into the IRWST. The injection from the reactor sump is also simulated. Note that the OSU facility models both AP1000 primary and secondary sumps. The primary sump collects the condensate return, the liquid break flow, and the liquid flow from the fourth-stage ADS; and will provide long-term injection to the reactor vessel. The secondary sump simulates the portions of containment that will remain dry during most events. This sump collects water only when the primary sump reaches its overflow level, and provides no injection to the reactor vessel.

The time period for the experimental simulations includes not only the IRWST injection, but also the draining of the IRWST and the sump injection to simulate the long-term cooling of the AP1000. This simulation could be from several hours to a day. The time scale for the OSU test facility is one-half; that is, events occur in half the normal time. To model the long-term cooling aspects of the transient, the two-phase flow from the break is separated in a swirl-vane separator and the liquid and vapor portions of the total flow are measured. The liquid fraction of the flow is discharged to the reactor primary sump as in the AP1000 plant. The vapor is discharged to the atmosphere. The capability exists to return a portion of the equivalent liquid flow to the IRWST and primary sump to simulate the condensate return from the passive containment to the IRWST and primary sump. A similar approach is also used for the fourth-stage ADS valve on the hot leg. The two-phase flow is separated in a swirl-vane separator, the two streams are measured, and the liquid phase is discharged into the primary sump while the vapor flow is discharged to the atmosphere. Again, the capability exists to return a portion of the liquid equivalent added to the IRWST and primary sump. In addition, all other steam vents from the facility are measured (for example, the IRWST vent), and a portion of the liquid equivalent may be added back to the facility. Note that not all of the steam discharge would be returned as liquid equivalent. A portion of the discharge would be removed to simulate the steam that is not available for recirculation because it provides containment pressurization. The IRWST and primary sump can be pressurized in the OSU facility to simulate the containment pressurization following a postulated LOCA.

A multi-tube passive residual heat removal (PRHR) heat exchanger is located in the IRWST. The heat exchanger uses the same C-tube design as the AP1000 and has two instrumented tubes to obtain wall heat 
fluxes during the tests. There are primary fluid thermocouples, wall thermocouples, and differential pressure drop measurements to determine when the heat exchanger begins to drain. The IRWST is also instrumented with strings of fluid thermocouples to determine the degree of mixing within the tank and to assess the temperature of the coolant that is delivered to the test vessel.

The reactor vessel for the OSU tests includes a 0.914-meter (3-foot) heated core consisting of forty-eight 0.025 -meter (1-inch) diameter heater rods. The heater rods have a top skewed power shape. The $1000 \mathrm{~kW}$ of electrical power available at the OSU test site will be used to simulate decay heat. Wall thermocouples are swaged inside the heater rods to measure the heater rod wall temperature.

Thermocouple rods in the heater rod bundle measure the axial coolant temperature distribution. The scaled flow volume in the core is preserved as well as the flow volume in the test vessel upper plenum. There are simulated reactor internals in the upper plenum to preserve the flow area and to correctly scale the fluid volume. The reactor vessel includes an annular downcomer into which the four cold legs and the two DVI lines are connected. The hot legs penetrate the reactor annulus and connect with the loops. The AP1000 reactor vessel neutron reflector is simulated using a ceramic liner to reduce the metal heat release to the coolant.

There were no special/unique requirements for the test other than those specified in the Initial Conditions. The specified conditions were verified on the control board prior to test implementation.

\subsection{Instrumentation}

The instrumentation has been designed to calculate a transient mass and energy balance on the test facility. All two-phase flow streams exiting the facility are separated, and each component is measured separately as a single-phase flow using conventional measurement devices, such as magnetic flow meters and vortex flow meters. Note that magnetic flow meters are not designed for two-phase flow and will indicate erratically. Also, the vortex flow meters are referenced to $212^{\circ} \mathrm{F}$ and the LDPs are referenced to $60^{\circ} \mathrm{F}$. All vertical components have differential pressure cells that act as level instruments to measure the transient mass change in the component. The hot and cold leg diameters are sufficiently large in the OSU test facility so that a narrow-range differential pressure cell can be used to determine if the flow becomes stratified.

Single flow measurements are made of the CMT, accumulator, IRWST, and sump flows into the reactor vessel through the DVI lines.

Various types of instrumentation are provided in the test facility; for example, thermocouples for coolant and wall temperatures, flow meters, pressure transducers, differential pressure transducers, and weigh tanks.

\subsection{References}

3-1 APP-LTCT-T2R-005, OSU APEX-1000 Test Facility Description Report

3-2 APP-LTCT-T2R-004, Scaling Assessment for the Design of the OSU APEX-1000 Test Facility 


\subsection{OREGON STATE UNIVERSITY TESTING PROGRAM MATRIX}

The test matrix for the OSU test facility is shown in Table 4-1. To satisfy the test objectives, several transients will be performed to provide data on the AP1000 passive safety system response for a range of break sizes, locations, orientations, and single failure assumptions. The break size orifices are scaled based on simulating a 0.5 -inch, 1-inch, 2 -inch, or larger pipe break.

The designation for this test is DBA-06-D, which identifies the test as a design basis inadvertent ADS actuation. The test matrix may be adjusted for future tests based on results and insights gained with each test. 


\begin{tabular}{|c|c|c|}
\hline \multicolumn{3}{|c|}{$\begin{array}{c}\text { TABLE 4-1 } \\
\text { OSU TEST MATRIX (AS SPECIFIED JUNE 3, 2004) }\end{array}$} \\
\hline Test Title & Break Location and Size & Single Failure Assumed \\
\hline DBA-01-D & $\begin{array}{l}\text { Double-ended DVI Line break with } \\
\text { continuation into long-term cooling }\end{array}$ & Fail 1 of 2 lines in one ADS- 4 train \\
\hline DBA-02-D & $\begin{array}{l}\text { Double-ended DVI Line break with } \\
\text { continuation into long-term cooling }\end{array}$ & $\begin{array}{l}\text { Fail } 1 \text { of } 2 \text { lines in one ADS- } 4 \text { train } \\
\text { (Adjusted ADS-4 Resistance) }\end{array}$ \\
\hline DBA-03-D & $\begin{array}{l}\text { Double-ended DVI Line break with } \\
\text { continuation into long-term cooling }\end{array}$ & $\begin{array}{l}\text { Fail } 1 \text { of } 2 \text { lines in one ADS- } 4 \text { train } \\
\text { (Failure location sensitivity) }\end{array}$ \\
\hline TR-01-D & $\begin{array}{l}\text { Transition Test ADS-4 opening, } 85 \text { psig initial } \\
\text { pressure and decay power } 480 \mathrm{sec}\end{array}$ & $\begin{array}{l}\text { Fail } 1 \text { of } 2 \text { lines in one ADS-4 train } \\
\text { (No ADS 1-3) }\end{array}$ \\
\hline TR-02-D & $\begin{array}{l}\text { Transition Test ADS-4 opening, } 100 \text { psig initial } \\
\text { pressure with prototypic plant conditions }\end{array}$ & $\begin{array}{l}\text { Fail } 1 \text { of } 2 \text { lines in one ADS-4 train } \\
\qquad(\text { No ADS 1-3) }\end{array}$ \\
\hline DBA-04-D & 2-inch Cold Leg Break & $\begin{array}{l}\text { Fail } 1 \text { of } 2 \text { lines in one ADS- } 4 \text { train } \\
\text { (Failure on non-pressurizer hot leg) }\end{array}$ \\
\hline ADS-01-D & $\begin{array}{l}\text { ADS-4 Two-Phase Characterization Test at } \\
\text { low-power conditions (steady-state points) }\end{array}$ & $\begin{array}{l}\text { Various ADS-4 line configurations } \\
\qquad \text { (No ADS 1-3) }\end{array}$ \\
\hline ADS-02-D & $\begin{array}{l}\text { ADS-4 Two-Phase Characterization Test at } \\
\text { high-power conditions (steady-state points) }\end{array}$ & $\begin{array}{l}\text { Various ADS-4 line configurations } \\
\text { (No ADS 1-3) }\end{array}$ \\
\hline DBA-03R-D & Repeat of Test DBA-03-D & $\begin{array}{l}\text { Fail } 1 \text { of } 2 \text { lines in one ADS- } 4 \text { train } \\
\text { (Failure on pressurizer hot leg) }\end{array}$ \\
\hline DBA-05-D & Inadvertent ADS-1 Actuation & $\begin{array}{l}\text { Fail } 1 \text { of } 2 \text { lines in one ADS- } 4 \text { train } \\
\text { (Failure on non-pressurizer hot leg) }\end{array}$ \\
\hline DBA-06-D & 0.5-inch Cold Leg Break & $\begin{array}{l}\text { Fail } 1 \text { of } 2 \text { lines in one ADS- } 4 \text { train } \\
\text { (Failure on non-pressurizer hot leg) }\end{array}$ \\
\hline
\end{tabular}




\subsection{TEST PROCEDURE}

The test was performed per a written procedure. There were no special/unique requirements for the test other than those specified in the initial conditions in Table 6-1. The specified conditions were checked on the control board before test implementation.

The appropriate prerequisites were completed and initial conditions were satisfied. The required break simulation piping and break instrumentation were installed per P\&ID drawing OSU 600904, Rev. 1. A break spool insert simulating the 0.5 -inch cold leg break in AP1000 was installed in the break spool in cold leg 3, which is a cold leg without a CMT balance line. Flow from the break is directed horizontally into the break separator. The 100-percent flow nozzle was installed in the ADS 4-2 (on hot leg 2), and the 50-percent flow nozzle was installed in ADS 4-1 (on hot leg 1). Flow nozzles that simulate full flow for ADS-1, ADS-2, and ADS-3 were installed. As per the AP600 tests, ADS-3 has been scaled for full flow from all three stages, and ADS-1 and ADS-2 are closed when ADS-3 is opened.

Fill and vent was performed per APEX Operations Manual Procedure OP-B.2. Instruments were checked for required calibration.

With the break valve TS-202 closed, flow was used to warm up the bypass line by opening isolation valves RCS-901 and RCS-902. After the appropriate prerequisites were completed and the test facility achieved specified initial conditions, the CMT warmup bypass line isolation valves RCS-901 and RCS- 902 were closed to maintain the $<80^{\circ} \mathrm{F}$ condition at the top of the CMT-1. With the CMT balance line valves (RCS-529 and RCS-530) placed in the open and automatic mode, both CMTs reached the Reactor Coolant System pressure.

Once all other initial conditions were satisfied, a break through TS-202 was initiated. The transient continued through ADS actuation; and CMT, accumulator, IRWST, and sump injection. Per Westinghouse instructions, ADS 4-2 was actuated automatically according to the actuation logic and ADS 4-1 was actuated manually after a 30-second delay. All other actions were automatic and required no operator action. 


\subsection{TEST RESULTS}

The test results for test DBA-06-D are provided in the following subsections.

\subsection{Initial and Boundary Conditions}

Table 6-1 provides a comparison of the specified and actual conditions for test DBA-06-D. The values in this table were averaged over approximately 2 minutes preceding the test. Test initial conditions were achieved for the steam generator pressure, pressurizer pressure, pressurizer level, steam generator 01 narrow-range level, and steam generator 02 narrow-range level. Test initial conditions for the hot leg temperature were found to be acceptable, and the results will not be adversely affected.

The actual power decay curves are provided in data plots in Appendix B. The measured maximum power was $863 \mathrm{~kW}$, which was less than the facility maximum power of $1000 \mathrm{~kW}$. The programmed decay heat curve was adjusted to account for this difference, and the differences between the actual and specified power decay are considered acceptable.

PT-501 and PT-502 pressure instruments indicate the pressure changes in the CMT-1 and CMT-2. CMT-1 (PT-501) and CMT-2 (PT-502) confirm that 1 minute after the test button was pushed, both CMTs reach Reactor Coolant System pressure.

The sequence of events is shown in Table 6-2. This table compares the actual sequence of events with the specified timing. As can be seen in this table, all the events occurred at or very near to when the event was planned.

\subsection{Inoperable Instruments}

Table 6-3 lists the instrumentation channels considered inoperable for the DBA-06-D test.

\subsection{Key Data Plots}

Table 6-4 lists the instrumentation channels sorted by component and includes the instrument number, units, and quick look (QL) plot number. The selection of channels was based on projecting an overall picture of the test results, which would then be examined by referring to the detailed data plots or tapes.

\subsection{Test Evaluation}

The following observations were made during the test:

1. The peak power before the test was initiated was $933 \mathrm{~kW}$. The decay heat curve was adjusted for this value. 


\begin{tabular}{|c|c|c|c|}
\hline \multicolumn{4}{|c|}{$\begin{array}{c}\text { TABLE 6-1 } \\
\text { ACTUAL TEST INITIAL CONDITIONS }\end{array}$} \\
\hline Conditions & Instrument No. & Actual & Comment \\
\hline Pressurizer Pressure & PT-604 & 370 psig & \\
\hline Hot Leg Temperature \#1 & $\begin{array}{l}\text { TF-141* } \\
\text { TF-205 } \\
\text { TF-143 }\end{array}$ & $\begin{array}{l}412^{\circ} \mathrm{F} \\
413^{\circ} \mathrm{F} \\
413^{\circ} \mathrm{F}\end{array}$ & \\
\hline Hot Leg Temperature \#2 & $\begin{array}{l}\text { TF-140* } \\
\text { TF-206 } \\
\text { TF-142 }\end{array}$ & $\begin{array}{l}414^{\circ} \mathrm{F} \\
413^{\circ} \mathrm{F} \\
415^{\circ} \mathrm{F}\end{array}$ & \\
\hline $\begin{array}{l}\text { Steam Generator Pressure } \\
\quad \# 1 \\
\# 2 \\
\text { Header }\end{array}$ & $\begin{array}{l}\text { PT-301* } \\
\text { PT-302* } \\
\text { PT-002 }\end{array}$ & $\begin{array}{l}245 \text { psig } \\
246 \text { psig } \\
205 \text { psig }\end{array}$ & \\
\hline Pressurizer Level & $\begin{array}{l}\text { LDP-601 uncompensated } \\
\text { LDP-601 } \\
\text { Compensated by SC-608 }\end{array}$ & $\begin{array}{l}26.5 \text { inches } \\
31.9 \text { inches }\end{array}$ & $\begin{array}{l}415^{\circ} \mathrm{F} \text { used for density } \\
\text { compensation }\end{array}$ \\
\hline $\begin{array}{l}\text { Steam Generator Level } \\
\# 1 \mathrm{NR}\end{array}$ & $\begin{array}{l}\text { LDP-303 uncompensated } \\
\text { LDP-303 compensated by } \\
\text { average of TF-305 and } \\
\text { TF-307 }\end{array}$ & $\begin{array}{l}20.5 \text { inches } \\
24.7 \text { inches }\end{array}$ & $\begin{array}{l}400^{\circ} \mathrm{F} \text { used for density } \\
\text { compensation }\end{array}$ \\
\hline $\begin{array}{l}\text { Steam Generator Level } \\
\text { \#2 NR }\end{array}$ & $\begin{array}{l}\text { LDP-304 uncompensated } \\
\text { LDP-304 compensated by } \\
\text { average of TF-306 and } \\
\text { TF-308 }\end{array}$ & $\begin{array}{l}21.7 \text { inches } \\
26.2 \text { inches }\end{array}$ & $\begin{array}{l}400^{\circ} \mathrm{F} \text { used for density } \\
\text { compensation }\end{array}$ \\
\hline IRWST Temperature & TF-701 & $70^{\circ} \mathrm{F}$ & Accepted $\left(<80^{\circ} \mathrm{F}\right)$ \\
\hline $\begin{array}{l}\text { CMT Temperature } \\
\text { \#1 } \\
\# 2\end{array}$ & $\begin{array}{l}\text { TF-513 } \\
\text { TF-516 }\end{array}$ & $\begin{array}{l}72.5^{\circ} \mathrm{F} \\
73^{\circ} \mathrm{F}\end{array}$ & Accepted $\left(<80^{\circ} \mathrm{F}\right)$ \\
\hline $\begin{array}{l}\text { Accumulator } \\
\text { Temperature } \\
\# 1 \\
\# 2\end{array}$ & $\begin{array}{l}\text { TF-403 } \\
\text { TF-404 }\end{array}$ & $\begin{array}{l}76.9^{\circ} \mathrm{F} \\
76.5^{\circ} \mathrm{F}\end{array}$ & Accepted $\left(<80^{\circ} \mathrm{F}\right)$ \\
\hline IRWST Level & LDP-701 & 91.9 inches & \\
\hline
\end{tabular}




\begin{tabular}{||c|c|c|c||}
\hline \multicolumn{5}{|c||}{ TABLE 6-1 (Continued) } \\
\multicolumn{4}{||c||}{ ACTUAL TEST INITIAL CONDITIONS } \\
\hline \hline Conditions & Instrument No. & Actual & Comment \\
\hline Accumulator Level & & & \\
$\# 1$ & LDP-401 & 36.3 inches & \\
$\# 2$ & LDP-402 & 36.9 inches & \\
\hline Accumulator Pressure & & & \\
$\# 1$ & PT-401 & $188.0 \mathrm{psig}$ & \\
$\# 2$ & PT-402 & $186.3 \mathrm{psig}$ & \\
\hline CMT Level & & & \\
$\# 1$ & LDP-507 & 57.5 inches & \\
$\# 2$ & LDP-502 & 57.5 inches & \\
\hline CMT Pressure & & & \\
$\# 1$ & PT-501 & $372.8 \mathrm{psig}$ & \\
$\# 2$ & PT-502 & & \\
\hline
\end{tabular}

* These instruments are used to establish initial conditions at the test site. 


\begin{tabular}{|c|c|c|}
\hline \multicolumn{3}{|c|}{$\begin{array}{c}\text { TABLE 6-2 } \\
\text { SEQUENCE OF EVENTS }\end{array}$} \\
\hline Event & Setpoint & Actual Time (sec) \\
\hline PB Depressed & $\mathrm{N} / \mathrm{A}$ & -120 \\
\hline Break Valve(s) Open & 0 & 0 \\
\hline Feed Pump Trip & $3.6 \mathrm{sec}$ & 185 \\
\hline CMT \#1 Outlet Valve Open (RCS-535) & $6.1 \mathrm{sec}$ & 188 \\
\hline CMT \#2 Outlet Valve Open (RCS-536) & $6.1 \mathrm{sec}$ & 188 \\
\hline PRHR HX Outlet Valve Open (RCS-804) & $6.1 \mathrm{sec}$ & 188 \\
\hline Reactor Coolant Pump \#1 Trip & $8.6 \mathrm{sec}$ & 242 \\
\hline Reactor Coolant Pump \#2 Trip & $8.6 \mathrm{sec}$ & 242 \\
\hline Reactor Coolant Pump \#3 Trip & $8.6 \mathrm{sec}$ & 242 \\
\hline Reactor Coolant Pump \#4 Trip & $8.6 \mathrm{sec}$ & 242 \\
\hline CMT \#1 Level Low (LDP-507) & 41 inches & 3500 \\
\hline CMT \#2 Level Low (LDP-502) & 41 inches & 3820 \\
\hline ADS \#1 Actuation (RCS-601) & CMT Level Low $+15 \mathrm{sec}$ & 3715 \\
\hline ADS \#2 Actuation (RCS-602) & CMT Level Low $+62 \mathrm{sec}$ & 3762 \\
\hline ADS \#3 Actuation (RCS-603) & CMT Level Low $+122 \mathrm{sec}$ & 3822 \\
\hline Low Reactor Pressure (P-107) & 40 psig & 4072 \\
\hline IRWST Valve Actuation (RCS-711) & $\begin{array}{l}\text { Low Reactor Pressure } \\
\qquad(<40 \mathrm{psig})\end{array}$ & 4072 \\
\hline IRWST Valve Actuation (RCS-712) & $\begin{array}{l}\text { Low Reactor Pressure } \\
\qquad(<40 \text { psig })\end{array}$ & 4072 \\
\hline CMT \#1 Low Low Level (LDP-507) & 17.14 inches & 3990 \\
\hline CMT \#2 Low Low Level (LDP-502) & 17.14 inches & 4040 \\
\hline ADS 4-1 Actuation (RCS-615) & $\begin{array}{l}\text { CMT Low Low (17.14") and } \\
\text { CMT Low }\left(41^{\prime \prime}\right)+180 \mathrm{sec}\end{array}$ & 4028 \\
\hline ADS 4-2 Actuation (RCS-616) & $\begin{array}{l}\text { CMT Low Low (17.14") and } \\
\text { CMT Low (41") + } 180 \mathrm{sec}\end{array}$ & 4058 \\
\hline Sump Valve Actuation (CSS-909) & IRWST Level Low Low & 12905 \\
\hline
\end{tabular}




\begin{tabular}{|c|c|c|}
\hline \multicolumn{3}{|c|}{$\begin{array}{c}\text { TABLE 6-2 (Continued) } \\
\text { SEQUENCE OF EVENTS }\end{array}$} \\
\hline Event & Setpoint & Actual Time (sec) \\
\hline Sump Valve Actuation (CSS-910) & IRWST Level Low Low & 12905 \\
\hline $\begin{array}{l}\text { Accumulator Injection } \\
\# 1 \text { (FMM-401) }\end{array}$ & N/A & 3825 \\
\hline $\begin{array}{l}\text { Accumulator Injection } \\
\text { \#2 (FMM-402) }\end{array}$ & N/A & 3830 \\
\hline $\begin{array}{l}\text { IRWST Injection } \\
\text { DVI \#1 (FMM-701) }\end{array}$ & N/A & 4500 \\
\hline $\begin{array}{l}\text { IRWST Injection } \\
\text { DVI \#2 (FMM-702) }\end{array}$ & N/A & 4500 \\
\hline $\begin{array}{l}\text { Accumulator Empty } \\
\text { \#1 (LDP-401) }\end{array}$ & $\mathrm{N} / \mathrm{A}$ & 4220 down to 1.2 -inch level \\
\hline $\begin{array}{l}\text { Accumulator Empty } \\
\text { \#2 (LDP-402) }\end{array}$ & N/A & 4260 down to 0.1 -inch level \\
\hline $\begin{array}{l}\text { CMT Empty } \\
\# 1 \text { (LDP-507) }\end{array}$ & N/A & 4100 down to 0.1 -inch level \\
\hline $\begin{array}{l}\text { CMT Empty } \\
\text { \#2 (LDP-502) }\end{array}$ & N/A & 4200 \\
\hline Sump Injection DVI \# 1 (FMM-901) & $\mathrm{N} / \mathrm{A}$ & 12905 \\
\hline Sump Injection DVI \# 2 (FMM-902) & N/A & 12905 \\
\hline
\end{tabular}




\begin{tabular}{|c|c|c|}
\hline \multicolumn{3}{|c|}{$\begin{array}{c}\text { TABLE 6-3 } \\
\text { INOPERABLE INSTRUMENTS FOR DBA-06-D TEST }\end{array}$} \\
\hline Instrument Number & Instrument Type & Inoperable Description \\
\hline $\begin{array}{l}\text { TW-202 } \\
\text { TW-204 } \\
\text { TW-205 } \\
\text { TW-206 } \\
\text { TW-209 } \\
\text { TW-803 } \\
\text { TW-804 }\end{array}$ & Thermocouple & Inoperative \\
\hline TH-603 & $\begin{array}{l}\text { Thermocouple measuring heater } \\
\text { temperature }\end{array}$ & Inoperative \\
\hline FMM-202 & Magnetic flow meter & Inoperative \\
\hline $\begin{array}{l}\text { TF-170 } \\
\text { TF-221 } \\
\text { TF-509 } \\
\text { TF-512 }\end{array}$ & $\begin{array}{l}\text { Thermocouple measuring fluid } \\
\text { temperature }\end{array}$ & Inoperative \\
\hline FVM-905 & Vortex flow meter & Erratic \\
\hline
\end{tabular}




\begin{tabular}{|c|c|c|c|c|}
\hline \multicolumn{5}{|c|}{$\begin{array}{c}\text { TABLE 6-4 } \\
\text { DATA PLOTS FOR QL REPORTS FOR DBA-06-D BY COMPONENT }\end{array}$} \\
\hline Component & Channel & Units & QL-Plot & Comment \\
\hline $\begin{array}{l}\text { Reactor Vessel } \\
\text { Pressure }\end{array}$ & PT-107 & psig & 1 & \\
\hline Reactor Vessel Level & LDP-127 & inch of $\mathrm{H}_{2} \mathrm{O}$ & 2 & \\
\hline $\begin{array}{l}\text { Reactor Vessel } \\
\text { Downcomer Level }\end{array}$ & LDP-140 & inch of $\mathrm{H}_{2} \mathrm{O}$ & 3 & \\
\hline $\begin{array}{l}\text { Cold Leg \#1 Fluid } \\
\text { Temperature }\end{array}$ & TF-107 & ${ }^{\circ} \mathrm{F}$ & 4 & \\
\hline $\begin{array}{l}\text { Cold Leg \#2 Fluid } \\
\text { Temperature }\end{array}$ & TF-108 & ${ }^{\circ} \mathrm{F}$ & 5 & \\
\hline $\begin{array}{l}\text { Cold Leg \#3 Fluid } \\
\text { Temperature }\end{array}$ & TF-103 & ${ }^{\circ} \mathrm{F}$ & 6 & \\
\hline $\begin{array}{l}\text { Cold Leg \#4 Fluid } \\
\text { Temperature }\end{array}$ & TF-104 & ${ }^{\circ} \mathrm{F}$ & 7 & \\
\hline $\begin{array}{l}\text { Reactor Vessel Fluid } \\
\text { Temperature Upper } \\
\text { Head }\end{array}$ & TF-120 & ${ }^{\circ} \mathrm{F}$ & 8 & \\
\hline $\begin{array}{l}\text { Reactor Coolant } \\
\text { System Hot Leg \#1 } \\
\text { Temperature }\end{array}$ & TF-143 & ${ }^{\circ} \mathrm{F}$ & 9 & \\
\hline $\begin{array}{l}\text { Reactor Coolant } \\
\text { System Hot Leg \#2 } \\
\text { Temperature }\end{array}$ & TF-142 & ${ }^{\circ} \mathrm{F}$ & 10 & \\
\hline Pressurizer Pressure & $\begin{array}{l}\text { PT-604 (WR) and PT-603 } \\
\text { (LP Indication) }\end{array}$ & psig & 11,12 & \\
\hline $\begin{array}{l}\text { Pressurizer Liquid } \\
\text { Level }\end{array}$ & LDP-601 & inch of $\mathrm{H}_{2} \mathrm{O}$ & 13 & $\begin{array}{c}\text { Sharp decrease followed } \\
\text { by rapid refill }\end{array}$ \\
\hline $\begin{array}{l}\text { Steam Generator \#1 } \\
\text { Tube Level }\end{array}$ & LDP-215 & inch of $\mathrm{H}_{2} \mathrm{O}$ & 14 & \\
\hline $\begin{array}{l}\text { Steam Generator \#1 } \\
\text { Secondary Pressure }\end{array}$ & PT-301 & psig & 15 & \\
\hline $\begin{array}{l}\text { Steam Generator \#1 } \\
\text { Feed Flow Rate }\end{array}$ & FMM-001 & gpm & 16 & \\
\hline
\end{tabular}




\begin{tabular}{|c|c|c|c|c|}
\hline \multicolumn{5}{|c|}{$\begin{array}{l}\text { TABLE 6-4 (Continued) } \\
\text { DATA PLOTS FOR QL REPORTS FOR DBA-06-D BY COMPONENT }\end{array}$} \\
\hline Component & Channel & Units & QL-Plot & Comment \\
\hline $\begin{array}{l}\text { Steam Generator \#2 } \\
\text { Tube Level }\end{array}$ & LDP-218 & inch of $\mathrm{H}_{2} \mathrm{O}$ & 17 & \\
\hline $\begin{array}{l}\text { Steam Generator \#2 } \\
\text { Secondary Pressure }\end{array}$ & PT-302 & psig & 18 & \\
\hline $\begin{array}{l}\text { Accumulators \#1 and } \\
\text { \#2 Pressure }\end{array}$ & PT-401 and PT-402 & psig & 19,20 & \\
\hline $\begin{array}{l}\text { Accumulators \#1 and } \\
\text { \#2 Liquid Level }\end{array}$ & LDP-401 and LDP-402 & inch of $\mathrm{H}_{2} \mathrm{O}$ & 21,22 & \\
\hline $\begin{array}{l}\text { Accumulators \#1 and } \\
\text { \#2 Flow Rate }\end{array}$ & FMM-401 and FMM-402 & gpm & 23,24 & \\
\hline $\begin{array}{l}\text { Accumulators \#1 and } \\
\text { \#2 Liquid Discharge } \\
\text { Temperature }\end{array}$ & TF-401 and TF-402 & ${ }^{\circ} \mathrm{F}$ & 25,26 & \\
\hline $\begin{array}{l}\text { CMT \#1 and \#2 Liquid } \\
\text { Level }\end{array}$ & LDP-507 and LDP-502 & inch of $\mathrm{H}_{2} \mathrm{O}$ & 27,28 & \\
\hline $\begin{array}{l}\text { CMT \#1 and \#2 Flow } \\
\text { Rate }\end{array}$ & FMM-501 and FMM-504 & gpm & 29,30 & \\
\hline $\begin{array}{l}\text { CMT \#1 and \#2 Liquid } \\
\text { Temperature }\end{array}$ & $\begin{array}{l}\text { TF-501 and TF-529; and } \\
\text { TF-504 and TF-532 }\end{array}$ & ${ }^{\circ} \mathrm{F}$ & $31-34$ & \\
\hline PRHR Inlet Flow Rate & FMM-802 & gpm & 35 & \\
\hline PRHR Liquid Level & LDP-802 & inch of $\mathrm{H}_{2} \mathrm{O}$ & 36 & \\
\hline $\begin{array}{l}\text { PRHR Outlet Flow } \\
\text { Rate }\end{array}$ & FMM-804 & gpm & 37 & \\
\hline IRWST Liquid Level & LDP-701 & inch of $\mathrm{H}_{2} \mathrm{O}$ & 38 & \\
\hline $\begin{array}{l}\text { IRWST Discharge Line } \\
\# 1 \text { and \#2 Flow Rate }\end{array}$ & FMM-701 and FMM-702 & gpm & 39,40 & \\
\hline $\begin{array}{l}\text { IRWST Fluid } \\
\text { Temperature }\end{array}$ & TF-701 and TF-709 & ${ }^{\circ} \mathrm{F}$ & 41,42 & \\
\hline $\begin{array}{l}\text { ADS 1-3 Separator } \\
\text { Pressure }\end{array}$ & PT-605 & psig & 43 & \\
\hline $\begin{array}{l}\text { ADS 1-3 Separator } \\
\text { Steam Flow Rate }\end{array}$ & FVM-601 & $\mathrm{scfm}$ & 44 & \\
\hline
\end{tabular}




\begin{tabular}{|c|c|c|c|c|}
\hline \multicolumn{5}{|c|}{$\begin{array}{c}\text { TABLE 6-4 (Continued) } \\
\text { DATA PLOTS FOR QL REPORTS FOR DBA-06-D BY COMPONENT }\end{array}$} \\
\hline Component & Channel & Units & QL-Plot & Comment \\
\hline $\begin{array}{l}\text { ADS 1-3 Separator } \\
\text { Liquid Flow Rate }\end{array}$ & FMM-601 & gpm & 45 & \\
\hline $\begin{array}{l}\text { ADS } 4-1 \text { and } 4-2 \\
\text { Separator Pressure }\end{array}$ & PT-611 and PT-610 & psig & 46,47 & \\
\hline $\begin{array}{l}\text { ADS 4-1 Separator } \\
\text { Steam Flow Rate }\end{array}$ & FVM-603 & $\operatorname{scfm}$ & 48 & \\
\hline $\begin{array}{l}\text { ADS 4-2 Separator } \\
\text { Steam Flow Rate }\end{array}$ & FVM-602 & $\operatorname{scfm}$ & 49 & \\
\hline $\begin{array}{l}\text { ADS 4-1 Separator } \\
\text { Liquid Flow Rate }\end{array}$ & FMM-603 & gpm & 50 & \\
\hline $\begin{array}{l}\text { ADS 4-2 Separator } \\
\text { Liquid Flow Rate }\end{array}$ & FMM-602 & gpm & 51 & \\
\hline Primary Sump Pressure & PT-901 & psig & 52 & \\
\hline $\begin{array}{l}\text { Primary Sump Liquid } \\
\text { Level }\end{array}$ & LDP-901 & inch of $\mathrm{H}_{2} \mathrm{O}$ & 53 & \\
\hline $\begin{array}{l}\text { Primary Sump } \\
\text { Injection Flow Rate }\end{array}$ & FMM-901 and FMM-902 & gpm & 54,55 & \\
\hline $\begin{array}{l}\text { Secondary Sump } \\
\text { Liquid Level }\end{array}$ & LDP-902 & inch of $\mathrm{H}_{2} \mathrm{O}$ & 56 & \\
\hline $\begin{array}{l}\text { Break Separator } \\
\text { Pressure }\end{array}$ & PT-905 & psig & 57 & \\
\hline $\begin{array}{l}\text { Break Separator Liquid } \\
\text { Level }\end{array}$ & LDP-905 & inch of $\mathrm{H}_{2} \mathrm{O}$ & 58 & \\
\hline $\begin{array}{l}\text { Break Separator Flow } \\
\text { to Primary Sump }\end{array}$ & FMM-905 & gpm & 59 & \\
\hline $\begin{array}{l}\text { BAMS Steam Flow } \\
\text { Rate }\end{array}$ & FVM-901 & $\mathrm{scfm}$ & 60 & \\
\hline $\begin{array}{l}\text { BAMS Steam Flow } \\
\text { Rate }\end{array}$ & FVM-902 & $\mathrm{scfm}$ & 61 & \\
\hline $\begin{array}{l}\text { BAMS/Primary Sump } \\
\text { Steam Flow Rate }\end{array}$ & FVM-903 & $\mathrm{scfm}$ & 62 & \\
\hline
\end{tabular}




\begin{tabular}{|c|c|c|c|c|}
\hline \multicolumn{5}{|c|}{$\begin{array}{l}\text { TABLE 6-4 (Continued) } \\
\text { DATA PLOTS FOR QL REPORTS FOR DBA-06-D BY COMPONENT }\end{array}$} \\
\hline Component & Channel & Units & QL-Plot & Comment \\
\hline $\begin{array}{l}\text { BAMS/Separator } \\
\text { Steam Flow Rate - } \\
\text { 6-inch Pipe }\end{array}$ & FVM-905 & $\operatorname{scfm}$ & 63 & \\
\hline $\begin{array}{l}\text { BAMS/Exhaust Line } \\
\text { Temperature }\end{array}$ & TF-916 and SC-917 & ${ }^{\circ} \mathrm{F}$ & 64,65 & \\
\hline $\begin{array}{l}\text { Pressurizer Heater } \\
\text { Input Power }\end{array}$ & KW-601 & $\mathrm{kW}$ & 66 & \\
\hline $\begin{array}{l}\text { Core Power Input } \\
\text { Power }\end{array}$ & $\begin{array}{l}\text { KW-101, KW-102, } \\
\text { KW-103, and KW-104 }\end{array}$ & $\mathrm{kW}$ & $67-70$ & \\
\hline $\begin{array}{l}\text { Reactor Vessel Liquid } \\
\text { Level Between Top of } \\
\text { Vessel - Upper Support } \\
\text { Plate }\end{array}$ & LDP-115 & inch of $\mathrm{H}_{2} \mathrm{O}$ & 71 & \\
\hline $\begin{array}{l}\text { Reactor Vessel Liquid } \\
\text { Level Between Bottom } \\
\text { of Upper Support } \\
\text { Plate - Upper Core } \\
\text { Spacer Grid }\end{array}$ & LDP-139 & inch of $\mathrm{H}_{2} \mathrm{O}$ & 72 & \\
\hline $\begin{array}{l}\text { Inner Core } \\
\text { Thermocouple } \\
\text { Measuring Heater } \\
\text { Temperature }\end{array}$ & TH-103-4 & ${ }^{\circ} \mathrm{F}$ & 73 & \\
\hline $\begin{array}{l}\text { Outer Core } \\
\text { Thermocouple } \\
\text { Measuring Heater } \\
\text { Temperature }\end{array}$ & TH-309-4 & ${ }^{\circ} \mathrm{F}$ & 74 & \\
\hline
\end{tabular}




\subsection{CONCLUSIONS}

The DBA-06-D tests were successfully completed, and the data was logged in the DAS. All critical instruments were found to operate properly with the exception of those noted in Section 6.4. The test was acceptable. 
APPENDIX A

DATA PLOTS 


\section{AP1000 Test Data Oregon State University Test DBA-06 June 3, 2004 \\ $-P T-107$ \\ 196 \\ 0 \\ o RX Up Head Press.}

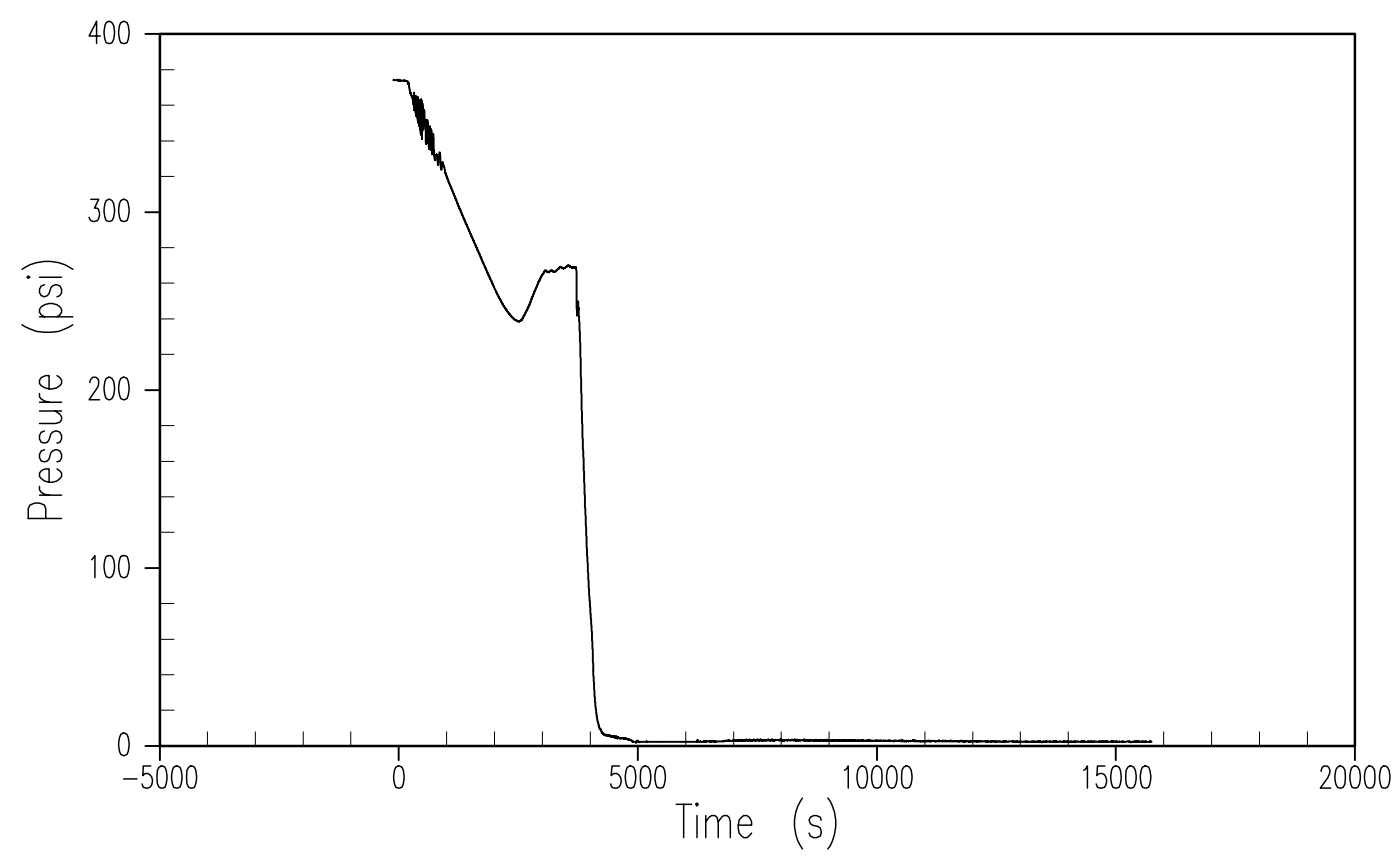

Figure A-1 Reactor Vessel Pressure 


\section{AP1000 Test Data Oregon State University Test DBA-06 June 3, 2004}

$\longrightarrow \operatorname{LDP}-127$

126

o Reactor WR Level

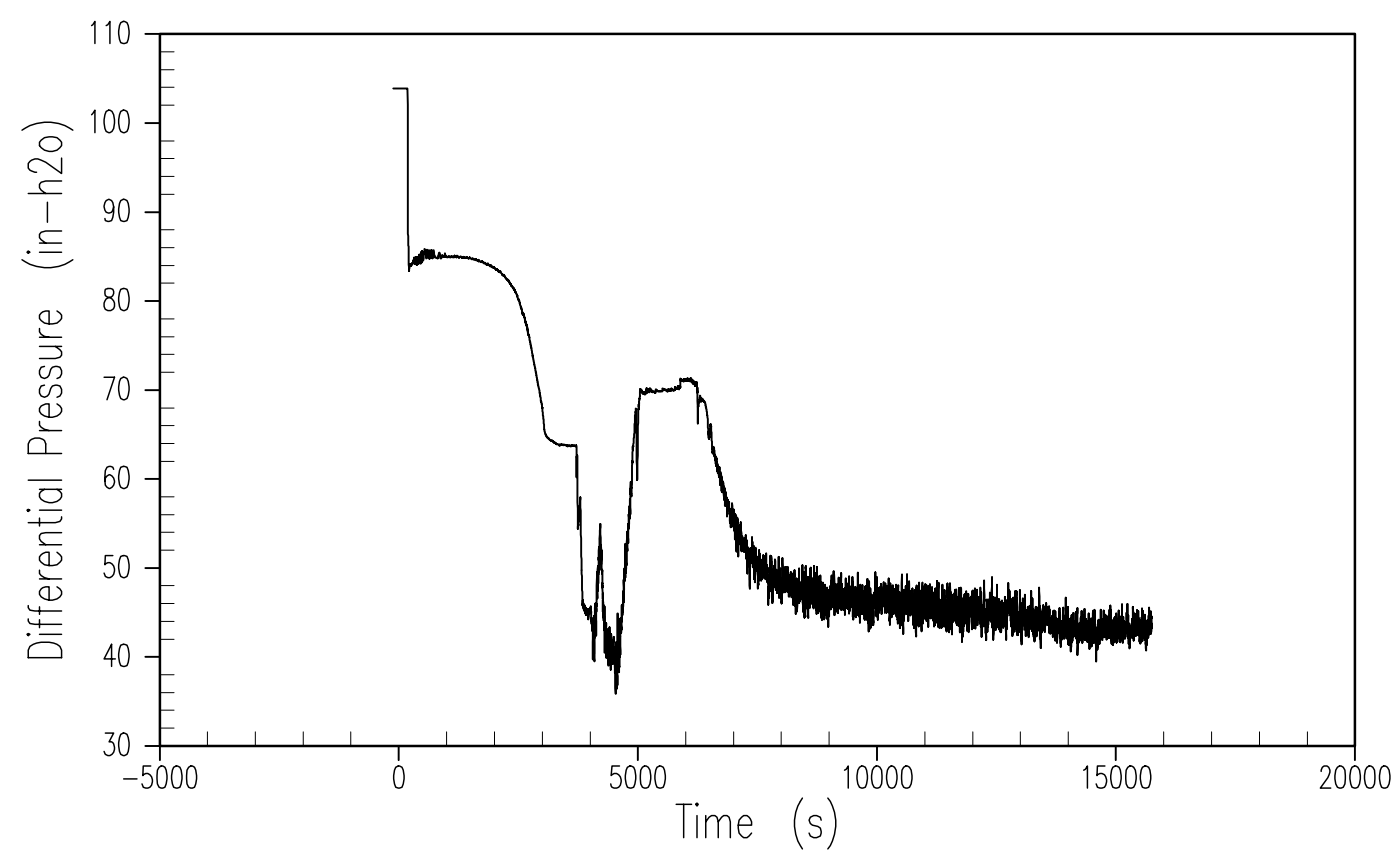

Figure A-2 Reactor Vessel Level 

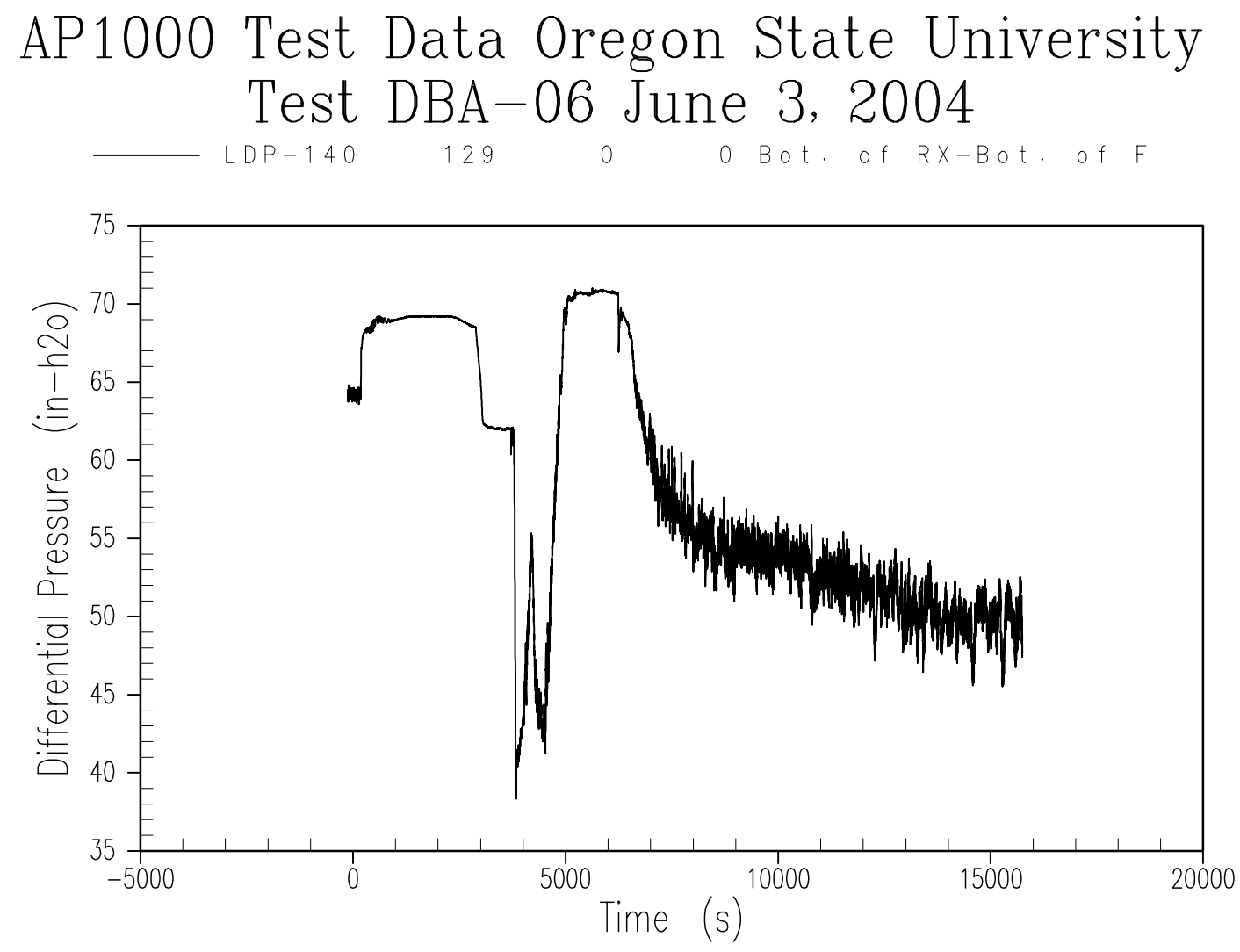

Figure A-3 Reactor Vessel Downcomer Level 

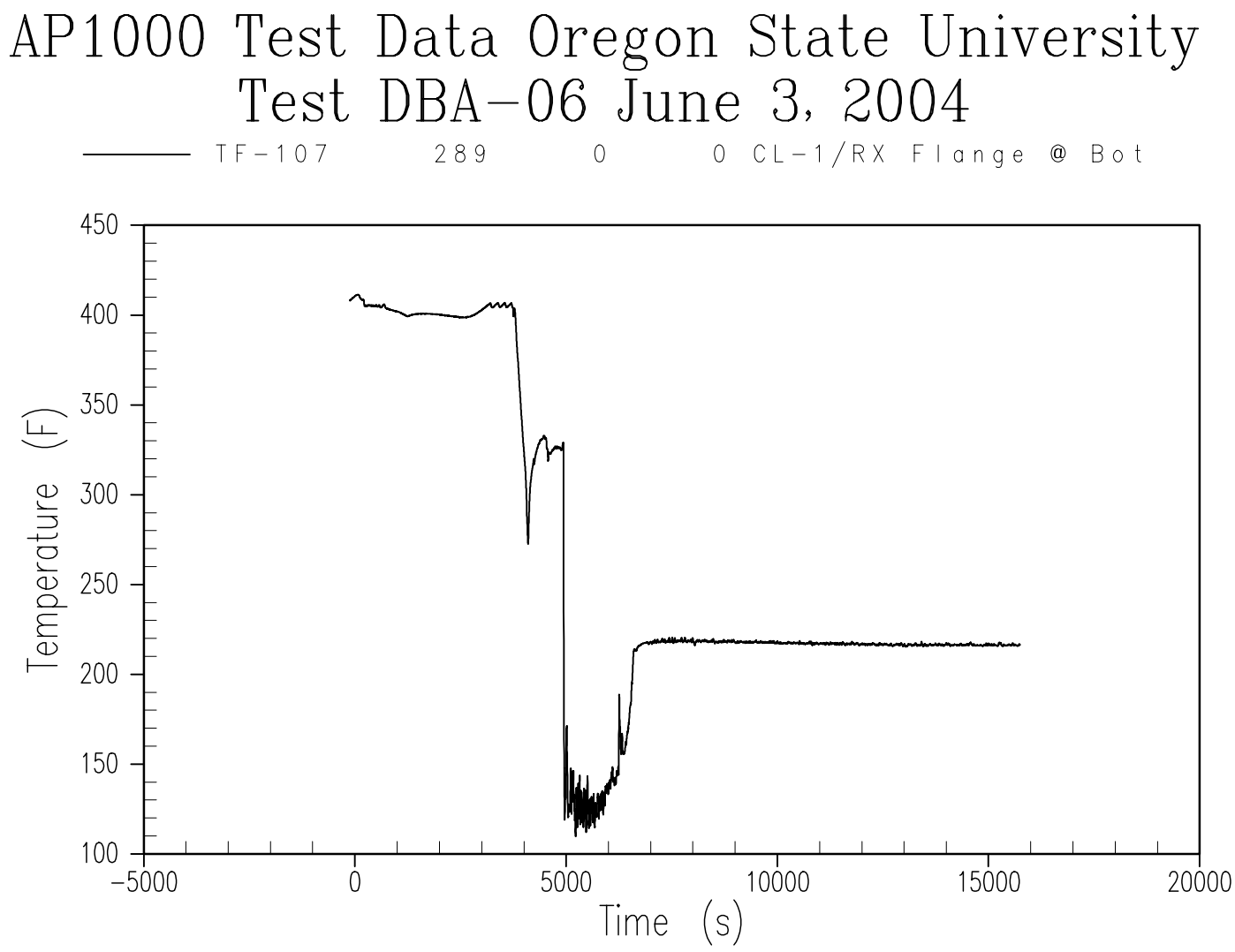

Figure A-4 Cold Leg 1 Fluid Temperature 

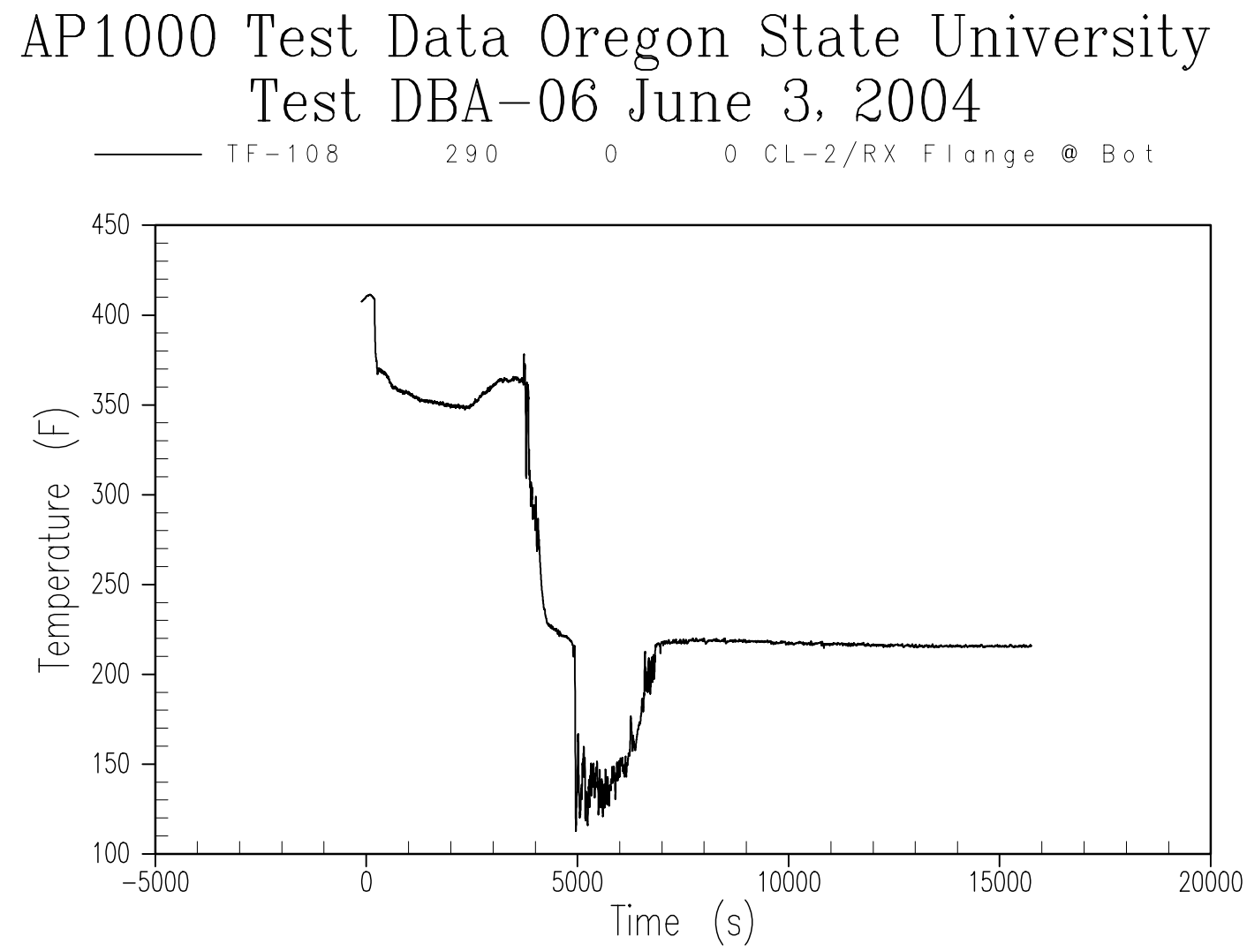

Figure A-5 Cold Leg 2 Fluid Temperature 

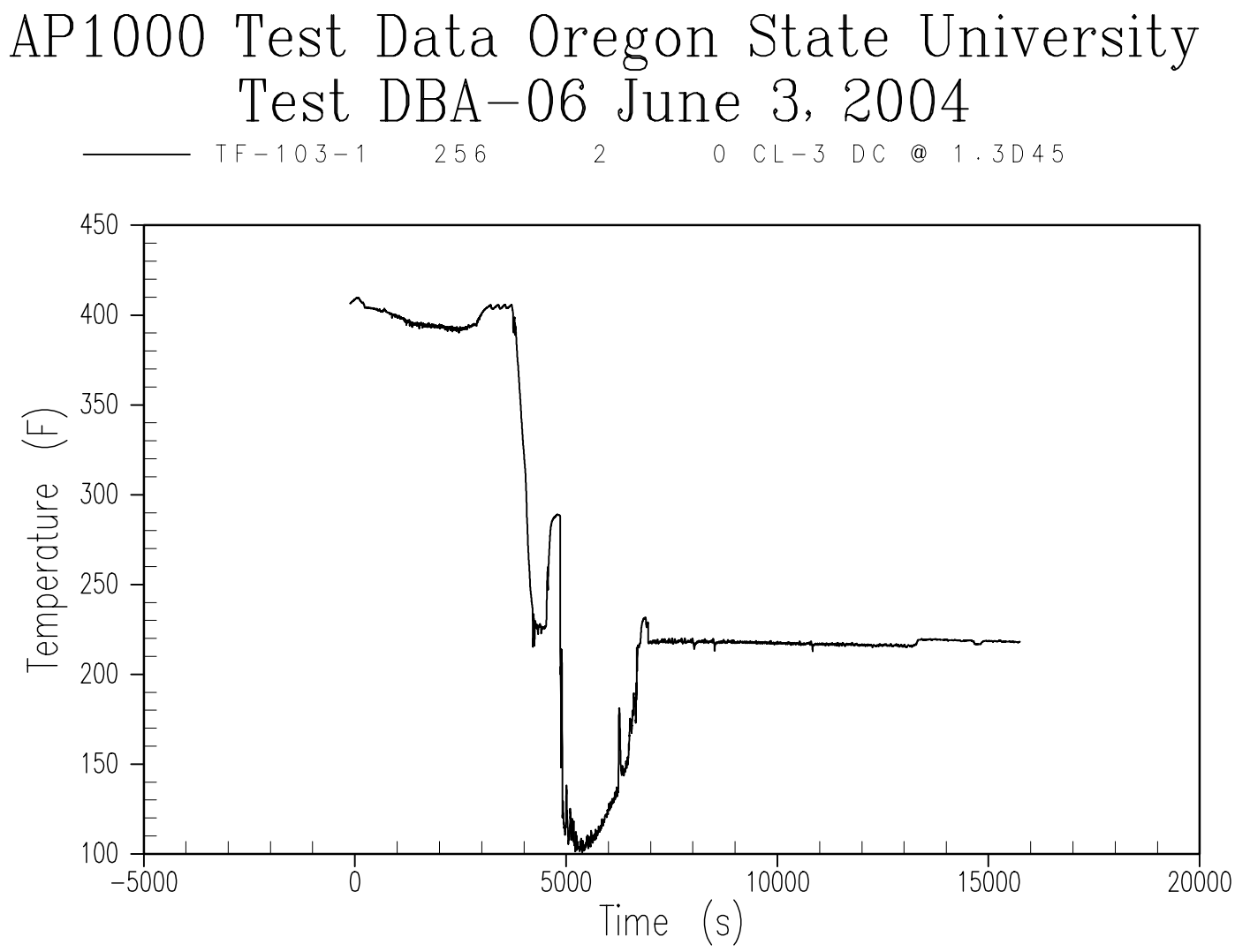

Figure A-6 Cold Leg 3 Fluid Temperature 

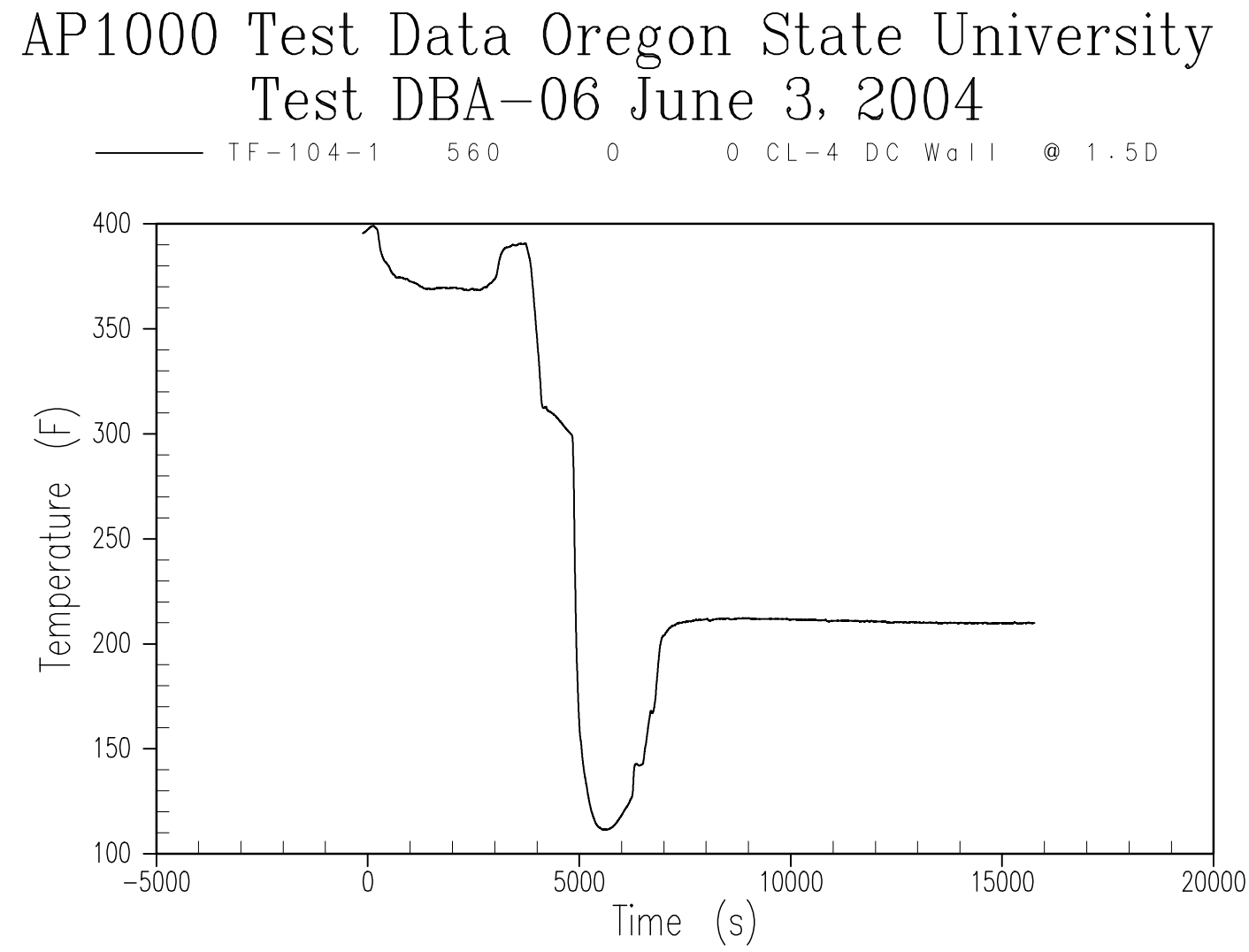

Figure A-7 Cold Leg 4 Fluid Temperature 

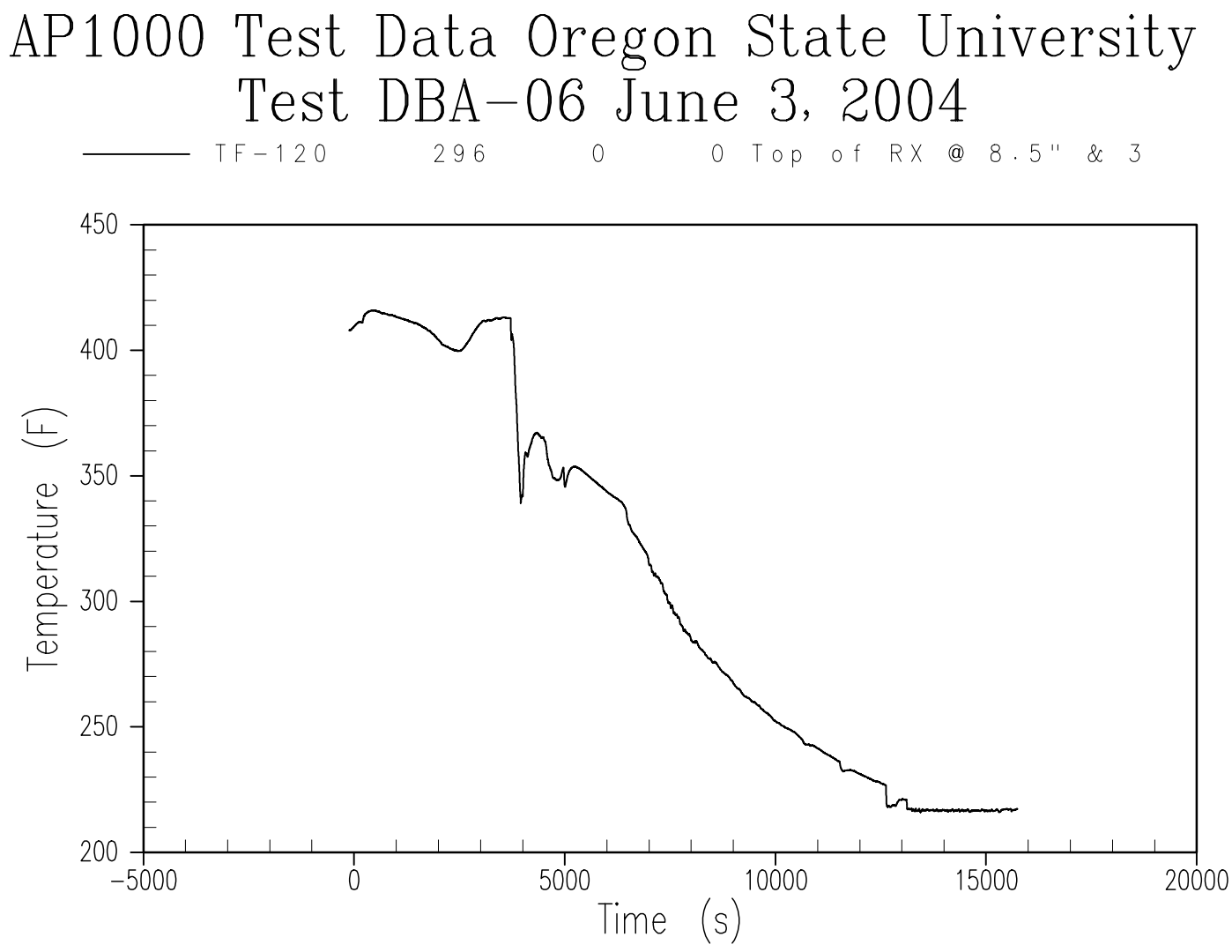

Figure A-8 Reactor Vessel Fluid Temperature Upper Head 

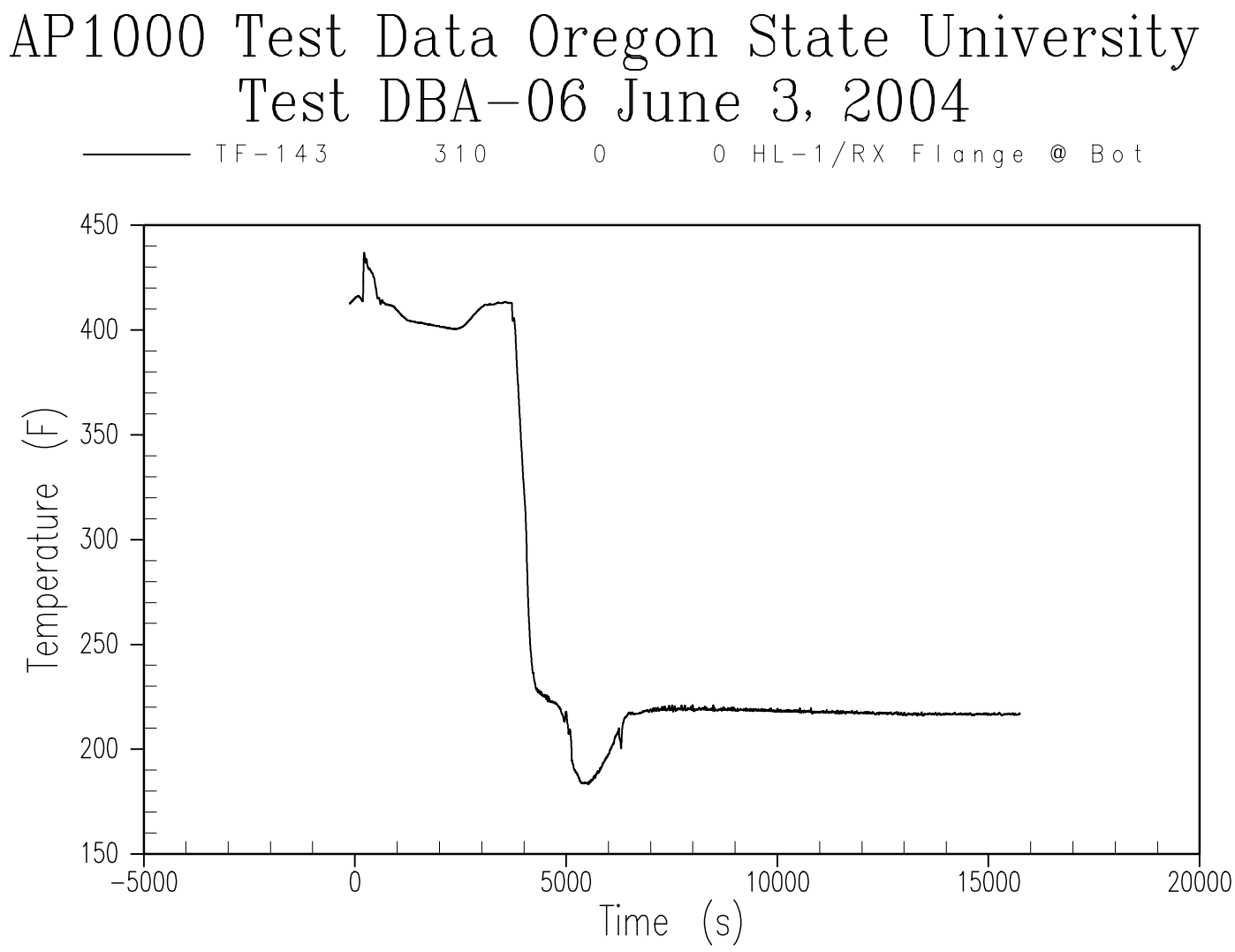

Figure A-9 Reactor Coolant System Hot Leg 1 Temperature 

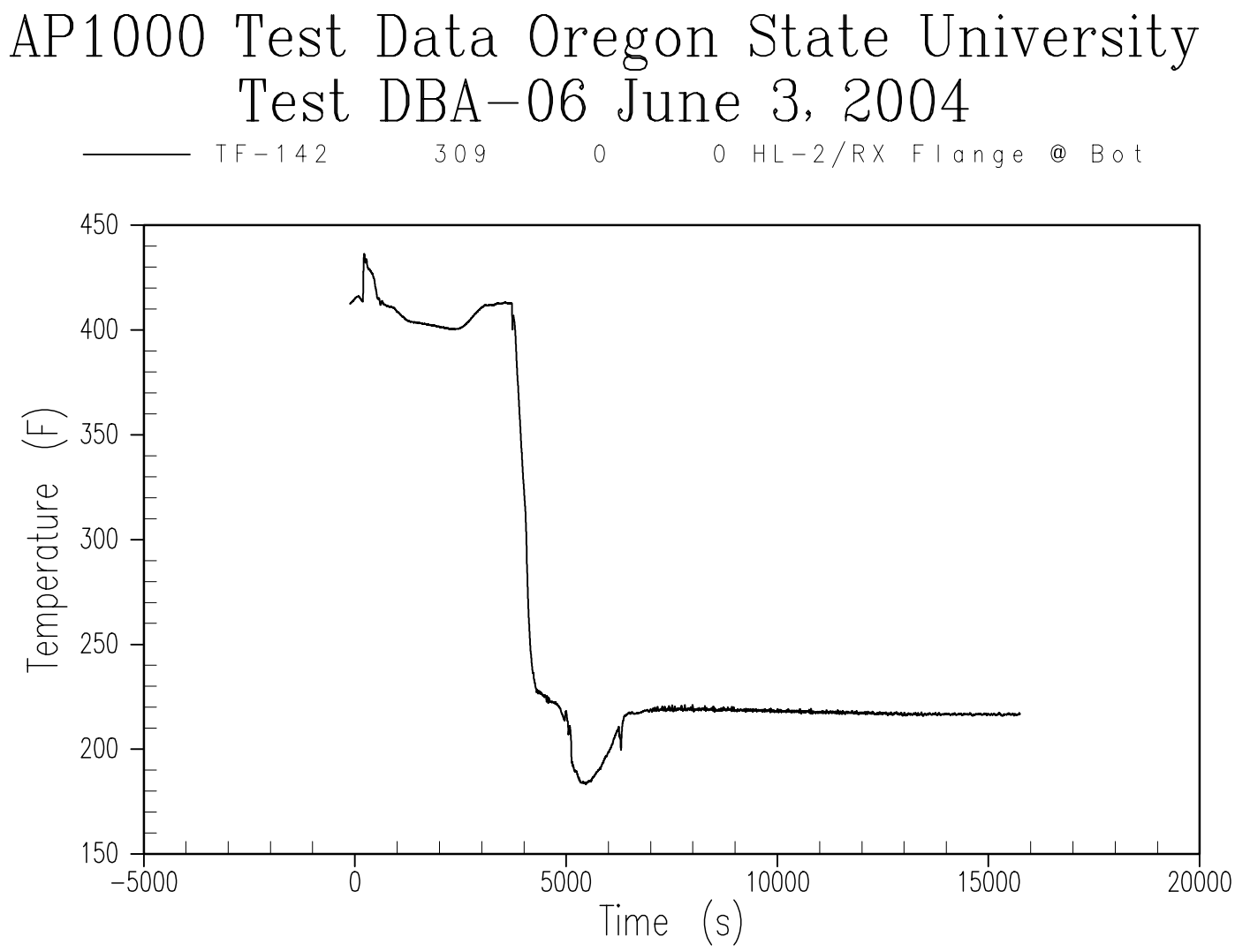

Figure A-10 Reactor Coolant System Hot Leg 2 Temperature 

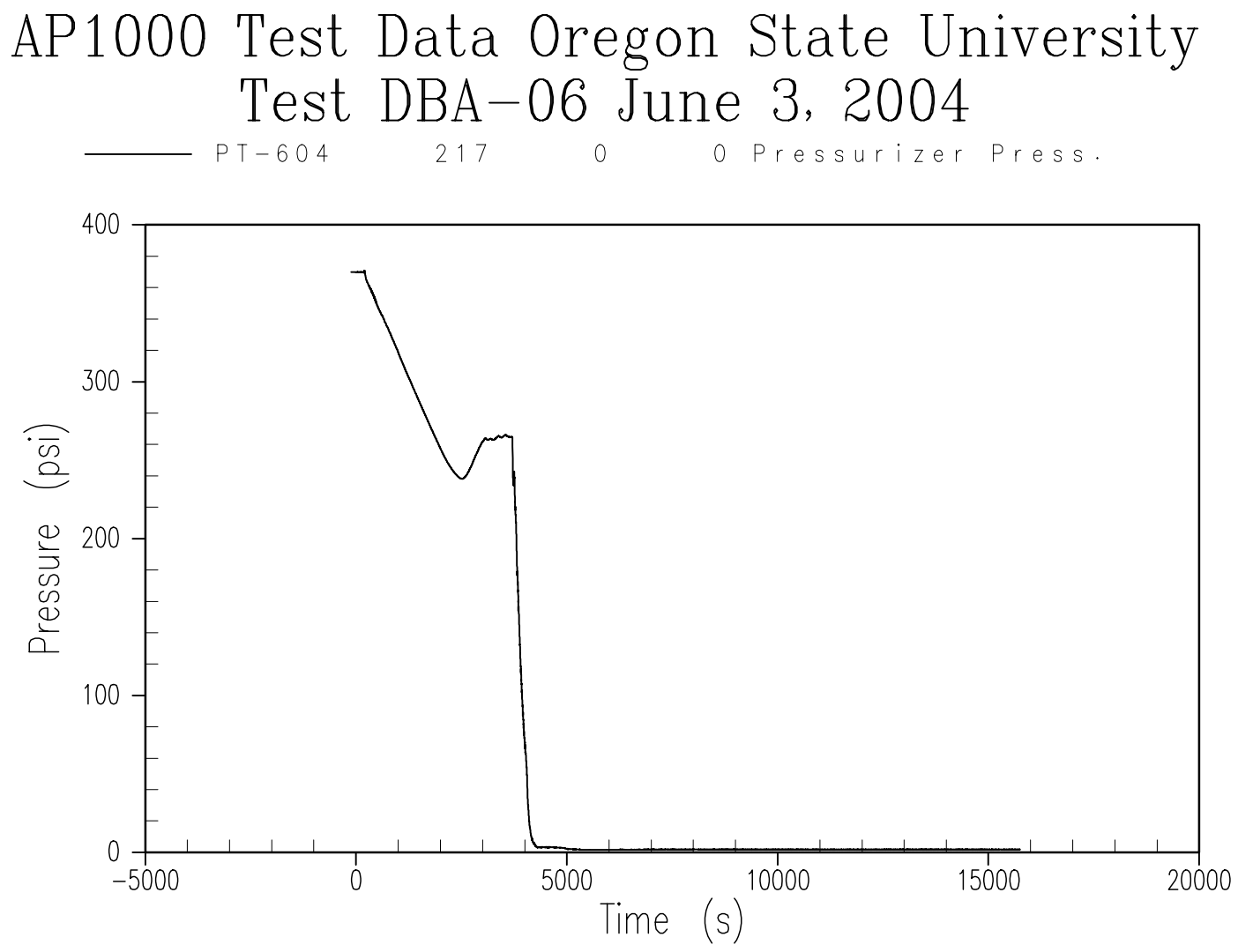

Figure A-11 Pressurizer Pressure - Wide Range 

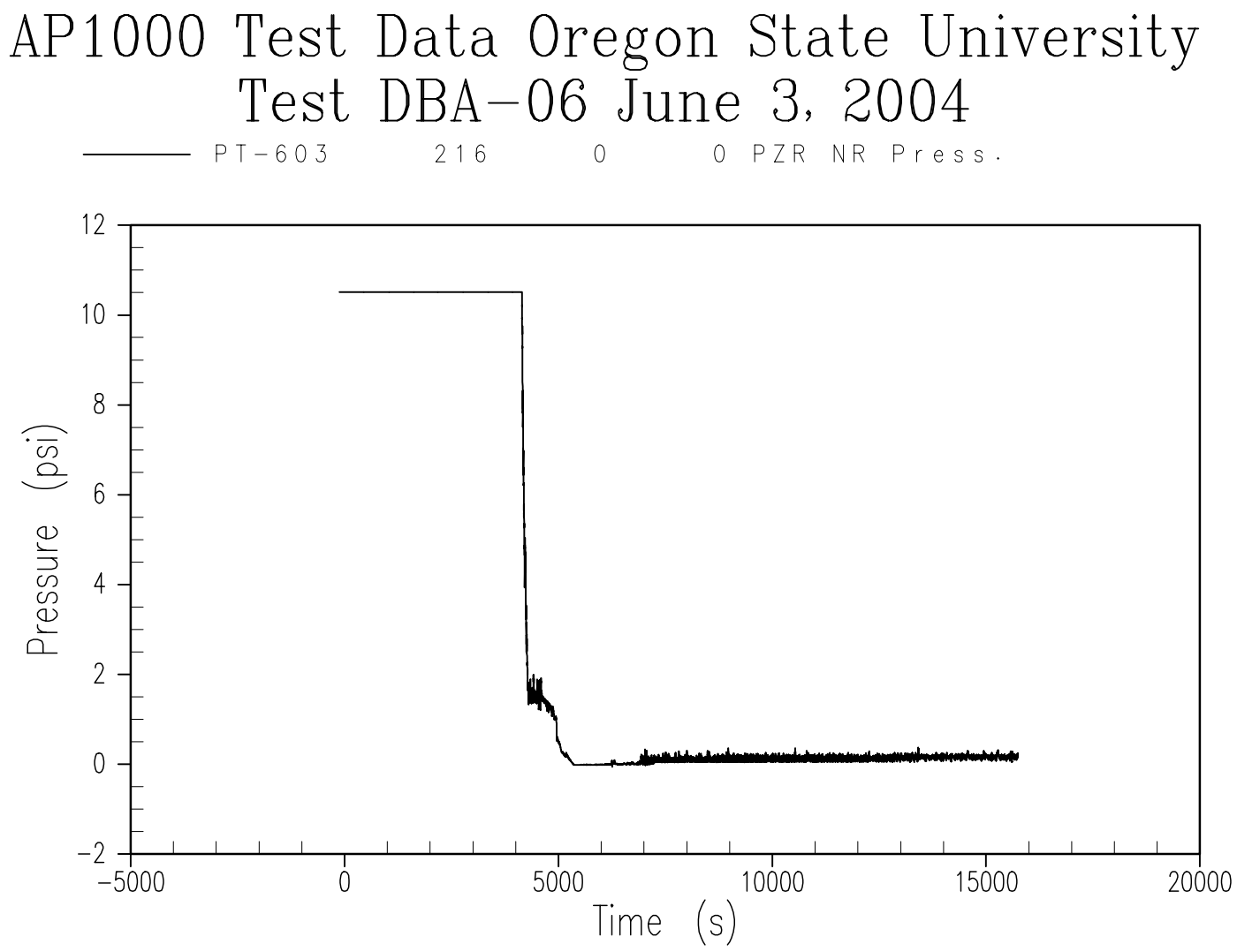

Figure A-12 Pressurizer Pressure - Narrow Range 

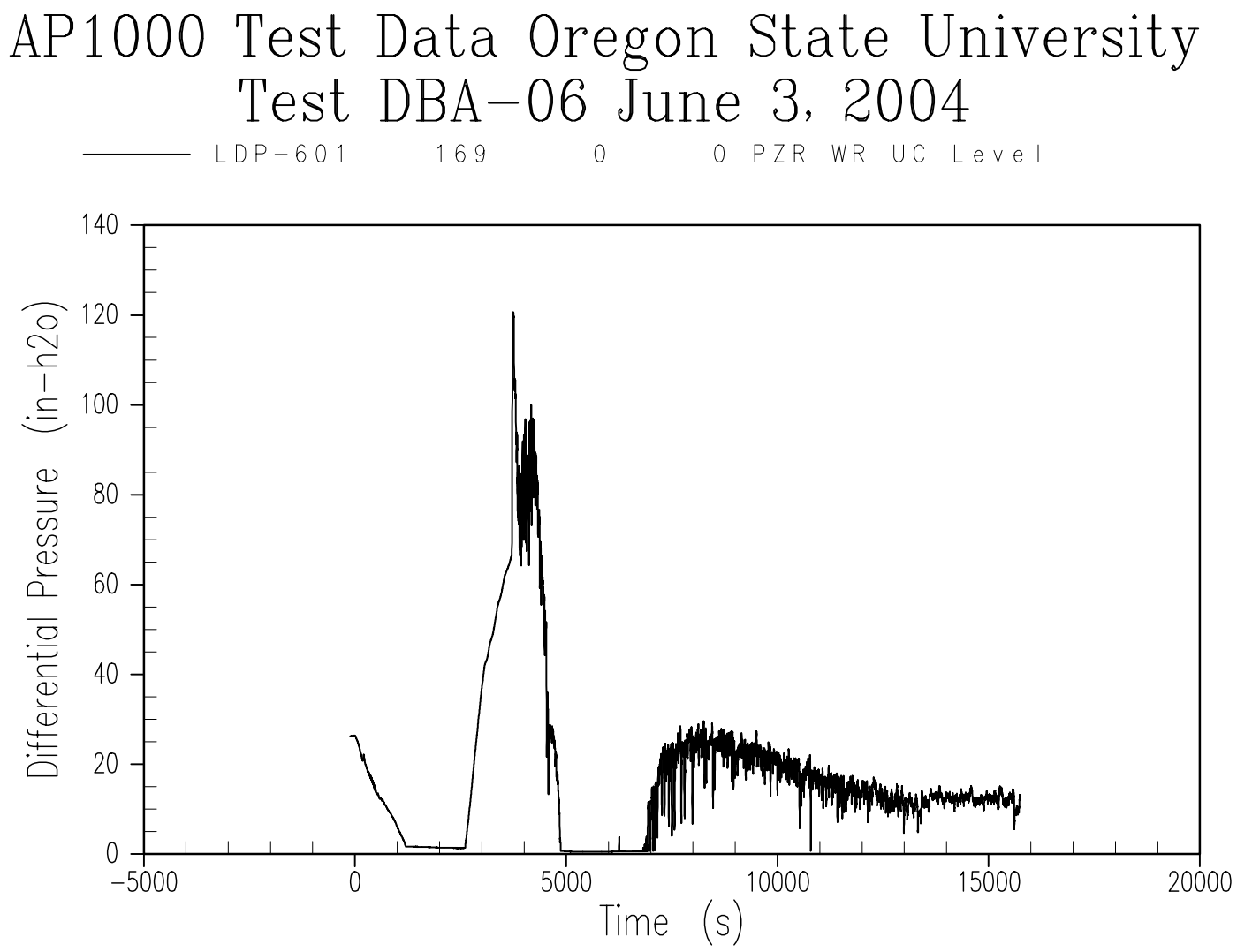

Figure A-13 Pressurizer Liquid Level 

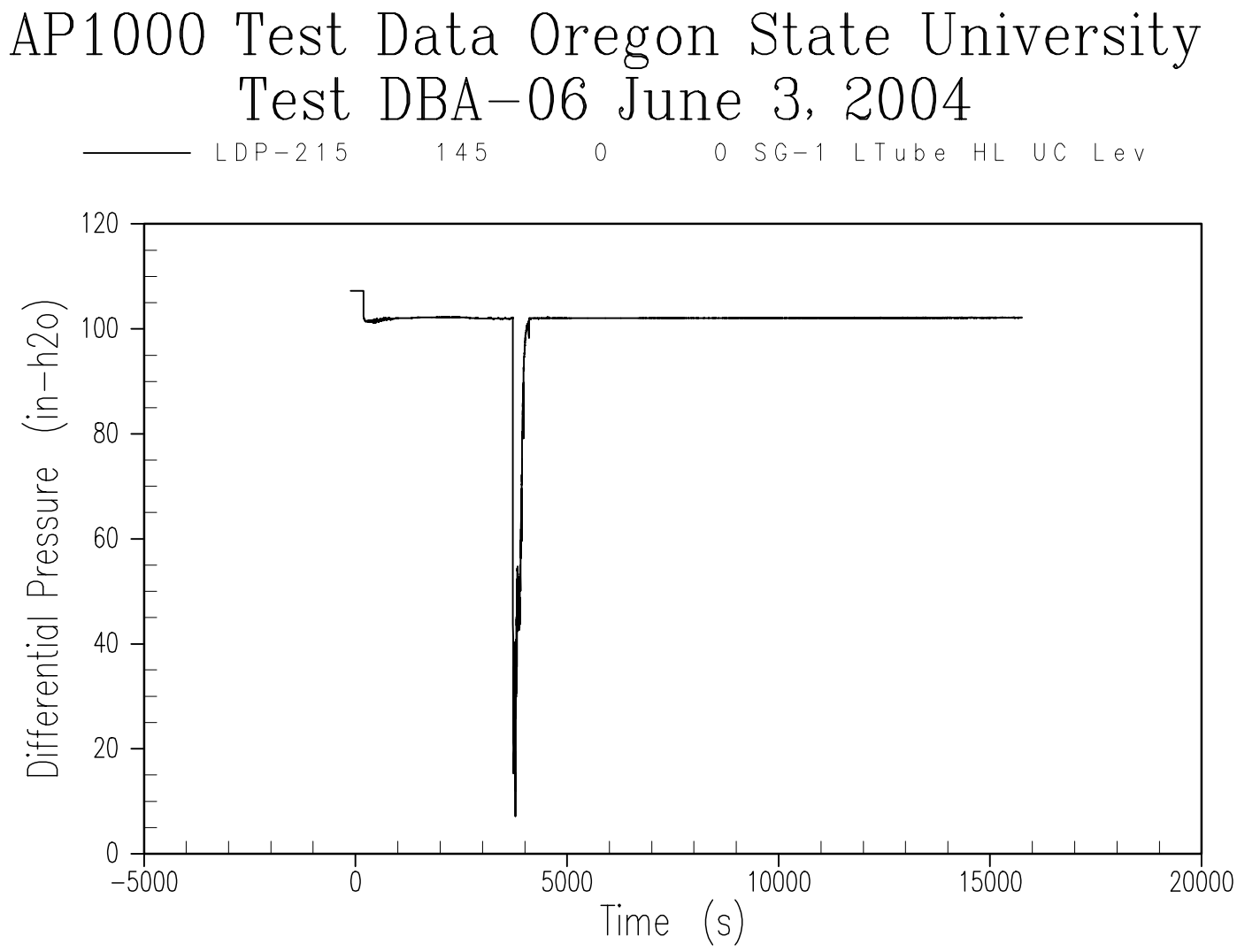

Figure A-14 Steam Generator 1 Tube Level 


\section{AP1000 Test Data Oregon State University Test DBA-06 June 3, 2004

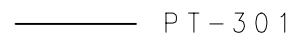

$$
209
$$ \\ 0 \\ o SG-1 Press.}

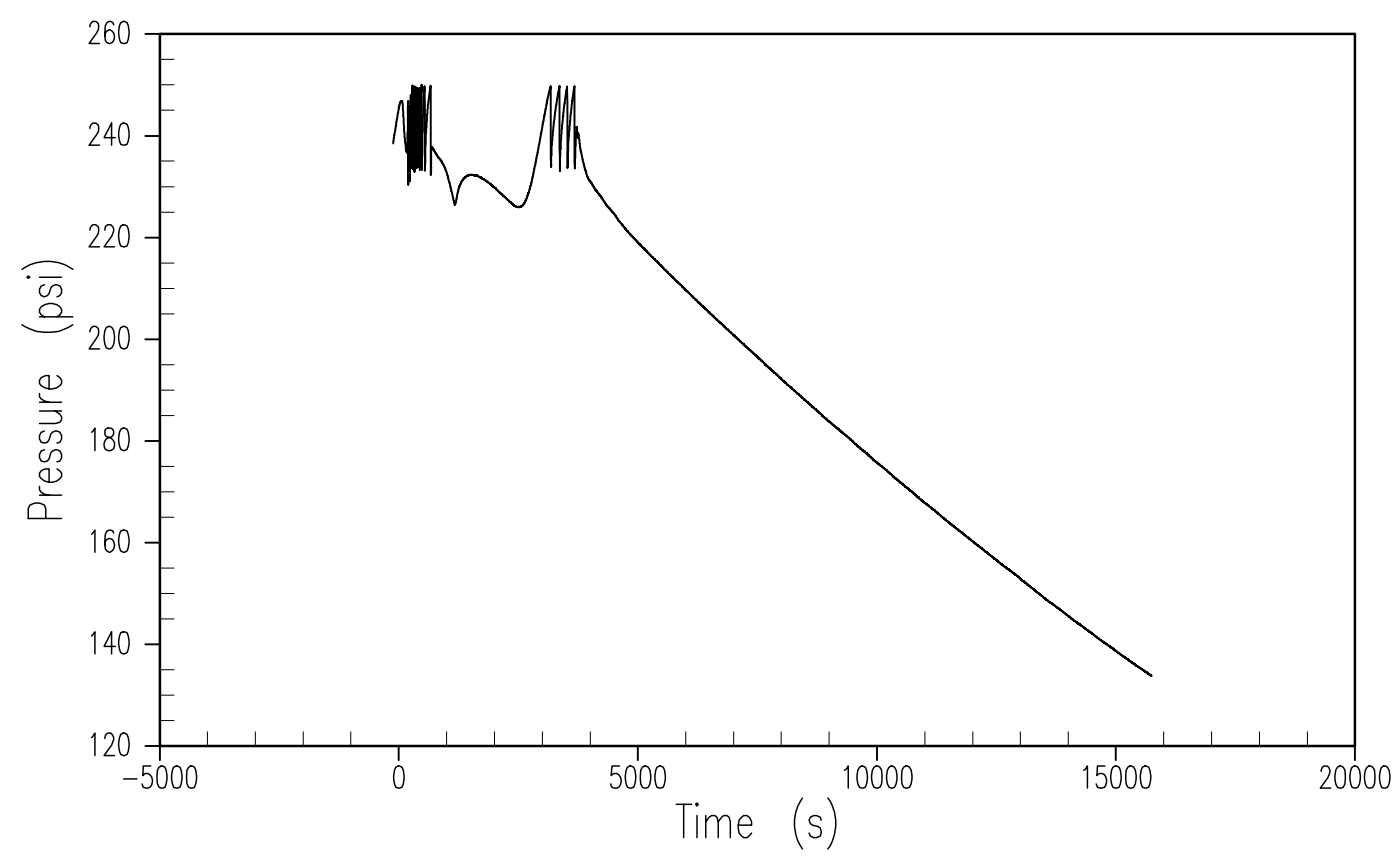

Figure A-15 Steam Generator 1 Secondary Pressure 

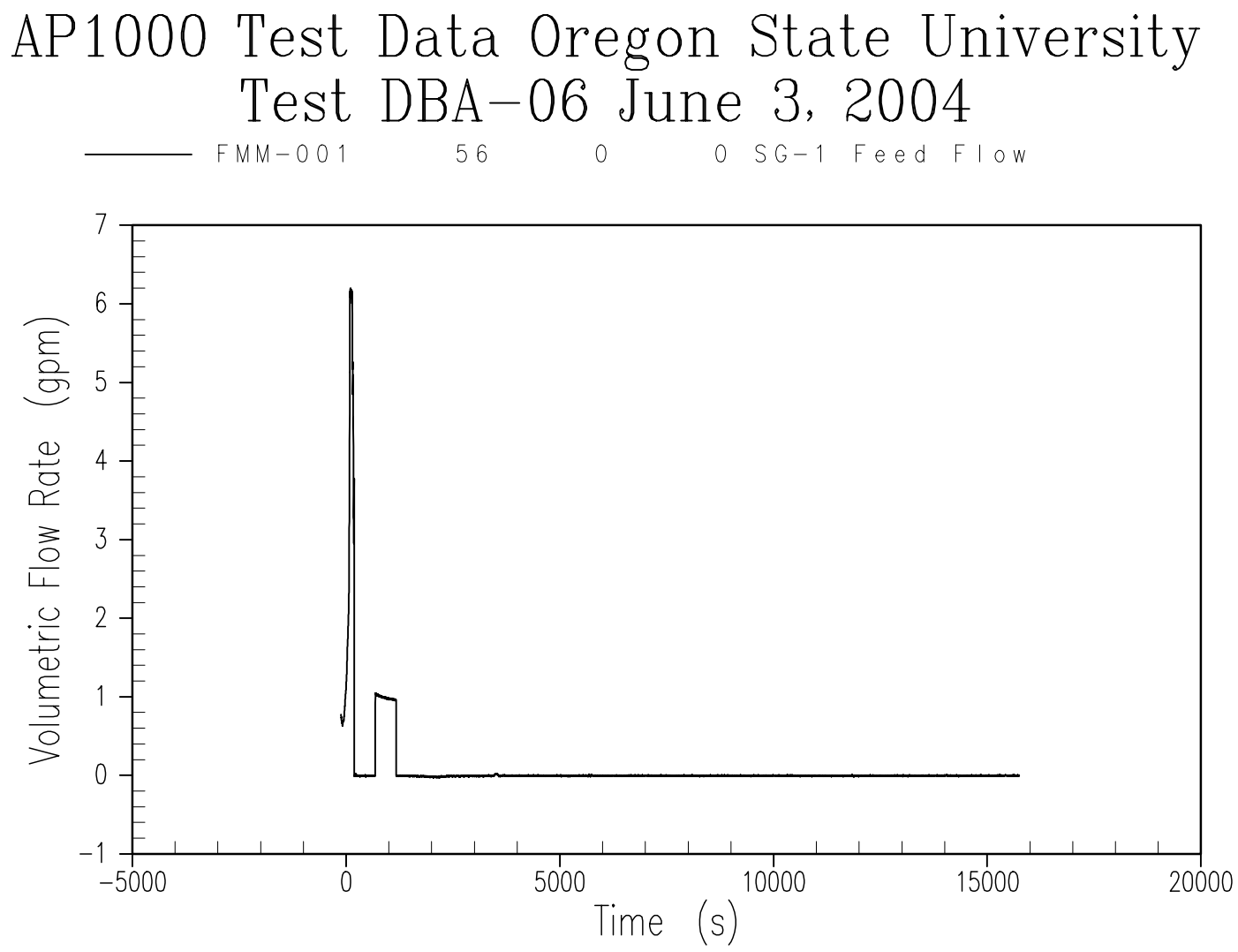

Figure A-16 Steam Generator 1 Feed Flow Rate 

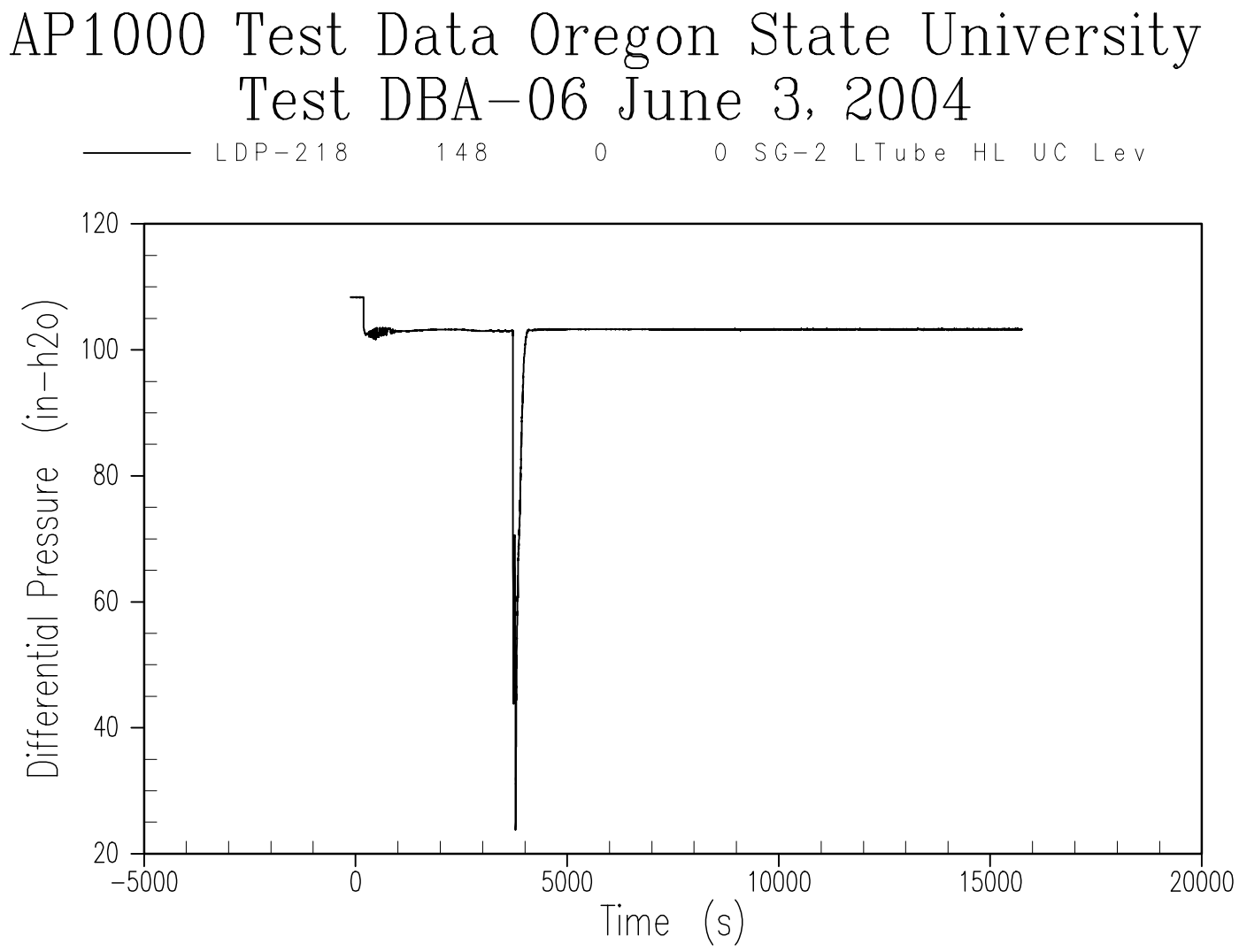

Figure A-17 Steam Generator 2 Tube Level 

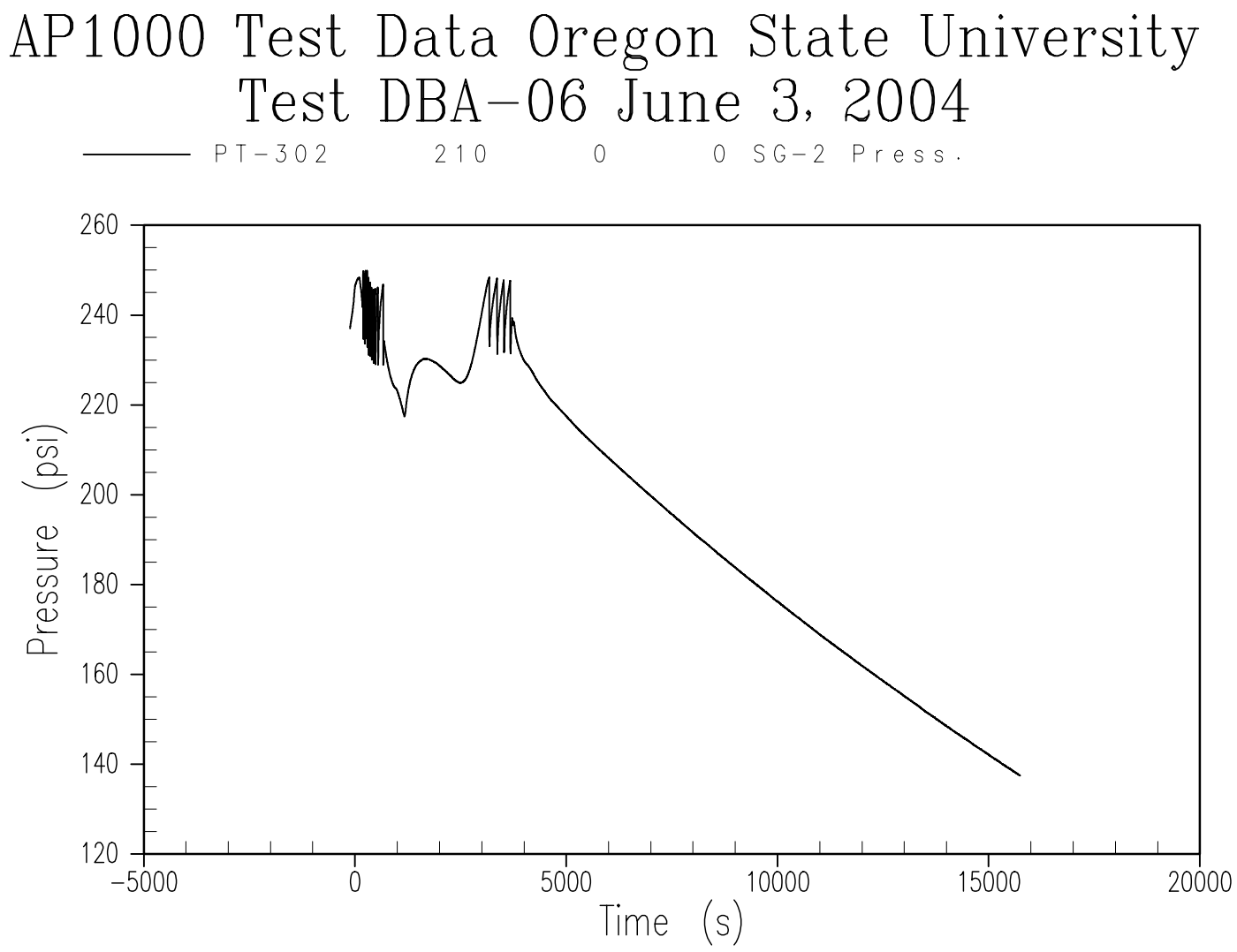

Figure A-18 Steam Generator 2 Secondary Pressure 


\section{AP1000 Test Data Oregon State University Test DBA-06 June 3, 2004 \\ $\longrightarrow P T-401$

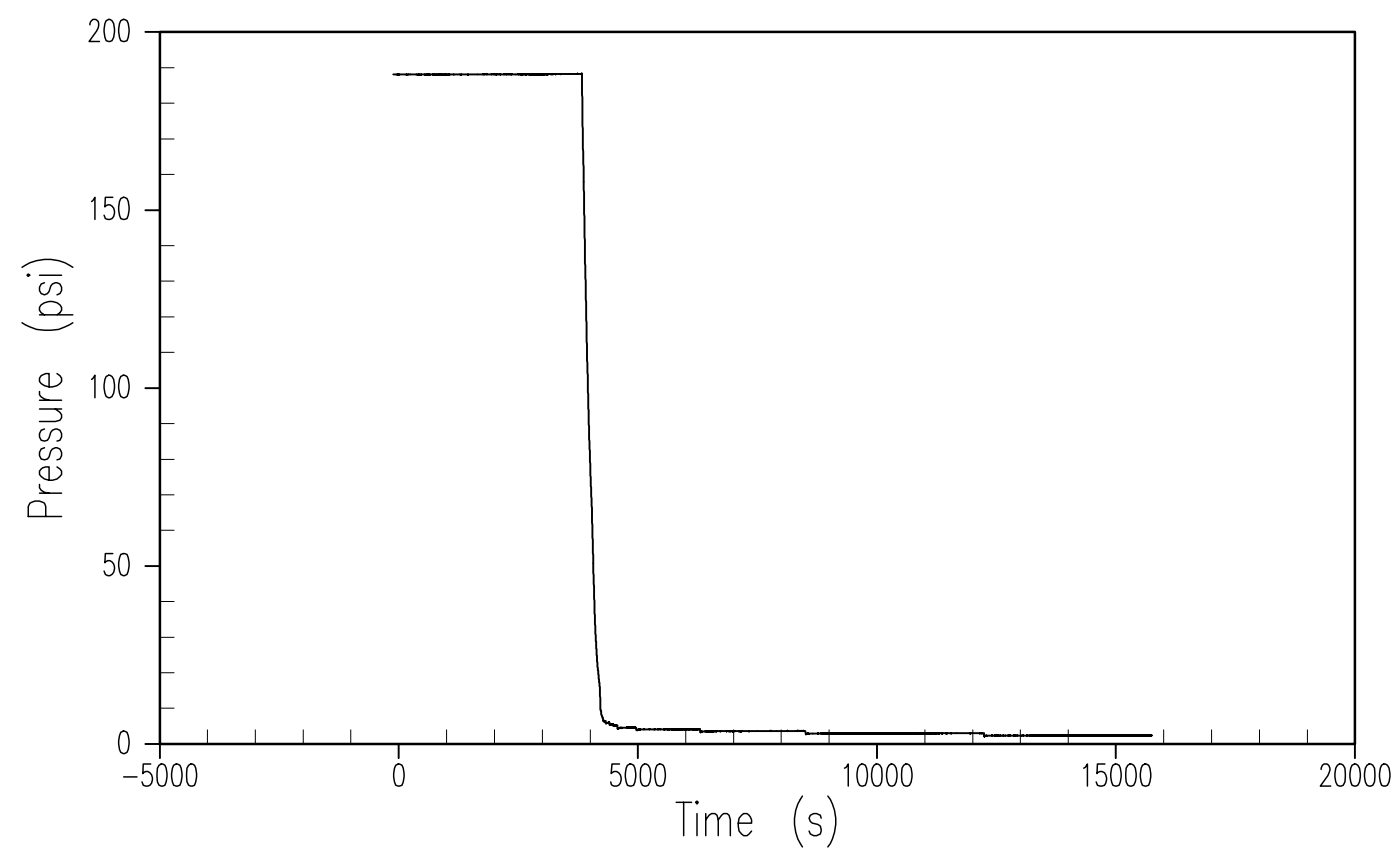

Figure A-19 Accumulator 1 Pressure 


\section{AP1000 Test Data Oregon State University Test DBA-06 June 3, 2004 \\ $\longrightarrow P T-402$

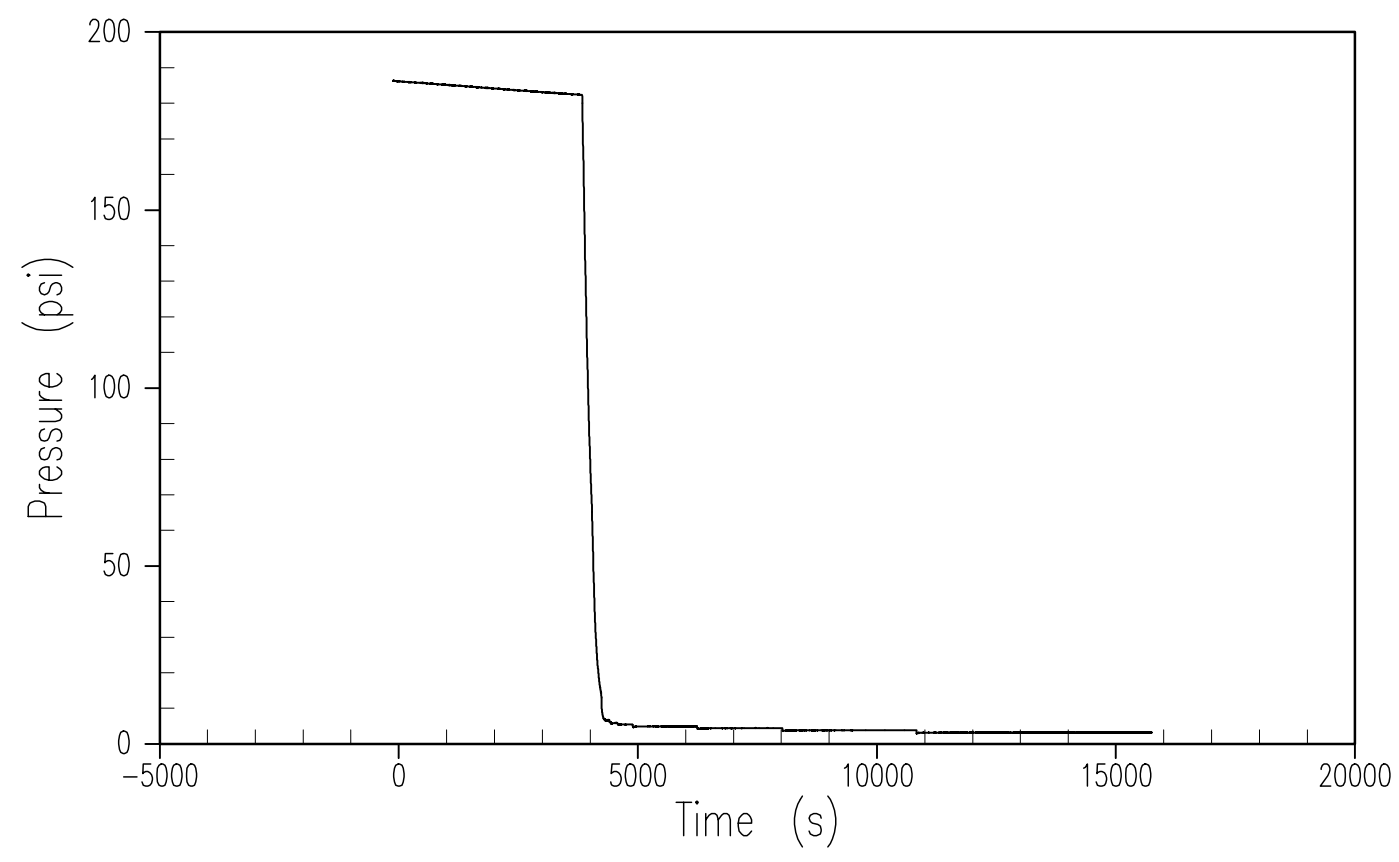

Figure A-20 Accumulator 2 Pressure 


\section{AP1000 Test Data Oregon State University Test DBA-06 June 3, 2004 \\ $\longrightarrow$ LDP -401

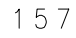 \\ 0 \\ 0 ACC-1 UC LeveI}

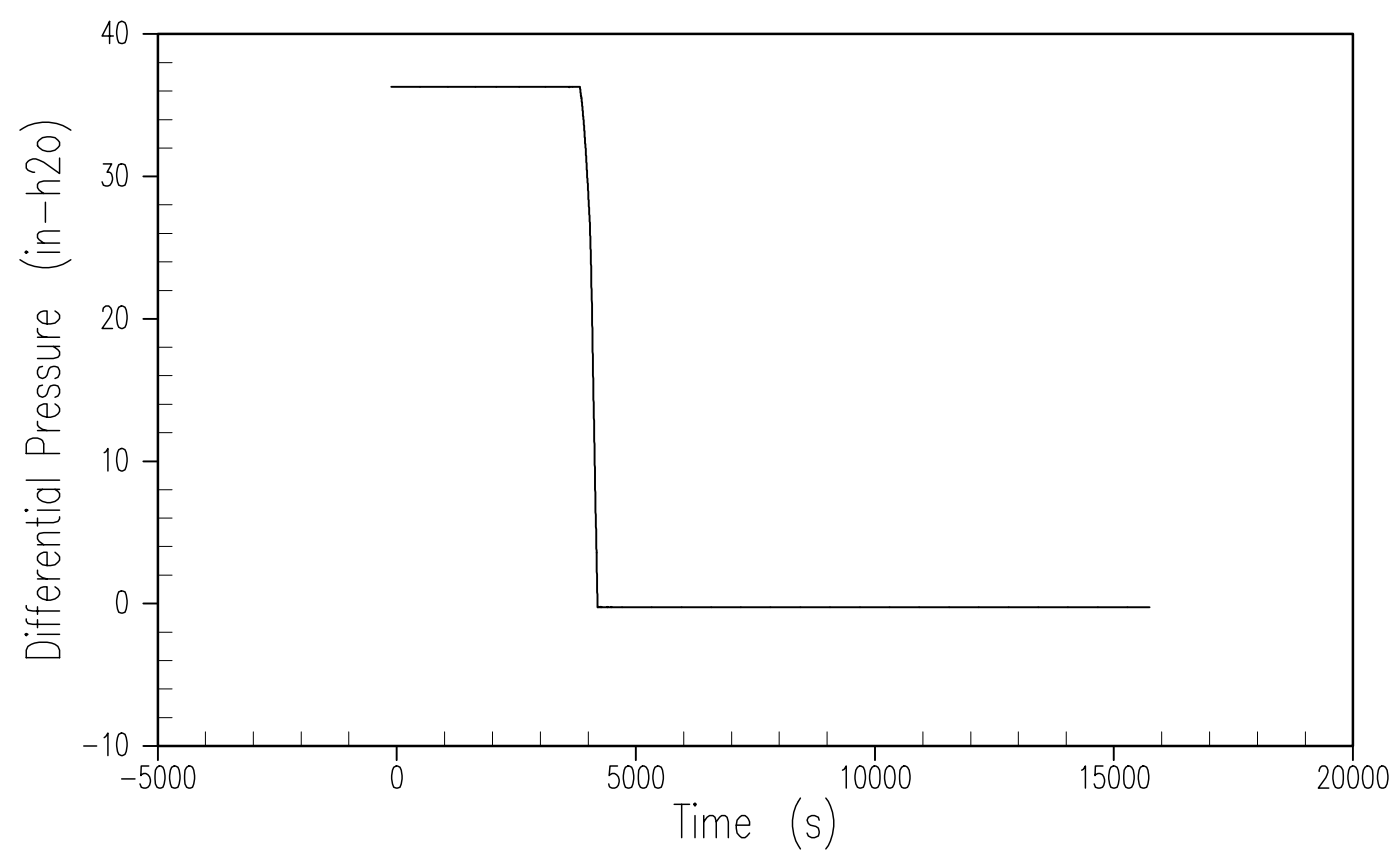

Figure A-21 Accumulator 1 Liquid Level 


\section{AP1000 Test Data Oregon State University Test DBA-06 June 3, 2004 \\ $\longrightarrow$ LDP -402

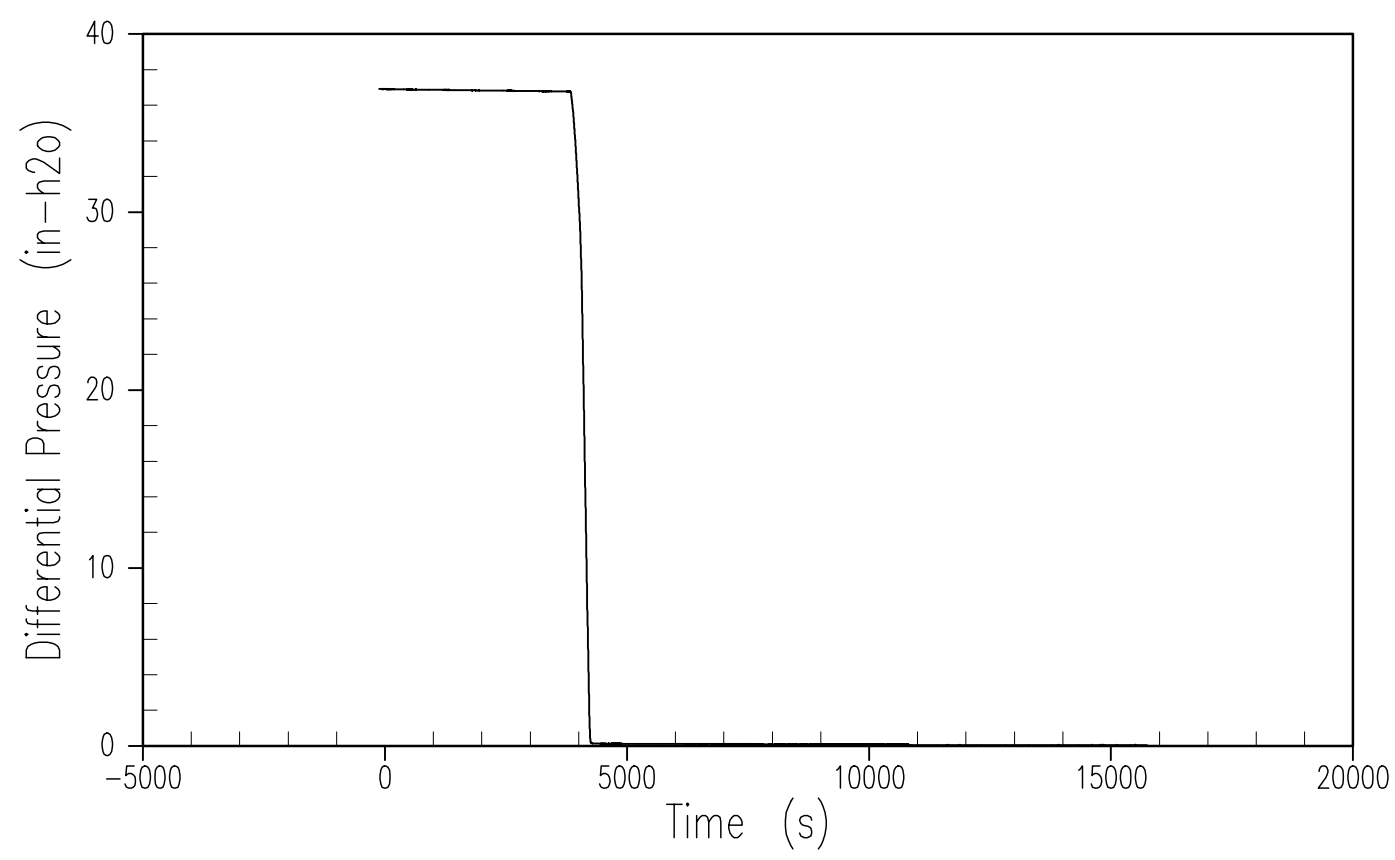

Figure A-22 Accumulator 2 Liquid Level 

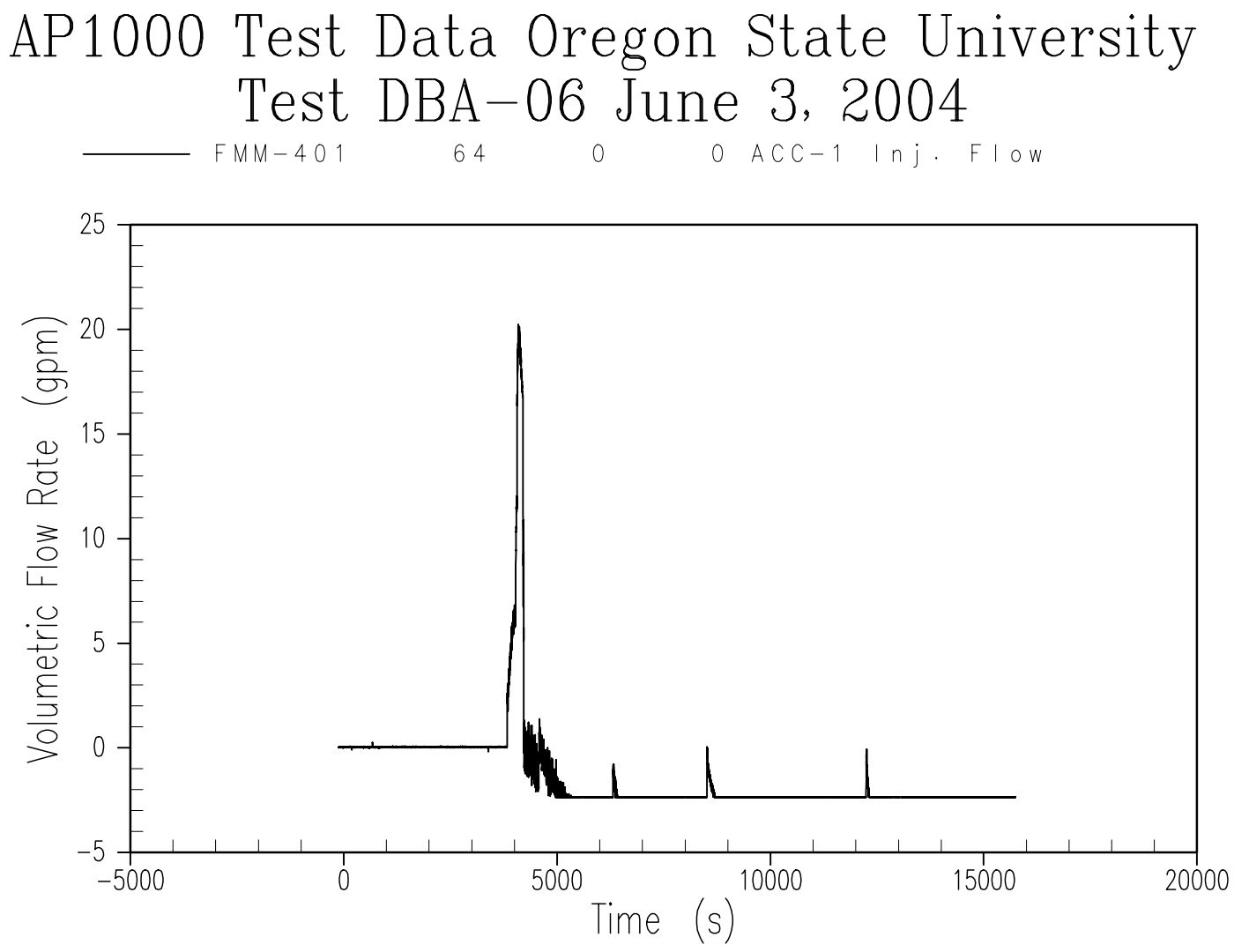

Figure A-23 Accumulator 1 Flow Rate 

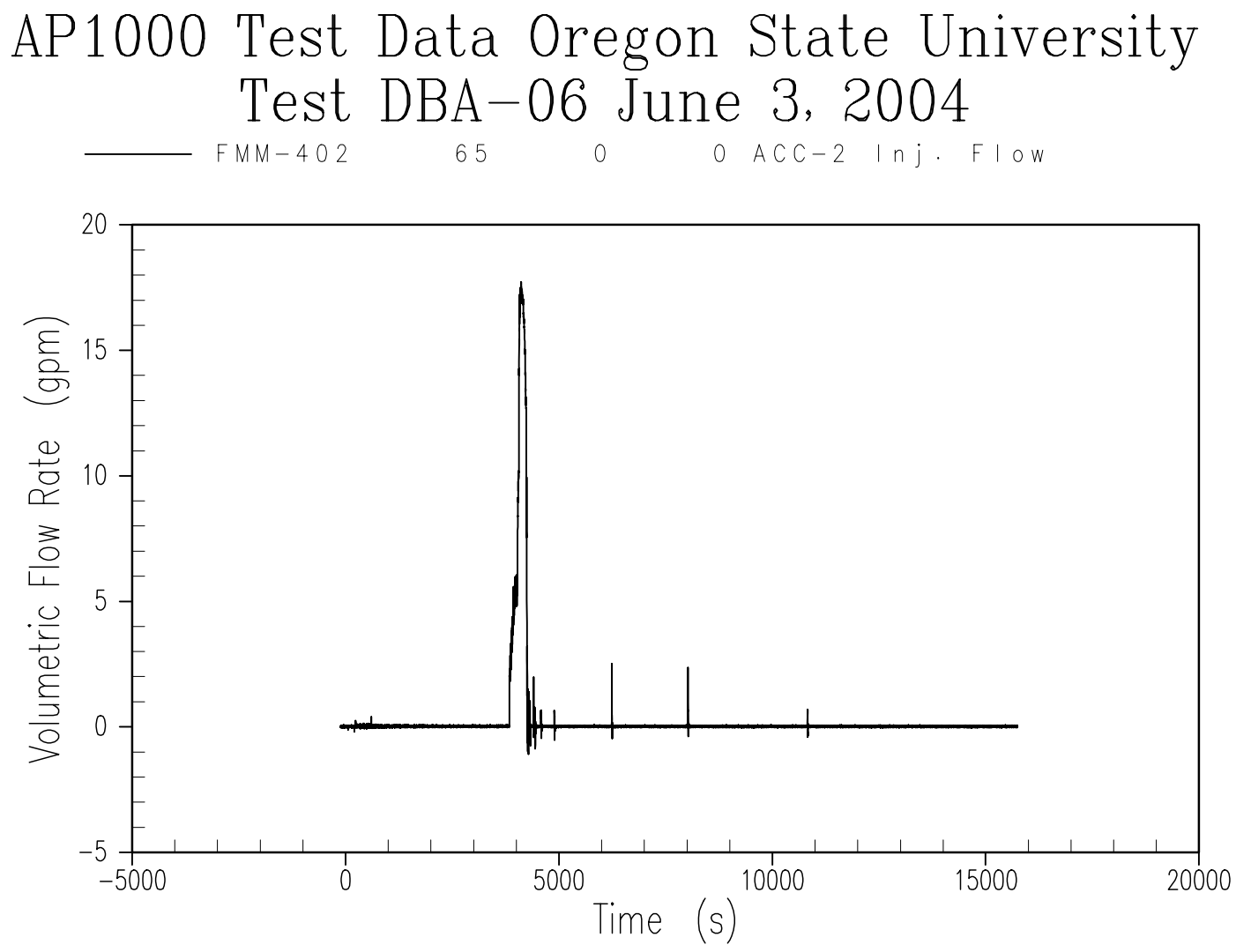

Figure A-24 Accumulator 2 Flow Rate 

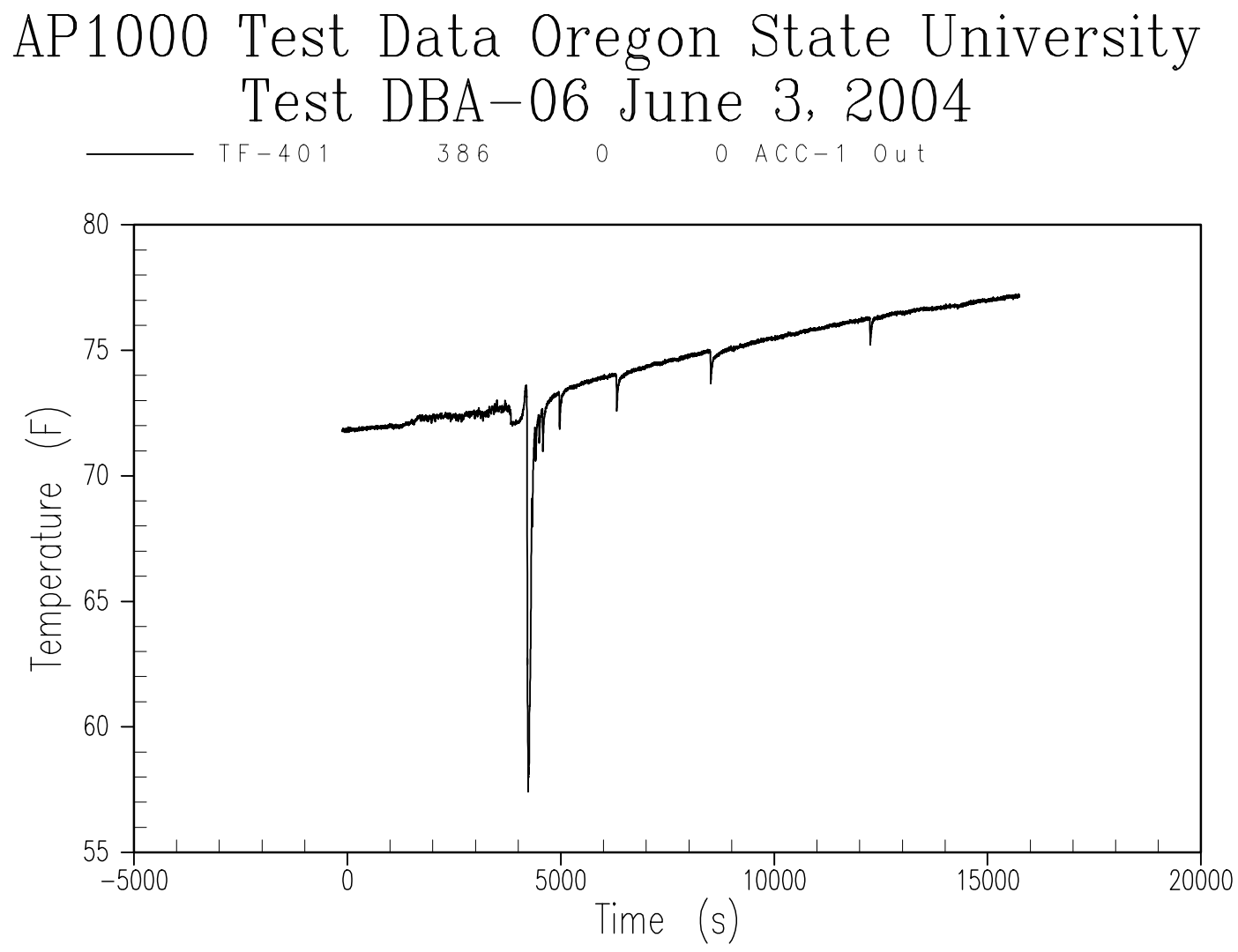

Figure A-25 Accumulator 1 Liquid Discharge Temperature 

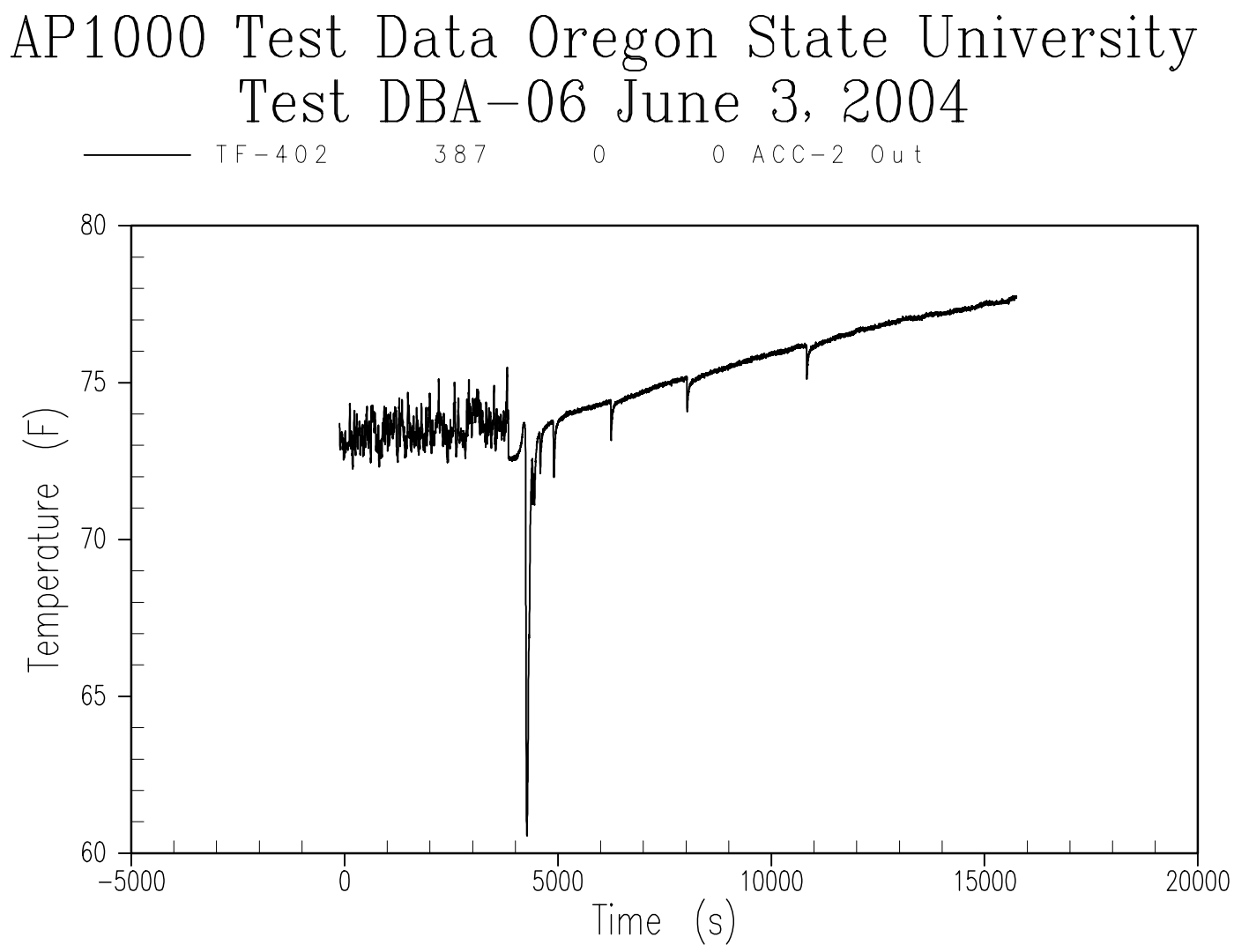

Figure A-26 Accumulator 2 Liquid Discharge Temperature 

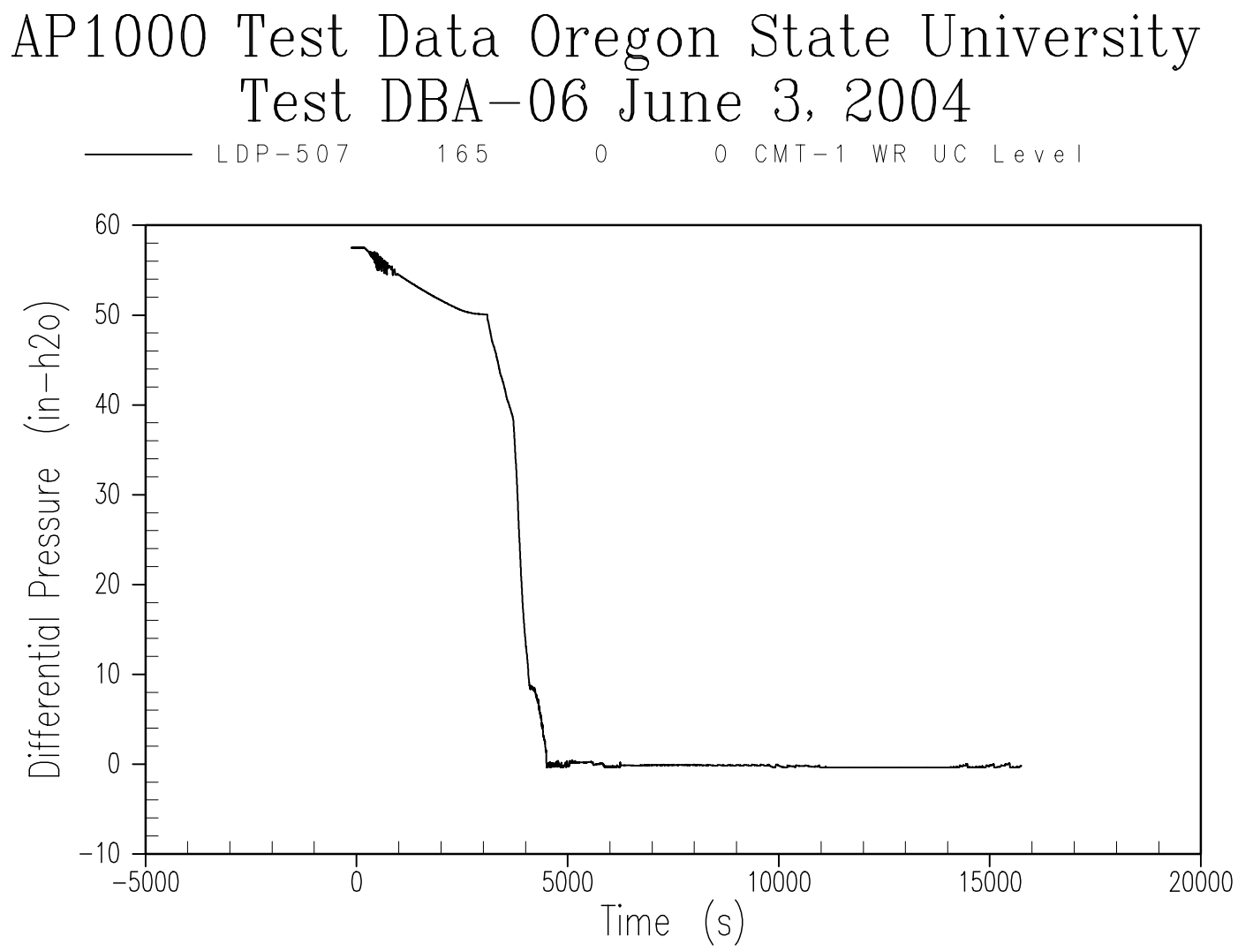

Figure A-27 Core Makeup Tank 1 Liquid Level 


\section{AP1000 Test Data Oregon State University Test DBA-06 June 3, 2004 \\ $\longrightarrow$ LDP -502

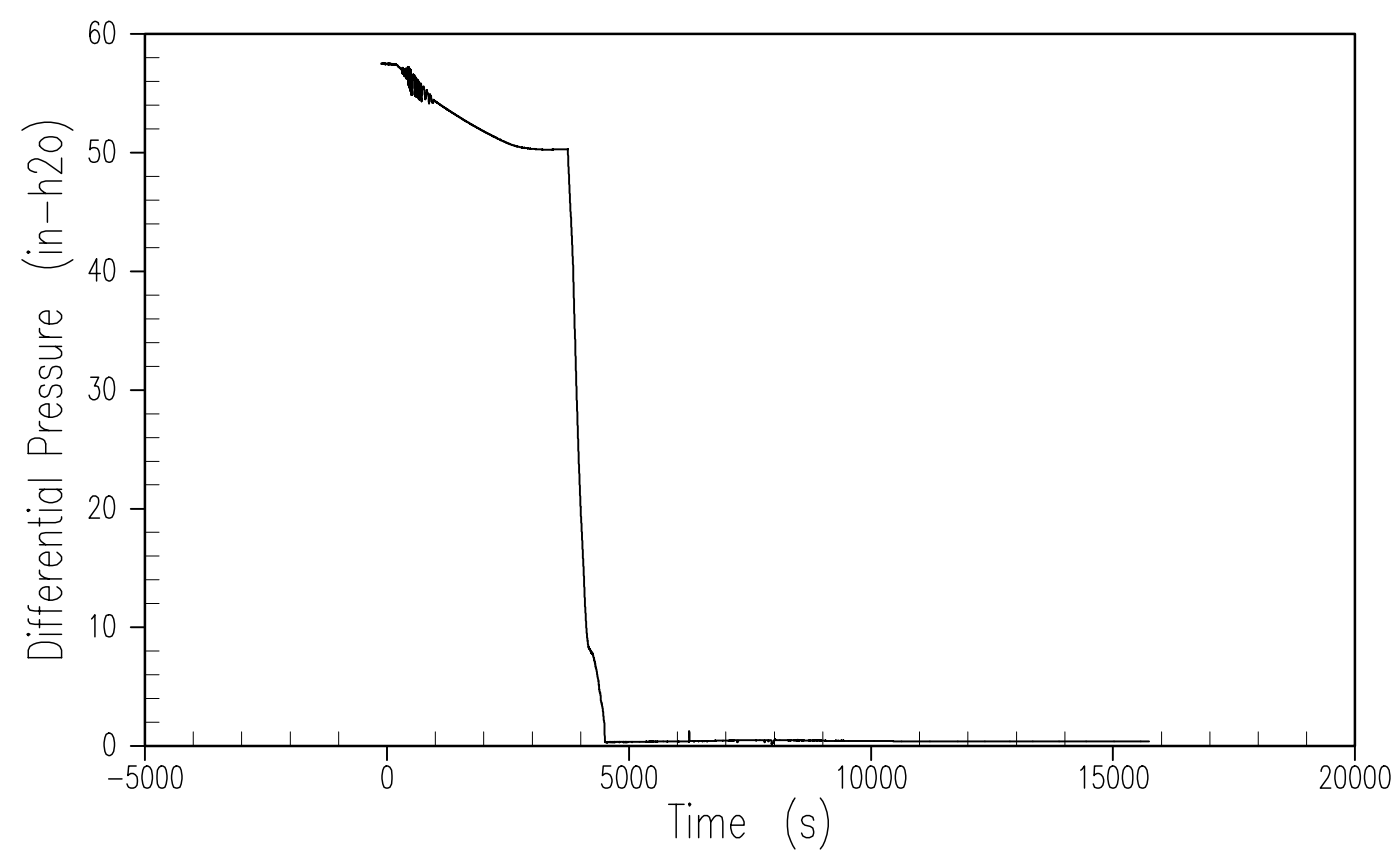

Figure A-28 Core Makeup Tank 2 Liquid Level 

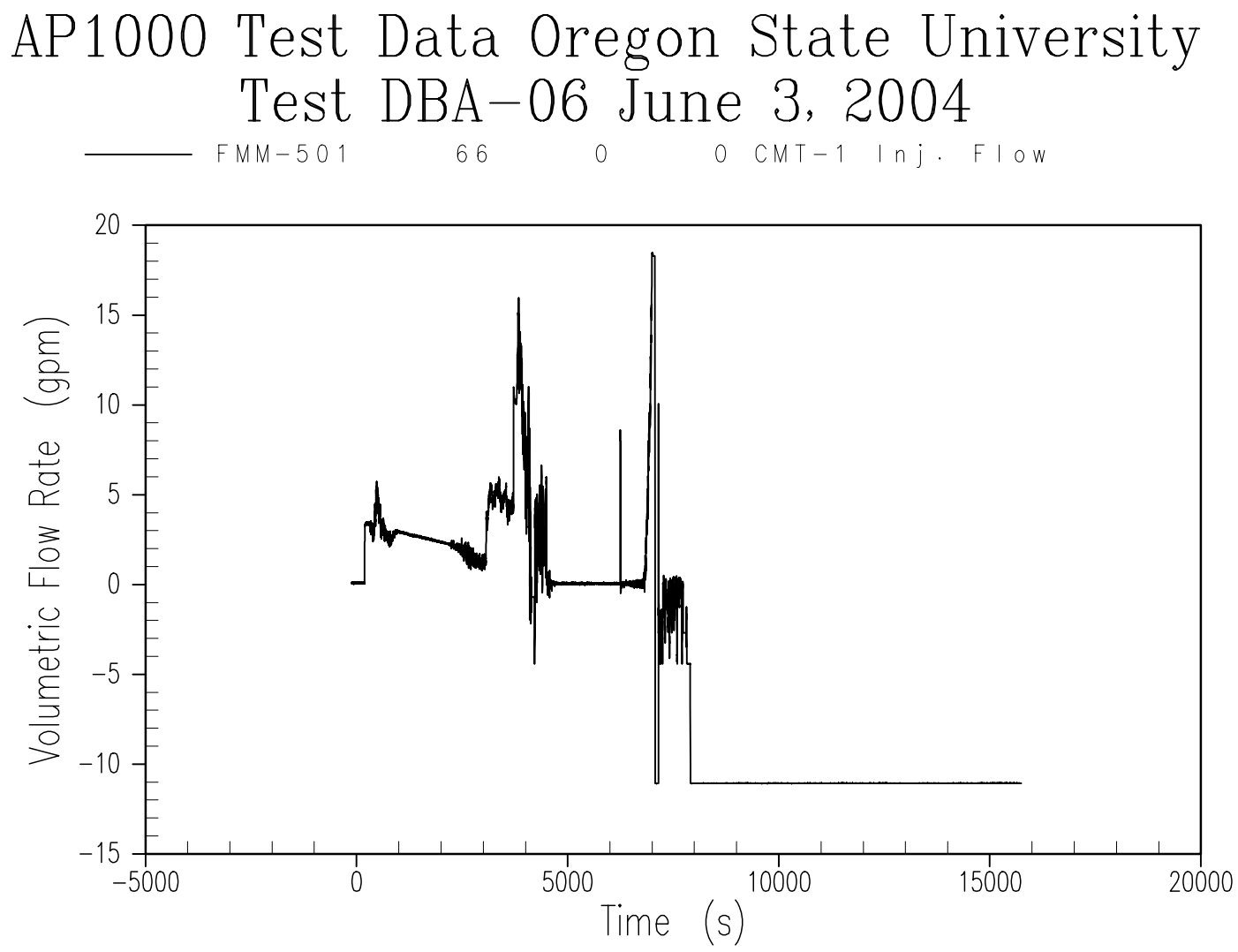

Figure A-29 Core Makeup Tank 1 Flow Rate 


\section{AP1000 Test Data Oregon State University Test DBA-06 June 3, 2004 \\ $\longrightarrow F M M-504$

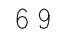 \\ 0 \\ o CMT-2 Inj. FI OW}

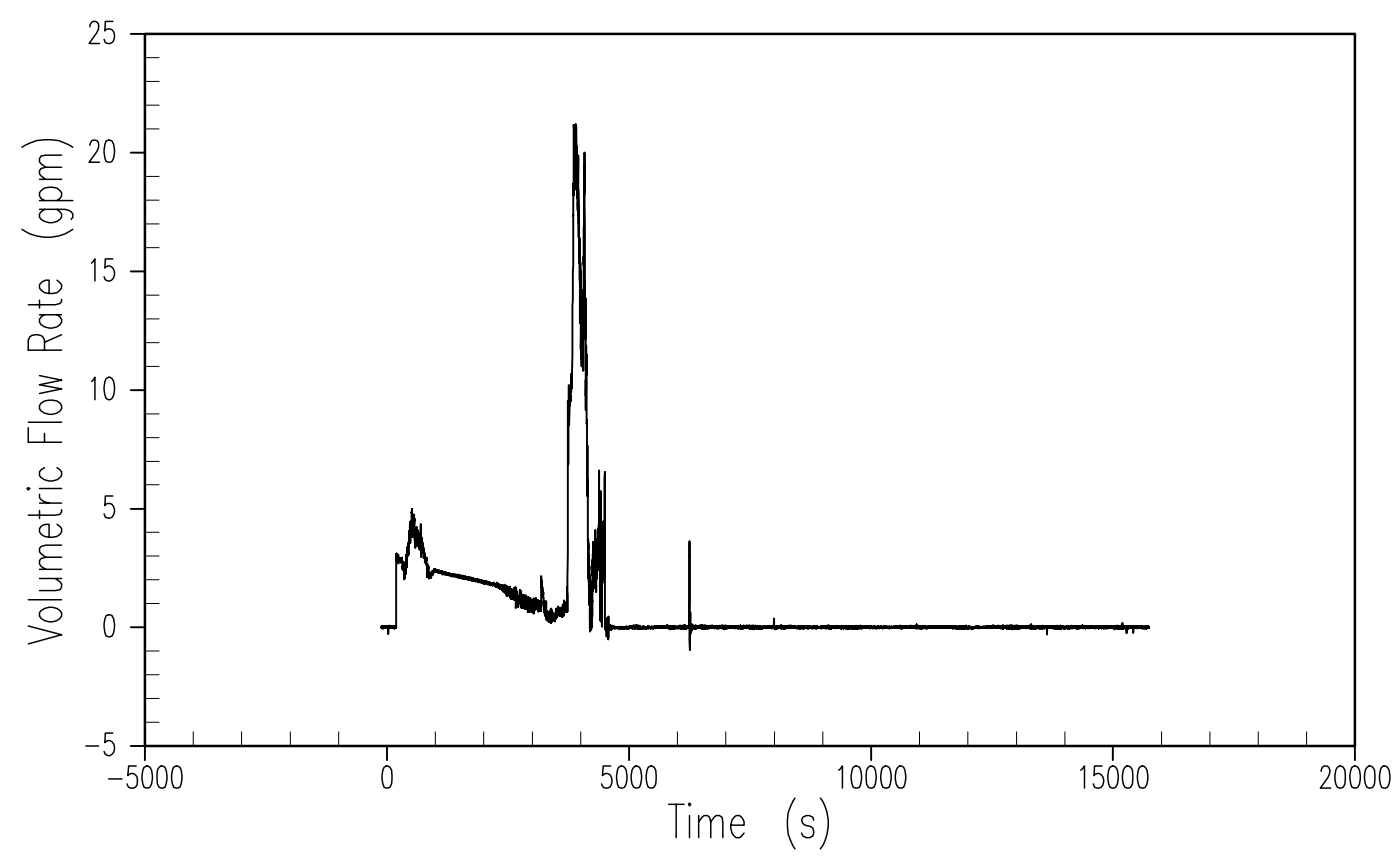

Figure A-30 Core Makeup Tank 2 Flow Rate 

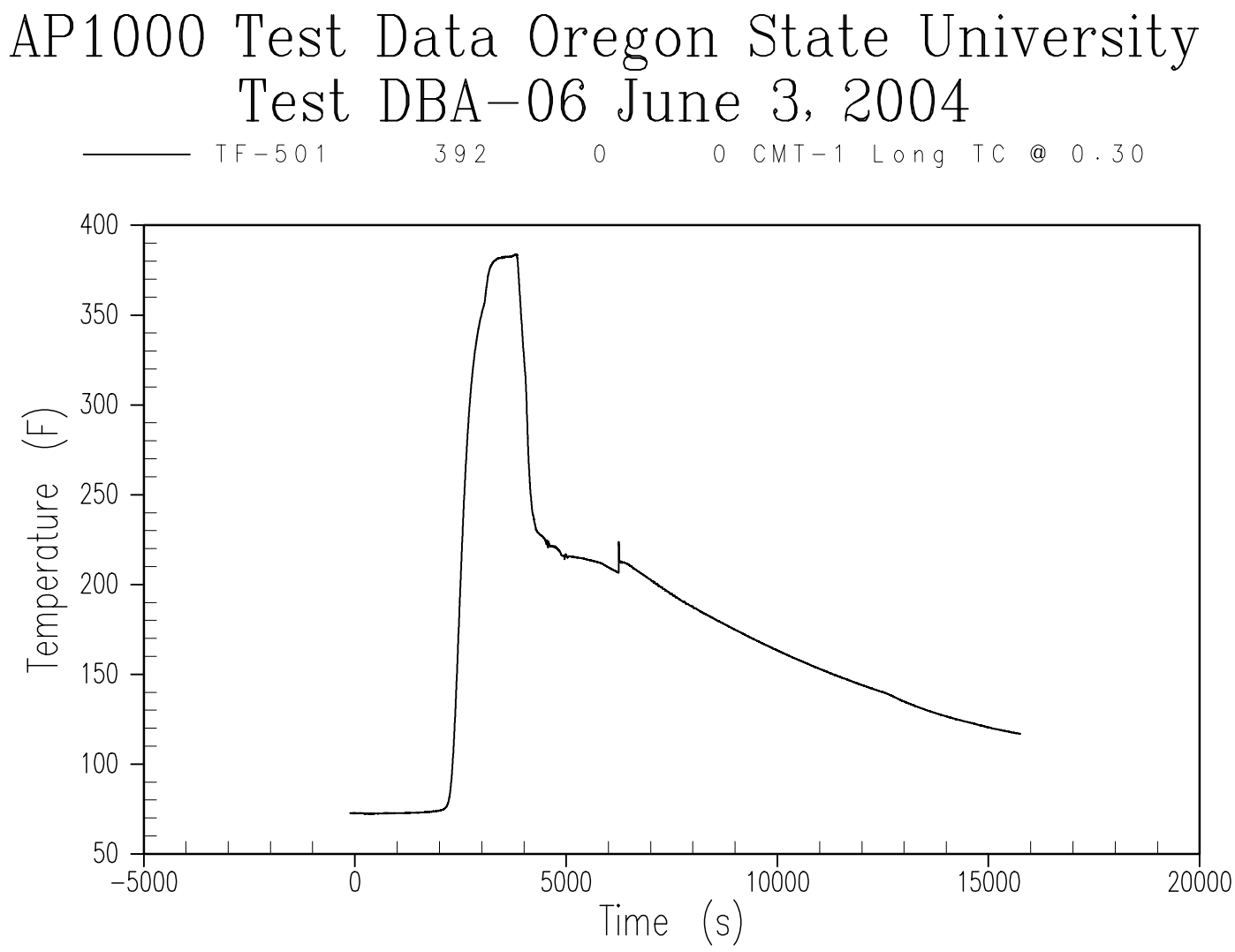

Figure A-31 Core Makeup Tank 1 Liquid Temperature - Bottom 

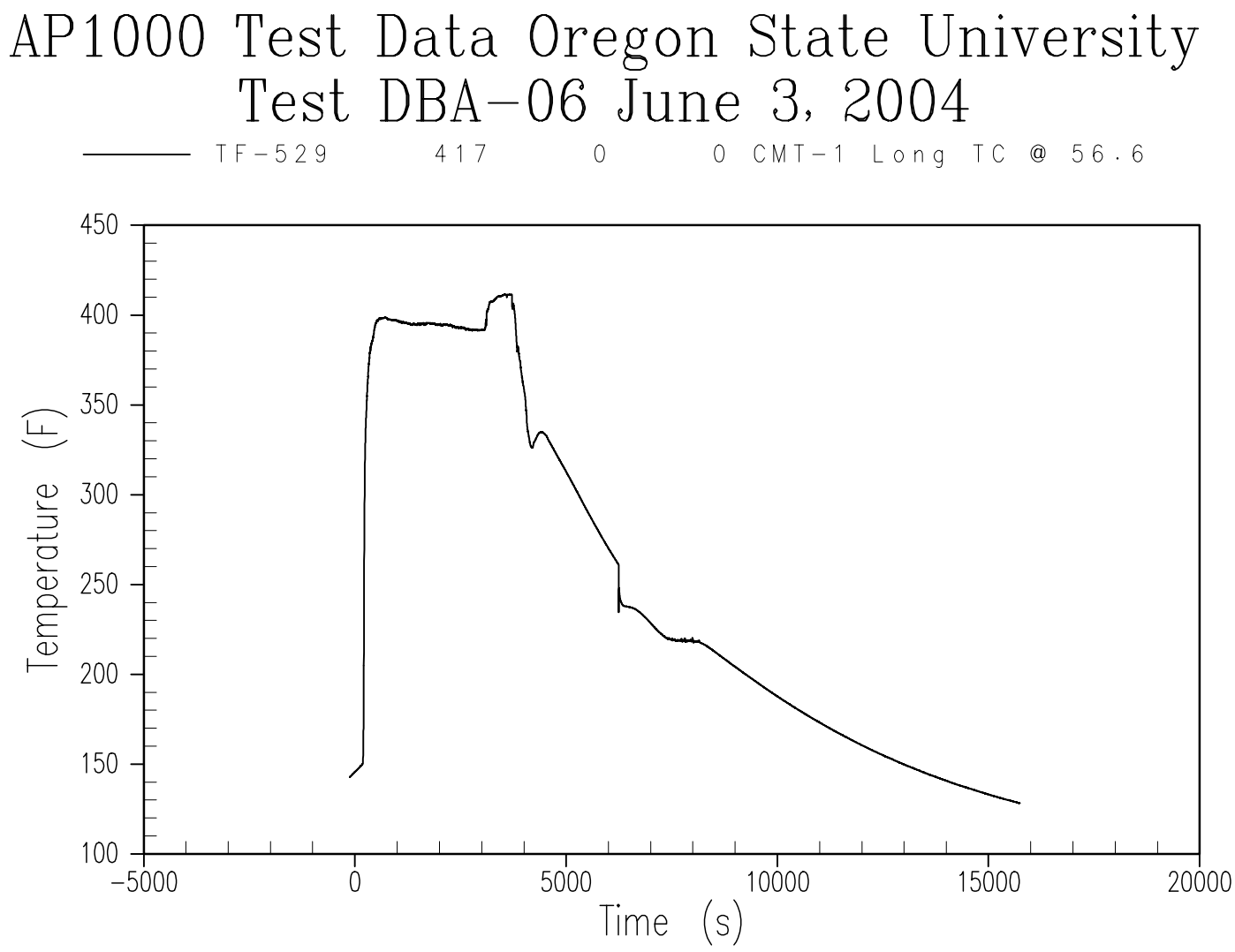

Figure A-32 Core Makeup Tank 1 Liquid Temperature - Top 

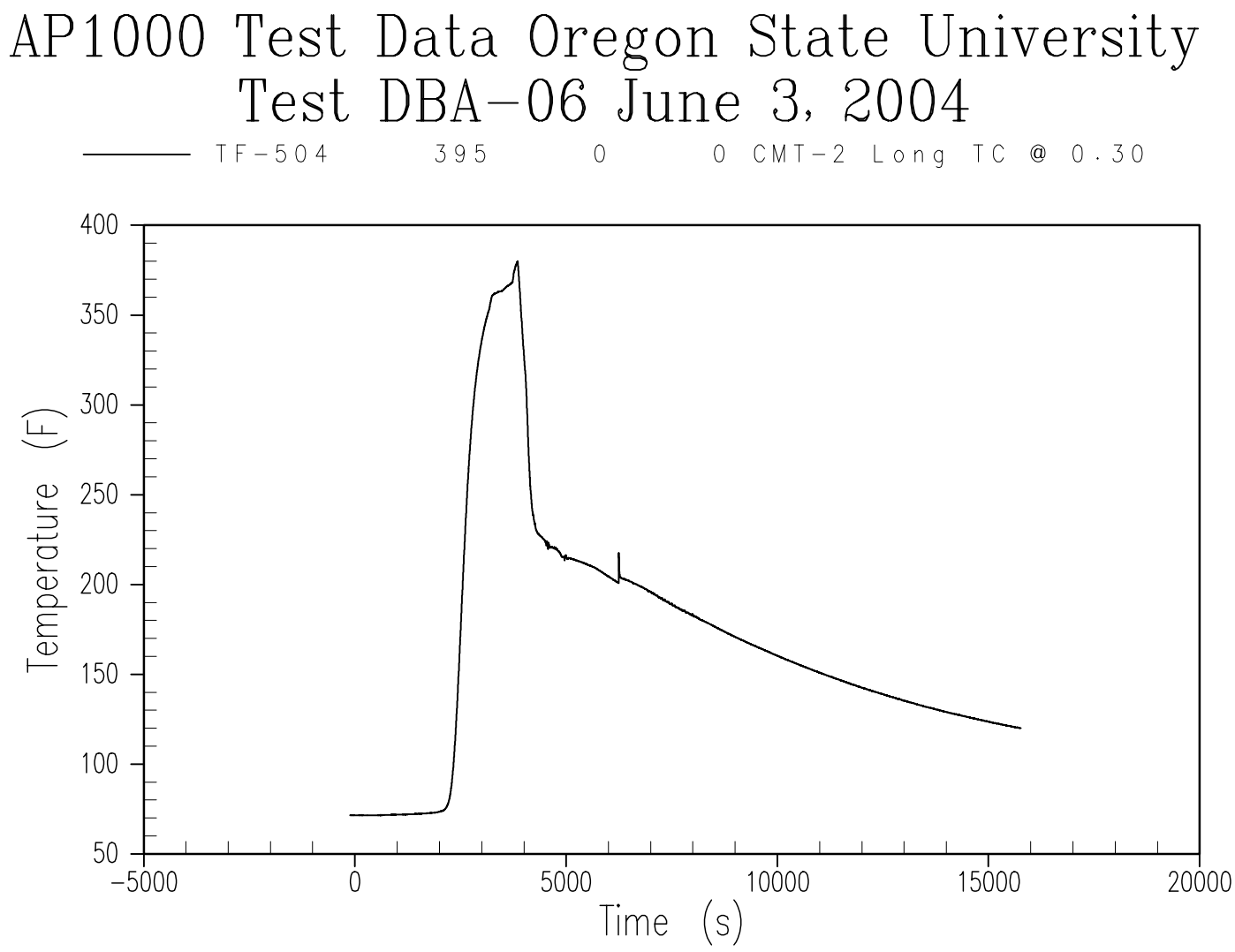

Figure A-33 Core Makeup Tank 2 Liquid Temperature - Bottom 

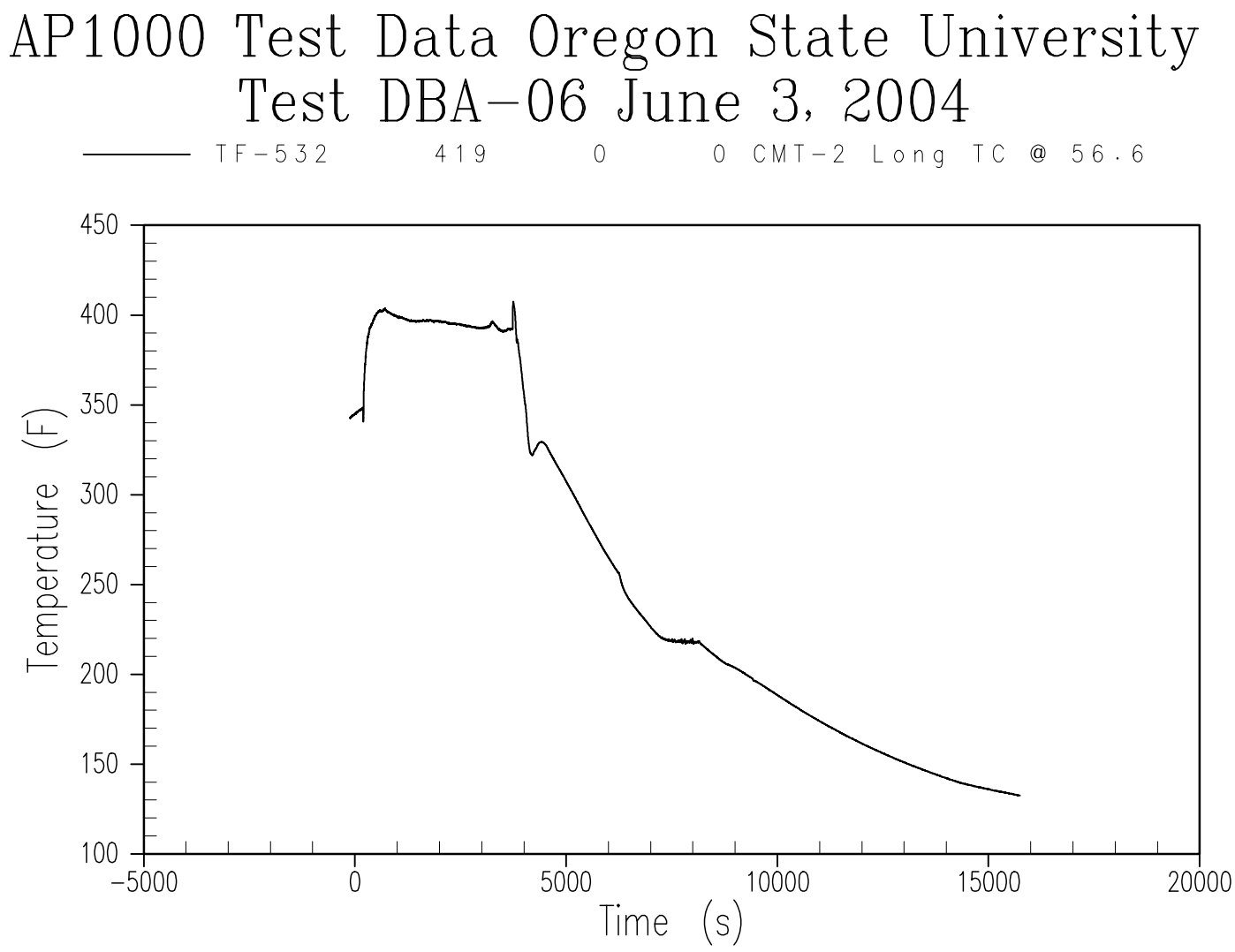

Figure A-34 Core Makeup Tank 2 Liquid Temperature - Top 


\section{AP1000 Test Data Oregon State University Test DBA-06 June 3, 2004 \\ $\longrightarrow$ FMM-802

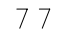 \\ O PRHR I $\mathrm{F}$ I OW}

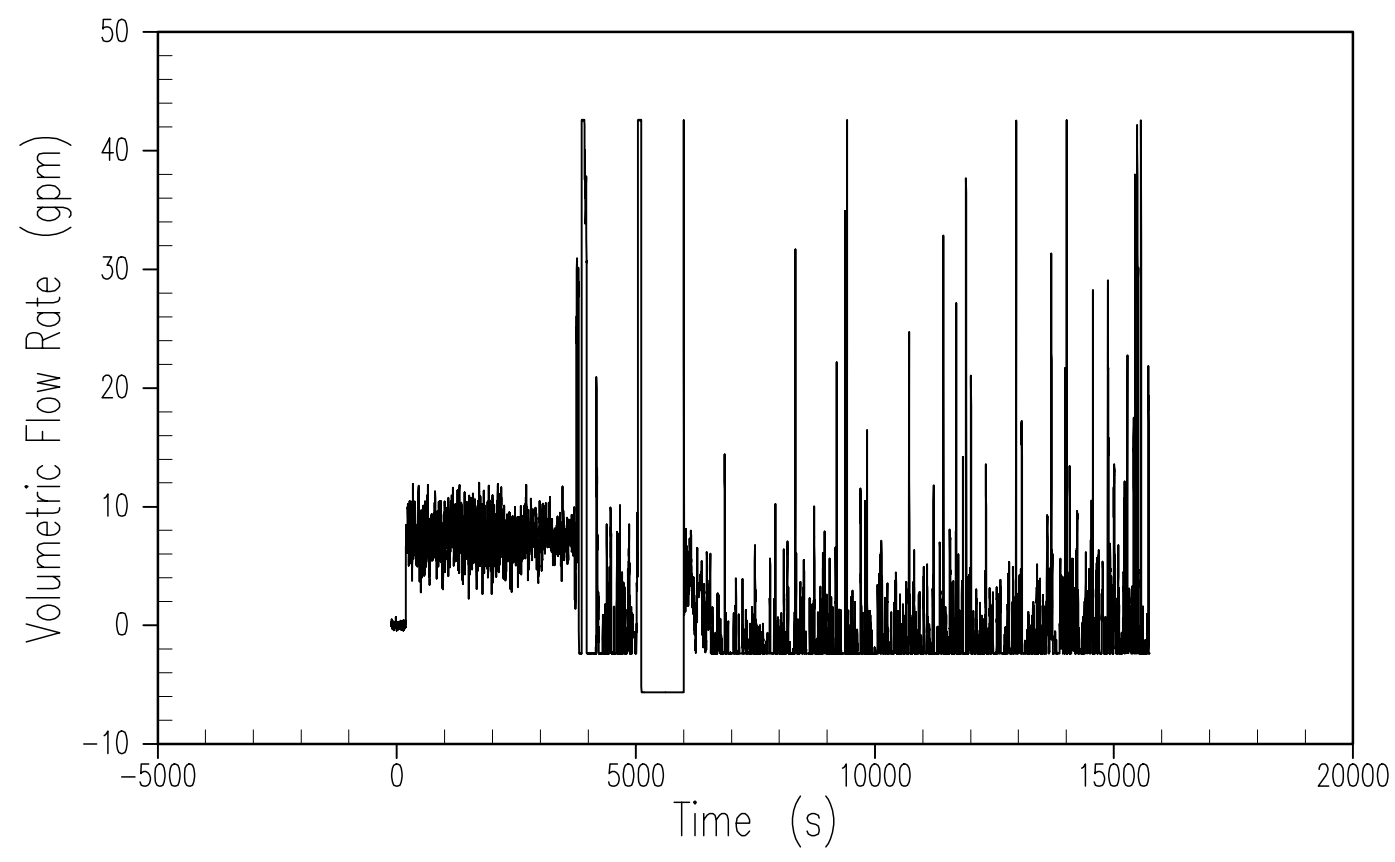

Figure A-35 Passive Residual Heat Removal Inlet Flow Rate 

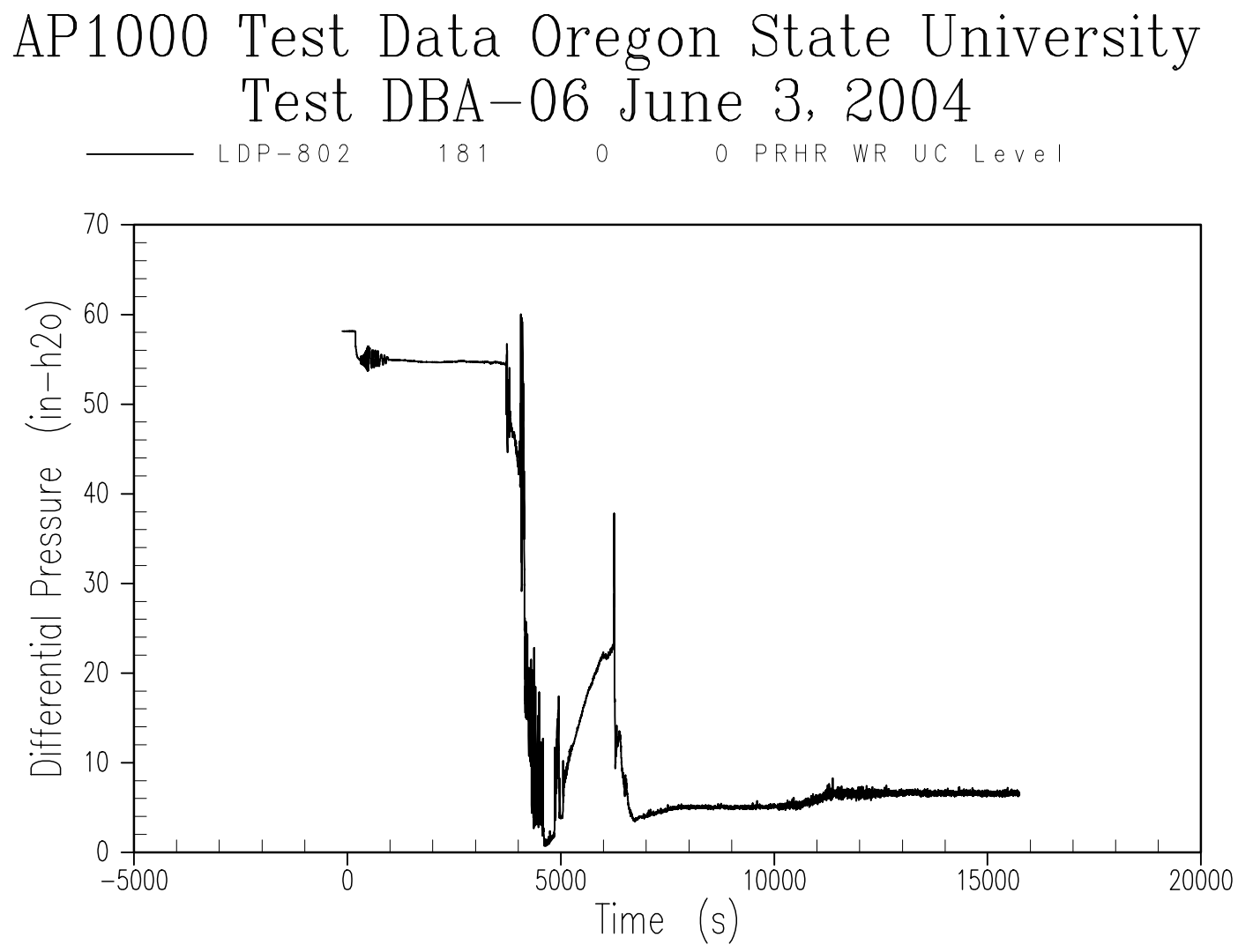

Figure A-36 Passive Residual Heat Removal Liquid Level 


\section{AP1000 Test Data Oregon State University Test DBA-06 June 3, 2004 \\ $\longrightarrow$ FMM-804

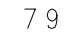 \\ 0 \\ o PRHR Out FIOW}

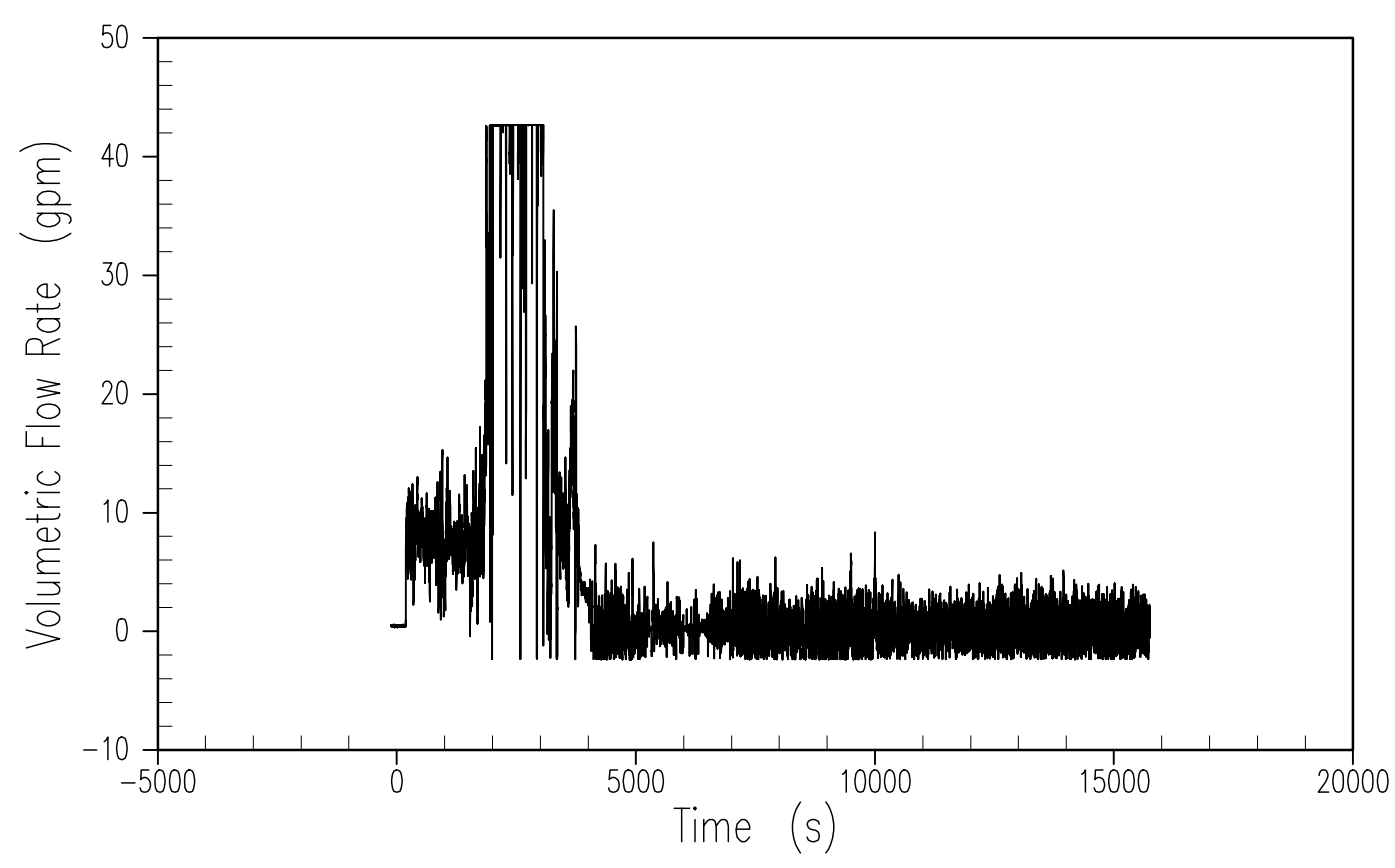

Figure A-37 Passive Residual Heat Removal Outlet Flow Rate 


\section{AP1000 Test Data Oregon State University Test DBA-06 June 3, 2004 \\ $\longrightarrow$ LDP -701 \\ 179 \\ o I RWST UC Leve I}

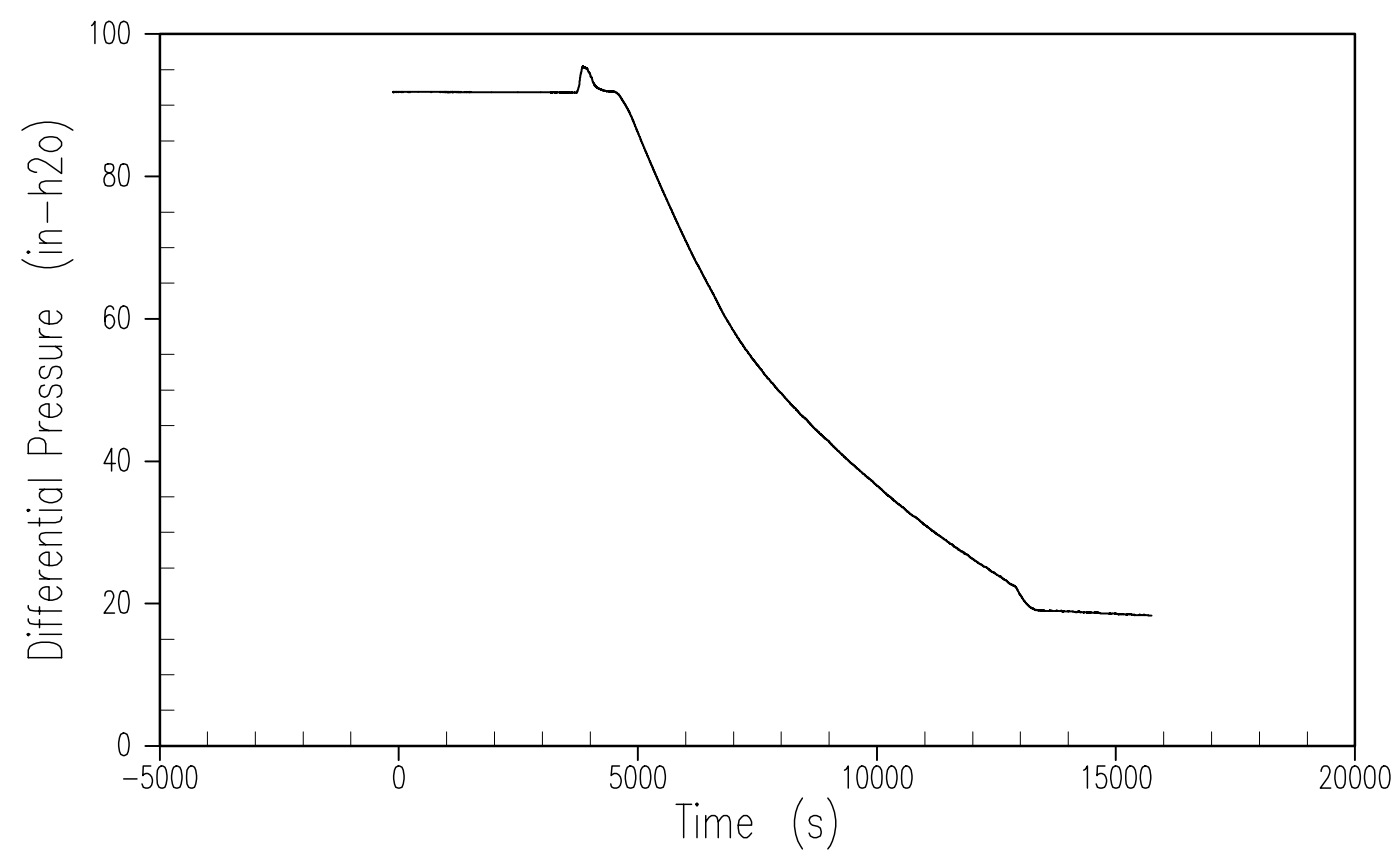

Figure A-38 IRWST Liquid Level 

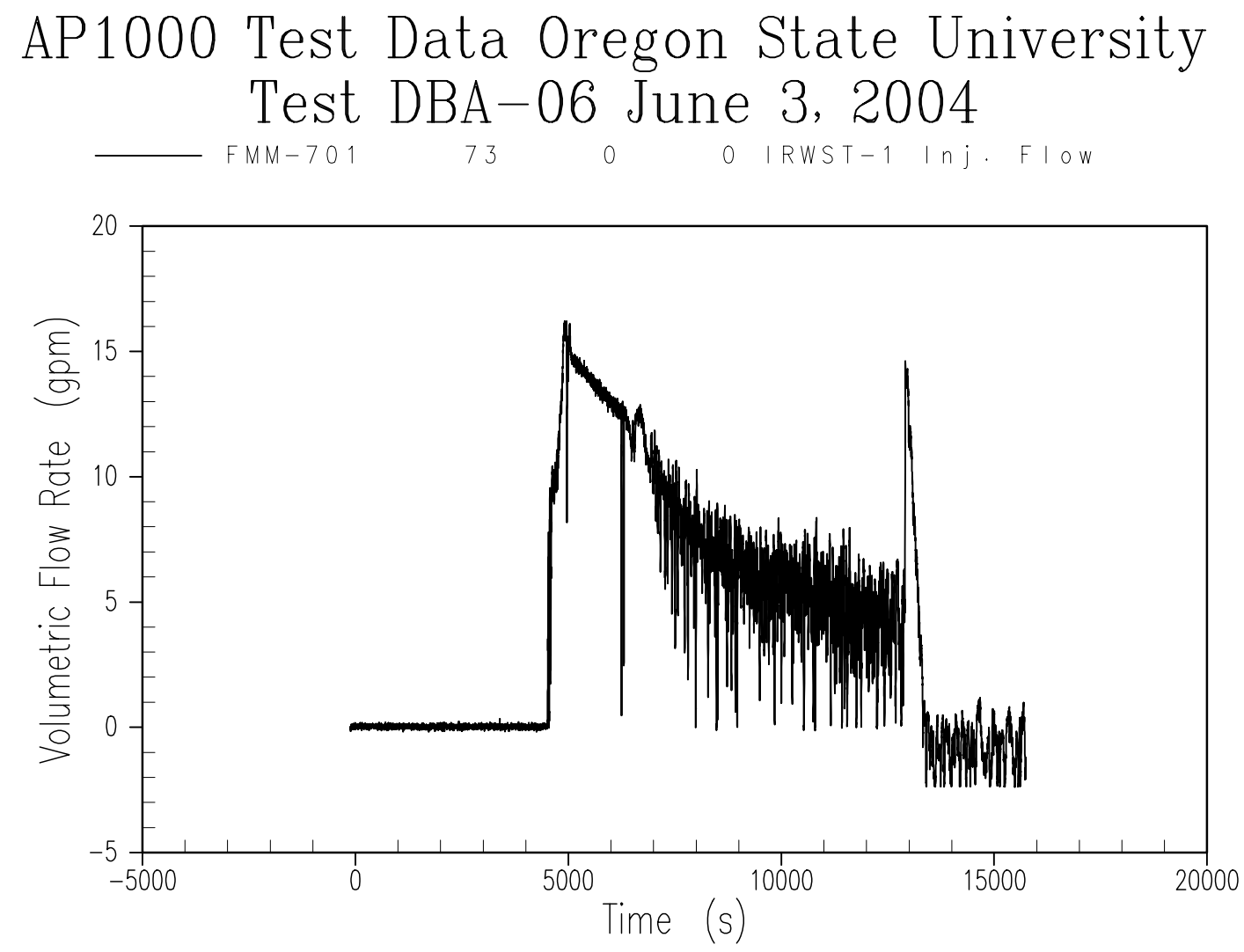

Figure A-39 IRWST Discharge Line 1 Flow Rate 

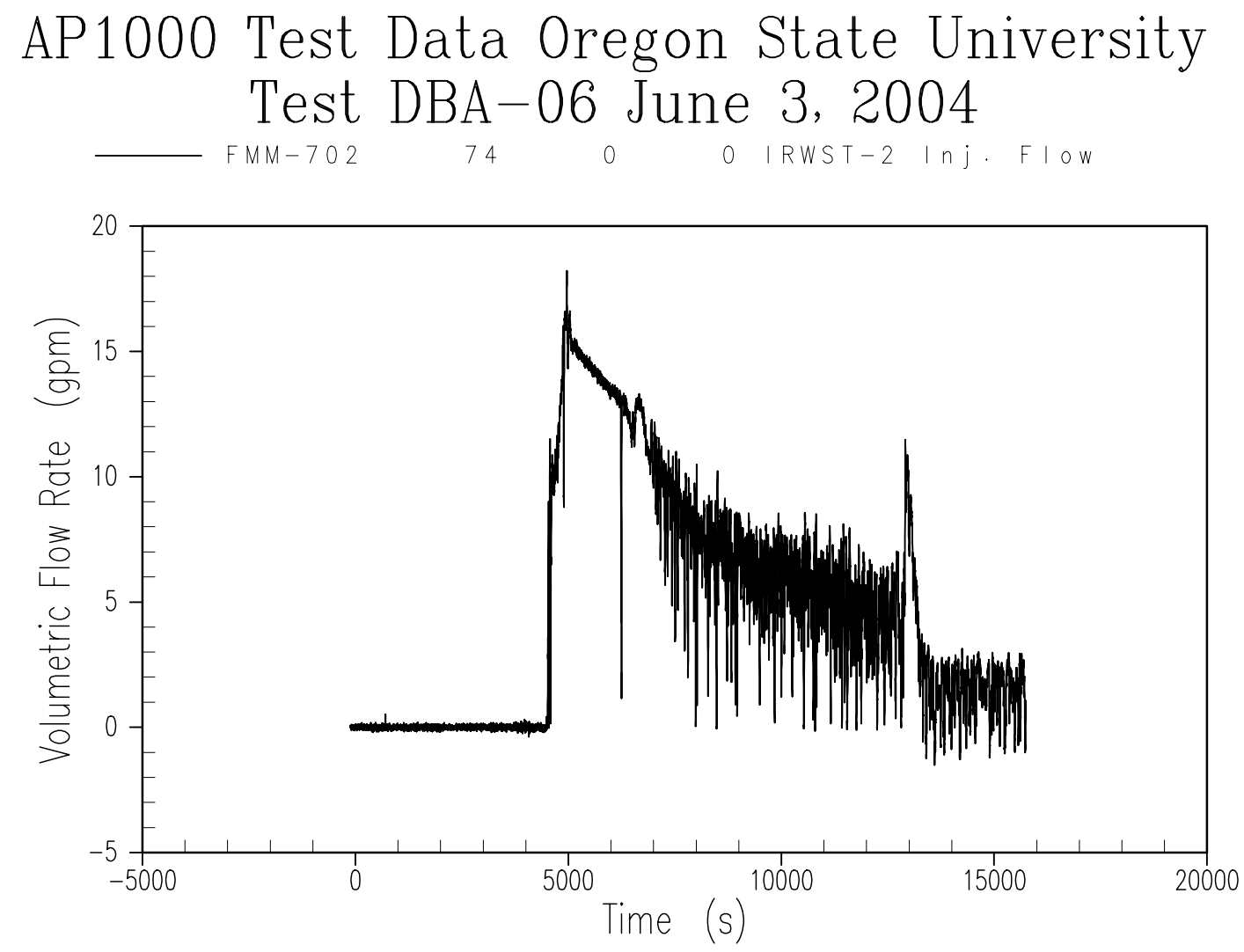

Figure A-40 IRWST Discharge Line 2 Flow Rate 

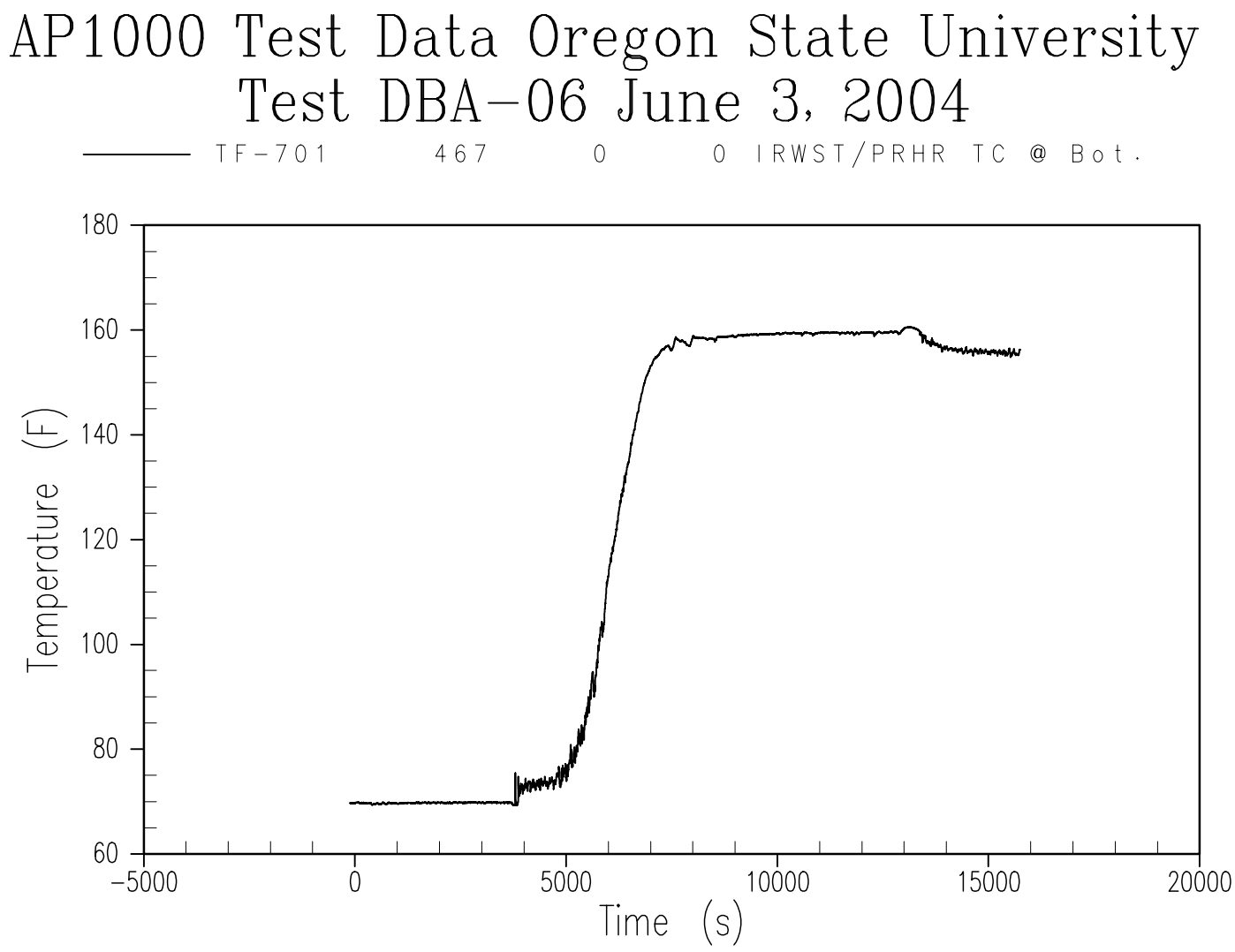

Figure A-41 IRWST Fluid Temperature - Bottom 

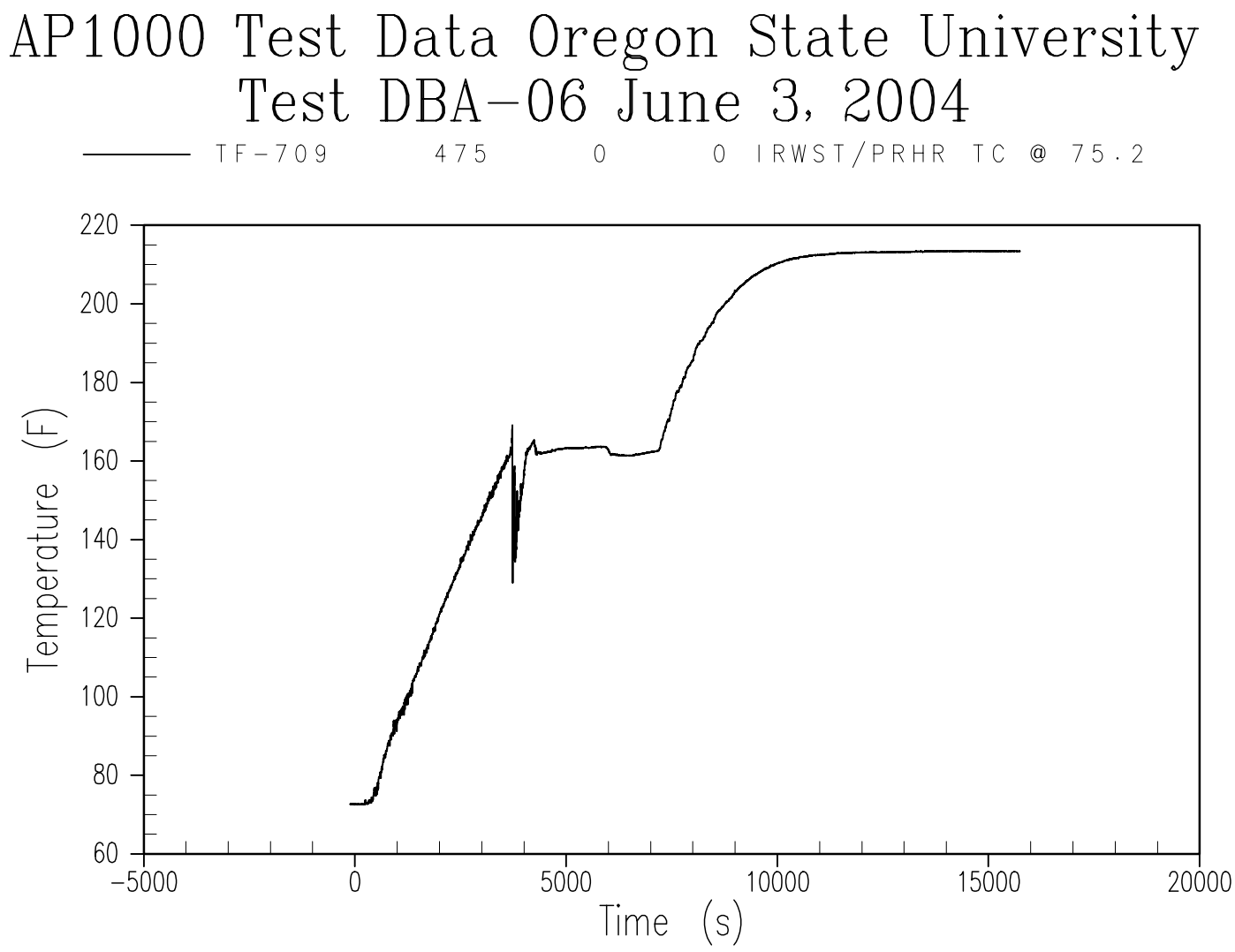

Figure A-42 IRWST Fluid Temperature - Top 

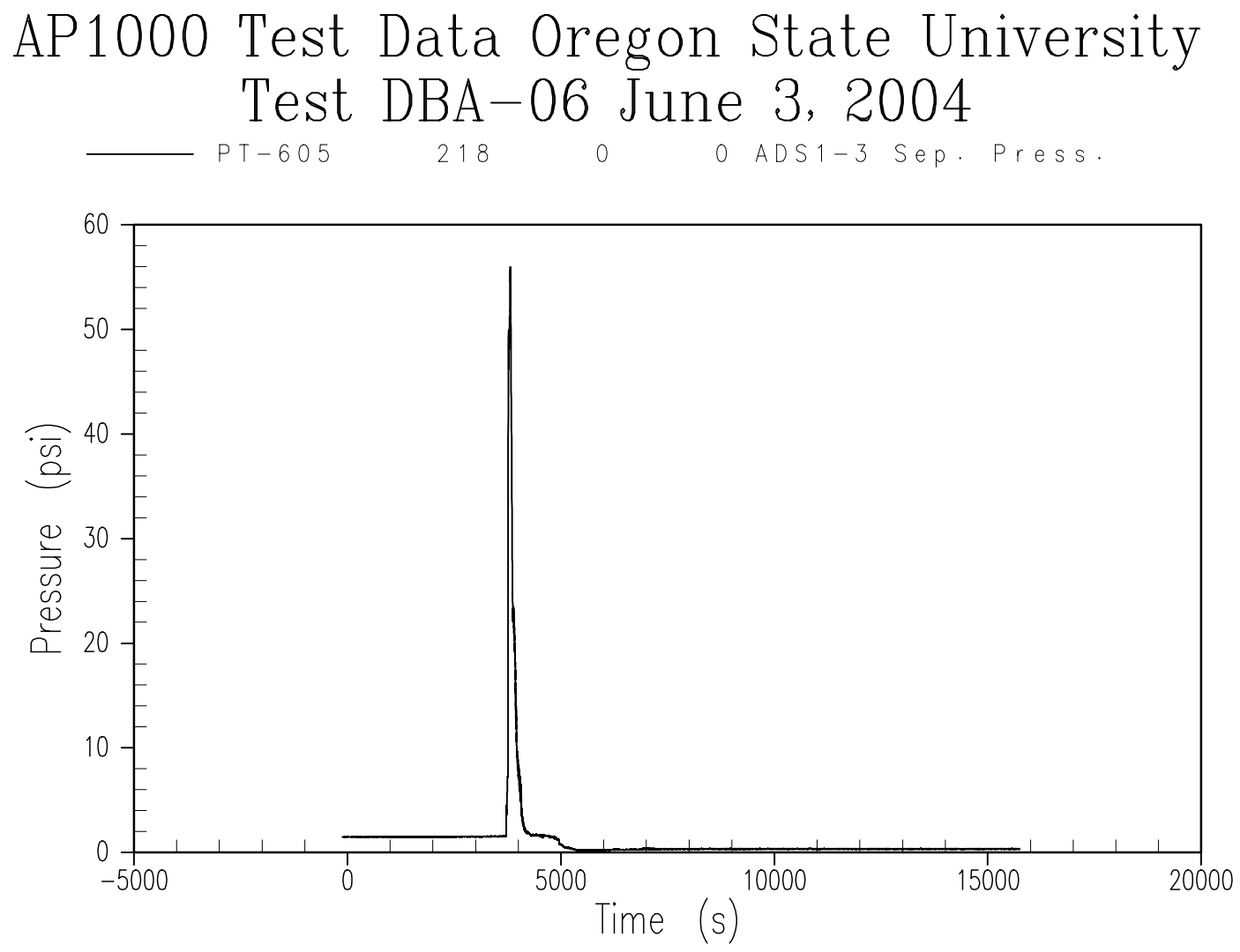

Figure A-43 ADS 1-3 Separator Pressure 

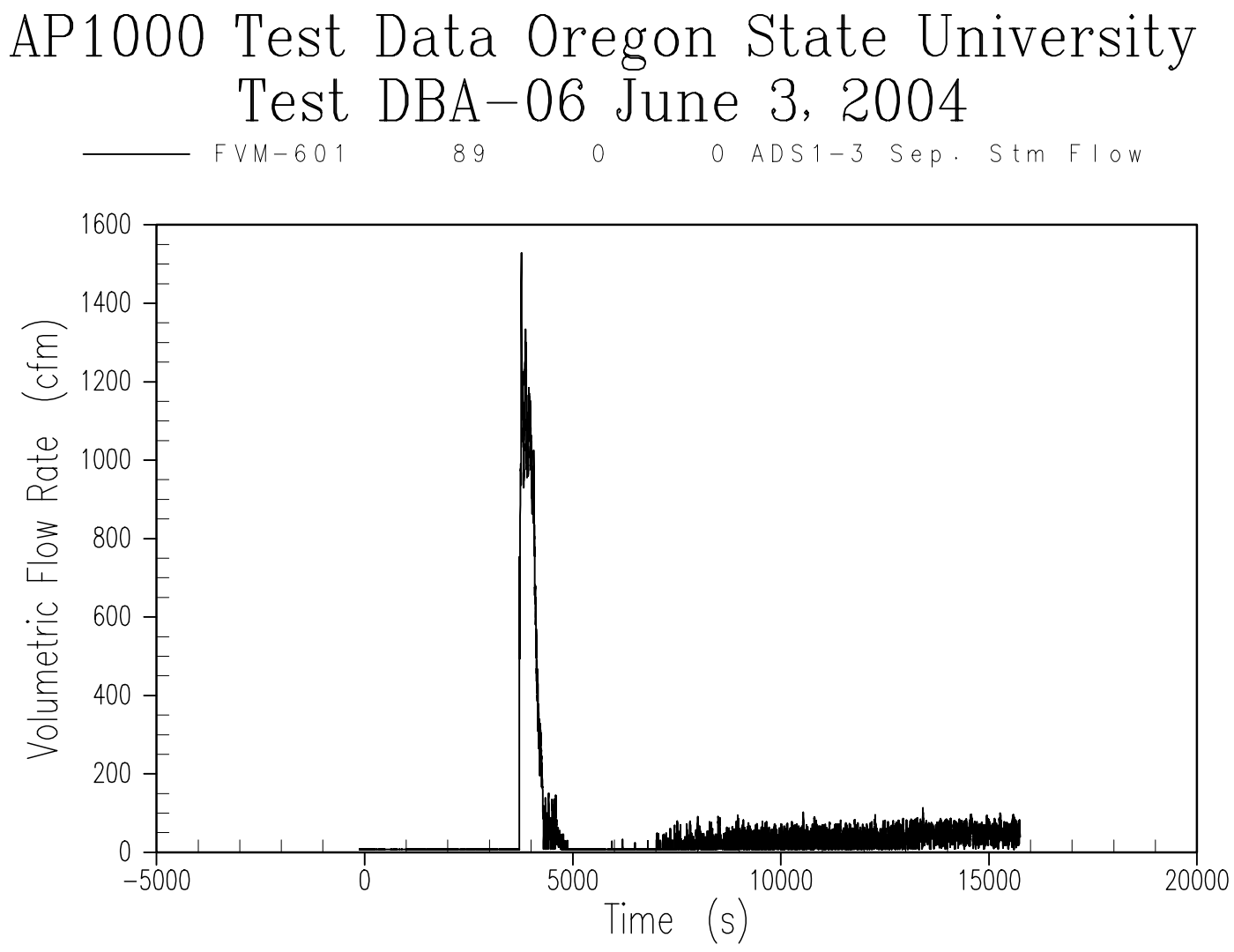

Figure A-44 ADS 1-3 Separator Steam Flow Rate 

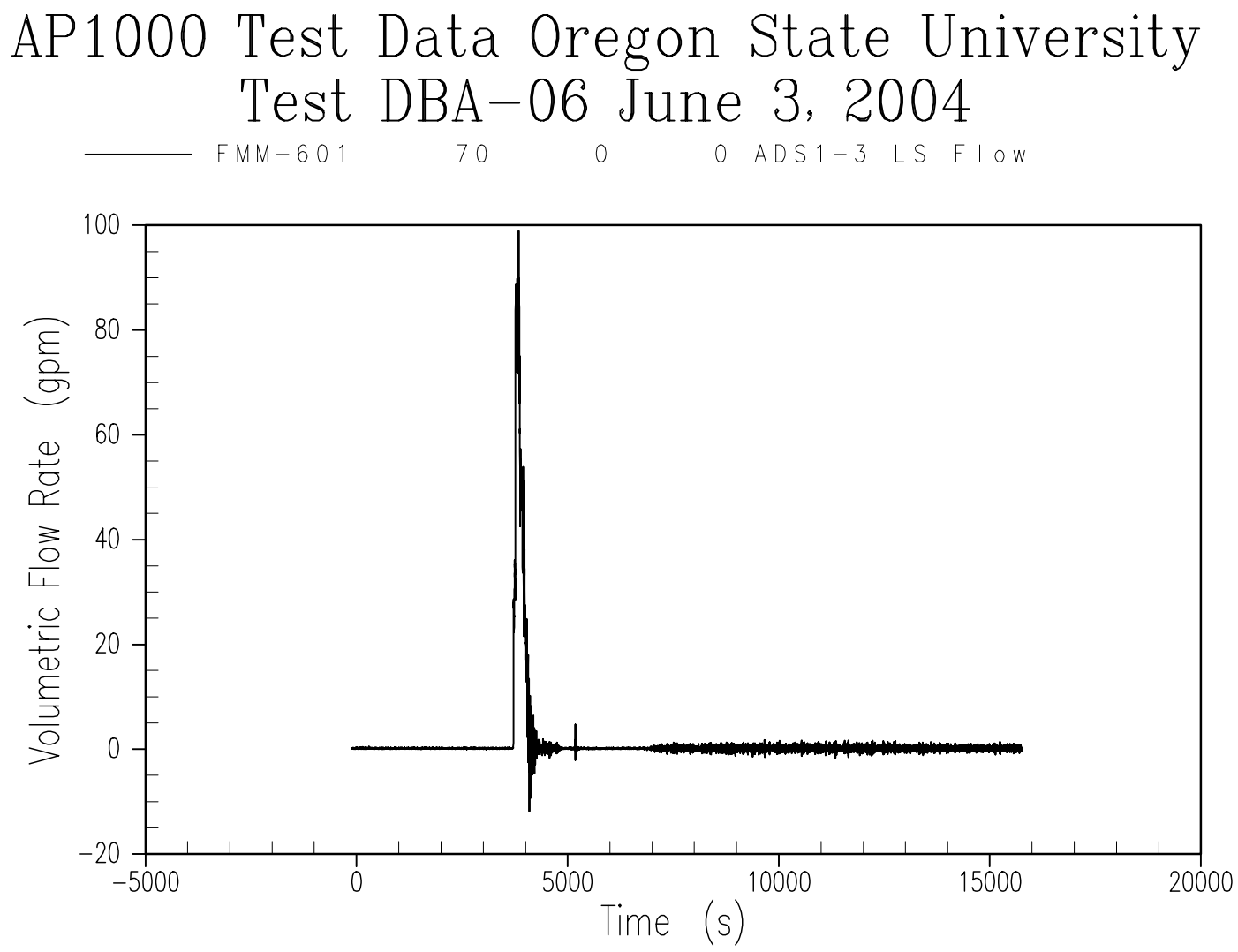

Figure A-45 ADS 1-3 Separator Liquid Flow Rate 

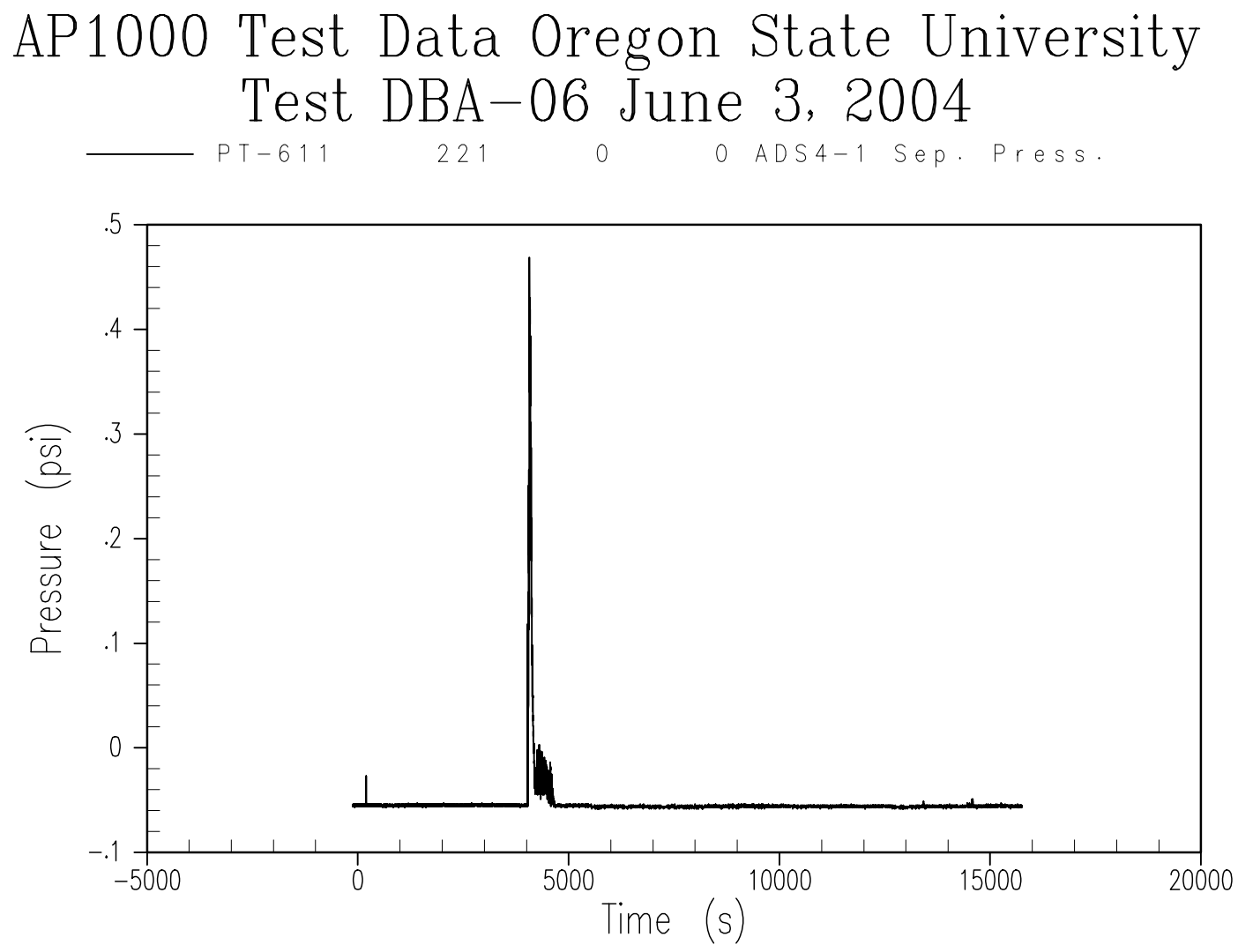

Figure A-46 ADS 4-1 Separator Pressure 

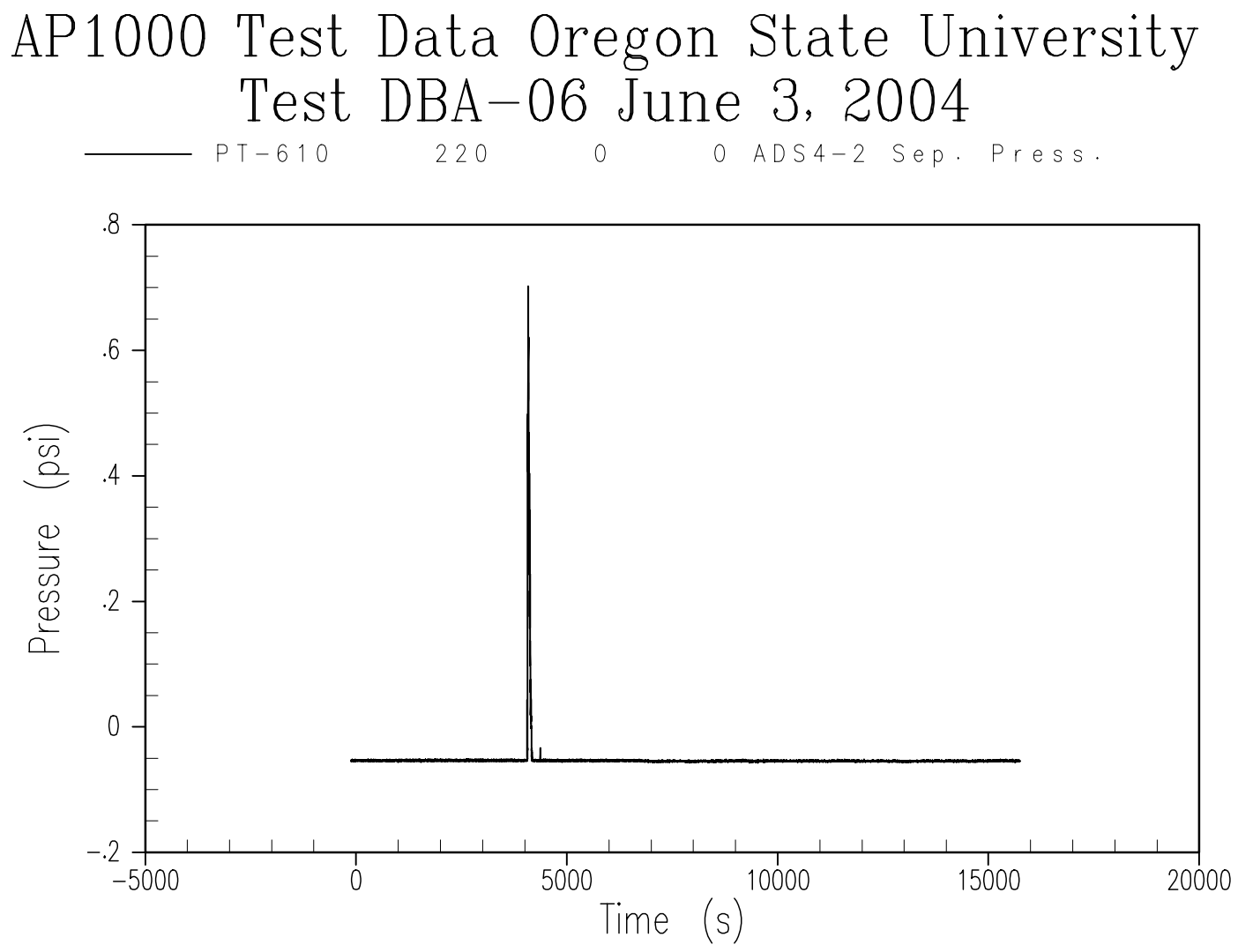

Figure A-47 ADS 4-2 Separator Pressure 

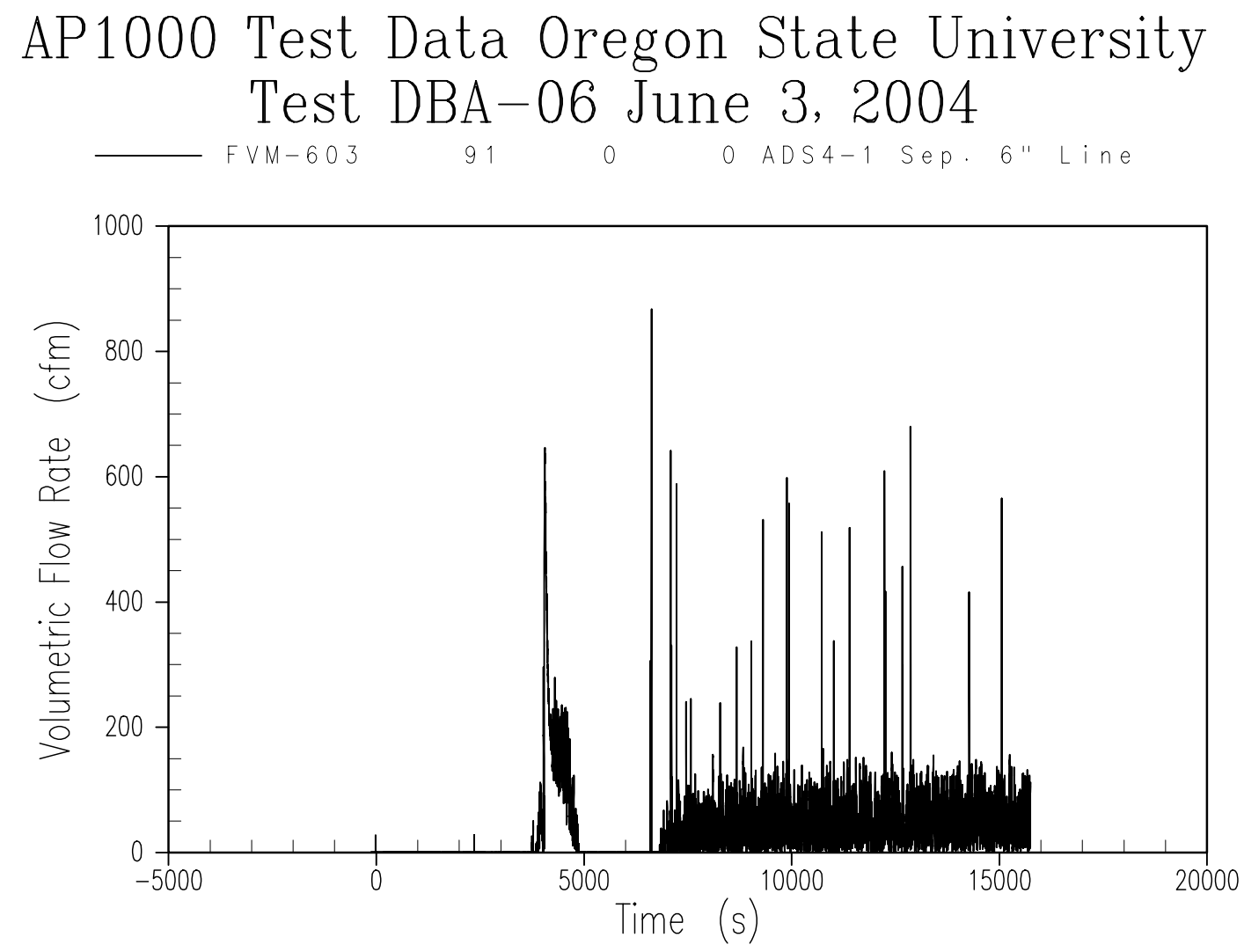

Figure A-48 ADS 4-1 Separator Steam Flow Rate 

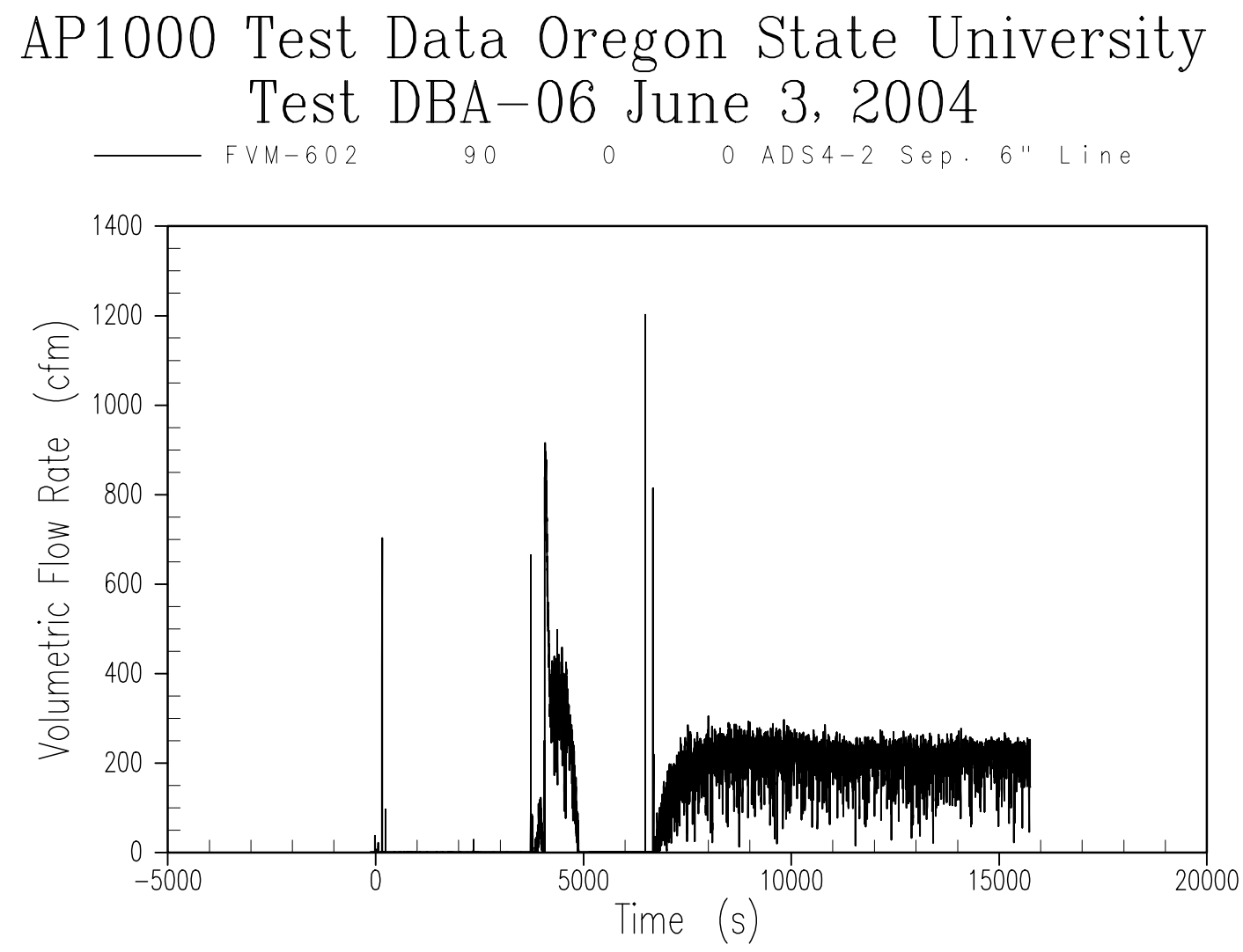

Figure A-49 ADS 4-2 Separator Steam Flow Rate 


\section{AP1000 Test Data Oregon State University Test DBA-06 June 3, 2004 \\ $\longrightarrow$ FMM-603

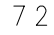 \\ 0 \\ O ADS4-1 LS F I OW}

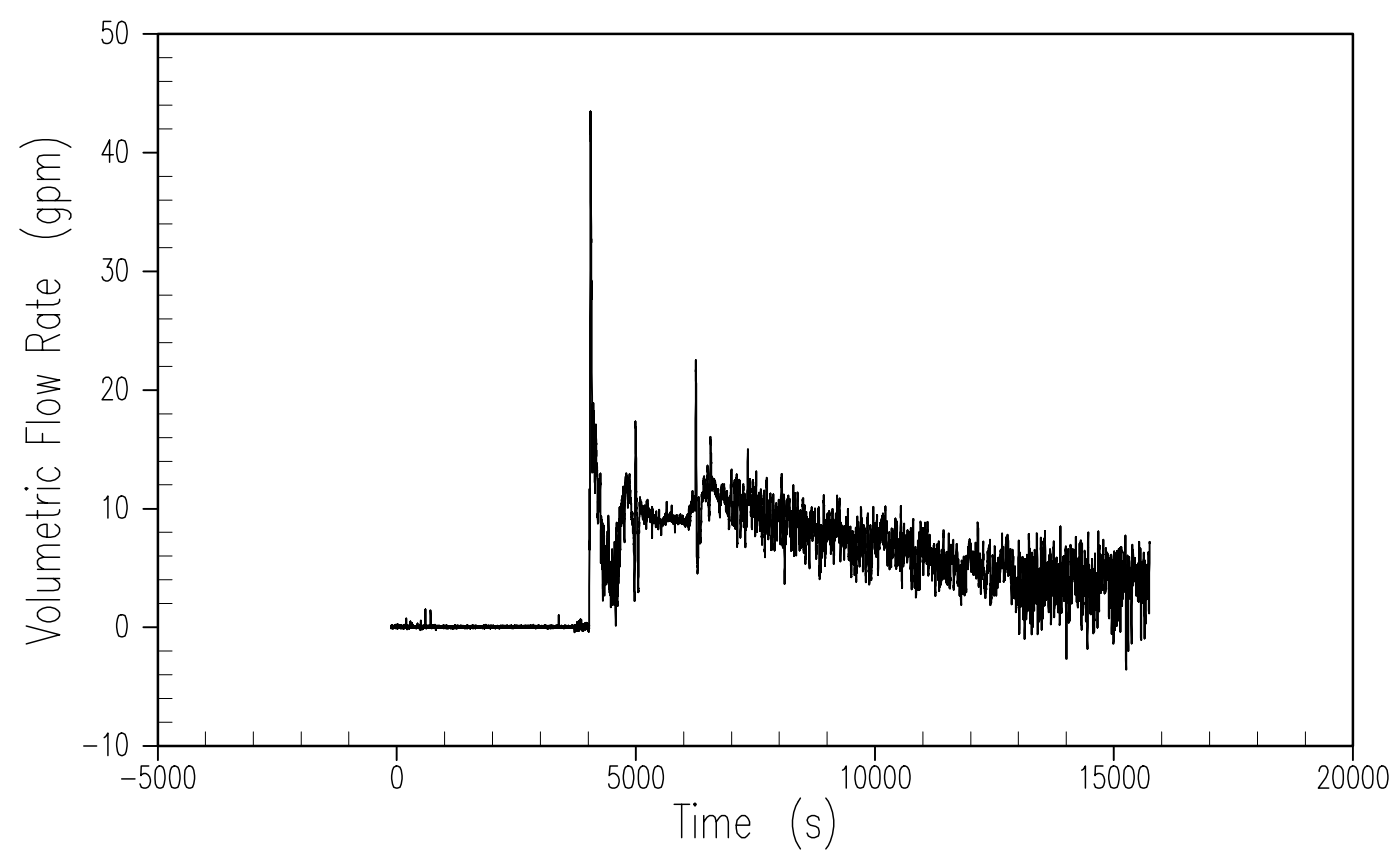

Figure A-50 ADS 4-1 Separator Liquid Flow Rate 

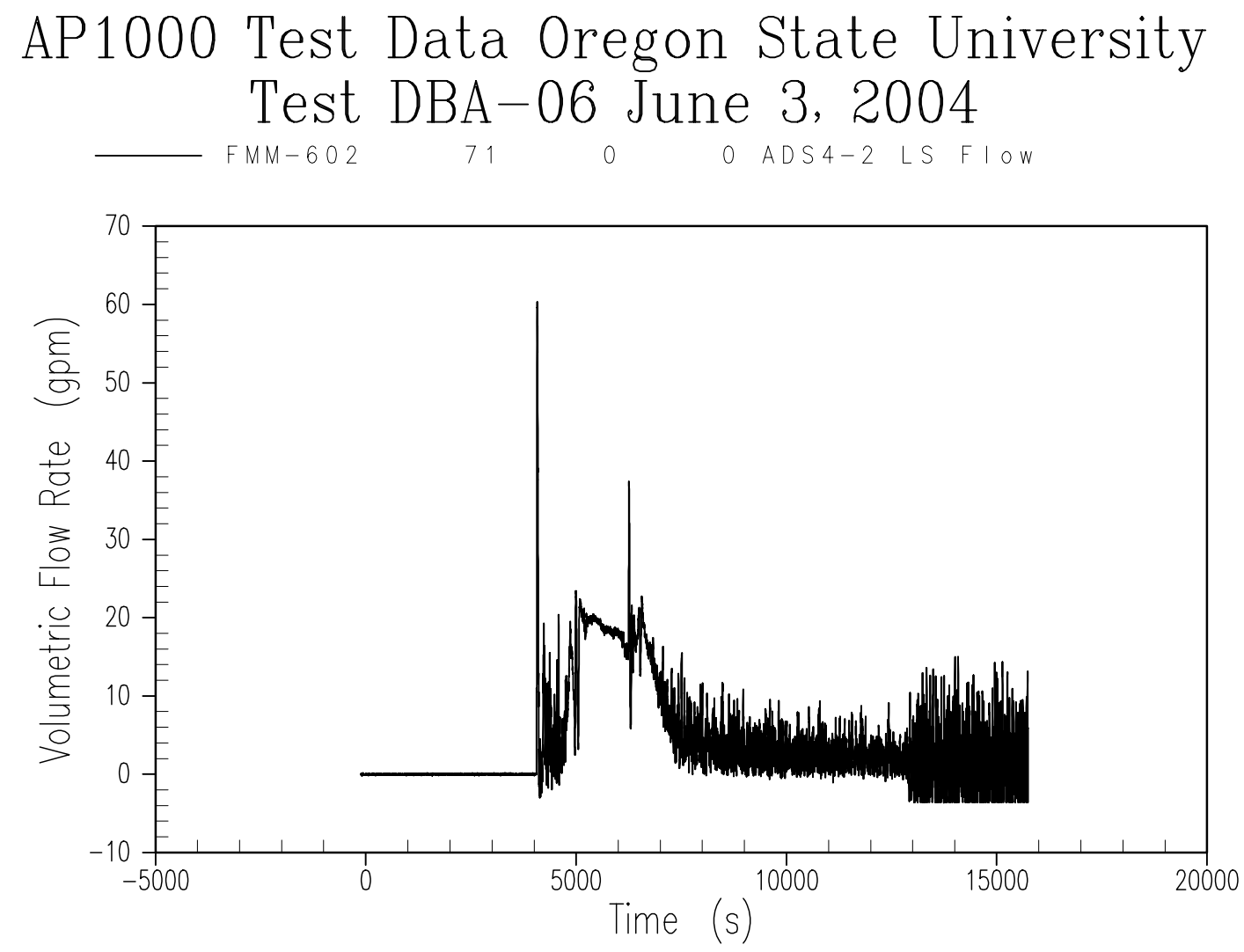

Figure A-51 ADS 4-2 Separator Liquid Flow Rate 

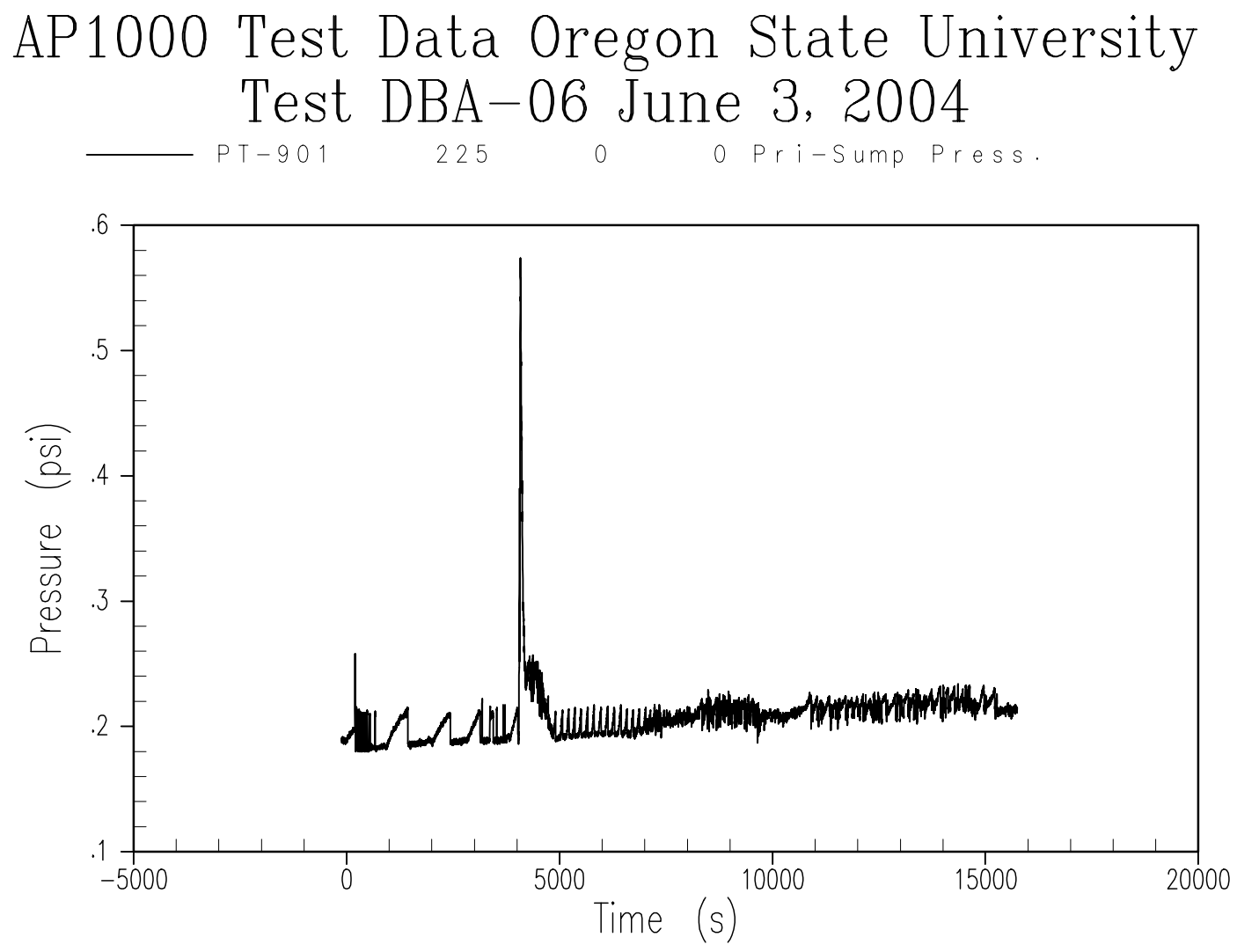

Figure A-52 Primary Sump Pressure 


\section{AP1000 Test Data Oregon State University Test DBA-06 June 3, 2004 \\ L LDP-901 \\ 182 \\ 0 \\ o Pri-Sump UC LeveI}

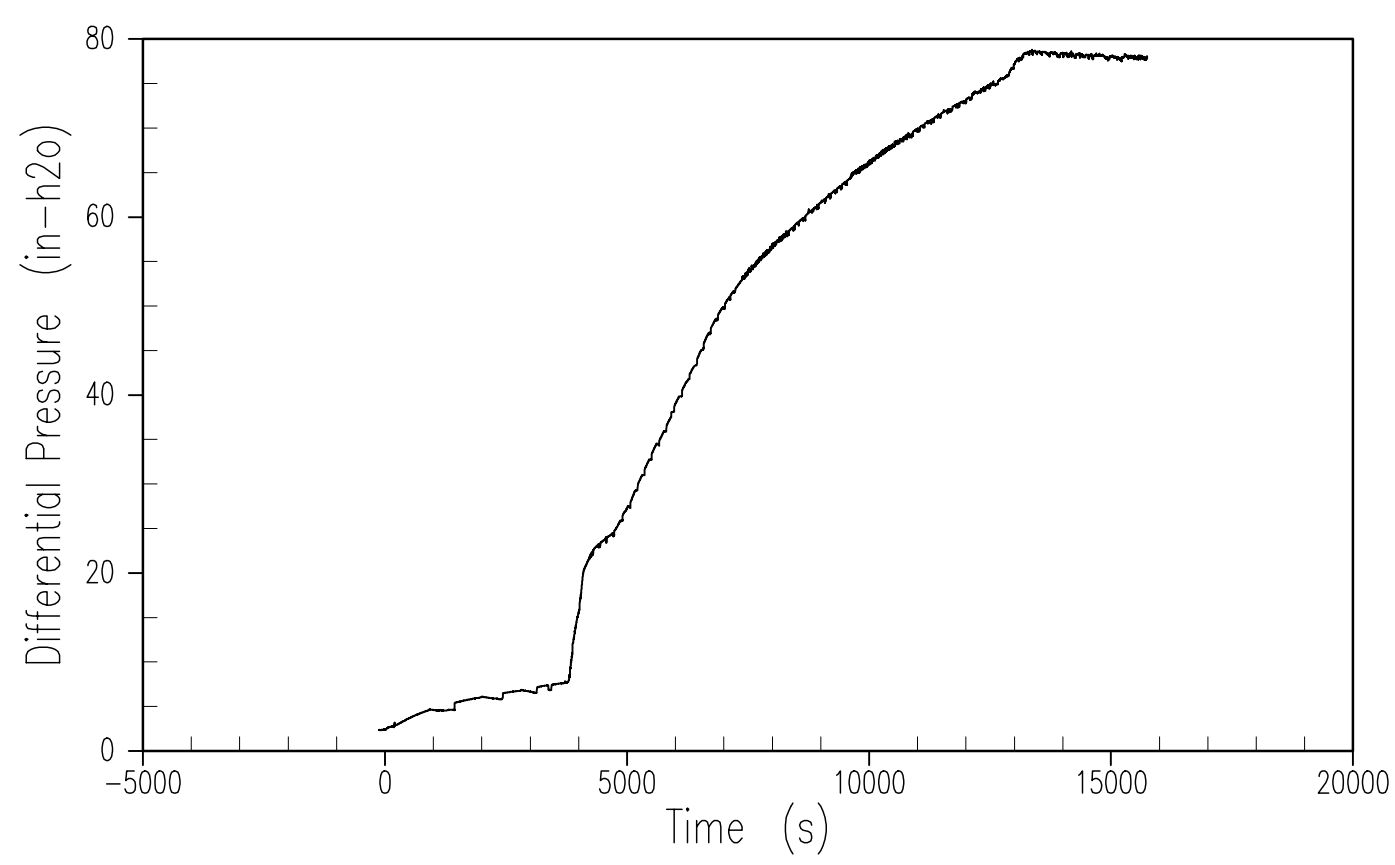

Figure A-53 Primary Sump Liquid Level 

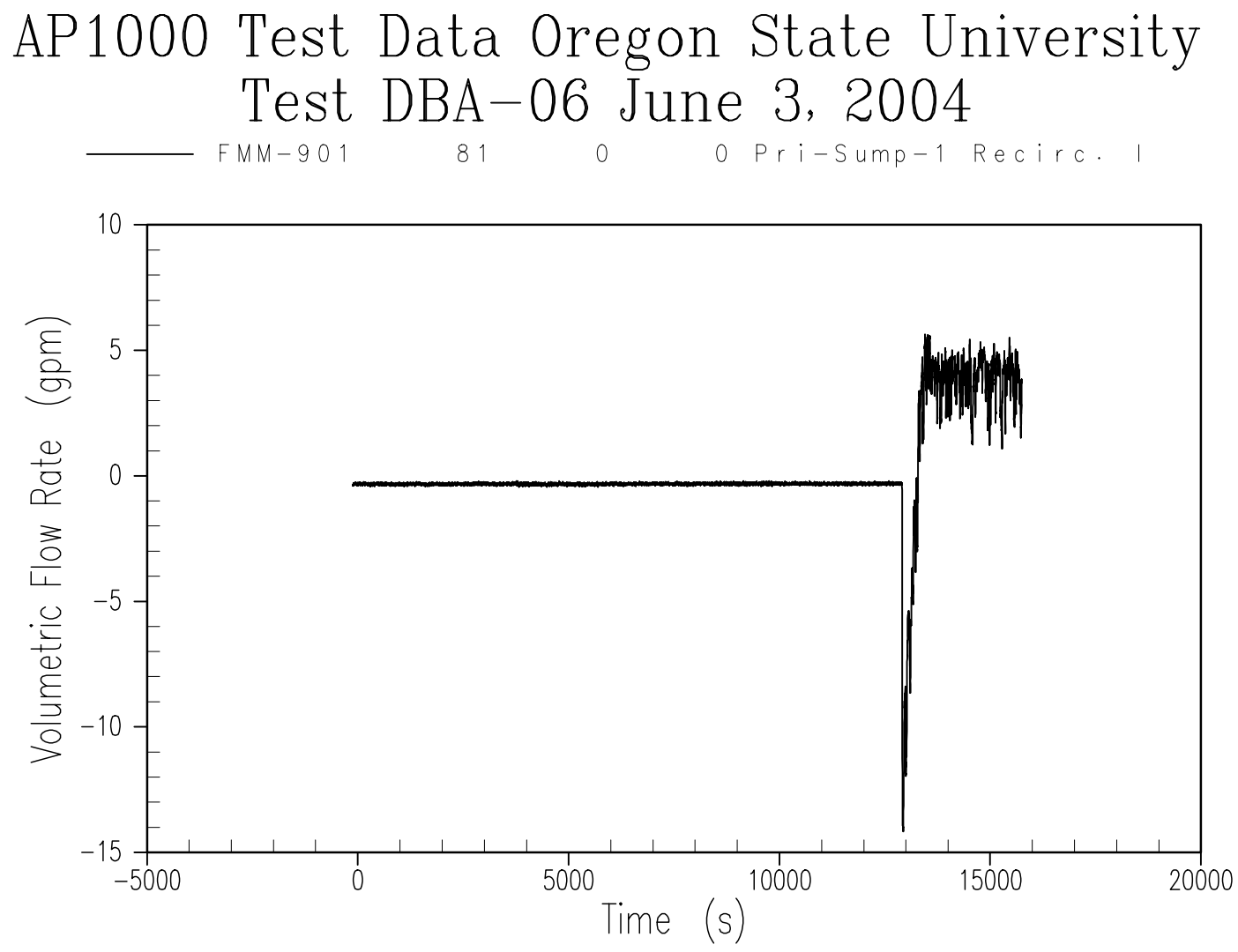

Figure A-54 Primary Sump 1 Injection Flow Rate 

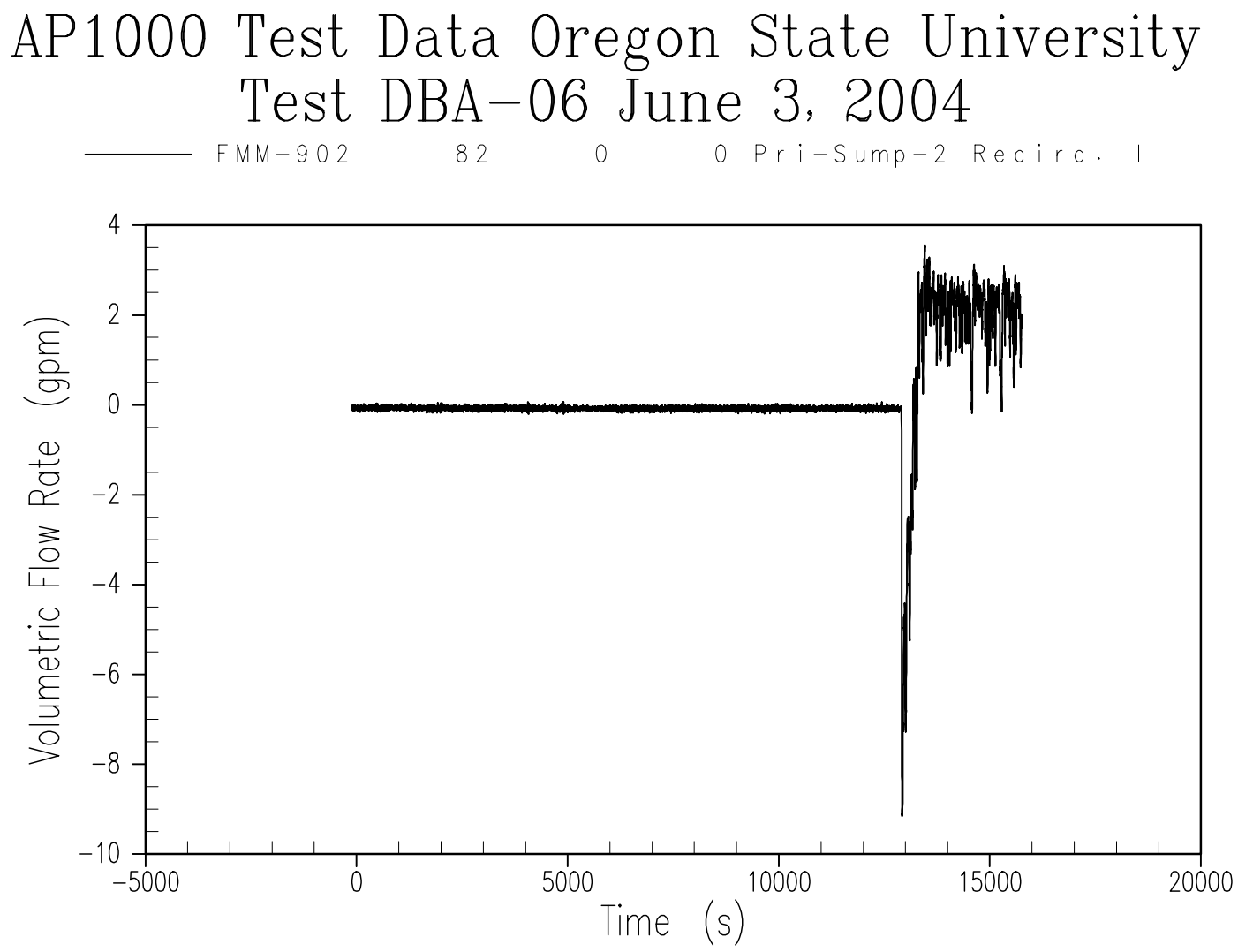

Figure A-55 Primary Sump 2 Injection Flow Rate 

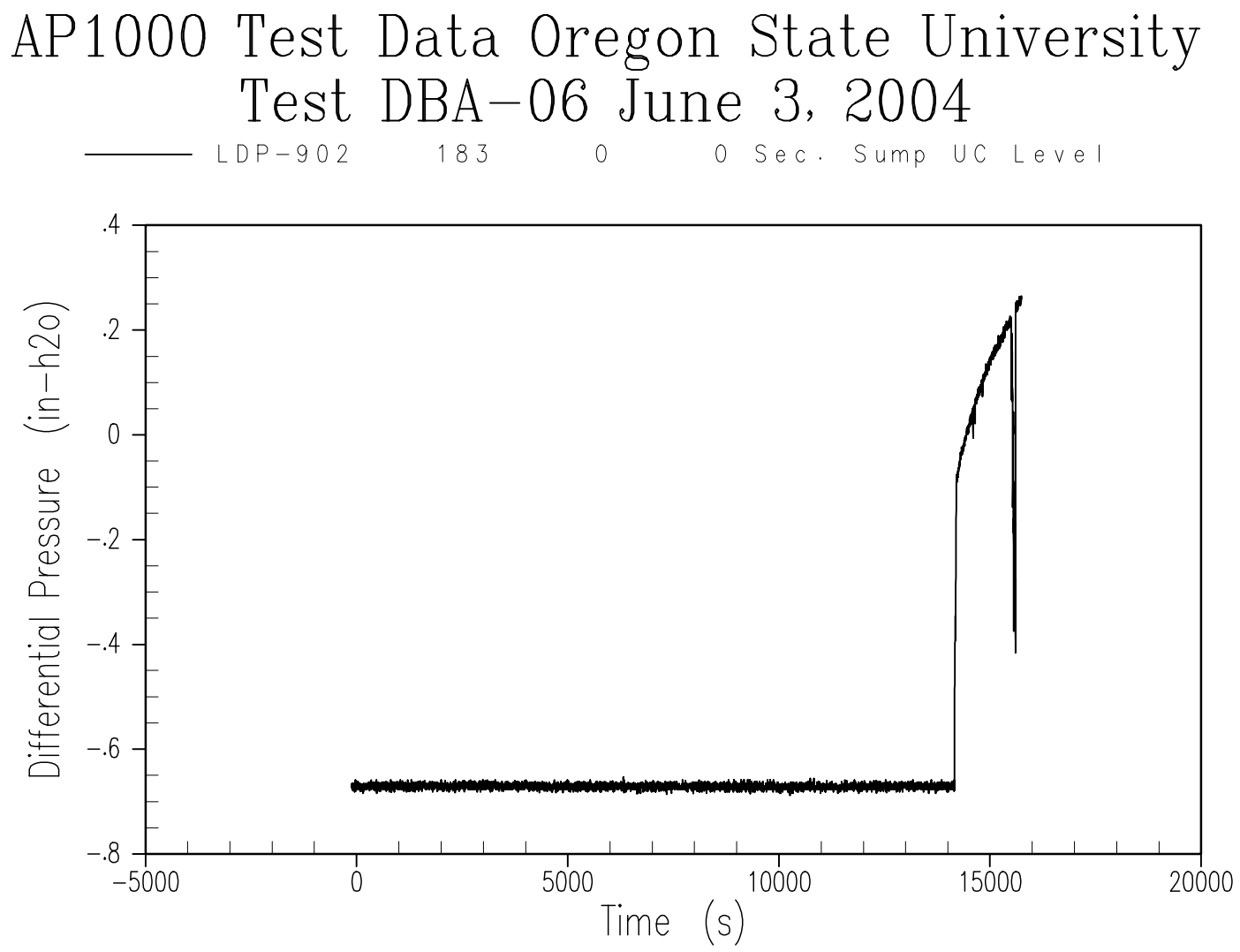

Figure A-56 Secondary Sump Liquid Level 


\section{AP1000 Test Data Oregon State University Test DBA-06 June 3, 2004 \\ - P T 905 \\ o Break Sep. Press.}

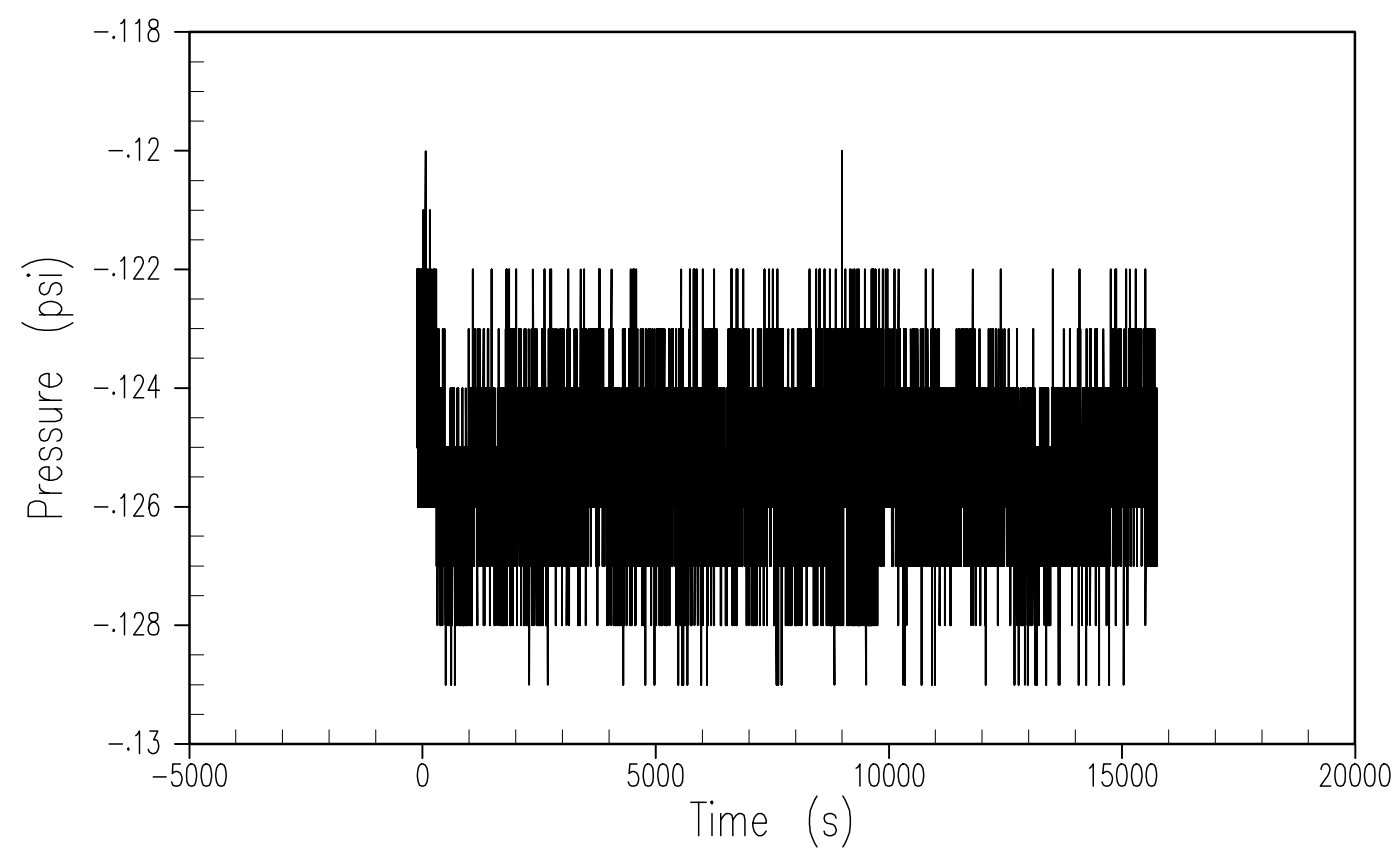

Figure A-57 Break Separator Pressure 

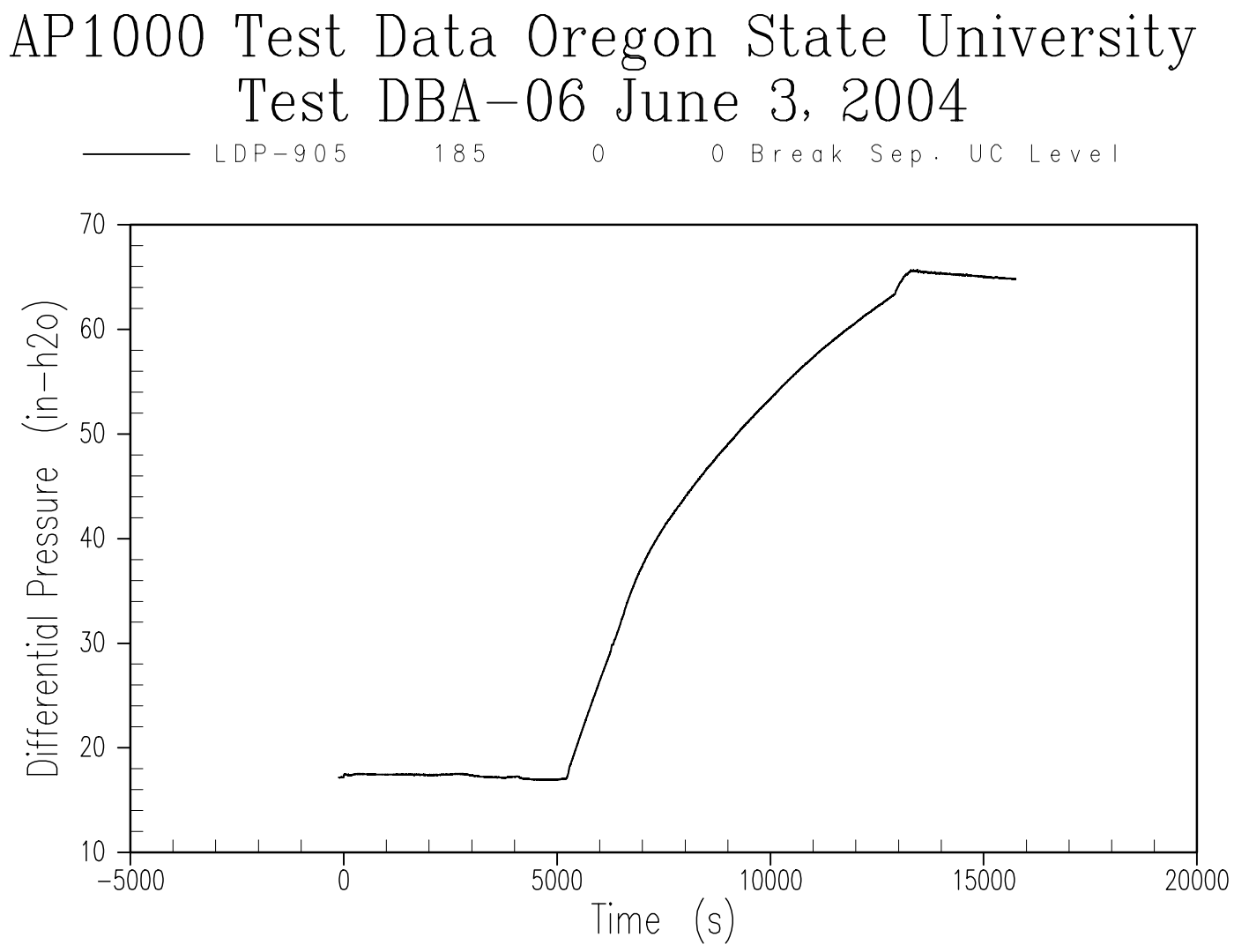

Figure A-58 Break Separator Liquid Level 

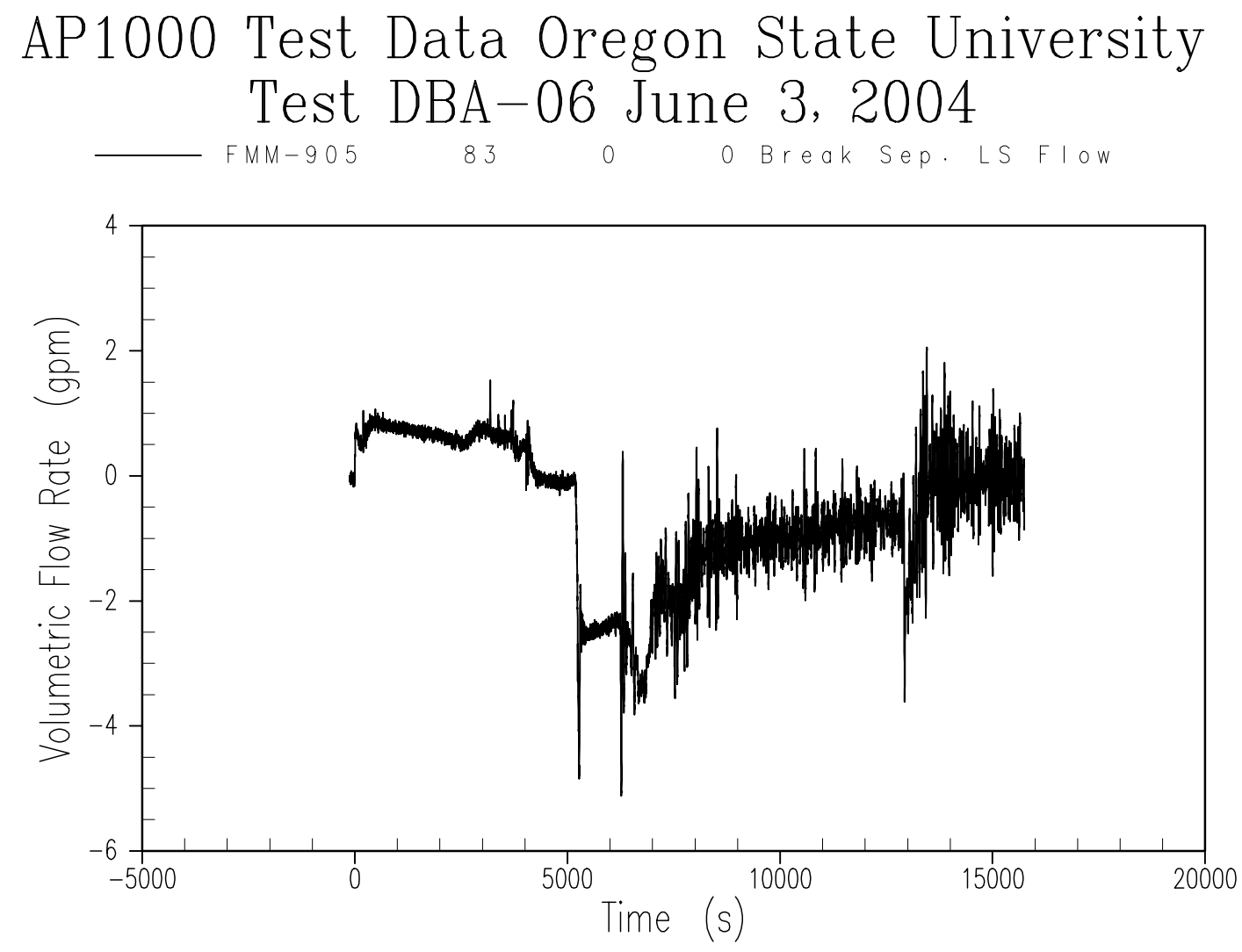

Figure A-59 Break Separator Flow to Primary Sump 

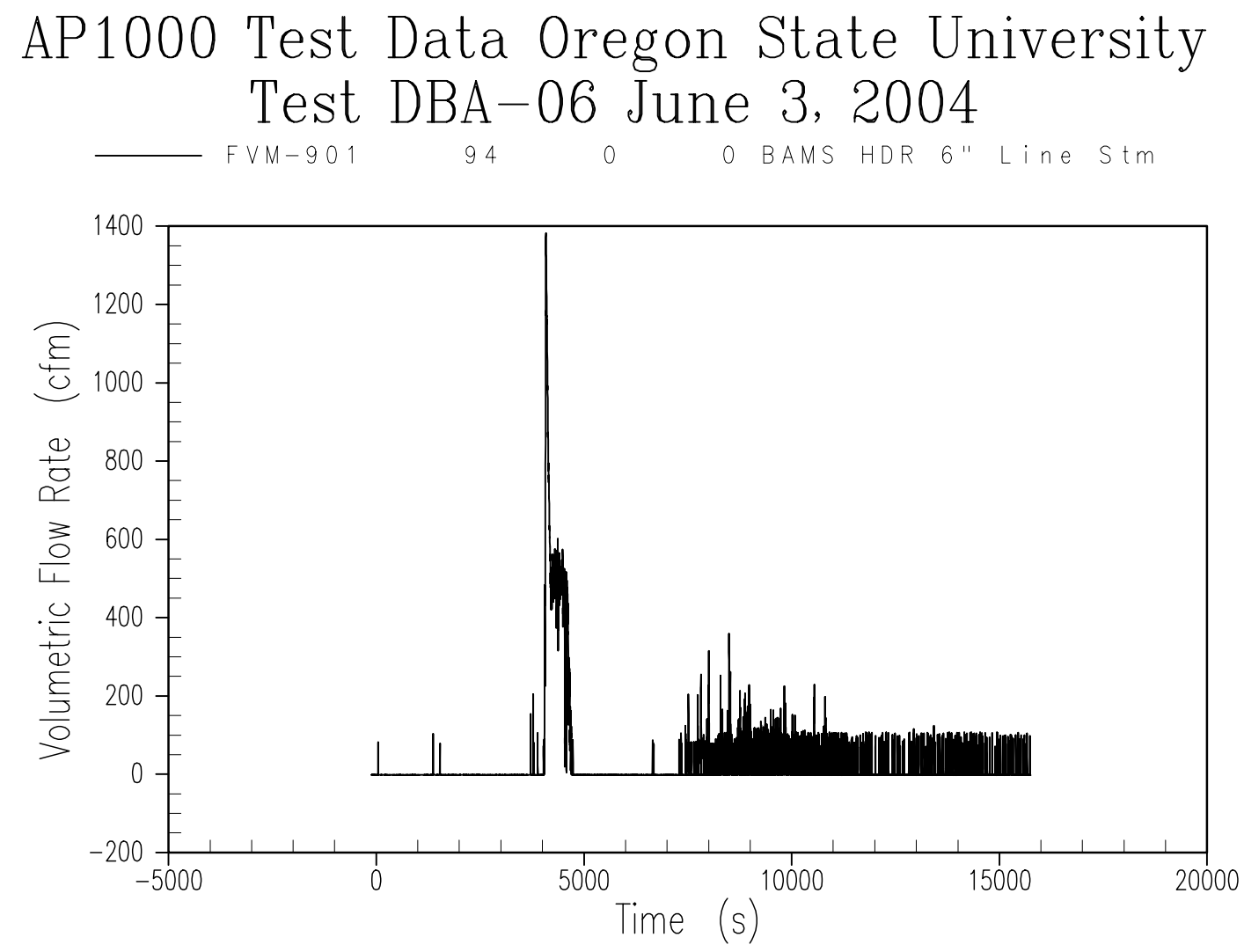

Figure A-60 BAMS Steam Flow Rate - 6-inch Line 


\section{AP1000 Test Data Oregon State University Test DBA-06 June 3, 2004 \\ $\longrightarrow$ FVM-902

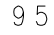 \\ o BAMS HDR 10" Line st}

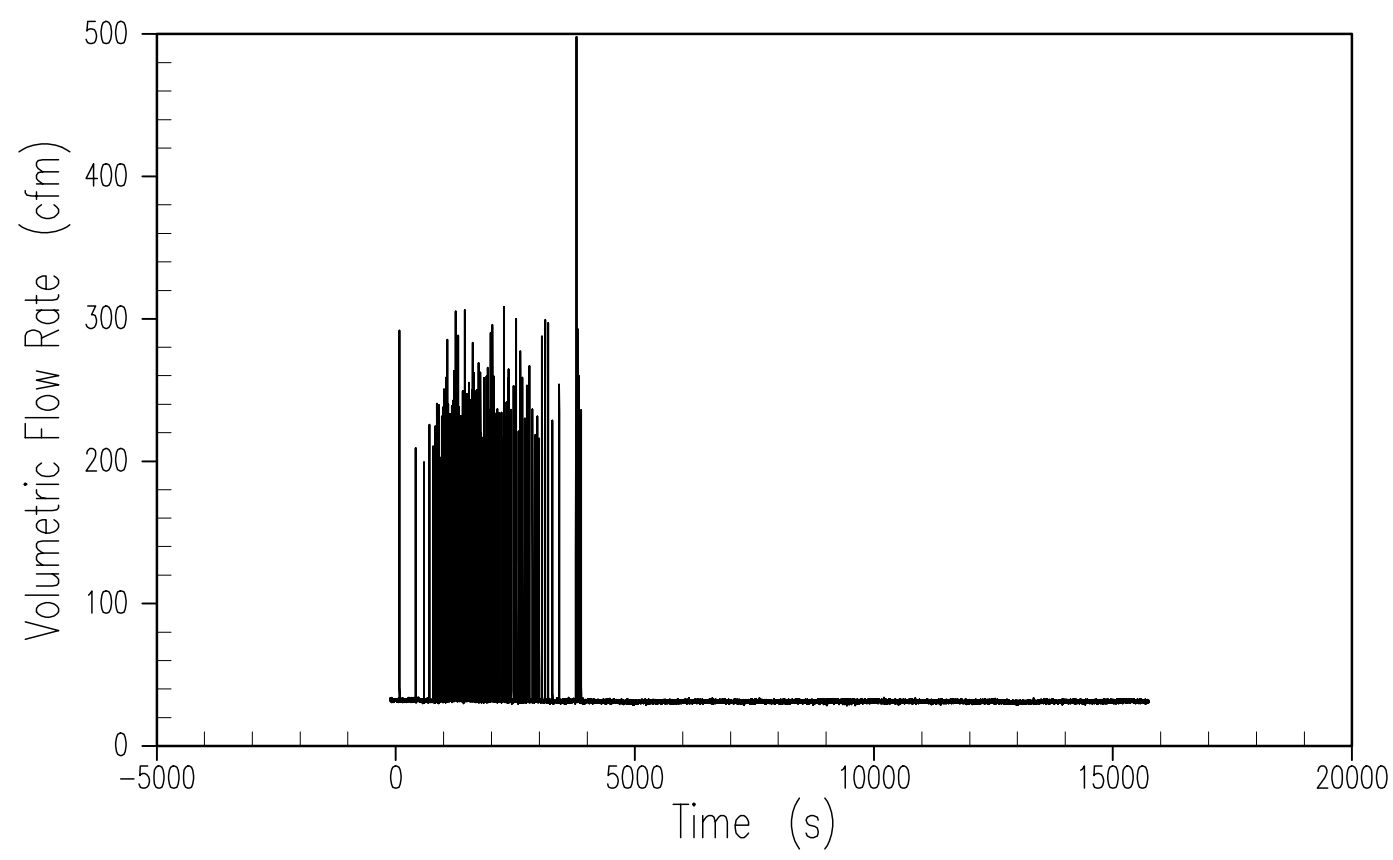

Figure A-61 BAMS Steam Flow Rate - 10-inch Line 


\section{AP1000 Test Data Oregon State University Test DBA-06 June 3, 2004 \\ FVM-903 \\ 96 \\ 0 \\ o Pri-Sump Stm Exhaust}

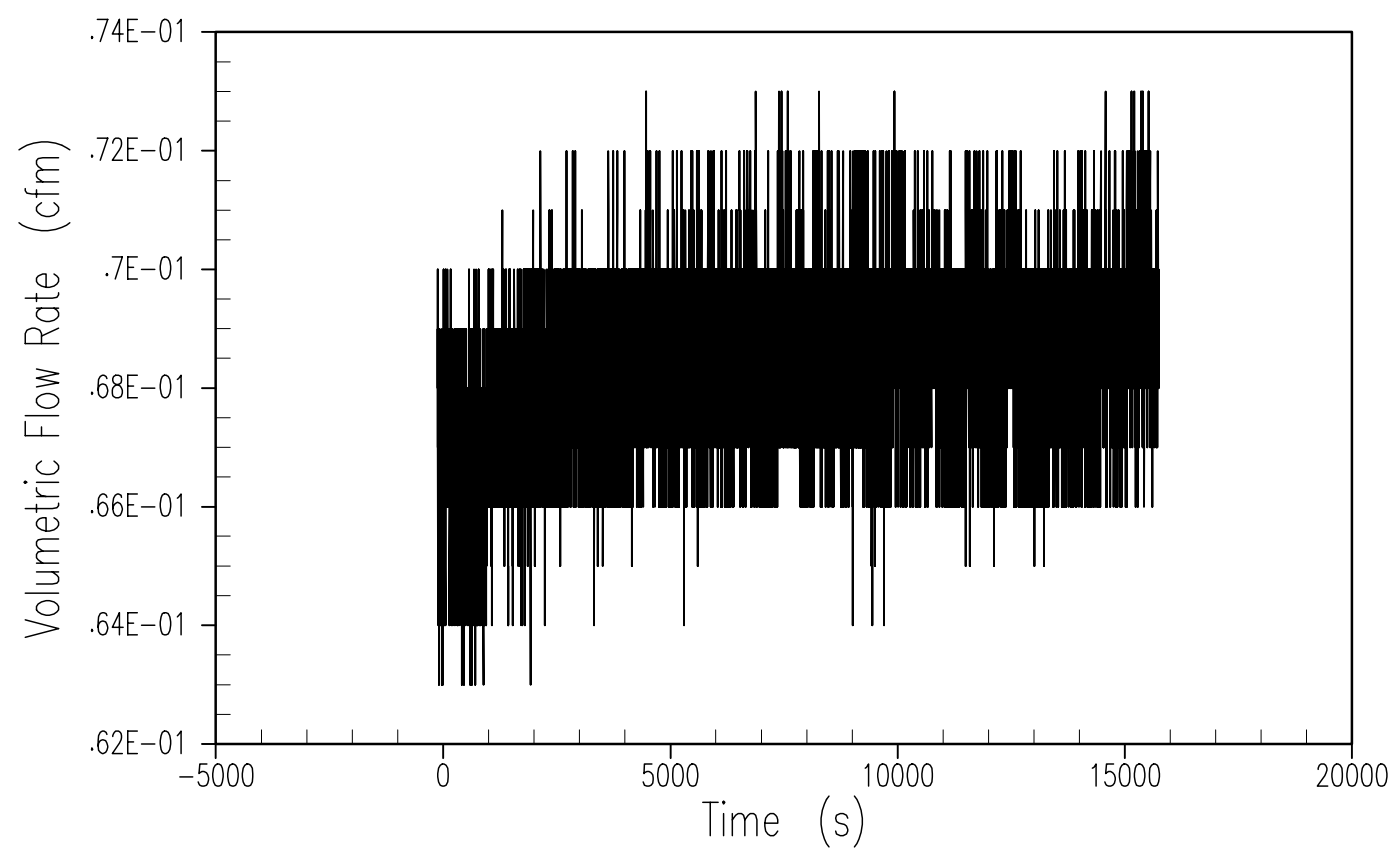

Figure A-62 BAMS/Primary Sump Steam Flow Rate 

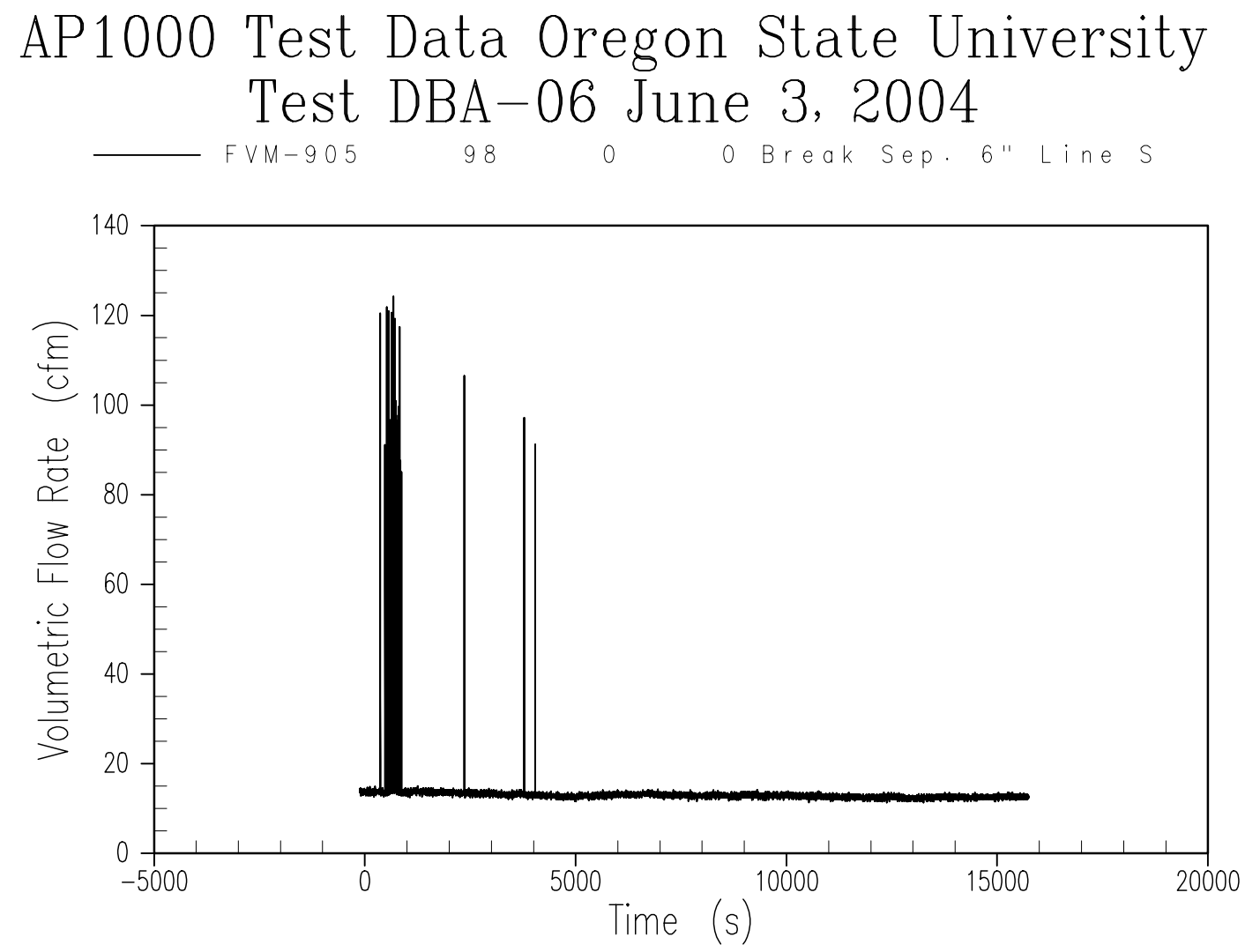

Figure A-63 BAMS/Separator Steam Flow Rate - 6-inch Pipe 

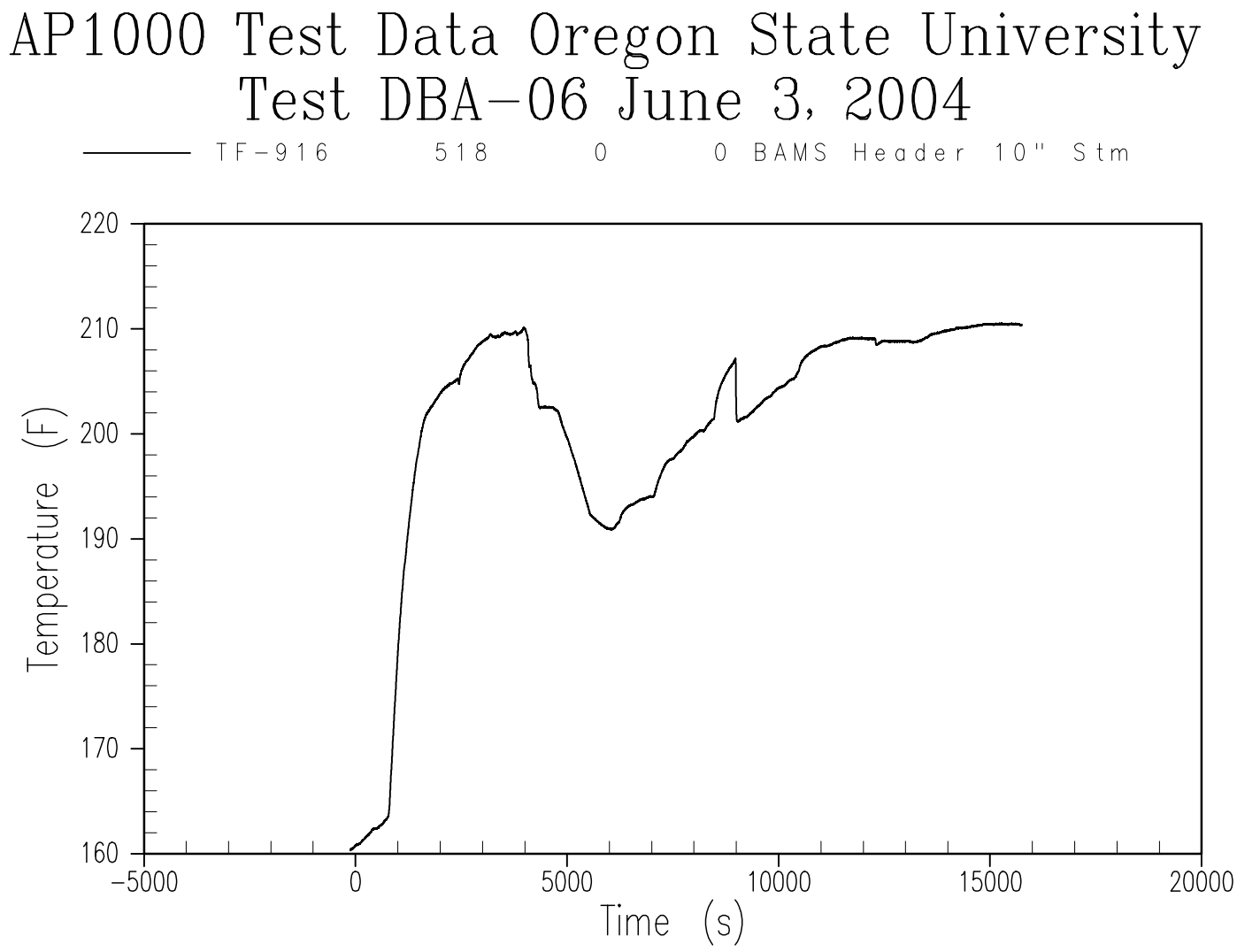

Figure A-64 BAMS/Exhaust Line Temperature - 10-inch Line 

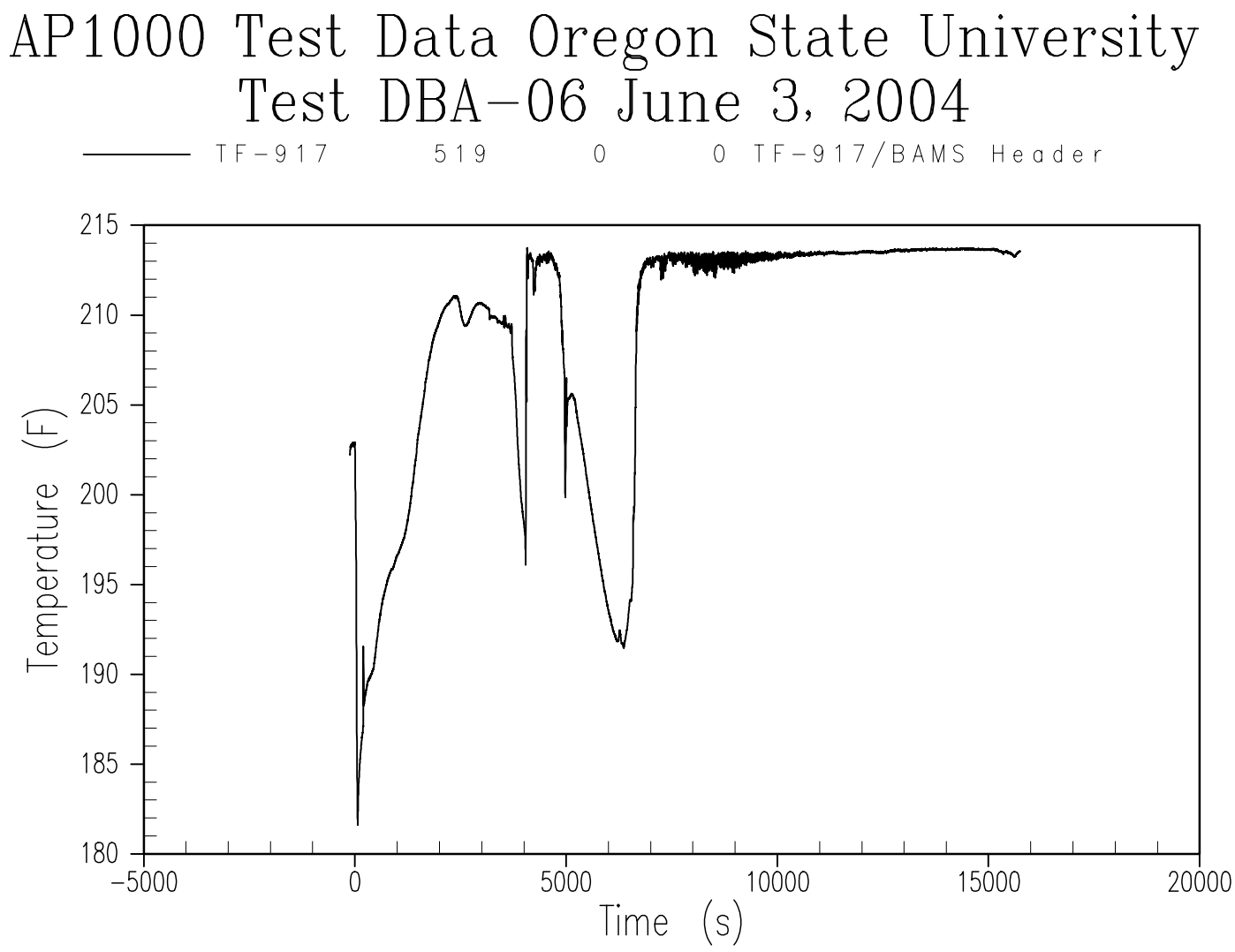

Figure A-65 BAMS/Exhaust Line Temperature - Header 

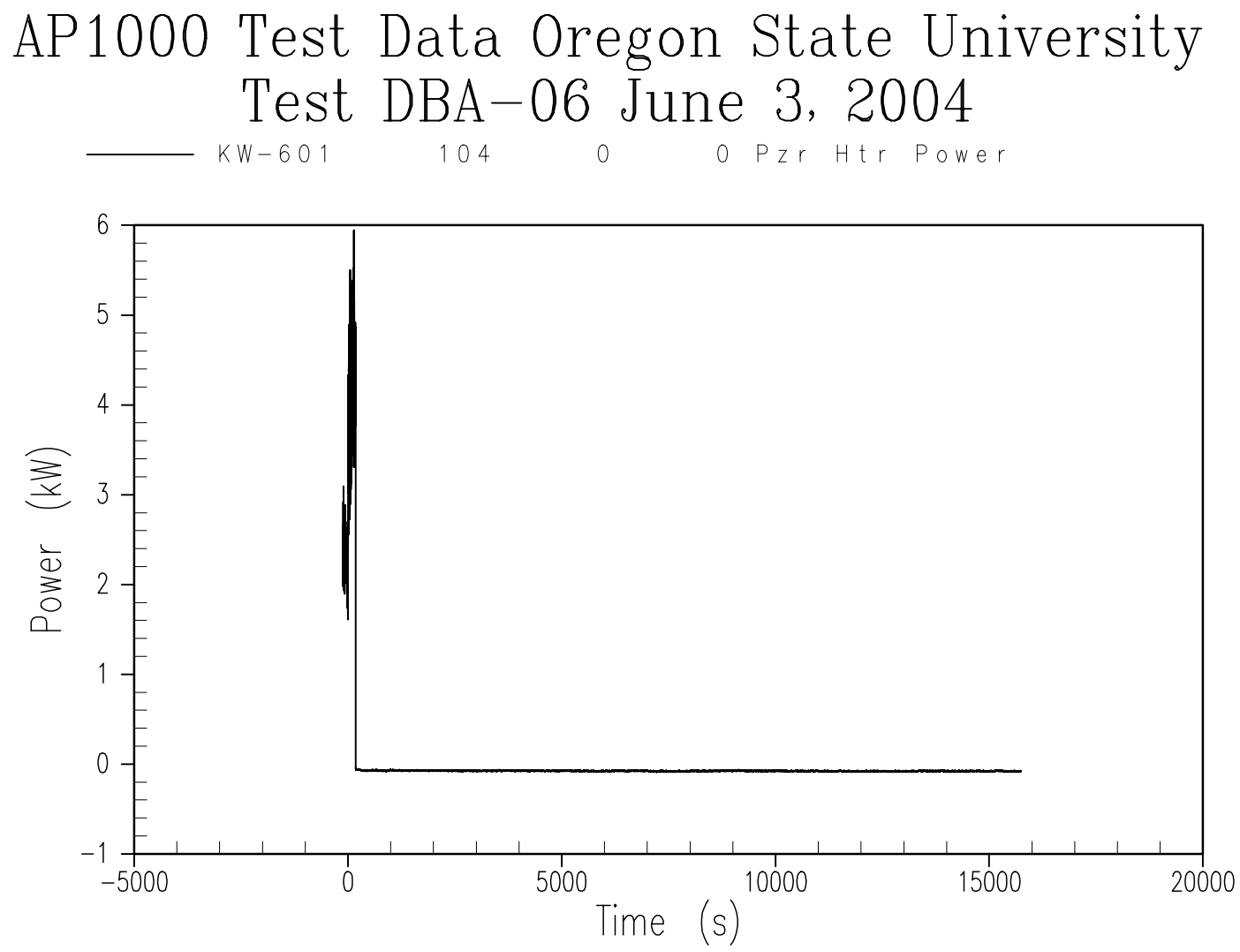

Figure A-66 Pressurizer Heater Input Power 

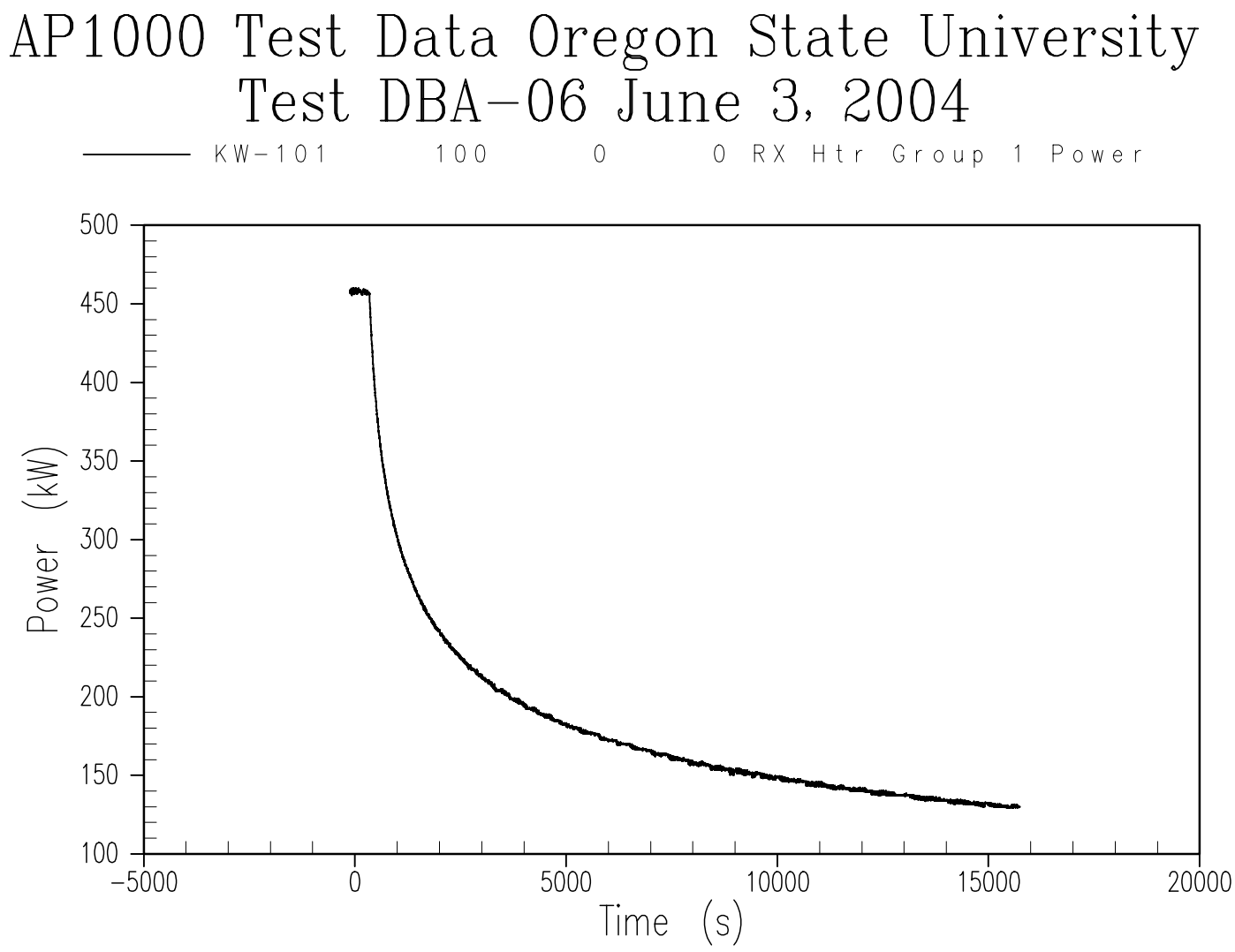

Figure A-67 Core Power Group 1 Input Power 

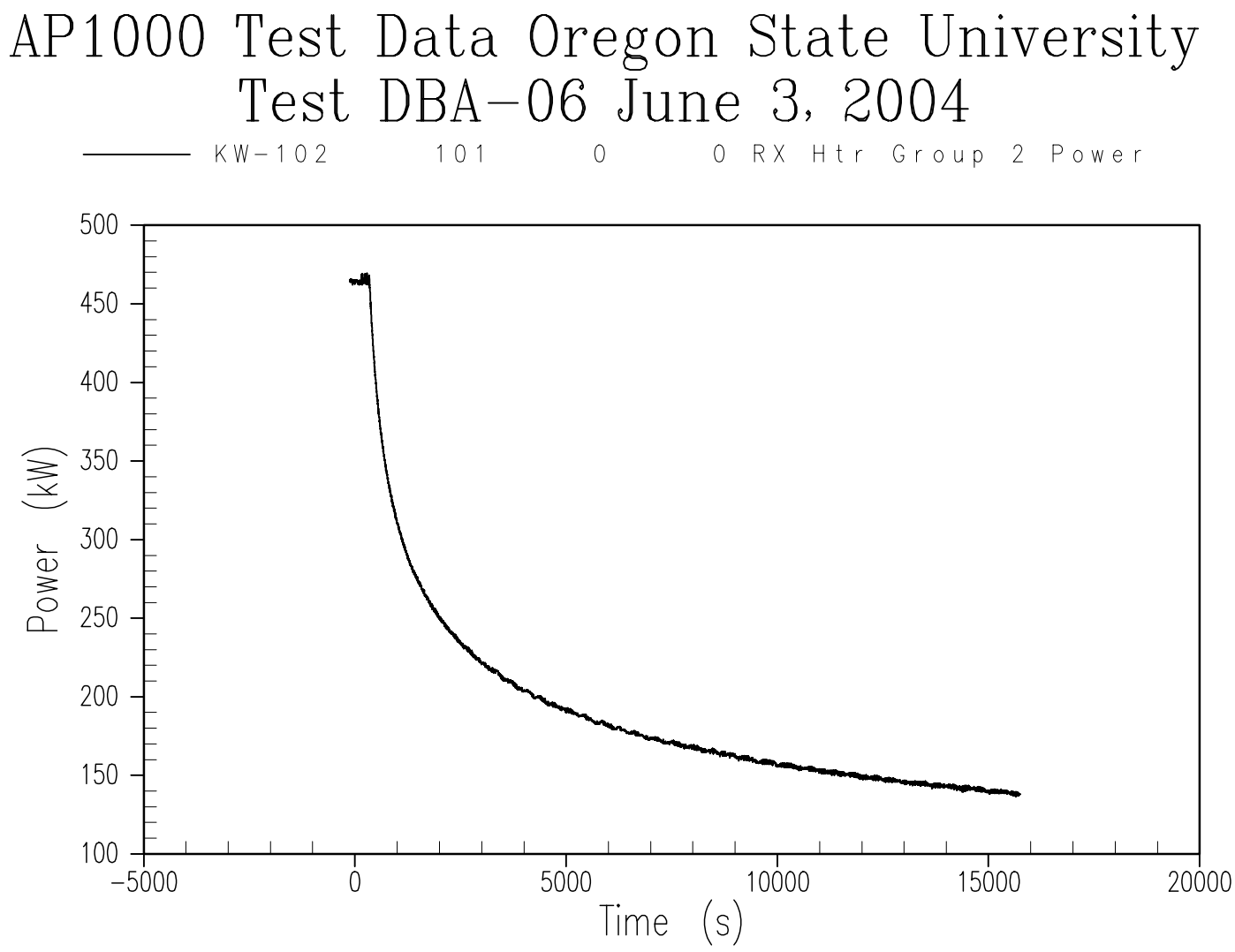

Figure A-68 Core Power Group 2 Input Power 

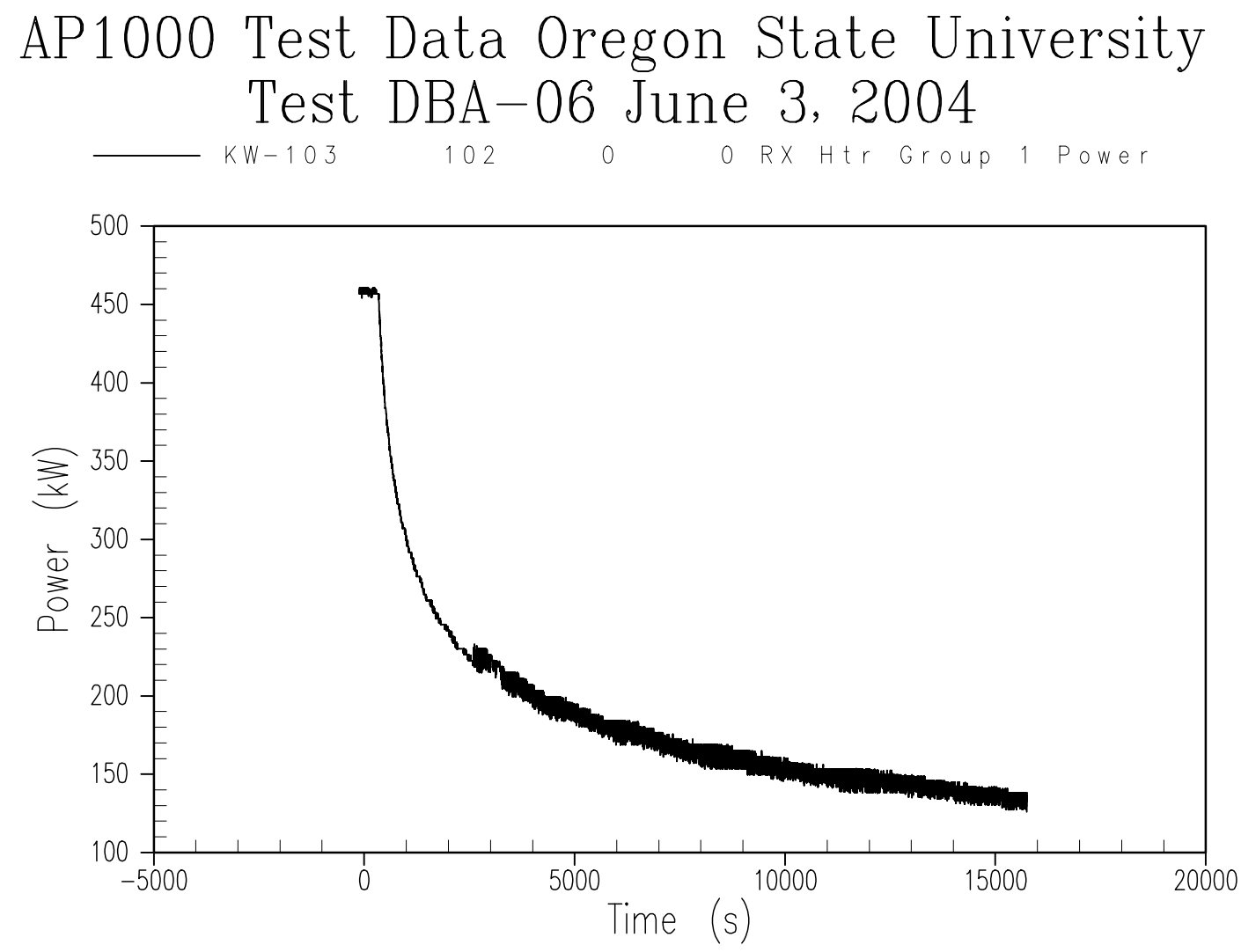

Figure A-69 Core Power Group 1 Alternate Input Power 

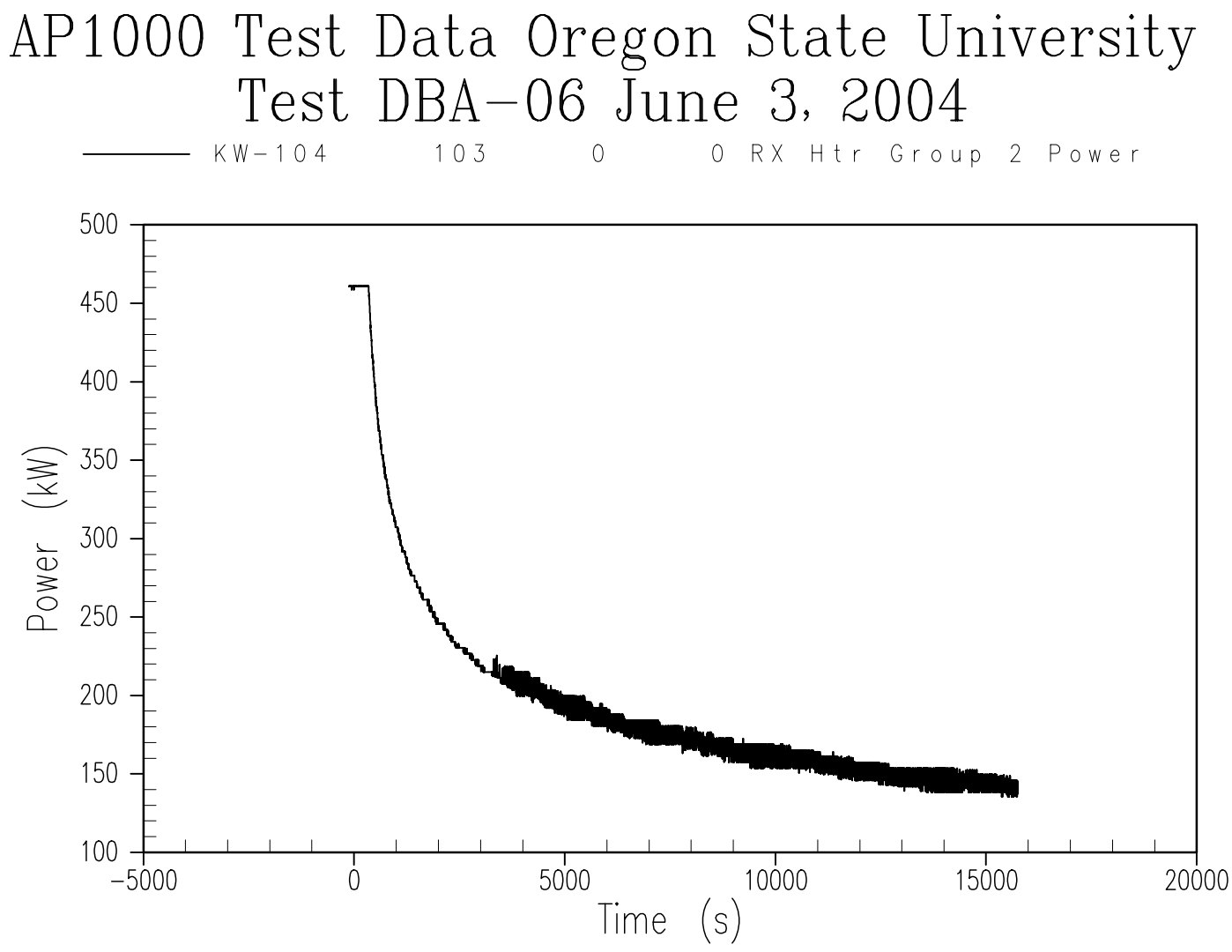

Figure A-70 Core Power Group 2 Alternate Input Power 

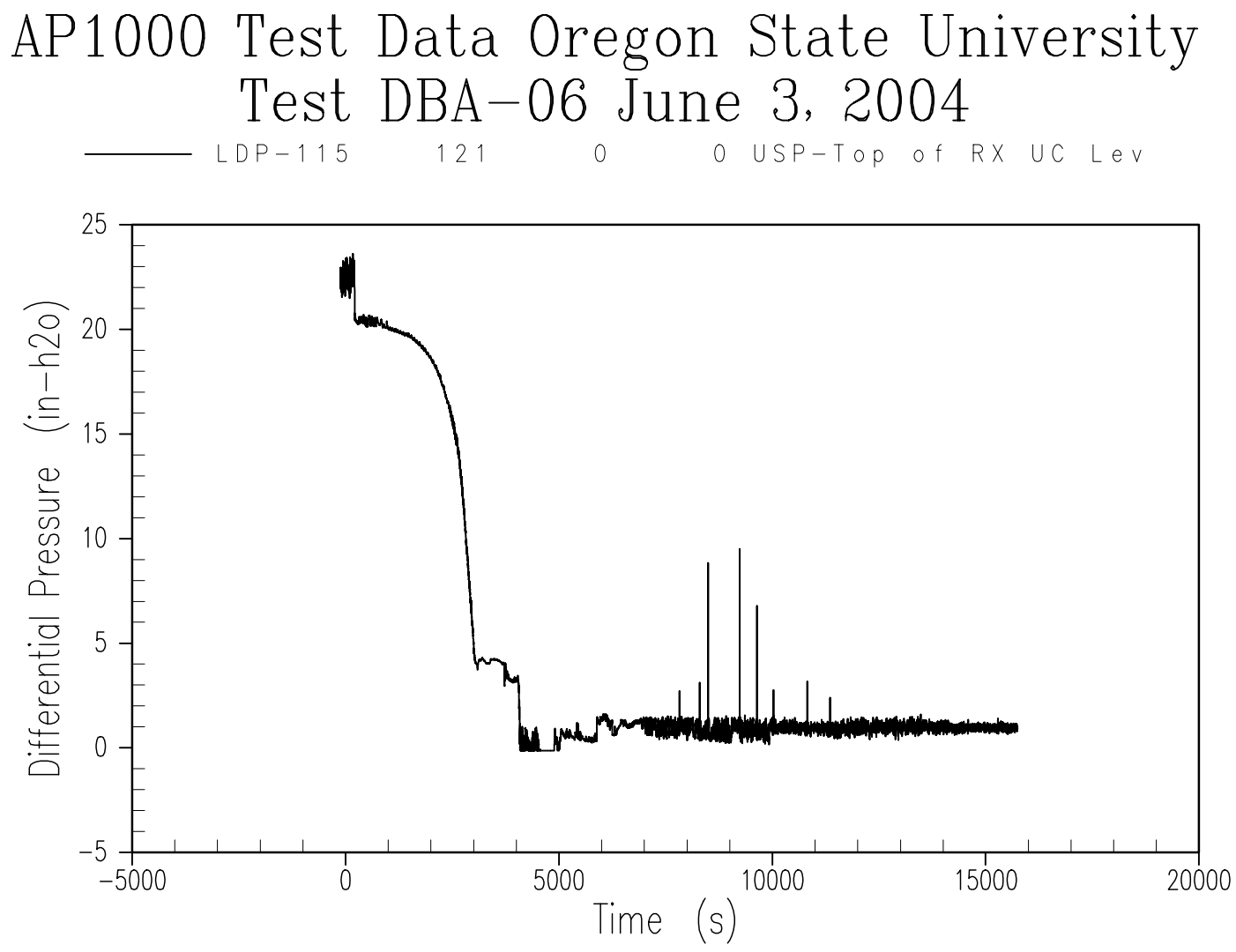

Figure A-71 Reactor Vessel Liquid Level Between Top of Vessel - Upper Support Plate 

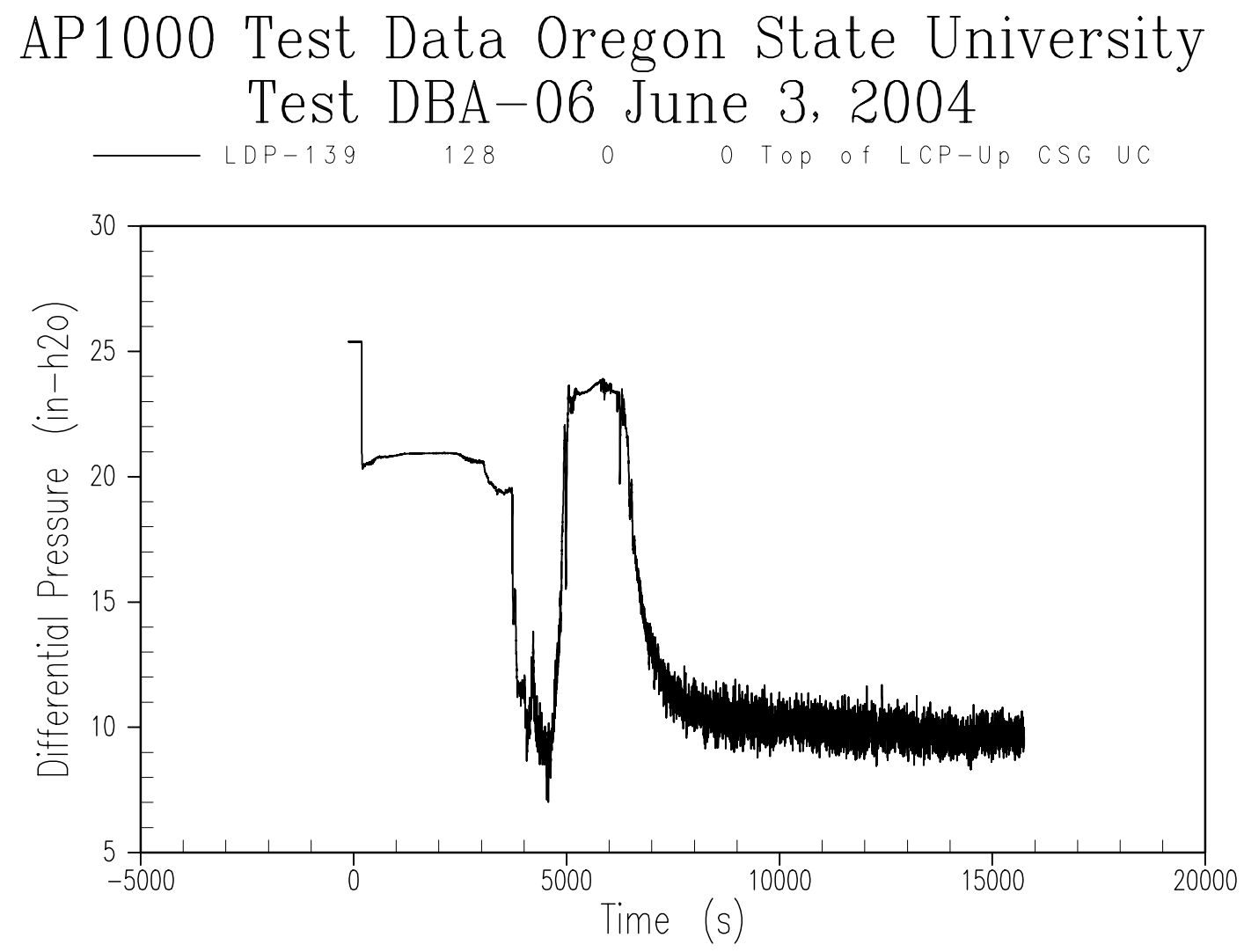

Figure A-72 Reactor Vessel Liquid Level Between Bottom of Upper Support Plate Upper Core Spacer Grid 

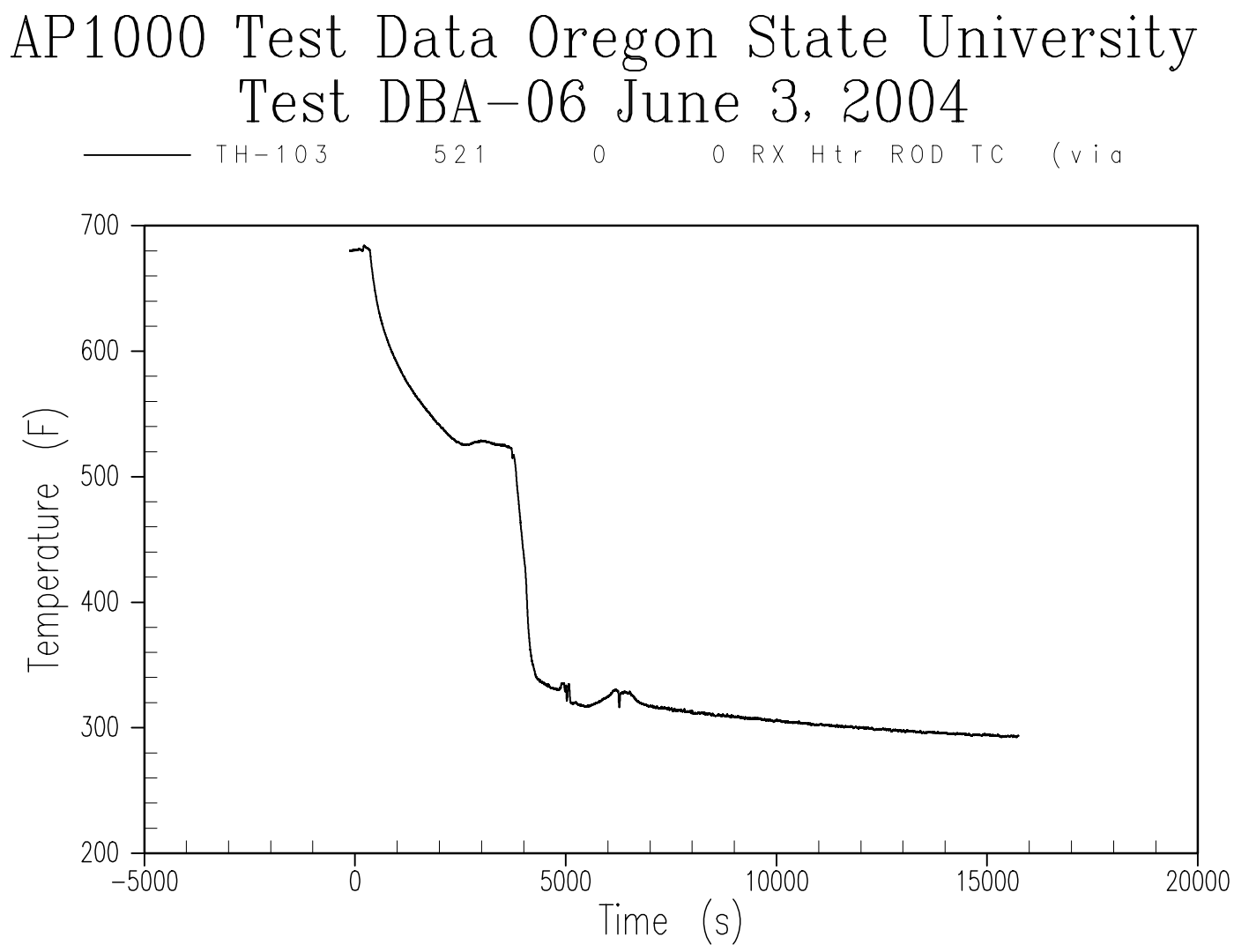

Figure A-73 Inner Core Thermocouple Measuring Heater Temperature 

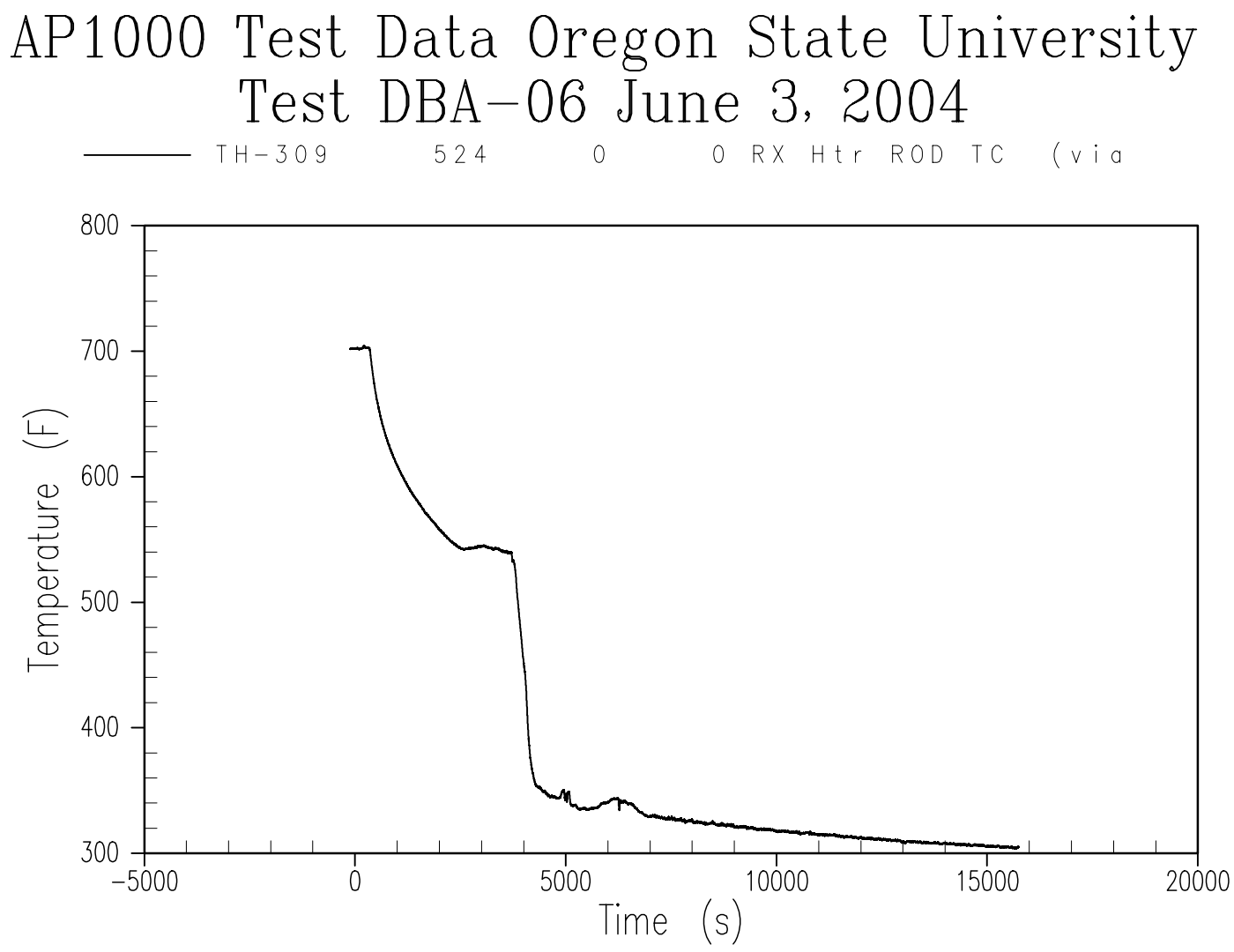

Figure A-74 Outer Core Thermocouple Measuring Heater Temperature 
APPENDIX B

TEST DATA 